\title{
Mechanism and Origin of Ligand-Controlled Chemo- and Regioselectivity in Palladium-Catalyzed Methoxycarbonylation of Alkynes
}

\author{
Ling Zhu, ${ }^{\dagger}, \#$ Ling-Jun Liu, ${ }^{\dagger, \#}$ Yuan-Ye Jiang, ${ }^{\dagger}, *$ Peng Liu,${ }^{\dagger}$ Xia Fan, ${ }^{\dagger}$ Qi Zhang, ${ }^{\dagger}$ Yulei \\ Zhao ${ }^{\dagger}$ Siwei $\mathrm{Bi}^{\dagger}, *$ \\ ${ }^{\dagger}$ School of Chemistry and Chemical Engineering, Qufu Normal University, Qufu 273165, People's Republic of China \\ Institute of Industry \& Equipment Technology, Hefei University of Technology, Hefei 230009, People's Republic of \\ China
}

\section{Corresponding Authors}

*E-mail: yuanyejiang@qfnu.edu.cn (Y.-Y.J.).

*E-mail: siweibi@126.com (S.B.). 


\section{Table of Contents}

Test of calculation methods for solution-phase single-point energy ......................................................... 3

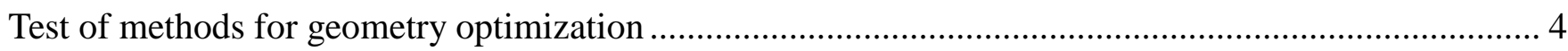

NPA charge and ESP maps of different Pd catalyst precoursors ........................................................... 5

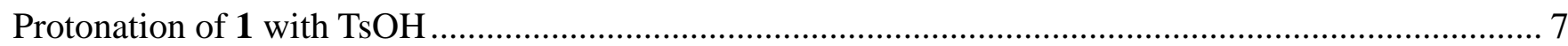

Hydrometallation of $\mathbf{a} 1$ with $\mathbf{L} \mathbf{1}$ from double protonated catalysts...................................................... 8

Hydrometallation and methanolysis of P2a with $\mathbf{L} \mathbf{1}$ from double protonated catalysts ........................... 9

Hydrometallation and methanolysis of $\mathbf{a} \mathbf{1}$ with $\mathbf{L} \mathbf{2}$ from double protonated catalysts............................. 9

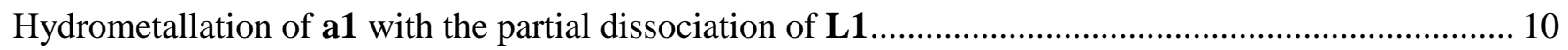

Less stable intermediates and transition states in the methanolysis stage of a1 with $\mathbf{L 1}$.........................11

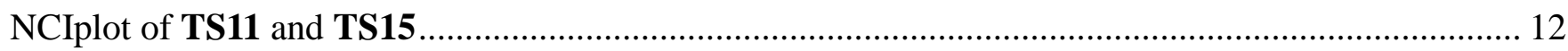

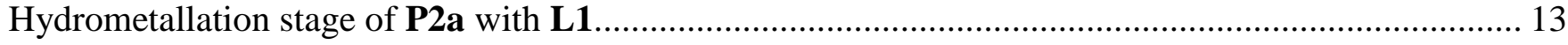

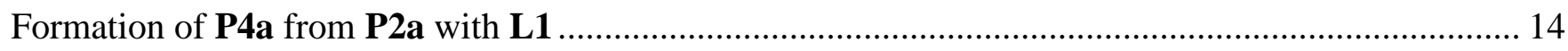

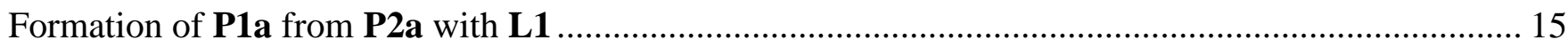

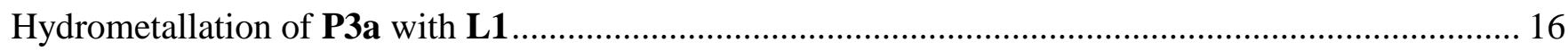

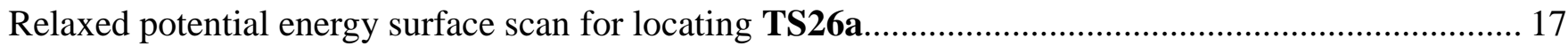

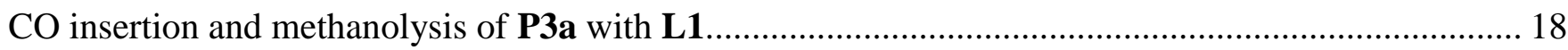

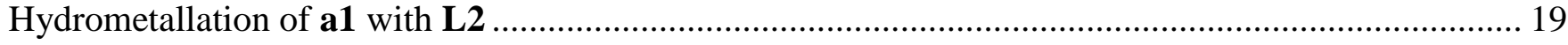

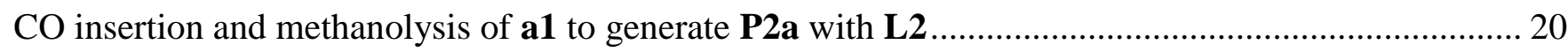

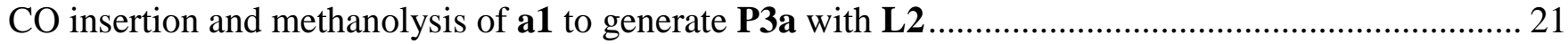

Hydrometallation stage of P2a with L2

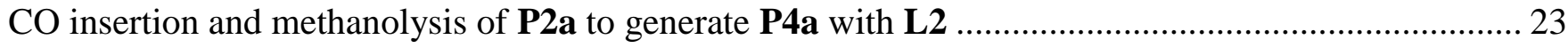

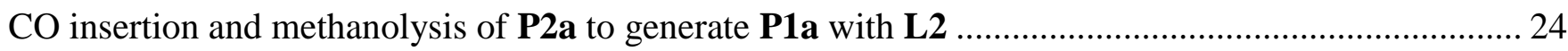

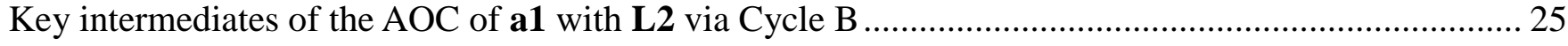

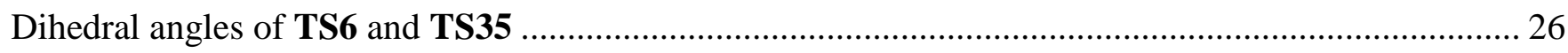

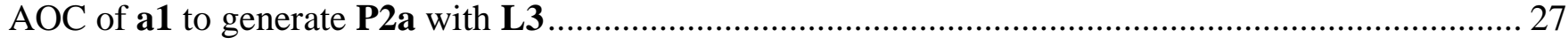

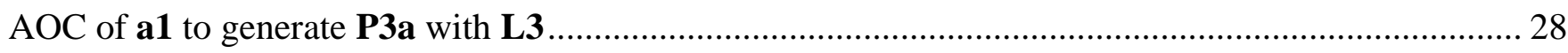

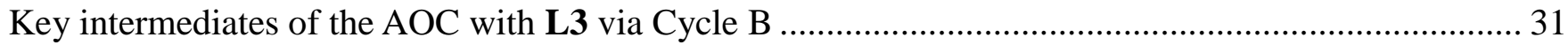

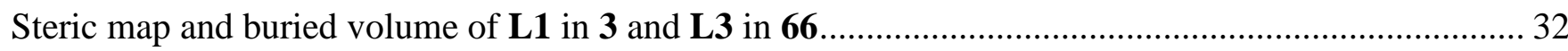

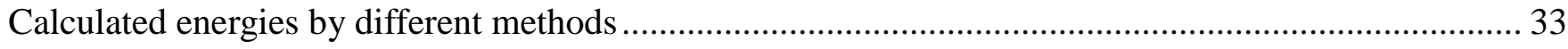

Cartesian coordinates of optimized structures by B3LYP and M06-L ................................................... 41 
M06-L/BS2/SMD//B3LYP/BS1

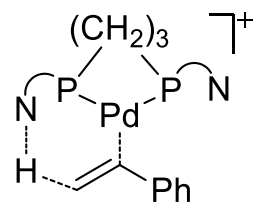

TS6

0.0

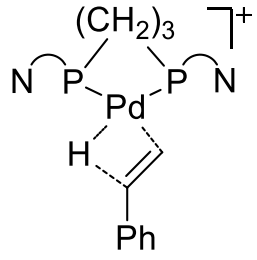

TS4

0.5<smiles>CC=NP(C1=C(c2ccccc2)CNCP1C)P1CNCP1</smiles>

TS7

2.1<smiles>CP(C)P(C)[Pb]1C=CC(c2ccccc2)P1PC[I-]</smiles>

TS5

3.2

M06-L-D3/BS2/SMD//B3LYP/BS1<smiles>CCNCP1P2CNC=C(c3ccccc3)P1P2C</smiles>

TS6

0.0

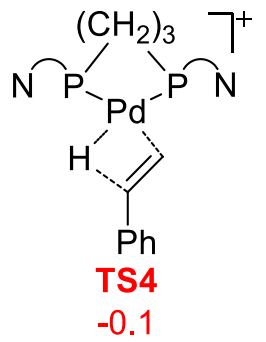

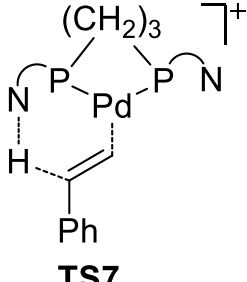

TS7<smiles>CP(C)P(C)P(CN)C1CC=C(c2ccccc2)P1P(C)CN</smiles>

TS5

M06-L/BS2/SMD//B3LYP/BS1

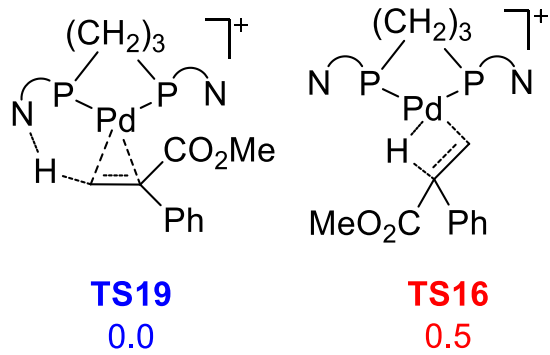

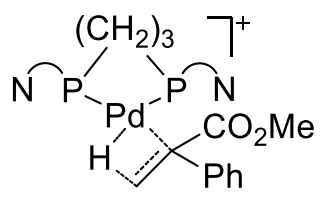

TS17

2.5

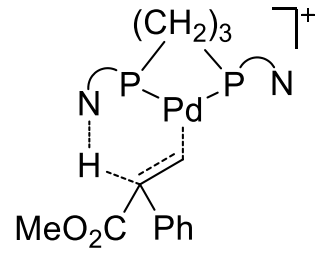

TS18

4.8

M06-L-D3/BS2/SMD//B3LYP/BS1<smiles>COC(=O)C1(c2ccccc2)C2CNCP(C)P2C1P(C)PC[I-]</smiles>

TS19

0.0

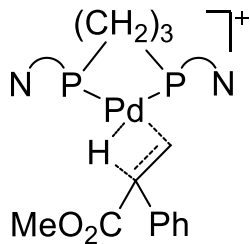

TS16

0.7

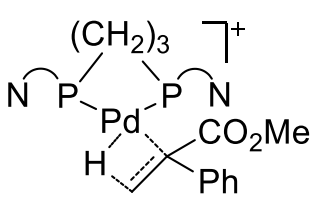

TS17

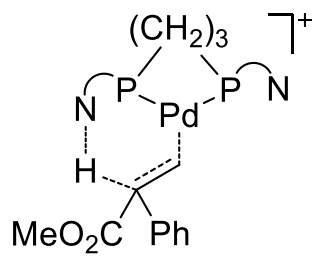

TS18

4.9

Figure S1. Calculated relative Gibbs free energies by different methods (in $\mathrm{kcal} / \mathrm{mol}$ ). The relative energies of TS6 and TS19 were set to be $0.0 \mathrm{kcal} / \mathrm{mol}$.

Geometry optimization and thermodynamic analysis were performed at the level of B3LYP/BS1 whereas solution-phase single-point energies were calculated with M06-L/BS2 and M06-L-D3/BS2, respectively. 

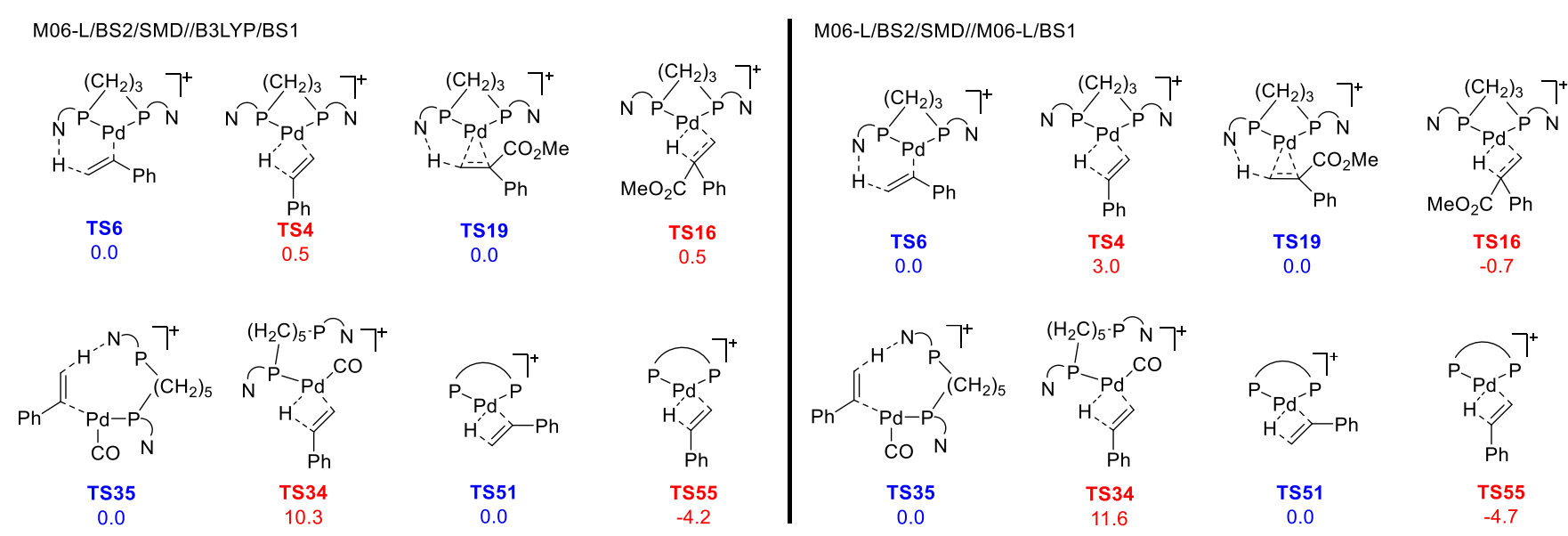

Figure S2. Calculated relative Gibbs free energies of hydrometallation transition states with different methods (in kcal/mol). The relative energies of TS6, TS19, TS35 and TS51 were set to be $0.0 \mathrm{kcal} / \mathrm{mol}$ for comparison.

Compared with the results from B3LYP-geometry optimization (left, Figure S2), M06-L-geometryoptimization predicted the similar selectivity for most cases (right, Figure S2). It should be pointed out that for the methoxycarbonylation of $\mathbf{a 1}$ with L1, B3LYP-geometry optimization show better performance as both P2a and P3a cumulated at the initial period. Consistent with this phenomenon, TS6 is energetically close to TS4 with B3LYP-geometry optimization, whereas TS4 is remarkably less stable than TS6 with M06-L geometry optimization. 


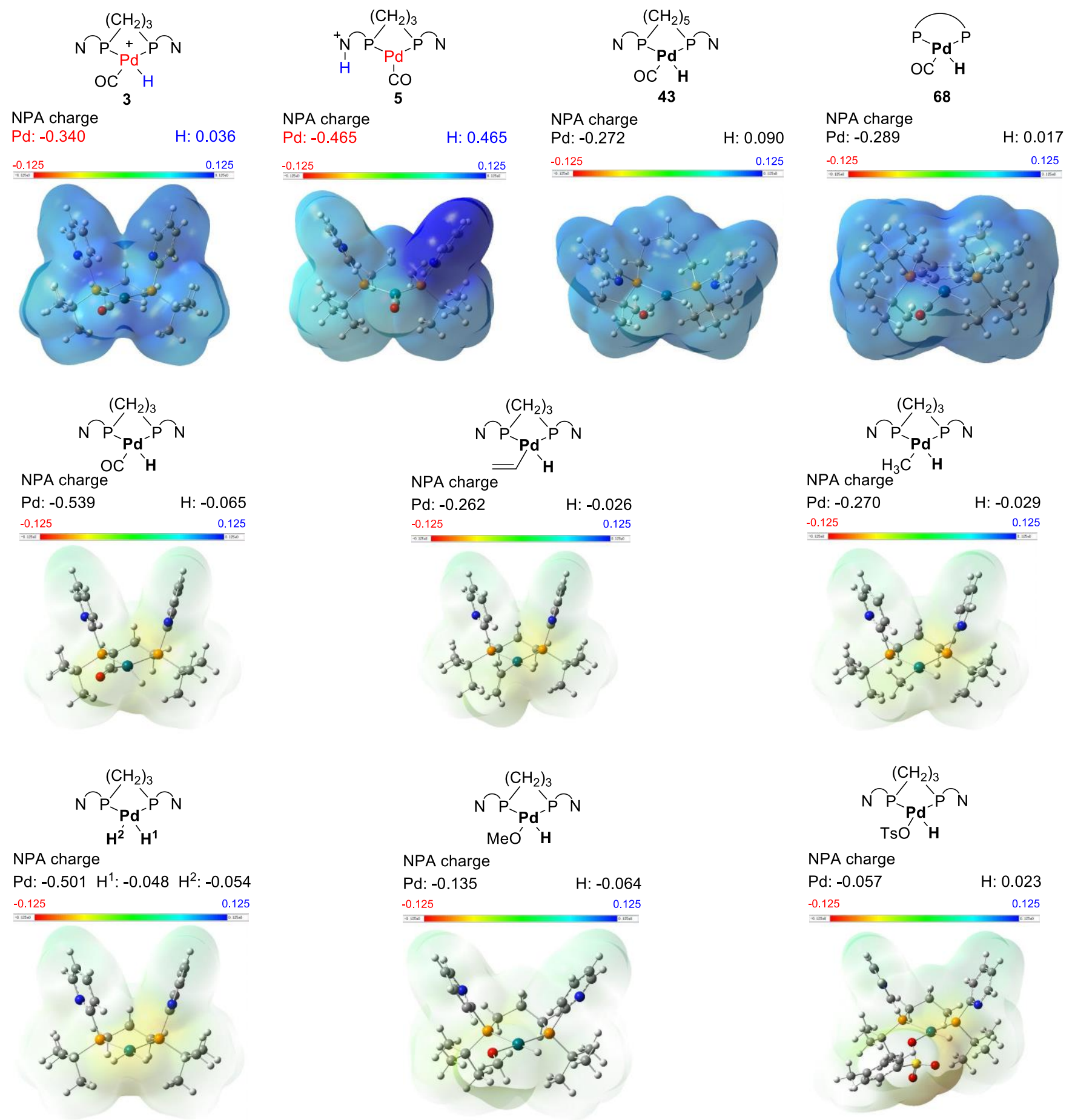

Figure S3. Natural population analysis charge of $\mathrm{Pd}$ and the lighted $\mathrm{H}$ atoms, and the electrostatic potential maps (isovalue $=0.0004$ ) of palladium complexes.

The natural population analysis (NPA) charges of $\mathbf{3}$ and $\mathbf{5}$ shows that the H attached at the pyridyl nitrogen in $\mathbf{5}$ is significantly positive charged $(0.465)$, indicating that it resembles a proton. The positive- 
charged electronic surface potential (ESP) area mainly spreads around the H-attached pyridyl group, also supporting that 5 contains a typical proton. By contrast, the NPA charge of the $\mathrm{H}$ atom attached to the Pd atom in $\mathbf{3}$ is small (0.036) and the positive-charged ESP of $\mathbf{3}$ uniformly spreads around the whole complex. The NPA charge of Pd increases from 5 (-0.465) to 3 (-0.340), suggesting that electron transfer from Pd to $\mathrm{H}$ occurs when the proton in $\mathbf{5}$ binds to $\mathrm{Pd}$ atom. To further probe character of the $\mathrm{H}$ atom attached to $\mathrm{Pd}$ in 3, the NPA charge and ESP of neutral complexes (L1)Pd(OTs)H, (L1)Pd(CO)H, (L1)Pd(H)H, (L1) $(\mathrm{Pd})\left(\mathrm{CH}_{3}\right) \mathrm{H}$ and $(\mathbf{L 1}) \mathrm{Pd}\left(\mathrm{CH}=\mathrm{CH}_{2}\right) \mathrm{H}$ were calculated for comparison. In the neutral complexes, the NPA charges of the $\mathrm{H}$ atoms attached to $\mathrm{Pd}$ vary between -0.065 and -0.026 except for that in (L1) $\mathrm{Pd}(\mathrm{OTs}) \mathrm{H}$ (0.023). This is because the stronger electron-withdrawing effect of OTs diminishes the electron transfer from Pd to $\mathrm{H}$. Consistent with the negative NPA charges, the negative-charged ESP appears around these typical hydride ligands, which is remarkably different to that of $\mathbf{3}$. These results suggest that the $\mathrm{H}$ atom attached to the $\mathrm{Pd}$ atom in $\mathbf{3}$ still approaches a slightly positive-charged proton rather than a typical hydride. 


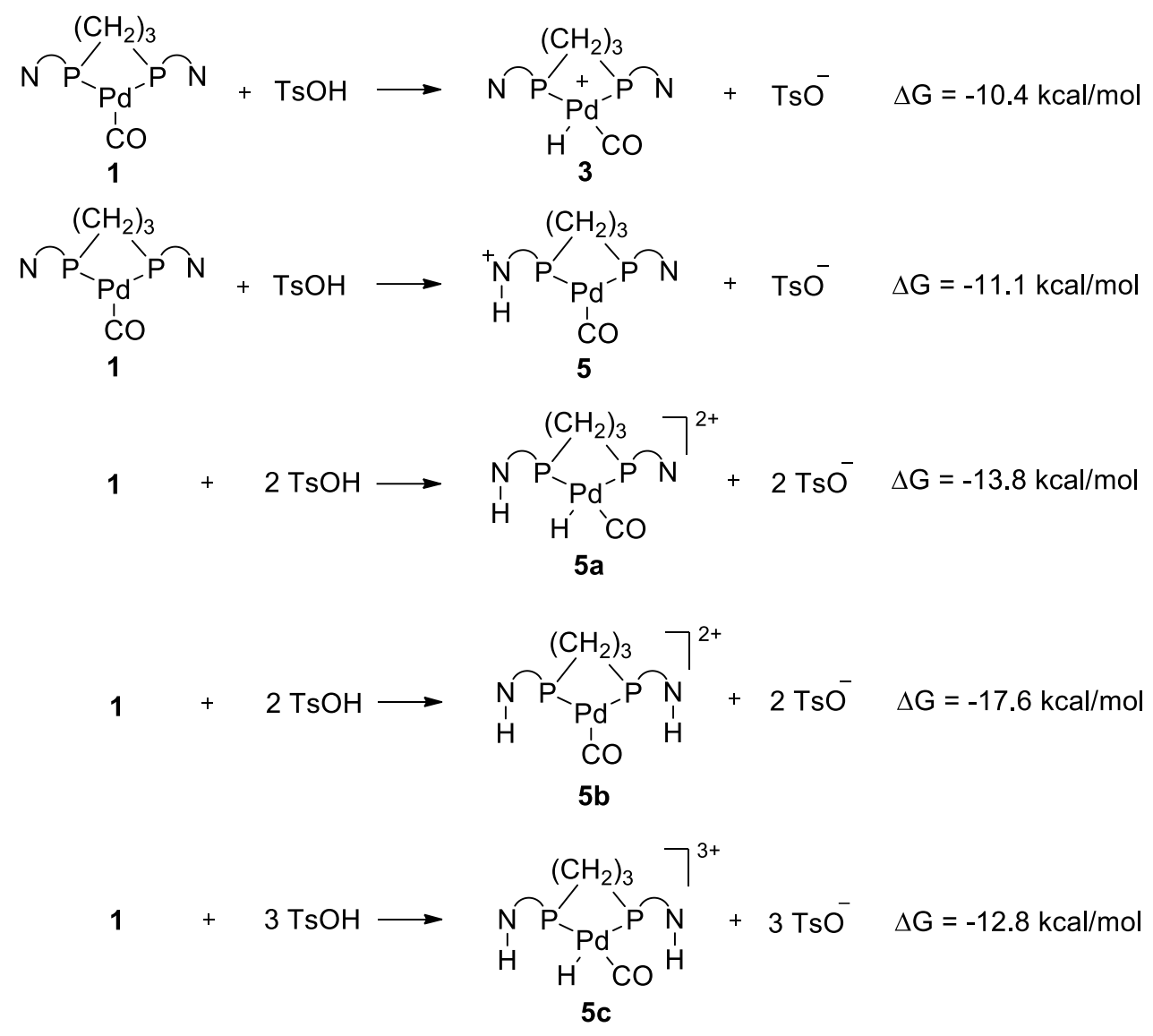

Figure S4. Calculated Gibbs free energies changes of the protonation of 1 with TsOH (in $\mathrm{kcal} / \mathrm{mol}$ ).

Double and triple protonation of $\mathbf{1}$ to by two or three TsOH to form $\mathbf{5 a}, \mathbf{5 b}$ and $\mathbf{5 c}$ were considered (Figure S4). At the condition that the concentration of every species is $1 \mathbf{M}$, formation of $\mathbf{5 a}, \mathbf{5 b}$ and $\mathbf{5 c}$ is indeed more thermodynamically favorable than formation of $\mathbf{5}$.

Then we examined the hydrometallation process from $\mathbf{5 a}, \mathbf{5 b}$ and $\mathbf{5 c}$ (Figure S5). Related transition states were failed to be located for $\mathbf{5 c}$ because there is no suitable coordination site left on Pd. From $\mathbf{5 a}$ and $\mathbf{5 b}$, we found that TS4-2H and TS6-2H are also energetically close, like the hydrometallation from monoprotonated catalysts via TS4 and TS6.

Furthermore, the selective-determining steps (hydrometallation and methanolysis) of P2a with L1 (Figure S6) as well as the hydrometallation of $\mathbf{a 1}$ with $\mathbf{L} 2$ for double protonated catalysts were tested (Figure S7). As shown in Figure S6, the relative energies arrange in the order of TS19-2H < TS16-2H < TS21-2H, leading to P1a formation. In Figure S7, TS35-2H is significantly energetically lower than TS34-2H, making P2a be the major product.

These results indicate that the double-protonated catalysts show very similar selectivity to the mono- 
protonated catalysts though the double-protonated pathway are more energetically favored in the tested steps. However, it should be noted that the concentration of every species is set to be $1 \mathrm{M}$ in the calculations whereas the real concentration of $\mathrm{TsOH}$ is much lower and not favored for the multiprotonation of catalysts. Anyway, we cannot fully exclude or agree with the possibility of the participation of double-protonated catalyst just according to the matter of concentrations because the calculations themselves have intrinsic errors.

hydrometallation of a1 with L1<smiles>CP(CN)P(C)p1c(-c2ccccc2)cp1P(C)C</smiles>

TS4

20.3

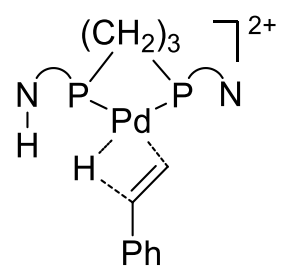

TS4-2H

16.3

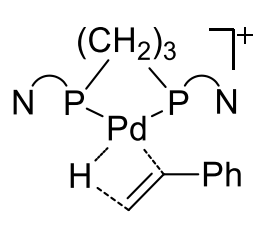

TS5

23.0

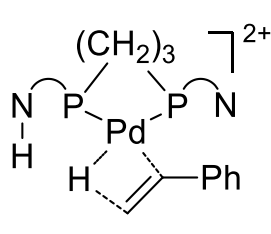

TS5-2H

19.0<smiles>CP1P2CNC=C(c3ccccc3)P1P2CPN</smiles>

TS6

19.8<smiles>C[PH]1(C)PNCC=C(c2ccccc2)P1PC[P+]</smiles>

TS6-2H

15.5

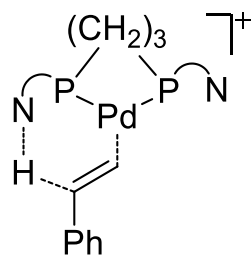

TS7

21.9

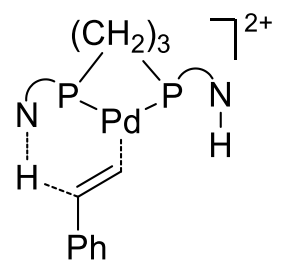

TS7-2H

16.0

Figure S5. Calculated Gibbs free energies changes of the hydrometallation of a1 with $\mathbf{L 1}$ from double protonated catalysts (in $\mathrm{kcal} / \mathrm{mol}$ ). 
hydrometallation and methanolysis of P2a with L1
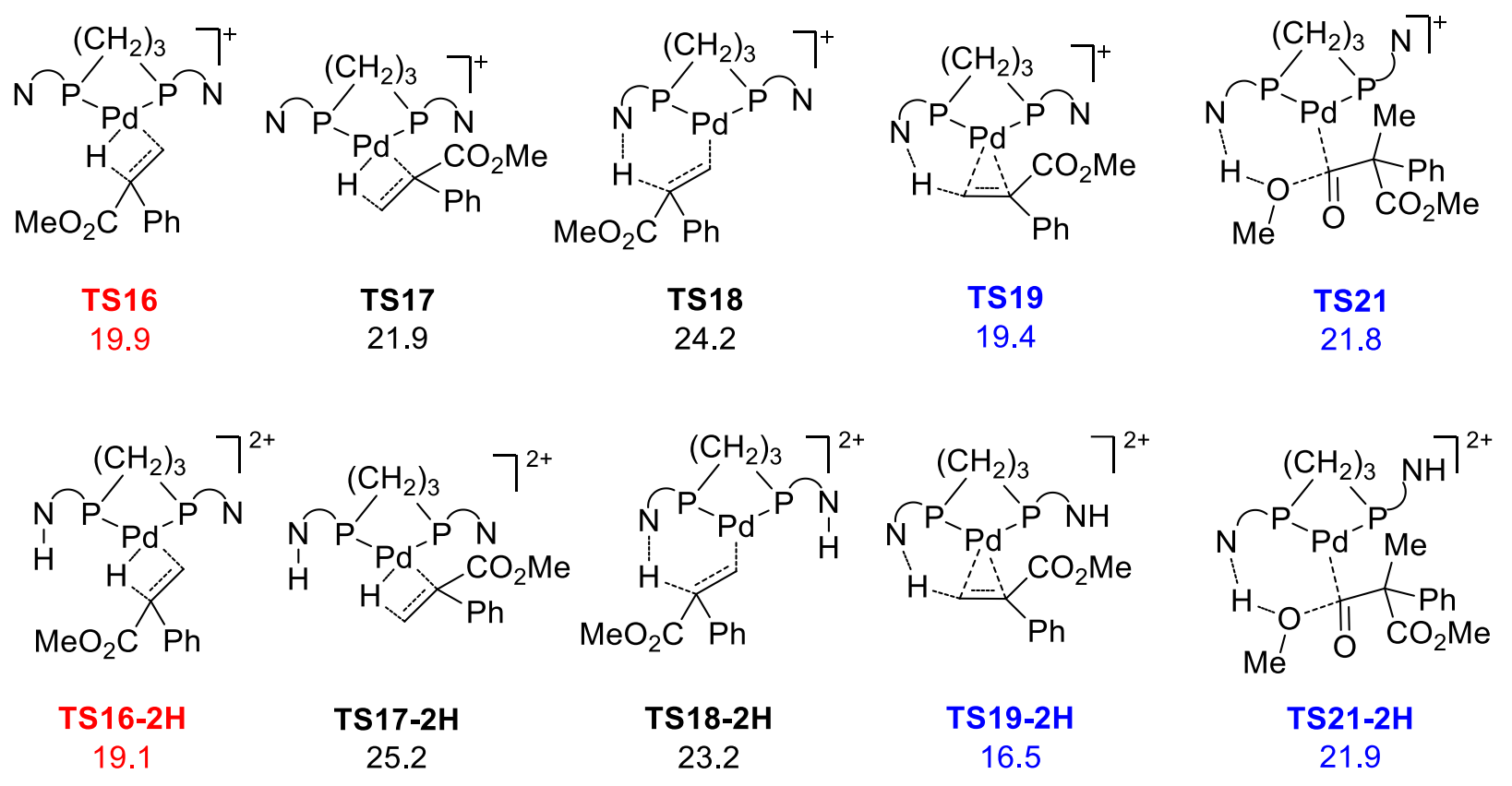

Figure S6. Calculated Gibbs free energies changes of the hydrometallation and methanolysis of P2a with $\mathbf{L 1}$ from double protonated catalysts (in $\mathrm{kcal} / \mathrm{mol}$ ).

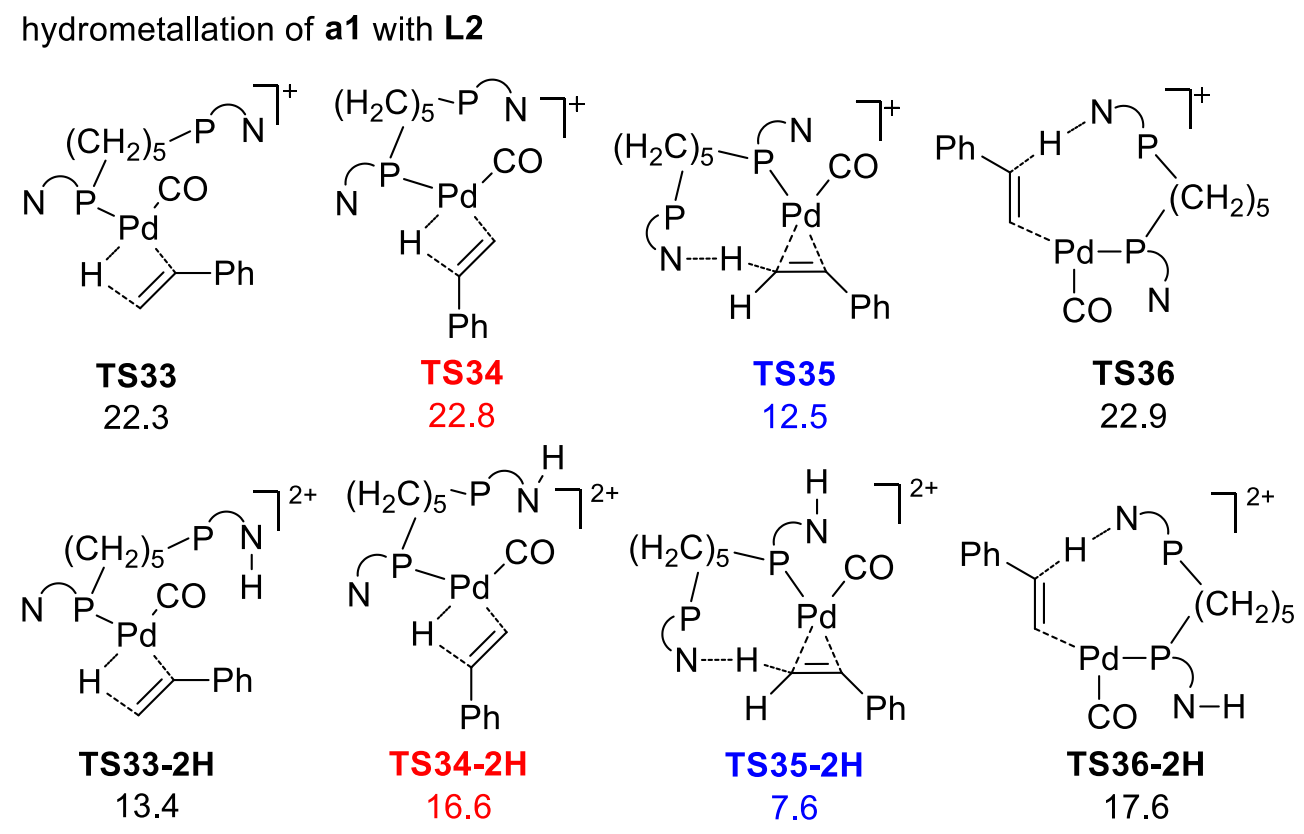

Figure S7. Calculated Gibbs free energies changes of the hydrometallation and methanolysis of a1 with $\mathbf{L} 2$ from double protonated catalysts (in $\mathrm{kcal} / \mathrm{mol}$ ). 
Anti-Markovnikov selective

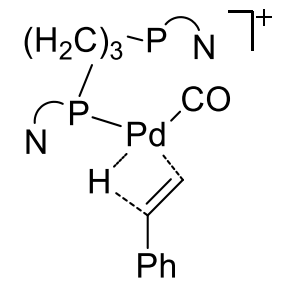

TS4a

26.1

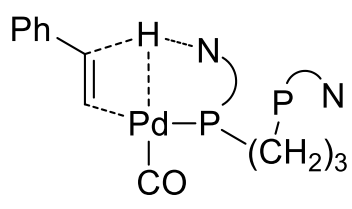

TS7a

26.5

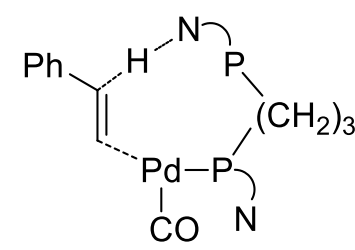

TS7b

33.3

\section{Markovnikov selective}

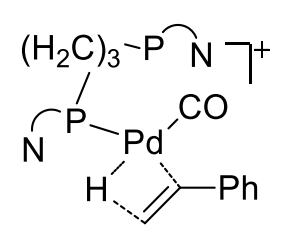

TS5a

24.8<smiles>C[P@]1(C=O)CP(CN)CPCNC=C1c1ccccc1</smiles>

TS6a

21.1<smiles>CP(CPCN)P1CNC[C]=C(c2ccccc2)P1C=O</smiles>

TS6b

20.5

Scheme S1. Hydrometallation of alkyne a1 with the partial dissociation of L1 
intermediates and transition states in P2a formation

(12b

intermediates and transition states in P3a formation

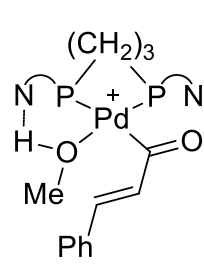

20b

$-24.1$

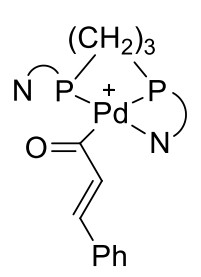

20a $-21.9$<smiles>CP(C)[P+](CN)C(=O)/C=C/c1ccccc1</smiles>

20c

$-19.2$

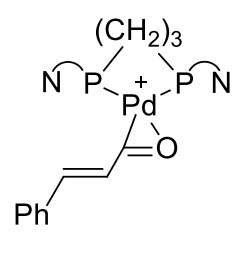

20-0 $-22.8$<smiles>CC(=O)[P+](CP)(C(=O)/C=C/c1ccccc1)P(C)C</smiles>

20-CO $-34.9$

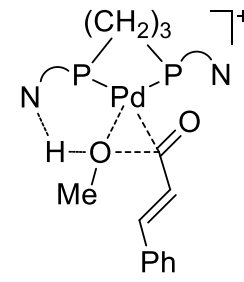

TS13a

$-2.2$

Scheme S2. Less stable intermediates and transition states in the methanolysis stage of a1 with L1 

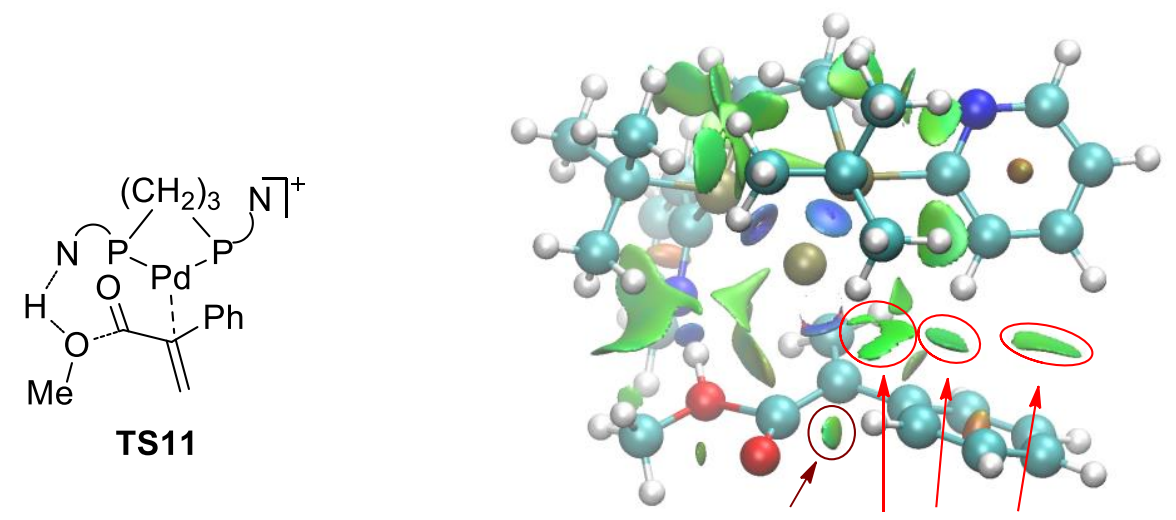

van der Waals interaction van der Waals interaction between phenyl and carbonyl between phenyl and L1
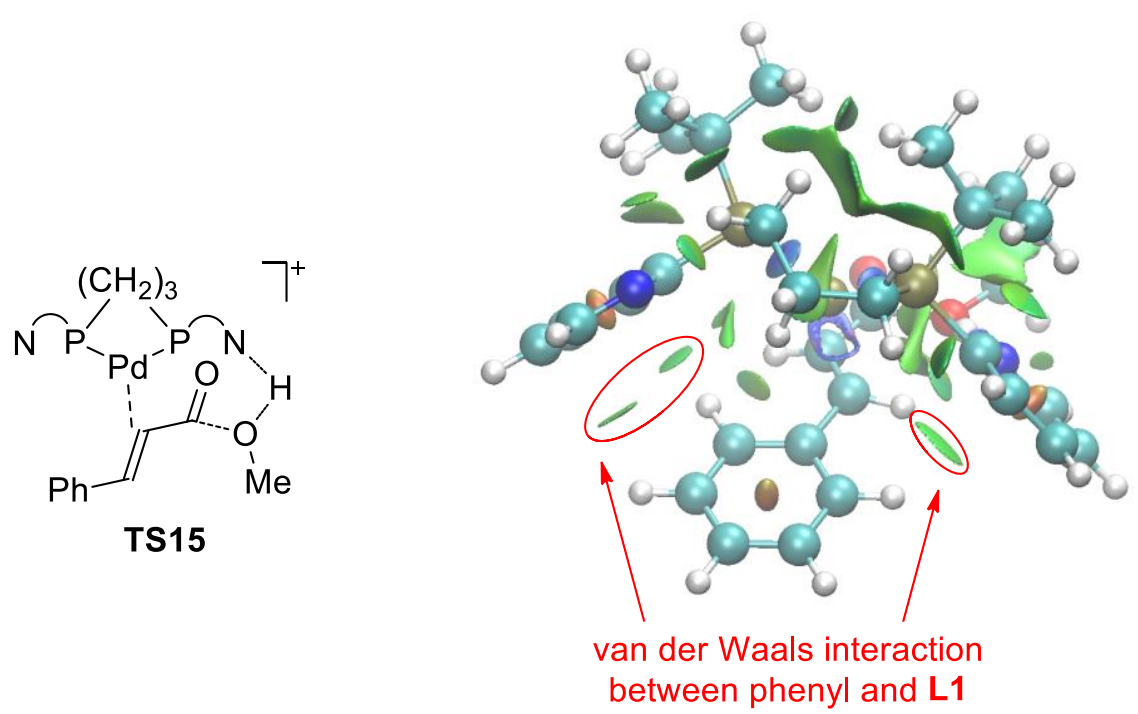

Figure S8. NCIplot of TS11 and TS15. Red areas indicate destabilizing interaction. Blue areas indicate stabilizing interaction. Green areas indicate van der Waals interaction. 
hydrometallation without dissociation of phosphine ligand

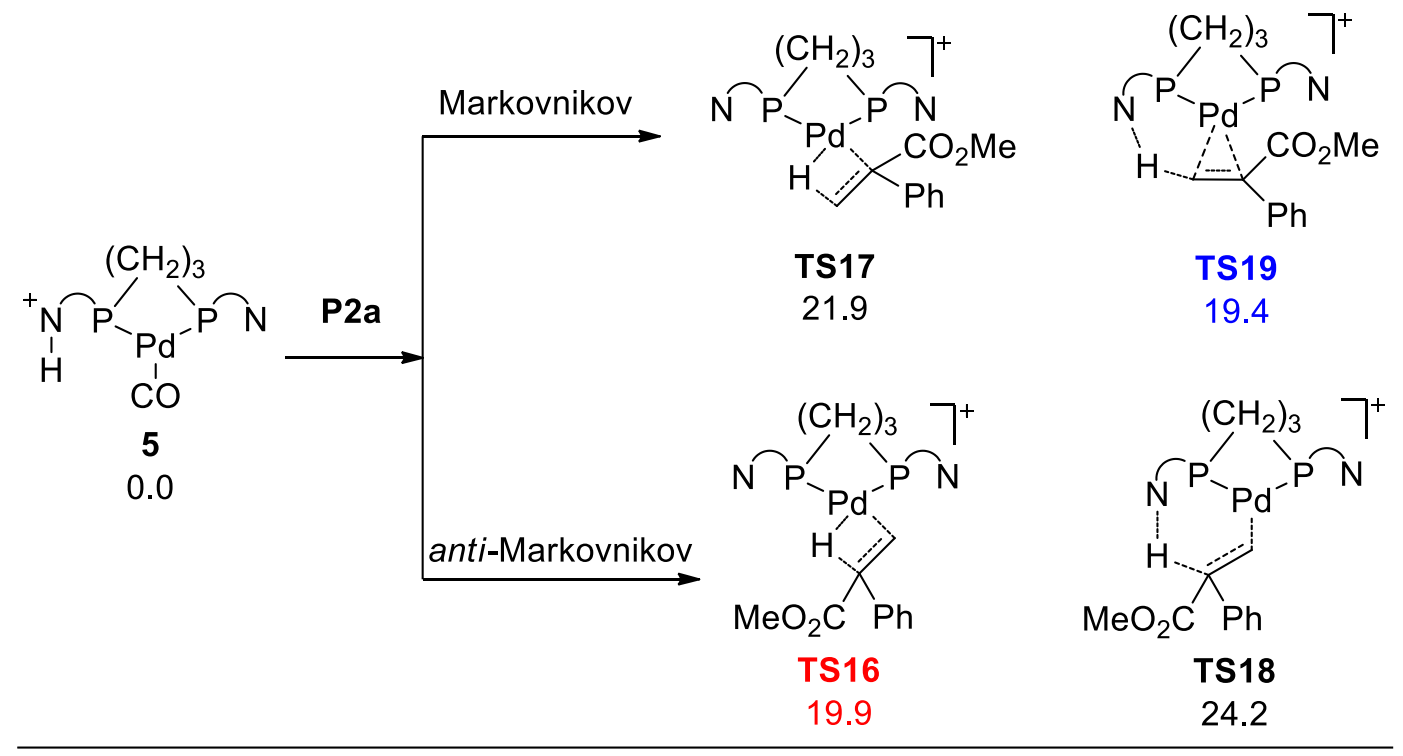

hydrometallation with dissociation of one phosphine ligand

anti-Markovnikov

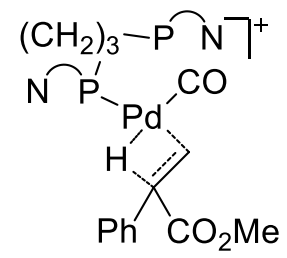

TS16a

29.0

Markovnikov

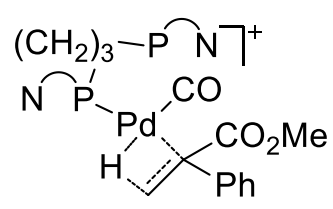

TS17a

24.5

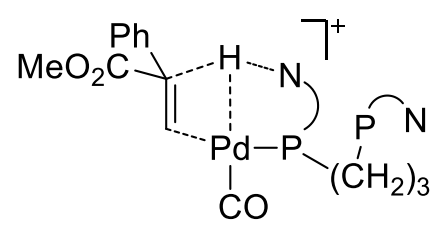

TS18a

28.1

TS19a

22.0

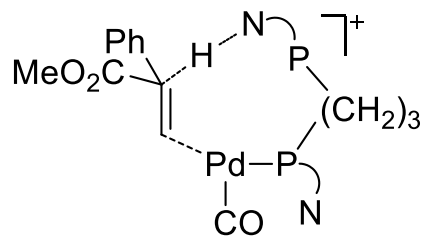

TS18b

40.6

Scheme S3. Calculated results of hydrometallation stage of P2a in the presence L1 
<smiles>COC(=O)C1(c2ccccc2)C2CNCP(C)P2C1PC[I-]</smiles>

TS19

19.4

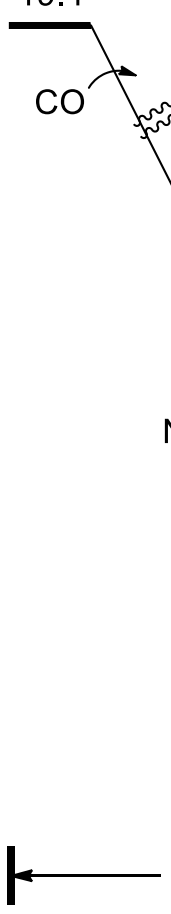

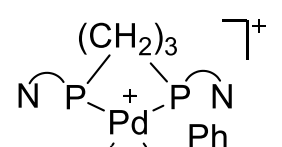

$\mathrm{Pd}^{-P h}$

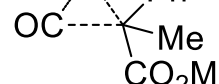

$\mathrm{Me}$
$\mathrm{CO}_{2} \mathrm{Me}$

TS20

17.8

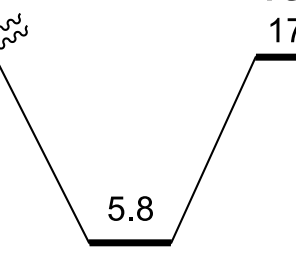

$\left(\mathrm{CH}_{2}\right)_{3}$<smiles>NCP1CCP(N)P1[PH2+]</smiles><smiles>CC(C)([Te]O[Te][O-])c1ccccc1</smiles>
$\mathrm{MeO}_{2} \mathrm{C} \mathrm{Me}$

25

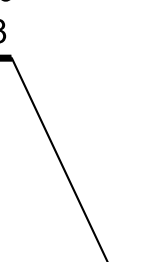

$\left(\mathrm{CH}_{2}\right)_{3}$ $\widehat{\mathrm{N}}-\mathrm{Pdg}^{+}-\mathrm{PN}$ $\mathrm{H} \mathrm{O}^{\prime} \mathrm{Pd}^{-} \mathrm{P}=\mathrm{O}$ $\mathrm{Me}_{\mathrm{Me}}^{\mathrm{f}} \underset{\mathrm{CO}_{2} \mathrm{Me}}{\mathrm{Ph}}$ $\mathrm{MeOH} 27$
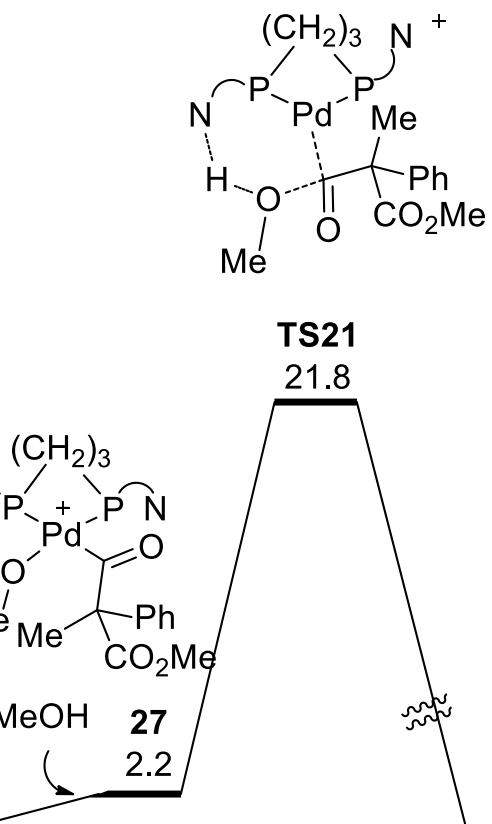

TS21

21.8

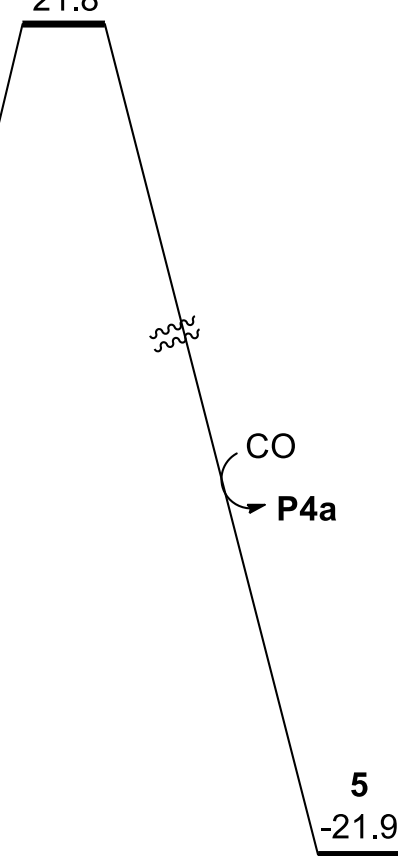

$-21.9$

CO insertion
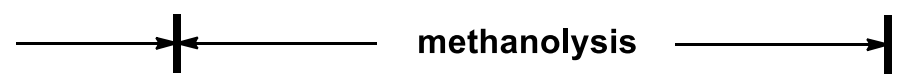

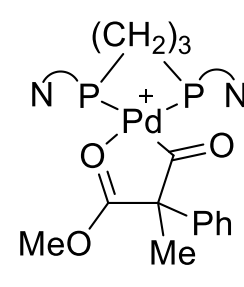

26

$-8.5$

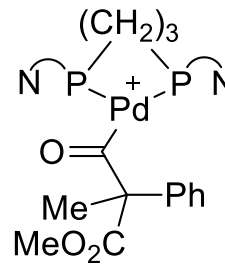

26a

1.4

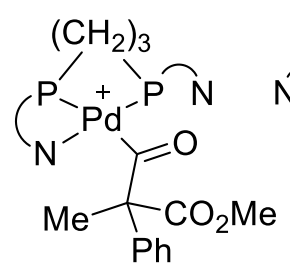

26b<smiles>COC(=O)C(C)(c1ccccc1)C1O[P+]1(P(C)C)P(C)N</smiles>

26-0

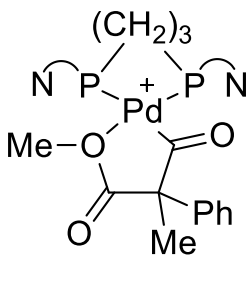

26-MeO

$-0.6$

Figure S9. Calculated solution-phase Gibbs free energy changes of the formation of P4a from P2a in the presence of $\mathbf{L 1}(\mathrm{kcal} / \mathrm{mol})$. 


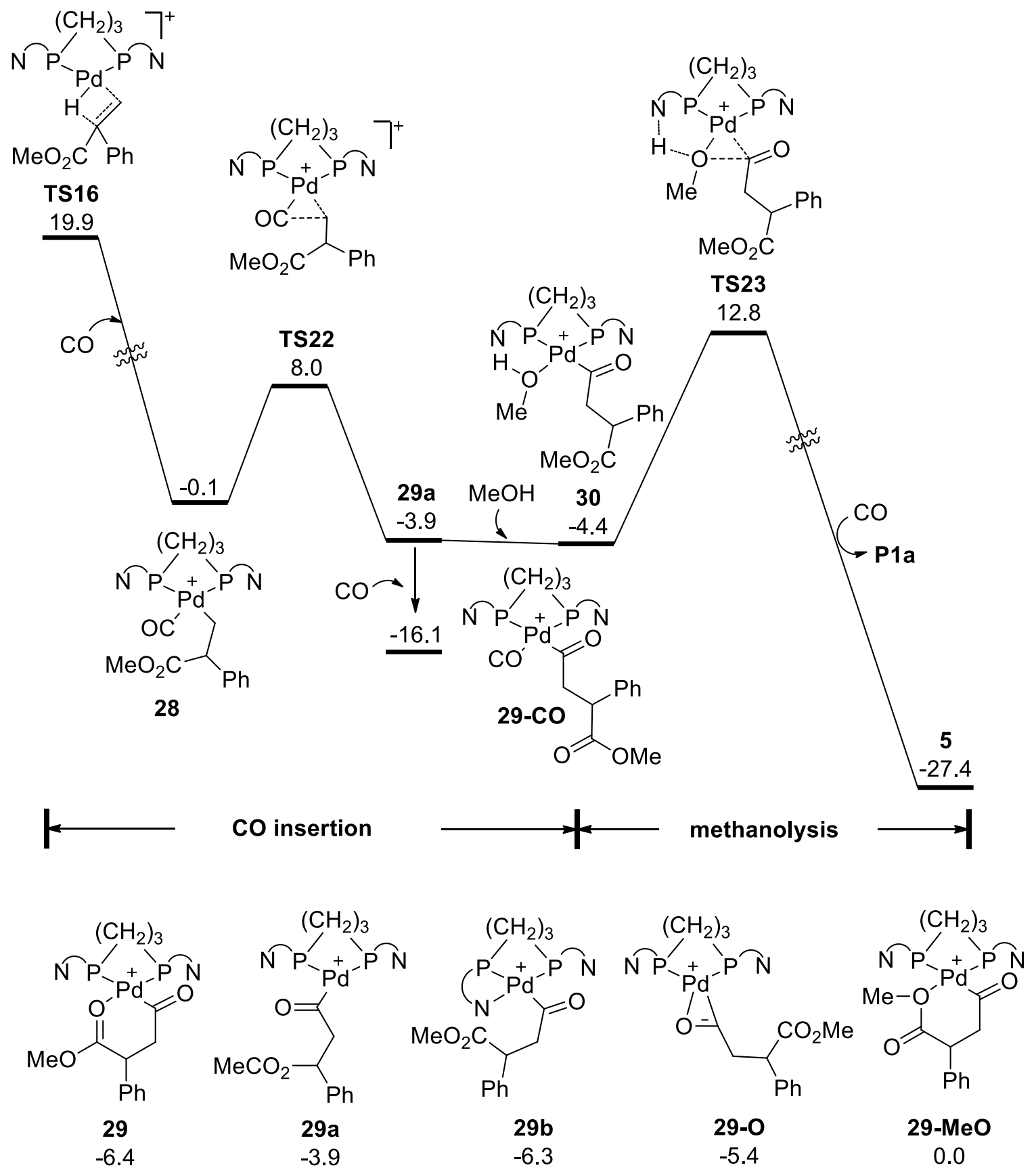

Figure S10. Calculated solution-phase Gibbs free energy changes of the formation of P1a from P2a in the presence of $\mathbf{L} \mathbf{1}(\mathrm{kcal} / \mathrm{mol})$. 
hydrometallation without dissociation of phosphine ligand

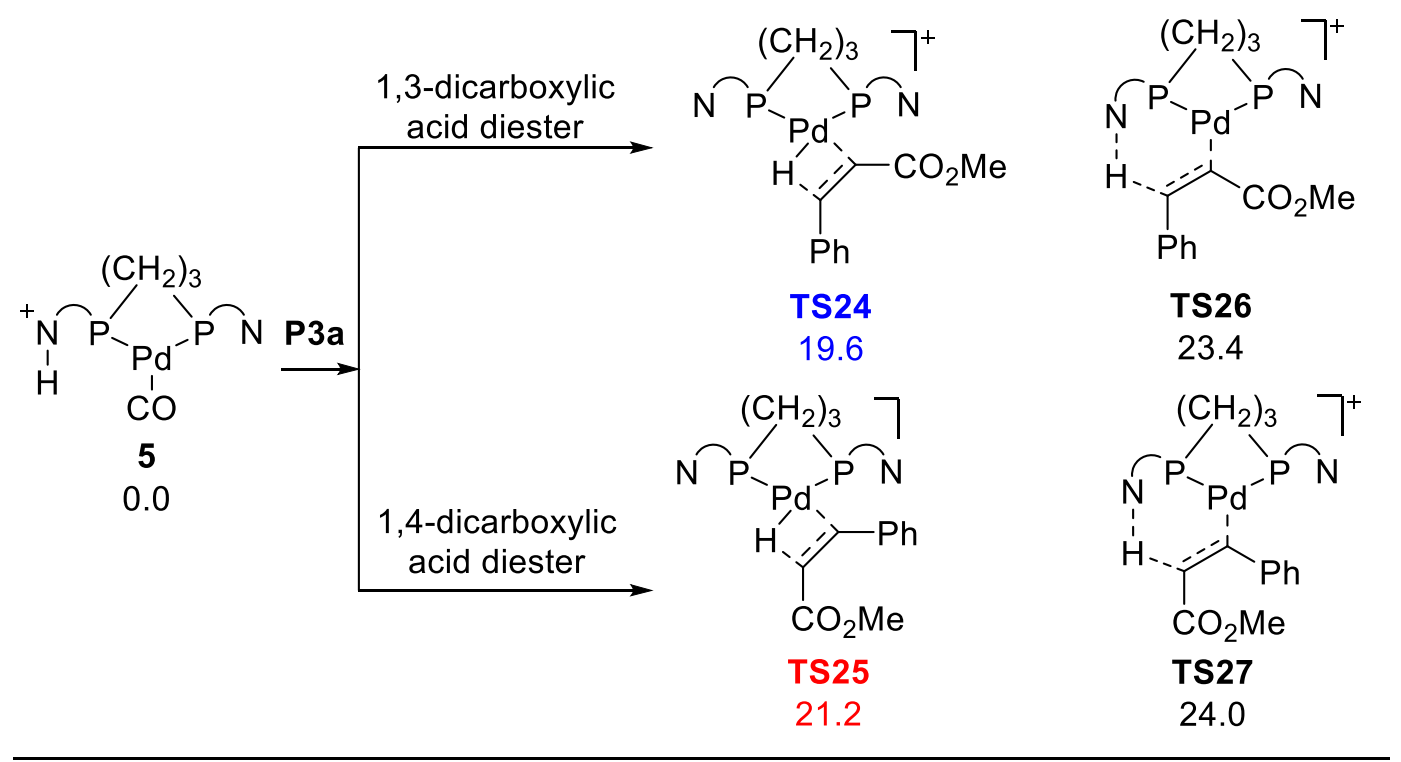

hydrometallation with dissociation of one phosphine ligand

1,3-dicarboxylic acid diester<smiles>[3H]CCOC(=O)C1C(P)=CP1P(C)CC</smiles>

TS24a

24.9

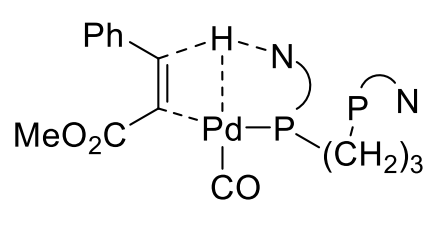

TS26a

28.4

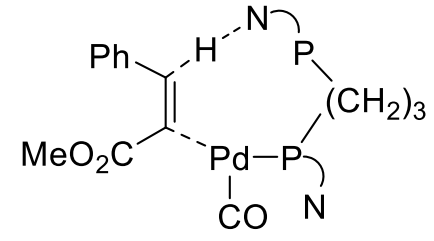

TS26b 43.5

1,4-dicarboxylic acid diester

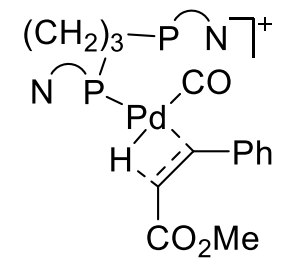

TS25a

26.4<smiles>COC(=O)C1=C(c2ccccc2)P2(C=O)(CCN)CNC12</smiles>

TS27a

29.0

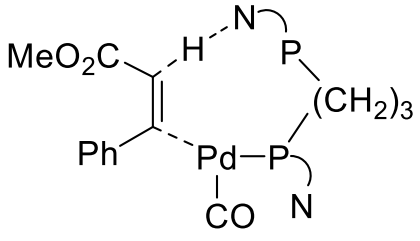

TS27b

41.7

Scheme S4. Calculation results of hydrometallation stage of P3a in the presence L1

It should be pointed out that the unrestricted geometry optimization to locate TS26a was failed. Its energy was estimated by performing frequency analysis and single-point energy calculation based on the maximum point in relaxed potential energy surface scan (Figure S11). 


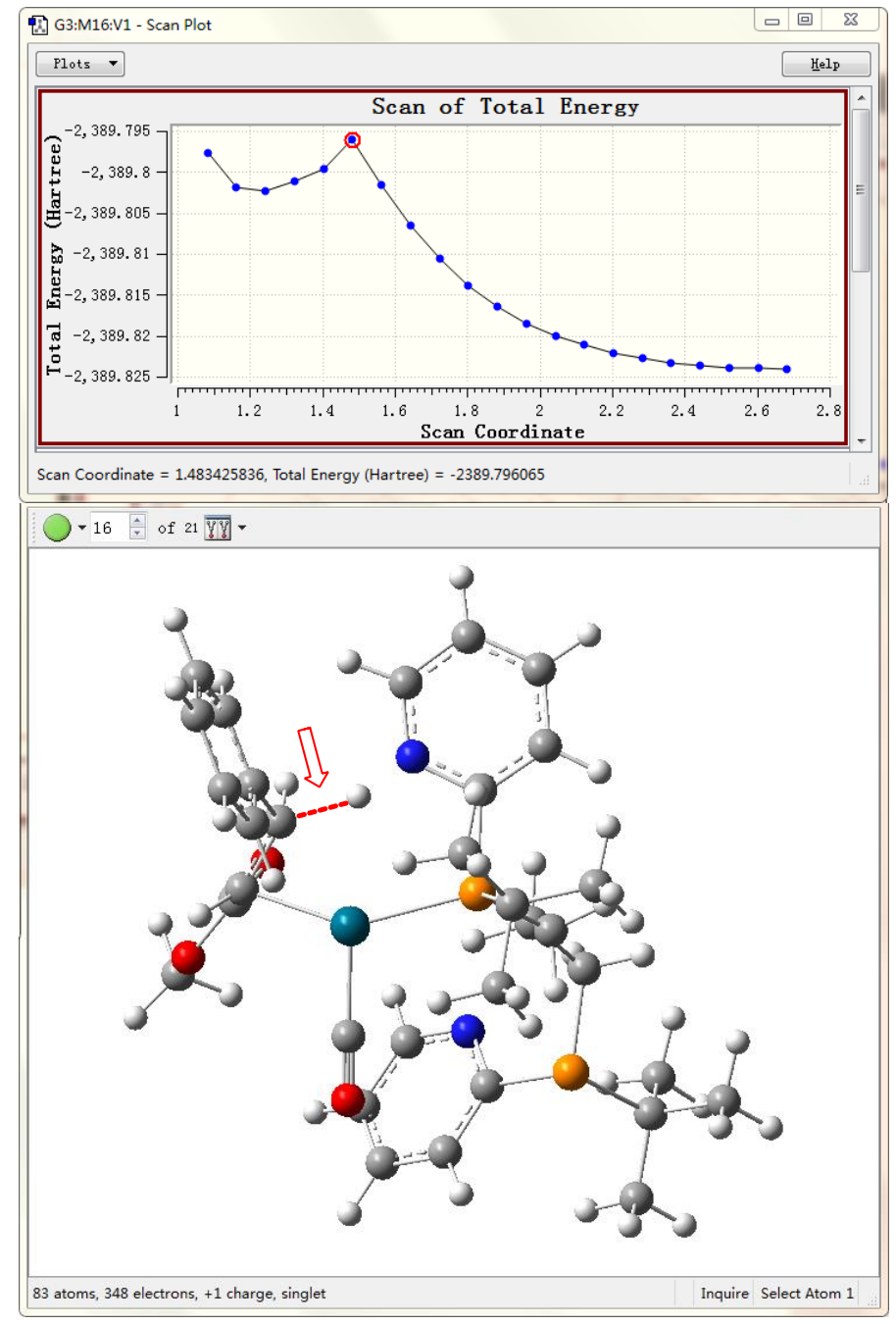

Figure S11. Relaxed potential energy surface scan of the $\mathrm{C}-\mathrm{H}$ interatomic distance (labeled by the red line) for estimating the energy of the ligand-assisted electrophilic addition transition state TS26a. 

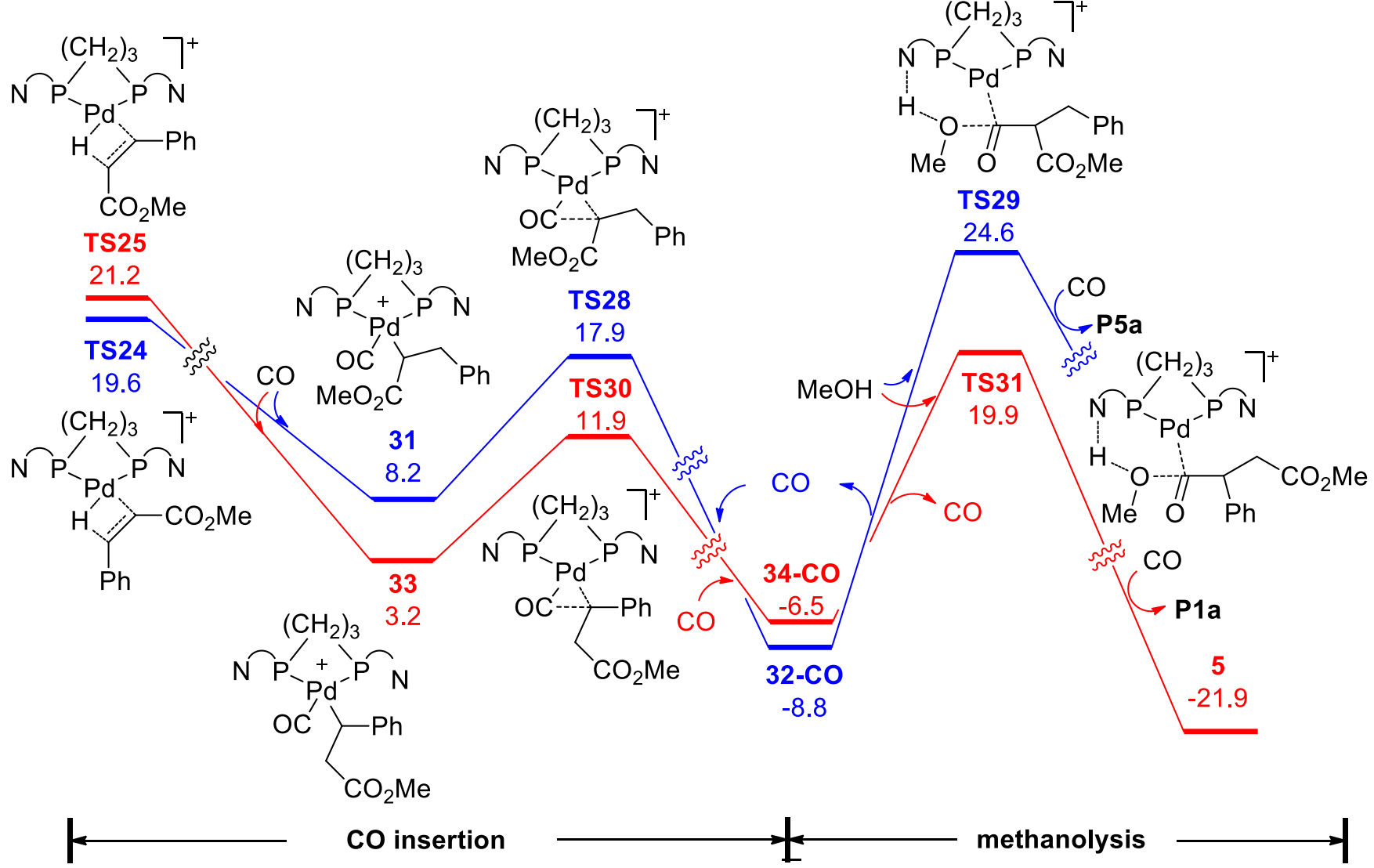

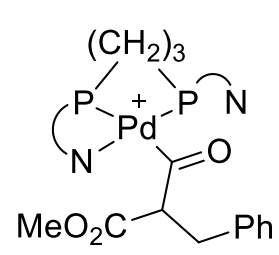

32

0.4

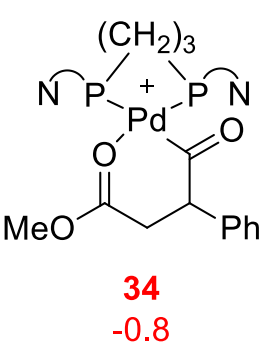

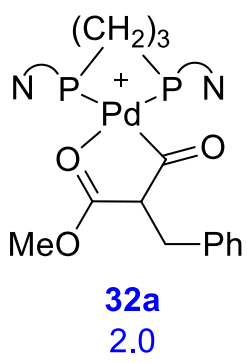

$\left(\mathrm{CH}_{2}\right)_{3}$

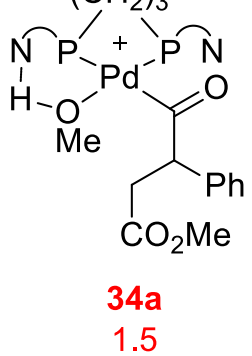

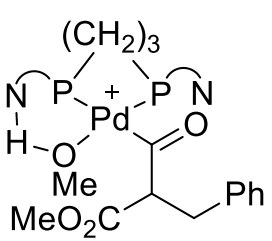

32b

5.5

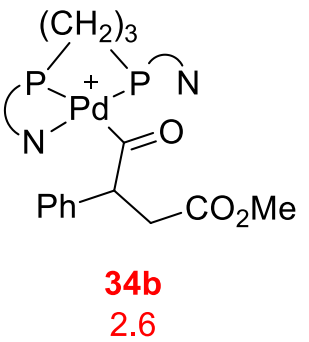

$\left(\mathrm{CH}_{2}\right)_{3}$ $\widehat{N} \mathrm{P}^{-1}+\mathrm{P}^{-}-\mathrm{P} \mathrm{N}$ $\mathrm{OC}^{\mathrm{Pd}} \mathrm{Pd}$<smiles>COC(=O)C(Cc1ccccc1)C(C)=O</smiles>

32-CO

$-8.8$

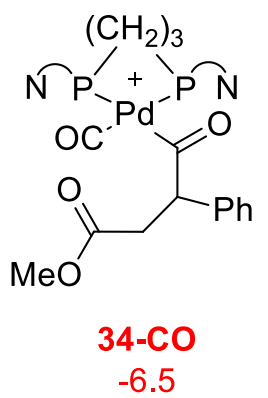

Figure S12. Calculated solution-phase Gibbs free energy changes of the $\mathrm{CO}$ insertion and methanolysis stages in the AOC of P3a with $\mathbf{L 1}(\mathrm{kcal} / \mathrm{mol})$. 


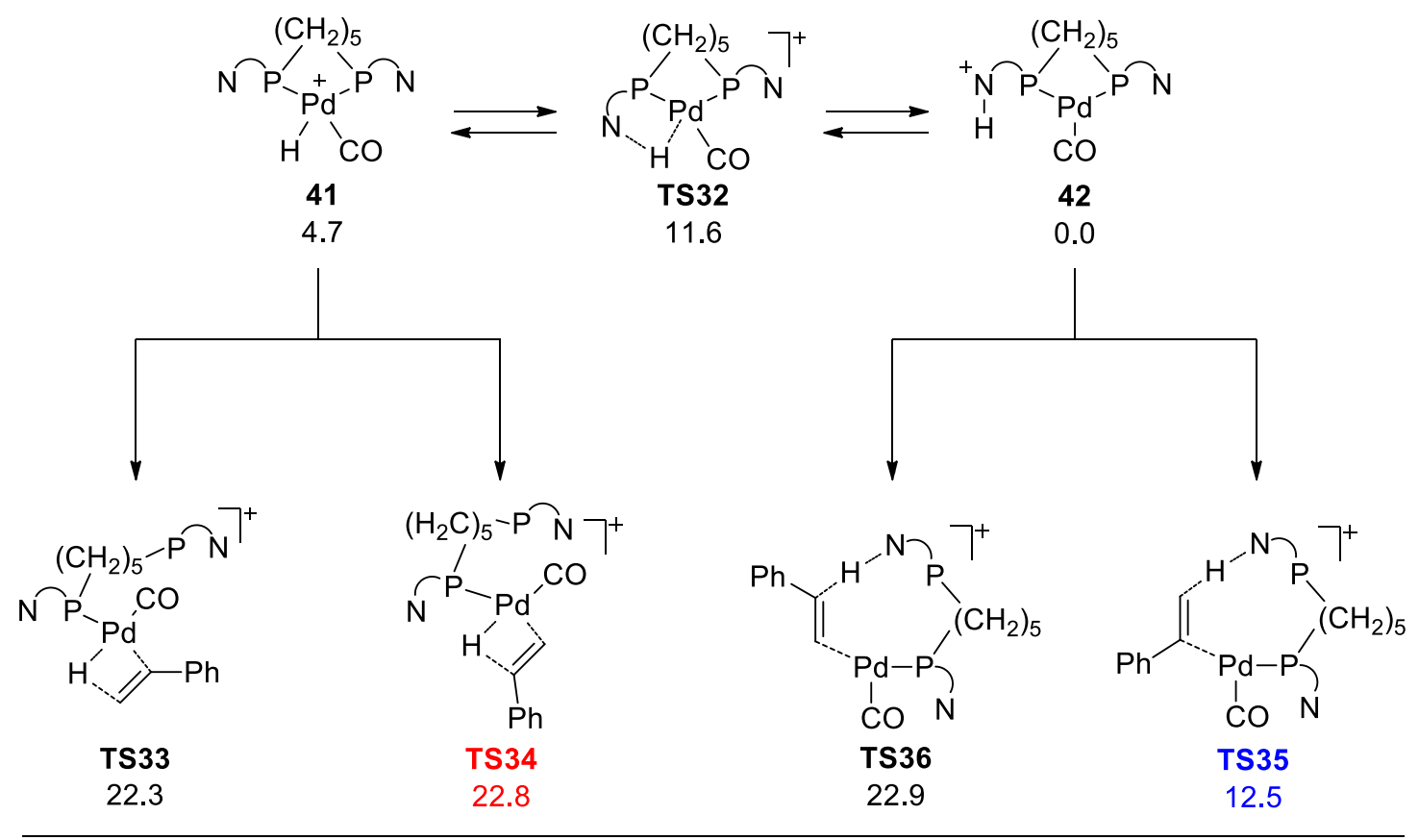

other less favored transition states

Markovnikov selective

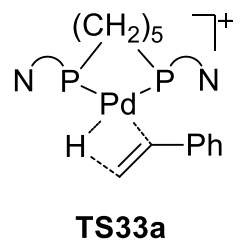

31.6

anti-Markovnikov selective

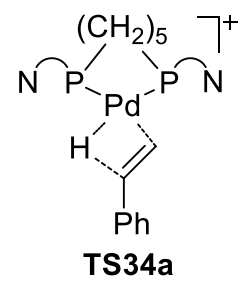

30.3

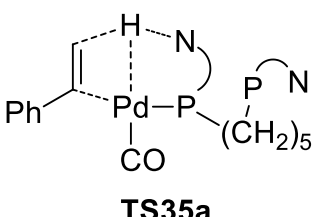

18.4

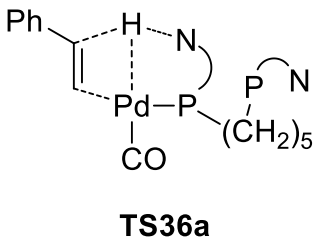

24.4<smiles>CCNCCPP1C(P)=CCNCP1C</smiles>

23.3<smiles>CCNPPC1=C(c2ccccc2)CNCP1C</smiles>

TS36b 25.9

Scheme S5. Calculation results of hydrometallation stage of a1 in the presence L2 

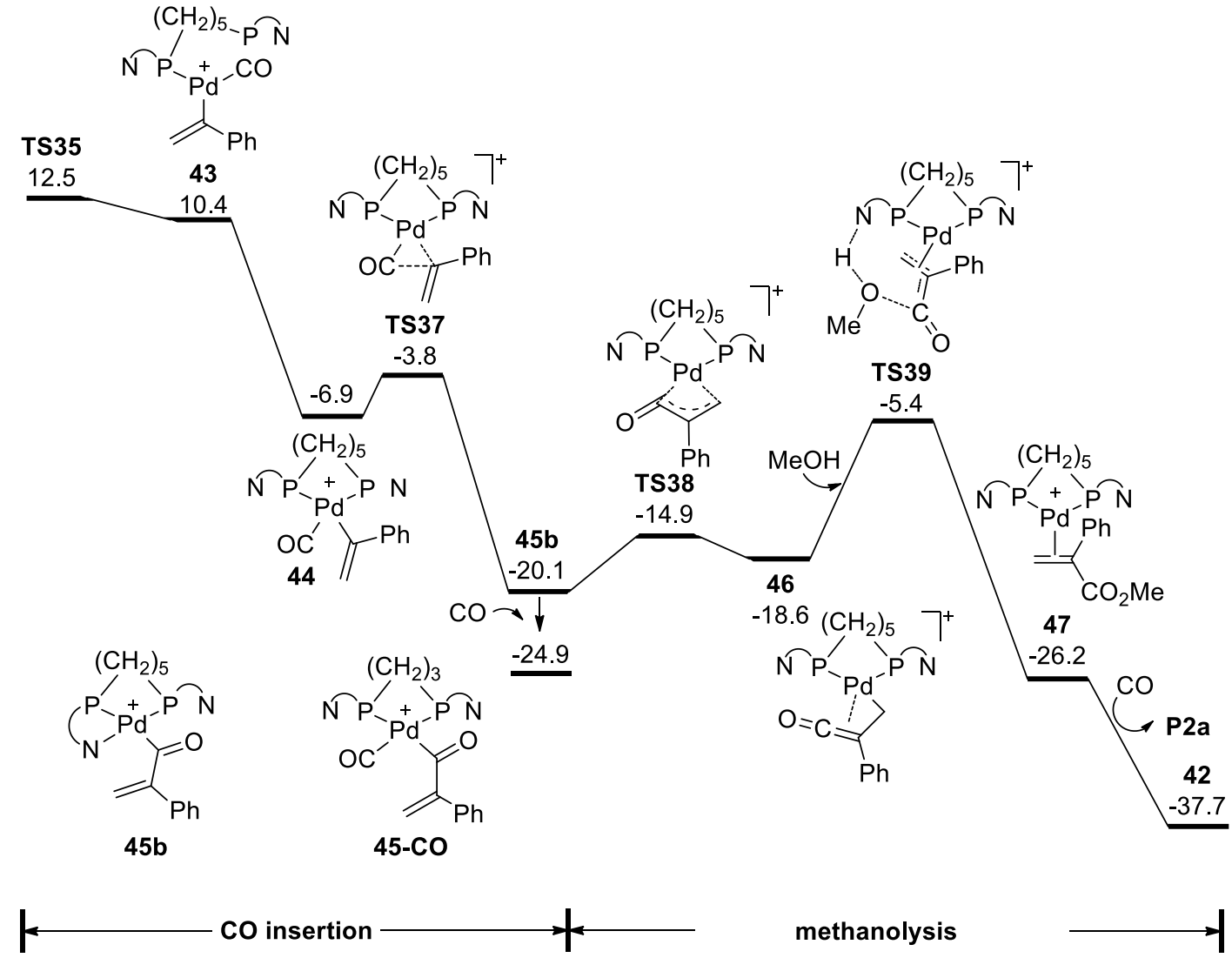<smiles>C=C(C(=O)C(P)(P)[Pb]1CCCP(N)P1P)c1ccccc1</smiles>

45

$-22.6$

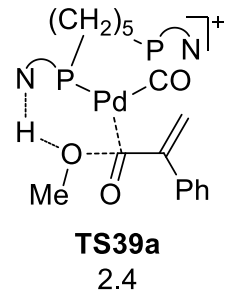

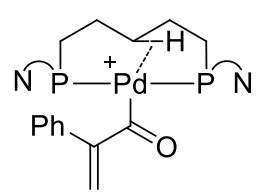

$45 a$

$-21.5$

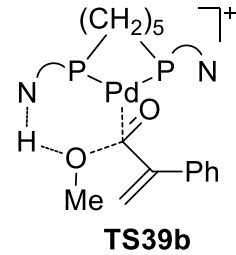

4.9

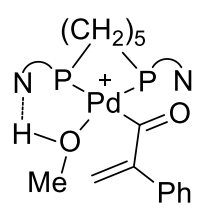

45c

$-12.8$

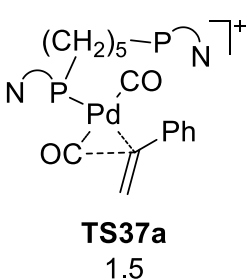<smiles>C=C(C1=[P+](P)[Ge](C)(P(C)N)O1)c1ccccc1</smiles>

45-0

$-12.8$

Figure S13. Calculated solution-phase Gibbs free energy changes of the $\mathrm{CO}$ insertion and methanolysis stages in the AOC of a1 to generate P2a with $\mathbf{L} 2(\mathrm{kcal} / \mathrm{mol})$. 


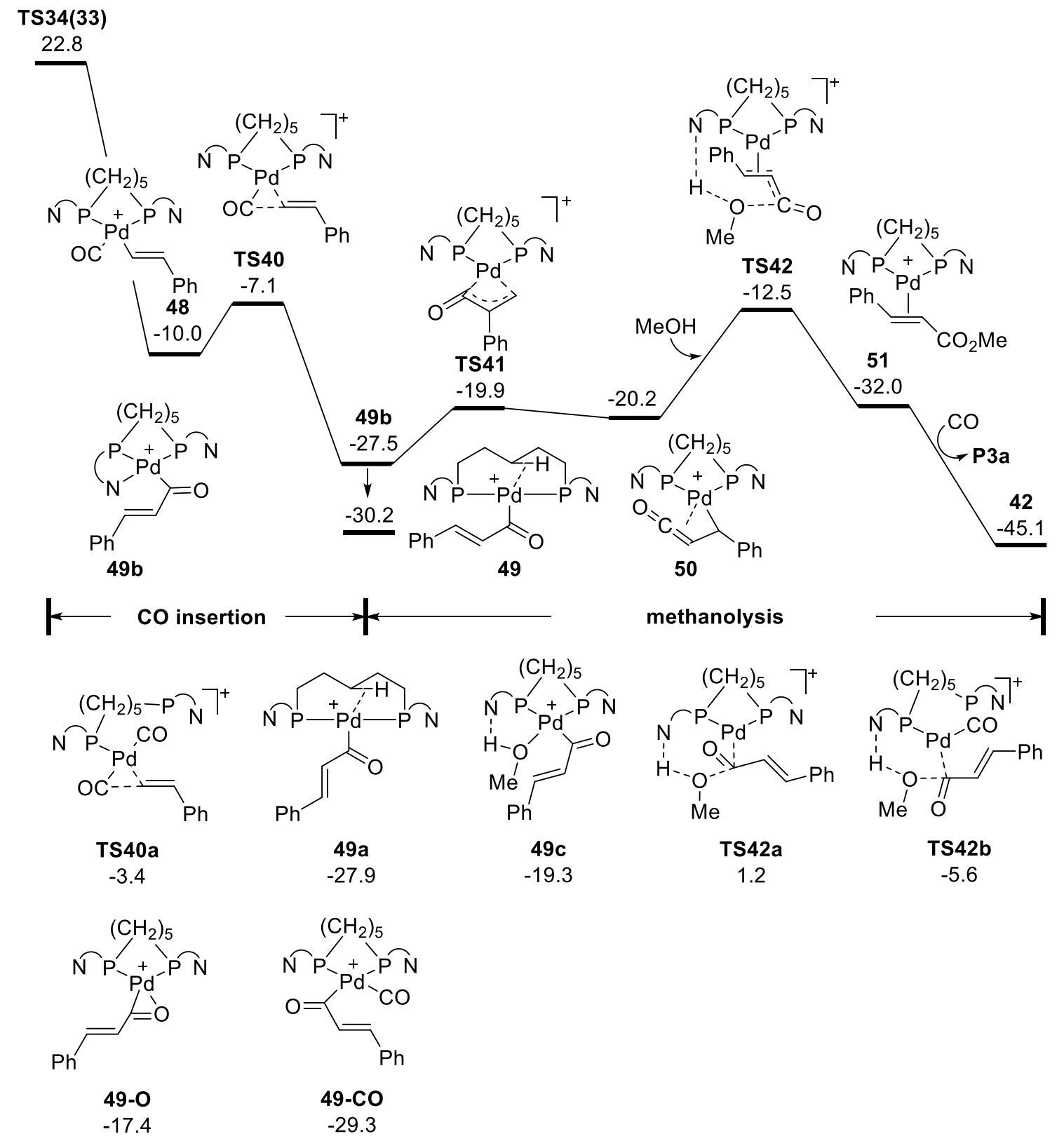

Figure S14. Calculated solution-phase Gibbs free energy changes of the CO insertion and methanolysis stages in the AOC of a1 to generate P3a with $\mathbf{L} 2(\mathrm{kcal} / \mathrm{mol})$. 


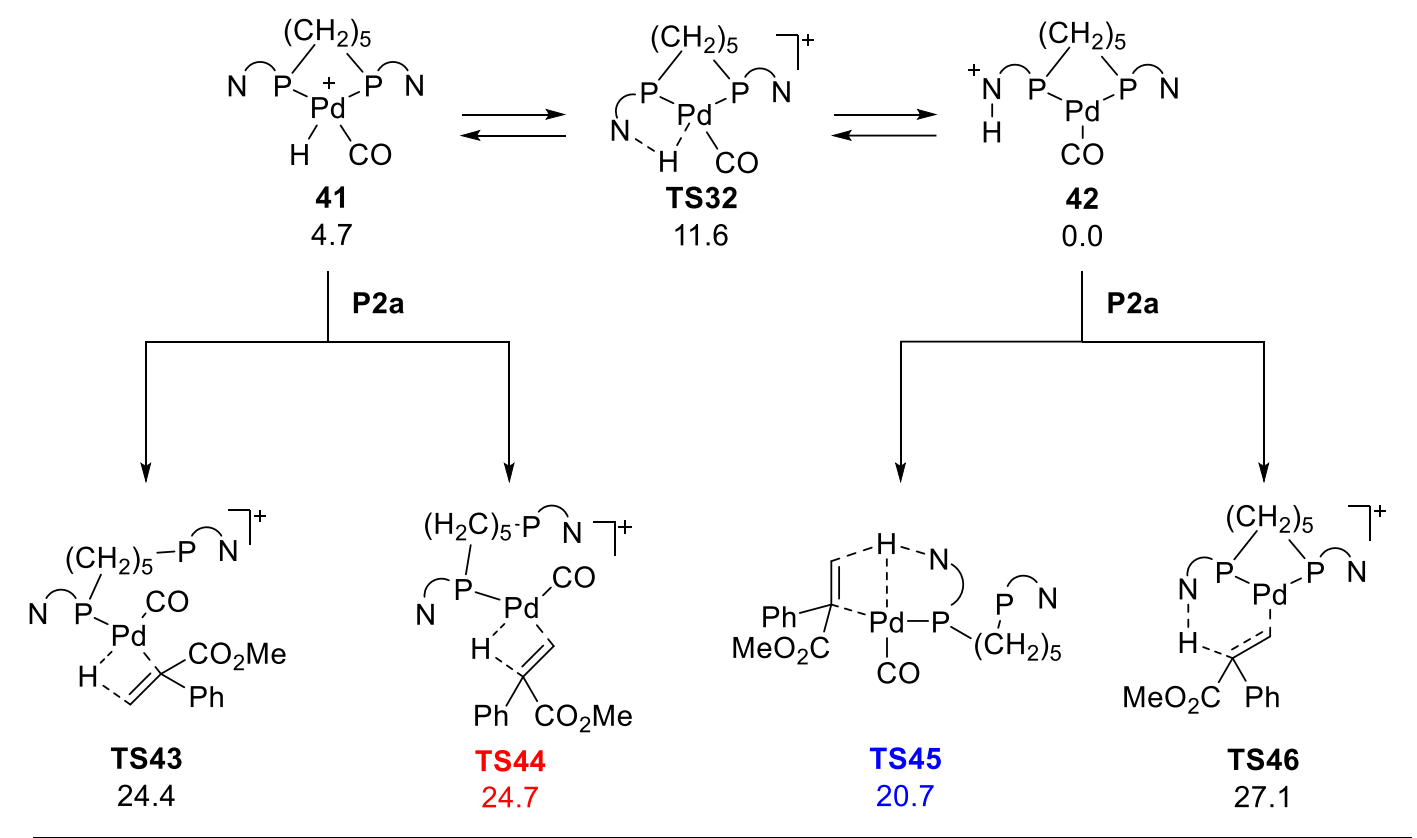

other less favored transition states

Markovnikov selective

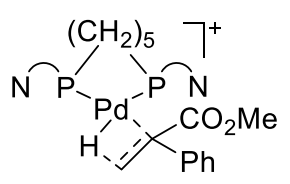

TS43

31.0

anti-Markovnikov selective<smiles>[3H]CPP(C)[Pb]1C(P(C)P(C)CN)=CC1(C(=O)OC)c1ccccc1</smiles>

TS44a

28.4

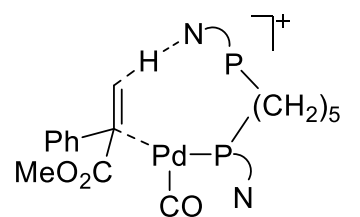

TS45a

21.9

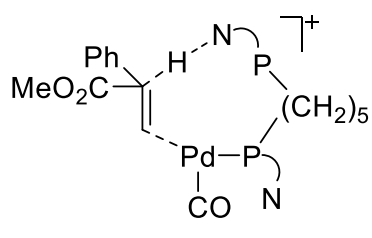

TS46a

27.4

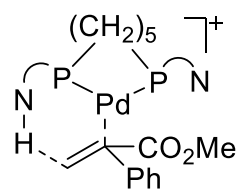

TS45b

26.8

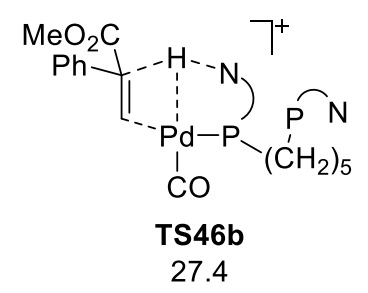

Scheme S6. Calculation results of hydrometallation stage of P2a in the presence L2 

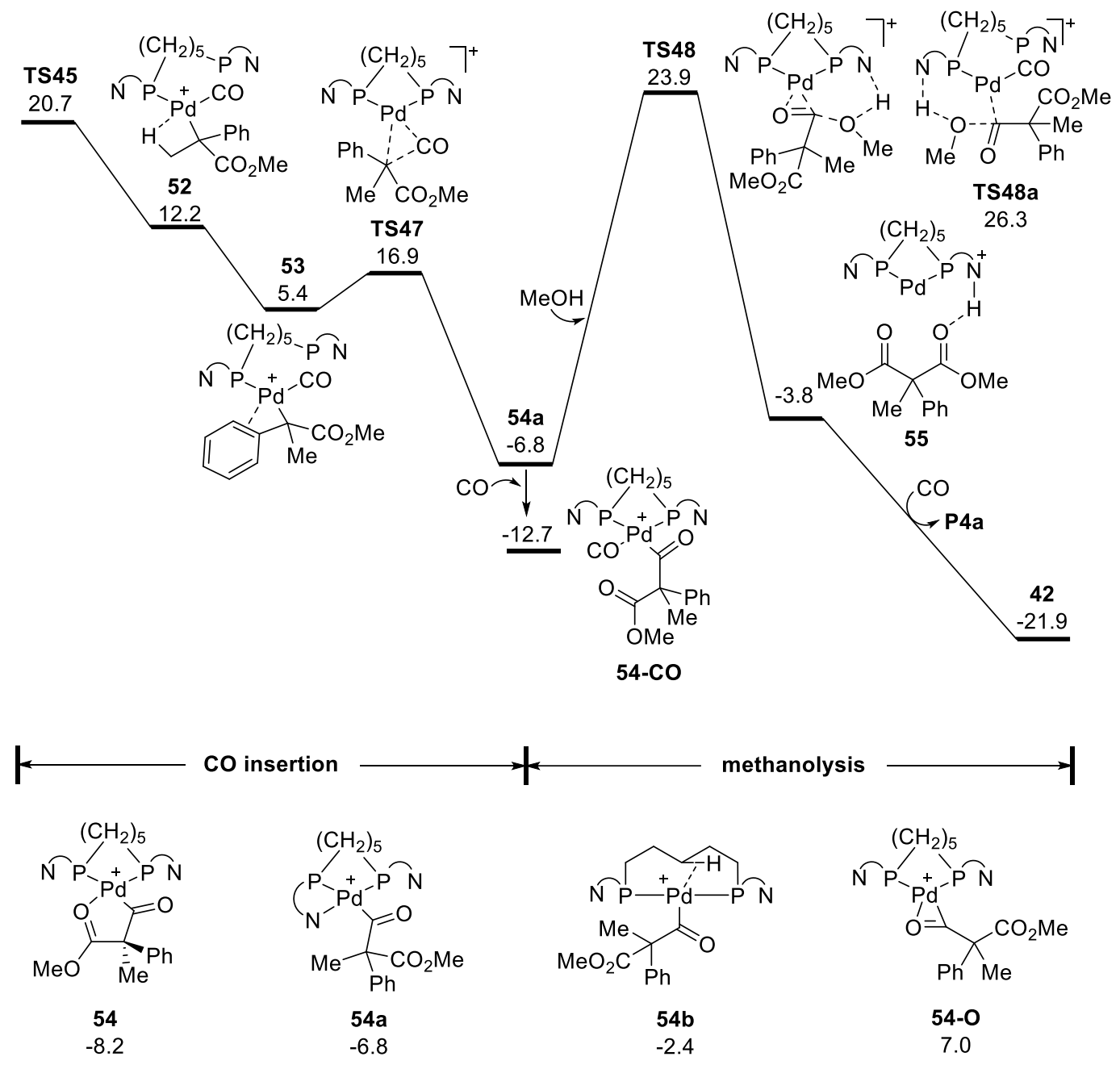

Figure S15. Calculated solution-phase Gibbs free energy changes of the CO insertion and methanolysis stages in the AOC of P2a to generate P4a with $\mathbf{L 2}(\mathrm{kcal} / \mathrm{mol})$. 

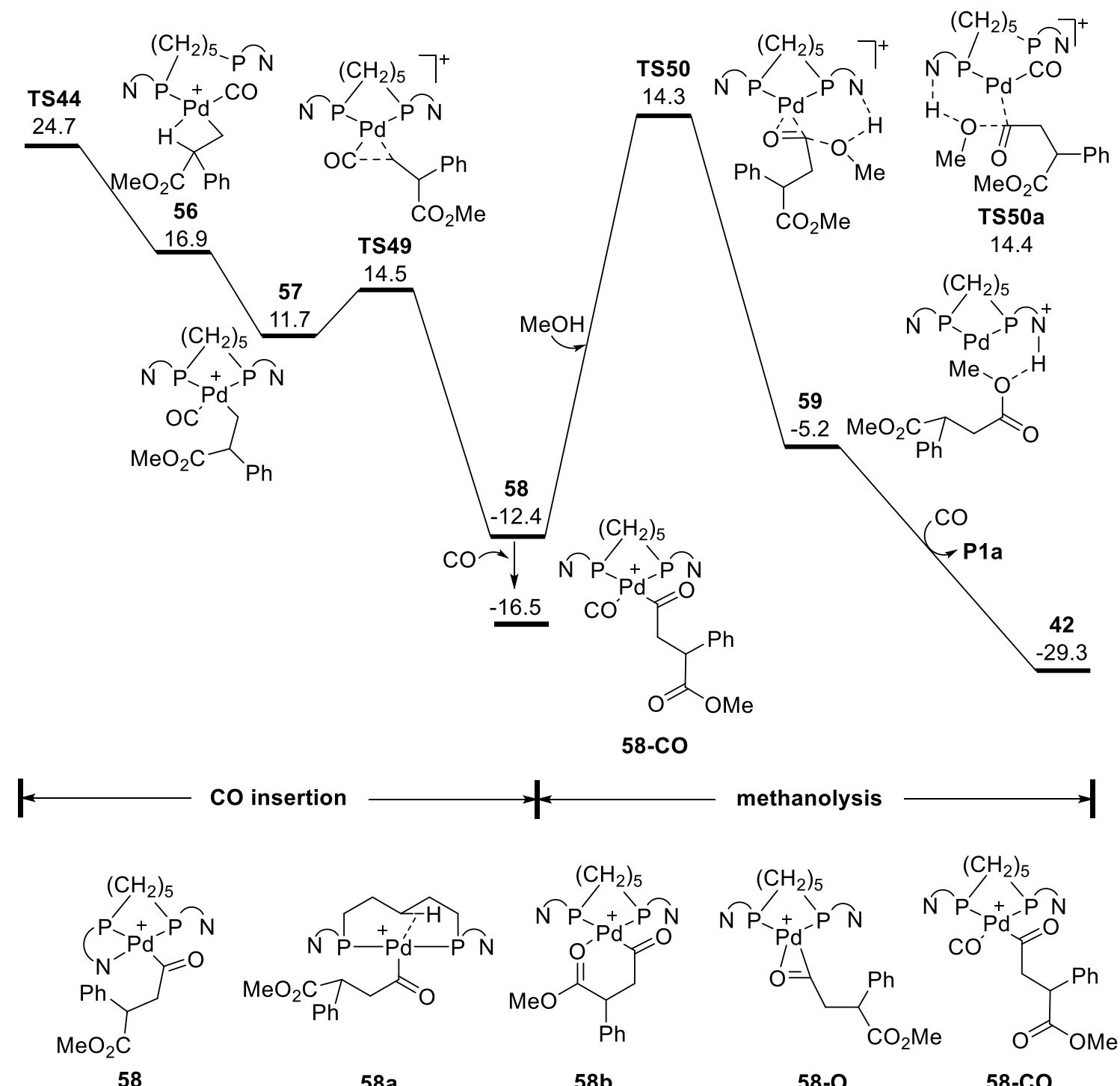

CO insertion
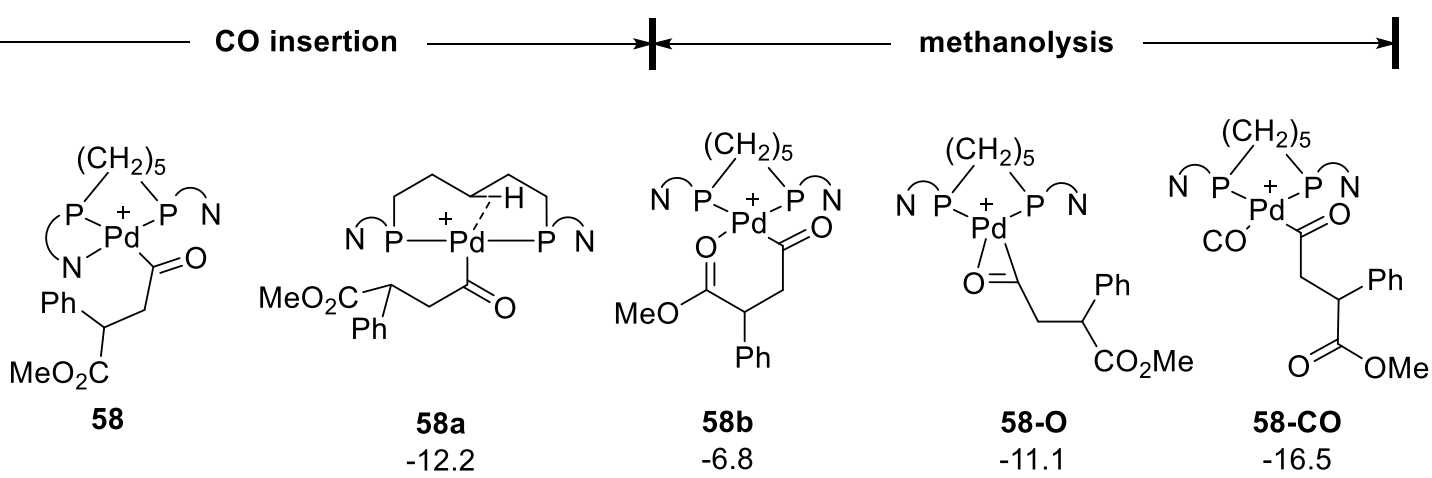

$-12.2$

$58 b$

58-0

$-11.1$

58-CO

$-16.5$

Figure S16. Calculated solution-phase Gibbs free energy changes of the $\mathrm{CO}$ insertion and methanolysis stages in the AOC of P2a to generate P1a with $\mathbf{L 2}(\mathrm{kcal} / \mathrm{mol})$. 

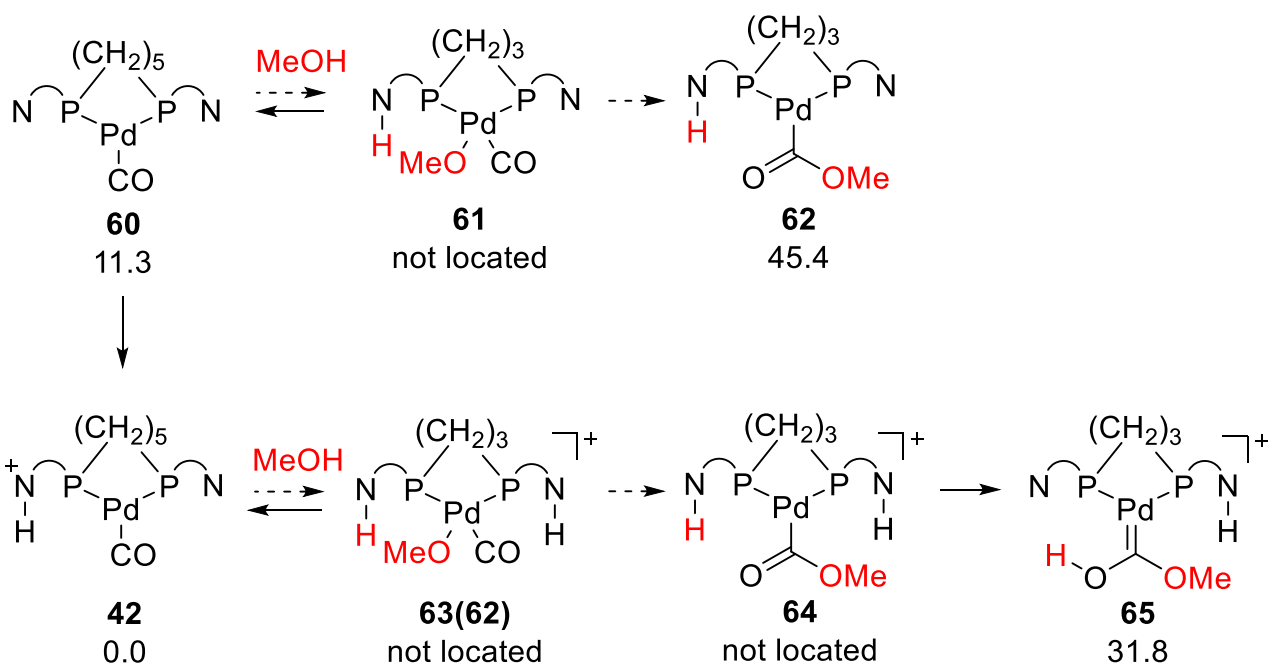

Scheme S7. Calculated relative energies of some key intermediates of the AOC of a1 in the presence of $\mathrm{L} 2$ via Cycle B (in kcal/mol). 


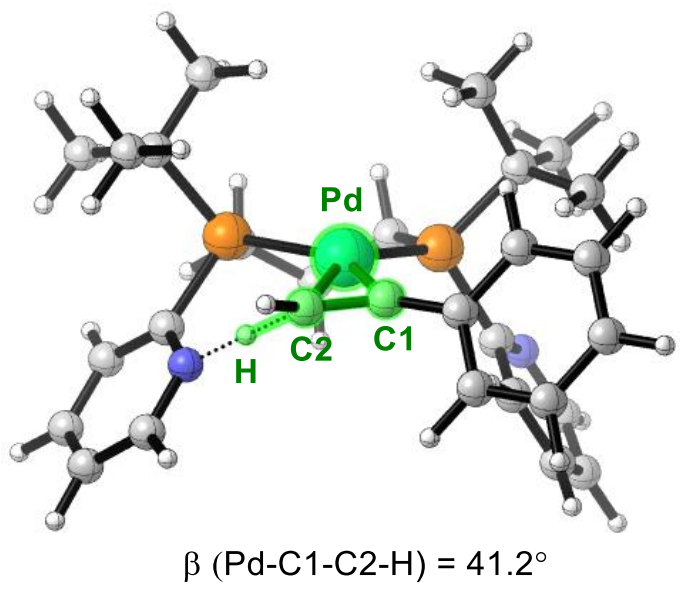

TS6

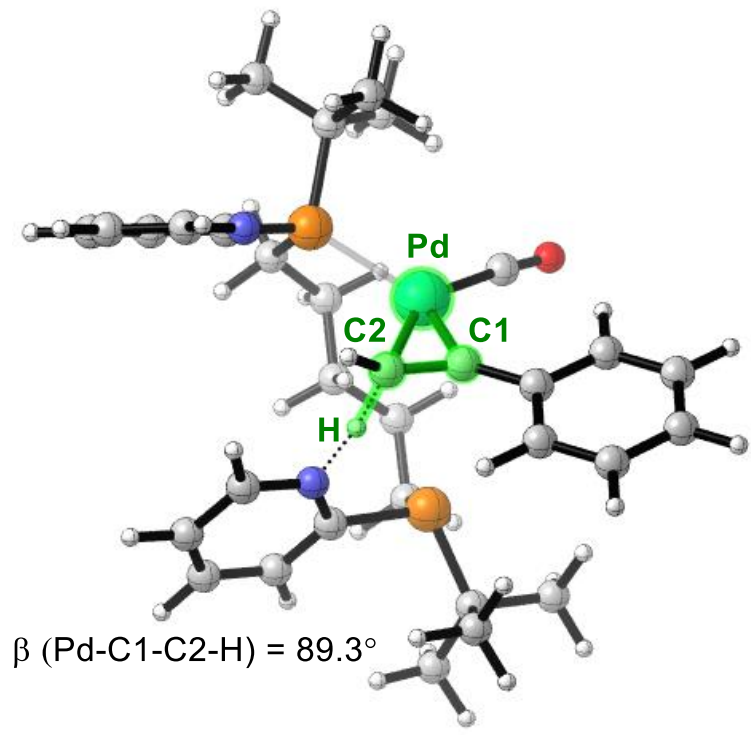

TS35

Figure S17. Dihedral angles in the ligand-assisted electrophilic addition transition states TS6 and TS35. 


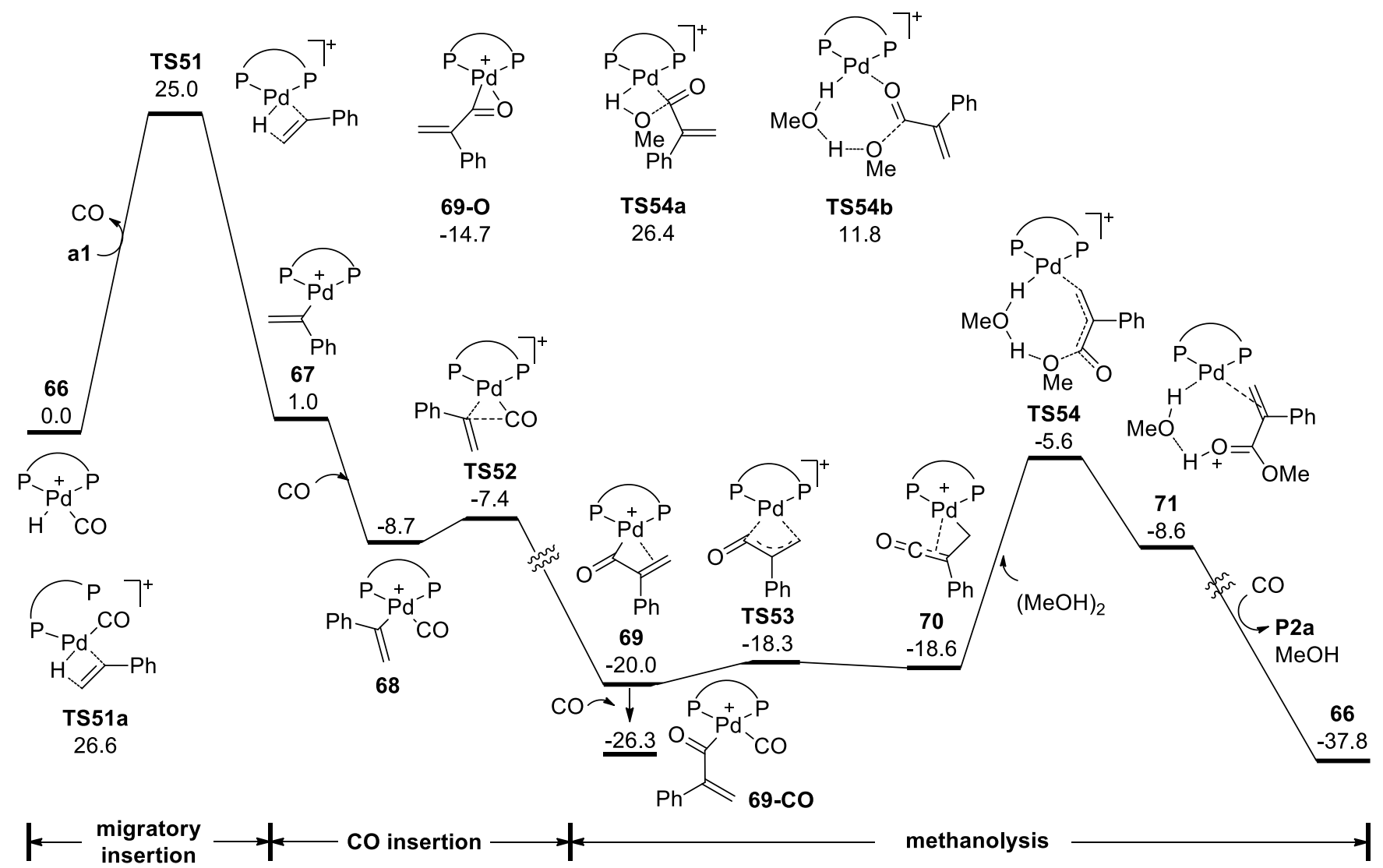

Figure S18. Calculated solution-phase Gibbs free energy changes of the AOC of a1 to generate P2a with L3 (kcal/mol). 


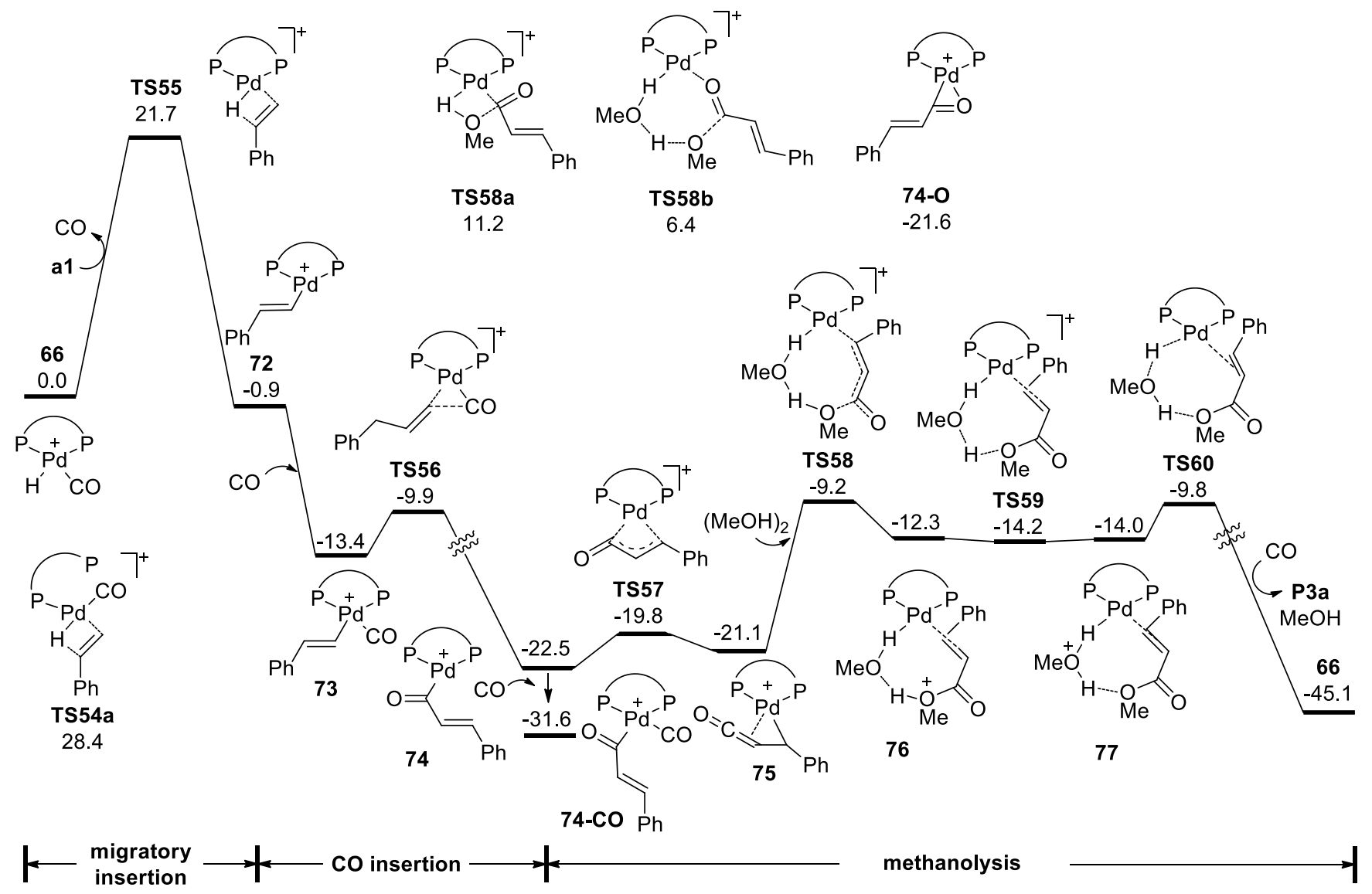

Figure S19. Calculated solution-phase Gibbs free energy changes of the AOC of a1 to generate P3a with $\mathbf{L 3}(\mathrm{kcal} / \mathrm{mol})$. 

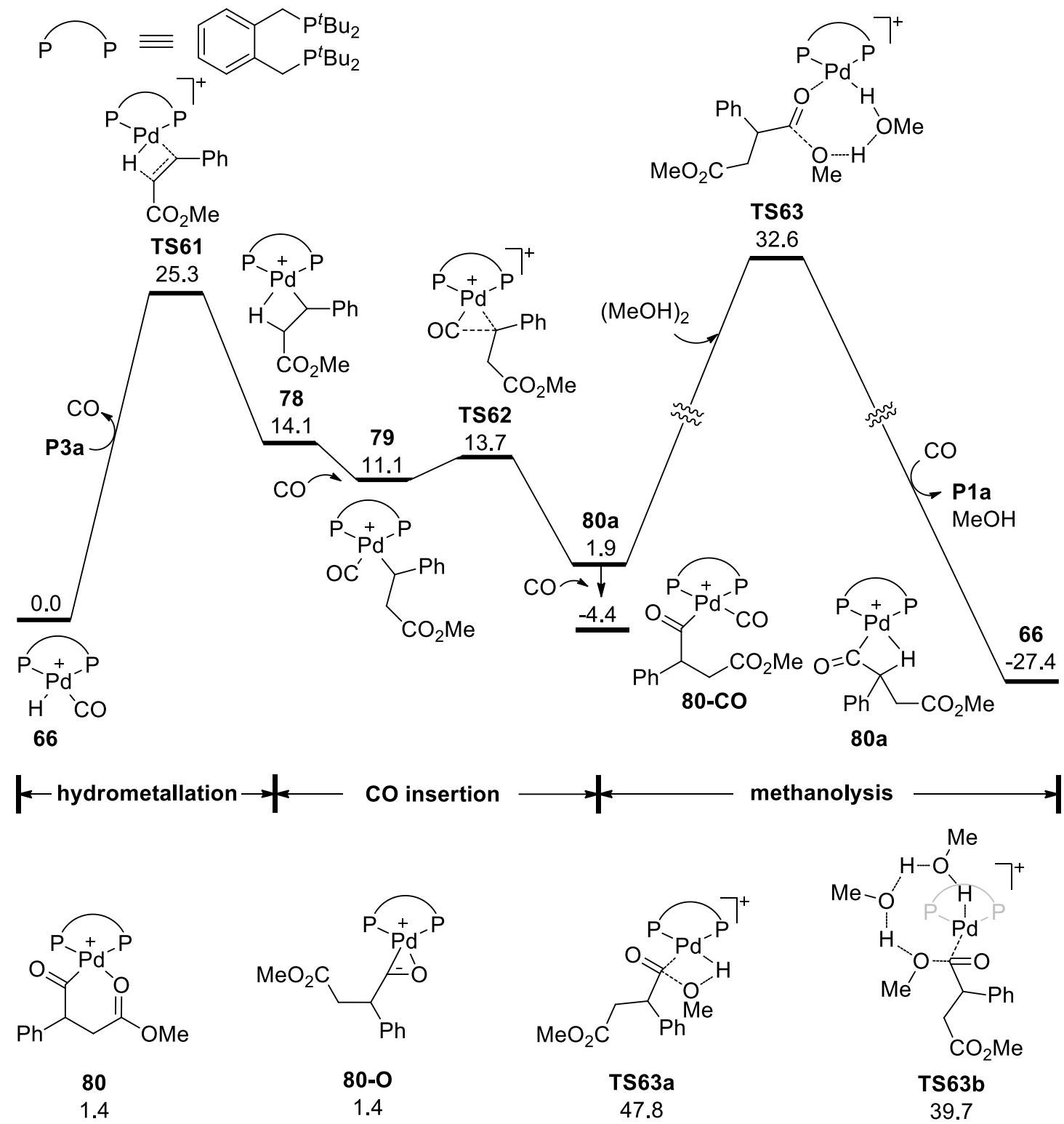

Figure S20. Calculated solution-phase Gibbs free energy changes of the the AOC of P3a to generate P1a with $\mathbf{L 3}(\mathrm{kcal} / \mathrm{mol})$. 


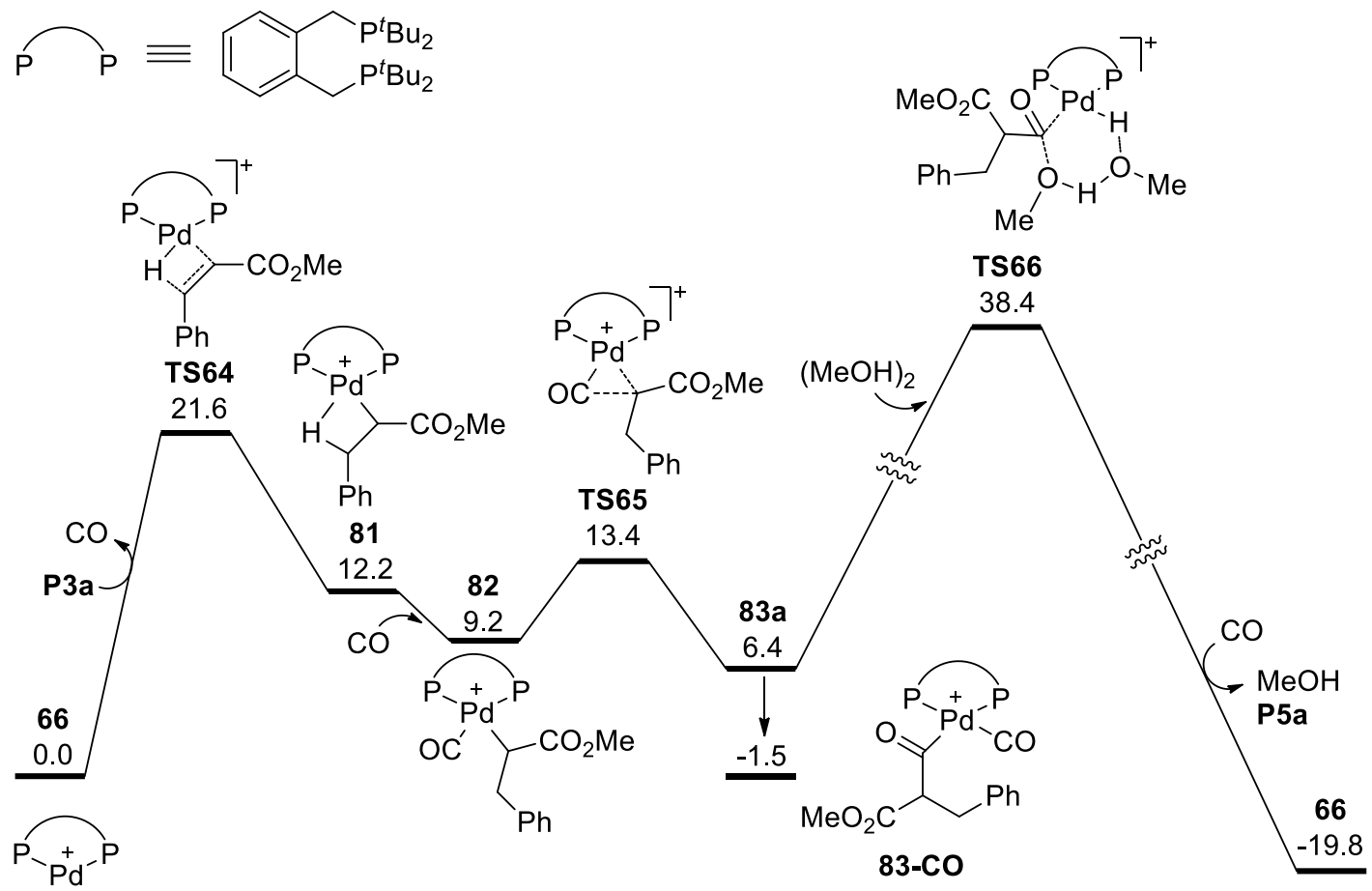

$\mathrm{H}^{\prime}$ 'CO

$\leftarrow$ hydrometallation $\rightarrow+$ co insertion $\longrightarrow$ methanolysis
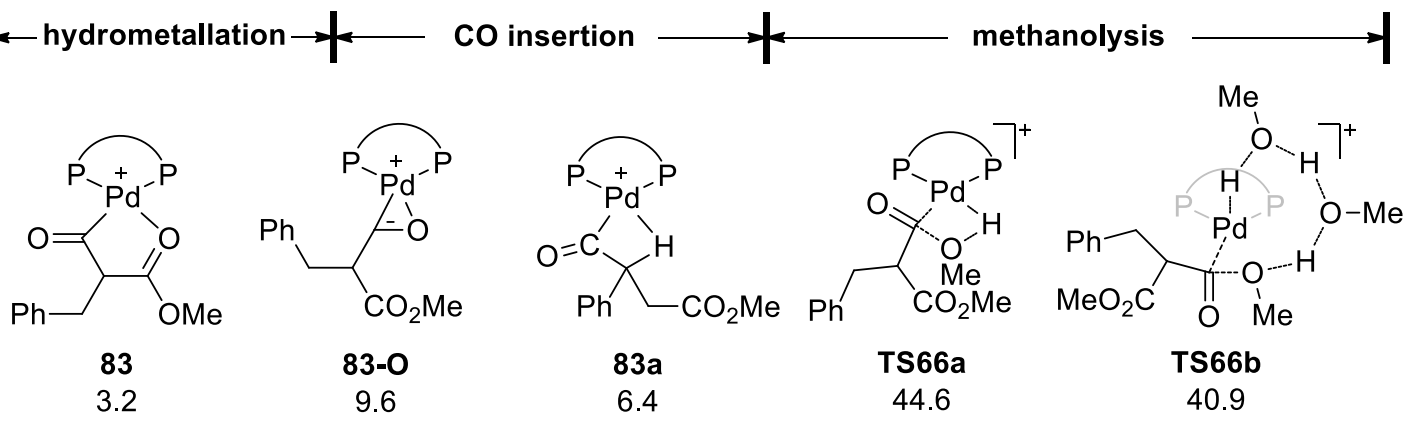

Figure S21. Calculated solution-phase Gibbs free energy changes of the the AOC of P3a to generate P5a with $\mathbf{L} \mathbf{3}(\mathrm{kcal} / \mathrm{mol})$. 


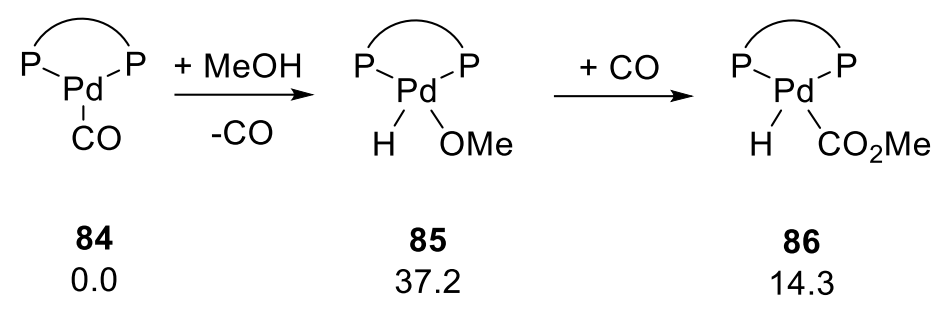

Scheme S8. Calculated relative energies of key intermediates of the AOC with L3 via Cycle B (in $\mathrm{kcal} / \mathbf{m o l}$ ) 


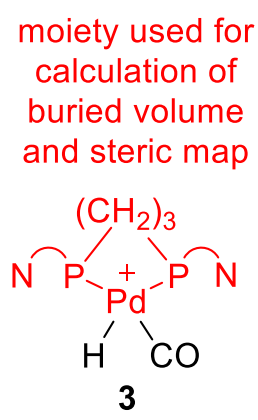

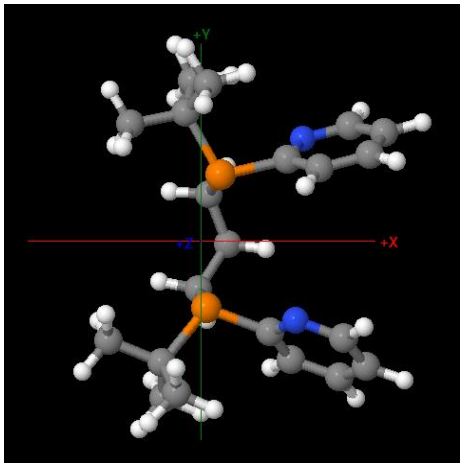

orientation used for generating steric map of L1

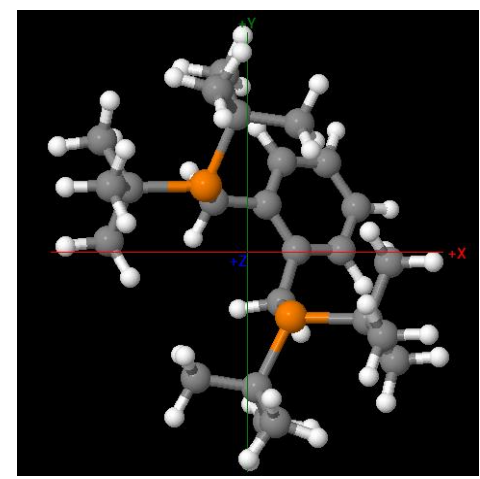

orientation used for generating steric map of L3

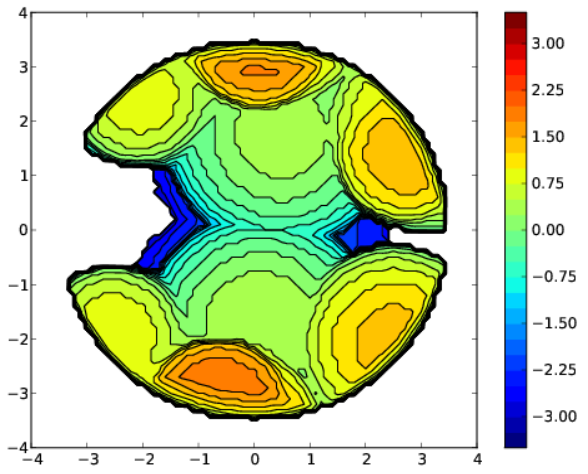

steric map of L1

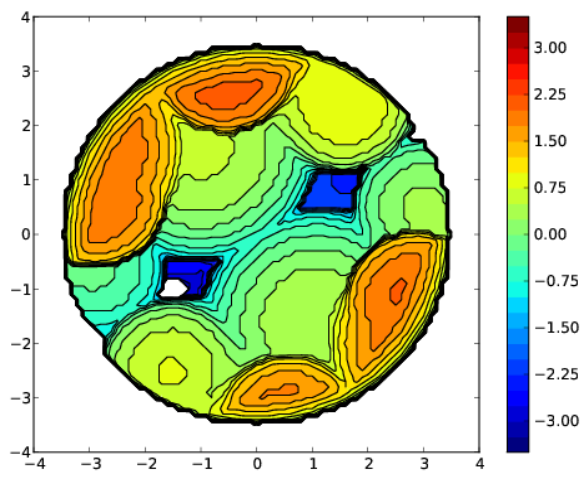

steric map of L3

moiety used for calculation of buried volume and steric map<smiles>O=S(=O)(O)[O+]1PCCCP1</smiles>

Figure S22. Steric map and buried volume of $\mathbf{L 1}$ in $\mathbf{3}$ and $\mathbf{L 3}$ in $\mathbf{6 6}$.

Buried volume and steric map were calculated by using the web tool (https://www.molnac.unisa.it/OMtools/sambvca2.1/index.html). Pd atom was used to help assigning the orientation and then deleted in the calculations. The parameters, i.e. Bondi radii (scaled by 1.17), sphere radius (3.5), distance of the coordination point from the center of the sphere (0.0), mesh spacing for numerical integration (0.1) and $\mathrm{H}$ atoms in the calculations (included) were used. 
Table S1. Calculated thermodynamic corrections for Gibbs free energies ( $\Delta G_{\text {cor }}$ in hartree), solution-phase single-point energies ( $\Delta E_{\text {sol }}$ in hartree) and Solution-phase Gibbs free energies $\left(\Delta G_{\text {sol }}\right.$ in hartree)

\begin{tabular}{|c|c|c|c|}
\hline \multicolumn{4}{|c|}{ Computational level: M06-L/BS2/SMD//B3LYP/BS1 } \\
\hline species & $\Delta \mathbf{G}_{\text {cor }}$ & $\Delta \mathbf{E}_{\text {sol }}$ & $\Delta \mathbf{G}_{\text {sol }}$ \\
\hline a1 & 0.079097 & -308.4328948 & -308.3507699 \\
\hline $\mathrm{TsOH}$ & 0.103604 & -895.4666804 & -895.3600486 \\
\hline $\mathrm{TsO}^{-}$ & 0.092963 & -895.031352 & -894.9353612 \\
\hline $\mathrm{CO}$ & -0.014102 & -113.3211046 & -113.3321788 \\
\hline $\mathrm{MeOH}$ & 0.02869 & -115.7476047 & -115.7158868 \\
\hline$(\mathrm{MeOH})_{2}$ & 0.07268 & -231.5000626 & -231.4243548 \\
\hline$(\mathrm{MeOH})_{3}$ & 0.12197 & -347.2552419 & -347.1302441 \\
\hline P1a & 0.197137 & -766.7507611 & -766.5505963 \\
\hline P2a & 0.139185 & -537.6011391 & -537.4589263 \\
\hline P3a & 0.138524 & -537.6112852 & -537.4697334 \\
\hline P4a & 0.19842 & -766.743264 & -766.5418162 \\
\hline P5a & 0.196542 & -766.7499542 & -766.5503843 \\
\hline 1 & 0.433252 & -1853.260969 & -1852.824689 \\
\hline 2 & 0.555851 & -2748.740863 & -2748.181984 \\
\hline TS1 & 0.558487 & -2748.733372 & -2748.171857 \\
\hline 3 & 0.44428 & -1853.713182 & -1853.265874 \\
\hline 4 & 0.558746 & -2748.750667 & -2748.188893 \\
\hline TS2 & 0.557059 & -2748.756742 & -2748.196655 \\
\hline 5 & 0.446793 & -1853.716918 & -1853.267097 \\
\hline $5 \mathbf{a}$ & 0.4569 & -1854.156027 & -1853.696099 \\
\hline $5 \mathbf{b}$ & 0.45778 & -1854.16285 & -1853.702042 \\
\hline $5 c$ & 0.470212 & -1854.592326 & -1854.119086 \\
\hline 6 & 0.538253 & -2048.798058 & -2048.256777 \\
\hline 7 & 0.539295 & -2048.797922 & -2048.255599 \\
\hline 8 & 0.545381 & -2048.819384 & -2048.270975 \\
\hline 9 & 0.547684 & -2048.819457 & -2048.268745 \\
\hline TS3 & 0.44278 & -1853.694067 & -1853.248259 \\
\hline TS4 & 0.539449 & -2048.795832 & -2048.253356 \\
\hline TS4a & 0.539302 & -2162.118676 & -2161.576346 \\
\hline TS4-2H & 0.552239 & -2049.239696 & -2048.684429 \\
\hline TS5 & 0.538649 & -2048.790672 & -2048.248995 \\
\hline TS5a & 0.540122 & -2162.12156 & -2161.57841 \\
\hline TS5-2H & 0.551851 & -2049.234987 & -2048.680108 \\
\hline TS6 & 0.542397 & -2048.79957 & -2048.254146 \\
\hline TS6a & 0.543007 & -2162.130244 & -2161.584209 \\
\hline
\end{tabular}




\begin{tabular}{|c|c|c|c|}
\hline TS6b & 0.540011 & -2162.128263 & -2161.585224 \\
\hline TS6-2H & 0.55554 & -2049.244256 & -2048.685688 \\
\hline TS7 & 0.54287 & -2048.796632 & -2048.250734 \\
\hline TS7a & 0.540683 & -2162.119334 & -2161.575623 \\
\hline TS7b & 0.544304 & -2162.112075 & -2161.564743 \\
\hline TS7-2H & 0.555432 & -2049.243379 & -2048.684919 \\
\hline 10 & 0.547077 & -2048.84157 & -2048.291465 \\
\hline 11 & 0.551306 & -2162.193222 & -2161.638889 \\
\hline TS8 & 0.550887 & -2162.186842 & -2161.632927 \\
\hline 12 & 0.551794 & -2162.210593 & -2161.655771 \\
\hline $12 \mathbf{a}$ & 0.555357 & -2162.208594 & -2161.650209 \\
\hline $12 \mathrm{~b}$ & 0.555909 & -2162.206876 & -2161.64794 \\
\hline $12 \mathrm{c}$ & 0.605884 & -2277.969267 & -2277.360356 \\
\hline 12d & 0.552903 & -2162.198866 & -2161.642935 \\
\hline $12-O$ & 0.553801 & -2162.196928 & -2161.640099 \\
\hline 12-CO & 0.55881 & -2275.558354 & -2274.996516 \\
\hline 13 & 0.605672 & -2277.970169 & -2277.361469 \\
\hline TS9 & 0.606437 & -2277.943229 & -2277.333764 \\
\hline TS9a & 0.604906 & -2277.939654 & -2277.33172 \\
\hline 14 & 0.603737 & -2277.954129 & -2277.347364 \\
\hline TS10 & 0.553322 & -2162.211304 & -2161.654954 \\
\hline 15 & 0.551999 & -2162.208724 & -2161.653697 \\
\hline TS11 & 0.607191 & -2277.94013 & -2277.329911 \\
\hline 16 & 0.608874 & -2277.979041 & -2277.367139 \\
\hline 17 & 0.546214 & -2048.840363 & -2048.291121 \\
\hline 18 & 0.550563 & -2162.196571 & -2161.64298 \\
\hline TS12 & 0.549777 & -2162.18857 & -2161.635765 \\
\hline 19 & 0.552123 & -2162.210364 & -2161.655213 \\
\hline 20 & 0.552102 & -2162.215453 & -2161.660323 \\
\hline $20 a$ & 0.553181 & -2162.209003 & -2161.652794 \\
\hline $20 b$ & 0.602276 & -2277.977519 & -2277.372215 \\
\hline $20 \mathrm{c}$ & 0.549293 & -2162.200711 & -2161.64839 \\
\hline $20-O$ & 0.553757 & -2162.210774 & -2161.653989 \\
\hline $20-\mathrm{CO}$ & 0.558929 & -2275.56755 & -2275.005593 \\
\hline 21 & 0.605537 & -2277.975695 & -2277.36713 \\
\hline TS13 & 0.60437 & -2277.946596 & -2277.339198 \\
\hline TS13a & 0.604364 & -2277.944678 & -2277.337286 \\
\hline 22 & 0.601317 & -2277.961605 & -2277.357261 \\
\hline TS14 & 0.553706 & -2162.214571 & -2161.657837 \\
\hline 23 & 0.55299 & -2162.213186 & -2161.657168 \\
\hline
\end{tabular}




\begin{tabular}{|c|c|c|c|}
\hline TS15 & 0.604940 & -2277.948589 & -2277.340621 \\
\hline 24 & 0.608394 & -2278.004639 & -2277.393217 \\
\hline TS16 & 0.601831 & -2277.966962 & -2277.362103 \\
\hline TS16a & 0.598621 & -2391.281479 & -2390.67983 \\
\hline TS16-2H & 0.613699 & -2278.409197 & -2277.79247 \\
\hline TS17 & 0.602625 & -2277.964591 & -2277.358938 \\
\hline TS17a & 0.597999 & -2391.287936 & -2390.686909 \\
\hline TS17-2H & 0.616432 & -2278.402218 & -2277.782758 \\
\hline TS18 & 0.603125 & -2277.961368 & -2277.355215 \\
\hline TS18a & 0.599133 & -2391.283484 & -2390.681323 \\
\hline TS18b & 0.607886 & -2391.272212 & -2390.661298 \\
\hline TS18-2H & 0.615789 & -2278.404754 & -2277.785937 \\
\hline TS19 & 0.605111 & -2277.971085 & -2277.362946 \\
\hline TS19a & 0.599608 & -2391.293563 & -2390.690927 \\
\hline TS19b & 0.605491 & -2391.277919 & -2390.6694 \\
\hline TS19-2H & 0.617697 & -2278.417254 & -2277.796529 \\
\hline 25 & 0.614191 & -2391.334027 & -2390.716808 \\
\hline TS20 & 0.612059 & -2391.312755 & -2390.697668 \\
\hline 26 & 0.614179 & -2391.356825 & -2390.739618 \\
\hline $26 a$ & 0.61251 & -2391.339402 & -2390.723864 \\
\hline $26 \mathrm{~b}$ & 0.614267 & -2391.342771 & -2390.725476 \\
\hline 26-O & 0.613255 & -2391.339168 & -2390.722885 \\
\hline 26-CO & 0.620907 & -2504.696781 & -2504.072846 \\
\hline 26-MeO & 0.61751 & -2391.347465 & -2390.726927 \\
\hline 27 & 0.666917 & -2507.108364 & -2506.438419 \\
\hline TS21 & 0.66639 & -2507.076595 & -2506.407177 \\
\hline TS21-2H & 0.681671 & -2507.529172 & -2506.844473 \\
\hline TS21-b1 & 0.554668 & -2048.090947 & -2047.533251 \\
\hline TS21-c1 & 0.629905 & -2279.169732 & -2278.536799 \\
\hline TS21-d1 & 0.591726 & -2276.013755 & -2275.419001 \\
\hline TS21-e1 & 0.661456 & -2393.706904 & -2393.04242 \\
\hline TS21-f1 & 0.631798 & -2616.2705 & -2615.635674 \\
\hline TS21-g1 & 0.687542 & -2244.672582 & -2243.982012 \\
\hline b1 & 0.029589 & -78.59424792 & -78.56163108 \\
\hline c1 & 0.101983 & -309.6842125 & -309.5792016 \\
\hline d1 & 0.065205 & -306.5197634 & -306.4515305 \\
\hline e1 & 0.132587 & -424.2294637 & -424.0938488 \\
\hline f1 & 0.102803 & -646.7869054 & -646.6810746 \\
\hline g1 & 0.160308 & -275.1952928 & -275.031957 \\
\hline 28 & 0.61271 & -2391.34191 & -2390.726173 \\
\hline
\end{tabular}




\begin{tabular}{|c|c|c|c|}
\hline TS22 & 0.613018 & -2391.32939 & -2390.713344 \\
\hline 29 & 0.617053 & -2391.356229 & -2390.736148 \\
\hline $29 a$ & 0.610877 & -2391.346187 & -2390.732282 \\
\hline 29-O & 0.611563 & -2391.349225 & -2390.734634 \\
\hline 29-CO & 0.618624 & -2504.70551 & -2504.083858 \\
\hline 29-MeO & 0.613024 & -2391.342028 & -2390.725976 \\
\hline 30 & 0.665772 & -2507.117645 & -2506.448845 \\
\hline TS23 & 0.665798 & -2507.090409 & -2506.421583 \\
\hline TS23-c1 & 0.625302 & -2279.166369 & -2278.538039 \\
\hline TS23-d1 & 0.593208 & -2276.015851 & -2275.419615 \\
\hline TS23-e1 & 0.657196 & -2393.705811 & -2393.045587 \\
\hline TS23-f1 & 0.628949 & -2616.281216 & -2615.649239 \\
\hline TS23-g1 & 0.688033 & -2244.68298 & -2243.991919 \\
\hline TS24 & 0.603889 & -2277.981429 & -2277.374512 \\
\hline TS24a & 0.602462 & -2391.303695 & -2390.698205 \\
\hline TS25 & 0.60271 & -2277.977588 & -2277.37185 \\
\hline TS25a & 0.599477 & -2391.298352 & -2390.695848 \\
\hline TS26 & 0.60298 & -2277.974396 & -2277.368388 \\
\hline TS26a & 0.604186 & -2391.299862 & -2390.692648 \\
\hline TS26b & 0.605208 & -2391.276735 & -2390.668499 \\
\hline TS27 & 0.604309 & -2277.974823 & -2277.367486 \\
\hline TS27a & 0.599224 & -2391.293823 & -2390.691571 \\
\hline TS27b & 0.604812 & -2391.279214 & -2390.671374 \\
\hline 31 & 0.612517 & -2391.340311 & -2390.724766 \\
\hline TS28 & 0.612083 & -2391.324502 & -2390.709391 \\
\hline 32 & 0.613441 & -2391.353681 & -2390.737212 \\
\hline $32 a$ & 0.616076 & -2391.353785 & -2390.734681 \\
\hline 32b & 0.665732 & -2507.113673 & -2506.444913 \\
\hline 32-CO & 0.617574 & -2504.704743 & -2504.084141 \\
\hline TS29 & 0.667578 & -2507.085221 & -2506.414615 \\
\hline 33 & 0.611609 & -2391.347447 & -2390.73281 \\
\hline TS30 & 0.610544 & -2391.332507 & -2390.718935 \\
\hline 34 & 0.616412 & -2391.35851 & -2390.73907 \\
\hline $34 a$ & 0.666174 & -2507.120487 & -2506.451285 \\
\hline 34b & 0.617291 & -2391.354069 & -2390.73375 \\
\hline 34-CO & 0.61918 & -2504.702582 & -2504.080375 \\
\hline TS31 & 0.665539 & -2507.09055 & -2506.421983 \\
\hline 37 & 0.486462 & -1968.966253 & -1968.476763 \\
\hline 40 & 0.500004 & -1969.429633 & -1968.926601 \\
\hline 41 & 0.50069 & -1932.33969 & -1931.835973 \\
\hline
\end{tabular}




\begin{tabular}{|c|c|c|c|}
\hline TS32 & 0.498398 & -1932.326364 & -1931.824939 \\
\hline 42 & 0.504263 & -1932.350766 & -1931.843475 \\
\hline TS33 & 0.594999 & -2240.756753 & -2240.158727 \\
\hline TS33a & 0.595733 & -2127.410514 & -2126.811753 \\
\hline TS33-2H & 0.602535 & -2241.203088 & -2240.597525 \\
\hline TS34 & 0.592914 & -2240.753917 & -2240.157975 \\
\hline TS34a & 0.595508 & -2127.412293 & -2126.813758 \\
\hline TS34-2H & 0.604072 & -2241.199522 & -2240.592422 \\
\hline TS35 & 0.595848 & -2240.773167 & -2240.174291 \\
\hline TS35a & 0.593676 & -2240.761661 & -2240.164957 \\
\hline TS35b & 0.597255 & -2127.425286 & -2126.825003 \\
\hline TS35-2H & 0.610836 & -2241.220641 & -2240.606777 \\
\hline 43 & 0.60014 & -2240.780782 & -2240.177615 \\
\hline TS36 & 0.598714 & -2240.759478 & -2240.157737 \\
\hline TS36a & 0.594334 & -2240.752752 & -2240.15539 \\
\hline TS36b & 0.597918 & -2127.421734 & -2126.820788 \\
\hline TS36-2H & 0.612137 & -2241.206023 & -2240.590858 \\
\hline 44 & 0.607933 & -2240.816167 & -2240.205206 \\
\hline TS37 & 0.609086 & -2240.812364 & -2240.20025 \\
\hline TS37a & 0.60456 & -2354.131553 & -2353.523965 \\
\hline $45 \mathrm{~b}$ & 0.609312 & -2240.8386 & -2240.226261 \\
\hline 45 & 0.608194 & -2240.841525 & -2240.230303 \\
\hline $45 a$ & 0.608419 & -2240.839891 & -2240.228444 \\
\hline $45 c$ & 0.66137 & -2356.594911 & -2355.930513 \\
\hline $45-O$ & 0.606945 & -2240.82457 & -2240.214597 \\
\hline 45-CO & 0.614799 & -2354.183892 & -2353.566065 \\
\hline TS38 & 0.61289 & -2240.833931 & -2240.218013 \\
\hline 46 & 0.608858 & -2240.835834 & -2240.223948 \\
\hline TS39 & 0.663273 & -2356.585091 & -2355.91879 \\
\hline TS39a & 0.655317 & -2469.896893 & -2469.238548 \\
\hline TS39b & 0.658774 & -2356.56411 & -2355.902308 \\
\hline 47 & 0.665496 & -2356.620416 & -2355.951892 \\
\hline 48 & 0.605967 & -2240.81911 & -2240.210116 \\
\hline TS40 & 0.607343 & -2240.815852 & -2240.205481 \\
\hline TS40a & 0.606715 & -2354.141584 & -2353.531841 \\
\hline 49b & 0.61033 & -2240.851408 & -2240.23805 \\
\hline $49 a$ & 0.607275 & -2240.849023 & -2240.23872 \\
\hline 49 & 0.606112 & -2240.851447 & -2240.242307 \\
\hline $49 c$ & 0.660021 & -2356.60384 & -2355.940791 \\
\hline 49-O & 0.611195 & -2240.836157 & -2240.221934 \\
\hline
\end{tabular}




\begin{tabular}{|c|c|c|c|}
\hline 49-CO & 0.607921 & -2354.179101 & -2353.568152 \\
\hline TS41 & 0.610191 & -2240.839191 & -2240.225972 \\
\hline 50 & 0.610989 & -2240.8404 & -2240.226383 \\
\hline TS42 & 0.662638 & -2356.595705 & -2355.930039 \\
\hline TS42a & 0.658471 & -2356.56974 & -2355.908241 \\
\hline TS42b & 0.65206 & -2469.906336 & -2469.251248 \\
\hline 51 & 0.665874 & -2356.630028 & -2355.961126 \\
\hline TS43 & 0.66013 & -2469.926729 & -2469.263571 \\
\hline TS43a & 0.660066 & -2356.583875 & -2355.920782 \\
\hline TS44 & 0.655868 & -2469.921979 & -2469.263083 \\
\hline TS44a & 0.656637 & -2356.584583 & -2355.924918 \\
\hline TS45 & 0.653155 & -2469.925666 & -2469.269483 \\
\hline TS45a & 0.658466 & -2469.928985 & -2469.267491 \\
\hline TS45b & 0.659647 & -2356.590264 & -2355.927589 \\
\hline TS46 & 0.659088 & -2356.589207 & -2355.927091 \\
\hline TS46a & 0.662598 & -2469.924292 & -2469.258666 \\
\hline TS46b & 0.654156 & -2469.915869 & -2469.258685 \\
\hline 52 & 0.655407 & -2469.941473 & -2469.283039 \\
\hline 53 & 0.66189 & -2469.958779 & -2469.293861 \\
\hline TS47 & 0.665375 & -2469.943894 & -2469.275491 \\
\hline $54 a$ & 0.672444 & -2469.988787 & -2469.313316 \\
\hline 54 & 0.66961 & -2469.988074 & -2469.315436 \\
\hline $54 \mathrm{~b}$ & 0.669559 & -2469.978837 & -2469.30625 \\
\hline 54-O & 0.670773 & -2469.965117 & -2469.291316 \\
\hline 54-CO & 0.674741 & -2583.332606 & -2582.654837 \\
\hline TS48 & 0.723641 & -2585.706799 & -2584.98013 \\
\hline TS48a & 0.719665 & -2699.031398 & -2698.308705 \\
\hline 55 & 0.718284 & -2585.745581 & -2585.024269 \\
\hline 56 & 0.65678 & -2469.935332 & -2469.275524 \\
\hline 57 & 0.668656 & -2469.955403 & -2469.283719 \\
\hline TS49 & 0.666732 & -2469.949045 & -2469.279285 \\
\hline 58 & 0.668908 & -2469.994151 & -2469.322215 \\
\hline $58 \mathrm{a}$ & 0.664977 & -2469.989786 & -2469.321781 \\
\hline $58 \mathrm{~b}$ & 0.67111 & -2469.987319 & -2469.313181 \\
\hline $58-0$ & 0.671009 & -2469.994145 & -2469.320108 \\
\hline 58-CO & 0.672551 & -2583.336554 & -2582.660975 \\
\hline TS50 & 0.716496 & -2585.715037 & -2584.995514 \\
\hline TS50a & 0.715431 & -2699.046167 & -2698.327708 \\
\hline 59 & 0.716482 & -2585.746143 & -2585.026633 \\
\hline 60 & 0.485317 & -1931.889198 & -1931.400853 \\
\hline
\end{tabular}




\begin{tabular}{|c|c|c|c|}
\hline 62 & 0.538275 & -2047.603732 & -2047.062429 \\
\hline 65 & 0.556038 & -2048.067819 & -2047.508753 \\
\hline 66 & 0.584858 & -1865.77385 & -1865.185965 \\
\hline TS51 & 0.680255 & -2060.848061 & -2060.164778 \\
\hline TS51a & 0.674919 & -2174.172218 & -2173.494272 \\
\hline 67 & 0.686315 & -2060.892369 & -2060.203026 \\
\hline 68 & 0.694348 & -2174.247929 & -2173.550553 \\
\hline TS52 & 0.692027 & -2174.243548 & -2173.548493 \\
\hline 69 & 0.69212 & -2174.263793 & -2173.568645 \\
\hline 69-0 & 0.692738 & -2174.255886 & -2173.56012 \\
\hline 69-CO & 0.698508 & -2287.612291 & -2286.910756 \\
\hline TS53 & 0.695422 & -2174.264362 & -2173.565912 \\
\hline 70 & 0.694742 & -2174.264218 & -2173.566449 \\
\hline TS54 & 0.793473 & -2405.766517 & -2404.970016 \\
\hline TS54a & 0.73822 & -2289.951725 & -2289.210477 \\
\hline TS54b & 0.789267 & -2405.734582 & -2404.942287 \\
\hline 71 & 0.792223 & -2405.77011 & -2404.974859 \\
\hline TS55 & 0.6792 & -2060.85218 & -2060.169952 \\
\hline TS55a & 0.676889 & -2174.171438 & -2173.491521 \\
\hline 72 & 0.683742 & -2060.892774 & -2060.206005 \\
\hline 73 & 0.690429 & -2174.25151 & -2173.558054 \\
\hline TS56 & 0.692164 & -2174.2477 & -2173.552508 \\
\hline 74 & 0.693022 & -2174.268595 & -2173.572545 \\
\hline 74-O & 0.694579 & -2174.268688 & -2173.571081 \\
\hline 74-CO & 0.697599 & -2287.619903 & -2286.919276 \\
\hline TS57 & 0.693846 & -2174.265084 & -2173.56821 \\
\hline 75 & 0.692612 & -2174.265989 & -2173.570349 \\
\hline TS58 & 0.794188 & -2405.772956 & -2404.97574 \\
\hline TS58a & 0.738699 & -2289.976519 & -2289.234792 \\
\hline TS58b & 0.791686 & -2405.745586 & -2404.950873 \\
\hline 76 & 0.795752 & -2405.779413 & -2404.980633 \\
\hline TS59 & 0.794053 & -2405.780761 & -2404.98368 \\
\hline 77 & 0.79546 & -2405.781829 & -2404.983341 \\
\hline TS60 & 0.792833 & -2405.77256 & -2404.976699 \\
\hline TS61 & 0.744958 & -2290.032234 & -2289.284248 \\
\hline 78 & 0.745448 & -2290.050501 & -2289.302026 \\
\hline 79 & 0.753591 & -2403.39558 & -2402.638961 \\
\hline TS62 & 0.752462 & -2403.390344 & -2402.634854 \\
\hline 80a & 0.751062 & -2403.40778 & -2402.65369 \\
\hline 80 & 0.755945 & -2403.41347 & -2402.654498 \\
\hline
\end{tabular}




\begin{tabular}{|c|c|c|c|}
\hline $80-0$ & 0.754547 & -2403.412621 & -2402.655046 \\
\hline $80-\mathrm{CO}$ & 0.758228 & -2516.75713 & -2515.995874 \\
\hline TS63 & 0.848251 & -2634.880409 & -2634.029131 \\
\hline TS63a & 0.799685 & -2519.099234 & -2518.296521 \\
\hline TS63b & 0.902689 & -2750.629382 & -2749.723666 \\
\hline TS64 & 0.742811 & -2290.035916 & -2289.290077 \\
\hline 81 & 0.746978 & -2290.05507 & -2289.305064 \\
\hline 82 & 0.752486 & -2403.397652 & -2402.642138 \\
\hline TS65 & 0.751596 & -2403.389957 & -2402.635333 \\
\hline 83a & 0.752774 & -2403.402315 & -2402.646513 \\
\hline 83 & 0.752105 & -2403.40672 & -2402.651588 \\
\hline 83-O & 0.752745 & -2403.403899 & -2402.648126 \\
\hline 83-CO & 0.759284 & -2516.753594 & -2515.991282 \\
\hline TS66 & 0.850084 & -2634.873046 & -2634.019934 \\
\hline TS66a & 0.798465 & -2519.102956 & -2518.301464 \\
\hline TS66b & 0.900476 & -2750.625252 & -2749.721748 \\
\hline 84 & 0.572689 & -1865.319663 & -1864.743947 \\
\hline 85 & 0.61739 & -1867.688716 & -1867.068299 \\
\hline 86 & 0.623627 & -1981.063779 & -1980.437124 \\
\hline \multicolumn{4}{|c|}{ Computational Level: M06-L-D3/BS2/SMD//B3LYP/BS1 } \\
\hline TS4 & 0.539449 & -2048.805338 & -2048.262861 \\
\hline TS5 & 0.538649 & -2048.800449 & -2048.258772 \\
\hline TS6 & 0.542397 & -2048.808055 & -2048.26263 \\
\hline TS7 & 0.54287 & -2048.80555 & -2048.259652 \\
\hline TS16 & 0.601831 & -2277.97836 & -2277.373501 \\
\hline TS17 & 0.602625 & -2277.97618 & -2277.370527 \\
\hline TS18 & 0.603125 & -2277.972903 & -2277.36675 \\
\hline TS19 & 0.605111 & -2277.982705 & -2277.374566 \\
\hline \multicolumn{4}{|c|}{ Computational Level: M06-L/BS2/SMD//M06-L/BS1 } \\
\hline TS4 & 0.54451 & -2048.801256 & -2048.253718 \\
\hline TS6 & 0.543552 & -2048.8051 & -2048.25852 \\
\hline TS16 & 0.606706 & -2277.973649 & -2277.363915 \\
\hline TS19 & 0.610257 & -2277.976045 & -2277.36276 \\
\hline TS34 & 0.600949 & -2240.761361 & -2240.157384 \\
\hline TS35 & 0.602211 & -2240.781185 & -2240.175946 \\
\hline TS51 & 0.684899 & -2060.85528 & -2060.167353 \\
\hline TS55 & 0.682924 & -2060.860051 & -2060.174099 \\
\hline
\end{tabular}


H

Cartesian coordinates of optimized structures (in angstrom)

B3LYP Geometry optimization

a1

C

C

C

C

C

C

H

$\mathrm{H}$

H

$\mathrm{H}$

H

C

C

H

$\mathrm{TsOH}$

H

S

$\mathrm{O}$

O

O

C

C

C

C

H

C

H

C

H

H

C

$\mathrm{H}$

H $\begin{array}{lll}-1.51263700 & -1.20867800 \quad-0.00001300\end{array}$

$\begin{array}{lll}-0.11993400 & -1.21339400 & 0.00001100\end{array}$

$\begin{array}{lll}0.59431500 & -0.00002100 & 0.00003200\end{array}$

$-0.11991400$

$-1.51260300$

$-2.05306300$

0.42840700

0.42847000

$-2.05302800$

$-3.29920200$

2.02419100

3.23447000

4.29965100

1.21338400

1.20869900

0.00001200

$-2.15085400$

$-2.14988400$

2.14984900

2.15087700

0.00003500

$-0.00001300$

0.00000300

0.00002600

0.00001300

$-0.00001500$

$-0.00002500$

$-0.00002300$

0.00002200

0.00002400

$-0.00002500$

$-0.00004500$

0.00001800

$-0.00050800$

$$
\begin{array}{rrr}
-2.90266200 & 0.68182900 & -1.61544000 \\
-1.90072600 & -0.02060700 & 0.13109200 \\
-2.34501000 & -1.28778300 & 0.69546700 \\
-2.39084900 & 1.26030300 & 0.64447400 \\
-2.26150000 & -0.03613100 & -1.47825500 \\
-0.12112300 & -0.00896500 & 0.07381600 \\
0.55512100 & 1.21168400 & 0.08320500 \\
0.56898900 & -1.22118600 & 0.02192100 \\
1.94710400 & 1.20987700 & 0.03256600 \\
-0.00225600 & 2.14009900 & 0.14256600 \\
1.96038000 & -1.20072700 & -0.03189900 \\
\hline 0.02190900 & -2.15737300 & 0.03265900 \\
\hline 2.67003800 & 0.00941000 & -0.02632400 \\
\hline 2.48148500 & 2.15599100 & 0.04217000 \\
2.50503800 & -2.14001700 & -0.07518300 \\
4.17922300 & 0.01723700 & -0.04930700 \\
4.56862900 & 0.94143800 & -0.48529300 \\
4.58481700 & -0.06173500 & 0.96693600 \\
\hline & & \\
\hline
\end{array}
$$

$-2.21309400$

0.00007200

4.57513300

$-0.82560800$

$-0.62325600$

$\mathrm{TsO}^{-}$

$\mathrm{O}$

$\mathrm{O}$

$\mathrm{O}$

C

C

C

C

$\mathrm{H}$

C

$\mathrm{H}$

C

$\mathrm{H}$

H

$\mathrm{H}$

C

H

H

H

$\mathrm{CO}$

O

C

\section{$\mathrm{MeOH}$}

C

H

H

H

H

O

H

$(\mathrm{MeOH})_{2}$

O

H

C

H

H

H

\begin{tabular}{rrr}
1.99171800 & -0.00006700 & 0.00464900 \\
2.36152400 & -1.25136300 & -0.71110100 \\
2.36243300 & 1.25666200 & -0.70092200 \\
2.31747700 & -0.00620200 & 1.45606400 \\
0.16911400 & 0.00047300 & -0.03427400 \\
-0.53821200 & 1.20444300 & -0.02533300 \\
-0.53855400 & -1.20385200 & -0.02531700 \\
-1.93347900 & 1.20188600 & 0.00170600 \\
0.02286800 & 2.13321600 & -0.05495800 \\
-1.93335000 & -1.20140500 & 0.00169700 \\
0.02250700 & -2.13264000 & -0.05510400 \\
-2.65496700 & 0.00047700 & 0.01615900 \\
\hline-2.47442400 & 2.14761900 & 0.00837600 \\
-2.47418500 & -2.14724800 & 0.00837200 \\
-4.16748500 & -0.00036000 & 0.00828200 \\
-4.57149200 & 0.90783300 & 0.46901200 \\
-4.57047900 & -0.04964800 & -1.01300700 \\
-4.57215400 & -0.86081900 & 0.55308100 \\
\hline
\end{tabular}

$\begin{array}{lll}0.00000000 & 0.00000000 & 0.48759700\end{array}$

$\begin{array}{lll}0.00000000 & 0.00000000 & -0.65013000\end{array}$

$\begin{array}{rrr}0.66117200 & -0.01951000 & 0.00000200 \\ 1.08240200 & 0.98935600 & -0.00066500 \\ 1.03840500 & -0.54360700 & -0.89156800 \\ 1.03850900 & -0.54248300 & 0.89219200 \\ -0.74888300 & 0.12198600 & 0.00000300 \\ -1.13528500 & -0.76209900 & 0.00001000\end{array}$

$\begin{array}{rrr}1.19569600 & 0.25027600 & 0.59697400 \\ 0.40831900 & 0.50894600 & 0.08649300 \\ 2.17229700 & -0.22583200 & -0.30458800 \\ 2.51038000 & 0.54333400 & -1.01760000 \\ 3.04158300 & -0.53823200 & 0.28275500 \\ 1.83342100 & -1.09776000 & -0.88765800\end{array}$




\begin{tabular}{|c|c|c|c|c|c|c|c|}
\hline $\mathrm{O}$ & -1.43489300 & 0.66218100 & -0.35103400 & $\mathrm{H}$ & -0.46135400 & 1.22647100 & -1.28660300 \\
\hline $\mathrm{H}$ & -1.67482700 & 1.30551400 & 0.32886300 & $\mathrm{C}$ & 0.39013200 & 0.56945100 & 0.62900800 \\
\hline $\mathrm{C}$ & -1.87882000 & -0.62502300 & 0.08808900 & $\mathrm{O}$ & -2.41865400 & 0.67581600 & 1.24467000 \\
\hline $\mathrm{H}$ & -2.95147400 & -0.62762800 & 0.31858300 & $\mathrm{C}$ & -2.04542900 & 1.05503800 & 0.15458500 \\
\hline $\mathrm{H}$ & -1.70298000 & -1.31561400 & -0.73958100 & $\mathrm{O}$ & -2.87829700 & 1.27748700 & -0.88333200 \\
\hline \multirow[t]{2}{*}{$\mathrm{H}$} & -1.31170700 & -0.97309200 & 0.95962400 & $\mathrm{C}$ & -4.27179800 & 1.04641000 & -0.61334100 \\
\hline & & & & $\mathrm{H}$ & -4.79268400 & 1.27026300 & -1.54409000 \\
\hline$(\mathrm{MeOH})_{3}$ & & & & $\mathrm{H}$ & -4.43775500 & 0.00701600 & -0.32006900 \\
\hline $\mathrm{O}$ & 0.55619000 & 1.40857100 & -0.48799900 & $\mathrm{H}$ & -4.62222400 & 1.69939700 & 0.18994400 \\
\hline $\mathrm{H}$ & 0.85639300 & 0.49358100 & -0.68055800 & $\mathrm{C}$ & 1.80228800 & 1.14450300 & 0.58299900 \\
\hline $\mathrm{C}$ & 1.55748600 & 2.07019300 & 0.27056000 & $\mathrm{O}$ & 2.55873400 & 1.18596200 & 1.52680500 \\
\hline $\mathrm{H}$ & 2.49347700 & 2.17555000 & -0.29503300 & $\mathrm{O}$ & 2.13337100 & 1.59446000 & -0.65284200 \\
\hline $\mathrm{H}$ & 1.18809700 & 3.07228800 & 0.50452100 & $\mathrm{C}$ & 3.46940100 & 2.11333100 & -0.77722600 \\
\hline $\mathrm{H}$ & 1.78028100 & 1.55697400 & 1.21752700 & $\mathrm{H}$ & 3.56451600 & 2.43703000 & -1.81360300 \\
\hline $\mathrm{O}$ & 0.64786300 & -1.33843100 & -0.59508800 & $\mathrm{H}$ & 3.61907100 & 2.95412400 & -0.09581100 \\
\hline $\mathrm{H}$ & -0.22512400 & -1.17183400 & -0.17580900 & $\mathrm{H}$ & 4.20429500 & 1.33831000 & -0.54648400 \\
\hline $\mathrm{C}$ & 1.42721400 & -2.13761500 & 0.28204300 & $\mathrm{H}$ & 0.08627500 & 0.64384200 & 1.67526600 \\
\hline $\mathrm{H}$ & 0.99048000 & -3.13641400 & 0.41931600 & & & & \\
\hline $\mathrm{H}$ & 2.41586000 & -2.25923900 & -0.16927200 & P2a & & & \\
\hline $\mathrm{H}$ & 1.55353800 & -1.67861800 & 1.27346900 & $\mathrm{C}$ & -2.30338600 & -1.36185500 & -0.71292200 \\
\hline $\mathrm{C}$ & -2.74252100 & 0.07365300 & -0.10890000 & $\mathrm{C}$ & -1.02683600 & -0.80697600 & -0.77838700 \\
\hline $\mathrm{H}$ & -3.34570900 & -0.81076300 & 0.11343600 & $\mathrm{C}$ & -0.72620400 & 0.38263700 & -0.09376200 \\
\hline $\mathrm{H}$ & -2.63259200 & 0.15296100 & -1.19949300 & $\mathrm{C}$ & -1.73958800 & 0.98910300 & 0.66643300 \\
\hline $\mathrm{H}$ & -3.28692300 & 0.95767800 & 0.25025700 & $\mathrm{C}$ & -3.01611200 & 0.43133500 & 0.73476900 \\
\hline $\mathrm{O}$ & -1.49429600 & -0.07887300 & 0.55375600 & $\mathrm{C}$ & -3.30332700 & -0.74609100 & 0.04331900 \\
\hline \multirow[t]{2}{*}{$\mathrm{H}$} & -0.91890500 & 0.68032000 & 0.31406600 & $\mathrm{H}$ & -2.51840400 & -2.27796000 & -1.25583100 \\
\hline & & & & $\mathrm{H}$ & -0.25772600 & -1.29427900 & -1.36742300 \\
\hline P1a & & & & $\mathrm{H}$ & -1.51443400 & 1.89521200 & 1.22129800 \\
\hline $\mathrm{C}$ & 0.40148300 & -0.91110600 & 0.24666500 & $\mathrm{H}$ & -3.78288600 & 0.91349000 & 1.33455600 \\
\hline $\mathrm{C}$ & -0.13729800 & -1.86100200 & 1.12322300 & $\mathrm{H}$ & -4.29598300 & -1.18387200 & 0.09670200 \\
\hline $\mathrm{C}$ & 0.92336300 & -1.34354700 & -0.98130200 & $\mathrm{C}$ & 0.60621200 & 1.03711900 & -0.20150700 \\
\hline $\mathrm{C}$ & -0.15445100 & -3.21432400 & 0.78185100 & $\mathrm{C}$ & 0.74057900 & 2.35539400 & -0.41943800 \\
\hline $\mathrm{H}$ & -0.55679100 & -1.53345400 & 2.06947800 & $\mathrm{H}$ & -0.12351600 & 2.99546900 & -0.56226100 \\
\hline $\mathrm{C}$ & 0.90727600 & -2.69632200 & -1.32181400 & $\mathrm{H}$ & 1.72764900 & 2.80153100 & -0.46965500 \\
\hline $\mathrm{H}$ & 1.35141500 & -0.61893500 & -1.66764300 & $\mathrm{C}$ & 1.88666500 & 0.26324900 & -0.04365700 \\
\hline $\mathrm{C}$ & 0.36893100 & -3.63647500 & -0.44090600 & $\mathrm{O}$ & 1.67712900 & -1.01859900 & 0.32799900 \\
\hline $\mathrm{H}$ & -0.57728900 & -3.93779200 & 1.47319800 & $\mathrm{O}$ & 2.99722500 & 0.72897500 & -0.20658600 \\
\hline $\mathrm{H}$ & 1.31840000 & -3.01607000 & -2.27534000 & $\mathrm{C}$ & 2.86677600 & -1.79913800 & 0.52306800 \\
\hline $\mathrm{H}$ & 0.35789000 & -4.68985900 & -0.70600700 & $\mathrm{H}$ & 3.48458600 & -1.36535300 & 1.31331500 \\
\hline $\mathrm{C}$ & -0.61076300 & 1.38284000 & -0.21682100 & $\mathrm{H}$ & 2.52192500 & -2.79316600 & 0.80737900 \\
\hline $\mathrm{H}$ & -0.47380800 & 2.45705700 & -0.03616600 & $\mathrm{H}$ & 3.45528500 & -1.84274200 & -0.39687900 \\
\hline
\end{tabular}




\begin{tabular}{|c|c|c|c|c|c|c|c|}
\hline P3a & & & & $\mathrm{H}$ & -0.79213600 & -0.42038200 & -1.89589600 \\
\hline $\mathrm{C}$ & -3.46482500 & -1.24087700 & -0.00000500 & $\mathrm{C}$ & -3.83969800 & -0.52249900 & -0.39237900 \\
\hline $\mathrm{C}$ & -2.07255900 & -1.29992100 & -0.00001900 & $\mathrm{H}$ & -4.16915800 & -0.31008100 & 1.72702800 \\
\hline $\mathrm{C}$ & -1.29476800 & -0.12758800 & -0.00001400 & $\mathrm{H}$ & -3.18205800 & -0.69847200 & -2.43972400 \\
\hline $\mathrm{C}$ & -1.96176900 & 1.11317500 & 0.00000700 & $\mathrm{H}$ & -4.89257800 & -0.64471600 & -0.63012900 \\
\hline $\mathrm{C}$ & -3.35128900 & 1.17246800 & 0.00002100 & $\mathrm{H}$ & 0.40007000 & -0.71575100 & 2.59326700 \\
\hline $\mathrm{C}$ & -4.10942500 & -0.00331800 & 0.00001500 & $\mathrm{C}$ & 0.86150700 & 1.18489200 & -0.30530400 \\
\hline $\mathrm{H}$ & -4.04501800 & -2.15901800 & -0.00000900 & $\mathrm{O}$ & 1.25056300 & 1.11302400 & -1.45178100 \\
\hline $\mathrm{H}$ & -1.57232400 & -2.26499800 & -0.00003400 & $\mathrm{O}$ & 0.73879300 & 2.34300200 & 0.36825000 \\
\hline $\mathrm{H}$ & -1.38985000 & 2.03575100 & 0.00001100 & $\mathrm{C}$ & 1.08047400 & 3.52542900 & -0.37714900 \\
\hline $\mathrm{H}$ & -3.84814500 & 2.13835300 & 0.00003600 & $\mathrm{H}$ & 0.92507000 & 4.35836300 & 0.30807300 \\
\hline $\mathrm{H}$ & -5.19429600 & 0.04800400 & 0.00002500 & $\mathrm{H}$ & 2.12186000 & 3.48390400 & -0.70478900 \\
\hline $\mathrm{C}$ & 0.16449900 & -0.25634900 & -0.00002700 & $\mathrm{H}$ & 0.43654200 & 3.62222200 & -1.25447600 \\
\hline $\mathrm{H}$ & 0.54245000 & -1.27632400 & -0.00004400 & $\mathrm{H}$ & 0.16628400 & 1.03796900 & 2.42095400 \\
\hline $\mathrm{C}$ & 1.07141300 & 0.73857200 & -0.00000400 & & & & \\
\hline $\mathrm{H}$ & 0.79063900 & 1.786668000 & 0.00002700 & P5a & & & \\
\hline $\mathrm{C}$ & 2.53165500 & 0.53217300 & 0.00000000 & $\mathrm{H}$ & 0.14155700 & -2.01929500 & -1.03532500 \\
\hline $\mathrm{O}$ & 2.89049500 & -0.77750300 & 0.00001900 & $\mathrm{C}$ & 1.70941700 & -0.66550700 & -0.43614400 \\
\hline $\mathrm{O}$ & 3.34148300 & 1.44093200 & -0.00003000 & $\mathrm{C}$ & 2.36139500 & 0.49850500 & -0.86422400 \\
\hline $\mathrm{C}$ & 4.30373600 & -1.01886000 & 0.00002600 & $\mathrm{C}$ & 2.40880500 & -1.55698700 & 0.38954300 \\
\hline $\mathrm{H}$ & 4.77125300 & -0.58473900 & 0.88778500 & $\mathrm{C}$ & 3.67508400 & 0.76755900 & -0.47567100 \\
\hline $\mathrm{H}$ & 4.41822000 & -2.10310600 & 0.00010300 & $\mathrm{H}$ & 1.83467700 & 1.19523100 & -1.51141800 \\
\hline \multirow[t]{2}{*}{$\mathrm{H}$} & 4.77123800 & -0.58486900 & -0.88780500 & $\mathrm{C}$ & 3.72088300 & -1.29154500 & 0.78103100 \\
\hline & & & & $\mathrm{H}$ & 1.92225000 & -2.47126100 & 0.72245600 \\
\hline P4a & & & & $\mathrm{C}$ & 4.35846300 & -0.12631900 & 0.34954400 \\
\hline $\mathrm{C}$ & 1.19421500 & -1.24459600 & 0.02047800 & $\mathrm{H}$ & 4.16521400 & 1.67364900 & -0.82099000 \\
\hline $\mathrm{O}$ & 0.73866800 & -2.24730100 & -0.47588500 & $\mathrm{H}$ & 4.24743300 & -1.99643900 & 1.41838500 \\
\hline $\mathrm{C}$ & 3.37070400 & -2.11189800 & -0.21806800 & $\mathrm{H}$ & 5.38129100 & 0.08032600 & 0.65112400 \\
\hline $\mathrm{H}$ & 3.12235300 & -3.05401300 & 0.27674500 & $\mathrm{C}$ & -0.75645400 & -0.54240500 & 0.22958400 \\
\hline $\mathrm{H}$ & 4.38344300 & -1.79952400 & 0.03613100 & $\mathrm{H}$ & -0.52483100 & -1.03461900 & 1.18046500 \\
\hline $\mathrm{H}$ & 3.26800700 & -2.24014900 & -1.29822000 & $\mathrm{C}$ & 0.27969700 & -0.95149300 & -0.84326200 \\
\hline $\mathrm{O}$ & 2.51230300 & -1.05565300 & 0.24351800 & $\mathrm{O}$ & -2.40683600 & -1.77153900 & -1.07604400 \\
\hline $\mathrm{C}$ & 0.38842300 & -0.02360300 & 0.52649500 & $\mathrm{C}$ & -2.15968600 & -0.99534200 & -0.18068300 \\
\hline $\mathrm{C}$ & 0.69774000 & 0.17129300 & 2.02680800 & $\mathrm{O}$ & -3.09724700 & -0.44207700 & 0.61432200 \\
\hline $\mathrm{H}$ & 1.76720900 & 0.32756000 & 2.17147000 & $\mathrm{C}$ & -4.45443600 & -0.82698000 & 0.33188300 \\
\hline $\mathrm{C}$ & -1.11033200 & -0.20851000 & 0.22486500 & $\mathrm{H}$ & -5.06707500 & -0.29404200 & 1.05837800 \\
\hline $\mathrm{C}$ & -2.07984100 & -0.17717200 & 1.23335200 & $\mathrm{H}$ & -4.72912600 & -0.54297800 & -0.68685100 \\
\hline $\mathrm{C}$ & -1.52983700 & -0.39630800 & -1.10182500 & $\mathrm{H}$ & -4.57691400 & -1.90700700 & 0.44358800 \\
\hline $\mathrm{C}$ & -3.43410900 & -0.33529700 & 0.92747100 & $\mathrm{C}$ & -0.73858400 & 0.95173000 & 0.54191700 \\
\hline $\mathrm{H}$ & -1.79334300 & -0.03243600 & 2.26848800 & $\mathrm{O}$ & -0.52141100 & 1.43747800 & 1.62680900 \\
\hline
\end{tabular}




\begin{tabular}{|c|c|c|c|c|c|c|c|}
\hline $\mathrm{O}$ & -0.99182200 & 1.67708800 & -0.57212200 & $\mathrm{C}$ & 4.02487700 & -1.62485200 & 0.69073900 \\
\hline $\mathrm{C}$ & -1.01079700 & 3.10224500 & -0.38195500 & $\mathrm{H}$ & 4.80558100 & -2.39471000 & 0.66296100 \\
\hline H & -1.23003900 & 3.52717600 & -1.36135900 & $\mathrm{H}$ & 4.50827900 & -0.67086700 & 0.92345400 \\
\hline $\mathrm{H}$ & -1.78204800 & 3.38108400 & 0.34014600 & $\mathrm{H}$ & 3.33676400 & -1.86894700 & 1.50652800 \\
\hline $\mathrm{H}$ & -0.04230600 & 3.45297300 & -0.01736500 & $\mathrm{C}$ & -2.61783200 & 1.24427600 & -0.06609600 \\
\hline \multirow[t]{2}{*}{$\mathrm{H}$} & 0.03572100 & -0.43316700 & -1.77433200 & $\mathrm{C}$ & -2.61442800 & 1.67359700 & 1.26973600 \\
\hline & & & & $\mathrm{C}$ & -3.73906600 & 3.14322600 & -0.73046400 \\
\hline 1 & & & & $\mathrm{C}$ & -3.21810100 & 2.88829900 & 1.59194400 \\
\hline $\mathrm{C}$ & -1.31289600 & -0.06013200 & -2.26660100 & $\mathrm{H}$ & -2.13678300 & 1.05908500 & 2.02641400 \\
\hline $\mathrm{H}$ & -1.22176300 & -1.03171100 & -2.76699300 & $\mathrm{C}$ & -3.79305000 & 3.64388900 & 0.57106900 \\
\hline $\mathrm{H}$ & -2.12813400 & 0.48923400 & -2.74526400 & $\mathrm{H}$ & -4.17137000 & 3.70646500 & -1.55597800 \\
\hline $\mathrm{C}$ & 0.00000400 & 0.73451200 & -2.43454600 & $\mathrm{H}$ & -3.23154100 & 3.24107300 & 2.61943400 \\
\hline $\mathrm{H}$ & 0.00000600 & 1.14491500 & -3.45225600 & $\mathrm{H}$ & -4.26752600 & 4.59942500 & 0.77152800 \\
\hline $\mathrm{H}$ & 0.00000500 & 1.60976700 & -1.77265200 & $\mathrm{C}$ & 2.61783800 & 1.24427000 & -0.06609100 \\
\hline $\mathrm{C}$ & 1.31290400 & -0.06013400 & -2.26659900 & $\mathrm{C}$ & 2.61444000 & 1.67358800 & 1.26974100 \\
\hline $\mathrm{H}$ & 2.12814300 & 0.48923100 & -2.74526000 & $\mathrm{C}$ & 3.73907100 & 3.14322200 & -0.73045800 \\
\hline $\mathrm{H}$ & 1.22177000 & -1.03171200 & -2.76699100 & $\mathrm{C}$ & 3.21811500 & 2.88828800 & 1.59195100 \\
\hline$P$ & 1.81323200 & -0.38373400 & -0.49725700 & $\mathrm{H}$ & 2.13679700 & 1.05907300 & 2.02641900 \\
\hline$P$ & -1.81322800 & -0.38373100 & -0.49726000 & $\mathrm{C}$ & 3.79306100 & 3.64388100 & 0.57107700 \\
\hline $\mathrm{C}$ & -3.29429100 & -1.58310500 & -0.66436800 & $\mathrm{H}$ & 4.17137300 & 3.70646300 & -1.55597200 \\
\hline $\mathrm{C}$ & 3.29429200 & -1.58311300 & -0.66436100 & $\mathrm{H}$ & 3.23155800 & 3.24105900 & 2.61944200 \\
\hline $\mathrm{C}$ & -2.70660900 & -2.97810000 & -0.95779400 & $\mathrm{H}$ & 4.26753900 & 4.59941600 & 0.77153700 \\
\hline H & -3.51623200 & -3.71697600 & -1.00507300 & $\mathrm{~N}$ & -3.17154600 & 1.97421900 & -1.05045000 \\
\hline $\mathrm{H}$ & -2.17887300 & -3.01121800 & -1.91714400 & $\mathrm{~N}$ & 3.17154900 & 1.97421600 & -1.05044600 \\
\hline H & -2.00823900 & -3.28674400 & -0.17336200 & $\mathrm{Pd}$ & 0.00000000 & -0.98747300 & 0.99596100 \\
\hline $\mathrm{C}$ & -4.27479900 & -1.17758600 & -1.77826500 & $\mathrm{C}$ & -0.00002300 & -1.72290500 & 2.77346700 \\
\hline $\mathrm{H}$ & -5.12695500 & -1.86939800 & -1.78727000 & o & -0.00005200 & -2.15966500 & 3.84614800 \\
\hline $\mathrm{H}$ & -4.66198300 & -0.16520100 & -1.63549300 & & & & \\
\hline $\mathrm{H}$ & -3.80935600 & -1.22129300 & -2.76739800 & 2 & & & \\
\hline $\mathrm{C}$ & -4.02486300 & -1.62486700 & 0.69073700 & $\mathrm{C}$ & 3.01429600 & -0.79295500 & 2.09485100 \\
\hline $\mathrm{H}$ & -4.50826500 & -0.67088700 & 0.92347200 & $\mathrm{H}$ & 2.39939400 & -0.83399600 & 3.00042800 \\
\hline $\mathrm{H}$ & -4.80556600 & -2.39472700 & 0.66295400 & $\mathrm{H}$ & 3.90147200 & -1.41016400 & 2.26074000 \\
\hline $\mathrm{H}$ & -3.33674200 & -1.86897400 & 1.50651600 & $\mathrm{C}$ & 3.44249500 & 0.66533400 & 1.82745900 \\
\hline $\mathrm{C}$ & 2.70660600 & -2.97811200 & -0.95775600 & $\mathrm{H}$ & 4.27721400 & 0.88854200 & 2.50378200 \\
\hline $\mathrm{H}$ & 2.00824100 & -3.28674100 & -0.17331300 & $\mathrm{H}$ & 3.85635400 & 0.76221900 & 0.81577000 \\
\hline $\mathrm{H}$ & 2.17886300 & -3.01124700 & -1.91710200 & $\mathrm{C}$ & 2.36322900 & 1.74201900 & 2.06881900 \\
\hline $\mathrm{H}$ & 3.51622800 & -3.71699000 & -1.00502600 & $\mathrm{H}$ & 2.84981700 & 2.70835500 & 2.22350500 \\
\hline $\mathrm{C}$ & 4.27479000 & -1.17761400 & -1.77827400 & $\mathrm{H}$ & 1.79836800 & 1.49933800 & 2.97597600 \\
\hline $\mathrm{H}$ & 4.66197800 & -0.16522800 & -1.63552300 & $\mathrm{P}$ & 1.13140900 & 1.97397600 & 0.68511200 \\
\hline $\mathrm{H}$ & 5.12694400 & -1.86942900 & -1.78727700 & $\mathrm{P}$ & 2.04440700 & -1.58191000 & 0.71074300 \\
\hline $\mathrm{H}$ & 3.80933700 & -1.22133600 & -2.76740100 & $\mathrm{C}$ & 1.24603500 & -3.12010600 & 1.51877600 \\
\hline
\end{tabular}




\begin{tabular}{|c|c|c|c|c|c|c|c|}
\hline $\mathrm{C}$ & -0.19314400 & 3.11218700 & 1.47273200 & $\mathrm{H}$ & 2.35246400 & 4.04974400 & -3.69195700 \\
\hline $\mathrm{C}$ & 0.16901500 & -2.62244600 & 2.50416600 & $\mathrm{H}$ & 4.09772100 & 5.52923000 & -2.64300000 \\
\hline $\mathrm{H}$ & -0.36121700 & -3.48623500 & 2.92323200 & $\mathrm{~N}$ & 4.61495500 & -2.52688200 & 0.26533700 \\
\hline $\mathrm{H}$ & 0.59954000 & -2.07052300 & 3.34619300 & $\mathrm{~N}$ & 3.02405700 & 3.92415400 & 0.12841700 \\
\hline $\mathrm{H}$ & -0.57484000 & -1.98536400 & 2.01791500 & $\mathrm{Pd}$ & 0.61944300 & -0.05231600 & -0.57364300 \\
\hline $\mathrm{C}$ & 2.27471900 & -3.99021100 & 2.26140300 & $\mathrm{H}$ & -1.70261000 & 0.30491100 & -0.72294600 \\
\hline $\mathrm{H}$ & 1.76369900 & -4.84983300 & 2.71328200 & $\mathrm{C}$ & -0.06937500 & -0.52121900 & -2.30354500 \\
\hline $\mathrm{H}$ & 3.04892700 & -4.37470200 & 1.59221800 & $\mathrm{O}$ & -0.25999000 & -0.83974200 & -3.40052300 \\
\hline $\mathrm{H}$ & 2.77199700 & -3.44257800 & 3.06776300 & $\mathrm{~S}$ & -3.26690100 & -0.90659200 & 0.17414500 \\
\hline $\mathrm{C}$ & 0.56211100 & -3.94053500 & 0.40836800 & $\mathrm{O}$ & -2.69175400 & 0.38967500 & -0.62761800 \\
\hline $\mathrm{H}$ & 1.28012400 & -4.30385700 & -0.33412900 & $\mathrm{O}$ & -2.97822800 & -0.75023800 & 1.60302700 \\
\hline $\mathrm{H}$ & 0.07759400 & -4.81749500 & 0.85465900 & $\mathrm{O}$ & -2.84082700 & -2.12361600 & -0.51959500 \\
\hline $\mathrm{H}$ & -0.20909600 & -3.35922300 & -0.10512600 & $\mathrm{C}$ & -5.00689800 & -0.63582900 & -0.10370800 \\
\hline $\mathrm{C}$ & -1.02264800 & 2.25480700 & 2.45070000 & $\mathrm{C}$ & -5.75558800 & 0.04533300 & 0.85574800 \\
\hline $\mathrm{H}$ & -1.48676500 & 1.39393300 & 1.96255300 & $\mathrm{C}$ & -5.59528100 & -1.12597100 & -1.27048000 \\
\hline $\mathrm{H}$ & -0.42189800 & 1.88830100 & 3.28986700 & $\mathrm{C}$ & -7.11621700 & 0.24477300 & 0.63094100 \\
\hline $\mathrm{H}$ & -1.82577400 & 2.86979400 & 2.87571100 & $\mathrm{H}$ & -5.27747100 & 0.39574000 & 1.76369600 \\
\hline $\mathrm{C}$ & 0.42306300 & 4.30646700 & 2.22434900 & $\mathrm{C}$ & -6.95619200 & -0.91542500 & -1.47709600 \\
\hline $\mathrm{H}$ & 1.05153700 & 4.92341000 & 1.57707300 & $\mathrm{H}$ & -4.99683200 & -1.67354600 & -1.99032700 \\
\hline $\mathrm{H}$ & -0.38293300 & 4.93913900 & 2.61737900 & $\mathrm{C}$ & -7.73515700 & -0.22493600 & -0.53637500 \\
\hline $\mathrm{H}$ & 1.03159500 & 3.98713700 & 3.07556300 & $\mathrm{H}$ & -7.70816200 & 0.76889400 & 1.37654600 \\
\hline $\mathrm{C}$ & -1.11248800 & 3.62706800 & 0.34905600 & $\mathrm{H}$ & -7.42348200 & -1.29841200 & -2.38037300 \\
\hline $\mathrm{H}$ & -1.89902200 & 4.25507600 & 0.78525500 & $\mathrm{C}$ & -9.20335900 & 0.02327900 & -0.78518100 \\
\hline $\mathrm{H}$ & -0.56809900 & 4.23761600 & -0.37829500 & $\mathrm{H}$ & -9.76232500 & 0.09469700 & 0.15222300 \\
\hline $\mathrm{H}$ & -1.60174300 & 2.80654600 & -0.18270900 & $\mathrm{H}$ & -9.35348500 & 0.96501000 & -1.32772000 \\
\hline $\mathrm{C}$ & 3.42834500 & -2.29596800 & -0.32221300 & $\mathrm{H}$ & -9.64766900 & -0.77371000 & -1.38839700 \\
\hline $\mathrm{C}$ & 3.20585800 & -2.56485800 & -1.68156700 & & & & \\
\hline
\end{tabular}

\section{TS1}

imaginary frequency $=-270.25 \mathrm{~cm}^{-1}$

C

H

C

H

$\mathrm{H}$

$\mathrm{H}$

C

C

C

C

H

C

H
$5.60498200 \quad-3.03378600 \quad-0.47861500$

$4.24038100 \quad-3.10667200 \quad-2.44272400$

$2.24205600 \quad-2.33905700 \quad-2.12455100$

$5.47053700 \quad-3.34612200 \quad-1.83174600$

$\begin{array}{lll}6.55135500 & -3.19847000 & 0.03411600\end{array}$

$4.08982900 \quad-3.32796600 \quad-3.49561700$

6.30830300

2.09007200

1.82518400

3.71260200

2.54144000

1.08024800

3.50906500

$-3.75781300 \quad-2.38576400$

$3.13259800 \quad-0.42686400$

$3.14644500-1.80435600$

$4.75012200 \quad-0.66792800$

$4.01928300 \quad-2.62253500$

$2.47062000 \quad-2.21150600$

$4.84014800 \quad-2.04554400$

$5.36921500 \quad-0.17952800$
$3.08719800-0.53776800$

2.08504600

$\begin{array}{lll}2.50565600 & -0.59978500 & 3.01068900\end{array}$

$\begin{array}{lll}4.02535300 & -1.07775100 & 2.23561500\end{array}$

$\begin{array}{lll}3.38430700 & 0.93832600 & 1.75276800\end{array}$

$\begin{array}{lll}4.21393800 & 1.25861700 \quad 2.39491100\end{array}$

$\begin{array}{lll}3.75784200 & 1.03437300 & 0.72548900\end{array}$

$\begin{array}{lll}2.22093600 & 1.91818600 & 1.99754400\end{array}$

$\begin{array}{lll}2.61700200 & 2.93390700 \quad 2.07645100\end{array}$

$\begin{array}{lll}1.72667200 & 1.67467000 & 2.94413700\end{array}$

$\begin{array}{lll}0.89591300 & 2.00462300 & 0.69401700\end{array}$

$2.15395300 \quad-1.43939100 \quad 0.75675500$

$\begin{array}{lll}1.37663900 & -2.95466900 & 1.61812400\end{array}$ 


\begin{tabular}{|c|c|c|c|c|c|c|c|}
\hline $\mathrm{C}$ & -0.50375500 & 2.98379200 & 1.54311500 & $\mathrm{H}$ & 1.58508000 & 4.07956200 & -3.80228100 \\
\hline $\mathrm{C}$ & 0.28689600 & -2.43813900 & 2.58052300 & $\mathrm{H}$ & 3.22985500 & 5.75462200 & -2.89600300 \\
\hline $\mathrm{H}$ & -0.20288900 & -3.29958000 & 3.05047900 & $\mathrm{~N}$ & 4.77477700 & -2.19072000 & 0.30800700 \\
\hline $\mathrm{H}$ & 0.70309700 & -1.82859200 & 3.38971100 & $\mathrm{~N}$ & 2.52742400 & 4.09928800 & -0.03795400 \\
\hline $\mathrm{H}$ & -0.48655700 & -1.86274800 & 2.06250100 & $\mathrm{Pd}$ & 0.46533200 & -0.03028000 & -0.46057300 \\
\hline $\mathrm{C}$ & 2.43179800 & -3.76670000 & 2.38948200 & $\mathrm{H}$ & -0.88470800 & 0.70582000 & -0.94231900 \\
\hline $\mathrm{H}$ & 1.94475400 & -4.62250400 & 2.87261900 & $\mathrm{C}$ & 0.09544600 & -1.02484600 & -2.12375900 \\
\hline $\mathrm{H}$ & 3.21528400 & -4.15550100 & 1.73277400 & $\mathrm{O}$ & 0.00189500 & -1.49987700 & -3.16030700 \\
\hline $\mathrm{H}$ & 2.91291200 & -3.17662900 & 3.17568000 & $\mathrm{~S}$ & -2.86018800 & -0.73443700 & -0.34274300 \\
\hline $\mathrm{C}$ & 0.70963900 & -3.83463700 & 0.54273100 & $\mathrm{O}$ & -2.52615200 & 0.60502700 & -0.99491600 \\
\hline $\mathrm{H}$ & 1.43376000 & -4.22017100 & -0.18240900 & $\mathrm{O}$ & -2.37987700 & -0.79088800 & 1.06610300 \\
\hline $\mathrm{H}$ & 0.24469500 & -4.69962100 & 1.03019700 & $\mathrm{O}$ & -2.45136500 & -1.88225600 & -1.18970100 \\
\hline $\mathrm{H}$ & -0.07975300 & -3.29759000 & 0.00851400 & $\mathrm{C}$ & -4.65637000 & -0.75266000 & -0.27562400 \\
\hline $\mathrm{C}$ & -1.20348600 & 2.02623000 & 2.52896200 & $\mathrm{C}$ & -5.31597300 & -0.30974900 & 0.87040700 \\
\hline $\mathrm{H}$ & -1.62107700 & 1.14840000 & 2.02772600 & $\mathrm{C}$ & -5.38079500 & -1.19394700 & -1.38426500 \\
\hline $\mathrm{H}$ & -0.52764200 & 1.68812900 & 3.32296400 & $\mathrm{C}$ & -6.71026500 & -0.30152900 & 0.89860400 \\
\hline $\mathrm{H}$ & -2.02511400 & 2.56433200 & 3.01789400 & $\mathrm{H}$ & -4.73561600 & 0.00255800 & 1.73174400 \\
\hline $\mathrm{C}$ & 0.05319300 & 4.20841900 & 2.29543100 & $\mathrm{C}$ & -6.77328400 & -1.17958700 & -1.34201200 \\
\hline $\mathrm{H}$ & 0.61324400 & 4.88034000 & 1.63948300 & $\mathrm{H}$ & -4.85127000 & -1.56168000 & -2.25658900 \\
\hline $\mathrm{H}$ & -0.78842900 & 4.77122500 & 2.71666200 & $\mathrm{C}$ & -7.46027800 & -0.72872900 & -0.20531300 \\
\hline $\mathrm{H}$ & 0.70470600 & 3.92602700 & 3.12754400 & $\mathrm{H}$ & -7.22475200 & 0.03559000 & 1.79539300 \\
\hline $\mathrm{C}$ & -1.50863400 & 3.44718100 & 0.47182000 & $\mathrm{H}$ & -7.33758000 & -1.53080500 & -2.20275300 \\
\hline $\mathrm{H}$ & -2.31947600 & 3.99413600 & 0.96821900 & $\mathrm{C}$ & -8.97029400 & -0.68900800 & -0.18029000 \\
\hline $\mathrm{H}$ & -1.04931200 & 4.13141000 & -0.24938000 & $\mathrm{H}$ & -9.35596500 & -0.74794300 & 0.84185100 \\
\hline $\mathrm{H}$ & -1.95400600 & 2.60564600 & -0.06410500 & $\mathrm{H}$ & -9.34888800 & 0.24318800 & -0.61867400 \\
\hline $\mathrm{C}$ & 3.55599200 & -2.14616400 & -0.25451400 & $\mathrm{H}$ & -9.40268200 & -1.51447300 & -0.75439800 \\
\hline $\mathrm{C}$ & 3.32359900 & -2.60675900 & -1.55933900 & & & & \\
\hline $\mathrm{C}$ & 5.78907600 & -2.69515200 & -0.40410700 & 3 & & & \\
\hline $\mathrm{C}$ & 4.38447300 & -3.14300800 & -2.28744600 & $\mathrm{C}$ & 1.20371600 & 0.24851800 & -2.33916900 \\
\hline $\mathrm{H}$ & 2.33447600 & -2.54388200 & -1.99669900 & $\mathrm{H}$ & 1.15574400 & 1.25355300 & -2.77304700 \\
\hline $\mathrm{C}$ & 5.64839100 & -3.18820400 & -1.70147100 & $\mathrm{H}$ & 2.01950700 & -0.29130200 & -2.82655500 \\
\hline $\mathrm{H}$ & 6.75951100 & -2.70572600 & 0.08898200 & $\mathrm{C}$ & -0.11327400 & -0.50462200 & -2.59423600 \\
\hline $\mathrm{H}$ & 4.22542600 & -3.51075200 & -3.29699700 & $\mathrm{H}$ & -0.13356100 & -0.76497800 & -3.65847200 \\
\hline $\mathrm{H}$ & 6.50561800 & -3.58904300 & -2.23279900 & $\mathrm{H}$ & -0.11501600 & -1.46089900 & -2.05844300 \\
\hline $\mathrm{C}$ & 1.65058200 & 3.20291200 & -0.51994600 & $\mathrm{C}$ & -1.40550400 & 0.28179100 & -2.29950500 \\
\hline $\mathrm{C}$ & 1.28912900 & 3.15959300 & -1.87376000 & $\mathrm{H}$ & -2.24099600 & -0.18844200 & -2.82631700 \\
\hline $\mathrm{C}$ & 3.06744400 & 4.97884600 & -0.88964500 & $\mathrm{H}$ & -1.32617200 & 1.30067900 & -2.69518100 \\
\hline $\mathrm{C}$ & 1.85124800 & 4.08804500 & -2.74927200 & $\mathrm{P}$ & -1.87742000 & 0.42355000 & -0.51134300 \\
\hline $\mathrm{H}$ & 0.58846200 & 2.40802100 & -2.22098100 & $\mathrm{P}$ & 1.69861200 & 0.41413700 & -0.56144700 \\
\hline $\mathrm{C}$ & 2.76209300 & 5.01788700 & -2.25069100 & $\mathrm{C}$ & 3.24272900 & 1.53369100 & -0.58501000 \\
\hline $\mathrm{H}$ & 3.77703600 & 5.68456200 & -0.46160500 & $\mathrm{C}$ & -3.45054300 & 1.49569100 & -0.48005900 \\
\hline
\end{tabular}




\begin{tabular}{|c|c|c|c|c|c|c|c|}
\hline $\mathrm{C}$ & 2.75898700 & 2.99062000 & -0.72818600 & $\mathrm{H}$ & -3.20151400 & -4.74722700 & 1.38918800 \\
\hline $\mathrm{H}$ & 3.62577000 & 3.66041700 & -0.74326300 & $\mathrm{~N}$ & 2.94739800 & -1.93579400 & -1.16121800 \\
\hline $\mathrm{H}$ & 2.20777100 & 3.15438600 & -1.66015600 & $\mathrm{~N}$ & -1.93826000 & -1.61957800 & 1.24019700 \\
\hline $\mathrm{H}$ & 2.11778200 & 3.29532400 & 0.10502700 & $\mathrm{Pd}$ & -0.14004000 & 1.11330200 & 0.93167800 \\
\hline $\mathrm{C}$ & 4.17615400 & 1.17056700 & -1.75697700 & $\mathrm{H}$ & -1.39840700 & 1.51049100 & 1.77881400 \\
\hline $\mathrm{H}$ & 5.08692600 & 1.77527900 & -1.68212400 & $\mathrm{C}$ & 0.82899100 & 1.85185600 & 2.47555800 \\
\hline $\mathrm{H}$ & 4.46584900 & 0.11720000 & -1.74172100 & $\mathrm{O}$ & 1.28570200 & 2.29887300 & 3.41852700 \\
\hline $\mathrm{H}$ & 3.72235900 & 1.38749600 & -2.72774300 & & & & \\
\hline $\mathrm{C}$ & 3.99878500 & 1.35699000 & 0.74468800 & 4 & & & \\
\hline $\mathrm{H}$ & 4.39119000 & 0.34304600 & 0.86007100 & $\mathrm{C}$ & -4.16422400 & -0.38095000 & -0.32084400 \\
\hline $\mathrm{H}$ & 4.84990500 & 2.04611500 & 0.76640000 & $\mathrm{H}$ & -4.59288500 & -0.41746100 & -1.32953900 \\
\hline $\mathrm{H}$ & 3.37759100 & 1.58591000 & 1.61526700 & $\mathrm{H}$ & -4.87811900 & -0.84322100 & 0.36655100 \\
\hline $\mathrm{C}$ & -3.01358700 & 2.96078700 & -0.68233300 & $\mathrm{C}$ & -3.96268200 & 1.08643300 & 0.10798700 \\
\hline $\mathrm{H}$ & -2.33350400 & 3.29139000 & 0.10831000 & $\mathrm{H}$ & -4.96283200 & 1.50817500 & 0.27172900 \\
\hline $\mathrm{H}$ & -2.52585400 & 3.12180700 & -1.64950800 & $\mathrm{H}$ & -3.47331400 & 1.11334200 & 1.08852800 \\
\hline $\mathrm{H}$ & -3.89848100 & 3.60564800 & -0.65463400 & $\mathrm{C}$ & -3.23482700 & 2.02409500 & -0.88658000 \\
\hline $\mathrm{C}$ & -4.42914000 & 1.07785200 & -1.59627400 & $\mathrm{H}$ & -3.55313700 & 3.05693300 & -0.70769200 \\
\hline $\mathrm{H}$ & -4.74283100 & 0.03402200 & -1.50271500 & $\mathrm{H}$ & -3.54776900 & 1.78472600 & -1.90937000 \\
\hline $\mathrm{H}$ & -5.33196600 & 1.69258400 & -1.51720200 & $\mathrm{P}$ & -1.36585100 & 1.89264600 & -0.88659200 \\
\hline $\mathrm{H}$ & -4.01985000 & 1.23601600 & -2.59775400 & $\mathrm{P}$ & -2.61825700 & -1.43028500 & -0.32885000 \\
\hline $\mathrm{C}$ & -4.14016300 & 1.33543600 & 0.88696400 & $\mathrm{C}$ & -3.23868400 & -3.13120900 & -0.94624800 \\
\hline $\mathrm{H}$ & -5.01051000 & 1.99934500 & 0.92227900 & $\mathrm{C}$ & -0.80991300 & 3.28937000 & -2.07914000 \\
\hline $\mathrm{H}$ & -4.49343000 & 0.31366700 & 1.05156200 & $\mathrm{C}$ & -3.43473800 & -3.01336300 & -2.47061700 \\
\hline $\mathrm{H}$ & -3.48272300 & 1.60904600 & 1.71718200 & $\mathrm{H}$ & -3.71731700 & -3.99008800 & -2.88274500 \\
\hline $\mathrm{C}$ & 2.30711600 & -1.29276000 & -0.17121500 & $\mathrm{H}$ & -4.23152400 & -2.30750400 & -2.73036600 \\
\hline $\mathrm{C}$ & 2.10996400 & -1.85396800 & 1.09569700 & $\mathrm{H}$ & -2.51448800 & -2.68628500 & -2.96468300 \\
\hline $\mathrm{C}$ & 3.42527200 & -3.16016500 & -0.91174800 & $\mathrm{C}$ & -4.54971200 & -3.58289800 & -0.27927300 \\
\hline $\mathrm{C}$ & 2.61745400 & -3.13059100 & 1.34589900 & $\mathrm{H}$ & -4.80118200 & -4.59653400 & -0.61694400 \\
\hline $\mathrm{H}$ & 1.56589500 & -1.30941600 & 1.86043800 & $\mathrm{H}$ & -4.47243700 & -3.59556400 & 0.81083700 \\
\hline $\mathrm{C}$ & 3.28995500 & -3.79752700 & 0.32463600 & $\mathrm{H}$ & -5.38915000 & -2.93400900 & -0.54787000 \\
\hline $\mathrm{H}$ & 3.93740000 & -3.65186700 & -1.73564800 & $\mathrm{C}$ & -2.12440800 & -4.15612500 & -0.66161700 \\
\hline $\mathrm{H}$ & 2.48622900 & -3.59381300 & 2.31910300 & $\mathrm{H}$ & -1.97569700 & -4.30886100 & 0.41169500 \\
\hline $\mathrm{H}$ & 3.69937500 & -4.79112300 & 0.47391600 & $\mathrm{H}$ & -2.39490600 & -5.12383600 & -1.10169400 \\
\hline $\mathrm{C}$ & -2.33859600 & -1.29283900 & -0.00068100 & $\mathrm{H}$ & -1.17097700 & -3.84029100 & -1.09740000 \\
\hline $\mathrm{C}$ & -3.05091900 & -2.17470700 & -0.82350200 & $\mathrm{C}$ & -1.20986500 & 2.83980700 & -3.49872200 \\
\hline $\mathrm{C}$ & -2.25539200 & -2.83321400 & 1.70474300 & $\mathrm{H}$ & -0.80409600 & 1.84953200 & -3.72860700 \\
\hline $\mathrm{C}$ & -3.37147200 & -3.43977800 & -0.32747900 & $\mathrm{H}$ & -2.29651400 & 2.80565000 & -3.63168900 \\
\hline $\mathrm{H}$ & -3.35229600 & -1.89196400 & -1.82605300 & $\mathrm{H}$ & -0.81299900 & 3.55128100 & -4.23307600 \\
\hline $\mathrm{C}$ & -2.97100600 & -3.77653700 & 0.96303200 & $\mathrm{C}$ & -1.43463700 & 4.65970000 & -1.76704000 \\
\hline $\mathrm{H}$ & -1.92053800 & -3.06062000 & 2.71390800 & $\mathrm{H}$ & -1.18196700 & 5.00772700 & -0.76055600 \\
\hline $\mathrm{H}$ & -3.92443300 & -4.14489500 & -0.94038200 & $\mathrm{H}$ & -1.05417700 & 5.40567200 & -2.47592400 \\
\hline
\end{tabular}




\begin{tabular}{|c|c|c|c|c|c|c|c|}
\hline $\mathrm{H}$ & -2.52503200 & 4.65129300 & -1.86429200 & $\mathrm{H}$ & 7.82165600 & -0.11013700 & 0.88365400 \\
\hline $\mathrm{C}$ & 0.72549000 & 3.38480400 & -1.99680300 & $\mathrm{H}$ & 5.59749900 & -2.22232500 & -2.12722200 \\
\hline $\mathrm{H}$ & 1.08603400 & 4.09940000 & -2.74686700 & $\mathrm{C}$ & 8.15130300 & -1.61740500 & -1.37567500 \\
\hline $\mathrm{H}$ & 1.06319000 & 3.73522700 & -1.01689600 & $\mathrm{H}$ & 8.08279600 & -1.50281500 & -2.46227200 \\
\hline $\mathrm{H}$ & 1.19806000 & 2.41797600 & -2.19218500 & $\mathrm{H}$ & 8.99448700 & -1.01737500 & -1.02279200 \\
\hline $\mathrm{C}$ & -2.35641200 & -1.67820600 & 1.49805400 & $\mathrm{H}$ & 8.38796400 & -2.66983600 & -1.17673700 \\
\hline $\mathrm{C}$ & -1.04461200 & -1.77054300 & 1.98349400 & & & & \\
\hline $\mathrm{C}$ & -3.22296300 & -1.89908300 & 3.61786900 & \multirow{2}{*}{\multicolumn{4}{|c|}{$\begin{array}{l}\text { TS2 } \\
\text { imaginary frequency }=-348.67 \mathrm{~cm}^{-1}\end{array}$}} \\
\hline $\mathrm{C}$ & -0.84209800 & -1.94173300 & 3.35299400 & & & & \\
\hline $\mathrm{H}$ & -0.19580000 & -1.69288000 & 1.31247700 & $\mathrm{C}$ & -4.13062900 & -0.24412000 & -0.31992300 \\
\hline $\mathrm{C}$ & -1.95408600 & -2.01046300 & 4.19056200 & $\mathrm{H}$ & -4.56910400 & -0.27650000 & -1.32443600 \\
\hline $\mathrm{H}$ & -4.11457300 & -1.93986300 & 4.24202700 & $\mathrm{H}$ & -4.85665300 & -0.66815700 & 0.37949100 \\
\hline $\mathrm{H}$ & 0.16965600 & -2.00540200 & 3.74185100 & $\mathrm{C}$ & -3.87245500 & 1.21982800 & 0.08985100 \\
\hline $\mathrm{H}$ & -1.84887600 & -2.13892100 & 5.26361600 & $\mathrm{H}$ & -4.85558000 & 1.68503100 & 0.23795900 \\
\hline $\mathrm{C}$ & -0.93365800 & 2.59625500 & 0.78936300 & $\mathrm{H}$ & -3.39275300 & 1.23863500 & 1.07545100 \\
\hline $\mathrm{C}$ & -1.78873600 & 3.40941700 & 1.54622800 & $\mathrm{C}$ & -3.09866000 & 2.11672000 & -0.90870300 \\
\hline $\mathrm{C}$ & 0.68046900 & 2.70787600 & 2.46913700 & $\mathrm{H}$ & -3.37616300 & 3.16460700 & -0.74836000 \\
\hline $\mathrm{C}$ & -1.38119800 & 3.87096800 & 2.79766600 & $\mathrm{H}$ & -3.40841200 & 1.87501800 & -1.93174000 \\
\hline $\mathrm{H}$ & -2.76404900 & 3.68343000 & 1.16304500 & $\mathrm{P}$ & -1.23919900 & 1.89876400 & -0.89760000 \\
\hline $\mathrm{C}$ & -0.12158600 & 3.51643600 & 3.27166000 & $\mathrm{P}$ & -2.62770400 & -1.35566300 & -0.32965500 \\
\hline $\mathrm{H}$ & 1.66774700 & 2.38432400 & 2.79087400 & $\mathrm{C}$ & -3.32227700 & -3.03870500 & -0.91356500 \\
\hline $\mathrm{H}$ & -2.04102400 & 4.49951900 & 3.38915100 & $\mathrm{C}$ & -0.58591700 & 3.29265800 & -2.04468900 \\
\hline $\mathrm{H}$ & 0.23673600 & 3.84842700 & 4.24011800 & $\mathrm{C}$ & -3.55146500 & -2.93335800 & -2.43405500 \\
\hline $\mathrm{N}$ & -3.43197800 & -1.73511500 & 2.30592500 & $\mathrm{H}$ & -3.87725200 & -3.90502900 & -2.82543000 \\
\hline $\mathrm{N}$ & 0.28371700 & 2.25531000 & 1.26921800 & $\mathrm{H}$ & -4.32999700 & -2.20416200 & -2.68455300 \\
\hline $\mathrm{Pd}$ & -0.73787300 & -0.37455500 & -1.41484800 & $\mathrm{H}$ & -2.63315400 & -2.64495200 & -2.95478800 \\
\hline $\mathrm{H}$ & 1.43784900 & 1.30449600 & 0.60644800 & $\mathrm{C}$ & -4.63192400 & -3.43369800 & -0.20893300 \\
\hline $\mathrm{C}$ & 0.68943100 & -1.05662400 & -2.51657300 & $\mathrm{H}$ & -4.92987700 & -4.44051100 & -0.52853600 \\
\hline $\mathrm{O}$ & 1.58455900 & -1.48598100 & -3.11311400 & $\mathrm{H}$ & -4.52781800 & -3.43839800 & 0.87898500 \\
\hline$S$ & 2.95901400 & -0.08020900 & 1.40378000 & $\mathrm{H}$ & -5.45307800 & -2.75657800 & -0.46416900 \\
\hline $\mathrm{O}$ & 2.15955800 & -1.29321700 & 1.62292200 & $\mathrm{C}$ & -2.23771400 & -4.09843600 & -0.64141300 \\
\hline $\mathrm{O}$ & 3.27860900 & 0.75389100 & 2.57447600 & $\mathrm{H}$ & -2.06668400 & -4.24149400 & 0.42981200 \\
\hline $\mathrm{O}$ & 2.28508200 & 0.81982700 & 0.26752700 & $\mathrm{H}$ & -2.55311600 & -5.06172100 & -1.06052800 \\
\hline $\mathrm{C}$ & 4.47401400 & -0.50961400 & 0.56770000 & $\mathrm{H}$ & -1.28493700 & -3.82332600 & -1.10535400 \\
\hline $\mathrm{C}$ & 5.68531000 & -0.08264500 & 1.10673000 & $\mathrm{C}$ & -1.00658700 & 2.90328300 & -3.47624400 \\
\hline $\mathrm{C}$ & 4.43032500 & -1.28725000 & -0.59287300 & $\mathrm{H}$ & -0.67376700 & 1.89139800 & -3.72667900 \\
\hline $\mathrm{C}$ & 6.87328000 & -0.44099400 & 0.46858900 & $\mathrm{H}$ & -2.09161300 & 2.95367900 & -3.61608200 \\
\hline $\mathrm{H}$ & 5.68768500 & 0.52145500 & 2.00703300 & $\mathrm{H}$ & -0.55189900 & 3.59992400 & -4.19064500 \\
\hline $\mathrm{C}$ & 5.62556000 & -1.62715800 & -1.21828100 & $\mathrm{C}$ & -1.11922900 & 4.69494000 & -1.70781200 \\
\hline $\mathrm{H}$ & 3.48099400 & -1.60789600 & -1.00745900 & $\mathrm{H}$ & -0.83129800 & 5.01718600 & -0.70220400 \\
\hline $\mathrm{C}$ & 6.86342400 & -1.21515300 & -0.69793200 & $\mathrm{H}$ & -0.70051300 & 5.42280500 & -2.41366700 \\
\hline
\end{tabular}




\begin{tabular}{|c|c|c|c|c|c|c|c|}
\hline $\mathrm{H}$ & -2.20908500 & 4.75891100 & -1.79118500 & $\mathrm{H}$ & 7.70033400 & -0.35955300 & 0.98537000 \\
\hline $\mathrm{C}$ & 0.95171700 & 3.27748600 & -1.94645400 & $\mathrm{H}$ & 5.45278600 & -1.99994500 & -2.28884800 \\
\hline $\mathrm{H}$ & 1.37033100 & 3.97257500 & -2.68418000 & $\mathrm{C}$ & 8.01431400 & -1.57179200 & -1.44976300 \\
\hline $\mathrm{H}$ & 1.30456300 & 3.59499100 & -0.96001700 & $\mathrm{H}$ & 8.01964300 & -1.17675600 & -2.47171600 \\
\hline $\mathrm{H}$ & 1.35738100 & 2.28126200 & -2.14415700 & $\mathrm{H}$ & 8.88695500 & -1.16890200 & -0.92833500 \\
\hline $\mathrm{C}$ & -2.34419200 & -1.58431600 & 1.49489800 & $\mathrm{H}$ & 8.14403400 & -2.65808300 & -1.52542500 \\
\hline $\mathrm{C}$ & -1.02937100 & -1.74065000 & 1.95400100 & & & & \\
\hline $\mathrm{C}$ & -3.17551400 & -1.72405400 & 3.63472800 & 5 & & & \\
\hline $\mathrm{C}$ & -0.80562800 & -1.90289700 & 3.32161500 & $\mathrm{C}$ & 1.35942600 & 0.73366200 & -2.15182600 \\
\hline $\mathrm{H}$ & -0.18800500 & -1.71989200 & 1.26934200 & $\mathrm{H}$ & 1.22527800 & 1.80085100 & -2.36044500 \\
\hline $\mathrm{C}$ & -1.90219600 & -1.89797400 & 4.18221300 & $\mathrm{H}$ & 2.21227800 & 0.38043900 & -2.73638400 \\
\hline $\mathrm{H}$ & -4.05406300 & -1.70579200 & 4.27831100 & $\mathrm{C}$ & 0.10344700 & -0.04935400 & -2.59241500 \\
\hline $\mathrm{H}$ & 0.21215300 & -2.01459600 & 3.68245900 & $\mathrm{H}$ & 0.16416200 & -0.18295700 & -3.67869900 \\
\hline $\mathrm{H}$ & -1.78175800 & -2.01756500 & 5.25477800 & $\mathrm{H}$ & 0.12120500 & -1.06338900 & -2.17332400 \\
\hline $\mathrm{C}$ & -0.81504500 & 2.57381700 & 0.79660500 & $\mathrm{C}$ & -1.25455700 & 0.63177800 & -2.30550300 \\
\hline $\mathrm{C}$ & -1.61641800 & 3.45101000 & 1.53682500 & $\mathrm{H}$ & -2.02824400 & 0.21701700 & -2.96063800 \\
\hline $\mathrm{C}$ & 0.76411300 & 2.55764500 & 2.55952100 & $\mathrm{H}$ & -1.18879200 & 1.69713400 & -2.55617500 \\
\hline $\mathrm{C}$ & -1.21001500 & 3.88143700 & 2.79997100 & $\mathrm{P}$ & -1.80160900 & 0.56743200 & -0.52366400 \\
\hline $\mathrm{H}$ & -2.55379900 & 3.80142600 & 1.12378900 & $\mathrm{P}$ & 1.82705200 & 0.55513600 & -0.35422600 \\
\hline $\mathrm{C}$ & 0.00145400 & 3.43290000 & 3.32196700 & $\mathrm{C}$ & 3.15070500 & 1.90336000 & -0.08062400 \\
\hline $\mathrm{H}$ & 1.71116400 & 2.12944100 & 2.88572400 & $\mathrm{C}$ & -3.21964800 & 1.84300000 & -0.38389700 \\
\hline $\mathrm{H}$ & -1.83618500 & 4.56388600 & 3.36744200 & $\mathrm{C}$ & 2.41341200 & 3.25589300 & -0.01408600 \\
\hline $\mathrm{H}$ & 0.35042700 & 3.74254200 & 4.30036600 & $\mathrm{H}$ & 3.13524100 & 4.05630100 & 0.18440100 \\
\hline $\mathrm{N}$ & -3.40475200 & -1.56831600 & 2.32491400 & $\mathrm{H}$ & 1.90874700 & 3.50263900 & -0.95419800 \\
\hline N & 0.34947300 & 2.15113000 & 1.34604800 & $\mathrm{H}$ & 1.67042000 & 3.26820600 & 0.79029200 \\
\hline $\mathrm{Pd}$ & -0.74315800 & -0.37717800 & -1.47288400 & $\mathrm{C}$ & 4.20073900 & 1.92182300 & -1.20549600 \\
\hline $\mathrm{H}$ & 1.09509600 & 1.35531000 & 0.84416800 & $\mathrm{H}$ & 4.96377400 & 2.67507200 & -0.97616700 \\
\hline $\mathrm{C}$ & 0.54665400 & -1.06293400 & -2.73830500 & $\mathrm{H}$ & 4.70473400 & 0.95762200 & -1.31352500 \\
\hline $\mathrm{O}$ & 1.35317900 & -1.48682400 & -3.45079500 & $\mathrm{H}$ & 3.76430600 & 2.18527200 & -2.17336500 \\
\hline S & 2.82068200 & -0.24284100 & 1.46661600 & $\mathrm{C}$ & 3.83406500 & 1.63455200 & 1.27340600 \\
\hline $\mathrm{O}$ & 2.16893400 & -1.52555800 & 1.80031000 & $\mathrm{H}$ & 4.40562000 & 0.70203800 & 1.26575800 \\
\hline $\mathrm{O}$ & 3.16632900 & 0.62846300 & 2.61807600 & $\mathrm{H}$ & 4.53437900 & 2.44850100 & 1.49223500 \\
\hline $\mathrm{O}$ & 2.00870700 & 0.54408700 & 0.41205200 & $\mathrm{H}$ & 3.10867700 & 1.58876000 & 2.09226200 \\
\hline $\mathrm{C}$ & 4.34192800 & -0.60105300 & 0.59206900 & $\mathrm{C}$ & -2.52053100 & 3.21716100 & -0.29716300 \\
\hline $\mathrm{C}$ & 5.56126800 & -0.29036900 & 1.18827600 & $\mathrm{H}$ & -1.83164600 & 3.26449000 & 0.55173200 \\
\hline $\mathrm{C}$ & 4.29633400 & -1.22256200 & -0.65971100 & $\mathrm{H}$ & -1.96434800 & 3.46220100 & -1.20778200 \\
\hline $\mathrm{C}$ & 6.74777400 & -0.60391100 & 0.52187900 & $\mathrm{H}$ & -3.27794900 & 3.99690600 & -0.16100500 \\
\hline $\mathrm{H}$ & 5.57067800 & 0.19644400 & 2.15712600 & $\mathrm{C}$ & -4.19155300 & 1.81607200 & -1.57548600 \\
\hline $\mathrm{C}$ & 5.48755600 & -1.52403600 & -1.31184700 & $\mathrm{H}$ & -4.72113800 & 0.86139500 & -1.65800100 \\
\hline $\mathrm{H}$ & 3.34263700 & -1.45374800 & -1.12165300 & $\mathrm{H}$ & -4.95365300 & 2.59105600 & -1.43524500 \\
\hline $\mathrm{C}$ & 6.73146500 & -1.22374100 & -0.73235300 & $\mathrm{H}$ & -3.69440900 & 2.02236900 & -2.52757700 \\
\hline
\end{tabular}




\begin{tabular}{|c|c|c|c|c|c|c|c|}
\hline $\mathrm{C}$ & -3.97985200 & 1.57575400 & 0.92834700 & $\mathrm{C}$ & -3.24194200 & -1.57411200 & -0.66248100 \\
\hline $\mathrm{H}$ & -4.70664200 & 2.37833000 & 1.09372200 & $\mathrm{C}$ & 3.23792800 & -1.78863300 & -0.44096500 \\
\hline $\mathrm{H}$ & -4.54080200 & 0.63531900 & 0.89601200 & $\mathrm{C}$ & -2.64969900 & -2.97982300 & -0.88650100 \\
\hline $\mathrm{H}$ & -3.30563100 & 1.55223800 & 1.78996800 & $\mathrm{H}$ & -3.46676400 & -3.70618600 & -0.94713700 \\
\hline $\mathrm{C}$ & 2.78669600 & -1.04154200 & -0.40033600 & $\mathrm{H}$ & -2.08510400 & -3.05600900 & -1.82127300 \\
\hline $\mathrm{C}$ & 2.97255000 & -1.77757500 & 0.77881700 & $\mathrm{H}$ & -1.99873800 & -3.28750100 & -0.06092100 \\
\hline $\mathrm{C}$ & 3.98510400 & -2.58055600 & -1.62343600 & $\mathrm{C}$ & -4.17048700 & -1.18346200 & -1.83047700 \\
\hline $\mathrm{C}$ & 3.71909600 & -2.95478600 & 0.72718000 & $\mathrm{H}$ & -5.03277700 & -1.85859200 & -1.83162600 \\
\hline $\mathrm{H}$ & 2.53942400 & -1.42856800 & 1.71041000 & $\mathrm{H}$ & -4.55096200 & -0.16282200 & -1.73467000 \\
\hline $\mathrm{C}$ & 4.23700000 & -3.36866600 & -0.49916100 & $\mathrm{H}$ & -3.68347700 & -1.28199500 & -2.80369900 \\
\hline $\mathrm{H}$ & 4.36608300 & -2.87258100 & -2.59988700 & $\mathrm{C}$ & -4.03734300 & -1.54751700 & 0.65503700 \\
\hline $\mathrm{H}$ & 3.88915900 & -3.53857300 & 1.62711500 & $\mathrm{H}$ & -4.48922700 & -0.57123500 & 0.84837900 \\
\hline $\mathrm{H}$ & 4.81984400 & -4.27951900 & -0.58853600 & $\mathrm{H}$ & -4.85121600 & -2.27645500 & 0.58652200 \\
\hline $\mathrm{C}$ & -2.70214300 & -1.07962900 & -0.45176200 & $\mathrm{H}$ & -3.43053800 & -1.83251000 & 1.51916100 \\
\hline $\mathrm{C}$ & -3.49839000 & -1.71387900 & -1.40507800 & $\mathrm{C}$ & 2.54727000 & -3.15144600 & -0.66563100 \\
\hline $\mathrm{C}$ & -3.07313900 & -2.91119000 & 1.04838400 & $\mathrm{H}$ & 1.82684000 & -3.37730000 & 0.12643600 \\
\hline $\mathrm{C}$ & -4.08700100 & -2.94835100 & -1.11807000 & $\mathrm{H}$ & 2.04123600 & -3.21853500 & -1.63378500 \\
\hline $\mathrm{H}$ & -3.66179300 & -1.24320200 & -2.36686500 & $\mathrm{H}$ & 3.31181700 & -3.93495000 & -0.64961800 \\
\hline $\mathrm{C}$ & -3.87902000 & -3.55557300 & 0.12467800 & $\mathrm{C}$ & 4.25668600 & -1.50490300 & -1.56274900 \\
\hline $\mathrm{H}$ & -2.84124300 & -3.30744600 & 2.03022400 & $\mathrm{H}$ & 4.74706200 & -0.53166400 & -1.44933500 \\
\hline $\mathrm{H}$ & -4.71044600 & -3.43573300 & -1.86137900 & $\mathrm{H}$ & 5.04762000 & -2.25957500 & -1.50890100 \\
\hline $\mathrm{H}$ & -4.32405900 & -4.51234200 & 0.37071500 & $\mathrm{H}$ & 3.82141300 & -1.57390300 & -2.56308200 \\
\hline $\mathrm{N}$ & 3.28197200 & -1.44181800 & -1.58272400 & $\mathrm{C}$ & 3.94707300 & -1.78063000 & 0.92454900 \\
\hline $\mathrm{N}$ & -2.52200200 & -1.72495800 & 0.72886300 & $\mathrm{H}$ & 4.68929000 & -2.58506500 & 0.93011600 \\
\hline $\mathrm{Pd}$ & -0.11336300 & 0.41816900 & 1.16269700 & $\mathrm{H}$ & 4.48455000 & -0.84642300 & 1.11347200 \\
\hline $\mathrm{H}$ & -1.87940500 & -1.22910400 & 1.38653300 & $\mathrm{H}$ & 3.25980700 & -1.96933200 & 1.75385700 \\
\hline $\mathrm{C}$ & -0.07161700 & 0.07522600 & 3.06509900 & $\mathrm{C}$ & -2.49582400 & 1.32991100 & -0.17373200 \\
\hline \multirow[t]{2}{*}{$\mathrm{O}$} & -0.01232400 & -0.10258400 & 4.20246800 & $\mathrm{C}$ & -2.79594100 & 1.71739000 & 1.13839500 \\
\hline & & & & $\mathrm{C}$ & -3.28083500 & 3.31760200 & -1.02492800 \\
\hline $5 \mathbf{a}$ & & & & $\mathrm{C}$ & -3.38467300 & 2.96701500 & 1.34540600 \\
\hline $\mathrm{C}$ & -1.21459700 & -0.13519600 & -2.30596500 & $\mathrm{H}$ & -2.59197200 & 1.06125400 & 1.97797900 \\
\hline $\mathrm{H}$ & -1.16165100 & -1.12671600 & -2.76733800 & $\mathrm{C}$ & -3.63571100 & 3.78290600 & 0.24390700 \\
\hline $\mathrm{H}$ & -2.00412000 & 0.43065600 & -2.80563300 & $\mathrm{H}$ & -3.45352900 & 3.92678500 & -1.90871200 \\
\hline $\mathrm{C}$ & 0.11396800 & 0.61359700 & -2.50156900 & $\mathrm{H}$ & -3.64432300 & 3.29286300 & 2.34786800 \\
\hline $\mathrm{H}$ & 0.15832800 & 0.92781700 & -3.54988300 & $\mathrm{H}$ & -4.09479000 & 4.75899700 & 0.35903400 \\
\hline $\mathrm{H}$ & 0.11751000 & 1.54036200 & -1.91531000 & $\mathrm{C}$ & 2.64519000 & 1.12497100 & 0.03028400 \\
\hline $\mathrm{C}$ & 1.37834500 & -0.23219500 & -2.23697800 & $\mathrm{C}$ & 2.71966800 & 1.55092700 & 1.35470900 \\
\hline $\mathrm{H}$ & 2.23620400 & 0.16885900 & -2.79085600 & $\mathrm{C}$ & 3.86018200 & 3.08996400 & -0.63364700 \\
\hline $\mathrm{H}$ & 1.24095700 & -1.23967100 & -2.64637800 & $\mathrm{C}$ & 3.37716100 & 2.74255900 & 1.68133800 \\
\hline$P$ & 1.83604300 & -0.48880600 & -0.45938900 & $\mathrm{H}$ & 2.25685100 & 0.94536800 & 2.12498800 \\
\hline$P$ & -1.78903000 & -0.34352300 & -0.55679500 & $\mathrm{C}$ & 3.95339900 & 3.52489800 & 0.67697600 \\
\hline
\end{tabular}


$\begin{array}{llll}4.27082700 & 3.62974100 & -1.47951500 & \text { C }\end{array}$

$\begin{array}{llll}3.43400200 & 3.05970200 & 2.71822900 & \mathrm{H}\end{array}$

$4.46351600 \quad 4.45520100 \quad 0.89934700$

$-2.72245200$

$2.11851200 \quad-1.23439600$

$\begin{array}{lll}3.22613600 & 1.92566200 & -0.90726500\end{array}$

0.04461900

1.25548800

$-1.01772900$

$-0.90165900$

1.01435300

$-1.20658600$

1.98098100

$-1.38203000$

2.60775700

$-1.53284400$

$-1.66173400$

3.58010700

3.18265300

$1.64056200 \quad-1.88179700$

$5 \mathbf{b}$

$\mathrm{C}$

$\mathrm{H}$

$\mathrm{H}$

C

$\mathrm{H}$

$\mathrm{H}$

C

$\mathrm{H}$

$\mathrm{H}$

\section{$\begin{array}{lll}-1.30243100 & -0.63987600 & -2.16021900\end{array}$}

$\begin{array}{lll}-1.17137300 & -1.71503000 & -2.32369700\end{array}$

$\begin{array}{lll}-2.08804800 & -0.36152800 & -2.87499700\end{array}$

$\begin{array}{lll}0.01139000 & 0.09691500 & -2.52910700\end{array}$

$\begin{array}{lll}0.01841600 & 0.21429800 & -3.61837200\end{array}$

$$
0.01291600
$$

$1.11295000-2.11453300$

$\begin{array}{lll}1.31685900 & -0.64728500 & -2.14432700\end{array}$

2.10809600

$-0.37055400 \quad-2.85250900$

$\begin{array}{lll}1.18169000 & -1.72271500 & -2.30334100\end{array}$

1.83771800

$-0.47535300 \quad-0.34756900$

$\begin{array}{lll}-1.84178000 & -0.48283600 & -0.36784000\end{array}$

$\begin{array}{lll}-3.00481300 & -1.98545300 & -0.08541500\end{array}$

$\begin{array}{lll}2.98559900 & -1.98000600 & -0.02735900\end{array}$

$\begin{array}{lll}-2.08981800 & -3.22710100 & -0.03798900\end{array}$

$\begin{array}{lll}-2.70250600 & -4.10850400 & 0.17824000\end{array}$

$\begin{array}{lll}-1.58124200 & -3.41932200 & -0.98813300\end{array}$

$\begin{array}{lll}-1.33743700 & -3.14255100 & 0.75173600\end{array}$

$\begin{array}{lll}-4.05596600 & -2.13405800 & -1.19894000\end{array}$

$\begin{array}{lll}-4.67602300 & -3.01271600 & -0.99097200\end{array}$

$\begin{array}{lll}-4.73308500 & -1.27472500 & -1.24932800\end{array}$

$\begin{array}{lll}-3.60805000 & -2.28816600 & -2.18542600\end{array}$

$\begin{array}{lll}-3.69365300 & -1.81941600 & 1.28221700\end{array}$

$\begin{array}{lll}-4.41201000 & -0.99441200 & 1.29023700\end{array}$

$\begin{array}{lll}-4.25409700 & -2.73238100 & 1.50849000\end{array}$

$\begin{array}{lll}-2.97068200 & -1.66526900 & 2.08894200\end{array}$

$2.05666700 \quad-3.20918900$

0.05996200

$1.30997000 \quad-3.09338000$

0.85137300

$1.53963500-3.41992100$

$-0.88176000$

$\begin{array}{lll}2.65964000 & -4.09207000 & 0.29668800\end{array}$
$\mathrm{C}$

$\mathrm{H}$

$\mathrm{H}$

C

$\mathrm{H}$

$\mathrm{H}$

H

C

$\mathrm{C}$

C

C

$\mathrm{H}$

$\mathrm{C}$

H

H

H

$\mathrm{C}$

C

C

C

H

C

$\mathrm{H}$

H

$\mathrm{H}$

N

N

Pd

C

O

$\mathrm{H}$

H

$5 c$

C

H

H

C

H

H

\begin{tabular}{rrr}
4.03273200 & -2.17774100 & -1.13699700 \\
4.72760100 & -1.33410600 & -1.20935200 \\
4.63721700 & -3.06141600 & -0.90574000 \\
3.58175900 & -2.35044700 & -2.11911900 \\
3.67971000 & -1.77653300 & 1.33281100 \\
4.24043600 & -2.68287100 & 1.58368600 \\
4.39725100 & -0.95061800 & 1.31563900 \\
2.95948000 & -1.59867800 & 2.13697100 \\
-3.04918900 & 0.94598800 & -0.40421800 \\
-3.43873100 & 1.57291300 & 0.78418100 \\
-4.43443000 & 2.49927400 & -1.61673700 \\
-4.33945200 & 2.63663400 & 0.76702500 \\
-3.01075000 & 1.22167900 & 1.71597100 \\
\hline-4.83993500 & 3.11646700 & -0.45153500 \\
-4.76227700 & 2.79886600 & -2.60535000 \\
-4.64271200 & 3.10260400 & 1.69951400 \\
-5.52675700 & 3.95351400 & -0.49582900 \\
\hline 3.06173900 & 0.94378400 & -0.39277700 \\
\hline 3.34658000 & 1.66521400 & 0.76969900 \\
\hline 4.60199600 & 2.35725400 & -1.58781300 \\
\hline 4.27050900 & 2.71053300 & 0.75237400 \\
\hline 2.82090600 & 1.39886900 & 1.67972200 \\
\hline 4.90264700 & 3.07082500 & -0.44478300 \\
\hline 5.03729000 & 2.56110600 & -2.55926700 \\
4.49030600 & 3.25266200 & 1.66697800 \\
\hline 5.61194400 & 3.88882700 & -0.49171800 \\
-3.57800200 & 1.44761900 & -1.55374000 \\
3.71675800 & 1.33135200 & -1.52186600 \\
-0.03460700 & 0.53412300 & 2.99063100 \\
\hline .05209700 & 0.87674600 & 4.08497700 \\
\hline 32689900 & 0.99935800 & -2.42937100 \\
\hline & & -2.37659300 \\
\hline
\end{tabular}

$\begin{array}{rrr}1.22377700 & -0.30285600 & 2.26361000 \\ 1.13728400 & -1.34042600 & 2.60319900 \\ 1.99574300 & 0.15076900 & 2.89296700 \\ -0.10942600 & 0.43966100 & 2.50490400 \\ -0.15336400 & 0.66953100 & 3.57442100 \\ -0.11861500 & 1.40942600 & 1.99271700\end{array}$




\begin{tabular}{|c|c|c|c|c|c|c|c|}
\hline $\mathrm{C}$ & -1.38042900 & -0.37614400 & 2.17289900 & $\mathrm{C}$ & -3.00596000 & 1.96770500 & -0.84053900 \\
\hline $\mathrm{H}$ & -2.20538100 & -0.01468100 & 2.79527000 & $\mathrm{C}$ & -4.90997100 & 2.31976200 & 1.12632500 \\
\hline $\mathrm{H}$ & -1.24067000 & -1.42451500 & 2.45829900 & $\mathrm{C}$ & -3.92474300 & 3.02517400 & -0.93409100 \\
\hline $\mathrm{P}$ & -1.91537200 & -0.39717700 & 0.38745200 & $\mathrm{H}$ & -2.26002500 & 1.82236900 & -1.61245200 \\
\hline $\mathrm{P}$ & 1.81371900 & -0.37431200 & 0.49096900 & $\mathrm{C}$ & -4.88700700 & 3.20617300 & 0.05915800 \\
\hline $\mathrm{C}$ & 2.92845200 & -1.92528400 & 0.34654400 & $\mathrm{H}$ & -5.62287000 & 2.37868300 & 1.94208100 \\
\hline $\mathrm{C}$ & -3.02623800 & -1.93227700 & 0.13089600 & $\mathrm{H}$ & -3.88536700 & 3.70024200 & -1.78399400 \\
\hline $\mathrm{C}$ & 1.97004400 & -3.13470100 & 0.40360700 & $\mathrm{H}$ & -5.61126300 & 4.01240700 & 0.01525600 \\
\hline $\mathrm{H}$ & 2.55269500 & -4.04782500 & 0.24471000 & $\mathrm{~N}$ & 3.96449600 & 1.27404200 & 1.29923700 \\
\hline $\mathrm{H}$ & 1.47440800 & -3.24166900 & 1.37354100 & $\mathrm{~N}$ & -4.00947400 & 1.31195000 & 1.17928900 \\
\hline $\mathrm{H}$ & 1.20910400 & -3.09533300 & -0.38239100 & $\mathrm{Pd}$ & -0.10160600 & -0.28541200 & -1.14555100 \\
\hline $\mathrm{C}$ & 3.96502800 & -2.02268000 & 1.48433100 & $\mathrm{H}$ & -1.34302400 & -0.28240700 & -2.08394300 \\
\hline $\mathrm{H}$ & 4.44781500 & -3.00409400 & 1.43021900 & $\mathrm{C}$ & 0.89246400 & -0.29474900 & -2.88110700 \\
\hline $\mathrm{H}$ & 4.77415100 & -1.29252900 & 1.37862600 & $\mathrm{O}$ & 1.36818200 & -0.31417200 & -3.90897300 \\
\hline $\mathrm{H}$ & 3.52503500 & -1.94972500 & 2.48398800 & $\mathrm{H}$ & -4.08469400 & 0.67420500 & 1.96744500 \\
\hline $\mathrm{C}$ & 3.64378700 & -1.88832300 & -1.01762600 & $\mathrm{H}$ & 4.06275100 & 0.59243100 & 2.04741900 \\
\hline $\mathrm{H}$ & 4.30601100 & -1.02373200 & -1.12673400 & & & & \\
\hline $\mathrm{H}$ & 4.26747000 & -2.78298500 & -1.11198300 & 6 & & & \\
\hline $\mathrm{H}$ & 2.94161600 & -1.90212600 & -1.85560300 & $\mathrm{C}$ & -0.53376000 & 3.10164800 & -0.66504500 \\
\hline $\mathrm{C}$ & -2.07233800 & -3.14536400 & 0.10165300 & $\mathrm{H}$ & -0.91577400 & 3.73673200 & 0.14211500 \\
\hline $\mathrm{H}$ & -1.34747100 & -3.07275500 & -0.71546900 & $\mathrm{H}$ & 0.00015400 & 3.74240900 & -1.37136700 \\
\hline $\mathrm{H}$ & -1.53652900 & -3.29311500 & 1.04468600 & $\mathrm{C}$ & -1.69955200 & 2.40925100 & -1.39127900 \\
\hline $\mathrm{H}$ & -2.66644600 & -4.04858500 & -0.07062500 & $\mathrm{H}$ & -2.20273100 & 3.17088900 & -1.99782000 \\
\hline $\mathrm{C}$ & -4.04673700 & -2.08237400 & 1.27857200 & $\mathrm{H}$ & -1.32238100 & 1.67039200 & -2.10841000 \\
\hline $\mathrm{H}$ & -4.81112000 & -1.29833800 & 1.26772400 & $\mathrm{C}$ & -2.77226600 & 1.78141800 & -0.48454400 \\
\hline $\mathrm{H}$ & -4.58636000 & -3.02398900 & 1.13450500 & $\mathrm{H}$ & -3.68153200 & 1.61254300 & -1.06774700 \\
\hline $\mathrm{H}$ & -3.58576000 & -2.13605700 & 2.26909500 & $\mathrm{H}$ & -3.01932500 & 2.46138800 & 0.33804600 \\
\hline $\mathrm{C}$ & -3.75944300 & -1.80540500 & -1.21726600 & $\mathrm{P}$ & -2.36778200 & 0.13164200 & 0.25101600 \\
\hline $\mathrm{H}$ & -4.36021300 & -2.70781900 & -1.36878700 & $\mathrm{P}$ & 0.73615700 & 1.95639400 & 0.05635800 \\
\hline $\mathrm{H}$ & -4.44697400 & -0.95473500 & -1.24706000 & $\mathrm{C}$ & 1.89054200 & 3.10413400 & 1.05239800 \\
\hline $\mathrm{H}$ & -3.07016000 & -1.73275600 & -2.06315500 & $\mathrm{C}$ & -3.76869700 & -0.21374600 & 1.49464100 \\
\hline $\mathrm{C}$ & 2.97149200 & 1.09622500 & 0.37579000 & $\mathrm{C}$ & 1.19775600 & 3.39774300 & 2.39867800 \\
\hline $\mathrm{C}$ & 2.89652800 & 2.03630300 & -0.64574700 & $\mathrm{H}$ & 1.83867200 & 4.04925800 & 3.00323300 \\
\hline $\mathrm{C}$ & 4.86316300 & 2.28417900 & 1.27201600 & $\mathrm{H}$ & 0.24211700 & 3.91627300 & 2.26782800 \\
\hline $\mathrm{C}$ & 3.81076300 & 3.09940400 & -0.71288400 & $\mathrm{H}$ & 1.01156600 & 2.48605600 & 2.97413800 \\
\hline $\mathrm{H}$ & 2.12053100 & 1.94228400 & -1.39552200 & $\mathrm{C}$ & 2.16660700 & 4.42796200 & 0.31368200 \\
\hline $\mathrm{C}$ & 4.80625600 & 3.22691400 & 0.25561400 & $\mathrm{H}$ & 2.89779200 & 5.00802600 & 0.88867600 \\
\hline $\mathrm{H}$ & 5.60201900 & 2.30039200 & 2.06638200 & $\mathrm{H}$ & 2.57245300 & 4.26310800 & -0.68690700 \\
\hline $\mathrm{H}$ & 3.74207100 & 3.82175700 & -1.52100400 & $\mathrm{H}$ & 1.26701900 & 5.04252100 & 0.21995100 \\
\hline $\mathrm{H}$ & 5.52874700 & 4.03547600 & 0.23152900 & $\mathrm{C}$ & 3.22170800 & 2.36656900 & 1.29642200 \\
\hline $\mathrm{C}$ & -3.04664300 & 1.08748100 & 0.23430500 & $\mathrm{H}$ & 3.76476500 & 2.19636300 & 0.36190700 \\
\hline
\end{tabular}




\begin{tabular}{|c|c|c|c|c|c|c|c|}
\hline $\mathrm{H}$ & 3.86042600 & 2.98076400 & 1.94105100 & $\mathrm{C}$ & 4.56329900 & -3.24931600 & 0.08766100 \\
\hline $\mathrm{H}$ & 3.07692900 & 1.40247000 & 1.79239000 & $\mathrm{H}$ & 4.29965100 & -1.20675500 & 0.72810100 \\
\hline $\mathrm{C}$ & -3.45386700 & 0.57125300 & 2.78308800 & $\mathrm{C}$ & 4.02874200 & -4.54145700 & 0.09754600 \\
\hline $\mathrm{H}$ & -2.51423800 & 0.23749300 & 3.23370400 & $\mathrm{H}$ & 2.41098700 & -5.80808700 & 0.75716700 \\
\hline $\mathrm{H}$ & -3.39106500 & 1.65057600 & 2.60930800 & $\mathrm{H}$ & 5.51026900 & -3.05591000 & -0.40617900 \\
\hline $\mathrm{H}$ & -4.25600200 & 0.40529600 & 3.51061100 & $\mathrm{H}$ & 4.56117500 & -5.34937900 & -0.39499300 \\
\hline $\mathrm{C}$ & -5.12591200 & 0.23592500 & 0.91285400 & $\mathrm{C}$ & 1.37946500 & -0.52996200 & 2.66018400 \\
\hline $\mathrm{H}$ & -5.33991300 & -0.23780200 & -0.04863400 & $\mathrm{H}$ & 1.19153300 & 0.07639400 & 3.52488800 \\
\hline $\mathrm{H}$ & -5.91783700 & -0.04892200 & 1.61404900 & $\mathrm{H}$ & -0.95560800 & -1.33264700 & 1.68825400 \\
\hline $\mathrm{H}$ & -5.18276500 & 1.31956000 & 0.78205600 & & & & \\
\hline $\mathrm{C}$ & -3.82274900 & -1.72017300 & 1.80503000 & 7 & & & \\
\hline $\mathrm{H}$ & -4.58575500 & -1.89465600 & 2.57133900 & $\mathrm{C}$ & -2.45308900 & -0.12424400 & -2.00625400 \\
\hline $\mathrm{H}$ & -4.09596000 & -2.31078400 & 0.92603900 & $\mathrm{H}$ & -1.97327800 & -0.39416300 & -2.95353900 \\
\hline $\mathrm{H}$ & -2.87139500 & -2.09439400 & 2.19531200 & $\mathrm{H}$ & -3.50236800 & -0.42594800 & -2.07384300 \\
\hline $\mathrm{C}$ & 1.69436600 & 1.49124500 & -1.46815800 & $\mathrm{C}$ & -2.35189200 & 1.39480400 & -1.78891200 \\
\hline $\mathrm{C}$ & 2.18055500 & 0.18879400 & -1.63170800 & $\mathrm{H}$ & -3.07887000 & 1.87132600 & -2.45640800 \\
\hline $\mathrm{C}$ & 2.57003300 & 2.14552200 & -3.49299300 & $\mathrm{H}$ & -2.66701100 & 1.66602000 & -0.77371800 \\
\hline $\mathrm{C}$ & 2.89897000 & -0.12083600 & -2.78812600 & $\mathrm{C}$ & -0.97651500 & 2.00476300 & -2.11185400 \\
\hline $\mathrm{H}$ & 1.99445800 & -0.56316700 & -0.87259100 & $\mathrm{H}$ & -1.07686500 & 3.08505500 & -2.24759200 \\
\hline $\mathrm{C}$ & 3.10001700 & 0.87619100 & -3.73958700 & $\mathrm{H}$ & -0.58787200 & 1.58085100 & -3.04404900 \\
\hline $\mathrm{H}$ & 2.70076600 & 2.94934100 & -4.21410700 & $\mathrm{P}$ & 0.32976100 & 1.81330700 & -0.81339500 \\
\hline $\mathrm{H}$ & 3.28911600 & -1.12274600 & -2.94099200 & $\mathrm{P}$ & -1.69876800 & -1.16854700 & -0.66662900 \\
\hline $\mathrm{H}$ & 3.64886300 & 0.68199600 & -4.65518200 & $\mathrm{C}$ & -1.60898000 & -2.91273700 & -1.43550800 \\
\hline $\mathrm{C}$ & -2.63189900 & -1.02711200 & -1.17199300 & $\mathrm{C}$ & 1.95712100 & 2.31905400 & -1.66190100 \\
\hline $\mathrm{C}$ & -1.87725000 & -2.19710100 & -1.31370700 & $\mathrm{C}$ & -0.33063200 & -2.94800300 & -2.29901800 \\
\hline $\mathrm{C}$ & -3.85388800 & -1.48759700 & -3.06366000 & $\mathrm{H}$ & -0.21828700 & -3.94513500 & -2.73962100 \\
\hline $\mathrm{C}$ & -2.15286800 & -3.03832900 & -2.39329900 & $\mathrm{H}$ & -0.37047800 & -2.23200600 & -3.12704000 \\
\hline $\mathrm{H}$ & -1.09980000 & -2.43574800 & -0.59580400 & $\mathrm{H}$ & 0.56543600 & -2.73460500 & -1.70724400 \\
\hline $\mathrm{C}$ & -3.15930000 & -2.67898400 & -3.28702000 & $\mathrm{C}$ & -2.83715300 & -3.23680100 & -2.30861900 \\
\hline $\mathrm{H}$ & -4.64442300 & -1.17101000 & -3.74005900 & $\mathrm{H}$ & -2.74164200 & -4.26338000 & -2.68131300 \\
\hline $\mathrm{H}$ & -1.58878200 & -3.95552600 & -2.53335800 & $\mathrm{H}$ & -3.76732800 & -3.15840000 & -1.74315100 \\
\hline $\mathrm{H}$ & -3.40407400 & -3.30147300 & -4.14125000 & $\mathrm{H}$ & -2.90926500 & -2.58249000 & -3.18171400 \\
\hline $\mathrm{N}$ & 1.88290200 & 2.45244100 & -2.38718700 & $\mathrm{C}$ & -1.49754400 & -3.95489100 & -0.30592000 \\
\hline $\mathrm{N}$ & -3.60112500 & -0.67501000 & -2.03134800 & $\mathrm{H}$ & -2.38065000 & -3.95394700 & 0.33956100 \\
\hline $\mathrm{Pd}$ & -0.24034100 & -0.05869000 & 1.13616000 & $\mathrm{H}$ & -1.41655800 & -4.95395600 & -0.74869000 \\
\hline $\mathrm{C}$ & 1.99267500 & -1.42118900 & 2.06890000 & $\mathrm{H}$ & -0.60865700 & -3.79922800 & 0.31119900 \\
\hline $\mathrm{C}$ & 2.66374800 & -2.46453000 & 1.37671700 & $\mathrm{C}$ & 2.43348400 & 1.11866000 & -2.50336700 \\
\hline $\mathrm{C}$ & 2.13387100 & -3.77340500 & 1.38642100 & $\mathrm{H}$ & 2.62056000 & 0.24083200 & -1.87709800 \\
\hline $\mathrm{C}$ & 3.88820600 & -2.21066400 & 0.72106100 & $\mathrm{H}$ & 1.71248800 & 0.84230200 & -3.27993100 \\
\hline $\mathrm{C}$ & 2.81629900 & -4.80138900 & 0.74547600 & $\mathrm{H}$ & 3.36967000 & 1.38159600 & -3.00819100 \\
\hline $\mathrm{H}$ & 1.19916700 & -3.96481900 & 1.90320700 & $\mathrm{C}$ & 1.73660700 & 3.55235100 & -2.56307400 \\
\hline
\end{tabular}




\begin{tabular}{|c|c|c|c|c|c|c|c|}
\hline $\mathrm{H}$ & 1.31743500 & 4.39642100 & -2.00973400 & $\mathrm{H}$ & -0.19156700 & -2.31884500 & 2.19879000 \\
\hline $\mathrm{H}$ & 2.70347900 & 3.86294300 & -2.97400400 & $\mathrm{H}$ & 1.67555700 & 0.49528500 & 0.79218100 \\
\hline $\mathrm{H}$ & 1.07929200 & 3.33757000 & -3.40958200 & & & & \\
\hline $\mathrm{C}$ & 3.01218200 & 2.65345100 & -0.59259000 & 8 & & & \\
\hline $\mathrm{H}$ & 3.95733300 & 2.89085500 & -1.09290200 & $\mathrm{C}$ & -2.76346800 & 1.78992400 & 0.63308500 \\
\hline $\mathrm{H}$ & 2.72871700 & 3.52292700 & 0.00740500 & $\mathrm{H}$ & -2.76804300 & 2.60626400 & -0.09784400 \\
\hline $\mathrm{H}$ & 3.19710000 & 1.81086500 & 0.08051300 & $\mathrm{H}$ & -3.76427600 & 1.72501300 & 1.06759400 \\
\hline $\mathrm{C}$ & -3.08223800 & -1.19745000 & 0.57505300 & $\mathrm{C}$ & -1.73856600 & 2.07990200 & 1.75084500 \\
\hline $\mathrm{C}$ & -2.86255800 & -0.81465600 & 1.90365100 & $\mathrm{H}$ & -2.21335800 & 2.74627800 & 2.48051700 \\
\hline $\mathrm{C}$ & -5.31190500 & -1.59212800 & 0.98660200 & $\mathrm{H}$ & -1.50034000 & 1.16016300 & 2.29906400 \\
\hline $\mathrm{C}$ & -3.93686900 & -0.83492200 & 2.79694100 & $\mathrm{C}$ & -0.44393500 & 2.78024600 & 1.28931900 \\
\hline $\mathrm{H}$ & -1.87752500 & -0.49091700 & 2.22388200 & $\mathrm{H}$ & 0.09750900 & 3.16529300 & 2.15937000 \\
\hline $\mathrm{C}$ & -5.18596500 & -1.23729900 & 2.33301700 & $\mathrm{H}$ & -0.70699300 & 3.65589900 & 0.68657000 \\
\hline $\mathrm{H}$ & -6.27374700 & -1.90234900 & 0.58435500 & $\mathrm{P}$ & 0.69591600 & 1.73176700 & 0.23886100 \\
\hline $\mathrm{H}$ & -3.79800000 & -0.53908300 & 3.83252700 & $\mathrm{P}$ & -2.46890600 & 0.18580700 & -0.26933400 \\
\hline $\mathrm{H}$ & -6.04890100 & -1.27097800 & 2.98975800 & $\mathrm{C}$ & -3.62309900 & 0.25249400 & -1.78523200 \\
\hline $\mathrm{C}$ & -0.09033900 & 3.17573900 & 0.37520300 & $\mathrm{C}$ & 1.57300600 & 2.99426000 & -0.90608500 \\
\hline $\mathrm{C}$ & 0.13687900 & 3.04864100 & 1.75033400 & $\mathrm{C}$ & -2.95381100 & 1.16889100 & -2.82851100 \\
\hline $\mathrm{C}$ & -0.90736200 & 5.31065800 & 0.62614600 & $\mathrm{H}$ & -3.57007300 & 1.20143600 & -3.73434300 \\
\hline $\mathrm{C}$ & -0.18051500 & 4.12644000 & 2.57939800 & $\mathrm{H}$ & -2.85198100 & 2.19902700 & -2.47017400 \\
\hline $\mathrm{H}$ & 0.55121600 & 2.13064500 & 2.15260700 & $\mathrm{H}$ & -1.96164000 & 0.79927400 & -3.10661500 \\
\hline $\mathrm{C}$ & -0.71315500 & 5.28038600 & 2.00932800 & $\mathrm{C}$ & -5.02604600 & 0.78229100 & -1.43938700 \\
\hline $\mathrm{H}$ & -1.32305400 & 6.19198700 & 0.14324500 & $\mathrm{H}$ & -5.65798500 & 0.73737100 & -2.33436300 \\
\hline $\mathrm{H}$ & -0.01591700 & 4.06227100 & 3.65078200 & $\mathrm{H}$ & -5.50640900 & 0.19427500 & -0.65409500 \\
\hline $\mathrm{H}$ & -0.97692600 & 6.13995600 & 2.61651600 & $\mathrm{H}$ & -5.00128600 & 1.82572300 & -1.11140400 \\
\hline $\mathrm{N}$ & -4.29168300 & -1.57025200 & 0.12297300 & $\mathrm{C}$ & -3.72434800 & -1.17434800 & -2.35805800 \\
\hline $\mathrm{N}$ & -0.60563800 & 4.28528600 & -0.17822600 & $\mathrm{H}$ & -4.22204700 & -1.85908500 & -1.66506400 \\
\hline $\mathrm{Pd}$ & 0.39843000 & -0.23594400 & 0.26165200 & $\mathrm{H}$ & -4.31249600 & -1.15338100 & -3.28246800 \\
\hline $\mathrm{C}$ & 1.89315000 & -2.05720400 & 1.43851300 & $\mathrm{H}$ & -2.73727400 & -1.58100000 & -2.59972200 \\
\hline $\mathrm{C}$ & 3.28835700 & -2.16625700 & 1.18167600 & $\mathrm{C}$ & 0.51694800 & 3.42515800 & -1.94634400 \\
\hline $\mathrm{C}$ & 3.75067400 & -2.94064500 & 0.09731800 & $\mathrm{H}$ & 0.11251900 & 2.56651100 & -2.48993100 \\
\hline $\mathrm{C}$ & 4.21554100 & -1.53826500 & 2.03993600 & $\mathrm{H}$ & -0.31660100 & 3.97521500 & -1.49813500 \\
\hline $\mathrm{C}$ & 5.11745200 & -3.07856100 & -0.12213100 & $\mathrm{H}$ & 0.98804000 & 4.09404100 & -2.67520500 \\
\hline $\mathrm{H}$ & 3.03422900 & -3.43111900 & -0.55355200 & $\mathrm{C}$ & 2.11488400 & 4.22564000 & -0.16025900 \\
\hline $\mathrm{C}$ & 5.57897500 & -1.68410500 & 1.80990100 & $\mathrm{H}$ & 2.91956200 & 3.96641300 & 0.53485200 \\
\hline $\mathrm{H}$ & 3.85450600 & -0.94825600 & 2.87589500 & $\mathrm{H}$ & 2.53644100 & 4.92915400 & -0.88712700 \\
\hline $\mathrm{C}$ & 6.03048600 & -2.45129300 & 0.73079900 & $\mathrm{H}$ & 1.33674200 & 4.76156600 & 0.39122300 \\
\hline $\mathrm{H}$ & 5.47271600 & -3.67742700 & -0.95454500 & $\mathrm{C}$ & 2.72120300 & 2.27136300 & -1.63372200 \\
\hline $\mathrm{H}$ & 6.29222400 & -1.20387100 & 2.47205100 & $\mathrm{H}$ & 3.18273300 & 2.95861000 & -2.35131500 \\
\hline $\mathrm{H}$ & 7.09628000 & -2.56277900 & 0.55659500 & $\mathrm{H}$ & 3.50961800 & 1.94232300 & -0.94821200 \\
\hline $\mathrm{C}$ & 0.68665200 & -1.97283100 & 1.69212300 & $\mathrm{H}$ & 2.35692300 & 1.40140700 & -2.18714600 \\
\hline
\end{tabular}




\begin{tabular}{|c|c|c|c|c|c|c|c|}
\hline $\mathrm{C}$ & -3.30017700 & -1.00385800 & 0.90243800 & $\mathrm{H}$ & -2.21335800 & 2.74627800 & 2.48051700 \\
\hline $\mathrm{C}$ & -2.80710200 & -2.30461000 & 1.06447700 & $\mathrm{H}$ & -1.50034000 & 1.16016300 & 2.29906400 \\
\hline $\mathrm{C}$ & -5.00746900 & -1.39548700 & 2.39765900 & $\mathrm{C}$ & -0.44393500 & 2.78024600 & 1.28931900 \\
\hline $\mathrm{C}$ & -3.47297800 & -3.17355200 & 1.93054400 & $\mathrm{H}$ & 0.09750900 & 3.16529300 & 2.15937000 \\
\hline $\mathrm{H}$ & -1.91756500 & -2.61635700 & 0.52830100 & $\mathrm{H}$ & -0.70699300 & 3.65589900 & 0.68657000 \\
\hline $\mathrm{C}$ & -4.59739000 & -2.71321800 & 2.61184800 & $\mathrm{P}$ & 0.69591600 & 1.73176700 & 0.23886100 \\
\hline $\mathrm{H}$ & -5.87618900 & -0.99535500 & 2.91633400 & $\mathrm{P}$ & -2.46890600 & 0.18580700 & -0.26933400 \\
\hline $\mathrm{H}$ & -3.11601800 & -4.18945800 & 2.07274800 & $\mathrm{C}$ & -3.62309900 & 0.25249400 & -1.78523200 \\
\hline $\mathrm{H}$ & -5.14478800 & -3.35197000 & 3.29724100 & $\mathrm{C}$ & 1.57300600 & 2.99426000 & -0.90608500 \\
\hline $\mathrm{C}$ & 2.06456100 & 1.28188500 & 1.45087400 & $\mathrm{C}$ & -2.95381100 & 1.16889100 & -2.82851100 \\
\hline $\mathrm{C}$ & 2.61410200 & 2.02013800 & 2.49877900 & $\mathrm{H}$ & -3.57007300 & 1.20143600 & -3.73434300 \\
\hline $\mathrm{C}$ & 3.59275700 & -0.50087800 & 1.92975400 & $\mathrm{H}$ & -2.85198100 & 2.19902700 & -2.47017400 \\
\hline $\mathrm{C}$ & 3.65582500 & 1.48545700 & 3.26200600 & $\mathrm{H}$ & -1.96164000 & 0.79927400 & -3.10661500 \\
\hline $\mathrm{H}$ & 2.23347000 & 3.01015300 & 2.71762800 & $\mathrm{C}$ & -5.02604600 & 0.78229100 & -1.43938700 \\
\hline $\mathrm{C}$ & 4.15981700 & 0.21442400 & 2.97249900 & $\mathrm{H}$ & -5.65798500 & 0.73737100 & -2.33436300 \\
\hline $\mathrm{H}$ & 3.90242100 & -1.49425100 & 1.62360500 & $\mathrm{H}$ & -5.50640900 & 0.19427500 & -0.65409500 \\
\hline $\mathrm{H}$ & 4.07809600 & 2.06344800 & 4.07834800 & $\mathrm{H}$ & -5.00128600 & 1.82572300 & -1.11140400 \\
\hline $\mathrm{H}$ & 4.97115100 & -0.21838900 & 3.54546400 & $\mathrm{C}$ & -3.72434800 & -1.17434800 & -2.35805800 \\
\hline $\mathrm{N}$ & -4.38045200 & -0.55639000 & 1.56543200 & $\mathrm{H}$ & -4.22204700 & -1.85908500 & -1.66506400 \\
\hline $\mathrm{N}$ & 2.57947100 & 0.04651500 & 1.23289400 & $\mathrm{H}$ & -4.31249600 & -1.15338100 & -3.28246800 \\
\hline $\mathrm{Pd}$ & -0.13894500 & -0.29745500 & -0.62668100 & $\mathrm{H}$ & -2.73727400 & -1.58100000 & -2.59972200 \\
\hline $\mathrm{C}$ & 1.23528200 & -1.90352600 & -1.13878200 & $\mathrm{C}$ & 0.51694800 & 3.42515800 & -1.94634400 \\
\hline $\mathrm{C}$ & 2.63493100 & -2.29536600 & -1.08310100 & $\mathrm{H}$ & 0.11251900 & 2.56651100 & -2.48993100 \\
\hline $\mathrm{C}$ & 3.63227300 & -1.59470600 & -1.78918400 & $\mathrm{H}$ & -0.31660100 & 3.97521500 & -1.49813500 \\
\hline $\mathrm{C}$ & 3.02501600 & -3.38579300 & -0.27502500 & $\mathrm{H}$ & 0.98804000 & 4.09404100 & -2.67520500 \\
\hline $\mathrm{C}$ & 4.96962400 & -1.97890400 & -1.70113900 & $\mathrm{C}$ & 2.11488400 & 4.22564000 & -0.16025900 \\
\hline $\mathrm{H}$ & 3.34452800 & -0.76174200 & -2.42253200 & $\mathrm{H}$ & 2.91956200 & 3.96641300 & 0.53485200 \\
\hline $\mathrm{C}$ & 4.36701900 & -3.75644300 & -0.18090300 & $\mathrm{H}$ & 2.53644100 & 4.92915400 & -0.88712700 \\
\hline $\mathrm{H}$ & 2.26368500 & -3.94090200 & 0.26493800 & $\mathrm{H}$ & 1.33674200 & 4.76156600 & 0.39122300 \\
\hline $\mathrm{C}$ & 5.34536600 & -3.05444300 & -0.89177200 & $\mathrm{C}$ & 2.72120300 & 2.27136300 & -1.63372200 \\
\hline $\mathrm{H}$ & 5.72118900 & -1.43872800 & -2.26947900 & $\mathrm{H}$ & 3.18273300 & 2.95861000 & -2.35131500 \\
\hline $\mathrm{H}$ & 4.64649500 & -4.60608300 & 0.43574200 & $\mathrm{H}$ & 3.50961800 & 1.94232300 & -0.94821200 \\
\hline $\mathrm{H}$ & 6.38768000 & -3.35045300 & -0.82455100 & $\mathrm{H}$ & 2.35692300 & 1.40140700 & -2.18714600 \\
\hline $\mathrm{C}$ & 0.01837100 & -2.22954500 & -1.33767200 & $\mathrm{C}$ & -3.30017700 & -1.00385800 & 0.90243800 \\
\hline $\mathrm{H}$ & -0.65669900 & -2.98538300 & -1.70439000 & $\mathrm{C}$ & -2.80710200 & -2.30461000 & 1.06447700 \\
\hline $\mathrm{H}$ & 2.11084900 & -0.49823000 & 0.47698800 & $\mathrm{C}$ & -5.00746900 & -1.39548700 & 2.39765900 \\
\hline 9 & & & & $\mathrm{C}$ & -3.47297800 & -3.17355200 & 1.93054400 \\
\hline $\mathrm{C}$ & -2.76346800 & 1.78992400 & 0.63308500 & $\mathrm{H}$ & -1.91756500 & -2.61635700 & 0.52830100 \\
\hline $\mathrm{H}$ & -2.76804300 & 2.60626400 & -0.09784400 & $\mathrm{C}$ & -4.59739000 & -2.71321800 & 2.61184800 \\
\hline $\mathrm{H}$ & -3.76427600 & 1.72501300 & 1.06759400 & $\mathrm{H}$ & -5.87618900 & -0.99535500 & 2.91633400 \\
\hline $\mathrm{C}$ & -1.73856600 & 2.07990200 & 1.75084500 & $\mathrm{H}$ & -3.11601800 & -4.18945800 & 2.07274800 \\
\hline
\end{tabular}




\begin{tabular}{|c|c|c|c|c|c|c|c|}
\hline $\mathrm{H}$ & -5.14478800 & -3.35197000 & 3.29724100 & $\mathrm{P}$ & 1.82185500 & 0.57523100 & -0.17011700 \\
\hline $\mathrm{C}$ & 2.06456100 & 1.28188500 & 1.45087400 & $\mathrm{C}$ & 2.99798500 & 1.88874400 & 0.55413600 \\
\hline $\mathrm{C}$ & 2.61410200 & 2.02013800 & 2.49877900 & $\mathrm{C}$ & -2.79521000 & 2.12438100 & -0.04207300 \\
\hline $\mathrm{C}$ & 3.59275700 & -0.50087800 & 1.92975400 & $\mathrm{C}$ & 2.13934600 & 3.09215400 & 0.98903900 \\
\hline $\mathrm{C}$ & 3.65582500 & 1.48545700 & 3.26200600 & $\mathrm{H}$ & 2.78326000 & 3.85433200 & 1.44206100 \\
\hline $\mathrm{H}$ & 2.23347000 & 3.01015300 & 2.71762800 & $\mathrm{H}$ & 1.62515000 & 3.56415400 & 0.14558100 \\
\hline $\mathrm{C}$ & 4.15981700 & 0.21442400 & 2.97249900 & $\mathrm{H}$ & 1.39130200 & 2.80307700 & 1.73489500 \\
\hline $\mathrm{H}$ & 3.90242100 & -1.49425100 & 1.62360500 & $\mathrm{C}$ & 4.05894100 & 2.33223700 & -0.46912500 \\
\hline $\mathrm{H}$ & 4.07809600 & 2.06344800 & 4.07834800 & $\mathrm{H}$ & 4.74625400 & 3.04013100 & 0.00845900 \\
\hline $\mathrm{H}$ & 4.97115100 & -0.21838900 & 3.54546400 & $\mathrm{H}$ & 4.64877600 & 1.49055400 & -0.84089000 \\
\hline $\mathrm{N}$ & -4.38045200 & -0.55639000 & 1.56543200 & $\mathrm{H}$ & 3.61381400 & 2.84004300 & -1.32959000 \\
\hline $\mathrm{N}$ & 2.57947100 & 0.04651500 & 1.23289400 & $\mathrm{C}$ & 3.68156800 & 1.28156500 & 1.79419500 \\
\hline $\mathrm{Pd}$ & -0.13894500 & -0.29745500 & -0.62668100 & $\mathrm{H}$ & 4.32423500 & 0.43527900 & 1.53623400 \\
\hline $\mathrm{C}$ & 1.23528200 & -1.90352600 & -1.13878200 & $\mathrm{H}$ & 4.31227300 & 2.04265800 & 2.26677500 \\
\hline $\mathrm{C}$ & 2.63493100 & -2.29536600 & -1.08310100 & $\mathrm{H}$ & 2.95226000 & 0.94839700 & 2.54032800 \\
\hline $\mathrm{C}$ & 3.63227300 & -1.59470600 & -1.78918400 & $\mathrm{C}$ & -1.80153600 & 3.29723600 & 0.07819600 \\
\hline $\mathrm{C}$ & 3.02501600 & -3.38579300 & -0.27502500 & $\mathrm{H}$ & -1.03342100 & 3.10093800 & 0.83159500 \\
\hline $\mathrm{C}$ & 4.96962400 & -1.97890400 & -1.70113900 & $\mathrm{H}$ & -1.30625800 & 3.52864900 & -0.87039000 \\
\hline $\mathrm{H}$ & 3.34452800 & -0.76174200 & -2.42253200 & $\mathrm{H}$ & -2.34716300 & 4.19644200 & 0.38409600 \\
\hline $\mathrm{C}$ & 4.36701900 & -3.75644300 & -0.18090300 & $\mathrm{C}$ & -3.87483800 & 2.44564000 & -1.09104300 \\
\hline $\mathrm{H}$ & 2.26368500 & -3.94090200 & 0.26493800 & $\mathrm{H}$ & -4.61907600 & 1.64818700 & -1.17011100 \\
\hline $\mathrm{C}$ & 5.34536600 & -3.05444300 & -0.89177200 & $\mathrm{H}$ & -4.40654000 & 3.35468100 & -0.78848200 \\
\hline $\mathrm{H}$ & 5.72118900 & -1.43872800 & -2.26947900 & $\mathrm{H}$ & -3.45635800 & 2.63332500 & -2.08456000 \\
\hline $\mathrm{H}$ & 4.64649500 & -4.60608300 & 0.43574200 & $\mathrm{C}$ & -3.44979500 & 1.86463700 & 1.32837900 \\
\hline $\mathrm{H}$ & 6.38768000 & -3.35045300 & -0.82455100 & $\mathrm{H}$ & -3.97845100 & 2.76831900 & 1.65035500 \\
\hline $\mathrm{C}$ & 0.01837100 & -2.22954500 & -1.33767200 & $\mathrm{H}$ & -4.18260100 & 1.05317200 & 1.28808200 \\
\hline $\mathrm{H}$ & -0.65669900 & -2.98538300 & -1.70439000 & $\mathrm{H}$ & -2.70631100 & 1.61822800 & 2.09244200 \\
\hline \multirow[t]{2}{*}{$\mathrm{H}$} & 2.11084900 & -0.49823000 & 0.47698800 & $\mathrm{C}$ & 2.92701500 & -0.85542900 & -0.62480200 \\
\hline & & & & $\mathrm{C}$ & 3.15003000 & -1.90059100 & 0.28120300 \\
\hline \multicolumn{4}{|c|}{ TS3 } & $\mathrm{C}$ & 4.30167600 & -1.83349100 & -2.19104800 \\
\hline \multicolumn{4}{|c|}{ imaginary frequency $=-727.19 \mathrm{~cm}^{-1}$} & $\mathrm{C}$ & 4.00458400 & -2.94022000 & -0.08777400 \\
\hline $\mathrm{C}$ & 1.35352800 & 1.19863800 & -1.86179700 & $\mathrm{H}$ & 2.66657800 & -1.89861500 & 1.25174400 \\
\hline $\mathrm{H}$ & 1.06132400 & 2.25112300 & -1.77641000 & $\mathrm{C}$ & 4.59320300 & -2.90950100 & -1.35036300 \\
\hline $\mathrm{H}$ & 2.25858900 & 1.14702200 & -2.47082400 & $\mathrm{H}$ & 4.73636800 & -1.77504100 & -3.18653800 \\
\hline $\mathrm{C}$ & 0.23889200 & 0.37866100 & -2.55251100 & $\mathrm{H}$ & 4.20118800 & -3.75964900 & 0.59702400 \\
\hline $\mathrm{H}$ & 0.38450700 & 0.46138000 & -3.63543500 & $\mathrm{H}$ & 5.25950200 & -3.69866600 & -1.68254700 \\
\hline $\mathrm{H}$ & 0.34909900 & -0.68751700 & -2.32200700 & $\mathrm{C}$ & -3.03364200 & -0.81315300 & -0.56732500 \\
\hline $\mathrm{C}$ & -1.20646600 & 0.83929900 & -2.26694800 & $\mathrm{C}$ & -3.99448600 & -1.08209600 & -1.54236900 \\
\hline $\mathrm{H}$ & -1.89539300 & 0.32261600 & -2.94312300 & $\mathrm{C}$ & -3.72541200 & -2.65269800 & 0.70051300 \\
\hline $\mathrm{H}$ & -1.29676800 & 1.90763100 & -2.48901400 & $\mathrm{C}$ & -4.83522700 & -2.18670000 & -1.37266900 \\
\hline $\mathrm{P}$ & -1.79049100 & 0.57771800 & -0.51478900 & $\mathrm{H}$ & -4.09032800 & -0.45168600 & -2.41950100 \\
\hline
\end{tabular}




\begin{tabular}{|c|c|c|c|c|c|c|c|}
\hline C & -4.70483800 & -2.98376000 & -0.23550900 & $\mathrm{H}$ & 2.19664900 & 1.66591700 & -4.15945200 \\
\hline $\mathrm{H}$ & -3.56710000 & -3.23435000 & 1.60399700 & $\mathrm{C}$ & 2.24017500 & 3.61281000 & -2.19310400 \\
\hline $\mathrm{H}$ & -5.58451000 & -2.42010500 & -2.12268200 & $\mathrm{H}$ & 2.58307500 & 4.06785600 & -1.25861700 \\
\hline $\mathrm{H}$ & -5.33988000 & -3.84829000 & -0.07659500 & $\mathrm{H}$ & 3.01932500 & 3.78220900 & -2.94406400 \\
\hline $\mathrm{N}$ & 3.49174200 & -0.82652400 & -1.84256300 & $\mathrm{H}$ & 1.34617400 & 4.14388600 & -2.53172600 \\
\hline $\mathrm{N}$ & -2.92183800 & -1.60091100 & 0.51567700 & $\mathrm{C}$ & 3.33708500 & 1.40502500 & -1.69382400 \\
\hline $\mathrm{P}$ & -0.21752000 & -0.23745500 & 1.06240400 & $\mathrm{H}$ & 4.05161500 & 1.58795300 & -2.50394200 \\
\hline $\mathrm{H}$ & -1.72756500 & -1.06557500 & 1.23050100 & $\mathrm{H}$ & 3.77137800 & 1.79122800 & -0.76873700 \\
\hline c & 0.24917400 & -1.19236600 & 2.69345400 & $\mathrm{H}$ & 3.22262900 & 0.32229500 & -1.59095600 \\
\hline \multirow[t]{2}{*}{$\mathrm{O}$} & 0.51530500 & -1.73865400 & 3.66414700 & $\mathrm{C}$ & -3.12026400 & -0.50342400 & 1.16314500 \\
\hline & & & & $\mathrm{C}$ & -2.42633800 & -1.17870000 & 2.17394500 \\
\hline \multirow{2}{*}{\multicolumn{4}{|c|}{$\begin{array}{l}\text { TS4 } \\
\text { imaginary frequency }=-201.12 \mathrm{~cm}^{-1}\end{array}$}} & $\mathrm{C}$ & -4.97782000 & -0.26248600 & 2.50041900 \\
\hline & & & & $\mathrm{C}$ & -3.06733500 & -1.39802900 & 3.39576800 \\
\hline c & -2.87411800 & 1.57917800 & -0.79921100 & $\mathrm{H}$ & -1.40723200 & -1.51243400 & 2.01177000 \\
\hline $\mathrm{H}$ & -2.97799100 & 1.71408500 & -1.88099400 & $\mathrm{C}$ & -4.36956300 & -0.93537600 & 3.56379900 \\
\hline $\mathrm{H}$ & -3.87325400 & 1.65886400 & -0.36120000 & $\mathrm{H}$ & -5.99106500 & 0.12155300 & 2.59516400 \\
\hline c & -1.96029200 & 2.67751400 & -0.23196800 & $\mathrm{H}$ & -2.55395800 & -1.91660000 & 4.19990000 \\
\hline $\mathrm{H}$ & -2.53181400 & 3.61263800 & -0.23838700 & $\mathrm{H}$ & -4.90575000 & -1.08210200 & 4.49552000 \\
\hline $\mathrm{H}$ & -1.72566500 & 2.48081400 & 0.82136200 & $\mathrm{C}$ & 1.45721000 & 2.36431300 & 0.90378900 \\
\hline c & -0.67294000 & 2.91357500 & -1.04800600 & $\mathrm{C}$ & 1.18223900 & 3.62673300 & 1.44446600 \\
\hline $\mathrm{H}$ & -0.31569600 & 3.93729800 & -0.90698400 & $\mathrm{C}$ & 2.86766300 & 1.85558000 & 2.65244900 \\
\hline $\mathrm{H}$ & -0.89292200 & 2.81858300 & -2.11682300 & $\mathrm{C}$ & 1.78822800 & 3.99039800 & 2.64928300 \\
\hline $\mathrm{P}$ & 0.72179300 & 1.74672300 & -0.68617000 & $\mathrm{H}$ & 0.51299300 & 4.32067200 & 0.95042300 \\
\hline $\mathrm{P}$ & -2.33266400 & -0.17326700 & -0.48971900 & $\mathrm{C}$ & 2.64875700 & 3.08983700 & 3.26910900 \\
\hline c & -3.32524600 & -1.17714900 & -1.77687600 & $\mathrm{H}$ & 3.53355000 & 1.12339800 & 3.10357800 \\
\hline c & 2.00715100 & 2.09553600 & -2.04667300 & $\mathrm{H}$ & 1.58762800 & 4.96203400 & 3.09025600 \\
\hline c & -2.60073100 & -1.01841800 & -3.12928700 & $\mathrm{H}$ & 3.13898000 & 3.32904200 & 4.20680800 \\
\hline $\mathrm{H}$ & -3.12763000 & -1.60192100 & -3.89260800 & $\mathrm{~N}$ & -4.37288500 & -0.04569400 & 1.32797100 \\
\hline $\mathrm{H}$ & -2.58512400 & 0.02113500 & -3.47325800 & $\mathrm{~N}$ & 2.28638400 & 1.49179000 & 1.50463400 \\
\hline $\mathrm{H}$ & -1.56854300 & -1.38012700 & -3.08154100 & $\mathrm{Pd}$ & 0.08641000 & -0.49363900 & -0.42582400 \\
\hline c & -4.77925900 & -0.67801300 & -1.89456800 & $\mathrm{C}$ & 1.27747700 & -2.48930300 & 0.04903200 \\
\hline $\mathrm{H}$ & -5.31101000 & -1.31483300 & -2.61080900 & $\mathrm{C}$ & 2.67268600 & -2.82662300 & 0.19646000 \\
\hline $\mathrm{H}$ & -5.30628800 & -0.72047000 & -0.93930600 & $\mathrm{C}$ & 3.05911800 & -4.13852600 & -0.14350900 \\
\hline $\mathrm{H}$ & -4.83768500 & 0.34723900 & -2.26913900 & $\mathrm{C}$ & 3.62455500 & -1.92831600 & 0.70894000 \\
\hline c & -3.33124200 & -2.66436700 & -1.37523700 & $\mathrm{C}$ & 4.38035200 & -4.53980200 & 0.03464700 \\
\hline $\mathrm{r}$ & -3.76281400 & -2.82553200 & -0.38273600 & $\mathrm{H}$ & 2.32394800 & -4.82864800 & -0.54433800 \\
\hline $\mathrm{H}$ & -3.94141900 & -3.22428300 & -2.09230200 & $\mathrm{C}$ & 4.94222800 & -2.34347400 & 0.88232600 \\
\hline $\mathrm{H}$ & -2.32803200 & -3.09633600 & -1.40434700 & $\mathrm{H}$ & 3.31977900 & -0.91695700 & 0.96303400 \\
\hline C & 1.46125700 & 1.50629600 & -3.36335900 & $\mathrm{C}$ & 5.32318000 & -3.64557900 & 0.54724200 \\
\hline $\mathrm{H}$ & 1.28709700 & 0.42906400 & -3.27878600 & $\mathrm{H}$ & 4.67409900 & -5.55016000 & -0.23182200 \\
\hline $\mathrm{H}$ & 0.52971100 & 1.98291100 & -3.68477100 & $\mathrm{H}$ & 5.67648800 & -1.64834000 & 1.27761700 \\
\hline
\end{tabular}




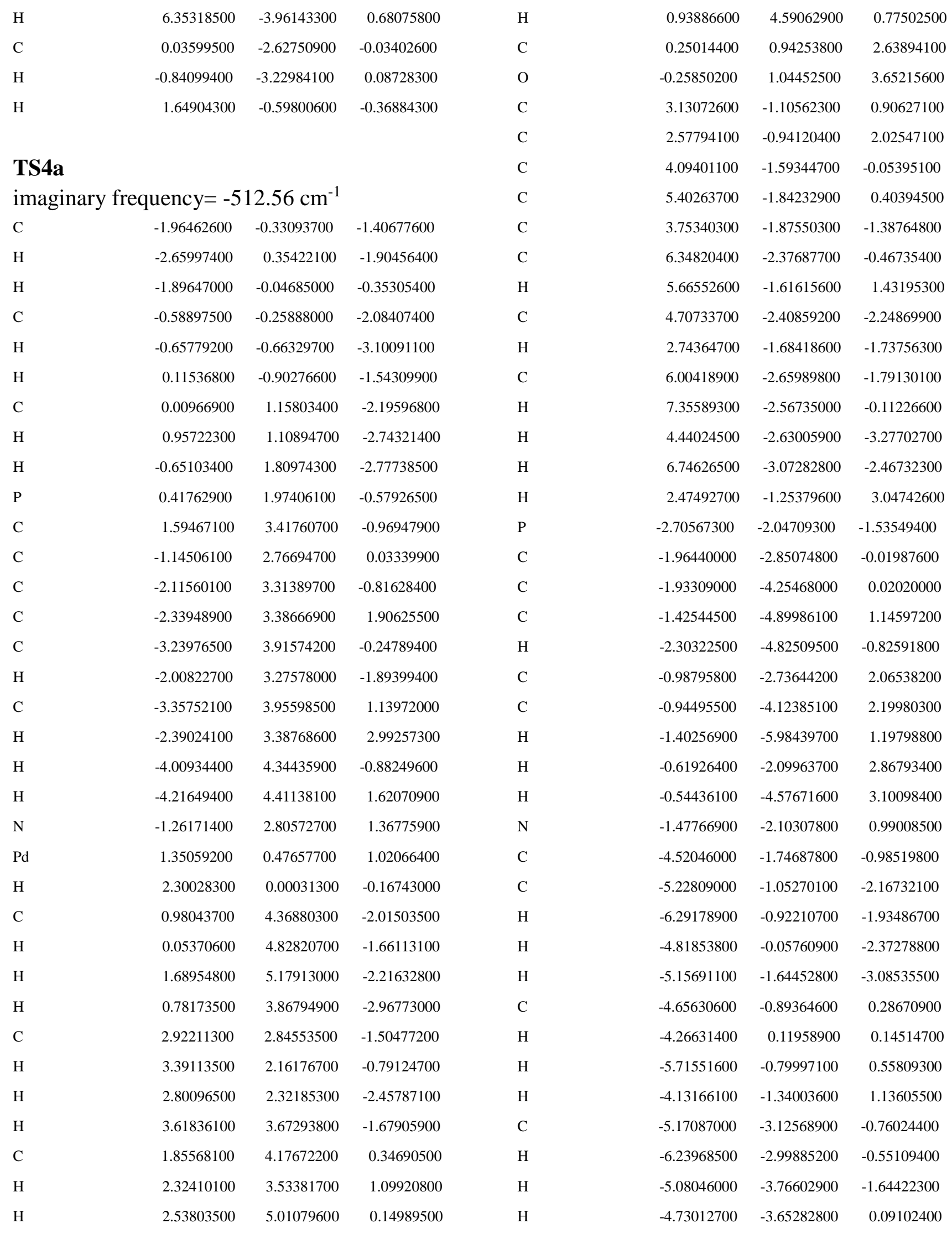


TS4-2H

imaginary frequency $=-536.52 \mathrm{~cm}^{-1}$

$\mathrm{C}$

$\mathrm{H}$

$\mathrm{H}$

C

$\mathrm{H}$

$\mathrm{H}$

C

$\mathrm{H}$

$\mathrm{H}$

P

P

C

C

C

$\mathrm{H}$

$\mathrm{H}$

$\mathrm{H}$

C

$\mathrm{H}$

$\mathrm{H}$

$\mathrm{H}$

C

$\mathrm{H}$

$\mathrm{H}$

$\mathrm{H}$

C

$\mathrm{H}$

$\mathrm{H}$

$\mathrm{H}$

C

$\mathrm{H}$

$\mathrm{H}$

$\mathrm{H}$

C

$\mathrm{H}$

$\mathrm{H}$

$\mathrm{H}$

C

C

$$
2.90933500 \quad-1.46020600 \quad-0.89240900
$$$$
2.94096800 \quad-1.59581200 \quad-1.97812300
$$$$
3.94062700 \quad-1.46206600 \quad-0.52762500
$$$$
\begin{array}{lll}
2.13648800 & -2.62023100 & -0.24558300
\end{array}
$$$$
2.78366200 \quad-3.50346400 \quad-0.27408800
$$$$
1.95365300 \quad-2.42357300
$$$$
0.82962200-2.99694100
$$$$
0.54119600-4.02571200
$$$$
\begin{array}{lll}
0.98527500 & -2.96635000 & -2.05601100
\end{array}
$$$$
-0.60032600 \quad-1.87171500 \quad-0.63155000
$$$$
\begin{array}{lll}
2.29525300 & 0.25874300 & -0.54129100
\end{array}
$$$$
3.08618000
$$$$
-1.94439800
$$$$
2.30084100
$$$$
1.32047100 \quad-1.91890500
$$$$
-2.26366000-1.92588700
$$$$
1.05750400-3.21937600
$$$$
2.72349300
$$$$
2.36576300
$$$$
1.66943400 \quad-4.02332300
$$$$
0.01436400 \quad-3.54542800
$$$$
1.24391900
$$$$
4.57061200
$$$$
1.32682200-3.11916700
$$$$
0.94385600-2.10542400
$$$$
5.00117200
$$$$
5.14834200
$$$$
1.60292400-2.86696300
$$$$
1.06765100-1.18687700
$$$$
4.70273700
$$$$
2.98081700
$$$$
-0.08266900 \quad-2.45607000
$$$$
2.81231900-1.54947500
$$$$
3.43957000 \quad 3.03904200 \quad-0.58251900
$$$$
3.51090300
$$$$
3.40011100 \quad-2.30589200
$$$$
1.94492100 \quad 3.16072600 \quad-1.54832600
$$$$
-1.48664900
$$$$
-1.56180600 \quad-3.22316100
$$$$
-1.37141600
$$$$
-0.54581100
$$$$
-0.48255000 \quad-3.08365700
$$$$
-1.96461100 \quad-3.61089400
$$$$
\begin{array}{lll}
-2.24597600 & -1.72141900 & -3.99567000
\end{array}
$$$$
\begin{array}{lll}
-2.09130200 & -3.77862400 & -2.16201800
\end{array}
$$$$
\begin{array}{lll}
-2.44022700 & -4.30956500 & -1.27107600
\end{array}
$$$$
-2.84646800
$$$$
-1.16808600
$$$$
-3.28649700
$$$$
-4.03215500
$$$$
-3.66511600
$$$$
-3.22442000
$$$$
3.19351800
$$$$
2.65004200
$$

$$
-3.93764900-2.93869500
$$$$
-4.24537700 \quad-2.51512300
$$$$
-1.67306600 \quad-1.45733400
$$$$
-1.82897300 \quad-2.24343700
$$$$
-2.16512100 \quad-0.55535800
$$$$
-0.59623200 \quad-1.27360200
$$$$
0.66382800 \quad 1.03579300
$$$$
1.51572700 \quad 2.00361100
$$

C

\section{TS5}

imaginary frequency $=-221.41 \mathrm{~cm}^{-1}$

$\begin{array}{lrrr}\mathrm{C} & -0.07432800 & 2.00104100 & 2.09872200 \\ \mathrm{H} & -0.15881000 & 1.52132700 & 3.07978900 \\ \mathrm{H} & 0.48633800 & 2.92943200 & 2.23703100\end{array}$

\begin{tabular}{rrr}
5.12971700 & 0.39169700 & 2.25002300 \\
3.41348800 & 1.81182000 & 3.13616100 \\
1.65873100 & 1.93636300 & 1.87765100 \\
4.67808300 & 1.24270400 & 3.26301300 \\
6.10790400 & -0.07841100 & 2.31369500 \\
3.02576900 & 2.47542600 & 3.90308000 \\
5.30479200 & 1.44636800 & 4.12468800 \\
-1.27062200 & -2.55412700 & 0.97996200 \\
-1.36788800 & -3.89371600 & 1.35134200 \\
-2.25492900 & -1.92832400 & 3.07717500 \\
-1.91601400 & -4.24467700 & 2.59043000 \\
-1.02018300 & -4.66767500 & 0.67861100 \\
-2.36897000 & -3.25313200 & 3.46468000 \\
-2.56932700 & -1.09166700 & 3.69022800 \\
-1.98911200 & -5.29121500 & 2.87038900 \\
-2.79693200 & -3.49763900 & 4.43000400 \\
-4.40730900 & 0.10636800 & 1.16076700 \\
-1.71800600 & -1.63263300 & 1.87452300 \\
-0.12494800 & 0.42005500 & -0.30977300 \\
-1.50014400 & 2.44283000 & 0.04325700 \\
-2.87706000 & 2.83319100 & 0.16622700 \\
-3.75498600 & 2.74990800 & -0.93398300 \\
-3.32326500 & 3.38161900 & 1.38680500 \\
-5.05741000 & 3.22086400 & -0.81212600 \\
-3.40331000 & 2.33835800 & -1.87485200 \\
-4.62873500 & 3.85315700 & 1.49252400 \\
-2.64010000 & 3.45572200 & 2.22701800 \\
-5.49463700 & 3.77156600 & 0.39803000 \\
-5.73138400 & 3.17005700 & -1.66099300 \\
-4.96955500 & 4.29056300 & 2.42528300 \\
\hline .54603700 & 3.23491700 & 0.03557100 \\
\hline & -0.65038500 & 1.59665700
\end{tabular}




\begin{tabular}{|c|c|c|c|c|c|c|c|}
\hline $\mathrm{C}$ & -1.47923600 & 2.31606000 & 1.55839500 & $\mathrm{H}$ & 2.05163000 & 2.85229700 & -3.47680300 \\
\hline $\mathrm{H}$ & -1.84075400 & 3.20866500 & 2.08142900 & $\mathrm{H}$ & 2.95370200 & 4.96642500 & -2.46326400 \\
\hline $\mathrm{H}$ & -1.43884800 & 2.58929800 & 0.49672500 & $\mathrm{C}$ & -3.49317200 & 0.28710200 & -0.83315500 \\
\hline $\mathrm{C}$ & -2.50388600 & 1.19197000 & 1.80000500 & $\mathrm{C}$ & -4.36867700 & 1.37930000 & -0.81532600 \\
\hline $\mathrm{H}$ & -3.52139900 & 1.59225900 & 1.80249900 & $\mathrm{C}$ & -4.09223800 & -0.24156500 & -2.99441700 \\
\hline $\mathrm{H}$ & -2.34825800 & 0.76889200 & 2.79770800 & $\mathrm{C}$ & -5.12183700 & 1.65456700 & -1.95935700 \\
\hline $\mathrm{P}$ & -2.43002200 & -0.22108500 & 0.60289800 & $\mathrm{H}$ & -4.47274700 & 2.01049700 & 0.05892700 \\
\hline $\mathrm{P}$ & 1.00653800 & 0.92800500 & 1.02546000 & $\mathrm{C}$ & -4.98600000 & 0.82975400 & -3.07168600 \\
\hline $\mathrm{C}$ & 2.47301800 & 0.49750300 & 2.17617100 & $\mathrm{H}$ & -3.95686300 & -0.91166900 & -3.84015800 \\
\hline $\mathrm{C}$ & -3.35608700 & -1.63738400 & 1.47779800 & $\mathrm{H}$ & -5.80336600 & 2.49958500 & -1.97375400 \\
\hline $\mathrm{C}$ & 2.10216200 & -0.81889000 & 2.88794000 & $\mathrm{H}$ & -5.55098100 & 1.00796500 & -3.98041700 \\
\hline $\mathrm{H}$ & 2.90602100 & -1.09911500 & 3.57811500 & $\mathrm{~N}$ & 2.10883400 & 3.34342100 & 0.37310800 \\
\hline $\mathrm{H}$ & 1.18476000 & -0.72416800 & 3.48001400 & $\mathrm{~N}$ & -3.35446400 & -0.50383900 & -1.91120900 \\
\hline $\mathrm{H}$ & 1.96654900 & -1.63936500 & 2.17672900 & $\mathrm{Pd}$ & -0.29395900 & -0.79514400 & -0.18525700 \\
\hline $\mathrm{C}$ & 2.74761000 & 1.60000700 & 3.21833900 & $\mathrm{C}$ & 1.36139600 & -1.98556400 & -1.41981200 \\
\hline $\mathrm{H}$ & 3.64521200 & 1.32482900 & 3.78421200 & $\mathrm{C}$ & 2.79023600 & -2.09232000 & -1.28963800 \\
\hline $\mathrm{H}$ & 2.92838800 & 2.56741200 & 2.74433800 & $\mathrm{C}$ & 3.64262500 & -1.31403000 & -2.09551400 \\
\hline $\mathrm{H}$ & 1.93462500 & 1.70984800 & 3.94090900 & $\mathrm{C}$ & 3.34271100 & -3.04109400 & -0.40724400 \\
\hline $\mathrm{C}$ & 3.73861300 & 0.29913900 & 1.32289200 & $\mathrm{C}$ & 5.02230700 & -1.48482900 & -2.01470500 \\
\hline $\mathrm{H}$ & 4.06731200 & 1.23835300 & 0.87045600 & $\mathrm{H}$ & 3.21848100 & -0.58934600 & -2.78225200 \\
\hline $\mathrm{H}$ & 4.54849600 & -0.05887700 & 1.96854500 & $\mathrm{C}$ & 4.72373400 & -3.20529400 & -0.33871700 \\
\hline $\mathrm{H}$ & 3.59799400 & -0.43754900 & 0.53171200 & $\mathrm{H}$ & 2.68476900 & -3.64818100 & 0.20611900 \\
\hline $\mathrm{C}$ & -2.47589700 & -2.11901200 & 2.64972000 & $\mathrm{C}$ & 5.56580000 & -2.42775500 & -1.13811700 \\
\hline $\mathrm{H}$ & -1.49548200 & -2.45995900 & 2.30207000 & $\mathrm{H}$ & 5.67468800 & -0.88563700 & -2.64217000 \\
\hline $\mathrm{H}$ & -2.32364900 & -1.34779100 & 3.41118700 & $\mathrm{H}$ & 5.14323700 & -3.94398800 & 0.33705600 \\
\hline $\mathrm{H}$ & -2.96901700 & -2.96404700 & 3.14238400 & $\mathrm{H}$ & 6.64182100 & -2.55909500 & -1.08149800 \\
\hline $\mathrm{C}$ & -4.72036000 & -1.14177000 & 1.99808500 & $\mathrm{C}$ & 0.21412700 & -2.19836200 & -1.85554500 \\
\hline $\mathrm{H}$ & -5.35741200 & -0.77126300 & 1.18918500 & $\mathrm{H}$ & -0.47488100 & -2.66192500 & -2.53322000 \\
\hline $\mathrm{H}$ & -5.24600100 & -1.97770500 & 2.47225400 & $\mathrm{H}$ & -1.34826600 & -1.64597000 & -0.95002900 \\
\hline
\end{tabular}

$\begin{array}{lrrc}\mathrm{C} & 2.57626200 & 0.48201200 & -0.82969200 \\ \mathrm{H} & 3.52309600 & -0.01080500 & -0.58090000 \\ \mathrm{H} & 1.91347500 & 0.41075100 & 0.03725300 \\ \mathrm{C} & 1.94826200 & -0.19146100 & -2.05811300 \\ \mathrm{H} & 2.57176100 & -0.00159900 & -2.93973000 \\ \mathrm{H} & 0.97261200 & 0.26315700 & -2.26796600 \\ \mathrm{C} & 1.77711800 & -1.72000100 & -1.94404200 \\ \mathrm{H} & 1.40299000 & -2.11327500 & -2.89570800 \\ \mathrm{H} & 2.74440900 & -2.20207200 & -1.76693600\end{array}$




\begin{tabular}{|c|c|c|c|c|c|c|c|}
\hline$P$ & 0.56181600 & -2.29177600 & -0.66232100 & C & 4.06164600 & 2.74396900 & 0.35425500 \\
\hline $\mathrm{C}$ & 0.14506300 & -4.10530700 & -1.06760100 & $\mathrm{C}$ & 5.44957400 & 2.11948200 & 0.10176400 \\
\hline $\mathrm{C}$ & 1.46863400 & -2.34925200 & 0.95574300 & $\mathrm{H}$ & 6.13692300 & 2.41146800 & 0.90481900 \\
\hline $\mathrm{C}$ & 2.82730600 & -2.67428200 & 1.06491700 & $\mathrm{H}$ & 5.41805600 & 1.02459600 & 0.08659900 \\
\hline $\mathrm{C}$ & 1.26738800 & -2.13380800 & 3.24216900 & $\mathrm{H}$ & 5.87910900 & 2.45903200 & -0.84598600 \\
\hline $\mathrm{C}$ & 3.40371100 & -2.72044600 & 2.33555900 & $\mathrm{C}$ & 3.50002000 & 2.25679900 & 1.69986100 \\
\hline $\mathrm{H}$ & 3.42943100 & -2.88712500 & 0.18935200 & $\mathrm{H}$ & 3.44927300 & 1.16443100 & 1.75092600 \\
\hline $\mathrm{C}$ & 2.61208600 & -2.44584000 & 3.44847300 & $\mathrm{H}$ & 4.15242900 & 2.59127800 & 2.51648900 \\
\hline $\mathrm{H}$ & 0.61212500 & -1.90567700 & 4.07957100 & $\mathrm{H}$ & 2.49752500 & 2.64974300 & 1.89074200 \\
\hline $\mathrm{H}$ & 4.45500800 & -2.96634800 & 2.44956800 & $\mathrm{C}$ & 4.20686600 & 4.27799800 & 0.37378300 \\
\hline $\mathrm{H}$ & 3.02215400 & -2.46733200 & 4.45245400 & $\mathrm{H}$ & 4.91589200 & 4.57018900 & 1.15741200 \\
\hline $\mathrm{N}$ & 0.70891700 & -2.08574800 & 2.02764600 & $\mathrm{H}$ & 4.58996500 & 4.66146500 & -0.57802800 \\
\hline $\mathrm{Pd}$ & -1.39816000 & -0.93952100 & -0.55947300 & $\mathrm{H}$ & 3.25700800 & 4.77698500 & 0.58695700 \\
\hline $\mathrm{H}$ & -1.37810100 & -1.14852600 & -2.12165400 & $\mathrm{C}$ & -4.22275900 & 0.81554400 & -0.15058200 \\
\hline $\mathrm{C}$ & 1.42640200 & -4.95362800 & -1.18563600 & $\mathrm{C}$ & -4.92940100 & 0.09739000 & 0.83189200 \\
\hline $\mathrm{H}$ & 1.99964900 & -4.96464900 & -0.25492400 & $\mathrm{C}$ & -4.41847400 & 2.20452000 & -0.28022200 \\
\hline $\mathrm{H}$ & 1.14680900 & -5.98914600 & -1.40820500 & $\mathrm{C}$ & -5.82583400 & 0.76133300 & 1.66384400 \\
\hline $\mathrm{H}$ & 2.07898500 & -4.61294000 & -1.99532300 & $\mathrm{H}$ & -4.78266200 & -0.97394700 & 0.92660400 \\
\hline $\mathrm{C}$ & -0.63505800 & -4.15410600 & -2.39586600 & $\mathrm{C}$ & -5.31399400 & 2.85722200 & 0.56327500 \\
\hline $\mathrm{H}$ & -1.57297100 & -3.59416700 & -2.33665700 & $\mathrm{H}$ & -3.87179300 & 2.75423800 & -1.03902600 \\
\hline $\mathrm{H}$ & -0.05546200 & -3.77293500 & -3.24233400 & $\mathrm{C}$ & -6.01777800 & 2.13988900 & 1.53391200 \\
\hline $\mathrm{H}$ & -0.88535200 & -5.19648100 & -2.62052400 & $\mathrm{H}$ & -6.37651100 & 0.20315600 & 2.41420800 \\
\hline $\mathrm{C}$ & -0.74587800 & -4.64846200 & 0.06723100 & $\mathrm{H}$ & -5.46795600 & 3.92658200 & 0.45821200 \\
\hline $\mathrm{H}$ & -1.66690800 & -4.06560400 & 0.17078600 & $\mathrm{H}$ & -6.71649100 & 2.65325100 & 2.18693300 \\
\hline $\mathrm{H}$ & -1.02862400 & -5.68136700 & -0.16320700 & $\mathrm{H}$ & -2.90093000 & -0.38559400 & -3.21228700 \\
\hline
\end{tabular}

C

$\mathrm{C}$

C

$\mathrm{P}$

C

C

C

$\mathrm{H}$

C

C

$\mathrm{H}$

$\mathrm{H}$

$\mathrm{H}$

$\mathrm{N}$
$-0.23327100 \quad-4.65081600 \quad 1.03272000$

$\begin{array}{lll}-1.48729700 & -0.54609400 & 1.42567400\end{array}$

$\begin{array}{lll}-1.65955400 & -0.19783700 & 2.49494800\end{array}$

$\begin{array}{lll}-2.84416600 & -0.24484300 & -2.15004700\end{array}$

$\begin{array}{lll}-3.33260700 & 0.14601500 & -1.06480900\end{array}$

$\begin{array}{lll}2.97412400 & 2.28289100 & -1.16021500\end{array}$

1.34042300

$3.07826800-0.72578900$

$\begin{array}{lll}1.09773000 & 4.37109600 & -1.21968400\end{array}$

$-0.08899300$

$5.02213300-0.89030500$

$\begin{array}{lll}1.83629500 & 4.85136900 & -1.85369500\end{array}$

$-0.70810300$

3.06098900

0.33178300

$-1.01506100$

4.35459400

$-0.08983700$

$-0.28739300$

6.02631600

$-1.25336700$

$-1.41324200$

2.50972800

0.95083700

$-1.95155400$

4.81862400

0.20249100

$\begin{array}{lll}0.43370700 & 2.42683700 & 0.02868000\end{array}$
TS5-2H

imaginary frequency $=-420.96 \mathrm{~cm}^{-1}$

$\begin{array}{lrrr}\mathrm{C} & -0.27674200 & 1.54534900 & 2.43455700 \\ \mathrm{H} & -0.18113200 & 0.88596800 & 3.30380300 \\ \mathrm{H} & 0.12743100 & 2.52179900 & 2.70939500 \\ \mathrm{C} & -1.75727500 & 1.71988200 & 2.06998600 \\ \mathrm{H} & -2.20428500 & 2.38223500 & 2.81918700 \\ \mathrm{H} & -1.86157500 & 2.24743000 & 1.11390200 \\ \mathrm{C} & -2.57831100 & 0.41743300 & 2.07908800 \\ \mathrm{H} & -3.64476400 & 0.63401800 & 2.19370000 \\ \mathrm{H} & -2.29871400 & -0.19702500 & 2.94255100 \\ \mathrm{P} & -2.35005700 & -0.65411200 & 0.59095900 \\ \mathrm{P} & 0.86816300 & 0.91097300 & 1.11276800 \\ \mathrm{C} & 2.50649400 & 0.65466700 & 2.07111600 \\ \mathrm{C} & -3.23278100 & -2.30573000 & 0.96552500\end{array}$




\begin{tabular}{|c|c|c|c|c|c|c|c|}
\hline $\mathrm{C}$ & 2.45368800 & -0.75328700 & 2.69475100 & $\mathrm{H}$ & -5.32236600 & 1.90365400 & -3.61948900 \\
\hline $\mathrm{H}$ & 3.36827000 & -0.92826600 & 3.27152800 & $\mathrm{~N}$ & 0.94006700 & 3.61124600 & 0.70866200 \\
\hline $\mathrm{H}$ & 1.61198500 & -0.87116900 & 3.38605400 & $\mathrm{~N}$ & -2.90949400 & 0.33325800 & -1.92087000 \\
\hline $\mathrm{H}$ & 2.38556200 & -1.53391900 & 1.93160000 & $\mathrm{Pd}$ & -0.18148400 & -0.87358200 & -0.29676900 \\
\hline $\mathrm{C}$ & 2.68340100 & 1.71761600 & 3.17419600 & $\mathrm{C}$ & 1.77368200 & -1.90512600 & -1.55333600 \\
\hline $\mathrm{H}$ & 3.67929700 & 1.59295200 & 3.61334300 & $\mathrm{C}$ & 3.17964400 & -1.87568000 & -1.34156300 \\
\hline $\mathrm{H}$ & 2.62073800 & 2.73627500 & 2.78168200 & $\mathrm{C}$ & 3.99691500 & -1.06572700 & -2.16249900 \\
\hline $\mathrm{H}$ & 1.95987100 & 1.61136300 & 3.98581200 & $\mathrm{C}$ & 3.77105000 & -2.74314200 & -0.39607100 \\
\hline $\mathrm{C}$ & 3.68189100 & 0.76553500 & 1.08453500 & $\mathrm{C}$ & 5.37943000 & -1.12642100 & -2.03187600 \\
\hline $\mathrm{H}$ & 3.79464600 & 1.78456900 & 0.70509000 & $\mathrm{H}$ & 3.54094600 & -0.41579400 & -2.90211500 \\
\hline $\mathrm{H}$ & 4.60803700 & 0.50480300 & 1.60763200 & $\mathrm{C}$ & 5.15551300 & -2.79397200 & -0.27995100 \\
\hline $\mathrm{H}$ & 3.58227200 & 0.08894100 & 0.23559100 & $\mathrm{H}$ & 3.14183400 & -3.37868200 & 0.21823900 \\
\hline $\mathrm{C}$ & -2.25120500 & -3.10696700 & 1.84873100 & $\mathrm{C}$ & 5.95906400 & -1.98667600 & -1.09246600 \\
\hline $\mathrm{H}$ & -1.30074900 & -3.29041500 & 1.33850400 & $\mathrm{H}$ & 6.00865100 & -0.51294400 & -2.66833500 \\
\hline $\mathrm{H}$ & -2.04576300 & -2.61338600 & 2.80371600 & $\mathrm{H}$ & 5.61145200 & -3.46875000 & 0.43708800 \\
\hline $\mathrm{H}$ & -2.69946700 & -4.07904600 & 2.07888600 & $\mathrm{H}$ & 7.03947100 & -2.03379700 & -0.99944400 \\
\hline $\mathrm{C}$ & -4.56104000 & -2.08459600 & 1.71507500 & $\mathrm{C}$ & 0.63322200 & -2.13329400 & -2.01006300 \\
\hline $\mathrm{H}$ & -5.29736000 & -1.54346100 & 1.11346100 & $\mathrm{H}$ & 0.11345700 & -2.69363700 & -2.76628600 \\
\hline $\mathrm{H}$ & -4.99773400 & -3.06313700 & 1.93957400 & $\mathrm{H}$ & -0.92230700 & -1.88367300 & -1.22494300 \\
\hline $\mathrm{H}$ & -4.43021400 & -1.56677700 & 2.66844000 & $\mathrm{H}$ & -1.95993200 & -0.03337700 & -2.04669900 \\
\hline $\mathrm{C}$ & -3.49407500 & -3.06316700 & -0.34844200 & & & & \\
\hline $\mathrm{H}$ & -3.94754500 & -4.03091200 & -0.11189200 & \multirow{2}{*}{\multicolumn{4}{|c|}{$\begin{array}{l}\text { TS6 } \\
\text { imaginary frequency }=-1302.92 \mathrm{~cm}^{-1}\end{array}$}} \\
\hline $\mathrm{H}$ & -4.19171200 & -2.53317700 & -1.00521600 & & & & \\
\hline $\mathrm{H}$ & -2.57136600 & -3.26675600 & -0.90002400 & $\mathrm{C}$ & 0.23436200 & 3.01908300 & 0.18647700 \\
\hline $\mathrm{C}$ & 1.11431400 & 2.43796500 & 0.08221300 & $\mathrm{H}$ & 0.57967900 & 3.42137700 & -0.77238300 \\
\hline $\mathrm{C}$ & 1.49762400 & 2.34736000 & -1.26170400 & $\mathrm{H}$ & -0.36774100 & 3.79030600 & 0.67313200 \\
\hline $\mathrm{C}$ & 1.15589000 & 4.73757500 & 0.01761300 & $\mathrm{C}$ & 1.44091900 & 2.67660000 & 1.08500600 \\
\hline $\mathrm{C}$ & 1.72546000 & 3.52768400 & -1.97291500 & $\mathrm{H}$ & 1.75397300 & 3.59693300 & 1.59100500 \\
\hline $\mathrm{H}$ & 1.63012800 & 1.37760300 & -1.73127400 & $\mathrm{H}$ & 1.14194200 & 1.98560800 & 1.88286600 \\
\hline $\mathrm{C}$ & 1.55254500 & 4.74788100 & -1.32229900 & $\mathrm{C}$ & 2.67264600 & 2.13047800 & 0.33436400 \\
\hline $\mathrm{H}$ & 1.00755600 & 5.66837400 & 0.55967300 & $\mathrm{H}$ & 3.55505800 & 2.16792500 & 0.98061300 \\
\hline $\mathrm{H}$ & 2.03623200 & 3.49491400 & -3.01293300 & $\mathrm{H}$ & 2.89234700 & 2.78009900 & -0.52024300 \\
\hline $\mathrm{H}$ & 1.72089800 & 5.68917600 & -1.83463700 & $\mathrm{P}$ & 2.43035800 & 0.41675000 & -0.35018200 \\
\hline $\mathrm{C}$ & -3.43015900 & 0.21636100 & -0.66991600 & $\mathrm{P}$ & -0.93944900 & 1.61577700 & -0.16669300 \\
\hline $\mathrm{C}$ & -4.69960800 & 0.75260200 & -0.46250400 & $\mathrm{C}$ & -2.08159800 & 2.29910800 & -1.53619100 \\
\hline $\mathrm{C}$ & -3.54066700 & 0.91173000 & -2.96486400 & $\mathrm{C}$ & 3.67586000 & 0.24840500 & -1.78248800 \\
\hline $\mathrm{C}$ & -5.39015700 & 1.36018200 & -1.51749500 & $\mathrm{C}$ & -1.37326800 & 2.05597300 & -2.88313400 \\
\hline $\mathrm{H}$ & -5.15629200 & 0.69942600 & 0.51776800 & $\mathrm{H}$ & -2.00581800 & 2.41994800 & -3.70087300 \\
\hline $\mathrm{C}$ & -4.80909500 & 1.43841300 & -2.78585900 & $\mathrm{H}$ & -0.41949500 & 2.59108000 & -2.94792600 \\
\hline $\mathrm{H}$ & -3.00446100 & 0.93706100 & -3.90649000 & $\mathrm{H}$ & -1.17807600 & 0.99183200 & -3.04943400 \\
\hline $\mathrm{H}$ & -6.38060900 & 1.77118200 & -1.34689000 & $\mathrm{C}$ & -2.36648000 & 3.80230600 & -1.35165800 \\
\hline
\end{tabular}




\begin{tabular}{|c|c|c|c|c|c|c|c|}
\hline $\mathrm{H}$ & -3.09726200 & 4.11948800 & -2.10474500 & $\mathrm{C}$ & -0.93281800 & -2.15876500 & -0.49345700 \\
\hline $\mathrm{H}$ & -2.78137400 & 4.02234000 & -0.36459200 & $\mathrm{H}$ & 1.39642300 & -2.06352400 & 0.45219300 \\
\hline $\mathrm{H}$ & -1.47132300 & 4.41465500 & -1.49128600 & C & -2.28496300 & -2.65834200 & -0.62038800 \\
\hline $\mathrm{C}$ & -3.41236800 & 1.52450200 & -1.49662700 & $\mathrm{C}$ & -2.78758600 & -3.57914200 & 0.32285900 \\
\hline $\mathrm{H}$ & -3.98906600 & 1.75612100 & -0.59682600 & $\mathrm{C}$ & -3.11059100 & -2.26598300 & -1.69233000 \\
\hline $\mathrm{H}$ & -4.01997700 & 1.81251100 & -2.36213300 & C & -4.08112700 & -4.08012600 & 0.19958800 \\
\hline $\mathrm{H}$ & -3.26886300 & 0.44193300 & -1.53644400 & $\mathrm{H}$ & -2.15419300 & -3.89053700 & 1.14792000 \\
\hline C & 3.11044500 & 1.09011800 & -2.94660300 & $\mathrm{C}$ & -4.39281000 & -2.79148100 & -1.82488400 \\
\hline $\mathrm{H}$ & 2.10390300 & 0.76586300 & -3.22765400 & $\mathrm{H}$ & -2.72282600 & -1.57293200 & -2.43149700 \\
\hline $\mathrm{H}$ & 3.07711800 & 2.15945500 & -2.71358200 & $\mathrm{C}$ & -4.88542200 & -3.69104100 & -0.87503800 \\
\hline $\mathrm{H}$ & 3.75882200 & 0.97189800 & -3.82171400 & $\mathrm{H}$ & -4.45886000 & -4.78398900 & 0.93512100 \\
\hline $\mathrm{C}$ & 5.08712700 & 0.73744600 & -1.41354500 & $\mathrm{H}$ & -5.01128800 & -2.49779500 & -2.66751800 \\
\hline $\mathrm{H}$ & 5.54199100 & 0.12763100 & -0.62721600 & $\mathrm{H}$ & -5.89037400 & -4.08972400 & -0.97399500 \\
\hline $\mathrm{H}$ & 5.73627500 & 0.66257600 & -2.29334900 & $\mathrm{H}$ & 0.70900100 & -3.53848400 & -0.65407100 \\
\hline $\mathrm{H}$ & 5.09681200 & 1.78362600 & -1.09305000 & & & & \\
\hline C & 3.72046100 & -1.23305700 & -2.20340000 & \multirow{2}{*}{\multicolumn{4}{|c|}{$\begin{array}{l}\text { TS6a } \\
\text { imaginary frequency }=-1325.09 \mathrm{~cm}^{-1}\end{array}$}} \\
\hline $\mathrm{H}$ & 4.36185000 & -1.33799400 & -3.08529600 & & & & \\
\hline H & 4.13846300 & -1.87186400 & -1.41855000 & $\mathrm{C}$ & -0.45818600 & -2.03834500 & -1.12186600 \\
\hline $\mathrm{H}$ & 2.72695200 & -1.61024400 & -2.46433900 & $\mathrm{H}$ & -0.00507300 & -2.19501500 & -2.10873000 \\
\hline C & -1.98723100 & 1.64449200 & 1.37309900 & $\mathrm{H}$ & -0.43640400 & -0.95846300 & -0.93487800 \\
\hline C & -2.55852900 & 0.46861000 & 1.87383800 & C & 0.36781200 & -2.79638500 & -0.06866600 \\
\hline $\mathrm{C}$ & -2.94581100 & 2.90643200 & 3.04228100 & $\mathrm{H}$ & 0.26639400 & -3.87465100 & -0.24288800 \\
\hline $\mathrm{C}$ & -3.36475600 & 0.54803100 & 3.01045600 & $\mathrm{H}$ & -0.03521900 & -2.60989200 & 0.93244700 \\
\hline $\mathrm{H}$ & -2.37743200 & -0.47959800 & 1.38034300 & $\mathrm{C}$ & 1.87424300 & -2.45954200 & -0.10852300 \\
\hline C & -3.56281500 & 1.78981500 & 3.61028500 & $\mathrm{H}$ & 2.43446500 & -3.20421200 & 0.46739400 \\
\hline $\mathrm{H}$ & -3.07382500 & 3.89338100 & 3.48162800 & $\mathrm{H}$ & 2.22329600 & -2.53659900 & -1.14404500 \\
\hline $\mathrm{H}$ & -3.82685700 & -0.34561500 & 3.41939300 & $\mathrm{P}$ & 2.34060900 & -0.74394600 & 0.48141000 \\
\hline $\mathrm{H}$ & -4.17817100 & 1.89762500 & 4.49744500 & C & 2.88193200 & -0.93967000 & 2.30280400 \\
\hline $\mathrm{C}$ & 3.12368700 & -0.69103000 & 1.00009800 & C & 3.93643700 & -0.40237100 & -0.40344200 \\
\hline $\mathrm{C}$ & 4.23099900 & -0.40786700 & 1.80447100 & C & 4.74991300 & -1.38990500 & -0.97547500 \\
\hline C & 2.83631000 & -2.74751200 & 2.10647700 & $\mathrm{C}$ & 5.39878000 & 1.26069200 & -1.04007800 \\
\hline $\mathrm{C}$ & 4.63554100 & -1.32606700 & 2.77664600 & C & 5.92982800 & -1.00209600 & -1.61278300 \\
\hline $\mathrm{H}$ & 4.77799100 & 0.51837300 & 1.67584200 & $\mathrm{H}$ & 4.48234600 & -2.43926200 & -0.92171900 \\
\hline C & 3.93049500 & -2.51882700 & 2.93372900 & C & 6.26467000 & 0.34935200 & -1.64787300 \\
\hline $\mathrm{H}$ & 2.23147400 & -3.64704300 & 2.17324800 & $\mathrm{H}$ & 5.62388900 & 2.32478600 & -1.04217200 \\
\hline $\mathrm{H}$ & 5.49419700 & -1.10921500 & 3.40455600 & $\mathrm{H}$ & 6.57601200 & -1.74638800 & -2.06852800 \\
\hline $\mathrm{H}$ & 4.21377800 & -3.25239800 & 3.67990200 & $\mathrm{H}$ & 7.17163000 & 0.69446300 & -2.13296500 \\
\hline N & -2.17652500 & 2.84347100 & 1.94922600 & $\mathrm{~N}$ & 4.25857700 & 0.90261200 & -0.43859400 \\
\hline N & 2.46189300 & -1.85269600 & 1.18307900 & $\mathrm{Pc}$ & 0.62357000 & 0.97783100 & 0.05302900 \\
\hline $\mathrm{Pd}$ & 0.20486300 & -0.42822000 & -0.51863200 & $\mathrm{C}$ & 4.12847300 & -1.83070700 & 2.44800500 \\
\hline C & 0.29929300 & -2.57996200 & -0.33533600 & $\mathrm{H}$ & 4.99986600 & -1.40346100 & 1.94579500 \\
\hline
\end{tabular}




\begin{tabular}{|c|c|c|c|c|c|c|c|}
\hline $\mathrm{H}$ & 4.37563700 & -1.93179800 & 3.51100900 & C & -3.00901900 & 3.34463800 & 1.22699400 \\
\hline $\mathrm{H}$ & 3.97034600 & -2.84204900 & 2.05870300 & $\mathrm{C}$ & -2.02159200 & 5.30669300 & -0.51182600 \\
\hline $\mathrm{C}$ & 1.71334700 & -1.55398800 & 3.09652900 & $\mathrm{H}$ & -0.39173500 & 3.94648800 & -0.88322900 \\
\hline $\mathrm{H}$ & 0.79566100 & -0.96899900 & 2.98510000 & $\mathrm{C}$ & -3.71150600 & 4.52887600 & 1.05246100 \\
\hline $\mathrm{H}$ & 1.50650000 & -2.58648400 & 2.79788600 & $\mathrm{H}$ & -3.38168100 & 2.57624000 & 1.89681300 \\
\hline $\mathrm{H}$ & 1.97165800 & -1.57051000 & 4.16157700 & $\mathrm{C}$ & -3.21741300 & 5.51087000 & 0.18470800 \\
\hline $\mathrm{C}$ & 3.18543500 & 0.47307000 & 2.84092300 & $\mathrm{H}$ & -1.64594400 & 6.07200100 & -1.18323200 \\
\hline $\mathrm{H}$ & 2.29832200 & 1.11327300 & 2.80620600 & $\mathrm{H}$ & -4.64086700 & 4.69473600 & 1.58799200 \\
\hline $\mathrm{H}$ & 3.51230300 & 0.40167500 & 3.88487600 & $\mathrm{H}$ & -3.76879800 & 6.43657000 & 0.04977200 \\
\hline $\mathrm{H}$ & 3.97653100 & 0.96623800 & 2.26995500 & $\mathrm{H}$ & -0.93702500 & 0.73269000 & 2.53361900 \\
\hline $\mathrm{C}$ & 1.44908000 & 1.88947600 & -1.48868000 & & & & \\
\hline $\mathrm{O}$ & 1.90714300 & 2.43522800 & -2.38400400 & \multirow{2}{*}{\multicolumn{4}{|c|}{$\begin{array}{l}\text { TS6b } \\
\text { imaginary frequency }=-1188.86 \mathrm{~cm}^{-1}\end{array}$}} \\
\hline $\mathrm{C}$ & -0.98886000 & 0.78811800 & 1.44656000 & & & & \\
\hline $\mathrm{C}$ & -1.06845100 & 1.90975000 & 0.73953500 & $\mathrm{C}$ & 3.29833200 & -0.79207800 & 1.03582000 \\
\hline$P$ & -2.19197400 & -2.70324500 & -1.30443100 & $\mathrm{H}$ & 3.77400200 & -1.77935000 & 1.04690600 \\
\hline $\mathrm{C}$ & -3.01501800 & -2.27301800 & 0.31689500 & $\mathrm{H}$ & 3.52512900 & -0.28865000 & 1.97833300 \\
\hline $\mathrm{C}$ & -4.08054300 & -3.11390400 & 0.68094300 & $\mathrm{C}$ & 1.76999300 & -0.94499700 & 0.91586800 \\
\hline $\mathrm{C}$ & -4.77532000 & -2.92048600 & 1.87173500 & $\mathrm{H}$ & 1.38824800 & -1.40170800 & 1.83787200 \\
\hline $\mathrm{H}$ & -4.34293800 & -3.93172900 & 0.01885100 & $\mathrm{H}$ & 1.31371500 & 0.04930100 & 0.85979700 \\
\hline $\mathrm{C}$ & -3.31618900 & -1.09280300 & 2.32781800 & $\mathrm{C}$ & 1.34182200 & -1.79117400 & -0.29591900 \\
\hline $\mathrm{C}$ & -4.38067300 & -1.88866000 & 2.72258200 & $\mathrm{H}$ & 1.74166000 & -2.80820400 & -0.21447300 \\
\hline $\mathrm{H}$ & -5.60052900 & -3.57376600 & 2.13758200 & $\mathrm{H}$ & 1.77237100 & -1.36274000 & -1.20828200 \\
\hline $\mathrm{H}$ & -2.95994100 & -0.27331600 & 2.94387200 & $\mathrm{P}$ & -0.48895500 & -1.84089400 & -0.58862200 \\
\hline $\mathrm{H}$ & -4.87706800 & -1.70409500 & 3.66844400 & $\mathrm{P}$ & 4.04699900 & 0.18813000 & -0.37747600 \\
\hline N & -2.66283300 & -1.27186800 & 1.16449100 & $\mathrm{C}$ & 5.92872700 & -0.00236100 & -0.06004200 \\
\hline $\mathrm{C}$ & -3.03770000 & -1.48903200 & -2.52572300 & $\mathrm{C}$ & -0.75286900 & -3.02231100 & -2.06019300 \\
\hline $\mathrm{C}$ & -2.32427300 & -1.68368800 & -3.88058300 & $\mathrm{C}$ & 6.30544700 & -1.44615900 & -0.45095900 \\
\hline $\mathrm{H}$ & -2.84929100 & -1.10927100 & -4.65246000 & $\mathrm{H}$ & 7.39041300 & -1.57884600 & -0.36404100 \\
\hline $\mathrm{H}$ & -1.28969200 & -1.32760700 & -3.85988600 & $\mathrm{H}$ & 5.83759300 & -2.19036900 & 0.20224100 \\
\hline $\mathrm{H}$ & -2.32192700 & -2.73300200 & -4.19340300 & $\mathrm{H}$ & 6.02388500 & -1.67211800 & -1.48475600 \\
\hline $\mathrm{C}$ & -2.97835400 & -0.01157000 & -2.10833600 & $\mathrm{C}$ & 6.34263200 & 0.27585700 & 1.39454800 \\
\hline $\mathrm{H}$ & -1.95125400 & 0.35491200 & -2.02098000 & $\mathrm{H}$ & 7.43269300 & 0.19501200 & 1.49026400 \\
\hline $\mathrm{H}$ & -3.47906900 & 0.60318200 & -2.86652700 & $\mathrm{H}$ & 6.05309500 & 1.27989100 & 1.71705400 \\
\hline $\mathrm{H}$ & -3.48803200 & 0.16788700 & -1.15648800 & $\mathrm{H}$ & 5.90137100 & -0.44152300 & 2.09317800 \\
\hline $\mathrm{C}$ & -4.50688800 & -1.93506600 & -2.66439000 & $\mathrm{C}$ & 6.65849100 & 0.97192600 & -1.00511600 \\
\hline $\mathrm{H}$ & -5.00367300 & -1.32489200 & -3.42737500 & $\mathrm{H}$ & 6.45199000 & 2.01660800 & -0.75473400 \\
\hline $\mathrm{H}$ & -4.58665100 & -2.98235400 & -2.97275800 & $\mathrm{H}$ & 7.74158700 & 0.82247000 & -0.92219400 \\
\hline $\mathrm{H}$ & -5.06623100 & -1.80773100 & -1.73187300 & $\mathrm{H}$ & 6.37974900 & 0.80746400 & -2.05165000 \\
\hline $\mathrm{H}$ & -1.73731700 & -0.31652700 & 1.06517800 & $\mathrm{C}$ & -0.24841300 & -2.26809300 & -3.30954900 \\
\hline $\mathrm{C}$ & -1.79422100 & 3.12215900 & 0.53212600 & $\mathrm{H}$ & -0.76982600 & -1.31528700 & -3.44560600 \\
\hline $\mathrm{C}$ & -1.31734300 & 4.12046800 & -0.34606800 & $\mathrm{H}$ & 0.82716800 & -2.07088100 & -3.27175500 \\
\hline
\end{tabular}




\begin{tabular}{|c|c|c|c|c|c|c|c|}
\hline $\mathrm{H}$ & -0.43585700 & -2.88150100 & -4.19768700 & $\mathrm{H}$ & -7.53420400 & 3.04570600 & 1.26580900 \\
\hline $\mathrm{C}$ & 0.00078300 & -4.35576600 & -1.91014200 & $\mathrm{H}$ & -4.54756700 & 5.25438300 & -0.91265500 \\
\hline $\mathrm{H}$ & -0.32998400 & -4.92215900 & -1.03421400 & $\mathrm{H}$ & -6.73987700 & 5.16616500 & 0.24812000 \\
\hline $\mathrm{H}$ & -0.19884200 & -4.97770200 & -2.78993700 & $\mathrm{H}$ & -4.53808400 & -1.20268700 & -0.40481900 \\
\hline $\mathrm{H}$ & 1.08464400 & -4.22135900 & -1.85206500 & $\mathrm{C}$ & -0.61079000 & 1.74084200 & -0.69196800 \\
\hline $\mathrm{C}$ & -2.26335600 & -3.29067300 & -2.19999800 & $\mathrm{O}$ & 0.10007100 & 2.62534400 & -0.83600100 \\
\hline $\mathrm{H}$ & -2.43703300 & -3.91209400 & -3.08531200 & & & & \\
\hline $\mathrm{H}$ & -2.66836200 & -3.83110200 & -1.33807900 & \multirow{2}{*}{\multicolumn{4}{|c|}{$\begin{array}{l}\text { TS6-2H } \\
\text { imaginary frequency }=-1177.03 \mathrm{~cm}^{-1}\end{array}$}} \\
\hline $\mathrm{H}$ & -2.83046800 & -2.36375000 & -2.32697900 & & & & \\
\hline $\mathrm{C}$ & 3.69569900 & 1.91813800 & 0.23083700 & $\mathrm{C}$ & -0.14942000 & -3.04081500 & 0.58118800 \\
\hline $\mathrm{C}$ & 3.80154100 & 2.98770100 & -0.67339100 & $\mathrm{H}$ & -0.51414200 & -3.58624500 & -0.29559200 \\
\hline $\mathrm{C}$ & 3.05365100 & 3.35189900 & 1.91881600 & $\mathrm{H}$ & 0.42012700 & -3.78457200 & 1.15024800 \\
\hline $\mathrm{C}$ & 3.54498100 & 4.28371100 & -0.22969800 & $\mathrm{C}$ & -1.35696700 & -2.57243200 & 1.42779200 \\
\hline $\mathrm{H}$ & 4.07627300 & 2.79987700 & -1.70671600 & $\mathrm{H}$ & -1.66057500 & -3.41827700 & 2.05440700 \\
\hline $\mathrm{C}$ & 3.16352800 & 4.47464500 & 1.09794100 & $\mathrm{H}$ & -1.06423000 & -1.77458200 & 2.12145200 \\
\hline $\mathrm{H}$ & 2.74612400 & 3.45738300 & 2.95785200 & $\mathrm{C}$ & -2.59147600 & -2.14816900 & 0.60372300 \\
\hline $\mathrm{H}$ & 3.63320500 & 5.12662300 & -0.90905000 & $\mathrm{H}$ & -3.47847800 & -2.17179700 & 1.24255400 \\
\hline $\mathrm{H}$ & 2.94922800 & 5.46392300 & 1.48915700 & $\mathrm{H}$ & -2.77122700 & -2.88293200 & -0.18886600 \\
\hline $\mathrm{C}$ & -1.13496100 & -2.76986100 & 0.90840400 & $\mathrm{P}$ & -2.42953900 & -0.48466400 & -0.22219700 \\
\hline $\mathrm{C}$ & -0.43950900 & -3.79231700 & 1.56329000 & $\mathrm{P}$ & 0.96517200 & -1.69395300 & -0.09764400 \\
\hline $\mathrm{C}$ & -2.89643100 & -2.96719700 & 2.44950500 & $\mathrm{C}$ & 1.87385600 & -2.55290800 & -1.55356900 \\
\hline $\mathrm{C}$ & -1.00313000 & -4.40526100 & 2.68428100 & $\mathrm{C}$ & -3.70549800 & -0.46384800 & -1.63680900 \\
\hline $\mathrm{H}$ & 0.53384800 & -4.10550100 & 1.20538500 & $\mathrm{C}$ & 0.82647800 & -2.70965200 & -2.67681900 \\
\hline $\mathrm{C}$ & -2.25432800 & -3.99021800 & 3.13888500 & $\mathrm{H}$ & 1.32163800 & -3.11599100 & -3.56502400 \\
\hline $\mathrm{H}$ & -3.86861300 & -2.58673400 & 2.74958100 & $\mathrm{H}$ & 0.02141300 & -3.40279100 & -2.41514000 \\
\hline $\mathrm{H}$ & -0.46723600 & -5.19790300 & 3.19719400 & $\mathrm{H}$ & 0.38448000 & -1.74747800 & -2.95177000 \\
\hline $\mathrm{H}$ & -2.72098200 & -4.43993300 & 4.00794500 & $\mathrm{C}$ & 2.45427200 & -3.92655700 & -1.17256600 \\
\hline $\mathrm{N}$ & 3.31349100 & 2.10410900 & 1.50730100 & $\mathrm{H}$ & 2.89997100 & -4.38304500 & -2.06280100 \\
\hline $\mathrm{N}$ & -2.33997700 & -2.38662600 & 1.37807400 & $\mathrm{H}$ & 3.25789700 & -3.84951000 & -0.43215800 \\
\hline $\mathrm{Pd}$ & -1.81726800 & 0.21742100 & -0.51830200 & $\mathrm{H}$ & 1.69502300 & -4.62569200 & -0.80862000 \\
\hline $\mathrm{C}$ & -3.77822600 & -0.54008600 & 0.00694800 & $\mathrm{C}$ & 2.99804000 & -1.62496900 & -2.04815800 \\
\hline $\mathrm{C}$ & -3.76648800 & 0.77389200 & -0.08202200 & $\mathrm{H}$ & 3.76387700 & -1.44584600 & -1.28609000 \\
\hline $\mathrm{H}$ & -2.99487800 & -1.36473400 & 0.73891400 & $\mathrm{H}$ & 3.49699800 & -2.09276300 & -2.90324000 \\
\hline $\mathrm{C}$ & -4.54236200 & 1.96957500 & 0.01526900 & $\mathrm{H}$ & 2.60040900 & -0.66394600 & -2.38434600 \\
\hline $\mathrm{C}$ & -5.79321400 & 1.93375500 & 0.67825100 & $\mathrm{C}$ & -3.13777700 & -1.36776300 & -2.75115300 \\
\hline $\mathrm{C}$ & -4.09906300 & 3.18511500 & -0.54891200 & $\mathrm{H}$ & -2.16112500 & -1.01757400 & -3.09860600 \\
\hline $\mathrm{C}$ & -6.57582400 & 3.07811500 & 0.75749900 & $\mathrm{H}$ & -3.04500500 & -2.41337600 & -2.43932400 \\
\hline $\mathrm{H}$ & -6.12974300 & 1.00233500 & 1.12199100 & $\mathrm{H}$ & -3.82100900 & -1.34981600 & -3.60668300 \\
\hline $\mathrm{C}$ & -4.89045400 & 4.32432800 & -0.47104900 & $\mathrm{C}$ & -5.09335800 & -0.96784900 & -1.20310000 \\
\hline $\mathrm{H}$ & -3.13539000 & 3.21381800 & -1.04596000 & $\mathrm{H}$ & -5.54607900 & -0.32829400 & -0.44039600 \\
\hline $\mathrm{C}$ & -6.12663200 & 4.27253400 & 0.18231100 & $\mathrm{H}$ & -5.76302500 & -0.95393400 & -2.06990600 \\
\hline
\end{tabular}




\begin{tabular}{|c|c|c|c|c|c|c|c|}
\hline $\mathrm{H}$ & -5.07289200 & -1.99737900 & -0.83232300 & $\mathrm{H}$ & 2.24648100 & -3.55589200 & 1.77834600 \\
\hline $\mathrm{C}$ & -3.80488800 & 0.98369900 & -2.15560600 & & & & \\
\hline $\mathrm{H}$ & -4.46096600 & 1.00573700 & -3.03199500 & \multirow{2}{*}{\multicolumn{4}{|c|}{$\begin{array}{l}\text { TS7 } \\
\text { imaginary frequency }=-1409.82 \mathrm{~cm}^{-1}\end{array}$}} \\
\hline $\mathrm{H}$ & -4.23522700 & 1.66059700 & -1.41111200 & & & & \\
\hline $\mathrm{H}$ & -2.82896700 & 1.37302900 & -2.46164500 & $\mathrm{C}$ & -2.85345900 & 1.72585900 & 0.24012400 \\
\hline C & 2.30050900 & -1.58495900 & 1.22628300 & $\mathrm{H}$ & -2.78698600 & 2.42489600 & -0.60101700 \\
\hline C & 2.94554500 & -0.37840300 & 1.49718500 & $\mathrm{H}$ & -3.90023300 & 1.67403000 & 0.55066000 \\
\hline C & 3.63841000 & -2.63200700 & 2.93188500 & $\mathrm{C}$ & -1.98914300 & 2.21881000 & 1.42428800 \\
\hline C & 3.94313200 & -0.30744500 & 2.47361000 & $\mathrm{H}$ & -2.59450000 & 2.91388800 & 2.01713300 \\
\hline $\mathrm{H}$ & 2.64973400 & 0.50228300 & 0.93825200 & $\mathrm{H}$ & -1.74963000 & 1.38652900 & 2.09720500 \\
\hline $\mathrm{C}$ & 4.29224900 & -1.44557400 & 3.20766500 & $\mathrm{C}$ & -0.70165700 & 2.97355800 & 1.03158700 \\
\hline $\mathrm{H}$ & 3.83741300 & -3.56502200 & 3.44655100 & $\mathrm{H}$ & -0.24609300 & 3.41488400 & 1.92342400 \\
\hline $\mathrm{H}$ & 4.44137800 & 0.63745900 & 2.66749600 & $\mathrm{H}$ & -0.96327900 & 3.81008300 & 0.37478500 \\
\hline $\mathrm{H}$ & 5.05283900 & -1.41757900 & 3.97921500 & $P$ & 0.55246200 & 1.94153100 & 0.11218400 \\
\hline $\mathrm{C}$ & -3.11465000 & 0.69218700 & 1.07184900 & $\mathrm{P}$ & -2.41277800 & 0.02618700 & -0.37156700 \\
\hline $\mathrm{C}$ & -4.20936800 & 0.41846200 & 1.89772700 & C & -3.37789500 & -0.23089600 & -1.98893600 \\
\hline $\mathrm{C}$ & -2.90880000 & 2.82587300 & 2.03007800 & $\mathrm{C}$ & 1.42095600 & 3.14393900 & -1.08504400 \\
\hline $\mathrm{C}$ & -4.65072700 & 1.38537100 & 2.80565600 & C & -2.62569600 & 0.52468500 & -3.10258600 \\
\hline $\mathrm{H}$ & -4.72615700 & -0.53136200 & 1.83202300 & $\mathrm{H}$ & -3.13745600 & 0.36294300 & -4.05804200 \\
\hline $\mathrm{C}$ & -3.99364400 & 2.61263900 & 2.87550900 & $\mathrm{H}$ & -2.60255300 & 1.60569100 & -2.92769100 \\
\hline $\mathrm{H}$ & -2.34863600 & 3.75647300 & 2.03186100 & $\mathrm{H}$ & -1.59634900 & 0.16650200 & -3.20493000 \\
\hline $\mathrm{H}$ & -5.50261600 & 1.18005200 & 3.44644900 & C & -4.82627200 & 0.28258500 & -1.88710400 \\
\hline $\mathrm{H}$ & -4.30871300 & 3.38716200 & 3.56566200 & $\mathrm{H}$ & -5.35198100 & 0.04960300 & -2.82035000 \\
\hline N & 2.68998600 & -2.66131200 & 1.96550000 & $\mathrm{H}$ & -5.37168300 & -0.18167500 & -1.06271200 \\
\hline N & -2.49432800 & 1.88601700 & 1.17014600 & $\mathrm{H}$ & -4.86808900 & 1.36752400 & -1.75298600 \\
\hline Pd & -0.17472300 & 0.32599900 & -0.49922600 & $\mathrm{C}$ & -3.37118700 & -1.73945300 & -2.30385100 \\
\hline C & -0.51111600 & 2.43599300 & -0.59046200 & $\mathrm{H}$ & -3.92139100 & -2.31695800 & -1.55542700 \\
\hline $\mathrm{C}$ & 0.78947600 & 2.20008900 & -0.61895900 & $\mathrm{H}$ & -3.85502800 & -1.90642900 & -3.27247200 \\
\hline $\mathrm{H}$ & -1.41160100 & 2.11924500 & 0.35380000 & $\mathrm{H}$ & -2.35217800 & -2.13347000 & -2.36912000 \\
\hline C & 2.04043500 & 2.89275800 & -0.70846700 & C & 0.40011100 & 3.47117500 & -2.19502800 \\
\hline $\mathrm{C}$ & 2.10331800 & 4.26287600 & -0.35028300 & $\mathrm{H}$ & 0.05537300 & 2.56709400 & -2.70558100 \\
\hline $\mathrm{C}$ & 3.21297800 & 2.25969500 & -1.17830200 & $\mathrm{H}$ & -0.47448700 & 4.01032000 & -1.81724700 \\
\hline $\mathrm{C}$ & 3.29426800 & 4.96775900 & -0.46858600 & $\mathrm{H}$ & 0.87662800 & 4.11682700 & -2.94084800 \\
\hline $\mathrm{H}$ & 1.20751600 & 4.75458800 & 0.01488400 & $\mathrm{C}$ & 1.89066200 & 4.43810600 & -0.39738800 \\
\hline $\mathrm{C}$ & 4.39807900 & 2.97432000 & -1.30921000 & $\mathrm{H}$ & 2.65187100 & 4.24569400 & 0.36415600 \\
\hline $\mathrm{H}$ & 3.16694400 & 1.21542700 & -1.46545100 & $\mathrm{H}$ & 2.34288600 & 5.09881500 & -1.14565000 \\
\hline $\mathrm{C}$ & 4.44133000 & 4.32690500 & -0.95169900 & $\mathrm{H}$ & 1.06713400 & 4.99108000 & 0.06462000 \\
\hline $\mathrm{H}$ & 3.33251600 & 6.01734600 & -0.19533700 & C & 2.62502400 & 2.41176000 & -1.70722700 \\
\hline $\mathrm{H}$ & 5.28799700 & 2.48770700 & -1.69578700 & $\mathrm{H}$ & 3.09281800 & 3.05887900 & -2.45716300 \\
\hline $\mathrm{H}$ & 5.36826500 & 4.88307400 & -1.05228800 & $\mathrm{H}$ & 3.38922600 & 2.17138100 & -0.96117900 \\
\hline $\mathrm{H}$ & -1.04720900 & 3.11424200 & -1.25462400 & $\mathrm{H}$ & 2.32154900 & 1.48673700 & -2.20725600 \\
\hline
\end{tabular}




\begin{tabular}{|c|c|c|c|c|c|c|c|}
\hline $\mathrm{C}$ & -3.26414800 & -1.03107100 & 0.90084700 & $\mathrm{H}$ & 3.33155800 & 0.62060100 & -2.24043700 \\
\hline $\mathrm{C}$ & -2.67578300 & -2.20295700 & 1.38845700 & $\mathrm{C}$ & 1.58712000 & 0.91458200 & -1.01864400 \\
\hline $\mathrm{C}$ & -5.12826000 & -1.33192200 & 2.21642700 & $\mathrm{H}$ & 1.00326800 & 1.16790200 & -1.91247600 \\
\hline $\mathrm{C}$ & -3.37568700 & -2.96158500 & 2.32913500 & $\mathrm{H}$ & 1.42491300 & -0.15503500 & -0.84453300 \\
\hline $\mathrm{H}$ & -1.69192500 & -2.50170600 & 1.04445700 & $\mathrm{C}$ & 1.07042200 & 1.73170100 & 0.17995800 \\
\hline $\mathrm{C}$ & -4.62714400 & -2.52099000 & 2.75225700 & $\mathrm{H}$ & 1.12781000 & 2.80573100 & -0.03071100 \\
\hline $\mathrm{H}$ & -6.09873900 & -0.95045100 & 2.52615900 & $\mathrm{H}$ & 1.70975600 & 1.55054700 & 1.05165200 \\
\hline $\mathrm{H}$ & -2.94660000 & -3.87685400 & 2.72598800 & $\mathrm{P}$ & -0.64620900 & 1.27230200 & 0.70030700 \\
\hline $\mathrm{H}$ & -5.20470900 & -3.07681400 & 3.48349100 & $\mathrm{P}$ & 4.20738700 & 0.56615500 & 0.06240200 \\
\hline $\mathrm{C}$ & 1.86021800 & 1.50799100 & 1.38404000 & $\mathrm{C}$ & 5.93646900 & 1.11616200 & -0.56298800 \\
\hline $\mathrm{C}$ & 2.31027200 & 2.34048500 & 2.41136200 & $\mathrm{C}$ & -1.11964000 & 2.39762200 & 2.15592500 \\
\hline $\mathrm{C}$ & 3.32446700 & -0.18664600 & 2.09070900 & $\mathrm{C}$ & 6.00368300 & 2.64797000 & -0.39504600 \\
\hline $\mathrm{C}$ & 3.28902900 & 1.87931300 & 3.29573600 & $\mathrm{H}$ & 7.00587800 & 3.00335300 & -0.66273500 \\
\hline $\mathrm{H}$ & 1.90445200 & 3.33886200 & 2.52624700 & $\mathrm{H}$ & 5.29325700 & 3.16945800 & -1.04496300 \\
\hline $\mathrm{C}$ & 3.80796200 & 0.59594000 & 3.13541600 & $\mathrm{H}$ & 5.80851500 & 2.95111800 & 0.63890100 \\
\hline $\mathrm{H}$ & 3.68198600 & -1.19357100 & 1.90186800 & $\mathrm{C}$ & 6.21253400 & 0.73040300 & -2.02500500 \\
\hline $\mathrm{H}$ & 3.64030700 & 2.51857800 & 4.09979500 & $\mathrm{H}$ & 7.23101100 & 1.03094800 & -2.30100700 \\
\hline $\mathrm{H}$ & 4.56674700 & 0.20310700 & 3.80270000 & $\mathrm{H}$ & 6.12859300 & -0.34820300 & -2.18427600 \\
\hline $\mathrm{N}$ & -4.46824400 & -0.59876300 & 1.31326300 & $\mathrm{H}$ & 5.52851600 & 1.22812900 & -2.71874000 \\
\hline $\mathrm{N}$ & 2.37968600 & 0.26763100 & 1.25607800 & $\mathrm{C}$ & 6.99211700 & 0.47054700 & 0.35520200 \\
\hline $\mathrm{Pc}$ & -0.09787300 & -0.26180700 & -0.49736800 & $\mathrm{H}$ & 7.01414800 & -0.61817500 & 0.25125300 \\
\hline $\mathrm{C}$ & 1.52835300 & -1.76780200 & -0.51623200 & $\mathrm{H}$ & 7.98753100 & 0.84512600 & 0.08894900 \\
\hline $\mathrm{C}$ & 2.88815500 & -2.32039600 & -0.67041000 & $\mathrm{H}$ & 6.81776700 & 0.71291400 & 1.40915400 \\
\hline $\mathrm{C}$ & 3.94956100 & -1.51738700 & -1.12142600 & $\mathrm{C}$ & -0.24823500 & 1.95620800 & 3.35154800 \\
\hline $\mathrm{C}$ & 3.14234800 & -3.67183900 & -0.36897100 & $\mathrm{H}$ & -0.39455600 & 0.89898200 & 3.59377700 \\
\hline $\mathrm{C}$ & 5.22537400 & -2.05611700 & -1.28930200 & $\mathrm{H}$ & 0.81791700 & 2.12783500 & 3.17618100 \\
\hline $\mathrm{H}$ & 3.76482100 & -0.47577100 & -1.36687300 & $\mathrm{H}$ & -0.53128100 & 2.54033600 & 4.23387400 \\
\hline $\mathrm{C}$ & 4.42044400 & -4.20289400 & -0.52863700 & $\mathrm{C}$ & -0.88886100 & 3.88975900 & 1.85894300 \\
\hline $\mathrm{H}$ & 2.33117500 & -4.29789100 & -0.01001200 & $\mathrm{H}$ & -1.50863600 & 4.24681900 & 1.03124400 \\
\hline $\mathrm{C}$ & 5.46602500 & -3.39840400 & -0.99050300 & $\mathrm{H}$ & -1.16344600 & 4.47570600 & 2.74311600 \\
\hline $\mathrm{H}$ & 6.03105400 & -1.42754400 & -1.65708100 & $\mathrm{H}$ & 0.15851100 & 4.11413400 & 1.63680100 \\
\hline $\mathrm{H}$ & 4.60024600 & -5.24816200 & -0.29542500 & $\mathrm{C}$ & -2.60509000 & 2.14769400 & 2.47977500 \\
\hline $\mathrm{H}$ & 6.45990900 & -3.81611100 & -1.11789800 & $\mathrm{H}$ & -2.88587800 & 2.73074700 & 3.36353900 \\
\hline $\mathrm{C}$ & 0.32118000 & -2.21609100 & -0.73135100 & $\mathrm{H}$ & -3.26207000 & 2.45805700 & 1.66060500 \\
\hline $\mathrm{H}$ & -0.17720100 & -3.13037000 & -1.02143600 & $\mathrm{H}$ & -2.79915500 & 1.09354600 & 2.70279000 \\
\hline \multirow[t]{2}{*}{$\mathrm{H}$} & 1.81684400 & -0.55143800 & 0.31167100 & $\mathrm{C}$ & 4.19621300 & -1.26781900 & -0.28472800 \\
\hline & & & & $\mathrm{C}$ & 4.70198500 & -2.14586400 & 0.68866300 \\
\hline \multicolumn{4}{|c|}{ TS7a } & $\mathrm{C}$ & 3.64659400 & -3.03794600 & -1.65750000 \\
\hline \multicolumn{4}{|c|}{ imaginary frequency $=-1275.81 \mathrm{~cm}^{-1}$} & $\mathrm{C}$ & 4.69279800 & -3.51728400 & 0.44075000 \\
\hline $\mathrm{C}$ & 3.07911300 & 1.14964000 & -1.31871200 & $\mathrm{H}$ & 5.09190700 & -1.75471600 & 1.62330400 \\
\hline $\mathrm{H}$ & 3.26743300 & 2.21861000 & -1.47139000 & $\mathrm{C}$ & 4.15466700 & -3.97817200 & -0.76072400 \\
\hline
\end{tabular}




\begin{tabular}{|c|c|c|c|c|c|c|c|}
\hline $\mathrm{H}$ & 3.20775900 & -3.35794100 & -2.60102700 & $\mathrm{C}$ & 1.31600900 & 1.40864700 & -1.85813400 \\
\hline $\mathrm{H}$ & 5.09084700 & -4.21267200 & 1.17405900 & $\mathrm{H}$ & 1.77151600 & 1.34414600 & -2.85156900 \\
\hline $\mathrm{H}$ & 4.12234800 & -5.03668100 & -0.99762100 & $\mathrm{H}$ & 1.50806800 & 2.42202400 & -1.48940000 \\
\hline $\mathrm{C}$ & -1.70548800 & 1.78794800 & -0.74847000 & $\mathrm{P}$ & 2.20816900 & 0.23055700 & -0.71181400 \\
\hline $\mathrm{C}$ & -1.50738200 & 2.95609900 & -1.49050800 & $\mathrm{C}$ & 2.95700900 & -1.11605900 & -1.84110800 \\
\hline $\mathrm{C}$ & -3.47196100 & 1.16325400 & -2.14832000 & C & 3.67786600 & 1.20443500 & -0.13434500 \\
\hline $\mathrm{C}$ & -2.32602900 & 3.21751300 & -2.59120200 & $\mathrm{C}$ & 4.21045800 & 2.30187700 & -0.82523800 \\
\hline $\mathrm{H}$ & -0.71914300 & 3.64895800 & -1.22096200 & $\mathrm{C}$ & 5.29658800 & 1.36689700 & 1.49915600 \\
\hline C & -3.32478600 & 2.30639600 & -2.92942100 & $\mathrm{C}$ & 5.33156300 & 2.94623500 & -0.29931600 \\
\hline $\mathrm{H}$ & -4.22993100 & 0.41637800 & -2.35816900 & $\mathrm{H}$ & 3.77363000 & 2.64501300 & -1.75636000 \\
\hline $\mathrm{H}$ & -2.17917400 & 4.11879400 & -3.17819400 & $\mathrm{C}$ & 5.88886500 & 2.47237500 & 0.88604200 \\
\hline $\mathrm{H}$ & -3.97529700 & 2.46847000 & -3.78152200 & $\mathrm{H}$ & 5.69825400 & 0.96381000 & 2.42603100 \\
\hline $\mathrm{N}$ & 3.66465900 & -1.71659000 & -1.43663600 & $\mathrm{H}$ & 5.76225900 & 3.80095600 & -0.81218000 \\
\hline $\mathrm{N}$ & -2.67957600 & 0.92223100 & -1.09346100 & $\mathrm{H}$ & 6.75948400 & 2.94406300 & 1.32923500 \\
\hline $\mathrm{Pd}$ & -1.24246300 & -1.08735200 & 0.86077300 & $\mathrm{~N}$ & 4.21575000 & 0.74928100 & 1.00905200 \\
\hline C & -3.27797500 & -1.59363500 & 0.09266700 & $\mathrm{Pd}$ & 0.96261500 & -0.87480900 & 1.13508700 \\
\hline $\mathrm{C}$ & -4.69860900 & -1.42297300 & -0.28279400 & $\mathrm{C}$ & 3.95090400 & -0.53529700 & -2.86263400 \\
\hline $\mathrm{C}$ & -5.44685300 & -0.34226400 & 0.20990100 & $\mathrm{H}$ & 4.81162200 & -0.06596100 & -2.37925800 \\
\hline C & -5.31609800 & -2.36382300 & -1.12499200 & $\mathrm{H}$ & 4.32867100 & -1.34799900 & -3.49321400 \\
\hline C & -6.79731600 & -0.21808500 & -0.11593700 & $\mathrm{H}$ & 3.48834000 & 0.19852300 & -3.53101700 \\
\hline $\mathrm{H}$ & -4.97685200 & 0.38328100 & 0.86704500 & $\mathrm{C}$ & 1.79967700 & -1.81792600 & -2.57923900 \\
\hline C & -6.66350200 & -2.22686600 & -1.45496900 & $\mathrm{H}$ & 1.05257700 & -2.22055000 & -1.88805400 \\
\hline $\mathrm{H}$ & -4.73682900 & -3.19463300 & -1.51528300 & $\mathrm{H}$ & 1.29467900 & -1.15267700 & -3.28665000 \\
\hline C & -7.40722600 & -1.15647500 & -0.95132200 & $\mathrm{H}$ & 2.20188100 & -2.65775800 & -3.15702800 \\
\hline $\mathrm{H}$ & -7.37351600 & 0.60991700 & 0.28556600 & $\mathrm{C}$ & 3.68061200 & -2.12995800 & -0.93209000 \\
\hline $\mathrm{H}$ & -7.13356600 & -2.96013500 & -2.10308200 & $\mathrm{H}$ & 2.98900500 & -2.59884900 & -0.22449600 \\
\hline $\mathrm{H}$ & -8.45736700 & -1.05606400 & -1.20712100 & $\mathrm{H}$ & 4.11665300 & -2.92238800 & -1.55101300 \\
\hline C & -2.54879400 & -2.57877800 & 0.51419800 & $\mathrm{H}$ & 4.48792300 & -1.66618000 & -0.35864000 \\
\hline $\mathrm{H}$ & -2.55368900 & -3.63564200 & 0.72601800 & $\mathrm{C}$ & 2.06927600 & -0.38275100 & 2.67519400 \\
\hline $\mathrm{H}$ & -2.75429000 & -0.32824400 & -0.31699100 & $\mathrm{O}$ & 2.61026400 & -0.14114100 & 3.65174300 \\
\hline C & 0.31744800 & -1.95988700 & 1.61320500 & C & -0.96099700 & -2.07416300 & 0.74213400 \\
\hline \multirow[t]{2}{*}{$\mathrm{O}$} & 1.23519500 & -2.45227300 & 2.07889700 & $\mathrm{C}$ & -0.21442100 & -2.34706400 & 1.79231500 \\
\hline & & & & $\mathrm{P}$ & -2.82478300 & 1.41407300 & -0.85789400 \\
\hline \multirow{2}{*}{\multicolumn{4}{|c|}{$\begin{array}{l}\text { TS7b } \\
\text { imaginary frequency }=-1246.54 \mathrm{~cm}^{-1}\end{array}$}} & $\mathrm{C}$ & -3.20247600 & 1.04427700 & 0.92555500 \\
\hline & & & & $\mathrm{C}$ & -4.21382200 & 1.64855500 & 1.68790400 \\
\hline $\mathrm{C}$ & -0.96713600 & 1.70593100 & -0.73045300 & $\mathrm{C}$ & -4.46640800 & 1.22951800 & 2.99215900 \\
\hline $\mathrm{H}$ & -0.76436500 & 2.77269300 & -0.58285600 & $\mathrm{H}$ & -4.80003700 & 2.45253600 & 1.26712600 \\
\hline $\mathrm{H}$ & -0.61380800 & 1.19625000 & 0.17193100 & $\mathrm{C}$ & -2.74575600 & -0.39091700 & 2.73215400 \\
\hline C & -0.20556800 & 1.18230000 & -1.95812800 & $\mathrm{C}$ & -3.70984900 & 0.19475300 & 3.53970100 \\
\hline $\mathrm{H}$ & -0.56354700 & 1.69249200 & -2.85935600 & $\mathrm{H}$ & -5.24623300 & 1.71126400 & 3.57400400 \\
\hline $\mathrm{H}$ & -0.42822100 & 0.12024800 & -2.10429000 & $\mathrm{H}$ & -2.13922100 & -1.22538900 & 3.07590100 \\
\hline
\end{tabular}




\begin{tabular}{|c|c|c|c|c|c|c|c|}
\hline $\mathrm{H}$ & -3.86965000 & -0.16154600 & 4.55066500 & $P$ & -2.38670000 & 0.09577000 & -0.39663100 \\
\hline $\mathrm{N}$ & -2.50763400 & 0.01380500 & 1.47716300 & $\mathrm{C}$ & -3.36351300 & -0.15977700 & -2.01541800 \\
\hline $\mathrm{C}$ & -3.53374600 & 3.14134300 & -1.27097900 & $\mathrm{C}$ & 1.46765800 & 3.09964500 & -1.11865100 \\
\hline $\mathrm{C}$ & -2.91711400 & 3.49599700 & -2.64465100 & $\mathrm{C}$ & -2.64434700 & 0.69658000 & -3.08041600 \\
\hline $\mathrm{H}$ & -3.37820100 & 4.41775200 & -3.01666500 & $\mathrm{H}$ & -3.12070600 & 0.52260000 & -4.05084200 \\
\hline $\mathrm{H}$ & -1.83998900 & 3.67719400 & -2.58219100 & $\mathrm{H}$ & -2.71337300 & 1.76963400 & -2.87635700 \\
\hline $\mathrm{H}$ & -3.09615200 & 2.71396100 & -3.38996600 & $\mathrm{H}$ & -1.58920200 & 0.42333200 & -3.17374100 \\
\hline $\mathrm{C}$ & -3.19673900 & 4.26492800 & -0.27446900 & $\mathrm{C}$ & -4.84003200 & 0.26334400 & -1.91144300 \\
\hline $\mathrm{H}$ & -2.11693500 & 4.40646800 & -0.16697700 & $\mathrm{H}$ & -5.30664200 & 0.16196700 & -2.89705900 \\
\hline $\mathrm{H}$ & -3.61009800 & 5.21166200 & -0.64188800 & $\mathrm{H}$ & -5.41600500 & -0.38133100 & -1.23858500 \\
\hline $\mathrm{H}$ & -3.60849500 & 4.09678100 & 0.72280900 & $\mathrm{H}$ & -4.96511500 & 1.30884500 & -1.61248900 \\
\hline $\mathrm{C}$ & -5.05998600 & 3.00625900 & -1.46738500 & $\mathrm{C}$ & -3.25947300 & -1.64942600 & -2.39570700 \\
\hline $\mathrm{H}$ & -5.46530600 & 3.95801800 & -1.82906100 & $\mathrm{H}$ & -3.77039300 & -2.29997300 & -1.67796700 \\
\hline $\mathrm{H}$ & -5.29783200 & 2.23818100 & -2.20965000 & $\mathrm{H}$ & -3.73724500 & -1.80317100 & -3.36863300 \\
\hline $\mathrm{H}$ & -5.59836200 & 2.75528700 & -0.54990700 & $\mathrm{H}$ & -2.21833600 & -1.97184000 & -2.48226800 \\
\hline $\mathrm{H}$ & -1.57934700 & -0.88274600 & 0.90857600 & $\mathrm{C}$ & 0.41578300 & 3.42048300 & -2.20044600 \\
\hline $\mathrm{C}$ & -1.66240800 & -2.95234700 & -0.21811400 & $\mathrm{H}$ & 0.04223100 & 2.51289800 & -2.68461700 \\
\hline C & -1.48491100 & -4.34968500 & -0.16169900 & $\mathrm{H}$ & -0.43547200 & 3.98673300 & -1.80804100 \\
\hline C & -2.51026100 & -2.41948100 & -1.20244500 & $\mathrm{H}$ & 0.87874100 & 4.04196000 & -2.97405000 \\
\hline $\mathrm{C}$ & -2.14218200 & -5.18329200 & -1.06042900 & $\mathrm{C}$ & 1.97806600 & 4.39863200 & -0.46918500 \\
\hline $\mathrm{H}$ & -0.82091100 & -4.77628500 & 0.58441400 & $\mathrm{H}$ & 2.76088600 & 4.20886700 & 0.26986900 \\
\hline $\mathrm{C}$ & -3.16716800 & -3.25898400 & -2.10203000 & $\mathrm{H}$ & 2.41629600 & 5.03507100 & -1.24557500 \\
\hline $\mathrm{H}$ & -2.66046200 & -1.34618800 & -1.26958900 & $\mathrm{H}$ & 1.17953900 & 4.97705500 & 0.00562500 \\
\hline C & -2.98711000 & -4.64085700 & -2.03415900 & $\mathrm{C}$ & 2.63961400 & 2.33146300 & -1.75870200 \\
\hline $\mathrm{H}$ & -1.99342700 & -6.25748200 & -1.00527000 & $\mathrm{H}$ & 3.09465000 & 2.95455800 & -2.53555700 \\
\hline $\mathrm{H}$ & -3.82059200 & -2.83072800 & -2.85616200 & $\mathrm{H}$ & 3.42175200 & 2.09677300 & -1.03023700 \\
\hline $\mathrm{H}$ & -3.49734100 & -5.29318000 & -2.73619800 & $\mathrm{H}$ & 2.30739100 & 1.40181600 & -2.23173800 \\
\hline \multirow[t]{2}{*}{$\mathrm{H}$} & -0.09063900 & -3.06742400 & 2.59078100 & $\mathrm{C}$ & -3.28570700 & -0.97694200 & 0.86074300 \\
\hline & & & & $\mathrm{C}$ & -2.71408100 & -2.13241000 & 1.38798200 \\
\hline \multirow{2}{*}{\multicolumn{4}{|c|}{$\begin{array}{l}\text { TS7-2H } \\
\text { imaginary frequency }=-1343.11 \mathrm{~cm}^{-1}\end{array}$}} & $\mathrm{C}$ & -5.24302500 & -1.38214200 & 2.20000100 \\
\hline & & & & $\mathrm{C}$ & -3.41388000 & -2.91531400 & 2.31286000 \\
\hline $\mathrm{C}$ & -2.79207300 & 1.80708100 & 0.22957900 & $\mathrm{H}$ & -1.71159400 & -2.39878700 & 1.07087600 \\
\hline $\mathrm{H}$ & -2.64847600 & 2.47947800 & -0.62368300 & $\mathrm{C}$ & -4.69344500 & -2.53704500 & 2.72813200 \\
\hline $\mathrm{H}$ & -3.85454600 & 1.89589000 & 0.48408900 & $\mathrm{H}$ & -6.22599100 & -1.00733100 & 2.46182000 \\
\hline C & -1.92551800 & 2.27013100 & 1.43144800 & $\mathrm{H}$ & -2.95773500 & -3.81582100 & 2.71269100 \\
\hline $\mathrm{H}$ & -2.52178800 & 2.97777500 & 2.01762100 & $\mathrm{H}$ & -5.25681500 & -3.11979700 & 3.44771600 \\
\hline $\mathrm{H}$ & -1.71167300 & 1.42933300 & 2.10285400 & $\mathrm{C}$ & 1.94478600 & 1.49814200 & 1.38827500 \\
\hline $\mathrm{C}$ & -0.61418400 & 2.99092600 & 1.05419000 & $\mathrm{C}$ & 2.44318600 & 2.35991600 & 2.36818800 \\
\hline H & -0.15869700 & 3.39910800 & 1.96103100 & $\mathrm{C}$ & 3.38909200 & -0.20005500 & 2.10387300 \\
\hline $\mathrm{H}$ & -0.84710600 & 3.84924900 & 0.41570400 & $\mathrm{C}$ & 3.44245000 & 1.90859100 & 3.23599800 \\
\hline$P$ & 0.62953500 & 1.93922900 & 0.13629500 & $\mathrm{H}$ & 2.06832800 & 3.37316800 & 2.45752700 \\
\hline
\end{tabular}




\begin{tabular}{|c|c|c|c|c|c|c|c|}
\hline $\mathrm{C}$ & 3.92617500 & 0.60886100 & 3.10330200 & $\mathrm{H}$ & 0.65378500 & 0.59389600 & -2.77281800 \\
\hline $\mathrm{H}$ & 3.72528800 & -1.21925200 & 1.94309900 & $\mathrm{C}$ & 2.87505300 & -2.09819400 & -2.61912200 \\
\hline $\mathrm{H}$ & 3.83748200 & 2.56877000 & 4.00183700 & $\mathrm{H}$ & 3.44538200 & -1.85604900 & -3.52300300 \\
\hline $\mathrm{H}$ & 4.70114900 & 0.22321100 & 3.75624000 & $\mathrm{H}$ & 3.57140900 & -2.51964300 & -1.88913900 \\
\hline $\mathrm{N}$ & -4.53628700 & -0.65812200 & 1.30119800 & $\mathrm{H}$ & 2.15153100 & -2.87294400 & -2.88817900 \\
\hline $\mathrm{N}$ & 2.42542800 & 0.24037500 & 1.28295000 & $\mathrm{C}$ & 3.25342300 & 0.28002300 & -1.87235800 \\
\hline $\mathrm{Pd}$ & -0.10262600 & -0.27849200 & -0.42809600 & $\mathrm{H}$ & 4.04174200 & -0.05238500 & -1.19170100 \\
\hline $\mathrm{C}$ & 1.50728500 & -1.81528800 & -0.45584200 & $\mathrm{H}$ & 3.72631800 & 0.51847300 & -2.83146400 \\
\hline $\mathrm{C}$ & 2.86457900 & -2.36788900 & -0.64203400 & $\mathrm{H}$ & 2.81821300 & 1.20220900 & -1.47881300 \\
\hline $\mathrm{C}$ & 3.92601200 & -1.55409200 & -1.07105900 & $\mathrm{C}$ & -2.94462600 & -1.45761300 & -2.99535100 \\
\hline $\mathrm{C}$ & 3.09666600 & -3.73738000 & -0.41448300 & $\mathrm{H}$ & -2.01645300 & -0.95287400 & -3.27935200 \\
\hline $\mathrm{C}$ & 5.18810900 & -2.10413000 & -1.29374900 & $\mathrm{H}$ & -2.73850300 & -2.52627600 & -2.87764700 \\
\hline $\mathrm{H}$ & 3.75547300 & -0.49889200 & -1.26016700 & $\mathrm{H}$ & -3.64630500 & -1.35632500 & -3.83043500 \\
\hline $\mathrm{C}$ & 4.36246700 & -4.27758300 & -0.62757900 & $\mathrm{C}$ & -4.88502100 & -1.55482200 & -1.38089100 \\
\hline $\mathrm{H}$ & 2.28357600 & -4.37023100 & -0.07275200 & $\mathrm{H}$ & -5.39060900 & -1.09136300 & -0.52918700 \\
\hline $\mathrm{C}$ & 5.41021000 & -3.46427400 & -1.06942000 & $\mathrm{H}$ & -5.56979600 & -1.49471900 & -2.23460400 \\
\hline $\mathrm{H}$ & 5.99696800 & -1.47160900 & -1.64601700 & $\mathrm{H}$ & -4.73222500 & -2.61595800 & -1.16102400 \\
\hline $\mathrm{H}$ & 4.53094000 & -5.33569200 & -0.45332300 & $\mathrm{C}$ & -3.83528700 & 0.66546900 & -1.97946600 \\
\hline $\mathrm{H}$ & 6.39396300 & -3.89004100 & -1.23934400 & $\mathrm{H}$ & -4.53237200 & 0.77524500 & -2.81740400 \\
\hline $\mathrm{C}$ & 0.29424100 & -2.24610800 & -0.66138900 & $\mathrm{H}$ & -4.28658300 & 1.15311300 & -1.11028300 \\
\hline $\mathrm{H}$ & -0.22251800 & -3.14367200 & -0.96854000 & $\mathrm{H}$ & -2.91702600 & 1.20190100 & -2.23830600 \\
\hline $\mathrm{H}$ & 1.78734000 & -0.63884000 & 0.31183300 & $\mathrm{C}$ & 2.62048800 & -1.66891600 & 0.73894400 \\
\hline \multirow[t]{2}{*}{$\mathrm{H}$} & -4.98091700 & 0.17442400 & 0.92527200 & $\mathrm{C}$ & 3.36931100 & -0.73466500 & 1.46472100 \\
\hline & & & & $\mathrm{C}$ & 3.79819900 & -3.42701200 & 1.64583100 \\
\hline 10 & & & & $\mathrm{C}$ & 4.38112700 & -1.20082500 & 2.30557400 \\
\hline $\mathrm{C}$ & 0.38288300 & -2.76234700 & -0.70260000 & $\mathrm{H}$ & 3.15165900 & 0.32270700 & 1.38907800 \\
\hline $\mathrm{H}$ & -0.10997700 & -2.72120700 & -1.67964500 & $\mathrm{C}$ & 4.60268600 & -2.57280000 & 2.40186400 \\
\hline $\mathrm{H}$ & 1.15723800 & -3.53000100 & -0.74485900 & $\mathrm{H}$ & 3.93395500 & -4.50510800 & 1.69397100 \\
\hline $\mathrm{C}$ & -0.62862100 & -3.12523700 & 0.41593400 & $\mathrm{H}$ & 4.98130700 & -0.50065900 & 2.87887600 \\
\hline $\mathrm{H}$ & -0.57860100 & -4.20580600 & 0.58690600 & $\mathrm{H}$ & 5.37578100 & -2.97720300 & 3.04676100 \\
\hline $\mathrm{H}$ & -0.33436000 & -2.66109700 & 1.36384200 & $\mathrm{C}$ & -3.00119000 & -0.06682200 & 1.12207800 \\
\hline $\mathrm{C}$ & -2.09999700 & -2.77482200 & 0.09777200 & $\mathrm{C}$ & -4.12948100 & -0.28456200 & 1.90927800 \\
\hline $\mathrm{H}$ & -2.74934800 & -3.04338700 & 0.93722000 & $\mathrm{C}$ & -2.41412900 & 1.82672100 & 2.36144600 \\
\hline $\mathrm{H}$ & -2.43787100 & -3.36002200 & -0.76441100 & $\mathrm{C}$ & -4.40503800 & 0.61389100 & 2.94591800 \\
\hline$P$ & -2.29696100 & -0.97531800 & -0.33292500 & $\mathrm{H}$ & -4.77877500 & -1.13280900 & 1.72323600 \\
\hline $\mathrm{P}$ & 1.29184500 & -1.15240500 & -0.46122200 & $\mathrm{C}$ & -3.54439100 & 1.68725300 & 3.16863500 \\
\hline $\mathrm{C}$ & 2.19471600 & -0.81509300 & -2.10276700 & $\mathrm{H}$ & -1.68435900 & 2.61485200 & 2.51628300 \\
\hline $\mathrm{C}$ & -3.57142700 & -0.83250900 & -1.73267400 & $\mathrm{H}$ & -5.28167500 & 0.47377900 & 3.57084000 \\
\hline $\mathrm{C}$ & 1.15429400 & -0.31406100 & -3.12304500 & $\mathrm{H}$ & -3.73119200 & 2.40116100 & 3.96343700 \\
\hline $\mathrm{H}$ & 1.66009800 & -0.07875100 & -4.06614200 & $\mathrm{~N}$ & 2.83113200 & -2.99165300 & 0.82988200 \\
\hline $\mathrm{H}$ & 0.39387400 & -1.06989700 & -3.34453100 & $\mathrm{~N}$ & -2.15721600 & 0.97113400 & 1.36540700 \\
\hline
\end{tabular}




\begin{tabular}{|c|c|c|c|c|c|c|c|}
\hline $\mathrm{Pd}$ & -0.32473800 & 0.37415100 & 0.24318000 & $\mathrm{H}$ & 3.44765900 & -0.45766500 & 3.21271800 \\
\hline $\mathrm{C}$ & 1.18684800 & 1.75546400 & 2.38416400 & $\mathrm{H}$ & 1.70331700 & -0.23455900 & 3.32598200 \\
\hline $\mathrm{C}$ & 0.90507300 & 1.77288700 & 1.07441800 & $\mathrm{C}$ & -1.87351900 & -2.30594100 & 2.75283800 \\
\hline $\mathrm{H}$ & 0.96281300 & 0.90418800 & 3.02111300 & $\mathrm{H}$ & -0.88501300 & -2.50638000 & 2.32669900 \\
\hline $\mathrm{C}$ & 1.27175300 & 2.88051800 & 0.16622300 & $\mathrm{H}$ & -1.76056900 & -1.56311400 & 3.54846000 \\
\hline $\mathrm{C}$ & 2.55514300 & 3.46024900 & 0.22507300 & $\mathrm{H}$ & -2.22557500 & -3.23005700 & 3.22420800 \\
\hline $\mathrm{C}$ & 0.35917000 & 3.38891000 & -0.77984300 & $\mathrm{C}$ & -4.25609000 & -1.57714000 & 2.31038100 \\
\hline $\mathrm{C}$ & 2.90622100 & 4.51135000 & -0.62104100 & $\mathrm{H}$ & -4.99356700 & -1.25900900 & 1.56742200 \\
\hline $\mathrm{H}$ & 3.27775400 & 3.07663300 & 0.93921300 & $\mathrm{H}$ & -4.63660400 & -2.49220600 & 2.77762200 \\
\hline $\mathrm{C}$ & 0.71223800 & 4.43886200 & -1.62585000 & $\mathrm{H}$ & -4.19955700 & -0.81522500 & 3.09276100 \\
\hline $\mathrm{H}$ & -0.64173700 & 2.96810600 & -0.83388100 & $\mathrm{C}$ & -3.03719400 & -3.00589400 & 0.64008100 \\
\hline $\mathrm{C}$ & 1.98793200 & 5.00328200 & -1.55114300 & $\mathrm{H}$ & -3.47601400 & -3.87830700 & 1.13651500 \\
\hline $\mathrm{H}$ & 3.89991300 & 4.94489000 & -0.55599600 & $\mathrm{H}$ & -3.69172300 & -2.73141900 & -0.19088700 \\
\hline $\mathrm{H}$ & -0.01073100 & 4.82256300 & -2.33983000 & $\mathrm{H}$ & -2.07330500 & -3.31958600 & 0.23158300 \\
\hline $\mathrm{H}$ & 2.26330900 & 5.81997700 & -2.21128300 & $\mathrm{C}$ & 1.34444800 & 2.79538500 & -0.23059100 \\
\hline \multirow[t]{2}{*}{$\mathrm{H}$} & 1.65559400 & 2.61186500 & 2.87170300 & $\mathrm{C}$ & 2.15185100 & 2.73853500 & -1.37466800 \\
\hline & & & & $\mathrm{C}$ & 0.88550200 & 5.02465500 & -0.57973400 \\
\hline 11 & & & & $\mathrm{C}$ & 2.32259700 & 3.90050300 & -2.12825600 \\
\hline $\mathrm{C}$ & -0.01478800 & 2.05161900 & 2.18293900 & $\mathrm{H}$ & 2.62512600 & 1.81152600 & -1.67077100 \\
\hline $\mathrm{H}$ & 0.08772500 & 1.42443400 & 3.07519300 & $\mathrm{C}$ & 1.67822500 & 5.06889200 & -1.72732600 \\
\hline $\mathrm{H}$ & 0.42795900 & 3.02329300 & 2.40350000 & $\mathrm{H}$ & 0.36129600 & 5.91206500 & -0.23228400 \\
\hline $\mathrm{C}$ & -1.50208800 & 2.25721400 & 1.85186400 & $\mathrm{H}$ & 2.94874500 & 3.88878200 & -3.01534100 \\
\hline $\mathrm{H}$ & -1.87219200 & 3.05773400 & 2.50202900 & $\mathrm{H}$ & 1.78265300 & 5.99295200 & -2.28610100 \\
\hline $\mathrm{H}$ & -1.62384200 & 2.62950500 & 0.82966900 & $\mathrm{C}$ & -3.40265400 & 0.13511300 & -0.50854800 \\
\hline $\mathrm{C}$ & -2.36951400 & 1.01483000 & 2.10375900 & $\mathrm{C}$ & -4.51089100 & 0.97252300 & -0.33312700 \\
\hline $\mathrm{H}$ & -3.42306500 & 1.29488500 & 2.18477000 & $\mathrm{C}$ & -3.93452500 & -0.20873800 & -2.72811200 \\
\hline $\mathrm{H}$ & -2.10174000 & 0.57113400 & 3.06841700 & $\mathrm{C}$ & -5.34752100 & 1.21716800 & -1.42452000 \\
\hline $\mathrm{P}$ & -2.18841800 & -0.31073600 & 0.82231200 & $\mathrm{H}$ & -4.73087800 & 1.42842400 & 0.62490000 \\
\hline $\mathrm{P}$ & 1.06814800 & 1.31277000 & 0.86704200 & $\mathrm{C}$ & -5.05830300 & 0.61650800 & -2.64667500 \\
\hline $\mathrm{C}$ & 2.70819800 & 1.01383500 & 1.81002700 & $\mathrm{H}$ & -3.67143300 & -0.70055800 & -3.66165500 \\
\hline $\mathrm{C}$ & -2.88500800 & -1.87307700 & 1.67155300 & $\mathrm{H}$ & -6.21147000 & 1.86560700 & -1.31542800 \\
\hline $\mathrm{C}$ & 3.00310900 & 2.19870700 & 2.75403700 & $\mathrm{H}$ & -5.68203100 & 0.78068700 & -3.51886300 \\
\hline $\mathrm{H}$ & 3.99324500 & 2.04780200 & 3.19797300 & $\mathrm{~N}$ & 0.72113500 & 3.91874400 & 0.15583100 \\
\hline $\mathrm{H}$ & 3.02477000 & 3.15530900 & 2.22282400 & $\mathrm{~N}$ & -3.12127900 & -0.43910500 & -1.69206800 \\
\hline $\mathrm{H}$ & 2.28898200 & 2.27501700 & 3.57705600 & $\mathrm{Pd}$ & 0.01370900 & -0.54524000 & -0.30724600 \\
\hline $\mathrm{C}$ & 3.88582100 & 0.86686600 & 0.83059700 & $\mathrm{C}$ & 2.63139500 & -2.02797900 & -1.01822100 \\
\hline $\mathrm{H}$ & 4.78349700 & 0.61313500 & 1.40542800 & $\mathrm{C}$ & 2.20727900 & -3.03156500 & -0.12764000 \\
\hline $\mathrm{H}$ & 3.72934800 & 0.07072800 & 0.10287400 & $\mathrm{C}$ & 3.96236700 & -2.08649400 & -1.48342400 \\
\hline $\mathrm{H}$ & 4.09181100 & 1.80026800 & 0.30064200 & $\mathrm{C}$ & 3.06050200 & -4.06513400 & 0.25980600 \\
\hline $\mathrm{C}$ & 2.54089300 & -0.28603500 & 2.62211100 & $\mathrm{H}$ & 1.19287200 & -3.00671300 & 0.26103600 \\
\hline $\mathrm{H}$ & 2.39831700 & -1.15285800 & 1.97154800 & $\mathrm{C}$ & 4.81493500 & -3.11634700 & -1.09472200 \\
\hline
\end{tabular}




\begin{tabular}{|c|c|c|c|c|c|c|c|}
\hline $\mathrm{H}$ & 4.33130800 & -1.31084200 & -2.14733600 & $\mathrm{H}$ & -0.63641100 & -3.32203400 & 2.72702000 \\
\hline $\mathrm{C}$ & 4.36827700 & -4.11336200 & -0.22338400 & $\mathrm{H}$ & -0.96082100 & -4.84935500 & 1.90704500 \\
\hline $\mathrm{H}$ & 2.70220500 & -4.83234400 & 0.93979500 & $\mathrm{C}$ & -3.29529100 & -3.43118200 & 1.85244500 \\
\hline $\mathrm{H}$ & 5.83390200 & -3.13829200 & -1.46971100 & $\mathrm{H}$ & -4.18097800 & -2.97294500 & 1.40174100 \\
\hline $\mathrm{H}$ & 5.03539700 & -4.91403900 & 0.08024600 & $\mathrm{H}$ & -3.47972500 & -4.50997500 & 1.89959500 \\
\hline $\mathrm{C}$ & 1.80845700 & -0.35686400 & -2.67219000 & $\mathrm{H}$ & -3.20000400 & -3.07653100 & 2.88211400 \\
\hline $\mathrm{H}$ & 2.58102500 & -0.62873400 & -3.39192800 & $\mathrm{C}$ & -2.19278200 & -3.85352400 & -0.36125900 \\
\hline $\mathrm{H}$ & 1.10635100 & 0.40501300 & -2.99686600 & $\mathrm{H}$ & -2.31034300 & -4.93291900 & -0.21493900 \\
\hline $\mathrm{C}$ & 1.72104400 & -0.95417400 & -1.47539200 & $\mathrm{H}$ & -3.08273700 & -3.49137400 & -0.88256000 \\
\hline $\mathrm{C}$ & -0.67111800 & -1.93297500 & -1.54689900 & $\mathrm{H}$ & -1.33018000 & -3.70352100 & -1.01181100 \\
\hline \multirow[t]{2}{*}{$\mathrm{O}$} & -0.95432700 & -2.72089500 & -2.31842500 & $\mathrm{C}$ & -0.49470800 & 2.80031000 & -0.06742400 \\
\hline & & & & $\mathrm{C}$ & -0.42922700 & 2.80306700 & -1.46643200 \\
\hline \multicolumn{4}{|c|}{ TS8 } & $\mathrm{C}$ & -1.82501300 & 4.67684000 & -0.05385400 \\
\hline \multicolumn{4}{|c|}{ imaginary frequency $=-283.31 \mathrm{~cm}^{-1}$} & $\mathrm{C}$ & -1.09398200 & 3.81152300 & -2.16534400 \\
\hline $\mathrm{C}$ & -0.52540900 & 1.54355700 & 2.52657600 & $\mathrm{H}$ & 0.13171800 & 2.03657700 & -1.98966500 \\
\hline $\mathrm{H}$ & 0.07986100 & 1.01087600 & 3.26873100 & $\mathrm{C}$ & -1.80737000 & 4.76920100 & -1.44715200 \\
\hline $\mathrm{H}$ & -0.58281000 & 2.59184600 & 2.82547200 & $\mathrm{H}$ & -2.37439000 & 5.40191500 & 0.54249100 \\
\hline $\mathrm{C}$ & -1.95313700 & 0.97630000 & 2.49597300 & $\mathrm{H}$ & -1.05604300 & 3.84633900 & -3.25006400 \\
\hline $\mathrm{H}$ & -2.44037600 & 1.28966900 & 3.42666300 & $\mathrm{H}$ & -2.34058100 & 5.56995700 & -1.94880700 \\
\hline $\mathrm{H}$ & -2.52613400 & 1.44959800 & 1.69176300 & $\mathrm{C}$ & -3.04194000 & -0.71253900 & -0.35403700 \\
\hline $\mathrm{C}$ & -2.07426400 & -0.55608900 & 2.42131500 & $\mathrm{C}$ & -4.33802000 & -0.39976100 & 0.07485800 \\
\hline $\mathrm{H}$ & -3.08509200 & -0.85144200 & 2.71693200 & $\mathrm{C}$ & -3.57159900 & -0.22257400 & -2.53919200 \\
\hline $\mathrm{H}$ & -1.39560500 & -1.02336400 & 3.14469600 & $\mathrm{C}$ & -5.27502000 & 0.01853600 & -0.87216900 \\
\hline $\mathrm{P}$ & -1.70421300 & -1.31959200 & 0.77144700 & $\mathrm{H}$ & -4.61890400 & -0.47729400 & 1.11932000 \\
\hline $\mathrm{P}$ & 0.40888800 & 1.51394400 & 0.92325000 & $\mathrm{C}$ & -4.88819800 & 0.10618500 & -2.20680200 \\
\hline $\mathrm{C}$ & 2.08587600 & 2.31693900 & 1.36203700 & $\mathrm{H}$ & -3.23139900 & -0.16509500 & -3.57047600 \\
\hline $\mathrm{C}$ & -2.02293200 & -3.18587200 & 1.01716500 & $\mathrm{H}$ & -6.28724800 & 0.26784500 & -0.56877200 \\
\hline $\mathrm{C}$ & 1.89274300 & 3.58156900 & 2.22169000 & $\mathrm{H}$ & -5.58416700 & 0.42350200 & -2.97617800 \\
\hline $\mathrm{H}$ & 2.86739500 & 4.06394600 & 2.35746500 & $\mathrm{~N}$ & -1.18443100 & 3.71887300 & 0.62726300 \\
\hline $\mathrm{H}$ & 1.22296500 & 4.30686800 & 1.75235900 & $\mathrm{~N}$ & -2.66181600 & -0.61665300 & -1.64109300 \\
\hline $\mathrm{H}$ & 1.50540600 & 3.35176300 & 3.21778100 & $\mathrm{Pd}$ & 0.38020800 & -0.67983800 & -0.21925600 \\
\hline $\mathrm{C}$ & 2.80646600 & 2.69552100 & 0.05464400 & $\mathrm{C}$ & 3.48895600 & -0.97523400 & -1.01828600 \\
\hline $\mathrm{H}$ & 3.79879800 & 3.09099400 & 0.29823200 & $\mathrm{C}$ & 3.65114100 & -1.89561000 & 0.03288100 \\
\hline $\mathrm{H}$ & 2.94686000 & 1.83470000 & -0.60233500 & $\mathrm{C}$ & 4.63789500 & -0.32551600 & -1.51303800 \\
\hline $\mathrm{H}$ & 2.26848300 & 3.47242900 & -0.49613800 & $\mathrm{C}$ & 4.91231400 & -2.17165900 & 0.56016000 \\
\hline $\mathrm{C}$ & 2.91661700 & 1.27668600 & 2.13905600 & $\mathrm{H}$ & 2.78377000 & -2.40586700 & 0.44121400 \\
\hline $\mathrm{H}$ & 3.10278100 & 0.37561200 & 1.54953300 & $\mathrm{C}$ & 5.89535800 & -0.59840500 & -0.98270300 \\
\hline $\mathrm{H}$ & 3.88855100 & 1.71342700 & 2.39497100 & $\mathrm{H}$ & 4.54837700 & 0.40317700 & -2.31232100 \\
\hline $\mathrm{H}$ & 2.43838800 & 0.98488500 & 3.07998100 & $\mathrm{C}$ & 6.03971000 & -1.52479900 & 0.05402700 \\
\hline $\mathrm{C}$ & -0.79943200 & -3.77808100 & 1.74478300 & $\mathrm{H}$ & 5.01199700 & -2.89284200 & 1.36550700 \\
\hline $\mathrm{H}$ & 0.11603500 & -3.66672200 & 1.15701900 & $\mathrm{H}$ & 6.76635400 & -0.08563600 & -1.37933400 \\
\hline
\end{tabular}




\begin{tabular}{|c|c|c|c|c|c|c|c|}
\hline $\mathrm{H}$ & 7.02222600 & -1.73688700 & 0.46380000 & $\mathrm{H}$ & 0.79812700 & -3.24094100 & 3.57103800 \\
\hline $\mathrm{C}$ & 1.89027300 & -0.12429400 & -2.77927600 & $\mathrm{C}$ & 1.86040100 & -3.75133100 & 0.29361300 \\
\hline $\mathrm{H}$ & 2.67766200 & 0.23798500 & -3.43835200 & $\mathrm{H}$ & 2.74796800 & -4.32423100 & 0.58523100 \\
\hline $\mathrm{H}$ & 0.87485400 & -0.02965400 & -3.15138500 & $\mathrm{H}$ & 1.08239300 & -4.46821600 & 0.01646500 \\
\hline $\mathrm{C}$ & 2.15752100 & -0.70636800 & -1.59745000 & $\mathrm{H}$ & 2.11132100 & -3.16916100 & -0.59326800 \\
\hline $\mathrm{C}$ & 0.87308200 & -2.15728400 & -1.32808600 & $\mathrm{C}$ & -2.82435700 & 1.55551900 & -0.33403500 \\
\hline \multirow[t]{2}{*}{$\mathrm{O}$} & 0.97471900 & -3.17917000 & -1.86334900 & $\mathrm{C}$ & -2.81939500 & 0.98699900 & -1.61242400 \\
\hline & & & & $\mathrm{C}$ & -5.06768300 & 2.04419900 & -0.47772000 \\
\hline 12 & & & & $\mathrm{C}$ & -4.01130800 & 0.96800600 & -2.34069900 \\
\hline $\mathrm{C}$ & -1.90006500 & 1.01602800 & 2.32421700 & $\mathrm{H}$ & -1.91130100 & 0.55100400 & -2.01491900 \\
\hline $\mathrm{H}$ & -1.17721300 & 1.30816500 & 3.09448600 & $\mathrm{C}$ & -5.15773300 & 1.51084100 & -1.76663800 \\
\hline $\mathrm{H}$ & -2.82844200 & 1.56117600 & 2.51449900 & $\mathrm{H}$ & -5.94342700 & 2.46838600 & 0.00833500 \\
\hline $\mathrm{C}$ & -2.17724100 & -0.49310200 & 2.40423000 & $\mathrm{H}$ & -4.04117000 & 0.53473000 & -3.33595800 \\
\hline $\mathrm{H}$ & -2.78069500 & -0.66080400 & 3.30385700 & $\mathrm{H}$ & -6.10488400 & 1.51921200 & -2.29582500 \\
\hline $\mathrm{H}$ & -2.80810800 & -0.81040900 & 1.56544400 & $\mathrm{C}$ & -1.19147900 & -2.76293600 & -0.05268400 \\
\hline $\mathrm{C}$ & -0.93534500 & -1.39550800 & 2.51806400 & $\mathrm{C}$ & -1.96275000 & -3.79810200 & 0.49267500 \\
\hline $\mathrm{H}$ & -1.23227600 & -2.35861400 & 2.94247700 & $\mathrm{C}$ & -2.03855400 & -3.15221600 & -2.15674500 \\
\hline $\mathrm{H}$ & -0.21756900 & -0.95891800 & 3.22275400 & $\mathrm{C}$ & -2.79689900 & -4.53190700 & -0.35201500 \\
\hline $\mathrm{P}$ & -0.01231400 & -1.73135800 & 0.93794400 & $\mathrm{H}$ & -1.92112100 & -4.03207900 & 1.55067800 \\
\hline$P$ & -1.27515900 & 1.63246600 & 0.68759400 & $\mathrm{C}$ & -2.83302500 & -4.20860900 & -1.70629300 \\
\hline $\mathrm{C}$ & -0.94365000 & 3.48992400 & 0.99263700 & $\mathrm{H}$ & -2.04335900 & -2.86591500 & -3.20594400 \\
\hline $\mathrm{C}$ & 1.42531600 & -2.87397300 & 1.48353600 & $\mathrm{H}$ & -3.40404200 & -5.34054700 & 0.04336900 \\
\hline $\mathrm{C}$ & -1.96849700 & 4.11353700 & 1.96187800 & $\mathrm{H}$ & -3.46278100 & -4.75544900 & -2.40017100 \\
\hline $\mathrm{H}$ & -1.77521000 & 5.19040200 & 2.02908800 & $\mathrm{~N}$ & -3.93239000 & 2.06713400 & 0.22845900 \\
\hline $\mathrm{H}$ & -2.99436000 & 3.96987300 & 1.61668300 & $\mathrm{~N}$ & -1.23916300 & -2.43732200 & -1.35636100 \\
\hline $\mathrm{H}$ & -1.88149400 & 3.70709000 & 2.97318100 & $\mathrm{Pd}$ & 0.49807300 & 0.24432700 & -0.31262100 \\
\hline $\mathrm{C}$ & -1.01348400 & 4.23175200 & -0.35540500 & $\mathrm{C}$ & 3.65371600 & 1.01083600 & -1.05971400 \\
\hline $\mathrm{H}$ & -0.70637000 & 5.27328900 & -0.20923900 & $\mathrm{C}$ & 4.65026600 & 0.02594100 & -1.18268900 \\
\hline $\mathrm{H}$ & -0.35208500 & 3.79558300 & -1.10848000 & $\mathrm{C}$ & 3.97456900 & 2.23314700 & -0.44412100 \\
\hline $\mathrm{H}$ & -2.02957000 & 4.24012200 & -0.75856100 & $\mathrm{C}$ & 5.93771000 & 0.26209700 & -0.70428000 \\
\hline $\mathrm{C}$ & 0.47062300 & 3.60723100 & 1.59429000 & $\mathrm{H}$ & 4.42459900 & -0.91687600 & -1.67125600 \\
\hline $\mathrm{H}$ & 1.23516100 & 3.20056300 & 0.92636800 & $\mathrm{C}$ & 5.26192800 & 2.46349200 & 0.03215000 \\
\hline $\mathrm{H}$ & 0.70281100 & 4.66279600 & 1.77545200 & $\mathrm{H}$ & 3.22353300 & 3.00872900 & -0.33855900 \\
\hline $\mathrm{H}$ & 0.55366100 & 3.08789100 & 2.55515800 & $\mathrm{C}$ & 6.24631200 & 1.47883500 & -0.09398200 \\
\hline $\mathrm{C}$ & 2.58539900 & -1.95787600 & 1.92341200 & $\mathrm{H}$ & 6.69998800 & -0.50270200 & -0.81410200 \\
\hline $\mathrm{H}$ & 2.94564000 & -1.33156700 & 1.10257600 & $\mathrm{H}$ & 5.49867500 & 3.41260800 & 0.50271300 \\
\hline $\mathrm{H}$ & 2.30342600 & -1.30060900 & 2.75352700 & $\mathrm{H}$ & 7.24904900 & 1.66222900 & 0.27909500 \\
\hline $\mathrm{H}$ & 3.42293300 & -2.57492000 & 2.26755500 & $\mathrm{C}$ & 1.30024400 & 1.70781900 & -1.85916600 \\
\hline $\mathrm{C}$ & 1.02965500 & -3.79307000 & 2.65668400 & $\mathrm{H}$ & 1.49619100 & 2.75578900 & -1.66955000 \\
\hline $\mathrm{H}$ & 0.18252500 & -4.44092200 & 2.41243000 & $\mathrm{H}$ & 0.51073600 & 1.49574800 & -2.57584100 \\
\hline $\mathrm{H}$ & 1.87712600 & -4.44983400 & 2.88244600 & $\mathrm{C}$ & 2.31548300 & 0.76449900 & -1.64981200 \\
\hline
\end{tabular}




\begin{tabular}{|c|c|c|c|c|c|c|c|}
\hline $\mathrm{C}$ & 1.83757100 & -0.59599000 & -1.72825600 & $\mathrm{C}$ & 1.38548000 & 2.56623000 & -0.82044100 \\
\hline \multirow[t]{2}{*}{$\mathrm{O}$} & 1.96310600 & -1.62543800 & -2.30183500 & $\mathrm{C}$ & 2.67536000 & 2.23085400 & -0.38420400 \\
\hline & & & & $\mathrm{C}$ & 2.22396000 & 3.76526400 & -2.59805900 \\
\hline $12 \mathbf{a}$ & & & & $\mathrm{C}$ & 3.76925400 & 2.71644100 & -1.10020100 \\
\hline $\mathrm{C}$ & -1.51315300 & 2.56006800 & -1.02599300 & $\mathrm{H}$ & 2.82789700 & 1.61172800 & 0.49172600 \\
\hline $\mathrm{H}$ & -2.35107400 & 2.86057200 & -0.38884000 & $\mathrm{C}$ & 3.54346700 & 3.49804400 & -2.23211500 \\
\hline $\mathrm{H}$ & -1.12850700 & 3.45423400 & -1.52131000 & $\mathrm{H}$ & 2.00204800 & 4.36699700 & -3.47647200 \\
\hline $\mathrm{C}$ & -1.98502200 & 1.55126500 & -2.10368400 & $\mathrm{H}$ & 4.77936000 & 2.48243600 & -0.77799300 \\
\hline $\mathrm{H}$ & -2.27540600 & 2.12099000 & -2.99330500 & $\mathrm{H}$ & 4.36562300 & 3.89194700 & -2.82028600 \\
\hline $\mathrm{H}$ & -1.15348400 & 0.91117200 & -2.41902900 & $\mathrm{C}$ & -2.35846600 & -2.05993800 & -0.79654500 \\
\hline $\mathrm{C}$ & -3.19906000 & 0.68131400 & -1.70452500 & $\mathrm{C}$ & -2.98595700 & -2.96472700 & -1.65120000 \\
\hline $\mathrm{H}$ & -3.51535000 & 0.06146800 & -2.55014600 & $\mathrm{C}$ & -0.57983600 & -3.53248300 & -0.44511800 \\
\hline $\mathrm{H}$ & -4.04521200 & 1.33351400 & -1.45971500 & $\mathrm{C}$ & -2.38329000 & -4.20568600 & -1.88018300 \\
\hline $\mathrm{P}$ & -2.87037800 & -0.37589300 & -0.20530800 & $\mathrm{H}$ & -3.92557300 & -2.71108700 & -2.12865900 \\
\hline $\mathrm{P}$ & -0.13276200 & 1.99263200 & 0.09377100 & $\mathrm{C}$ & -1.17220600 & -4.49995500 & -1.25760400 \\
\hline $\mathrm{C}$ & -0.23258400 & 3.09061900 & 1.64703500 & $\mathrm{H}$ & 0.38139400 & -3.70086900 & 0.02852100 \\
\hline $\mathrm{C}$ & -4.51836900 & -0.60366900 & 0.71540900 & $\mathrm{H}$ & -2.85758800 & -4.93163700 & -2.53337900 \\
\hline $\mathrm{C}$ & -1.49297900 & 2.69222600 & 2.43901000 & $\mathrm{H}$ & -0.68051000 & -5.45527500 & -1.40516800 \\
\hline $\mathrm{H}$ & -1.55010900 & 3.30360700 & 3.34628800 & $\mathrm{~N}$ & 1.16655500 & 3.31659800 & -1.91009900 \\
\hline $\mathrm{H}$ & -2.41506100 & 2.86366500 & 1.87514200 & $\mathrm{~N}$ & -1.15720500 & -2.34419000 & -0.22354900 \\
\hline $\mathrm{H}$ & -1.45994900 & 1.64140900 & 2.74295400 & $\mathrm{Pd}$ & -0.45396900 & -0.30042900 & 0.41041000 \\
\hline $\mathrm{C}$ & -0.29990700 & 4.57730700 & 1.24755700 & $\mathrm{C}$ & 3.71161100 & -1.85971700 & 0.21791200 \\
\hline $\mathrm{H}$ & -0.31650600 & 5.18916000 & 2.15660300 & $\mathrm{C}$ & 3.84912800 & -2.59989700 & 1.40457700 \\
\hline $\mathrm{H}$ & 0.56827100 & 4.88423000 & 0.65770100 & $\mathrm{C}$ & 4.84737700 & -1.66139700 & -0.58715300 \\
\hline $\mathrm{H}$ & -1.20219300 & 4.81501500 & 0.67664700 & $\mathrm{C}$ & 5.08254400 & -3.14399800 & 1.75948300 \\
\hline $\mathrm{C}$ & 1.00897600 & 2.83265900 & 2.52356800 & $\mathrm{H}$ & 2.99273800 & -2.75238400 & 2.05140800 \\
\hline $\mathrm{H}$ & 1.92585900 & 3.18996900 & 2.04805600 & $\mathrm{C}$ & 6.07979000 & -2.20360600 & -0.22782200 \\
\hline $\mathrm{H}$ & 0.89630800 & 3.38469600 & 3.46330500 & $\mathrm{H}$ & 4.76540100 & -1.06268300 & -1.48976500 \\
\hline $\mathrm{H}$ & 1.12951900 & 1.77319600 & 2.76711900 & $\mathrm{C}$ & 6.20053000 & -2.94942300 & 0.94630900 \\
\hline $\mathrm{C}$ & -4.86292300 & 0.74958300 & 1.37052500 & $\mathrm{H}$ & 5.17007400 & -3.71996500 & 2.67573700 \\
\hline $\mathrm{H}$ & -4.08701600 & 1.07007100 & 2.07122200 & $\mathrm{H}$ & 6.94682200 & -2.03633700 & -0.85985600 \\
\hline $\mathrm{H}$ & -5.01790900 & 1.54596000 & 0.63507600 & $\mathrm{H}$ & 7.16089000 & -3.36934600 & 1.22945300 \\
\hline $\mathrm{H}$ & -5.79725700 & 0.64663300 & 1.93313800 & $\mathrm{C}$ & 2.00542000 & -1.28284000 & -1.50161100 \\
\hline $\mathrm{C}$ & -5.66036000 & -1.03682800 & -0.22180700 & $\mathrm{H}$ & 2.61633100 & -1.70758100 & -2.29136600 \\
\hline $\mathrm{H}$ & -5.48723600 & -2.02753900 & -0.65060500 & $\mathrm{H}$ & 1.06449700 & -0.82251900 & -1.78619500 \\
\hline $\mathrm{H}$ & -6.59313500 & -1.09434000 & 0.35112200 & $\mathrm{C}$ & 2.39830100 & -1.30898200 & -0.21471700 \\
\hline $\mathrm{H}$ & -5.82449100 & -0.32607200 & -1.03760000 & $\mathrm{C}$ & 1.48578500 & -0.70523400 & 0.82424400 \\
\hline $\mathrm{C}$ & -4.29595300 & -1.66177700 & 1.81362000 & $\mathrm{O}$ & 1.79434900 & -0.56583600 & 1.98548000 \\
\hline $\mathrm{H}$ & -5.20788600 & -1.75674500 & 2.41308500 & & & & \\
\hline $\mathrm{H}$ & -4.07289000 & -2.64772800 & 1.39495700 & $12 b$ & & & \\
\hline $\mathrm{H}$ & -3.48078000 & -1.38241200 & 2.48871000 & $\mathrm{C}$ & 0.85387000 & -2.03547600 & 1.73938000 \\
\hline
\end{tabular}




\begin{tabular}{|c|c|c|c|c|c|c|c|}
\hline $\mathrm{H}$ & 0.36783200 & -2.99921800 & 1.55657300 & $\mathrm{C}$ & 5.40985500 & 0.62636200 & 1.37113500 \\
\hline $\mathrm{H}$ & 1.75085700 & -2.21935200 & 2.33499000 & $\mathrm{H}$ & 4.91370100 & -0.31359300 & 3.25529700 \\
\hline $\mathrm{C}$ & -0.09183600 & -1.08713400 & 2.52203200 & $\mathrm{H}$ & 5.56416000 & 1.40694600 & -0.63786300 \\
\hline $\mathrm{H}$ & 0.11113800 & -1.21745700 & 3.59091900 & $\mathrm{H}$ & 6.33055200 & 1.07161000 & 1.73309900 \\
\hline $\mathrm{H}$ & 0.14112200 & -0.04087500 & 2.29848300 & $\mathrm{C}$ & -2.88267200 & 0.44428100 & 0.23132500 \\
\hline $\mathrm{C}$ & -1.60339600 & -1.34034300 & 2.31499300 & $\mathrm{C}$ & -3.97893600 & 1.07235200 & 0.81927800 \\
\hline $\mathrm{H}$ & -2.18922000 & -0.64817300 & 2.92881100 & $\mathrm{C}$ & -2.60077100 & 2.21140200 & -1.27042000 \\
\hline $\mathrm{H}$ & -1.85103100 & -2.35154500 & 2.65682600 & $\mathrm{C}$ & -4.40543500 & 2.30259700 & 0.30908100 \\
\hline $\mathrm{P}$ & -2.11601400 & -1.22053600 & 0.52570000 & $\mathrm{H}$ & -4.49691300 & 0.61290300 & 1.65312800 \\
\hline$P$ & 1.47543900 & -1.40151300 & 0.09816800 & $\mathrm{C}$ & -3.72099500 & 2.87001300 & -0.76250800 \\
\hline $\mathrm{C}$ & 2.02250400 & -2.93326600 & -0.89494000 & $\mathrm{H}$ & -2.00962500 & 2.63187300 & -2.07678600 \\
\hline $\mathrm{C}$ & -3.49868500 & -2.49788300 & 0.23736500 & $\mathrm{H}$ & -5.26589000 & 2.80427000 & 0.74100500 \\
\hline $\mathrm{C}$ & 0.76660800 & -3.73324300 & -1.28674300 & $\mathrm{H}$ & -4.03263500 & 3.81532100 & -1.19287100 \\
\hline $\mathrm{H}$ & 1.06637900 & -4.58681800 & -1.90450500 & $\mathrm{~N}$ & 3.47590500 & -0.72730100 & 1.83536100 \\
\hline $\mathrm{H}$ & 0.24203800 & -4.13503400 & -0.41493700 & $\mathrm{~N}$ & -2.19168900 & 1.03390300 & -0.78226800 \\
\hline $\mathrm{H}$ & 0.06673700 & -3.12586600 & -1.86865500 & $\mathrm{Pd}$ & -0.28634100 & -0.12979300 & -0.79717000 \\
\hline $\mathrm{C}$ & 2.96686300 & -3.80951800 & -0.04924600 & $\mathrm{C}$ & 0.61007900 & 3.15807200 & -0.26726900 \\
\hline $\mathrm{H}$ & 3.28881600 & -4.66700200 & -0.65106300 & $\mathrm{C}$ & 1.05581200 & 2.57032100 & 0.92846100 \\
\hline $\mathrm{H}$ & 3.86431700 & -3.26728400 & 0.26056700 & $\mathrm{C}$ & -0.13657100 & 4.34687200 & -0.18408300 \\
\hline $\mathrm{H}$ & 2.47906200 & -4.20302600 & 0.84738500 & $\mathrm{C}$ & 0.76550400 & 3.15222800 & 2.16358400 \\
\hline $\mathrm{C}$ & 2.73940200 & -2.46548200 & -2.17697500 & $\mathrm{H}$ & 1.66916700 & 1.67893100 & 0.89009900 \\
\hline $\mathrm{H}$ & 3.68883900 & -1.97162300 & -1.95533600 & $\mathrm{C}$ & -0.42638900 & 4.92870300 & 1.04934800 \\
\hline $\mathrm{H}$ & 2.96701400 & -3.34336900 & -2.79174200 & $\mathrm{H}$ & -0.49458800 & 4.81614700 & -1.09604300 \\
\hline $\mathrm{H}$ & 2.12322700 & -1.78435600 & -2.77016200 & $\mathrm{C}$ & 0.02148800 & 4.33147700 & 2.22964900 \\
\hline $\mathrm{C}$ & -2.85632700 & -3.89723500 & 0.32125400 & $\mathrm{H}$ & 1.13889600 & 2.69248000 & 3.07416300 \\
\hline $\mathrm{H}$ & -2.06551200 & -4.02607100 & -0.42214300 & $\mathrm{H}$ & -1.00041000 & 5.84978100 & 1.08858100 \\
\hline $\mathrm{H}$ & -2.44214200 & -4.11190300 & 1.31191200 & $\mathrm{H}$ & -0.19967200 & 4.78624300 & 3.19045900 \\
\hline $\mathrm{H}$ & -3.62364000 & -4.65394100 & 0.12447300 & $\mathrm{C}$ & 1.34206700 & 3.36862700 & -2.62755500 \\
\hline $\mathrm{C}$ & -4.62946500 & -2.38264700 & 1.27545800 & $\mathrm{H}$ & 1.44407500 & 4.44393000 & -2.52050700 \\
\hline $\mathrm{H}$ & -5.17444700 & -1.43962200 & 1.18640100 & $\mathrm{H}$ & 1.57150000 & 2.92927400 & -3.59238600 \\
\hline $\mathrm{H}$ & -5.35425500 & -3.18802600 & 1.10881100 & $\mathrm{C}$ & 0.96354100 & 2.59138000 & -1.59428100 \\
\hline $\mathrm{H}$ & -4.26578600 & -2.48129600 & 2.30274700 & $\mathrm{C}$ & 0.87912000 & 1.10071400 & -1.89249500 \\
\hline $\mathrm{C}$ & -4.05177600 & -2.27389200 & -1.18366400 & $\mathrm{O}$ & 1.35537900 & 0.62623600 & -2.90013600 \\
\hline $\mathrm{H}$ & -4.79770100 & -3.04461000 & -1.40677500 & & & & \\
\hline $\mathrm{H}$ & -4.54337100 & -1.30173700 & -1.28485300 & $12 c$ & & & \\
\hline H & -3.26536700 & -2.34157500 & -1.94202300 & $\mathrm{C}$ & 0.77718300 & 2.87601000 & 1.16689500 \\
\hline $\mathrm{C}$ & 3.06642200 & -0.55130700 & 0.57003100 & $\mathrm{H}$ & 1.32655400 & 3.60154400 & 0.56018200 \\
\hline $\mathrm{C}$ & 3.78643300 & 0.22065800 & -0.35433100 & $\mathrm{H}$ & 0.13456200 & 3.43038300 & 1.85581200 \\
\hline $\mathrm{C}$ & 4.61947100 & -0.14766500 & 2.22142500 & $\mathrm{C}$ & 1.77244900 & 2.02826200 & 1.97858400 \\
\hline $\mathrm{C}$ & 4.98168000 & 0.81045000 & 0.05764300 & $\mathrm{H}$ & 2.09155900 & 2.63782500 & 2.83183300 \\
\hline $\mathrm{H}$ & 3.42572500 & 0.36025400 & -1.36645600 & $\mathrm{H}$ & 1.27478800 & 1.15129800 & 2.40922200 \\
\hline
\end{tabular}




\begin{tabular}{|c|c|c|c|c|c|c|c|}
\hline $\mathrm{C}$ & 3.03989900 & 1.61904100 & 1.20158800 & $\mathrm{C}$ & 3.78961900 & -1.26442900 & 2.40281400 \\
\hline $\mathrm{H}$ & 3.86722000 & 1.43310100 & 1.89131400 & $\mathrm{C}$ & 2.99272500 & -3.62762800 & 1.29297100 \\
\hline $\mathrm{H}$ & 3.36339500 & 2.45684100 & 0.57486200 & $\mathrm{C}$ & 4.00973300 & -2.45029800 & 3.10627900 \\
\hline $\mathrm{P}$ & 2.81107000 & 0.16298200 & 0.06736000 & $\mathrm{H}$ & 4.10401100 & -0.32222600 & 2.83420300 \\
\hline $\mathrm{P}$ & -0.41431100 & 1.98793200 & 0.04137200 & $\mathrm{C}$ & 3.61043100 & -3.65862100 & 2.54214600 \\
\hline $\mathrm{C}$ & -0.60301500 & 3.15326900 & -1.46196200 & $\mathrm{H}$ & 2.65241200 & -4.54316200 & 0.81606500 \\
\hline $\mathrm{C}$ & 4.33837500 & 0.23551100 & -1.09039000 & $\mathrm{H}$ & 4.48981200 & -2.42534700 & 4.07984300 \\
\hline $\mathrm{C}$ & 0.72438600 & 3.13008400 & -2.24342500 & $\mathrm{H}$ & 3.76370400 & -4.60241900 & 3.05376800 \\
\hline $\mathrm{H}$ & 0.64456700 & 3.80603500 & -3.10184400 & $\mathrm{~N}$ & -1.94186900 & 2.73179700 & 2.18523900 \\
\hline $\mathrm{H}$ & 1.57702600 & 3.46612800 & -1.64557900 & $\mathrm{~N}$ & 2.77174400 & -2.49220000 & 0.61646100 \\
\hline $\mathrm{H}$ & 0.94103600 & 2.12771700 & -2.62499000 & $\mathrm{Pd}$ & 0.34235200 & -0.15278600 & -0.54697000 \\
\hline $\mathrm{C}$ & -0.92382100 & 4.58636900 & -0.99225200 & $\mathrm{C}$ & -3.60248800 & -2.14278000 & -0.09119000 \\
\hline $\mathrm{H}$ & -1.05359700 & 5.22428300 & -1.87391100 & $\mathrm{C}$ & -3.88770500 & -2.80729800 & -1.29559000 \\
\hline $\mathrm{H}$ & -1.85181600 & 4.63076000 & -0.41521200 & $\mathrm{C}$ & -4.61337500 & -2.04969900 & 0.88227100 \\
\hline $\mathrm{H}$ & -0.12463900 & 5.02543300 & -0.38900300 & $\mathrm{C}$ & -5.14156900 & -3.37920100 & -1.50665400 \\
\hline $\mathrm{C}$ & -1.72632500 & 2.65563800 & -2.39253100 & $\mathrm{H}$ & -3.12723700 & -2.88502900 & -2.06309200 \\
\hline $\mathrm{H}$ & -2.71314100 & 2.76228300 & -1.93603500 & $\mathrm{C}$ & -5.86655600 & -2.62090600 & 0.66719300 \\
\hline $\mathrm{H}$ & -1.72526100 & 3.27185900 & -3.29859600 & $\mathrm{H}$ & -4.41647000 & -1.51217700 & 1.80540900 \\
\hline $\mathrm{H}$ & -1.58943600 & 1.61374200 & -2.69181500 & $\mathrm{C}$ & -6.13400500 & -3.28961700 & -0.52871400 \\
\hline $\mathrm{C}$ & 4.17122100 & 1.45199400 & -2.02257700 & $\mathrm{H}$ & -5.34278300 & -3.89848400 & -2.43881200 \\
\hline $\mathrm{H}$ & 3.25732700 & 1.38542600 & -2.61946400 & $\mathrm{H}$ & -6.63478500 & -2.53847500 & 1.43042000 \\
\hline $\mathrm{H}$ & 4.16130400 & 2.40254200 & -1.48071100 & $\mathrm{H}$ & -7.11019800 & -3.73322400 & -0.69952600 \\
\hline $\mathrm{H}$ & 5.01860800 & 1.48786600 & -2.71612000 & $\mathrm{C}$ & -1.67510500 & -1.66856200 & 1.40097500 \\
\hline $\mathrm{C}$ & 5.64434400 & 0.36685900 & -0.28492500 & $\mathrm{H}$ & -2.13128600 & -2.24196400 & 2.20125600 \\
\hline $\mathrm{H}$ & 5.79912900 & -0.47986500 & 0.39024900 & $\mathrm{H}$ & -0.72263400 & -1.18963500 & 1.60827300 \\
\hline $\mathrm{H}$ & 6.49290200 & 0.39198000 & -0.97812400 & $\mathrm{C}$ & -2.26232800 & -1.56009000 & 0.19542300 \\
\hline $\mathrm{H}$ & 5.68045800 & 1.28902300 & 0.30271400 & $\mathrm{C}$ & -1.54989900 & -0.80421700 & -0.89652900 \\
\hline $\mathrm{C}$ & 4.38170400 & -1.04243600 & -1.94768900 & $\mathrm{O}$ & -1.96283900 & -0.71033400 & -2.02893500 \\
\hline $\mathrm{H}$ & 5.23730800 & -0.98566100 & -2.62981500 & $\mathrm{O}$ & 0.77361100 & -2.25958000 & -1.17956100 \\
\hline $\mathrm{H}$ & 4.49681400 & -1.94715200 & -1.34578400 & $\mathrm{H}$ & 1.60029700 & -2.49293100 & -0.67355400 \\
\hline $\mathrm{H}$ & 3.48093800 & -1.14688700 & -2.55918300 & $\mathrm{C}$ & 0.81680800 & -2.67090600 & -2.55173000 \\
\hline $\mathrm{C}$ & -2.02082400 & 2.13573700 & 0.98765900 & $\mathrm{H}$ & 1.68860400 & -2.26330900 & -3.07459800 \\
\hline $\mathrm{C}$ & -3.23164300 & 1.64785400 & 0.47513800 & $\mathrm{H}$ & 0.83487500 & -3.76384800 & -2.61205800 \\
\hline $\mathrm{C}$ & -3.05965400 & 2.87457400 & 2.90767900 & $\mathrm{H}$ & -0.09034400 & -2.30070000 & -3.03029600 \\
\hline $\mathrm{C}$ & -4.39334200 & 1.81376200 & 1.22889700 & & & & \\
\hline $\mathrm{H}$ & -3.27822500 & 1.14725700 & -0.48343600 & $12 d$ & & & \\
\hline $\mathrm{C}$ & -4.31081700 & 2.43895300 & 2.47185300 & $\mathrm{C}$ & 1.08469200 & 0.98451600 & -2.44820300 \\
\hline $\mathrm{H}$ & -2.94529900 & 3.36158100 & 3.87341700 & $\mathrm{H}$ & 0.21644100 & 0.77045600 & -3.08119800 \\
\hline $\mathrm{H}$ & -5.34316000 & 1.45017500 & 0.84925300 & $\mathrm{H}$ & 1.63676900 & 1.81139100 & -2.89940300 \\
\hline $\mathrm{H}$ & -5.18911300 & 2.58485100 & 3.09179500 & $\mathrm{C}$ & 2.02616200 & -0.23184400 & -2.37520800 \\
\hline $\mathrm{C}$ & 3.16948100 & -1.31404200 & 1.14902300 & $\mathrm{H}$ & 2.61562700 & -0.22540500 & -3.29940500 \\
\hline
\end{tabular}




\begin{tabular}{|c|c|c|c|c|c|c|c|}
\hline $\mathrm{H}$ & 2.75623800 & -0.10708400 & -1.56908700 & $\mathrm{C}$ & 2.03419300 & -2.32092900 & 0.42932500 \\
\hline $\mathrm{C}$ & 1.35977500 & -1.61188100 & -2.28189600 & $\mathrm{C}$ & 1.95620500 & -2.12598600 & 1.81466500 \\
\hline $\mathrm{H}$ & 2.11862100 & -2.38077900 & -2.44598400 & $\mathrm{C}$ & 4.22310200 & -3.01204800 & 0.58270600 \\
\hline $\mathrm{H}$ & 0.59264800 & -1.71581200 & -3.05696200 & $\mathrm{C}$ & 3.07628300 & -2.40754900 & 2.59828400 \\
\hline $\mathrm{P}$ & 0.56062500 & -1.98598000 & -0.65287900 & $\mathrm{H}$ & 1.04032200 & -1.75942800 & 2.26877400 \\
\hline $\mathrm{P}$ & 0.45804900 & 1.67951500 & -0.84325200 & $\mathrm{C}$ & 4.23538500 & -2.85993500 & 1.97116100 \\
\hline $\mathrm{C}$ & -0.51639800 & 3.24577900 & -1.34980200 & $\mathrm{H}$ & 5.11012500 & -3.35694600 & 0.05596500 \\
\hline $\mathrm{C}$ & -0.26017200 & -3.68847600 & -0.91057500 & $\mathrm{H}$ & 3.04344500 & -2.27185000 & 3.67513300 \\
\hline $\mathrm{C}$ & 0.22927500 & 4.01290900 & -2.46346300 & $\mathrm{H}$ & 5.13173500 & -3.08807200 & 2.53831000 \\
\hline $\mathrm{H}$ & -0.29294200 & 4.96029200 & -2.63765900 & $\mathrm{~N}$ & 2.96491300 & 2.69927400 & -0.90891200 \\
\hline $\mathrm{H}$ & 1.26245500 & 4.24221700 & -2.19131800 & $\mathrm{~N}$ & 3.15149800 & -2.75360200 & -0.17626600 \\
\hline $\mathrm{H}$ & 0.23271300 & 3.46785700 & -3.41058000 & $\mathrm{Pd}$ & -0.67518900 & -0.02509600 & 0.34784500 \\
\hline $\mathrm{C}$ & -0.66968400 & 4.16941800 & -0.12749300 & $\mathrm{C}$ & -3.68760300 & 0.36184600 & 0.96974000 \\
\hline $\mathrm{H}$ & -1.26082400 & 5.04409000 & -0.42060800 & $\mathrm{C}$ & -3.26356500 & -0.97539400 & 1.09927000 \\
\hline $\mathrm{H}$ & -1.19004400 & 3.68765900 & 0.70188800 & $\mathrm{C}$ & -4.85048000 & 0.63015600 & 0.23188100 \\
\hline $\mathrm{H}$ & 0.29631100 & 4.53022400 & 0.23574700 & $\mathrm{C}$ & -3.99595000 & -2.01577700 & 0.51839100 \\
\hline $\mathrm{C}$ & -1.89958800 & 2.80152600 & -1.86265300 & $\mathrm{H}$ & -2.42894800 & -1.21912000 & 1.76320500 \\
\hline $\mathrm{H}$ & -2.48072100 & 2.29399200 & -1.08874200 & $\mathrm{C}$ & -5.57342500 & -0.40831700 & -0.35301800 \\
\hline $\mathrm{H}$ & -2.46306700 & 3.68501400 & -2.18308200 & $\mathrm{H}$ & -5.17978100 & 1.65676700 & 0.10440500 \\
\hline $\mathrm{H}$ & -1.82453700 & 2.13384400 & -2.72723300 & $\mathrm{C}$ & -5.15178900 & -1.73332200 & -0.20896900 \\
\hline $\mathrm{C}$ & -1.47939900 & -3.48025200 & -1.83141000 & $\mathrm{H}$ & -3.67881900 & -3.04377100 & 0.65716000 \\
\hline $\mathrm{H}$ & -2.18295000 & -2.74701400 & -1.42731300 & $\mathrm{H}$ & -6.46868800 & -0.18339600 & -0.92449800 \\
\hline $\mathrm{H}$ & -1.18637200 & -3.15623900 & -2.83483500 & $\mathrm{H}$ & -5.72523000 & -2.53943000 & -0.65533700 \\
\hline $\mathrm{H}$ & -2.01317100 & -4.43099100 & -1.94499900 & $\mathrm{C}$ & -3.46079100 & 2.53134200 & 2.21086600 \\
\hline $\mathrm{C}$ & 0.70666100 & -4.70362100 & -1.54843400 & $\mathrm{H}$ & -4.53000500 & 2.71734400 & 2.18635200 \\
\hline $\mathrm{H}$ & 1.62031500 & -4.83211000 & -0.96332400 & $\mathrm{H}$ & -2.83539600 & 3.26467900 & 2.70806500 \\
\hline $\mathrm{H}$ & 0.20717900 & -5.67736300 & -1.61271900 & $\mathrm{C}$ & -2.92521300 & 1.43222600 & 1.65156100 \\
\hline $\mathrm{H}$ & 0.99215600 & -4.41807800 & -2.56443900 & $\mathrm{C}$ & -1.43161300 & 1.27737500 & 1.68862500 \\
\hline $\mathrm{C}$ & -0.71859500 & -4.20968600 & 0.46472500 & $\mathrm{O}$ & -0.72346900 & 1.69922800 & 2.57002100 \\
\hline $\mathrm{H}$ & -1.27374500 & -5.14527800 & 0.33342400 & & & & \\
\hline $\mathrm{H}$ & 0.12833100 & -4.41984800 & 1.12419600 & $12-0$ & & & \\
\hline $\mathrm{H}$ & -1.37897500 & -3.50094500 & 0.97488500 & $\mathrm{C}$ & 3.45793000 & 0.02490700 & 1.27568500 \\
\hline $\mathrm{C}$ & 2.02357400 & 2.28250300 & -0.04699200 & $\mathrm{H}$ & 3.45569900 & -0.01783000 & 2.36999200 \\
\hline $\mathrm{C}$ & 2.19277700 & 2.30704400 & 1.34163800 & $\mathrm{H}$ & 4.42167600 & -0.35064200 & 0.92107100 \\
\hline $\mathrm{C}$ & 4.11679500 & 3.16080300 & -0.40920600 & $\mathrm{C}$ & 3.25264500 & 1.47461200 & 0.80699800 \\
\hline $\mathrm{C}$ & 3.40078300 & 2.79289800 & 1.84828000 & $\mathrm{H}$ & 4.16411700 & 2.03200100 & 1.05188000 \\
\hline $\mathrm{H}$ & 1.40233400 & 1.97024300 & 2.00194700 & $\mathrm{H}$ & 3.16748000 & 1.51079200 & -0.28553800 \\
\hline $\mathrm{C}$ & 4.38251300 & 3.22676400 & 0.96074100 & $\mathrm{C}$ & 2.06888100 & 2.19832400 & 1.48210000 \\
\hline $\mathrm{H}$ & 4.85689000 & 3.48893000 & -1.13544200 & $\mathrm{H}$ & 2.25710900 & 3.27516700 & 1.51352100 \\
\hline $\mathrm{H}$ & 3.56701700 & 2.83030000 & 2.92067800 & $\mathrm{H}$ & 1.99679000 & 1.87898200 & 2.52678600 \\
\hline $\mathrm{H}$ & 5.33456200 & 3.60899900 & 1.31397200 & $\mathrm{P}$ & 0.37904000 & 1.95048300 & 0.74352400 \\
\hline
\end{tabular}




\begin{tabular}{|c|c|c|c|c|c|c|c|}
\hline $\mathrm{P}$ & 2.17295800 & -1.14602900 & 0.64210700 & $\mathrm{C}$ & 0.21465200 & 4.54364600 & -3.05672500 \\
\hline $\mathrm{C}$ & 2.24749100 & -2.67642100 & 1.76991600 & $\mathrm{H}$ & -1.67847400 & 3.51734500 & -3.25795400 \\
\hline $\mathrm{C}$ & -0.77149000 & 2.78239300 & 2.02110100 & $\mathrm{H}$ & 2.14025000 & 5.35802200 & -2.51752800 \\
\hline $\mathrm{C}$ & 3.70033400 & -3.13999200 & 1.98175100 & $\mathrm{H}$ & 0.10540700 & 5.11279000 & -3.97366100 \\
\hline $\mathrm{H}$ & 3.70067100 & -4.05298200 & 2.58823100 & $\mathrm{~N}$ & 4.23198600 & -1.59731700 & -1.11154200 \\
\hline $\mathrm{H}$ & 4.20182300 & -3.36435000 & 1.03687300 & $\mathrm{~N}$ & -0.70261200 & 2.90907400 & -1.54697100 \\
\hline $\mathrm{H}$ & 4.29843100 & -2.39465400 & 2.51403900 & $\mathrm{Pd}$ & -0.06828500 & -0.28326900 & 0.25928000 \\
\hline $\mathrm{C}$ & 1.42235000 & -3.80062100 & 1.11452200 & $\mathrm{O}$ & -1.51320800 & -1.82944200 & -0.30724600 \\
\hline $\mathrm{H}$ & 1.40115000 & -4.66605100 & 1.78596300 & $\mathrm{C}$ & -1.96202200 & -0.68728200 & -0.33498700 \\
\hline $\mathrm{H}$ & 0.38680100 & -3.49622500 & 0.93189900 & $\mathrm{C}$ & -3.31847700 & -0.27779300 & -0.71540700 \\
\hline $\mathrm{H}$ & 1.85842000 & -4.12981300 & 0.16713400 & $\mathrm{C}$ & -4.45791000 & -1.22336700 & -0.55169900 \\
\hline $\mathrm{C}$ & 1.60510100 & -2.29171300 & 3.11785800 & $\mathrm{C}$ & -5.69305400 & -0.71766900 & -0.11062200 \\
\hline $\mathrm{H}$ & 0.56481100 & -1.97310300 & 2.99432900 & $\mathrm{C}$ & -4.34760500 & -2.59405200 & -0.83659900 \\
\hline $\mathrm{H}$ & 1.61003200 & -3.16320100 & 3.78199200 & $\mathrm{C}$ & -6.79627100 & -1.55813300 & 0.02412200 \\
\hline $\mathrm{H}$ & 2.15228200 & -1.49352100 & 3.62999200 & $\mathrm{H}$ & -5.78113800 & 0.33426100 & 0.14524500 \\
\hline $\mathrm{C}$ & -0.78628100 & 1.89684800 & 3.28426200 & $\mathrm{C}$ & -5.45480900 & -3.43053900 & -0.70152800 \\
\hline $\mathrm{H}$ & -1.12462100 & 0.88008000 & 3.06037200 & $\mathrm{H}$ & -3.40414300 & -3.00470300 & -1.17738800 \\
\hline $\mathrm{H}$ & 0.19107400 & 1.83581700 & 3.77205700 & $\mathrm{C}$ & -6.68031500 & -2.91738200 & -0.27245000 \\
\hline $\mathrm{H}$ & -1.48230900 & 2.32759700 & 4.01232100 & $\mathrm{H}$ & -7.74216800 & -1.15308800 & 0.37049300 \\
\hline $\mathrm{C}$ & -0.26714200 & 4.19868000 & 2.36444100 & $\mathrm{H}$ & -5.35924700 & -4.48646400 & -0.93532300 \\
\hline $\mathrm{H}$ & -0.19220500 & 4.83516000 & 1.47733000 & $\mathrm{H}$ & -7.53798000 & -3.57401700 & -0.16345500 \\
\hline $\mathrm{H}$ & -0.98204800 & 4.67149300 & 3.04676800 & $\mathrm{C}$ & -3.43435000 & 0.96141800 & -1.23769400 \\
\hline $\mathrm{H}$ & 0.70218600 & 4.19106700 & 2.86984400 & $\mathrm{H}$ & -2.57866100 & 1.62948500 & -1.29201300 \\
\hline $\mathrm{C}$ & -2.19885800 & 2.87241900 & 1.45582700 & $\mathrm{H}$ & -4.38160500 & 1.29426900 & -1.65105500 \\
\hline $\mathrm{H}$ & -2.84733200 & 3.32721300 & 2.21294900 & & & & \\
\hline $\mathrm{H}$ & -2.24815100 & 3.49034400 & 0.55612600 & 12-CO & & & \\
\hline $\mathrm{H}$ & -2.60142300 & 1.88588900 & 1.21836800 & $\mathrm{C}$ & -3.12605800 & 0.69437500 & -1.76792500 \\
\hline $\mathrm{C}$ & 2.89932600 & -1.68238700 & -0.98302700 & $\mathrm{H}$ & -2.96690100 & 0.33847700 & -2.79184400 \\
\hline $\mathrm{C}$ & 2.06929800 & -2.15685500 & -2.00761000 & $\mathrm{H}$ & -4.20461000 & 0.79060000 & -1.61399200 \\
\hline $\mathrm{C}$ & 4.78260900 & -1.99026300 & -2.26643900 & $\mathrm{C}$ & -2.44816900 & 2.05780000 & -1.56885800 \\
\hline $\mathrm{C}$ & 2.65500200 & -2.57074500 & -3.20479800 & $\mathrm{H}$ & -3.06654200 & 2.80772800 & -2.07546500 \\
\hline $\mathrm{H}$ & 0.99357600 & -2.20269600 & -1.86933700 & $\mathrm{H}$ & -2.44222000 & 2.33666300 & -0.51008300 \\
\hline $\mathrm{C}$ & 4.03938400 & -2.48611000 & -3.33975600 & $\mathrm{C}$ & -1.03551800 & 2.16633000 & -2.16529500 \\
\hline $\mathrm{H}$ & 5.86482600 & -1.90491600 & -2.33417200 & $\mathrm{H}$ & -0.78535200 & 3.22253700 & -2.30115400 \\
\hline $\mathrm{H}$ & 2.03989500 & -2.94759300 & -4.01634100 & $\mathrm{H}$ & -1.01677000 & 1.70666400 & -3.16029500 \\
\hline $\mathrm{H}$ & 4.53712100 & -2.79256800 & -4.25378300 & $\mathrm{P}$ & 0.37572300 & 1.43718900 & -1.18364900 \\
\hline $\mathrm{C}$ & 0.38249200 & 3.03879400 & -0.76341500 & $\mathrm{P}$ & -2.57257300 & -0.63187500 & -0.59765500 \\
\hline $\mathrm{C}$ & 1.42812500 & 3.91494800 & -1.08078700 & $\mathrm{C}$ & -3.33308800 & -2.23465900 & -1.30783300 \\
\hline $\mathrm{C}$ & -0.78127900 & 3.64966400 & -2.65730300 & $\mathrm{C}$ & 1.85534300 & 1.65139000 & -2.37487100 \\
\hline $\mathrm{C}$ & 1.33969900 & 4.67552100 & -2.24905000 & $\mathrm{C}$ & -4.71718200 & -1.97747600 & -1.93745100 \\
\hline $\mathrm{H}$ & 2.29834400 & 4.01233800 & -0.44392200 & $\mathrm{H}$ & -5.13727500 & -2.93592300 & -2.26340100 \\
\hline
\end{tabular}




\begin{tabular}{|c|c|c|c|c|c|c|c|}
\hline $\mathrm{H}$ & -5.40931100 & -1.52436900 & -1.22443500 & $\mathrm{~N}$ & -0.38475000 & 2.22334900 & 1.22574000 \\
\hline $\mathrm{H}$ & -4.65813500 & -1.33374800 & -2.81906200 & $\mathrm{Pd}$ & -0.10886400 & -0.69663700 & -0.07769300 \\
\hline $\mathrm{C}$ & -3.48252000 & -3.27172300 & -0.17865600 & $\mathrm{C}$ & 4.32990700 & -1.51082500 & 0.59190700 \\
\hline $\mathrm{H}$ & -3.86580000 & -4.20617900 & -0.60307200 & $\mathrm{C}$ & 4.61689900 & -1.32047700 & 1.95443200 \\
\hline $\mathrm{H}$ & -2.53363400 & -3.50733000 & 0.30920600 & $\mathrm{C}$ & 5.39923600 & -1.63294300 & -0.31330700 \\
\hline $\mathrm{H}$ & -4.19067600 & -2.94113000 & 0.58570900 & $\mathrm{C}$ & 5.93812500 & -1.27434200 & 2.39579000 \\
\hline $\mathrm{C}$ & -2.36054600 & -2.76356700 & -2.38138000 & $\mathrm{H}$ & 3.80980500 & -1.21845200 & 2.66983900 \\
\hline $\mathrm{H}$ & -1.38112200 & -3.01182800 & -1.95962800 & $\mathrm{C}$ & 6.71891100 & -1.58122700 & 0.13148300 \\
\hline $\mathrm{H}$ & -2.77269600 & -3.67503300 & -2.82855100 & $\mathrm{H}$ & 5.19264500 & -1.75283700 & -1.37305100 \\
\hline $\mathrm{H}$ & -2.20919100 & -2.04423400 & -3.19351700 & $\mathrm{C}$ & 6.99200900 & -1.40336700 & 1.48880900 \\
\hline $\mathrm{C}$ & 1.79186500 & 0.45617000 & -3.35205400 & $\mathrm{H}$ & 6.14443500 & -1.13837200 & 3.45305200 \\
\hline $\mathrm{H}$ & 1.91854900 & -0.49681200 & -2.83210600 & $\mathrm{H}$ & 7.53196000 & -1.67311100 & -0.58227900 \\
\hline $\mathrm{H}$ & 0.85161000 & 0.42254700 & -3.91256800 & $\mathrm{H}$ & 8.01946300 & -1.36098400 & 1.83718500 \\
\hline $\mathrm{H}$ & 2.60128400 & 0.54903800 & -4.08452400 & $\mathrm{C}$ & 2.56530900 & -2.50944300 & -0.84853600 \\
\hline $\mathrm{C}$ & 1.76999800 & 2.96243000 & -3.18837100 & $\mathrm{H}$ & 3.26302300 & -3.24679900 & -1.23089500 \\
\hline $\mathrm{H}$ & 1.72877100 & 3.85828000 & -2.56305900 & $\mathrm{H}$ & 1.55589500 & -2.52536800 & -1.24791800 \\
\hline $\mathrm{H}$ & 2.66953200 & 3.04264100 & -3.80826600 & $\mathrm{C}$ & 2.93321100 & -1.61369200 & 0.08664600 \\
\hline $\mathrm{H}$ & 0.91243100 & 2.98120100 & -3.86462500 & $\mathrm{C}$ & 1.89986600 & -0.65890300 & 0.62209600 \\
\hline $\mathrm{C}$ & 3.19625600 & 1.61067500 & -1.61569900 & $\mathrm{O}$ & 2.09346600 & 0.12199000 & 1.51603600 \\
\hline $\mathrm{H}$ & 4.00935800 & 1.71044700 & -2.34320400 & $\mathrm{C}$ & -0.19211800 & -2.36417900 & 0.95909600 \\
\hline $\mathrm{H}$ & 3.30258700 & 2.42118900 & -0.89216500 & $\mathrm{O}$ & -0.24635800 & -3.29514100 & 1.61711100 \\
\hline $\mathrm{H}$ & 3.35200900 & 0.66983700 & -1.08907000 & & & & \\
\hline $\mathrm{C}$ & -3.57124100 & -0.21983800 & 0.91327700 & 13 & & & \\
\hline $\mathrm{C}$ & -2.99738500 & -0.23108400 & 2.18916300 & $\mathrm{C}$ & -1.32654300 & -2.07308500 & -1.75501900 \\
\hline $\mathrm{C}$ & -5.62686400 & 0.37053200 & 1.76095500 & $\mathrm{H}$ & -1.01701600 & -3.06494100 & -1.40948700 \\
\hline $\mathrm{C}$ & -3.80551700 & 0.06581900 & 3.28889300 & $\mathrm{H}$ & -2.20318400 & -2.19733300 & -2.39248800 \\
\hline $\mathrm{H}$ & -1.94265800 & -0.44779800 & 2.31565800 & $\mathrm{C}$ & -0.19568900 & -1.40631600 & -2.56640100 \\
\hline $\mathrm{C}$ & -5.14754300 & 0.36926200 & 3.07372600 & $\mathrm{H}$ & -0.35740800 & -1.64120500 & -3.62413200 \\
\hline $\mathrm{H}$ & -6.66648100 & 0.61137500 & 1.55085800 & $\mathrm{H}$ & -0.25140700 & -0.31508900 & -2.49264800 \\
\hline $\mathrm{H}$ & -3.39164900 & 0.06085900 & 4.29288900 & $\mathrm{C}$ & 1.21453900 & -1.89859800 & -2.19664400 \\
\hline $\mathrm{H}$ & -5.81230000 & 0.60584400 & 3.89791000 & $\mathrm{H}$ & 1.92716800 & -1.55571400 & -2.95016300 \\
\hline $\mathrm{C}$ & 0.46057900 & 2.60596600 & 0.25183100 & $\mathrm{H}$ & 1.23360500 & -2.99222400 & -2.23716900 \\
\hline $\mathrm{C}$ & 1.23870600 & 3.76383500 & 0.34394300 & $\mathrm{P}$ & 1.79290500 & -1.38726400 & -0.49134500 \\
\hline $\mathrm{C}$ & -0.47441000 & 2.98168000 & 2.32331800 & $\mathrm{P}$ & -1.91293200 & -1.12777400 & -0.27076800 \\
\hline $\mathrm{C}$ & 1.14538100 & 4.54023100 & 1.50093000 & $\mathrm{C}$ & -2.95050400 & -2.40138600 & 0.71532700 \\
\hline H & 1.90277300 & 4.06327600 & -0.45682500 & $\mathrm{C}$ & 2.77484200 & -2.90448600 & 0.14555100 \\
\hline $\mathrm{C}$ & 0.27546900 & 4.14388400 & 2.51306400 & $\mathrm{C}$ & -1.97228500 & -3.32678700 & 1.46343800 \\
\hline $\mathrm{H}$ & -1.17430900 & 2.64180300 & 3.08301400 & $\mathrm{H}$ & -2.54437400 & -4.07455600 & 2.02403100 \\
\hline $\mathrm{H}$ & 1.74436800 & 5.43979600 & 1.60419800 & $\mathrm{H}$ & -1.30672000 & -3.86995400 & 0.78608600 \\
\hline $\mathrm{H}$ & 0.17565800 & 4.71751800 & 3.42836000 & $\mathrm{H}$ & -1.36244300 & -2.76640000 & 2.17893000 \\
\hline N & -4.86215500 & 0.08479200 & 0.70150200 & $\mathrm{C}$ & -3.82730400 & -3.22010800 & -0.25412400 \\
\hline
\end{tabular}




\begin{tabular}{|c|c|c|c|c|c|c|c|}
\hline $\mathrm{H}$ & -4.46453800 & -3.89140800 & 0.33249500 & $\mathrm{C}$ & -0.42575500 & 2.64890900 & -0.82530000 \\
\hline $\mathrm{H}$ & -4.48512300 & -2.58249800 & -0.85217000 & $\mathrm{C}$ & 0.74017600 & 4.02127200 & 0.78221500 \\
\hline $\mathrm{H}$ & -3.23907900 & -3.84194000 & -0.93395800 & $\mathrm{C}$ & 0.36481400 & 3.17974700 & -1.84716200 \\
\hline $\mathrm{C}$ & -3.85579000 & -1.69669900 & 1.74355000 & $\mathrm{H}$ & -1.21424200 & 1.94696800 & -1.06977700 \\
\hline $\mathrm{H}$ & -4.62743400 & -1.09173500 & 1.26086700 & $\mathrm{C}$ & 1.52808000 & 4.55190200 & -0.23877100 \\
\hline $\mathrm{H}$ & -4.37047900 & -2.46593100 & 2.33053100 & $\mathrm{H}$ & 0.89130800 & 4.34310600 & 1.80821200 \\
\hline $\mathrm{H}$ & -3.29026300 & -1.06869300 & 2.43440300 & $\mathrm{C}$ & 1.34489500 & 4.13062700 & -1.55799400 \\
\hline $\mathrm{C}$ & 1.76400700 & -4.03628800 & 0.41210700 & $\mathrm{H}$ & 0.19734900 & 2.86576800 & -2.87365200 \\
\hline $\mathrm{H}$ & 1.00764900 & -3.73603300 & 1.14255200 & $\mathrm{H}$ & 2.28087400 & 5.29905200 & -0.00448700 \\
\hline $\mathrm{H}$ & 1.25559300 & -4.37260000 & -0.49734500 & $\mathrm{H}$ & 1.95178900 & 4.54973100 & -2.35506200 \\
\hline $\mathrm{H}$ & 2.29485100 & -4.90395900 & 0.81942300 & $\mathrm{C}$ & -1.74749500 & 3.39283600 & 2.45043200 \\
\hline $\mathrm{C}$ & 3.83587300 & -3.37292300 & -0.86716600 & $\mathrm{H}$ & -1.70471900 & 4.46916300 & 2.31608500 \\
\hline $\mathrm{H}$ & 4.59682000 & -2.60889900 & -1.04935000 & $\mathrm{H}$ & -2.32765500 & 3.00365400 & 3.28070000 \\
\hline $\mathrm{H}$ & 4.34827900 & -4.25520500 & -0.46680900 & $\mathrm{C}$ & -1.10449600 & 2.55920700 & 1.61318200 \\
\hline $\mathrm{H}$ & 3.40074000 & -3.66223400 & -1.82879100 & $\mathrm{C}$ & -1.24387600 & 1.07601500 & 1.90661000 \\
\hline $\mathrm{C}$ & 3.45695500 & -2.52653900 & 1.47330600 & $\mathrm{O}$ & -1.85413300 & 0.66062400 & 2.86433000 \\
\hline $\mathrm{H}$ & 4.00937900 & -3.39306800 & 1.85338000 & $\mathrm{O}$ & 1.47925000 & 0.81122800 & 2.11796700 \\
\hline $\mathrm{H}$ & 4.16901700 & -1.70530800 & 1.35726000 & $\mathrm{H}$ & 2.29944500 & 0.76759400 & 1.54364100 \\
\hline $\mathrm{H}$ & 2.72378600 & -2.24440300 & 2.23458200 & $\mathrm{C}$ & 1.73605300 & 0.42329900 & 3.47292900 \\
\hline $\mathrm{C}$ & -3.15072800 & 0.03802400 & -1.03662600 & $\mathrm{H}$ & 2.13332000 & -0.59516800 & 3.54118800 \\
\hline $\mathrm{C}$ & -3.84148700 & 0.99117800 & -0.27315600 & $\mathrm{H}$ & 2.44658800 & 1.11988000 & 3.93029800 \\
\hline $\mathrm{C}$ & -4.20597900 & 0.72281900 & -2.96623200 & $\mathrm{H}$ & 0.79157400 & 0.47420000 & 4.01631200 \\
\hline $\mathrm{C}$ & -4.76078700 & 1.82166300 & -0.91369300 & & & & \\
\hline $\mathrm{H}$ & -3.67241000 & 1.08366300 & 0.79257400 & \multirow{2}{*}{\multicolumn{4}{|c|}{$\begin{array}{l}\text { TS9 } \\
\text { imaginary frequency }=-287.18 \mathrm{~cm}^{-1}\end{array}$}} \\
\hline $\mathrm{C}$ & -4.94956100 & 1.68892000 & -2.28820100 & & & & \\
\hline $\mathrm{H}$ & -4.31766200 & 0.58857800 & -4.03978800 & $\mathrm{C}$ & -1.41787700 & -2.23756500 & -1.69354500 \\
\hline $\mathrm{H}$ & -5.31643400 & 2.56162500 & -0.34578400 & $\mathrm{H}$ & -1.18707900 & -3.23500400 & -1.30579600 \\
\hline $\mathrm{H}$ & -5.65156500 & 2.31774900 & -2.82549900 & $\mathrm{H}$ & -2.29690100 & -2.32163500 & -2.33613400 \\
\hline $\mathrm{C}$ & 3.13979200 & -0.13169800 & -0.80706900 & $\mathrm{C}$ & -0.23134700 & -1.69874500 & -2.52488700 \\
\hline $\mathrm{C}$ & 3.88521500 & -0.03092800 & -1.98746900 & $\mathrm{H}$ & -0.37920700 & -2.00846300 & -3.56579200 \\
\hline $\mathrm{C}$ & 4.34619100 & 1.61584100 & 0.13845300 & $\mathrm{H}$ & -0.23301000 & -0.60253400 & -2.53877700 \\
\hline $\mathrm{C}$ & 4.88754200 & 0.93534700 & -2.08635200 & $\mathrm{C}$ & 1.15569300 & -2.22423100 & -2.10125800 \\
\hline $\mathrm{H}$ & 3.70522400 & -0.69951300 & -2.82023100 & $\mathrm{H}$ & 1.89136600 & -2.00513700 & -2.88172600 \\
\hline $\mathrm{C}$ & 5.12623900 & 1.77820200 & -1.00431400 & $\mathrm{H}$ & 1.11349200 & -3.31635700 & -2.02257400 \\
\hline $\mathrm{H}$ & 4.49027000 & 2.25099400 & 1.00829600 & $\mathrm{P}$ & 1.75559300 & -1.61735900 & -0.43529100 \\
\hline H & 5.47411100 & 1.02144600 & -2.99599200 & $\mathrm{P}$ & -1.92479100 & -1.17015100 & -0.25336000 \\
\hline $\mathrm{H}$ & 5.89440200 & 2.54289800 & -1.03891500 & $\mathrm{C}$ & -3.00648500 & -2.30429600 & 0.83471400 \\
\hline N & -3.32836200 & -0.08730400 & -2.36069300 & $\mathrm{C}$ & 3.02071600 & -2.95121200 & 0.12136000 \\
\hline N & 3.38256900 & 0.69010500 & 0.23751200 & $\mathrm{C}$ & -2.07200600 & -3.32184800 & 1.51960100 \\
\hline $\mathrm{Pd}$ & -0.07403700 & -0.16428300 & 0.83570700 & $\mathrm{H}$ & -2.66219600 & -3.96393300 & 2.18344400 \\
\hline $\mathrm{C}$ & -0.24914200 & 3.06305300 & 0.50541500 & $\mathrm{H}$ & -1.56693200 & -3.97648300 & 0.80144300 \\
\hline
\end{tabular}




\begin{tabular}{|c|c|c|c|c|c|c|c|}
\hline $\mathrm{H}$ & -1.31170700 & -2.81951700 & 2.12620100 & $\mathrm{Pd}$ & 0.00430200 & -0.39010800 & 0.82748900 \\
\hline $\mathrm{C}$ & -4.07228600 & -3.03773700 & 0.00035800 & $\mathrm{C}$ & -0.10063200 & 3.15592500 & 0.59643900 \\
\hline $\mathrm{H}$ & -4.69383800 & -3.64667800 & 0.66723100 & $\mathrm{C}$ & -0.16483500 & 2.68722700 & -0.72565700 \\
\hline $\mathrm{H}$ & -4.73155800 & -2.34213000 & -0.52563900 & $\mathrm{C}$ & 0.64822500 & 4.31555200 & 0.86129200 \\
\hline $\mathrm{H}$ & -3.63011500 & -3.71089600 & -0.74010500 & $\mathrm{C}$ & 0.50150300 & 3.36050700 & -1.75293700 \\
\hline $\mathrm{C}$ & -3.68311600 & -1.43992300 & 1.91725700 & $\mathrm{H}$ & -0.76855300 & 1.81519000 & -0.95033900 \\
\hline $\mathrm{H}$ & -4.39282700 & -0.72811100 & 1.48612500 & $\mathrm{C}$ & 1.31517900 & 4.98736900 & -0.16502100 \\
\hline $\mathrm{H}$ & -4.24706300 & -2.09379200 & 2.59250400 & $\mathrm{H}$ & 0.69622900 & 4.69135500 & 1.87901400 \\
\hline $\mathrm{H}$ & -2.95291100 & -0.88801300 & 2.51652000 & $\mathrm{C}$ & 1.24460100 & 4.51052800 & -1.47741600 \\
\hline $\mathrm{C}$ & 2.17084500 & -4.15602100 & 0.57907900 & $\mathrm{H}$ & 0.41714400 & 2.99991000 & -2.77456500 \\
\hline $\mathrm{H}$ & 1.46082500 & -3.87706200 & 1.36340300 & $\mathrm{H}$ & 1.87479700 & 5.89147400 & 0.05789900 \\
\hline H & 1.61226200 & -4.61301100 & -0.24386400 & $\mathrm{H}$ & 1.74557300 & 5.04376500 & -2.28032900 \\
\hline $\mathrm{H}$ & 2.83297600 & -4.92839100 & 0.98567000 & $\mathrm{C}$ & -1.83555700 & 3.16442900 & 2.35447400 \\
\hline $\mathrm{C}$ & 4.00063800 & -3.38680300 & -0.98079700 & $\mathrm{H}$ & -2.13353000 & 4.16416100 & 2.05446500 \\
\hline $\mathrm{H}$ & 4.67219700 & -2.57684000 & -1.28039700 & $\mathrm{H}$ & -2.34633700 & 2.70788200 & 3.19553700 \\
\hline $\mathrm{H}$ & 4.63193500 & -4.19854900 & -0.60098500 & $\mathrm{C}$ & -0.85560400 & 2.51133300 & 1.70878100 \\
\hline $\mathrm{H}$ & 3.49099300 & -3.76715300 & -1.87113100 & $\mathrm{C}$ & -0.52527300 & 1.13377400 & 2.21926700 \\
\hline $\mathrm{C}$ & 3.80043700 & -2.39956700 & 1.32859200 & $\mathrm{O}$ & -0.95854400 & 0.64321700 & 3.24264200 \\
\hline $\mathrm{H}$ & 4.47484700 & -3.17398400 & 1.71044900 & $\mathrm{O}$ & 1.20266400 & 1.06033300 & 2.07375300 \\
\hline $\mathrm{H}$ & 4.41882400 & -1.53520600 & 1.06367300 & $\mathrm{H}$ & 2.15736200 & 0.92785400 & 0.74804700 \\
\hline H & 3.13207200 & -2.11487200 & 2.14666300 & $\mathrm{C}$ & 1.82598300 & 0.60912900 & 3.28139900 \\
\hline $\mathrm{C}$ & -3.13958000 & -0.01909000 & -1.07611500 & $\mathrm{H}$ & 2.63688700 & -0.08732700 & 3.04423900 \\
\hline $\mathrm{C}$ & -3.55845000 & 1.15020100 & -0.42631600 & $\mathrm{H}$ & 2.23501800 & 1.47461000 & 3.81528900 \\
\hline $\mathrm{C}$ & -4.48032200 & 0.43251800 & -2.89257700 & $\mathrm{H}$ & 1.09327300 & 0.10991900 & 3.92133600 \\
\hline $\mathrm{C}$ & -4.49658700 & 1.96844700 & -1.05614800 & & & & \\
\hline $\mathrm{H}$ & -3.15244100 & 1.41733200 & 0.54252100 & \multirow{2}{*}{\multicolumn{4}{|c|}{$\begin{array}{l}\text { TS9a } \\
\text { imaginary frequency }=-245.86 \mathrm{~cm}^{-1}\end{array}$}} \\
\hline $\mathrm{C}$ & -4.97069300 & 1.60488200 & -2.31523700 & & & & \\
\hline $\mathrm{H}$ & -4.81768800 & 0.11762600 & -3.87785400 & $\mathrm{C}$ & 1.55265600 & 2.83121800 & 1.04214300 \\
\hline $\mathrm{H}$ & -4.84504700 & 2.87612100 & -0.57246400 & $\mathrm{H}$ & 2.34340600 & 3.22683300 & 0.39647200 \\
\hline $\mathrm{H}$ & -5.69771900 & 2.21290800 & -2.84343600 & $\mathrm{H}$ & 1.17449800 & 3.65628500 & 1.65129100 \\
\hline $\mathrm{C}$ & 2.85718800 & -0.16865300 & -0.91386700 & $\mathrm{C}$ & 2.12844300 & 1.74090200 & 1.97234200 \\
\hline $\mathrm{C}$ & 3.69263700 & -0.08555100 & -2.02833900 & $\mathrm{H}$ & 2.56447400 & 2.24579200 & 2.84245200 \\
\hline $\mathrm{C}$ & 3.53432000 & 2.03388100 & -0.28683500 & $\mathrm{H}$ & 1.32349800 & 1.11128800 & 2.36981100 \\
\hline $\mathrm{C}$ & 4.46279300 & 1.05823900 & -2.25706500 & $\mathrm{C}$ & 3.25051500 & 0.86487100 & 1.37143000 \\
\hline $\mathrm{H}$ & 3.74651300 & -0.91682800 & -2.71954500 & $\mathrm{H}$ & 3.81442700 & 0.38260000 & 2.17647700 \\
\hline $\mathrm{C}$ & 4.38653500 & 2.13557200 & -1.37514100 & $\mathrm{H}$ & 3.96840100 & 1.50901700 & 0.85006700 \\
\hline $\mathrm{H}$ & 3.37383700 & 2.83116200 & 0.42895200 & $\mathrm{P}$ & 2.70569200 & -0.39660200 & 0.10230800 \\
\hline $\mathrm{H}$ & 5.11530800 & 1.10595400 & -3.12346300 & $\mathrm{P}$ & 0.13204100 & 2.30186800 & -0.04550200 \\
\hline $\mathrm{H}$ & 4.96054100 & 3.04131200 & -1.52951300 & $\mathrm{C}$ & 0.17352800 & 3.50793200 & -1.52389800 \\
\hline N & -3.58944600 & -0.36640700 & -2.29289500 & $\mathrm{C}$ & 4.33783900 & -0.93855400 & -0.75811000 \\
\hline N & 2.81939200 & 0.91197300 & -0.08608100 & $\mathrm{C}$ & 1.39016700 & 3.13892700 & -2.39623800 \\
\hline
\end{tabular}




\begin{tabular}{|c|c|c|c|c|c|c|c|}
\hline $\mathrm{H}$ & 1.42473100 & 3.80215700 & -3.26815500 & $\mathrm{~N}$ & -1.11899400 & 3.63987800 & 2.01168000 \\
\hline $\mathrm{H}$ & 2.34053800 & 3.25486700 & -1.86449600 & $\mathrm{~N}$ & 1.28079100 & -2.67135800 & 0.75456800 \\
\hline $\mathrm{H}$ & 1.32104400 & 2.10797400 & -2.75875200 & $\mathrm{Pd}$ & 0.41377100 & 0.07655800 & -0.67699500 \\
\hline $\mathrm{C}$ & 0.27882300 & 4.97055400 & -1.05420100 & $\mathrm{C}$ & -3.57895400 & -1.98358600 & -0.10836700 \\
\hline $\mathrm{H}$ & 0.26258700 & 5.63305600 & -1.92758800 & $\mathrm{C}$ & -3.69714200 & -2.87261600 & -1.18930700 \\
\hline $\mathrm{H}$ & -0.55587700 & 5.25357500 & -0.40708600 & $\mathrm{C}$ & -4.68106600 & -1.82487100 & 0.74995900 \\
\hline $\mathrm{H}$ & 1.20867900 & 5.16719500 & -0.51210100 & $\mathrm{C}$ & -4.86949300 & -3.59914100 & -1.38922200 \\
\hline $\mathrm{C}$ & -1.11132600 & 3.31093000 & -2.35284600 & $\mathrm{H}$ & -2.86915400 & -2.99820700 & -1.87767000 \\
\hline $\mathrm{H}$ & -2.00102300 & 3.64076400 & -1.80991500 & $\mathrm{C}$ & -5.85309200 & -2.55158700 & 0.54907000 \\
\hline $\mathrm{H}$ & -1.04407400 & 3.91473800 & -3.26517500 & $\mathrm{H}$ & -4.62666500 & -1.10811900 & 1.56360500 \\
\hline $\mathrm{H}$ & -1.25585400 & 2.26706500 & -2.64840800 & $\mathrm{C}$ & -5.95064900 & -3.44472600 & -0.51951800 \\
\hline $\mathrm{C}$ & 4.68998500 & 0.21339000 & -1.72345300 & $\mathrm{H}$ & -4.94059000 & -4.28388900 & -2.22909500 \\
\hline $\mathrm{H}$ & 3.87504700 & 0.42082700 & -2.42379900 & $\mathrm{H}$ & -6.69562700 & -2.40926400 & 1.21933800 \\
\hline $\mathrm{H}$ & 4.93349000 & 1.14110500 & -1.19612500 & $\mathrm{H}$ & -6.86615000 & -4.00564500 & -0.68095100 \\
\hline $\mathrm{H}$ & 5.57311500 & -0.06503800 & -2.30921700 & $\mathrm{C}$ & -1.89845600 & -0.92416500 & 1.38303000 \\
\hline $\mathrm{C}$ & 5.50955000 & -1.19765000 & 0.20296600 & $\mathrm{H}$ & -2.44489300 & -1.25645100 & 2.25978400 \\
\hline $\mathrm{H}$ & 5.31506900 & -2.03984600 & 0.87406200 & $\mathrm{H}$ & -1.01914900 & -0.30867800 & 1.53847500 \\
\hline $\mathrm{H}$ & 6.40403700 & -1.45283000 & -0.37722900 & $\mathrm{C}$ & -2.31845300 & -1.23107500 & 0.14689600 \\
\hline $\mathrm{H}$ & 5.75902500 & -0.32042800 & 0.80691100 & $\mathrm{C}$ & -1.56120400 & -0.78832800 & -1.09353900 \\
\hline $\mathrm{C}$ & 4.03838200 & -2.20812800 & -1.57676500 & $\mathrm{O}$ & -2.07175700 & -0.37257100 & -2.11316000 \\
\hline $\mathrm{H}$ & 4.91920800 & -2.47507500 & -2.17092500 & $\mathrm{O}$ & -0.40463600 & -1.95863900 & -1.29059200 \\
\hline $\mathrm{H}$ & 3.80608200 & -3.06802100 & -0.93939800 & $\mathrm{H}$ & 0.70820900 & -2.37320000 & -0.08072500 \\
\hline $\mathrm{H}$ & 3.20688900 & -2.05650200 & -2.27282500 & $\mathrm{C}$ & -0.14543300 & -2.30848700 & -2.66063400 \\
\hline $\mathrm{C}$ & -1.34373600 & 2.84927800 & 0.95089900 & $\mathrm{H}$ & 0.93079100 & -2.28206700 & -2.85364200 \\
\hline $\mathrm{C}$ & -2.63113800 & 2.43202400 & 0.58112500 & $\mathrm{H}$ & -0.52778000 & -3.31813900 & -2.84506700 \\
\hline $\mathrm{C}$ & -2.17099900 & 4.05117800 & 2.73115200 & $\mathrm{H}$ & -0.65458300 & -1.60110800 & -3.32044000 \\
\hline $\mathrm{C}$ & -3.71951500 & 2.88201100 & 1.32744100 & & & & \\
\hline $\mathrm{H}$ & -2.77365300 & 1.77258600 & -0.26765500 & 14 & & & \\
\hline $\mathrm{C}$ & -3.48862300 & 3.70770300 & 2.42723500 & $\mathrm{C}$ & -3.15441500 & -0.66693000 & -1.48513700 \\
\hline $\mathrm{H}$ & -1.94699800 & 4.68688800 & 3.58519500 & $\mathrm{H}$ & -3.81298800 & -1.45606700 & -1.10633400 \\
\hline $\mathrm{H}$ & -4.72887300 & 2.58703700 & 1.05622000 & $\mathrm{H}$ & -3.73079200 & -0.05497600 & -2.18335100 \\
\hline $\mathrm{H}$ & -4.30542700 & 4.07626400 & 3.03910100 & $\mathrm{C}$ & -1.97015500 & -1.29748200 & -2.25243500 \\
\hline $\mathrm{C}$ & 2.31533400 & -1.89378500 & 1.17740400 & $\mathrm{H}$ & -2.33942600 & -1.54243800 & -3.25574400 \\
\hline $\mathrm{C}$ & 2.96017200 & -2.28664800 & 2.35177100 & $\mathrm{H}$ & -1.18103500 & -0.55141600 & -2.40584300 \\
\hline $\mathrm{C}$ & 0.86297700 & -3.78267700 & 1.39025100 & $\mathrm{C}$ & -1.37618800 & -2.60812600 & -1.67981600 \\
\hline $\mathrm{C}$ & 2.55707700 & -3.43680000 & 3.03484600 & $\mathrm{H}$ & -0.94518600 & -3.19924000 & -2.49525300 \\
\hline $\mathrm{H}$ & 3.78257100 & -1.69486700 & 2.73303600 & $\mathrm{H}$ & -2.18157800 & -3.22252000 & -1.26094200 \\
\hline $\mathrm{C}$ & 1.49591300 & -4.20297200 & 2.54815100 & $\mathrm{P}$ & -0.13099300 & -2.42978900 & -0.28815900 \\
\hline $\mathrm{H}$ & 0.01453100 & -4.29094800 & 0.94707800 & $\mathrm{P}$ & -2.70667500 & 0.43816000 & -0.04094600 \\
\hline $\mathrm{H}$ & 3.07098200 & -3.73317900 & 3.94412800 & $\mathrm{C}$ & -4.37622500 & 0.72338900 & 0.84585900 \\
\hline $\mathrm{H}$ & 1.15765500 & -5.09777500 & 3.05681700 & $\mathrm{C}$ & 0.22151900 & -4.25198800 & 0.22440400 \\
\hline
\end{tabular}




\begin{tabular}{|c|c|c|c|c|c|c|c|}
\hline $\mathrm{C}$ & -4.72521100 & -0.57970800 & 1.59260200 & $\mathrm{H}$ & 4.77093200 & -0.73616600 & -2.78601000 \\
\hline $\mathrm{H}$ & -5.65077600 & -0.43699300 & 2.16234300 & $\mathrm{~N}$ & -2.86800600 & 2.19282400 & -2.17446800 \\
\hline $\mathrm{H}$ & -4.89209300 & -1.41975600 & 0.91045300 & $\mathrm{~N}$ & 2.25955300 & -1.13528100 & -0.62469300 \\
\hline $\mathrm{H}$ & -3.93608200 & -0.86197400 & 2.29726000 & $\mathrm{Pd}$ & -0.89528900 & -0.69723500 & 0.93259000 \\
\hline $\mathrm{C}$ & -5.50618500 & 1.09449800 & -0.13130200 & $\mathrm{C}$ & 3.21104500 & 2.44355300 & 0.65698800 \\
\hline $\mathrm{H}$ & -6.42570100 & 1.29135900 & 0.43293500 & $\mathrm{C}$ & 2.92328500 & 2.86483200 & -0.65193900 \\
\hline $\mathrm{H}$ & -5.27099400 & 1.99180100 & -0.71017700 & $\mathrm{C}$ & 4.53339400 & 2.08213700 & 0.97279800 \\
\hline $\mathrm{H}$ & -5.72391700 & 0.28672400 & -0.83611400 & $\mathrm{C}$ & 3.92947100 & 2.93042900 & -1.61865400 \\
\hline $\mathrm{C}$ & -4.18146000 & 1.85175300 & 1.87658200 & $\mathrm{H}$ & 1.90378900 & 3.13853800 & -0.90839400 \\
\hline $\mathrm{H}$ & -3.97548200 & 2.81356600 & 1.39788300 & $\mathrm{C}$ & 5.53883800 & 2.15314800 & 0.00721900 \\
\hline $\mathrm{H}$ & -5.09814900 & 1.96780900 & 2.46592300 & $\mathrm{H}$ & 4.77693000 & 1.76490200 & 1.98153900 \\
\hline $\mathrm{H}$ & -3.36442600 & 1.62864100 & 2.57126400 & $\mathrm{C}$ & 5.24030300 & 2.57721800 & -1.29216600 \\
\hline $\mathrm{C}$ & -0.98149100 & -4.66702500 & 1.09786700 & $\mathrm{H}$ & 3.68890200 & 3.26494400 & -2.62353500 \\
\hline $\mathrm{H}$ & -1.11527100 & -3.98954500 & 1.94670000 & $\mathrm{H}$ & 6.55916600 & 1.89389300 & 0.27431600 \\
\hline $\mathrm{H}$ & -1.91773400 & -4.69271300 & 0.53150400 & $\mathrm{H}$ & 6.02682900 & 2.64388900 & -2.03807100 \\
\hline $\mathrm{H}$ & -0.81228100 & -5.67572800 & 1.49149200 & $\mathrm{C}$ & 1.38997100 & 3.54425600 & 1.92495900 \\
\hline $\mathrm{C}$ & 0.37571500 & -5.22924800 & -0.95320800 & $\mathrm{H}$ & 1.55473100 & 4.46694700 & 1.37882600 \\
\hline $\mathrm{H}$ & 1.23527300 & -4.98595600 & -1.58611200 & $\mathrm{H}$ & 0.62196400 & 3.52730500 & 2.69083700 \\
\hline $\mathrm{H}$ & 0.54481600 & -6.24009100 & -0.56374500 & $\mathrm{C}$ & 2.14050700 & 2.45497400 & 1.69255300 \\
\hline $\mathrm{H}$ & -0.51800600 & -5.27474300 & -1.58171300 & $\mathrm{C}$ & 1.85412300 & 1.25747300 & 2.54941700 \\
\hline $\mathrm{C}$ & 1.49748500 & -4.26953000 & 1.08529600 & $\mathrm{O}$ & 1.07409100 & 1.23814900 & 3.47477200 \\
\hline $\mathrm{H}$ & 1.65607300 & -5.27792000 & 1.48331000 & $\mathrm{O}$ & 2.57794200 & 0.15183300 & 2.18711100 \\
\hline $\mathrm{H}$ & 2.39109500 & -4.00625200 & 0.50818600 & $\mathrm{H}$ & 1.95991900 & -0.78041100 & 0.29447400 \\
\hline $\mathrm{H}$ & 1.41784600 & -3.58687000 & 1.93651700 & $\mathrm{C}$ & 2.46491300 & -0.95499300 & 3.11439400 \\
\hline $\mathrm{C}$ & -2.39598400 & 2.05228700 & -0.92503600 & $\mathrm{H}$ & 3.17638900 & -1.70316300 & 2.76501400 \\
\hline $\mathrm{C}$ & -1.68560900 & 3.07447500 & -0.27725000 & $\mathrm{H}$ & 2.71915300 & -0.62544700 & 4.12305000 \\
\hline $\mathrm{C}$ & -2.65986400 & 3.35421400 & -2.80798600 & $\mathrm{H}$ & 1.44678300 & -1.35044000 & 3.10980300 \\
\hline $\mathrm{C}$ & -1.49061800 & 4.28627200 & -0.93901100 & & & & \\
\hline $\mathrm{H}$ & -1.29837300 & 2.91549400 & 0.72380500 & \multirow{2}{*}{\multicolumn{4}{|c|}{$\begin{array}{l}\text { TS10 } \\
\text { imaginary frequency }=-30.49 \mathrm{~cm}^{-1}\end{array}$}} \\
\hline $\mathrm{C}$ & -1.98642900 & 4.43313000 & -2.23435200 & & & & \\
\hline $\mathrm{H}$ & -3.05532800 & 3.42433000 & -3.81938600 & $\mathrm{C}$ & 0.69385700 & 1.37763300 & 2.57550200 \\
\hline $\mathrm{H}$ & -0.96155400 & 5.10132300 & -0.45320800 & $\mathrm{H}$ & 0.05632100 & 0.80276200 & 3.25655700 \\
\hline $\mathrm{H}$ & -1.85637300 & 5.35650600 & -2.78930700 & $\mathrm{H}$ & 0.82651300 & 2.35967400 & 3.03837900 \\
\hline $\mathrm{C}$ & 1.41997400 & -1.99484700 & -1.26326900 & $\mathrm{C}$ & 2.06551200 & 0.69112000 & 2.44016200 \\
\hline $\mathrm{C}$ & 1.80428200 & -2.40794700 & -2.53981900 & $\mathrm{H}$ & 2.63281000 & 0.91629000 & 3.35068900 \\
\hline $\mathrm{C}$ & 3.42865500 & -0.68453700 & -1.12285100 & $\mathrm{H}$ & 2.64084800 & 1.13559200 & 1.61966900 \\
\hline $\mathrm{C}$ & 3.00710800 & -1.96681100 & -3.09645800 & $\mathrm{C}$ & 2.03607000 & -0.83954100 & 2.30080700 \\
\hline $\mathrm{H}$ & 1.16364300 & -3.07938300 & -3.09741900 & $\mathrm{H}$ & 3.04256000 & -1.22758200 & 2.47622100 \\
\hline $\mathrm{C}$ & 3.83528800 & -1.09983700 & -2.37926000 & $\mathrm{H}$ & 1.37062800 & -1.27592500 & 3.05441500 \\
\hline $\mathrm{H}$ & 3.98628500 & 0.00384300 & -0.49851200 & $\mathrm{P}$ & 1.52706000 & -1.49812400 & 0.64112800 \\
\hline $\mathrm{H}$ & 3.29798200 & -2.30119700 & -4.08772700 & $\mathrm{P}$ & -0.25465900 & 1.59123100 & 0.98990900 \\
\hline
\end{tabular}




\begin{tabular}{|c|c|c|c|c|c|c|c|}
\hline $\mathrm{C}$ & -1.87840000 & 2.43428800 & 1.54157200 & $\mathrm{H}$ & 6.26678100 & -1.17561800 & 0.02985500 \\
\hline $\mathrm{C}$ & 1.55443500 & -3.39531700 & 0.86980300 & $\mathrm{H}$ & 4.00741400 & -0.03749400 & -3.45502400 \\
\hline $\mathrm{C}$ & -2.71238000 & 1.38070400 & 2.29947400 & $\mathrm{H}$ & 6.23137200 & -0.38718200 & -2.33951500 \\
\hline $\mathrm{H}$ & -3.67979000 & 1.81692900 & 2.57240900 & $\mathrm{~N}$ & 0.71251000 & 2.67704900 & -1.27373500 \\
\hline $\mathrm{H}$ & -2.23258100 & 1.05517900 & 3.22787400 & $\mathrm{~N}$ & 4.21523900 & -1.27805200 & 0.21676700 \\
\hline $\mathrm{H}$ & -2.90855200 & 0.49774800 & 1.68306200 & $\mathrm{Pd}$ & -0.42543800 & -0.43559800 & -0.30226100 \\
\hline $\mathrm{C}$ & -1.61833100 & 3.65048000 & 2.45093800 & $\mathrm{C}$ & -3.49984200 & -1.26875000 & -1.06098000 \\
\hline $\mathrm{H}$ & -2.58035200 & 4.09687300 & 2.72646700 & $\mathrm{C}$ & -4.59630700 & -0.51598200 & -1.51289200 \\
\hline $\mathrm{H}$ & -1.03551500 & 4.42557300 & 1.94546200 & $\mathrm{C}$ & -3.70376900 & -2.26811000 & -0.09582200 \\
\hline $\mathrm{H}$ & -1.11257100 & 3.38163700 & 3.38245800 & $\mathrm{C}$ & -5.87355600 & -0.76399400 & -1.01228800 \\
\hline $\mathrm{C}$ & -2.65202000 & 2.89494300 & 0.29267800 & $\mathrm{H}$ & -4.45577900 & 0.24916100 & -2.27121300 \\
\hline $\mathrm{H}$ & -2.07200900 & 3.58211700 & -0.32934300 & $\mathrm{C}$ & -4.98308000 & -2.51395400 & 0.39769600 \\
\hline $\mathrm{H}$ & -3.56097900 & 3.41757700 & 0.61077600 & $\mathrm{H}$ & -2.86523800 & -2.84985600 & 0.27258300 \\
\hline $\mathrm{H}$ & -2.96221700 & 2.05096300 & -0.32626500 & $\mathrm{C}$ & -6.07023600 & -1.76255200 & -0.05692500 \\
\hline $\mathrm{C}$ & 0.21990400 & -3.80562300 & 1.52208600 & $\mathrm{H}$ & -6.71470000 & -0.18205400 & -1.37551500 \\
\hline $\mathrm{H}$ & -0.63661000 & -3.54357700 & 0.89479700 & $\mathrm{H}$ & -5.13138100 & -3.29151500 & 1.14060700 \\
\hline $\mathrm{H}$ & 0.07811700 & -3.33874100 & 2.50264700 & $\mathrm{H}$ & -7.06534700 & -1.95690600 & 0.33060700 \\
\hline $\mathrm{H}$ & 0.20810200 & -4.89071200 & 1.67396400 & $\mathrm{C}$ & -1.06991300 & -1.95751600 & -1.71206600 \\
\hline $\mathrm{C}$ & 2.72637600 & -3.84660900 & 1.76528900 & $\mathrm{H}$ & -1.26686300 & -2.97450700 & -1.39303600 \\
\hline $\mathrm{H}$ & 3.68852200 & -3.48727600 & 1.39359200 & $\mathrm{H}$ & -0.34301000 & -1.86025400 & -2.51504100 \\
\hline $\mathrm{H}$ & 2.75278600 & -4.94218900 & 1.78071300 & $\mathrm{C}$ & -2.15658000 & -1.03360000 & -1.66059600 \\
\hline $\mathrm{H}$ & 2.60877200 & -3.51195100 & 2.79944300 & $\mathrm{C}$ & -1.80318300 & 0.28337700 & -1.99331400 \\
\hline $\mathrm{C}$ & 1.69419400 & -4.05737300 & -0.51409900 & $\mathrm{O}$ & -1.83649000 & 1.26352200 & -2.63644000 \\
\hline $\mathrm{H}$ & 1.61051800 & -5.14401200 & -0.40185000 & & & & \\
\hline $\mathrm{H}$ & 2.66663800 & -3.84655100 & -0.96735500 & 15 & & & \\
\hline $\mathrm{H}$ & 0.91562900 & -3.73738500 & -1.21102700 & $\mathrm{C}$ & 0.67707600 & 1.44672100 & 2.63563600 \\
\hline $\mathrm{C}$ & 0.71845700 & 2.86473400 & 0.05773200 & $\mathrm{H}$ & 0.13933300 & 0.83702600 & 3.37027900 \\
\hline $\mathrm{C}$ & 1.40113700 & 3.92879300 & 0.66283000 & $\mathrm{H}$ & 0.74989800 & 2.44883600 & 3.06871100 \\
\hline $\mathrm{C}$ & 1.36622200 & 3.55729700 & -2.04023700 & $\mathrm{C}$ & 2.09060100 & 0.87195500 & 2.42367400 \\
\hline $\mathrm{C}$ & 2.08850500 & 4.83409600 & -0.14745500 & $\mathrm{H}$ & 2.68514600 & 1.13498300 & 3.30620800 \\
\hline $\mathrm{H}$ & 1.40262700 & 4.05637900 & 1.73945000 & $\mathrm{H}$ & 2.58566600 & 1.36570100 & 1.57922800 \\
\hline $\mathrm{C}$ & 2.06846400 & 4.65045200 & -1.52780900 & $\mathrm{C}$ & 2.17528800 & -0.65746100 & 2.26920600 \\
\hline $\mathrm{H}$ & 1.32776900 & 3.37751500 & -3.11220700 & $\mathrm{H}$ & 3.21120000 & -0.96763900 & 2.42733000 \\
\hline $\mathrm{H}$ & 2.62661400 & 5.66691500 & 0.29504300 & $\mathrm{H}$ & 1.55632300 & -1.15086500 & 3.02713800 \\
\hline $\mathrm{H}$ & 2.58542400 & 5.33195400 & -2.19524900 & $\mathrm{P}$ & 1.70170000 & -1.34730500 & 0.61093700 \\
\hline $\mathrm{C}$ & 3.02613700 & -1.10172500 & -0.38285000 & $\mathrm{P}$ & -0.37883600 & 1.50354800 & 1.10883900 \\
\hline $\mathrm{C}$ & 2.90476200 & -0.64988000 & -1.70164600 & $\mathrm{C}$ & -2.07156700 & 2.13372800 & 1.72572700 \\
\hline $\mathrm{C}$ & 5.32281500 & -1.02166800 & -0.48811300 & $\mathrm{C}$ & 1.88750100 & -3.23713800 & 0.81244100 \\
\hline $\mathrm{C}$ & 4.06760500 & -0.38760100 & -2.42892300 & $\mathrm{C}$ & -2.70156100 & 1.02246400 & 2.59022500 \\
\hline $\mathrm{H}$ & 1.92473400 & -0.49446400 & -2.13958600 & $\mathrm{H}$ & -3.70312300 & 1.33688500 & 2.90459600 \\
\hline $\mathrm{C}$ & 5.30211100 & -0.57901300 & -1.81335100 & $\mathrm{H}$ & -2.12721400 & 0.82280500 & 3.50027900 \\
\hline
\end{tabular}




\begin{tabular}{|c|c|c|c|c|c|c|c|}
\hline $\mathrm{H}$ & -2.80839200 & 0.08610100 & 2.03366700 & $\mathrm{Pd}$ & -0.32171700 & -0.50472200 & -0.24198200 \\
\hline $\mathrm{C}$ & -1.93065300 & 3.42766600 & 2.54900100 & $\mathrm{C}$ & -3.33509200 & -1.43504400 & -1.08676100 \\
\hline $\mathrm{H}$ & -2.92621700 & 3.76578900 & 2.85799000 & $\mathrm{C}$ & -4.53791600 & -1.00253200 & -1.66531800 \\
\hline $\mathrm{H}$ & -1.47633800 & 4.23667900 & 1.96957100 & $\mathrm{C}$ & -3.37950500 & -2.26105200 & 0.04712000 \\
\hline $\mathrm{H}$ & -1.34432600 & 3.28112600 & 3.46088100 & $\mathrm{C}$ & -5.76181900 & -1.38424300 & -1.11583300 \\
\hline $\mathrm{C}$ & -2.97043400 & 2.39552100 & 0.50256000 & $\mathrm{H}$ & -4.52513900 & -0.38319400 & -2.55853700 \\
\hline $\mathrm{H}$ & -2.54562900 & 3.14117700 & -0.17445400 & $\mathrm{C}$ & -4.60601900 & -2.65069700 & 0.58346400 \\
\hline $\mathrm{H}$ & -3.94177600 & 2.76821900 & 0.84653200 & $\mathrm{H}$ & -2.45646400 & -2.59260900 & 0.51177300 \\
\hline $\mathrm{H}$ & -3.15459800 & 1.47817000 & -0.06382300 & $\mathrm{C}$ & -5.80018200 & -2.21111600 & 0.00752600 \\
\hline $\mathrm{C}$ & 0.60644800 & -3.76663500 & 1.48559900 & $\mathrm{H}$ & -6.68422500 & -1.04450800 & -1.57627200 \\
\hline $\mathrm{H}$ & -0.28081500 & -3.57364300 & 0.87631900 & $\mathrm{H}$ & -4.62744400 & -3.29573400 & 1.45655900 \\
\hline $\mathrm{H}$ & 0.44523600 & -3.32512200 & 2.47484300 & $\mathrm{H}$ & -6.75307500 & -2.51442500 & 0.42937500 \\
\hline $\mathrm{H}$ & 0.69124500 & -4.85033600 & 1.62361300 & $\mathrm{C}$ & -0.82089500 & -1.89890200 & -1.70929300 \\
\hline $\mathrm{C}$ & 3.11351700 & -3.59912800 & 1.67558600 & $\mathrm{H}$ & -1.00405700 & -2.93089700 & -1.42371800 \\
\hline $\mathrm{H}$ & 4.03427200 & -3.15736500 & 1.28799800 & $\mathrm{H}$ & -0.15201200 & -1.80134300 & -2.56278300 \\
\hline $\mathrm{H}$ & 3.23105300 & -4.68877200 & 1.67585900 & $\mathrm{C}$ & -2.01779700 & -1.05321000 & -1.69295000 \\
\hline $\mathrm{H}$ & 2.99242900 & -3.28691800 & 2.71635700 & $\mathrm{C}$ & -1.95044100 & 0.12754700 & -2.37426600 \\
\hline $\mathrm{C}$ & 2.04736800 & -3.86758900 & -0.58423100 & $\mathrm{O}$ & -1.96819700 & 1.07426500 & -3.04100200 \\
\hline
\end{tabular}

$\mathrm{H}$

H

C

C

C

C

H

C

H

H

H

C

C

C

C

$\mathrm{H}$

C

H

H

H

N

N
$2.06071100 \quad-4.95855400 \quad-0.48445700$

$2.98535700 \quad-3.56841900 \quad-1.05992900$

$1.22435200 \quad-3.60796200 \quad-1.25475600$

0.32563900

2.89028200

0.10239300

1.06432300

0.48667900

3.96169100

0.61963300

$3.72881300-2.03822400$

1.52792700

4.94460700

$-0.25723400$

1.27586900

4.03810300

1.68026000

1.23254900

4.83079000

0.23114700

3.60425600

$-1.61329500$

2.10558100

5.78448600

$-3.08807000$

1.56991900

5.57326300

0.11690300

3.14513800

$-0.8169020$

$-2.32884200$

2.97177600

$-0.3964150$

$-0.43286900$

5.42251700

$-0.51724600$

$-1.75605600$

4.09855800

$-0.03182600$

$-0.55768700$

1.97804600

$-0.34778600$

$-2.49566400$

5.34997900

$-0.0945380$

$-2.18722700$

6.38047200

$-0.5733830$

3.99800600

0.2974730

$-1.88744800$

$-0.04564500$

6.25257700

0.18023900

$-3.52550100$

0.04953300

2.77299500

$-2.42312700$

4.34968600

$-0.87057600$

$-1.21046900$

0.15924900

\section{TS11}

imaginary frequency $=-73.20 \mathrm{~cm}^{-1}$

C

C

C

C

C

$\mathrm{H}$

$\mathrm{H}$

$\mathrm{H}$

$\mathrm{H}$

N

$\mathrm{P}$

Pd

$\mathrm{H}$

C

O

C

C

C

$\mathrm{H}$

H

$\begin{array}{rrr}3.39434600 & -0.04499700 & -1.15947400 \\ 4.17443400 & -0.43548800 & -2.25329600 \\ 5.14100500 & 0.43437000 & -2.75879800 \\ 5.31804400 & 1.67710900 & -2.15419500 \\ 4.50570000 & 2.00046500 & -1.07140700 \\ 4.04401500 & -1.41453800 & -2.69915900 \\ 5.75115400 & 0.13968100 & -3.60712000 \\ 6.05796100 & 2.38402000 & -2.51242600 \\ 4.59348100 & 2.96547700 & -0.57860600 \\ 3.56337300 & 1.17382500 & -0.58723100 \\ 2.07294000 & -1.07562700 & -0.35406500 \\ 0.11776900 & 0.17931600 & 0.06449500 \\ 1.52907600 & 2.25710400 & -1.07019200 \\ 0.40348700 & 2.77700400 & 1.37909700 \\ 0.19394100 & 3.15323800 & 2.47596100 \\ 0.48927700 & 1.98671000 & -0.94743100 \\ -0.27265500 & 2.47745400 & 0.15905500 \\ 2.88276500 & 2.88397900 & 2.35263800 \\ 3.94006900 & 2.83603000 & 2.08751600 \\ 2.65562700 & 2.16449800 & 3.14141400\end{array}$




\begin{tabular}{|c|c|c|c|c|c|c|c|}
\hline $\mathrm{H}$ & 2.62725400 & 3.88884600 & 2.68477800 & $\mathrm{C}$ & -3.31531000 & -1.26003500 & 2.38504000 \\
\hline $\mathrm{O}$ & 2.12469500 & 2.59695900 & 1.15691900 & $\mathrm{H}$ & -4.09525000 & -1.00088400 & 1.66406000 \\
\hline $\mathrm{H}$ & 2.58762300 & 1.90414700 & 0.53986400 & $\mathrm{H}$ & -3.81224500 & -1.58805000 & 3.30507700 \\
\hline $\mathrm{C}$ & 3.06428100 & -1.81342600 & 1.12763000 & $\mathrm{H}$ & -2.74649800 & -0.35347800 & 2.61853700 \\
\hline $\mathrm{C}$ & 3.29756500 & -0.70563400 & 2.17055500 & $\mathrm{C}$ & -1.39038100 & -2.75478200 & 2.97048800 \\
\hline $\mathrm{H}$ & 3.92030300 & 0.10176400 & 1.77775600 & $\mathrm{H}$ & -0.73976300 & -3.58474400 & 2.67755200 \\
\hline $\mathrm{H}$ & 2.34999000 & -0.28406600 & 2.52098500 & $\mathrm{H}$ & -0.76493900 & -1.89890300 & 3.24637800 \\
\hline $\mathrm{H}$ & 3.81846200 & -1.12947800 & 3.03647200 & $\mathrm{H}$ & -1.93061200 & -3.07272500 & 3.86964400 \\
\hline $\mathrm{C}$ & 2.24523700 & -2.93662000 & 1.78587500 & $\mathrm{H}$ & -0.03977500 & 1.88172500 & -1.89264900 \\
\hline $\mathrm{H}$ & 2.08853300 & -3.78932600 & 1.11967600 & $\mathrm{C}$ & -1.68109200 & 2.97911200 & 0.05980600 \\
\hline $\mathrm{H}$ & 2.79640900 & -3.30867600 & 2.65682500 & $\mathrm{C}$ & -2.13297700 & 3.52339000 & -1.15397600 \\
\hline $\mathrm{H}$ & 1.27638100 & -2.57980300 & 2.13832500 & $\mathrm{C}$ & -2.56873500 & 2.94688100 & 1.15035900 \\
\hline $\mathrm{C}$ & 4.41773400 & -2.38044500 & 0.65837300 & $\mathrm{C}$ & -3.43872300 & 3.99893900 & -1.28123800 \\
\hline $\mathrm{H}$ & 4.92579600 & -2.84271100 & 1.51252900 & $\mathrm{H}$ & -1.45549000 & 3.59881900 & -1.99857200 \\
\hline $\mathrm{H}$ & 4.30484900 & -3.15605200 & -0.10587400 & $\mathrm{C}$ & -3.86878200 & 3.43220300 & 1.02245900 \\
\hline $\mathrm{H}$ & 5.07983700 & -1.60592100 & 0.26309500 & $\mathrm{H}$ & -2.24222100 & 2.54113700 & 2.10178700 \\
\hline $\mathrm{C}$ & 1.73075500 & -2.53192400 & -1.47772300 & $\mathrm{C}$ & -4.31340300 & 3.95567600 & -0.19478400 \\
\hline $\mathrm{H}$ & 2.14988600 & -2.31161500 & -2.46455600 & $\mathrm{H}$ & -3.76310100 & 4.42222000 & -2.22736100 \\
\hline $\mathrm{H}$ & 2.26168700 & -3.41360300 & -1.10423600 & $\mathrm{H}$ & -4.53712400 & 3.40031200 & 1.87773700 \\
\hline $\mathrm{C}$ & 0.22737600 & -2.84776500 & -1.67563100 & $\mathrm{H}$ & -5.32508700 & 4.33794700 & -0.28955600 \\
\hline $\mathrm{H}$ & -0.23923000 & -2.01756100 & -2.21629400 & & & & \\
\hline $\mathrm{H}$ & 0.18280800 & -3.70395600 & -2.35863900 & 16 & & & \\
\hline $\mathrm{C}$ & -0.62486300 & -3.19877300 & -0.42901200 & $\mathrm{C}$ & 3.41486800 & -0.38346100 & -1.13959500 \\
\hline $\mathrm{H}$ & -1.42754300 & -3.86775900 & -0.74449500 & $\mathrm{C}$ & 4.20093300 & -0.93061300 & -2.16313000 \\
\hline $\mathrm{H}$ & -0.03176000 & -3.71943800 & 0.32722700 & $\mathrm{C}$ & 5.13714600 & -0.15225500 & -2.83639300 \\
\hline $\mathrm{P}$ & -1.43900200 & -1.71256800 & 0.36332900 & $\mathrm{C}$ & 5.30597500 & 1.20072500 & -2.50093100 \\
\hline $\mathrm{C}$ & -2.77939100 & -1.37833300 & -0.89447400 & $\mathrm{C}$ & 4.51119600 & 1.72796200 & -1.50477500 \\
\hline $\mathrm{C}$ & -3.43051400 & -0.13704700 & -0.92035900 & $\mathrm{H}$ & 4.09556700 & -1.97916300 & -2.41122200 \\
\hline $\mathrm{C}$ & -4.43640400 & 0.07462400 & -1.86422800 & $\mathrm{H}$ & 5.74990000 & -0.59817000 & -3.61367000 \\
\hline $\mathrm{H}$ & -3.15772100 & 0.64515600 & -0.22108000 & $\mathrm{H}$ & 6.02781700 & 1.82849400 & -3.00866400 \\
\hline $\mathrm{C}$ & -4.03673600 & -2.14651900 & -2.66374500 & $\mathrm{H}$ & 4.54644000 & 2.76425100 & -1.18992200 \\
\hline $\mathrm{C}$ & -4.74811300 & -0.94889600 & -2.75695800 & $\mathrm{~N}$ & 3.60509400 & 0.94608500 & -0.87523900 \\
\hline $\mathrm{H}$ & -4.95638700 & 1.02691300 & -1.90028100 & $\mathrm{P}$ & 2.07203300 & -1.17606600 & -0.13315500 \\
\hline $\mathrm{H}$ & -4.24526800 & -2.96841300 & -3.34554700 & $\mathrm{Pd}$ & 0.16893300 & 0.18534000 & 0.01796700 \\
\hline $\mathrm{H}$ & -5.51856800 & -0.82730600 & -3.51133600 & $\mathrm{H}$ & 1.51269000 & 2.20967800 & -1.34957700 \\
\hline $\mathrm{N}$ & -3.07660100 & -2.36402800 & -1.75657200 & $\mathrm{C}$ & 0.63427200 & 3.02145200 & 1.07548100 \\
\hline $\mathrm{C}$ & -2.40342100 & -2.39017700 & 1.86906000 & $\mathrm{O}$ & 0.22686800 & 3.57625700 & 2.07094100 \\
\hline $\mathrm{C}$ & -3.24845900 & -3.62441300 & 1.50555700 & $\mathrm{C}$ & 0.46392500 & 2.00398300 & -1.18113400 \\
\hline $\mathrm{H}$ & -3.82757800 & -3.93766700 & 2.38234800 & $\mathrm{C}$ & -0.19575000 & 2.52156700 & -0.06097900 \\
\hline $\mathrm{H}$ & -3.95241900 & -3.41712700 & 0.69572700 & $\mathrm{C}$ & 2.80602100 & 3.43164100 & 1.93439500 \\
\hline $\mathrm{H}$ & -2.62782900 & -4.47361700 & 1.20495400 & $\mathrm{H}$ & 3.84392800 & 3.22888700 & 1.66529400 \\
\hline
\end{tabular}




\begin{tabular}{|c|c|c|c|c|c|c|c|}
\hline $\mathrm{H}$ & 2.57375700 & 2.99655500 & 2.90810100 & $\mathrm{H}$ & -2.71560600 & -4.16895400 & 1.71175400 \\
\hline $\mathrm{H}$ & 2.62822200 & 4.50772400 & 1.97707900 & $\mathrm{C}$ & -3.50597000 & -0.80588800 & 2.20618100 \\
\hline $\mathrm{O}$ & 2.00263300 & 2.82822000 & 0.89648900 & $\mathrm{H}$ & -4.24838100 & -0.70956900 & 1.40936300 \\
\hline $\mathrm{H}$ & 2.98513200 & 1.39638800 & -0.18437900 & $\mathrm{H}$ & -4.04813900 & -0.96287900 & 3.14578200 \\
\hline $\mathrm{C}$ & 3.05525100 & -1.57136400 & 1.48171700 & $\mathrm{H}$ & -2.96578000 & 0.14281100 & 2.28490300 \\
\hline $\mathrm{C}$ & 3.41245500 & -0.25311700 & 2.19314000 & $\mathrm{C}$ & -1.60977900 & -2.12199200 & 3.18000600 \\
\hline $\mathrm{H}$ & 4.16298300 & 0.32566800 & 1.64549400 & $\mathrm{H}$ & -0.91090200 & -2.95817300 & 3.07625800 \\
\hline $\mathrm{H}$ & 2.53016900 & 0.37337500 & 2.35230400 & $\mathrm{H}$ & -1.03593800 & -1.20412300 & 3.34611500 \\
\hline $\mathrm{H}$ & 3.84649400 & -0.48174200 & 3.17271400 & $\mathrm{H}$ & -2.20325000 & -2.30857500 & 4.08256400 \\
\hline $\mathrm{C}$ & 2.12064300 & -2.38275300 & 2.39710100 & $\mathrm{H}$ & -0.10873500 & 1.79365600 & -2.08081200 \\
\hline $\mathrm{H}$ & 1.85130700 & -3.35188400 & 1.96770700 & $\mathrm{C}$ & -1.63394400 & 2.91904500 & -0.07923000 \\
\hline $\mathrm{H}$ & 2.63733400 & -2.58244800 & 3.34249900 & $\mathrm{C}$ & -2.21586700 & 3.36933100 & -1.27791800 \\
\hline $\mathrm{H}$ & 1.20462600 & -1.83412000 & 2.62415100 & $\mathrm{C}$ & -2.43806300 & 2.88439400 & 1.07594600 \\
\hline $\mathrm{C}$ & 4.33686000 & -2.37571700 & 1.19925600 & $\mathrm{C}$ & -3.55964000 & 3.74290300 & -1.33002300 \\
\hline $\mathrm{H}$ & 4.82637500 & -2.61596700 & 2.15012400 & $\mathrm{H}$ & -1.60659800 & 3.45560400 & -2.17236300 \\
\hline $\mathrm{H}$ & 4.13641000 & -3.32577900 & 0.69500100 & $\mathrm{C}$ & -3.77755700 & 3.26289900 & 1.02274100 \\
\hline $\mathrm{H}$ & 5.05614700 & -1.81324200 & 0.59650900 & $\mathrm{H}$ & -2.00648200 & 2.56442600 & 2.01618200 \\
\hline $\mathrm{C}$ & 1.77075300 & -2.84167900 & -0.93383200 & $\mathrm{C}$ & -4.34838800 & 3.68847600 & -0.18024300 \\
\hline $\mathrm{H}$ & 2.31876300 & -2.89589700 & -1.87952600 & $\mathrm{H}$ & -3.98133000 & 4.09789300 & -2.26606800 \\
\hline $\mathrm{H}$ & 2.18569700 & -3.62452800 & -0.29114800 & $\mathrm{H}$ & -4.37861800 & 3.22771000 & 1.92657000 \\
\hline $\mathrm{C}$ & 0.28033400 & -3.13297700 & -1.23170500 & $\mathrm{H}$ & -5.39115100 & 3.98906800 & -0.21564800 \\
\hline $\mathrm{H}$ & -0.09310100 & -2.40295400 & -1.95785100 & & & & \\
\hline $\mathrm{H}$ & 0.25749800 & -4.10000800 & -1.74749100 & 17 & & & \\
\hline $\mathrm{C}$ & -0.68558300 & -3.21873400 & -0.02503600 & $\mathrm{C}$ & -1.87901900 & -2.60640400 & -0.06099900 \\
\hline $\mathrm{H}$ & -1.48912300 & -3.91139400 & -0.28083000 & $\mathrm{H}$ & -2.47010500 & -2.45987300 & -0.97171800 \\
\hline $\mathrm{H}$ & -0.17478300 & -3.60968000 & 0.86097700 & $\mathrm{H}$ & -1.76373700 & -3.68573100 & 0.07646100 \\
\hline $\mathrm{P}$ & -1.48799800 & -1.58976600 & 0.42536100 & $\mathrm{C}$ & -2.59598800 & -1.99431400 & 1.17175300 \\
\hline $\mathrm{C}$ & -2.74896500 & -1.46458700 & -0.94456200 & $\mathrm{H}$ & -3.22305800 & -2.77169800 & 1.62179200 \\
\hline $\mathrm{C}$ & -3.35870400 & -0.23248100 & -1.22145700 & $\mathrm{H}$ & -1.86248200 & -1.72299200 & 1.93947400 \\
\hline $\mathrm{C}$ & -4.31674000 & -0.17014400 & -2.23327800 & $\mathrm{C}$ & -3.51493400 & -0.78414900 & 0.87905700 \\
\hline $\mathrm{H}$ & -3.08900000 & 0.65381300 & -0.65885100 & $\mathrm{H}$ & -3.94583400 & -0.40561000 & 1.81152800 \\
\hline $\mathrm{C}$ & -3.94925900 & -2.50571700 & -2.61063000 & $\mathrm{H}$ & -4.35393900 & -1.10604400 & 0.25310300 \\
\hline $\mathrm{C}$ & -4.62095400 & -1.32905900 & -2.94566400 & $\mathrm{P}$ & -2.63254500 & 0.57587500 & -0.03443400 \\
\hline $\mathrm{H}$ & -4.80795800 & 0.77134600 & -2.45949800 & $\mathrm{P}$ & -0.15596400 & -1.95135700 & -0.36907000 \\
\hline $\mathrm{H}$ & -4.15177200 & -3.43022000 & -3.14762200 & $\mathrm{C}$ & 0.41804500 & -2.61103400 & -2.05086400 \\
\hline $\mathrm{H}$ & -5.35573300 & -1.32671200 & -3.74417100 & $\mathrm{C}$ & -3.82230200 & 1.27773700 & -1.33456400 \\
\hline $\mathrm{N}$ & -3.03650200 & -2.58103000 & -1.63370100 & $\mathrm{C}$ & -0.17592300 & -1.67958800 & -3.12758800 \\
\hline $\mathrm{C}$ & -2.55131400 & -1.99119600 & 1.96799700 & $\mathrm{H}$ & 0.15547300 & -2.02138500 & -4.11426500 \\
\hline $\mathrm{C}$ & -3.36031500 & -3.28866700 & 1.79076900 & $\mathrm{H}$ & -1.27115100 & -1.69753400 & -3.12951500 \\
\hline $\mathrm{H}$ & -4.00563800 & -3.43523300 & 2.66526600 & $\mathrm{H}$ & 0.15522700 & -0.64501600 & -2.99706900 \\
\hline $\mathrm{H}$ & -3.99989900 & -3.25717600 & 0.90488100 & $\mathrm{C}$ & -0.04997800 & -4.05909100 & -2.29986000 \\
\hline
\end{tabular}




\begin{tabular}{|c|c|c|c|c|c|c|c|}
\hline $\mathrm{H}$ & 0.34212000 & -4.38746200 & -3.26917400 & $\mathrm{C}$ & 3.76051600 & 2.00551500 & -0.53490100 \\
\hline $\mathrm{H}$ & 0.31407900 & -4.73857500 & -1.52843800 & $\mathrm{C}$ & 4.78459400 & 1.24526000 & 0.06001500 \\
\hline $\mathrm{H}$ & -1.14057900 & -4.13579500 & -2.34999400 & $\mathrm{C}$ & 4.11638400 & 3.19375200 & -1.19689200 \\
\hline $\mathrm{C}$ & 1.95720400 & -2.53067900 & -2.09186000 & $\mathrm{C}$ & 6.11106200 & 1.65992000 & -0.00980800 \\
\hline $\mathrm{H}$ & 2.41705100 & -3.21312900 & -1.37258000 & $\mathrm{H}$ & 4.54214100 & 0.32513800 & 0.58351700 \\
\hline $\mathrm{H}$ & 2.29836000 & -2.82204000 & -3.09128700 & $\mathrm{C}$ & 5.44537600 & 3.60893600 & -1.26862800 \\
\hline $\mathrm{H}$ & 2.31803100 & -1.51582800 & -1.89936300 & $\mathrm{H}$ & 3.33939200 & 3.79394800 & -1.66485300 \\
\hline $\mathrm{C}$ & -4.05531900 & 0.17945600 & -2.39167000 & $\mathrm{C}$ & 6.44891400 & 2.84310200 & -0.67467900 \\
\hline $\mathrm{H}$ & -3.11913200 & -0.13154000 & -2.86499400 & $\mathrm{H}$ & 6.88713000 & 1.05962200 & 0.45618700 \\
\hline $\mathrm{H}$ & -4.54848800 & -0.70633600 & -1.97836800 & $\mathrm{H}$ & 5.69621000 & 4.52896700 & -1.78829300 \\
\hline $\mathrm{H}$ & -4.70948000 & 0.57253200 & -3.17741300 & $\mathrm{H}$ & 7.48512200 & 3.16285100 & -0.72744100 \\
\hline $\mathrm{C}$ & -5.16160100 & 1.70356000 & -0.70510300 & $\mathrm{C}$ & 1.79684600 & 0.49614200 & 0.01615900 \\
\hline $\mathrm{H}$ & -5.03418800 & 2.51699200 & 0.01491900 & $\mathrm{H}$ & 2.42505900 & -0.27471600 & 0.45647700 \\
\hline $\mathrm{H}$ & -5.82931100 & 2.07072700 & -1.49284300 & $\mathrm{H}$ & 1.67304400 & 2.35287400 & -0.95870900 \\
\hline $\mathrm{H}$ & -5.67339800 & 0.87392800 & -0.20743800 & & & & \\
\hline $\mathrm{C}$ & -3.13567500 & 2.48846900 & -1.99644500 & 18 & & & \\
\hline $\mathrm{H}$ & -3.78069700 & 2.87595200 & -2.79248500 & $\mathrm{C}$ & 0.94188200 & 2.60058900 & -1.59327900 \\
\hline $\mathrm{H}$ & -2.96399600 & 3.30289600 & -1.28656300 & $\mathrm{H}$ & 0.98869800 & 2.31901200 & -2.65061700 \\
\hline $\mathrm{H}$ & -2.17625800 & 2.21593500 & -2.44706500 & $\mathrm{H}$ & 0.62485300 & 3.64630000 & -1.54892200 \\
\hline $\mathrm{C}$ & 0.77824200 & -2.83365300 & 0.97178800 & $\mathrm{C}$ & 2.32945800 & 2.44706300 & -0.94802000 \\
\hline $\mathrm{C}$ & 1.26878400 & -2.16174600 & 2.09625600 & $\mathrm{H}$ & 2.92345100 & 3.32254300 & -1.23313700 \\
\hline $\mathrm{C}$ & 1.48574600 & -4.86772000 & 1.77868200 & $\mathrm{H}$ & 2.25876300 & 2.48015900 & 0.14622600 \\
\hline $\mathrm{C}$ & 1.89600200 & -2.90905600 & 3.09704500 & $\mathrm{C}$ & 3.09632800 & 1.19594400 & -1.41174400 \\
\hline $\mathrm{H}$ & 1.16108000 & -1.08653400 & 2.18231000 & $\mathrm{H}$ & 4.16512700 & 1.31579100 & -1.21666600 \\
\hline $\mathrm{C}$ & 2.01360700 & -4.28644100 & 2.93516400 & $\mathrm{H}$ & 2.99866800 & 1.09477500 & -2.49776800 \\
\hline $\mathrm{H}$ & 1.55138800 & -5.94134100 & 1.61801200 & $\mathrm{P}$ & 2.51589500 & -0.39943100 & -0.66147100 \\
\hline $\mathrm{H}$ & 2.28605900 & -2.41901700 & 3.98402900 & $\mathrm{P}$ & -0.44870200 & 1.65279400 & -0.80295400 \\
\hline $\mathrm{H}$ & 2.49733800 & -4.90411900 & 3.68452000 & $\mathrm{C}$ & -1.90800500 & 1.85252700 & -2.02121700 \\
\hline $\mathrm{C}$ & -2.20690600 & 1.97297700 & 1.10612400 & $\mathrm{C}$ & 3.18496700 & -1.74416600 & -1.83664100 \\
\hline $\mathrm{C}$ & -2.95711100 & 2.69345800 & 2.03195600 & $\mathrm{C}$ & -1.82678600 & 3.19495600 & -2.77837700 \\
\hline $\mathrm{C}$ & -0.28653600 & 3.21391000 & 1.58407300 & $\mathrm{H}$ & -2.71777100 & 3.28141500 & -3.41022500 \\
\hline $\mathrm{C}$ & -2.32980400 & 3.72223100 & 2.74367000 & $\mathrm{H}$ & -1.81100000 & 4.04553500 & -2.09397100 \\
\hline $\mathrm{H}$ & -4.00371800 & 2.46232000 & 2.19754700 & $\mathrm{H}$ & -0.95859600 & 3.25553000 & -3.43991500 \\
\hline $\mathrm{C}$ & -0.98237400 & 3.99200500 & 2.51171000 & $\mathrm{C}$ & -3.24371800 & 1.79750200 & -1.25405900 \\
\hline $\mathrm{H}$ & 0.77133500 & 3.35741500 & 1.38672500 & $\mathrm{H}$ & -4.06134500 & 1.86498500 & -1.98038500 \\
\hline H & -2.88971700 & 4.30412400 & 3.46930900 & $\mathrm{H}$ & -3.36910300 & 0.86586800 & -0.70192300 \\
\hline $\mathrm{H}$ & -0.46771500 & 4.78210300 & 3.04754700 & $\mathrm{H}$ & -3.34610000 & 2.63961400 & -0.56473100 \\
\hline N & 0.87428600 & -4.16474100 & 0.81985100 & $\mathrm{C}$ & -1.81706600 & 0.68118100 & -3.02006000 \\
\hline $\mathrm{N}$ & -0.88696700 & 2.23244900 & 0.90167700 & $\mathrm{H}$ & -1.93576900 & -0.28345600 & -2.52024400 \\
\hline $\mathrm{Pd}$ & -0.21636000 & 0.32294100 & -0.11175400 & $\mathrm{H}$ & -2.61802000 & 0.77965400 & -3.76107600 \\
\hline $\mathrm{C}$ & 2.33553200 & 1.61694400 & -0.49476600 & $\mathrm{H}$ & -0.86788600 & 0.67745800 & -3.56823400 \\
\hline
\end{tabular}




\begin{tabular}{|c|c|c|c|c|c|c|c|}
\hline $\mathrm{C}$ & 2.35970500 & -1.67556400 & -3.13817100 & $\mathrm{H}$ & -6.57260900 & -4.12342200 & -0.69230000 \\
\hline $\mathrm{H}$ & 1.29100800 & -1.81926700 & -2.94802800 & $\mathrm{H}$ & -6.65265300 & -1.03309300 & 2.29734100 \\
\hline $\mathrm{H}$ & 2.49050100 & -0.72926500 & -3.67239300 & $\mathrm{H}$ & -7.81227000 & -2.83658300 & 1.03909400 \\
\hline $\mathrm{H}$ & 2.68749900 & -2.47314600 & -3.81376700 & $\mathrm{C}$ & -2.65066800 & -1.76051300 & -0.02499800 \\
\hline $\mathrm{C}$ & 4.67924000 & -1.51836100 & -2.13744900 & $\mathrm{H}$ & -2.25480000 & -2.41925300 & -0.79957200 \\
\hline $\mathrm{H}$ & 5.28986800 & -1.55733400 & -1.23046300 & $\mathrm{C}$ & -1.85256300 & -0.81573900 & 0.49818400 \\
\hline $\mathrm{H}$ & 5.03310600 & -2.31303900 & -2.80351800 & $\mathrm{C}$ & 0.29866100 & -2.31459100 & 0.83447500 \\
\hline $\mathrm{H}$ & 4.86700300 & -0.56682600 & -2.64304500 & $\mathrm{O}$ & 0.28069100 & -3.30473600 & 1.39614200 \\
\hline $\mathrm{C}$ & 2.99569500 & -3.12868800 & -1.18931000 & $\mathrm{H}$ & -2.20735900 & -0.15380400 & 1.28566700 \\
\hline $\mathrm{H}$ & 3.40183600 & -3.89182600 & -1.86211400 & & & & \\
\hline $\mathrm{H}$ & 3.51625800 & -3.21782200 & -0.23242200 & \multirow{2}{*}{\multicolumn{4}{|c|}{$\begin{array}{l}\text { TS12 } \\
\text { imaginary frequency }=-258.54 \mathrm{~cm}^{-1}\end{array}$}} \\
\hline $\mathrm{H}$ & 1.94017900 & -3.36545600 & -1.03156700 & & & & \\
\hline $\mathrm{C}$ & -0.78595500 & 2.72681200 & 0.67264700 & $\mathrm{C}$ & -1.10959200 & 1.91913800 & 2.31067700 \\
\hline $\mathrm{C}$ & -0.72822800 & 2.23649900 & 1.98100100 & $\mathrm{H}$ & -0.79783300 & 1.45287300 & 3.25215600 \\
\hline $\mathrm{C}$ & -1.28050900 & 4.85014200 & 1.40434800 & $\mathrm{H}$ & -1.07790200 & 3.00309300 & 2.44628000 \\
\hline $\mathrm{C}$ & -0.96507300 & 3.12235700 & 3.03579200 & $\mathrm{C}$ & -2.54832900 & 1.50263100 & 1.96353500 \\
\hline $\mathrm{H}$ & -0.49712800 & 1.19355900 & 2.16856400 & $\mathrm{H}$ & -3.21158200 & 2.02345600 & 2.66366500 \\
\hline $\mathrm{C}$ & -1.25257000 & 4.45277600 & 2.74453200 & $\mathrm{H}$ & -2.82204300 & 1.87389200 & 0.96889800 \\
\hline $\mathrm{H}$ & -1.49411200 & 5.88228700 & 1.13642500 & $\mathrm{C}$ & -2.85203700 & -0.00258200 & 2.08281300 \\
\hline $\mathrm{H}$ & -0.92487500 & 2.77505500 & 4.06374500 & $\mathrm{H}$ & -3.93194100 & -0.15105800 & 2.17235100 \\
\hline $\mathrm{H}$ & -1.44655100 & 5.17387000 & 3.53155000 & $\mathrm{H}$ & -2.41274900 & -0.39960200 & 3.00539600 \\
\hline $\mathrm{C}$ & 3.47420500 & -0.57179800 & 0.91836300 & $\mathrm{P}$ & -2.22500400 & -1.05884300 & 0.69181200 \\
\hline $\mathrm{C}$ & 4.71190100 & 0.03182800 & 1.17199500 & $\mathrm{P}$ & 0.17831800 & 1.53557700 & 1.03033900 \\
\hline $\mathrm{C}$ & 3.47256200 & -1.58553300 & 2.99133200 & $\mathrm{C}$ & 1.82072100 & 2.06459600 & 1.84166900 \\
\hline $\mathrm{C}$ & 5.33174500 & -0.19719800 & 2.40248500 & $\mathrm{C}$ & -2.68626900 & -2.84032500 & 1.19272100 \\
\hline $\mathrm{H}$ & 5.19536200 & 0.66375100 & 0.43620700 & $\mathrm{C}$ & 1.66080800 & 3.36266800 & 2.65707100 \\
\hline $\mathrm{C}$ & 4.70397200 & -1.02160200 & 3.33233000 & $\mathrm{H}$ & 2.64779900 & 3.66896100 & 3.02215200 \\
\hline $\mathrm{H}$ & 2.94816900 & -2.23794800 & 3.68560500 & $\mathrm{H}$ & 1.25223800 & 4.17842000 & 2.05603700 \\
\hline $\mathrm{H}$ & 6.29066100 & 0.26089300 & 2.62475300 & $\mathrm{H}$ & 1.02071100 & 3.22617100 & 3.53300700 \\
\hline $\mathrm{H}$ & 5.15109200 & -1.22559000 & 4.29939700 & $\mathrm{C}$ & 2.86856600 & 2.28404900 & 0.73345700 \\
\hline $\mathrm{N}$ & -1.04733200 & 4.01329200 & 0.38823900 & $\mathrm{H}$ & 3.83273900 & 2.51821900 & 1.19826500 \\
\hline $\mathrm{N}$ & 2.86587800 & -1.36324800 & 1.82043400 & $\mathrm{H}$ & 3.00803000 & 1.39431800 & 0.11570400 \\
\hline $\mathrm{Pd}$ & 0.12340200 & -0.56035400 & -0.07416400 & $\mathrm{H}$ & 2.60292700 & 3.12399700 & 0.08517700 \\
\hline $\mathrm{C}$ & -4.06648200 & -2.02266800 & 0.30124200 & $\mathrm{C}$ & 2.26476500 & 0.91457300 & 2.76794400 \\
\hline $\mathrm{C}$ & -4.73756700 & -3.03986000 & -0.40008100 & $\mathrm{H}$ & 2.42524100 & -0.01364400 & 2.21310800 \\
\hline $\mathrm{C}$ & -4.78214800 & -1.30441900 & 1.27744400 & $\mathrm{H}$ & 3.20984300 & 1.18530500 & 3.25197800 \\
\hline $\mathrm{C}$ & -6.07515200 & -3.33256600 & -0.13895000 & $\mathrm{H}$ & 1.53808700 & 0.72088300 & 3.56440600 \\
\hline $\mathrm{H}$ & -4.20060800 & -3.60650400 & -1.15699100 & $\mathrm{C}$ & -1.66132300 & -3.30240200 & 2.24830000 \\
\hline $\mathrm{C}$ & -6.11739500 & -1.59652900 & 1.53867400 & $\mathrm{H}$ & -0.64053400 & -3.28792100 & 1.85363700 \\
\hline $\mathrm{H}$ & -4.29210500 & -0.51578400 & 1.84088600 & $\mathrm{H}$ & -1.68820100 & -2.68870100 & 3.15492500 \\
\hline $\mathrm{C}$ & -6.77063200 & -2.61125000 & 0.83194900 & $\mathrm{H}$ & -1.88924500 & -4.33150600 & 2.54687800 \\
\hline
\end{tabular}




\begin{tabular}{|c|c|c|c|c|c|c|c|}
\hline $\mathrm{C}$ & -4.11095600 & -2.90868800 & 1.77675400 & $\mathrm{H}$ & 2.81641900 & -2.14091000 & 0.63179500 \\
\hline $\mathrm{H}$ & -4.86377200 & -2.54526500 & 1.07050400 & $\mathrm{C}$ & 1.99916000 & -0.91039700 & -0.85899300 \\
\hline $\mathrm{H}$ & -4.35210300 & -3.95470800 & 1.99556000 & $\mathrm{C}$ & 0.52749500 & -2.20281100 & -1.06657200 \\
\hline $\mathrm{H}$ & -4.21031000 & -2.35307400 & 2.71313600 & $\mathrm{O}$ & 0.56932000 & -3.12618100 & -1.76175100 \\
\hline $\mathrm{C}$ & -2.60530800 & -3.75041300 & -0.04759800 & $\mathrm{H}$ & 2.14007100 & -0.32460000 & -1.76446700 \\
\hline $\mathrm{H}$ & -2.82199100 & -4.78002900 & 0.25782400 & & & & \\
\hline $\mathrm{H}$ & -3.33701800 & -3.47013800 & -0.80990600 & 19 & & & \\
\hline $\mathrm{H}$ & -1.61600600 & -3.74580100 & -0.50819100 & $\mathrm{C}$ & -0.65708500 & 1.97490700 & 2.31109600 \\
\hline $\mathrm{C}$ & -0.19227200 & 2.81990900 & -0.25859700 & $\mathrm{H}$ & -0.26893600 & 1.48656200 & 3.21197000 \\
\hline $\mathrm{C}$ & -0.09605800 & 2.52215100 & -1.62276800 & $\mathrm{H}$ & -0.61498400 & 3.05605900 & 2.46589100 \\
\hline $\mathrm{C}$ & -0.80561500 & 4.99234300 & -0.69968500 & $\mathrm{C}$ & -2.11526500 & 1.55266900 & 2.06649300 \\
\hline $\mathrm{C}$ & -0.36623100 & 3.53174700 & -2.54884600 & $\mathrm{H}$ & -2.72607700 & 2.05102900 & 2.82806100 \\
\hline $\mathrm{H}$ & 0.17261500 & 1.52250500 & -1.94758900 & $\mathrm{H}$ & -2.47685200 & 1.94325100 & 1.10899600 \\
\hline $\mathrm{C}$ & -0.72569300 & 4.79322200 & -2.08054000 & $\mathrm{C}$ & -2.40819200 & 0.04541000 & 2.18277800 \\
\hline $\mathrm{H}$ & -1.08994200 & 5.96124000 & -0.29540200 & $\mathrm{H}$ & -3.47612000 & -0.08589600 & 2.36878400 \\
\hline $\mathrm{H}$ & -0.29960700 & 3.33342500 & -3.61435900 & $\mathrm{H}$ & -1.86042100 & -0.38430100 & 3.02897500 \\
\hline $\mathrm{H}$ & -0.94519700 & 5.60748400 & -2.76315200 & $\mathrm{P}$ & -2.05431900 & -1.01278900 & 0.69965300 \\
\hline $\mathrm{C}$ & -3.29448300 & -0.62747600 & -0.75638200 & $\mathrm{P}$ & 0.49467100 & 1.59498500 & 0.91038600 \\
\hline $\mathrm{C}$ & -4.62724800 & -0.21060300 & -0.64910700 & $\mathrm{C}$ & 2.23578200 & 1.99361500 & 1.57079600 \\
\hline $\mathrm{C}$ & -3.37266200 & -0.51703900 & -3.05447400 & $\mathrm{C}$ & -2.40482700 & -2.79178400 & 1.30000700 \\
\hline $\mathrm{C}$ & -5.34184600 & 0.05908500 & -1.81810400 & $\mathrm{C}$ & 2.27029600 & 3.32920300 & 2.33623300 \\
\hline $\mathrm{H}$ & -5.10561600 & -0.09678100 & 0.31725500 & $\mathrm{H}$ & 3.30383600 & 3.54640600 & 2.63029900 \\
\hline $\mathrm{C}$ & -4.70598300 & -0.09960200 & -3.04655600 & $\mathrm{H}$ & 1.90911900 & 4.16169500 & 1.72774500 \\
\hline $\mathrm{H}$ & -2.84065900 & -0.65185900 & -3.99334000 & $\mathrm{H}$ & 1.67258600 & 3.29507500 & 3.25136300 \\
\hline $\mathrm{H}$ & -6.37635100 & 0.38441900 & -1.76530000 & $\mathrm{C}$ & 3.19996600 & 2.05390400 & 0.37098100 \\
\hline $\mathrm{H}$ & -5.22412900 & 0.09594900 & -3.97945600 & $\mathrm{H}$ & 4.22096700 & 2.21425000 & 0.73485800 \\
\hline $\mathrm{N}$ & -0.54773800 & 4.03283000 & 0.19595000 & $\mathrm{H}$ & 3.19937700 & 1.12094000 & -0.20198100 \\
\hline $\mathrm{N}$ & -2.67426300 & -0.76981700 & -1.94198100 & $\mathrm{H}$ & 2.95793500 & 2.87805500 & -0.30620100 \\
\hline $\mathrm{Pd}$ & 0.06067900 & -0.69810000 & 0.03953200 & $\mathrm{C}$ & 2.64908300 & 0.83841100 & 2.50495200 \\
\hline $\mathrm{C}$ & 4.46307800 & -1.39460100 & -0.53736300 & $\mathrm{H}$ & 2.66324600 & -0.12234200 & 1.97988800 \\
\hline $\mathrm{C}$ & 5.37530200 & -2.12657800 & 0.24674600 & $\mathrm{H}$ & 3.65947600 & 1.02352900 & 2.88687000 \\
\hline $\mathrm{C}$ & 4.96550800 & -0.59706300 & -1.58509700 & $\mathrm{H}$ & 1.98728700 & 0.74724300 & 3.37248500 \\
\hline $\mathrm{C}$ & 6.74349700 & -2.06838400 & -0.00630700 & $\mathrm{C}$ & -1.19451100 & -3.25474100 & 2.13368900 \\
\hline $\mathrm{H}$ & 5.00186100 & -2.74705100 & 1.05742000 & $\mathrm{H}$ & -0.27988500 & -3.28080400 & 1.53245900 \\
\hline $\mathrm{C}$ & 6.33136000 & -0.54106600 & -1.83747000 & $\mathrm{H}$ & -1.01651400 & -2.61457500 & 3.00411000 \\
\hline $\mathrm{H}$ & 4.28560700 & -0.02146100 & -2.20572200 & $\mathrm{H}$ & -1.37879300 & -4.26816900 & 2.50684900 \\
\hline $\mathrm{C}$ & 7.22490200 & -1.27551100 & -1.04950700 & $\mathrm{C}$ & -3.68466100 & -2.82291400 & 2.16154000 \\
\hline $\mathrm{H}$ & 7.43223000 & -2.64108500 & 0.60687400 & $\mathrm{H}$ & -4.54725900 & -2.40695500 & 1.63354100 \\
\hline $\mathrm{H}$ & 6.70481700 & 0.07514100 & -2.64956300 & $\mathrm{H}$ & -3.91470100 & -3.86621200 & 2.40464400 \\
\hline $\mathrm{H}$ & 8.29078200 & -1.22836300 & -1.25030100 & $\mathrm{H}$ & -3.56731300 & -2.28724300 & 3.10673100 \\
\hline $\mathrm{C}$ & 3.03694200 & -1.49827600 & -0.22141300 & $\mathrm{C}$ & -2.59110400 & -3.72484800 & 0.08889400 \\
\hline
\end{tabular}




\begin{tabular}{|c|c|c|c|c|c|c|c|}
\hline $\mathrm{H}$ & -2.73186900 & -4.74810700 & 0.45463100 & 20 & & & \\
\hline $\mathrm{H}$ & -3.47672600 & -3.46155800 & -0.49586000 & $\mathrm{C}$ & -3.74212100 & -0.01304300 & 0.48677900 \\
\hline $\mathrm{H}$ & -1.73017800 & -3.72568600 & -0.58137400 & $\mathrm{H}$ & -4.22315700 & -0.01772700 & 1.47121900 \\
\hline $\mathrm{C}$ & 0.09668100 & 2.96759400 & -0.27942300 & $\mathrm{H}$ & -4.50522800 & 0.29242900 & -0.23676700 \\
\hline $\mathrm{C}$ & 0.20560400 & 2.76470600 & -1.66123800 & $\mathrm{C}$ & -3.22871600 & -1.43381600 & 0.16149900 \\
\hline $\mathrm{C}$ & -0.58006500 & 5.14526000 & -0.58558300 & $\mathrm{H}$ & -4.09609600 & -2.10263700 & 0.16211500 \\
\hline $\mathrm{C}$ & -0.09140000 & 3.82195600 & -2.52278400 & $\mathrm{H}$ & -2.83363700 & -1.46413700 & -0.85994300 \\
\hline $\mathrm{H}$ & 0.51184800 & 1.79821500 & -2.05019200 & $\mathrm{C}$ & -2.18874900 & -1.99463400 & 1.16701200 \\
\hline $\mathrm{C}$ & -0.49280300 & 5.03866900 & -1.97577800 & $\mathrm{H}$ & -2.36968300 & -3.06176300 & 1.30730800 \\
\hline $\mathrm{H}$ & -0.89450700 & 6.07702000 & -0.12073200 & $\mathrm{H}$ & -2.28425700 & -1.50141800 & 2.14003700 \\
\hline $\mathrm{H}$ & -0.01548500 & 3.69494400 & -3.59856100 & $\mathrm{P}$ & -0.40238400 & -1.85755100 & 0.64915800 \\
\hline $\mathrm{H}$ & -0.73802100 & 5.88730500 & -2.60570800 & $\mathrm{P}$ & -2.35913900 & 1.23401300 & 0.55521300 \\
\hline $\mathrm{C}$ & -3.44562700 & -0.57473700 & -0.44985400 & $\mathrm{C}$ & -2.83342800 & 2.55167600 & 1.83924900 \\
\hline $\mathrm{C}$ & -3.38292900 & -0.87431200 & -1.81659100 & $\mathrm{C}$ & 0.60519000 & -2.22390400 & 2.22317700 \\
\hline $\mathrm{C}$ & -5.54334500 & 0.34098500 & -0.66007300 & $\mathrm{C}$ & -4.24937000 & 3.11520400 & 1.62073600 \\
\hline $\mathrm{C}$ & -4.47687100 & -0.54079000 & -2.61672900 & $\mathrm{H}$ & -4.48409800 & 3.82366200 & 2.42378100 \\
\hline $\mathrm{H}$ & -2.51426900 & -1.36808800 & -2.23705600 & $\mathrm{H}$ & -4.33167000 & 3.66196900 & 0.67739400 \\
\hline $\mathrm{C}$ & -5.57789900 & 0.08178800 & -2.03152600 & $\mathrm{H}$ & -5.01775700 & 2.33599800 & 1.64105200 \\
\hline $\mathrm{H}$ & -6.38222300 & 0.82448300 & -0.16470800 & $\mathrm{C}$ & -1.79041000 & 3.68250700 & 1.74852200 \\
\hline $\mathrm{H}$ & -4.46595100 & -0.76529300 & -3.67905400 & $\mathrm{H}$ & -1.99523900 & 4.42910000 & 2.52376600 \\
\hline $\mathrm{H}$ & -6.44665600 & 0.36091400 & -2.61848800 & $\mathrm{H}$ & -0.77300200 & 3.30980300 & 1.90474100 \\
\hline $\mathrm{N}$ & -0.29317300 & 4.13881800 & 0.24818000 & $\mathrm{H}$ & -1.82287500 & 4.19433000 & 0.78196600 \\
\hline $\mathrm{N}$ & -4.50386500 & 0.02063300 & 0.12026600 & $\mathrm{C}$ & -2.74442200 & 1.88058900 & 3.22564000 \\
\hline $\mathrm{Pd}$ & 0.09173600 & -0.60318100 & -0.20084300 & $\mathrm{H}$ & -1.74397600 & 1.48291800 & 3.42180700 \\
\hline $\mathrm{C}$ & 3.98549200 & -1.81494700 & -0.70000200 & $\mathrm{H}$ & -2.96470000 & 2.62390700 & 3.99971000 \\
\hline $\mathrm{C}$ & 4.71130200 & -2.32437800 & 0.39615400 & $\mathrm{H}$ & -3.46912400 & 1.06865700 & 3.34551400 \\
\hline $\mathrm{C}$ & 4.68983100 & -1.16941800 & -1.73861100 & $\mathrm{C}$ & 0.47650900 & -1.01337300 & 3.16842400 \\
\hline $\mathrm{C}$ & 6.09574300 & -2.19455600 & 0.45558300 & $\mathrm{H}$ & 0.84582900 & -0.09465400 & 2.70147300 \\
\hline $\mathrm{H}$ & 4.18010200 & -2.82951400 & 1.19857800 & $\mathrm{H}$ & -0.55624300 & -0.84317300 & 3.48833700 \\
\hline $\mathrm{C}$ & 6.07148700 & -1.04119900 & -1.67637100 & $\mathrm{H}$ & 1.07056700 & -1.19589100 & 4.07081500 \\
\hline $\mathrm{H}$ & 4.15741700 & -0.77271800 & -2.59738600 & $\mathrm{C}$ & 0.08244600 & -3.49728600 & 2.91633900 \\
\hline $\mathrm{C}$ & 6.77765600 & -1.55302100 & -0.58049100 & $\mathrm{H}$ & 0.11570000 & -4.36786200 & 2.25567700 \\
\hline $\mathrm{H}$ & 6.64212300 & -2.59483200 & 1.30348700 & $\mathrm{H}$ & 0.71282700 & -3.71540900 & 3.78601000 \\
\hline $\mathrm{H}$ & 6.60456100 & -0.54626100 & -2.48184400 & $\mathrm{H}$ & -0.94305100 & -3.38178600 & 3.27761400 \\
\hline $\mathrm{H}$ & 7.85783800 & -1.45273400 & -0.53886200 & $\mathrm{C}$ & 2.08448500 & -2.41352300 & 1.83648800 \\
\hline $\mathrm{C}$ & 2.54199000 & -1.99563700 & -0.71075800 & $\mathrm{H}$ & 2.67907000 & -2.53648600 & 2.74848600 \\
\hline $\mathrm{H}$ & 2.13513100 & -2.52144300 & 0.15297000 & $\mathrm{H}$ & 2.23537000 & -3.30746700 & 1.22569500 \\
\hline $\mathrm{C}$ & 1.66582400 & -1.65552700 & -1.70208000 & $\mathrm{H}$ & 2.48336700 & -1.55174600 & 1.29283100 \\
\hline $\mathrm{C}$ & 0.21538400 & -1.99026800 & -1.60805900 & $\mathrm{C}$ & -2.29814000 & 2.08480200 & -1.09381200 \\
\hline $\mathrm{O}$ & -0.49576000 & -2.59344100 & -2.35790600 & $\mathrm{C}$ & -3.28827500 & 2.74346500 & -1.82085500 \\
\hline $\mathrm{H}$ & 1.98789700 & -1.17549800 & -2.62332200 & $\mathrm{C}$ & -0.71846200 & 2.48728800 & -2.76755400 \\
\hline
\end{tabular}




\begin{tabular}{|c|c|c|c|c|c|c|c|}
\hline $\mathrm{C}$ & -2.95632200 & 3.30637900 & -3.05698800 & $\mathrm{H}$ & 4.13871500 & 2.30605400 & 0.00977700 \\
\hline $\mathrm{H}$ & -4.29955400 & 2.81355200 & -1.43647800 & $\mathrm{H}$ & 2.88414800 & 1.57267800 & -0.95823800 \\
\hline $\mathrm{C}$ & -1.65231300 & 3.18638700 & -3.53259500 & $\mathrm{C}$ & 2.26882300 & 2.10890900 & 1.07503800 \\
\hline $\mathrm{H}$ & 0.29962200 & 2.33673700 & -3.11089600 & $\mathrm{H}$ & 2.37374300 & 3.19108900 & 1.19053000 \\
\hline $\mathrm{H}$ & -3.70867700 & 3.82936400 & -3.63928300 & $\mathrm{H}$ & 2.42626200 & 1.65558800 & 2.05964400 \\
\hline $\mathrm{H}$ & -1.35948500 & 3.61030600 & -4.48684000 & $\mathrm{P}$ & 0.48381200 & 1.84509900 & 0.58923400 \\
\hline $\mathrm{C}$ & -0.20429200 & -3.36350000 & -0.42725600 & $\mathrm{P}$ & 2.62989200 & -1.11975100 & 0.55914800 \\
\hline $\mathrm{C}$ & 0.94033000 & -3.57149100 & -1.20717100 & $\mathrm{C}$ & 3.24755000 & -2.35405400 & 1.86283000 \\
\hline $\mathrm{C}$ & -1.13453900 & -5.34524000 & -1.13904500 & $\mathrm{C}$ & -0.56037300 & 2.34314500 & 2.09841300 \\
\hline $\mathrm{C}$ & 1.02740100 & -4.73731100 & -1.96796700 & $\mathrm{C}$ & 4.67702700 & -2.84882900 & 1.57851400 \\
\hline $\mathrm{H}$ & 1.74132200 & -2.84425600 & -1.22933200 & $\mathrm{H}$ & 4.99926000 & -3.51197700 & 2.38976800 \\
\hline $\mathrm{C}$ & -0.02988300 & -5.64390800 & -1.93776800 & $\mathrm{H}$ & 4.73289600 & -3.42541100 & 0.65091400 \\
\hline $\mathrm{H}$ & -1.98352500 & -6.02332000 & -1.08945500 & $\mathrm{H}$ & 5.39945600 & -2.02873700 & 1.52343100 \\
\hline $\mathrm{H}$ & 1.90401500 & -4.92618800 & -2.57986400 & $\mathrm{C}$ & 2.26688300 & -3.54305500 & 1.88050300 \\
\hline $\mathrm{H}$ & -0.00630700 & -6.55900800 & -2.52005800 & $\mathrm{H}$ & 2.56330300 & -4.24394000 & 2.66857900 \\
\hline $\mathrm{N}$ & -1.03411000 & 1.95364100 & -1.58012900 & $\mathrm{H}$ & 1.24135700 & -3.22165500 & 2.08877600 \\
\hline $\mathrm{N}$ & -1.22406400 & -4.23536100 & -0.39693400 & $\mathrm{H}$ & 2.26919000 & -4.09136600 & 0.93362900 \\
\hline $\mathrm{Pd}$ & -0.23098700 & 0.24702900 & -0.36036000 & $\mathrm{C}$ & 3.19863900 & -1.63039100 & 3.22452700 \\
\hline $\mathrm{C}$ & 2.95132600 & 0.90072200 & -0.28870600 & $\mathrm{H}$ & 2.19167800 & -1.26867700 & 3.45565500 \\
\hline $\mathrm{H}$ & 2.19205500 & 0.98936600 & 0.48995600 & $\mathrm{H}$ & 3.48970700 & -2.33141000 & 4.01449200 \\
\hline $\mathrm{C}$ & 2.58066900 & 0.28683000 & -1.43549000 & $\mathrm{H}$ & 3.89080000 & -0.78376100 & 3.27594100 \\
\hline $\mathrm{C}$ & 1.22347200 & -0.23156500 & -1.68571400 & $\mathrm{C}$ & -0.57704000 & 1.14163500 & 3.06473200 \\
\hline $\mathrm{O}$ & 0.90542400 & -0.77050300 & -2.72283800 & $\mathrm{H}$ & -1.00139900 & 0.24746800 & 2.59761000 \\
\hline $\mathrm{H}$ & 3.26745200 & 0.14947100 & -2.26872300 & $\mathrm{H}$ & 0.42486200 & 0.89077300 & 3.42918200 \\
\hline $\mathrm{C}$ & 4.25284000 & 1.46669900 & 0.04814800 & $\mathrm{H}$ & -1.18890500 & 1.39028800 & 3.93919600 \\
\hline $\mathrm{C}$ & 5.35477900 & 1.44203900 & -0.83139600 & $\mathrm{C}$ & 0.01000300 & 3.57975400 & 2.82034600 \\
\hline $\mathrm{C}$ & 4.41660700 & 2.06532100 & 1.31253800 & $\mathrm{H}$ & 0.10746300 & 4.43504400 & 2.14969200 \\
\hline $\mathrm{C}$ & 6.57206600 & 1.99570100 & -0.45305700 & $\mathrm{H}$ & -0.66829100 & 3.85365200 & 3.63685200 \\
\hline $\mathrm{H}$ & 5.25846900 & 0.98830900 & -1.81266500 & $\mathrm{H}$ & 0.98903300 & 3.37910200 & 3.26549700 \\
\hline $\mathrm{C}$ & 5.63640100 & 2.62072400 & 1.68999300 & $\mathrm{C}$ & -1.99045000 & 2.63684200 & 1.60407600 \\
\hline $\mathrm{H}$ & 3.57493800 & 2.09230600 & 2.00020100 & $\mathrm{H}$ & -2.63541400 & 2.83085100 & 2.46829500 \\
\hline $\mathrm{C}$ & 6.71742600 & 2.58674000 & 0.80743500 & $\mathrm{H}$ & -2.02197000 & 3.52046500 & 0.96111400 \\
\hline $\mathrm{H}$ & 7.41257000 & 1.96920800 & -1.13959600 & $\mathrm{H}$ & -2.41959300 & 1.79431400 & 1.05255100 \\
\hline $\mathrm{H}$ & 5.74463200 & 3.07829200 & 2.66841500 & $\mathrm{C}$ & 2.51870100 & -2.03914400 & -1.04908300 \\
\hline \multirow[t]{2}{*}{$\mathrm{H}$} & 7.67056300 & 3.01815800 & 1.09748900 & $\mathrm{C}$ & 3.50025200 & -2.64310700 & -1.83261000 \\
\hline & & & & $\mathrm{C}$ & 0.85224100 & -2.61338100 & -2.58376400 \\
\hline $20 \mathbf{a}$ & & & & $\mathrm{C}$ & 3.12038400 & -3.26911200 & -3.02427000 \\
\hline $\mathrm{C}$ & 3.92544700 & 0.20729400 & 0.37915400 & $\mathrm{H}$ & 4.54018500 & -2.62379300 & -1.52602900 \\
\hline $\mathrm{H}$ & 4.45944400 & 0.26700800 & 1.33404600 & $\mathrm{C}$ & 1.77935300 & -3.26043300 & -3.40200900 \\
\hline $\mathrm{H}$ & 4.66557800 & -0.06868300 & -0.37923900 & $\mathrm{H}$ & -0.19611000 & -2.53967100 & -2.85349000 \\
\hline $\mathrm{C}$ & 3.31238400 & 1.58799100 & 0.05088100 & $\mathrm{H}$ & 3.86504400 & -3.75257200 & -3.64910200 \\
\hline
\end{tabular}




\begin{tabular}{|c|c|c|c|c|c|c|c|}
\hline $\mathrm{H}$ & 1.45078400 & -3.73149100 & -4.32196200 & $\mathrm{P}$ & -2.53403900 & -0.23186500 & -0.56010600 \\
\hline $\mathrm{C}$ & 0.28792800 & 3.19857600 & -0.67099000 & $\mathrm{P}$ & 0.28879300 & 2.17737600 & -0.32026000 \\
\hline $\mathrm{C}$ & -0.23076100 & 2.95990300 & -1.94752700 & $\mathrm{C}$ & 0.06362900 & 3.85218300 & 0.57728000 \\
\hline $\mathrm{C}$ & 0.61864400 & 5.42914600 & -1.12803600 & $\mathrm{C}$ & -4.33821100 & 0.07428000 & 0.00652900 \\
\hline $\mathrm{C}$ & -0.32105400 & 4.03628100 & -2.83553900 & $\mathrm{C}$ & -1.36572300 & 3.88238400 & 1.15319500 \\
\hline $\mathrm{H}$ & -0.54732500 & 1.96810700 & -2.24643500 & $\mathrm{H}$ & -1.52459200 & 4.83826300 & 1.66460700 \\
\hline $\mathrm{C}$ & 0.10485800 & 5.29452400 & -2.42098800 & $\mathrm{H}$ & -2.13487800 & 3.79784700 & 0.37966100 \\
\hline $\mathrm{H}$ & 0.97070300 & 6.39351500 & -0.76842000 & $\mathrm{H}$ & -1.51668900 & 3.08234000 & 1.88459200 \\
\hline $\mathrm{H}$ & -0.71737700 & 3.88559200 & -3.83518100 & $\mathrm{C}$ & 0.26750000 & 5.01392500 & -0.41626700 \\
\hline $\mathrm{H}$ & 0.05088300 & 6.15556000 & -3.07906700 & $\mathrm{H}$ & 0.18822100 & 5.96062900 & 0.12977000 \\
\hline $\mathrm{N}$ & 1.21445500 & -2.02542000 & -1.43654800 & $\mathrm{H}$ & 1.25685100 & 4.98563100 & -0.88265300 \\
\hline $\mathrm{N}$ & 0.71601700 & 4.40859800 & -0.27055500 & $\mathrm{H}$ & -0.48558300 & 5.03126200 & -1.20830200 \\
\hline $\mathrm{Pd}$ & 0.34710400 & -0.36221900 & -0.18503900 & $\mathrm{C}$ & 1.06593300 & 3.99608600 & 1.73825500 \\
\hline $\mathrm{C}$ & -3.77268700 & -0.88613300 & -0.83192500 & $\mathrm{H}$ & 2.09692500 & 4.06595500 & 1.38251500 \\
\hline $\mathrm{H}$ & -3.86668800 & -0.67491300 & -1.89635800 & $\mathrm{H}$ & 0.84690100 & 4.92994100 & 2.26836800 \\
\hline $\mathrm{C}$ & -2.54138600 & -0.70205800 & -0.29167200 & $\mathrm{H}$ & 0.98954200 & 3.17809100 & 2.45740900 \\
\hline $\mathrm{C}$ & -1.42083700 & -0.25519500 & -1.13822300 & $\mathrm{C}$ & -4.48834100 & 1.58345500 & 0.28248400 \\
\hline $\mathrm{O}$ & -1.48385700 & -0.04539000 & -2.33264500 & $\mathrm{H}$ & -3.77634400 & 1.92947300 & 1.03729600 \\
\hline $\mathrm{H}$ & -2.33875000 & -0.87185900 & 0.75998000 & $\mathrm{H}$ & -4.36136800 & 2.19339500 & -0.61744900 \\
\hline $\mathrm{C}$ & -4.99077600 & -1.32115600 & -0.16395100 & $\mathrm{H}$ & -5.49752500 & 1.78042900 & 0.66085500 \\
\hline $\mathrm{C}$ & -5.04250600 & -1.66393900 & 1.20342600 & $\mathrm{C}$ & -5.35495400 & -0.37378300 & -1.05878500 \\
\hline $\mathrm{C}$ & -6.17484100 & -1.40459500 & -0.92319800 & $\mathrm{H}$ & -5.28923000 & -1.44620500 & -1.26345200 \\
\hline $\mathrm{C}$ & -6.23652500 & -2.07261500 & 1.78408800 & $\mathrm{H}$ & -6.36955000 & -0.17148100 & -0.69682200 \\
\hline $\mathrm{H}$ & -4.14461600 & -1.61338800 & 1.81149900 & $\mathrm{H}$ & -5.23597400 & 0.16532500 & -2.00336400 \\
\hline $\mathrm{C}$ & -7.37102400 & -1.81374200 & -0.33965300 & $\mathrm{C}$ & -4.58409900 & -0.69548200 & 1.31736900 \\
\hline $\mathrm{H}$ & -6.14775400 & -1.14377600 & -1.97779400 & $\mathrm{H}$ & -5.60684700 & -0.50260500 & 1.65980600 \\
\hline $\mathrm{C}$ & -7.40426600 & -2.14837400 & 1.01517600 & $\mathrm{H}$ & -4.47477800 & -1.77602800 & 1.19313300 \\
\hline $\mathrm{H}$ & -6.26336300 & -2.33510500 & 2.83719100 & $\mathrm{H}$ & -3.90442300 & -0.36744000 & 2.10924100 \\
\hline $\mathrm{H}$ & -8.27427600 & -1.87186700 & -0.93875400 & $\mathrm{C}$ & 2.03576100 & 2.17237000 & -0.97543600 \\
\hline \multirow[t]{2}{*}{$\mathrm{H}$} & -8.33497700 & -2.46849800 & 1.47357700 & $\mathrm{C}$ & 3.14956600 & 2.08563500 & -0.12817900 \\
\hline & & & & $\mathrm{C}$ & 3.38252500 & 2.24960500 & -2.84305600 \\
\hline $20 b$ & & & & $\mathrm{C}$ & 4.42397500 & 2.10526300 & -0.69384900 \\
\hline $\mathrm{C}$ & -0.68461500 & 2.39268900 & -1.88702000 & $\mathrm{H}$ & 3.03090000 & 2.00408100 & 0.94444300 \\
\hline $\mathrm{H}$ & -1.58154300 & 2.96892800 & -1.63841000 & $\mathrm{C}$ & 4.54819600 & 2.18767800 & -2.07957400 \\
\hline $\mathrm{H}$ & -0.05768300 & 3.00860700 & -2.53352900 & $\mathrm{H}$ & 3.43153600 & 2.30796900 & -3.92812100 \\
\hline $\mathrm{C}$ & -1.07446200 & 1.10668400 & -2.64211900 & $\mathrm{H}$ & 5.30365900 & 2.05182300 & -0.05970200 \\
\hline $\mathrm{H}$ & -1.10539600 & 1.34565600 & -3.71099400 & $\mathrm{H}$ & 5.52066700 & 2.20104400 & -2.56031700 \\
\hline $\mathrm{H}$ & -0.30022400 & 0.33910800 & -2.53336800 & $\mathrm{C}$ & -2.50818300 & -2.07033400 & -0.89446100 \\
\hline $\mathrm{C}$ & -2.45753900 & 0.55226400 & -2.25381200 & $\mathrm{C}$ & -2.82124400 & -2.67626200 & -2.11702300 \\
\hline $\mathrm{H}$ & -2.80208600 & -0.15192500 & -3.01444700 & $\mathrm{C}$ & -2.14104100 & -4.15305100 & 0.07417400 \\
\hline $\mathrm{H}$ & -3.18248600 & 1.37276900 & -2.25919900 & $\mathrm{C}$ & -2.78438500 & -4.06768000 & -2.22429800 \\
\hline
\end{tabular}




\begin{tabular}{|c|c|c|c|c|c|c|c|}
\hline H & -3.10115900 & -2.08451400 & -2.97941900 & $\mathrm{H}$ & 2.09226700 & -0.65941800 & -2.91944900 \\
\hline $\mathrm{C}$ & -2.44006500 & -4.82604600 & -1.10874200 & $\mathrm{P}$ & 1.54057600 & -1.59958200 & -0.75490100 \\
\hline H & -1.86110100 & -4.69870800 & 0.97159500 & $\mathrm{P}$ & 0.68848000 & 2.04625400 & -0.75360700 \\
\hline $\mathrm{H}$ & -3.02696400 & -4.54790500 & -3.16743100 & $\mathrm{C}$ & -0.50749300 & 3.25701700 & -1.60265900 \\
\hline $\mathrm{H}$ & -2.39987800 & -5.90902800 & -1.14807500 & $\mathrm{C}$ & 1.10692200 & -3.23926900 & -1.62859000 \\
\hline $\mathrm{N}$ & 2.15466400 & 2.24654300 & -2.30950300 & $\mathrm{C}$ & 0.22076100 & 4.49205500 & -2.16347700 \\
\hline $\mathrm{N}$ & -2.16981000 & -2.81754400 & 0.18052400 & $\mathrm{H}$ & -0.51697600 & 5.18207100 & -2.58937000 \\
\hline $\mathrm{Pd}$ & -0.43938300 & 0.29164300 & 0.86420200 & $\mathrm{H}$ & 0.77916600 & 5.02592600 & -1.39109000 \\
\hline $\mathrm{C}$ & 2.39850800 & -1.34031400 & 0.66757100 & $\mathrm{H}$ & 0.91920800 & 4.22715600 & -2.96252800 \\
\hline $\mathrm{H}$ & 1.65229300 & -1.15906200 & -0.10641500 & $\mathrm{C}$ & -1.55723900 & 3.68984200 & -0.56074600 \\
\hline $\mathrm{C}$ & 2.27322700 & -0.64039600 & 1.81603400 & $\mathrm{H}$ & -2.30661400 & 4.32779700 & -1.04243600 \\
\hline $\mathrm{C}$ & 1.18386600 & 0.31010700 & 2.08449500 & $\mathrm{H}$ & -2.08452900 & 2.83055600 & -0.12980600 \\
\hline $\mathrm{O}$ & 1.10523000 & 0.95073600 & 3.11173000 & $\mathrm{H}$ & -1.11181000 & 4.26389700 & 0.25684700 \\
\hline $\mathrm{O}$ & -0.89313500 & -1.49223600 & 2.14065800 & $\mathrm{C}$ & -1.20670800 & 2.49095200 & -2.74389300 \\
\hline $\mathrm{H}$ & -1.46417700 & -2.06986400 & 1.56009100 & $\mathrm{H}$ & -1.75072700 & 1.61639200 & -2.37017200 \\
\hline C & -1.35938900 & -1.43268500 & 3.49461000 & $\mathrm{H}$ & -1.93070700 & 3.15100600 & -3.23468100 \\
\hline $\mathrm{H}$ & -0.73963400 & -0.70833000 & 4.02351300 & $\mathrm{H}$ & -0.50386100 & 2.15334800 & -3.51239200 \\
\hline $\mathrm{H}$ & -2.40757800 & -1.11931100 & 3.55296700 & $\mathrm{C}$ & 0.02557000 & -2.94621700 & -2.68640700 \\
\hline $\mathrm{H}$ & -1.24830700 & -2.41319600 & 3.96942100 & $\mathrm{H}$ & -0.89291200 & -2.56351600 & -2.23070400 \\
\hline $\mathrm{C}$ & 3.42722600 & -2.31715500 & 0.32491100 & $\mathrm{H}$ & 0.36246600 & -2.22669600 & -3.43984900 \\
\hline $\mathrm{C}$ & 3.41992300 & -2.87505700 & -0.96815900 & $\mathrm{H}$ & -0.22450200 & -3.87437300 & -3.21211900 \\
\hline $\mathrm{C}$ & 4.42723400 & -2.73174100 & 1.22839800 & $\mathrm{C}$ & 2.36219700 & -3.82945200 & -2.30599800 \\
\hline $\mathrm{C}$ & 4.37872900 & -3.80950600 & -1.35155800 & $\mathrm{H}$ & 3.18865800 & -3.95912700 & -1.60236700 \\
\hline $\mathrm{H}$ & 2.65389900 & -2.56533100 & -1.67476800 & $\mathrm{H}$ & 2.10895100 & -4.81539900 & -2.71138500 \\
\hline $\mathrm{C}$ & 5.38286100 & -3.66568200 & 0.84534200 & $\mathrm{H}$ & 2.71310200 & -3.21582500 & -3.13978900 \\
\hline $\mathrm{H}$ & 4.45177000 & -2.32757700 & 2.23539400 & $\mathrm{C}$ & 0.56927000 & -4.24524600 & -0.59424200 \\
\hline $\mathrm{C}$ & 5.36318600 & -4.20712400 & -0.44511700 & $\mathrm{H}$ & 0.28388900 & -5.16577300 & -1.11505200 \\
\hline $\mathrm{H}$ & 4.35896800 & -4.22728700 & -2.35344700 & $\mathrm{H}$ & 1.32481300 & -4.50815800 & 0.15098200 \\
\hline $\mathrm{H}$ & 6.14534700 & -3.97713200 & 1.55261700 & $\mathrm{H}$ & -0.31373700 & -3.87623100 & -0.06811800 \\
\hline $\mathrm{H}$ & 6.11150200 & -4.93681800 & -0.73904400 & $\mathrm{C}$ & 1.42329100 & 2.98537100 & 0.66912000 \\
\hline \multirow[t]{2}{*}{$\mathrm{H}$} & 2.97897700 & -0.74189800 & 2.63824200 & $\mathrm{C}$ & 0.97666600 & 2.75482600 & 1.97626200 \\
\hline & & & & $\mathrm{C}$ & 2.92562900 & 4.57193400 & 1.38714500 \\
\hline 20c & & & & $\mathrm{C}$ & 1.54419000 & 3.48870600 & 3.01939200 \\
\hline $\mathrm{C}$ & 2.14051300 & 1.88494400 & -1.89840400 & $\mathrm{H}$ & 0.20659100 & 2.01411400 & 2.17067600 \\
\hline $\mathrm{H}$ & 1.75639100 & 1.77323900 & -2.91816200 & $\mathrm{C}$ & 2.53926300 & 4.41669100 & 2.72087100 \\
\hline $\mathrm{H}$ & 2.68308600 & 2.83302300 & -1.84359400 & $\mathrm{H}$ & 3.70257400 & 5.28300200 & 1.11530600 \\
\hline $\mathrm{C}$ & 3.09424400 & 0.72500500 & -1.56132200 & $\mathrm{H}$ & 1.21819500 & 3.33357600 & 4.04344800 \\
\hline $\mathrm{H}$ & 4.02522500 & 0.90792300 & -2.11010900 & $\mathrm{H}$ & 3.01231500 & 5.00675800 & 3.49881100 \\
\hline $\mathrm{H}$ & 3.37646600 & 0.75197800 & -0.50293000 & $\mathrm{C}$ & 2.72625700 & -2.03351900 & 0.60436200 \\
\hline $\mathrm{C}$ & 2.61992100 & -0.68754400 & -1.95997700 & $\mathrm{C}$ & 2.27828100 & -2.28796200 & 1.90643800 \\
\hline $\mathrm{H}$ & 3.49792500 & -1.32727800 & -2.07455600 & $\mathrm{C}$ & 4.91529100 & -2.42201400 & 1.18307800 \\
\hline
\end{tabular}




\begin{tabular}{|c|c|c|c|c|c|c|c|}
\hline $\mathrm{C}$ & 3.22705800 & -2.62828700 & 2.87283100 & $\mathrm{C}$ & -5.59711900 & -0.62821100 & -1.24236900 \\
\hline $\mathrm{H}$ & 1.22307100 & -2.23125800 & 2.15061600 & $\mathrm{H}$ & -6.23481700 & -1.06764700 & -2.01780900 \\
\hline $\mathrm{C}$ & 4.57038900 & -2.69542300 & 2.50859000 & $\mathrm{H}$ & -5.85858500 & -1.10991800 & -0.29574200 \\
\hline $\mathrm{H}$ & 5.95264900 & -2.46329000 & 0.85899300 & $\mathrm{H}$ & -5.85268100 & 0.43399300 & -1.17586700 \\
\hline $\mathrm{H}$ & 2.91781400 & -2.83675200 & 3.89261300 & $\mathrm{C}$ & -3.82595000 & -2.36354200 & -1.72195400 \\
\hline $\mathrm{H}$ & 5.33862700 & -2.95358900 & 3.22998100 & $\mathrm{H}$ & -4.44875600 & -2.79363800 & -2.51430700 \\
\hline $\mathrm{N}$ & 2.38464500 & 3.87703800 & 0.38002900 & $\mathrm{H}$ & -2.77913600 & -2.55969100 & -1.97327200 \\
\hline $\mathrm{N}$ & 4.01727900 & -2.09726400 & 0.24529300 & $\mathrm{H}$ & -4.05649600 & -2.89345200 & -0.79353400 \\
\hline $\mathrm{Pd}$ & -0.18814700 & -0.17783000 & -0.07915100 & $\mathrm{C}$ & -3.80174100 & -0.17042400 & -2.95934200 \\
\hline $\mathrm{C}$ & -5.08011900 & -0.84370000 & 0.82295000 & $\mathrm{H}$ & -2.75853300 & -0.32329100 & -3.25335400 \\
\hline $\mathrm{C}$ & -6.04008400 & -0.91832500 & 1.85399600 & $\mathrm{H}$ & -4.43540400 & -0.60281300 & -3.74156300 \\
\hline $\mathrm{C}$ & -5.50550800 & -0.47725600 & -0.47275700 & $\mathrm{H}$ & -4.00242800 & 0.90602900 & -2.94085500 \\
\hline $\mathrm{C}$ & -7.37958000 & -0.63835100 & 1.60194500 & $\mathrm{C}$ & 0.28299500 & 2.14012100 & -3.07596200 \\
\hline $\mathrm{H}$ & -5.72392900 & -1.20135700 & 2.85429000 & $\mathrm{H}$ & 0.22192400 & 1.05627500 & -3.21553800 \\
\hline $\mathrm{C}$ & -6.84256700 & -0.19842700 & -0.72121300 & $\mathrm{H}$ & -0.73379000 & 2.54588200 & -3.09105900 \\
\hline $\mathrm{H}$ & -4.78834300 & -0.41214200 & -1.28514400 & $\mathrm{H}$ & 0.81303300 & 2.56379000 & -3.93621800 \\
\hline $\mathrm{C}$ & -7.78277800 & -0.27827200 & 0.31438200 & $\mathrm{C}$ & 1.14839900 & 4.03922400 & -1.65076000 \\
\hline $\mathrm{H}$ & -8.10729100 & -0.70143300 & 2.40453300 & $\mathrm{H}$ & 1.62570600 & 4.33588900 & -0.71501400 \\
\hline $\mathrm{H}$ & -7.16022100 & 0.08106100 & -1.72076800 & $\mathrm{H}$ & 1.75150400 & 4.41839600 & -2.48367300 \\
\hline $\mathrm{H}$ & -8.82716000 & -0.05955000 & 0.11407900 & $\mathrm{H}$ & 0.17385100 & 4.53103300 & -1.70993000 \\
\hline $\mathrm{C}$ & -3.70156700 & -1.15646800 & 1.14912300 & $\mathrm{C}$ & 2.45747600 & 1.90183700 & -1.84151100 \\
\hline $\mathrm{H}$ & -3.51083100 & -1.41569600 & 2.18995700 & $\mathrm{H}$ & 2.99668900 & 2.33615500 & -2.69045400 \\
\hline $\mathrm{C}$ & -2.62677500 & -1.18849400 & 0.31192000 & $\mathrm{H}$ & 3.03478100 & 2.12603500 & -0.93901300 \\
\hline $\mathrm{C}$ & -1.29793200 & -1.54211700 & 0.84668900 & $\mathrm{H}$ & 2.43096200 & 0.81975400 & -1.98849800 \\
\hline $\mathrm{O}$ & -1.02984900 & -2.16497800 & 1.84202800 & $\mathrm{C}$ & -3.25503200 & -1.04208700 & 1.26302700 \\
\hline \multirow[t]{2}{*}{$\mathrm{H}$} & -2.70922000 & -0.96047200 & -0.74593400 & $\mathrm{C}$ & -4.35459300 & -0.83822400 & 2.10599100 \\
\hline & & & & $\mathrm{C}$ & -2.42619200 & -2.69843800 & 2.63404200 \\
\hline 20-O & & & & $\mathrm{C}$ & -4.46957000 & -1.61554400 & 3.25995700 \\
\hline $\mathrm{C}$ & -3.53483100 & 1.59849300 & 0.02330000 & $\mathrm{H}$ & -5.10984900 & -0.09514300 & 1.87485800 \\
\hline $\mathrm{H}$ & -3.76032000 & 2.06744300 & -0.94097300 & $\mathrm{C}$ & -3.48936800 & -2.56738700 & 3.53040400 \\
\hline $\mathrm{H}$ & -4.47778000 & 1.56094900 & 0.57662000 & $\mathrm{H}$ & -1.63969500 & -3.42856100 & 2.80992300 \\
\hline $\mathrm{C}$ & -2.50813900 & 2.45688900 & 0.78864700 & $\mathrm{H}$ & -5.31196200 & -1.47917700 & 3.93135900 \\
\hline $\mathrm{H}$ & -3.02675400 & 3.35414400 & 1.14536200 & $\mathrm{H}$ & -3.54095200 & -3.19425300 & 4.41443700 \\
\hline $\mathrm{H}$ & -2.16550300 & 1.93525400 & 1.69078100 & $\mathrm{C}$ & 1.07387600 & 1.96848200 & 1.21836000 \\
\hline $\mathrm{C}$ & -1.31399800 & 2.92786500 & -0.06016200 & $\mathrm{C}$ & 1.44080500 & 0.88175000 & 2.02001900 \\
\hline $\mathrm{H}$ & -0.84120800 & 3.79417500 & 0.41235800 & $\mathrm{C}$ & 2.06608400 & 3.46371500 & 2.65749800 \\
\hline $\mathrm{H}$ & -1.66862000 & 3.25020800 & -1.04494100 & $\mathrm{C}$ & 2.15917500 & 1.12892000 & 3.19314900 \\
\hline $\mathrm{P}$ & 0.08134400 & 1.72450900 & -0.33362500 & $\mathrm{H}$ & 1.15613700 & -0.12577800 & 1.73554000 \\
\hline $\mathrm{P}$ & -2.93985700 & -0.12548000 & -0.31579700 & $\mathrm{C}$ & 2.48385500 & 2.44344700 & 3.51731600 \\
\hline $\mathrm{C}$ & -4.12065700 & -0.85401400 & -1.61392900 & $\mathrm{H}$ & 2.29238900 & 4.50381600 & 2.88115700 \\
\hline $\mathrm{C}$ & 1.04401600 & 2.50560500 & -1.78464200 & $\mathrm{H}$ & 2.45234800 & 0.30837800 & 3.84123100 \\
\hline
\end{tabular}




\begin{tabular}{|c|c|c|c|c|c|c|c|}
\hline $\mathrm{H}$ & 3.03897900 & 2.68251800 & 4.41838000 & $\mathrm{H}$ & 0.15834800 & -5.22495400 & 0.66501900 \\
\hline $\mathrm{N}$ & -2.30053900 & -1.95399900 & 1.52955200 & $\mathrm{H}$ & -0.35442500 & -3.81819400 & -0.26103300 \\
\hline $\mathrm{N}$ & 1.37190300 & 3.23943800 & 1.53706500 & $\mathrm{H}$ & 1.30961500 & -4.40789000 & -0.39946700 \\
\hline $\mathrm{Pd}$ & -0.56893200 & -0.47182400 & -0.68401300 & $\mathrm{C}$ & -0.35371800 & -3.12040800 & 2.37903200 \\
\hline $\mathrm{C}$ & 3.29097300 & -1.31662000 & -0.42918100 & $\mathrm{H}$ & -1.14325000 & -2.60077100 & 1.82592100 \\
\hline $\mathrm{H}$ & 2.95817700 & -0.35954300 & -0.03538700 & $\mathrm{H}$ & -0.75945000 & -4.08137500 & 2.71414100 \\
\hline $\mathrm{C}$ & 2.34490900 & -2.11826300 & -0.98092900 & $\mathrm{H}$ & -0.12437400 & -2.53810300 & 3.27679600 \\
\hline $\mathrm{C}$ & 0.95179600 & -1.75676500 & -1.01103300 & $\mathrm{C}$ & -2.04573100 & 2.21640000 & 2.04155500 \\
\hline $\mathrm{O}$ & 0.02377800 & -2.51154500 & -1.29997200 & $\mathrm{H}$ & -2.51090700 & 1.53006000 & 1.33086600 \\
\hline $\mathrm{H}$ & 2.57555700 & -3.10496400 & -1.37566200 & $\mathrm{H}$ & -1.69221300 & 1.63951900 & 2.90311200 \\
\hline $\mathrm{C}$ & 4.70874700 & -1.59347100 & -0.27654300 & $\mathrm{H}$ & -2.82339600 & 2.89662100 & 2.40660800 \\
\hline $\mathrm{C}$ & 5.31878100 & -2.77615300 & -0.74723000 & $\mathrm{C}$ & -0.36302500 & 4.05538600 & 2.41237200 \\
\hline $\mathrm{C}$ & 5.51047000 & -0.63251500 & 0.37318400 & $\mathrm{H}$ & 0.51177900 & 4.58820400 & 2.03224700 \\
\hline $\mathrm{C}$ & 6.68088800 & -2.98273400 & -0.57140400 & $\mathrm{H}$ & -1.14507600 & 4.79390400 & 2.62126600 \\
\hline $\mathrm{H}$ & 4.72702600 & -3.53239800 & -1.25272900 & $\mathrm{H}$ & -0.09798900 & 3.58881500 & 3.36476500 \\
\hline $\mathrm{C}$ & 6.87470300 & -0.84249500 & 0.54935600 & $\mathrm{C}$ & -1.45302800 & 3.81522400 & 0.17190000 \\
\hline $\mathrm{H}$ & 5.05042600 & 0.28085100 & 0.74118000 & $\mathrm{H}$ & -2.25964800 & 4.47724800 & 0.50587000 \\
\hline $\mathrm{C}$ & 7.46202700 & -2.01829900 & 0.07710500 & $\mathrm{H}$ & -0.67910300 & 4.44109900 & -0.28084800 \\
\hline $\mathrm{H}$ & 7.14013400 & -3.89516900 & -0.93824700 & $\mathrm{H}$ & -1.86149600 & 3.16654600 & -0.60392700 \\
\hline $\mathrm{H}$ & 7.47951700 & -0.09402300 & 1.05134800 & $\mathrm{C}$ & 2.90127000 & -2.02613800 & -0.28303000 \\
\hline \multirow[t]{2}{*}{$\mathrm{H}$} & 8.52621100 & -2.18572000 & 0.21206000 & $\mathrm{C}$ & 4.24656700 & -2.14182200 & 0.08588400 \\
\hline & & & & $\mathrm{C}$ & 3.40494100 & -2.43456500 & -2.49659800 \\
\hline 20-CO & & & & $\mathrm{C}$ & 5.19358700 & -2.41155200 & -0.90468500 \\
\hline $\mathrm{C}$ & 2.29863300 & -0.93067300 & 2.38470700 & $\mathrm{H}$ & 4.56148700 & -2.02801600 & 1.11677200 \\
\hline $\mathrm{H}$ & 1.60926700 & -1.09604400 & 3.21991100 & $\mathrm{C}$ & 4.76870000 & -2.56284200 & -2.22175400 \\
\hline $\mathrm{H}$ & 3.20494500 & -1.49285000 & 2.62762200 & $\mathrm{H}$ & 3.03007400 & -2.54646900 & -3.51128300 \\
\hline $\mathrm{C}$ & 2.62235600 & 0.56773900 & 2.27827500 & $\mathrm{H}$ & 6.24413800 & -2.50327200 & -0.64613400 \\
\hline $\mathrm{H}$ & 3.29464200 & 0.81580700 & 3.10740000 & $\mathrm{H}$ & 5.47111000 & -2.77252500 & -3.02140800 \\
\hline $\mathrm{H}$ & 3.19171400 & 0.78156500 & 1.36651500 & $\mathrm{C}$ & 1.68228200 & 2.82350300 & -0.13277800 \\
\hline $\mathrm{C}$ & 1.40660800 & 1.50440500 & 2.38794000 & $\mathrm{C}$ & 1.77096700 & 2.74918700 & -1.52727500 \\
\hline $\mathrm{H}$ & 1.76330600 & 2.48956500 & 2.69284700 & $\mathrm{C}$ & 3.38476100 & 4.36591700 & -0.02033900 \\
\hline $\mathrm{H}$ & 0.71144000 & 1.14380500 & 3.15407800 & $\mathrm{C}$ & 2.72794000 & 3.53834500 & -2.17063200 \\
\hline $\mathrm{P}$ & 0.44433700 & 1.81862400 & 0.82867600 & $\mathrm{H}$ & 1.09619000 & 2.11755300 & -2.09317200 \\
\hline $\mathrm{P}$ & 1.50531900 & -1.66914000 & 0.88036100 & $\mathrm{C}$ & 3.55146400 & 4.36193300 & -1.40746400 \\
\hline $\mathrm{C}$ & 0.88574500 & -3.37120700 & 1.49595000 & $\mathrm{H}$ & 4.00712800 & 4.99518300 & 0.61199700 \\
\hline $\mathrm{C}$ & -0.91761200 & 3.04005000 & 1.39099500 & $\mathrm{H}$ & 2.82138300 & 3.50792000 & -3.25200900 \\
\hline $\mathrm{C}$ & 1.97859300 & -4.09547900 & 2.30589500 & $\mathrm{H}$ & 4.30687800 & 4.98996100 & -1.86821500 \\
\hline $\mathrm{H}$ & 1.60072600 & -5.07554200 & 2.61802400 & $\mathrm{~N}$ & 2.49122500 & -2.16482000 & -1.55812500 \\
\hline $\mathrm{H}$ & 2.88154600 & -4.26855900 & 1.71250400 & $\mathrm{~N}$ & 2.47534400 & 3.61393100 & 0.60915900 \\
\hline $\mathrm{H}$ & 2.25775700 & -3.55424500 & 3.21385000 & $\mathrm{Pd}$ & -0.11285600 & -0.21656700 & -0.40952100 \\
\hline $\mathrm{C}$ & 0.48236300 & -4.24580600 & 0.29542000 & $\mathrm{C}$ & -3.44604000 & -0.18277300 & -0.62096000 \\
\hline
\end{tabular}




\begin{tabular}{|c|c|c|c|c|c|c|c|}
\hline $\mathrm{H}$ & -2.77032500 & -0.72413100 & 0.04238300 & $\mathrm{C}$ & -1.99885200 & 3.20289000 & -1.73334900 \\
\hline $\mathrm{C}$ & -2.88340200 & 0.66250600 & -1.51496100 & $\mathrm{H}$ & -2.59147900 & 3.50625400 & -0.86605700 \\
\hline $\mathrm{C}$ & -1.43853200 & 0.86136400 & -1.66581200 & $\mathrm{H}$ & -2.26771300 & 3.86776900 & -2.56184600 \\
\hline $\mathrm{O}$ & -0.95671400 & 1.54775200 & -2.53910100 & $\mathrm{H}$ & -2.28177000 & 2.19104900 & -2.02571500 \\
\hline $\mathrm{H}$ & -3.47893900 & 1.24364900 & -2.21594900 & $\mathrm{C}$ & 3.93067100 & 0.88892300 & -2.16855900 \\
\hline $\mathrm{C}$ & -4.86178000 & -0.46819500 & -0.42765900 & $\mathrm{H}$ & 2.90119900 & 1.01514500 & -2.51705200 \\
\hline $\mathrm{C}$ & -5.87890100 & 0.12717500 & -1.20277200 & $\mathrm{H}$ & 4.22653000 & 1.79102300 & -1.62366900 \\
\hline $\mathrm{C}$ & -5.23118800 & -1.38148600 & 0.57942500 & $\mathrm{H}$ & 4.57534900 & 0.82305400 & -3.05208600 \\
\hline $\mathrm{C}$ & -7.21342600 & -0.18320300 & -0.97227400 & $\mathrm{C}$ & 5.54602500 & -0.52503900 & -0.83894400 \\
\hline $\mathrm{H}$ & -5.62426200 & 0.83119100 & -1.98839200 & $\mathrm{H}$ & 5.70902200 & -1.45064400 & -0.27955000 \\
\hline $\mathrm{C}$ & -6.56884400 & -1.69111800 & 0.80936700 & $\mathrm{H}$ & 6.21750300 & -0.54421800 & -1.70506200 \\
\hline $\mathrm{H}$ & -4.45700200 & -1.84798900 & 1.18364300 & $\mathrm{H}$ & 5.85512400 & 0.31629800 & -0.21073000 \\
\hline $\mathrm{C}$ & -7.56295900 & -1.09206700 & 0.03342200 & $\mathrm{C}$ & 3.72029900 & -1.60794300 & -2.19703000 \\
\hline $\mathrm{H}$ & -7.98665200 & 0.28078300 & -1.57652100 & $\mathrm{H}$ & 4.41170300 & -1.67099300 & -3.04476300 \\
\hline $\mathrm{H}$ & -6.83640100 & -2.39686100 & 1.58944800 & $\mathrm{H}$ & 3.78815900 & -2.55033000 & -1.64586300 \\
\hline $\mathrm{H}$ & -8.60737200 & -1.33134000 & 0.20844900 & $\mathrm{H}$ & 2.70816500 & -1.51319600 & -2.60097500 \\
\hline $\mathrm{C}$ & -0.49296600 & -1.63797800 & -1.74679600 & $\mathrm{C}$ & -1.08670200 & 2.51630400 & 1.34485600 \\
\hline \multirow[t]{2}{*}{$\mathrm{O}$} & -0.69508500 & -2.38584200 & -2.58172200 & $\mathrm{C}$ & -2.34157800 & 1.90404600 & 1.45900400 \\
\hline & & & & $\mathrm{C}$ & -1.43461000 & 3.74115100 & 3.26358200 \\
\hline 21 & & & & $\mathrm{C}$ & -3.16181200 & 2.25450600 & 2.53180500 \\
\hline $\mathrm{C}$ & 1.64346500 & 2.87696600 & 0.56006700 & $\mathrm{H}$ & -2.67341100 & 1.17618800 & 0.72795300 \\
\hline $\mathrm{H}$ & 2.31020800 & 3.02983000 & -0.29522200 & $\mathrm{C}$ & -2.70154700 & 3.18934200 & 3.45708200 \\
\hline $\mathrm{H}$ & 1.37553500 & 3.85619700 & 0.95809400 & $\mathrm{H}$ & -1.03584500 & 4.47364600 & 3.96191200 \\
\hline $\mathrm{C}$ & 2.34278000 & 2.06348400 & 1.67064100 & $\mathrm{H}$ & -4.14262500 & 1.80144300 & 2.64122500 \\
\hline $\mathrm{H}$ & 2.79806300 & 2.77374600 & 2.36941000 & $\mathrm{H}$ & -3.30459800 & 3.48687500 & 4.30851700 \\
\hline $\mathrm{H}$ & 1.61027900 & 1.50106600 & 2.26010200 & $\mathrm{C}$ & 3.21354100 & -1.81726900 & 1.11920300 \\
\hline $\mathrm{C}$ & 3.45843300 & 1.12731100 & 1.17522400 & $\mathrm{C}$ & 4.20876000 & -1.91689800 & 2.09909800 \\
\hline $\mathrm{H}$ & 4.00495400 & 0.73697200 & 2.03709800 & $\mathrm{C}$ & 2.62481000 & -4.04183000 & 1.45014100 \\
\hline $\mathrm{H}$ & 4.18118400 & 1.70244200 & 0.58798000 & $\mathrm{C}$ & 4.39624000 & -3.12783100 & 2.76707500 \\
\hline $\mathrm{P}$ & 2.84488700 & -0.28656400 & 0.11834600 & $\mathrm{H}$ & 4.84292300 & -1.07156100 & 2.33742300 \\
\hline $\mathrm{P}$ & 0.05981600 & 2.14898100 & -0.07983200 & $\mathrm{C}$ & 3.58984400 & -4.21492400 & 2.43992300 \\
\hline $\mathrm{C}$ & -0.48524200 & 3.33753000 & -1.47697200 & $\mathrm{H}$ & 1.97050800 & -4.85959200 & 1.15929700 \\
\hline $\mathrm{C}$ & 4.09280600 & -0.39290000 & -1.32720900 & $\mathrm{H}$ & 5.16533100 & -3.21760000 & 3.52819300 \\
\hline $\mathrm{C}$ & 0.29071300 & 2.94451500 & -2.74992400 & $\mathrm{H}$ & 3.70088800 & -5.17345400 & 2.93480000 \\
\hline $\mathrm{H}$ & 0.01799600 & 3.62937200 & -3.56102600 & $\mathrm{~N}$ & -0.64296600 & 3.41919500 & 2.23322200 \\
\hline $\mathrm{H}$ & 1.37454000 & 3.02341900 & -2.61286000 & $\mathrm{~N}$ & 2.43795000 & -2.87959700 & 0.80930700 \\
\hline $\mathrm{H}$ & 0.04443100 & 1.92819500 & -3.06820300 & $\mathrm{Pd}$ & 0.37396700 & -0.12982400 & -0.57889100 \\
\hline $\mathrm{C}$ & -0.17449100 & 4.79716200 & -1.08804700 & $\mathrm{C}$ & -3.71607900 & -1.20163100 & -1.11564000 \\
\hline $\mathrm{H}$ & -0.58212600 & 5.45828600 & -1.86091300 & $\mathrm{H}$ & -3.88685800 & -0.86021300 & -2.13593900 \\
\hline $\mathrm{H}$ & -0.63228300 & 5.07943400 & -0.13527700 & $\mathrm{C}$ & -2.48015900 & -0.97741800 & -0.60498900 \\
\hline $\mathrm{H}$ & 0.89874400 & 4.99425000 & -1.02723000 & $\mathrm{C}$ & -1.45169300 & -0.30760800 & -1.41760700 \\
\hline
\end{tabular}




\begin{tabular}{|c|c|c|c|c|c|c|c|}
\hline $\mathrm{O}$ & -1.55076000 & -0.03801600 & -2.59715300 & $\mathrm{H}$ & -1.47950700 & 4.81994300 & 0.04316900 \\
\hline $\mathrm{O}$ & 0.33356700 & -2.37030700 & -0.79463200 & $\mathrm{H}$ & 0.24493500 & 5.17116600 & -0.14886800 \\
\hline $\mathrm{H}$ & 1.11716600 & -2.67924500 & -0.25431400 & $\mathrm{C}$ & -1.69340500 & 3.01028600 & -2.09351500 \\
\hline $\mathrm{C}$ & 0.19420600 & -3.13684500 & -1.99748600 & $\mathrm{H}$ & -2.59013800 & 3.00364200 & -1.46681300 \\
\hline $\mathrm{H}$ & -0.62175100 & -2.70691100 & -2.57856400 & $\mathrm{H}$ & -1.86119700 & 3.73827700 & -2.89552500 \\
\hline $\mathrm{H}$ & 1.10843600 & -3.11701000 & -2.60045600 & $\mathrm{H}$ & -1.57918800 & 2.02491600 & -2.55541400 \\
\hline $\mathrm{H}$ & -0.05479000 & -4.17464700 & -1.75159200 & $\mathrm{C}$ & 4.90868500 & 1.07255500 & -1.26115900 \\
\hline $\mathrm{C}$ & -4.85360600 & -1.83667200 & -0.46270800 & $\mathrm{H}$ & 4.13391700 & 1.22270400 & -2.01926200 \\
\hline C & -6.06883500 & -1.92288800 & -1.16997200 & $\mathrm{H}$ & 4.93960400 & 1.95912400 & -0.62022500 \\
\hline C & -4.79469100 & -2.37121100 & 0.84132800 & $\mathrm{H}$ & 5.87429900 & 1.01427000 & -1.77561700 \\
\hline $\mathrm{C}$ & -7.18945200 & -2.51838900 & -0.59631500 & $\mathrm{C}$ & 5.79087700 & -0.39791400 & 0.59172700 \\
\hline $\mathrm{H}$ & -6.12640700 & -1.51738000 & -2.17653500 & $\mathrm{H}$ & 5.70110000 & -1.34074900 & 1.14006100 \\
\hline $\mathrm{C}$ & -5.91353400 & -2.96539100 & 1.41222300 & $\mathrm{H}$ & 6.76587100 & -0.41228200 & 0.09083500 \\
\hline $\mathrm{H}$ & -3.86838600 & -2.32582700 & 1.40583100 & $\mathrm{H}$ & 5.81196000 & 0.42470100 & 1.31248500 \\
\hline $\mathrm{C}$ & -7.11435400 & -3.04037900 & 0.69615900 & $\mathrm{C}$ & 4.69521200 & -1.42107100 & -1.43548600 \\
\hline $\mathrm{H}$ & -8.11803500 & -2.57634800 & -1.15543900 & $\mathrm{H}$ & 5.67036200 & -1.47524900 & -1.93195700 \\
\hline $\mathrm{H}$ & -5.85479400 & -3.37527700 & 2.41592300 & $\mathrm{H}$ & 4.53976700 & -2.37805000 & -0.92565700 \\
\hline $\mathrm{H}$ & -7.98569300 & -3.50651300 & 1.14600800 & $\mathrm{H}$ & 3.93553600 & -1.31202700 & -2.21533000 \\
\hline \multirow[t]{2}{*}{ H } & -2.19788600 & -1.27233900 & 0.39905800 & $\mathrm{C}$ & -1.56273900 & 2.18771700 & 1.18009300 \\
\hline & & & & $\mathrm{C}$ & -2.73942000 & 1.48098800 & 0.89965900 \\
\hline \multirow{2}{*}{\multicolumn{4}{|c|}{$\begin{array}{l}\text { TS13 } \\
\text { imaginary frequency }=-308.60 \mathrm{~cm}^{-1}\end{array}$}} & $\mathrm{C}$ & -2.46675200 & 3.04947100 & 3.11442200 \\
\hline & & & & $\mathrm{C}$ & -3.81761200 & 1.59362700 & 1.77741200 \\
\hline $\mathrm{C}$ & 1.22530900 & 2.82303200 & 1.10935600 & $\mathrm{H}$ & -2.80184300 & 0.84911000 & 0.02268900 \\
\hline $\mathrm{H}$ & 2.01034700 & 3.22049200 & 0.45763700 & $\mathrm{C}$ & -3.68203200 & 2.39458800 & 2.91028700 \\
\hline $\mathrm{H}$ & 0.77187700 & 3.65929600 & 1.64404700 & $\mathrm{H}$ & -2.31724500 & 3.67865500 & 3.98952100 \\
\hline $\mathrm{C}$ & 1.82525800 & 1.83717700 & 2.13936000 & $\mathrm{H}$ & -4.74003900 & 1.05545600 & 1.58106500 \\
\hline $\mathrm{H}$ & 2.07242100 & 2.40151400 & 3.04558300 & $\mathrm{H}$ & -4.49233500 & 2.50926900 & 3.62284500 \\
\hline $\mathrm{H}$ & 1.07650100 & 1.09839800 & 2.44850700 & $\mathrm{C}$ & 2.70911500 & -1.71438300 & 1.17414600 \\
\hline $\mathrm{C}$ & 3.12106400 & 1.13116800 & 1.69045300 & $\mathrm{C}$ & 3.31162200 & -2.10165000 & 2.37317000 \\
\hline $\mathrm{H}$ & 3.60021400 & 0.65032500 & 2.54947700 & $\mathrm{C}$ & 1.62723500 & -3.84038900 & 1.07549200 \\
\hline $\mathrm{H}$ & 3.83187700 & 1.88480200 & 1.33304500 & $\mathrm{C}$ & 3.07243400 & -3.36705100 & 2.91382200 \\
\hline$P$ & 2.91577100 & -0.07401300 & 0.27595100 & $\mathrm{H}$ & 3.97460100 & -1.41486100 & 2.88365700 \\
\hline$P$ & -0.10065500 & 2.09137100 & 0.02469800 & $\mathrm{C}$ & 2.22124000 & -4.25670800 & 2.25548900 \\
\hline C & -0.43902200 & 3.42301300 & -1.29752100 & $\mathrm{H}$ & 0.93994200 & -4.45115000 & 0.50084600 \\
\hline $\mathrm{C}$ & 4.68354700 & -0.22927800 & -0.46168300 & $\mathrm{H}$ & 3.55179800 & -3.65732800 & 3.84367800 \\
\hline $\mathrm{C}$ & 0.77373700 & 3.44695900 & -2.24994400 & $\mathrm{H}$ & 2.01529500 & -5.24546500 & 2.64785200 \\
\hline $\mathrm{H}$ & 0.58634300 & 4.16467800 & -3.05686600 & $\mathrm{~N}$ & -1.42875400 & 2.95548600 & 2.27390200 \\
\hline $\mathrm{H}$ & 1.69392400 & 3.76043800 & -1.74585400 & $\mathrm{~N}$ & 1.87662500 & -2.61310600 & 0.58037500 \\
\hline $\mathrm{H}$ & 0.94434000 & 2.46625100 & -2.70572000 & $\mathrm{Pd}$ & 0.69279400 & 0.05339200 & -0.81944800 \\
\hline $\mathrm{C}$ & -0.64868700 & 4.81195400 & -0.66766500 & $\mathrm{C}$ & -3.24127200 & -1.41296200 & -1.40328500 \\
\hline $\mathrm{H}$ & -0.88007400 & 5.53490800 & -1.45865200 & $\mathrm{H}$ & -3.42805700 & -1.04398100 & -2.41025700 \\
\hline
\end{tabular}




\begin{tabular}{|c|c|c|c|c|c|c|c|}
\hline $\mathrm{C}$ & -1.95357200 & -1.45225800 & -0.99983000 & $\mathrm{C}$ & -0.21417700 & -5.05793700 & -0.51682400 \\
\hline $\mathrm{C}$ & -0.89128600 & -0.95508800 & -1.88463300 & $\mathrm{H}$ & -0.06385700 & -6.01952800 & -0.01205800 \\
\hline $\mathrm{O}$ & -0.96489700 & -0.57794800 & -3.03289200 & $\mathrm{H}$ & -1.25855200 & -5.01193100 & -0.83810700 \\
\hline $\mathrm{O}$ & 0.42250900 & -2.07721500 & -1.58625700 & $\mathrm{H}$ & 0.41619900 & -5.05727900 & -1.41118500 \\
\hline $\mathrm{H}$ & 1.32592500 & -2.34489600 & -0.30283500 & $\mathrm{C}$ & -0.70944300 & -4.02642600 & 1.73335000 \\
\hline $\mathrm{C}$ & 1.03703800 & -2.50852300 & -2.80488400 & $\mathrm{H}$ & -1.77795300 & -4.08046400 & 1.50841500 \\
\hline $\mathrm{H}$ & 0.82903000 & -1.79489400 & -3.60686100 & $\mathrm{H}$ & -0.44200600 & -4.94938100 & 2.26071500 \\
\hline $\mathrm{H}$ & 2.12021700 & -2.59277600 & -2.66543800 & $\mathrm{H}$ & -0.54025100 & -3.18683500 & 2.41469600 \\
\hline $\mathrm{H}$ & 0.63075300 & -3.48668800 & -3.08715400 & $\mathrm{C}$ & 5.01136500 & -0.89836400 & -0.24086400 \\
\hline $\mathrm{C}$ & -4.42538200 & -1.81907000 & -0.64541700 & $\mathrm{H}$ & 4.53562900 & -1.39621000 & 0.60955900 \\
\hline $\mathrm{C}$ & -5.66866100 & -1.84466600 & -1.30519200 & $\mathrm{H}$ & 4.87704100 & -1.52718500 & -1.12655800 \\
\hline $\mathrm{C}$ & -4.38572800 & -2.18169300 & 0.71619900 & $\mathrm{H}$ & 6.08759900 & -0.84902700 & -0.04142000 \\
\hline $\mathrm{C}$ & -6.82911700 & -2.22882900 & -0.63639000 & $\mathrm{C}$ & 5.15428900 & 1.18539100 & -1.65614800 \\
\hline $\mathrm{H}$ & -5.71708900 & -1.56443500 & -2.35409100 & $\mathrm{H}$ & 4.84169900 & 2.22417300 & -1.79992500 \\
\hline $\mathrm{C}$ & -5.54541700 & -2.56186400 & 1.38422800 & $\mathrm{H}$ & 6.23834500 & 1.19920900 & -1.49345600 \\
\hline $\mathrm{H}$ & -3.44429300 & -2.15727300 & 1.25717400 & $\mathrm{H}$ & 4.97620100 & 0.63537600 & -2.58476600 \\
\hline $\mathrm{C}$ & -6.77162600 & -2.58923700 & 0.71078500 & $\mathrm{C}$ & 4.74219600 & 1.35918400 & 0.82492000 \\
\hline $\mathrm{H}$ & -7.77692800 & -2.24530800 & -1.16564400 & $\mathrm{H}$ & 5.82227800 & 1.40654400 & 1.00255700 \\
\hline $\mathrm{H}$ & -5.49747400 & -2.83727000 & 2.43377200 & $\mathrm{H}$ & 4.38401100 & 2.39041500 & 0.73588000 \\
\hline $\mathrm{H}$ & -7.67433900 & -2.88712100 & 1.23536200 & $\mathrm{H}$ & 4.28409400 & 0.90634700 & 1.71005700 \\
\hline \multirow[t]{2}{*}{$\mathrm{H}$} & -1.64814200 & -1.82167100 & -0.02810800 & $\mathrm{C}$ & -1.96931300 & -2.22685700 & -0.81079000 \\
\hline & & & & $\mathrm{C}$ & -2.95886800 & -2.00440400 & 0.15834800 \\
\hline \multicolumn{4}{|c|}{ TS13a } & $\mathrm{C}$ & -3.55353800 & -2.47633500 & -2.46317900 \\
\hline \multicolumn{4}{|c|}{ imaginary frequency $=-267.22 \mathrm{~cm}^{-1}$} & $\mathrm{C}$ & -4.29746900 & -2.04821500 & -0.22837000 \\
\hline $\mathrm{C}$ & 0.65517500 & -2.30118500 & -2.00696000 & $\mathrm{H}$ & -2.68546200 & -1.79526500 & 1.18582300 \\
\hline $\mathrm{H}$ & 1.57935000 & -2.87659200 & -1.89056800 & $\mathrm{C}$ & -4.60571500 & -2.28892000 & -1.56675300 \\
\hline $\mathrm{H}$ & -0.01699000 & -2.87021400 & -2.65303400 & $\mathrm{H}$ & -3.75013700 & -2.65858900 & -3.51771800 \\
\hline $\mathrm{C}$ & 0.95703200 & -0.94121900 & -2.67502000 & $\mathrm{H}$ & -5.08466100 & -1.88916400 & 0.50245500 \\
\hline $\mathrm{H}$ & 0.92998600 & -1.09103500 & -3.76067900 & $\mathrm{H}$ & -5.63357400 & -2.32615200 & -1.91227000 \\
\hline $\mathrm{H}$ & 0.15947800 & -0.22044100 & -2.45848400 & $\mathrm{C}$ & 1.99506400 & 2.11901700 & -0.81057600 \\
\hline $\mathrm{C}$ & 2.33939600 & -0.32943200 & -2.35584100 & $\mathrm{C}$ & 2.15307000 & 2.94179300 & -1.92769600 \\
\hline $\mathrm{H}$ & 2.58196200 & 0.43846800 & -3.09780700 & $\mathrm{C}$ & 0.87399000 & 3.91477400 & 0.30189700 \\
\hline $\mathrm{H}$ & 3.10596500 & -1.10493600 & -2.46914200 & $\mathrm{C}$ & 1.67604100 & 4.25482400 & -1.91909100 \\
\hline $\mathrm{P}$ & 2.56389000 & 0.33216100 & -0.61963700 & $\mathrm{H}$ & 2.65265100 & 2.55691100 & -2.80744200 \\
\hline $\mathrm{P}$ & -0.16772300 & -2.20610800 & -0.33732400 & $\mathrm{C}$ & 1.02944000 & 4.75555100 & -0.78756700 \\
\hline $\mathrm{C}$ & 0.14107800 & -3.91561500 & 0.45238300 & $\mathrm{H}$ & 0.36566400 & 4.19980000 & 1.21566300 \\
\hline $\mathrm{C}$ & 4.46835900 & 0.53246200 & -0.44498400 & $\mathrm{H}$ & 1.80973700 & 4.88399100 & -2.79372900 \\
\hline $\mathrm{C}$ & 1.63284300 & -3.98660600 & 0.83740600 & $\mathrm{H}$ & 0.64444300 & 5.76759700 & -0.75094300 \\
\hline $\mathrm{H}$ & 1.83643800 & -4.94977000 & 1.31909300 & $\mathrm{~N}$ & -2.26437900 & -2.45076200 & -2.10130200 \\
\hline $\mathrm{H}$ & 2.29581500 & -3.91221100 & -0.03101900 & $\mathrm{~N}$ & 1.34871500 & 2.65499300 & 0.26162500 \\
\hline $\mathrm{H}$ & 1.89911000 & -3.19317800 & 1.54335300 & $\mathrm{Pd}$ & 0.76228000 & -0.44843700 & 0.87689800 \\
\hline
\end{tabular}




\begin{tabular}{|c|c|c|c|c|c|c|c|}
\hline $\mathrm{C}$ & -2.09896100 & 1.30908600 & 0.73679500 & $\mathrm{H}$ & 1.32378200 & 3.39296200 & -2.57046100 \\
\hline $\mathrm{H}$ & -1.41096700 & 0.98531200 & -0.04425500 & $\mathrm{C}$ & -0.57126300 & 5.35745500 & -0.39592500 \\
\hline $\mathrm{C}$ & -1.83100500 & 0.91961000 & 1.99321500 & $\mathrm{H}$ & -0.68932200 & 6.21621200 & -1.06780100 \\
\hline $\mathrm{C}$ & -0.65412400 & 0.12500300 & 2.45111600 & $\mathrm{H}$ & -1.49334300 & 5.25121500 & 0.18065600 \\
\hline $\mathrm{O}$ & -0.66701700 & -0.65183000 & 3.38544200 & $\mathrm{H}$ & 0.23548500 & 5.59886300 & 0.30250600 \\
\hline $\mathrm{O}$ & 0.61922100 & 1.20085700 & 2.43968100 & $\mathrm{C}$ & -1.37972200 & 3.86120900 & -2.25849600 \\
\hline $\mathrm{H}$ & 1.13179800 & 2.05161400 & 1.10601000 & $\mathrm{H}$ & -2.35905800 & 3.75656200 & -1.78233800 \\
\hline $\mathrm{C}$ & 1.48615700 & 1.08120700 & 3.57853500 & $\mathrm{H}$ & -1.43373300 & 4.71601800 & -2.94227600 \\
\hline $\mathrm{H}$ & 1.31957800 & 0.11928100 & 4.06946500 & $\mathrm{H}$ & -1.19209800 & 2.96501400 & -2.85996600 \\
\hline $\mathrm{H}$ & 2.52868100 & 1.15630500 & 3.25533100 & $\mathrm{C}$ & 4.90506500 & 0.79392800 & -1.15330900 \\
\hline $\mathrm{H}$ & 1.25883200 & 1.88882500 & 4.28273200 & $\mathrm{H}$ & 4.20504800 & 0.85832900 & -1.99180200 \\
\hline $\mathrm{C}$ & -3.25020300 & 2.09742800 & 0.27979000 & $\mathrm{H}$ & 4.91277400 & 1.76074600 & -0.64073800 \\
\hline $\mathrm{C}$ & -3.47514100 & 2.22376800 & -1.10314900 & $\mathrm{H}$ & 5.91006500 & 0.63511700 & -1.56068100 \\
\hline $\mathrm{C}$ & -4.14118800 & 2.74107900 & 1.16054200 & $\mathrm{C}$ & 5.55295400 & -0.42779100 & 0.95928600 \\
\hline $\mathrm{C}$ & -4.55630700 & 2.95448600 & -1.59313600 & $\mathrm{H}$ & 5.33769200 & -1.24701200 & 1.65322200 \\
\hline $\mathrm{H}$ & -2.79981700 & 1.72908700 & -1.79733500 & $\mathrm{H}$ & 6.56052500 & -0.60021900 & 0.56305200 \\
\hline $\mathrm{C}$ & -5.22050400 & 3.47061500 & 0.67162000 & $\mathrm{H}$ & 5.58840600 & 0.50654300 & 1.52617600 \\
\hline $\mathrm{H}$ & -3.98489300 & 2.67831200 & 2.23320300 & $\mathrm{C}$ & 4.59723700 & -1.69020300 & -0.99878100 \\
\hline $\mathrm{C}$ & -5.43383500 & 3.58016100 & -0.70644600 & $\mathrm{H}$ & 5.59830400 & -1.82542600 & -1.42302700 \\
\hline $\mathrm{H}$ & -4.71555700 & 3.03308200 & -2.66451300 & $\mathrm{H}$ & 4.39689900 & -2.56237200 & -0.36623600 \\
\hline $\mathrm{H}$ & -5.89736500 & 3.95957700 & 1.36587200 & $\mathrm{H}$ & 3.88366800 & -1.68602700 & -1.82869300 \\
\hline $\mathrm{H}$ & -6.27699200 & 4.15101000 & -1.08330400 & $\mathrm{C}$ & -1.77650400 & 2.37821100 & 0.66148200 \\
\hline $\mathrm{H}$ & -2.51315900 & 1.13608500 & 2.81358800 & $\mathrm{C}$ & -2.72634800 & 1.48506000 & 0.14747000 \\
\hline & & & & $\mathrm{C}$ & -3.22918500 & 3.06863000 & 2.30918700 \\
\hline 22 & & & & $\mathrm{C}$ & -3.98238600 & 1.41229500 & 0.75055300 \\
\hline $\mathrm{C}$ & 0.95264100 & 3.05977000 & 1.28964800 & $\mathrm{H}$ & -2.47694000 & 0.85952800 & -0.70363800 \\
\hline $\mathrm{H}$ & 1.74669500 & 3.70601300 & 0.89988000 & $\mathrm{C}$ & -4.24210300 & 2.22216200 & 1.85478700 \\
\hline $\mathrm{H}$ & 0.31303700 & 3.67271000 & 1.93160500 & $\mathrm{H}$ & -3.39079300 & 3.70985000 & 3.17350800 \\
\hline $\mathrm{C}$ & 1.57635000 & 1.92999800 & 2.14104300 & $\mathrm{H}$ & -4.73768200 & 0.73177600 & 0.36971400 \\
\hline $\mathrm{H}$ & 1.81018500 & 2.36629800 & 3.11996900 & $\mathrm{H}$ & -5.20269800 & 2.19904400 & 2.35920800 \\
\hline $\mathrm{H}$ & 0.83186700 & 1.14849800 & 2.33731400 & $\mathrm{C}$ & 2.41623800 & -1.54917200 & 1.43758300 \\
\hline $\mathrm{C}$ & 2.89508100 & 1.30551600 & 1.61820600 & $\mathrm{C}$ & 2.76226600 & -1.74786500 & 2.77836000 \\
\hline $\mathrm{H}$ & 3.50114400 & 0.96115900 & 2.46297100 & $\mathrm{C}$ & 1.40069500 & -3.72554700 & 1.42787800 \\
\hline $\mathrm{H}$ & 3.49224000 & 2.08146600 & 1.12604700 & $\mathrm{C}$ & 2.43492300 & -2.93710000 & 3.42962000 \\
\hline $\mathrm{P}$ & 2.72851000 & -0.05640900 & 0.33676600 & $\mathrm{H}$ & 3.29995800 & -0.97250100 & 3.30914300 \\
\hline $\mathrm{P}$ & -0.10883700 & 2.52401400 & -0.15924600 & $\mathrm{C}$ & 1.75039800 & -3.94932300 & 2.74631700 \\
\hline $\mathrm{C}$ & -0.25959100 & 4.10031100 & -1.22746200 & $\mathrm{H}$ & 0.85858000 & -4.42891200 & 0.80716700 \\
\hline $\mathrm{C}$ & 4.55089200 & -0.37182800 & -0.20578200 & $\mathrm{H}$ & 2.72059300 & -3.08073200 & 4.46727600 \\
\hline $\mathrm{C}$ & 1.07883700 & 4.27629500 & -1.97174200 & $\mathrm{H}$ & 1.48680700 & -4.88390600 & 3.22661300 \\
\hline $\mathrm{H}$ & 1.01092000 & 5.13651700 & -2.64783800 & $\mathrm{~N}$ & -2.02468000 & 3.15209900 & 1.73180900 \\
\hline $\mathrm{H}$ & 1.91249500 & 4.46785400 & -1.28797800 & $\mathrm{~N}$ & 1.72786500 & -2.55721000 & 0.83726800 \\
\hline
\end{tabular}




\begin{tabular}{|c|c|c|c|c|c|c|c|}
\hline $\mathrm{Pd}$ & 0.97414800 & 0.58525900 & -0.91501100 & $\mathrm{H}$ & -3.45606700 & 3.16054700 & -3.25092300 \\
\hline $\mathrm{C}$ & -2.98642300 & -2.49987500 & -1.40011100 & $\mathrm{H}$ & -3.02743100 & 3.54895200 & -1.57738400 \\
\hline $\mathrm{H}$ & -3.20396400 & -2.42297600 & -2.46446200 & $\mathrm{H}$ & -1.83728300 & 3.74643900 & -2.87559300 \\
\hline C & -1.70267100 & -2.76693400 & -1.07848100 & $\mathrm{C}$ & -3.45709600 & 0.79165100 & -2.00542700 \\
\hline $\mathrm{C}$ & -0.71978600 & -2.96692700 & -2.15114600 & $\mathrm{H}$ & -4.14237300 & 0.80786300 & -2.86034600 \\
\hline $\mathrm{O}$ & -0.91504900 & -2.87935500 & -3.34255500 & $\mathrm{H}$ & -3.19913600 & -0.25033100 & -1.80320800 \\
\hline $\mathrm{O}$ & 0.52531200 & -3.31583700 & -1.64950600 & $\mathrm{H}$ & -3.99986800 & 1.17812900 & -1.13847600 \\
\hline $\mathrm{H}$ & 1.39758400 & -2.42277600 & -0.13208300 & $\mathrm{C}$ & -1.55834400 & 1.13973700 & -3.63015000 \\
\hline C & 1.49960900 & -3.63316600 & -2.67166500 & $\mathrm{H}$ & -1.27334400 & 0.08545900 & -3.55825200 \\
\hline $\mathrm{H}$ & 1.69900100 & -2.75886300 & -3.29411000 & $\mathrm{H}$ & -2.26430600 & 1.24132800 & -4.46208600 \\
\hline $\mathrm{H}$ & 2.39956300 & -3.94245200 & -2.13979700 & $\mathrm{H}$ & -0.66393900 & 1.71464200 & -3.89195500 \\
\hline $\mathrm{H}$ & 1.13072800 & -4.44344500 & -3.30242700 & $\mathrm{C}$ & 4.28255500 & -0.17347300 & -2.14983000 \\
\hline C & -4.12221900 & -2.31362000 & -0.50257000 & $\mathrm{H}$ & 3.47949500 & -0.64276100 & -2.72341500 \\
\hline $\mathrm{C}$ & -5.37335500 & -1.99673000 & -1.06631000 & $\mathrm{H}$ & 4.34448900 & 0.88068800 & -2.43669800 \\
\hline $\mathrm{C}$ & -4.03201200 & -2.44420600 & 0.89809600 & $\mathrm{H}$ & 5.22439700 & -0.64402700 & -2.45353100 \\
\hline C & -6.49602100 & -1.81178700 & -0.26113200 & $\mathrm{C}$ & 5.23741300 & 0.36149900 & 0.12228300 \\
\hline $\mathrm{H}$ & -5.45915900 & -1.90002600 & -2.14523000 & $\mathrm{H}$ & 5.13982900 & 0.27049600 & 1.20778300 \\
\hline C & -5.15250100 & -2.26307000 & 1.70065900 & $\mathrm{H}$ & 6.19075700 & -0.09912900 & -0.16024500 \\
\hline $\mathrm{H}$ & -3.08188500 & -2.69477100 & 1.35978300 & $\mathrm{H}$ & 5.30767900 & 1.42234100 & -0.13424200 \\
\hline $\mathrm{C}$ & -6.38835300 & -1.94571500 & 1.12457500 & $\mathrm{C}$ & 4.12357000 & -1.85201100 & -0.26993300 \\
\hline $\mathrm{H}$ & -7.45297200 & -1.57194300 & -0.71404000 & $\mathrm{H}$ & 5.09853900 & -2.26881800 & -0.54546200 \\
\hline $\mathrm{H}$ & -5.06907100 & -2.37213900 & 2.77773200 & $\mathrm{H}$ & 3.97492600 & -2.02242300 & 0.79942900 \\
\hline $\mathrm{H}$ & -7.26242000 & -1.81031600 & 1.75434100 & $\mathrm{H}$ & 3.36310000 & -2.41774000 & -0.81360500 \\
\hline \multirow[t]{2}{*}{$\mathrm{H}$} & -1.36569300 & -2.87004700 & -0.05262900 & $\mathrm{C}$ & -1.88166800 & 1.87592100 & 0.63320800 \\
\hline & & & & $\mathrm{C}$ & -2.36640700 & 0.87094100 & 1.47786900 \\
\hline \multirow{2}{*}{\multicolumn{3}{|c|}{$\begin{array}{l}\text { TS14 } \\
\text { imaginary frequency }=-69.15 \mathrm{~cm}^{-1}\end{array}$}} & & $\mathrm{C}$ & -2.75454900 & 3.52996000 & 1.97168900 \\
\hline & & & & $\mathrm{C}$ & -3.08626700 & 1.24524600 & 2.61404800 \\
\hline $\mathrm{C}$ & 0.14986600 & 2.93172700 & -1.10448500 & $\mathrm{H}$ & -2.18744000 & -0.17415100 & 1.25253300 \\
\hline $\mathrm{H}$ & 0.51981000 & 2.94569600 & -2.13587000 & $\mathrm{C}$ & -3.28573100 & 2.60010100 & 2.86896000 \\
\hline $\mathrm{H}$ & -0.49796200 & 3.79977600 & -0.96546400 & $\mathrm{H}$ & -2.88480700 & 4.59705500 & 2.13781200 \\
\hline C & 1.32258900 & 3.03607500 & -0.11404700 & $\mathrm{H}$ & -3.48037000 & 0.48831500 & 3.28545500 \\
\hline $\mathrm{H}$ & 1.64817800 & 4.08277900 & -0.11277900 & $\mathrm{H}$ & -3.83673700 & 2.93629600 & 3.74113300 \\
\hline $\mathrm{H}$ & 0.97502900 & 2.83734700 & 0.90601200 & $\mathrm{C}$ & 2.30109200 & 0.18237200 & 1.69216200 \\
\hline C & 2.56037700 & 2.18182800 & -0.44545400 & $\mathrm{C}$ & 2.78401000 & 1.13730400 & 2.59601500 \\
\hline $\mathrm{H}$ & 3.42120100 & 2.55415300 & 0.11830200 & $\mathrm{C}$ & 1.64571300 & -1.20192500 & 3.41408600 \\
\hline $\mathrm{H}$ & 2.81600500 & 2.30361200 & -1.50396800 & $\mathrm{C}$ & 2.67623300 & 0.88677500 & 3.96518200 \\
\hline$P$ & 2.38719200 & 0.35565300 & -0.15350400 & $\mathrm{H}$ & 3.24006800 & 2.05724300 & 2.24841600 \\
\hline$P$ & -0.95149400 & 1.44890200 & -0.91720800 & $\mathrm{C}$ & 2.09849900 & -0.30788400 & 4.38652000 \\
\hline $\mathrm{C}$ & -2.21975700 & 1.64670700 & -2.33343900 & $\mathrm{H}$ & 1.19081700 & -2.14644900 & 3.70433100 \\
\hline $\mathrm{C}$ & 4.09798500 & -0.35426300 & -0.62867900 & $\mathrm{H}$ & 3.04223800 & 1.61201200 & 4.68562100 \\
\hline $\mathrm{C}$ & -2.65139900 & 3.11636500 & -2.50800000 & $\mathrm{H}$ & 1.99758600 & -0.54637900 & 5.44004100 \\
\hline
\end{tabular}




\begin{tabular}{|c|c|c|c|c|c|c|c|}
\hline $\mathrm{N}$ & -2.06811800 & 3.18376200 & 0.87660300 & $\mathrm{H}$ & -3.17437200 & -0.47515800 & -1.82429000 \\
\hline $\mathrm{N}$ & 1.73116000 & -0.96944800 & 2.09864900 & $\mathrm{H}$ & -4.05805600 & 0.93774100 & -1.23388200 \\
\hline $\mathrm{Pd}$ & 0.28537300 & -0.63001600 & -0.87683400 & $\mathrm{C}$ & -1.55196500 & 0.90906900 & -3.66019000 \\
\hline $\mathrm{C}$ & -2.16831200 & -2.60533900 & -0.14100600 & $\mathrm{H}$ & -1.21624200 & -0.12389100 & -3.52632500 \\
\hline $\mathrm{C}$ & -3.53195300 & -2.74859700 & -0.45551200 & $\mathrm{H}$ & -2.23930600 & 0.93322100 & -4.51335200 \\
\hline $\mathrm{C}$ & -1.75482300 & -2.84019400 & 1.18534200 & $\mathrm{H}$ & -0.68116500 & 1.51665800 & -3.92786800 \\
\hline $\mathrm{C}$ & -4.45425900 & -3.11750200 & 0.52197600 & $\mathrm{C}$ & 3.97804200 & -0.15017800 & -2.34809000 \\
\hline $\mathrm{H}$ & -3.86557900 & -2.59030800 & -1.47739600 & $\mathrm{H}$ & 3.15499700 & -0.74658500 & -2.75248200 \\
\hline $\mathrm{C}$ & -2.67931200 & -3.21236600 & 2.15903100 & $\mathrm{H}$ & 3.88809300 & 0.86770600 & -2.74009000 \\
\hline $\mathrm{H}$ & -0.71092400 & -2.70576400 & 1.45646600 & $\mathrm{H}$ & 4.91268700 & -0.56508900 & -2.74170300 \\
\hline $\mathrm{C}$ & -4.03161500 & -3.35230400 & 1.83206400 & $\mathrm{C}$ & 5.19932200 & 0.65164600 & -0.28956800 \\
\hline $\mathrm{H}$ & -5.50114200 & -3.23137400 & 0.25818400 & $\mathrm{H}$ & 5.23580500 & 0.68243000 & 0.80355400 \\
\hline $\mathrm{H}$ & -2.34564600 & -3.39407300 & 3.17641300 & $\mathrm{H}$ & 6.13598000 & 0.19940500 & -0.63481900 \\
\hline $\mathrm{H}$ & -4.74884700 & -3.64599600 & 2.59217500 & $\mathrm{H}$ & 5.18049200 & 1.67803000 & -0.66654000 \\
\hline $\mathrm{C}$ & -1.21176000 & -2.24528900 & -1.20435900 & $\mathrm{C}$ & 4.17757300 & -1.64131000 & -0.32834100 \\
\hline $\mathrm{H}$ & -1.64741700 & -2.03053100 & -2.17666900 & $\mathrm{H}$ & 5.10592500 & -2.05261600 & -0.73981700 \\
\hline $\mathrm{C}$ & 0.10669200 & -2.81766800 & -1.19991000 & $\mathrm{H}$ & 4.23039800 & -1.71489500 & 0.76059800 \\
\hline $\mathrm{C}$ & 1.01721800 & -2.41928800 & -2.15446800 & $\mathrm{H}$ & 3.35601300 & -2.27813300 & -0.66791000 \\
\hline $\mathrm{O}$ & 1.74269400 & -2.41538900 & -3.07033600 & $\mathrm{C}$ & -2.03188800 & 1.84081000 & 0.55147900 \\
\hline \multirow[t]{2}{*}{$\mathrm{H}$} & 0.48763900 & -3.44220300 & -0.39599000 & $\mathrm{C}$ & -2.48615200 & 0.86544100 & 1.44565300 \\
\hline & & & & $\mathrm{C}$ & -3.02345000 & 3.52517600 & 1.76417600 \\
\hline 23 & & & & $\mathrm{C}$ & -3.25216400 & 1.27009200 & 2.54058200 \\
\hline $\mathrm{C}$ & -0.00354800 & 2.90072400 & -1.18166400 & $\mathrm{H}$ & -2.24839600 & -0.18004600 & 1.29013400 \\
\hline $\mathrm{H}$ & 0.37585100 & 2.89539700 & -2.20959800 & $\mathrm{C}$ & -3.52779600 & 2.62530000 & 2.70630700 \\
\hline $\mathrm{H}$ & -0.69621400 & 3.73903700 & -1.08081800 & $\mathrm{H}$ & -3.21308400 & 4.59200600 & 1.85992800 \\
\hline $\mathrm{C}$ & 1.15372300 & 3.09744100 & -0.18628300 & $\mathrm{H}$ & -3.62266100 & 0.53535000 & 3.24903400 \\
\hline $\mathrm{H}$ & 1.41347300 & 4.16221700 & -0.20044200 & $\mathrm{H}$ & -4.11670300 & 2.98456700 & 3.54382800 \\
\hline $\mathrm{H}$ & 0.81607500 & 2.89211900 & 0.83628700 & $\mathrm{C}$ & 2.43811400 & 0.33917700 & 1.67532200 \\
\hline $\mathrm{C}$ & 2.43877900 & 2.31109300 & -0.50351400 & $\mathrm{C}$ & 2.96358000 & 1.32122700 & 2.52421600 \\
\hline $\mathrm{H}$ & 3.28100600 & 2.74128600 & 0.04648400 & $\mathrm{C}$ & 1.98427100 & -1.04457800 & 3.46204400 \\
\hline $\mathrm{H}$ & 2.68229000 & 2.42360300 & -1.56613700 & $\mathrm{C}$ & 2.98357000 & 1.08535800 & 3.90030300 \\
\hline$P$ & 2.34324200 & 0.48726100 & -0.17169100 & $\mathrm{H}$ & 3.35298300 & 2.25292100 & 2.13020100 \\
\hline $\mathrm{P}$ & -1.03890600 & 1.37601900 & -0.94988100 & $\mathrm{C}$ & 2.48708100 & -0.12240300 & 4.38256100 \\
\hline $\mathrm{C}$ & -2.27333100 & 1.44341700 & -2.40737400 & $\mathrm{H}$ & 1.58986400 & -2.00017000 & 3.80073400 \\
\hline $\mathrm{C}$ & 4.01469100 & -0.18645400 & -0.80649700 & $\mathrm{H}$ & 3.38396000 & 1.83262900 & 4.57873400 \\
\hline $\mathrm{C}$ & -2.76904800 & 2.88070800 & -2.66437000 & $\mathrm{H}$ & 2.48508800 & -0.34988200 & 5.44332100 \\
\hline $\mathrm{H}$ & -3.54958900 & 2.85075900 & -3.43329200 & $\mathrm{~N}$ & -2.29228300 & 3.14926400 & 0.70869600 \\
\hline $\mathrm{H}$ & -3.19579300 & 3.33627800 & -1.76739500 & $\mathrm{~N}$ & 1.94701800 & -0.82677600 & 2.14211100 \\
\hline $\mathrm{H}$ & -1.97417100 & 3.53294100 & -3.03556400 & $\mathrm{Pd}$ & 0.25912700 & -0.60769200 & -0.76950800 \\
\hline $\mathrm{C}$ & -3.47713200 & 0.54445900 & -2.07263300 & $\mathrm{C}$ & -2.01892700 & -2.61498100 & 0.05795100 \\
\hline $\mathrm{H}$ & -4.14173700 & 0.49479300 & -2.94247900 & $\mathrm{C}$ & -3.36878300 & -2.85970800 & -0.25286000 \\
\hline
\end{tabular}




\begin{tabular}{|c|c|c|c|c|c|c|c|}
\hline $\mathrm{C}$ & -1.60902800 & -2.73352200 & 1.40118300 & $\mathrm{H}$ & -0.57924700 & 1.60917200 & 2.86540300 \\
\hline $\mathrm{C}$ & -4.28113400 & -3.20514500 & 0.74294000 & $\mathrm{C}$ & 0.22894400 & 2.88009400 & 0.43604500 \\
\hline $\mathrm{H}$ & -3.70052500 & -2.79590400 & -1.28562200 & $\mathrm{C}$ & -1.12941400 & 3.22212600 & 0.58252300 \\
\hline $\mathrm{C}$ & -2.52200000 & -3.08481100 & 2.39343700 & $\mathrm{C}$ & 0.99798000 & 3.59386700 & -0.50633200 \\
\hline $\mathrm{H}$ & -0.57687200 & -2.52620800 & 1.67224500 & $\mathrm{C}$ & -1.69432400 & 4.23544800 & -0.18766900 \\
\hline $\mathrm{C}$ & -3.86174300 & -3.32055400 & 2.06965500 & $\mathrm{H}$ & -1.74891000 & 2.68959600 & 1.29468600 \\
\hline $\mathrm{H}$ & -5.31705500 & -3.39603500 & 0.48003100 & $\mathrm{C}$ & 0.43235600 & 4.60667400 & -1.27417000 \\
\hline $\mathrm{H}$ & -2.18831000 & -3.17603800 & 3.42291700 & $\mathrm{H}$ & 2.05182500 & 3.35343600 & -0.62292400 \\
\hline $\mathrm{H}$ & -4.56972500 & -3.59875900 & 2.84411200 & $\mathrm{C}$ & -0.91870200 & 4.93066400 & -1.11872800 \\
\hline $\mathrm{C}$ & -1.07364500 & -2.25944000 & -1.02389900 & $\mathrm{H}$ & -2.74381500 & 4.48255800 & -0.06128200 \\
\hline $\mathrm{H}$ & -1.51910300 & -2.23475800 & -2.01605500 & $\mathrm{H}$ & 1.04349900 & 5.15002500 & -1.98857000 \\
\hline $\mathrm{C}$ & 0.28610600 & -2.81993400 & -0.95520100 & $\mathrm{H}$ & -1.36151300 & 5.72247600 & -1.71498200 \\
\hline $\mathrm{C}$ & 1.05199700 & -2.93290400 & -2.06536400 & $\mathrm{C}$ & 2.23979800 & -2.67716000 & -1.02993800 \\
\hline $\mathrm{O}$ & 1.69139800 & -3.07621200 & -3.01904300 & $\mathrm{C}$ & 2.87584700 & -2.92359400 & 0.35015000 \\
\hline \multirow[t]{2}{*}{$\mathrm{H}$} & 0.66820800 & -3.32131600 & -0.06975600 & $\mathrm{H}$ & 3.72343200 & -2.25728500 & 0.53271500 \\
\hline & & & & $\mathrm{H}$ & 2.14545100 & -2.79620400 & 1.15547000 \\
\hline \multirow{2}{*}{\multicolumn{4}{|c|}{$\begin{array}{l}\text { TS15 } \\
\text { imaginary frequency }=-119.82 \mathrm{~cm}^{-1}\end{array}$}} & $\mathrm{H}$ & 3.25296500 & -3.95143700 & 0.39649300 \\
\hline & & & & $\mathrm{C}$ & 1.12178400 & -3.70997900 & -1.24594100 \\
\hline $\mathrm{C}$ & 3.02549900 & 0.11038100 & -1.06074300 & $\mathrm{H}$ & 0.65856000 & -3.62678200 & -2.23308600 \\
\hline $\mathrm{C}$ & 3.66517200 & 0.61227200 & -2.19978600 & $\mathrm{H}$ & 1.55000200 & -4.71638400 & -1.17690000 \\
\hline $\mathrm{C}$ & 4.83667000 & 1.35789400 & -2.06848100 & $\mathrm{H}$ & 0.34556600 & -3.62894300 & -0.48455700 \\
\hline $\mathrm{C}$ & 5.36174600 & 1.58003800 & -0.79617900 & $\mathrm{C}$ & 3.30324700 & -2.84240200 & -2.13170700 \\
\hline $\mathrm{C}$ & 4.67487600 & 1.05878500 & 0.29510700 & $\mathrm{H}$ & 3.67185200 & -3.87467900 & -2.12055300 \\
\hline $\mathrm{H}$ & 3.26125700 & 0.40915900 & -3.18488200 & $\mathrm{H}$ & 2.89966400 & -2.65714100 & -3.13235000 \\
\hline $\mathrm{H}$ & 5.33709200 & 1.74938800 & -2.94894900 & $\mathrm{H}$ & 4.16454400 & -2.18606800 & -1.98290200 \\
\hline $\mathrm{H}$ & 6.27262500 & 2.14873000 & -0.64716800 & $\mathrm{C}$ & 0.66568400 & -0.75251500 & -2.73169600 \\
\hline $\mathrm{H}$ & 5.03160100 & 1.22080100 & 1.30889300 & $\mathrm{H}$ & 1.12613100 & 0.08564300 & -3.26479800 \\
\hline $\mathrm{N}$ & 3.53453400 & 0.35596000 & 0.17584000 & $\mathrm{H}$ & 0.87380400 & -1.64991100 & -3.32354800 \\
\hline $\mathrm{P}$ & 1.46965900 & -0.90803200 & -1.04740000 & $\mathrm{C}$ & -0.86184200 & -0.48663900 & -2.67492200 \\
\hline $\mathrm{Pd}$ & -0.02242400 & -0.07256300 & 0.59423700 & $\mathrm{H}$ & -1.03442500 & 0.50327400 & -2.23950500 \\
\hline $\mathrm{H}$ & 1.95386900 & 1.80008500 & 1.09454400 & $\mathrm{H}$ & -1.19568400 & -0.41124400 & -3.71643100 \\
\hline $\mathrm{C}$ & 1.07566300 & 0.38116700 & 3.23828500 & $\mathrm{C}$ & -1.76773000 & -1.53356900 & -1.97144000 \\
\hline $\mathrm{O}$ & 0.93316800 & -0.14781900 & 4.28833000 & $\mathrm{H}$ & -2.73997600 & -1.53118100 & -2.46791500 \\
\hline $\mathrm{C}$ & 0.87854100 & 1.81571300 & 1.21449800 & $\mathrm{H}$ & -1.34964500 & -2.54029600 & -2.06028900 \\
\hline $\mathrm{C}$ & 0.33043300 & 1.25045400 & 2.39805500 & $\mathrm{P}$ & -2.07344900 & -1.18664200 & -0.15633100 \\
\hline $\mathrm{C}$ & 3.47216900 & -0.71801500 & 3.38719400 & $\mathrm{C}$ & -3.40016800 & 0.12456000 & -0.27723900 \\
\hline $\mathrm{H}$ & 4.35159900 & -0.11970500 & 3.63575900 & $\mathrm{C}$ & -3.81542500 & 0.81988900 & 0.86976700 \\
\hline $\mathrm{H}$ & 3.75228100 & -1.61555600 & 2.83479600 & $\mathrm{C}$ & -4.83811500 & 1.76036100 & 0.75700700 \\
\hline $\mathrm{H}$ & 2.93982100 & -0.99200000 & 4.29709000 & $\mathrm{H}$ & -3.34564700 & 0.62457000 & 1.82820900 \\
\hline $\mathrm{O}$ & 2.57340500 & 0.07711600 & 2.58130300 & $\mathrm{C}$ & -4.89829700 & 1.28419300 & -1.58784200 \\
\hline $\mathrm{H}$ & 2.84359400 & 0.10663000 & 1.54133200 & $\mathrm{C}$ & -5.39323700 & 2.00290900 & -0.49968800 \\
\hline
\end{tabular}




\begin{tabular}{|c|c|c|c|c|c|c|c|}
\hline $\mathrm{H}$ & -5.19175800 & 2.29675300 & 1.63289000 & $\mathrm{H}$ & 3.47153300 & 2.59476300 & -0.79324900 \\
\hline $\mathrm{H}$ & -5.29621800 & 1.44934600 & -2.58687800 & $\mathrm{H}$ & 4.76938800 & 1.90644500 & 0.18568100 \\
\hline $\mathrm{H}$ & -6.18784900 & 2.72891800 & -0.63686900 & $\mathrm{H}$ & 3.57101800 & 0.83692500 & -0.54111000 \\
\hline $\mathrm{N}$ & -3.93332500 & 0.36037500 & -1.48509600 & $\mathrm{C}$ & -1.52236400 & -2.74838500 & 2.94899100 \\
\hline $\mathrm{C}$ & -3.06239700 & -2.69947300 & 0.49364200 & $\mathrm{H}$ & -0.64156400 & -3.00414600 & 2.35342700 \\
\hline $\mathrm{C}$ & -4.26761200 & -3.02113900 & -0.40896300 & $\mathrm{H}$ & -1.20242100 & -2.11117000 & 3.78038100 \\
\hline $\mathrm{H}$ & -4.82653600 & -3.86125500 & 0.02001800 & $\mathrm{H}$ & -1.91726100 & -3.67411600 & 3.38204400 \\
\hline $\mathrm{H}$ & -4.95423500 & -2.17526100 & -0.49688500 & $\mathrm{C}$ & -3.85103800 & -1.78283700 & 2.95893900 \\
\hline $\mathrm{H}$ & -3.96000700 & -3.31447200 & -1.41704100 & $\mathrm{H}$ & -4.65449200 & -1.30142600 & 2.39196400 \\
\hline C & -3.54552900 & -2.38292500 & 1.92263500 & $\mathrm{H}$ & -4.25591200 & -2.72719300 & 3.33972200 \\
\hline $\mathrm{H}$ & -4.29138300 & -1.58452700 & 1.93825000 & $\mathrm{H}$ & -3.61442700 & -1.16236900 & 3.82751300 \\
\hline $\mathrm{H}$ & -4.01700800 & -3.27637400 & 2.34751600 & $\mathrm{C}$ & -3.01629600 & -3.02834400 & 0.93758100 \\
\hline $\mathrm{H}$ & -2.71682200 & -2.09996800 & 2.58088900 & $\mathrm{H}$ & -3.35429300 & -3.98225400 & 1.35716400 \\
\hline $\mathrm{C}$ & -2.12420900 & -3.91830900 & 0.55729000 & $\mathrm{H}$ & -3.84828100 & -2.62551200 & 0.34942200 \\
\hline $\mathrm{H}$ & -1.78716900 & -4.23495800 & -0.43377300 & $\mathrm{H}$ & -2.18816200 & -3.24499200 & 0.25883500 \\
\hline $\mathrm{H}$ & -1.24801800 & -3.72559300 & 1.18544800 & $\mathrm{C}$ & 0.62651500 & 2.89919300 & -0.48908400 \\
\hline $\mathrm{H}$ & -2.66431300 & -4.76464400 & 0.99700800 & $\mathrm{C}$ & 0.82169700 & 2.61884200 & -1.85049100 \\
\hline & & & & $\mathrm{C}$ & -0.11681000 & 5.02515700 & -0.97540000 \\
\hline 24 & & & & $\mathrm{C}$ & 0.54776700 & 3.61198700 & -2.79036500 \\
\hline $\mathrm{C}$ & 0.14884200 & 2.30309300 & 2.28065800 & $\mathrm{H}$ & 1.18719000 & 1.64389500 & -2.15635400 \\
\hline $\mathrm{H}$ & 0.58351000 & 1.82784500 & 3.16738300 & $\mathrm{C}$ & 0.06756000 & 4.84396100 & -2.34668300 \\
\hline $\mathrm{H}$ & 0.38333600 & 3.36813700 & 2.32153200 & $\mathrm{H}$ & -0.49639300 & 5.96889600 & -0.58888100 \\
\hline $\mathrm{C}$ & -1.38230000 & 2.15593900 & 2.30727900 & $\mathrm{H}$ & 0.70765300 & 3.42794500 & -3.84898500 \\
\hline $\mathrm{H}$ & -1.75924500 & 2.85455900 & 3.06386100 & $\mathrm{H}$ & -0.15923100 & 5.64634700 & -3.04112000 \\
\hline $\mathrm{H}$ & -1.80739300 & 2.50455200 & 1.35827000 & $\mathrm{C}$ & -3.08530200 & 0.11273800 & 0.13951600 \\
\hline $\mathrm{C}$ & -1.93317900 & 0.76400000 & 2.67894900 & $\mathrm{C}$ & -4.30323600 & 0.73084300 & 0.43105600 \\
\hline $\mathrm{H}$ & -2.97124900 & 0.85391800 & 3.01410100 & $\mathrm{C}$ & -3.55364300 & 0.37888600 & -2.19501600 \\
\hline $\mathrm{H}$ & -1.37476300 & 0.36135400 & 3.53290100 & $\mathrm{C}$ & -5.14621400 & 1.15938500 & -0.59551600 \\
\hline$P$ & -1.79173800 & -0.52492100 & 1.34161800 & $\mathrm{H}$ & -4.59219200 & 0.87587200 & 1.46423400 \\
\hline$P$ & 1.03558000 & 1.60973700 & 0.79361400 & $\mathrm{C}$ & -4.77154700 & 0.97878000 & -1.93003200 \\
\hline $\mathrm{C}$ & 2.87184100 & 1.95112400 & 1.21876500 & $\mathrm{H}$ & -3.17002200 & 0.20695500 & -3.19230800 \\
\hline $\mathrm{C}$ & -2.61974900 & -2.08569800 & 2.08832500 & $\mathrm{H}$ & -6.09356200 & 1.63239700 & -0.35581700 \\
\hline C & 3.30783000 & 0.87582000 & 2.23449800 & $\mathrm{H}$ & -5.40433000 & 1.30190400 & -2.74791400 \\
\hline $\mathrm{H}$ & 4.36194200 & 1.02922100 & 2.49309100 & $\mathrm{~N}$ & 0.15631600 & 4.08337300 & -0.06267800 \\
\hline $\mathrm{H}$ & 2.73613000 & 0.93085100 & 3.16739500 & $\mathrm{~N}$ & -2.76729900 & -0.02183900 & -1.17537300 \\
\hline $\mathrm{H}$ & 3.20767500 & -0.13288500 & 1.82443600 & $\mathrm{Pd}$ & 0.26080700 & -0.59850300 & 0.14806900 \\
\hline $\mathrm{C}$ & 3.08210200 & 3.35662100 & 1.81238100 & $\mathrm{C}$ & 1.63157600 & -1.64111900 & -1.25898500 \\
\hline $\mathrm{H}$ & 4.15654600 & 3.52738300 & 1.94717500 & $\mathrm{H}$ & 1.62402200 & -1.06817700 & -2.18335000 \\
\hline $\mathrm{H}$ & 2.70046700 & 4.14527600 & 1.15753200 & $\mathrm{C}$ & 0.49532100 & -2.45101100 & -1.00353900 \\
\hline $\mathrm{H}$ & 2.61264500 & 3.46974900 & 2.79321600 & $\mathrm{C}$ & -0.58559400 & -2.65022400 & -1.97415400 \\
\hline $\mathrm{C}$ & 3.70703200 & 1.81154100 & -0.06663700 & $\mathrm{O}$ & -1.30637800 & -3.62744900 & -2.03228800 \\
\hline
\end{tabular}




\begin{tabular}{|c|c|c|c|c|c|c|c|}
\hline $\mathrm{O}$ & -0.74816100 & -1.59740900 & -2.86944500 & $\mathrm{H}$ & -4.76523100 & -0.15761000 & -2.65654700 \\
\hline $\mathrm{H}$ & -1.84778800 & -0.43528300 & -1.40538300 & $\mathrm{C}$ & -3.23866300 & -2.92121700 & -1.19773900 \\
\hline $\mathrm{C}$ & -1.49934100 & -1.94546100 & -4.04952300 & $\mathrm{H}$ & -3.85157200 & -2.96634100 & -0.29313800 \\
\hline $\mathrm{H}$ & -2.51947500 & -2.24802300 & -3.79876200 & $\mathrm{H}$ & -3.66477400 & -3.62626200 & -1.91989100 \\
\hline $\mathrm{H}$ & -1.49449300 & -1.05359300 & -4.67884800 & $\mathrm{H}$ & -2.23590400 & -3.27521100 & -0.95066700 \\
\hline $\mathrm{H}$ & -1.01832300 & -2.77138800 & -4.57845900 & $\mathrm{C}$ & 1.40876300 & 1.70451000 & -3.22783100 \\
\hline $\mathrm{C}$ & 2.97969500 & -1.96586300 & -0.74479600 & $\mathrm{H}$ & 1.42054400 & 0.61814700 & -3.09318600 \\
\hline $\mathrm{C}$ & 4.10583000 & -1.59645200 & -1.50232400 & $\mathrm{H}$ & 0.43418200 & 1.98752300 & -3.63844800 \\
\hline $\mathrm{C}$ & 3.19359200 & -2.67738000 & 0.45190400 & $\mathrm{H}$ & 2.16086500 & 1.95842200 & -3.98344100 \\
\hline $\mathrm{C}$ & 5.39578300 & -1.92399700 & -1.08509200 & $\mathrm{C}$ & 1.75217800 & 3.97545500 & -2.16719400 \\
\hline $\mathrm{H}$ & 3.96378900 & -1.06087400 & -2.43800200 & $\mathrm{H}$ & 1.90257100 & 4.54346300 & -1.24532700 \\
\hline $\mathrm{C}$ & 4.48150900 & -3.00529900 & 0.86804700 & $\mathrm{H}$ & 2.57832800 & 4.21252000 & -2.84670000 \\
\hline $\mathrm{H}$ & 2.34549000 & -2.96999700 & 1.06437400 & $\mathrm{H}$ & 0.83207400 & 4.32918700 & -2.63947200 \\
\hline $\mathrm{C}$ & 5.58995800 & -2.63008100 & 0.10248800 & $\mathrm{C}$ & 3.11034300 & 2.00448700 & -1.39583500 \\
\hline $\mathrm{H}$ & 6.24783200 & -1.63364900 & -1.69270400 & $\mathrm{H}$ & 3.86680300 & 2.22593500 & -2.15690200 \\
\hline $\mathrm{H}$ & 4.62274900 & -3.55671200 & 1.79313100 & $\mathrm{H}$ & 3.39701700 & 2.53512500 & -0.48367400 \\
\hline $\mathrm{H}$ & 6.59246900 & -2.88946800 & 0.42838100 & $\mathrm{H}$ & 3.14953900 & 0.92918000 & -1.19538000 \\
\hline $\mathrm{H}$ & 0.59128000 & -3.30948200 & -0.34576600 & $\mathrm{C}$ & -3.21053900 & -0.55721400 & 1.03418700 \\
\hline & & & & $\mathrm{C}$ & -2.51491900 & -1.12550400 & 2.10683600 \\
\hline & & & & $\mathrm{C}$ & -5.12954000 & -0.36331400 & 2.28855900 \\
\hline & uency $=-5$ & $38.43 \mathrm{~cm}^{-1}$ & & $\mathrm{C}$ & -3.18556400 & -1.31495400 & 3.31707300 \\
\hline $\mathrm{C}$ & -3.08976000 & 1.36459300 & -1.09601400 & $\mathrm{H}$ & -1.47331500 & -1.40542000 & 1.99634700 \\
\hline $\mathrm{H}$ & -3.05404200 & 1.43827300 & -2.18844100 & $\mathrm{C}$ & -4.51995900 & -0.92856900 & 3.41184400 \\
\hline $\mathrm{H}$ & -4.14151600 & 1.33415200 & -0.79901000 & $\mathrm{H}$ & -6.16863100 & -0.04399200 & 2.32398500 \\
\hline $\mathrm{C}$ & -2.40881200 & 2.58974400 & -0.46525400 & $\mathrm{H}$ & -2.67301000 & -1.75366000 & 4.16792800 \\
\hline $\mathrm{H}$ & -3.10023300 & 3.43397400 & -0.56669900 & $\mathrm{H}$ & -5.08068900 & -1.05501200 & 4.33197300 \\
\hline $\mathrm{H}$ & -2.27691700 & 2.45055600 & 0.61457500 & $\mathrm{C}$ & 0.92401700 & 2.81245000 & 0.94290000 \\
\hline $\mathrm{C}$ & -1.08379900 & 3.00583200 & -1.12492500 & $\mathrm{C}$ & 1.71929300 & 2.13607600 & 1.87597700 \\
\hline $\mathrm{H}$ & -0.84066000 & 4.03306700 & -0.84215200 & $\mathrm{C}$ & 0.90921500 & 4.72057800 & 2.22828400 \\
\hline $\mathrm{H}$ & -1.17683600 & 2.96450400 & -2.21535200 & $\mathrm{C}$ & 2.12097000 & 2.81496600 & 3.02754300 \\
\hline $\mathrm{P}$ & 0.39248800 & 2.00205800 & -0.64350100 & $\mathrm{H}$ & 2.01988300 & 1.10829500 & 1.70639400 \\
\hline $\mathrm{P}$ & -2.39772900 & -0.28884600 & -0.61552700 & $\mathrm{C}$ & 1.70735300 & 4.13249400 & 3.21178500 \\
\hline $\mathrm{C}$ & -3.24075800 & -1.51075100 & -1.81863800 & $\mathrm{H}$ & 0.56346200 & 5.74627900 & 2.33419100 \\
\hline $\mathrm{C}$ & 1.73365500 & 2.45287100 & -1.91891000 & $\mathrm{H}$ & 2.74358500 & 2.31962900 & 3.76650400 \\
\hline $\mathrm{C}$ & -2.43007900 & -1.49978700 & -3.13071200 & $\mathrm{H}$ & 1.99190800 & 4.69592800 & 4.09418900 \\
\hline $\mathrm{H}$ & -2.89286800 & -2.18857500 & -3.84624800 & $\mathrm{~N}$ & -4.49562700 & -0.17776800 & 1.12572800 \\
\hline $\mathrm{H}$ & -2.42148500 & -0.50904000 & -3.59766900 & $\mathrm{~N}$ & 0.52561100 & 4.08205600 & 1.11667900 \\
\hline $\mathrm{H}$ & -1.39217500 & -1.81366700 & -2.98711900 & $\mathrm{Pd}$ & 0.01816200 & -0.32686700 & -0.44874100 \\
\hline $\mathrm{C}$ & -4.69964900 & -1.09948400 & -2.10594800 & $\mathrm{H}$ & 1.61541600 & -0.42597400 & -0.27707000 \\
\hline $\mathrm{H}$ & -5.16189400 & -1.87217700 & -2.73076900 & $\mathrm{C}$ & 2.32865600 & -2.31602200 & 1.04348900 \\
\hline $\mathrm{H}$ & -5.28710300 & -1.00491800 & -1.18989600 & $\mathrm{C}$ & 1.85871600 & -1.70055300 & 2.21313200 \\
\hline
\end{tabular}




\begin{tabular}{|c|c|c|c|c|c|c|c|}
\hline $\mathrm{C}$ & 3.40544900 & -3.20988800 & 1.13734600 & $\mathrm{H}$ & 6.58938300 & -2.49592100 & 2.59962500 \\
\hline $\mathrm{C}$ & 2.45151500 & -1.96480800 & 3.44736900 & $\mathrm{H}$ & 6.32497000 & -1.05768100 & 1.60255500 \\
\hline $\mathrm{H}$ & 1.02833800 & -1.00165400 & 2.14878400 & $\mathrm{H}$ & 5.12488300 & -1.53508500 & 2.81108800 \\
\hline $\mathrm{C}$ & 3.99420700 & -3.47586600 & 2.37402400 & $\mathrm{C}$ & 6.18161000 & -3.39902600 & 0.05976400 \\
\hline $\mathrm{H}$ & 3.78448800 & -3.70362900 & 0.25037700 & $\mathrm{H}$ & 6.77004600 & -2.62042000 & -0.43439800 \\
\hline $\mathrm{C}$ & 3.52325900 & -2.85530100 & 3.53144300 & $\mathrm{H}$ & 6.87986000 & -4.03118800 & 0.62102600 \\
\hline $\mathrm{H}$ & 2.07784100 & -1.47385300 & 4.34110900 & $\mathrm{H}$ & 5.71796900 & -4.02224800 & -0.71276500 \\
\hline $\mathrm{H}$ & 4.82497000 & -4.17248200 & 2.42910400 & $\mathrm{C}$ & -2.54889100 & 0.28647900 & 2.85852400 \\
\hline $\mathrm{H}$ & 3.98873000 & -3.06190800 & 4.49003500 & $\mathrm{H}$ & -3.13407500 & -0.19262300 & 2.06748900 \\
\hline $\mathrm{C}$ & 0.25060000 & -2.52137000 & -0.36283200 & $\mathrm{H}$ & -1.79283800 & -0.42345600 & 3.20659000 \\
\hline $\mathrm{H}$ & -0.10485100 & -2.84001100 & -1.33559400 & $\mathrm{H}$ & -3.22702900 & 0.47251100 & 3.69851000 \\
\hline $\mathrm{H}$ & -0.24346100 & -2.92648500 & 0.51289900 & $\mathrm{C}$ & -1.19249600 & 2.28251100 & 3.58845700 \\
\hline $\mathrm{C}$ & 1.60469500 & -2.13181400 & -0.26283700 & $\mathrm{H}$ & -0.70859300 & 3.22010800 & 3.30574600 \\
\hline $\mathrm{C}$ & 2.35522700 & -2.13089600 & -1.60004100 & $\mathrm{H}$ & -1.91435600 & 2.50112900 & 4.38301400 \\
\hline $\mathrm{O}$ & 1.81067100 & -2.33184500 & -2.66433800 & $\mathrm{H}$ & -0.43080600 & 1.62066000 & 4.00967900 \\
\hline $\mathrm{O}$ & 3.65597700 & -1.85539800 & -1.45536500 & $\mathrm{C}$ & -3.05630000 & 2.55955000 & 1.90330400 \\
\hline $\mathrm{C}$ & 4.43367200 & -1.84622300 & -2.67621300 & $\mathrm{H}$ & -3.77671300 & 2.72700000 & 2.71142800 \\
\hline $\mathrm{H}$ & 4.07859300 & -1.05710700 & -3.34185100 & $\mathrm{H}$ & -2.67200700 & 3.53642600 & 1.59725600 \\
\hline $\mathrm{H}$ & 5.45859700 & -1.65710600 & -2.36225800 & $\mathrm{H}$ & -3.60014000 & 2.12189100 & 1.05922600 \\
\hline $\mathrm{H}$ & 4.35107700 & -2.80958700 & -3.18242200 & $\mathrm{C}$ & 4.81390800 & -0.46453700 & -0.75443000 \\
\hline & & & & $\mathrm{C}$ & 5.48654700 & -0.65150500 & -1.97356000 \\
\hline & & & & $\mathrm{C}$ & 5.52012100 & 1.72793400 & -0.65289200 \\
\hline & |uency $=-5$ & $38.05 \mathrm{~cm}^{-1}$ & & $\mathrm{C}$ & 6.22074100 & 0.40119500 & -2.51627500 \\
\hline $\mathrm{C}$ & 2.83477500 & -0.95559500 & 1.25089100 & $\mathrm{H}$ & 5.42737300 & -1.60727600 & -2.48492800 \\
\hline $\mathrm{H}$ & 2.47360300 & -1.70091100 & 1.96951500 & $\mathrm{C}$ & 6.24211600 & 1.62158300 & -1.84148000 \\
\hline $\mathrm{H}$ & 3.47025600 & -0.23913700 & 1.77854900 & $\mathrm{H}$ & 5.50090500 & 2.66763300 & -0.10323900 \\
\hline $\mathrm{C}$ & 1.64479500 & -0.21968500 & 0.61860800 & $\mathrm{H}$ & 6.75837000 & 0.27421600 & -3.45148600 \\
\hline $\mathrm{H}$ & 2.03136800 & 0.56986100 & -0.03571200 & $\mathrm{H}$ & 6.79599000 & 2.47178500 & -2.22624500 \\
\hline $\mathrm{H}$ & 1.07246800 & -0.91543000 & -0.00940900 & $\mathrm{C}$ & -0.03783200 & 2.97408300 & 0.52339000 \\
\hline $\mathrm{C}$ & 0.71885200 & 0.39885000 & 1.68022000 & $\mathrm{C}$ & -0.62089400 & 3.75433500 & -0.48313800 \\
\hline $\mathrm{H}$ & 1.26851700 & 1.11850800 & 2.29400400 & $\mathrm{C}$ & 1.56149500 & 4.56812300 & 0.94498000 \\
\hline $\mathrm{H}$ & 0.31728200 & -0.38134900 & 2.33583800 & $\mathrm{C}$ & -0.06540800 & 5.00453800 & -0.76214800 \\
\hline$P$ & -0.72734600 & 1.31317300 & 0.97533300 & $\mathrm{H}$ & -1.48779600 & 3.39689100 & -1.03070000 \\
\hline$P$ & 3.83010300 & -1.88060100 & -0.04117500 & $\mathrm{C}$ & 1.04975100 & 5.41971200 & -0.03733100 \\
\hline $\mathrm{C}$ & 5.13355000 & -2.80641700 & 1.02148000 & $\mathrm{H}$ & 2.43059800 & 4.85621500 & 1.53163400 \\
\hline $\mathrm{C}$ & -1.93941300 & 1.62709200 & 2.40740200 & $\mathrm{H}$ & -0.49483600 & 5.63758400 & -1.53259500 \\
\hline $\mathrm{C}$ & 4.39393900 & -3.96452100 & 1.72182700 & $\mathrm{H}$ & 1.51735800 & 6.38054700 & -0.22472100 \\
\hline $\mathrm{H}$ & 5.11203500 & -4.57346800 & 2.28393900 & $\mathrm{~N}$ & 4.82452000 & 0.71849900 & -0.11306200 \\
\hline $\mathrm{H}$ & 3.64540000 & -3.60810700 & 2.43723700 & $\mathrm{~N}$ & 1.03184400 & 3.37213200 & 1.22681200 \\
\hline $\mathrm{H}$ & 3.89325100 & -4.61956900 & 1.00128900 & $\mathrm{Pd}$ & -1.61638500 & 0.21894100 & -0.97951100 \\
\hline $\mathrm{C}$ & 5.82764700 & -1.91550600 & 2.06388000 & $\mathrm{H}$ & -2.83264000 & -0.29763000 & -0.05505800 \\
\hline
\end{tabular}




\begin{tabular}{|c|c|c|c|c|c|c|c|}
\hline $\mathrm{C}$ & -2.86281000 & -0.91792400 & -2.38484400 & $\mathrm{H}$ & -2.68035100 & -2.82359700 & -3.78389600 \\
\hline $\mathrm{H}$ & -2.27889400 & -1.75252300 & -2.75847100 & $\mathrm{H}$ & -2.41214600 & -1.08678800 & -3.70075900 \\
\hline $\mathrm{H}$ & -3.19084600 & -0.16477900 & -3.09567500 & $\mathrm{H}$ & -1.20799400 & -2.20260600 & -3.03119200 \\
\hline $\mathrm{C}$ & -3.62941900 & -1.09230300 & -1.20441700 & $\mathrm{C}$ & -4.54271400 & -1.80440700 & -2.03894000 \\
\hline $\mathrm{C}$ & -4.81665500 & -0.11800100 & -1.07731200 & $\mathrm{H}$ & -4.94249600 & -2.67399700 & -2.57143800 \\
\hline $\mathrm{O}$ & -5.06274200 & 0.73032900 & -1.90399900 & $\mathrm{H}$ & -5.09949100 & -1.70764200 & -1.10244300 \\
\hline $\mathrm{O}$ & -5.49405700 & -0.30501300 & 0.05739300 & $\mathrm{H}$ & -4.74852800 & -0.92788100 & -2.65798100 \\
\hline $\mathrm{C}$ & -6.63419100 & 0.56639300 & 0.25927800 & $\mathrm{C}$ & -2.83604600 & -3.37519200 & -1.08813700 \\
\hline $\mathrm{H}$ & -7.34717600 & 0.44647500 & -0.55821500 & $\mathrm{H}$ & -3.38710500 & -3.42359100 & -0.14537700 \\
\hline $\mathrm{H}$ & -7.07021000 & 0.25293100 & 1.20585900 & $\mathrm{H}$ & -3.22074600 & -4.17263300 & -1.73233300 \\
\hline $\mathrm{H}$ & -6.30734500 & 1.60711100 & 0.30429400 & $\mathrm{H}$ & -1.78610900 & -3.60004600 & -0.89137600 \\
\hline $\mathrm{C}$ & -3.65884800 & -2.43686100 & -0.52835000 & $\mathrm{C}$ & 1.59536100 & 1.76356800 & -3.22137500 \\
\hline $\mathrm{C}$ & -2.47393000 & -3.03766900 & -0.08052900 & $\mathrm{H}$ & 1.70430500 & 0.68720600 & -3.05792900 \\
\hline $\mathrm{C}$ & -4.86116800 & -3.15475000 & -0.44795600 & $\mathrm{H}$ & 0.65863100 & 1.93994300 & -3.75910000 \\
\hline $\mathrm{C}$ & -2.48667800 & -4.33056400 & 0.43885400 & $\mathrm{H}$ & 2.40621900 & 2.08473700 & -3.88382900 \\
\hline $\mathrm{H}$ & -1.53807400 & -2.48517400 & -0.12958800 & $\mathrm{C}$ & 1.56880800 & 4.07799200 & -2.18815000 \\
\hline $\mathrm{C}$ & -4.86894300 & -4.45127900 & 0.06630900 & $\mathrm{H}$ & 1.60372700 & 4.68093600 & -1.27390600 \\
\hline $\mathrm{H}$ & -5.78932700 & -2.71175100 & -0.79050200 & $\mathrm{H}$ & 2.42060600 & 4.38982100 & -2.80098500 \\
\hline $\mathrm{C}$ & -3.68612800 & -5.04159800 & 0.51266900 & $\mathrm{H}$ & 0.66588800 & 4.33265200 & -2.74897700 \\
\hline $\mathrm{H}$ & -1.56219300 & -4.77964700 & 0.78813900 & $\mathrm{C}$ & 3.03470800 & 2.27525200 & -1.21475200 \\
\hline $\mathrm{H}$ & -5.80496300 & -4.99839700 & 0.11797600 & $\mathrm{H}$ & 3.84282100 & 2.55668500 & -1.89764200 \\
\hline $\mathrm{H}$ & -3.69879900 & -6.04807100 & 0.91881300 & $\mathrm{H}$ & 3.17200400 & 2.85911500 & -0.29952500 \\
\hline $\mathrm{C}$ & -0.19971700 & 0.72120100 & -2.29004500 & $\mathrm{H}$ & 3.16295000 & 1.21505900 & -0.97826800 \\
\hline $\mathrm{O}$ & 0.60864900 & 1.00631500 & -3.03695400 & $\mathrm{C}$ & -3.14978800 & -0.72089200 & 0.92448800 \\
\hline & & & & $\mathrm{C}$ & -2.69549900 & -1.60017300 & 1.91666500 \\
\hline \multirow{2}{*}{\multicolumn{4}{|c|}{$\begin{array}{l}\text { TS16-2H } \\
\text { imaginary frequency }=-364.29 \mathrm{~cm}^{-1}\end{array}$}} & $\mathrm{C}$ & -4.87971500 & 0.00446100 & 2.25916900 \\
\hline & & & & $\mathrm{C}$ & -3.39974600 & -1.67212500 & 3.12003100 \\
\hline $\mathrm{C}$ & -3.10270800 & 0.90991300 & -1.45055200 & $\mathrm{H}$ & -1.82374100 & -2.22455700 & 1.75388600 \\
\hline $\mathrm{H}$ & -2.97718800 & 0.86896600 & -2.53771000 & $\mathrm{C}$ & -4.51568200 & -0.85610200 & 3.29685500 \\
\hline $\mathrm{H}$ & -4.16775900 & 0.82219300 & -1.22941800 & $\mathrm{H}$ & -5.74043100 & 0.66139200 & 2.35907200 \\
\hline $\mathrm{C}$ & -2.61512300 & 2.26528600 & -0.91396600 & $\mathrm{H}$ & -3.08374500 & -2.35637600 & 3.90156700 \\
\hline $\mathrm{H}$ & -3.37249100 & 3.01025600 & -1.18163200 & $\mathrm{H}$ & -5.09416300 & -0.88261100 & 4.21416000 \\
\hline $\mathrm{H}$ & -2.59027800 & 2.24946400 & 0.18208600 & $\mathrm{C}$ & 0.48764100 & 2.84843900 & 0.81489500 \\
\hline $\mathrm{C}$ & -1.27702300 & 2.75248200 & -1.50849800 & $\mathrm{C}$ & 1.27254700 & 2.32077500 & 1.84007400 \\
\hline $\mathrm{H}$ & -1.19955800 & 3.84646800 & -1.45975700 & $\mathrm{C}$ & 0.05367600 & 4.77470300 & 2.18754200 \\
\hline $\mathrm{H}$ & -1.24882800 & 2.52957400 & -2.58124800 & $\mathrm{C}$ & 1.45915000 & 3.03392200 & 3.02765600 \\
\hline$P$ & 0.24538300 & 1.95626500 & -0.81148400 & $\mathrm{H}$ & 1.72439100 & 1.34409000 & 1.70769600 \\
\hline $\mathrm{P}$ & -2.31522400 & -0.61277700 & -0.73462200 & $\mathrm{C}$ & 0.84125900 & 4.27565700 & 3.20856500 \\
\hline $\mathrm{C}$ & -3.03399900 & -2.02625500 & -1.80479200 & $\mathrm{H}$ & -0.47176500 & 5.72158000 & 2.23698600 \\
\hline $\mathrm{C}$ & 1.69120200 & 2.56782200 & -1.90572600 & $\mathrm{H}$ & 2.08054600 & 2.61629700 & 3.81386100 \\
\hline $\mathrm{C}$ & -2.28100300 & -2.02435300 & -3.15060800 & $\mathrm{H}$ & 0.96105400 & 4.84555400 & 4.12268600 \\
\hline
\end{tabular}




\begin{tabular}{|c|c|c|c|c|c|c|c|}
\hline $\mathrm{N}$ & -4.21637200 & 0.07423600 & 1.09738700 & $\mathrm{C}$ & -1.96584600 & 1.75224300 & -2.34726900 \\
\hline $\mathrm{N}$ & -0.08744100 & 4.06121300 & 1.04500000 & $\mathrm{C}$ & 3.59583800 & -1.46808200 & -1.49506300 \\
\hline $\mathrm{Pd}$ & 0.10905200 & -0.36009600 & -0.48290200 & $\mathrm{C}$ & -1.60324000 & 0.84561800 & -3.54171600 \\
\hline $\mathrm{H}$ & 1.68021100 & -0.25051500 & -0.23364000 & $\mathrm{H}$ & -2.37029500 & 0.95331700 & -4.31773500 \\
\hline $\mathrm{C}$ & 2.49940600 & -2.08714600 & 1.22939800 & $\mathrm{H}$ & -0.64914500 & 1.13159500 & -3.99744400 \\
\hline $\mathrm{C}$ & 1.80214000 & -1.55773100 & 2.32965000 & $\mathrm{H}$ & -1.55035800 & -0.21010600 & -3.26346800 \\
\hline C & 3.69971300 & -2.77728500 & 1.45992100 & $\mathrm{C}$ & -2.09390400 & 3.21421100 & -2.82068100 \\
\hline $\mathrm{C}$ & 2.29251800 & -1.70734400 & 3.62536400 & $\mathrm{H}$ & -2.94838600 & 3.28339400 & -3.50382300 \\
\hline $\mathrm{H}$ & 0.86345100 & -1.03207700 & 2.16210200 & $\mathrm{H}$ & -2.26288300 & 3.90274900 & -1.99000500 \\
\hline C & 4.18332500 & -2.93126300 & 2.76050700 & $\mathrm{H}$ & -1.21332700 & 3.55121100 & -3.37338800 \\
\hline $\mathrm{H}$ & 4.25160100 & -3.20713400 & 0.63384900 & $\mathrm{C}$ & -3.30735500 & 1.31209800 & -1.73724600 \\
\hline C & 3.48825300 & -2.39658900 & 3.84474500 & $\mathrm{H}$ & -3.59866200 & 1.94439500 & -0.89297700 \\
\hline $\mathrm{H}$ & 1.74044000 & -1.29460500 & 4.46472600 & $\mathrm{H}$ & -4.09198500 & 1.39894600 & -2.49746500 \\
\hline $\mathrm{H}$ & 5.10817800 & -3.47568400 & 2.92194500 & $\mathrm{H}$ & -3.29108000 & 0.27275600 & -1.40409000 \\
\hline $\mathrm{H}$ & 3.87228300 & -2.51741600 & 4.85259000 & $\mathrm{C}$ & 2.85659800 & -1.77309000 & -2.81378900 \\
\hline C & 0.58933500 & -2.52416900 & -0.34784900 & $\mathrm{H}$ & 1.85096300 & -2.16726400 & -2.63729600 \\
\hline $\mathrm{H}$ & 0.32438700 & -2.84642700 & -1.34895800 & $\mathrm{H}$ & 2.76871800 & -0.89002600 & -3.45502000 \\
\hline $\mathrm{H}$ & 0.06068800 & -2.96814200 & 0.48702300 & $\mathrm{H}$ & 3.42090400 & -2.52575600 & -3.37536900 \\
\hline C & 1.89219900 & -2.03584200 & -0.13948800 & $\mathrm{C}$ & 5.01313400 & -0.93257900 & -1.78998300 \\
\hline $\mathrm{C}$ & 2.73272300 & -1.92567300 & -1.42355200 & $\mathrm{H}$ & 5.53604700 & -0.62761400 & -0.88040400 \\
\hline $\mathrm{O}$ & 2.25327700 & -2.07559100 & -2.52670200 & $\mathrm{H}$ & 5.59471100 & -1.72877000 & -2.26731500 \\
\hline $\mathrm{O}$ & 4.00710100 & -1.62222000 & -1.17983100 & $\mathrm{H}$ & 4.99967000 & -0.08442000 & -2.47902500 \\
\hline C & 4.87975800 & -1.57026500 & -2.34106300 & $\mathrm{C}$ & 3.70389300 & -2.74982500 & -0.64991700 \\
\hline $\mathrm{H}$ & 4.55236800 & -0.78116500 & -3.02008300 & $\mathrm{H}$ & 4.26192600 & -3.50190000 & -1.21772400 \\
\hline $\mathrm{H}$ & 5.87053000 & -1.35934200 & -1.94395700 & $\mathrm{H}$ & 4.23661300 & -2.58125100 & 0.29018500 \\
\hline $\mathrm{H}$ & 4.86231900 & -2.52840400 & -2.86244500 & $\mathrm{H}$ & 2.72300300 & -3.17962100 & -0.42179400 \\
\hline \multirow[t]{2}{*}{$\mathrm{H}$} & -0.66785700 & 4.46289400 & 0.31372900 & $\mathrm{C}$ & -1.12665000 & 2.49629800 & 0.46241000 \\
\hline & & & & $\mathrm{C}$ & -1.39902600 & 1.84270600 & 1.66854800 \\
\hline \multirow{2}{*}{\multicolumn{4}{|c|}{$\begin{array}{l}\text { TS17 } \\
\text { imaginary frequency }=-138.46 \mathrm{~cm}^{-1}\end{array}$}} & $\mathrm{C}$ & -1.59260200 & 4.55078100 & 1.38829300 \\
\hline & & & & $\mathrm{C}$ & -1.78957600 & 2.60466000 & 2.77220100 \\
\hline $\mathrm{C}$ & 0.74021800 & 2.67341500 & -1.68243500 & $\mathrm{H}$ & -1.31548300 & 0.76419300 & 1.73936300 \\
\hline $\mathrm{H}$ & 0.88066700 & 2.46129600 & -2.74790400 & $\mathrm{C}$ & -1.88930200 & 3.98619300 & 2.63219500 \\
\hline $\mathrm{H}$ & 0.31853700 & 3.67772900 & -1.59629500 & $\mathrm{H}$ & -1.65543900 & 5.62652100 & 1.23957500 \\
\hline $\mathrm{C}$ & 2.09155500 & 2.62814400 & -0.95572500 & $\mathrm{H}$ & -2.01155900 & 2.12268400 & 3.71951300 \\
\hline $\mathrm{H}$ & 2.63415500 & 3.54499300 & -1.21300200 & $\mathrm{H}$ & -2.18611900 & 4.61913800 & 3.46200800 \\
\hline $\mathrm{H}$ & 1.95157100 & 2.66710200 & 0.13139700 & $\mathrm{C}$ & 3.34199200 & 0.00548100 & 1.12803700 \\
\hline $\mathrm{C}$ & 2.99065600 & 1.44628200 & -1.34582500 & $\mathrm{C}$ & 2.74054200 & -0.52139200 & 2.27610400 \\
\hline $\mathrm{H}$ & 4.02294100 & 1.64420500 & -1.04319000 & $\mathrm{C}$ & 5.12750600 & 0.80774900 & 2.33479400 \\
\hline $\mathrm{H}$ & 2.97603600 & 1.30153400 & -2.43111600 & $\mathrm{C}$ & 3.39200100 & -0.36446800 & 3.50139500 \\
\hline$P$ & 2.57244400 & -0.16792700 & -0.55127400 & $\mathrm{H}$ & 1.78626100 & -1.03241700 & 2.20949600 \\
\hline$P$ & -0.58552000 & 1.53920000 & -1.03847800 & $\mathrm{C}$ & 4.60912100 & 0.31156100 & 3.53388000 \\
\hline
\end{tabular}




\begin{tabular}{|c|c|c|c|c|c|c|c|}
\hline $\mathrm{H}$ & 6.07289300 & 1.34493900 & 2.31909000 & $\mathrm{P}$ & 0.51988800 & 2.01139500 & -0.62075300 \\
\hline $\mathrm{H}$ & 2.95251300 & -0.75991300 & 4.41210100 & $\mathrm{P}$ & -3.65304500 & -1.81223400 & -0.60746900 \\
\hline $\mathrm{H}$ & 5.14801300 & 0.45913500 & 4.46384200 & $\mathrm{C}$ & -4.96834600 & -2.39656900 & -1.87887900 \\
\hline $\mathrm{N}$ & -1.21769200 & 3.83017400 & 0.32642000 & $\mathrm{C}$ & 1.29435700 & 3.33319300 & -1.74435400 \\
\hline $\mathrm{N}$ & 4.51236600 & 0.66286700 & 1.15611100 & $\mathrm{C}$ & -4.19810700 & -3.05934000 & -3.03950600 \\
\hline Pd & 0.30216600 & -0.69555400 & -0.40230500 & $\mathrm{H}$ & -4.90900900 & -3.48147700 & -3.75994500 \\
\hline $\mathrm{H}$ & 1.11152600 & -1.94718900 & 0.05601700 & $\mathrm{H}$ & -3.57228200 & -2.34540000 & -3.58554800 \\
\hline $\mathrm{C}$ & -1.70573800 & -2.25859900 & 0.09925600 & $\mathrm{H}$ & -3.55765100 & -3.87437700 & -2.68665400 \\
\hline C & -0.49213300 & -2.93363700 & 0.08577700 & $\mathrm{C}$ & -5.86021900 & -1.26453800 & -2.41416200 \\
\hline $\mathrm{H}$ & -0.04755700 & -3.27603600 & 1.01197100 & $\mathrm{H}$ & -6.61498000 & -1.67753000 & -3.09526500 \\
\hline $\mathrm{H}$ & -0.18239900 & -3.43538000 & -0.82380600 & $\mathrm{H}$ & -6.38601200 & -0.74447800 & -1.60860000 \\
\hline $\mathrm{C}$ & -2.43175200 & -1.97365700 & 1.37154000 & $\mathrm{H}$ & -5.28730500 & -0.52096200 & -2.97647600 \\
\hline C & -3.40303800 & -0.96067200 & 1.47002400 & $\mathrm{C}$ & -5.83864200 & -3.46461100 & -1.18896600 \\
\hline C & -2.18016300 & -2.75362600 & 2.51786700 & $\mathrm{H}$ & -6.44148200 & -3.04102800 & -0.38042300 \\
\hline C & -4.08131600 & -0.72854100 & 2.66496600 & $\mathrm{H}$ & -6.52994400 & -3.90327200 & -1.91849800 \\
\hline $\mathrm{H}$ & -3.63243900 & -0.34883900 & 0.60767500 & $\mathrm{H}$ & -5.23227000 & -4.27824600 & -0.77600000 \\
\hline C & -2.85400800 & -2.51716700 & 3.71441500 & $\mathrm{C}$ & 2.07744700 & 2.60236000 & -2.85479800 \\
\hline $\mathrm{H}$ & -1.48371000 & -3.58433900 & 2.47056300 & $\mathrm{H}$ & 2.89694800 & 1.99729700 & -2.45294300 \\
\hline $\mathrm{C}$ & -3.80806300 & -1.50165300 & 3.79485400 & $\mathrm{H}$ & 1.43178100 & 1.95839400 & -3.46140500 \\
\hline $\mathrm{H}$ & -4.82971500 & 0.05699300 & 2.71005100 & $\mathrm{H}$ & 2.51452100 & 3.34642000 & -3.52958900 \\
\hline $\mathrm{H}$ & -2.64856100 & -3.14332900 & 4.57728900 & $\mathrm{C}$ & 0.20856800 & 4.23179600 & -2.37035900 \\
\hline $\mathrm{H}$ & -4.34329600 & -1.32520700 & 4.72280800 & $\mathrm{H}$ & -0.42666700 & 4.69407300 & -1.61256000 \\
\hline C & -2.40441800 & -2.28159600 & -1.24127000 & $\mathrm{H}$ & 0.70135200 & 5.02568300 & -2.94266200 \\
\hline $\mathrm{O}$ & -1.82161100 & -2.41163300 & -2.30293400 & $\mathrm{H}$ & -0.42935700 & 3.67808600 & -3.06494700 \\
\hline $\mathrm{O}$ & -3.73846900 & -2.22809500 & -1.12723100 & $\mathrm{C}$ & 2.26063000 & 4.18348100 & -0.89866700 \\
\hline C & -4.48252300 & -2.39543700 & -2.35505900 & $\mathrm{H}$ & 2.75692300 & 4.91028600 & -1.55037300 \\
\hline $\mathrm{H}$ & -4.23458300 & -3.35318600 & -2.81627900 & $\mathrm{H}$ & 1.73956500 & 4.74160800 & -0.11588600 \\
\hline $\mathrm{H}$ & -5.53052400 & -2.36637800 & -2.06153000 & $\mathrm{H}$ & 3.04237400 & 3.57420000 & -0.43324500 \\
\hline \multirow[t]{2}{*}{$\mathrm{H}$} & -4.25174300 & -1.58875400 & -3.05338900 & $\mathrm{C}$ & -4.68643700 & -0.94462100 & 0.68210600 \\
\hline & & & & C & -5.20451800 & -1.69268200 & 1.75227900 \\
\hline \multirow{2}{*}{\multicolumn{4}{|c|}{$\begin{array}{l}\text { TS17a } \\
\text { imaginary frequency }=-554.22 \mathrm{~cm}^{-1}\end{array}$}} & $\mathrm{C}$ & -5.61141500 & 0.98918100 & 1.53094800 \\
\hline & & & & $\mathrm{C}$ & -5.97675000 & -1.05404300 & 2.72051400 \\
\hline C & -2.90395300 & -0.32458200 & -1.46550700 & $\mathrm{H}$ & -4.99853100 & -2.75639600 & 1.82038500 \\
\hline $\mathrm{H}$ & -2.57688700 & -0.66052000 & -2.45652000 & $\mathrm{C}$ & -6.18945700 & 0.31984200 & 2.60952200 \\
\hline $\mathrm{H}$ & -3.65979900 & 0.45485300 & -1.59242800 & $\mathrm{H}$ & -5.74393900 & 2.06379300 & 1.41572800 \\
\hline C & -1.70759700 & 0.23814800 & -0.68315000 & $\mathrm{H}$ & -6.39729500 & -1.61601900 & 3.54946600 \\
\hline $\mathrm{H}$ & -2.06233100 & 0.63478400 & 0.27383900 & $\mathrm{H}$ & -6.78081000 & 0.86233900 & 3.34007300 \\
\hline $\mathrm{H}$ & -1.00528000 & -0.57130100 & -0.44745600 & $\mathrm{C}$ & -0.17366600 & 2.86623900 & 0.86738700 \\
\hline C & -0.99289800 & 1.35567400 & -1.46334300 & $\mathrm{C}$ & 0.29059900 & 2.63751000 & 2.16547300 \\
\hline $\mathrm{H}$ & -1.66597300 & 2.20730300 & -1.60197300 & $\mathrm{C}$ & -1.74731900 & 4.37033100 & 1.60535100 \\
\hline $\mathrm{H}$ & -0.68495300 & 1.00358100 & -2.45514300 & $\mathrm{C}$ & -0.31306400 & 3.32819700 & 3.21926200 \\
\hline
\end{tabular}




\begin{tabular}{|c|c|c|c|c|c|c|c|}
\hline $\mathrm{H}$ & 1.09184100 & 1.93122300 & 2.35009200 & $\mathrm{H}$ & -2.22337800 & 2.12735100 & 1.35117200 \\
\hline $\mathrm{C}$ & -1.34867700 & 4.21394900 & 2.93613200 & $\mathrm{C}$ & -2.97247200 & 0.13186400 & 1.90877700 \\
\hline $\mathrm{H}$ & -2.55595000 & 5.04873700 & 1.34397300 & $\mathrm{H}$ & -4.06147700 & 0.23423900 & 2.00621600 \\
\hline $\mathrm{H}$ & 0.01981800 & 3.17147000 & 4.24068500 & $\mathrm{H}$ & -2.70638100 & -0.61870600 & 2.66130100 \\
\hline $\mathrm{H}$ & -1.84549500 & 4.77059000 & 3.72370600 & $\mathrm{P}$ & -2.55524900 & -0.64396500 & 0.28187200 \\
\hline $\mathrm{N}$ & -4.88083600 & 0.38342700 & 0.58567300 & $\mathrm{P}$ & 0.51541800 & 0.80611800 & 1.58479400 \\
\hline $\mathrm{N}$ & -1.18061600 & 3.71100800 & 0.59006800 & $\mathrm{C}$ & 1.95536600 & 0.39203000 & 2.77868400 \\
\hline $\mathrm{Pd}$ & 2.14842600 & 0.32950200 & -0.15514000 & $\mathrm{C}$ & -3.44907000 & -2.33492400 & 0.27841600 \\
\hline $\mathrm{H}$ & 2.71130600 & 1.44327700 & 0.82052200 & $\mathrm{C}$ & 1.63255100 & -0.95387600 & 3.46031000 \\
\hline $\mathrm{C}$ & 4.00188600 & -0.83739400 & 0.65718600 & $\mathrm{H}$ & 2.46741400 & -1.22562300 & 4.11589400 \\
\hline $\mathrm{C}$ & 4.07128400 & 0.46581600 & 1.18807500 & $\mathrm{H}$ & 0.74286700 & -0.89855600 & 4.09543300 \\
\hline $\mathrm{C}$ & 1.49843800 & -1.06342800 & -1.45526300 & $\mathrm{H}$ & 1.49517300 & -1.76041500 & 2.73512500 \\
\hline $\mathrm{O}$ & 1.11489600 & -1.79207300 & -2.24056500 & $\mathrm{C}$ & 2.12406900 & 1.50211600 & 3.83587500 \\
\hline $\mathrm{H}$ & 4.73482400 & 1.18108800 & 0.71303100 & $\mathrm{H}$ & 3.00155200 & 1.26773100 & 4.44846500 \\
\hline $\mathrm{H}$ & 3.84274300 & 0.62656200 & 2.23574200 & $\mathrm{H}$ & 2.28944400 & 2.48501200 & 3.38581600 \\
\hline $\mathrm{C}$ & 3.58174900 & -2.00209600 & 1.48693900 & $\mathrm{H}$ & 1.27044100 & 1.57164500 & 4.51422100 \\
\hline $\mathrm{C}$ & 2.99929500 & -3.14639900 & 0.91063200 & $\mathrm{C}$ & 3.25898400 & 0.26301900 & 1.97509200 \\
\hline $\mathrm{C}$ & 3.77943400 & -1.98644700 & 2.87998400 & $\mathrm{H}$ & 3.53546800 & 1.19636500 & 1.47771100 \\
\hline $\mathrm{C}$ & 2.60789900 & -4.22285100 & 1.70009800 & $\mathrm{H}$ & 4.07327400 & 0.00326000 & 2.66027200 \\
\hline $\mathrm{H}$ & 2.85661800 & -3.19623200 & -0.16296200 & $\mathrm{H}$ & 3.19926700 & -0.52478600 & 1.22256300 \\
\hline $\mathrm{C}$ & 3.38927800 & -3.06697600 & 3.66974600 & $\mathrm{C}$ & -2.61028700 & -3.25123500 & 1.19694900 \\
\hline $\mathrm{H}$ & 4.27791500 & -1.14547200 & 3.35171200 & $\mathrm{H}$ & -1.57940600 & -3.35381600 & 0.84563800 \\
\hline $\mathrm{C}$ & 2.79708000 & -4.18602700 & 3.08431200 & $\mathrm{H}$ & -2.58976700 & -2.90376700 & 2.23427600 \\
\hline $\mathrm{H}$ & 2.15482800 & -5.09259800 & 1.23475200 & $\mathrm{H}$ & -3.06529800 & -4.24715700 & 1.20218000 \\
\hline $\mathrm{H}$ & 3.56194300 & -3.03771200 & 4.74102400 & $\mathrm{C}$ & -4.88656100 & -2.19544200 & 0.81906000 \\
\hline $\mathrm{H}$ & 2.49408000 & -5.02796900 & 3.69876200 & $\mathrm{H}$ & -5.50346400 & -1.52828900 & 0.20678300 \\
\hline $\mathrm{C}$ & 4.78973900 & -1.00745100 & -0.63227200 & $\mathrm{H}$ & -5.36478400 & -3.17972800 & 0.79071000 \\
\hline $\mathrm{O}$ & 5.02457600 & -0.08240800 & -1.38792000 & $\mathrm{H}$ & -4.91909600 & -1.85807100 & 1.85818000 \\
\hline $\mathrm{O}$ & 5.21284500 & -2.25884600 & -0.81339500 & $\mathrm{C}$ & -3.48630100 & -2.91976600 & -1.14409000 \\
\hline $\mathrm{C}$ & 6.01926500 & -2.48769200 & -1.99394000 & $\mathrm{H}$ & -3.93554700 & -3.91676900 & -1.09587500 \\
\hline $\mathrm{H}$ & 6.27337200 & -3.54559200 & -1.96601500 & $\mathrm{H}$ & -4.09915000 & -2.32481300 & -1.82832300 \\
\hline $\mathrm{H}$ & 5.44928500 & -2.24594800 & -2.89340200 & $\mathrm{H}$ & -2.48653100 & -3.03511800 & -1.57182700 \\
\hline \multirow[t]{2}{*}{$\mathrm{H}$} & 6.91825300 & -1.86985600 & -1.95865800 & $\mathrm{C}$ & 0.91754600 & 2.45950600 & 0.83144800 \\
\hline & & & & $\mathrm{C}$ & 1.79469500 & 2.55322400 & -0.25658300 \\
\hline \multirow{2}{*}{\multicolumn{4}{|c|}{$\begin{array}{l}\text { TS17-2H } \\
\text { imaginary frequency }=-16.15 \mathrm{~cm}^{-1}\end{array}$}} & $\mathrm{C}$ & 0.62128600 & 4.74103000 & 0.88335500 \\
\hline & & & & $\mathrm{C}$ & 2.09560000 & 3.81830600 & -0.76422100 \\
\hline $\mathrm{C}$ & -0.83030200 & 1.28246800 & 2.78072100 & $\mathrm{H}$ & 2.23806800 & 1.66561600 & -0.69294600 \\
\hline $\mathrm{H}$ & -0.84038500 & 0.53703400 & 3.58165700 & $\mathrm{C}$ & 1.50235500 & 4.93697700 & -0.18241500 \\
\hline $\mathrm{H}$ & -0.51651900 & 2.23676700 & 3.20719400 & $\mathrm{H}$ & 0.13090700 & 5.58688900 & 1.35957300 \\
\hline $\mathrm{C}$ & -2.24717800 & 1.45831500 & 2.22014000 & $\mathrm{H}$ & 2.78272200 & 3.92199900 & -1.59825000 \\
\hline $\mathrm{H}$ & -2.83756700 & 1.97891500 & 2.98257000 & $\mathrm{H}$ & 1.71107000 & 5.93899900 & -0.54200700 \\
\hline
\end{tabular}




\begin{tabular}{|c|c|c|c|c|c|c|c|}
\hline $\mathrm{C}$ & -3.51557600 & 0.41568100 & -0.92877900 & $\mathrm{H}$ & -3.96651400 & -1.48699500 & -1.30872500 \\
\hline $\mathrm{C}$ & -3.28674200 & 0.38746200 & -2.30436000 & $\mathrm{C}$ & -2.02715500 & -1.61953500 & -2.26745100 \\
\hline $\mathrm{C}$ & -5.23529300 & 2.05854300 & -1.29129400 & $\mathrm{H}$ & -2.61416600 & -2.00824900 & -3.10717000 \\
\hline $\mathrm{C}$ & -4.04767300 & 1.17908800 & -3.16829200 & $\mathrm{H}$ & -1.77190700 & -0.58842100 & -2.53836600 \\
\hline $\mathrm{H}$ & -2.50094700 & -0.25107000 & -2.68859600 & $\mathrm{C}$ & -0.74368200 & -2.47312000 & -2.18553700 \\
\hline $\mathrm{C}$ & -5.03346700 & 2.03065100 & -2.65842900 & $\mathrm{H}$ & -0.22265800 & -2.44246000 & -3.14782700 \\
\hline $\mathrm{H}$ & -5.96746400 & 2.68986500 & -0.80098600 & $\mathrm{H}$ & -1.01419300 & -3.51947700 & -2.01083300 \\
\hline $\mathrm{H}$ & -3.86589700 & 1.13847000 & -4.23787000 & $\mathrm{P}$ & 0.42522600 & -1.95306600 & -0.82708100 \\
\hline $\mathrm{H}$ & -5.63047500 & 2.66426700 & -3.30403500 & $\mathrm{P}$ & -2.54537900 & -0.28634800 & 0.21520800 \\
\hline$N$ & 0.33050500 & 3.53268300 & 1.38295100 & $\mathrm{C}$ & -3.62408500 & -0.62545200 & 1.74611800 \\
\hline $\mathrm{N}$ & -4.48984000 & 1.26013800 & -0.49005200 & $\mathrm{C}$ & 0.97998700 & -3.56327000 & 0.03204700 \\
\hline Pd & -0.32341600 & -0.71931800 & -0.24780200 & $\mathrm{C}$ & -2.96164400 & -1.76687400 & 2.54178600 \\
\hline $\mathrm{H}$ & -1.02231300 & -1.45747800 & -1.42084200 & $\mathrm{H}$ & -3.53507800 & -1.95023900 & 3.45741700 \\
\hline $\mathrm{C}$ & 2.03862900 & -1.66847800 & -1.38235800 & $\mathrm{H}$ & -2.94476000 & -2.70547300 & 1.97859600 \\
\hline $\mathrm{C}$ & 0.88299400 & -2.02674400 & -2.04617500 & $\mathrm{H}$ & -1.93635800 & -1.51672400 & 2.83306300 \\
\hline $\mathrm{H}$ & 0.63170900 & -1.59723400 & -3.00791400 & $\mathrm{C}$ & -5.06224500 & -1.01879700 & 1.35889100 \\
\hline $\mathrm{H}$ & 0.40491700 & -2.96701600 & -1.80025000 & $\mathrm{H}$ & -5.65506900 & -1.14072000 & 2.27279400 \\
\hline $\mathrm{C}$ & 2.99770100 & -0.66112500 & -1.89375100 & $\mathrm{H}$ & -5.54647700 & -0.26013100 & 0.73983400 \\
\hline $\mathrm{C}$ & 4.30160800 & -0.53666800 & -1.37249100 & $\mathrm{H}$ & -5.09776800 & -1.96981300 & 0.81951400 \\
\hline $\mathrm{C}$ & 2.63280400 & 0.18867900 & -2.96235600 & $\mathrm{C}$ & -3.64212500 & 0.65336700 & 2.60620200 \\
\hline $\mathrm{C}$ & 5.20263800 & 0.38336600 & -1.90808600 & $\mathrm{H}$ & -4.13001200 & 1.48686400 & 2.09296400 \\
\hline $\mathrm{H}$ & 4.63060400 & -1.18316600 & -0.57149700 & $\mathrm{H}$ & -4.20298000 & 0.45737600 & 3.52656700 \\
\hline $\mathrm{C}$ & 3.53286900 & 1.10535700 & -3.49241400 & $\mathrm{H}$ & -2.63361700 & 0.96576100 & 2.89311600 \\
\hline $\mathrm{H}$ & 1.63600800 & 0.14126700 & -3.38771100 & $\mathrm{C}$ & -0.24628700 & -4.12727100 & 0.77716000 \\
\hline $\mathrm{C}$ & 4.82708700 & 1.20603200 & -2.96907100 & $\mathrm{H}$ & -0.64330400 & -3.40992400 & 1.50024000 \\
\hline $\mathrm{H}$ & 6.20551800 & 0.44608400 & -1.49810800 & $\mathrm{H}$ & -1.05283500 & -4.42012000 & 0.09681600 \\
\hline $\mathrm{H}$ & 3.23064800 & 1.73554400 & -4.32313400 & $\mathrm{H}$ & 0.05201700 & -5.02650700 & 1.32722000 \\
\hline $\mathrm{H}$ & 5.53406800 & 1.91318500 & -3.39134000 & $\mathrm{C}$ & 1.51465300 & -4.60505900 & -0.96674100 \\
\hline $\mathrm{C}$ & 2.33547500 & -2.62233700 & -0.23457700 & $\mathrm{H}$ & 2.42623900 & -4.26544400 & -1.46628300 \\
\hline $\mathrm{O}$ & 1.48583800 & -2.93554500 & 0.58363500 & $\mathrm{H}$ & 1.76653500 & -5.52363600 & -0.42473500 \\
\hline $\mathrm{O}$ & 3.55727000 & -3.13981300 & -0.28312600 & $\mathrm{H}$ & 0.77807800 & -4.87378800 & -1.73038200 \\
\hline $\mathrm{C}$ & 3.87152600 & -4.16192200 & 0.70246400 & $\mathrm{C}$ & 2.07587500 & -3.20526100 & 1.05510400 \\
\hline $\mathrm{H}$ & 3.17884000 & -4.99851500 & 0.60143300 & $\mathrm{H}$ & 2.37016800 & -4.11244600 & 1.59479300 \\
\hline $\mathrm{H}$ & 4.88990700 & -4.46967300 & 0.47530500 & $\mathrm{H}$ & 2.97350800 & -2.80885300 & 0.56975200 \\
\hline $\mathrm{H}$ & 3.80250600 & -3.74442100 & 1.70824100 & $\mathrm{H}$ & 1.73009000 & -2.47643500 & 1.79441100 \\
\hline \multirow[t]{2}{*}{$\mathrm{H}$} & -4.67061000 & 1.30596200 & 0.50884100 & $\mathrm{C}$ & -3.34696300 & 1.16782600 & -0.63171600 \\
\hline & & & & $\mathrm{C}$ & -2.83311900 & 2.46318500 & -0.50432800 \\
\hline \multicolumn{4}{|c|}{$\mathbf{T S}$} & $\mathrm{C}$ & -5.07640100 & 1.91088700 & -1.95720600 \\
\hline \multicolumn{4}{|c|}{ imaginary frequency $=-1283.73 \mathrm{~cm}^{-1}$} & $\mathrm{C}$ & -3.49937600 & 3.51526300 & -1.13552900 \\
\hline $\mathrm{C}$ & -2.92739400 & -1.63701500 & -1.00778200 & $\mathrm{H}$ & -1.93256700 & 2.63980500 & 0.07005400 \\
\hline $\mathrm{H}$ & -2.86106300 & -2.60133800 & -0.49258000 & $\mathrm{C}$ & -4.64476700 & 3.23691800 & -1.87730500 \\
\hline
\end{tabular}




\begin{tabular}{|c|c|c|c|c|c|c|c|}
\hline $\mathrm{H}$ & -5.96288000 & 1.65044000 & -2.53128500 & $\mathrm{C}$ & 3.36953700 & -1.35352300 & -0.08497200 \\
\hline $\mathrm{H}$ & -3.12604700 & 4.53139800 & -1.05022900 & $\mathrm{H}$ & 3.49846500 & -2.32341900 & -0.57897100 \\
\hline $\mathrm{H}$ & -5.19298200 & 4.02222000 & -2.38713100 & $\mathrm{H}$ & 3.77302900 & -1.42144100 & 0.92911000 \\
\hline $\mathrm{C}$ & 1.96966500 & -1.31745600 & -1.64826700 & $\mathrm{C}$ & 1.87744700 & -0.99559600 & -0.02477800 \\
\hline $\mathrm{C}$ & 2.56595600 & -1.82744900 & -2.80371500 & $\mathrm{H}$ & 1.76391900 & -0.02247300 & 0.46614600 \\
\hline C & 3.67368400 & 0.26353800 & -1.41480300 & $\mathrm{H}$ & 1.47480500 & -0.89152800 & -1.04006300 \\
\hline C & 3.75251600 & -1.25445400 & -3.26777000 & $\mathrm{C}$ & 1.07033600 & -2.05626700 & 0.74572900 \\
\hline $\mathrm{H}$ & 2.11661000 & -2.65910600 & -3.33476400 & $\mathrm{H}$ & 1.44563700 & -2.15414400 & 1.77043000 \\
\hline C & 4.32243100 & -0.19895200 & -2.55833200 & $\mathrm{H}$ & 1.19009100 & -3.03823000 & 0.27249200 \\
\hline $\mathrm{H}$ & 4.05719400 & 1.08769400 & -0.82402900 & $\mathrm{P}$ & -0.75236500 & -1.71999900 & 0.77179200 \\
\hline $\mathrm{H}$ & 4.22564600 & -1.63291800 & -4.16864900 & $\mathrm{P}$ & 4.34428900 & -0.09563000 & -1.07310200 \\
\hline $\mathrm{H}$ & 5.24463400 & 0.26890000 & -2.88461200 & $\mathrm{C}$ & 6.08992900 & -0.89339000 & -1.13175900 \\
\hline $\mathrm{N}$ & -4.44850600 & 0.89679200 & -1.35136100 & $\mathrm{C}$ & -1.58107700 & -3.14606000 & 1.71850600 \\
\hline $\mathrm{N}$ & 2.52434500 & -0.27958200 & -0.99141000 & $\mathrm{C}$ & 6.00731300 & -2.05914000 & -2.13878800 \\
\hline $\mathrm{Pd}$ & -0.20139800 & -0.08416300 & 0.50370600 & $\mathrm{H}$ & 7.00091500 & -2.50476200 & -2.26778600 \\
\hline C & 1.45117100 & 1.31383900 & 1.29687000 & $\mathrm{H}$ & 5.33898200 & -2.85707500 & -1.79846900 \\
\hline $\mathrm{C}$ & 0.09311900 & 1.52931800 & 1.78832700 & $\mathrm{H}$ & 5.66273100 & -1.72163200 & -3.12157000 \\
\hline $\mathrm{H}$ & 1.67161200 & 0.26351500 & 0.13862300 & $\mathrm{C}$ & 6.58440000 & -1.40547100 & 0.23088800 \\
\hline $\mathrm{H}$ & -0.37733500 & 2.47282600 & 1.53302100 & $\mathrm{H}$ & 7.60501800 & -1.79567700 & 0.13170900 \\
\hline $\mathrm{H}$ & -0.13525000 & 1.15102500 & 2.78224100 & $\mathrm{H}$ & 6.60188900 & -0.61215100 & 0.98371200 \\
\hline $\mathrm{C}$ & 2.02129100 & 2.47035500 & 0.49423000 & $\mathrm{H}$ & 5.96264100 & -2.22037100 & 0.61385700 \\
\hline $\mathrm{C}$ & 1.44814900 & 2.80699600 & -0.74183600 & C & 7.07058900 & 0.16223900 & -1.67821200 \\
\hline $\mathrm{C}$ & 3.03963300 & 3.29127000 & 1.00297900 & $\mathrm{H}$ & 7.20686800 & 0.99464500 & -0.98168000 \\
\hline C & 1.87999300 & 3.92628300 & -1.45410800 & $\mathrm{H}$ & 8.05358400 & -0.29742600 & -1.83534000 \\
\hline $\mathrm{H}$ & 0.65508300 & 2.18240300 & -1.14633600 & $\mathrm{H}$ & 6.73765400 & 0.56815600 & -2.63971700 \\
\hline C & 3.47356000 & 4.40920600 & 0.28805200 & $\mathrm{C}$ & -1.56640000 & -4.37488800 & 0.78478200 \\
\hline H & 3.49589800 & 3.06130300 & 1.95869900 & $\mathrm{H}$ & -2.12135800 & -4.18802900 & -0.13906000 \\
\hline C & 2.89896400 & 4.73118700 & -0.94251000 & $\mathrm{H}$ & -0.55162500 & -4.69292100 & 0.52487800 \\
\hline $\mathrm{H}$ & 1.42217000 & 4.16609600 & -2.40950700 & $\mathrm{H}$ & -2.04782200 & -5.21579400 & 1.29554600 \\
\hline $\mathrm{H}$ & 4.25999600 & 5.03351400 & 0.70165300 & $\mathrm{C}$ & -0.85338500 & -3.47971300 & 3.03268400 \\
\hline $\mathrm{H}$ & 3.23999100 & 5.60144900 & -1.49474900 & $\mathrm{H}$ & -0.84432200 & -2.63377500 & 3.72604500 \\
\hline $\mathrm{C}$ & 2.32392400 & 0.53159700 & 2.26123100 & $\mathrm{H}$ & -1.38064100 & -4.29976100 & 3.53272900 \\
\hline $\mathrm{O}$ & 1.89614600 & -0.11218700 & 3.19853400 & $\mathrm{H}$ & 0.17633800 & -3.80971900 & 2.86812700 \\
\hline $\mathrm{O}$ & 3.63108400 & 0.56970400 & 1.93064100 & $\mathrm{C}$ & -3.03823200 & -2.73546500 & 2.00855300 \\
\hline $\mathrm{C}$ & 4.51338300 & -0.16901300 & 2.80324100 & $\mathrm{H}$ & -3.56712900 & -3.58484300 & 2.45468400 \\
\hline $\mathrm{H}$ & 4.27893600 & -1.23512600 & 2.76907000 & $\mathrm{H}$ & -3.09322900 & -1.90580200 & 2.72041800 \\
\hline $\mathrm{H}$ & 5.51816500 & 0.01733800 & 2.42616300 & $\mathrm{H}$ & -3.57895700 & -2.44700900 & 1.10141600 \\
\hline \multirow[t]{2}{*}{$\mathrm{H}$} & 4.41488100 & 0.18419500 & 3.83135600 & $\mathrm{C}$ & 4.54405100 & 1.23090600 & 0.22631000 \\
\hline & & & & $\mathrm{C}$ & 5.00251600 & 2.49923000 & -0.16858100 \\
\hline \multicolumn{4}{|c|}{ TS18a } & $\mathrm{C}$ & 4.35143300 & 1.93943200 & 2.41249900 \\
\hline \multicolumn{4}{|c|}{ imaginary frequency $=-1111.82 \mathrm{~cm}^{-1}$} & $\mathrm{C}$ & 5.16061000 & 3.49820700 & 0.78942500 \\
\hline
\end{tabular}




\begin{tabular}{|c|c|c|c|c|c|c|c|}
\hline $\mathrm{H}$ & 5.22764400 & 2.69281000 & -1.21277700 & $\mathrm{H}$ & -6.93090200 & 0.87177100 & 0.65632000 \\
\hline $\mathrm{C}$ & 4.83118100 & 3.21433300 & 2.11477300 & & & & \\
\hline $\mathrm{H}$ & 4.07282600 & 1.68307600 & 3.43377400 & \multirow{2}{*}{\multicolumn{4}{|c|}{$\begin{array}{l}\text { TS18b } \\
\text { imaginary frequency }=-1258.62 \mathrm{~cm}^{-1}\end{array}$}} \\
\hline $\mathrm{H}$ & 5.52744500 & 4.48063700 & 0.50644200 & & & & \\
\hline $\mathrm{H}$ & 4.93634400 & 3.95924600 & 2.89691500 & $\mathrm{C}$ & -0.14863400 & 1.99024000 & 0.53042000 \\
\hline $\mathrm{C}$ & -0.93865500 & -0.21003700 & 1.84035800 & $\mathrm{H}$ & -0.83118700 & 2.61045300 & -0.06390900 \\
\hline $\mathrm{C}$ & -0.23785300 & 0.02750100 & 3.02659200 & $\mathrm{H}$ & 0.11933500 & 1.13902000 & -0.10312700 \\
\hline $\mathrm{C}$ & -2.07255700 & 1.81754000 & 2.05133200 & $\mathrm{C}$ & -0.86498400 & 1.54018800 & 1.82370000 \\
\hline $\mathrm{C}$ & -0.48170200 & 1.20593600 & 3.73486200 & $\mathrm{H}$ & -0.76271000 & 2.32665400 & 2.58141100 \\
\hline $\mathrm{H}$ & 0.49210300 & -0.68635700 & 3.39004500 & $\mathrm{H}$ & -0.37179200 & 0.65021400 & 2.22692400 \\
\hline $\mathrm{C}$ & -1.41865200 & 2.11404400 & 3.24532500 & $\mathrm{C}$ & -2.38255000 & 1.28495900 & 1.65948300 \\
\hline $\mathrm{H}$ & -2.80047100 & 2.48904000 & 1.60937500 & $\mathrm{H}$ & -2.84773800 & 1.22354400 & 2.64927900 \\
\hline $\mathrm{H}$ & 0.05672600 & 1.41008000 & 4.65510300 & $\mathrm{H}$ & -2.83061300 & 2.14935700 & 1.15821100 \\
\hline $\mathrm{H}$ & -1.63311300 & 3.04101600 & 3.76554600 & $\mathrm{P}$ & -2.88290200 & -0.23460100 & 0.67493000 \\
\hline $\mathrm{N}$ & 4.20893200 & 0.96575900 & 1.50319200 & $\mathrm{C}$ & -3.57226100 & -1.49398100 & 1.93001900 \\
\hline N & -1.82659600 & 0.68684100 & 1.37501400 & $\mathrm{C}$ & -4.36257000 & 0.31189700 & -0.29828600 \\
\hline $\mathrm{Pd}$ & -1.89842100 & -1.07762100 & -1.27118200 & $\mathrm{C}$ & -5.24607800 & 1.31823100 & 0.11557900 \\
\hline $\mathrm{C}$ & -3.30142500 & 0.77215900 & -1.19074700 & $\mathrm{C}$ & -5.58791500 & -0.06907900 & -2.21196100 \\
\hline $\mathrm{C}$ & -3.27612800 & 0.01344000 & -2.42463900 & $\mathrm{C}$ & -6.33734700 & 1.62814300 & -0.69736700 \\
\hline $\mathrm{H}$ & -2.37287800 & 0.32743700 & -0.06455900 & $\mathrm{H}$ & -5.09910100 & 1.84368000 & 1.05288700 \\
\hline $\mathrm{C}$ & -1.34454000 & -2.39804500 & -2.61092200 & $\mathrm{C}$ & -6.51559000 & 0.92173400 & -1.88494300 \\
\hline $\mathrm{O}$ & -0.98911600 & -3.16447100 & -3.37695000 & $\mathrm{H}$ & -5.68896700 & -0.64293600 & -3.13010400 \\
\hline $\mathrm{H}$ & -2.80167200 & 0.46752000 & -3.28912300 & $\mathrm{H}$ & -7.03688800 & 2.40466300 & -0.40292300 \\
\hline $\mathrm{H}$ & -4.09497500 & -0.67225900 & -2.62597000 & $\mathrm{H}$ & -7.35017300 & 1.13016700 & -2.54594400 \\
\hline $\mathrm{C}$ & -2.75738200 & 2.18727300 & -1.29351300 & $\mathrm{~N}$ & -4.53181900 & -0.36713200 & -1.44542800 \\
\hline $\mathrm{C}$ & -3.61980300 & 3.28679600 & -1.42411300 & $\mathrm{Pd}$ & -0.95295900 & -0.93462100 & -0.74335200 \\
\hline $\mathrm{C}$ & -1.37507100 & 2.39986600 & -1.38386800 & $\mathrm{C}$ & -4.85196900 & -0.98465300 & 2.61535100 \\
\hline $\mathrm{C}$ & -3.10253800 & 4.56647600 & -1.62818300 & $\mathrm{H}$ & -5.67554200 & -0.85239600 & 1.90946600 \\
\hline $\mathrm{H}$ & -4.69304600 & 3.14654000 & -1.37004200 & $\mathrm{H}$ & -5.17163700 & -1.72024600 & 3.36233800 \\
\hline $\mathrm{C}$ & -0.85990900 & 3.67958700 & -1.59362900 & $\mathrm{H}$ & -4.69649400 & -0.03801300 & 3.14370700 \\
\hline $\mathrm{H}$ & -0.69528200 & 1.55583800 & -1.28904500 & $\mathrm{C}$ & -2.48347000 & -1.76290100 & 2.98688700 \\
\hline $\mathrm{C}$ & -1.72372400 & 4.76852700 & -1.71270900 & $\mathrm{H}$ & -1.54700700 & -2.08591900 & 2.52782200 \\
\hline $\mathrm{H}$ & -3.78323800 & 5.40616800 & -1.73061500 & $\mathrm{H}$ & -2.28292100 & -0.88132700 & 3.60508600 \\
\hline $\mathrm{H}$ & 0.21426300 & 3.82189700 & -1.66259600 & $\mathrm{H}$ & -2.83105000 & -2.55553200 & 3.65953500 \\
\hline $\mathrm{H}$ & -1.32704500 & 5.76598100 & -1.87454400 & $\mathrm{C}$ & -3.87278600 & -2.78869300 & 1.14844100 \\
\hline $\mathrm{C}$ & -4.52184700 & 0.42908500 & -0.33373300 & $\mathrm{H}$ & -2.96605600 & -3.20690300 & 0.70055000 \\
\hline $\mathrm{O}$ & -5.03601100 & -0.67001000 & -0.33758600 & $\mathrm{H}$ & -4.28612100 & -3.53709200 & 1.83406700 \\
\hline $\mathrm{O}$ & -4.92943400 & 1.44287300 & 0.44030900 & $\mathrm{H}$ & -4.60233700 & -2.62682700 & 0.34970900 \\
\hline $\mathrm{C}$ & -6.08095000 & 1.16584800 & 1.27410800 & $\mathrm{C}$ & -1.66357200 & -0.43599700 & -2.42352800 \\
\hline $\mathrm{H}$ & -5.85123200 & 0.36246600 & 1.97678600 & $\mathrm{O}$ & -2.03163700 & -0.14833200 & -3.46545600 \\
\hline $\mathrm{H}$ & -6.28742100 & 2.09696900 & 1.79920500 & $\mathrm{C}$ & 1.93399100 & -1.39002900 & -0.55821600 \\
\hline
\end{tabular}




\begin{tabular}{|c|c|c|c|c|c|c|c|}
\hline $\mathrm{P}$ & 1.30551000 & 3.12502900 & 0.83660500 & $\mathrm{O}$ & 2.11696200 & -2.37967400 & 1.60269100 \\
\hline $\mathrm{C}$ & 2.74858700 & 2.01628200 & 1.29532800 & $\mathrm{C}$ & 1.50436600 & -2.72295400 & 2.86055500 \\
\hline $\mathrm{C}$ & 3.64091200 & 2.62177400 & 2.19324200 & $\mathrm{H}$ & 0.72261000 & -3.47134200 & 2.71670500 \\
\hline $\mathrm{C}$ & 4.79527400 & 1.96784100 & 2.61937500 & $\mathrm{H}$ & 2.31063200 & -3.13268500 & 3.46796500 \\
\hline $\mathrm{H}$ & 3.40972900 & 3.61718000 & 2.55609600 & $\mathrm{H}$ & 1.07832200 & -1.83916200 & 3.34098800 \\
\hline $\mathrm{C}$ & 4.12658900 & 0.10351400 & 1.29051700 & & & & \\
\hline $\mathrm{C}$ & 5.04270300 & 0.67767700 & 2.15970200 & \multirow{2}{*}{\multicolumn{4}{|c|}{$\begin{array}{l}\text { TS18-2H } \\
\text { imaginary frequency }=-1233.70 \mathrm{~cm}^{-1}\end{array}$}} \\
\hline $\mathrm{H}$ & 5.47981900 & 2.45571100 & 3.30636200 & & & & \\
\hline $\mathrm{H}$ & 4.25275300 & -0.90120300 & 0.90798600 & $\mathrm{C}$ & -2.88313300 & -1.66872400 & -1.06252400 \\
\hline $\mathrm{H}$ & 5.91890400 & 0.11724000 & 2.46481000 & $\mathrm{H}$ & -2.78301200 & -2.63182500 & -0.54950900 \\
\hline $\mathrm{N}$ & 3.02374700 & 0.75403100 & 0.86292200 & $\mathrm{H}$ & -3.92903200 & -1.61904700 & -1.38461800 \\
\hline $\mathrm{C}$ & 1.78670700 & 3.72924300 & -0.91893200 & $\mathrm{C}$ & -1.95260600 & -1.61295100 & -2.30609500 \\
\hline $\mathrm{C}$ & 0.68600800 & 4.72654000 & -1.34135000 & $\mathrm{H}$ & -2.52433700 & -1.98357500 & -3.16386400 \\
\hline $\mathrm{H}$ & 0.95851500 & 5.18140000 & -2.30058200 & $\mathrm{H}$ & -1.69274800 & -0.57496800 & -2.54679300 \\
\hline $\mathrm{H}$ & -0.28665900 & 4.24357100 & -1.48084400 & $\mathrm{C}$ & -0.66815500 & -2.46536300 & -2.21900700 \\
\hline $\mathrm{H}$ & 0.56627800 & 5.53403700 & -0.61224500 & $\mathrm{H}$ & -0.14028400 & -2.40753200 & -3.17585500 \\
\hline $\mathrm{C}$ & 1.91400900 & 2.61269400 & -1.96573500 & $\mathrm{H}$ & -0.94129300 & -3.51607700 & -2.08041000 \\
\hline $\mathrm{H}$ & 0.97322000 & 2.07248600 & -2.10768800 & $\mathrm{P}$ & 0.49484300 & -1.98385900 & -0.83674500 \\
\hline $\mathrm{H}$ & 2.19162900 & 3.04465500 & -2.93504000 & $\mathrm{P}$ & -2.52103000 & -0.35991400 & 0.22331800 \\
\hline $\mathrm{H}$ & 2.69044700 & 1.89052800 & -1.69604600 & $\mathrm{C}$ & -3.64683900 & -0.70627400 & 1.72663000 \\
\hline $\mathrm{C}$ & 3.12270000 & 4.48845700 & -0.79695500 & $\mathrm{C}$ & 1.01626300 & -3.61246700 & 0.00768700 \\
\hline $\mathrm{H}$ & 3.36241700 & 4.96090200 & -1.75630800 & $\mathrm{C}$ & -2.98776700 & -1.88738500 & 2.47160800 \\
\hline $\mathrm{H}$ & 3.07360900 & 5.28007800 & -0.04207300 & $\mathrm{H}$ & -3.54752900 & -2.08009200 & 3.39280800 \\
\hline $\mathrm{H}$ & 3.95376900 & 3.82328000 & -0.54364800 & $\mathrm{H}$ & -3.00296800 & -2.81166800 & 1.88601300 \\
\hline $\mathrm{H}$ & 2.36358300 & -0.03459200 & 0.14312300 & $\mathrm{H}$ & -1.95390600 & -1.66556000 & 2.75258300 \\
\hline $\mathrm{C}$ & 0.89322100 & -1.24045000 & -1.67446000 & $\mathrm{C}$ & -5.09620900 & -1.07441000 & 1.35560700 \\
\hline $\mathrm{H}$ & 0.78165400 & -2.16921200 & -2.24656900 & $\mathrm{H}$ & -5.62919600 & -1.36457700 & 2.26728800 \\
\hline $\mathrm{H}$ & 1.11277100 & -0.42864300 & -2.37006300 & $\mathrm{H}$ & -5.65574000 & -0.22605500 & 0.94525900 \\
\hline $\mathrm{C}$ & 3.23921500 & -1.93692200 & -1.08183400 & $\mathrm{H}$ & -5.16276100 & -1.92478500 & 0.66993500 \\
\hline $\mathrm{C}$ & 3.96805900 & -1.17307300 & -2.01379300 & $\mathrm{C}$ & -3.63134900 & 0.54585200 & 2.62588200 \\
\hline $\mathrm{C}$ & 3.74316600 & -3.20552800 & -0.73983300 & $\mathrm{H}$ & -4.10410000 & 1.41019200 & 2.14756400 \\
\hline $\mathrm{C}$ & 5.15534700 & -1.64630200 & -2.57050900 & $\mathrm{H}$ & -4.19412400 & 0.33393100 & 3.54063500 \\
\hline $\mathrm{H}$ & 3.59490800 & -0.19691000 & -2.31209300 & $\mathrm{H}$ & -2.61629100 & 0.82457500 & 2.92082800 \\
\hline $\mathrm{C}$ & 4.93790900 & -3.67434700 & -1.28887300 & $\mathrm{C}$ & -0.22311100 & -4.16857900 & 0.73582000 \\
\hline $\mathrm{H}$ & 3.20207300 & -3.82878000 & -0.03705400 & $\mathrm{H}$ & -0.60603800 & -3.46202200 & 1.47743200 \\
\hline $\mathrm{C}$ & 5.65021400 & -2.89967700 & -2.20560500 & $\mathrm{H}$ & -1.03215800 & -4.43621500 & 0.04700300 \\
\hline $\mathrm{H}$ & 5.69439100 & -1.03483400 & -3.28842500 & $\mathrm{H}$ & 0.05626600 & -5.08496800 & 1.26604600 \\
\hline $\mathrm{H}$ & 5.30501200 & -4.65710800 & -1.00700800 & $\mathrm{C}$ & 1.54216100 & -4.64580400 & -1.00497900 \\
\hline $\mathrm{H}$ & 6.57558200 & -3.27064400 & -2.63537700 & $\mathrm{H}$ & 2.45967800 & -4.31128900 & -1.49581000 \\
\hline $\mathrm{C}$ & 1.34131100 & -1.85726100 & 0.64366400 & $\mathrm{H}$ & 1.78313300 & -5.57181800 & -0.47169700 \\
\hline $\mathrm{O}$ & 0.10006700 & -1.67250100 & 0.90654900 & $\mathrm{H}$ & 0.80648600 & -4.90196600 & -1.77370800 \\
\hline
\end{tabular}




\begin{tabular}{|c|c|c|c|c|c|c|c|}
\hline $\mathrm{C}$ & 2.11232000 & -3.27937200 & 1.03929500 & $\mathrm{C}$ & 2.33609500 & 0.56250400 & 2.24194400 \\
\hline $\mathrm{H}$ & 2.38116400 & -4.19471300 & 1.57736500 & $\mathrm{O}$ & 1.89594200 & -0.23953900 & 3.04123000 \\
\hline $\mathrm{H}$ & 3.02229500 & -2.90673800 & 0.55920000 & $\mathrm{O}$ & 3.63399100 & 0.83213700 & 2.07981000 \\
\hline $\mathrm{H}$ & 1.78271300 & -2.54358300 & 1.77908300 & $\mathrm{C}$ & 4.54139100 & 0.14302400 & 2.97995700 \\
\hline $\mathrm{C}$ & -3.29804200 & 1.14307600 & -0.60410900 & $\mathrm{H}$ & 4.46370200 & -0.93616700 & 2.83743100 \\
\hline $\mathrm{C}$ & -2.62509500 & 2.35498600 & -0.73589700 & $\mathrm{H}$ & 5.53449600 & 0.50152700 & 2.71599500 \\
\hline $\mathrm{C}$ & -5.19440500 & 2.12408300 & -1.71549900 & $\mathrm{H}$ & 4.30083200 & 0.39107600 & 4.01475700 \\
\hline $\mathrm{C}$ & -3.24242500 & 3.45130300 & -1.34950300 & $\mathrm{H}$ & -5.07934100 & 0.21948000 & -1.02542700 \\
\hline $\mathrm{H}$ & -1.61024100 & 2.42723500 & -0.36412500 & & & & \\
\hline C & -4.54358700 & 3.33873600 & -1.84499000 & \multirow{2}{*}{\multicolumn{4}{|c|}{$\begin{array}{l}\text { TS19 } \\
\text { imaginary frequency }=-1362.42 \mathrm{~cm}^{-1}\end{array}$}} \\
\hline $\mathrm{H}$ & -6.20172200 & 1.93874200 & -2.07091300 & & & & \\
\hline $\mathrm{H}$ & -2.70483500 & 4.39014700 & -1.44138900 & $\mathrm{C}$ & 0.19169900 & -2.98438200 & -1.04929000 \\
\hline $\mathrm{H}$ & -5.04712400 & 4.16987500 & -2.32501000 & $\mathrm{H}$ & 0.71014200 & -3.53524800 & -0.25662300 \\
\hline $\mathrm{C}$ & 2.04896800 & -1.32772600 & -1.61330200 & $\mathrm{H}$ & -0.47185000 & -3.68085400 & -1.56379500 \\
\hline $\mathrm{C}$ & 2.67770000 & -1.84209100 & -2.74919700 & $\mathrm{C}$ & 1.20065400 & -2.41782300 & -2.07254400 \\
\hline $\mathrm{C}$ & 3.75485900 & 0.23584900 & -1.31683800 & $\mathrm{H}$ & 1.37696400 & -3.18455400 & -2.83516500 \\
\hline $\mathrm{C}$ & 3.88741600 & -1.28074300 & -3.16887100 & $\mathrm{H}$ & 0.76675600 & -1.56506200 & -2.60635500 \\
\hline $\mathrm{H}$ & 2.24175700 & -2.66959200 & -3.29779000 & $\mathrm{C}$ & 2.57410800 & -2.03707900 & -1.48626500 \\
\hline $\mathrm{C}$ & 4.44238200 & -0.23410200 & -2.43529500 & $\mathrm{H}$ & 3.25648500 & -1.75090800 & -2.29245200 \\
\hline $\mathrm{H}$ & 4.12703000 & 1.05800300 & -0.71540700 & $\mathrm{H}$ & 3.01830100 & -2.90973500 & -0.99622500 \\
\hline $\mathrm{H}$ & 4.38953400 & -1.66341300 & -4.05195900 & $\mathrm{P}$ & 2.48109100 & -0.66493800 & -0.22958900 \\
\hline $\mathrm{H}$ & 5.38307800 & 0.22144400 & -2.72413300 & $\mathrm{P}$ & -0.91242800 & -1.71983500 & -0.24205000 \\
\hline $\mathrm{N}$ & -4.56265200 & 1.09010200 & -1.11398000 & $\mathrm{C}$ & -1.88038200 & -2.71810900 & 1.06932700 \\
\hline $\mathrm{N}$ & 2.58505400 & -0.29421400 & -0.93318900 & $\mathrm{C}$ & 3.65849100 & -1.14866500 & 1.19142600 \\
\hline $\mathrm{Pd}$ & -0.20399300 & -0.11395100 & 0.50035500 & $\mathrm{C}$ & -0.96663500 & -2.89090800 & 2.29864800 \\
\hline $\mathrm{C}$ & 1.43867200 & 1.32633300 & 1.26666900 & $\mathrm{H}$ & -1.50883400 & -3.43901600 & 3.07792600 \\
\hline $\mathrm{C}$ & 0.08784400 & 1.50801300 & 1.78529500 & $\mathrm{H}$ & -0.06711100 & -3.47088300 & 2.06714500 \\
\hline $\mathrm{H}$ & 1.66413000 & 0.29400700 & 0.18676200 & $\mathrm{H}$ & -0.65657700 & -1.92789400 & 2.71647900 \\
\hline $\mathrm{H}$ & -0.41338400 & 2.44072500 & 1.54704800 & $\mathrm{C}$ & -2.29895200 & -4.09719000 & 0.52389000 \\
\hline $\mathrm{H}$ & -0.12499900 & 1.10697600 & 2.77433400 & $\mathrm{H}$ & -2.92779000 & -4.59783000 & 1.26915200 \\
\hline $\mathrm{C}$ & 1.95517600 & 2.50837000 & 0.46150400 & $\mathrm{H}$ & -2.87923600 & -4.01572400 & -0.39960100 \\
\hline $\mathrm{C}$ & 1.48151200 & 2.73352300 & -0.83954100 & $\mathrm{H}$ & -1.44114500 & -4.74776700 & 0.33346900 \\
\hline $\mathrm{C}$ & 2.81019300 & 3.45906800 & 1.03969100 & $\mathrm{C}$ & -3.13926400 & -1.92597200 & 1.46881500 \\
\hline $\mathrm{C}$ & 1.85423700 & 3.87536300 & -1.55188500 & $\mathrm{H}$ & -3.85823600 & -1.86623500 & 0.64763300 \\
\hline $\mathrm{H}$ & 0.83427400 & 1.99282500 & -1.30562700 & $\mathrm{H}$ & -3.63349000 & -2.44321800 & 2.29944700 \\
\hline $\mathrm{C}$ & 3.18318500 & 4.59903800 & 0.32595700 & $\mathrm{H}$ & -2.90688900 & -0.91014400 & 1.79749900 \\
\hline $\mathrm{H}$ & 3.18604500 & 3.31264300 & 2.04557800 & $\mathrm{C}$ & 3.02534100 & -2.35778800 & 1.90806400 \\
\hline $\mathrm{C}$ & 2.70925000 & 4.81213400 & -0.97011000 & $\mathrm{H}$ & 2.03325800 & -2.11699000 & 2.29883200 \\
\hline $\mathrm{H}$ & 1.48851100 & 4.02607100 & -2.56359300 & $\mathrm{H}$ & 2.94348900 & -3.23665300 & 1.26016900 \\
\hline $\mathrm{H}$ & 3.84406100 & 5.32493100 & 0.78940600 & $\mathrm{H}$ & 3.65847600 & -2.64159500 & 2.75584900 \\
\hline $\mathrm{H}$ & 3.00679500 & 5.69857700 & -1.52121700 & C & 5.06440500 & -1.51008800 & 0.68079100 \\
\hline
\end{tabular}




$\begin{array}{lrrrrrrr}\mathrm{H} & 5.56511300 & -0.65635600 & 0.21515700 & \mathrm{H} & -2.25763900 & 3.58340800 & -2.81725100 \\ \mathrm{H} & 5.68294100 & -1.82397400 & 1.52910100 & \mathrm{H} & -5.00138700 & 3.84896900 & 0.48064600 \\ \mathrm{H} & 5.05287800 & -2.33945600 & -0.03354400 & \mathrm{H} & -4.46632500 & 4.30256000 & -1.90731800 \\ \mathrm{C} & 3.73571600 & 0.03659700 & 2.17367600 & \mathrm{C} & -1.01736400 & 1.29921400 & 2.62469800 \\ \mathrm{H} & 4.37157600 & -0.24315900 & 3.02092800 & \mathrm{O} & -0.10155500 & 1.02017600 & 3.38525700 \\ \mathrm{H} & 4.18089900 & 0.92505100 & 1.71458300 & \mathrm{O} & -2.30925900 & 1.10251300 & 2.96469500 \\ \mathrm{H} & 2.75128600 & 0.30245200 & 2.57012700 & \mathrm{C} & -2.53068100 & 0.60106100 & 4.29981600 \\ \mathrm{C} & -2.14637800 & -1.42450700 & -1.60611900 & \mathrm{H} & -3.61296100 & 0.56117700 & 4.41670600 \\ \mathrm{C} & -3.03872300 & -0.34679500 & -1.54534500 & \mathrm{H} & -2.09439400 & -0.39328000 & 4.41447000 \\ \mathrm{C} & -3.03017100 & -2.13535700 & -3.60898900 & \mathrm{H} & -2.08541100 & 1.27270700 & 5.03610100\end{array}$

\section{TS19a} imaginary frequency $=-1236.90 \mathrm{~cm}^{-1}$

\begin{tabular}{lrrr}
$\mathrm{C}$ & -3.27213800 & -0.10951200 & -1.48211700 \\
$\mathrm{H}$ & -3.10558400 & -0.35684600 & -2.53683500 \\
$\mathrm{H}$ & -3.93828200 & 0.75612900 & -1.42659100 \\
$\mathrm{C}$ & -1.93280100 & 0.22281400 & -0.80797500 \\
$\mathrm{H}$ & -2.11150800 & 0.44354300 & 0.25027100 \\
$\mathrm{H}$ & -1.26828000 & -0.64902100 & -0.84300500 \\
$\mathrm{C}$ & -1.24285900 & 1.42776200 & -1.47396000 \\
$\mathrm{H}$ & -1.88610900 & 2.31375000 & -1.42968700 \\
$\mathrm{H}$ & -1.06678600 & 1.22196100 & -2.53640400 \\
$\mathrm{P}$ & 0.41679000 & 1.81621400 & -0.74572500 \\
$\mathrm{P}$ & -4.08866300 & -1.59515000 & -0.68302300 \\
$\mathrm{C}$ & -5.60726100 & -1.89308200 & -1.81952000 \\
$\mathrm{C}$ & 1.10981400 & 3.33836400 & -1.65133300 \\
$\mathrm{C}$ & -5.06515500 & -2.50286500 & -3.12832200 \\
$\mathrm{H}$ & -5.90214900 & -2.77465700 & -3.78253700 \\
$\mathrm{H}$ & -4.43649900 & -1.80070900 & -3.68569700 \\
$\mathrm{H}$ & -4.48067300 & -3.40941700 & -2.94058900 \\
$\mathrm{C}$ & -6.41743900 & -0.62280700 & -2.12212600 \\
$\mathrm{H}$ & -7.29417500 & -0.87808600 & -2.73056300 \\
$\mathrm{H}$ & -6.77457400 & -0.13947200 & -1.20850000 \\
$\mathrm{H}$ & -5.83514500 & 0.11140500 & -2.68699400 \\
$\mathrm{H}$ & -6.50772600 & -2.93348400 & -1.12593700 \\
$\mathrm{H}$ & -6.95378000 & -2.54183500 & -0.20706100 \\
$\mathrm{H}$ & -7.32978400 & -3.21256700 & -1.79577100 \\
$\mathrm{H}$ & -5.95669600 & -3.84742600 & -0.87902400 \\
$\mathrm{H}$ & 1.45199800 & 2.89276100 & -3.08887000 \\
$\mathrm{H}$ & 2.18401500 & 2.07996400 & -3.10042100 \\
\hline & & & -3.65104200
\end{tabular}




\begin{tabular}{|c|c|c|c|c|c|c|c|}
\hline $\mathrm{H}$ & 1.89197600 & 3.73920300 & -3.62707100 & $\mathrm{H}$ & 3.45160600 & -1.95702100 & 3.22025500 \\
\hline $\mathrm{C}$ & 0.10964000 & 4.50744600 & -1.69139900 & $\mathrm{C}$ & 3.39603100 & -4.58529700 & 0.38232300 \\
\hline $\mathrm{H}$ & -0.14448500 & 4.86865200 & -0.69065100 & $\mathrm{H}$ & 3.64280200 & -2.93867100 & -0.96688900 \\
\hline $\mathrm{H}$ & 0.56513500 & 5.34582100 & -2.23000600 & $\mathrm{C}$ & 3.25764900 & -4.99034500 & 1.71327300 \\
\hline $\mathrm{H}$ & -0.81451500 & 4.25044400 & -2.21745000 & $\mathrm{H}$ & 3.17979000 & -4.33562000 & 3.76526700 \\
\hline $\mathrm{C}$ & 2.39840900 & 3.76641500 & -0.92070900 & $\mathrm{H}$ & 3.39535900 & -5.32303000 & -0.41426300 \\
\hline $\mathrm{H}$ & 2.86100100 & 4.59578700 & -1.46692200 & $\mathrm{H}$ & 3.15096800 & -6.04306700 & 1.95574000 \\
\hline $\mathrm{H}$ & 2.19467600 & 4.11941500 & 0.09531000 & $\mathrm{C}$ & 4.88896300 & -0.30970900 & -0.01875400 \\
\hline $\mathrm{H}$ & 3.13247600 & 2.95590300 & -0.86444900 & $\mathrm{O}$ & 5.09410900 & 0.87309100 & -0.23915100 \\
\hline $\mathrm{C}$ & -4.84977700 & -0.76300100 & 0.80645900 & $\mathrm{O}$ & 5.69349700 & -1.29512600 & -0.42788800 \\
\hline $\mathrm{C}$ & -5.33872300 & -1.56691600 & 1.85022200 & $\mathrm{C}$ & 6.86121700 & -0.88510300 & -1.17273900 \\
\hline $\mathrm{C}$ & -5.42692900 & 1.14928500 & 1.95898800 & $\mathrm{H}$ & 7.40299600 & -1.80458800 & -1.38772600 \\
\hline $\mathrm{C}$ & -5.91560200 & -0.96403300 & 2.96574100 & $\mathrm{H}$ & 6.56506300 & -0.38366000 & -2.09699300 \\
\hline $\mathrm{H}$ & -5.26092000 & -2.64752000 & 1.78297800 & $\mathrm{H}$ & 7.47100400 & -0.20524900 & -0.57480900 \\
\hline $\mathrm{C}$ & -5.96667100 & 0.42865500 & 3.02364900 & & & & \\
\hline $\mathrm{H}$ & -5.43848700 & 2.23845800 & 1.97278300 & \multirow{2}{*}{\multicolumn{4}{|c|}{$\begin{array}{l}\text { TS19b } \\
\text { imaginary frequency }=-1391.89 \mathrm{~cm}^{-1}\end{array}$}} \\
\hline $\mathrm{H}$ & -6.31139200 & -1.56866500 & 3.77662500 & & & & \\
\hline $\mathrm{H}$ & -6.40627400 & 0.94488200 & 3.87100600 & $\mathrm{C}$ & 2.26161500 & -1.33975800 & 1.68621600 \\
\hline $\mathrm{C}$ & 0.03733000 & 2.38976400 & 0.98338600 & $\mathrm{H}$ & 2.27637400 & -1.43349900 & 2.77783100 \\
\hline $\mathrm{C}$ & -1.05385500 & 3.17793400 & 1.35946700 & $\mathrm{H}$ & 1.61346500 & -0.49454600 & 1.44437500 \\
\hline $\mathrm{C}$ & 0.79584300 & 2.31617900 & 3.19134900 & $\mathrm{C}$ & 1.73612900 & -2.64379700 & 1.05851400 \\
\hline $\mathrm{C}$ & -1.20389700 & 3.53593300 & 2.70104600 & $\mathrm{H}$ & 2.28871100 & -3.48349000 & 1.49373000 \\
\hline $\mathrm{H}$ & -1.78023900 & 3.49992800 & 0.62261700 & $\mathrm{H}$ & 1.97454400 & -2.65916900 & -0.00809300 \\
\hline $\mathrm{C}$ & -0.26131600 & 3.10671600 & 3.63467200 & $\mathrm{C}$ & 0.22853700 & -2.89539400 & 1.26630500 \\
\hline $\mathrm{H}$ & 1.55420300 & 1.94139000 & 3.87263600 & $\mathrm{H}$ & 0.03236000 & -3.97276200 & 1.28577200 \\
\hline $\mathrm{H}$ & -2.04848700 & 4.14299800 & 3.01145500 & $\mathrm{H}$ & -0.07666000 & -2.50400200 & 2.24282200 \\
\hline $\mathrm{H}$ & -0.34453700 & 3.36601400 & 4.68415200 & $\mathrm{P}$ & -0.92121500 & -2.12587300 & 0.01041800 \\
\hline $\mathrm{N}$ & -4.88196500 & 0.58154100 & 0.87458000 & $\mathrm{C}$ & -0.99910400 & -3.35511400 & -1.46063300 \\
\hline N & 0.92319700 & 1.96961500 & 1.90399700 & $\mathrm{C}$ & -2.57892500 & -2.32858800 & 0.83307200 \\
\hline $\mathrm{Pd}$ & 2.08218700 & 0.09328900 & -0.39641700 & $\mathrm{C}$ & -2.80005500 & -3.10303400 & 1.98002900 \\
\hline $\mathrm{C}$ & 3.10742400 & 0.21395100 & 1.61828300 & $\mathrm{C}$ & -4.81322900 & -1.75619500 & 0.72043900 \\
\hline $\mathrm{C}$ & 3.68681300 & -0.80252300 & 0.76029300 & $\mathrm{C}$ & -4.09253600 & -3.17840600 & 2.50287700 \\
\hline $\mathrm{H}$ & 1.93077700 & 1.02130400 & 1.36477600 & $\mathrm{H}$ & -1.99470300 & -3.64665400 & 2.45871900 \\
\hline $\mathrm{C}$ & 2.03341800 & -0.89476900 & -2.09666900 & $\mathrm{C}$ & -5.12309100 & -2.49360800 & 1.86440400 \\
\hline $\mathrm{O}$ & 1.91119400 & -1.42451000 & -3.10102300 & $\mathrm{H}$ & -5.58563700 & -1.20907000 & 0.18451100 \\
\hline $\mathrm{H}$ & 3.73096400 & 1.09347200 & 1.77319200 & $\mathrm{H}$ & -4.28692600 & -3.76894900 & 3.39310000 \\
\hline $\mathrm{H}$ & 2.62067800 & -0.17186200 & 2.51265400 & $\mathrm{H}$ & -6.14106800 & -2.52618600 & 2.23774500 \\
\hline $\mathrm{C}$ & 3.52782800 & -2.25544400 & 1.07770100 & $\mathrm{~N}$ & -3.57623200 & -1.66742700 & 0.22052400 \\
\hline $\mathrm{C}$ & 3.40285500 & -2.68025400 & 2.41215000 & $\mathrm{Pd}$ & -0.81911700 & 0.32497200 & -0.49699200 \\
\hline $\mathrm{C}$ & 3.53250900 & -3.23696100 & 0.06973500 & $\mathrm{C}$ & -1.56321600 & -4.72190600 & -1.03046600 \\
\hline $\mathrm{C}$ & 3.26683400 & -4.03300100 & 2.72620700 & $\mathrm{H}$ & -2.59721000 & -4.65111500 & -0.68363900 \\
\hline
\end{tabular}




\begin{tabular}{|c|c|c|c|c|c|c|c|}
\hline $\mathrm{H}$ & -1.55034300 & -5.39885800 & -1.89228100 & $\mathrm{C}$ & -0.85483600 & 3.28193800 & 2.11279200 \\
\hline $\mathrm{H}$ & -0.96726500 & -5.19539000 & -0.24389900 & $\mathrm{C}$ & -2.78990400 & 3.03169000 & 0.69778500 \\
\hline $\mathrm{C}$ & 0.41694100 & -3.54141400 & -2.04044800 & $\mathrm{C}$ & -1.66886200 & 3.75278600 & 3.14301300 \\
\hline $\mathrm{H}$ & 0.89329100 & -2.58664400 & -2.28638300 & $\mathrm{H}$ & 0.21769400 & 3.25217800 & 2.27112100 \\
\hline $\mathrm{H}$ & 1.07305600 & -4.09637400 & -1.36469800 & $\mathrm{C}$ & -3.60157800 & 3.49797100 & 1.72634200 \\
\hline $\mathrm{H}$ & 0.34520100 & -4.12038400 & -2.96808400 & $\mathrm{H}$ & -3.24012600 & 2.76691800 & -0.25079300 \\
\hline $\mathrm{C}$ & -1.90814000 & -2.74295000 & -2.54377700 & $\mathrm{C}$ & -3.04635200 & 3.85752000 & 2.95772500 \\
\hline $\mathrm{H}$ & -1.48910900 & -1.81255900 & -2.93985500 & $\mathrm{H}$ & -1.22010900 & 4.04823600 & 4.08653700 \\
\hline $\mathrm{H}$ & -1.99381000 & -3.44877900 & -3.37769300 & $\mathrm{H}$ & -4.67210600 & 3.58307000 & 1.56595000 \\
\hline $\mathrm{H}$ & -2.91425500 & -2.53904600 & -2.16987800 & $\mathrm{H}$ & -3.68114900 & 4.22352900 & 3.75875100 \\
\hline $\mathrm{C}$ & -2.57598200 & 0.40550600 & -1.40349100 & $\mathrm{C}$ & -0.54950000 & 3.05774500 & -1.59673600 \\
\hline $\mathrm{O}$ & -3.50285300 & 0.55224800 & -2.05451900 & $\mathrm{O}$ & 0.24003800 & 2.79383300 & -2.48964200 \\
\hline $\mathrm{C}$ & 0.80873200 & 1.83829400 & 0.09754400 & $\mathrm{O}$ & -1.53693800 & 3.94893500 & -1.73038800 \\
\hline $\mathrm{P}$ & 4.02312100 & -1.09731200 & 1.11083300 & $\mathrm{C}$ & -1.63728900 & 4.59167200 & -3.01986500 \\
\hline $\mathrm{C}$ & 3.72596400 & -0.49061900 & -0.63308900 & $\mathrm{H}$ & -2.46884100 & 5.28860000 & -2.92979300 \\
\hline $\mathrm{C}$ & 4.75751100 & -0.74880500 & -1.54974500 & $\mathrm{H}$ & -0.71088100 & 5.12044500 & -3.25139400 \\
\hline $\mathrm{C}$ & 4.61784600 & -0.40978700 & -2.89369500 & $\mathrm{H}$ & -1.83393700 & 3.85114600 & -3.79845300 \\
\hline $\mathrm{H}$ & 5.66079800 & -1.23405900 & -1.19578100 & $\mathrm{H}$ & 1.03917500 & 1.79373300 & 1.15632000 \\
\hline $\mathrm{C}$ & 2.43243500 & 0.40290700 & -2.37360700 & & & & \\
\hline
\end{tabular}

C

$\mathrm{H}$

H

H

N

C

C

H

H

H

C

$\mathrm{H}$

H

H

C

$\mathrm{H}$

H

$\mathrm{H}$

H

H

C

C
$2.43243500 \quad 0.40290700 \quad-2.37360700$

$3.42520900 \quad 0.17170700 \quad-3.31854300$

$\begin{array}{lll}5.42213900 & -0.60678500 & -3.59589600\end{array}$

1.50360200

3.26169600

2.57542200

4.70335700

4.91234200

5.40262400

3.96604800

5.54821600

3.80463100

2.83391400

4.28009000

3.63850800

6.07344400

6.55536800

6.74413500

5.97977400

1.40390500

1.60289200

$-0.50181200$

$-1.39676900$
$0.90144900-2.63709300$

$0.45103900-4.35333500$

$0.08259600-1.07203400$

$0.41568500 \quad 2.06055400$

$-0.06974800 \quad 3.51135100$

$0.71929000 \quad 4.09317100$

$-0.30085400 \quad 4.01177500$

$-0.95942400 \quad 3.55521400$

$1.65890400 \quad 2.05188000$

$1.46112000 \quad 2.51736700$

$2.46420200 \quad 2.62534200$

$2.03554600 \quad 1.03822900$

$0.75750900 \quad 1.44165400$

$\begin{array}{ll}1.54640300 & 2.03054300\end{array}$

$-0.10784100$

1.43624400

$-0.39407200$

$-0.47882200$

$-0.22836800$

0.86988200
0.41510300

\section{TS19-2H}

imaginary frequency $=-1277.42 \mathrm{~cm}^{-1}$

C

$\mathrm{H}$

$\mathrm{H}$

C

H

$\mathrm{H}$

C

$\mathrm{H}$

H

P

P

C

C

C

$\mathrm{H}$

H

$\mathrm{H}$

C

H

H

$$
\begin{array}{rrr}
0.16969000 & -2.83765800 & -1.34009100 \\
0.72234700 & -3.43152400 & -0.60349600 \\
-0.43901400 & -3.58181300 & -1.86936600 \\
1.16517700 & -2.15004500 & -2.31365600 \\
1.31502000 & -2.81615800 & -3.17093100 \\
0.74385800 & -1.22081300 & -2.71779400 \\
2.55614000 & -1.86697800 & -1.71163200 \\
3.22695100 & -1.51645400 & -2.50132900 \\
2.98424800 & -2.79995900 & -1.33184400 \\
2.52880500 & -0.62913600 & -0.31584300 \\
-0.91078700 & -1.68916300 & -0.33373600 \\
-1.85080600 & -2.84307200 & 0.87805200 \\
3.71865400 & -1.27445900 & 1.02517200 \\
-0.86108200 & -3.14167300 & 2.02362900 \\
-1.35581000 & -3.78699200 & 2.75731100 \\
0.02912900 & -3.67673300 & 1.67887600 \\
-0.54142400 & -2.22997100 & 2.53710400 \\
-2.29529100 & -4.14989600 & 0.19681500 \\
-2.86495000 & -4.74519700 & 0.91842400 \\
-2.95707300 & -3.97252100 & -0.65802500
\end{array}
$$




\begin{tabular}{|c|c|c|c|c|c|c|c|}
\hline $\mathrm{H}$ & -1.45445500 & -4.76767600 & -0.12946500 & $\mathrm{H}$ & 1.16497100 & 2.24342200 & 2.14392800 \\
\hline $\mathrm{C}$ & -3.08240700 & -2.10334100 & 1.43328800 & $\mathrm{H}$ & 0.61348400 & 3.22043700 & 0.70587700 \\
\hline $\mathrm{H}$ & -3.86658600 & -1.98593700 & 0.67995500 & $\mathrm{C}$ & -1.87553400 & 2.39764000 & 0.57625700 \\
\hline $\mathrm{H}$ & -3.50867300 & -2.70313100 & 2.24431200 & $\mathrm{C}$ & -1.58283100 & 2.79443400 & -0.74914200 \\
\hline $\mathrm{H}$ & -2.83922200 & -1.11923300 & 1.84243700 & $\mathrm{C}$ & -3.15877600 & 2.69684100 & 1.07383700 \\
\hline $\mathrm{C}$ & 3.08586300 & -2.54094700 & 1.63585300 & $\mathrm{C}$ & -2.51661900 & 3.46499500 & -1.53360500 \\
\hline $\mathrm{H}$ & 2.10665500 & -2.33114100 & 2.07547000 & $\mathrm{H}$ & -0.60567100 & 2.57307400 & -1.16948800 \\
\hline $\mathrm{H}$ & 2.98676500 & -3.35790700 & 0.91235900 & $\mathrm{C}$ & -4.09689200 & 3.36544600 & 0.28085700 \\
\hline $\mathrm{H}$ & 3.73451400 & -2.90759700 & 2.43817800 & $\mathrm{H}$ & -3.42054500 & 2.43253800 & 2.08789000 \\
\hline $\mathrm{C}$ & 5.11054300 & -1.59912800 & 0.45368200 & $\mathrm{C}$ & -3.78492800 & 3.75678100 & -1.02108300 \\
\hline $\mathrm{H}$ & 5.61165800 & -0.71175200 & 0.05824400 & $\mathrm{H}$ & -2.25199300 & 3.77019900 & -2.54191500 \\
\hline $\mathrm{H}$ & 5.74026800 & -1.99047000 & 1.26003500 & $\mathrm{H}$ & -5.07002600 & 3.60137500 & 0.70102100 \\
\hline $\mathrm{H}$ & 5.08167900 & -2.36474600 & -0.32824100 & $\mathrm{H}$ & -4.51024000 & 4.29683500 & -1.62199100 \\
\hline $\mathrm{C}$ & 3.82897100 & -0.18667400 & 2.11327700 & $\mathrm{C}$ & -0.97988300 & 1.06198100 & 2.71060000 \\
\hline $\mathrm{H}$ & 4.47028300 & -0.55754100 & 2.91960500 & $\mathrm{O}$ & -0.03624500 & 0.66020800 & 3.37390100 \\
\hline $\mathrm{H}$ & 4.28602500 & 0.73223400 & 1.73366100 & $\mathrm{O}$ & -2.25643900 & 0.89765800 & 3.09810300 \\
\hline $\mathrm{H}$ & 2.85601800 & 0.05752100 & 2.55103600 & $\mathrm{C}$ & -2.44313100 & 0.32452600 & 4.41842500 \\
\hline $\mathrm{C}$ & -2.22378800 & -1.22182900 & -1.57798300 & $\mathrm{H}$ & -3.51959200 & 0.32457000 & 4.58060100 \\
\hline $\mathrm{C}$ & -3.14937900 & -0.20820500 & -1.32418400 & $\mathrm{H}$ & -2.04300900 & -0.69014700 & 4.45395100 \\
\hline $\mathrm{C}$ & -3.22783700 & -1.53959500 & -3.74277000 & $\mathrm{H}$ & -1.93755300 & 0.93739000 & 5.16592200 \\
\hline $\mathrm{C}$ & -4.11862800 & 0.12053400 & -2.27402800 & $\mathrm{H}$ & -1.65278800 & -2.58379900 & -2.99566900 \\
\hline $\mathrm{H}$ & -3.09752600 & 0.33765600 & -0.39054700 & & & & \\
\hline $\mathrm{C}$ & -4.16061600 & -0.54975500 & -3.50187900 & 25 & & & \\
\hline $\mathrm{H}$ & -3.17545500 & -2.11042700 & -4.66262800 & $\mathrm{C}$ & 0.88925200 & 2.32665800 & -2.11584600 \\
\hline $\mathrm{H}$ & -4.82729300 & 0.91285200 & -2.05681900 & $\mathrm{H}$ & 0.66510500 & 1.83738900 & -3.07058200 \\
\hline $\mathrm{H}$ & -4.89628400 & -0.30823000 & -4.26010500 & $\mathrm{H}$ & 0.70435900 & 3.39488300 & -2.25363400 \\
\hline $\mathrm{C}$ & 3.33740300 & 0.90953300 & -0.97807700 & $\mathrm{C}$ & 2.36408700 & 2.11817900 & -1.74854900 \\
\hline $\mathrm{C}$ & 4.43959900 & 0.96467100 & -1.83428000 & $\mathrm{H}$ & 2.95087300 & 2.82833000 & -2.34264300 \\
\hline $\mathrm{C}$ & 3.27113400 & 3.24207300 & -0.84696400 & $\mathrm{H}$ & 2.55542000 & 2.38716800 & -0.70407600 \\
\hline $\mathrm{C}$ & 4.95624900 & 2.21166900 & -2.19957000 & $\mathrm{C}$ & 2.91718700 & 0.72122100 & -2.04951500 \\
\hline $\mathrm{H}$ & 4.90055400 & 0.05706400 & -2.20723200 & $\mathrm{H}$ & 4.00078200 & 0.73237400 & -1.91470800 \\
\hline $\mathrm{C}$ & 4.36941700 & 3.37107300 & -1.69507800 & $\mathrm{H}$ & 2.70137900 & 0.44540600 & -3.08763300 \\
\hline $\mathrm{H}$ & 2.76987600 & 4.10947300 & -0.42681000 & $\mathrm{P}$ & 2.28266700 & -0.63608900 & -0.96591200 \\
\hline $\mathrm{H}$ & 5.81207300 & 2.27276400 & -2.86453600 & $\mathrm{P}$ & -0.40436800 & 1.77786500 & -0.88709300 \\
\hline $\mathrm{H}$ & 4.74662200 & 4.35465400 & -1.95191200 & $\mathrm{C}$ & -2.02898300 & 2.22723900 & -1.80548000 \\
\hline $\mathrm{N}$ & -2.31204700 & -1.83967500 & -2.78987600 & $\mathrm{C}$ & 3.09756100 & -2.21202800 & -1.69009700 \\
\hline $\mathrm{N}$ & 2.77483600 & 2.04298100 & -0.51573700 & $\mathrm{C}$ & -2.34141900 & 1.04536300 & -2.74682600 \\
\hline $\mathrm{Pd}$ & 0.38924100 & 0.17721600 & 0.39255900 & $\mathrm{H}$ & -3.24033700 & 1.28549900 & -3.32558900 \\
\hline $\mathrm{C}$ & 0.57123300 & 2.26729200 & 1.23303000 & $\mathrm{H}$ & -1.53581800 & 0.85998600 & -3.46601000 \\
\hline $\mathrm{C}$ & -0.78902100 & 1.75565100 & 1.38281600 & $\mathrm{H}$ & -2.53910400 & 0.12342700 & -2.19553800 \\
\hline $\mathrm{H}$ & 1.56308300 & 1.81900900 & 0.31377700 & $\mathrm{C}$ & -1.88602900 & 3.51753500 & -2.64225600 \\
\hline
\end{tabular}




\begin{tabular}{|c|c|c|c|c|c|c|c|}
\hline $\mathrm{H}$ & -2.86726300 & 3.74307400 & -3.07526800 & $\mathrm{O}$ & -0.54761200 & -3.57992800 & -1.12444300 \\
\hline $\mathrm{H}$ & -1.58397800 & 4.36683800 & -2.02786300 & $\mathrm{C}$ & -0.39948600 & -2.52667600 & -0.70598300 \\
\hline $\mathrm{H}$ & -1.18980400 & 3.40569100 & -3.47724000 & $\mathrm{C}$ & -1.70443100 & 0.38633600 & 2.10512000 \\
\hline $\mathrm{C}$ & -3.17481200 & 2.41523800 & -0.79631000 & $\mathrm{H}$ & -0.65302100 & 0.48183300 & 2.37309900 \\
\hline $\mathrm{H}$ & -2.98942400 & 3.26530900 & -0.13661200 & $\mathrm{H}$ & -2.24102700 & 0.21680100 & 3.04927200 \\
\hline $\mathrm{H}$ & -4.09663600 & 2.61866400 & -1.35301000 & $\mathrm{H}$ & -2.05866400 & 1.32573200 & 1.68305200 \\
\hline $\mathrm{H}$ & -3.35322000 & 1.52095800 & -0.20034300 & $\mathrm{C}$ & -3.31545600 & -0.94000000 & 0.61121400 \\
\hline $\mathrm{C}$ & 2.36052600 & -2.59020000 & -2.99024100 & $\mathrm{C}$ & -3.61614400 & -1.70559600 & -0.52944800 \\
\hline $\mathrm{H}$ & 1.31027400 & -2.83956900 & -2.82520500 & $\mathrm{C}$ & -4.39929600 & -0.35070300 & 1.29273900 \\
\hline $\mathrm{H}$ & 2.40974100 & -1.79350000 & -3.73925900 & $\mathrm{C}$ & -4.92616600 & -1.88360100 & -0.96612400 \\
\hline $\mathrm{H}$ & 2.83980500 & -3.47274200 & -3.42818300 & $\mathrm{H}$ & -2.81443000 & -2.15918900 & -1.09895500 \\
\hline $\mathrm{C}$ & 4.58562900 & -1.94919700 & -2.00612900 & $\mathrm{C}$ & -5.71358400 & -0.52824000 & 0.85891900 \\
\hline $\mathrm{H}$ & 5.13594400 & -1.57365400 & -1.14009600 & $\mathrm{H}$ & -4.22643200 & 0.24118400 & 2.18407200 \\
\hline $\mathrm{H}$ & 5.04672000 & -2.89433800 & -2.31399000 & $\mathrm{C}$ & -5.98578800 & -1.29510000 & -0.27278800 \\
\hline $\mathrm{H}$ & 4.71630200 & -1.24287000 & -2.82960400 & $\mathrm{H}$ & -5.11829800 & -2.47877600 & -1.85392600 \\
\hline $\mathrm{C}$ & 3.00486700 & -3.35375900 & -0.66018400 & $\mathrm{H}$ & -6.52465000 & -0.06587500 & 1.41356700 \\
\hline $\mathrm{H}$ & 3.40548900 & -4.26926100 & -1.10888900 & $\mathrm{H}$ & -7.00762500 & -1.43000500 & -0.61341800 \\
\hline $\mathrm{H}$ & 3.59471000 & -3.13936400 & 0.23485500 & $\mathrm{C}$ & -1.36036200 & -2.02579200 & 1.84704400 \\
\hline $\mathrm{H}$ & 1.98060800 & -3.56897500 & -0.34802200 & $\mathrm{O}$ & -0.31304400 & -2.02383600 & 2.48110900 \\
\hline $\mathrm{C}$ & -0.09817900 & 3.02279600 & 0.46096800 & $\mathrm{O}$ & -2.12004100 & -3.12194500 & 1.70766500 \\
\hline $\mathrm{C}$ & 0.75788400 & 2.72206500 & 1.52796800 & $\mathrm{C}$ & -1.64403600 & -4.31572900 & 2.36477000 \\
\hline $\mathrm{C}$ & -0.40893000 & 5.17140500 & 1.22212100 & $\mathrm{H}$ & -2.41972000 & -5.06209700 & 2.20154100 \\
\hline $\mathrm{C}$ & 1.02922500 & 3.71761100 & 2.46852900 & $\mathrm{H}$ & -0.69804800 & -4.64354400 & 1.92652800 \\
\hline $\mathrm{H}$ & 1.20174400 & 1.73719700 & 1.62341200 & $\mathrm{H}$ & -1.50041400 & -4.13083700 & 3.43080700 \\
\hline
\end{tabular}

$\mathrm{H}$

H

$\mathrm{H}$

C

C

C

C

$\mathrm{H}$

C

$\mathrm{H}$

H

H

N

N

Pd

C
0.43135500

$-0.89426200$

1.69186200

0.60827500

3.18831100

2.68898800

5.05017300

3.44026900

1.74225400

4.64333300

5.98011500

3.08353200

5.25609900

$-0.66583300$

4.34426600

$-0.15787100$

$-1.91972600$
4.96632800

6.13217000

1.06697700

$3.51572400 \quad 3.30461700$

5.76665500

3.02940200

$-0.32051900$

0.62943100

$-0.77925200$

1.85584000

$0.58631600 \quad 1.63169700$

$-0.53344800$

3.00862700

$-1.30635700 \quad 1.92461400$

0.16014100

2.89858800

1.13548300

1.50248600

$-0.88089900$

3.97358200

$0.37137000 \quad 3.76880000$

4.22846100

0.30813500

$0.35444400 \quad 0.51898200$

$-0.61290300$

$-0.31478700$

$-0.79377100 \quad 1.15859700$

\section{TS20}

imaginary frequency $=-115.75 \mathrm{~cm}^{-1}$

C

H

$\mathrm{H}$

C

$\mathrm{H}$

H

C

H

$\mathrm{H}$

$\mathrm{P}$

$\mathrm{P}$

C

C

C

$\begin{array}{rrr}1.83855800 & 2.86245300 & -1.24529200 \\ 1.72831700 & 2.88728300 & -2.33487500 \\ 1.97793100 & 3.89698400 & -0.91771000 \\ 3.07115300 & 2.02412200 & -0.87704900 \\ 3.95685200 & 2.62298100 & -1.11843100 \\ 3.12399100 & 1.85341400 & 0.20469600 \\ 3.20400600 & 0.69886800 & -1.64549800 \\ 4.23077000 & 0.33378900 & -1.55686300 \\ 2.99295500 & 0.85351700 & -2.70932900 \\ 2.15976200 & -0.71329200 & -1.05412600 \\ 0.21238100 & 2.29255100 & -0.53285900 \\ -1.07613700 & 3.24128500 & -1.57023500 \\ 2.43567700 & -2.10111000 & -2.33672900 \\ -1.18181300 & 2.50013300 & -2.92098500\end{array}$




\begin{tabular}{|c|c|c|c|c|c|c|c|}
\hline $\mathrm{H}$ & -1.93440500 & 2.99821000 & -3.54203700 & $\mathrm{~N}$ & -0.14391000 & 4.27371500 & 1.34778700 \\
\hline $\mathrm{H}$ & -0.24195000 & 2.51365400 & -3.48240100 & $\mathrm{~N}$ & 4.39306300 & -1.15617600 & 0.41626400 \\
\hline $\mathrm{H}$ & -1.48973600 & 1.45708500 & -2.79614400 & $\mathrm{Pd}$ & -0.08277100 & -0.16491200 & -0.46796000 \\
\hline C & -0.66046100 & 4.70621800 & -1.81879400 & $\mathrm{C}$ & -2.31818900 & -0.97778000 & 0.94930600 \\
\hline $\mathrm{H}$ & -1.44530700 & 5.19096800 & -2.41095500 & $\mathrm{O}$ & -1.13898700 & -2.97813900 & -0.79442300 \\
\hline $\mathrm{H}$ & -0.54729700 & 5.25248600 & -0.88180100 & $\mathrm{C}$ & -0.87688700 & -1.90251000 & -0.45691800 \\
\hline $\mathrm{H}$ & 0.26726000 & 4.78500200 & -2.39224500 & $\mathrm{C}$ & -2.20707300 & 0.32153800 & 1.75601800 \\
\hline C & -2.44205800 & 3.20731300 & -0.85883100 & $\mathrm{H}$ & -1.24514600 & 0.41850000 & 2.25770800 \\
\hline $\mathrm{H}$ & -2.40146500 & 3.69588300 & 0.11739500 & $\mathrm{H}$ & -2.98003000 & 0.28292400 & 2.53887100 \\
\hline $\mathrm{H}$ & -3.17076200 & 3.75030300 & -1.47124400 & $\mathrm{H}$ & -2.40821500 & 1.20233800 & 1.15114500 \\
\hline $\mathrm{H}$ & -2.82095600 & 2.18840100 & -0.74017900 & $\mathrm{C}$ & -3.57369800 & -1.06097800 & 0.12577200 \\
\hline $\mathrm{C}$ & 1.44750900 & -1.87132500 & -3.49712200 & $\mathrm{C}$ & -3.60933400 & -0.58162800 & -1.19170000 \\
\hline $\mathrm{H}$ & 0.40710900 & -1.93832200 & -3.16725000 & $\mathrm{C}$ & -4.77819500 & -1.50648900 & 0.69985600 \\
\hline $\mathrm{H}$ & 1.59390700 & -0.89953000 & -3.98085500 & $\mathrm{C}$ & -4.79692600 & -0.55251100 & -1.92247100 \\
\hline $\mathrm{H}$ & 1.60546500 & -2.64050600 & -4.26106800 & $\mathrm{H}$ & -2.68525800 & -0.24204400 & -1.65501100 \\
\hline C & 3.88641000 & -2.07046000 & -2.86245300 & $\mathrm{C}$ & -5.96729300 & -1.47194800 & -0.02758900 \\
\hline $\mathrm{H}$ & 4.61721400 & -2.13327600 & -2.05224100 & $\mathrm{H}$ & -4.78749700 & -1.88162000 & 1.71653300 \\
\hline $\mathrm{H}$ & 4.03276600 & -2.93552300 & -3.51874700 & $\mathrm{C}$ & -5.98331900 & -0.99871300 & -1.34046700 \\
\hline $\mathrm{H}$ & 4.09787200 & -1.17604700 & -3.45391100 & $\mathrm{H}$ & -4.79178000 & -0.18791000 & -2.94529300 \\
\hline C & 2.17264200 & -3.46665500 & -1.67353400 & $\mathrm{H}$ & -6.88501400 & -1.82066800 & 0.43641500 \\
\hline $\mathrm{H}$ & 2.27726400 & -4.24859700 & -2.43363900 & $\mathrm{H}$ & -6.91021900 & -0.98247500 & -1.90520000 \\
\hline $\mathrm{H}$ & 2.89619300 & -3.67816100 & -0.88179900 & $\mathrm{C}$ & -1.90357100 & -2.13272400 & 1.83981300 \\
\hline $\mathrm{H}$ & 1.16655700 & -3.54980500 & -1.26029900 & $\mathrm{O}$ & -0.91309500 & -2.08254000 & 2.55423500 \\
\hline C & 0.29162900 & 3.01694700 & 1.17536300 & $\mathrm{O}$ & -2.68764500 & -3.20999900 & 1.74069900 \\
\hline C & 0.84526500 & 2.26027100 & 2.21749000 & $\mathrm{C}$ & -2.29321400 & -4.35705400 & 2.52448500 \\
\hline $\mathrm{C}$ & -0.04433000 & 4.81614000 & 2.56716900 & $\mathrm{H}$ & -3.06450300 & -5.10478700 & 2.34791100 \\
\hline $\mathrm{C}$ & 0.95972400 & 2.84252000 & 3.48132300 & $\mathrm{H}$ & -1.31928700 & -4.72232100 & 2.19118200 \\
\hline $\mathrm{H}$ & 1.17576300 & 1.24065700 & 2.04519200 & $\mathrm{H}$ & -2.23887700 & -4.09448600 & 3.58265700 \\
\hline C & 0.50305500 & 4.14553400 & 3.66342900 & & & & \\
\hline $\mathrm{H}$ & -0.41604100 & 5.83307900 & 2.66932600 & 26 & & & \\
\hline $\mathrm{H}$ & 1.38966700 & 2.28358600 & 4.30697300 & $\mathrm{C}$ & 1.38806000 & -1.87015200 & 2.27616500 \\
\hline $\mathrm{H}$ & 0.56420700 & 4.63580800 & 4.62927100 & $\mathrm{H}$ & 0.81066300 & -1.48845000 & 3.12583400 \\
\hline C & 3.05388600 & -1.23298400 & 0.48915400 & $\mathrm{H}$ & 1.58703600 & -2.92833800 & 2.45583300 \\
\hline C & 2.37601400 & -1.68421300 & 1.62768300 & $\mathrm{C}$ & 2.73647500 & -1.13920500 & 2.17444300 \\
\hline C & 5.10560500 & -1.54051200 & 1.48085300 & $\mathrm{H}$ & 3.38988800 & -1.56806000 & 2.94304400 \\
\hline C & 3.13425600 & -2.08177800 & 2.73263000 & $\mathrm{H}$ & 3.22373500 & -1.36307500 & 1.21842200 \\
\hline $\mathrm{H}$ & 1.29219100 & -1.72753400 & 1.66774300 & $\mathrm{C}$ & 2.68369100 & 0.37878900 & 2.41388300 \\
\hline $\mathrm{C}$ & 4.52297900 & -2.01171600 & 2.66071600 & $\mathrm{H}$ & 3.69364900 & 0.76074500 & 2.58863500 \\
\hline $\mathrm{H}$ & 6.18651900 & -1.46682100 & 1.38614500 & $\mathrm{H}$ & 2.11330100 & 0.58885600 & 3.32658500 \\
\hline $\mathrm{H}$ & 2.63879200 & -2.43814700 & 3.63058900 & $P$ & 1.87748100 & 1.32513300 & 1.03827400 \\
\hline $\mathrm{H}$ & 5.14800600 & -2.31007900 & 3.49591800 & $\mathrm{P}$ & 0.27139700 & -1.86521500 & 0.78812900 \\
\hline
\end{tabular}




\begin{tabular}{|c|c|c|c|c|c|c|c|}
\hline $\mathrm{C}$ & -1.21958400 & -2.92609000 & 1.37211600 & $\mathrm{H}$ & 2.86271800 & 1.32557600 & -3.59189400 \\
\hline $\mathrm{C}$ & 1.83210000 & 3.13306900 & 1.65133600 & $\mathrm{H}$ & 6.41701900 & 1.20975200 & -1.16523200 \\
\hline $\mathrm{C}$ & -2.18714700 & -1.99690100 & 2.12865700 & $\mathrm{H}$ & 5.35475600 & 1.25909400 & -3.44173800 \\
\hline $\mathrm{H}$ & -3.03239800 & -2.58682900 & 2.50048900 & $\mathrm{~N}$ & 1.85497700 & -3.97843900 & 0.16481300 \\
\hline $\mathrm{H}$ & -1.71340800 & -1.52618100 & 2.99742300 & $\mathrm{~N}$ & 2.55548800 & 1.31009000 & -1.55305400 \\
\hline $\mathrm{H}$ & -2.58556900 & -1.20836900 & 1.48660900 & $\mathrm{Pd}$ & -0.15908900 & 0.30142000 & -0.01407600 \\
\hline $\mathrm{C}$ & -0.75128100 & -4.05963400 & 2.31126600 & $\mathrm{C}$ & -1.59132500 & -0.25856400 & -1.33261200 \\
\hline $\mathrm{H}$ & -1.61532000 & -4.69743500 & 2.52856400 & $\mathrm{O}$ & -1.57493600 & -1.21445700 & -2.06506000 \\
\hline $\mathrm{H}$ & 0.02203500 & -4.68122500 & 1.85402000 & $\mathrm{C}$ & -2.72089600 & 0.84244700 & -1.52782300 \\
\hline $\mathrm{H}$ & -0.38044100 & -3.68430600 & 3.26815800 & $\mathrm{C}$ & -2.09956100 & 2.17651300 & -1.16521700 \\
\hline $\mathrm{C}$ & -1.92621200 & -3.55960100 & 0.15896700 & $\mathrm{O}$ & -1.01275800 & 2.27956500 & -0.57927400 \\
\hline $\mathrm{H}$ & -1.26790000 & -4.24794700 & -0.37831800 & $\mathrm{O}$ & -2.80001200 & 3.22598000 & -1.53662800 \\
\hline $\mathrm{H}$ & -2.78321100 & -4.13775100 & 0.52262000 & $\mathrm{C}$ & -2.26345900 & 4.54036400 & -1.24304300 \\
\hline $\mathrm{H}$ & -2.29710500 & -2.81982900 & -0.54897800 & $\mathrm{H}$ & -1.30909200 & 4.67556000 & -1.75498700 \\
\hline $\mathrm{C}$ & 0.60801700 & 3.26179200 & 2.58044700 & $\mathrm{H}$ & -3.00756500 & 5.23962800 & -1.61873600 \\
\hline $\mathrm{H}$ & -0.32094200 & 3.03819000 & 2.04806100 & $\mathrm{H}$ & -2.12824400 & 4.65734100 & -0.16651100 \\
\hline $\mathrm{H}$ & 0.67400300 & 2.60046100 & 3.45108300 & $\mathrm{C}$ & -3.17708400 & 0.86875200 & -3.00357800 \\
\hline $\mathrm{H}$ & 0.54446200 & 4.28843300 & 2.95914200 & $\mathrm{H}$ & -4.03255800 & 1.53578200 & -3.12167200 \\
\hline $\mathrm{C}$ & 3.11517700 & 3.51751600 & 2.41057400 & $\mathrm{H}$ & -2.36628500 & 1.21555500 & -3.65080200 \\
\hline $\mathrm{H}$ & 4.00974900 & 3.39915700 & 1.79109500 & $\mathrm{H}$ & -3.44764300 & -0.13419100 & -3.33000800 \\
\hline $\mathrm{H}$ & 3.05718200 & 4.57434500 & 2.69545200 & $\mathrm{C}$ & -3.85946300 & 0.49083900 & -0.55527800 \\
\hline $\mathrm{H}$ & 3.24836100 & 2.94197900 & 3.33074900 & $\mathrm{C}$ & -4.75016700 & -0.54459800 & -0.87609000 \\
\hline $\mathrm{C}$ & 1.66223300 & 4.06487200 & 0.43542300 & $\mathrm{C}$ & -4.00932200 & 1.14815800 & 0.67480900 \\
\hline $\mathrm{H}$ & 1.54506100 & 5.09481400 & 0.79275000 & $\mathrm{C}$ & -5.77672800 & -0.89430400 & 0.00088300 \\
\hline $\mathrm{H}$ & 2.53729700 & 4.04166900 & -0.21998800 & $\mathrm{H}$ & -4.64759000 & -1.08463800 & -1.81071800 \\
\hline $\mathrm{H}$ & 0.78221500 & 3.80528000 & -0.15590400 & $\mathrm{C}$ & -5.04064500 & 0.80070500 & 1.54892800 \\
\hline $\mathrm{C}$ & 1.23570600 & -2.92423100 & -0.39252800 & $\mathrm{H}$ & -3.32616000 & 1.94140100 & 0.96430800 \\
\hline $\mathrm{C}$ & 1.30515400 & -2.63488100 & -1.75895800 & $\mathrm{C}$ & -5.92938200 & -0.22077200 & 1.21416200 \\
\hline $\mathrm{C}$ & 2.56204200 & -4.78738700 & -0.63187000 & $\mathrm{H}$ & -6.46029300 & -1.69354200 & -0.26890700 \\
\hline $\mathrm{C}$ & 2.04781200 & -3.48749500 & -2.57853200 & $\mathrm{H}$ & -5.14753100 & 1.33182100 & 2.48989800 \\
\hline $\mathrm{H}$ & 0.77997800 & -1.78047000 & -2.16591200 & $\mathrm{H}$ & -6.73376300 & -0.48960800 & 1.89171800 \\
\hline $\mathrm{C}$ & 2.68901800 & -4.58445800 & -2.00819800 & & & & \\
\hline $\mathrm{H}$ & 3.04892000 & -5.63107200 & -0.14788500 & $26 a$ & & & \\
\hline $\mathrm{H}$ & 2.11964300 & -3.29605600 & -3.64496000 & $\mathrm{C}$ & -1.71954000 & 3.13819800 & 0.59422300 \\
\hline $\mathrm{H}$ & 3.27567900 & -5.27149600 & -2.60915800 & $\mathrm{H}$ & -1.76626100 & 3.89131200 & -0.19926200 \\
\hline $\mathrm{C}$ & 3.12353900 & 1.29089600 & -0.33138400 & $\mathrm{H}$ & -2.53183900 & 3.33969000 & 1.29797800 \\
\hline $\mathrm{C}$ & 4.51181900 & 1.25299100 & -0.15071400 & $\mathrm{C}$ & -0.36111200 & 3.22116700 & 1.30806400 \\
\hline $\mathrm{C}$ & 3.35782700 & 1.30425100 & -2.62370900 & $\mathrm{H}$ & -0.36962400 & 4.12442500 & 1.92843900 \\
\hline $\mathrm{C}$ & 5.33729200 & 1.23950800 & -1.27660200 & $\mathrm{H}$ & -0.23333800 & 2.38793400 & 2.00848900 \\
\hline $\mathrm{H}$ & 4.94697100 & 1.23503400 & 0.84237900 & $\mathrm{C}$ & 0.84236700 & 3.33626500 & 0.35515200 \\
\hline $\mathrm{C}$ & 4.75163100 & 1.26748600 & -2.53987500 & $\mathrm{H}$ & 1.64980400 & 3.87362200 & 0.85701600 \\
\hline
\end{tabular}




\begin{tabular}{|c|c|c|c|c|c|c|c|}
\hline $\mathrm{H}$ & 0.55623100 & 3.90366100 & -0.53551800 & $\mathrm{C}$ & 4.69968500 & 0.16601800 & 2.06757400 \\
\hline $\mathrm{P}$ & 1.64191100 & 1.77232300 & -0.24327100 & $\mathrm{H}$ & 3.69356100 & -0.41984400 & 0.26018000 \\
\hline $\mathrm{P}$ & -2.09003400 & 1.47944100 & -0.14935400 & $\mathrm{C}$ & 4.76402800 & 1.12239500 & 3.07941500 \\
\hline $\mathrm{C}$ & -3.29635300 & 1.82754900 & -1.58463200 & $\mathrm{H}$ & 3.89470800 & 2.97078100 & 3.79407400 \\
\hline $\mathrm{C}$ & 2.59574900 & 2.32642900 & -1.81054700 & $\mathrm{H}$ & 5.36226700 & -0.69388200 & 2.06913500 \\
\hline $\mathrm{C}$ & -2.48911000 & 2.48458300 & -2.72140600 & $\mathrm{H}$ & 5.47589900 & 1.03682800 & 3.89353400 \\
\hline $\mathrm{H}$ & -3.15096400 & 2.67374600 & -3.57397200 & $\mathrm{~N}$ & -3.70337000 & 1.52323300 & 2.07557700 \\
\hline $\mathrm{H}$ & -2.06114700 & 3.44821600 & -2.42687600 & $\mathrm{~N}$ & 2.98238500 & 2.36455700 & 2.04681700 \\
\hline $\mathrm{H}$ & -1.67589300 & 1.83828500 & -3.06718100 & $\mathrm{Pd}$ & -0.00738400 & 0.10370500 & -0.51457400 \\
\hline $\mathrm{C}$ & -4.44132100 & 2.75401300 & -1.13639200 & $\mathrm{C}$ & 0.95881300 & -2.52237900 & 0.29424300 \\
\hline $\mathrm{H}$ & -5.13156000 & 2.90361200 & -1.97470500 & $\mathrm{O}$ & 2.22462300 & -1.48029600 & -1.51401600 \\
\hline $\mathrm{H}$ & -5.01019800 & 2.33061100 & -0.30477800 & $\mathrm{C}$ & 1.37246400 & -1.35905500 & -0.67901500 \\
\hline $\mathrm{H}$ & -4.08040800 & 3.73989000 & -0.83096200 & $\mathrm{C}$ & 0.99609700 & -2.00501700 & 1.74898000 \\
\hline $\mathrm{C}$ & -3.87073100 & 0.48828100 & -2.08219500 & $\mathrm{H}$ & 1.98979700 & -1.62862400 & 1.99068700 \\
\hline $\mathrm{H}$ & -4.47533200 & -0.00960000 & -1.31894800 & $\mathrm{H}$ & 0.77723100 & -2.81631200 & 2.44653000 \\
\hline $\mathrm{H}$ & -4.51750500 & 0.67237300 & -2.94732800 & $\mathrm{H}$ & 0.26092900 & -1.21014500 & 1.90058300 \\
\hline $\mathrm{H}$ & -3.08160800 & -0.20089600 & -2.40000900 & $\mathrm{C}$ & -0.43789500 & -2.99900500 & -0.12875500 \\
\hline $\mathrm{C}$ & 1.57260100 & 2.48982300 & -2.95041100 & $\mathrm{C}$ & -0.99209000 & -2.63865400 & -1.37001400 \\
\hline $\mathrm{H}$ & 1.10401000 & 1.53505000 & -3.20788300 & $\mathrm{C}$ & -1.18172000 & -3.84322000 & 0.70966300 \\
\hline $\mathrm{H}$ & 0.78247000 & 3.20823600 & -2.71121300 & $\mathrm{C}$ & -2.26352600 & -3.08870900 & -1.75035100 \\
\hline $\mathrm{H}$ & 2.08928800 & 2.85971300 & -3.84280500 & $\mathrm{H}$ & -0.40465200 & -2.07837400 & -2.09496200 \\
\hline $\mathrm{C}$ & 3.30149400 & 3.66919800 & -1.51908100 & $\mathrm{C}$ & -2.44043600 & -4.30228000 & 0.32389800 \\
\hline $\mathrm{H}$ & 3.97071400 & 3.60275000 & -0.65609400 & $\mathrm{H}$ & -0.77554500 & -4.15821300 & 1.66522300 \\
\hline $\mathrm{H}$ & 3.91314400 & 3.92993100 & -2.38988600 & $\mathrm{C}$ & -2.99140200 & -3.92162500 & -0.90488900 \\
\hline $\mathrm{H}$ & 2.60423800 & 4.49350300 & -1.35341000 & $\mathrm{H}$ & -2.66390800 & -2.80694400 & -2.71926100 \\
\hline $\mathrm{C}$ & 3.65720500 & 1.28958300 & -2.22153900 & $\mathrm{H}$ & -2.99143800 & -4.96713800 & 0.98270500 \\
\hline $\mathrm{H}$ & 4.13886700 & 1.64171900 & -3.14055200 & $\mathrm{H}$ & -3.96933200 & -4.28646800 & -1.20267300 \\
\hline $\mathrm{H}$ & 4.44055700 & 1.18645900 & -1.46617400 & $\mathrm{C}$ & 2.04968500 & -3.61061500 & 0.13470500 \\
\hline $\mathrm{H}$ & 3.22744800 & 0.30767000 & -2.41803400 & $\mathrm{O}$ & 3.07403400 & -3.60893900 & 0.78104600 \\
\hline $\mathrm{C}$ & -3.13950900 & 0.69958800 & 1.17989100 & $\mathrm{O}$ & 1.74162300 & -4.49971700 & -0.81212700 \\
\hline $\mathrm{C}$ & -3.32069800 & -0.68917700 & 1.21749200 & $\mathrm{C}$ & 2.74668600 & -5.50446900 & -1.07680200 \\
\hline $\mathrm{C}$ & -4.46910600 & 0.99256900 & 3.03570500 & $\mathrm{H}$ & 2.32673300 & -6.13527600 & -1.85839300 \\
\hline $\mathrm{C}$ & -4.12803900 & -1.23202500 & 2.21830200 & $\mathrm{H}$ & 3.67006000 & -5.02951200 & -1.41440300 \\
\hline $\mathrm{H}$ & -2.85018600 & -1.33128900 & 0.48123900 & $\mathrm{H}$ & 2.94722800 & -6.08495400 & -0.17413900 \\
\hline $\mathrm{C}$ & -4.71435800 & -0.37751300 & 3.14913500 & & & & \\
\hline $\mathrm{H}$ & -4.90524900 & 1.69452900 & 3.74287500 & $26 b$ & & & \\
\hline $\mathrm{H}$ & -4.28925000 & -2.30444800 & 2.26786500 & $\mathrm{C}$ & 1.21743300 & 2.82121700 & 0.80288400 \\
\hline $\mathrm{H}$ & -5.34481900 & -0.75624100 & 3.94675700 & $\mathrm{H}$ & 1.99764200 & 3.09306300 & 0.08458100 \\
\hline $\mathrm{C}$ & 2.92225900 & 1.44425800 & 1.07436800 & $\mathrm{H}$ & 0.75673400 & 3.74018500 & 1.16650300 \\
\hline $\mathrm{C}$ & 3.76078900 & 0.32055100 & 1.04706400 & $\mathrm{C}$ & 1.81644900 & 2.04851900 & 2.00601600 \\
\hline $\mathrm{C}$ & 3.88219400 & 2.20224100 & 3.02480600 & $\mathrm{H}$ & 1.97729300 & 2.75626600 & 2.82566700 \\
\hline
\end{tabular}




\begin{tabular}{|c|c|c|c|c|c|c|c|}
\hline $\mathrm{H}$ & 1.09872700 & 1.31425700 & 2.38584300 & $\mathrm{C}$ & 2.99851600 & -1.52721100 & 0.80553400 \\
\hline $\mathrm{C}$ & 3.16403600 & 1.35734500 & 1.70562700 & $\mathrm{C}$ & 3.81606300 & -2.19791000 & 1.71452200 \\
\hline $\mathrm{H}$ & 3.52113600 & 0.80620500 & 2.58171800 & $\mathrm{C}$ & 1.84653800 & -3.48230100 & 0.29575100 \\
\hline $\mathrm{H}$ & 3.92304600 & 2.11226300 & 1.47460500 & $\mathrm{C}$ & 3.63008800 & -3.57045200 & 1.90076600 \\
\hline $\mathrm{P}$ & 3.01971600 & 0.22778300 & 0.23549700 & $\mathrm{H}$ & 4.58526400 & -1.66426700 & 2.26200300 \\
\hline $\mathrm{P}$ & -0.12073500 & 1.92202100 & -0.13351500 & $\mathrm{C}$ & 2.64521300 & -4.22627900 & 1.16530400 \\
\hline $\mathrm{C}$ & -0.41255000 & 2.95248600 & -1.71111400 & $\mathrm{H}$ & 1.06037300 & -3.95258200 & -0.28415000 \\
\hline $\mathrm{C}$ & 4.60611900 & 0.40044200 & -0.80180500 & $\mathrm{H}$ & 4.25442500 & -4.11873800 & 2.59947200 \\
\hline $\mathrm{C}$ & 0.79523600 & 2.74488800 & -2.64554400 & $\mathrm{H}$ & 2.48441800 & -5.29417100 & 1.26529400 \\
\hline $\mathrm{H}$ & 0.63051200 & 3.30702300 & -3.57147700 & $\mathrm{~N}$ & -1.33724200 & 3.01784900 & 2.03569900 \\
\hline $\mathrm{H}$ & 1.72845800 & 3.11174400 & -2.20732800 & $\mathrm{~N}$ & 2.00190000 & -2.16096800 & 0.13179800 \\
\hline $\mathrm{H}$ & 0.92120200 & 1.69046400 & -2.91074200 & $\mathrm{Pd}$ & 0.71064900 & -0.26150000 & -0.49359900 \\
\hline $\mathrm{C}$ & -0.56054300 & 4.44430200 & -1.35066100 & $\mathrm{C}$ & -0.91167500 & -1.12754400 & -1.35054500 \\
\hline $\mathrm{H}$ & -0.78175600 & 5.00808900 & -2.26394600 & $\mathrm{O}$ & -0.69962500 & -1.18723000 & -2.53848800 \\
\hline $\mathrm{H}$ & -1.38086100 & 4.61905900 & -0.64863900 & $\mathrm{C}$ & -2.15152700 & -1.93214400 & -0.77705300 \\
\hline $\mathrm{H}$ & 0.35404400 & 4.86372600 & -0.92234200 & $\mathrm{C}$ & -3.30636200 & -1.45129700 & -1.68288900 \\
\hline $\mathrm{C}$ & -1.68663600 & 2.46267300 & -2.42388100 & $\mathrm{O}$ & -3.68635400 & -0.29611700 & -1.71693900 \\
\hline $\mathrm{H}$ & -2.58631000 & 2.64674100 & -1.83333500 & $\mathrm{O}$ & -3.82782200 & -2.42455000 & -2.43090500 \\
\hline $\mathrm{H}$ & -1.79253800 & 3.01620100 & -3.36372500 & $\mathrm{C}$ & -4.87893800 & -2.02921700 & -3.34188000 \\
\hline $\mathrm{H}$ & -1.64575700 & 1.39897000 & -2.66773400 & $\mathrm{H}$ & -4.50039900 & -1.29122100 & -4.05159900 \\
\hline $\mathrm{C}$ & 4.62813600 & 1.82517600 & -1.39011800 & $\mathrm{H}$ & -5.17561200 & -2.94332700 & -3.85328100 \\
\hline $\mathrm{H}$ & 3.75680900 & 2.01630800 & -2.02227700 & $\mathrm{H}$ & -5.71825800 & -1.60249300 & -2.78924000 \\
\hline $\mathrm{H}$ & 4.67662100 & 2.59978400 & -0.61785500 & $\mathrm{C}$ & -1.82673900 & -3.43199700 & -0.99984300 \\
\hline $\mathrm{H}$ & 5.52119800 & 1.93916500 & -2.01418600 & $\mathrm{H}$ & -2.70665000 & -4.03342200 & -0.76414400 \\
\hline $\mathrm{C}$ & 5.86872100 & 0.16359600 & 0.04664700 & $\mathrm{H}$ & -1.53355800 & -3.62362800 & -2.03335000 \\
\hline $\mathrm{H}$ & 5.91647100 & -0.85760600 & 0.43477900 & $\mathrm{H}$ & -1.02565900 & -3.73736400 & -0.32385600 \\
\hline $\mathrm{H}$ & 6.75550600 & 0.31253100 & -0.58022700 & $\mathrm{C}$ & -2.49395600 & -1.71319000 & 0.69638200 \\
\hline $\mathrm{H}$ & 5.94574400 & 0.86091100 & 0.88693300 & $\mathrm{C}$ & -1.48788000 & -1.65384900 & 1.67069800 \\
\hline $\mathrm{C}$ & 4.53279500 & -0.62689000 & -1.94793500 & $\mathrm{C}$ & -3.82939200 & -1.71434600 & 1.12565100 \\
\hline $\mathrm{H}$ & 5.40651400 & -0.50374800 & -2.59723600 & $\mathrm{C}$ & -1.80436000 & -1.56532500 & 3.02746400 \\
\hline $\mathrm{H}$ & 4.54056200 & -1.65672400 & -1.57905500 & $\mathrm{H}$ & -0.44542600 & -1.67929100 & 1.37092000 \\
\hline $\mathrm{H}$ & 3.63733900 & -0.48926700 & -2.56183100 & $\mathrm{C}$ & -4.14822600 & -1.63567400 & 2.48169900 \\
\hline $\mathrm{C}$ & -1.58512900 & 2.23826800 & 0.97149300 & $\mathrm{H}$ & -4.63668400 & -1.77784600 & 0.40277000 \\
\hline $\mathrm{C}$ & -2.85310100 & 1.71801500 & 0.68956700 & $\mathrm{C}$ & -3.13690600 & -1.55431200 & 3.43936500 \\
\hline $\mathrm{C}$ & -2.35406500 & 3.31898300 & 2.85131800 & $\mathrm{H}$ & -1.00667300 & -1.51440100 & 3.76321100 \\
\hline $\mathrm{C}$ & -3.90624500 & 2.05307700 & 1.54189700 & $\mathrm{H}$ & -5.19014900 & -1.64361800 & 2.78775600 \\
\hline $\mathrm{H}$ & -3.02640400 & 1.07086700 & -0.16239700 & $\mathrm{H}$ & -3.38455100 & -1.49266500 & 4.49463500 \\
\hline $\mathrm{C}$ & -3.65829100 & 2.86874800 & 2.64347200 & & & & \\
\hline $\mathrm{H}$ & -2.11293600 & 3.95040900 & 3.70369200 & 26-O & & & \\
\hline $\mathrm{H}$ & -4.90356100 & 1.67293600 & 1.34444900 & $\mathrm{C}$ & -1.52185400 & 1.37009100 & 2.73993000 \\
\hline $\mathrm{H}$ & -4.45000000 & 3.14938800 & 3.33038900 & $\mathrm{H}$ & -1.50572100 & 0.64033700 & 3.55680900 \\
\hline
\end{tabular}




\begin{tabular}{|c|c|c|c|c|c|c|c|}
\hline $\mathrm{H}$ & -1.30901100 & 2.35495700 & 3.16136600 & $\mathrm{H}$ & -0.61016500 & 5.74583200 & 1.22358700 \\
\hline $\mathrm{C}$ & -2.91468700 & 1.41119900 & 2.09062300 & $\mathrm{H}$ & 1.30851600 & 3.92504800 & -2.17724400 \\
\hline $\mathrm{H}$ & -3.57745800 & 1.93688700 & 2.78756300 & $\mathrm{H}$ & 0.44304400 & 5.99035700 & -1.02737300 \\
\hline $\mathrm{H}$ & -2.89422700 & 2.02588900 & 1.18327300 & $\mathrm{C}$ & -3.41791000 & 0.02746700 & -1.13368200 \\
\hline $\mathrm{C}$ & -3.53975000 & 0.03483000 & 1.80246100 & $\mathrm{C}$ & -4.65029600 & 0.68940000 & -1.19519600 \\
\hline $\mathrm{H}$ & -4.61643400 & 0.13631200 & 1.63652800 & $\mathrm{C}$ & -2.97160000 & 0.51111500 & -3.34015800 \\
\hline $\mathrm{H}$ & -3.42357300 & -0.61645200 & 2.67616800 & $\mathrm{C}$ & -5.03371200 & 1.28425300 & -2.39886900 \\
\hline$P$ & -2.75996100 & -0.83396900 & 0.36600200 & $\mathrm{H}$ & -5.30407200 & 0.74414400 & -0.33185900 \\
\hline$P$ & -0.06690800 & 1.01004700 & 1.64392600 & $\mathrm{C}$ & -4.18100100 & 1.19307200 & -3.49568100 \\
\hline $\mathrm{C}$ & 1.39839100 & 0.94936700 & 2.86078800 & $\mathrm{H}$ & -2.27899400 & 0.41748000 & -4.17340500 \\
\hline $\mathrm{C}$ & -3.50654000 & -2.58440600 & 0.34245000 & $\mathrm{H}$ & -5.98328300 & 1.80534700 & -2.47472500 \\
\hline $\mathrm{C}$ & 1.37332700 & -0.42697200 & 3.55434700 & $\mathrm{H}$ & -4.44009800 & 1.63851500 & -4.45043400 \\
\hline $\mathrm{H}$ & 2.20029300 & -0.47891600 & 4.27182900 & $\mathrm{~N}$ & -0.35134000 & 3.70188300 & 1.32255800 \\
\hline $\mathrm{H}$ & 0.44880300 & -0.58671700 & 4.11952100 & $\mathrm{~N}$ & -2.58758600 & -0.05404400 & -2.19037200 \\
\hline $\mathrm{H}$ & 1.49955100 & -1.24275000 & 2.83956200 & $\mathrm{Pd}$ & -0.35230000 & -0.80432100 & 0.21233900 \\
\hline $\mathrm{C}$ & 1.29415600 & 2.07766300 & 3.90741300 & $\mathrm{C}$ & 1.30594700 & -1.62406000 & -0.64162500 \\
\hline $\mathrm{H}$ & 2.20885000 & 2.07490700 & 4.51099800 & $\mathrm{O}$ & 0.45779700 & -2.36071800 & -1.11700400 \\
\hline $\mathrm{H}$ & 1.19798500 & 3.06506200 & 3.44799200 & $\mathrm{C}$ & 2.79545100 & -1.72456800 & -1.04072500 \\
\hline $\mathrm{H}$ & 0.45518000 & 1.93262700 & 4.59262400 & $\mathrm{C}$ & 3.54042500 & -2.07452900 & 0.25518200 \\
\hline $\mathrm{C}$ & 2.70116800 & 1.11897400 & 2.06204700 & $\mathrm{O}$ & 2.97827900 & -2.33253300 & 1.30071800 \\
\hline $\mathrm{H}$ & 2.78812100 & 2.12006900 & 1.63035700 & $\mathrm{O}$ & 4.86127500 & -2.16786000 & 0.07150200 \\
\hline $\mathrm{H}$ & 3.55327800 & 0.96988800 & 2.73465500 & $\mathrm{C}$ & 5.62840800 & -2.62895400 & 1.20963100 \\
\hline $\mathrm{H}$ & 2.77898700 & 0.38790000 & 1.25676300 & $\mathrm{H}$ & 5.29115300 & -3.62231600 & 1.51106700 \\
\hline $\mathrm{C}$ & -2.90510000 & -3.36116500 & 1.53155800 & $\mathrm{H}$ & 6.66120500 & -2.65660000 & 0.86694100 \\
\hline $\mathrm{H}$ & -1.81329000 & -3.41205600 & 1.47186300 & $\mathrm{H}$ & 5.51461000 & -1.93864700 & 2.04761600 \\
\hline $\mathrm{H}$ & -3.17792300 & -2.92658100 & 2.49864500 & $\mathrm{C}$ & 3.00941300 & -2.91884600 & -2.01325400 \\
\hline $\mathrm{H}$ & -3.28761700 & -4.38782000 & 1.51970700 & $\mathrm{H}$ & 4.06384800 & -2.96464400 & -2.28954300 \\
\hline $\mathrm{C}$ & -5.04092800 & -2.53925400 & 0.45706700 & $\mathrm{H}$ & 2.71978200 & -3.86193600 & -1.54240400 \\
\hline $\mathrm{H}$ & -5.49647900 & -1.97261800 & -0.36051500 & $\mathrm{H}$ & 2.41076800 & -2.79056500 & -2.91573100 \\
\hline $\mathrm{H}$ & -5.43347400 & -3.56099100 & 0.40437700 & $\mathrm{C}$ & 3.17428200 & -0.39970500 & -1.72401800 \\
\hline $\mathrm{H}$ & -5.37652700 & -2.11260700 & 1.40702900 & $\mathrm{C}$ & 2.43427800 & -0.01313700 & -2.85477100 \\
\hline $\mathrm{C}$ & -3.09793300 & -3.26773100 & -0.97737900 & $\mathrm{C}$ & 4.22003900 & 0.42686500 & -1.29353100 \\
\hline $\mathrm{H}$ & -3.47510000 & -4.29656800 & -0.97427700 & $\mathrm{C}$ & 2.74150600 & 1.15966100 & -3.54475300 \\
\hline $\mathrm{H}$ & -3.51912000 & -2.76064200 & -1.84936900 & $\mathrm{H}$ & 1.61792300 & -0.63518200 & -3.21116600 \\
\hline $\mathrm{H}$ & -2.01209600 & -3.30776000 & -1.09906600 & $\mathrm{C}$ & 4.52654900 & 1.60069800 & -1.98597000 \\
\hline $\mathrm{C}$ & 0.10924700 & 2.60202300 & 0.70463300 & $\mathrm{H}$ & 4.82117500 & 0.14864600 & -0.43721400 \\
\hline $\mathrm{C}$ & 0.70856200 & 2.63449900 & -0.55913600 & $\mathrm{C}$ & 3.79325700 & 1.97085600 & -3.11334600 \\
\hline $\mathrm{C}$ & -0.22408400 & 4.87692700 & 0.69532600 & $\mathrm{H}$ & 2.16646800 & 1.43015500 & -4.42538900 \\
\hline $\mathrm{C}$ & 0.84108500 & 3.86770900 & -1.19936100 & $\mathrm{H}$ & 5.35026200 & 2.22022200 & -1.64447000 \\
\hline $\mathrm{H}$ & 1.06138000 & 1.72283200 & -1.02779500 & $\mathrm{H}$ & 4.04579300 & 2.87621600 & -3.65707400 \\
\hline $\mathrm{C}$ & 0.36493900 & 5.01237800 & -0.56389600 & & & & \\
\hline
\end{tabular}




\begin{tabular}{|c|c|c|c|c|c|c|c|}
\hline 26-CO & & & & $\mathrm{C}$ & 2.49891100 & 3.24593400 & 2.51404900 \\
\hline $\mathrm{C}$ & 2.09868000 & 1.36911500 & -2.36407600 & $\mathrm{H}$ & 0.62165300 & 2.35151900 & 1.91019900 \\
\hline $\mathrm{H}$ & 1.51698900 & 1.14842500 & -3.26622200 & $\mathrm{C}$ & 3.75238800 & 3.66117400 & 2.06906500 \\
\hline $\mathrm{H}$ & 2.66529900 & 2.28302700 & -2.54277300 & $\mathrm{H}$ & 5.03592100 & 3.79983500 & 0.33224600 \\
\hline $\mathrm{C}$ & 3.11003300 & 0.24696700 & -2.09532900 & $\mathrm{H}$ & 2.21154600 & 3.36472300 & 3.55444000 \\
\hline $\mathrm{H}$ & 3.89467900 & 0.34559000 & -2.85460400 & $\mathrm{H}$ & 4.47238200 & 4.10942000 & 2.74583300 \\
\hline $\mathrm{H}$ & 3.60847200 & 0.41316100 & -1.13523700 & $\mathrm{C}$ & 2.82730500 & -1.97794600 & 0.60660800 \\
\hline $\mathrm{C}$ & 2.54753700 & -1.17804400 & -2.19482400 & $\mathrm{C}$ & 4.19748200 & -2.15813600 & 0.37783000 \\
\hline $\mathrm{H}$ & 3.36047900 & -1.89078400 & -2.36164500 & $\mathrm{C}$ & 3.11355000 & -2.17355000 & 2.88561800 \\
\hline $\mathrm{H}$ & 1.89108300 & -1.25659900 & -3.06772700 & $\mathrm{C}$ & 5.04053100 & -2.34793500 & 1.47468500 \\
\hline $\mathrm{P}$ & 1.56286000 & -1.71683700 & -0.72285400 & $\mathrm{H}$ & 4.60713200 & -2.15453200 & -0.62561600 \\
\hline $\mathrm{P}$ & 0.89105100 & 1.82314000 & -1.02352600 & $\mathrm{C}$ & 4.49170200 & -2.35833600 & 2.75456600 \\
\hline $\mathrm{C}$ & -0.00050000 & 3.33654300 & -1.80704100 & $\mathrm{H}$ & 2.64295100 & -2.17554300 & 3.86604500 \\
\hline $\mathrm{C}$ & 0.95346300 & -3.46516900 & -1.20666400 & $\mathrm{H}$ & 6.10717300 & -2.48741000 & 1.32691500 \\
\hline $\mathrm{C}$ & -1.02710800 & 2.80411300 & -2.82321700 & $\mathrm{H}$ & 5.11114600 & -2.50314600 & 3.63332400 \\
\hline $\mathrm{H}$ & -1.58272900 & 3.64475900 & -3.25356100 & $\mathrm{~N}$ & 3.22539900 & 2.94564500 & -0.16677800 \\
\hline $\mathrm{H}$ & -0.55118900 & 2.27267400 & -3.65369100 & $\mathrm{~N}$ & 2.29882900 & -1.97831900 & 1.84294600 \\
\hline $\mathrm{H}$ & -1.74606100 & 2.12501600 & -2.35942300 & $\mathrm{Pd}$ & -0.19748100 & -0.07554000 & 0.12156700 \\
\hline $\mathrm{C}$ & 1.01128300 & 4.25456000 & -2.52660800 & $\mathrm{C}$ & -1.76983600 & 1.08324500 & 0.87111400 \\
\hline $\mathrm{H}$ & 0.48723000 & 5.16615300 & -2.83452600 & $\mathrm{O}$ & -1.56587700 & 1.97352200 & 1.64960300 \\
\hline $\mathrm{H}$ & 1.83865800 & 4.55009400 & -1.87617900 & $\mathrm{C}$ & -3.26990100 & 0.72405200 & 0.48220300 \\
\hline $\mathrm{H}$ & 1.42439400 & 3.80113500 & -3.43053300 & $\mathrm{C}$ & -3.89596600 & 0.26663300 & 1.81772500 \\
\hline $\mathrm{C}$ & -0.69992900 & 4.16390100 & -0.71295500 & $\mathrm{O}$ & -3.28889400 & -0.36301600 & 2.66178500 \\
\hline $\mathrm{H}$ & 0.01912600 & 4.58939700 & -0.00777500 & $\mathrm{O}$ & -5.18261900 & 0.60567000 & 1.91682800 \\
\hline $\mathrm{H}$ & -1.22503600 & 4.99886100 & -1.19008000 & $\mathrm{C}$ & -5.85832200 & 0.17210800 & 3.12014200 \\
\hline $\mathrm{H}$ & -1.43131200 & 3.59402900 & -0.14235300 & $\mathrm{H}$ & -5.36934200 & 0.59607100 & 3.99915100 \\
\hline $\mathrm{C}$ & 0.00768700 & -3.31772900 & -2.41579500 & $\mathrm{H}$ & -6.87820500 & 0.54103800 & 3.02615500 \\
\hline $\mathrm{H}$ & -0.81407800 & -2.62384700 & -2.21505300 & $\mathrm{H}$ & -5.84395300 & -0.91747600 & 3.18830700 \\
\hline $\mathrm{H}$ & 0.53057600 & -2.99062600 & -3.31922800 & $\mathrm{C}$ & -3.92764200 & 2.04160700 & 0.01200800 \\
\hline $\mathrm{H}$ & -0.43646200 & -4.29415500 & -2.63981900 & $\mathrm{H}$ & -4.96713200 & 1.85982700 & -0.26308100 \\
\hline $\mathrm{C}$ & 2.13783000 & -4.38098000 & -1.57010800 & $\mathrm{H}$ & -3.89994400 & 2.77723100 & 0.81702400 \\
\hline $\mathrm{H}$ & 2.82509700 & -4.51761500 & -0.73066200 & $\mathrm{H}$ & -3.40718400 & 2.46559200 & -0.84823800 \\
\hline $\mathrm{H}$ & 1.74970000 & -5.36919400 & -1.84127100 & $\mathrm{C}$ & -3.41582300 & -0.41608600 & -0.54017200 \\
\hline $\mathrm{H}$ & 2.70565500 & -4.01342900 & -2.42946300 & $\mathrm{C}$ & -3.56371900 & -0.14174000 & -1.90934300 \\
\hline $\mathrm{C}$ & 0.17731600 & -4.07947600 & -0.02679800 & $\mathrm{C}$ & -3.46039600 & -1.76227900 & -0.13378500 \\
\hline $\mathrm{H}$ & -0.08568000 & -5.11330300 & -0.27735800 & $\mathrm{C}$ & -3.74079800 & -1.16901000 & -2.83815100 \\
\hline $\mathrm{H}$ & 0.76347900 & -4.09909900 & 0.89572000 & $\mathrm{H}$ & -3.57561400 & 0.88134200 & -2.26470400 \\
\hline $\mathrm{H}$ & -0.75697800 & -3.54521300 & 0.16231300 & $\mathrm{C}$ & -3.64989800 & -2.78893400 & -1.06046700 \\
\hline $\mathrm{C}$ & 2.01969800 & 2.54736400 & 0.26803800 & $\mathrm{H}$ & -3.38056700 & -2.01420900 & 0.91768400 \\
\hline $\mathrm{C}$ & 1.61011800 & 2.67354400 & 1.60192300 & $\mathrm{C}$ & -3.78925500 & -2.49825600 & -2.41815600 \\
\hline $\mathrm{C}$ & 4.06823600 & 3.48930600 & 0.71994500 & $\mathrm{H}$ & -3.86432500 & -0.92348600 & -3.88878200 \\
\hline
\end{tabular}




\begin{tabular}{|c|c|c|c|c|c|c|c|}
\hline $\mathrm{H}$ & -3.70452300 & -3.81673500 & -0.71375800 & $\mathrm{H}$ & 2.95245700 & -3.45818700 & -0.02188100 \\
\hline $\mathrm{H}$ & -3.94956600 & -3.29557100 & -3.13745300 & $\mathrm{H}$ & 1.19957300 & -3.54484400 & -0.27207900 \\
\hline $\mathrm{C}$ & -0.65718300 & -1.30564700 & 1.63436800 & $\mathrm{C}$ & -0.15079000 & 3.24808700 & 0.38419800 \\
\hline \multirow[t]{2}{*}{$\mathrm{O}$} & -0.79260200 & -1.95785200 & 2.55522400 & $\mathrm{C}$ & 0.30743800 & 3.02762500 & 1.68627400 \\
\hline & & & & $\mathrm{C}$ & 0.02523600 & 5.52948900 & 0.62114200 \\
\hline 26-МeO & & & & $\mathrm{C}$ & 0.63379400 & 4.13514000 & 2.47199500 \\
\hline $\mathrm{C}$ & 0.58466400 & 2.27936800 & -2.19761100 & $\mathrm{H}$ & 0.39475000 & 2.02196900 & 2.07697000 \\
\hline $\mathrm{H}$ & 0.16642800 & 1.83515100 & -3.10638000 & $\mathrm{C}$ & 0.48643800 & 5.41097200 & 1.93452700 \\
\hline $\mathrm{H}$ & 0.48123400 & 3.36444400 & -2.28868900 & $\mathrm{H}$ & -0.09857800 & 6.50737200 & 0.16164600 \\
\hline $\mathrm{C}$ & 2.06854200 & 1.91008800 & -2.08031500 & $\mathrm{H}$ & 0.99209800 & 3.99873100 & 3.48776900 \\
\hline $\mathrm{H}$ & 2.61247100 & 2.53397800 & -2.79900100 & $\mathrm{H}$ & 0.72407700 & 6.29818700 & 2.51202800 \\
\hline $\mathrm{H}$ & 2.46265700 & 2.18260100 & -1.09393500 & $\mathrm{C}$ & 3.54378600 & -0.52830500 & 0.05992600 \\
\hline $\mathrm{C}$ & 2.36644300 & 0.43789800 & -2.41363100 & $\mathrm{C}$ & 4.67607100 & 0.21786800 & -0.29296300 \\
\hline $\mathrm{H}$ & 3.39921000 & 0.32288600 & -2.75332200 & $\mathrm{C}$ & 4.46391900 & -1.04344400 & 2.11522900 \\
\hline $\mathrm{H}$ & 1.73918200 & 0.12348500 & -3.25375700 & $\mathrm{C}$ & 5.72937700 & 0.32367400 & 0.61775000 \\
\hline $\mathrm{P}$ & 2.04504100 & -0.74525900 & -1.01915700 & $\mathrm{H}$ & 4.75035400 & 0.71077900 & -1.25431700 \\
\hline $\mathrm{P}$ & -0.54703500 & 1.85433700 & -0.78206800 & $\mathrm{C}$ & 5.62680500 & -0.31942900 & 1.84780300 \\
\hline $\mathrm{C}$ & -2.27494300 & 2.16559600 & -1.55541500 & $\mathrm{H}$ & 4.34172000 & -1.56264800 & 3.06337700 \\
\hline $\mathrm{C}$ & 2.25681600 & -2.45656800 & -1.85150300 & $\mathrm{H}$ & 6.61470300 & 0.89872500 & 0.36376800 \\
\hline $\mathrm{C}$ & -2.62916100 & 0.90552300 & -2.36946000 & $\mathrm{H}$ & 6.42095200 & -0.26423400 & 2.58463700 \\
\hline $\mathrm{H}$ & -3.60757700 & 1.05087100 & -2.84026800 & $\mathrm{~N}$ & -0.28030300 & 4.47572000 & -0.14316400 \\
\hline $\mathrm{H}$ & -1.90981700 & 0.71931800 & -3.17426700 & $\mathrm{~N}$ & 3.44402600 & -1.14158500 & 1.25486500 \\
\hline $\mathrm{H}$ & -2.68740600 & 0.01205900 & -1.74464800 & $\mathrm{Pd}$ & -0.11287700 & -0.21568100 & 0.26501900 \\
\hline $\mathrm{C}$ & -2.25534800 & 3.39393100 & -2.49470200 & $\mathrm{C}$ & -1.70663000 & 0.04591900 & 1.49894200 \\
\hline $\mathrm{H}$ & -3.27609100 & 3.54952500 & -2.86134900 & $\mathrm{O}$ & -1.97579000 & 1.03733000 & 2.11674400 \\
\hline $\mathrm{H}$ & -1.93115700 & 4.29867600 & -1.97872400 & $\mathrm{C}$ & -2.47145000 & -1.31414600 & 1.76477700 \\
\hline $\mathrm{H}$ & -1.62158800 & 3.24303700 & -3.37157400 & $\mathrm{C}$ & -1.44124300 & -2.33478400 & 2.25144600 \\
\hline $\mathrm{C}$ & -3.32856800 & 2.40896300 & -0.46040200 & $\mathrm{O}$ & -1.67177100 & -3.27304200 & 2.96603500 \\
\hline $\mathrm{H}$ & -3.07788400 & 3.26937400 & 0.16546300 & $\mathrm{O}$ & -0.16594900 & -2.04703400 & 1.81829800 \\
\hline $\mathrm{H}$ & -4.28736900 & 2.61945900 & -0.94673000 & $\mathrm{C}$ & 0.87563100 & -2.87345800 & 2.42852900 \\
\hline $\mathrm{H}$ & -3.47237600 & 1.54391600 & 0.18545900 & $\mathrm{H}$ & 0.78053500 & -2.80641600 & 3.51299200 \\
\hline $\mathrm{C}$ & 1.09277400 & -2.63683700 & -2.84813300 & $\mathrm{H}$ & 0.74527600 & -3.91045300 & 2.11791400 \\
\hline $\mathrm{H}$ & 0.11730300 & -2.53042900 & -2.36233000 & $\mathrm{H}$ & 1.81221100 & -2.44914900 & 2.07564200 \\
\hline $\mathrm{H}$ & 1.13968900 & -1.93146800 & -3.68283600 & $\mathrm{C}$ & -3.47510100 & -1.10933100 & 2.91928900 \\
\hline $\mathrm{H}$ & 1.14347300 & -3.64398100 & -3.27690500 & $\mathrm{H}$ & -4.02005900 & -2.03825800 & 3.09329300 \\
\hline $\mathrm{C}$ & 3.60576900 & -2.56326200 & -2.58635200 & $\mathrm{H}$ & -2.94998000 & -0.83442700 & 3.83588400 \\
\hline $\mathrm{H}$ & 4.45361400 & -2.41526800 & -1.91104400 & $\mathrm{H}$ & -4.17582500 & -0.30673900 & 2.69114300 \\
\hline $\mathrm{H}$ & 3.70052800 & -3.56630400 & -3.01772400 & $\mathrm{C}$ & -3.14036400 & -1.80863000 & 0.46978300 \\
\hline $\mathrm{H}$ & 3.69189000 & -1.85021300 & -3.41121900 & $\mathrm{C}$ & -4.43374900 & -1.38237100 & 0.13431200 \\
\hline $\mathrm{C}$ & 2.16910000 & -3.55488100 & -0.77741400 & $\mathrm{C}$ & -2.48335000 & -2.69025400 & -0.40304100 \\
\hline $\mathrm{H}$ & 2.28213600 & -4.53384200 & -1.25660900 & $\mathrm{C}$ & -5.05188000 & -1.82822100 & -1.03527300 \\
\hline
\end{tabular}




\begin{tabular}{|c|c|c|c|c|c|c|c|}
\hline $\mathrm{H}$ & -4.97630200 & -0.70968700 & 0.78829300 & $\mathrm{H}$ & 5.42583700 & 2.14111300 & 0.34955900 \\
\hline $\mathrm{C}$ & -3.10262000 & -3.13792100 & -1.57125900 & $\mathrm{C}$ & 4.19659400 & 0.18685900 & -2.27085300 \\
\hline $\mathrm{H}$ & -1.48955100 & -3.05752300 & -0.16594200 & $\mathrm{H}$ & 4.95303300 & 0.51716900 & -2.99145400 \\
\hline $\mathrm{C}$ & -4.39019400 & -2.70727100 & -1.89306000 & $\mathrm{H}$ & 4.50201300 & -0.79605900 & -1.90069500 \\
\hline $\mathrm{H}$ & -6.05739300 & -1.49163600 & -1.26876000 & $\mathrm{H}$ & 3.24996300 & 0.08113900 & -2.80791100 \\
\hline $\mathrm{H}$ & -2.58229600 & -3.83582500 & -2.22044300 & $\mathrm{C}$ & -2.00504800 & 2.08719100 & 1.21343700 \\
\hline \multirow[t]{2}{*}{$\mathrm{H}$} & -4.87628700 & -3.06097700 & -2.79689000 & $\mathrm{C}$ & -3.18315500 & 1.33456600 & 1.18392200 \\
\hline & & & & $\mathrm{C}$ & -2.64759400 & 3.16707000 & 3.14175700 \\
\hline 27 & & & & $\mathrm{C}$ & -4.12711900 & 1.54605500 & 2.19015500 \\
\hline $\mathrm{C}$ & 0.58547400 & 3.07971500 & 0.57353300 & $\mathrm{H}$ & -3.37567300 & 0.61337100 & 0.39911300 \\
\hline $\mathrm{H}$ & 1.26846700 & 3.35403700 & -0.23568900 & $\mathrm{C}$ & -3.85945000 & 2.47680300 & 3.19094300 \\
\hline $\mathrm{H}$ & 0.06853000 & 3.98236100 & 0.89859200 & $\mathrm{H}$ & -2.39411200 & 3.90021900 & 3.90442500 \\
\hline $\mathrm{C}$ & 1.34410900 & 2.46906400 & 1.77481700 & $\mathrm{H}$ & -5.05802600 & 0.98713400 & 2.18567400 \\
\hline $\mathrm{H}$ & 1.45189300 & 3.24130500 & 2.54320800 & $\mathrm{H}$ & -4.56658100 & 2.66842600 & 3.99136600 \\
\hline $\mathrm{H}$ & 0.75356600 & 1.67461500 & 2.24203500 & $\mathrm{C}$ & 3.59487300 & -0.83999500 & 0.90228200 \\
\hline $\mathrm{C}$ & 2.74862600 & 1.94082700 & 1.44150900 & $\mathrm{C}$ & 4.42819700 & -0.77855300 & 2.02592900 \\
\hline $\mathrm{H}$ & 3.19841300 & 1.54155800 & 2.35349200 & $\mathrm{C}$ & 3.92933400 & -3.13748200 & 0.74709000 \\
\hline $\mathrm{H}$ & 3.38831800 & 2.76662200 & 1.11581500 & $\mathrm{C}$ & 5.01404800 & -1.94837500 & 2.51190600 \\
\hline$P$ & 2.75394400 & 0.62731600 & 0.10937800 & $\mathrm{H}$ & 4.63653200 & 0.16558100 & 2.51406700 \\
\hline$P$ & -0.71586200 & 1.95797500 & -0.12755200 & $\mathrm{C}$ & 4.76290000 & -3.15476600 & 1.86280500 \\
\hline $\mathrm{C}$ & -1.44465500 & 2.90166800 & -1.62438700 & $\mathrm{H}$ & 3.70143900 & -4.05184800 & 0.20537000 \\
\hline $\mathrm{C}$ & 4.08377400 & 1.22738500 & -1.14220200 & $\mathrm{H}$ & 5.66227700 & -1.91224800 & 3.38217900 \\
\hline $\mathrm{C}$ & -0.49960100 & 2.69166000 & -2.82371700 & $\mathrm{H}$ & 5.19811100 & -4.08646500 & 2.20700700 \\
\hline $\mathrm{H}$ & -0.89751900 & 3.23193600 & -3.69031300 & $\mathrm{~N}$ & -1.74006100 & 2.98508600 & 2.17595600 \\
\hline $\mathrm{H}$ & 0.50374900 & 3.08631500 & -2.63250600 & $\mathrm{~N}$ & 3.35883000 & -2.01699700 & 0.28109800 \\
\hline $\mathrm{H}$ & -0.42162800 & 1.63464500 & -3.09215500 & $\mathrm{Pd}$ & 0.36518200 & -0.08377300 & -0.66643000 \\
\hline $\mathrm{C}$ & -1.55863400 & 4.40430700 & -1.29421800 & $\mathrm{C}$ & -1.28164600 & -0.97419500 & -1.43628300 \\
\hline $\mathrm{H}$ & -2.06312200 & 4.90746300 & -2.12674600 & $\mathrm{O}$ & -1.47948900 & -0.79745600 & -2.61080600 \\
\hline $\mathrm{H}$ & -2.15089700 & 4.58606500 & -0.39229100 & $\mathrm{O}$ & 1.32185500 & -1.98648200 & -1.45068300 \\
\hline $\mathrm{H}$ & -0.58282900 & 4.88132700 & -1.16988300 & $\mathrm{H}$ & 2.16111700 & -2.09511100 & -0.91433900 \\
\hline $\mathrm{C}$ & -2.84465900 & 2.36080400 & -1.97476300 & $\mathrm{C}$ & 1.50258100 & -2.34089600 & -2.82923700 \\
\hline H & -3.57459400 & 2.58275900 & -1.19295700 & $\mathrm{H}$ & 0.69447600 & -1.88610300 & -3.40270200 \\
\hline $\mathrm{H}$ & -3.18357000 & 2.85879500 & -2.89027100 & $\mathrm{H}$ & 2.46188600 & -1.97956700 & -3.21101500 \\
\hline $\mathrm{H}$ & -2.85399900 & 1.28642700 & -2.16276500 & $\mathrm{H}$ & 1.45389000 & -3.42925800 & -2.94370400 \\
\hline $\mathrm{C}$ & 3.61332100 & 2.56322300 & -1.74982300 & $\mathrm{C}$ & -2.07119400 & -2.13437200 & -0.68820400 \\
\hline $\mathrm{H}$ & 2.64103900 & 2.46870100 & -2.24188400 & $\mathrm{C}$ & -3.53876200 & -1.90048700 & -1.10511400 \\
\hline $\mathrm{H}$ & 3.55658000 & 3.36867600 & -1.01168700 & $\mathrm{O}$ & -4.12173000 & -0.84297200 & -0.95050700 \\
\hline $\mathrm{H}$ & 4.33506600 & 2.87966700 & -2.51115400 & $\mathrm{O}$ & -4.10627800 & -2.97603200 & -1.65273600 \\
\hline $\mathrm{C}$ & 5.45625400 & 1.41806000 & -0.47146400 & $\mathrm{C}$ & -5.47612500 & -2.81810800 & -2.08604800 \\
\hline $\mathrm{H}$ & 5.86186500 & 0.47842800 & -0.08730600 & $\mathrm{H}$ & -5.54305900 & -2.02838600 & -2.83668500 \\
\hline $\mathrm{H}$ & 6.16604100 & 1.80265500 & -1.21277100 & $\mathrm{H}$ & -5.75729700 & -3.78133700 & -2.50835800 \\
\hline
\end{tabular}




\begin{tabular}{|c|c|c|c|c|c|c|c|}
\hline $\mathrm{H}$ & -6.11534800 & -2.56497000 & -1.23777000 & $\mathrm{H}$ & -3.27613400 & 2.64115600 & -1.09797600 \\
\hline $\mathrm{C}$ & -1.53548300 & -3.46966500 & -1.27028400 & $\mathrm{H}$ & -2.90375600 & 3.18324000 & -2.73750200 \\
\hline $\mathrm{H}$ & -2.09791300 & -4.30024600 & -0.84033800 & $\mathrm{H}$ & -2.41437600 & 1.56545700 & -2.20701000 \\
\hline $\mathrm{H}$ & -1.65720300 & -3.48942700 & -2.35478100 & $\mathrm{C}$ & 4.29894800 & 1.72669500 & -2.10126100 \\
\hline $\mathrm{H}$ & -0.48085700 & -3.58982400 & -1.02671100 & $\mathrm{H}$ & 3.37886100 & 1.66024200 & -2.68987000 \\
\hline $\mathrm{C}$ & -1.92004800 & -2.13186900 & 0.83621600 & $\mathrm{H}$ & 4.29310200 & 2.67759100 & -1.55951600 \\
\hline $\mathrm{C}$ & -0.64096200 & -2.26823100 & 1.40260000 & $\mathrm{H}$ & 5.14226000 & 1.76004800 & -2.79981600 \\
\hline $\mathrm{C}$ & -3.02000000 & -2.08577700 & 1.70290800 & $\mathrm{C}$ & 5.77057300 & 0.65065400 & -0.35407000 \\
\hline $\mathrm{C}$ & -0.46810600 & -2.32869600 & 2.78581500 & $\mathrm{H}$ & 5.96583700 & -0.22750400 & 0.26904400 \\
\hline $\mathrm{H}$ & 0.22689500 & -2.36060700 & 0.75844600 & $\mathrm{H}$ & 6.61645100 & 0.74396100 & -1.04508100 \\
\hline $\mathrm{C}$ & -2.84909800 & -2.15923900 & 3.08746600 & $\mathrm{H}$ & 5.77750900 & 1.54011300 & 0.28271700 \\
\hline $\mathrm{H}$ & -4.02483900 & -1.98643900 & 1.30801400 & $\mathrm{C}$ & 4.51790400 & -0.77112300 & -2.01319600 \\
\hline $\mathrm{C}$ & -1.57331200 & -2.27366000 & 3.63660000 & $\mathrm{H}$ & 5.37385300 & -0.72341700 & -2.69538400 \\
\hline $\mathrm{H}$ & 0.53174100 & -2.43646700 & 3.19701400 & $\mathrm{H}$ & 4.64482600 & -1.67098100 & -1.40095700 \\
\hline $\mathrm{H}$ & -3.72033300 & -2.12801300 & 3.73491400 & $\mathrm{H}$ & 3.61725900 & -0.88548400 & -2.62278500 \\
\hline \multirow[t]{2}{*}{$\mathrm{H}$} & -1.44180400 & -2.33035300 & 4.71283500 & $\mathrm{C}$ & -1.66781400 & 2.29690300 & 1.38164500 \\
\hline & & & & $\mathrm{C}$ & -2.81393500 & 1.49411400 & 1.43639100 \\
\hline \multicolumn{4}{|c|}{ TS21 } & $\mathrm{C}$ & -2.22496500 & 3.35556700 & 3.34886200 \\
\hline \multicolumn{4}{|c|}{ imaginary frequency $=-278.14 \mathrm{~cm}^{-1}$} & $\mathrm{C}$ & -3.69669200 & 1.66965200 & 2.50269000 \\
\hline $\mathrm{C}$ & 0.94029200 & 3.17689300 & 0.63867100 & $\mathrm{H}$ & -3.01823100 & 0.76021600 & 0.66575100 \\
\hline $\mathrm{H}$ & 1.54759400 & 3.51092700 & -0.20939200 & $\mathrm{C}$ & -3.40136400 & 2.61697000 & 3.48102400 \\
\hline $\mathrm{H}$ & 0.48844600 & 4.05663800 & 1.09845600 & $\mathrm{H}$ & -1.95195900 & 4.10095600 & 4.09329000 \\
\hline $\mathrm{C}$ & 1.82017800 & 2.46140500 & 1.68952000 & $\mathrm{H}$ & -4.59849700 & 1.06790500 & 2.56513900 \\
\hline $\mathrm{H}$ & 2.10377300 & 3.19036500 & 2.45653600 & $\mathrm{H}$ & -4.05939400 & 2.78238000 & 4.32802700 \\
\hline $\mathrm{H}$ & 1.24770400 & 1.68906600 & 2.21567400 & $\mathrm{C}$ & 3.15722100 & -1.04498700 & 0.99449100 \\
\hline $\mathrm{C}$ & 3.12663000 & 1.86975400 & 1.12848000 & $\mathrm{C}$ & 4.05085400 & -1.16979500 & 2.06059500 \\
\hline $\mathrm{H}$ & 3.78725800 & 1.56549200 & 1.94633800 & $\mathrm{C}$ & 2.44628700 & -3.29520200 & 1.37995300 \\
\hline $\mathrm{H}$ & 3.66391000 & 2.65040600 & 0.57902500 & $\mathrm{C}$ & 4.14275200 & -2.36562200 & 2.77643400 \\
\hline $\mathrm{P}$ & 2.87859500 & 0.47016700 & -0.08728800 & $\mathrm{H}$ & 4.67931700 & -0.33135800 & 2.33238900 \\
\hline $\mathrm{P}$ & -0.45792100 & 2.14344500 & -0.03091100 & $\mathrm{C}$ & 3.33223000 & -3.44934800 & 2.43304600 \\
\hline $\mathrm{C}$ & -1.18048400 & 3.21209800 & -1.43848200 & $\mathrm{H}$ & 1.76067400 & -4.06747800 & 1.05146000 \\
\hline $\mathrm{C}$ & 4.47054700 & 0.51112100 & -1.16415200 & $\mathrm{H}$ & 4.84501200 & -2.44938000 & 3.60018100 \\
\hline $\mathrm{C}$ & -0.18871600 & 3.15659700 & -2.61882800 & $\mathrm{H}$ & 3.37622900 & -4.38819100 & 2.97203600 \\
\hline $\mathrm{H}$ & -0.58706300 & 3.74777300 & -3.45167100 & $\mathrm{~N}$ & -1.37457800 & 3.20730800 & 2.32607000 \\
\hline $\mathrm{H}$ & 0.79115800 & 3.57547500 & -2.36542100 & $\mathrm{~N}$ & 2.38673900 & -2.12980500 & 0.70704400 \\
\hline $\mathrm{H}$ & -0.04907500 & 2.13020700 & -2.97288900 & $\mathrm{Pd}$ & 0.49307800 & 0.08867700 & -0.74048300 \\
\hline $\mathrm{C}$ & -1.39296600 & 4.66924900 & -0.98786300 & $\mathrm{C}$ & -0.93039400 & -1.28918400 & -1.57882500 \\
\hline $\mathrm{H}$ & -1.86375100 & 5.23069200 & -1.80338100 & $\mathrm{O}$ & -1.03264700 & -0.96047800 & -2.74662300 \\
\hline $\mathrm{H}$ & -2.04911000 & 4.73835700 & -0.11571700 & $\mathrm{O}$ & 0.50623000 & -2.14623200 & -1.25124900 \\
\hline $\mathrm{H}$ & -0.45253500 & 5.17331100 & -0.74697400 & $\mathrm{H}$ & 1.64678900 & -2.06732200 & -0.05259100 \\
\hline $\mathrm{C}$ & -2.52187400 & 2.60454600 & -1.88810400 & $\mathrm{C}$ & 1.14280100 & -2.60206900 & -2.45393900 \\
\hline
\end{tabular}




\begin{tabular}{|c|c|c|c|c|c|c|c|}
\hline $\mathrm{H}$ & 0.93485300 & -1.92306700 & -3.28393300 & $\mathrm{C}$ & 4.34536200 & 0.82903800 & -1.18099300 \\
\hline $\mathrm{H}$ & 2.22324600 & -2.65670300 & -2.28784200 & $\mathrm{C}$ & -0.11977300 & 3.20222700 & -2.57231600 \\
\hline $\mathrm{H}$ & 0.76682100 & -3.59979800 & -2.70594500 & $\mathrm{H}$ & -0.50314700 & 3.76047000 & -3.43292900 \\
\hline $\mathrm{C}$ & -2.07888700 & -2.11918900 & -0.89338400 & $\mathrm{H}$ & 0.78220100 & 3.72067500 & -2.23251000 \\
\hline $\mathrm{C}$ & -3.35895500 & -1.41184500 & -1.40644600 & $\mathrm{H}$ & 0.15515200 & 2.20231800 & -2.92085500 \\
\hline $\mathrm{O}$ & -3.75593700 & -0.34492300 & -0.98345800 & C & -1.58172700 & 4.56300600 & -1.01553000 \\
\hline $\mathrm{O}$ & -3.98174200 & -2.10011300 & -2.36827200 & $\mathrm{H}$ & -1.98794800 & 5.12775200 & -1.86149100 \\
\hline C & -5.15025300 & -1.47039800 & -2.93561400 & $\mathrm{H}$ & -2.35315500 & 4.55684600 & -0.23835600 \\
\hline $\mathrm{H}$ & -4.87214600 & -0.53156000 & -3.41868200 & $\mathrm{H}$ & -0.71514200 & 5.12156800 & -0.64923700 \\
\hline $\mathrm{H}$ & -5.53575500 & -2.18035300 & -3.66578900 & $\mathrm{C}$ & -2.46391500 & 2.45177000 & -2.08421100 \\
\hline $\mathrm{H}$ & -5.89015400 & -1.27079800 & -2.15785300 & $\mathrm{H}$ & -3.30524200 & 2.43461800 & -1.38720300 \\
\hline C & -1.96859500 & -3.55912100 & -1.46541500 & $\mathrm{H}$ & -2.78640700 & 3.01594700 & -2.96584300 \\
\hline $\mathrm{H}$ & -2.86194000 & -4.13348700 & -1.21519400 & $\mathrm{H}$ & -2.25534900 & 1.42689600 & -2.39819300 \\
\hline $\mathrm{H}$ & -1.87417400 & -3.53847700 & -2.55214700 & $\mathrm{C}$ & 3.97257700 & 2.07219200 & -2.01684600 \\
\hline $\mathrm{H}$ & -1.10126500 & -4.05599700 & -1.02965800 & $\mathrm{H}$ & 3.02745300 & 1.93526800 & -2.55104100 \\
\hline C & -2.14800700 & -2.19758800 & 0.63499900 & $\mathrm{H}$ & 3.90732200 & 2.98403900 & -1.41402400 \\
\hline $\mathrm{C}$ & -1.13896800 & -1.75779500 & 1.49630100 & $\mathrm{H}$ & 4.75406200 & 2.24307300 & -2.76470800 \\
\hline C & -3.28046000 & -2.80245000 & 1.20712100 & $\mathrm{C}$ & 5.68567600 & 1.05545600 & -0.46156500 \\
\hline C & -1.25097200 & -1.91705000 & 2.88078200 & $\mathrm{H}$ & 6.01855400 & 0.16779400 & 0.08345100 \\
\hline $\mathrm{H}$ & -0.27581400 & -1.24148700 & 1.08724600 & $\mathrm{H}$ & 6.45605400 & 1.27575900 & -1.20866700 \\
\hline C & -3.39668400 & -2.96373600 & 2.58609200 & $\mathrm{H}$ & 5.66112100 & 1.90424900 & 0.22864100 \\
\hline $\mathrm{H}$ & -4.08863300 & -3.15005800 & 0.56888600 & $\mathrm{C}$ & 4.44392900 & -0.39324800 & -2.11129100 \\
\hline C & -2.37675900 & -2.52437100 & 3.43305600 & $\mathrm{H}$ & 5.23665700 & -0.22137400 & -2.84695600 \\
\hline $\mathrm{H}$ & -0.45909000 & -1.55012900 & 3.52882600 & $\mathrm{H}$ & 4.70353100 & -1.30854000 & -1.56893200 \\
\hline $\mathrm{H}$ & -4.28528000 & -3.43237000 & 2.99839600 & $\mathrm{H}$ & 3.51493900 & -0.56027200 & -2.66374800 \\
\hline \multirow[t]{2}{*}{$\mathrm{H}$} & -2.46550000 & -2.64648900 & 4.50835000 & $\mathrm{C}$ & -1.86381700 & 2.12342000 & 1.22634300 \\
\hline & & & & $\mathrm{C}$ & -3.01332700 & 1.33484300 & 1.14110400 \\
\hline \multirow{2}{*}{\multicolumn{4}{|c|}{$\begin{array}{l}\text { TS21-2H } \\
\text { imaginary frequency }=-297.13 \mathrm{~cm}^{-1}\end{array}$}} & C & -2.64602500 & 2.96994900 & 3.34210900 \\
\hline & & & & $\mathrm{C}$ & -3.97656900 & 1.38774100 & 2.14921000 \\
\hline C & 0.78780600 & 3.13824300 & 0.72246600 & $\mathrm{H}$ & -3.15571400 & 0.67166900 & 0.29084200 \\
\hline $\mathrm{H}$ & 1.42248100 & 3.46915600 & -0.10580500 & $\mathrm{C}$ & -3.79427700 & 2.20952000 & 3.26885700 \\
\hline $\mathrm{H}$ & 0.35851500 & 4.07160400 & 1.10828600 & $\mathrm{H}$ & -2.41093100 & 3.63479900 & 4.16488400 \\
\hline C & 1.65643700 & 2.43748000 & 1.80355100 & $\mathrm{H}$ & -4.86743500 & 0.77437600 & 2.06308300 \\
\hline $\mathrm{H}$ & 1.87780300 & 3.16151900 & 2.59629900 & $\mathrm{H}$ & -4.52428300 & 2.25865300 & 4.06817400 \\
\hline $\mathrm{H}$ & 1.10585100 & 1.61809700 & 2.28228400 & $\mathrm{C}$ & 3.36892500 & -0.97669200 & 0.98648700 \\
\hline C & 3.01266000 & 1.91588400 & 1.28699400 & $\mathrm{C}$ & 4.37302800 & -1.06254500 & 1.95205300 \\
\hline $\mathrm{H}$ & 3.62567200 & 1.59401400 & 2.13341700 & $\mathrm{C}$ & 2.92973600 & -3.30564900 & 1.28314400 \\
\hline $\mathrm{H}$ & 3.55541800 & 2.73907700 & 0.81152100 & $\mathrm{C}$ & 4.65655300 & -2.28189200 & 2.57539900 \\
\hline$P$ & 2.87056000 & 0.54941600 & 0.01182600 & $\mathrm{H}$ & 4.94533500 & -0.18149600 & 2.21484500 \\
\hline$P$ & -0.52324600 & 2.06394900 & -0.07371500 & $\mathrm{C}$ & 3.92951700 & -3.42390400 & 2.23561300 \\
\hline C & -1.22232000 & 3.14509300 & -1.49356300 & $\mathrm{H}$ & 2.30512100 & -4.13254300 & 0.96454100 \\
\hline
\end{tabular}




\begin{tabular}{|c|c|c|c|c|c|c|c|}
\hline $\mathrm{H}$ & 5.44556400 & -2.33803800 & 3.31911600 & $\mathrm{H}$ & 1.11777800 & -3.01453200 & 1.33740700 \\
\hline H & 4.12688900 & -4.38390800 & 2.69813300 & $\mathrm{H}$ & 2.02854900 & -1.91534400 & 2.37070200 \\
\hline $\mathrm{N}$ & -1.73938000 & 2.90814700 & 2.33496300 & $\mathrm{C}$ & -0.10288600 & -1.53392100 & 2.34914000 \\
\hline $\mathrm{N}$ & 2.68011800 & -2.11654300 & 0.69998800 & $\mathrm{H}$ & -0.08765400 & -1.95894300 & 3.35993100 \\
\hline $\mathrm{Pd}$ & 0.51060100 & 0.03312800 & -0.63771300 & $\mathrm{H}$ & -0.11732600 & -0.44552300 & 2.48408300 \\
\hline $\mathrm{C}$ & -0.88358900 & -1.32063700 & -1.50884200 & $\mathrm{C}$ & -1.41688100 & -2.01475700 & 1.68871900 \\
\hline $\mathrm{O}$ & -0.93206000 & -1.03412600 & -2.68096200 & $\mathrm{H}$ & -2.21535000 & -2.05717800 & 2.43650900 \\
\hline $\mathrm{O}$ & 0.67225000 & -2.17348800 & -1.09794100 & $\mathrm{H}$ & -1.28742900 & -3.04287000 & 1.33095400 \\
\hline $\mathrm{H}$ & 1.86855700 & -2.09127200 & 0.00733200 & $P$ & -1.96627300 & -1.03135100 & 0.19839200 \\
\hline $\mathrm{C}$ & 1.31006700 & -2.62915700 & -2.30006100 & $P$ & 1.76098900 & -0.95518600 & 0.18642900 \\
\hline $\mathrm{H}$ & 1.03589600 & -2.00352400 & -3.15343400 & $\mathrm{C}$ & 2.53244100 & -2.22725000 & -1.01597700 \\
\hline $\mathrm{H}$ & 2.39839900 & -2.60329900 & -2.17700000 & $\mathrm{C}$ & -3.40710500 & -2.05567200 & -0.55896800 \\
\hline $\mathrm{H}$ & 0.99639000 & -3.65931700 & -2.50143500 & $\mathrm{C}$ & 1.39240100 & -3.10624300 & -1.56517200 \\
\hline $\mathrm{C}$ & -2.01832900 & -2.16716100 & -0.82309400 & $\mathrm{H}$ & 1.80286900 & -3.82731400 & -2.28113100 \\
\hline $\mathrm{C}$ & -3.28824400 & -1.57575100 & -1.49636100 & $\mathrm{H}$ & 0.88983000 & -3.68142200 & -0.78039100 \\
\hline $\mathrm{O}$ & -3.75628200 & -0.48918200 & -1.19941700 & $\mathrm{H}$ & 0.64232500 & -2.50265200 & -2.08557200 \\
\hline $\mathrm{O}$ & -3.79167600 & -2.36593000 & -2.43254700 & $\mathrm{C}$ & 3.58655700 & -3.10069700 & -0.31116100 \\
\hline $\mathrm{C}$ & -4.93849400 & -1.86318900 & -3.16383000 & $\mathrm{H}$ & 4.01322700 & -3.80566300 & -1.03447000 \\
\hline $\mathrm{H}$ & -4.65986800 & -0.96197200 & -3.71260100 & $\mathrm{H}$ & 4.40806300 & -2.50364800 & 0.09367100 \\
\hline $\mathrm{H}$ & -5.21456400 & -2.66359200 & -3.84721200 & $\mathrm{H}$ & 3.16235900 & -3.68962000 & 0.50741000 \\
\hline $\mathrm{H}$ & -5.75443800 & -1.63820800 & -2.47511000 & $\mathrm{C}$ & 3.18311400 & -1.46644200 & -2.18812200 \\
\hline $\mathrm{C}$ & -1.80524700 & -3.65004200 & -1.22901300 & $\mathrm{H}$ & 4.04845000 & -0.88220200 & -1.86486600 \\
\hline $\mathrm{H}$ & -2.68951100 & -4.23083200 & -0.95883700 & $\mathrm{H}$ & 3.53777100 & -2.18898300 & -2.93201000 \\
\hline $\mathrm{H}$ & -1.65092700 & -3.74621400 & -2.30483200 & $\mathrm{H}$ & 2.47731500 & -0.79099900 & -2.68178400 \\
\hline H & -0.94419600 & -4.05114600 & -0.69568400 & $\mathrm{C}$ & -2.73241300 & -3.23363900 & -1.29353900 \\
\hline $\mathrm{C}$ & -2.17086000 & -2.08484300 & 0.69704200 & $\mathrm{H}$ & -2.00567500 & -2.88944900 & -2.03517600 \\
\hline $\mathrm{C}$ & -1.11186400 & -1.79595800 & 1.56594300 & $\mathrm{H}$ & -2.22485000 & -3.91882900 & -0.60741500 \\
\hline $\mathrm{C}$ & -3.40872900 & -2.43159400 & 1.26479300 & $\mathrm{H}$ & -3.49814200 & -3.81396500 & -1.82009900 \\
\hline $\mathrm{C}$ & -1.28563200 & -1.82921300 & 2.95301200 & $\mathrm{C}$ & -4.42060700 & -2.59202800 & 0.46459300 \\
\hline $\mathrm{H}$ & -0.13989100 & -1.53995800 & 1.15970800 & $\mathrm{H}$ & -4.96556700 & -1.78916500 & 0.96999300 \\
\hline $\mathrm{C}$ & -3.58514200 & -2.47179100 & 2.64751800 & $\mathrm{H}$ & -5.16736000 & -3.20398300 & -0.05465000 \\
\hline $\mathrm{H}$ & -4.24796000 & -2.68627100 & 0.62373200 & $\mathrm{H}$ & -3.95507100 & -3.23017400 & 1.22141300 \\
\hline $\mathrm{C}$ & -2.52236400 & -2.16663900 & 3.50125900 & $\mathrm{C}$ & -4.12137700 & -1.16116500 & -1.58932500 \\
\hline $\mathrm{H}$ & -0.44612200 & -1.60521300 & 3.60614900 & $\mathrm{H}$ & -4.87592200 & -1.74976500 & -2.12268400 \\
\hline $\mathrm{H}$ & -4.54972400 & -2.75686500 & 3.05667800 & $\mathrm{H}$ & -4.64196400 & -0.32061600 & -1.11823600 \\
\hline $\mathrm{H}$ & -2.65454000 & -2.20826900 & 4.57805900 & $\mathrm{H}$ & -3.42585300 & -0.76480300 & -2.33635900 \\
\hline \multirow[t]{2}{*}{$\mathrm{H}$} & -0.92097400 & 3.50236600 & 2.41058600 & $\mathrm{C}$ & 3.24429200 & -0.05397600 & 0.87137300 \\
\hline & & & & $\mathrm{C}$ & 3.84847900 & 0.96942600 & 0.12347800 \\
\hline \multirow{2}{*}{\multicolumn{4}{|c|}{$\begin{array}{l}\text { TS21-b1 } \\
\text { imaginary frequency }=-280.40 \mathrm{~cm}^{-1}\end{array}$}} & $\mathrm{C}$ & 4.77160000 & 0.20185900 & 2.57574500 \\
\hline & & & & $\mathrm{C}$ & 4.97610800 & 1.60315200 & 0.64556400 \\
\hline $\mathrm{C}$ & 1.20315500 & -1.97021800 & 1.65425600 & $\mathrm{H}$ & 3.44249400 & 1.26411200 & -0.83870700 \\
\hline
\end{tabular}




\begin{tabular}{|c|c|c|c|c|c|c|c|}
\hline C & 5.45054200 & 1.21456000 & 1.89792100 & $\mathrm{H}$ & -2.00839200 & -2.13824300 & 2.80202900 \\
\hline $\mathrm{H}$ & 5.10305600 & -0.12602700 & 3.55866700 & $\mathrm{H}$ & -1.36133800 & -3.45246700 & 1.84403100 \\
\hline $\mathrm{H}$ & 5.47086200 & 2.39037500 & 0.08433700 & $\mathrm{P}$ & -1.85785400 & -1.59964500 & 0.37965700 \\
\hline $\mathrm{H}$ & 6.32121100 & 1.68415300 & 2.34353200 & $\mathrm{P}$ & 1.86380500 & -1.31916400 & 0.23437200 \\
\hline $\mathrm{C}$ & -2.88565800 & 0.39687200 & 1.01199000 & $\mathrm{C}$ & 3.00697400 & -2.35172300 & -0.89714900 \\
\hline $\mathrm{C}$ & -3.67317500 & 0.34105900 & 2.16449900 & $\mathrm{C}$ & -3.22212100 & -2.79496900 & -0.25216000 \\
\hline $\mathrm{C}$ & -3.37970800 & 2.72630300 & 0.83503800 & $\mathrm{C}$ & 2.11999900 & -3.35968100 & -1.65702600 \\
\hline $\mathrm{C}$ & -4.32215400 & 1.48285200 & 2.63961600 & $\mathrm{H}$ & 2.73626900 & -3.89909400 & -2.38502000 \\
\hline $\mathrm{H}$ & -3.78246500 & -0.59805500 & 2.69196000 & $\mathrm{H}$ & 1.67251300 & -4.10951800 & -0.99649800 \\
\hline $\mathrm{C}$ & -4.18365800 & 2.69591700 & 1.96238700 & $\mathrm{H}$ & 1.31926100 & -2.85484600 & -2.20719000 \\
\hline $\mathrm{H}$ & -3.18922500 & 3.62375000 & 0.25754600 & $\mathrm{C}$ & 4.08632000 & -3.09768300 & -0.09146200 \\
\hline $\mathrm{H}$ & -4.93497000 & 1.42402300 & 3.53389300 & $\mathrm{H}$ & 4.72455200 & -3.66151300 & -0.78156500 \\
\hline H & -4.67462600 & 3.59940900 & 2.30392100 & $\mathrm{H}$ & 4.73129800 & -2.40936500 & 0.46248800 \\
\hline $\mathrm{N}$ & 3.69759600 & -0.42435500 & 2.07776800 & $\mathrm{H}$ & 3.66105100 & -3.81709600 & 0.61576600 \\
\hline $\mathrm{N}$ & -2.76624200 & 1.60780600 & 0.40216900 & C & 3.67080200 & -1.40579000 & -1.91805200 \\
\hline $\mathrm{Pd}$ & -0.04203500 & 0.21411100 & -0.72241800 & $\mathrm{H}$ & 4.30681400 & -0.66215500 & -1.43355600 \\
\hline $\mathrm{C}$ & 0.71393900 & 2.14343300 & -1.30125100 & $\mathrm{H}$ & 4.29738200 & -2.00150800 & -2.59229100 \\
\hline $\mathrm{O}$ & 1.36050100 & 2.17613300 & -2.32279000 & $\mathrm{H}$ & 2.92946100 & -0.87746200 & -2.52321900 \\
\hline $\mathrm{O}$ & -0.99601200 & 2.12646200 & -1.52806200 & $\mathrm{C}$ & -2.46546100 & -4.02857000 & -0.79031600 \\
\hline $\mathrm{H}$ & -2.09241800 & 1.73643200 & -0.42126700 & $\mathrm{H}$ & -1.72924300 & -3.75149900 & -1.55108900 \\
\hline $\mathrm{C}$ & -1.39212500 & 2.10915800 & -2.90461700 & $\mathrm{H}$ & -1.95148700 & -4.58442000 & 0.00045700 \\
\hline $\mathrm{H}$ & -0.51747500 & 1.92942800 & -3.53653300 & $\mathrm{H}$ & -3.18227800 & -4.71647900 & -1.25216600 \\
\hline $\mathrm{H}$ & -2.13229700 & 1.31988500 & -3.07514200 & $\mathrm{C}$ & -4.23575000 & -3.22588800 & 0.82102200 \\
\hline $\mathrm{H}$ & -1.82443100 & 3.08170600 & -3.16578800 & $\mathrm{H}$ & -4.84917400 & -2.38966700 & 1.16899700 \\
\hline $\mathrm{C}$ & 0.88955000 & 3.04120700 & -0.08565400 & $\mathrm{H}$ & -4.92162500 & -3.96600800 & 0.39251700 \\
\hline $\mathrm{C}$ & 0.91558400 & 4.52590600 & -0.48184300 & $\mathrm{H}$ & -3.75909200 & -3.69424000 & 1.68756300 \\
\hline H & 1.10026200 & 5.14972300 & 0.39763000 & $\mathrm{C}$ & -3.95343900 & -2.10786000 & -1.41964900 \\
\hline $\mathrm{H}$ & 1.70572800 & 4.71520700 & -1.21225800 & $\mathrm{H}$ & -4.67406500 & -2.80644400 & -1.85887500 \\
\hline $\mathrm{H}$ & -0.03651800 & 4.82939800 & -0.92684100 & $\mathrm{H}$ & -4.51508900 & -1.22496700 & -1.09592200 \\
\hline $\mathrm{H}$ & 0.10014300 & 2.83774000 & 0.64221000 & $\mathrm{H}$ & -3.25916400 & -1.80954100 & -2.21084200 \\
\hline \multirow[t]{2}{*}{$\mathrm{H}$} & 1.83767800 & 2.75623000 & 0.37964700 & $\mathrm{C}$ & 3.03318800 & -0.25795200 & 1.22050800 \\
\hline & & & & $\mathrm{C}$ & 3.37081900 & -0.48022000 & 2.56356400 \\
\hline \multirow{2}{*}{\multicolumn{4}{|c|}{$\begin{array}{l}\text { TS21-c1 } \\
\text { imaginary frequency }=-301.15 \mathrm{~cm}^{-1}\end{array}$}} & $\mathrm{C}$ & 4.40905600 & 1.59589200 & 1.13290600 \\
\hline & & & & $\mathrm{C}$ & 4.26620400 & 0.38858700 & 3.19050900 \\
\hline $\mathrm{C}$ & 1.24654400 & -2.54232500 & 1.50512700 & $\mathrm{H}$ & 2.95862000 & -1.31351100 & 3.11917200 \\
\hline $\mathrm{H}$ & 0.87586100 & -3.40661700 & 0.94440700 & $\mathrm{C}$ & 4.80487600 & 1.44620700 & 2.46325900 \\
\hline $\mathrm{H}$ & 2.08338400 & -2.91622500 & 2.10140300 & $\mathrm{H}$ & 4.79952300 & 2.41132900 & 0.52798900 \\
\hline $\mathrm{C}$ & 0.12823900 & -1.99985000 & 2.43275800 & $\mathrm{H}$ & 4.54106200 & 0.23280100 & 4.22959300 \\
\hline $\mathrm{H}$ & 0.26994300 & -2.42452700 & 3.43352100 & $\mathrm{H}$ & 5.50817100 & 2.14149500 & 2.90912700 \\
\hline $\mathrm{H}$ & 0.21447200 & -0.91344700 & 2.55372600 & $\mathrm{C}$ & -2.84448300 & -0.10983700 & 0.96177900 \\
\hline $\mathrm{C}$ & -1.30493800 & -2.36889900 & 1.99560300 & $\mathrm{C}$ & -3.67895800 & -0.04227200 & 2.07751000 \\
\hline
\end{tabular}




\begin{tabular}{|c|c|c|c|c|c|c|c|}
\hline $\mathrm{C}$ & -3.36416200 & 2.16767100 & 0.47654000 & $\mathrm{C}$ & -0.12886600 & 1.35521400 & -2.54306100 \\
\hline $\mathrm{C}$ & -4.37051600 & 1.13479200 & 2.37878400 & $\mathrm{H}$ & -0.06036500 & 1.59805800 & -3.61005900 \\
\hline $\mathrm{H}$ & -3.79664300 & -0.91167500 & 2.71147500 & $\mathrm{H}$ & -0.01729000 & 0.26639500 & -2.47572300 \\
\hline $\mathrm{C}$ & -4.21513300 & 2.25753600 & 1.56776500 & $\mathrm{C}$ & -1.54384500 & 1.77785900 & -2.08569800 \\
\hline $\mathrm{H}$ & -3.14367900 & 2.99658100 & -0.18496800 & $\mathrm{H}$ & -2.27579200 & 1.51288400 & -2.85539000 \\
\hline $\mathrm{H}$ & -5.02442400 & 1.17115200 & 3.24468800 & $\mathrm{H}$ & -1.58354600 & 2.86941800 & -1.99562400 \\
\hline $\mathrm{H}$ & -4.72784700 & 3.18860600 & 1.77752500 & $\mathrm{P}$ & -2.06896100 & 1.10880000 & -0.42140900 \\
\hline $\mathrm{N}$ & 3.53959800 & 0.77999400 & 0.52658300 & $\mathrm{P}$ & 1.62794100 & 1.29893500 & -0.23070100 \\
\hline $\mathrm{N}$ & -2.72297500 & 1.01679600 & 0.20591700 & $\mathrm{C}$ & 2.30198900 & 2.76815700 & 0.78820600 \\
\hline $\mathrm{Pd}$ & -0.03704100 & -0.43679700 & -0.81733500 & $\mathrm{C}$ & -3.50580300 & 2.25000800 & 0.13812500 \\
\hline $\mathrm{C}$ & 0.61803800 & 1.09897700 & -2.12773800 & $\mathrm{C}$ & 1.10528300 & 3.64643900 & 1.20447600 \\
\hline $\mathrm{O}$ & 0.93935200 & 0.60568500 & -3.18411700 & $\mathrm{H}$ & 1.46931700 & 4.48592100 & 1.80748300 \\
\hline $\mathrm{O}$ & -1.13204800 & 1.22525300 & -1.94723000 & $\mathrm{H}$ & 0.57398100 & 4.07268900 & 0.34732400 \\
\hline $\mathrm{H}$ & -2.05493800 & 1.04506800 & -0.63273800 & $\mathrm{H}$ & 0.39042000 & 3.08188600 & 1.81135200 \\
\hline $\mathrm{C}$ & -1.82648700 & 0.89474700 & -3.15163100 & $\mathrm{C}$ & 3.31012300 & 3.59916800 & -0.02804800 \\
\hline $\mathrm{H}$ & -2.14843700 & 1.81884300 & -3.64688000 & $\mathrm{H}$ & 4.17376400 & 3.00473100 & -0.33925600 \\
\hline $\mathrm{H}$ & -1.17243600 & 0.33979900 & -3.83072400 & $\mathrm{H}$ & 2.86158100 & 4.03936600 & -0.92353500 \\
\hline $\mathrm{H}$ & -2.70831200 & 0.28484600 & -2.92377800 & $\mathrm{C}$ & 2.97955200 & 2.23043200 & 2.06484400 \\
\hline $\mathrm{C}$ & 1.24314500 & 2.37105500 & -1.51851200 & $\mathrm{H}$ & 3.90838100 & 1.69972300 & 1.84168500 \\
\hline $\mathrm{C}$ & 1.76512700 & 3.25184600 & -2.67435700 & $\mathrm{H}$ & 3.24037500 & 3.07597500 & 2.71157500 \\
\hline $\mathrm{H}$ & 2.26539100 & 4.13599500 & -2.26980000 & $\mathrm{H}$ & 2.32446600 & 1.56131100 & 2.63148900 \\
\hline $\mathrm{H}$ & 2.47618000 & 2.68770400 & -3.28010000 & $\mathrm{C}$ & -2.84024900 & 3.56600300 & 0.59484000 \\
\hline $\mathrm{H}$ & 0.96114800 & 3.58378500 & -3.33679300 & $\mathrm{H}$ & -2.10751900 & 3.39775700 & 1.38904600 \\
\hline $\mathrm{C}$ & 0.39141300 & 3.13276700 & -0.51419100 & $\mathrm{H}$ & -2.34276300 & 4.09140700 & -0.22671600 \\
\hline $\mathrm{C}$ & -0.46983000 & 4.17015000 & -0.90736200 & $\mathrm{H}$ & -3.61102500 & 4.23894700 & 0.98664300 \\
\hline $\mathrm{C}$ & 0.51304400 & 2.86194400 & 0.85649800 & $\mathrm{C}$ & -4.54529600 & 2.54760000 & -0.95604500 \\
\hline $\mathrm{C}$ & -1.17503700 & 4.91723400 & 0.03764000 & $\mathrm{H}$ & -5.10329200 & 1.65448400 & -1.25157700 \\
\hline $\mathrm{H}$ & -0.57677700 & 4.41430600 & -1.95910400 & $\mathrm{H}$ & -5.27756900 & 3.26620900 & -0.57002600 \\
\hline $\mathrm{C}$ & -0.20031000 & 3.59938200 & 1.80672400 & $\mathrm{H}$ & -4.09848400 & 2.99310100 & -1.84997500 \\
\hline $\mathrm{H}$ & 1.19617400 & 2.08326400 & 1.18009200 & $\mathrm{C}$ & -4.19043800 & 1.58659300 & 1.34781500 \\
\hline $\mathrm{C}$ & -1.04405600 & 4.63436100 & 1.40172400 & $\mathrm{H}$ & -4.94975000 & 2.26448000 & 1.75290500 \\
\hline $\mathrm{H}$ & -1.80989200 & 5.73639300 & -0.28992700 & $\mathrm{H}$ & -4.69933100 & 0.65522200 & 1.07768900 \\
\hline $\mathrm{H}$ & -0.07163200 & 3.38353600 & 2.86397600 & $\mathrm{H}$ & -3.47696800 & 1.37383800 & 2.15025800 \\
\hline $\mathrm{H}$ & -1.57414700 & 5.23139800 & 2.13848300 & $\mathrm{C}$ & 3.15886000 & 0.38102800 & -0.76895500 \\
\hline \multirow[t]{2}{*}{$\mathrm{H}$} & 2.11275000 & 1.97759900 & -0.97943200 & $\mathrm{C}$ & 3.87167600 & -0.40156400 & 0.15224700 \\
\hline & & & & $\mathrm{C}$ & 4.63087900 & -0.15562600 & -2.45591800 \\
\hline \multicolumn{4}{|c|}{ TS21-d1 } & $\mathrm{C}$ & 5.02251800 & -1.06020200 & -0.27482400 \\
\hline \multicolumn{4}{|c|}{ imaginary frequency $=-273.22 \mathrm{~cm}^{-1}$} & $\mathrm{H}$ & 3.52841500 & -0.49211000 & 1.17671400 \\
\hline $\mathrm{C}$ & 1.04629400 & 2.06186500 & -1.83211800 & $\mathrm{C}$ & 5.41368000 & -0.93849600 & -1.60825000 \\
\hline $\mathrm{H}$ & 0.78644000 & 3.10698400 & -1.63565000 & $\mathrm{H}$ & 4.89663000 & -0.03802600 & -3.50442100 \\
\hline $\mathrm{H}$ & 1.91652000 & 2.05414100 & -2.49402000 & $\mathrm{H}$ & 5.60045300 & -1.66213300 & 0.42020100 \\
\hline
\end{tabular}




\begin{tabular}{|c|c|c|c|c|c|c|c|}
\hline $\mathrm{H}$ & 6.30061900 & -1.43704800 & -1.98510700 & $\mathrm{C}$ & -0.03507500 & 2.40514800 & 2.18905300 \\
\hline $\mathrm{C}$ & -2.97124600 & -0.47078000 & -0.89689500 & $\mathrm{H}$ & -0.18419100 & 2.98697800 & 3.10552000 \\
\hline $\mathrm{C}$ & -3.81471500 & -0.65184600 & -1.99469100 & $\mathrm{H}$ & -0.17655700 & 1.35714200 & 2.47774400 \\
\hline $\mathrm{C}$ & -3.35823700 & -2.73795300 & -0.26738300 & $\mathrm{C}$ & 1.42296400 & 2.63775500 & 1.74636000 \\
\hline $\mathrm{C}$ & -4.44658400 & -1.87887500 & -2.20912100 & $\mathrm{H}$ & 2.10349100 & 2.43050400 & 2.57835900 \\
\hline $\mathrm{H}$ & -3.98202400 & 0.16721900 & -2.68215100 & $\mathrm{H}$ & 1.55351600 & 3.69692000 & 1.50043100 \\
\hline $\mathrm{C}$ & -4.22372200 & -2.93889700 & -1.32980800 & $\mathrm{P}$ & 1.92603300 & 1.67544000 & 0.21817900 \\
\hline $\mathrm{H}$ & -3.07821400 & -3.51094800 & 0.43658600 & $\mathrm{P}$ & -1.76963300 & 1.42913100 & 0.13136100 \\
\hline $\mathrm{H}$ & -5.10653700 & -2.00549000 & -3.06183200 & $\mathrm{C}$ & -2.82760600 & 2.26996900 & -1.21594500 \\
\hline $\mathrm{H}$ & -4.69139200 & -3.90574800 & -1.47210200 & $\mathrm{C}$ & 3.18345900 & 2.82706000 & -0.66446500 \\
\hline $\mathrm{N}$ & 3.53191900 & 0.49579400 & -2.05144900 & $\mathrm{C}$ & -1.86481000 & 2.88152900 & -2.25387100 \\
\hline $\mathrm{N}$ & -2.78011700 & -1.53882800 & -0.07900000 & $\mathrm{H}$ & -2.44797000 & 3.35671200 & -3.05123000 \\
\hline $\mathrm{Pd}$ & -0.12730600 & 0.16699900 & 0.80556900 & $\mathrm{H}$ & -1.22001500 & 3.65311400 & -1.81959000 \\
\hline $\mathrm{C}$ & 0.71942300 & -1.36549100 & 1.99078700 & $\mathrm{H}$ & -1.23338000 & 2.11299800 & -2.71044100 \\
\hline $\mathrm{O}$ & 1.19844700 & -0.99853000 & 3.04562900 & $\mathrm{C}$ & -3.73677700 & 3.36311300 & -0.62671600 \\
\hline $\mathrm{O}$ & -1.00316100 & -1.51555100 & 1.99871900 & $\mathrm{H}$ & -4.37541500 & 3.76595800 & -1.42155000 \\
\hline $\mathrm{H}$ & -2.07140800 & -1.49210600 & 0.70799500 & $\mathrm{H}$ & -4.38673000 & 2.97801000 & 0.16306800 \\
\hline $\mathrm{C}$ & -1.57593700 & -1.19590600 & 3.27156200 & $\mathrm{H}$ & -3.16292200 & 4.19852600 & -0.21521800 \\
\hline $\mathrm{H}$ & -0.84959400 & -0.66186300 & 3.89043000 & $\mathrm{C}$ & -3.67894400 & 1.18702100 & -1.90680900 \\
\hline $\mathrm{H}$ & -2.46638000 & -0.57268700 & 3.13473700 & $\mathrm{H}$ & -4.40591100 & 0.73838400 & -1.22340000 \\
\hline $\mathrm{H}$ & -1.85755700 & -2.12641100 & 3.77813000 & $\mathrm{H}$ & -4.24059500 & 1.64544000 & -2.72897100 \\
\hline $\mathrm{C}$ & 1.22048100 & -2.64281300 & 1.28260500 & $\mathrm{H}$ & -3.05412100 & 0.39540800 & -2.32974700 \\
\hline $\mathrm{C}$ & 0.90008800 & -3.88389800 & 2.14231400 & $\mathrm{C}$ & 2.33612200 & 3.96210000 & -1.27912200 \\
\hline $\mathrm{H}$ & 1.41087500 & -4.76643000 & 1.74599900 & $\mathrm{H}$ & 1.56036000 & 3.57373500 & -1.94620300 \\
\hline $\mathrm{H}$ & 1.24212300 & -3.71452700 & 3.16544700 & $\mathrm{H}$ & 1.85546000 & 4.58614300 & -0.51935600 \\
\hline $\mathrm{H}$ & -0.17505900 & -4.07101700 & 2.15076100 & $\mathrm{H}$ & 2.98819700 & 4.61741200 & -1.86726600 \\
\hline $\mathrm{C}$ & 0.72899400 & -2.84931700 & -0.13966000 & $\mathrm{C}$ & 4.25867000 & 3.41620300 & 0.26423500 \\
\hline $\mathrm{O}$ & -0.42796600 & -3.02428200 & -0.47948700 & $\mathrm{H}$ & 4.93242800 & 2.64791400 & 0.65513600 \\
\hline $\mathrm{O}$ & 1.75820500 & -2.88579100 & -1.00139000 & $\mathrm{H}$ & 4.87581000 & 4.12308800 & -0.30245200 \\
\hline $\mathrm{C}$ & 1.43179800 & -3.16449900 & -2.37577500 & $\mathrm{H}$ & 3.83264500 & 3.96839400 & 1.10709500 \\
\hline $\mathrm{H}$ & 0.80655800 & -2.36941900 & -2.78923300 & $\mathrm{C}$ & 3.85494400 & 2.03464500 & -1.80047700 \\
\hline $\mathrm{H}$ & 2.38617700 & -3.20611600 & -2.89825400 & $\mathrm{H}$ & 4.52592000 & 2.69777900 & -2.35755200 \\
\hline $\mathrm{H}$ & 0.90360200 & -4.11705800 & -2.45744600 & $\mathrm{H}$ & 4.46216700 & 1.20337800 & -1.42549000 \\
\hline $\mathrm{H}$ & 2.30359900 & -2.51591000 & 1.23425600 & $\mathrm{H}$ & 3.11988400 & 1.64104700 & -2.50788500 \\
\hline \multirow[t]{2}{*}{$\mathrm{H}$} & 3.68191000 & 4.42415300 & 0.59083100 & $\mathrm{C}$ & -3.02216300 & 0.73069400 & 1.32783800 \\
\hline & & & & $\mathrm{C}$ & -3.51778000 & -0.56935300 & 1.16667000 \\
\hline \multicolumn{4}{|c|}{ TS21-e1 } & $\mathrm{C}$ & -4.33488800 & 1.07406000 & 3.18843800 \\
\hline \multicolumn{4}{|c|}{ imaginary frequency $=-257.53 \mathrm{~cm}^{-1}$} & $\mathrm{C}$ & -4.47656300 & -1.03605900 & 2.06809900 \\
\hline $\mathrm{C}$ & -1.10224100 & 2.83692600 & 1.15464500 & $\mathrm{H}$ & -3.14479300 & -1.20488500 & 0.37091800 \\
\hline $\mathrm{H}$ & -0.69763000 & 3.58929200 & 0.46891400 & $\mathrm{C}$ & -4.89813000 & -0.20039400 & 3.09981400 \\
\hline $\mathrm{H}$ & -1.95164600 & 3.27933800 & 1.67810700 & $\mathrm{H}$ & -4.62954500 & 1.75532400 & 3.98409900 \\
\hline
\end{tabular}




\begin{tabular}{|c|c|c|c|c|c|c|c|}
\hline $\mathrm{H}$ & -4.87884000 & -2.03984600 & 1.96711800 & $\mathrm{H}$ & -3.34043400 & -3.83904700 & -2.28776900 \\
\hline $\mathrm{H}$ & -5.63828500 & -0.52340200 & 3.82459700 & $\mathrm{H}$ & -4.18216900 & -2.54697800 & -1.40999800 \\
\hline $\mathrm{C}$ & 3.03155100 & 0.33036400 & 0.92364900 & & & & \\
\hline $\mathrm{C}$ & 3.92040200 & 0.44398700 & 1.99285200 & \multirow{2}{*}{\multicolumn{4}{|c|}{$\begin{array}{l}\text { TS21-f1 } \\
\text { imaginary frequency }=-254.38 \mathrm{~cm}^{-1}\end{array}$}} \\
\hline $\mathrm{C}$ & 3.66754700 & -1.96112200 & 0.68524500 & & & & \\
\hline $\mathrm{C}$ & 4.69354000 & -0.64855900 & 2.39503000 & $\mathrm{C}$ & -0.05508800 & -3.09086600 & 1.38940500 \\
\hline $\mathrm{H}$ & 4.01510600 & 1.39078500 & 2.50941000 & $\mathrm{H}$ & -0.58627400 & -3.78781900 & 0.73168700 \\
\hline $\mathrm{C}$ & 4.57063000 & -1.86946600 & 1.73239900 & $\mathrm{H}$ & 0.60284300 & -3.69731600 & 2.01808000 \\
\hline $\mathrm{H}$ & 3.47078100 & -2.87338500 & 0.13669700 & $\mathrm{C}$ & -1.06284400 & -2.33414300 & 2.28848500 \\
\hline $\mathrm{H}$ & 5.38762200 & -0.54381100 & 3.22325100 & $\mathrm{H}$ & -1.19539500 & -2.91738100 & 3.20749100 \\
\hline $\mathrm{H}$ & 5.14932300 & -2.73765500 & 2.02402300 & $\mathrm{H}$ & -0.63794500 & -1.37614100 & 2.59887000 \\
\hline $\mathrm{N}$ & -3.42071300 & 1.53602200 & 2.32744900 & $\mathrm{C}$ & -2.46875700 & -2.15253800 & 1.68453800 \\
\hline $\mathrm{N}$ & 2.94590000 & -0.88638700 & 0.31622100 & $\mathrm{H}$ & -3.15914400 & -1.79673800 & 2.45611200 \\
\hline $\mathrm{Pd}$ & 0.10815800 & 0.27098400 & -0.73168700 & $\mathrm{H}$ & -2.85139100 & -3.12798000 & 1.36395900 \\
\hline $\mathrm{C}$ & -0.46126000 & -1.42464400 & -1.90351500 & $\mathrm{P}$ & -2.54614300 & -1.06253800 & 0.16755100 \\
\hline $\mathrm{O}$ & -0.90261600 & -1.04477300 & -2.97230300 & $\mathrm{P}$ & 1.03941400 & -2.01786500 & 0.30118800 \\
\hline $\mathrm{O}$ & 1.22974400 & -1.40590800 & -1.79225000 & $\mathrm{C}$ & 1.77114900 & -3.23743000 & -0.96402900 \\
\hline $\mathrm{H}$ & 2.24822500 & -1.03864000 & -0.46257000 & $\mathrm{C}$ & -4.12808300 & -1.66671300 & -0.73977500 \\
\hline $\mathrm{C}$ & 1.85826700 & -1.12519500 & -3.04975000 & $\mathrm{C}$ & 0.61566100 & -3.63941700 & -1.90981900 \\
\hline $\mathrm{H}$ & 1.19148200 & -0.54632200 & -3.69384600 & $\mathrm{H}$ & 1.01116600 & -4.29671200 & -2.69237100 \\
\hline $\mathrm{H}$ & 2.78085700 & -0.56345900 & -2.87575000 & $\mathrm{H}$ & -0.17839400 & -4.19107100 & -1.39629000 \\
\hline $\mathrm{H}$ & 2.10188900 & -2.07033100 & -3.54902800 & $\mathrm{H}$ & 0.17598200 & -2.76403400 & -2.39779000 \\
\hline $\mathrm{C}$ & -0.86335400 & -2.81293500 & -1.27386400 & $\mathrm{C}$ & 2.34903200 & -4.51329500 & -0.31874900 \\
\hline $\mathrm{C}$ & -0.64583600 & -3.85260900 & -2.38908900 & $\mathrm{H}$ & 2.75563000 & -5.15925800 & -1.10542200 \\
\hline $\mathrm{H}$ & -0.99359600 & -4.83639600 & -2.06284200 & $\mathrm{H}$ & 3.15711400 & -4.31240600 & 0.38798700 \\
\hline $\mathrm{H}$ & -1.18259600 & -3.56051900 & -3.29100500 & $\mathrm{H}$ & 1.58121000 & -5.08887500 & 0.20711900 \\
\hline $\mathrm{H}$ & 0.41248400 & -3.91838300 & -2.64803200 & $\mathrm{C}$ & 2.83204800 & -2.50615000 & -1.81723300 \\
\hline $\mathrm{C}$ & -0.10674200 & -3.15913000 & 0.01159500 & $\mathrm{H}$ & 3.69131600 & -2.15836700 & -1.24082800 \\
\hline $\mathrm{C}$ & -0.36624500 & -2.43730900 & 1.18742800 & $\mathrm{H}$ & 3.20831400 & -3.19807500 & -2.57954300 \\
\hline $\mathrm{C}$ & 0.80208800 & -4.22468600 & 0.08194400 & $\mathrm{H}$ & 2.40434700 & -1.64187600 & -2.33089900 \\
\hline $\mathrm{C}$ & 0.26381700 & -2.76587300 & 2.38980400 & $\mathrm{C}$ & -3.75005900 & -3.02434200 & -1.37093300 \\
\hline $\mathrm{H}$ & -1.08547400 & -1.62979400 & 1.16649000 & $\mathrm{H}$ & -2.87438600 & -2.93983900 & -2.02175100 \\
\hline $\mathrm{C}$ & 1.42783800 & -4.56188900 & 1.28599600 & $\mathrm{H}$ & -3.54894400 & -3.79574700 & -0.62113000 \\
\hline $\mathrm{H}$ & 1.01570300 & -4.82266500 & -0.79586300 & $\mathrm{H}$ & -4.58768700 & -3.38064500 & -1.98066600 \\
\hline $\mathrm{C}$ & 1.16364500 & -3.83188900 & 2.44663800 & $\mathrm{C}$ & -5.34859500 & -1.83506000 & 0.18104700 \\
\hline $\mathrm{H}$ & 0.02883500 & -2.20253000 & 3.28879200 & $\mathrm{H}$ & -5.69118600 & -0.88008300 & 0.59071800 \\
\hline $\mathrm{H}$ & 2.10331800 & -5.41267300 & 1.31734700 & $\mathrm{H}$ & -6.18138500 & -2.25042800 & -0.39817400 \\
\hline $\mathrm{H}$ & 1.63355100 & -4.10578400 & 3.38695300 & $\mathrm{H}$ & -5.15990900 & -2.52334600 & 1.01010700 \\
\hline $\mathrm{O}$ & -2.23997400 & -2.72885900 & -0.88828300 & $\mathrm{C}$ & -4.45571700 & -0.66942900 & -1.86482100 \\
\hline $\mathrm{C}$ & -3.24551700 & -2.81797100 & -1.90249300 & $\mathrm{H}$ & -5.32141300 & -1.03142300 & -2.43031900 \\
\hline $\mathrm{H}$ & -3.06037500 & -2.12713800 & -2.72919300 & $\mathrm{H}$ & -4.71436900 & 0.32307400 & -1.47983300 \\
\hline
\end{tabular}




\begin{tabular}{|c|c|c|c|c|c|c|c|}
\hline $\mathrm{H}$ & -3.62368300 & -0.56769600 & -2.56696600 & $\mathrm{H}$ & 0.92990300 & 4.77659200 & -1.10747900 \\
\hline $\mathrm{C}$ & 2.33353400 & -1.56463700 & 1.56676500 & $\mathrm{C}$ & 0.15559700 & 4.15148500 & 2.13426500 \\
\hline $\mathrm{C}$ & 3.69636700 & -1.88389200 & 1.52719600 & $\mathrm{H}$ & 0.36917300 & 2.23193600 & 3.09845000 \\
\hline $\mathrm{C}$ & 2.59817200 & -0.47074400 & 3.58381400 & $\mathrm{H}$ & 0.09847000 & 5.91313200 & 0.88810800 \\
\hline $\mathrm{C}$ & 4.51926900 & -1.46576400 & 2.57352400 & $\mathrm{H}$ & -0.19494300 & 4.66340000 & 3.02608100 \\
\hline $\mathrm{H}$ & 4.12033500 & -2.44074700 & 0.70358500 & $\mathrm{C}$ & 3.11932000 & 1.60605900 & -1.11741400 \\
\hline $\mathrm{C}$ & 3.96446600 & -0.74671500 & 3.62866900 & F & 3.14094900 & 0.64200800 & -0.16599300 \\
\hline $\mathrm{H}$ & 2.12215000 & 0.09060800 & 4.38517500 & $\mathrm{~F}$ & 3.71132100 & 1.08324500 & -2.20585200 \\
\hline $\mathrm{H}$ & 5.57906600 & -1.70186800 & 2.55921000 & F & 3.89878700 & 2.61453400 & -0.67718200 \\
\hline $\mathrm{H}$ & 4.56878700 & -0.40289500 & 4.46133900 & & & & \\
\hline $\mathrm{C}$ & -3.07765100 & 0.59780000 & 0.87208000 & \multirow{2}{*}{\multicolumn{4}{|c|}{$\begin{array}{l}\text { TS21-g1 } \\
\text { imaginary frequency }=-292.97 \mathrm{~cm}^{-1}\end{array}$}} \\
\hline $\mathrm{C}$ & -3.94425500 & 0.82028900 & 1.94188200 & & & & \\
\hline $\mathrm{C}$ & -2.79297600 & 2.96193100 & 0.65679000 & $\mathrm{C}$ & 0.60991100 & -2.93876800 & 0.86720300 \\
\hline $\mathrm{C}$ & -4.24512600 & 2.12140700 & 2.35517600 & $\mathrm{H}$ & 0.09535400 & -3.54737900 & 0.11656300 \\
\hline $\mathrm{H}$ & -4.39035000 & -0.02357300 & 2.45260900 & $\mathrm{H}$ & 1.36935100 & -3.56511800 & 1.33721100 \\
\hline $\mathrm{C}$ & -3.66414900 & 3.21017800 & 1.70591100 & $\mathrm{C}$ & -0.39184600 & -2.46170000 & 1.94528000 \\
\hline $\mathrm{H}$ & -2.26013500 & 3.73638400 & 0.11831000 & $\mathrm{H}$ & -0.33967300 & -3.15579900 & 2.79139700 \\
\hline $\mathrm{H}$ & -4.92816000 & 2.28035000 & 3.18395600 & $\mathrm{H}$ & -0.09490600 & -1.48828500 & 2.34687200 \\
\hline $\mathrm{H}$ & -3.86670800 & 4.23012800 & 2.00975800 & $\mathrm{C}$ & -1.86111100 & -2.43095000 & 1.48127000 \\
\hline $\mathrm{N}$ & 1.80017300 & -0.85850500 & 2.58217000 & $\mathrm{H}$ & -2.52077300 & -2.27105000 & 2.33993600 \\
\hline $\mathrm{N}$ & -2.53858400 & 1.69715100 & 0.27359400 & $\mathrm{H}$ & -2.12699400 & -3.41256900 & 1.07464200 \\
\hline $\mathrm{Pd}$ & -0.33235700 & -0.39521900 & -0.73477800 & $\mathrm{P}$ & -2.22487900 & -1.19267900 & 0.12281200 \\
\hline $\mathrm{C}$ & 0.82764500 & 0.92125300 & -1.93274500 & $\mathrm{P}$ & 1.49353200 & -1.57145200 & -0.04751800 \\
\hline $\mathrm{O}$ & 1.07419500 & 0.34975800 & -2.97729900 & $\mathrm{C}$ & 2.26968400 & -2.46161400 & -1.55322600 \\
\hline $\mathrm{O}$ & -0.76512400 & 1.53734200 & -1.83390500 & $\mathrm{C}$ & -3.71440300 & -1.97907900 & -0.81434200 \\
\hline $\mathrm{H}$ & -1.83688400 & 1.58194900 & -0.50821600 & $\mathrm{C}$ & 1.10989600 & -2.90010700 & -2.47289900 \\
\hline $\mathrm{C}$ & -1.44500300 & 1.48592600 & -3.09412400 & $\mathrm{H}$ & 1.52494700 & -3.36345300 & -3.37510500 \\
\hline $\mathrm{H}$ & -1.02796900 & 0.69790500 & -3.72667200 & $\mathrm{H}$ & 0.45477100 & -3.64014300 & -2.00345600 \\
\hline $\mathrm{H}$ & -2.50925500 & 1.29751500 & -2.92528200 & $\mathrm{H}$ & 0.50253000 & -2.04367200 & -2.78341300 \\
\hline $\mathrm{H}$ & -1.32833100 & 2.44941000 & -3.60382300 & $\mathrm{C}$ & 3.09781500 & -3.68704000 & -1.12621100 \\
\hline $\mathrm{C}$ & 1.69242000 & 2.12621400 & -1.41732200 & $\mathrm{H}$ & 3.54244500 & -4.15278000 & -2.01363400 \\
\hline $\mathrm{C}$ & 1.83501500 & 3.07341900 & -2.63605400 & $\mathrm{H}$ & 3.91488700 & -3.41296000 & -0.45262600 \\
\hline $\mathrm{H}$ & 2.52639100 & 3.88753200 & -2.40862800 & $\mathrm{H}$ & 2.48774100 & -4.44790800 & -0.63045200 \\
\hline $\mathrm{H}$ & 2.21688300 & 2.51543600 & -3.48937900 & $\mathrm{C}$ & 3.15341600 & -1.47386100 & -2.34184100 \\
\hline $\mathrm{H}$ & 0.86526700 & 3.49528800 & -2.90349300 & $\mathrm{H}$ & 4.07878000 & -1.23725600 & -1.81185800 \\
\hline $\mathrm{C}$ & 1.12789800 & 2.83249800 & -0.17557500 & $\mathrm{H}$ & 3.44324600 & -1.93752100 & -3.29186500 \\
\hline $\mathrm{C}$ & 0.95489800 & 2.14235900 & 1.03593900 & $\mathrm{H}$ & 2.62484500 & -0.54350800 & -2.57017200 \\
\hline $\mathrm{C}$ & 0.79910300 & 4.19745800 & -0.20199800 & $\mathrm{C}$ & -3.11784600 & -3.14586800 & -1.63006600 \\
\hline $\mathrm{C}$ & 0.47383900 & 2.79347500 & 2.17428400 & $\mathrm{H}$ & -2.32036500 & -2.80897800 & -2.29905900 \\
\hline $\mathrm{H}$ & 1.21226500 & 1.09501100 & 1.11053200 & $\mathrm{H}$ & -2.72042400 & -3.94358500 & -0.99473500 \\
\hline $\mathrm{C}$ & 0.32016600 & 4.85062100 & 0.93664100 & $\mathrm{H}$ & -3.90637500 & -3.59052800 & -2.24743800 \\
\hline
\end{tabular}




\begin{tabular}{|c|c|c|c|c|c|c|c|}
\hline $\mathrm{C}$ & -4.83217200 & -2.50046600 & 0.10476900 & $\mathrm{H}$ & 0.02936700 & 4.59611900 & -1.55646200 \\
\hline $\mathrm{H}$ & -5.33469300 & -1.69204100 & 0.64432200 & $\mathrm{C}$ & 2.75025700 & 2.74126900 & -0.35107500 \\
\hline $\mathrm{H}$ & -5.59543700 & -2.99911700 & -0.50392800 & $\mathrm{H}$ & 2.82359500 & 1.76270700 & 0.12927300 \\
\hline $\mathrm{H}$ & -4.47208400 & -3.23397400 & 0.83225400 & $\mathrm{H}$ & 3.10072000 & 3.46669200 & 0.39246400 \\
\hline $\mathrm{C}$ & -4.28418300 & -0.93106500 & -1.78698900 & $\mathrm{C}$ & 3.70059500 & 2.79280300 & -1.55418100 \\
\hline $\mathrm{H}$ & -5.08049000 & -1.38671600 & -2.38578900 & $\mathrm{H}$ & 3.40993100 & 2.08982500 & -2.33701300 \\
\hline $\mathrm{H}$ & -4.72390600 & -0.07403900 & -1.26535700 & $\mathrm{H}$ & 3.74201100 & 3.79099200 & -1.99933400 \\
\hline $\mathrm{H}$ & -3.52053600 & -0.56484900 & -2.47957300 & $\mathrm{H}$ & 4.71745700 & 2.54614100 & -1.22963800 \\
\hline $\mathrm{C}$ & 2.89202400 & -1.25441500 & 1.15193900 & $\mathrm{C}$ & 0.50539800 & 3.28347800 & 0.76145900 \\
\hline $\mathrm{C}$ & 4.00821700 & -0.46616700 & 0.83053400 & $\mathrm{H}$ & -0.56117400 & 3.29032300 & 0.51507600 \\
\hline $\mathrm{C}$ & 3.67716900 & -1.57465100 & 3.29829500 & $\mathrm{C}$ & 0.81500300 & 4.65910900 & 1.38759700 \\
\hline $\mathrm{C}$ & 4.99354300 & -0.26328500 & 1.79554200 & $\mathrm{H}$ & 0.50115800 & 5.49345400 & 0.75628100 \\
\hline $\mathrm{H}$ & 4.10903400 & -0.01770700 & -0.14929200 & $\mathrm{H}$ & 0.28398800 & 4.75244600 & 2.34089900 \\
\hline $\mathrm{C}$ & 4.82974000 & -0.82716900 & 3.05986300 & $\mathrm{H}$ & 1.88077200 & 4.78431500 & 1.60512600 \\
\hline $\mathrm{H}$ & 3.50221700 & -2.02613900 & 4.27276700 & $\mathrm{C}$ & 0.74251100 & 2.19412700 & 1.82067100 \\
\hline $\mathrm{H}$ & 5.87294700 & 0.32963000 & 1.56192300 & $\mathrm{H}$ & 0.07324500 & 2.35014900 & 2.67473000 \\
\hline $\mathrm{H}$ & 5.56976200 & -0.69149700 & 3.84152000 & $\mathrm{H}$ & 0.56340900 & 1.18892200 & 1.42194300 \\
\hline $\mathrm{C}$ & -3.06665700 & 0.20194700 & 1.06337500 & $\mathrm{H}$ & 1.76622200 & 2.22078400 & 2.20667600 \\
\hline $\mathrm{C}$ & -3.90339700 & 0.08877500 & 2.17668000 & & & & \\
\hline $\mathrm{C}$ & -3.39040100 & 2.56819500 & 1.11128800 & b1 & & & \\
\hline $\mathrm{C}$ & -4.48766000 & 1.22162300 & 2.74633400 & $\mathrm{C}$ & 0.00000000 & 0.66526500 & 0.00000000 \\
\hline $\mathrm{H}$ & -4.10602400 & -0.88878500 & 2.59532900 & $\mathrm{H}$ & 0.92362000 & 1.23828500 & 0.00000000 \\
\hline $\mathrm{C}$ & -4.23724500 & 2.48357400 & 2.20313400 & $\mathrm{H}$ & -0.92357800 & 1.23833400 & 0.00000000 \\
\hline $\mathrm{H}$ & -3.11741900 & 3.50234300 & 0.63439400 & $\mathrm{C}$ & 0.00000000 & -0.66526500 & 0.00000000 \\
\hline $\mathrm{H}$ & -5.14020300 & 1.11839400 & 3.60785200 & $\mathrm{H}$ & -0.92362000 & -1.23828500 & 0.00000000 \\
\hline $\mathrm{H}$ & -4.67780900 & 3.38174900 & 2.61919300 & $\mathrm{H}$ & 0.92357800 & -1.23833400 & 0.00000000 \\
\hline $\mathrm{N}$ & 2.73348400 & -1.79137000 & 2.37353700 & & & & \\
\hline $\mathrm{N}$ & -2.83791300 & 1.45638300 & 0.58738900 & c1 & & & \\
\hline Pd & -0.17917200 & 0.00942400 & -0.64356900 & $\mathrm{C}$ & 2.97674500 & -0.33529900 & -0.00025200 \\
\hline $\mathrm{C}$ & 0.65521000 & 1.87309100 & -1.45823300 & $\mathrm{H}$ & 2.83792200 & -1.41245500 & -0.00075000 \\
\hline $\mathrm{O}$ & 1.03838700 & 1.59522500 & -2.57280000 & $\mathrm{H}$ & 4.00327200 & 0.01623500 & -0.00014000 \\
\hline $\mathrm{O}$ & -1.05549500 & 2.01602500 & -1.35834400 & $\mathrm{C}$ & 1.95487200 & 0.52965400 & 0.00018400 \\
\hline $\mathrm{H}$ & -2.13376500 & 1.60848100 & -0.20074200 & $\mathrm{H}$ & 2.18692500 & 1.59449300 & 0.00059900 \\
\hline $\mathrm{C}$ & -1.67587200 & 2.03371100 & -2.65007000 & $\mathrm{C}$ & 0.51518800 & 0.22076000 & 0.00012600 \\
\hline $\mathrm{H}$ & -1.03192900 & 1.55442400 & -3.39173000 & $\mathrm{C}$ & -0.40653400 & 1.28181700 & 0.00000100 \\
\hline $\mathrm{H}$ & -2.63248700 & 1.50312400 & -2.60467600 & $\mathrm{C}$ & 0.00934900 & -1.09260400 & 0.00015700 \\
\hline $\mathrm{H}$ & -1.85127400 & 3.07338100 & -2.95054300 & $\mathrm{C}$ & -1.78100300 & 1.04615300 & -0.00012200 \\
\hline $\mathrm{C}$ & 1.24545000 & 3.04059400 & -0.59948500 & $\mathrm{H}$ & -0.03517700 & 2.30381400 & -0.00003100 \\
\hline $\mathrm{C}$ & 1.07757500 & 4.28463700 & -1.51247800 & $\mathrm{C}$ & -1.36183400 & -1.32983400 & 0.00005100 \\
\hline $\mathrm{H}$ & 1.66761900 & 5.12124400 & -1.13008400 & $\mathrm{H}$ & 0.69437400 & -1.93498200 & 0.00031500 \\
\hline $\mathrm{H}$ & 1.41264200 & 4.07071900 & -2.52804500 & $\mathrm{C}$ & -2.26513800 & -0.26205800 & -0.00009200 \\
\hline
\end{tabular}




\begin{tabular}{|c|c|c|c|c|c|c|c|}
\hline $\mathrm{H}$ & -2.47232100 & 1.88420500 & -0.00021600 & $\mathrm{C}$ & 2.39892900 & -1.35518900 & -0.31914300 \\
\hline $\mathrm{H}$ & -1.73011100 & -2.35201400 & 0.00009200 & $\mathrm{C}$ & 1.05465300 & -0.99758200 & -0.41170800 \\
\hline \multirow[t]{2}{*}{$\mathrm{H}$} & -3.33474300 & -0.45082500 & -0.00018200 & $\mathrm{C}$ & 0.64216900 & 0.31449300 & -0.12084200 \\
\hline & & & & $\mathrm{C}$ & 1.61410200 & 1.24640500 & 0.28261000 \\
\hline d1 & & & & $\mathrm{C}$ & 2.95718700 & 0.88704800 & 0.37085200 \\
\hline $\mathrm{C}$ & -1.31763000 & -0.64707300 & 0.00005800 & $\mathrm{C}$ & 3.35595000 & -0.41633000 & 0.06838100 \\
\hline $\mathrm{C}$ & -2.49100700 & -0.01152600 & 0.00003800 & $\mathrm{H}$ & 2.69808100 & -2.37259500 & -0.55400000 \\
\hline $\mathrm{H}$ & -3.43269300 & -0.55069500 & -0.00015800 & $\mathrm{H}$ & 0.32550300 & -1.73566100 & -0.72353000 \\
\hline $\mathrm{H}$ & -2.52354900 & 1.07398400 & -0.00033000 & $\mathrm{H}$ & 1.30785900 & 2.25245100 & 0.55236800 \\
\hline $\mathrm{C}$ & -0.04351400 & 0.11620800 & 0.00001100 & $\mathrm{H}$ & 3.69038600 & 1.62236000 & 0.68957200 \\
\hline $\mathrm{O}$ & 1.01671200 & -0.72584900 & -0.00007400 & $\mathrm{H}$ & 4.40185000 & -0.69943900 & 0.14246500 \\
\hline $\mathrm{O}$ & 0.06055300 & 1.32681100 & 0.00002300 & $\mathrm{C}$ & -0.77794200 & 0.73732100 & -0.23835300 \\
\hline $\mathrm{C}$ & 2.30246900 & -0.08782200 & 0.00002400 & $\mathrm{C}$ & -1.16082700 & 1.95415000 & -0.64407900 \\
\hline $\mathrm{H}$ & 2.42143300 & 0.53944400 & 0.88730600 & $\mathrm{H}$ & -0.43276500 & 2.69313200 & -0.95987900 \\
\hline $\mathrm{H}$ & 3.03357700 & -0.89620800 & 0.00146200 & $\mathrm{H}$ & -2.20600400 & 2.23557200 & -0.67982200 \\
\hline $\mathrm{H}$ & 2.42259400 & 0.53719300 & -0.88871300 & $\mathrm{C}$ & -1.84342300 & -0.27026100 & 0.13411500 \\
\hline \multirow[t]{2}{*}{$\mathrm{H}$} & -1.24138700 & -1.73014200 & 0.00006200 & $\mathrm{~F}$ & -1.57957800 & -0.85591200 & 1.32083400 \\
\hline & & & & $\mathrm{F}$ & -1.93013300 & -1.26850600 & -0.78536200 \\
\hline e1 & & & & $\mathrm{F}$ & -3.07136700 & 0.28040100 & 0.22028600 \\
\hline $\mathrm{C}$ & -2.11554900 & -1.36820600 & 0.18542000 & & & & \\
\hline $\mathrm{C}$ & -0.74038600 & -1.13841200 & 0.20261000 & g1 & & & \\
\hline $\mathrm{C}$ & -0.22908600 & 0.15681400 & 0.02123000 & $\mathrm{C}$ & -0.27531600 & 0.43805400 & -0.31050100 \\
\hline $\mathrm{C}$ & -1.13180200 & 1.20956000 & -0.20096600 & $\mathrm{C}$ & -0.16420600 & 1.72459800 & -0.65935200 \\
\hline $\mathrm{C}$ & -2.50547600 & 0.97806800 & -0.21529800 & $\mathrm{H}$ & 0.74411300 & 2.29799300 & -0.50500700 \\
\hline $\mathrm{C}$ & -3.00437000 & -0.31189200 & -0.01961000 & $\mathrm{H}$ & -0.99458500 & 2.25585500 & -1.11751900 \\
\hline $\mathrm{H}$ & -2.49297100 & -2.37620600 & 0.33340500 & $\mathrm{C}$ & -1.58550000 & -0.30424600 & -0.50414500 \\
\hline $\mathrm{H}$ & -0.05113500 & -1.95972200 & 0.36095100 & $\mathrm{H}$ & -1.39267400 & -1.30295900 & -0.91795200 \\
\hline $\mathrm{H}$ & -0.75458000 & 2.21012000 & -0.38654300 & $\mathrm{H}$ & -2.19702800 & 0.22464000 & -1.24372600 \\
\hline $\mathrm{H}$ & -3.18744300 & 1.80494400 & -0.39261300 & $\mathrm{C}$ & -2.39357700 & -0.45492800 & 0.79824600 \\
\hline $\mathrm{H}$ & -4.07543300 & -0.49222800 & -0.03558400 & $\mathrm{H}$ & -3.32129600 & -1.00859300 & 0.61902600 \\
\hline $\mathrm{C}$ & 1.23623100 & 0.39470700 & 0.06364300 & $\mathrm{H}$ & -1.82995500 & -0.99540600 & 1.56599100 \\
\hline $\mathrm{C}$ & 1.80608300 & 1.56914400 & 0.38628300 & $\mathrm{H}$ & -2.65543500 & 0.52606700 & 1.20703500 \\
\hline $\mathrm{H}$ & 1.18879700 & 2.41092600 & 0.66886700 & $\mathrm{C}$ & 0.87093400 & -0.36836400 & 0.29333500 \\
\hline $\mathrm{H}$ & 2.87671500 & 1.72195300 & 0.39694000 & $\mathrm{H}$ & 0.43283200 & -1.03331400 & 1.05214000 \\
\hline $\mathrm{O}$ & 1.92726300 & -0.74474600 & -0.24525200 & $\mathrm{C}$ & 1.51888800 & -1.27182000 & -0.77895500 \\
\hline $\mathrm{C}$ & 3.34213200 & -0.69852000 & -0.17366600 & $\mathrm{H}$ & 2.28243900 & -1.91758400 & -0.33156700 \\
\hline $\mathrm{H}$ & 3.68281300 & -0.42713100 & 0.83385000 & $\mathrm{H}$ & 0.78478800 & -1.91707400 & -1.27135300 \\
\hline $\mathrm{H}$ & 3.69308300 & -1.70161200 & -0.42144800 & $\mathrm{H}$ & 1.99870400 & -0.66184600 & -1.55198000 \\
\hline \multirow[t]{2}{*}{$\mathrm{H}$} & 3.75539700 & 0.01934200 & -0.89368500 & $\mathrm{C}$ & 1.94219100 & 0.47946200 & 0.99213100 \\
\hline & & & & $\mathrm{H}$ & 1.50267400 & 1.13639100 & 1.74876400 \\
\hline f1 & & & & $\mathrm{H}$ & 2.67725400 & -0.16624700 & 1.48412200 \\
\hline
\end{tabular}




\begin{tabular}{|c|c|c|c|c|c|c|c|}
\hline \multirow{2}{*}{$\mathrm{H}$} & 2.48767800 & 1.10553300 & 0.27746600 & $\mathrm{C}$ & -0.95994200 & 1.30927500 & 2.30953700 \\
\hline & & & & $\mathrm{C}$ & -1.04886600 & 3.84661700 & 3.32264000 \\
\hline 28 & & & & $\mathrm{C}$ & -1.35296000 & 1.49008200 & 3.63852800 \\
\hline $\mathrm{C}$ & 1.58327000 & 3.19494700 & -0.08186600 & $\mathrm{H}$ & -0.91456200 & 0.31731300 & 1.87427000 \\
\hline $\mathrm{H}$ & 1.88549100 & 3.48697500 & -1.09340400 & $\mathrm{C}$ & -1.40321900 & 2.78093300 & 4.15582000 \\
\hline $\mathrm{H}$ & 1.35471900 & 4.11097700 & 0.46921600 & $\mathrm{H}$ & -1.07100800 & 4.86953300 & 3.69119200 \\
\hline $\mathrm{C}$ & 2.72596800 & 2.44712200 & 0.61854200 & $\mathrm{H}$ & -1.61455000 & 0.63437000 & 4.25344600 \\
\hline $\mathrm{H}$ & 3.44669300 & 3.19819000 & 0.96098500 & $\mathrm{H}$ & -1.70554800 & 2.96755500 & 5.18089000 \\
\hline $\mathrm{H}$ & 2.36848000 & 1.94908000 & 1.52740700 & $\mathrm{C}$ & 2.84920900 & -1.06222000 & 0.94827000 \\
\hline $\mathrm{C}$ & 3.48833100 & 1.45846200 & -0.27349500 & $\mathrm{C}$ & 1.88515000 & -1.96215000 & 1.41787000 \\
\hline $\mathrm{H}$ & 4.41807600 & 1.16460600 & 0.21963300 & $\mathrm{C}$ & 4.23615300 & -1.49831400 & 2.73043000 \\
\hline $\mathrm{H}$ & 3.74390300 & 1.93071300 & -1.22851600 & $\mathrm{C}$ & 2.13892000 & -2.65208900 & 2.60520000 \\
\hline $\mathrm{P}$ & 2.59638700 & -0.12236100 & -0.63380300 & $\mathrm{H}$ & 0.95946800 & -2.12497300 & 0.87509400 \\
\hline $\mathrm{P}$ & -0.04423400 & 2.30958100 & -0.19869300 & $\mathrm{C}$ & 3.33665000 & -2.41804400 & 3.27618300 \\
\hline $\mathrm{C}$ & -1.11641700 & 3.50186000 & -1.26059600 & $\mathrm{H}$ & 5.18130600 & -1.28642100 & 3.22507000 \\
\hline $\mathrm{C}$ & 3.69870500 & -1.00005000 & -1.92598100 & $\mathrm{H}$ & 1.40626600 & -3.35333000 & 2.99178600 \\
\hline $\mathrm{C}$ & -0.88526700 & 3.13258700 & -2.73997000 & $\mathrm{H}$ & 3.57508400 & -2.93036600 & 4.20242700 \\
\hline $\mathrm{H}$ & -1.44554400 & 3.82906700 & -3.37341100 & $\mathrm{~N}$ & -0.66410800 & 3.68550600 & 2.05284300 \\
\hline $\mathrm{H}$ & 0.16994000 & 3.21550500 & -3.02396600 & $\mathrm{~N}$ & 4.00344900 & -0.83171300 & 1.59417100 \\
\hline $\mathrm{H}$ & -1.22225000 & 2.12027700 & -2.97661000 & $\mathrm{Pd}$ & 0.12218000 & 0.01863300 & -1.00525200 \\
\hline $\mathrm{C}$ & -0.69228500 & 4.97035100 & -1.03360400 & $\mathrm{H}$ & -2.56194300 & -1.99793100 & -1.83922600 \\
\hline $\mathrm{H}$ & -1.37829800 & 5.60895400 & -1.60119400 & $\mathrm{C}$ & -2.02664400 & -0.05037000 & -1.01446000 \\
\hline $\mathrm{H}$ & -0.75627800 & 5.25267400 & 0.01903200 & $\mathrm{H}$ & -2.32707600 & 0.45597800 & -1.93376700 \\
\hline $\mathrm{H}$ & 0.31591300 & 5.17984500 & -1.39885900 & $\mathrm{H}$ & -2.34034500 & 0.55127600 & -0.16445100 \\
\hline $\mathrm{C}$ & -2.60814000 & 3.37473900 & -0.88860200 & $\mathrm{C}$ & -2.72240200 & -1.42111900 & -0.92533200 \\
\hline $\mathrm{H}$ & -2.78760300 & 3.66410800 & 0.14992300 & $\mathrm{O}$ & -0.06506800 & -2.36684600 & -2.94823600 \\
\hline $\mathrm{H}$ & -3.17900300 & 4.06250000 & -1.52240200 & $\mathrm{C}$ & 0.02436100 & -1.56363200 & -2.14145000 \\
\hline $\mathrm{H}$ & -3.01435400 & 2.37611700 & -1.04374100 & $\mathrm{C}$ & -4.23592500 & -1.15705100 & -0.83938300 \\
\hline $\mathrm{C}$ & 3.34171300 & -0.43470500 & -3.31515900 & $\mathrm{O}$ & -4.73996300 & -0.26014600 & -0.19398300 \\
\hline $\mathrm{H}$ & 2.30916700 & -0.65443900 & -3.60176400 & $\mathrm{O}$ & -4.92512800 & -2.05634500 & -1.55450700 \\
\hline $\mathrm{H}$ & 3.48621000 & 0.64955400 & -3.37037800 & $\mathrm{C}$ & -6.36356500 & -1.93006500 & -1.50356500 \\
\hline $\mathrm{H}$ & 3.99599100 & -0.88720100 & -4.06844800 & $\mathrm{H}$ & -6.67215000 & -0.95428600 & -1.88454300 \\
\hline $\mathrm{C}$ & 5.19306600 & -0.75234800 & -1.63162700 & $\mathrm{H}$ & -6.74786000 & -2.73028300 & -2.13418400 \\
\hline $\mathrm{H}$ & 5.46620200 & -1.04310800 & -0.61410600 & $\mathrm{H}$ & -6.71728400 & -2.04265200 & -0.47658100 \\
\hline $\mathrm{H}$ & 5.78937800 & -1.35338100 & -2.32733200 & $\mathrm{C}$ & -2.31277800 & -2.28933400 & 0.26330900 \\
\hline $\mathrm{H}$ & 5.47877500 & 0.29212500 & -1.78067200 & $\mathrm{C}$ & -1.67467700 & -3.51998100 & 0.05569600 \\
\hline $\mathrm{C}$ & 3.43106900 & -2.51639900 & -1.87944200 & $\mathrm{C}$ & -2.60397700 & -1.89714300 & 1.57998300 \\
\hline $\mathrm{H}$ & 4.00105600 & -3.00215800 & -2.67887100 & $\mathrm{C}$ & -1.31397400 & -4.33197200 & 1.13504200 \\
\hline $\mathrm{H}$ & 3.75180200 & -2.95491000 & -0.93078200 & $\mathrm{H}$ & -1.48438800 & -3.86399800 & -0.95729900 \\
\hline $\mathrm{H}$ & 2.38025000 & -2.77161300 & -2.03461500 & $\mathrm{C}$ & -2.24594500 & -2.70793800 & 2.65796200 \\
\hline $\mathrm{C}$ & -0.62864400 & 2.43794700 & 1.55548000 & $\mathrm{H}$ & -3.15248700 & -0.97605000 & 1.75251000 \\
\hline
\end{tabular}




\begin{tabular}{|c|c|c|c|c|c|c|c|}
\hline $\mathrm{C}$ & -1.59610700 & -3.92634900 & 2.44060400 & $\mathrm{H}$ & 2.67014600 & -4.51009100 & -1.75213500 \\
\hline $\mathrm{H}$ & -0.83561700 & -5.28966200 & 0.95147000 & $\mathrm{H}$ & 2.72795300 & -3.77440500 & -0.14795500 \\
\hline $\mathrm{H}$ & -2.49828800 & -2.40143500 & 3.66927000 & $\mathrm{H}$ & 1.27428800 & -3.61931100 & -1.15326700 \\
\hline \multirow[t]{2}{*}{$\mathrm{H}$} & -1.33942800 & -4.56625100 & 3.27987800 & $\mathrm{C}$ & 0.46339800 & 2.67661100 & 1.03287100 \\
\hline & & & & $\mathrm{C}$ & -0.09990200 & 1.90409200 & 2.05318100 \\
\hline \multicolumn{4}{|c|}{ TS22 } & $\mathrm{C}$ & 1.12257700 & 4.30301200 & 2.51843400 \\
\hline \multicolumn{4}{|c|}{ imaginary frequency $=-238.93 \mathrm{~cm}^{-1}$} & $\mathrm{C}$ & -0.03904200 & 2.38434000 & 3.36309700 \\
\hline $\mathrm{C}$ & 2.06572300 & 2.66740800 & -1.34854400 & $\mathrm{H}$ & -0.57449800 & 0.95452600 & 1.83048400 \\
\hline $\mathrm{H}$ & 2.05900700 & 2.60510700 & -2.44273700 & $\mathrm{C}$ & 0.58191600 & 3.60727100 & 3.60350500 \\
\hline $\mathrm{H}$ & 2.15574900 & 3.71954900 & -1.07044800 & $\mathrm{H}$ & 1.61992900 & 5.25902600 & 2.66553000 \\
\hline $\mathrm{C}$ & 3.26785300 & 1.90328200 & -0.77173500 & $\mathrm{H}$ & -0.46770900 & 1.80928300 & 4.17834100 \\
\hline $\mathrm{H}$ & 4.15984300 & 2.50704400 & -0.97517200 & $\mathrm{H}$ & 0.65278700 & 4.01807900 & 4.60520200 \\
\hline $\mathrm{H}$ & 3.19956900 & 1.84713600 & 0.31983700 & $\mathrm{C}$ & 2.77354500 & -1.13131000 & 0.90719700 \\
\hline $\mathrm{C}$ & 3.53697500 & 0.51029200 & -1.35956700 & $\mathrm{C}$ & 1.80802700 & -1.60357100 & 1.80449200 \\
\hline $\mathrm{H}$ & 4.51920200 & 0.17301700 & -1.02029600 & $\mathrm{C}$ & 4.39329900 & -1.10319500 & 2.53737600 \\
\hline $\mathrm{H}$ & 3.55212600 & 0.55342100 & -2.45447100 & $\mathrm{C}$ & 2.18768600 & -1.83509300 & 3.12760400 \\
\hline $\mathrm{P}$ & 2.35986700 & -0.83284000 & -0.87471200 & $\mathrm{H}$ & 0.78872400 & -1.78750800 & 1.47985400 \\
\hline $\mathrm{P}$ & 0.40193600 & 2.12272100 & -0.73544900 & $\mathrm{C}$ & 3.50459900 & -1.58043600 & 3.50412400 \\
\hline $\mathrm{C}$ & -0.83391400 & 3.29850800 & -1.60474000 & $\mathrm{H}$ & 5.42843500 & -0.88911500 & 2.79312800 \\
\hline $\mathrm{C}$ & 2.96584100 & -2.37326600 & -1.82221400 & $\mathrm{H}$ & 1.46237100 & -2.20379400 & 3.84634200 \\
\hline $\mathrm{C}$ & -1.10510100 & 2.73053600 & -3.01253600 & $\mathrm{H}$ & 3.84254600 & -1.74361300 & 4.52214900 \\
\hline $\mathrm{H}$ & -1.80000400 & 3.39287400 & -3.54067700 & $\mathrm{~N}$ & 1.06976600 & 3.85366200 & 1.25989700 \\
\hline $\mathrm{H}$ & -0.19293900 & 2.67112900 & -3.61613500 & $\mathrm{~N}$ & 4.04297100 & -0.88182100 & 1.26489000 \\
\hline $\mathrm{H}$ & -1.55752800 & 1.73559300 & -2.97867000 & $\mathrm{Pd}$ & 0.03232900 & -0.30269100 & -1.06225100 \\
\hline $\mathrm{C}$ & -0.25515400 & 4.72301100 & -1.72673200 & $\mathrm{H}$ & -3.42211900 & -2.32168900 & -1.00725600 \\
\hline $\mathrm{H}$ & -1.02943000 & 5.37685900 & -2.14382600 & $\mathrm{C}$ & -2.30155900 & -0.49217700 & -1.32794500 \\
\hline $\mathrm{H}$ & 0.04575500 & 5.12864200 & -0.75795300 & $\mathrm{H}$ & -2.54859000 & -0.46836300 & -2.39028400 \\
\hline $\mathrm{H}$ & 0.60264600 & 4.76687300 & -2.40297100 & $\mathrm{H}$ & -2.31128800 & 0.52121900 & -0.93936400 \\
\hline $\mathrm{C}$ & -2.14212100 & 3.36576900 & -0.79018300 & $\mathrm{C}$ & -3.27201400 & -1.35989100 & -0.51183600 \\
\hline $\mathrm{H}$ & -1.97841000 & 3.79354200 & 0.20276400 & $\mathrm{O}$ & -1.12064300 & -2.96869000 & -1.95401200 \\
\hline $\mathrm{H}$ & -2.84676400 & 4.01972000 & -1.31584200 & $\mathrm{C}$ & -0.79906100 & -1.92865400 & -1.56335000 \\
\hline $\mathrm{H}$ & -2.64276500 & 2.40155200 & -0.67052400 & $\mathrm{C}$ & -4.62903300 & -0.63844200 & -0.50076100 \\
\hline $\mathrm{C}$ & 2.49090300 & -2.25459100 & -3.28390800 & $\mathrm{O}$ & -4.76249200 & 0.56656500 & -0.41107500 \\
\hline $\mathrm{H}$ & 1.40065500 & -2.23298500 & -3.36284300 & $\mathrm{O}$ & -5.63866500 & -1.51088100 & -0.57431600 \\
\hline $\mathrm{H}$ & 2.88942900 & -1.36307200 & -3.77927000 & $\mathrm{C}$ & -6.96823500 & -0.94652700 & -0.51277100 \\
\hline $\mathrm{H}$ & 2.84654500 & -3.12391400 & -3.84766600 & $\mathrm{H}$ & -7.12312000 & -0.25269500 & -1.34149500 \\
\hline $\mathrm{C}$ & 4.50585700 & -2.46440300 & -1.77821100 & $\mathrm{H}$ & -7.64627500 & -1.79512600 & -0.58669000 \\
\hline $\mathrm{H}$ & 4.89395700 & -2.44690800 & -0.75650700 & $\mathrm{H}$ & -7.11031100 & -0.41767400 & 0.43191300 \\
\hline $\mathrm{H}$ & 4.81046700 & -3.41069600 & -2.23874000 & $\mathrm{C}$ & -2.83222700 & -1.63018900 & 0.92764600 \\
\hline $\mathrm{H}$ & 4.98508300 & -1.66084800 & -2.34317100 & $\mathrm{C}$ & -2.39204700 & -2.90979100 & 1.29432300 \\
\hline $\mathrm{C}$ & 2.36608200 & -3.63336500 & -1.17004700 & $\mathrm{C}$ & -2.87996100 & -0.62579100 & 1.90679000 \\
\hline
\end{tabular}




\begin{tabular}{|c|c|c|c|c|c|c|c|}
\hline $\mathrm{C}$ & -1.99349800 & -3.17905200 & 2.60620400 & $\mathrm{H}$ & 2.00815100 & -4.98009700 & -2.80515500 \\
\hline $\mathrm{H}$ & -2.37897100 & -3.70643300 & 0.55568400 & $\mathrm{H}$ & 2.95791300 & -3.51337400 & -3.01977400 \\
\hline $\mathrm{C}$ & -2.48978900 & -0.89801900 & 3.21963700 & $\mathrm{C}$ & 0.08637700 & -4.16759400 & -1.18174200 \\
\hline $\mathrm{H}$ & -3.26007000 & 0.35778800 & 1.64707100 & $\mathrm{H}$ & -0.17381900 & -5.02270100 & -1.81522300 \\
\hline $\mathrm{C}$ & -2.04090500 & -2.17307600 & 3.57334000 & $\mathrm{H}$ & 0.55294900 & -4.55901700 & -0.27376100 \\
\hline $\mathrm{H}$ & -1.67070000 & -4.18070000 & 2.87464700 & $\mathrm{H}$ & -0.84464600 & -3.67701200 & -0.89498400 \\
\hline $\mathrm{H}$ & -2.56207500 & -0.11861300 & 3.97315400 & $\mathrm{C}$ & 2.10278400 & 2.20681500 & 1.18275500 \\
\hline $\mathrm{H}$ & -1.75690300 & -2.38746300 & 4.59949900 & $\mathrm{C}$ & 1.30441000 & 1.81569300 & 2.26505800 \\
\hline & & & & $\mathrm{C}$ & 3.69921500 & 3.08325000 & 2.58824400 \\
\hline 29 & & & & $\mathrm{C}$ & 1.74570700 & 2.09153000 & 3.56037800 \\
\hline $\mathrm{C}$ & 3.15789600 & 1.52180400 & -1.39864600 & $\mathrm{H}$ & 0.36246800 & 1.30367500 & 2.09222300 \\
\hline $\mathrm{H}$ & 3.00720400 & 1.63665300 & -2.47841500 & $\mathrm{C}$ & 2.96766300 & 2.73913300 & 3.72778800 \\
\hline $\mathrm{H}$ & 3.86582100 & 2.28730400 & -1.07131500 & $\mathrm{H}$ & 4.66010000 & 3.58508000 & 2.67878700 \\
\hline $\mathrm{C}$ & 3.73700700 & 0.13467200 & -1.08884500 & $\mathrm{H}$ & 1.14823500 & 1.80247900 & 4.41990800 \\
\hline $\mathrm{H}$ & 4.76466300 & 0.12115300 & -1.47032700 & $\mathrm{H}$ & 3.35278800 & 2.97196900 & 4.71507900 \\
\hline $\mathrm{H}$ & 3.82983300 & -0.01157800 & -0.00773900 & $\mathrm{C}$ & 2.16154600 & -2.39339900 & 0.68860000 \\
\hline $\mathrm{C}$ & 3.01449000 & -1.06060200 & -1.73250800 & $\mathrm{C}$ & 1.36026600 & -2.52017100 & 1.82954400 \\
\hline $\mathrm{H}$ & 3.70333800 & -1.90672200 & -1.73647700 & $\mathrm{C}$ & 3.98765300 & -3.28370600 & 1.76391600 \\
\hline $\mathrm{H}$ & 2.74845500 & -0.83494900 & -2.77125600 & $\mathrm{C}$ & 1.93382800 & -3.06678500 & 2.97963600 \\
\hline$P$ & 1.48702700 & -1.70880400 & -0.90131700 & $\mathrm{H}$ & 0.31985400 & -2.21515600 & 1.80994500 \\
\hline$P$ & 1.54614500 & 1.88314600 & -0.56003600 & $\mathrm{C}$ & 3.27151600 & -3.45473500 & 2.95077400 \\
\hline $\mathrm{C}$ & 1.02935200 & 3.59074300 & -1.23363200 & $\mathrm{H}$ & 5.03428300 & -3.57266600 & 1.70031400 \\
\hline $\mathrm{C}$ & 1.03933200 & -3.24575200 & -1.96429600 & $\mathrm{H}$ & 1.34092300 & -3.18675600 & 3.88148000 \\
\hline $\mathrm{C}$ & 0.35418100 & 3.35343900 & -2.59981100 & $\mathrm{H}$ & 3.75511400 & -3.88154400 & 3.82330500 \\
\hline $\mathrm{H}$ & 0.02426400 & 4.31291500 & -3.01574600 & $\mathrm{~N}$ & 3.28362600 & 2.82689100 & 1.34255700 \\
\hline $\mathrm{H}$ & 1.04151400 & 2.90910600 & -3.32778200 & $\mathrm{~N}$ & 3.45086200 & -2.76582900 & 0.65289500 \\
\hline $\mathrm{H}$ & -0.51824300 & 2.69940700 & -2.51018500 & $\mathrm{Pd}$ & -0.13100800 & -0.02415700 & -0.57893500 \\
\hline C & 2.23012700 & 4.54066900 & -1.39799800 & $\mathrm{H}$ & -4.48805800 & -0.21039600 & -1.75228600 \\
\hline $\mathrm{H}$ & 1.86227000 & 5.53062300 & -1.69314400 & $\mathrm{C}$ & -2.74827600 & -1.33685700 & -1.21389800 \\
\hline $\mathrm{H}$ & 2.79529100 & 4.65172500 & -0.46924900 & $\mathrm{H}$ & -3.25527700 & -2.30552800 & -1.16269100 \\
\hline $\mathrm{H}$ & 2.91793400 & 4.20501500 & -2.17898600 & $\mathrm{H}$ & -2.35764900 & -1.19219400 & -2.22450300 \\
\hline $\mathrm{C}$ & 0.01943000 & 4.20385300 & -0.24480700 & $\mathrm{C}$ & -3.79073600 & -0.22899800 & -0.90839400 \\
\hline $\mathrm{H}$ & 0.49316800 & 4.46432100 & 0.70579700 & $\mathrm{O}$ & -1.67227100 & -1.91835300 & 0.85292600 \\
\hline $\mathrm{H}$ & -0.38868900 & 5.12769700 & -0.67189800 & $\mathrm{C}$ & -1.59366100 & -1.35144400 & -0.20885300 \\
\hline $\mathrm{H}$ & -0.81166000 & 3.52346200 & -0.04384600 & $\mathrm{C}$ & -3.15755900 & 1.15169400 & -0.87149400 \\
\hline $\mathrm{C}$ & 0.36417100 & -2.75687600 & -3.25979200 & $\mathrm{O}$ & -1.97054700 & 1.40199000 & -0.63589800 \\
\hline $\mathrm{H}$ & -0.56572500 & -2.21806300 & -3.06070700 & $\mathrm{O}$ & -4.05105400 & 2.09589900 & -1.10204900 \\
\hline $\mathrm{H}$ & 1.01619000 & -2.10303800 & -3.84782600 & $\mathrm{C}$ & -3.64048400 & 3.48039500 & -1.02017100 \\
\hline $\mathrm{H}$ & 0.12077200 & -3.62122700 & -3.88772000 & $\mathrm{H}$ & -2.83690900 & 3.68000800 & -1.73020800 \\
\hline $\mathrm{C}$ & 2.31181400 & -4.04656600 & -2.31851600 & $\mathrm{H}$ & -4.53031800 & 4.05425800 & -1.27155900 \\
\hline $\mathrm{H}$ & 2.89977200 & -4.30440300 & -1.43429600 & $\mathrm{H}$ & -3.30868700 & 3.71105500 & -0.00618400 \\
\hline
\end{tabular}




\begin{tabular}{|c|c|c|c|c|c|c|c|}
\hline $\mathrm{C}$ & -4.62227700 & -0.46872200 & 0.35264600 & $\mathrm{H}$ & 2.04267100 & -3.86791900 & -3.31229100 \\
\hline $\mathrm{C}$ & -5.86104900 & -1.11122200 & 0.23264000 & $\mathrm{C}$ & 4.12145300 & -3.17111500 & -1.65300700 \\
\hline $\mathrm{C}$ & -4.18147800 & -0.07256300 & 1.62176100 & $\mathrm{H}$ & 4.68961500 & -3.08963800 & -0.72221100 \\
\hline $\mathrm{C}$ & -6.64050300 & -1.36474000 & 1.36085100 & $\mathrm{H}$ & 4.27305400 & -4.18127300 & -2.04903200 \\
\hline $\mathrm{H}$ & -6.22023400 & -1.41273500 & -0.74831700 & $\mathrm{H}$ & 4.54291600 & -2.47123900 & -2.37849300 \\
\hline $\mathrm{C}$ & -4.96551400 & -0.31878500 & 2.74934100 & $\mathrm{C}$ & 2.05925900 & -4.10279600 & -0.56517100 \\
\hline $\mathrm{H}$ & -3.22473700 & 0.42844000 & 1.73916600 & $\mathrm{H}$ & 2.20594600 & -5.04592000 & -1.10286200 \\
\hline $\mathrm{C}$ & -6.19462000 & -0.96722900 & 2.62256700 & $\mathrm{H}$ & 2.58822400 & -4.18201400 & 0.38836500 \\
\hline $\mathrm{H}$ & -7.59822800 & -1.86434400 & 1.25215200 & $\mathrm{H}$ & 0.99339400 & -4.00183200 & -0.35570100 \\
\hline $\mathrm{H}$ & -4.61424200 & -0.00397400 & 3.72732300 & $\mathrm{C}$ & 0.64790200 & 3.06871500 & 0.40260400 \\
\hline $\mathrm{H}$ & -6.80343900 & -1.15816400 & 3.50082500 & $\mathrm{C}$ & -0.19282400 & 2.73014900 & 1.46994400 \\
\hline & & & & $\mathrm{C}$ & 1.43824200 & 4.91902600 & 1.51545600 \\
\hline 29a & & & & $\mathrm{C}$ & -0.20265100 & 3.54921600 & 2.60009800 \\
\hline $\mathrm{C}$ & 2.43475000 & 2.24890400 & -1.70202100 & $\mathrm{H}$ & -0.82842600 & 1.85158000 & 1.41766300 \\
\hline $\mathrm{H}$ & 2.46814800 & 2.05311700 & -2.77936900 & $\mathrm{C}$ & 0.62989100 & 4.66579000 & 2.62675500 \\
\hline $\mathrm{H}$ & 2.67640300 & 3.30290300 & -1.54047400 & $\mathrm{H}$ & 2.10356500 & 5.77938600 & 1.49933600 \\
\hline $\mathrm{C}$ & 3.45967600 & 1.36719700 & -0.96859800 & $\mathrm{H}$ & -0.84681500 & 3.31221200 & 3.44128300 \\
\hline $\mathrm{H}$ & 4.45228500 & 1.77911800 & -1.18306500 & $\mathrm{H}$ & 0.65986700 & 5.32806500 & 3.48567800 \\
\hline $\mathrm{H}$ & 3.33918300 & 1.45657500 & 0.11660800 & $\mathrm{C}$ & 3.02292600 & -1.43721300 & 1.14411700 \\
\hline $\mathrm{C}$ & 3.49714000 & -0.11422600 & -1.39053200 & $\mathrm{C}$ & 2.24351300 & -1.87323900 & 2.22253200 \\
\hline $\mathrm{H}$ & 4.48254100 & -0.51781900 & -1.14732900 & $\mathrm{C}$ & 4.89261300 & -1.22838100 & 2.46289000 \\
\hline $\mathrm{H}$ & 3.34938500 & -0.20978400 & -2.47170800 & $\mathrm{C}$ & 2.85403100 & -1.98390400 & 3.47313300 \\
\hline $\mathrm{P}$ & 2.31392700 & -1.28025000 & -0.56339900 & $\mathrm{H}$ & 1.19993300 & -2.13143600 & 2.08304700 \\
\hline$P$ & 0.68900000 & 2.01669100 & -1.12615000 & $\mathrm{C}$ & 4.20195000 & -1.65334300 & 3.59970700 \\
\hline $\mathrm{C}$ & -0.39932800 & 2.91085900 & -2.40500500 & $\mathrm{H}$ & 5.94559400 & -0.96241200 & 2.51908500 \\
\hline $\mathrm{C}$ & 2.60849700 & -2.95502400 & -1.43270800 & $\mathrm{H}$ & 2.28324700 & -2.32312600 & 4.33224500 \\
\hline $\mathrm{C}$ & -0.45144400 & 2.02662200 & -3.66725100 & $\mathrm{H}$ & 4.71270600 & -1.72238600 & 4.55443500 \\
\hline $\mathrm{H}$ & -1.09395100 & 2.50159100 & -4.41697500 & $\mathrm{~N}$ & 1.45166900 & 4.14365800 & 0.42520300 \\
\hline $\mathrm{H}$ & 0.53457500 & 1.89378200 & -4.12418900 & $\mathrm{~N}$ & 4.32136600 & -1.12094300 & 1.25716600 \\
\hline $\mathrm{H}$ & -0.86765500 & 1.03575400 & -3.45501500 & $\mathrm{Pd}$ & 0.15643600 & -0.36697400 & -0.60105300 \\
\hline $\mathrm{C}$ & 0.15910500 & 4.30289200 & -2.75408000 & $\mathrm{H}$ & -3.40781200 & -2.80740500 & 0.59790900 \\
\hline $\mathrm{H}$ & -0.53054900 & 4.80329700 & -3.44367700 & $\mathrm{C}$ & -2.15388200 & -1.69674900 & -0.76283400 \\
\hline $\mathrm{H}$ & 0.27104200 & 4.93442600 & -1.86929600 & $\mathrm{H}$ & -2.15846900 & -2.45590000 & -1.55586900 \\
\hline $\mathrm{H}$ & 1.13122000 & 4.24284000 & -3.25159000 & $\mathrm{H}$ & -2.17506700 & -0.72973700 & -1.28627700 \\
\hline $\mathrm{C}$ & -1.81611900 & 3.04704300 & -1.81576600 & $\mathrm{C}$ & -3.40621800 & -1.81551100 & 0.13912400 \\
\hline $\mathrm{H}$ & -1.83143800 & 3.68714100 & -0.92894000 & $\mathrm{O}$ & -0.70536900 & -2.75957400 & 0.88533000 \\
\hline $\mathrm{H}$ & -2.47763100 & 3.49825800 & -2.56338300 & $\mathrm{C}$ & -0.84948800 & -1.95043600 & 0.01595900 \\
\hline $\mathrm{H}$ & -2.24378400 & 2.07525500 & -1.54505900 & $\mathrm{C}$ & -4.64102800 & -1.68861500 & -0.75512800 \\
\hline $\mathrm{C}$ & 1.87426900 & -2.92145300 & -2.78682700 & $\mathrm{O}$ & -4.68983700 & -0.98615300 & -1.74572800 \\
\hline $\mathrm{H}$ & 0.79419500 & -2.79773300 & -2.65858300 & $\mathrm{O}$ & -5.65389400 & -2.42408700 & -0.28881000 \\
\hline $\mathrm{H}$ & 2.23522300 & -2.11917500 & -3.43863600 & $\mathrm{C}$ & -6.89502900 & -2.32854700 & -1.02441900 \\
\hline
\end{tabular}




\begin{tabular}{|c|c|c|c|c|c|c|c|}
\hline $\mathrm{H}$ & -6.74797600 & -2.65074000 & -2.05722000 & $\mathrm{C}$ & 0.50762600 & 3.19224900 & -2.02046100 \\
\hline H & -7.58891400 & -2.98928100 & -0.50758600 & $\mathrm{H}$ & 1.24888900 & 2.46654600 & -1.67638300 \\
\hline H & -7.26031700 & -1.29957900 & -1.01591600 & $\mathrm{H}$ & 0.06013700 & 2.80016000 & -2.94015300 \\
\hline $\mathrm{C}$ & -3.44533200 & -0.76648100 & 1.24644800 & $\mathrm{H}$ & 1.03067100 & 4.11797600 & -2.28690700 \\
\hline $\mathrm{C}$ & -3.17531600 & -1.13510100 & 2.57173400 & $\mathrm{C}$ & -1.55644900 & 4.54728300 & -1.50666500 \\
\hline $\mathrm{C}$ & -3.75511900 & 0.57300000 & 0.96204700 & $\mathrm{H}$ & -2.39679700 & 4.71464400 & -0.82862100 \\
\hline $\mathrm{C}$ & -3.20980800 & -0.18465700 & 3.59314800 & $\mathrm{H}$ & -1.02779400 & 5.49867700 & -1.63552700 \\
\hline $\mathrm{H}$ & -2.94590900 & -2.17099800 & 2.80382800 & $\mathrm{H}$ & -1.94989900 & 4.26197700 & -2.48570600 \\
\hline $\mathrm{C}$ & -3.79457700 & 1.52172100 & 1.98591900 & $\mathrm{C}$ & 0.09023600 & 4.06665800 & 0.32390200 \\
\hline $\mathrm{H}$ & -4.00278600 & 0.86436800 & -0.05473900 & $\mathrm{H}$ & 0.73196000 & 4.91445700 & 0.05972700 \\
\hline $\mathrm{C}$ & -3.52162100 & 1.14550400 & 3.30347300 & $\mathrm{H}$ & -0.65969400 & 4.42640600 & 1.03359500 \\
\hline $\mathrm{H}$ & -3.00931200 & -0.48748600 & 4.61649500 & $\mathrm{H}$ & 0.70889600 & 3.32579000 & 0.83699300 \\
\hline $\mathrm{H}$ & -4.05724800 & 2.55004200 & 1.75556800 & $\mathrm{C}$ & -2.80742100 & -2.38395900 & 0.34349900 \\
\hline \multirow[t]{2}{*}{$\mathrm{H}$} & -3.57190400 & 1.87975400 & 4.10225800 & $\mathrm{C}$ & -2.26182900 & -2.59909800 & 1.61622200 \\
\hline & & & & $\mathrm{C}$ & -4.81776400 & -3.30771700 & 0.96966700 \\
\hline 29-O & & & & $\mathrm{C}$ & -3.05252100 & -3.21299600 & 2.58832200 \\
\hline $\mathrm{C}$ & -3.07244700 & -0.91430300 & -2.13217700 & $\mathrm{H}$ & -1.24278300 & -2.29540700 & 1.83563500 \\
\hline $\mathrm{H}$ & -2.62028300 & -0.80843500 & -3.12430700 & $\mathrm{C}$ & -4.35834300 & -3.57408700 & 2.26124300 \\
\hline $\mathrm{H}$ & -3.87214300 & -1.65652500 & -2.19668900 & $\mathrm{H}$ & -5.83217300 & -3.57015900 & 0.67769000 \\
\hline $\mathrm{C}$ & -3.66272900 & 0.42847500 & -1.67156100 & $\mathrm{H}$ & -2.65655800 & -3.40202400 & 3.58166600 \\
\hline $\mathrm{H}$ & -4.54535700 & 0.62464300 & -2.29123100 & $\mathrm{H}$ & -5.01083900 & -4.04941800 & 2.98614500 \\
\hline $\mathrm{H}$ & -4.04049600 & 0.34658800 & -0.64635300 & $\mathrm{C}$ & -2.44361300 & 2.23272900 & 1.00359200 \\
\hline $\mathrm{C}$ & -2.74381100 & 1.65599900 & -1.80649000 & $\mathrm{C}$ & -2.09691400 & 1.71808500 & 2.25747800 \\
\hline $\mathrm{H}$ & -3.35692300 & 2.55789700 & -1.73794900 & $\mathrm{C}$ & -4.26461500 & 3.33542300 & 1.87212400 \\
\hline H & -2.24600400 & 1.65928200 & -2.78289200 & $\mathrm{C}$ & -2.89048200 & 2.04966900 & 3.35764200 \\
\hline$P$ & -1.43238100 & 1.87026600 & -0.50940800 & $\mathrm{H}$ & -1.23209500 & 1.07069000 & 2.36122800 \\
\hline$P$ & -1.78712200 & -1.60478100 & -0.99624600 & $\mathrm{C}$ & -3.99555600 & 2.87509900 & 3.16371100 \\
\hline $\mathrm{C}$ & -0.98031300 & -3.05045700 & -1.93750700 & $\mathrm{H}$ & -5.12087100 & 3.97786300 & 1.67990800 \\
\hline $\mathrm{C}$ & -0.56103100 & 3.50602500 & -0.95456200 & $\mathrm{H}$ & -2.64999000 & 1.66759000 & 4.34518100 \\
\hline $\mathrm{C}$ & -0.08224500 & -2.44510300 & -3.03610700 & $\mathrm{H}$ & -4.64102400 & 3.15703200 & 3.98894200 \\
\hline $\mathrm{H}$ & 0.40903500 & -3.25622400 & -3.58546600 & $\mathrm{~N}$ & -4.06458700 & -2.72978100 & 0.02616400 \\
\hline $\mathrm{H}$ & -0.65086000 & -1.85921000 & -3.76525900 & $\mathrm{~N}$ & -3.51115800 & 3.02461100 & 0.81123900 \\
\hline $\mathrm{H}$ & 0.70113000 & -1.80519100 & -2.61647900 & $\mathrm{Pd}$ & -0.12276200 & -0.02095700 & -0.18990200 \\
\hline $\mathrm{C}$ & -2.04429400 & -3.97010500 & -2.56323600 & $\mathrm{H}$ & 4.82580900 & 1.90151900 & 0.73394800 \\
\hline $\mathrm{H}$ & -1.54630000 & -4.82282800 & -3.03908100 & $\mathrm{C}$ & 2.77310800 & 1.35791700 & 0.91769200 \\
\hline $\mathrm{H}$ & -2.74036800 & -4.36140700 & -1.81674700 & $\mathrm{H}$ & 2.71267800 & 1.39848700 & 2.01350300 \\
\hline $\mathrm{H}$ & -2.62665300 & -3.46038400 & -3.33602600 & $\mathrm{H}$ & 2.49590900 & 2.34180200 & 0.53933300 \\
\hline $\mathrm{C}$ & -0.10788000 & -3.84636500 & -0.94763500 & $\mathrm{C}$ & 4.22487600 & 1.01734100 & 0.48909500 \\
\hline $\mathrm{H}$ & -0.70559100 & -4.32781800 & -0.16831500 & $\mathrm{O}$ & 1.82551600 & -0.84748400 & 0.51785600 \\
\hline $\mathrm{H}$ & 0.42007700 & -4.63699100 & -1.49250400 & $\mathrm{C}$ & 1.72641400 & 0.36746900 & 0.48881900 \\
\hline $\mathrm{H}$ & 0.64631100 & -3.21435600 & -0.46757900 & $\mathrm{C}$ & 4.26073600 & 0.88468100 & -1.03180700 \\
\hline
\end{tabular}




\begin{tabular}{|c|c|c|c|c|c|c|c|}
\hline $\mathrm{O}$ & 3.26462300 & 0.76098800 & -1.72448200 & $\mathrm{H}$ & -3.32066800 & -3.60165800 & 1.17968100 \\
\hline $\mathrm{O}$ & 5.50518500 & 0.93383700 & -1.50487400 & $\mathrm{H}$ & -3.43577300 & -4.77249400 & -0.13663600 \\
\hline $\mathrm{C}$ & 5.65314900 & 0.80396500 & -2.93604200 & $\mathrm{H}$ & -1.95771300 & -3.84004400 & 0.07421200 \\
\hline $\mathrm{H}$ & 5.11582500 & 1.60596500 & -3.44638900 & $\mathrm{C}$ & 0.79533900 & 1.79360200 & -3.43529900 \\
\hline $\mathrm{H}$ & 6.72316600 & 0.87514600 & -3.12310100 & $\mathrm{H}$ & 1.22674600 & 0.83656400 & -3.13165800 \\
\hline $\mathrm{H}$ & 5.26583100 & -0.16079100 & -3.27034200 & $\mathrm{H}$ & -0.10360000 & 1.58363200 & -4.02435700 \\
\hline $\mathrm{C}$ & 4.85346000 & -0.16499200 & 1.21913100 & $\mathrm{H}$ & 1.50937700 & 2.29202300 & -4.10056600 \\
\hline C & 5.44688100 & 0.05444300 & 2.46983300 & $\mathrm{C}$ & -0.09116600 & 4.04252900 & -2.74345500 \\
\hline $\mathrm{C}$ & 4.85239000 & -1.46149600 & 0.68946800 & $\mathrm{H}$ & -0.43968200 & 4.68066300 & -1.92777800 \\
\hline C & 6.01622200 & -0.99979400 & 3.18331200 & $\mathrm{H}$ & 0.69753500 & 4.58090100 & -3.28114300 \\
\hline $\mathrm{H}$ & 5.47347100 & 1.05934900 & 2.88557400 & $\mathrm{H}$ & -0.91547200 & 3.89358600 & -3.44539500 \\
\hline $\mathrm{C}$ & 5.42792000 & -2.51580200 & 1.39960500 & $\mathrm{C}$ & 1.77592100 & 3.01613000 & -1.43500700 \\
\hline $\mathrm{H}$ & 4.40349200 & -1.65522800 & -0.28007200 & $\mathrm{H}$ & 2.53000400 & 3.42456000 & -2.11685500 \\
\hline C & 6.00825900 & -2.28932400 & 2.64843600 & $\mathrm{H}$ & 1.59337600 & 3.76503400 & -0.65975900 \\
\hline $\mathrm{H}$ & 6.47557600 & -0.81150400 & 4.14897700 & $\mathrm{H}$ & 2.20954900 & 2.14012900 & -0.94935000 \\
\hline $\mathrm{H}$ & 5.42499800 & -3.51455100 & 0.97349100 & $\mathrm{C}$ & -3.21442500 & -0.74972200 & 1.38436600 \\
\hline $\mathrm{H}$ & 6.45874400 & -3.11032500 & 3.19774400 & $\mathrm{C}$ & -2.24743500 & -1.06940300 & 2.34679400 \\
\hline & & & & $\mathrm{C}$ & -4.69650400 & -0.05316500 & 2.99893800 \\
\hline 29-CO & & & & C & -2.55388200 & -0.87222800 & 3.69405200 \\
\hline C & -4.02380900 & 0.18122700 & -1.23155700 & $\mathrm{H}$ & -1.28070200 & -1.46437100 & 2.04913000 \\
\hline $\mathrm{H}$ & -4.09982000 & -0.09926900 & -2.28822400 & $\mathrm{C}$ & -3.80292200 & -0.35370700 & 4.02956600 \\
\hline $\mathrm{H}$ & -5.00266900 & 0.03539500 & -0.76957300 & $\mathrm{H}$ & -5.67880700 & 0.35835800 & 3.21987400 \\
\hline $\mathrm{C}$ & -3.63843800 & 1.66015400 & -1.09954300 & $\mathrm{H}$ & -1.82873200 & -1.11739100 & 4.46420300 \\
\hline $\mathrm{H}$ & -4.48450600 & 2.24222200 & -1.48300900 & $\mathrm{H}$ & -4.08355600 & -0.18158000 & 5.06339600 \\
\hline $\mathrm{H}$ & -3.55412200 & 1.93527800 & -0.04380600 & $\mathrm{C}$ & -0.84701600 & 2.82085200 & 0.44617600 \\
\hline $\mathrm{C}$ & -2.39464800 & 2.12029500 & -1.87769800 & $\mathrm{C}$ & -0.22564100 & 2.43067600 & 1.63735400 \\
\hline $\mathrm{H}$ & -2.44426300 & 3.20563200 & -1.97857800 & $\mathrm{C}$ & -1.63212900 & 4.76467100 & 1.39028600 \\
\hline $\mathrm{H}$ & -2.38092700 & 1.68663900 & -2.88416300 & C & -0.32846900 & 3.27736500 & 2.74382100 \\
\hline$P$ & -0.74344700 & 1.78934000 & -1.09442500 & $\mathrm{H}$ & 0.33311300 & 1.50371800 & 1.69049500 \\
\hline$P$ & -2.87659000 & -1.02333100 & -0.42050900 & $\mathrm{C}$ & -1.04373600 & 4.46599800 & 2.62197800 \\
\hline $\mathrm{C}$ & -3.65322700 & -2.72980500 & -0.80790500 & $\mathrm{H}$ & -2.20091200 & 5.68179600 & 1.25465000 \\
\hline C & 0.49309100 & 2.70864400 & -2.23252300 & $\mathrm{H}$ & 0.14555500 & 3.00926500 & 3.68326300 \\
\hline C & -3.33522900 & -3.08197300 & -2.27532900 & $\mathrm{H}$ & -1.14822000 & 5.15104900 & 3.45690100 \\
\hline $\mathrm{H}$ & -3.77916100 & -4.05465700 & -2.51464100 & $\mathrm{~N}$ & -4.41708200 & -0.24644300 & 1.70429600 \\
\hline $\mathrm{H}$ & -3.75716800 & -2.35436100 & -2.97591800 & $\mathrm{~N}$ & -1.54047500 & 3.96463800 & 0.32233000 \\
\hline $\mathrm{H}$ & -2.26168200 & -3.15703900 & -2.46619000 & $\mathrm{Pd}$ & -0.38592700 & -0.61663000 & -0.73726800 \\
\hline $\mathrm{C}$ & -5.18233300 & -2.69731600 & -0.60733300 & $\mathrm{H}$ & 4.65044700 & -1.65916900 & -1.45996000 \\
\hline $\mathrm{H}$ & -5.57406900 & -3.71268700 & -0.73601800 & $\mathrm{C}$ & 2.76322800 & -0.70609500 & -1.26149300 \\
\hline $\mathrm{H}$ & -5.46365500 & -2.35228300 & 0.39077600 & $\mathrm{H}$ & 3.02111000 & 0.21817600 & -1.79104400 \\
\hline $\mathrm{H}$ & -5.68225900 & -2.06235900 & -1.34316500 & $\mathrm{H}$ & 2.36768600 & -1.39430900 & -2.01171800 \\
\hline C & -3.04741800 & -3.78541900 & 0.13703500 & $\mathrm{C}$ & 4.04722800 & -1.29068000 & -0.61976300 \\
\hline
\end{tabular}


C

C

O

O

C

$\mathrm{H}$

$\mathrm{H}$

$\mathrm{H}$

C

C

C

C

$\mathrm{H}$

C

$\mathrm{H}$

C

$\mathrm{H}$

$\mathrm{H}$

$\mathrm{H}$

C

O

\section{9-MeO}

C

$\mathrm{H}$

$\mathrm{H}$

C

$\mathrm{H}$

$\mathrm{H}$

C

$\mathrm{H}$

$\mathrm{H}$

P

P

C

C

C

$\mathrm{H}$

$\mathrm{H}$

H

$\begin{array}{rrr}1.80008500 & 0.03048500 & 0.83410600 \\ 1.64059600 & -0.34738500 & -0.29455500 \\ 3.68947600 & -2.53612100 & 0.18923300 \\ 2.54902800 & -2.91976600 & 0.38496800 \\ 4.77751500 & -3.17425700 & 0.62220300 \\ 4.55316900 & -4.38288800 & 1.37901900 \\ 4.01635700 & -5.11592100 & 0.77305500 \\ 5.54492800 & -4.74919100 & 1.63879900 \\ 3.97413500 & -4.16487600 & 2.27895100 \\ 4.91901400 & -0.28392400 & 0.12568600 \\ 5.86390800 & 0.45451100 & -0.59943300 \\ 4.80508900 & -0.06358900 & 1.50423300 \\ 6.66999300 & 1.40126300 & 0.03331200 \\ 5.97916000 & 0.28082500 & -1.66718000 \\ 5.61535800 & 0.87738900 & 2.13945300 \\ 4.08039200 & -0.62390200 & 2.08580400 \\ 6.54763100 & 1.61457300 & 1.40696200 \\ 7.40025200 & 1.96033600 & -0.54399100 \\ 5.52005200 & 1.03184200 & 3.21020300 \\ 7.17983400 & 2.34370000 & 1.90455200 \\ 0.06190600 & -2.51762400 & -1.08423300 \\ 0.24330600 & -3.58262700 & -1.44804400\end{array}$

3.79407300

3.92397600

4.65227900

3.75365300

4.76264200

3.57393600

2.79075100

3.15176400

2.76624900

1.04031100

2.29315000

2.66651000

0.18451800

2.92229400

3.00231400

3.85832400

2.11148900
$0.45637900 \quad-1.03407200$

$0.59140000 \quad-2.11300700$

$0.90407300 \quad-0.52698500$

$\begin{array}{ll}-1.04306800 & -0.69887800\end{array}$

$\begin{array}{ll}-1.43258000 & -0.87643800\end{array}$

$-1.18440900 \quad 0.37136200$

$-1.90703900-1.53656900$

$-2.93841700 \quad-1.52515200$

$-1.56940000 \quad-2.57856700$

$-2.03333900-0.92600600$

$1.42618700 \quad-0.53413500$

$3.21132700-1.11970900$

$-3.23781600 \quad-2.13564600$

$3.18997400-2.63965500$

$4.21983500-3.00537100$

$2.68519500-2.89335500$

$2.70841600 \quad-3.19333900$
C

$\mathrm{H}$

$\mathrm{H}$

$\mathrm{H}$

C

$\mathrm{H}$

$\mathrm{H}$

$\mathrm{H}$

C

$\mathrm{H}$

$\mathrm{H}$

$\mathrm{H}$

C

$\mathrm{H}$

$\mathrm{H}$

$\mathrm{H}$

C

$\mathrm{H}$

$\mathrm{H}$

$\mathrm{H}$

C

C

C

C

$\mathrm{H}$

C

$\mathrm{H}$

$\mathrm{H}$

$\mathrm{H}$

C

C

C

C

$\mathrm{H}$

C

$\mathrm{H}$

$\mathrm{H}$

$\mathrm{H}$

N

$\mathrm{N}$

$\mathrm{Pd}$

\begin{tabular}{|c|c|c|}
\hline 3.89675600 & 3.80087800 & -0.40418100 \\
\hline 4.08825900 & 4.80557200 & -0.79880400 \\
\hline 3.74330500 & 3.89091600 & 0.67361400 \\
\hline 4.79985100 & 3.20698500 & -0.56896700 \\
\hline 1.42961700 & 4.07607500 & -0.81344700 \\
\hline 1.20263600 & 4.09782200 & 0.25730900 \\
\hline 1.61639200 & 5.10903800 & -1.12754900 \\
\hline 0.54304600 & 3.72216500 & -1.34923200 \\
\hline-0.15699200 & -2.45300800 & -3.41772300 \\
\hline-0.80294000 & -1.59321000 & -3.21680500 \\
\hline 0.74135800 & -2.08827300 & -3.92634100 \\
\hline-0.67998600 & -3.11374300 & -4.11797400 \\
\hline 1.09762100 & -4.42982500 & -2.49327300 \\
\hline 1.44166100 & -4.96642200 & -1.60700500 \\
\hline 0.52812800 & -5.12697400 & -3.11827500 \\
\hline 1.97306300 & -4.11946700 & -3.06939700 \\
\hline-1.10124400 & -3.77135000 & -1.47662900 \\
\hline-1.62040200 & -4.42613500 & -2.18489200 \\
\hline-0.88570800 & -4.35813300 & -0.57955300 \\
\hline-1.79667000 & -2.97412800 & -1.20129800 \\
\hline 2.48300300 & 1.51206000 & 1.31443400 \\
\hline 1.38614500 & 1.82715000 & 2.12872700 \\
\hline 3.87066900 & 1.32207500 & 3.13910000 \\
\hline 1.57572800 & 1.90164800 & 3.50946600 \\
\hline 0.40516700 & 2.00437300 & 1.69804900 \\
\hline 2.84316300 & 1.64459300 & 4.02835600 \\
\hline 4.87204100 & 1.10621300 & 3.50521100 \\
\hline 0.74612800 & 2.14915200 & 4.16495800 \\
\hline 3.03608500 & 1.68663600 & 5.09521700 \\
\hline 1.28193400 & -2.96619200 & 0.66297600 \\
\hline 0.58688500 & -2.64666200 & 1.83343000 \\
\hline 2.42531100 & -4.67049900 & 1.70006100 \\
\hline 0.84590700 & -3.40228700 & 2.98052200 \\
\hline-0.13601500 & -1.84044200 & 1.84598200 \\
\hline 1.77992800 & -4.43248400 & 2.91668000 \\
\hline 3.16374000 & -5.46379100 & 1.60883400 \\
\hline 0.32341700 & -3.18372200 & 3.90695400 \\
\hline 2.01072500 & -5.04112800 & 3.78476400 \\
\hline 3.70318100 & 1.25928400 & 1.81261600 \\
\hline 2.18816400 & -3.95563700 & 0.59536800 \\
\hline 0.11432000 & 0.14548700 & -0.80291200 \\
\hline
\end{tabular}




\begin{tabular}{|c|c|c|c|c|c|c|c|}
\hline $\mathrm{H}$ & -4.39648600 & 1.06959300 & -1.69223700 & $\mathrm{H}$ & -3.17140200 & 4.15058600 & 1.73710000 \\
\hline $\mathrm{C}$ & -2.89638900 & -0.34846100 & -1.14522000 & $\mathrm{H}$ & -3.32131400 & 3.12634800 & 0.31095600 \\
\hline $\mathrm{H}$ & -3.70254500 & -0.90569900 & -0.65757500 & $\mathrm{H}$ & -2.71537200 & 2.44263200 & 1.83387800 \\
\hline $\mathrm{H}$ & -2.69374900 & -0.80154000 & -2.11770900 & $\mathrm{C}$ & -1.26197600 & 5.00868800 & -0.03258900 \\
\hline $\mathrm{C}$ & -3.36366900 & 1.12723900 & -1.33444700 & $\mathrm{H}$ & -1.68257000 & 5.82881800 & 0.56036000 \\
\hline $\mathrm{O}$ & -1.80042500 & -0.73476400 & 0.95095600 & $\mathrm{H}$ & -0.25254200 & 5.30360900 & -0.33251600 \\
\hline $\mathrm{C}$ & -1.68096500 & -0.50806400 & -0.22346200 & $\mathrm{H}$ & -1.87266300 & 4.91304700 & -0.93443500 \\
\hline $\mathrm{C}$ & -2.63953800 & 1.83846700 & -2.46828500 & $\mathrm{C}$ & -0.47627700 & 3.98324800 & 2.12821900 \\
\hline $\mathrm{O}$ & -1.31991100 & 1.48001400 & -2.57035300 & $\mathrm{H}$ & 0.50692100 & 4.41875600 & 1.93472600 \\
\hline $\mathrm{O}$ & -3.15437700 & 2.61848900 & -3.22741300 & $\mathrm{H}$ & -1.03409100 & 4.70704000 & 2.73301200 \\
\hline $\mathrm{C}$ & -3.37420700 & 1.94242600 & -0.04330900 & $\mathrm{H}$ & -0.34048300 & 3.07488100 & 2.72013100 \\
\hline $\mathrm{C}$ & -4.45499900 & 1.80844000 & 0.83774500 & $\mathrm{C}$ & -5.13760500 & 0.48157000 & 0.39498000 \\
\hline $\mathrm{C}$ & -2.32774800 & 2.80929900 & 0.29786900 & $\mathrm{H}$ & -4.59660600 & 0.91077800 & 1.24271700 \\
\hline $\mathrm{C}$ & -4.48332500 & 2.51348500 & 2.04026200 & $\mathrm{H}$ & -5.11164200 & 1.20032100 & -0.42982100 \\
\hline $\mathrm{H}$ & -5.28342000 & 1.15354000 & 0.57972400 & $\mathrm{H}$ & -6.18709400 & 0.37179900 & 0.69006800 \\
\hline $\mathrm{C}$ & -2.36015700 & 3.52359600 & 1.49858700 & $\mathrm{C}$ & -5.39005500 & -1.48590300 & -1.16674300 \\
\hline $\mathrm{H}$ & -1.49474500 & 2.95794400 & -0.38337200 & $\mathrm{H}$ & -5.01066100 & -2.46256600 & -1.48157600 \\
\hline $\mathrm{C}$ & -3.43483700 & 3.37352800 & 2.37481400 & $\mathrm{H}$ & -6.42961600 & -1.62530500 & -0.84832500 \\
\hline $\mathrm{H}$ & -5.33034300 & 2.40051100 & 2.70985200 & $\mathrm{H}$ & -5.40549900 & -0.82471300 & -2.03783000 \\
\hline $\mathrm{H}$ & -1.55396600 & 4.21131500 & 1.73767300 & $\mathrm{C}$ & -4.67426200 & -1.83609400 & 1.21930400 \\
\hline $\mathrm{H}$ & -3.46487200 & 3.93392900 & 3.30409600 & $\mathrm{H}$ & -5.72401200 & -1.91870700 & 1.52228100 \\
\hline $\mathrm{C}$ & -0.64339200 & 2.02004100 & -3.73482800 & $\mathrm{H}$ & -4.31153200 & -2.84306000 & 1.00066500 \\
\hline $\mathrm{H}$ & 0.31409000 & 1.50564800 & -3.77800000 & $\mathrm{H}$ & -4.11539700 & -1.44781100 & 2.07558600 \\
\hline $\mathrm{H}$ & -1.23456600 & 1.82178700 & -4.62964900 & $\mathrm{C}$ & 1.23348900 & 2.89217300 & -0.60225000 \\
\hline $\mathrm{H}$ & -0.50683400 & 3.09747800 & -3.62721300 & $\mathrm{C}$ & 2.23463900 & 3.08536900 & 0.36296200 \\
\hline & & & & $\mathrm{C}$ & 2.62075500 & 3.66124600 & -2.27678900 \\
\hline 30 & & & & $\mathrm{C}$ & 3.46386600 & 3.60671700 & -0.04121100 \\
\hline $\mathrm{C}$ & -1.34227900 & 2.30135000 & -1.79324200 & $\mathrm{H}$ & 2.06414100 & 2.83529000 & 1.40173700 \\
\hline $\mathrm{H}$ & -2.31286300 & 2.78492000 & -1.64749900 & $\mathrm{C}$ & 3.66676800 & 3.90326800 & -1.38757800 \\
\hline $\mathrm{H}$ & -0.73251000 & 2.96263800 & -2.41361000 & $\mathrm{H}$ & 2.73355900 & 3.87291000 & -3.33780800 \\
\hline $\mathrm{C}$ & -1.53428800 & 0.95548800 & -2.52136400 & $\mathrm{H}$ & 4.24866500 & 3.77873200 & 0.68945700 \\
\hline $\mathrm{H}$ & -1.60552200 & 1.17623800 & -3.59299000 & $\mathrm{H}$ & 4.60747100 & 4.31010100 & -1.74317500 \\
\hline $\mathrm{H}$ & -0.65684600 & 0.31167800 & -2.39738700 & $\mathrm{C}$ & -2.19007300 & -2.39913900 & -0.89555400 \\
\hline $\mathrm{C}$ & -2.82151000 & 0.20692600 & -2.12295900 & $\mathrm{C}$ & -2.15029200 & -2.95127800 & -2.18029700 \\
\hline $\mathrm{H}$ & -3.11181000 & -0.49606100 & -2.90826300 & $\mathrm{C}$ & -1.43112500 & -4.39494900 & 0.02150800 \\
\hline $\mathrm{H}$ & -3.64592500 & 0.92500300 & -2.05246800 & $\mathrm{C}$ & -1.73483800 & -4.27346600 & -2.34485600 \\
\hline $\mathrm{P}$ & -2.73294700 & -0.66557700 & -0.48000100 & $\mathrm{H}$ & -2.43661200 & -2.36945900 & -3.04741400 \\
\hline$P$ & -0.47672500 & 2.27608100 & -0.14508800 & $\mathrm{C}$ & -1.37321000 & -5.01643400 & -1.22431500 \\
\hline $\mathrm{C}$ & -1.25853300 & 3.72738600 & 0.82424500 & $\mathrm{H}$ & -1.14911400 & -4.92945200 & 0.92514900 \\
\hline $\mathrm{C}$ & -4.57685100 & -0.89886200 & 0.00192000 & $\mathrm{H}$ & -1.69951800 & -4.71435700 & -3.33649900 \\
\hline C & -2.70051700 & 3.32679800 & 1.18923500 & $\mathrm{H}$ & -1.04809600 & -6.04769400 & -1.30729300 \\
\hline
\end{tabular}




\begin{tabular}{|c|c|c|c|c|c|c|c|}
\hline N & 1.43307900 & 3.17189300 & -1.89844300 & $\mathrm{H}$ & -1.55848800 & 0.93742600 & -3.61809300 \\
\hline N & -1.82154200 & -3.12355700 & 0.18504700 & $\mathrm{H}$ & -0.64434800 & 0.14641300 & -2.35031500 \\
\hline $\mathrm{Pd}$ & -0.72783200 & 0.19803700 & 0.92333100 & $\mathrm{C}$ & -2.82181100 & 0.09158100 & -2.12505600 \\
\hline $\mathrm{C}$ & 1.00793400 & 0.34765700 & 1.92120300 & $\mathrm{H}$ & -3.09467700 & -0.64674000 & -2.88604000 \\
\hline $\mathrm{O}$ & 1.22161000 & 1.14048700 & 2.80248200 & $\mathrm{H}$ & -3.63964600 & 0.82236100 & -2.11662300 \\
\hline $\mathrm{O}$ & -0.94196400 & -1.63024500 & 2.24215700 & $\mathrm{P}$ & -2.83779700 & -0.68220100 & -0.42413100 \\
\hline $\mathrm{H}$ & -1.40576300 & -2.28582500 & 1.64793700 & $P$ & -0.56821000 & 2.23465500 & -0.17969700 \\
\hline C & -1.49924400 & -1.56748200 & 3.56005700 & $\mathrm{C}$ & -1.47426200 & 3.68950700 & 0.67610200 \\
\hline H & -1.46261400 & -2.55501700 & 4.03155300 & $\mathrm{C}$ & -4.68594000 & -1.13295100 & -0.12006400 \\
\hline $\mathrm{H}$ & -0.88802800 & -0.87467000 & 4.14038100 & $\mathrm{C}$ & -2.94523000 & 3.27788100 & 0.87767100 \\
\hline H & -2.53374900 & -1.20709700 & 3.55241000 & $\mathrm{H}$ & -3.48108600 & 4.08398400 & 1.39187600 \\
\hline $\mathrm{C}$ & 2.97847000 & -0.57206300 & 0.47745700 & $\mathrm{H}$ & -3.46407300 & 3.09847300 & -0.06978300 \\
\hline H & 3.47762000 & 0.39003200 & 0.62518600 & $\mathrm{H}$ & -3.02264200 & 2.37400500 & 1.49026000 \\
\hline $\mathrm{C}$ & 1.96228900 & -0.82253900 & 1.61541400 & $\mathrm{C}$ & -1.39746700 & 4.97831400 & -0.16327700 \\
\hline $\mathrm{H}$ & 1.37680300 & -1.71350500 & 1.38971000 & $\mathrm{H}$ & -1.91359400 & 5.78558800 & 0.36964700 \\
\hline $\mathrm{C}$ & 2.31827700 & -0.53294500 & -0.89791300 & $\mathrm{H}$ & -0.36628400 & 5.29812600 & -0.33245900 \\
\hline $\mathrm{O}$ & 1.21369200 & -0.97733700 & -1.16295400 & $\mathrm{H}$ & -1.88033600 & 4.87151500 & -1.13905300 \\
\hline $\mathrm{O}$ & 3.13738000 & 0.01768900 & -1.80263900 & $\mathrm{C}$ & -0.83510700 & 3.93128200 & 2.05786800 \\
\hline $\mathrm{C}$ & 2.68477800 & 0.01494300 & -3.17103800 & $\mathrm{H}$ & 0.19312000 & 4.29247100 & 1.97621200 \\
\hline $\mathrm{H}$ & 2.35988300 & -0.98538200 & -3.46365400 & $\mathrm{H}$ & -1.40945700 & 4.69928800 & 2.58860900 \\
\hline $\mathrm{H}$ & 1.86283100 & 0.72355000 & -3.29503800 & $\mathrm{H}$ & -0.83174300 & 3.02604000 & 2.67334500 \\
\hline H & 3.54519800 & 0.32705300 & -3.76172500 & $\mathrm{C}$ & -5.38957000 & 0.19711900 & 0.22275500 \\
\hline $\mathrm{C}$ & 4.05193900 & -1.66221900 & 0.48345300 & $\mathrm{H}$ & -4.92862100 & 0.69188200 & 1.08256000 \\
\hline $\mathrm{C}$ & 5.36197900 & -1.35994400 & 0.87080800 & $\mathrm{H}$ & -5.39121100 & 0.89882800 & -0.61719300 \\
\hline $\mathrm{C}$ & 3.73535000 & -2.97920200 & 0.12222600 & $\mathrm{H}$ & -6.43664400 & -0.00517600 & 0.47470200 \\
\hline $\mathrm{C}$ & 6.33984400 & -2.35542500 & 0.90070800 & $\mathrm{C}$ & -5.38228000 & -1.79745800 & -1.31885200 \\
\hline H & 5.61967200 & -0.34160900 & 1.15010400 & $\mathrm{H}$ & -4.93442500 & -2.76196700 & -1.57695200 \\
\hline C & 4.71342500 & -3.97344200 & 0.14909000 & $\mathrm{H}$ & -6.43182000 & -1.98987800 & -1.06680600 \\
\hline $\mathrm{H}$ & 2.72391600 & -3.22603300 & -0.19070900 & $\mathrm{H}$ & -5.37743900 & -1.16218900 & -2.20945900 \\
\hline C & 6.01804900 & -3.66444500 & 0.53954100 & $\mathrm{C}$ & -4.74537500 & -2.06150600 & 1.10741900 \\
\hline $\mathrm{H}$ & 7.35242200 & -2.10665000 & 1.20384400 & $\mathrm{H}$ & -5.79075200 & -2.22106200 & 1.39352200 \\
\hline $\mathrm{H}$ & 4.45769300 & -4.98940800 & -0.13723700 & $\mathrm{H}$ & -4.31351600 & -3.04690800 & 0.90414200 \\
\hline $\mathrm{H}$ & 6.77894000 & -4.43860800 & 0.56038300 & $\mathrm{H}$ & -4.23040400 & -1.62825200 & 1.97159400 \\
\hline \multirow[t]{2}{*}{$\mathrm{H}$} & 2.52109000 & -0.99613000 & 2.54066000 & $\mathrm{C}$ & 1.14162800 & 2.92746200 & -0.43348700 \\
\hline & & & & $\mathrm{C}$ & 2.05846900 & 2.89062400 & 0.62844500 \\
\hline \multirow{2}{*}{\multicolumn{4}{|c|}{$\begin{array}{l}\text { TS23 } \\
\text { imaginary frequency }=-255.45 \mathrm{~cm}^{-1}\end{array}$}} & $\mathrm{C}$ & 2.64253400 & 4.03531900 & -1.78060200 \\
\hline & & & & $\mathrm{C}$ & 3.30806200 & 3.48407000 & 0.45238500 \\
\hline $\mathrm{C}$ & -1.27134000 & 2.19287900 & -1.91089600 & $\mathrm{H}$ & 1.79794600 & 2.39766600 & 1.55969400 \\
\hline H & -2.20813300 & 2.76028800 & -1.91505300 & $\mathrm{C}$ & 3.60924300 & 4.07172600 & -0.77578200 \\
\hline $\mathrm{H}$ & -0.56124200 & 2.75529400 & -2.52523700 & $\mathrm{H}$ & 2.84172400 & 4.47327300 & -2.75655100 \\
\hline C & -1.50578200 & 0.79852700 & -2.53092200 & $\mathrm{H}$ & 4.03460300 & 3.48312700 & 1.25969100 \\
\hline
\end{tabular}




\begin{tabular}{|c|c|c|c|c|c|c|c|}
\hline $\mathrm{H}$ & 4.56966200 & 4.54290900 & -0.95697700 & $\mathrm{H}$ & 5.25701500 & -4.47362700 & -1.03180400 \\
\hline $\mathrm{C}$ & -2.10778000 & -2.38286000 & -0.74741200 & $\mathrm{H}$ & 7.25885200 & -3.81518500 & 0.28632100 \\
\hline $\mathrm{C}$ & -2.20822900 & -3.13848000 & -1.91730700 & $\mathrm{H}$ & 2.21674400 & -1.95917400 & 1.96761900 \\
\hline $\mathrm{C}$ & -0.86647400 & -4.16585200 & 0.24934400 & & & & \\
\hline $\mathrm{C}$ & -1.64354700 & -4.41269500 & -1.99216400 & \multirow{2}{*}{\multicolumn{4}{|c|}{$\begin{array}{l}\text { TS23-c1 } \\
\text { imaginary frequency }=-278.34 \mathrm{~cm}^{-1}\end{array}$}} \\
\hline $\mathrm{H}$ & -2.73044000 & -2.73033700 & -2.77292300 & & & & \\
\hline $\mathrm{C}$ & -0.97076000 & -4.94473200 & -0.88977700 & $\mathrm{C}$ & -0.73846900 & 2.10601400 & -2.11900600 \\
\hline $\mathrm{H}$ & -0.33766300 & -4.47548900 & 1.14332300 & $\mathrm{H}$ & -1.57839000 & 2.80185100 & -2.21254200 \\
\hline $\mathrm{H}$ & -1.73162300 & -4.98985800 & -2.90742800 & $\mathrm{H}$ & 0.06906800 & 2.49903400 & -2.74489400 \\
\hline $\mathrm{H}$ & -0.52406400 & -5.93142000 & -0.91520800 & $\mathrm{C}$ & -1.16072100 & 0.71638900 & -2.63883000 \\
\hline $\mathrm{N}$ & 1.43243100 & 3.48283300 & -1.61935900 & $\mathrm{H}$ & -1.17622700 & 0.77503100 & -3.73387800 \\
\hline $\mathrm{N}$ & -1.42152400 & -2.93846600 & 0.28857400 & $\mathrm{H}$ & -0.39631800 & -0.02999100 & -2.39063600 \\
\hline $\mathrm{Pd}$ & -0.93181600 & 0.21239800 & 0.92238400 & $\mathrm{C}$ & -2.55990700 & 0.21886100 & -2.20452000 \\
\hline $\mathrm{C}$ & 0.83606400 & -0.39185000 & 1.99800700 & $\mathrm{H}$ & -2.94349800 & -0.49713500 & -2.93839800 \\
\hline $\mathrm{O}$ & 1.20657800 & 0.32960200 & 2.89768600 & $\mathrm{H}$ & -3.26447700 & 1.05849500 & -2.21396000 \\
\hline $\mathrm{O}$ & -0.46295100 & -1.45286000 & 2.35378000 & $\mathrm{P}$ & -2.65547400 & -0.49169300 & -0.47975900 \\
\hline $\mathrm{H}$ & -1.20345100 & -2.35842500 & 1.14710000 & $\mathrm{P}$ & -0.08123100 & 2.19847100 & -0.36902200 \\
\hline $\mathrm{C}$ & -0.99087000 & -1.30052000 & 3.67651600 & $\mathrm{C}$ & -0.86466400 & 3.78580200 & 0.36386800 \\
\hline $\mathrm{H}$ & -0.71834400 & -2.18007400 & 4.27088200 & $\mathrm{C}$ & -4.53877600 & -0.74380100 & -0.16454600 \\
\hline $\mathrm{H}$ & -0.56091700 & -0.40899900 & 4.14332300 & $\mathrm{C}$ & -2.38397100 & 3.55877500 & 0.48566800 \\
\hline $\mathrm{H}$ & -2.08120900 & -1.20499700 & 3.64233200 & $\mathrm{H}$ & -2.84622500 & 4.44153800 & 0.94200800 \\
\hline $\mathrm{C}$ & 2.82844900 & -0.68470000 & 0.33671400 & $\mathrm{H}$ & -2.86637600 & 3.40993900 & -0.48594600 \\
\hline $\mathrm{H}$ & 3.10939900 & 0.28504300 & 0.75430300 & $\mathrm{H}$ & -2.60770400 & 2.69532500 & 1.11977400 \\
\hline $\mathrm{C}$ & 1.73471400 & -1.34270400 & 1.20097600 & $\mathrm{C}$ & -0.58464400 & 5.01463900 & -0.52081800 \\
\hline $\mathrm{H}$ & 1.13611500 & -1.99353000 & 0.56621900 & $\mathrm{H}$ & -1.05016800 & 5.89803100 & -0.06812600 \\
\hline $\mathrm{C}$ & 2.33588000 & -0.45164400 & -1.09079600 & $\mathrm{H}$ & 0.48555400 & 5.21568400 & -0.61538400 \\
\hline $\mathrm{O}$ & 1.37937200 & -1.01077500 & -1.60231600 & $\mathrm{H}$ & -0.99740400 & 4.90830200 & -1.52840600 \\
\hline $\mathrm{O}$ & 3.13547300 & 0.40079700 & -1.74208800 & $\mathrm{C}$ & -0.28591800 & 4.01614300 & 1.77374900 \\
\hline $\mathrm{C}$ & 2.86537300 & 0.59989700 & -3.14378300 & $\mathrm{H}$ & 0.77980100 & 4.25631700 & 1.74483200 \\
\hline $\mathrm{H}$ & 2.73718500 & -0.35988600 & -3.64770100 & $\mathrm{H}$ & -0.79824100 & 4.86657600 & 2.23812500 \\
\hline $\mathrm{H}$ & 1.96853300 & 1.20993700 & -3.27085100 & $\mathrm{H}$ & -0.42424400 & 3.14285200 & 2.41929900 \\
\hline $\mathrm{H}$ & 3.73455600 & 1.12756500 & -3.53442400 & $\mathrm{C}$ & -5.09687500 & 0.65808200 & 0.15907100 \\
\hline $\mathrm{C}$ & 4.08529800 & -1.55695000 & 0.29123700 & $\mathrm{H}$ & -4.59099000 & 1.10885400 & 1.01776600 \\
\hline $\mathrm{C}$ & 5.21950900 & -1.19209100 & 1.02570900 & $\mathrm{H}$ & -5.01574300 & 1.34695700 & -0.68758300 \\
\hline $\mathrm{C}$ & 4.11057900 & -2.74822600 & -0.44746600 & $\mathrm{H}$ & -6.16132300 & 0.57281100 & 0.40469900 \\
\hline $\mathrm{C}$ & 6.35609400 & -2.00213300 & 1.02683800 & $\mathrm{C}$ & -5.30897400 & -1.35020000 & -1.34887500 \\
\hline $\mathrm{H}$ & 5.21248600 & -0.27038600 & 1.60163100 & $\mathrm{H}$ & -4.97108800 & -2.36245200 & -1.59079500 \\
\hline $\mathrm{C}$ & 5.24796200 & -3.55650000 & -0.44977000 & $\mathrm{H}$ & -6.37127000 & -1.42410400 & -1.08799200 \\
\hline $\mathrm{H}$ & 3.24223100 & -3.03865600 & -1.03373000 & $\mathrm{H}$ & -5.24278800 & -0.73481800 & -2.25094200 \\
\hline $\mathrm{C}$ & 6.37354700 & -3.18664500 & 0.28886000 & $\mathrm{C}$ & -4.68678200 & -1.64326800 & 1.07705000 \\
\hline $\mathrm{H}$ & 7.22811700 & -1.70523700 & 1.60191600 & $\mathrm{H}$ & -5.74295400 & -1.70016700 & 1.36254300 \\
\hline
\end{tabular}




$\begin{array}{lrrrrrrr}\mathrm{H} & -4.34712500 & -2.66769800 & 0.89074900 & \mathrm{H} & 3.53775000 & -1.19327600 & -1.82950000 \\ \mathrm{H} & -4.13581700 & -1.24565800 & 1.93592100 & \mathrm{C} & 5.77279200 & -3.65518100 & -1.10672700 \\ \mathrm{C} & 1.69249500 & 2.71240100 & -0.63677200 & \mathrm{H} & 6.41491900 & -4.18882400 & 0.88149700 \\ \mathrm{C} & 2.58639400 & 2.72396900 & 0.44474800 & \mathrm{H} & 4.96155800 & -2.88198800 & -2.94878800 \\ \mathrm{C} & 3.31470500 & 3.49254100 & -2.07237000 & \mathrm{H} & 6.40563900 & -4.38893300 & -1.59698300 \\ \mathrm{C} & 3.89000900 & 3.17147700 & 0.22939600 & \mathrm{H} & 2.05864200 & -2.23106000 & 1.85069500 \\ \mathrm{H} & 2.27307600 & 2.37556300 & 1.42324800 & \mathrm{H} & 3.09308800 & 0.13198200 & 0.19636000\end{array}$

$$
4.26568900
$$

$$
3.56952900
$$

$$
4.60136100
$$$$
5.27080100
$$$$
-2.09055800
$$$$
-2.31514800
$$$$
-0.93663200
$$$$
-1.85433400
$$$$
-2.85399600
$$$$
-1.16031100
$$$$
-0.38594600
$$$$
-2.03852200
$$$$
-0.78937200
$$$$
2.05512600
$$$$
-1.39326500
$$$$
-0.67892900
$$$$
0.99407800
$$$$
1.32092700
$$$$
-0.38981800
$$$$
-1.09882100
$$$$
-0.98814100
$$$$
-0.81858800
$$$$
-0.52506000
$$$$
-2.06412700
$$$$
3.25080900
$$$$
3.72219500
$$$$
1.89151800
$$$$
1.37032600
$$$$
4.14903000
$$$$
4.97096100
$$$$
4.15584600
$$$$
5.77689400
$$$$
4.98850900
$$$$
4.96033700
$$

$-1.05422800$

$3.77749300 \quad-3.09087700$

$3.20106300 \quad 1.04937200$

$3.91410700-1.26726400$

$-2.26410200-0.76758400$

$-3.04633800 \quad-1.90287600$

$-4.10526600 \quad 0.22422600$

$-4.36340800 \quad-1.96244500$

$-2.62609600-2.74235000$

$-4.90939400 \quad-0.88080400$

$-4.42872200 \quad 1.10011100$

$-4.96122200 \quad-2.84981000$

$-5.92727200 \quad-0.89556200$

$3.08372900 \quad-1.87305200$

$-2.83827300$

0.25076700

0.29587500

0.83852700

$-0.41778200 \quad 2.02947800$

$0.25670400 \quad 2.97512700$

$-1.42030100$

2.27333100

$-2.25329600$

1.09635000

$-1.28032600$

3.56703300

$-2.19903000$

4.13999800

$-0.44170300$

4.09564600

$-1.09997300$

3.47129500

$-0.73020500$

0.84899300

$-0.35915800$

1.76484300

$-1.35036500$

1.21746800

$-1.68906000$

0.31804000

$-1.73913100$

0.16415600

$-2.59262900$

0.91353300

$-1.86055700-1.23216400$

$-3.54293300$

0.28520500

1.99746100

$-1.86477400$

TS23-d1

imaginary frequency $=-256.30 \mathrm{~cm}^{-1}$

C

$\mathrm{H}$

H

C

$\mathrm{H}$

$\mathrm{H}$

$\mathrm{C}$

$\mathrm{H}$

$\mathrm{H}$

P

P

C

C

$\mathrm{C}$

$\mathrm{H}$

H

H

$\mathrm{H}$

C

$\mathrm{H}$

$\mathrm{H}$

$\mathrm{H}$

C

$\mathrm{H}$

$\mathrm{H}$

H

$\mathrm{H}$

C

H

H

H

C

H

\begin{tabular}{rrr}
1.01911600 & 1.41024800 & -2.10075000 \\
0.80466500 & 2.46732200 & -2.28997500 \\
1.93018100 & 1.16790500 & -2.65655800 \\
-0.14764000 & 0.54046400 & -2.61583400 \\
-0.05028300 & 0.48926700 & -3.70752600 \\
-0.04746200 & -0.48754700 & -2.25245300 \\
-1.56897700 & 1.08240600 & -2.33534800 \\
-2.27527200 & 0.66196700 & -3.05846700 \\
-1.58546800 & 2.16621000 & -2.50201700 \\
-2.19636500 & 0.84190200 & -0.59154400 \\
1.47444600 & 1.21836400 & -0.29784100 \\
1.87795600 & 2.99336300 & 0.29648600 \\
-3.78078000 & 1.93348200 & -0.50073700 \\
0.56709600 & 3.80286000 & 0.29906700 \\
0.76939800 & 4.81693400 & 0.66237600 \\
0.12983300 & 3.89895400 & -0.70018000 \\
-0.17658600 & 3.34782500 & 0.96065500 \\
2.91720500 & 3.67429900 & -0.61318900 \\
3.12401800 & 4.68171900 & -0.23300000 \\
3.86384700 & 3.12821700 & -0.63489700 \\
2.56576200 & 3.78045400 & -1.64384000 \\
2.41402700 & 2.92375900 & 1.74029400 \\
3.39174300 & 2.43829000 & 1.79134300 \\
2.53531200 & 3.94184800 & 2.12787700 \\
1.72912700 & 2.38666800 & 2.40430800 \\
-3.28331400 & 3.38890400 & -0.37578400 \\
-2.64011300 & 3.52638000 & 0.49796100 \\
-2.73481000 & 3.72217700 & -1.26223100 \\
-4.14706900 & 4.05365300 & -0.26265500 \\
\hline .118737700 & 1.80412400 & -1.72074200 \\
\hline & 0.79550600 & -1.82408900 \\
\hline
\end{tabular}




\begin{tabular}{|c|c|c|c|c|c|c|c|}
\hline $\mathrm{H}$ & -5.55804400 & 2.48529600 & -1.60166600 & $\mathrm{O}$ & 2.69337800 & -3.01776100 & -1.21688700 \\
\hline $\mathrm{H}$ & -4.20696100 & 2.07419700 & -2.65528900 & C & 2.65061600 & -2.94904200 & -2.65590900 \\
\hline C & -4.54237400 & 1.55383400 & 0.78344700 & $\mathrm{H}$ & 1.82709400 & -3.55108300 & -3.04459400 \\
\hline $\mathrm{H}$ & -5.37937700 & 2.24581100 & 0.92770500 & $\mathrm{H}$ & 2.53472600 & -1.91128500 & -2.97638200 \\
\hline $\mathrm{H}$ & -4.96400800 & 0.54440000 & 0.73440400 & $\mathrm{H}$ & 3.60852600 & -3.34169700 & -2.99475600 \\
\hline $\mathrm{H}$ & -3.90358200 & 1.62094000 & 1.67052200 & $\mathrm{H}$ & 0.42500400 & -3.23591600 & 2.62927100 \\
\hline C & 3.16140000 & 0.42686000 & -0.37448500 & $\mathrm{H}$ & 1.96595600 & -4.03090500 & 1.08625200 \\
\hline C & 3.76425900 & -0.03154000 & 0.80789000 & & & & \\
\hline C & 5.00349100 & -0.16797900 & -1.62105200 & \multirow{2}{*}{\multicolumn{4}{|c|}{$\begin{array}{l}\text { TS23-e1 } \\
\text { imaginary frequency }=-275.10 \mathrm{~cm}^{-1}\end{array}$}} \\
\hline $\mathrm{C}$ & 5.05570600 & -0.55400600 & 0.74067900 & & & & \\
\hline $\mathrm{H}$ & 3.22741100 & 0.00591400 & 1.75061400 & $\mathrm{C}$ & 2.11935000 & 2.12719600 & 1.78592500 \\
\hline $\mathrm{C}$ & 5.69351800 & -0.62259400 & -0.49759000 & $\mathrm{H}$ & 3.16395900 & 2.36829300 & 1.56353100 \\
\hline $\mathrm{H}$ & 5.46004100 & -0.21414000 & -2.60762500 & $\mathrm{H}$ & 1.72893700 & 2.91941900 & 2.43128000 \\
\hline $\mathrm{H}$ & 5.55183400 & -0.90568700 & 1.64054700 & C & 2.04664900 & 0.77968100 & 2.53702600 \\
\hline $\mathrm{H}$ & 6.69714500 & -1.02294800 & -0.59562600 & $\mathrm{H}$ & 2.27892400 & 0.98209400 & 3.58932100 \\
\hline C & -2.94630200 & -0.88004900 & -0.66611900 & $\mathrm{H}$ & 1.02143200 & 0.39001500 & 2.52993000 \\
\hline $\mathrm{C}$ & -3.56388500 & -1.48674400 & -1.76173500 & $\mathrm{C}$ & 3.04245700 & -0.30813600 & 2.07040700 \\
\hline C & -3.40319700 & -2.83942000 & 0.62395900 & $\mathrm{H}$ & 3.19044400 & -1.03865500 & 2.87196700 \\
\hline C & -4.10833000 & -2.76752000 & -1.65316600 & $\mathrm{H}$ & 4.02286000 & 0.15222900 & 1.90106200 \\
\hline $\mathrm{H}$ & -3.62161700 & -0.95618800 & -2.70332200 & P & 2.60899900 & -1.16737500 & 0.46795700 \\
\hline C & -4.03870400 & -3.45552600 & -0.43992000 & $P$ & 1.14525400 & 2.25546800 & 0.19902000 \\
\hline $\mathrm{H}$ & -3.27319600 & -3.30123900 & 1.59587800 & C & 2.11643900 & 3.50608700 & -0.86704100 \\
\hline $\mathrm{H}$ & -4.58784000 & -3.22551900 & -2.51275500 & C & 4.20131300 & -2.12134700 & -0.01585700 \\
\hline $\mathrm{H}$ & -4.45290200 & -4.44975700 & -0.32289000 & $\mathrm{C}$ & 3.41949700 & 2.82082700 & -1.32449800 \\
\hline $\mathrm{N}$ & 3.76896900 & 0.35010800 & -1.56786000 & $\mathrm{H}$ & 3.98379500 & 3.50722700 & -1.96598700 \\
\hline $\mathrm{N}$ & -2.88668800 & -1.60259200 & 0.48605500 & $\mathrm{H}$ & 4.07054200 & 2.55345000 & -0.48575100 \\
\hline $\mathrm{Pd}$ & -0.28820500 & 0.28137500 & 0.90785300 & $\mathrm{H}$ & 3.21106400 & 1.91401500 & -1.90154200 \\
\hline C & 0.46734100 & -1.17069600 & 2.29935900 & $\mathrm{C}$ & 2.43871500 & 4.79019400 & -0.08093200 \\
\hline $\mathrm{O}$ & 1.19356600 & -0.75865000 & 3.17763300 & $\mathrm{H}$ & 2.96484700 & 5.49229800 & -0.73830500 \\
\hline $\mathrm{O}$ & -1.21600300 & -0.95758400 & 2.54046200 & $\mathrm{H}$ & 1.53520100 & 5.28810500 & 0.28095300 \\
\hline $\mathrm{H}$ & -2.32646600 & -1.24078900 & 1.30880700 & $\mathrm{H}$ & 3.08788600 & 4.60141700 & 0.77909500 \\
\hline $\mathrm{C}$ & -1.54252700 & -0.23756900 & 3.73457700 & C & 1.27308000 & 3.85126400 & -2.11063300 \\
\hline $\mathrm{H}$ & -2.00159000 & -0.92778100 & 4.45143000 & $\mathrm{H}$ & 0.37103900 & 4.41086300 & -1.84983900 \\
\hline $\mathrm{H}$ & -0.62991200 & 0.17673300 & 4.17358200 & $\mathrm{H}$ & 1.86633100 & 4.48273300 & -2.78170300 \\
\hline $\mathrm{H}$ & -2.24091000 & 0.57539800 & 3.50936400 & $\mathrm{H}$ & 0.97386600 & 2.95627600 & -2.66526200 \\
\hline C & 1.72392900 & -2.97324600 & 0.92625800 & C & 5.18400900 & -1.04826200 & -0.53193500 \\
\hline $\mathrm{H}$ & 2.61083000 & -2.41921300 & 1.24120600 & $\mathrm{H}$ & 4.75108200 & -0.45648100 & -1.34433300 \\
\hline $\mathrm{C}$ & 0.48352600 & -2.59767800 & 1.73970000 & $\mathrm{H}$ & 5.50613700 & -0.36334400 & 0.25876000 \\
\hline $\mathrm{H}$ & -0.40409300 & -2.77936400 & 1.13577700 & $\mathrm{H}$ & 6.08331300 & -1.54050600 & -0.91857000 \\
\hline $\mathrm{C}$ & 1.53914300 & -2.80199500 & -0.57091000 & $\mathrm{C}$ & 4.84837800 & -2.91321300 & 1.13256700 \\
\hline $\mathrm{O}$ & 0.48587900 & -2.55010300 & -1.13065500 & $\mathrm{H}$ & 4.20908700 & -3.72749900 & 1.48679500 \\
\hline
\end{tabular}




$\begin{array}{lrrrrrrr}\mathrm{H} & 5.77848900 & -3.37128700 & 0.77614700 & \mathrm{C} & -5.47060200 & 0.08404300 & -0.44120200 \\ \mathrm{H} & 5.10948900 & -2.27802300 & 1.98437300 & \mathrm{C} & -4.57779300 & -1.67003500 & 0.95418300 \\ \mathrm{C} & 3.83616400 & -3.07115700 & -1.17234200 & \mathrm{C} & -6.53235800 & 0.25382700 & 0.44905400 \\ \mathrm{H} & 4.74311100 & -3.56258000 & -1.54156100 & \mathrm{H} & -5.41441900 & 0.69601300 & -1.33874100 \\ \mathrm{H} & 3.14479200 & -3.86020500 & -0.85785200 & \mathrm{C} & -5.64063300 & -1.50558400 & 1.84251500 \\ \mathrm{H} & 3.38735900 & -2.53226500 & -2.01297200 & \mathrm{H} & -3.82488800 & -2.43106700 & 1.13836500 \\ \mathrm{C} & -0.36419600 & 3.20713600 & 0.73678400 & \mathrm{C} & -6.61918700 & -0.54034600 & 1.59377800 \\ \mathrm{C} & -1.46493300 & 3.31594100 & -0.12714100 & \mathrm{H} & -7.29705500 & 0.99682800 & 0.24246600 \\ \mathrm{C} & -1.41587300 & 4.47525600 & 2.34412600 & \mathrm{H} & -5.70926700 & -2.13231600 & 2.72713900 \\ \mathrm{C} & -2.56848100 & 4.06185400 & 0.28643100 & \mathrm{H} & -7.44840800 & -0.41416600 & 2.28331200 \\ \mathrm{H} & -1.45409600 & 2.82743100 & -1.09606400 & \mathrm{O} & -2.90922300 & -2.38043000 & -1.32540600 \\ \mathrm{C} & -2.54795500 & 4.65400800 & 1.54881400 & \mathrm{C} & -3.83285400 & -3.12197400 & -2.11728300 \\ \mathrm{H} & -1.36181600 & 4.91491200 & 3.33774200 & \mathrm{H} & -3.42791400 & -4.13127600 & -2.22222500 \\ \mathrm{H} & -3.43020000 & 4.17374200 & -0.36492800 & \mathrm{H} & -4.82105800 & -3.17913600 & -1.64472700 \\ \mathrm{H} & -3.38671900 & 5.23775200 & 1.91329300 & \mathrm{H} & -3.94195900 & -2.67750200 & -3.11586200 \\ \mathrm{C} & 1.47127300 & -2.54187100 & 1.07823700 & & \mathbf{T S 2 3 - f 1} & \\ \mathrm{C} & 1.58968900 & -3.25290400 & 2.27446100 & & & \end{array}$

$\begin{array}{lll}-0.52541100 & -3.75944700 & 0.59094300\end{array}$

$\begin{array}{lll}0.65019100 & -4.23004100 & 2.61514200\end{array}$

$2.41457000 \quad-3.04444900 \quad 2.94375800$

$\begin{array}{lll}-0.42673300 & -4.48850900 & 1.76650300\end{array}$

$-1.34927600 \quad-3.83310600 \quad-0.11203100$

$\begin{array}{lll}0.75651100 & -4.77952300 & 3.54554500\end{array}$

$\begin{array}{lll}-1.18033500 & -5.22734900 & 2.01226500\end{array}$

$\begin{array}{lll}-0.34386400 & 3.77486400 & 1.95154500\end{array}$

0.40950300

$-2.84147400$

0.28225500

0.90903600

$-1.03319400$

$0.17527800-0.79953000$

$0.06962000-1.67629500$

$-1.25433100$

$0.63305000 \quad-2.72319900$

$\begin{array}{lll}-0.16541500 & -1.46056400 & -1.84780100\end{array}$

$\begin{array}{lll}0.22874800 & -2.29521500 & -0.63725900\end{array}$

0.18424300

$-1.78217100 \quad-3.19332800$

$\begin{array}{lll}1.24597300 & -2.04229000 & -3.26425200\end{array}$

$-0.42729700$

$-2.62872800 \quad-3.52480000$

$-0.02034500$

$-0.92193000-3.83844200$

$-3.29824700$

$-1.01731900 \quad-1.14285500$

$-3.56568900$

$-0.58253600 \quad-2.11737300$

$-2.07077000$

$-0.24789500 \quad-0.60457300$

$-2.40664200$

0.71666700

$-0.21472900$

$-1.63024200$

$-0.79723500$

0.22990300

$-4.48045200$

$-0.87463600 \quad-0.19489600$

\section{TS23-f1}

imaginary frequency $=-277.07 \mathrm{~cm}^{-1}$

$\begin{array}{lrrr}\mathrm{C} & 3.30799900 & 1.23620300 & 1.29546400 \\ \mathrm{H} & 4.27130700 & 1.01094000 & 0.82643900 \\ \mathrm{H} & 3.43757200 & 2.13435700 & 1.90566400 \\ \mathrm{C} & 2.88408400 & 0.06516800 & 2.20855100 \\ \mathrm{H} & 3.41231500 & 0.18644400 & 3.16160700 \\ \mathrm{H} & 1.81658500 & 0.13412300 & 2.45004900 \\ \mathrm{C} & 3.23111800 & -1.35070400 & 1.69296100 \\ \mathrm{H} & 3.24437200 & -2.05586000 & 2.53023700 \\ \mathrm{H} & 4.25027300 & -1.34758500 & 1.28928100 \\ \mathrm{P} & 2.14594100 & -1.98772600 & 0.31106200 \\ \mathrm{P} & 2.10692200 & 1.71498000 & -0.05052500 \\ \mathrm{C} & 3.19541400 & 2.43450200 & -1.44335800 \\ \mathrm{C} & 3.10944600 & -3.47595900 & -0.42353400 \\ \mathrm{C} & 3.97736300 & 1.26862400 & -2.08040500 \\ \mathrm{H} & 4.59656200 & 1.65332500 & -2.89868500 \\ \mathrm{H} & 4.64974900 & 0.77450900 & -1.37139900 \\ \mathrm{H} & 3.29937200 & 0.51595000 & -2.49576600 \\ \mathrm{C} & 4.16912500 & 3.49741300 & -0.90158000 \\ \mathrm{H} & 4.75149600 & 3.91069900 & -1.73338700 \\ \mathrm{H} & 3.64290500 & 4.32676000 & -0.42101700 \\ \mathrm{H} & 4.87974500 & 3.08377200 & -0.17993800 \\ \mathrm{C} & 2.27934200 & 3.05983000 & -2.51434700 \\ \mathrm{H} & 1.77102300 & 3.95409200 & -2.14482400 \\ & & & \\ \mathrm{H} & & \end{array}$




\begin{tabular}{|c|c|c|c|c|c|c|c|}
\hline $\mathrm{H}$ & 2.88908100 & 3.36406600 & -3.37267500 & $\mathrm{H}$ & -2.10771800 & -2.09612100 & -2.83056600 \\
\hline $\mathrm{H}$ & 1.52184400 & 2.35445400 & -2.87057800 & $\mathrm{H}$ & -0.97638800 & -0.83372700 & -3.38087800 \\
\hline $\mathrm{C}$ & 4.24141900 & -2.85425800 & -1.26943200 & $\mathrm{C}$ & -3.39456000 & 1.10186600 & -0.32460000 \\
\hline $\mathrm{H}$ & 3.85271400 & -2.16761900 & -2.02738300 & $\mathrm{H}$ & -3.44073400 & 1.71516300 & -1.22989900 \\
\hline $\mathrm{H}$ & 4.97168200 & -2.31425200 & -0.65850200 & $\mathrm{C}$ & -1.89177300 & 0.88530600 & 0.00374800 \\
\hline $\mathrm{H}$ & 4.78309000 & -3.65371600 & -1.78695600 & $\mathrm{H}$ & -1.48408600 & 1.80749200 & 0.42406000 \\
\hline $\mathrm{C}$ & 3.70326000 & -4.43014600 & 0.62565700 & $\mathrm{H}$ & -1.76326200 & 0.09810700 & 0.74734300 \\
\hline $\mathrm{H}$ & 2.92936500 & -4.93923900 & 1.20788800 & $\mathrm{C}$ & -4.02212300 & 1.96725600 & 0.76453500 \\
\hline $\mathrm{H}$ & 4.28336000 & -5.20929500 & 0.11763700 & $\mathrm{~F}$ & -3.84142900 & 1.44372700 & 2.00608000 \\
\hline $\mathrm{H}$ & 4.38324300 & -3.92230400 & 1.31613000 & $\mathrm{~F}$ & -3.45261600 & 3.19723100 & 0.78013000 \\
\hline $\mathrm{C}$ & 2.14821200 & -4.24202500 & -1.35164500 & $\mathrm{~F}$ & -5.34073800 & 2.13353200 & 0.58190400 \\
\hline $\mathrm{H}$ & 2.69858800 & -5.03140600 & -1.87517700 & $\mathrm{C}$ & -4.15770100 & -0.18884500 & -0.57783700 \\
\hline $\mathrm{H}$ & 1.33602100 & -4.72614800 & -0.79899500 & $\mathrm{C}$ & -4.52211900 & -0.52633500 & -1.88701400 \\
\hline $\mathrm{H}$ & 1.70955800 & -3.58705400 & -2.11155500 & $\mathrm{C}$ & -4.49618700 & -1.06999000 & 0.46175700 \\
\hline $\mathrm{C}$ & 1.26178200 & 3.20349100 & 0.68778900 & $\mathrm{C}$ & -5.19877100 & -1.71768600 & -2.15939500 \\
\hline $\mathrm{C}$ & 0.14751200 & 3.76935500 & 0.04873100 & $\mathrm{H}$ & -4.27345700 & 0.14983600 & -2.70032000 \\
\hline $\mathrm{C}$ & 1.14571900 & 4.74431500 & 2.39400000 & $\mathrm{C}$ & -5.18072800 & -2.25741400 & 0.19153300 \\
\hline $\mathrm{C}$ & -0.46975100 & 4.87595900 & 0.63159100 & $\mathrm{H}$ & -4.25848300 & -0.80970400 & 1.48818900 \\
\hline $\mathrm{H}$ & -0.23076100 & 3.34874700 & -0.87705300 & $\mathrm{C}$ & -5.52903500 & -2.58898600 & -1.12088100 \\
\hline $\mathrm{C}$ & 0.03770800 & 5.37685500 & 1.82971100 & $\mathrm{H}$ & -5.47903700 & -1.95596800 & -3.18116600 \\
\hline $\mathrm{H}$ & 1.57038500 & 5.10124600 & 3.32978800 & $\mathrm{H}$ & -5.46820700 & -2.91138300 & 1.01093400 \\
\hline $\mathrm{H}$ & -1.33671800 & 5.32988400 & 0.16171800 & $\mathrm{H}$ & -6.06951400 & -3.50768600 & -1.32809800 \\
\hline $\mathrm{H}$ & -0.41395200 & 6.23223600 & 2.32094800 & & & & \\
\hline $\mathrm{C}$ & 0.76151200 & -2.83825600 & 1.26362000 & \multirow{2}{*}{\multicolumn{4}{|c|}{$\begin{array}{l}\text { TS23-g1 } \\
\text { imaginary frequency }=-284.03 \mathrm{~cm}^{-1}\end{array}$}} \\
\hline $\mathrm{C}$ & 0.88221800 & -3.55315900 & 2.45673500 & & & & \\
\hline $\mathrm{C}$ & -1.58308400 & -3.30558800 & 1.26283300 & $\mathrm{C}$ & -0.32861700 & -2.95794900 & 1.47311300 \\
\hline $\mathrm{C}$ & -0.23530500 & -4.15395000 & 3.04206000 & $\mathrm{H}$ & -0.94474600 & -3.76356800 & 1.06138800 \\
\hline $\mathrm{H}$ & 1.85135400 & -3.64479600 & 2.93019800 & $\mathrm{H}$ & 0.40052300 & -3.41683900 & 2.14748300 \\
\hline $\mathrm{C}$ & -1.48711000 & -4.03603100 & 2.43678400 & $\mathrm{C}$ & -1.21682200 & -1.98086000 & 2.27068900 \\
\hline $\mathrm{H}$ & -2.51634900 & -3.12472300 & 0.73839000 & $\mathrm{H}$ & -1.39338700 & -2.42995300 & 3.25537200 \\
\hline $\mathrm{H}$ & -0.12639700 & -4.71136200 & 3.96736800 & $\mathrm{H}$ & -0.67887300 & -1.04427000 & 2.46137900 \\
\hline $\mathrm{H}$ & -2.37288600 & -4.48738300 & 2.86770800 & $\mathrm{C}$ & -2.60883700 & -1.69020100 & 1.66414400 \\
\hline $\mathrm{N}$ & 1.74863200 & 3.68346100 & 1.84159000 & $\mathrm{H}$ & -3.29225800 & -1.35027600 & 2.44871000 \\
\hline $\mathrm{N}$ & -0.48460600 & -2.74229600 & 0.72310800 & $\mathrm{H}$ & -3.03710600 & -2.62304000 & 1.27969700 \\
\hline $\mathrm{Pd}$ & 0.84910600 & -0.13490400 & -0.70699100 & $\mathrm{P}$ & -2.61837400 & -0.50129200 & 0.22221200 \\
\hline $\mathrm{C}$ & -1.07719500 & 0.58936800 & -1.24841500 & $\mathrm{P}$ & 0.67787400 & -2.22358600 & 0.08047800 \\
\hline $\mathrm{O}$ & -1.20589000 & 1.14996500 & -2.31691100 & $\mathrm{C}$ & 0.75833100 & -3.60759200 & -1.23761400 \\
\hline $\mathrm{O}$ & -1.06587100 & -1.13558000 & -1.33099700 & $\mathrm{C}$ & -4.36427700 & -0.71026600 & -0.55888800 \\
\hline $\mathrm{H}$ & -0.65436600 & -2.13626400 & -0.14122400 & $\mathrm{C}$ & -0.64932000 & -3.76615800 & -1.84533200 \\
\hline $\mathrm{C}$ & -1.12154700 & -1.65022300 & -2.66743400 & $\mathrm{H}$ & -0.62121300 & -4.53476400 & -2.62617300 \\
\hline $\mathrm{H}$ & -0.34157700 & -2.40521700 & -2.81378600 & $\mathrm{H}$ & -1.39327300 & -4.08441800 & -1.10745400 \\
\hline
\end{tabular}




\begin{tabular}{|c|c|c|c|c|c|c|c|}
\hline $\mathrm{H}$ & -0.99290700 & -2.83273100 & -2.30274500 & $\mathrm{Pc}$ & -0.31887800 & -0.25685600 & -0.68467800 \\
\hline $\mathrm{C}$ & 1.22546800 & -4.94230900 & -0.62815100 & $\mathrm{C}$ & 1.25863700 & 1.13553900 & -1.21704400 \\
\hline $\mathrm{H}$ & 1.28138800 & -5.69966400 & -1.41897400 & $\mathrm{O}$ & 1.83376800 & 0.90484400 & -2.25482200 \\
\hline $\mathrm{H}$ & 2.21692700 & -4.86081300 & -0.17512200 & $\mathrm{O}$ & -0.27790900 & 1.89536600 & -1.40169900 \\
\hline $\mathrm{H}$ & 0.53649700 & -5.31733900 & 0.13428500 & $\mathrm{H}$ & -1.45489100 & 2.03009700 & -0.28245700 \\
\hline $\mathrm{C}$ & 1.73064200 & -3.17341200 & -2.35213300 & $\mathrm{C}$ & -0.66329000 & 2.08752600 & -2.76871200 \\
\hline $\mathrm{H}$ & 2.76411000 & -3.12943500 & -1.99919900 & $\mathrm{H}$ & -1.68345900 & 1.72219300 & -2.92814800 \\
\hline $\mathrm{H}$ & 1.69550300 & -3.90839800 & -3.16445300 & $\mathrm{H}$ & -0.61442700 & 3.15591400 & -3.00768100 \\
\hline $\mathrm{H}$ & 1.46707100 & -2.19627000 & -2.76886500 & $\mathrm{H}$ & 0.02351100 & 1.54593700 & -3.42560400 \\
\hline $\mathrm{C}$ & -4.30399700 & -2.03674900 & -1.34604400 & $\mathrm{C}$ & 2.82790500 & 2.93249100 & -0.27084400 \\
\hline $\mathrm{H}$ & -3.49596000 & -2.03645800 & -2.08325100 & $\mathrm{H}$ & 3.49270500 & 2.57257300 & -1.06696600 \\
\hline $\mathrm{H}$ & -4.17250100 & -2.90513000 & -0.69270400 & $\mathrm{C}$ & 1.85486900 & 1.75967800 & 0.03681000 \\
\hline $\mathrm{H}$ & -5.24830500 & -2.17800700 & -1.88353700 & $\mathrm{H}$ & 2.40170200 & 0.95516300 & 0.53661500 \\
\hline $\mathrm{C}$ & -5.51882200 & -0.75994500 & 0.45542700 & $\mathrm{H}$ & 1.05695700 & 2.06854800 & 0.71739600 \\
\hline $\mathrm{H}$ & -5.64376300 & 0.18701000 & 0.98887500 & $\mathrm{C}$ & 2.09632400 & 4.18447200 & -0.83331400 \\
\hline $\mathrm{H}$ & -6.45820000 & -0.95062000 & -0.07665400 & $\mathrm{H}$ & 1.35823200 & 3.81925200 & -1.55706900 \\
\hline $\mathrm{H}$ & -5.39807800 & -1.56170100 & 1.19007700 & $\mathrm{C}$ & 3.05578800 & 5.10693000 & -1.60246300 \\
\hline $\mathrm{C}$ & -4.57875700 & 0.45258000 & -1.54547800 & $\mathrm{H}$ & 2.51457800 & 5.95161200 & -2.04182700 \\
\hline $\mathrm{H}$ & -5.51135000 & 0.29486100 & -2.09831300 & $\mathrm{H}$ & 3.83287500 & 5.52395300 & -0.95244600 \\
\hline $\mathrm{H}$ & -4.66500400 & 1.41810300 & -1.03571500 & $\mathrm{H}$ & 3.55266200 & 4.56955300 & -2.41657200 \\
\hline $\mathrm{H}$ & -3.76846600 & 0.51793800 & -2.27898300 & $\mathrm{C}$ & 1.33270900 & 4.97932200 & 0.23997900 \\
\hline $\mathrm{C}$ & 2.40009400 & -2.22642700 & 0.79762600 & $\mathrm{H}$ & 0.69909500 & 5.74308800 & -0.22554100 \\
\hline $\mathrm{C}$ & 3.43949400 & -1.56654200 & 0.12358600 & $\mathrm{H}$ & 0.69411000 & 4.33410100 & 0.85494800 \\
\hline $\mathrm{C}$ & 3.83444200 & -2.92567400 & 2.45843800 & $\mathrm{H}$ & 2.01368600 & 5.49951100 & 0.92122400 \\
\hline $\mathrm{C}$ & 4.72859200 & -1.63271900 & 0.65309400 & $\mathrm{C}$ & 3.69944900 & 3.24682500 & 0.96667300 \\
\hline $\mathrm{H}$ & 3.24277400 & -1.01477200 & -0.78984900 & $\mathrm{H}$ & 4.17377500 & 4.22426000 & 0.82241200 \\
\hline $\mathrm{C}$ & 4.93471600 & -2.32559600 & 1.84555400 & $\mathrm{H}$ & 3.06054500 & 3.35285200 & 1.85379200 \\
\hline $\mathrm{H}$ & 3.94857200 & -3.46638800 & 3.39554900 & $\mathrm{C}$ & 4.80175100 & 2.21973000 & 1.25052500 \\
\hline $\mathrm{H}$ & 5.55486000 & -1.14470600 & 0.14501400 & $\mathrm{H}$ & 5.39921500 & 2.52122600 & 2.11665300 \\
\hline $\mathrm{H}$ & 5.91957300 & -2.39753000 & 2.29498900 & $\mathrm{H}$ & 4.40117500 & 1.22375600 & 1.46471900 \\
\hline $\mathrm{C}$ & -2.78998400 & 1.15556000 & 1.09803400 & $\mathrm{H}$ & 5.48081100 & 2.12879900 & 0.39534500 \\
\hline $\mathrm{C}$ & -3.53549300 & 1.42819800 & 2.24748000 & & & & \\
\hline $\mathrm{C}$ & -2.18289100 & 3.46352500 & 0.99349700 & \multicolumn{4}{|c|}{ TS24 } \\
\hline $\mathrm{C}$ & -3.61059500 & 2.72631600 & 2.75719300 & \multicolumn{4}{|c|}{ imaginary frequency $=-429.00 \mathrm{~cm}^{-1}$} \\
\hline $\mathrm{H}$ & -4.06561300 & 0.62558600 & 2.74388200 & $\mathrm{C}$ & -1.84402600 & 0.47924100 & -2.64365800 \\
\hline $\mathrm{C}$ & -2.93407800 & 3.76615400 & 2.11701400 & $\mathrm{H}$ & -1.16915400 & 0.18610300 & -3.45583700 \\
\hline $\mathrm{H}$ & -1.59795900 & 4.19488200 & 0.44843200 & $\mathrm{H}$ & -2.86884700 & 0.31949200 & -2.98796000 \\
\hline $\mathrm{H}$ & -4.19899000 & 2.92433500 & 3.64787400 & $\mathrm{C}$ & -1.65113100 & 1.96639500 & -2.30196300 \\
\hline $\mathrm{H}$ & -2.97436700 & 4.78479800 & 2.48401800 & $\mathrm{H}$ & -2.14324700 & 2.54837100 & -3.08955700 \\
\hline $\mathrm{N}$ & 2.59694300 & -2.88694300 & 1.94849000 & $\mathrm{H}$ & -2.18000000 & 2.22321500 & -1.37646000 \\
\hline $\mathrm{N}$ & -2.12693000 & 2.19970800 & 0.52951700 & $\mathrm{C}$ & -0.19292500 & 2.44588600 & -2.23534900 \\
\hline
\end{tabular}




\begin{tabular}{|c|c|c|c|c|c|c|c|}
\hline $\mathrm{H}$ & -0.16796400 & 3.53931500 & -2.22861200 & $\mathrm{C}$ & -0.54489900 & 5.23685000 & 1.05041400 \\
\hline $\mathrm{H}$ & 0.35779700 & 2.10082800 & -3.11732600 & $\mathrm{C}$ & -0.45549600 & 3.63057700 & 2.82801000 \\
\hline $\mathrm{P}$ & 0.76025700 & 1.92882500 & -0.73732800 & $\mathrm{H}$ & 0.19740000 & 1.66810700 & 2.19135800 \\
\hline $\mathrm{P}$ & -1.60156900 & -0.71022800 & -1.23691400 & $\mathrm{C}$ & -0.72733900 & 4.92813800 & 2.40099000 \\
\hline $\mathrm{C}$ & -1.82747300 & -2.42947500 & -2.04024700 & $\mathrm{H}$ & -0.75105300 & 6.23744900 & 0.67743400 \\
\hline $\mathrm{C}$ & 2.56008200 & 2.42490600 & -1.11020400 & $\mathrm{H}$ & -0.58864700 & 3.34800100 & 3.86792500 \\
\hline $\mathrm{C}$ & -0.46246400 & -2.84568200 & -2.62340200 & $\mathrm{H}$ & -1.07675900 & 5.68818500 & 3.09194700 \\
\hline $\mathrm{H}$ & -0.56021600 & -3.82135100 & -3.11245700 & $\mathrm{~N}$ & -4.28707500 & -0.35479200 & -0.95121100 \\
\hline $\mathrm{H}$ & -0.10020100 & -2.13830600 & -3.37718700 & $\mathrm{~N}$ & -0.11782400 & 4.34757200 & 0.14692500 \\
\hline $\mathrm{H}$ & 0.30296300 & -2.93467400 & -1.84733100 & $\mathrm{Pd}$ & 0.45414700 & -0.27511800 & 0.00465400 \\
\hline $\mathrm{C}$ & -2.88095800 & -2.40934000 & -3.16644500 & $\mathrm{H}$ & 1.78278900 & 0.10126500 & 0.79197900 \\
\hline $\mathrm{H}$ & -3.03117800 & -3.43603600 & -3.51978900 & $\mathrm{C}$ & 3.25635600 & -1.90763400 & 0.82639600 \\
\hline $\mathrm{H}$ & -3.84078700 & -2.02308100 & -2.81775000 & $\mathrm{C}$ & 3.34941800 & -2.63893900 & -0.37090100 \\
\hline $\mathrm{H}$ & -2.55817800 & -1.81676500 & -4.02677000 & $\mathrm{C}$ & 4.43843700 & -1.54082400 & 1.49167700 \\
\hline $\mathrm{C}$ & -2.26922600 & -3.42920200 & -0.95550200 & $\mathrm{C}$ & 4.59345500 & -2.99825200 & -0.88103200 \\
\hline $\mathrm{H}$ & -3.28019200 & -3.20998400 & -0.60065700 & $\mathrm{H}$ & 2.44962100 & -2.92291900 & -0.90737800 \\
\hline $\mathrm{H}$ & -2.28199800 & -4.43753500 & -1.38449300 & $\mathrm{C}$ & 5.68350400 & -1.90628000 & 0.98218200 \\
\hline $\mathrm{H}$ & -1.60380200 & -3.44345000 & -0.08959000 & $\mathrm{H}$ & 4.37829700 & -0.97808500 & 2.41976400 \\
\hline $\mathrm{C}$ & 3.16131600 & 1.35024800 & -2.03804400 & $\mathrm{C}$ & 5.76338700 & -2.63450400 & -0.20569000 \\
\hline $\mathrm{H}$ & 3.18459600 & 0.36672900 & -1.55932700 & $\mathrm{H}$ & 4.65367800 & -3.56474000 & -1.80513500 \\
\hline $\mathrm{H}$ & 2.61398300 & 1.26188900 & -2.98240500 & $\mathrm{H}$ & 6.58772900 & -1.62471500 & 1.51246100 \\
\hline $\mathrm{H}$ & 4.19190100 & 1.62709400 & -2.28656400 & $\mathrm{H}$ & 6.73159700 & -2.91949400 & -0.60543900 \\
\hline $\mathrm{C}$ & 2.61099800 & 3.80680600 & -1.79576500 & $\mathrm{C}$ & 0.72985000 & -2.17195800 & 1.21287600 \\
\hline $\mathrm{H}$ & 2.11348500 & 4.57874800 & -1.20389000 & $\mathrm{H}$ & 0.67921600 & -3.04102100 & 0.56817300 \\
\hline $\mathrm{H}$ & 3.65995100 & 4.09677900 & -1.92213200 & $\mathrm{C}$ & 1.96702600 & -1.53534100 & 1.44400800 \\
\hline $\mathrm{H}$ & 2.15721100 & 3.79199700 & -2.79020000 & $\mathrm{C}$ & -0.28215600 & -2.08231100 & 2.29813900 \\
\hline $\mathrm{C}$ & 3.35798300 & 2.47869400 & 0.20452200 & $\mathrm{O}$ & -0.36305400 & -1.16837400 & 3.10407600 \\
\hline $\mathrm{H}$ & 4.39969900 & 2.72826900 & -0.02403100 & $\mathrm{O}$ & -1.08954400 & -3.15700100 & 2.29758700 \\
\hline $\mathrm{H}$ & 2.97573300 & 3.24175100 & 0.88825700 & $\mathrm{C}$ & -2.08199300 & -3.21242600 & 3.34385500 \\
\hline $\mathrm{H}$ & 3.36208400 & 1.51473000 & 0.72333500 & $\mathrm{H}$ & -1.61225200 & -3.07820000 & 4.31971900 \\
\hline $\mathrm{C}$ & -3.14118900 & -0.38144600 & -0.24969000 & $\mathrm{H}$ & -2.83546900 & -2.43606800 & 3.19265200 \\
\hline $\mathrm{C}$ & -3.09354900 & -0.14662100 & 1.12898400 & $\mathrm{H}$ & -2.53268900 & -4.20035600 & 3.26305500 \\
\hline $\mathrm{C}$ & -5.42566300 & -0.10673300 & -0.29590700 & $\mathrm{H}$ & 2.03938000 & -1.00872700 & 2.39374000 \\
\hline
\end{tabular}

C

$\mathrm{H}$

C

$\mathrm{H}$

$\mathrm{H}$

$\mathrm{H}$

C

C $\begin{array}{lll}-4.28997200 & 0.11947400 & 1.80136100\end{array}$

$\begin{array}{lll}-2.15237100 & -0.16319900 & 1.66711100\end{array}$

$\begin{array}{lll}-5.48015700 & 0.13676000 & 1.07941100\end{array}$

$\begin{array}{lll}-6.33236300 & -0.09698400 & -0.89666800\end{array}$

$\begin{array}{lll}-4.28560600 & 0.31340900 & 2.86998200\end{array}$

$\begin{array}{lll}-6.43115400 & 0.33914500 & 1.56094500\end{array}$

$\begin{array}{lll}0.14802100 & 3.09916000 & 0.56478700\end{array}$

$-0.01104500 \quad 2.69130600 \quad 1.89474700$
TS24a

imaginary frequency $=-561.93 \mathrm{~cm}^{-1}$

$\begin{array}{llll}\mathrm{C} & -2.29094000 & 0.64278500 & -1.00452700 \\ \mathrm{H} & -2.80521400 & 1.57083100 & -1.27784900 \\ \mathrm{H} & -1.81750300 & 0.79320400 & -0.02978400 \\ \mathrm{C} & -1.25140600 & 0.29524900 & -2.08063500 \\ \mathrm{H} & -1.76343400 & 0.03514300 & -3.01452800\end{array}$




\begin{tabular}{|c|c|c|c|c|c|c|c|}
\hline $\mathrm{H}$ & -0.69189000 & -0.60050700 & -1.78465600 & $\mathrm{H}$ & -1.61426800 & -3.92139000 & 3.40788400 \\
\hline $\mathrm{C}$ & -0.25631200 & 1.42734800 & -2.40905100 & $\mathrm{~N}$ & -1.95971700 & -1.25092400 & 1.35779100 \\
\hline $\mathrm{H}$ & 0.40640700 & 1.10109900 & -3.21828000 & $\mathrm{C}$ & -5.02701300 & 0.25822400 & 0.03984700 \\
\hline $\mathrm{H}$ & -0.78779800 & 2.31071700 & -2.77875400 & $\mathrm{C}$ & -5.67762700 & 1.17141400 & -1.01981700 \\
\hline $\mathrm{P}$ & 0.86753000 & 1.92184700 & -1.01584300 & $\mathrm{H}$ & -6.54061100 & 1.68651300 & -0.58134400 \\
\hline $\mathrm{C}$ & 2.32377400 & 2.87227800 & -1.78818700 & $\mathrm{H}$ & -4.99269300 & 1.94444700 & -1.38478100 \\
\hline $\mathrm{C}$ & -0.05404900 & 3.16675900 & 0.00660300 & $\mathrm{H}$ & -6.03273000 & 0.59925700 & -1.88271700 \\
\hline $\mathrm{C}$ & -0.93875700 & 4.10590200 & -0.53989800 & $\mathrm{C}$ & -4.56015600 & 1.09270100 & 1.24377500 \\
\hline $\mathrm{C}$ & -0.37626800 & 3.99942800 & 2.13150700 & $\mathrm{H}$ & -3.86374400 & 1.88445000 & 0.94948300 \\
\hline $\mathrm{C}$ & -1.55510400 & 5.02112800 & 0.31542000 & $\mathrm{H}$ & -5.42448300 & 1.57674600 & 1.71597000 \\
\hline $\mathrm{H}$ & -1.14935400 & 4.13102400 & -1.60273500 & $\mathrm{H}$ & -4.06724800 & 0.47651800 & 2.00085300 \\
\hline $\mathrm{C}$ & -1.27012500 & 4.97103200 & 1.67809200 & $\mathrm{C}$ & -6.06714700 & -0.78267600 & 0.49655900 \\
\hline $\mathrm{H}$ & -0.12992500 & 3.91736400 & 3.18751500 & $\mathrm{H}$ & -6.94472200 & -0.26899500 & 0.90654500 \\
\hline $\mathrm{H}$ & -2.24651400 & 5.75878600 & -0.08023200 & $\mathrm{H}$ & -6.40901300 & -1.40690500 & -0.33600100 \\
\hline $\mathrm{H}$ & -1.72965300 & 5.66182700 & 2.37686900 & $\mathrm{H}$ & -5.67584000 & -1.43883200 & 1.27942700 \\
\hline $\mathrm{N}$ & 0.21692500 & 3.11814700 & 1.31858800 & $\mathrm{C}$ & 2.51669600 & -2.03026200 & -0.60287700 \\
\hline $\mathrm{Pd}$ & 1.57158300 & 0.03655100 & 0.26114500 & $\mathrm{H}$ & 3.50354600 & -1.81765800 & -1.01457400 \\
\hline $\mathrm{H}$ & 2.03971600 & -0.58545300 & -1.15333200 & $\mathrm{C}$ & 2.44827700 & -1.90117800 & 0.81129100 \\
\hline $\mathrm{C}$ & 1.81968400 & 3.98995100 & -2.72226700 & $\mathrm{H}$ & 1.71488400 & -2.46543800 & 1.37809300 \\
\hline $\mathrm{H}$ & 1.21584300 & 4.73013400 & -2.19061000 & $\mathrm{C}$ & 1.65672300 & -2.91348800 & -1.42316600 \\
\hline $\mathrm{H}$ & 2.68362900 & 4.51686800 & -3.14169900 & $\mathrm{C}$ & 2.16034600 & -3.36233600 & -2.65486200 \\
\hline $\mathrm{H}$ & 1.23735600 & 3.60065000 & -3.56301800 & $\mathrm{C}$ & 0.38410400 & -3.33876100 & -1.00579500 \\
\hline $\mathrm{C}$ & 3.19576800 & 1.87997300 & -2.58243400 & $\mathrm{C}$ & 1.41581200 & -4.23435200 & -3.44760300 \\
\hline $\mathrm{H}$ & 3.62462900 & 1.11180000 & -1.93251600 & $\mathrm{H}$ & 3.14412500 & -3.03805400 & -2.98434600 \\
\hline $\mathrm{H}$ & 2.64901300 & 1.38727000 & -3.39292000 & $\mathrm{C}$ & -0.35909300 & -4.20460300 & -1.80318800 \\
\hline $\mathrm{H}$ & 4.02690000 & 2.42739900 & -3.03989500 & $\mathrm{H}$ & -0.03282200 & -2.98718200 & -0.06723900 \\
\hline $\mathrm{C}$ & 3.15878900 & 3.47670600 & -0.64147600 & $\mathrm{C}$ & 0.15468400 & -4.65594400 & -3.02329400 \\
\hline $\mathrm{H}$ & 3.53764900 & 2.70461500 & 0.03579000 & $\mathrm{H}$ & 1.82002100 & -4.58326700 & -4.39252300 \\
\hline $\mathrm{H}$ & 4.02334900 & 3.99738900 & -1.06749800 & $\mathrm{H}$ & -1.34214700 & -4.52406000 & -1.47296500 \\
\hline $\mathrm{H}$ & 2.59142900 & 4.20202500 & -0.05220500 & $\mathrm{H}$ & -0.42858300 & -5.33180500 & -3.64095000 \\
\hline $\mathrm{C}$ & 1.05976700 & 0.59596600 & 2.11889400 & $\mathrm{C}$ & 3.70709800 & -1.51684800 & 1.52186300 \\
\hline $\mathrm{O}$ & 0.81490600 & 0.75787700 & 3.21837900 & $\mathrm{O}$ & 4.69021900 & -1.05657700 & 0.97267900 \\
\hline $\mathrm{P}$ & -3.60869400 & -0.68242500 & -0.85161100 & $\mathrm{O}$ & 3.60098200 & -1.74330400 & 2.84051200 \\
\hline $\mathrm{C}$ & -2.91154500 & -1.72840700 & 0.53229600 & $\mathrm{C}$ & 4.77090000 & -1.42403000 & 3.62681700 \\
\hline $\mathrm{C}$ & -3.41978300 & -3.03220700 & 0.67298800 & $\mathrm{H}$ & 4.99273900 & -0.35649500 & 3.55990900 \\
\hline $\mathrm{C}$ & -2.96486600 & -3.84201100 & 1.71170600 & $\mathrm{H}$ & 4.51805900 & -1.70263100 & 4.64838200 \\
\hline $\mathrm{H}$ & -4.16582300 & -3.39564500 & -0.02697100 & $\mathrm{H}$ & 5.63182400 & -1.99330700 & 3.27119300 \\
\hline $\mathrm{C}$ & -1.52792400 & -2.04103500 & 2.35318400 & & & & \\
\hline $\mathrm{C}$ & -1.99768400 & -3.33471400 & 2.57944400 & \multicolumn{4}{|c|}{ TS25 } \\
\hline $\mathrm{H}$ & -3.35579100 & -4.84704200 & 1.84095000 & \multicolumn{4}{|c|}{ imaginary frequency $=-565.44 \mathrm{~cm}^{-1}$} \\
\hline $\mathrm{H}$ & -0.77373600 & -1.61256800 & 3.01072900 & $\mathrm{C}$ & 0.53514800 & -1.76043000 & -2.66679000 \\
\hline
\end{tabular}




\begin{tabular}{|c|c|c|c|c|c|c|c|}
\hline $\mathrm{H}$ & 0.06901400 & -1.24305000 & -3.51237000 & $\mathrm{C}$ & 4.51254900 & -3.10811300 & 0.43606100 \\
\hline $\mathrm{H}$ & 1.37363100 & -2.34302500 & -3.05751700 & $\mathrm{H}$ & 4.81314400 & -3.74442700 & -1.61115200 \\
\hline $\mathrm{C}$ & -0.48114100 & -2.69878500 & -1.99675200 & $\mathrm{H}$ & 3.92843700 & -2.24434500 & 2.32816900 \\
\hline $\mathrm{H}$ & -0.56415200 & -3.59538600 & -2.62145800 & $\mathrm{H}$ & 5.28189900 & -3.75647300 & 0.84228500 \\
\hline $\mathrm{H}$ & -0.10672000 & -3.05127900 & -1.02777700 & $\mathrm{C}$ & -2.43386300 & -2.01068700 & 0.98069400 \\
\hline $\mathrm{C}$ & -1.89537200 & -2.11661300 & -1.85046900 & $\mathrm{C}$ & -2.05601400 & -1.64297100 & 2.27710600 \\
\hline $\mathrm{H}$ & -2.60845400 & -2.91875200 & -1.63994200 & $\mathrm{C}$ & -3.32699700 & -3.99570900 & 1.72612200 \\
\hline $\mathrm{H}$ & -2.19906000 & -1.62934300 & -2.78268300 & $\mathrm{C}$ & -2.34797900 & -2.51038500 & 3.33138900 \\
\hline $\mathrm{P}$ & -2.11566800 & -0.89982600 & -0.47529800 & $\mathrm{H}$ & -1.54242000 & -0.70370000 & 2.45018800 \\
\hline $\mathrm{P}$ & 1.30872700 & -0.48304200 & -1.55997900 & $\mathrm{C}$ & -2.99611400 & -3.71143500 & 3.05328000 \\
\hline $\mathrm{C}$ & 2.24238300 & 0.65852100 & -2.77509900 & $\mathrm{H}$ & -3.82935700 & -4.92467700 & 1.46646400 \\
\hline $\mathrm{C}$ & -3.77189200 & -0.03252300 & -0.84684500 & $\mathrm{H}$ & -2.06997600 & -2.25313200 & 4.34901900 \\
\hline $\mathrm{C}$ & 1.22642500 & 1.68977000 & -3.30804900 & $\mathrm{H}$ & -3.23894800 & -4.41756600 & 3.84029500 \\
\hline $\mathrm{H}$ & 1.73329600 & 2.36025100 & -4.01145000 & $\mathrm{~N}$ & 3.31524800 & -2.33155400 & -1.50070400 \\
\hline $\mathrm{H}$ & 0.40759600 & 1.21142600 & -3.85668600 & $\mathrm{~N}$ & -3.05580500 & -3.16899700 & 0.71014400 \\
\hline $\mathrm{H}$ & 0.79097900 & 2.30357700 & -2.51438800 & $\mathrm{Pd}$ & -0.31545100 & 0.56888100 & -0.05843200 \\
\hline $\mathrm{C}$ & 2.85198200 & -0.12996700 & -3.95235800 & $\mathrm{H}$ & -1.39930000 & 1.27301700 & 0.87275100 \\
\hline $\mathrm{H}$ & 3.42949600 & 0.56523000 & -4.57276700 & $\mathrm{C}$ & 0.96023800 & 2.29642500 & 0.89118600 \\
\hline $\mathrm{H}$ & 3.52026900 & -0.92376800 & -3.61197900 & $\mathrm{H}$ & 1.41184300 & 2.74794700 & 0.01319400 \\
\hline $\mathrm{H}$ & 2.08636000 & -0.57357000 & -4.59404800 & $\mathrm{C}$ & -0.39179500 & 2.61131800 & 1.11333000 \\
\hline $\mathrm{C}$ & 3.37339600 & 1.37694600 & -2.01760000 & $\mathrm{H}$ & -0.78012500 & 2.61724800 & 2.12731100 \\
\hline $\mathrm{H}$ & 4.13243300 & 0.67714700 & -1.65691500 & $\mathrm{C}$ & -1.06973800 & 3.56531800 & 0.16733000 \\
\hline $\mathrm{H}$ & 3.86509600 & 2.08380600 & -2.69487700 & $\mathrm{O}$ & -0.68912700 & 3.81507200 & -0.95813400 \\
\hline $\mathrm{H}$ & 2.99952800 & 1.94688700 & -1.16455900 & $\mathrm{O}$ & -2.14650800 & 4.10949100 & 0.75138900 \\
\hline $\mathrm{C}$ & -3.53361300 & 0.95967800 & -2.00332200 & $\mathrm{C}$ & -2.86216100 & 5.09018800 & -0.03478300 \\
\hline $\mathrm{H}$ & -2.78187000 & 1.71389100 & -1.75065000 & $\mathrm{H}$ & -3.23516000 & 4.63762300 & -0.95580300 \\
\hline $\mathrm{H}$ & -3.21609700 & 0.45784000 & -2.92288600 & $\mathrm{H}$ & -3.68491700 & 5.42162900 & 0.59619500 \\
\hline $\mathrm{H}$ & -4.47189800 & 1.47906700 & -2.22841200 & $\mathrm{H}$ & -2.20391500 & 5.92476400 & -0.28343300 \\
\hline $\mathrm{C}$ & -4.84357700 & -1.06656100 & -1.25030400 & $\mathrm{C}$ & 1.86546900 & 1.82133900 & 1.94953300 \\
\hline $\mathrm{H}$ & -4.99662100 & -1.82599300 & -0.47989200 & $\mathrm{C}$ & 3.24732500 & 2.05864200 & 1.82846000 \\
\hline $\mathrm{H}$ & -5.79462900 & -0.54547100 & -1.40653600 & $\mathrm{C}$ & 1.39871000 & 1.17097900 & 3.10973700 \\
\hline $\mathrm{H}$ & -4.59535800 & -1.57604000 & -2.18483100 & $\mathrm{C}$ & 4.13114000 & 1.66501500 & 2.83100100 \\
\hline $\mathrm{C}$ & -4.24469600 & 0.73227100 & 0.40215600 & $\mathrm{H}$ & 3.62646400 & 2.57327000 & 0.95082600 \\
\hline $\mathrm{H}$ & -5.19843800 & 1.22194500 & 0.17699800 & $\mathrm{C}$ & 2.28409300 & 0.77190400 & 4.10769200 \\
\hline $\mathrm{H}$ & -4.41085700 & 0.06877500 & 1.25596700 & $\mathrm{H}$ & 0.33755700 & 0.97438700 & 3.23208500 \\
\hline $\mathrm{H}$ & -3.54055600 & 1.51554700 & 0.69949000 & $\mathrm{C}$ & 3.65430200 & 1.01730700 & 3.97274200 \\
\hline $\mathrm{C}$ & 2.59676700 & -1.51844800 & -0.70745500 & $\mathrm{H}$ & 5.19186000 & 1.86943600 & 2.72336800 \\
\hline $\mathrm{C}$ & 2.78318000 & -1.45815200 & 0.67712900 & $\mathrm{H}$ & 1.90589700 & 0.27578200 & 4.99647300 \\
\hline $\mathrm{C}$ & 4.25103100 & -3.10179800 & -0.93715000 & $\mathrm{H}$ & 4.34204700 & 0.71372000 & 4.75591700 \\
\hline $\mathrm{C}$ & 3.76124500 & -2.27098900 & 1.25579000 & & & & \\
\hline $\mathrm{H}$ & 2.18323900 & -0.79468100 & 1.28839700 & TS25a & & & \\
\hline
\end{tabular}




\begin{tabular}{|c|c|c|c|c|c|c|c|}
\hline \multicolumn{4}{|c|}{ imaginary frequency $=-616.56 \mathrm{~cm}^{-1}$} & $\mathrm{C}$ & -3.06539500 & -4.34218500 & 0.92454000 \\
\hline $\mathrm{C}$ & -2.70370200 & 0.62280600 & -0.82917900 & $\mathrm{H}$ & -4.39790400 & -3.60911700 & -0.60953900 \\
\hline $\mathrm{H}$ & -3.26050300 & 1.56351300 & -0.90441500 & $\mathrm{C}$ & -1.63844900 & -2.64613900 & 1.82352900 \\
\hline $\mathrm{H}$ & -2.20170000 & 0.59969200 & 0.14206300 & $\mathrm{C}$ & -2.05249100 & -3.97799500 & 1.81150100 \\
\hline $\mathrm{C}$ & -1.69613700 & 0.53193400 & -1.98504800 & $\mathrm{H}$ & -3.41846400 & -5.36814000 & 0.87360900 \\
\hline $\mathrm{H}$ & -2.23727200 & 0.39950300 & -2.92937300 & $\mathrm{H}$ & -0.85093600 & -2.32635100 & 2.50350700 \\
\hline $\mathrm{H}$ & -1.06987100 & -0.36070300 & -1.86315600 & $\mathrm{H}$ & -1.59367000 & -4.70235900 & 2.47666400 \\
\hline $\mathrm{C}$ & -0.78711900 & 1.76749300 & -2.15923400 & $\mathrm{~N}$ & -2.16595300 & -1.69184700 & 1.04337800 \\
\hline $\mathrm{H}$ & -0.21135300 & 1.65839400 & -3.08472100 & $\mathrm{C}$ & -5.35977200 & -0.08379900 & 0.24600300 \\
\hline $\mathrm{H}$ & -1.39118600 & 2.67441200 & -2.27298600 & $\mathrm{C}$ & -6.09646700 & 1.01054100 & -0.55441300 \\
\hline$P$ & 0.48215400 & 2.02268700 & -0.82777600 & $\mathrm{H}$ & -6.95050900 & 1.37793200 & 0.02693600 \\
\hline $\mathrm{C}$ & 1.73157800 & 3.30020600 & -1.48971500 & $\mathrm{H}$ & -5.45821500 & 1.87445300 & -0.76935500 \\
\hline $\mathrm{C}$ & -0.37019900 & 2.87045800 & 0.58524600 & $\mathrm{H}$ & -6.47974600 & 0.62718800 & -1.50533300 \\
\hline $\mathrm{C}$ & -1.41450100 & 3.78821100 & 0.41131500 & $\mathrm{C}$ & -4.84966400 & 0.48836900 & 1.57854200 \\
\hline $\mathrm{C}$ & -0.40832200 & 3.15628200 & 2.87110300 & $\mathrm{H}$ & -4.20502000 & 1.36068300 & 1.43165900 \\
\hline $\mathrm{C}$ & -1.96154200 & 4.40084700 & 1.53997100 & $\mathrm{H}$ & -5.70016400 & 0.81235600 & 2.19168100 \\
\hline $\mathrm{H}$ & -1.79667400 & 4.02437400 & -0.57483700 & $\mathrm{H}$ & -4.28634400 & -0.25241300 & 2.15266500 \\
\hline $\mathrm{C}$ & -1.45034100 & 4.08196900 & 2.79619500 & $\mathrm{C}$ & -6.34495100 & -1.23732400 & 0.51781600 \\
\hline $\mathrm{H}$ & 0.01709700 & 2.87269000 & 3.83085200 & $\mathrm{H}$ & -7.21321400 & -0.85564700 & 1.06776900 \\
\hline $\mathrm{H}$ & -2.77317200 & 5.11435200 & 1.43561500 & $\mathrm{H}$ & -6.71326100 & -1.68433800 & -0.41183300 \\
\hline $\mathrm{H}$ & -1.84830800 & 4.53320600 & 3.69878900 & $\mathrm{H}$ & -5.89499100 & -2.02838400 & 1.12472500 \\
\hline $\mathrm{N}$ & 0.12002200 & 2.56270400 & 1.79458300 & $\mathrm{C}$ & 2.75982400 & -1.94548700 & -0.14242400 \\
\hline $\mathrm{Pd}$ & 1.51233700 & -0.02599900 & -0.16974200 & $\mathrm{H}$ & 2.07276500 & -2.65787300 & 0.30755100 \\
\hline $\mathrm{H}$ & 1.87353300 & -0.19011700 & -1.71945100 & $\mathrm{C}$ & 2.55247600 & -1.66347700 & -1.51245500 \\
\hline $\mathrm{C}$ & 1.02625800 & 4.60879700 & -1.89761800 & $\mathrm{H}$ & 3.39083700 & -1.32606800 & -2.11527900 \\
\hline $\mathrm{H}$ & 0.52172600 & 5.08648900 & -1.05388800 & $\mathrm{C}$ & 4.00329100 & -1.68157100 & 0.59619200 \\
\hline $\mathrm{H}$ & 1.77684000 & 5.31418200 & -2.27065700 & $\mathrm{C}$ & 5.02576700 & -0.84589400 & 0.10462100 \\
\hline $\mathrm{H}$ & 0.29821600 & 4.45562400 & -2.70012300 & $\mathrm{C}$ & 4.18118400 & -2.30118800 & 1.84803900 \\
\hline $\mathrm{C}$ & 2.45818000 & 2.70434900 & -2.71075800 & $\mathrm{C}$ & 6.18531000 & -0.63927300 & 0.84574500 \\
\hline $\mathrm{H}$ & 2.97512500 & 1.77246500 & -2.46339000 & $\mathrm{H}$ & 4.92046000 & -0.35702000 & -0.85938900 \\
\hline $\mathrm{H}$ & 1.78550300 & 2.51285000 & -3.55199800 & $\mathrm{C}$ & 5.34402100 & -2.09600700 & 2.58494500 \\
\hline $\mathrm{H}$ & 3.21275600 & 3.41861700 & -3.05757700 & $\mathrm{H}$ & 3.40367500 & -2.95450600 & 2.23576900 \\
\hline $\mathrm{C}$ & 2.75298400 & 3.57743100 & -0.36845600 & $\mathrm{C}$ & 6.34833800 & -1.26207400 & 2.08714900 \\
\hline $\mathrm{H}$ & 3.29420700 & 2.67001100 & -0.08076000 & $\mathrm{H}$ & 6.96701400 & 0.00386800 & 0.45382900 \\
\hline $\mathrm{H}$ & 3.48809700 & 4.30670400 & -0.72613500 & $\mathrm{H}$ & 5.46918600 & -2.58793100 & 3.54433600 \\
\hline $\mathrm{H}$ & 2.28297000 & 3.98942000 & 0.52837600 & $\mathrm{H}$ & 7.25591000 & -1.10123800 & 2.66041800 \\
\hline $\mathrm{C}$ & 1.13389600 & -0.06193200 & 1.81746800 & $\mathrm{C}$ & 1.55554300 & -2.43637800 & -2.34206100 \\
\hline $\mathrm{O}$ & 0.97612000 & -0.20897800 & 2.93494000 & $\mathrm{O}$ & 1.66889400 & -2.54484700 & -3.54144500 \\
\hline$P$ & -3.97044000 & -0.75804500 & -0.89602700 & $\mathrm{O}$ & 0.57261600 & -2.97081700 & -1.60329400 \\
\hline $\mathrm{C}$ & -3.15717300 & -2.03553800 & 0.19906000 & $\mathrm{C}$ & -0.39188600 & -3.76808800 & -2.33205500 \\
\hline $\mathrm{C}$ & -3.61622700 & -3.36105100 & 0.10187200 & $\mathrm{H}$ & 0.09999900 & -4.63855300 & -2.77072400 \\
\hline
\end{tabular}




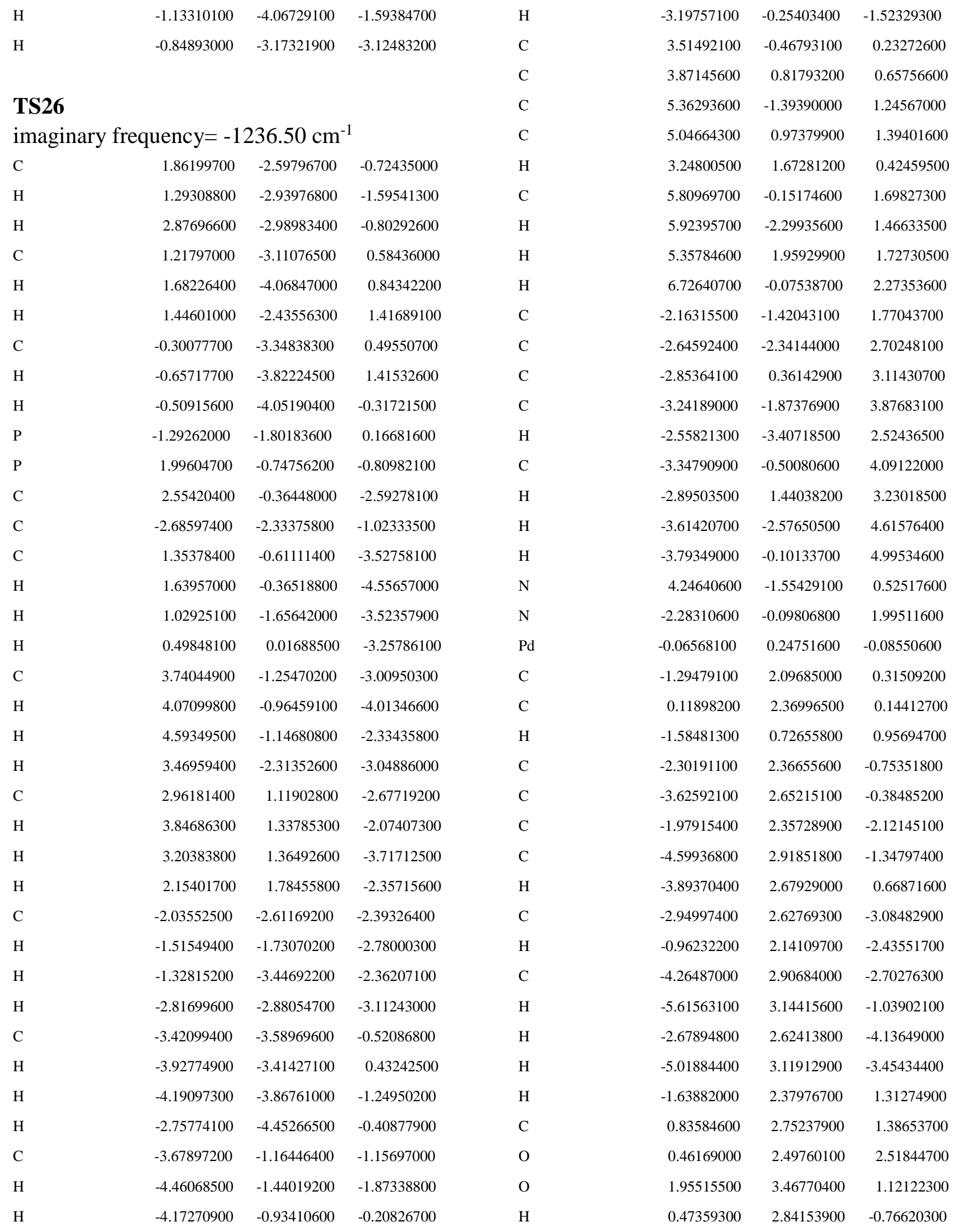




$\begin{array}{llll}\mathrm{C} & 2.66108900 & 3.95779700 & 2.27869500 \\ \mathrm{H} & 3.49267100 & 4.54301900 & 1.88716100 \\ \mathrm{H} & 2.00515600 & 4.58473000 & 2.88611500 \\ \mathrm{H} & 3.02221100 & 3.12718700 & 2.88999400\end{array}$

\section{TS26a} imaginary frequency $=-329.19 \mathrm{~cm}^{-1}$
C

H

H

C

H

H

C

H

H

P

P

C

C

C

H

H

H

C

H

H

H

C

H

H

H

C

H

H

H

C

H

H

H

C$$
3.16042200-1.47004500
$$

1.22771800

$3.37650300 \quad-2.53243100$

1.38785700

$3.51528200 \quad-0.90928400$

2.09524600

$1.64218200-1.25248200$

1.08623500

$1.16401700 \quad-1.42434100$

$1.45053700 \quad-0.20180300$

0.83768000

$1.00002700 \quad-2.17056000$

0.02943200

$1.04306300-3.21781500$

0.34875900

$1.56708200 \quad-2.10043600$

$-0.90686500$

$-0.73339500 \quad-1.68763500$

$-0.40885100$

$4.10129600 \quad-0.90662900$

$-0.29414200$

5.88558100

$-1.50497400$

0.06506800

$-1.37010400 \quad-2.95798400$

$-1.67735600$

$$
5.89462800
$$

6.91488900

$-3.03283700$

$-0.14765300$

$-0.02312100$

5.26407900

$-3.41458700$

0.57810200

5.55766500

$-3.55740100$

$-1.15363100$

$6.37862300 \quad-1.16725100$

1.48218500

7.42194800

$-1.48752200$

1.59567600

$6.33426000-0.09408600$

1.68774500

5.79630200

$-1.68105900$

2.25295200

6.81170400

$-0.85355200$

$-0.98033500$

6.87482200

0.23095700

$-0.85195900$

7.82581100

$-1.25660500$

$-0.87432900$

6.47786800

$-0.75685100$

$-1.05970200$

$-2.00313100$

$-1.06752900$

$-2.54309200 \quad-3.03306000$

0.33736000

$-1.09769200$

$-0.96513800$

$-1.38679900$

$-1.35616900$

0.11926100

$-1.53706600$

$-3.32979100$

$-2.57724500 \quad-3.02407300$

$-3.24039200 \quad-3.80609200$

$-4.40538700 \quad-1.34846000$

$-4.75047000 \quad-0.39987600$

$-5.06776700 \quad-2.12882700$

$-2.90485200$

$-4.54182600 \quad-1.32317100$

$-2.84603400 \quad-1.74267500$
2.05895100
$\mathrm{H}$

$\mathrm{H}$

$\mathrm{H}$

C

C

C

$\mathrm{H}$

C

$\mathrm{H}$

$\mathrm{H}$

C

C

H

$\mathrm{H}$

H

$\mathrm{H}$

$\mathrm{N}$

$\mathrm{N}$

Pd

C

H

C

C

C

C

$\mathrm{H}$

C

H

C

H

H

H

H

C

O

\begin{tabular}{|c|c|c|}
\hline-3.27628000 & -3.46732200 & -2.56480600 \\
\hline-3.38108800 & -3.20197600 & -0.82347800 \\
\hline-3.23883400 & -1.82046500 & -1.92362000 \\
\hline 4.19032900 & 0.92341200 & 0.06873400 \\
\hline 4.49963400 & 1.80741400 & -0.97858000 \\
\hline 3.97899200 & 2.67908900 & 1.54926500 \\
\hline 4.57255400 & 3.17452900 & -0.71693600 \\
\hline 4.67829200 & 1.42381200 & -1.97829400 \\
\hline 4.30841900 & 3.62486000 & 0.57686900 \\
\hline 3.75480800 & 2.99008900 & 2.56803500 \\
\hline 4.82518100 & 3.87452700 & -1.50816000 \\
\hline 4.35634400 & 4.67919900 & 0.83015600 \\
\hline-1.67872800 & -2.03741100 & 1.16365900 \\
\hline-1.55190300 & -3.17723100 & 1.96075300 \\
\hline-3.28647500 & -1.16917600 & 2.65656200 \\
\hline-2.31678100 & -3.30416700 & 3.12172900 \\
\hline-0.85166300 & -3.95510900 & 1.68200900 \\
\hline-3.20556700 & -2.28822600 & 3.47374900 \\
\hline-3.94940400 & -0.33879200 & 2.86686400 \\
\hline-2.21695600 & -4.18699300 & 3.74540600 \\
\hline-3.81704200 & -2.35119900 & 4.36631100 \\
\hline 3.92238400 & 1.36240600 & 1.31146300 \\
\hline-2.53588800 & -1.06546300 & 1.54662400 \\
\hline-1.11062000 & 0.66542500 & -0.75963200 \\
\hline-2.85751900 & 1.33850200 & 0.48744200 \\
\hline-2.14905000 & 2.40710000 & -0.17520000 \\
\hline-2.53701500 & -0.04437200 & 0.91812600 \\
\hline-4.26637900 & 1.04449200 & 0.04920000 \\
\hline-5.32185300 & 1.17358100 & 0.96551800 \\
\hline-4.56865200 & 0.68110600 & -1.27177100 \\
\hline-6.64139900 & 0.93924300 & 0.57450000 \\
\hline-5.11590200 & 1.50286000 & 1.98211400 \\
\hline-5.88722200 & 0.45288500 & -1.66547500 \\
\hline-3.76304600 & 0.58868900 & -1.99659900 \\
\hline-6.92777000 & 0.57402600 & -0.74159100 \\
\hline-7.44506200 & 1.05510800 & 1.29553100 \\
\hline-6.10230400 & 0.17952700 & -2.69417800 \\
\hline-7.95335300 & 0.39264500 & -1.04730600 \\
\hline-2.71156500 & 1.44402300 & 1.56838800 \\
\hline-1.19390400 & 3.21111500 & 0.64249700 \\
\hline-0.83568900 & 2.91352000 & 1.76909600 \\
\hline
\end{tabular}




\begin{tabular}{|c|c|c|c|c|c|c|c|}
\hline $\mathrm{O}$ & -0.77870600 & 4.30355800 & -0.02063300 & $\mathrm{C}$ & -3.47397500 & -0.93929800 & -2.58286700 \\
\hline $\mathrm{H}$ & -2.58379900 & 2.90509800 & -1.03708400 & $\mathrm{H}$ & -2.93103600 & -0.00825700 & -2.77182300 \\
\hline $\mathrm{C}$ & 0.16238800 & 5.14058900 & 0.68080900 & $\mathrm{H}$ & -3.92583800 & -1.25379900 & -3.53023300 \\
\hline H & 0.31101600 & 6.00980000 & 0.04166300 & $\mathrm{H}$ & -4.28340700 & -0.73341800 & -1.87806500 \\
\hline $\mathrm{H}$ & -0.24033100 & 5.43764400 & 1.65125800 & $\mathrm{C}$ & -2.87813200 & 1.63725500 & -0.48678100 \\
\hline $\mathrm{H}$ & 1.10498400 & 4.60737300 & 0.82982400 & $\mathrm{O}$ & -3.82765300 & 2.22880400 & -0.70850800 \\
\hline $\mathrm{C}$ & 0.25902600 & 1.27231100 & -2.04523700 & $\mathrm{C}$ & 0.90614200 & 1.83273900 & 0.63561600 \\
\hline \multirow[t]{2}{*}{$\mathrm{O}$} & 1.05424600 & 1.61333100 & -2.79027200 & $\mathrm{P}$ & 3.33473700 & -2.13701400 & -0.51862200 \\
\hline & & & & $\mathrm{C}$ & 2.99224800 & -0.68190200 & -1.63918800 \\
\hline \multirow{2}{*}{\multicolumn{4}{|c|}{$\begin{array}{l}\text { TS26b } \\
\text { imaginary frequency }=-1464.37 \mathrm{~cm}^{-1}\end{array}$}} & $\mathrm{C}$ & 3.73499700 & -0.68634900 & -2.83211100 \\
\hline & & & & $\mathrm{C}$ & 3.53534700 & 0.28462800 & -3.80860200 \\
\hline $\mathrm{C}$ & 1.75151500 & -2.19180100 & 0.46550000 & $\mathrm{H}$ & 4.45804600 & -1.47975600 & -2.98817400 \\
\hline $\mathrm{H}$ & 1.94095200 & -2.74509900 & 1.39159600 & $\mathrm{C}$ & 1.84119800 & 1.20777900 & -2.40191500 \\
\hline $\mathrm{H}$ & 1.39954500 & -1.19908800 & 0.75528600 & $\mathrm{C}$ & 2.55470100 & 1.24904400 & -3.59322500 \\
\hline $\mathrm{C}$ & 0.71266600 & -2.94089200 & -0.38817000 & $\mathrm{H}$ & 4.12169000 & 0.27601600 & -4.72230700 \\
\hline $\mathrm{H}$ & 1.03111200 & -3.98460000 & -0.48200500 & $\mathrm{H}$ & 1.10139800 & 1.97080300 & -2.18073300 \\
\hline $\mathrm{H}$ & 0.72241500 & -2.54324100 & -1.40688300 & $\mathrm{H}$ & 2.34174700 & 2.02527400 & -4.31954500 \\
\hline C & -0.72139000 & -2.91595700 & 0.17923100 & $\mathrm{~N}$ & 2.05113900 & 0.28226600 & -1.44189600 \\
\hline $\mathrm{H}$ & -1.23510000 & -3.85242700 & -0.06064900 & $\mathrm{C}$ & 4.70834300 & -1.53914300 & 0.66425500 \\
\hline $\mathrm{H}$ & -0.68648000 & -2.85033500 & 1.27220300 & $\mathrm{C}$ & 4.94707600 & -2.70375100 & 1.64946200 \\
\hline$P$ & -1.80049200 & -1.50787100 & -0.39846000 & $\mathrm{H}$ & 5.80313800 & -2.46512000 & 2.29095800 \\
\hline $\mathrm{C}$ & -2.53127600 & -2.05282600 & -2.08638000 & $\mathrm{H}$ & 4.08845600 & -2.87382300 & 2.30714000 \\
\hline C & -3.24594400 & -1.62803900 & 0.76775700 & $\mathrm{H}$ & 5.17246800 & -3.64001000 & 1.12880800 \\
\hline $\mathrm{C}$ & -3.51220400 & -2.74373200 & 1.57326700 & $\mathrm{C}$ & 4.37430700 & -0.25861700 & 1.43504800 \\
\hline $\mathrm{C}$ & -5.11281700 & -0.53084200 & 1.57260500 & $\mathrm{H}$ & 3.49319400 & -0.37810100 & 2.06906600 \\
\hline $\mathrm{C}$ & -4.62955800 & -2.71885600 & 2.41038900 & $\mathrm{H}$ & 5.21296000 & 0.01303300 & 2.08829900 \\
\hline $\mathrm{H}$ & -2.87491500 & -3.61966000 & 1.55021400 & $\mathrm{H}$ & 4.19908100 & 0.58583100 & 0.76112000 \\
\hline $\mathrm{C}$ & -5.44903500 & -1.59297500 & 2.41363000 & $\mathrm{C}$ & 5.98100500 & -1.33199400 & -0.18100700 \\
\hline $\mathrm{H}$ & -5.72419000 & 0.36808600 & 1.53805800 & $\mathrm{H}$ & 6.82273500 & -1.09800100 & 0.48095100 \\
\hline $\mathrm{H}$ & -4.85453100 & -3.57027100 & 3.04556900 & $\mathrm{H}$ & 6.24559900 & -2.23042100 & -0.74820000 \\
\hline $\mathrm{H}$ & -6.32605500 & -1.53301600 & 3.04918800 & $\mathrm{H}$ & 5.87739900 & -0.49857000 & -0.88295600 \\
\hline $\mathrm{N}$ & -4.03828400 & -0.54216700 & 0.77642600 & $\mathrm{H}$ & 1.16579000 & 0.72908400 & -0.31526800 \\
\hline $\mathrm{Pd}$ & -1.08284400 & 0.88293800 & -0.18305900 & $\mathrm{C}$ & -0.28558200 & 2.67285000 & 0.50508200 \\
\hline $\mathrm{C}$ & -3.31785600 & -3.37108000 & -1.96385800 & $\mathrm{H}$ & 1.73883200 & 2.28135300 & 0.08621300 \\
\hline $\mathrm{H}$ & -4.17192700 & -3.28033300 & -1.28773000 & $\mathrm{C}$ & 1.26696400 & 1.46272800 & 2.05166000 \\
\hline $\mathrm{H}$ & -3.70609500 & -3.64591900 & -2.95106000 & $\mathrm{C}$ & 2.31725000 & 2.14409700 & 2.68632700 \\
\hline $\mathrm{H}$ & -2.69461300 & -4.20384700 & -1.62405800 & $\mathrm{C}$ & 0.53145000 & 0.52958300 & 2.79369200 \\
\hline $\mathrm{C}$ & -1.38251000 & -2.22454700 & -3.10046800 & $\mathrm{C}$ & 2.62233600 & 1.89640800 & 4.02457800 \\
\hline $\mathrm{H}$ & -0.74065000 & -1.33865000 & -3.15273900 & $\mathrm{H}$ & 2.89324400 & 2.88026800 & 2.13161800 \\
\hline $\mathrm{H}$ & -0.75964500 & -3.09503200 & -2.87925700 & $\mathrm{C}$ & 0.83589700 & 0.27797700 & 4.13315300 \\
\hline $\mathrm{H}$ & -1.81068600 & -2.37909300 & -4.09724600 & $\mathrm{H}$ & -0.28989100 & -0.00258000 & 2.31733700 \\
\hline
\end{tabular}




\begin{tabular}{|c|c|c|c|c|c|c|c|}
\hline $\mathrm{C}$ & 1.88404800 & 0.96028300 & 4.75218900 & $\mathrm{H}$ & -1.72132600 & -2.52184500 & -2.28696400 \\
\hline $\mathrm{H}$ & 3.43523000 & 2.43740100 & 4.49949400 & $\mathrm{H}$ & -2.01173000 & -3.93241400 & -1.24550200 \\
\hline $\mathrm{H}$ & 0.25348200 & -0.44873600 & 4.69189300 & $\mathrm{H}$ & -3.22416400 & -3.44098100 & -2.42387400 \\
\hline $\mathrm{H}$ & 2.12235500 & 0.76675400 & 5.79345200 & $\mathrm{C}$ & -4.25788700 & -2.93732700 & 0.07365300 \\
\hline $\mathrm{H}$ & -0.83709600 & 2.90765300 & 1.41326700 & $\mathrm{H}$ & -4.81582500 & -2.30516400 & 0.77018500 \\
\hline $\mathrm{C}$ & -0.32819000 & 3.76363600 & -0.51537400 & $\mathrm{H}$ & -4.98055800 & -3.34553100 & -0.64204300 \\
\hline $\mathrm{O}$ & 0.40730100 & 3.85493500 & -1.48419500 & $\mathrm{H}$ & -3.84203100 & -3.78440400 & 0.62802000 \\
\hline $\mathrm{O}$ & -1.29638200 & 4.64663900 & -0.22710800 & $\mathrm{C}$ & -3.83347700 & -0.96291900 & -1.43680200 \\
\hline $\mathrm{C}$ & -1.44303200 & 5.74812500 & -1.14907400 & $\mathrm{H}$ & -4.57822500 & -1.34987500 & -2.14137700 \\
\hline $\mathrm{H}$ & -2.25227900 & 6.35576800 & -0.74780000 & $\mathrm{H}$ & -4.35525000 & -0.28451800 & -0.75397600 \\
\hline $\mathrm{H}$ & -0.51643600 & 6.32317100 & -1.20223100 & $\mathrm{H}$ & -3.10208300 & -0.38653100 & -2.01004000 \\
\hline \multirow[t]{2}{*}{$\mathrm{H}$} & -1.69489000 & 5.37794200 & -2.14557500 & $\mathrm{C}$ & 3.08998800 & -1.08663400 & 0.71186300 \\
\hline & & & & $\mathrm{C}$ & 3.65620400 & 0.18998600 & 0.80678100 \\
\hline \multicolumn{4}{|c|}{ TS27 } & $\mathrm{C}$ & 4.61681800 & -1.95153200 & 2.20211400 \\
\hline \multicolumn{4}{|c|}{ imaginary frequency $=-1356.92 \mathrm{~cm}^{-1}$} & $\mathrm{C}$ & 4.76218600 & 0.37041100 & 1.63877500 \\
\hline $\mathrm{C}$ & 1.07657800 & -3.06055400 & 0.26332100 & $\mathrm{H}$ & 3.24465800 & 1.02147400 & 0.25002300 \\
\hline $\mathrm{H}$ & 0.46894600 & -3.55601200 & -0.50118400 & $\mathrm{C}$ & 5.25541600 & -0.71919300 & 2.35277000 \\
\hline $\mathrm{H}$ & 1.97714700 & -3.65590100 & 0.41971200 & $\mathrm{H}$ & 4.96614100 & -2.82711800 & 2.74516500 \\
\hline $\mathrm{C}$ & 0.30765700 & -2.95368500 & 1.60250900 & $\mathrm{H}$ & 5.21999000 & 1.35072800 & 1.72727000 \\
\hline $\mathrm{H}$ & 0.54872700 & -3.83457600 & 2.20742200 & $\mathrm{H}$ & 6.11036000 & -0.62310800 & 3.01398400 \\
\hline $\mathrm{H}$ & 0.66485100 & -2.09336200 & 2.17925300 & $\mathrm{C}$ & -2.77723800 & -0.35291000 & 1.64089300 \\
\hline $\mathrm{C}$ & -1.22775000 & -2.89719100 & 1.47287500 & $\mathrm{C}$ & -3.56241600 & -0.76322700 & 2.72110800 \\
\hline $\mathrm{H}$ & -1.68160400 & -2.87098000 & 2.46854000 & $\mathrm{C}$ & -3.31918200 & 1.88707100 & 2.06063400 \\
\hline $\mathrm{H}$ & -1.58697600 & -3.81192900 & 0.99052300 & $\mathrm{C}$ & -4.22868200 & 0.19597200 & 3.48793900 \\
\hline $\mathrm{P}$ & -1.83056400 & -1.44830500 & 0.46345500 & $\mathrm{H}$ & -3.65805800 & -1.81566100 & 2.96252100 \\
\hline $\mathrm{P}$ & 1.63764400 & -1.41817400 & -0.40896300 & $\mathrm{C}$ & -4.11436900 & 1.54418400 & 3.15101100 \\
\hline $\mathrm{C}$ & 2.40957200 & -1.79867100 & -2.10904500 & $\mathrm{H}$ & -3.19146500 & 2.91662800 & 1.73984700 \\
\hline $\mathrm{C}$ & -3.18458600 & -2.14508600 & -0.69273800 & $\mathrm{H}$ & -4.83559700 & -0.10951900 & 4.33465800 \\
\hline $\mathrm{C}$ & 1.26332600 & -2.10800700 & -3.09179600 & $\mathrm{H}$ & -4.62288800 & 2.31527300 & 3.71877900 \\
\hline $\mathrm{H}$ & 1.68074000 & -2.28838800 & -4.08886000 & $\mathrm{~N}$ & 3.56092500 & -2.13823400 & 1.40172300 \\
\hline $\mathrm{H}$ & 0.70520300 & -3.00631800 & -2.80903800 & $\mathrm{~N}$ & -2.66573800 & 0.95957400 & 1.34915100 \\
\hline $\mathrm{H}$ & 0.56189200 & -1.27088700 & -3.16909400 & $\mathrm{Pd}$ & -0.18729900 & 0.11020000 & -0.34932700 \\
\hline $\mathrm{C}$ & 3.37915200 & -2.99325400 & -2.03028100 & $\mathrm{C}$ & -0.99758400 & 2.20581500 & -0.58183300 \\
\hline $\mathrm{H}$ & 3.84356600 & -3.14201600 & -3.01191500 & $\mathrm{C}$ & 0.40838200 & 2.05454300 & -0.97953700 \\
\hline $\mathrm{H}$ & 4.18004700 & -2.82946400 & -1.30414100 & $\mathrm{H}$ & -1.71089200 & 1.29532900 & 0.32732400 \\
\hline $\mathrm{H}$ & 2.86765800 & -3.92375300 & -1.76814300 & $\mathrm{H}$ & 0.55779500 & 1.94798000 & -2.05322600 \\
\hline $\mathrm{C}$ & 3.16286200 & -0.54514300 & -2.59237000 & $\mathrm{H}$ & -1.15452200 & 2.96797900 & 0.18365200 \\
\hline $\mathrm{H}$ & 4.00838100 & -0.29774300 & -1.94476400 & $\mathrm{C}$ & 1.48146100 & 2.79542800 & -0.28626800 \\
\hline $\mathrm{H}$ & 3.55860400 & -0.73024200 & -3.59718700 & $\mathrm{C}$ & 2.58822500 & 3.25398800 & -1.02542000 \\
\hline $\mathrm{H}$ & 2.50396200 & 0.32621300 & -2.65036000 & $\mathrm{C}$ & 1.43772000 & 3.08979000 & 1.09095500 \\
\hline $\mathrm{C}$ & -2.48082400 & -3.06122000 & -1.71430300 & $\mathrm{C}$ & 3.61122800 & 3.97932800 & -0.41423100 \\
\hline
\end{tabular}




\begin{tabular}{|c|c|c|c|c|c|c|c|}
\hline $\mathrm{H}$ & 2.63625800 & 3.05005600 & -2.09188500 & $\mathrm{H}$ & -6.62286800 & 0.84593000 & 2.23938600 \\
\hline C & 2.45729500 & 3.81607300 & 1.70056300 & $\mathrm{C}$ & 0.33499400 & -1.20401700 & 3.69833400 \\
\hline $\mathrm{H}$ & 0.60402000 & 2.73539200 & 1.69139600 & $\mathrm{H}$ & 0.70832000 & -0.19025700 & 3.52671100 \\
\hline $\mathrm{C}$ & 3.55022100 & 4.26451100 & 0.95150400 & $\mathrm{H}$ & -0.75876800 & -1.17376700 & 3.70649700 \\
\hline $\mathrm{H}$ & 4.44998300 & 4.33099600 & -1.00764600 & $\mathrm{H}$ & 0.65941400 & -1.51105000 & 4.69851000 \\
\hline $\mathrm{H}$ & 2.40222500 & 4.03311500 & 2.76335200 & C & 0.34828600 & -3.61341500 & 2.96093000 \\
\hline $\mathrm{H}$ & 4.34084700 & 4.83676200 & 1.42716000 & $\mathrm{H}$ & 0.76491700 & -4.36844500 & 2.28856800 \\
\hline C & -1.98942100 & 2.30096400 & -1.70193500 & $\mathrm{H}$ & 0.63715500 & -3.89726300 & 3.97918700 \\
\hline $\mathrm{O}$ & -1.87214300 & 1.78596300 & -2.79629500 & $\mathrm{H}$ & -0.74347000 & -3.66018700 & 2.91155300 \\
\hline $\mathrm{O}$ & -3.06758700 & 3.02286400 & -1.32422700 & $\mathrm{C}$ & 2.42998000 & -2.20887300 & 2.72301500 \\
\hline $\mathrm{C}$ & -4.08356300 & 3.19965700 & -2.33426900 & $\mathrm{H}$ & 2.75269500 & -2.52548800 & 3.72088200 \\
\hline $\mathrm{H}$ & -4.84958900 & 3.82173700 & -1.87301500 & $\mathrm{H}$ & 2.86871100 & -2.90437400 & 2.00013100 \\
\hline $\mathrm{H}$ & -3.66350900 & 3.69497500 & -3.21181000 & $\mathrm{H}$ & 2.84472800 & -1.21196900 & 2.54080300 \\
\hline \multirow[t]{2}{*}{$\mathrm{H}$} & -4.49849700 & 2.23460100 & -2.63365400 & $\mathrm{C}$ & -4.06931400 & 1.58304300 & -0.31600700 \\
\hline & & & & C & -4.22790400 & 2.80907800 & 0.35147200 \\
\hline \multirow{2}{*}{\multicolumn{4}{|c|}{$\begin{array}{l}\text { TS27a } \\
\text { imaginary frequency }=-1268.71 \mathrm{~cm}^{-1}\end{array}$}} & $\mathrm{C}$ & -3.50104400 & 2.66906100 & -2.26933700 \\
\hline & & & & $\mathrm{C}$ & -4.03466500 & 3.99721300 & -0.35114400 \\
\hline C & -3.55200400 & -1.21190800 & -0.57800700 & $\mathrm{H}$ & -4.49417000 & 2.82433800 & 1.40387600 \\
\hline $\mathrm{H}$ & -3.94530000 & -2.20830700 & -0.34534100 & $\mathrm{C}$ & -3.66200200 & 3.92963000 & -1.69342400 \\
\hline $\mathrm{H}$ & -3.86400800 & -0.94447100 & -1.58985600 & $\mathrm{H}$ & -3.19896100 & 2.57373000 & -3.31068500 \\
\hline C & -2.01304700 & -1.22688800 & -0.53131000 & $\mathrm{H}$ & -4.16395300 & 4.95645800 & 0.14185300 \\
\hline $\mathrm{H}$ & -1.64037700 & -1.85442800 & -1.35104000 & $\mathrm{H}$ & -3.49388500 & 4.82673000 & -2.28041000 \\
\hline $\mathrm{H}$ & -1.63845400 & -0.21601500 & -0.72871600 & $\mathrm{C}$ & 1.06301700 & -2.76841900 & -0.29190800 \\
\hline C & -1.45218700 & -1.74460900 & 0.80575500 & $\mathrm{C}$ & 0.61551100 & -4.08303200 & -0.45699400 \\
\hline $\mathrm{H}$ & -1.73697200 & -2.79093600 & 0.96747100 & $\mathrm{C}$ & 2.63934200 & -3.05859000 & -1.99434400 \\
\hline $\mathrm{H}$ & -1.88352400 & -1.16923000 & 1.63256300 & $\mathrm{C}$ & 1.22069700 & -4.89748200 & -1.41608800 \\
\hline$P$ & 0.38323200 & -1.55154000 & 0.94756600 & $\mathrm{H}$ & -0.19714700 & -4.46608300 & 0.14865600 \\
\hline$P$ & -4.30833100 & -0.00231500 & 0.64005800 & $\mathrm{C}$ & 2.25751900 & -4.38271400 & -2.19274900 \\
\hline $\mathrm{C}$ & -6.18518100 & -0.35046200 & 0.44920400 & $\mathrm{H}$ & 3.41038300 & -2.58523500 & -2.59305200 \\
\hline $\mathrm{C}$ & 0.89027900 & -2.20454400 & 2.66182100 & $\mathrm{H}$ & 0.88306600 & -5.91989000 & -1.55480100 \\
\hline $\mathrm{C}$ & -6.46113500 & -1.69602800 & 1.15081200 & $\mathrm{H}$ & 2.75223500 & -4.98420200 & -2.94721800 \\
\hline $\mathrm{H}$ & -7.53646800 & -1.90922800 & 1.12836600 & $\mathrm{~N}$ & -3.70116100 & 1.52133000 & -1.60841300 \\
\hline $\mathrm{H}$ & -5.95650000 & -2.53352100 & 0.65758500 & $\mathrm{~N}$ & 2.04941100 & -2.28477300 & -1.07103100 \\
\hline $\mathrm{H}$ & -6.14720200 & -1.67715800 & 2.19966300 & $\mathrm{Pd}$ & 1.44037600 & 0.54263500 & 0.30399900 \\
\hline C & -6.65530900 & -0.40965700 & -1.01345800 & $\mathrm{C}$ & 2.97647400 & 0.39480600 & -1.40074900 \\
\hline $\mathrm{H}$ & -7.74257100 & -0.55378600 & -1.04689400 & $\mathrm{C}$ & 2.96814900 & 1.66438300 & -0.68556300 \\
\hline $\mathrm{H}$ & -6.42403700 & 0.51247000 & -1.55388700 & $\mathrm{H}$ & 2.32451500 & -0.81717500 & -0.93141300 \\
\hline $\mathrm{H}$ & -6.20108500 & -1.24233900 & -1.55895500 & $\mathrm{H}$ & 2.46105600 & 2.48013600 & -1.19565900 \\
\hline C & -6.94501200 & 0.76306800 & 1.19565700 & $\mathrm{C}$ & 4.00409800 & 2.05990500 & 0.28064700 \\
\hline $\mathrm{H}$ & -6.81536500 & 1.73838800 & 0.71737600 & $\mathrm{C}$ & 4.14315500 & 3.42381200 & 0.60439300 \\
\hline $\mathrm{H}$ & -8.01823000 & 0.53833900 & 1.19600300 & $\mathrm{C}$ & 4.86286000 & 1.13118000 & 0.90285800 \\
\hline
\end{tabular}




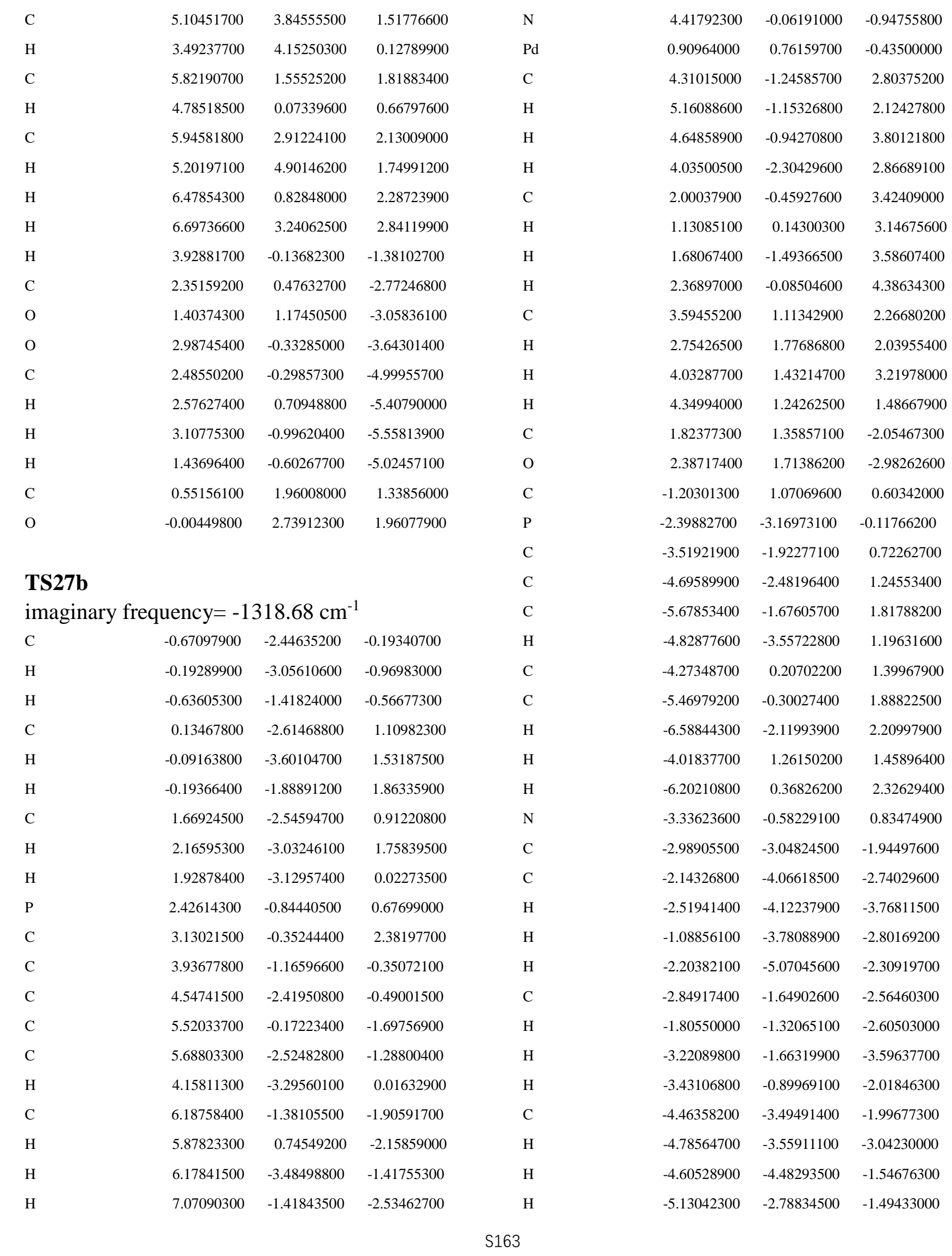




\begin{tabular}{|c|c|c|c|c|c|c|c|}
\hline $\mathrm{H}$ & -2.26233800 & 0.14193800 & 0.63072400 & $\mathrm{C}$ & -0.31359100 & 4.67368100 & 1.08341700 \\
\hline C & -1.10812400 & 1.56924400 & -0.76115000 & $\mathrm{H}$ & -0.93180400 & 5.57621900 & 1.14624100 \\
\hline $\mathrm{H}$ & -0.49701900 & 0.21671300 & 0.85291600 & $\mathrm{H}$ & -0.11637900 & 4.33881600 & 2.10400600 \\
\hline C & -1.33346900 & 2.02690500 & 1.74904800 & $\mathrm{H}$ & 0.63822100 & 4.96404500 & 0.63301400 \\
\hline $\mathrm{O}$ & -2.34616000 & 2.66091300 & 1.97917200 & $\mathrm{C}$ & -2.35752200 & 3.23666700 & 1.06047400 \\
\hline $\mathrm{O}$ & -0.24397700 & 2.07172900 & 2.54516000 & $\mathrm{H}$ & -2.11046300 & 2.70722200 & 1.98680800 \\
\hline $\mathrm{H}$ & -1.42301600 & 0.86057800 & -1.52878900 & $\mathrm{H}$ & -2.88354800 & 4.15370500 & 1.34873700 \\
\hline C & -1.27296300 & 2.97277200 & -1.18283000 & $\mathrm{H}$ & -3.04491700 & 2.64117600 & 0.46162900 \\
\hline C & -1.97497700 & 3.23299400 & -2.37435700 & $\mathrm{C}$ & 0.92090200 & -2.07133000 & -3.48417400 \\
\hline C & -0.73548100 & 4.06175600 & -0.46979400 & $\mathrm{H}$ & -0.05478800 & -2.22568600 & -3.01483400 \\
\hline C & -2.15817700 & 4.53772600 & -2.82695000 & $\mathrm{H}$ & 0.89321800 & -1.11155500 & -4.01055000 \\
\hline $\mathrm{H}$ & -2.38713800 & 2.40308900 & -2.94294300 & $\mathrm{H}$ & 1.05253300 & -2.85363800 & -4.23997500 \\
\hline C & -0.91140400 & 5.36330600 & -0.92736800 & $\mathrm{C}$ & 3.42693900 & -2.02316000 & -3.21224700 \\
\hline $\mathrm{H}$ & -0.15068500 & 3.88725800 & 0.42757800 & $\mathrm{H}$ & 4.27255000 & -1.95198500 & -2.52317600 \\
\hline $\mathrm{C}$ & -1.62781400 & 5.60726500 & -2.10356200 & $\mathrm{H}$ & 3.56675500 & -2.92079900 & -3.82485100 \\
\hline $\mathrm{H}$ & -2.70994300 & 4.71882400 & -3.74415800 & $\mathrm{H}$ & 3.45450400 & -1.16462200 & -3.88756700 \\
\hline $\mathrm{H}$ & -0.48443500 & 6.19160800 & -0.37037200 & $\mathrm{C}$ & 2.05606000 & -3.49597900 & -1.72746600 \\
\hline $\mathrm{H}$ & -1.76323800 & 6.62487300 & -2.45658700 & $\mathrm{H}$ & 2.11242100 & -4.30315500 & -2.46586000 \\
\hline C & -0.36219600 & 2.91572900 & 3.71420100 & $\mathrm{H}$ & 2.91159700 & -3.60393500 & -1.05574000 \\
\hline $\mathrm{H}$ & 0.58261900 & 2.81000000 & 4.24489400 & $\mathrm{H}$ & 1.14489600 & -3.65640500 & -1.14892700 \\
\hline $\mathrm{H}$ & -0.52428400 & 3.95313900 & 3.41558600 & $\mathrm{C}$ & 0.84325600 & 1.84006800 & 1.60567000 \\
\hline $\mathrm{H}$ & -1.19686600 & 2.58829300 & 4.33723100 & $\mathrm{C}$ & 0.24288100 & 1.05000500 & 2.59558500 \\
\hline & & & & C & 2.57508100 & 2.40784700 & 3.00717800 \\
\hline 31 & & & & $\mathrm{C}$ & 0.85167600 & 0.97152500 & 3.84891000 \\
\hline C & 1.33630800 & 2.78724600 & -1.14249500 & $\mathrm{H}$ & -0.67568700 & 0.51062000 & 2.38918200 \\
\hline $\mathrm{H}$ & 0.86676200 & 2.91319800 & -2.12498200 & $\mathrm{C}$ & 2.04209400 & 1.66386000 & 4.06224500 \\
\hline $\mathrm{H}$ & 1.54749300 & 3.78654000 & -0.75668700 & $\mathrm{H}$ & 3.50650500 & 2.95571700 & 3.13080300 \\
\hline $\mathrm{C}$ & 2.67089400 & 2.04632900 & -1.28942500 & $\mathrm{H}$ & 0.40332800 & 0.38061600 & 4.64214600 \\
\hline $\mathrm{H}$ & 3.34366900 & 2.72396500 & -1.82877500 & $\mathrm{H}$ & 2.54970200 & 1.63251000 & 5.02063500 \\
\hline $\mathrm{H}$ & 3.12462100 & 1.89705200 & -0.30785900 & $\mathrm{C}$ & 3.06462600 & -1.07423300 & 0.14736000 \\
\hline C & 2.64165700 & 0.73322800 & -2.07681400 & $\mathrm{C}$ & 2.64213800 & -1.63733100 & 1.35637000 \\
\hline $\mathrm{H}$ & 3.67025400 & 0.43368300 & -2.28662800 & $\mathrm{C}$ & 5.26344300 & -1.08678100 & 0.81793100 \\
\hline $\mathrm{H}$ & 2.12229700 & 0.85940700 & -3.03406600 & $\mathrm{C}$ & 3.60316200 & -1.93961400 & 2.32285300 \\
\hline$P$ & 1.86486800 & -0.70720800 & -1.21512900 & $\mathrm{H}$ & 1.59075200 & -1.83177100 & 1.53816700 \\
\hline$P$ & 0.01646100 & 2.07470700 & -0.04159900 & $\mathrm{C}$ & 4.94036300 & -1.66006800 & 2.05061300 \\
\hline C & -1.10085600 & 3.62626300 & 0.26583100 & $\mathrm{H}$ & 6.29545300 & -0.84946700 & 0.57011700 \\
\hline C & 2.07903200 & -2.14648000 & -2.47005600 & $\mathrm{H}$ & 3.31050900 & -2.38374000 & 3.26940600 \\
\hline $\mathrm{C}$ & -1.50691700 & 4.23338300 & -1.09236100 & $\mathrm{H}$ & 5.72036600 & -1.87719800 & 2.77281400 \\
\hline $\mathrm{H}$ & -2.17310900 & 5.08234000 & -0.90199500 & $\mathrm{~N}$ & 1.99113200 & 2.50462600 & 1.80646100 \\
\hline $\mathrm{H}$ & -0.64956500 & 4.61859600 & -1.65045300 & $\mathrm{~N}$ & 4.35184600 & -0.79959800 & -0.11838700 \\
\hline $\mathrm{H}$ & -2.06283800 & 3.52833300 & -1.71257400 & $\mathrm{Pd}$ & -0.53570000 & -0.29006000 & -0.59567700 \\
\hline
\end{tabular}




\begin{tabular}{|c|c|c|c|c|c|c|c|}
\hline $\mathrm{C}$ & -2.73271500 & -0.22318200 & -0.72181900 & $\mathrm{C}$ & -1.45717000 & 3.94576900 & -1.52531400 \\
\hline $\mathrm{C}$ & -3.56491400 & -0.13218000 & 0.55916000 & $\mathrm{H}$ & -2.10530000 & 4.82887400 & -1.55466000 \\
\hline $\mathrm{O}$ & -1.05237500 & -3.28083900 & -0.03926600 & $\mathrm{H}$ & -0.64146100 & 4.12318500 & -2.23327800 \\
\hline $\mathrm{C}$ & -0.88481100 & -2.18591000 & -0.31137600 & $\mathrm{H}$ & -2.05458700 & 3.09789600 & -1.86463900 \\
\hline $\mathrm{C}$ & -3.01948300 & 0.81076300 & -1.76248600 & $\mathrm{C}$ & -0.20268900 & 5.01992700 & 0.37744200 \\
\hline $\mathrm{O}$ & -3.69507800 & 1.81160300 & -1.61115200 & $\mathrm{H}$ & -0.92557800 & 5.84109600 & 0.43897300 \\
\hline $\mathrm{O}$ & -2.46173800 & 0.47106600 & -2.95347600 & $\mathrm{H}$ & 0.25218400 & 4.90615300 & 1.36483500 \\
\hline $\mathrm{C}$ & -2.76795800 & 1.33964800 & -4.06293200 & $\mathrm{H}$ & 0.57422200 & 5.32890000 & -0.32626900 \\
\hline $\mathrm{H}$ & -2.35968000 & 0.84454000 & -4.94312700 & $\mathrm{C}$ & -2.14151400 & 3.50173200 & 0.86145600 \\
\hline $\mathrm{H}$ & -3.84753800 & 1.46957100 & -4.15955100 & $\mathrm{H}$ & -1.82171900 & 3.37865000 & 1.90035800 \\
\hline $\mathrm{H}$ & -2.30231700 & 2.31932900 & -3.92765500 & $\mathrm{H}$ & -2.80225700 & 4.37545000 & 0.82954900 \\
\hline $\mathrm{H}$ & -2.90541000 & -1.18655500 & -1.20595500 & $\mathrm{H}$ & -2.73817100 & 2.64191700 & 0.55281000 \\
\hline $\mathrm{H}$ & -3.20901900 & 0.66503500 & 1.21154800 & $\mathrm{C}$ & 0.91663400 & -1.77411600 & -3.81146700 \\
\hline $\mathrm{H}$ & -4.58038100 & 0.16511300 & 0.25805000 & $\mathrm{H}$ & -0.11096600 & -1.84487900 & -3.44616200 \\
\hline $\mathrm{C}$ & -3.63586700 & -1.43346100 & 1.33095900 & $\mathrm{H}$ & 1.05445200 & -0.78265200 & -4.25597700 \\
\hline $\mathrm{C}$ & -4.29451500 & -2.54867300 & 0.79017400 & $\mathrm{H}$ & 1.03473900 & -2.50930600 & -4.61500100 \\
\hline $\mathrm{C}$ & -3.06306500 & -1.55350100 & 2.60401000 & $\mathrm{C}$ & 3.37428400 & -2.03851300 & -3.31366000 \\
\hline $\mathrm{C}$ & -4.35742100 & -3.75280400 & 1.49064100 & $\mathrm{H}$ & 4.15126500 & -2.11714200 & -2.54854800 \\
\hline $\mathrm{H}$ & -4.77621100 & -2.47015300 & -0.18219000 & $\mathrm{H}$ & 3.47653600 & -2.89828000 & -3.98486900 \\
\hline $\mathrm{C}$ & -3.12479500 & -2.75714000 & 3.31102100 & $\mathrm{H}$ & 3.55862600 & -1.14038700 & -3.90833800 \\
\hline $\mathrm{H}$ & -2.58932400 & -0.68656800 & 3.05990500 & $\mathrm{C}$ & 1.71030000 & -3.45625300 & -2.09044700 \\
\hline $\mathrm{C}$ & -3.76729600 & -3.86248200 & 2.75273400 & $\mathrm{H}$ & 1.73554200 & -4.20201300 & -2.89255000 \\
\hline $\mathrm{H}$ & -4.87741700 & -4.60238400 & 1.05800200 & $\mathrm{H}$ & 2.49163600 & -3.72063300 & -1.37297100 \\
\hline $\mathrm{H}$ & -2.68353000 & -2.82674400 & 4.30119600 & $\mathrm{H}$ & 0.74160500 & -3.54437400 & -1.59779600 \\
\hline $\mathrm{H}$ & -3.82223900 & -4.79775200 & 3.30133300 & $\mathrm{C}$ & 0.84681300 & 2.18040700 & 1.69983800 \\
\hline & & & & $\mathrm{C}$ & 0.10338300 & 1.56838900 & 2.71790600 \\
\hline & & & & $\mathrm{C}$ & 2.49439100 & 2.79272600 & 3.18610400 \\
\hline & uency $=-1$ & $36.00 \mathrm{~cm}^{-1}$ & & $\mathrm{C}$ & 0.59724700 & 1.59477900 & 4.02244400 \\
\hline $\mathrm{C}$ & 1.70711200 & 2.84301400 & -0.97913600 & $\mathrm{H}$ & -0.84597300 & 1.09273400 & 2.49970900 \\
\hline $\mathrm{H}$ & 1.36431200 & 3.11269100 & -1.98438000 & $\mathrm{C}$ & 1.82002600 & 2.21787000 & 4.26477400 \\
\hline $\mathrm{H}$ & 2.02110400 & 3.75807000 & -0.47464000 & $\mathrm{H}$ & 3.45188500 & 3.28786600 & 3.33151700 \\
\hline $\mathrm{C}$ & 2.92240500 & 1.91170400 & -1.06364700 & $\mathrm{H}$ & 0.03515600 & 1.13835400 & 4.83173300 \\
\hline $\mathrm{H}$ & 3.75248100 & 2.51265800 & -1.45381400 & $\mathrm{H}$ & 2.24393300 & 2.26206100 & 5.26245700 \\
\hline $\mathrm{H}$ & 3.23250400 & 1.60502300 & -0.06180100 & $\mathrm{C}$ & 2.77664100 & -1.33763400 & 0.08431600 \\
\hline $\mathrm{C}$ & 2.79198300 & 0.69458100 & -1.98721800 & $\mathrm{C}$ & 2.17006500 & -1.96514400 & 1.17810100 \\
\hline $\mathrm{H}$ & 3.78828900 & 0.28116600 & -2.15623800 & $\mathrm{C}$ & 4.87589600 & -1.62991900 & 0.97236200 \\
\hline $\mathrm{H}$ & 2.37570900 & 0.98198000 & -2.95976800 & $\mathrm{C}$ & 2.98696000 & -2.44709900 & 2.20253900 \\
\hline $\mathrm{P}$ & 1.78673000 & -0.72178900 & -1.35350600 & $\mathrm{H}$ & 1.09211700 & -2.07962900 & 1.22526900 \\
\hline $\mathrm{P}$ & 0.22003600 & 2.22776800 & -0.05308400 & $\mathrm{C}$ & 4.36582200 & -2.27603900 & 2.10121100 \\
\hline $\mathrm{C}$ & -0.95102500 & 3.75238800 & -0.08206500 & $\mathrm{H}$ & 5.94616700 & -1.47533900 & 0.85742900 \\
\hline $\mathrm{C}$ & 1.95345600 & -2.06568100 & -2.70897400 & $\mathrm{H}$ & 2.55211800 & -2.94606000 & 3.06323000 \\
\hline
\end{tabular}




\begin{tabular}{|c|c|c|c|c|c|c|c|}
\hline $\mathrm{H}$ & 5.03676700 & -2.63271300 & 2.87559400 & $\mathrm{P}$ & -1.21135400 & -1.98065800 & 0.32371400 \\
\hline $\mathrm{N}$ & 2.02339100 & 2.78141500 & 1.93268300 & $\mathrm{C}$ & -2.03702000 & -3.30543700 & -0.76544100 \\
\hline $\mathrm{N}$ & 4.10431500 & -1.17028200 & -0.01958500 & $\mathrm{C}$ & -4.28330600 & 1.98170500 & -0.57001100 \\
\hline $\mathrm{Pd}$ & -0.44193800 & -0.11994600 & -0.72495300 & $\mathrm{C}$ & -3.17640600 & -2.63416900 & -1.55629500 \\
\hline $\mathrm{C}$ & -2.91684000 & -0.59367100 & -0.52493600 & $\mathrm{H}$ & -3.63547100 & -3.37551800 & -2.21967300 \\
\hline $\mathrm{C}$ & -3.28639300 & -0.36175400 & 0.94047300 & $\mathrm{H}$ & -3.96771000 & -2.25060400 & -0.90478800 \\
\hline $\mathrm{O}$ & -1.47769500 & -2.93740200 & -1.06894600 & $\mathrm{H}$ & -2.80579600 & -1.81204500 & -2.17658400 \\
\hline $\mathrm{C}$ & -1.29338400 & -1.80331400 & -0.92499200 & $\mathrm{C}$ & -2.59333600 & -4.44330800 & 0.11324600 \\
\hline $\mathrm{C}$ & -3.29100000 & 0.47193500 & -1.51290600 & $\mathrm{H}$ & -3.00645200 & -5.22159600 & -0.53831600 \\
\hline $\mathrm{O}$ & -3.79360100 & 1.54216500 & -1.22849000 & $\mathrm{H}$ & -1.81729100 & -4.90586700 & 0.72936900 \\
\hline $\mathrm{O}$ & -3.07057500 & 0.05501500 & -2.77860100 & $\mathrm{H}$ & -3.39963900 & -4.10884800 & 0.77213200 \\
\hline $\mathrm{C}$ & -3.52753300 & 0.93668000 & -3.82694800 & $\mathrm{C}$ & -1.00079100 & -3.87068400 & -1.75723200 \\
\hline $\mathrm{H}$ & -3.39983000 & 0.37703700 & -4.75232200 & $\mathrm{H}$ & -0.19434900 & -4.40379800 & -1.24708900 \\
\hline $\mathrm{H}$ & -4.57620800 & 1.19781900 & -3.67372400 & $\mathrm{H}$ & -1.50422200 & -4.59191700 & -2.41064800 \\
\hline $\mathrm{H}$ & -2.92808900 & 1.84982600 & -3.84863400 & $\mathrm{H}$ & -0.56294500 & -3.09273600 & -2.38741800 \\
\hline $\mathrm{H}$ & -3.38143900 & -1.51288900 & -0.88138000 & $\mathrm{C}$ & -5.26672100 & 0.79728800 & -0.65922800 \\
\hline $\mathrm{H}$ & -2.72052800 & 0.47036800 & 1.36298900 & $\mathrm{H}$ & -4.85970500 & -0.02326700 & -1.25669000 \\
\hline $\mathrm{H}$ & -4.33794000 & -0.04290200 & 0.94667000 & $\mathrm{H}$ & -5.54554800 & 0.40692100 & 0.32501300 \\
\hline $\mathrm{C}$ & -3.13432900 & -1.61200700 & 1.78571500 & $\mathrm{H}$ & -6.18957700 & 1.13494800 & -1.14340200 \\
\hline $\mathrm{C}$ & -4.15770100 & -2.56914600 & 1.81389200 & $\mathrm{C}$ & -4.90350100 & 3.12007400 & 0.25975900 \\
\hline $\mathrm{C}$ & -1.97150400 & -1.85487400 & 2.52931200 & $\mathrm{H}$ & -4.26323400 & 4.00601300 & 0.28163700 \\
\hline $\mathrm{C}$ & -4.02037400 & -3.74190100 & 2.55717000 & $\mathrm{H}$ & -5.85391500 & 3.42050600 & -0.19611300 \\
\hline $\mathrm{H}$ & -5.07475900 & -2.39095100 & 1.25701800 & $\mathrm{H}$ & -5.11899700 & 2.81799100 & 1.28937000 \\
\hline $\mathrm{C}$ & -1.83184200 & -3.02603200 & 3.27780500 & $\mathrm{C}$ & -3.94606000 & 2.47726500 & -1.98991100 \\
\hline $\mathrm{H}$ & -1.17216100 & -1.11794700 & 2.53392000 & $\mathrm{H}$ & -4.86736100 & 2.80103900 & -2.48636600 \\
\hline $\mathrm{C}$ & -2.85525300 & -3.97459100 & 3.29072000 & $\mathrm{H}$ & -3.26227900 & 3.33119400 & -1.97652000 \\
\hline $\mathrm{H}$ & -4.82638000 & -4.46925800 & 2.57046600 & $\mathrm{H}$ & -3.49779400 & 1.68795300 & -2.60150000 \\
\hline $\mathrm{H}$ & -0.92977300 & -3.19267800 & 3.85997800 & $\mathrm{C}$ & 0.14182900 & -2.85588600 & 1.25427900 \\
\hline \multirow[t]{2}{*}{$\mathrm{H}$} & -2.75074000 & -4.88334700 & 3.87534200 & $\mathrm{C}$ & 1.37101200 & -3.15339600 & 0.65410500 \\
\hline & & & & $\mathrm{C}$ & 0.79446700 & -3.81977800 & 3.23859100 \\
\hline 32 & & & & $\mathrm{C}$ & 2.33832100 & -3.82001400 & 1.40741000 \\
\hline $\mathrm{C}$ & -2.43362900 & -1.60914900 & 1.68061200 & $\mathrm{H}$ & 1.59469300 & -2.87118100 & -0.36625400 \\
\hline $\mathrm{H}$ & -3.43403300 & -1.61189300 & 1.23559000 & $\mathrm{C}$ & 2.04683900 & -4.16326200 & 2.72589700 \\
\hline $\mathrm{H}$ & -2.36410400 & -2.45696900 & 2.36498900 & $\mathrm{H}$ & 0.52814900 & -4.06510400 & 4.26426400 \\
\hline $\mathrm{C}$ & -2.19422000 & -0.30020500 & 2.47522800 & $\mathrm{H}$ & 3.30190000 & -4.04643300 & 0.96280700 \\
\hline $\mathrm{H}$ & -2.46562200 & -0.48269200 & 3.52060600 & $\mathrm{H}$ & 2.76997200 & -4.67852900 & 3.34940500 \\
\hline $\mathrm{H}$ & -1.12861200 & -0.04557600 & 2.48572600 & $\mathrm{C}$ & -1.46336200 & 2.71761300 & 0.15684900 \\
\hline $\mathrm{C}$ & -3.02109600 & 0.91151500 & 1.99002600 & $\mathrm{C}$ & -1.47637700 & 3.96219200 & 0.78348600 \\
\hline $\mathrm{H}$ & -2.83524200 & 1.78185800 & 2.62796900 & $\mathrm{C}$ & 0.54326100 & 3.21337800 & -0.92201600 \\
\hline $\mathrm{H}$ & -4.08874800 & 0.68012000 & 2.07465800 & $\mathrm{C}$ & -0.43650700 & 4.85994600 & 0.52356700 \\
\hline $\mathrm{P}$ & -2.67861700 & 1.32199000 & 0.20661300 & $\mathrm{H}$ & -2.28015600 & 4.22939000 & 1.46027900 \\
\hline
\end{tabular}




\begin{tabular}{|c|c|c|c|c|c|c|c|}
\hline $\mathrm{C}$ & 0.57885300 & 4.48676400 & -0.35308000 & $\mathrm{H}$ & 0.11796300 & -4.15026900 & 0.90837900 \\
\hline $\mathrm{H}$ & 1.32743700 & 2.87859800 & -1.59161700 & $\mathrm{H}$ & 1.45132500 & -3.66921800 & -0.13948400 \\
\hline $\mathrm{H}$ & -0.42566400 & 5.83730600 & 0.99590800 & $\mathrm{P}$ & -0.47449700 & -2.19941300 & -0.30545000 \\
\hline $\mathrm{H}$ & 1.39906200 & 5.15668400 & -0.58542900 & $\mathrm{P}$ & 2.61327200 & -0.44025100 & 0.44284300 \\
\hline $\mathrm{N}$ & -0.14081700 & -3.18066400 & 2.52567700 & $\mathrm{C}$ & 4.23234400 & -0.33216400 & -0.56400000 \\
\hline $\mathrm{N}$ & -0.44493300 & 2.34481800 & -0.66686900 & $\mathrm{C}$ & -0.88746900 & -3.16222000 & -1.91666300 \\
\hline $\mathrm{Pd}$ & -0.71761200 & 0.08187600 & -0.68938100 & $\mathrm{C}$ & 4.12420400 & -1.36408700 & -1.70394800 \\
\hline $\mathrm{C}$ & 1.01182800 & -0.53432500 & -1.53685700 & $\mathrm{H}$ & 5.03415100 & -1.33016800 & -2.31353600 \\
\hline $\mathrm{O}$ & 0.92529200 & -1.17644500 & -2.55298600 & $\mathrm{H}$ & 4.02441800 & -2.38926800 & -1.33298300 \\
\hline $\mathrm{C}$ & 2.48824400 & 0.23694600 & 0.47018600 & $\mathrm{H}$ & 3.27359300 & -1.15066500 & -2.35938100 \\
\hline $\mathrm{H}$ & 1.47745800 & 0.36776800 & 0.87436000 & $\mathrm{C}$ & 5.45763700 & -0.63150500 & 0.32046200 \\
\hline $\mathrm{H}$ & 2.86947200 & -0.69229700 & 0.90877200 & $\mathrm{H}$ & 6.36784400 & -0.52366000 & -0.28101200 \\
\hline $\mathrm{C}$ & 2.37980000 & 0.03015800 & -1.04960400 & $\mathrm{H}$ & 5.53239400 & 0.06023500 & 1.16426200 \\
\hline $\mathrm{H}$ & 2.45023100 & 0.99429800 & -1.56606900 & $\mathrm{H}$ & 5.44952400 & -1.65005000 & 0.71762800 \\
\hline $\mathrm{C}$ & 3.47932900 & -0.87711200 & -1.60314300 & $\mathrm{C}$ & 4.36615900 & 1.07770700 & -1.16852500 \\
\hline $\mathrm{O}$ & 3.78735900 & -1.94453200 & -1.11457000 & $\mathrm{H}$ & 4.49073100 & 1.84081500 & -0.39484800 \\
\hline $\mathrm{O}$ & 4.04114100 & -0.35504000 & -2.70081800 & $\mathrm{H}$ & 5.25741400 & 1.10844900 & -1.80560600 \\
\hline $\mathrm{C}$ & 5.04785300 & -1.17268800 & -3.34087100 & $\mathrm{H}$ & 3.50044300 & 1.34291200 & -1.78040100 \\
\hline $\mathrm{H}$ & 4.60935600 & -2.11776300 & -3.66701000 & $\mathrm{C}$ & 0.12030000 & -2.71220500 & -2.99275100 \\
\hline $\mathrm{H}$ & 5.39262100 & -0.59197800 & -4.19479400 & $\mathrm{H}$ & 0.01458900 & -1.64826400 & -3.22637000 \\
\hline $\mathrm{H}$ & 5.86861600 & -1.37268600 & -2.64926900 & $\mathrm{H}$ & 1.15827000 & -2.89776000 & -2.69544200 \\
\hline $\mathrm{C}$ & 3.34783400 & 1.40939700 & 0.91266000 & $\mathrm{H}$ & -0.06152700 & -3.27910100 & -3.91275500 \\
\hline $\mathrm{C}$ & 4.41759200 & 1.89866100 & 0.14998300 & $\mathrm{C}$ & -0.76073700 & -4.68538200 & -1.69248500 \\
\hline $\mathrm{C}$ & 3.08773000 & 2.01127500 & 2.15339500 & $\mathrm{H}$ & -1.40834500 & -5.03007500 & -0.88376400 \\
\hline $\mathrm{C}$ & 5.20111800 & 2.95884300 & 0.61402100 & $\mathrm{H}$ & -1.07046400 & -5.18811000 & -2.61564500 \\
\hline $\mathrm{H}$ & 4.65514100 & 1.45547900 & -0.81281100 & $\mathrm{H}$ & 0.26489100 & -4.99983000 & -1.48339500 \\
\hline $\mathrm{C}$ & 3.87006400 & 3.06679500 & 2.62055100 & $\mathrm{C}$ & -2.32452800 & -2.85385500 & -2.37743000 \\
\hline $\mathrm{H}$ & 2.26708500 & 1.64043300 & 2.76403800 & $\mathrm{H}$ & -2.50369100 & -3.38378300 & -3.31992300 \\
\hline $\mathrm{C}$ & 4.93141900 & 3.54661400 & 1.84957700 & $\mathrm{H}$ & -3.06199800 & -3.20996200 & -1.65376700 \\
\hline $\mathrm{H}$ & 6.02709000 & 3.31983000 & 0.00805700 & $\mathrm{H}$ & -2.49527800 & -1.79117100 & -2.54401800 \\
\hline $\mathrm{H}$ & 3.65198100 & 3.51421300 & 3.58608800 & $\mathrm{C}$ & 2.77349300 & 0.86197400 & 1.77070500 \\
\hline \multirow[t]{2}{*}{$\mathrm{H}$} & 5.54345800 & 4.36745200 & 2.21086400 & $\mathrm{C}$ & 2.41792900 & 2.19358300 & 1.50880200 \\
\hline & & & & $\mathrm{C}$ & 3.35358600 & 1.37721000 & 3.93870500 \\
\hline $32 \mathbf{a}$ & & & & $\mathrm{C}$ & 2.55952600 & 3.13867800 & 2.52544600 \\
\hline $\mathrm{C}$ & 2.78443200 & -2.00430800 & 1.42324200 & $\mathrm{H}$ & 2.04857900 & 2.47742400 & 0.52979600 \\
\hline $\mathrm{H}$ & 3.39311900 & -2.70295700 & 0.84050300 & $\mathrm{C}$ & 3.03485600 & 2.72539500 & 3.76865200 \\
\hline $\mathrm{H}$ & 3.34226500 & -1.74018800 & 2.32570800 & $\mathrm{H}$ & 3.72528000 & 1.01147500 & 4.89327100 \\
\hline $\mathrm{C}$ & 1.45486400 & -2.67736500 & 1.79346500 & $\mathrm{H}$ & 2.30073800 & 4.17896600 & 2.35047300 \\
\hline $\mathrm{H}$ & 1.67986500 & -3.48354500 & 2.50084700 & $\mathrm{H}$ & 3.15602600 & 3.42483600 & 4.58909200 \\
\hline $\mathrm{H}$ & 0.80503000 & -1.98125700 & 2.33782400 & $\mathrm{C}$ & -1.95332800 & -2.33190900 & 0.80789700 \\
\hline $\mathrm{C}$ & 0.72405600 & -3.29783700 & 0.58942400 & $\mathrm{C}$ & -2.63029800 & -1.22492500 & 1.32206800 \\
\hline
\end{tabular}




\begin{tabular}{|c|c|c|c|c|c|c|c|}
\hline $\mathrm{C}$ & -3.35312800 & -3.79905600 & 1.89359600 & $\mathrm{H}$ & -2.62408500 & -2.03923900 & 3.16546900 \\
\hline $\mathrm{C}$ & -3.72634300 & -1.44741500 & 2.15867800 & $\mathrm{H}$ & -1.41109000 & -0.95770800 & 2.53577900 \\
\hline $\mathrm{H}$ & -2.32508000 & -0.21849400 & 1.07144500 & $\mathrm{C}$ & -3.37832700 & -0.75466300 & 1.62974000 \\
\hline $\mathrm{C}$ & -4.09886500 & -2.75634500 & 2.45011800 & $\mathrm{H}$ & -3.76288700 & -0.07157600 & 2.39046800 \\
\hline $\mathrm{H}$ & -3.60753900 & -4.83614500 & 2.09983300 & $\mathrm{H}$ & -4.21229900 & -1.40708400 & 1.35282600 \\
\hline $\mathrm{H}$ & -4.27837100 & -0.60473400 & 2.56290900 & $\mathrm{P}$ & -2.86990200 & 0.21174900 & 0.11275700 \\
\hline $\mathrm{H}$ & -4.94512300 & -2.97381900 & 3.09334900 & $\mathrm{P}$ & -0.22251800 & -2.39292300 & 0.35536900 \\
\hline $\mathrm{N}$ & 3.23022700 & 0.46517200 & 2.96690300 & $\mathrm{C}$ & -0.00673700 & -3.91783800 & -0.78553000 \\
\hline $\mathrm{N}$ & -2.30202100 & -3.59775300 & 1.09269600 & $\mathrm{C}$ & -4.33107400 & 0.01283000 & -1.10815500 \\
\hline $\mathrm{Pd}$ & 0.37679900 & -0.05843600 & -0.69886400 & $\mathrm{C}$ & -0.86586900 & -3.68210800 & -2.04232800 \\
\hline $\mathrm{C}$ & -1.26672500 & 2.23394200 & -1.62382900 & $\mathrm{H}$ & -0.78849400 & -4.55495800 & -2.70048700 \\
\hline $\mathrm{C}$ & -1.62802200 & 3.08108800 & -0.35504300 & $\mathrm{H}$ & -1.92581000 & -3.55319500 & -1.79981700 \\
\hline $\mathrm{O}$ & -2.49300900 & 0.20427700 & -1.50175000 & $\mathrm{H}$ & -0.51910800 & -2.80707000 & -2.59907300 \\
\hline $\mathrm{C}$ & -1.40657200 & 0.69917800 & -1.35506300 & $\mathrm{C}$ & -0.46938500 & -5.19074900 & -0.04582700 \\
\hline $\mathrm{C}$ & 0.14642200 & 2.54968400 & -2.01084300 & $\mathrm{H}$ & -0.25322700 & -6.05762100 & -0.67994000 \\
\hline $\mathrm{O}$ & 1.10066300 & 1.86005300 & -1.61864400 & $\mathrm{H}$ & 0.06302000 & -5.33492200 & 0.89947900 \\
\hline $\mathrm{O}$ & 0.29526000 & 3.62224700 & -2.75664300 & $\mathrm{H}$ & -1.54333500 & -5.19723100 & 0.15678000 \\
\hline $\mathrm{C}$ & 1.64206800 & 3.99302200 & -3.15396500 & $\mathrm{C}$ & 1.46751600 & -4.10100300 & -1.19706700 \\
\hline $\mathrm{H}$ & 1.52126500 & 4.88755600 & -3.76114100 & $\mathrm{H}$ & 2.10468000 & -4.33907500 & -0.34186500 \\
\hline $\mathrm{H}$ & 2.24841400 & 4.20096000 & -2.27092000 & $\mathrm{H}$ & 1.52298300 & -4.94759100 & -1.89059900 \\
\hline $\mathrm{H}$ & 2.09202300 & 3.18623400 & -3.73474800 & $\mathrm{H}$ & 1.87232700 & -3.22980700 & -1.71300200 \\
\hline $\mathrm{H}$ & -1.95202100 & 2.49821800 & -2.43477200 & $\mathrm{C}$ & -4.36147300 & -1.46181700 & -1.55541100 \\
\hline $\mathrm{H}$ & -0.90851400 & 2.84818900 & 0.43767400 & $\mathrm{H}$ & -3.41296200 & -1.76401700 & -2.00932500 \\
\hline $\mathrm{H}$ & -1.46617200 & 4.13040900 & -0.63227100 & $\mathrm{H}$ & -4.58872400 & -2.14732200 & -0.73292100 \\
\hline $\mathrm{C}$ & -3.03656200 & 2.90269300 & 0.17336200 & $\mathrm{H}$ & -5.14725800 & -1.59370200 & -2.30741800 \\
\hline $\mathrm{C}$ & -4.15682500 & 3.13430900 & -0.63648300 & $\mathrm{C}$ & -5.67805500 & 0.40072200 & -0.47278600 \\
\hline $\mathrm{C}$ & -3.23682000 & 2.56451600 & 1.51768600 & $\mathrm{H}$ & -5.71051000 & 1.45644100 & -0.18952200 \\
\hline $\mathrm{C}$ & -5.44523000 & 3.02136300 & -0.11666600 & $\mathrm{H}$ & -6.47989500 & 0.23391000 & -1.20118500 \\
\hline $\mathrm{H}$ & -4.02766600 & 3.41027600 & -1.67991500 & $\mathrm{H}$ & -5.91577100 & -0.20115800 & 0.40985500 \\
\hline $\mathrm{C}$ & -4.52735200 & 2.46042000 & 2.04385600 & $\mathrm{C}$ & -4.05908500 & 0.90058900 & -2.33605700 \\
\hline $\mathrm{H}$ & -2.37754200 & 2.40274300 & 2.16478500 & $\mathrm{H}$ & -4.87991400 & 0.78785300 & -3.05295700 \\
\hline $\mathrm{C}$ & -5.63491400 & 2.68443100 & 1.22594500 & $\mathrm{H}$ & -3.99155400 & 1.96072300 & -2.07578400 \\
\hline $\mathrm{H}$ & -6.30205800 & 3.20384100 & -0.75798300 & $\mathrm{H}$ & -3.13505400 & 0.60713900 & -2.84217200 \\
\hline $\mathrm{H}$ & -4.66380900 & 2.21956700 & 3.09443300 & $\mathrm{C}$ & 1.08303000 & -2.55106000 & 1.67392500 \\
\hline \multirow[t]{2}{*}{$\mathrm{H}$} & -6.63901000 & 2.60613100 & 1.63134900 & $\mathrm{C}$ & 2.43201600 & -2.27834900 & 1.41516000 \\
\hline & & & & $\mathrm{C}$ & 1.53792800 & -3.10219300 & 3.86138000 \\
\hline $32 b$ & & & & $\mathrm{C}$ & 3.35494300 & -2.44384100 & 2.44851000 \\
\hline $\mathrm{C}$ & -1.75885900 & -2.75049800 & 1.32920200 & $\mathrm{H}$ & 2.78047400 & -1.94475100 & 0.44540200 \\
\hline $\mathrm{H}$ & -2.53840100 & -3.04601400 & 0.61915400 & $\mathrm{C}$ & 2.90309100 & -2.86226700 & 3.69853800 \\
\hline $\mathrm{H}$ & -1.52277600 & -3.61620600 & 1.94865700 & $\mathrm{H}$ & 1.14207800 & -3.42784700 & 4.82076700 \\
\hline $\mathrm{C}$ & -2.24604800 & -1.59980500 & 2.23637400 & $\mathrm{H}$ & 4.40595400 & -2.24016900 & 2.26856900 \\
\hline
\end{tabular}




\begin{tabular}{|c|c|c|c|c|c|c|c|}
\hline $\mathrm{H}$ & 3.58593800 & -2.99926400 & 4.53050500 & $\mathrm{H}$ & 4.65091100 & 5.14471500 & -0.35159400 \\
\hline $\mathrm{C}$ & -2.99368200 & 1.99956200 & 0.63732400 & $\mathrm{H}$ & 2.39013000 & 4.84475100 & 3.29208600 \\
\hline $\mathrm{C}$ & -3.81558900 & 2.47734200 & 1.66483800 & $\mathrm{H}$ & 3.89222600 & 6.14770900 & 1.79303800 \\
\hline $\mathrm{C}$ & -2.24267400 & 4.15669500 & 0.19623600 & & & & \\
\hline $\mathrm{C}$ & -3.83045900 & 3.84295900 & 1.95372500 & 32-CO & & & \\
\hline $\mathrm{H}$ & -4.44879200 & 1.80457800 & 2.23022600 & $\mathrm{C}$ & -1.79532800 & -2.32511700 & 1.88805400 \\
\hline $\mathrm{C}$ & -3.02777200 & 4.70313800 & 1.20903900 & $\mathrm{H}$ & -2.67614000 & -2.87165300 & 1.53887700 \\
\hline $\mathrm{H}$ & -1.59824800 & 4.78503000 & -0.41273300 & $\mathrm{H}$ & -1.29955400 & -2.93133800 & 2.65003700 \\
\hline $\mathrm{H}$ & -4.46428600 & 4.22534400 & 2.74797100 & $\mathrm{C}$ & -2.23319500 & -0.98779100 & 2.50888200 \\
\hline $\mathrm{H}$ & -3.00639500 & 5.76991700 & 1.40244600 & $\mathrm{H}$ & -2.56965800 & -1.19598200 & 3.53063300 \\
\hline $\mathrm{N}$ & 0.64337400 & -2.95444500 & 2.87613000 & $\mathrm{H}$ & -1.38253700 & -0.30305600 & 2.60950400 \\
\hline $\mathrm{N}$ & -2.22443000 & 2.84571600 & -0.08268200 & $\mathrm{C}$ & -3.39979000 & -0.31781900 & 1.75935500 \\
\hline $\mathrm{Pd}$ & -0.46133300 & -0.29165500 & -0.69135500 & $\mathrm{H}$ & -3.93488100 & 0.37386300 & 2.41543300 \\
\hline $\mathrm{C}$ & 1.39400400 & -0.37426600 & -1.48262700 & $\mathrm{H}$ & -4.12989700 & -1.08177500 & 1.47375100 \\
\hline $\mathrm{O}$ & 1.60626500 & -1.04981700 & -2.45684900 & $\mathrm{P}$ & -2.89882900 & 0.57006700 & 0.20934900 \\
\hline $\mathrm{O}$ & -0.42395900 & 1.71384100 & -1.68925200 & $\mathrm{P}$ & -0.57661400 & -2.27629400 & 0.48486100 \\
\hline $\mathrm{H}$ & -1.11744700 & 2.24054400 & -1.18736300 & $\mathrm{C}$ & -1.01059000 & -3.83513600 & -0.53872800 \\
\hline $\mathrm{C}$ & -0.45348000 & 1.98438400 & -3.09675600 & $\mathrm{C}$ & -4.51654700 & 0.75692900 & -0.79044600 \\
\hline $\mathrm{H}$ & 0.29658100 & 1.35473500 & -3.57794900 & $\mathrm{C}$ & -2.33402500 & -3.55887200 & -1.27734400 \\
\hline $\mathrm{H}$ & -1.43240700 & 1.76688000 & -3.53564400 & $\mathrm{H}$ & -2.60558500 & -4.44150000 & -1.86667800 \\
\hline $\mathrm{H}$ & -0.20145100 & 3.03466200 & -3.27711200 & $\mathrm{H}$ & -3.16655300 & -3.35759600 & -0.59594300 \\
\hline $\mathrm{C}$ & 2.27149700 & 1.14551500 & 0.45701500 & $\mathrm{H}$ & -2.23751700 & -2.71467500 & -1.96775200 \\
\hline $\mathrm{H}$ & 1.21781000 & 1.04968200 & 0.74645300 & $\mathrm{C}$ & -1.15965900 & -5.05106100 & 0.39828000 \\
\hline $\mathrm{H}$ & 2.81920500 & 0.43167800 & 1.08179000 & $\mathrm{H}$ & -1.35975000 & -5.93833400 & -0.21277000 \\
\hline $\mathrm{C}$ & 2.41047000 & 0.70863500 & -1.00914800 & $\mathrm{H}$ & -0.24608600 & -5.24283900 & 0.96797800 \\
\hline $\mathrm{H}$ & 2.18257600 & 1.55451500 & -1.66589700 & $\mathrm{H}$ & -1.98798800 & -4.94913200 & 1.10399000 \\
\hline $\mathrm{C}$ & 3.80795100 & 0.18599000 & -1.33275100 & $\mathrm{C}$ & 0.08979400 & -4.12835500 & -1.57503900 \\
\hline $\mathrm{O}$ & 4.38500900 & -0.65111600 & -0.66718100 & $\mathrm{H}$ & 1.03900500 & -4.38973700 & -1.10055100 \\
\hline $\mathrm{O}$ & 4.30685000 & 0.74157400 & -2.44377300 & $\mathrm{H}$ & -0.22050900 & -4.99277900 & -2.17274700 \\
\hline $\mathrm{C}$ & 5.60904900 & 0.26548500 & -2.85381200 & $\mathrm{H}$ & 0.25865600 & -3.29120000 & -2.25416600 \\
\hline $\mathrm{H}$ & 5.56720600 & -0.80477100 & -3.06529500 & $\mathrm{C}$ & -4.97425400 & -0.64800300 & -1.23135000 \\
\hline $\mathrm{H}$ & 5.85288900 & 0.82776800 & -3.75367600 & $\mathrm{H}$ & -4.21685500 & -1.15118200 & -1.83942400 \\
\hline $\mathrm{H}$ & 6.34385300 & 0.45126700 & -2.06797800 & $\mathrm{H}$ & -5.22938400 & -1.29648200 & -0.38773800 \\
\hline $\mathrm{C}$ & 2.73441900 & 2.55801100 & 0.77715000 & $\mathrm{H}$ & -5.87700900 & -0.54965900 & -1.84403500 \\
\hline $\mathrm{C}$ & 3.58126700 & 3.30134500 & -0.05506700 & $\mathrm{C}$ & -5.60789100 & 1.42520600 & 0.06803700 \\
\hline $\mathrm{C}$ & 2.31436200 & 3.13838300 & 1.98465800 & $\mathrm{H}$ & -5.31876300 & 2.42932600 & 0.39133800 \\
\hline $\mathrm{C}$ & 3.99317500 & 4.58706400 & 0.30887900 & $\mathrm{H}$ & -6.52141300 & 1.52309400 & -0.52915300 \\
\hline $\mathrm{H}$ & 3.93500500 & 2.88714600 & -0.99414300 & $\mathrm{H}$ & -5.86348900 & 0.83652000 & 0.95418900 \\
\hline $\mathrm{C}$ & 2.72608400 & 4.41814900 & 2.35119200 & $\mathrm{C}$ & -4.24650300 & 1.60884300 & -2.04479100 \\
\hline $\mathrm{H}$ & 1.66100500 & 2.57452800 & 2.64750000 & $\mathrm{H}$ & -5.18305200 & 1.72296200 & -2.60180400 \\
\hline $\mathrm{C}$ & 3.56893400 & 5.15028300 & 1.51092700 & $\mathrm{H}$ & -3.87711100 & 2.60775400 & -1.80224100 \\
\hline
\end{tabular}




\begin{tabular}{|c|c|c|c|c|c|c|c|}
\hline $\mathrm{H}$ & -3.52785200 & 1.12750700 & -2.71436900 & C & 5.21282700 & 2.98314800 & 1.50146500 \\
\hline $\mathrm{C}$ & 1.02323800 & -2.68554700 & 1.36338000 & $\mathrm{H}$ & 4.83189500 & 0.87339800 & 1.28217300 \\
\hline $\mathrm{C}$ & 2.25025200 & -2.72560600 & 0.69017300 & $\mathrm{C}$ & 4.69230300 & 4.27265800 & 1.38388500 \\
\hline $\mathrm{C}$ & 2.02313200 & -3.25421200 & 3.35868200 & $\mathrm{H}$ & 2.96091000 & 5.45000500 & 0.87011000 \\
\hline $\mathrm{C}$ & 3.40032600 & -3.04821700 & 1.41240700 & $\mathrm{H}$ & 6.23552600 & 2.83774700 & 1.83673100 \\
\hline $\mathrm{H}$ & 2.33281000 & -2.50129500 & -0.36412500 & $\mathrm{H}$ & 5.30733100 & 5.13408700 & 1.62596600 \\
\hline $\mathrm{C}$ & 3.28853900 & -3.32189000 & 2.77336800 & $\mathrm{C}$ & -0.75895300 & 1.29000600 & -2.14600200 \\
\hline $\mathrm{H}$ & 1.88883000 & -3.45538800 & 4.41903200 & $\mathrm{O}$ & -0.76583100 & 2.02264300 & -3.01790900 \\
\hline $\mathrm{H}$ & 4.36101900 & -3.06280200 & 0.90886500 & & & & \\
\hline $\mathrm{H}$ & 4.15636500 & -3.57460300 & 3.37342800 & \multirow{2}{*}{\multicolumn{4}{|c|}{$\begin{array}{l}\text { TS29 } \\
\text { imaginary frequency }=-288.75 \mathrm{~cm}^{-1}\end{array}$}} \\
\hline $\mathrm{C}$ & -2.47425100 & 2.29053000 & 0.77058000 & & & & \\
\hline $\mathrm{C}$ & -2.78205400 & 2.82669900 & 2.02705700 & $\mathrm{C}$ & 0.41475800 & -3.20245400 & 1.26338500 \\
\hline $\mathrm{C}$ & -1.49881200 & 4.27438400 & 0.09958000 & $\mathrm{H}$ & 0.08056800 & -3.89693700 & 0.48522900 \\
\hline $\mathrm{C}$ & -2.41559200 & 4.14536100 & 2.30546400 & $\mathrm{H}$ & 1.09036700 & -3.74081500 & 1.92892800 \\
\hline $\mathrm{H}$ & -3.29773800 & 2.24493000 & 2.78132500 & $\mathrm{C}$ & -0.79160100 & -2.68829200 & 2.08339100 \\
\hline $\mathrm{C}$ & -1.76676600 & 4.88971000 & 1.32408900 & $\mathrm{H}$ & -0.88551900 & -3.30881400 & 2.98117300 \\
\hline $\mathrm{H}$ & -0.99415100 & 4.81641200 & -0.69707800 & $\mathrm{H}$ & -0.60852800 & -1.67180300 & 2.44785800 \\
\hline $\mathrm{H}$ & -2.64070500 & 4.58077600 & 3.27420200 & $\mathrm{C}$ & -2.14125500 & -2.75647400 & 1.34365200 \\
\hline $\mathrm{H}$ & -1.46954100 & 5.91828600 & 1.49803500 & $\mathrm{H}$ & -2.96415500 & -2.56775200 & 2.04023100 \\
\hline $\mathrm{N}$ & 0.91611200 & -2.94251600 & 2.67488500 & $\mathrm{H}$ & -2.29067500 & -3.77216700 & 0.96145200 \\
\hline $\mathrm{N}$ & -1.82886500 & 3.00622800 & -0.16915500 & $\mathrm{P}$ & -2.25046500 & -1.62190600 & -0.13943900 \\
\hline $\mathrm{Pd}$ & -0.71103500 & -0.20408300 & -0.81578100 & $\mathrm{P}$ & 1.41549000 & -1.86691000 & 0.43369400 \\
\hline $\mathrm{C}$ & 1.21279500 & -0.49171400 & -1.58871500 & $\mathrm{C}$ & 2.64338400 & -2.81919900 & -0.67464900 \\
\hline $\mathrm{O}$ & 1.41719100 & -1.29435100 & -2.45729700 & $\mathrm{C}$ & -3.52911800 & -2.46504900 & -1.29437600 \\
\hline $\mathrm{C}$ & 2.24299700 & 0.84244300 & 0.40257500 & $\mathrm{C}$ & 1.83685300 & -3.42994100 & -1.83896300 \\
\hline $\mathrm{H}$ & 1.19915900 & 1.03407300 & 0.68045300 & $\mathrm{H}$ & 2.52276000 & -3.95403400 & -2.51445000 \\
\hline $\mathrm{H}$ & 2.57210500 & -0.03797000 & 0.95839800 & $\mathrm{H}$ & 1.09667500 & -4.16171600 & -1.49938300 \\
\hline $\mathrm{C}$ & 2.28785000 & 0.51408300 & -1.10497700 & $\mathrm{H}$ & 1.32412400 & -2.65504400 & -2.41740400 \\
\hline $\mathrm{H}$ & 2.06713200 & 1.42586300 & -1.67557500 & $\mathrm{C}$ & 3.36904200 & -3.92658700 & 0.11211800 \\
\hline $\mathrm{C}$ & 3.66388300 & 0.00333000 & -1.53859400 & $\mathrm{H}$ & 4.08793000 & -4.42347200 & -0.54955900 \\
\hline $\mathrm{O}$ & 4.30130900 & -0.82949100 & -0.92641200 & $\mathrm{H}$ & 3.92629900 & -3.52587500 & 0.96387700 \\
\hline $\mathrm{O}$ & 4.05515200 & 0.57601700 & -2.67947400 & $\mathrm{H}$ & 2.68372300 & -4.69442500 & 0.48293000 \\
\hline $\mathrm{C}$ & 5.32962600 & 0.13733800 & -3.20081700 & $\mathrm{C}$ & 3.67354400 & -1.83089800 & -1.25438500 \\
\hline $\mathrm{H}$ & 5.30170300 & -0.93399300 & -3.40959000 & $\mathrm{H}$ & 4.30996400 & -1.39603700 & -0.48005100 \\
\hline $\mathrm{H}$ & 5.47918200 & 0.70627900 & -4.11683300 & $\mathrm{H}$ & 4.32654000 & -2.37142800 & -1.94956200 \\
\hline $\mathrm{H}$ & 6.12279500 & 0.34676500 & -2.48033400 & $\mathrm{H}$ & 3.19539900 & -1.01893100 & -1.80819400 \\
\hline $\mathrm{C}$ & 3.09814600 & 2.04130900 & 0.76251700 & $\mathrm{C}$ & -2.78256300 & -3.65619200 & -1.93339900 \\
\hline $\mathrm{C}$ & 2.58480400 & 3.34081700 & 0.65099400 & $\mathrm{H}$ & -1.87774400 & -3.33325100 & -2.45701100 \\
\hline $\mathrm{C}$ & 4.42129100 & 1.87520000 & 1.19333800 & $\mathrm{H}$ & -2.50337600 & -4.41870800 & -1.19950100 \\
\hline $\mathrm{C}$ & 3.37411000 & 4.44924900 & 0.95806700 & $\mathrm{H}$ & -3.43985800 & -4.13943900 & -2.66461800 \\
\hline $\mathrm{H}$ & 1.55503000 & 3.48458000 & 0.32932000 & $\mathrm{C}$ & -4.79309200 & -2.96220500 & -0.57300600 \\
\hline
\end{tabular}




\begin{tabular}{|c|c|c|c|c|c|c|c|}
\hline $\mathrm{H}$ & -5.39167800 & -2.13819100 & -0.17341400 & $\mathrm{C}$ & 3.01053600 & 2.24288800 & -1.46402900 \\
\hline $\mathrm{H}$ & -5.42746600 & -3.49831600 & -1.28840400 & $\mathrm{O}$ & 3.85115200 & 1.69195600 & -0.77908500 \\
\hline $\mathrm{H}$ & -4.56851800 & -3.65822100 & 0.24045000 & $\mathrm{O}$ & 3.27984300 & 2.88143700 & -2.60683000 \\
\hline $\mathrm{C}$ & -3.91698500 & -1.46631000 & -2.39961000 & $\mathrm{C}$ & 4.65247100 & 2.83113500 & -3.05041000 \\
\hline $\mathrm{H}$ & -4.58586400 & -1.95831300 & -3.11435700 & $\mathrm{H}$ & 4.94716900 & 1.79740500 & -3.24296100 \\
\hline $\mathrm{H}$ & -4.45282900 & -0.59631700 & -2.00410300 & $\mathrm{H}$ & 4.68230400 & 3.41640700 & -3.96807100 \\
\hline $\mathrm{H}$ & -3.04228900 & -1.11635600 & -2.95494600 & $\mathrm{H}$ & 5.31241900 & 3.26086600 & -2.29395000 \\
\hline $\mathrm{C}$ & 2.41953400 & -1.26869300 & 1.88894300 & $\mathrm{C}$ & 0.12103700 & 3.39408700 & 0.79634400 \\
\hline $\mathrm{C}$ & 3.30666900 & -0.18985400 & 1.76878100 & $\mathrm{C}$ & -0.37406700 & 4.45652500 & 0.02650700 \\
\hline $\mathrm{C}$ & 2.93735500 & -1.53596000 & 4.11773900 & $\mathrm{C}$ & -0.40496400 & 3.22091100 & 2.08772900 \\
\hline $\mathrm{C}$ & 4.04434400 & 0.19259500 & 2.88992700 & $\mathrm{C}$ & -1.35166100 & 5.32027900 & 0.53181900 \\
\hline $\mathrm{H}$ & 3.42602300 & 0.34363800 & 0.83216400 & $\mathrm{H}$ & 0.01297200 & 4.63971100 & -0.97086300 \\
\hline $\mathrm{C}$ & 3.85987200 & -0.48977300 & 4.09110500 & $\mathrm{C}$ & -1.37438900 & 4.08435800 & 2.60068000 \\
\hline $\mathrm{H}$ & 2.75539900 & -2.09094400 & 5.03592800 & $\mathrm{H}$ & -0.02500900 & 2.41282400 & 2.70867300 \\
\hline $\mathrm{H}$ & 4.74983400 & 1.01541100 & 2.81989200 & $\mathrm{C}$ & -1.85324800 & 5.14236300 & 1.82327300 \\
\hline $\mathrm{H}$ & 4.41105800 & -0.22171400 & 4.98658900 & $\mathrm{H}$ & -1.70344800 & 6.14630100 & -0.07987700 \\
\hline $\mathrm{C}$ & -3.20812600 & -0.15934000 & 0.54925100 & $\mathrm{H}$ & -1.74423200 & 3.93871400 & 3.61187600 \\
\hline $\mathrm{C}$ & -4.25538200 & -0.19871100 & 1.46947300 & $\mathrm{H}$ & -2.59348200 & 5.82912500 & 2.22349800 \\
\hline $\mathrm{C}$ & -3.38144300 & 2.22496300 & 0.50382100 & & & & \\
\hline $\mathrm{C}$ & -4.87618700 & 0.98102800 & 1.89062400 & 33 & & & \\
\hline $\mathrm{H}$ & -4.59189000 & -1.15231600 & 1.85602100 & $\mathrm{C}$ & 0.97687100 & 3.06956200 & -1.11366100 \\
\hline $\mathrm{C}$ & -4.43626200 & 2.21092700 & 1.40337100 & $\mathrm{H}$ & 1.03717400 & 3.01845300 & -2.20657900 \\
\hline $\mathrm{H}$ & -2.94535200 & 3.13262700 & 0.10181800 & $\mathrm{H}$ & 0.68307400 & 4.09089700 & -0.85595800 \\
\hline $\mathrm{H}$ & -5.69649600 & 0.93569800 & 2.60041100 & $\mathrm{C}$ & 2.34782500 & 2.76152200 & -0.49272600 \\
\hline $\mathrm{H}$ & -4.88336300 & 3.14498500 & 1.72087300 & $\mathrm{H}$ & 2.95105500 & 3.67381600 & -0.56350300 \\
\hline $\mathrm{N}$ & 2.23424600 & -1.92487200 & 3.04699200 & $\mathrm{H}$ & 2.25558200 & 2.55677300 & 0.58006300 \\
\hline $\mathrm{N}$ & -2.81933800 & 1.06885600 & 0.10528000 & $\mathrm{C}$ & 3.13936900 & 1.64409400 & -1.18599600 \\
\hline $\mathrm{Pd}$ & -0.10759100 & -0.50190200 & -0.74282100 & $\mathrm{H}$ & 4.16290600 & 1.63858600 & -0.80392800 \\
\hline $\mathrm{C}$ & 0.87598700 & 1.05126700 & -1.79886400 & $\mathrm{H}$ & 3.17685700 & 1.82543600 & -2.26575700 \\
\hline $\mathrm{O}$ & 1.23806300 & 0.61025200 & -2.87167100 & $\mathrm{P}$ & 2.48651800 & -0.06826300 & -0.92579200 \\
\hline $\mathrm{O}$ & -0.80653800 & 1.41649700 & -1.72703400 & $\mathrm{P}$ & -0.45552400 & 2.00592400 & -0.57475200 \\
\hline $\mathrm{H}$ & -2.00013900 & 1.15430300 & -0.55982700 & $\mathrm{C}$ & -1.90227100 & 2.66113900 & -1.65139600 \\
\hline $\mathrm{C}$ & -1.39609900 & 1.41022100 & -3.03253900 & $\mathrm{C}$ & 3.54223600 & -1.15042800 & -2.09746100 \\
\hline $\mathrm{H}$ & -0.90570100 & 0.67941600 & -3.68040600 & $\mathrm{C}$ & -1.82742200 & 1.91200000 & -2.99813500 \\
\hline $\mathrm{H}$ & -2.46032600 & 1.16685800 & -2.94593700 & $\mathrm{H}$ & -2.63139100 & 2.27127200 & -3.64998000 \\
\hline $\mathrm{H}$ & -1.29368200 & 2.40560300 & -3.48073200 & $\mathrm{H}$ & -0.88171200 & 2.09153700 & -3.52147900 \\
\hline $\mathrm{C}$ & 1.27310200 & 2.49830000 & 0.36589000 & $\mathrm{H}$ & -1.94677600 & 0.83169200 & -2.87952900 \\
\hline $\mathrm{H}$ & 1.15593700 & 1.51477800 & 0.83247000 & $\mathrm{C}$ & -1.77675500 & 4.17855800 & -1.91331600 \\
\hline $\mathrm{H}$ & 2.18950800 & 2.91536000 & 0.80076900 & $\mathrm{H}$ & -2.65570300 & 4.49511000 & -2.48618200 \\
\hline $\mathrm{C}$ & 1.51904000 & 2.30196800 & -1.13988600 & $\mathrm{H}$ & -1.75593900 & 4.74570900 & -0.98164100 \\
\hline $\mathrm{H}$ & 1.11744700 & 3.13466600 & -1.72078600 & $\mathrm{H}$ & -0.89826800 & 4.43291700 & -2.51176300 \\
\hline
\end{tabular}




\begin{tabular}{|c|c|c|c|c|c|c|c|}
\hline $\mathrm{C}$ & -3.25520500 & 2.39787700 & -0.96246000 & $\mathrm{O}$ & -4.78386000 & -0.61785700 & 0.37392000 \\
\hline $\mathrm{H}$ & -3.33625000 & 2.95093900 & -0.02466200 & $\mathrm{O}$ & -5.13122400 & -2.45811100 & -0.89302400 \\
\hline $\mathrm{H}$ & -4.05263700 & 2.75280200 & -1.62525300 & $\mathrm{C}$ & -6.50284200 & -2.49338700 & -0.44090300 \\
\hline $\mathrm{H}$ & -3.45299200 & 1.34488500 & -0.76071400 & $\mathrm{H}$ & -6.54085300 & -2.60099200 & 0.64507100 \\
\hline $\mathrm{C}$ & 3.02219600 & -0.94951100 & -3.53515400 & $\mathrm{H}$ & -7.02083900 & -1.57593400 & -0.72848900 \\
\hline $\mathrm{H}$ & 1.98170800 & -1.26350900 & -3.65199300 & $\mathrm{H}$ & -6.94839000 & -3.35782600 & -0.93078500 \\
\hline $\mathrm{H}$ & 3.10354600 & 0.09129500 & -3.86442500 & $\mathrm{O}$ & -0.19651900 & -2.80454900 & -2.75385300 \\
\hline $\mathrm{H}$ & 3.62558500 & -1.55251100 & -4.22262800 & $\mathrm{C}$ & -0.09667200 & -2.00216800 & -1.94536600 \\
\hline $\mathrm{C}$ & 5.02944600 & -0.74788800 & -2.02469500 & $\mathrm{H}$ & -3.02879400 & -0.85813200 & -1.91599400 \\
\hline $\mathrm{H}$ & 5.42082100 & -0.78871300 & -1.00523000 & $\mathrm{H}$ & -2.35869100 & 0.02439800 & 0.39388200 \\
\hline $\mathrm{H}$ & 5.61188800 & -1.44497400 & -2.63769600 & $\mathrm{C}$ & -1.52707800 & -1.79572700 & 1.08626700 \\
\hline $\mathrm{H}$ & 5.20654600 & 0.25603100 & -2.41910400 & $\mathrm{C}$ & -1.49962200 & -1.29960200 & 2.40721600 \\
\hline $\mathrm{C}$ & 3.40120700 & -2.62738500 & -1.68368800 & $\mathrm{C}$ & -1.16836100 & -3.14786000 & 0.88933700 \\
\hline $\mathrm{H}$ & 3.94086800 & -3.25260600 & -2.40326800 & $\mathrm{C}$ & -1.12349000 & -2.10879600 & 3.47698400 \\
\hline $\mathrm{H}$ & 3.83040700 & -2.81570100 & -0.69577700 & $\mathrm{H}$ & -1.81528000 & -0.27778800 & 2.59128600 \\
\hline $\mathrm{H}$ & 2.36246900 & -2.96803900 & -1.67552600 & $\mathrm{C}$ & -0.79085800 & -3.95715500 & 1.95845000 \\
\hline $\mathrm{C}$ & -0.67319500 & 2.61697300 & 1.16448100 & $\mathrm{H}$ & -1.20093500 & -3.58071200 & -0.10551900 \\
\hline $\mathrm{C}$ & -0.12566900 & 1.90719700 & 2.23940700 & $\mathrm{C}$ & -0.76085800 & -3.44023800 & 3.25760000 \\
\hline $\mathrm{C}$ & -1.38739100 & 4.31015200 & 2.54670500 & $\mathrm{H}$ & -1.13339300 & -1.70693400 & 4.48594800 \\
\hline $\mathrm{C}$ & -0.22295500 & 2.45659900 & 3.52062600 & $\mathrm{H}$ & -0.53182300 & -4.99653900 & 1.78021100 \\
\hline $\mathrm{H}$ & 0.36438500 & 0.95304800 & 2.07953300 & $\mathrm{H}$ & -0.47888900 & -4.07617300 & 4.09135800 \\
\hline $\mathrm{C}$ & -0.87055500 & 3.67822800 & 3.68118600 & & & & \\
\hline $\mathrm{H}$ & -1.89894300 & 5.26622200 & 2.62954800 & \multirow{2}{*}{\multicolumn{4}{|c|}{$\begin{array}{l}\text { TS30 } \\
\text { imaginary frequency }=-144.92 \mathrm{~cm}^{-1}\end{array}$}} \\
\hline $\mathrm{H}$ & 0.19807300 & 1.93501900 & 4.37493500 & & & & \\
\hline $\mathrm{H}$ & -0.97462400 & 4.13857300 & 4.65802800 & $\mathrm{C}$ & 1.69639300 & 2.12324700 & -2.19193900 \\
\hline $\mathrm{C}$ & 3.11295700 & -0.46399200 & 0.78187900 & $\mathrm{H}$ & 1.37187700 & 1.80378000 & -3.18898300 \\
\hline $\mathrm{C}$ & 2.46526500 & -1.41153200 & 1.58583100 & $\mathrm{H}$ & 1.84380200 & 3.20491200 & -2.21618900 \\
\hline $\mathrm{C}$ & 4.70462500 & -0.07422400 & 2.39731800 & $\mathrm{C}$ & 3.02910800 & 1.45551700 & -1.82156100 \\
\hline $\mathrm{C}$ & 2.99274100 & -1.68879300 & 2.84874700 & $\mathrm{H}$ & 3.80725900 & 1.93626700 & -2.42568000 \\
\hline $\mathrm{H}$ & 1.57230700 & -1.92117800 & 1.23829600 & $\mathrm{H}$ & 3.29326300 & 1.67596500 & -0.78163300 \\
\hline $\mathrm{C}$ & 4.13519100 & -1.00897600 & 3.26535000 & $\mathrm{C}$ & 3.13066200 & -0.05326800 & -2.08962000 \\
\hline $\mathrm{H}$ & 5.59425900 & 0.48076000 & 2.68641900 & $\mathrm{H}$ & 4.18059900 & -0.34882600 & -2.03251600 \\
\hline $\mathrm{H}$ & 2.51206400 & -2.42011100 & 3.49093600 & $\mathrm{H}$ & 2.76424000 & -0.28967600 & -3.09505500 \\
\hline $\mathrm{H}$ & 4.57858900 & -1.19192700 & 4.23858000 & $\mathrm{P}$ & 2.24473100 & -1.16097500 & -0.90028200 \\
\hline $\mathrm{N}$ & -1.28609500 & 3.80159100 & 1.31409500 & $\mathrm{P}$ & 0.29323100 & 1.83338700 & -1.01345700 \\
\hline $\mathrm{N}$ & 4.21099400 & 0.19608100 & 1.18314000 & $\mathrm{C}$ & -1.15898000 & 2.82599100 & -1.77555000 \\
\hline $\mathrm{Pd}$ & -0.00208500 & -0.37260100 & -0.82092200 & $\mathrm{C}$ & 2.54037600 & -2.92257800 & -1.57773800 \\
\hline $\mathrm{H}$ & -2.74483900 & -2.48651900 & -1.33282800 & $\mathrm{C}$ & -1.79610500 & 1.94478800 & -2.86839500 \\
\hline $\mathrm{C}$ & -1.96608000 & -0.89938500 & -0.02091700 & $\mathrm{H}$ & -2.59143300 & 2.50942300 & -3.36730200 \\
\hline $\mathrm{C}$ & -2.97927600 & -1.47110800 & -1.00903600 & $\mathrm{H}$ & -1.07280500 & 1.65296000 & -3.63755900 \\
\hline $\mathrm{C}$ & -4.38548400 & -1.45478000 & -0.40985800 & $\mathrm{H}$ & -2.25420600 & 1.04304900 & -2.45450500 \\
\hline
\end{tabular}




\begin{tabular}{|c|c|c|c|c|c|c|c|}
\hline $\mathrm{C}$ & -0.66310400 & 4.14726400 & -2.39698900 & $\mathrm{H}$ & -3.59841800 & -2.47809900 & -0.52748500 \\
\hline $\mathrm{H}$ & -1.53507600 & 4.72077000 & -2.73077600 & $\mathrm{C}$ & -2.45481100 & -0.74398500 & 0.19322800 \\
\hline $\mathrm{H}$ & -0.10839500 & 4.75805500 & -1.68076300 & $\mathrm{C}$ & -3.34377400 & -1.45828600 & -0.81781600 \\
\hline $\mathrm{H}$ & -0.03123900 & 3.98214700 & -3.27348200 & $\mathrm{C}$ & -4.62179000 & -0.65191200 & -1.04986600 \\
\hline $\mathrm{C}$ & -2.19523300 & 3.14689800 & -0.68099500 & $\mathrm{O}$ & -4.66588800 & 0.56164700 & -1.05882900 \\
\hline $\mathrm{H}$ & -1.76818900 & 3.75629400 & 0.12079700 & $\mathrm{O}$ & -5.66685600 & -1.45358300 & -1.28161200 \\
\hline $\mathrm{H}$ & -3.01375400 & 3.71995100 & -1.13004600 & $\mathrm{C}$ & -6.91767900 & -0.79037400 & -1.57270900 \\
\hline $\mathrm{H}$ & -2.65160100 & 2.25387100 & -0.24821400 & $\mathrm{H}$ & -7.22216400 & -0.17155500 & -0.72618600 \\
\hline $\mathrm{C}$ & 1.54047500 & -3.16449100 & -2.72563000 & $\mathrm{H}$ & -6.81604100 & -0.16221800 & -2.46010600 \\
\hline $\mathrm{H}$ & 0.50246200 & -3.11195300 & -2.38473600 & $\mathrm{H}$ & -7.63632800 & -1.58993100 & -1.74510400 \\
\hline $\mathrm{H}$ & 1.66890800 & -2.44983900 & -3.54548300 & $\mathrm{O}$ & -1.09588300 & -3.30874200 & 0.33624600 \\
\hline $\mathrm{H}$ & 1.70405600 & -4.16553100 & -3.13969500 & $\mathrm{C}$ & -0.83871600 & -2.21363600 & 0.06913000 \\
\hline $\mathrm{C}$ & 3.98435300 & -3.06614500 & -2.10308200 & $\mathrm{H}$ & -2.84522300 & -1.52257000 & -1.79284000 \\
\hline $\mathrm{H}$ & 4.72829400 & -2.80361800 & -1.34626000 & $\mathrm{H}$ & -2.35153200 & 0.29580000 & -0.09806900 \\
\hline $\mathrm{H}$ & 4.14763000 & -4.11177300 & -2.38680900 & $\mathrm{C}$ & -2.76569100 & -0.85295700 & 1.64059600 \\
\hline $\mathrm{H}$ & 4.16593700 & -2.45695900 & -2.99206300 & $\mathrm{C}$ & -2.58660400 & 0.27334800 & 2.46846700 \\
\hline $\mathrm{C}$ & 2.31305000 & -3.94595100 & -0.44877900 & $\mathrm{C}$ & -3.27042600 & -2.03064600 & 2.22477900 \\
\hline $\mathrm{H}$ & 2.42642000 & -4.95466000 & -0.86088300 & $\mathrm{C}$ & -2.89725200 & 0.22703200 & 3.82469300 \\
\hline $\mathrm{H}$ & 3.04724300 & -3.83482700 & 0.35381300 & $\mathrm{H}$ & -2.22691600 & 1.20112700 & 2.03260700 \\
\hline $\mathrm{H}$ & 1.31324300 & -3.88385100 & -0.01531000 & $\mathrm{C}$ & -3.58039800 & -2.07586600 & 3.58287600 \\
\hline $\mathrm{C}$ & 0.85552600 & 2.81380300 & 0.46102700 & $\mathrm{H}$ & -3.42303900 & -2.91972800 & 1.62255000 \\
\hline $\mathrm{C}$ & 0.62568400 & 2.35766800 & 1.76430100 & $\mathrm{C}$ & -3.39248700 & -0.95130800 & 4.38939300 \\
\hline $\mathrm{C}$ & 1.89485800 & 4.71566900 & 1.23234900 & $\mathrm{H}$ & -2.76776800 & 1.11312900 & 4.43909700 \\
\hline $\mathrm{C}$ & 1.05498400 & 3.14461200 & 2.83528100 & $\mathrm{H}$ & -3.97349200 & -2.99259800 & 4.01173800 \\
\hline $\mathrm{H}$ & 0.12388500 & 1.41097500 & 1.93540400 & $\mathrm{H}$ & -3.63839200 & -0.99008300 & 5.44605200 \\
\hline $\mathrm{C}$ & 1.70159700 & 4.34842100 & 2.56673100 & & & & \\
\hline $\mathrm{H}$ & 2.40068700 & 5.64534100 & 0.98163800 & 34 & & & \\
\hline $\mathrm{H}$ & 0.88890200 & 2.81997900 & 3.85813700 & $\mathrm{C}$ & 2.42826000 & 2.43340200 & -0.88902900 \\
\hline $\mathrm{H}$ & 2.05385500 & 4.99124300 & 3.36662100 & $\mathrm{H}$ & 2.60767500 & 2.09958900 & -1.91631700 \\
\hline $\mathrm{C}$ & 3.28476100 & -1.03083100 & 0.62938700 & $\mathrm{H}$ & 2.60621300 & 3.51059300 & -0.85147400 \\
\hline $\mathrm{C}$ & 2.73181600 & -1.21746800 & 1.90164900 & $\mathrm{C}$ & 3.37952700 & 1.72387100 & 0.09761500 \\
\hline $\mathrm{C}$ & 5.38528600 & -0.70269000 & 1.50407400 & $\mathrm{H}$ & 4.30139800 & 2.31358400 & 0.15693000 \\
\hline $\mathrm{C}$ & 3.57496500 & -1.14708400 & 3.01182200 & $\mathrm{H}$ & 2.95778900 & 1.73912000 & 1.11000000 \\
\hline $\mathrm{H}$ & 1.67113200 & -1.41553200 & 2.01699800 & $\mathrm{C}$ & 3.77837400 & 0.29460000 & -0.30364600 \\
\hline $\mathrm{C}$ & 4.92838100 & -0.88331800 & 2.81185000 & $\mathrm{H}$ & 4.52187300 & -0.09674700 & 0.39695400 \\
\hline $\mathrm{H}$ & 6.43380300 & -0.49173000 & 1.30694800 & $\mathrm{H}$ & 4.26825500 & 0.32722400 & -1.28085100 \\
\hline $\mathrm{H}$ & 3.17991000 & -1.29423600 & 4.01246600 & $\mathrm{P}$ & 2.37558100 & -0.94084800 & -0.42220100 \\
\hline $\mathrm{H}$ & 5.61934700 & -0.81668400 & 3.64562800 & $\mathrm{P}$ & 0.62190500 & 2.24220200 & -0.50184500 \\
\hline $\mathrm{N}$ & 1.48514800 & 3.97091800 & 0.19959200 & $\mathrm{C}$ & -0.29473700 & 3.19666900 & -1.87840800 \\
\hline $\mathrm{N}$ & 4.58755500 & -0.77538900 & 0.43199000 & $\mathrm{C}$ & 2.76088300 & -1.92825700 & -2.00659300 \\
\hline $\mathrm{Pd}$ & -0.03685700 & -0.56123500 & -0.44552100 & $\mathrm{C}$ & -0.47516700 & 2.24084200 & -3.07360800 \\
\hline
\end{tabular}




\begin{tabular}{|c|c|c|c|c|c|c|c|}
\hline $\mathrm{H}$ & -0.98876800 & 2.76879400 & -3.88484800 & $\mathrm{~N}$ & 1.12280000 & 4.48509200 & 0.93053800 \\
\hline $\mathrm{H}$ & 0.48321600 & 1.88931100 & -3.47011300 & $\mathrm{~N}$ & 2.47573700 & -3.36613000 & 0.87349100 \\
\hline $\mathrm{H}$ & -1.07472400 & 1.36478500 & -2.80787500 & $\mathrm{Pd}$ & 0.04089800 & 0.00090600 & -0.19003300 \\
\hline $\mathrm{C}$ & 0.50332200 & 4.43800800 & -2.33001900 & $\mathrm{C}$ & -1.93560500 & 0.40174900 & 0.13328800 \\
\hline $\mathrm{H}$ & -0.10127000 & 4.99320600 & -3.05615900 & $\mathrm{O}$ & -2.34021500 & 1.02349800 & 1.07990400 \\
\hline $\mathrm{H}$ & 0.73075900 & 5.10190200 & -1.49371500 & $\mathrm{C}$ & -2.31538100 & -1.58081800 & -1.52355600 \\
\hline $\mathrm{H}$ & 1.43969000 & 4.16954700 & -2.82689500 & $\mathrm{H}$ & -3.09612200 & -2.13479700 & -2.04592000 \\
\hline $\mathrm{C}$ & -1.66888000 & 3.64764600 & -1.34684200 & $\mathrm{H}$ & -1.56694700 & -1.26316700 & -2.25975900 \\
\hline $\mathrm{H}$ & -1.56944400 & 4.38140900 & -0.54286800 & $\mathrm{C}$ & -2.93044800 & -0.33942200 & -0.82266300 \\
\hline $\mathrm{H}$ & -2.22607600 & 4.12174100 & -2.16240200 & $\mathrm{H}$ & -3.10757800 & 0.39953000 & -1.61520500 \\
\hline $\mathrm{H}$ & -2.27175600 & 2.81809600 & -0.97053000 & $\mathrm{C}$ & -4.26056100 & -0.65494100 & -0.15883800 \\
\hline $\mathrm{C}$ & 2.70734300 & -0.93760800 & -3.18992000 & $\mathrm{C}$ & -5.45235500 & -0.24124700 & -0.76543900 \\
\hline $\mathrm{H}$ & 1.74498700 & -0.41633600 & -3.24118100 & $\mathrm{C}$ & -4.32804800 & -1.39425600 & 1.03070200 \\
\hline $\mathrm{H}$ & 3.50475200 & -0.18941300 & -3.16161400 & $\mathrm{C}$ & -6.68838700 & -0.56673400 & -0.20409700 \\
\hline $\mathrm{H}$ & 2.82974500 & -1.49705900 & -4.12384400 & $\mathrm{H}$ & -5.41681700 & 0.33838900 & -1.68489200 \\
\hline $\mathrm{C}$ & 4.14876300 & -2.59232800 & -1.93043500 & $\mathrm{C}$ & -5.56170700 & -1.71925700 & 1.59291700 \\
\hline $\mathrm{H}$ & 4.19085100 & -3.33652600 & -1.13234400 & $\mathrm{H}$ & -3.41628400 & -1.69868000 & 1.53841300 \\
\hline $\mathrm{H}$ & 4.35522500 & -3.10359300 & -2.87798000 & $\mathrm{C}$ & -6.74544800 & -1.30848200 & 0.97572700 \\
\hline $\mathrm{H}$ & 4.95146600 & -1.86529000 & -1.77316000 & $\mathrm{H}$ & -7.60317800 & -0.23795700 & -0.68775800 \\
\hline $\mathrm{C}$ & 1.67849000 & -3.00372100 & -2.22826300 & $\mathrm{H}$ & -5.59949400 & -2.28751200 & 2.51750800 \\
\hline $\mathrm{H}$ & 1.90889500 & -3.55299000 & -3.14845900 & $\mathrm{H}$ & -7.70527800 & -1.56057700 & 1.41593600 \\
\hline $\mathrm{H}$ & 1.64640800 & -3.71008300 & -1.39858600 & $\mathrm{C}$ & -1.59853900 & -2.48206200 & -0.55248200 \\
\hline $\mathrm{H}$ & 0.68946800 & -2.55229600 & -2.35588300 & $\mathrm{O}$ & -0.67912900 & -2.08020800 & 0.17430100 \\
\hline $\mathrm{C}$ & 0.51908200 & 3.28879200 & 1.03211300 & $\mathrm{O}$ & -2.01408100 & -3.72909800 & -0.54074400 \\
\hline $\mathrm{C}$ & -0.10351100 & 2.84707200 & 2.20292400 & $\mathrm{C}$ & -1.35719500 & -4.64685800 & 0.37392500 \\
\hline $\mathrm{C}$ & 1.11483100 & 5.28897500 & 1.99875500 & $\mathrm{H}$ & -0.27445200 & -4.57316700 & 0.26721200 \\
\hline $\mathrm{C}$ & -0.09794200 & 3.69480500 & 3.31419000 & $\mathrm{H}$ & -1.64693400 & -4.40551100 & 1.39862100 \\
\hline $\mathrm{H}$ & -0.60363600 & 1.88786900 & 2.23205200 & $\mathrm{H}$ & -1.72118400 & -5.63328600 & 0.09405800 \\
\hline $\mathrm{C}$ & 0.52042800 & 4.93787000 & 3.21400000 & & & & \\
\hline $\mathrm{H}$ & 1.60820700 & 6.25091200 & 1.87933700 & $34 \mathbf{a}$ & & & \\
\hline $\mathrm{H}$ & -0.57474400 & 3.38476900 & 4.23912800 & $\mathrm{C}$ & -0.75963300 & -3.22196900 & 0.36197900 \\
\hline $\mathrm{H}$ & 0.54613300 & 5.62587000 & 4.05264400 & $\mathrm{H}$ & -1.44083400 & -3.65264500 & -0.37833700 \\
\hline $\mathrm{C}$ & 2.69034500 & -2.05095500 & 1.03362200 & $\mathrm{H}$ & -0.17152000 & -4.03625700 & 0.78936700 \\
\hline $\mathrm{C}$ & 3.06439400 & -1.49833700 & 2.26857100 & $\mathrm{C}$ & -1.56542200 & -2.53381700 & 1.48352800 \\
\hline $\mathrm{C}$ & 2.65744200 & -4.17120900 & 1.92904500 & $\mathrm{H}$ & -1.79502200 & -3.29502200 & 2.23800000 \\
\hline $\mathrm{C}$ & 3.24001600 & -2.34626600 & 3.36069900 & $\mathrm{H}$ & -0.96484900 & -1.77054900 & 1.98954900 \\
\hline $\mathrm{H}$ & 3.21397800 & -0.42945300 & 2.38072000 & $\mathrm{C}$ & -2.90529700 & -1.94377900 & 1.00416200 \\
\hline $\mathrm{C}$ & 3.03860000 & -3.71509800 & 3.19042900 & $\mathrm{H}$ & -3.57405900 & -1.78089000 & 1.85304000 \\
\hline $\mathrm{H}$ & 2.49152500 & -5.23201000 & 1.75320200 & $\mathrm{H}$ & -3.41154600 & -2.67827600 & 0.36912200 \\
\hline $\mathrm{H}$ & 3.53240600 & -1.94365500 & 4.32584300 & $\mathrm{P}$ & -2.74797100 & -0.38359800 & -0.00300400 \\
\hline $\mathrm{H}$ & 3.17067400 & -4.41306900 & 4.01026700 & $\mathrm{P}$ & 0.46517400 & -2.16975300 & -0.55939100 \\
\hline
\end{tabular}




\begin{tabular}{|c|c|c|c|c|c|c|c|}
\hline $\mathrm{C}$ & 0.64686200 & -3.03998700 & -2.25330600 & $\mathrm{H}$ & -2.71205500 & 4.25910900 & 1.04975400 \\
\hline $\mathrm{C}$ & -4.33431900 & -0.38481900 & -1.08356300 & $\mathrm{H}$ & -4.19494700 & 1.86245700 & 4.29982200 \\
\hline $\mathrm{C}$ & -0.65070500 & -2.78784600 & -3.04498900 & $\mathrm{H}$ & -3.64017100 & 4.12992800 & 3.36667200 \\
\hline $\mathrm{H}$ & -0.56924800 & -3.26664000 & -4.02713800 & $\mathrm{~N}$ & 1.92446500 & -3.37526100 & 1.41796100 \\
\hline $\mathrm{H}$ & -1.53455100 & -3.20616700 & -2.55433900 & $\mathrm{~N}$ & -2.76863000 & 2.22107900 & 0.72669500 \\
\hline $\mathrm{H}$ & -0.81498300 & -1.71721300 & -3.20382500 & $\mathrm{Pd}$ & -0.28181300 & 0.05913300 & -0.70972900 \\
\hline $\mathrm{C}$ & 0.86740700 & -4.55339700 & -2.05799600 & $\mathrm{C}$ & 1.57675600 & 0.81406800 & -0.85851600 \\
\hline $\mathrm{H}$ & 1.00501500 & -5.01995700 & -3.04009500 & $\mathrm{O}$ & 2.36677800 & 0.56586200 & -1.72977100 \\
\hline $\mathrm{H}$ & 1.76262300 & -4.76338100 & -1.46572400 & $\mathrm{O}$ & -0.87944500 & 2.18912100 & -1.16602400 \\
\hline $\mathrm{H}$ & 0.01689500 & -5.04639900 & -1.57952200 & $\mathrm{H}$ & -1.70324900 & 2.32409400 & -0.61525400 \\
\hline $\mathrm{C}$ & 1.82870800 & -2.44712400 & -3.04517600 & $\mathrm{C}$ & -0.99651100 & 2.71386200 & -2.49418000 \\
\hline $\mathrm{H}$ & 2.79113700 & -2.71638800 & -2.60351400 & $\mathrm{H}$ & -1.36723000 & 3.74344100 & -2.45860600 \\
\hline $\mathrm{H}$ & 1.81266700 & -2.86547000 & -4.05789300 & $\mathrm{H}$ & -0.00021700 & 2.71607600 & -2.93863100 \\
\hline $\mathrm{H}$ & 1.77699800 & -1.35899400 & -3.12315100 & $\mathrm{H}$ & -1.66594800 & 2.11021900 & -3.11682000 \\
\hline $\mathrm{C}$ & -4.20550400 & -1.52723500 & -2.11032000 & $\mathrm{C}$ & 2.57218700 & 1.29428400 & 1.43662700 \\
\hline $\mathrm{H}$ & -3.32096500 & -1.40604700 & -2.74160700 & $\mathrm{H}$ & 3.43230800 & 0.68959300 & 1.13627900 \\
\hline $\mathrm{H}$ & -4.16350200 & -2.51609100 & -1.64337200 & $\mathrm{H}$ & 2.98184300 & 2.11326800 & 2.03988300 \\
\hline $\mathrm{H}$ & -5.08451700 & -1.52046300 & -2.76439100 & $\mathrm{C}$ & 1.82592600 & 1.90781100 & 0.23116100 \\
\hline $\mathrm{C}$ & -5.59723500 & -0.58546000 & -0.22537700 & $\mathrm{H}$ & 0.85073900 & 2.23687200 & 0.58939900 \\
\hline $\mathrm{H}$ & -5.72578700 & 0.21572500 & 0.50825600 & $\mathrm{C}$ & 2.55502100 & 3.10296200 & -0.36251700 \\
\hline $\mathrm{H}$ & -6.48005800 & -0.58039400 & -0.87506800 & $\mathrm{C}$ & 1.88231500 & 4.32610100 & -0.48070600 \\
\hline $\mathrm{H}$ & -5.59334500 & -1.54140200 & 0.30670300 & $\mathrm{C}$ & 3.88858800 & 3.02262200 & -0.78913900 \\
\hline $\mathrm{C}$ & -4.43116700 & 0.95018000 & -1.84425200 & $\mathrm{C}$ & 2.52667400 & 5.44852900 & -1.00372200 \\
\hline $\mathrm{H}$ & -5.31549300 & 0.92933200 & -2.49100900 & $\mathrm{H}$ & 0.84665000 & 4.39631600 & -0.15931300 \\
\hline $\mathrm{H}$ & -4.53054000 & 1.80629300 & -1.17279100 & $\mathrm{C}$ & 4.53278500 & 4.14305000 & -1.31306300 \\
\hline $\mathrm{H}$ & -3.55914000 & 1.11280000 & -2.48435200 & $\mathrm{H}$ & 4.42573200 & 2.08211700 & -0.72255300 \\
\hline $\mathrm{C}$ & 2.05028900 & -2.55779800 & 0.36168700 & $\mathrm{C}$ & 3.85479700 & 5.35934300 & -1.42163700 \\
\hline $\mathrm{C}$ & 3.29396300 & -2.04309600 & -0.03779100 & $\mathrm{H}$ & 1.99210600 & 6.39071900 & -1.08247000 \\
\hline $\mathrm{C}$ & 3.02485200 & -3.72011100 & 2.09897300 & $\mathrm{H}$ & 5.56605100 & 4.06591600 & -1.63822000 \\
\hline $\mathrm{C}$ & 4.43407800 & -2.41876600 & 0.67309100 & $\mathrm{H}$ & 4.35909400 & 6.23029900 & -1.82911000 \\
\hline $\mathrm{H}$ & 3.37212900 & -1.36476200 & -0.87721700 & $\mathrm{C}$ & 1.68966600 & 0.47175600 & 2.35798500 \\
\hline $\mathrm{C}$ & 4.30313900 & -3.27561000 & 1.76422300 & $\mathrm{O}$ & 0.47467500 & 0.39709500 & 2.29811200 \\
\hline $\mathrm{H}$ & 2.87263400 & -4.38607000 & 2.94561600 & $\mathrm{O}$ & 2.42767600 & -0.14432500 & 3.29223800 \\
\hline $\mathrm{H}$ & 5.40943900 & -2.04588400 & 0.37475800 & $\mathrm{C}$ & 1.70332500 & -0.89869300 & 4.28469800 \\
\hline $\mathrm{H}$ & 5.16444700 & -3.59215900 & 2.34293400 & $\mathrm{H}$ & 0.95723800 & -0.26878500 & 4.77364900 \\
\hline $\mathrm{C}$ & -3.07526900 & 0.99583500 & 1.20851600 & $\mathrm{H}$ & 1.21181000 & -1.75672900 & 3.82093300 \\
\hline $\mathrm{C}$ & -3.58874200 & 0.84107900 & 2.50083900 & $\mathrm{H}$ & 2.45346300 & -1.23046800 & 5.00135900 \\
\hline $\mathrm{C}$ & -2.97616300 & 3.30159600 & 1.49149500 & & & & \\
\hline $\mathrm{C}$ & -3.79669300 & 1.96942400 & 3.29538900 & $34 b$ & & & \\
\hline $\mathrm{H}$ & -3.82788000 & -0.13861200 & 2.89453700 & $\mathrm{C}$ & 1.96561000 & 2.37864500 & 0.79587300 \\
\hline $\mathrm{C}$ & -3.49173000 & 3.22782700 & 2.78341700 & $\mathrm{H}$ & 2.93049000 & 2.42046300 & 0.28072400 \\
\hline
\end{tabular}




\begin{tabular}{|c|c|c|c|c|c|c|c|}
\hline $\mathrm{H}$ & 1.73752300 & 3.38302000 & 1.15971300 & $\mathrm{H}$ & -1.20964700 & 5.42520400 & 2.31804900 \\
\hline $\mathrm{C}$ & 2.02680900 & 1.40049400 & 1.99814200 & $\mathrm{H}$ & -3.96608900 & 3.96278000 & -0.64705100 \\
\hline $\mathrm{H}$ & 2.30668600 & 1.97922100 & 2.88587600 & $\mathrm{H}$ & -3.46945300 & 5.52945300 & 1.25534300 \\
\hline $\mathrm{H}$ & 1.03700500 & 0.98339600 & 2.20779600 & $\mathrm{C}$ & 1.92400000 & -2.34678800 & 0.84070400 \\
\hline $\mathrm{C}$ & 3.06337700 & 0.26096500 & 1.86056300 & $\mathrm{C}$ & 2.26348800 & -3.32264700 & 1.77711100 \\
\hline $\mathrm{H}$ & 3.05971500 & -0.36329900 & 2.76024500 & $\mathrm{C}$ & 0.08622000 & -3.59338000 & 0.12077600 \\
\hline $\mathrm{H}$ & 4.06821400 & 0.69070900 & 1.77864800 & $\mathrm{C}$ & 1.48749100 & -4.48240000 & 1.85572500 \\
\hline $\mathrm{P}$ & 2.79116800 & -0.78462400 & 0.34151100 & $\mathrm{H}$ & 3.11835000 & -3.18558300 & 2.42890700 \\
\hline$P$ & 0.65981700 & 2.01662800 & -0.48734300 & $\mathrm{C}$ & 0.39990600 & -4.63178300 & 0.99800200 \\
\hline $\mathrm{C}$ & 1.25135100 & 2.84118600 & -2.10402100 & $\mathrm{H}$ & -0.77626500 & -3.64952400 & -0.53463600 \\
\hline $\mathrm{C}$ & 4.50812400 & -1.34285200 & -0.27775000 & $\mathrm{H}$ & 1.73856700 & -5.26132300 & 2.56911700 \\
\hline $\mathrm{C}$ & 2.49985300 & 2.09003600 & -2.60172100 & $\mathrm{H}$ & -0.21056800 & -5.52806400 & 1.01215400 \\
\hline $\mathrm{H}$ & 2.81248900 & 2.51582200 & -3.56165000 & $\mathrm{~N}$ & -0.50213500 & 3.92662000 & 1.08979200 \\
\hline $\mathrm{H}$ & 3.34534100 & 2.18806000 & -1.91505800 & $\mathrm{~N}$ & 0.82013700 & -2.47308700 & 0.05605500 \\
\hline $\mathrm{H}$ & 2.29508500 & 1.02590400 & -2.75457900 & $\mathrm{Pd}$ & 0.47811300 & -0.33020300 & -0.57411300 \\
\hline $\mathrm{C}$ & 1.58900500 & 4.32460200 & -1.86014900 & $\mathrm{C}$ & -1.49515600 & -0.37281600 & -0.97737100 \\
\hline $\mathrm{H}$ & 1.91445200 & 4.77572900 & -2.80452600 & $\mathrm{O}$ & -1.92648700 & 0.11477400 & -1.99294500 \\
\hline $\mathrm{H}$ & 0.72343200 & 4.89037600 & -1.50516700 & $\mathrm{C}$ & -3.16748700 & -0.10168200 & 0.90720800 \\
\hline $\mathrm{H}$ & 2.40076200 & 4.45412500 & -1.13851500 & $\mathrm{H}$ & -3.57876100 & 0.71862300 & 0.31228800 \\
\hline $\mathrm{C}$ & 0.14286300 & 2.72054200 & -3.16910100 & $\mathrm{H}$ & -4.03022000 & -0.60476900 & 1.35897800 \\
\hline $\mathrm{H}$ & -0.72729100 & 3.33475300 & -2.92568500 & $\mathrm{C}$ & -2.39708300 & -1.12886100 & 0.03946700 \\
\hline $\mathrm{H}$ & 0.53561300 & 3.08176400 & -4.12601900 & $\mathrm{H}$ & -1.74035300 & -1.66448500 & 0.72578900 \\
\hline $\mathrm{H}$ & -0.19049600 & 1.68815700 & -3.30599700 & $\mathrm{C}$ & -3.34448100 & -2.13479300 & -0.61076100 \\
\hline $\mathrm{C}$ & 5.21083300 & -0.10376800 & -0.86725100 & $\mathrm{C}$ & -3.50212200 & -3.38572000 & 0.00834700 \\
\hline $\mathrm{H}$ & 4.65102400 & 0.31835100 & -1.70527900 & $\mathrm{C}$ & -4.11579800 & -1.84842000 & -1.74654300 \\
\hline $\mathrm{H}$ & 5.37115800 & 0.68366700 & -0.12345300 & $\mathrm{C}$ & -4.39729600 & -4.33041400 & -0.49557300 \\
\hline $\mathrm{H}$ & 6.19822800 & -0.39686500 & -1.24062500 & $\mathrm{H}$ & -2.93076700 & -3.61535000 & 0.90520700 \\
\hline $\mathrm{C}$ & 5.37503300 & -1.94179600 & 0.84526000 & $\mathrm{C}$ & -5.00640700 & -2.79667800 & -2.25307600 \\
\hline $\mathrm{H}$ & 4.95451400 & -2.86911500 & 1.24209500 & $\mathrm{H}$ & -4.01116300 & -0.89154200 & -2.24322400 \\
\hline $\mathrm{H}$ & 6.36412600 & -2.18692400 & 0.44085800 & $\mathrm{C}$ & -5.15056600 & -4.03885500 & -1.63322200 \\
\hline $\mathrm{H}$ & 5.52914600 & -1.24469800 & 1.67471600 & $\mathrm{H}$ & -4.50647900 & -5.28989900 & 0.00154700 \\
\hline $\mathrm{C}$ & 4.28969700 & -2.38395400 & -1.39285000 & $\mathrm{H}$ & -5.59174800 & -2.56000400 & -3.13665100 \\
\hline $\mathrm{H}$ & 5.25891500 & -2.66329100 & -1.82043700 & $\mathrm{H}$ & -5.84572400 & -4.77158700 & -2.03144000 \\
\hline $\mathrm{H}$ & 3.82142600 & -3.29808400 & -1.01618900 & $\mathrm{C}$ & -2.33670500 & 0.45878400 & 2.04912800 \\
\hline $\mathrm{H}$ & 3.66924800 & -1.98968000 & -2.20386300 & $\mathrm{O}$ & -1.22242300 & 0.07809100 & 2.36002100 \\
\hline $\mathrm{C}$ & -0.76098700 & 3.09411900 & 0.07045100 & $\mathrm{O}$ & -3.01342600 & 1.40648800 & 2.71110900 \\
\hline $\mathrm{C}$ & -2.00564900 & 3.06811400 & -0.57776300 & $\mathrm{C}$ & -2.36354400 & 1.95144000 & 3.87852200 \\
\hline $\mathrm{C}$ & -1.46507700 & 4.76674800 & 1.49063400 & $\mathrm{H}$ & -2.14775100 & 1.15836400 & 4.59772100 \\
\hline $\mathrm{C}$ & -2.99759000 & 3.95399900 & -0.15592500 & $\mathrm{H}$ & -1.43370800 & 2.44962200 & 3.59691700 \\
\hline $\mathrm{H}$ & -2.19620900 & 2.37456200 & -1.38731000 & $\mathrm{H}$ & -3.07103400 & 2.66570500 & 4.29724300 \\
\hline $\mathrm{C}$ & -2.72655800 & 4.82393500 & 0.89844800 & & & & \\
\hline
\end{tabular}




\begin{tabular}{|c|c|c|c|c|c|c|c|}
\hline 34-CO & & & & $\mathrm{C}$ & -4.89358700 & 0.89966100 & 0.11372800 \\
\hline $\mathrm{C}$ & -0.33382100 & 3.51521100 & 0.08995800 & $\mathrm{H}$ & -3.38338900 & 0.20956200 & -1.25824200 \\
\hline $\mathrm{H}$ & 0.23298100 & 4.08551600 & -0.65154500 & $\mathrm{C}$ & -5.17687000 & 1.80264800 & 1.13739900 \\
\hline $\mathrm{H}$ & -1.22548200 & 4.08936400 & 0.34780200 & $\mathrm{H}$ & -4.34386600 & 3.40234000 & 2.33205600 \\
\hline $\mathrm{C}$ & 0.52010100 & 3.32929400 & 1.35429600 & $\mathrm{H}$ & -5.64320000 & 0.19147600 & -0.22652800 \\
\hline $\mathrm{H}$ & 0.44594600 & 4.25413500 & 1.93740500 & $\mathrm{H}$ & -6.14936500 & 1.82815300 & 1.61807900 \\
\hline $\mathrm{H}$ & 0.10757100 & 2.54120900 & 1.99328100 & $\mathrm{C}$ & 2.21795400 & 0.43684700 & 2.13031200 \\
\hline C & 2.01289000 & 3.08534900 & 1.06985500 & $\mathrm{C}$ & 3.03807000 & 0.55643000 & 3.25759200 \\
\hline $\mathrm{H}$ & 2.60649500 & 3.29198600 & 1.96624600 & $\mathrm{C}$ & 0.76076900 & -0.96511100 & 3.25553700 \\
\hline $\mathrm{H}$ & 2.35991300 & 3.77415400 & 0.29084700 & C & 2.69287200 & -0.14319300 & 4.41533100 \\
\hline$P$ & 2.39588400 & 1.34689200 & 0.52655400 & $\mathrm{H}$ & 3.92130500 & 1.18423900 & 3.24425800 \\
\hline$P$ & -0.97967100 & 1.99432800 & -0.76568800 & $\mathrm{C}$ & 1.53140600 & -0.91249100 & 4.41855900 \\
\hline C & -1.21379600 & 2.61568800 & -2.56560500 & $\mathrm{H}$ & -0.17162100 & -1.52119700 & 3.21353600 \\
\hline C & 4.21876100 & 1.37103000 & -0.02587100 & $\mathrm{H}$ & 3.31614100 & -0.07531800 & 5.30188000 \\
\hline $\mathrm{C}$ & 0.19086800 & 2.79904600 & -3.17626200 & $\mathrm{H}$ & 1.21951000 & -1.45688300 & 5.30367500 \\
\hline $\mathrm{H}$ & 0.08727600 & 3.14353600 & -4.21109900 & $\mathrm{~N}$ & -2.96129200 & 2.71989700 & 0.96266400 \\
\hline $\mathrm{H}$ & 0.79118700 & 3.54576600 & -2.64756800 & $\mathrm{~N}$ & 1.10323200 & -0.32037600 & 2.13087200 \\
\hline $\mathrm{H}$ & 0.74784500 & 1.85693700 & -3.19000100 & $\mathrm{Pd}$ & 0.54568300 & 0.06820900 & -0.66453400 \\
\hline $\mathrm{C}$ & -1.98001000 & 3.95420000 & -2.56946400 & $\mathrm{C}$ & -0.95097600 & -1.31297800 & -1.06302200 \\
\hline $\mathrm{H}$ & -2.14432400 & 4.26221000 & -3.60830200 & $\mathrm{O}$ & -1.76258500 & -1.27097800 & -1.94373800 \\
\hline $\mathrm{H}$ & -2.96165700 & 3.86446000 & -2.09480400 & $\mathrm{C}$ & -0.83431600 & -2.51176200 & -0.08243800 \\
\hline $\mathrm{H}$ & -1.43268600 & 4.76040400 & -2.07481100 & $\mathrm{H}$ & -0.35477800 & -2.15701400 & 0.83026000 \\
\hline $\mathrm{C}$ & -1.98898600 & 1.59010900 & -3.41233700 & $\mathrm{C}$ & 0.05947100 & -3.56608400 & -0.72793100 \\
\hline $\mathrm{H}$ & -3.02770200 & 1.49463100 & -3.08688800 & $\mathrm{C}$ & 1.19324400 & -4.02582900 & -0.04163300 \\
\hline $\mathrm{H}$ & -2.00787700 & 1.94135300 & -4.45020600 & $\mathrm{C}$ & -0.24380100 & -4.11347700 & -1.98391500 \\
\hline $\mathrm{H}$ & -1.53123900 & 0.60016300 & -3.39404800 & $\mathrm{C}$ & 2.00450000 & -5.01764400 & -0.59525600 \\
\hline $\mathrm{C}$ & 4.21436500 & 1.80326100 & -1.50901000 & $\mathrm{H}$ & 1.43311700 & -3.61238800 & 0.93459100 \\
\hline $\mathrm{H}$ & 3.67146300 & 1.09660500 & -2.14249800 & C & 0.57016400 & -5.10213600 & -2.53727900 \\
\hline $\mathrm{H}$ & 3.77342800 & 2.79624200 & -1.64853000 & $\mathrm{H}$ & -1.11257300 & -3.76198800 & -2.53264000 \\
\hline $\mathrm{H}$ & 5.24588300 & 1.85205300 & -1.87479500 & $\mathrm{C}$ & 1.69577100 & -5.55602200 & -1.84563300 \\
\hline $\mathrm{C}$ & 5.08651400 & 2.36048300 & 0.77764900 & $\mathrm{H}$ & 2.87449000 & -5.37010700 & -0.04923300 \\
\hline $\mathrm{H}$ & 5.14233100 & 2.11128800 & 1.84047600 & $\mathrm{H}$ & 0.32495500 & -5.51801400 & -3.50980300 \\
\hline $\mathrm{H}$ & 6.11034100 & 2.32732300 & 0.38836200 & $\mathrm{H}$ & 2.32696500 & -6.32597400 & -2.27851800 \\
\hline $\mathrm{H}$ & 4.73868300 & 3.39267400 & 0.68508700 & $\mathrm{C}$ & -2.23274500 & -3.05902300 & 0.27051100 \\
\hline C & 4.79974400 & -0.05058700 & 0.10240400 & $\mathrm{H}$ & -2.89633400 & -3.06145200 & -0.59722200 \\
\hline $\mathrm{H}$ & 5.81544400 & -0.06210500 & -0.30790100 & $\mathrm{H}$ & -2.13491900 & -4.10373500 & 0.58900400 \\
\hline $\mathrm{H}$ & 4.85840500 & -0.37692800 & 1.14446700 & $\mathrm{C}$ & -2.88811100 & -2.31755100 & 1.42222800 \\
\hline $\mathrm{H}$ & 4.21690900 & -0.79017000 & -0.45288000 & $\mathrm{O}$ & -2.30369700 & -1.62269700 & 2.23175000 \\
\hline $\mathrm{C}$ & -2.69171300 & 1.85672600 & -0.02688800 & $\mathrm{O}$ & -4.20345500 & -2.57324300 & 1.47077400 \\
\hline C & -3.62856000 & 0.91314600 & -0.47399700 & $\mathrm{C}$ & -4.90894300 & -2.03661100 & 2.60935800 \\
\hline C & -4.17184600 & 2.68609500 & 1.53174200 & $\mathrm{H}$ & -4.47377500 & -2.41266700 & 3.53780900 \\
\hline
\end{tabular}




\begin{tabular}{|c|c|c|c|c|c|c|c|}
\hline $\mathrm{H}$ & -4.86130200 & -0.94573500 & 2.60644500 & $\mathrm{H}$ & -3.06900100 & -4.92504100 & -1.72927200 \\
\hline $\mathrm{H}$ & -5.93708100 & -2.37974800 & 2.50189400 & $\mathrm{H}$ & -3.56554400 & -3.51225700 & -0.79974900 \\
\hline $\mathrm{C}$ & 1.74293200 & -1.14352700 & -1.69591600 & $\mathrm{H}$ & -2.37483300 & -3.34505000 & -2.10803400 \\
\hline \multirow[t]{2}{*}{$\mathrm{O}$} & 2.47130300 & -1.59134300 & -2.45410900 & $\mathrm{C}$ & 3.32222600 & 0.84587100 & 0.17840700 \\
\hline & & & & $\mathrm{C}$ & 3.26532300 & 1.93511300 & -0.70508200 \\
\hline \multirow{2}{*}{\multicolumn{4}{|c|}{$\begin{array}{l}\text { TS31 } \\
\text { imaginary frequency }=-250.26 \mathrm{~cm}^{-1}\end{array}$}} & $\mathrm{C}$ & 4.91173700 & 1.89434200 & 1.47178600 \\
\hline & & & & $\mathrm{C}$ & 4.09959000 & 3.02875000 & -0.47367800 \\
\hline $\mathrm{C}$ & 2.47718000 & -1.67781200 & 1.39159700 & $\mathrm{H}$ & 2.57461600 & 1.92736500 & -1.54195100 \\
\hline $\mathrm{H}$ & 2.92982800 & -2.64192900 & 1.13693500 & $\mathrm{C}$ & 4.94495300 & 3.00986000 & 0.63505700 \\
\hline $\mathrm{H}$ & 3.21274800 & -1.13902100 & 1.99724600 & $\mathrm{H}$ & 5.54716200 & 1.84454100 & 2.35362900 \\
\hline $\mathrm{C}$ & 1.19495400 & -1.90570900 & 2.21935100 & $\mathrm{H}$ & 4.08468500 & 3.88164700 & -1.14584100 \\
\hline $\mathrm{H}$ & 1.51648300 & -2.18902300 & 3.22936500 & $\mathrm{H}$ & 5.61013200 & 3.83879500 & 0.85316100 \\
\hline $\mathrm{H}$ & 0.64534100 & -0.96545300 & 2.33070500 & $\mathrm{C}$ & -2.31799200 & -1.82641000 & 1.10869400 \\
\hline $\mathrm{C}$ & 0.26235100 & -3.03983000 & 1.73023200 & $\mathrm{C}$ & -2.85852900 & -2.16369600 & 2.35111600 \\
\hline $\mathrm{H}$ & -0.34110700 & -3.40749800 & 2.56619000 & $\mathrm{C}$ & -4.03819500 & -0.18769200 & 0.84736500 \\
\hline $\mathrm{H}$ & 0.86488600 & -3.89501500 & 1.40125300 & $\mathrm{C}$ & -4.00099300 & -1.51768700 & 2.82843900 \\
\hline $\mathrm{P}$ & -0.82698000 & -2.61218400 & 0.27267200 & $\mathrm{H}$ & -2.38837300 & -2.93488600 & 2.94743700 \\
\hline $\mathrm{P}$ & 2.30230200 & -0.67329300 & -0.17509200 & $\mathrm{C}$ & -4.60969700 & -0.52054000 & 2.06445400 \\
\hline $\mathrm{C}$ & 3.37856300 & -1.58691600 & -1.46998700 & $\mathrm{H}$ & -4.40488000 & 0.60572000 & 0.20581700 \\
\hline $\mathrm{C}$ & -1.56838700 & -4.29519000 & -0.29833300 & $\mathrm{H}$ & -4.41368100 & -1.79349300 & 3.79400000 \\
\hline $\mathrm{C}$ & 2.71377300 & -2.94386300 & -1.76717400 & $\mathrm{H}$ & -5.49569800 & 0.00002800 & 2.40779100 \\
\hline $\mathrm{H}$ & 3.29609400 & -3.47441100 & -2.52925200 & $\mathrm{~N}$ & 4.12471200 & 0.83220300 & 1.25314900 \\
\hline $\mathrm{H}$ & 2.66833100 & -3.59273500 & -0.88641200 & $\mathrm{~N}$ & -2.93799700 & -0.83332700 & 0.41410400 \\
\hline $\mathrm{H}$ & 1.69782300 & -2.80896900 & -2.15017900 & $\mathrm{Pd}$ & 0.06724800 & -0.53588300 & -0.83079800 \\
\hline $\mathrm{C}$ & 4.81684700 & -1.80291000 & -0.96415800 & $\mathrm{C}$ & -0.45213800 & 1.47674000 & -1.35579600 \\
\hline $\mathrm{H}$ & 5.39450600 & -2.32602900 & -1.73535300 & $\mathrm{O}$ & 0.06922400 & 1.96227200 & -2.33603600 \\
\hline $\mathrm{H}$ & 5.32420500 & -0.85813500 & -0.75339700 & $\mathrm{O}$ & -1.77815800 & 0.38917300 & -1.66347900 \\
\hline $\mathrm{H}$ & 4.85544100 & -2.41397700 & -0.05758400 & $\mathrm{H}$ & -2.50244300 & -0.45259300 & -0.48163400 \\
\hline $\mathrm{C}$ & 3.40209800 & -0.75764100 & -2.76915700 & $\mathrm{C}$ & -2.03660100 & 0.16773600 & -3.05235700 \\
\hline $\mathrm{H}$ & 3.93265800 & 0.18929900 & -2.64233400 & $\mathrm{H}$ & -2.97523300 & 0.66534200 & -3.32066500 \\
\hline $\mathrm{H}$ & 3.92678000 & -1.32337600 & -3.54751800 & $\mathrm{H}$ & -1.22573100 & 0.59522300 & -3.65015000 \\
\hline $\mathrm{H}$ & 2.39185900 & -0.54026500 & -3.13082300 & $\mathrm{H}$ & -2.11247600 & -0.90542100 & -3.25850300 \\
\hline $\mathrm{C}$ & -0.43419400 & -5.02485800 & -1.04784100 & $\mathrm{C}$ & 0.08949500 & 3.06116000 & 0.57141100 \\
\hline $\mathrm{H}$ & -0.05716600 & -4.43599200 & -1.88858200 & $\mathrm{H}$ & 0.95629900 & 3.23867000 & -0.06957500 \\
\hline $\mathrm{H}$ & 0.40913600 & -5.27033300 & -0.39474000 & $\mathrm{H}$ & -0.28661300 & 4.05395700 & 0.83976800 \\
\hline $\mathrm{H}$ & -0.81927300 & -5.96973300 & -1.44733000 & $\mathrm{C}$ & -1.01479100 & 2.25299300 & -0.14877100 \\
\hline $\mathrm{C}$ & -2.08774400 & -5.18161700 & 0.84573800 & $\mathrm{H}$ & -1.37669300 & 1.52034600 & 0.57459300 \\
\hline $\mathrm{H}$ & -2.92969000 & -4.72568900 & 1.37502100 & $\mathrm{C}$ & -2.19677000 & 3.13955600 & -0.54700500 \\
\hline $\mathrm{H}$ & -2.44818800 & -6.13068200 & 0.43173000 & $\mathrm{C}$ & -3.24424500 & 3.30012700 & 0.37424200 \\
\hline $\mathrm{H}$ & -1.30813300 & -5.42537400 & 1.57388500 & $\mathrm{C}$ & -2.25795500 & 3.83983800 & -1.75874800 \\
\hline C & -2.70902700 & -3.98909500 & -1.28773200 & $\mathrm{C}$ & -4.32955100 & 4.13193000 & 0.09157900 \\
\hline
\end{tabular}




\begin{tabular}{|c|c|c|c|c|c|c|c|}
\hline $\mathrm{H}$ & -3.19447000 & 2.78938100 & 1.33417800 & $\mathrm{C}$ & -2.33621400 & -2.17536000 & 2.49141800 \\
\hline $\mathrm{C}$ & -3.34688100 & 4.66686400 & -2.04368800 & $\mathrm{H}$ & -1.40782000 & -2.57153100 & 2.06675700 \\
\hline $\mathrm{H}$ & -1.45690100 & 3.73046700 & -2.48159500 & $\mathrm{H}$ & -2.08628200 & -1.59277200 & 3.38391300 \\
\hline $\mathrm{C}$ & -4.38608800 & 4.81588500 & -1.12395400 & $\mathrm{H}$ & -2.94696400 & -3.02440600 & 2.82246000 \\
\hline $\mathrm{H}$ & -5.12488300 & 4.25141500 & 0.82220300 & $\mathrm{C}$ & -4.36107600 & -0.71952300 & 2.09355300 \\
\hline $\mathrm{H}$ & -3.37880000 & 5.20027800 & -2.98927100 & $\mathrm{H}$ & -4.96510400 & -0.17803800 & 1.36014500 \\
\hline $\mathrm{H}$ & -5.22814700 & 5.46330200 & -1.34888400 & $\mathrm{H}$ & -4.99584400 & -1.49852600 & 2.53561500 \\
\hline $\mathrm{C}$ & 0.55337800 & 2.40553600 & 1.86112800 & $\mathrm{H}$ & -4.09123900 & -0.02498700 & 2.89666500 \\
\hline $\mathrm{O}$ & 0.08932200 & 1.38823100 & 2.34545700 & $\mathrm{C}$ & -3.55898700 & -2.33182300 & 0.32250500 \\
\hline $\mathrm{O}$ & 1.52986400 & 3.12171900 & 2.43481500 & $\mathrm{H}$ & -4.19811900 & -3.11468400 & 0.75265700 \\
\hline $\mathrm{C}$ & 2.02042600 & 2.63041800 & 3.69856300 & $\mathrm{H}$ & -4.11112600 & -1.81306900 & -0.46202400 \\
\hline $\mathrm{H}$ & 1.19820800 & 2.51217200 & 4.40737700 & $\mathrm{H}$ & -2.68805200 & -2.80840500 & -0.13805000 \\
\hline $\mathrm{H}$ & 2.52552800 & 1.67228400 & 3.55918700 & $\mathrm{C}$ & 2.42692000 & 1.40652100 & -0.16399900 \\
\hline \multirow[t]{2}{*}{$\mathrm{H}$} & 2.72426600 & 3.38411700 & 4.04917900 & $\mathrm{C}$ & 2.74419900 & 2.71915400 & 0.21722400 \\
\hline & & & & $\mathrm{C}$ & 3.08712100 & 1.87538700 & -2.34481500 \\
\hline 37 & & & & $\mathrm{C}$ & 3.23944100 & 3.61976900 & -0.72513900 \\
\hline $\mathrm{C}$ & 1.07862500 & 1.14964100 & 2.39852100 & $\mathrm{H}$ & 2.60953100 & 3.03893400 & 1.24357300 \\
\hline $\mathrm{H}$ & 0.92190700 & 0.45784700 & 3.23443700 & $\mathrm{C}$ & 3.42085900 & 3.19326800 & -2.03817000 \\
\hline $\mathrm{H}$ & 1.82445800 & 1.87446100 & 2.74463300 & $\mathrm{H}$ & 3.20393900 & 1.49420800 & -3.35728200 \\
\hline $\mathrm{C}$ & -0.25128100 & 1.88003300 & 2.09084600 & $\mathrm{H}$ & 3.48298100 & 4.63789000 & -0.43389100 \\
\hline $\mathrm{H}$ & -0.35067200 & 2.69523600 & 2.81988700 & $\mathrm{H}$ & 3.80191900 & 3.85734800 & -2.80678300 \\
\hline $\mathrm{H}$ & -0.18525600 & 2.36569900 & 1.10914000 & $\mathrm{C}$ & -3.02292500 & 1.06997200 & -0.28596900 \\
\hline $\mathrm{C}$ & -1.53458900 & 1.01935700 & 2.18510400 & $\mathrm{C}$ & -2.95997300 & 2.47182400 & -0.18146100 \\
\hline $\mathrm{H}$ & -2.39106900 & 1.66738900 & 2.40211500 & $\mathrm{C}$ & -4.56909900 & 1.24045800 & -1.99495800 \\
\hline $\mathrm{H}$ & -1.43662400 & 0.34898100 & 3.04463500 & $\mathrm{C}$ & -3.73220900 & 3.25796200 & -1.03434200 \\
\hline$P$ & -1.91817700 & -0.07274600 & 0.69337200 & $\mathrm{H}$ & -2.31841800 & 2.94785700 & 0.55008700 \\
\hline$P$ & 1.71168600 & 0.10015400 & 0.97879000 & $\mathrm{C}$ & -4.56043500 & 2.63590900 & -1.96616400 \\
\hline $\mathrm{C}$ & 3.30429000 & -0.68683700 & 1.72286200 & $\mathrm{H}$ & -5.20495000 & 0.70859000 & -2.70141600 \\
\hline $\mathrm{C}$ & -3.12498200 & -1.36333000 & 1.44196700 & $\mathrm{H}$ & -3.68905200 & 4.34196800 & -0.96670800 \\
\hline $\mathrm{C}$ & 2.83262900 & -1.76567000 & 2.71874100 & $\mathrm{H}$ & -5.17960100 & 3.20734800 & -2.65032000 \\
\hline $\mathrm{H}$ & 3.69953300 & -2.31345400 & 3.10928000 & $\mathrm{~N}$ & 2.59746500 & 1.01014000 & -1.44801900 \\
\hline $\mathrm{H}$ & 2.30535600 & -1.33725000 & 3.57796000 & $\mathrm{~N}$ & -3.82177400 & 0.47681300 & -1.19437500 \\
\hline $\mathrm{H}$ & 2.16585500 & -2.48655000 & 2.23527000 & $\mathrm{Pd}$ & 0.03369500 & -0.96082100 & -0.28741100 \\
\hline $\mathrm{C}$ & 4.23122100 & 0.31314800 & 2.43330900 & $\mathrm{C}$ & 0.73061600 & -1.86841200 & -2.01565500 \\
\hline $\mathrm{H}$ & 5.12113700 & -0.20707500 & 2.81122600 & $\mathrm{O}$ & 1.69794200 & -1.30125600 & -2.72491100 \\
\hline H & 4.57668500 & 1.10047300 & 1.75577100 & $\mathrm{H}$ & 1.97776300 & -0.47995500 & -2.22605500 \\
\hline $\mathrm{H}$ & 3.74834300 & 0.79016300 & 3.29244300 & $\mathrm{O}$ & 0.40650800 & -3.05594100 & -2.51617400 \\
\hline $\mathrm{C}$ & 4.06441200 & -1.37326900 & 0.57245400 & $\mathrm{C}$ & 1.10294700 & -3.57536900 & -3.67530500 \\
\hline $\mathrm{H}$ & 4.45921200 & -0.64904200 & -0.14638000 & $\mathrm{H}$ & 2.17463900 & -3.65981300 & -3.48125300 \\
\hline $\mathrm{H}$ & 4.91315800 & -1.93852600 & 0.97767900 & $\mathrm{H}$ & 0.66955000 & -4.55948800 & -3.85296000 \\
\hline $\mathrm{H}$ & 3.41829300 & -2.06836900 & 0.02778700 & $\mathrm{H}$ & 0.94482200 & -2.92978300 & -4.54227500 \\
\hline
\end{tabular}




\begin{tabular}{|c|c|c|c|}
\hline $\mathrm{C}$ & 1.42843200 & -2.05761800 & 1.57486700 \\
\hline $\mathrm{H}$ & 1.50597300 & -3.07823100 & 1.18417400 \\
\hline $\mathrm{H}$ & 2.16740800 & -1.98303800 & 2.38014700 \\
\hline $\mathrm{C}$ & 0.01108400 & -1.84581200 & 2.15354200 \\
\hline $\mathrm{H}$ & -0.04668700 & -2.44253800 & 3.07148600 \\
\hline $\mathrm{H}$ & -0.11315700 & -0.80212500 & 2.46762000 \\
\hline $\mathrm{C}$ & -1.16314300 & -2.27615100 & 1.23516800 \\
\hline $\mathrm{H}$ & -1.98542600 & -2.64943100 & 1.85319500 \\
\hline $\mathrm{H}$ & -0.85338900 & -3.12405600 & 0.61301500 \\
\hline$P$ & -1.75451800 & -0.95125600 & 0.04777500 \\
\hline$P$ & 1.87025400 & -0.90512600 & 0.16274600 \\
\hline $\mathrm{C}$ & 3.10583600 & -1.90883500 & -0.89957200 \\
\hline $\mathrm{C}$ & -3.11044500 & -1.82737000 & -0.98259300 \\
\hline $\mathrm{C}$ & 2.24729900 & -2.92892500 & -1.67791400 \\
\hline $\mathrm{H}$ & 2.88682000 & -3.48002600 & -2.37643500 \\
\hline $\mathrm{H}$ & 1.77692300 & -3.66720600 & -1.01983900 \\
\hline $\mathrm{H}$ & 1.46171900 & -2.43286500 & -2.25577800 \\
\hline $\mathrm{C}$ & 4.18552600 & -2.64192000 & -0.08525100 \\
\hline $\mathrm{H}$ & 4.80513900 & -3.24038000 & -0.76319200 \\
\hline $\mathrm{H}$ & 4.85758100 & -1.94663900 & 0.42712900 \\
\hline $\mathrm{H}$ & 3.76387400 & -3.32800600 & 0.65567500 \\
\hline $\mathrm{C}$ & 3.76714700 & -0.94004100 & -1.89879900 \\
\hline $\mathrm{H}$ & 4.41244700 & -0.21005100 & -1.39794800 \\
\hline $\mathrm{H}$ & 4.39827400 & -1.50705900 & -2.59223900 \\
\hline $\mathrm{H}$ & 3.02167800 & -0.39491000 & -2.48529600 \\
\hline $\mathrm{C}$ & -2.36826200 & -2.72433100 & -1.99482800 \\
\hline $\mathrm{H}$ & -1.67270900 & -2.14413300 & -2.60941600 \\
\hline $\mathrm{H}$ & -1.80632000 & -3.52690500 & -1.50499700 \\
\hline $\mathrm{H}$ & -3.09406900 & -3.19983200 & -2.66458800 \\
\hline $\mathrm{C}$ & -4.08908600 & -2.67409600 & -0.15167400 \\
\hline $\mathrm{H}$ & -4.63209900 & -2.07253200 & 0.58329600 \\
\hline $\mathrm{H}$ & -4.83593700 & -3.12482300 & -0.81624000 \\
\hline $\mathrm{H}$ & -3.58973700 & -3.49454000 & 0.37329300 \\
\hline $\mathrm{C}$ & -3.88282500 & -0.73447500 & -1.74762700 \\
\hline $\mathrm{H}$ & -4.58334400 & -1.20530000 & -2.44672200 \\
\hline $\mathrm{H}$ & -4.46580500 & -0.09710700 & -1.07584800 \\
\hline $\mathrm{H}$ & -3.21074900 & -0.09342000 & -2.32714700 \\
\hline $\mathrm{C}$ & 2.99403700 & 0.35950900 & 1.01575500 \\
\hline $\mathrm{C}$ & 3.98681000 & 0.21831300 & 1.98578400 \\
\hline
\end{tabular}

\begin{tabular}{|c|c|c|}
\hline 3.39277000 & 2.71230300 & 0.96143200 \\
\hline 4.68723400 & 1.34144300 & 2.43429000 \\
\hline 4.21549400 & -0.76190400 & 2.38723200 \\
\hline 4.39405800 & 2.60622000 & 1.91455000 \\
\hline 3.08372000 & 3.64894500 & 0.51068900 \\
\hline 5.46307300 & 1.23147700 & 3.18603300 \\
\hline 4.92292500 & 3.49181700 & 2.24689400 \\
\hline-2.73599200 & 0.16469300 & 1.18908000 \\
\hline-3.40005500 & -0.26601800 & 2.34537800 \\
\hline-3.47160600 & 2.34150000 & 1.56486300 \\
\hline-4.11563800 & 0.64843900 & 3.11925600 \\
\hline-3.36774900 & -1.30758300 & 2.64109800 \\
\hline-4.15845800 & 1.98235800 & 2.72214800 \\
\hline-3.46874600 & 3.37058300 & 1.21337500 \\
\hline-4.63422800 & 0.31962300 & 4.01498200 \\
\hline-4.70381100 & 2.72823600 & 3.28995700 \\
\hline 2.74093200 & 1.60827100 & 0.56181700 \\
\hline-2.77562600 & 1.46690100 & 0.82422600 \\
\hline 0.16753300 & 0.32039600 & -0.80193000 \\
\hline-1.97007600 & 2.20179000 & -0.42385900 \\
\hline-0.47727700 & 2.11685800 & -1.59761300 \\
\hline-1.54962100 & 2.74287200 & -1.18058800 \\
\hline 1.92517300 & 1.62468600 & -0.14529500 \\
\hline 0.04118200 & 2.67221900 & -2.68072900 \\
\hline-0.63128600 & 3.79490600 & -3.31816400 \\
\hline-0.65764300 & 4.65570100 & -2.64727200 \\
\hline-0.03594500 & 4.01596600 & -4.20249700 \\
\hline-1.64971600 & 3.52070600 & -3.59796800 \\
\hline
\end{tabular}

41

C

$\mathrm{H}$

C

H

C

C

C

$\mathrm{H}$$$
\text { C }
$$

$\mathrm{H}$

$\mathrm{N}$

$\mathrm{N}$

Pd

$\mathrm{H}$

C

$\mathrm{O}$

H

C

$\mathrm{H}$

H

H

H

\begin{tabular}{|c|c|c|}
\hline 3.39277000 & 2.71230300 & 0.96143200 \\
\hline 4.68723400 & 1.34144300 & 2.43429000 \\
\hline 4.21549400 & -0.76190400 & 2.38723200 \\
\hline 4.39405800 & 2.60622000 & 1.91455000 \\
\hline 3.08372000 & 3.64894500 & 0.51068900 \\
\hline 5.46307300 & 1.23147700 & 3.18603300 \\
\hline 4.92292500 & 3.49181700 & 2.24689400 \\
\hline-2.73599200 & 0.16469300 & 1.18908000 \\
\hline-3.40005500 & -0.26601800 & 2.34537800 \\
\hline-3.47160600 & 2.34150000 & 1.56486300 \\
\hline-4.11563800 & 0.64843900 & 3.11925600 \\
\hline-3.36774900 & -1.30758300 & 2.64109800 \\
\hline-4.15845800 & 1.98235800 & 2.72214800 \\
\hline-3.46874600 & 3.37058300 & 1.21337500 \\
\hline-4.63422800 & 0.31962300 & 4.01498200 \\
\hline-4.70381100 & 2.72823600 & 3.28995700 \\
\hline 2.74093200 & 1.60827100 & 0.56181700 \\
\hline-2.77562600 & 1.46690100 & 0.82422600 \\
\hline 0.16753300 & 0.32039600 & -0.80193000 \\
\hline-1.97007600 & 2.20179000 & -0.42385900 \\
\hline-0.47727700 & 2.11685800 & -1.59761300 \\
\hline-1.54962100 & 2.74287200 & -1.18058800 \\
\hline 1.92517300 & 1.62468600 & -0.14529500 \\
\hline 0.04118200 & 2.67221900 & -2.68072900 \\
\hline-0.63128600 & 3.79490600 & -3.31816400 \\
\hline-0.65764300 & 4.65570100 & -2.64727200 \\
\hline-0.03594500 & 4.01596600 & -4.20249700 \\
\hline-1.64971600 & 3.52070600 & -3.59796800 \\
\hline
\end{tabular}

\begin{tabular}{|c|c|c|}
\hline 3.39277000 & 2.71230300 & 0.96143200 \\
\hline 4.68723400 & 1.34144300 & 2.43429000 \\
\hline 4.21549400 & -0.76190400 & 2.38723200 \\
\hline 4.39405800 & 2.60622000 & 1.91455000 \\
\hline 3.08372000 & 3.64894500 & 0.51068900 \\
\hline 5.46307300 & 1.23147700 & 3.18603300 \\
\hline 4.92292500 & 3.49181700 & 2.24689400 \\
\hline-2.73599200 & 0.16469300 & 1.18908000 \\
\hline-3.40005500 & -0.26601800 & 2.34537800 \\
\hline-3.47160600 & 2.34150000 & 1.56486300 \\
\hline-4.11563800 & 0.64843900 & 3.11925600 \\
\hline-3.36774900 & -1.30758300 & 2.64109800 \\
\hline-4.15845800 & 1.98235800 & 2.72214800 \\
\hline-3.46874600 & 3.37058300 & 1.21337500 \\
\hline-4.63422800 & 0.31962300 & 4.01498200 \\
\hline-4.70381100 & 2.72823600 & 3.28995700 \\
\hline 2.74093200 & 1.60827100 & 0.56181700 \\
\hline-2.77562600 & 1.46690100 & 0.82422600 \\
\hline 0.16753300 & 0.32039600 & -0.80193000 \\
\hline-1.97007600 & 2.20179000 & -0.42385900 \\
\hline-0.47727700 & 2.11685800 & -1.59761300 \\
\hline-1.54962100 & 2.74287200 & -1.18058800 \\
\hline 1.92517300 & 1.62468600 & -0.14529500 \\
\hline 0.04118200 & 2.67221900 & -2.68072900 \\
\hline-0.63128600 & 3.79490600 & -3.31816400 \\
\hline-0.65764300 & 4.65570100 & -2.64727200 \\
\hline-0.03594500 & 4.01596600 & -4.20249700 \\
\hline-1.64971600 & 3.52070600 & -3.59796800 \\
\hline
\end{tabular}

\begin{tabular}{|c|c|c|}
\hline 3.39277000 & 2.71230300 & 0.96143200 \\
\hline 4.68723400 & 1.34144300 & 2.43429000 \\
\hline 4.21549400 & -0.76190400 & 2.38723200 \\
\hline 4.39405800 & 2.60622000 & 1.91455000 \\
\hline 3.08372000 & 3.64894500 & 0.51068900 \\
\hline 5.46307300 & 1.23147700 & 3.18603300 \\
\hline 4.92292500 & 3.49181700 & 2.24689400 \\
\hline-2.73599200 & 0.16469300 & 1.18908000 \\
\hline-3.40005500 & -0.26601800 & 2.34537800 \\
\hline-3.47160600 & 2.34150000 & 1.56486300 \\
\hline-4.11563800 & 0.64843900 & 3.11925600 \\
\hline-3.36774900 & -1.30758300 & 2.64109800 \\
\hline-4.15845800 & 1.98235800 & 2.72214800 \\
\hline-3.46874600 & 3.37058300 & 1.21337500 \\
\hline-4.63422800 & 0.31962300 & 4.01498200 \\
\hline-4.70381100 & 2.72823600 & 3.28995700 \\
\hline 2.74093200 & 1.60827100 & 0.56181700 \\
\hline-2.77562600 & 1.46690100 & 0.82422600 \\
\hline 0.16753300 & 0.32039600 & -0.80193000 \\
\hline-1.97007600 & 2.20179000 & -0.42385900 \\
\hline-0.47727700 & 2.11685800 & -1.59761300 \\
\hline-1.54962100 & 2.74287200 & -1.18058800 \\
\hline 1.92517300 & 1.62468600 & -0.14529500 \\
\hline 0.04118200 & 2.67221900 & -2.68072900 \\
\hline-0.63128600 & 3.79490600 & -3.31816400 \\
\hline-0.65764300 & 4.65570100 & -2.64727200 \\
\hline-0.03594500 & 4.01596600 & -4.20249700 \\
\hline-1.64971600 & 3.52070600 & -3.59796800 \\
\hline
\end{tabular}

\begin{tabular}{|c|c|c|}
\hline 3.39277000 & 2.71230300 & 0.96143200 \\
\hline 4.68723400 & 1.34144300 & 2.43429000 \\
\hline 4.21549400 & -0.76190400 & 2.38723200 \\
\hline 4.39405800 & 2.60622000 & 1.91455000 \\
\hline 3.08372000 & 3.64894500 & 0.51068900 \\
\hline 5.46307300 & 1.23147700 & 3.18603300 \\
\hline 4.92292500 & 3.49181700 & 2.24689400 \\
\hline-2.73599200 & 0.16469300 & 1.18908000 \\
\hline-3.40005500 & -0.26601800 & 2.34537800 \\
\hline-3.47160600 & 2.34150000 & 1.56486300 \\
\hline-4.11563800 & 0.64843900 & 3.11925600 \\
\hline-3.36774900 & -1.30758300 & 2.64109800 \\
\hline-4.15845800 & 1.98235800 & 2.72214800 \\
\hline-3.46874600 & 3.37058300 & 1.21337500 \\
\hline-4.63422800 & 0.31962300 & 4.01498200 \\
\hline-4.70381100 & 2.72823600 & 3.28995700 \\
\hline 2.74093200 & 1.60827100 & 0.56181700 \\
\hline-2.77562600 & 1.46690100 & 0.82422600 \\
\hline 0.16753300 & 0.32039600 & -0.80193000 \\
\hline-1.97007600 & 2.20179000 & -0.42385900 \\
\hline-0.47727700 & 2.11685800 & -1.59761300 \\
\hline-1.54962100 & 2.74287200 & -1.18058800 \\
\hline 1.92517300 & 1.62468600 & -0.14529500 \\
\hline 0.04118200 & 2.67221900 & -2.68072900 \\
\hline-0.63128600 & 3.79490600 & -3.31816400 \\
\hline-0.65764300 & 4.65570100 & -2.64727200 \\
\hline-0.03594500 & 4.01596600 & -4.20249700 \\
\hline-1.64971600 & 3.52070600 & -3.59796800 \\
\hline
\end{tabular}

\begin{tabular}{|c|c|c|}
\hline 3.39277000 & 2.71230300 & 0.96143200 \\
\hline 4.68723400 & 1.34144300 & 2.43429000 \\
\hline 4.21549400 & -0.76190400 & 2.38723200 \\
\hline 4.39405800 & 2.60622000 & 1.91455000 \\
\hline 3.08372000 & 3.64894500 & 0.51068900 \\
\hline 5.46307300 & 1.23147700 & 3.18603300 \\
\hline 4.92292500 & 3.49181700 & 2.24689400 \\
\hline-2.73599200 & 0.16469300 & 1.18908000 \\
\hline-3.40005500 & -0.26601800 & 2.34537800 \\
\hline-3.47160600 & 2.34150000 & 1.56486300 \\
\hline-4.11563800 & 0.64843900 & 3.11925600 \\
\hline-3.36774900 & -1.30758300 & 2.64109800 \\
\hline-4.15845800 & 1.98235800 & 2.72214800 \\
\hline-3.46874600 & 3.37058300 & 1.21337500 \\
\hline-4.63422800 & 0.31962300 & 4.01498200 \\
\hline-4.70381100 & 2.72823600 & 3.28995700 \\
\hline 2.74093200 & 1.60827100 & 0.56181700 \\
\hline-2.77562600 & 1.46690100 & 0.82422600 \\
\hline 0.16753300 & 0.32039600 & -0.80193000 \\
\hline-1.97007600 & 2.20179000 & -0.42385900 \\
\hline-0.47727700 & 2.11685800 & -1.59761300 \\
\hline-1.54962100 & 2.74287200 & -1.18058800 \\
\hline 1.92517300 & 1.62468600 & -0.14529500 \\
\hline 0.04118200 & 2.67221900 & -2.68072900 \\
\hline-0.63128600 & 3.79490600 & -3.31816400 \\
\hline-0.65764300 & 4.65570100 & -2.64727200 \\
\hline-0.03594500 & 4.01596600 & -4.20249700 \\
\hline-1.64971600 & 3.52070600 & -3.59796800 \\
\hline
\end{tabular}

\begin{tabular}{|c|c|c|}
\hline 3.39277000 & 2.71230300 & 0.96143200 \\
\hline 4.68723400 & 1.34144300 & 2.43429000 \\
\hline 4.21549400 & -0.76190400 & 2.38723200 \\
\hline 4.39405800 & 2.60622000 & 1.91455000 \\
\hline 3.08372000 & 3.64894500 & 0.51068900 \\
\hline 5.46307300 & 1.23147700 & 3.18603300 \\
\hline 4.92292500 & 3.49181700 & 2.24689400 \\
\hline-2.73599200 & 0.16469300 & 1.18908000 \\
\hline-3.40005500 & -0.26601800 & 2.34537800 \\
\hline-3.47160600 & 2.34150000 & 1.56486300 \\
\hline-4.11563800 & 0.64843900 & 3.11925600 \\
\hline-3.36774900 & -1.30758300 & 2.64109800 \\
\hline-4.15845800 & 1.98235800 & 2.72214800 \\
\hline-3.46874600 & 3.37058300 & 1.21337500 \\
\hline-4.63422800 & 0.31962300 & 4.01498200 \\
\hline-4.70381100 & 2.72823600 & 3.28995700 \\
\hline 2.74093200 & 1.60827100 & 0.56181700 \\
\hline-2.77562600 & 1.46690100 & 0.82422600 \\
\hline 0.16753300 & 0.32039600 & -0.80193000 \\
\hline-1.97007600 & 2.20179000 & -0.42385900 \\
\hline-0.47727700 & 2.11685800 & -1.59761300 \\
\hline-1.54962100 & 2.74287200 & -1.18058800 \\
\hline 1.92517300 & 1.62468600 & -0.14529500 \\
\hline 0.04118200 & 2.67221900 & -2.68072900 \\
\hline-0.63128600 & 3.79490600 & -3.31816400 \\
\hline-0.65764300 & 4.65570100 & -2.64727200 \\
\hline-0.03594500 & 4.01596600 & -4.20249700 \\
\hline-1.64971600 & 3.52070600 & -3.59796800 \\
\hline
\end{tabular}

\begin{tabular}{|c|c|c|}
\hline 3.39277000 & 2.71230300 & 0.96143200 \\
\hline 4.68723400 & 1.34144300 & 2.43429000 \\
\hline 4.21549400 & -0.76190400 & 2.38723200 \\
\hline 4.39405800 & 2.60622000 & 1.91455000 \\
\hline 3.08372000 & 3.64894500 & 0.51068900 \\
\hline 5.46307300 & 1.23147700 & 3.18603300 \\
\hline 4.92292500 & 3.49181700 & 2.24689400 \\
\hline-2.73599200 & 0.16469300 & 1.18908000 \\
\hline-3.40005500 & -0.26601800 & 2.34537800 \\
\hline-3.47160600 & 2.34150000 & 1.56486300 \\
\hline-4.11563800 & 0.64843900 & 3.11925600 \\
\hline-3.36774900 & -1.30758300 & 2.64109800 \\
\hline-4.15845800 & 1.98235800 & 2.72214800 \\
\hline-3.46874600 & 3.37058300 & 1.21337500 \\
\hline-4.63422800 & 0.31962300 & 4.01498200 \\
\hline-4.70381100 & 2.72823600 & 3.28995700 \\
\hline 2.74093200 & 1.60827100 & 0.56181700 \\
\hline-2.77562600 & 1.46690100 & 0.82422600 \\
\hline 0.16753300 & 0.32039600 & -0.80193000 \\
\hline-1.97007600 & 2.20179000 & -0.42385900 \\
\hline-0.47727700 & 2.11685800 & -1.59761300 \\
\hline-1.54962100 & 2.74287200 & -1.18058800 \\
\hline 1.92517300 & 1.62468600 & -0.14529500 \\
\hline 0.04118200 & 2.67221900 & -2.68072900 \\
\hline-0.63128600 & 3.79490600 & -3.31816400 \\
\hline-0.65764300 & 4.65570100 & -2.64727200 \\
\hline-0.03594500 & 4.01596600 & -4.20249700 \\
\hline-1.64971600 & 3.52070600 & -3.59796800 \\
\hline
\end{tabular}

\begin{tabular}{|c|c|c|c|}
\hline & 3.39277000 & 2.71230300 & 0.96143200 \\
\hline & 4.68723400 & 1.34144300 & 2.43429000 \\
\hline & 4.21549400 & -0.76190400 & 2.38723200 \\
\hline & 4.39405800 & 2.60622000 & 1.91455000 \\
\hline & 3.08372000 & 3.64894500 & 0.51068900 \\
\hline & 5.46307300 & 1.23147700 & 3.18603300 \\
\hline & 4.92292500 & 3.49181700 & 2.24689400 \\
\hline & -2.73599200 & 0.16469300 & 1.18908000 \\
\hline & -3.40005500 & -0.26601800 & 2.34537800 \\
\hline & -3.47160600 & 2.34150000 & 1.56486300 \\
\hline & -4.11563800 & 0.64843900 & 3.11925600 \\
\hline & -3.36774900 & -1.30758300 & 2.64109800 \\
\hline & -4.15845800 & 1.98235800 & 2.72214800 \\
\hline & -3.46874600 & 3.37058300 & 1.21337500 \\
\hline & -4.63422800 & 0.31962300 & 4.01498200 \\
\hline & -4.70381100 & 2.72823600 & 3.28995700 \\
\hline & 2.74093200 & 1.60827100 & 0.56181700 \\
\hline & -2.77562600 & 1.46690100 & 0.82422600 \\
\hline & 0.16753300 & 0.32039600 & -0.80193000 \\
\hline & -1.97007600 & 2.20179000 & -0.42385900 \\
\hline & -0.47727700 & 2.11685800 & -1.59761300 \\
\hline & -1.54962100 & 2.74287200 & -1.18058800 \\
\hline & 1.92517300 & 1.62468600 & -0.14529500 \\
\hline & 0.04118200 & 2.67221900 & -2.68072900 \\
\hline & -0.63128600 & 3.79490600 & -3.31816400 \\
\hline & -0.65764300 & 4.65570100 & -2.64727200 \\
\hline & -0.03594500 & 4.01596600 & -4.20249700 \\
\hline & -1.64971600 & 3.52070600 & -3.59796800 \\
\hline
\end{tabular}




\begin{tabular}{|c|c|c|}
\hline 2.38213200 & 3.96637500 & -1.30361200 \\
\hline 4.11805600 & 2.44653000 & 0.19142900 \\
\hline 4.86072200 & 1.65770900 & 0.34037600 \\
\hline 4.61884100 & 3.27178200 & -0.32657700 \\
\hline 3.80645100 & 2.82268000 & 1.17041100 \\
\hline 3.42301100 & 1.53603600 & -2.05603300 \\
\hline 3.89526600 & 2.39378300 & -2.54719600 \\
\hline 4.16295600 & 0.73407000 & -1.99804900 \\
\hline 2.60203400 & 1.19331000 & -2.69235400 \\
\hline 3.32961200 & -0.84311600 & 0.25964900 \\
\hline 4.21613800 & -0.95574300 & 1.33867300 \\
\hline 4.28888100 & -2.64181600 & -0.80954000 \\
\hline 5.16761700 & -1.97792700 & 1.31769300 \\
\hline 4.17649500 & -0.26837800 & 2.17638700 \\
\hline 5.20698000 & -2.83971100 & 0.22484700 \\
\hline 4.28659400 & -3.28896500 & -1.68315900 \\
\hline 5.86418900 & -2.09397500 & 2.14232800 \\
\hline 5.92751400 & -3.64881400 & 0.16995200 \\
\hline 3.36759200 & -1.67312900 & -0.79268200 \\
\hline 0.11784000 & -0.25633600 & -0.97579000 \\
\hline 1.34716600 & -0.81841700 & -1.74699700 \\
\hline-0.91547800 & -1.24411100 & -2.36478700 \\
\hline-1.31091100 & -1.82808500 & -3.25935900 \\
\hline-0.01308100 & 0.15065100 & 3.71528500 \\
\hline 0.03582300 & -0.54923700 & 4.55701700 \\
\hline 0.20648100 & 1.14024500 & 4.13768600 \\
\hline-1.45894900 & 0.11228000 & 3.19076600 \\
\hline-1.67464600 & -0.90300200 & 2.83752600 \\
\hline-2.12098100 & 0.26719700 & 4.05020400 \\
\hline-1.85263100 & 1.15845800 & 2.11174700 \\
\hline-1.17858700 & 2.01910200 & 2.14592700 \\
\hline-2.84324000 & 1.56266800 & 2.34354900 \\
\hline-1.91074500 & 0.58453200 & 0.33075600 \\
\hline-2.83640200 & 2.00600800 & -0.56519800 \\
\hline-4.28322600 & 2.16963400 & -0.06485000 \\
\hline-4.33810600 & 2.38670600 & 1.00637900 \\
\hline-4.74460800 & 3.01605700 & -0.58592200 \\
\hline-4.89262200 & 1.28566500 & -0.26807400 \\
\hline-2.05673900 & 3.31415000 & -0.32678000 \\
\hline-2.10101800 & 3.64071900 & 0.71640700 \\
\hline-1.00671900 & 3.22619600 & -0.61930000 \\
\hline
\end{tabular}

$\begin{array}{rrr}-2.49983500 & 4.11142600 & -0.93382900 \\ -2.85027700 & 1.68260000 & -2.07161200 \\ -3.38769200 & 2.47334200 & -2.60695300 \\ -1.83666300 & 1.63303900 & -2.48240300 \\ -3.35475000 & 0.73536100 & -2.28351400 \\ -3.15040800 & -0.80030900 & 0.36417300 \\ -4.11503200 & -0.96858700 & 1.36661500 \\ -5.01126700 & -2.03408500 & 1.26724700 \\ -4.17728300 & -0.28545200 & 2.20550400 \\ -3.93886600 & -2.64303500 & -0.78328400 \\ -4.92582600 & -2.89052700 & 0.17195800 \\ -5.76483700 & -2.18750000 & 2.03373000 \\ -3.83484400 & -3.28186000 & -1.65739200 \\ -5.60222200 & -3.73071200 & 0.05687300 \\ -3.06867900 & -1.63165300 & -0.68734200\end{array}$

TS32 imaginary frequency $=-654.54 \mathrm{~cm}^{-1}$

H

$\mathrm{H}$

C

$\mathrm{H}$

H

$\mathrm{P}$

C

C

$\mathrm{H}$

H

H

C

H

$\mathrm{H}$

H

C

H

$\mathrm{H}$

H

C

$\mathrm{C}$

C

\begin{tabular}{rrr}
1.63340400 & 0.57762400 & 3.08921700 \\
2.31209000 & 0.82022200 & 3.91466500 \\
1.80838600 & -0.48390300 & 2.87225900 \\
2.06326600 & 1.45380600 & 1.88129000 \\
3.08586200 & 1.81085600 & 2.03745300 \\
1.44376400 & 2.35426700 & 1.81405900 \\
2.01930100 & 0.65271300 & 0.19740300 \\
2.86795000 & 1.90071000 & -0.97390900 \\
1.86926600 & 3.05029300 & -1.21105400 \\
0.94778400 & 2.68457000 & -1.67131600 \\
1.61133200 & 3.58121400 & -0.28896500 \\
2.31606500 & 3.78183200 & -1.89328100 \\
4.18395800 & 2.45943100 & -0.40361900 \\
4.91381500 & 1.67245800 & -0.19131400 \\
4.63633500 & 3.12434900 & -1.14773400 \\
4.03272300 & 3.05163300 & 0.50333100 \\
3.14474900 & 1.18219300 & -2.30946200 \\
3.53410500 & 1.90745800 & -3.03219900 \\
3.89198400 & 0.39021600 & -2.20318300 \\
2.23565200 & 0.74088900 & -2.72952800 \\
3.24883000 & -0.74817600 & 0.34093300 \\
4.42227300 & -0.79514700 & 1.09470700 \\
3.62599800 & -2.90818700 & -0.47661900 \\
& & \\
\hline
\end{tabular}




\begin{tabular}{|c|c|c|c|c|c|c|c|}
\hline $\mathrm{C}$ & 5.20609700 & -1.95234700 & 1.05096900 & $\mathrm{H}$ & -4.21481400 & -3.32509800 & -1.24681300 \\
\hline $\mathrm{H}$ & 4.72203400 & 0.04573700 & 1.70978000 & $\mathrm{H}$ & -5.74029000 & -3.56257300 & 0.71843900 \\
\hline $\mathrm{C}$ & 4.80856500 & -3.02512000 & 0.25270200 & $\mathrm{~N}$ & -3.29583300 & -1.61339900 & -0.55654200 \\
\hline $\mathrm{H}$ & 3.25503900 & -3.70670100 & -1.11264800 & & & & \\
\hline $\mathrm{H}$ & 6.11830000 & -2.01366300 & 1.63635000 & 42 & & & \\
\hline $\mathrm{H}$ & 5.39347100 & -3.93674700 & 0.20006300 & $\mathrm{C}$ & 2.20813900 & 1.48774400 & 0.27877300 \\
\hline $\mathrm{N}$ & 2.88372700 & -1.79853100 & -0.41130500 & $\mathrm{C}$ & 3.12029500 & 2.31790700 & 0.93715500 \\
\hline $\mathrm{Pd}$ & 0.13870300 & -0.45706900 & -0.79967100 & $\mathrm{C}$ & 2.99615200 & 3.70564400 & 0.87427200 \\
\hline $\mathrm{H}$ & 1.56808200 & -1.41244400 & -0.94596700 & $\mathrm{C}$ & 1.94748300 & 4.27932300 & 0.14972000 \\
\hline $\mathrm{C}$ & -0.57283800 & -1.82632900 & -2.00171400 & $\mathrm{C}$ & 1.04280100 & 3.43883300 & -0.47247300 \\
\hline $\mathrm{O}$ & -0.94536800 & -2.60978100 & -2.74577900 & $\mathrm{H}$ & 3.93070500 & 1.87279100 & 1.49983400 \\
\hline $\mathrm{C}$ & 0.19582500 & 0.74733200 & 3.61508800 & $\mathrm{H}$ & 3.71429000 & 4.33804400 & 1.38726300 \\
\hline $\mathrm{H}$ & 0.14733200 & 0.19700100 & 4.56151900 & $\mathrm{H}$ & 1.82311100 & 5.35333100 & 0.08012300 \\
\hline $\mathrm{H}$ & 0.02064300 & 1.79967700 & 3.87512800 & $\mathrm{H}$ & 0.18003000 & 3.78558300 & -1.02615000 \\
\hline $\mathrm{C}$ & -0.93001400 & 0.23483400 & 2.69134400 & $\mathrm{~N}$ & 1.19509100 & 2.10042600 & -0.38895700 \\
\hline $\mathrm{H}$ & -0.53223300 & -0.58505600 & 2.07744800 & $\mathrm{P}$ & 2.18311800 & -0.39258700 & 0.21211400 \\
\hline $\mathrm{H}$ & -1.71694300 & -0.21980600 & 3.30021500 & $\mathrm{C}$ & 3.76149800 & -0.75513300 & -0.82057500 \\
\hline $\mathrm{C}$ & -1.57712900 & 1.31434800 & 1.78707000 & $\mathrm{C}$ & 2.64348600 & -0.84062000 & 1.98042900 \\
\hline $\mathrm{H}$ & -0.87841400 & 2.13769200 & 1.60966400 & $\mathrm{H}$ & 3.38705200 & -1.64108700 & 1.91237900 \\
\hline $\mathrm{H}$ & -2.44827400 & 1.75945100 & 2.27981700 & $\mathrm{H}$ & 3.12977900 & -0.00410000 & 2.49381100 \\
\hline$P$ & -1.97662100 & 0.64686200 & 0.09577800 & $\mathrm{C}$ & 1.41463800 & -1.33988700 & 2.76338700 \\
\hline $\mathrm{C}$ & -2.97952900 & 2.00109600 & -0.80082300 & $\mathrm{H}$ & 1.75265300 & -1.78649800 & 3.70632100 \\
\hline $\mathrm{C}$ & -4.31794400 & 2.29915500 & -0.10104800 & $\mathrm{H}$ & 0.95552100 & -2.14892100 & 2.18343000 \\
\hline $\mathrm{H}$ & -4.18177200 & 2.64383800 & 0.92930200 & $\mathrm{C}$ & 0.35881900 & -0.25263900 & 3.03664400 \\
\hline $\mathrm{H}$ & -4.83610800 & 3.09859800 & -0.64327600 & $\mathrm{H}$ & 0.64677300 & 0.32933000 & 3.92081800 \\
\hline $\mathrm{H}$ & -4.97883500 & 1.42899700 & -0.08737400 & $\mathrm{H}$ & 0.36395700 & 0.45437800 & 2.19987400 \\
\hline $\mathrm{C}$ & -2.12840500 & 3.28407200 & -0.84671700 & $\mathrm{C}$ & -1.08431100 & -0.79150600 & 3.19782600 \\
\hline $\mathrm{H}$ & -1.96046200 & 3.70720600 & 0.14883000 & $\mathrm{H}$ & -1.39907500 & -0.71993300 & 4.24495200 \\
\hline $\mathrm{H}$ & -1.15740600 & 3.11336100 & -1.31997800 & $\mathrm{H}$ & -1.11457200 & -1.85895200 & 2.95177900 \\
\hline $\mathrm{H}$ & -2.65209200 & 4.04367700 & -1.43785800 & $\mathrm{C}$ & -2.12176200 & -0.03894400 & 2.33780800 \\
\hline $\mathrm{C}$ & -3.23839600 & 1.50031900 & -2.23610300 & $\mathrm{H}$ & -3.13128100 & -0.30696000 & 2.66248700 \\
\hline $\mathrm{H}$ & -3.81934400 & 2.25087400 & -2.78418400 & $\mathrm{H}$ & -2.01705900 & 1.03923700 & 2.50474300 \\
\hline $\mathrm{H}$ & -2.30273100 & 1.33714300 & -2.78078200 & $\mathrm{P}$ & -1.96562200 & -0.34419800 & 0.47864500 \\
\hline $\mathrm{H}$ & -3.80275500 & 0.56369000 & -2.24840600 & $\mathrm{C}$ & -3.50851500 & -1.41325900 & 0.05105000 \\
\hline $\mathrm{C}$ & -3.22838100 & -0.68720300 & 0.41590100 & $\mathrm{C}$ & -2.40633100 & 1.32305500 & -0.23784700 \\
\hline $\mathrm{C}$ & -4.05288000 & -0.73253800 & 1.54817700 & $\mathrm{C}$ & -3.44874200 & 2.13436900 & 0.23865200 \\
\hline $\mathrm{C}$ & -4.96962500 & -1.77738700 & 1.67297700 & $\mathrm{C}$ & -2.02126400 & 2.79748000 & -1.98305300 \\
\hline $\mathrm{H}$ & -3.99386100 & 0.03353500 & 2.31326800 & $\mathrm{C}$ & -3.77929200 & 3.30412900 & -0.44472800 \\
\hline $\mathrm{C}$ & -4.18843100 & -2.60274200 & -0.43399800 & $\mathrm{H}$ & -4.00168900 & 1.85097700 & 1.12668500 \\
\hline $\mathrm{C}$ & -5.04222100 & -2.73394300 & 0.66289600 & $\mathrm{C}$ & -3.06942600 & 3.63275700 & -1.59910500 \\
\hline $\mathrm{H}$ & -5.61764700 & -1.83769400 & 2.54228700 & $\mathrm{H}$ & -1.43106700 & 3.02407800 & -2.86960000 \\
\hline
\end{tabular}




\begin{tabular}{|c|c|c|c|c|c|c|c|}
\hline $\mathrm{H}$ & -4.58792600 & 3.93698700 & -0.09137600 & $\mathrm{H}$ & 2.92411700 & -2.40563500 & 4.52014700 \\
\hline $\mathrm{H}$ & -3.31217700 & 4.51457600 & -2.18272900 & $\mathrm{H}$ & 2.59512600 & -0.13767200 & 3.53240800 \\
\hline $\mathrm{N}$ & -1.67222000 & 1.68648200 & -1.31242700 & $\mathrm{~N}$ & 2.50700600 & -0.79474300 & 1.57838300 \\
\hline $\mathrm{Pd}$ & 0.10223200 & -1.27415500 & -0.49089400 & P & 2.36399800 & -1.36039100 & -1.05191200 \\
\hline $\mathrm{C}$ & -4.84999700 & -0.85723700 & 0.56048000 & $\mathrm{C}$ & 4.17821200 & -1.30774600 & -1.67424800 \\
\hline $\mathrm{H}$ & -5.11556600 & 0.08681800 & 0.07838900 & $\mathrm{C}$ & 1.66723700 & -2.92135300 & -1.85781500 \\
\hline $\mathrm{H}$ & -5.64294500 & -1.57402200 & 0.31805700 & $\mathrm{H}$ & 2.44544700 & -3.43958000 & -2.42619100 \\
\hline $\mathrm{H}$ & -4.87548000 & -0.71603700 & 1.64555300 & $\mathrm{H}$ & 1.32944800 & -3.61826000 & -1.08338300 \\
\hline $\mathrm{C}$ & -3.25606800 & -2.79724300 & 0.68447800 & $\mathrm{C}$ & 0.48265600 & -2.57050800 & -2.78105100 \\
\hline $\mathrm{H}$ & -2.31551700 & -3.23526500 & 0.33783200 & $\mathrm{H}$ & 0.25022900 & -3.42732100 & -3.42636200 \\
\hline $\mathrm{H}$ & -3.23543200 & -2.75305700 & 1.77891200 & $\mathrm{H}$ & 0.77579300 & -1.75286100 & -3.45244900 \\
\hline $\mathrm{H}$ & -4.06712800 & -3.47834500 & 0.40273400 & $\mathrm{C}$ & -0.77524100 & -2.16253000 & -1.99869000 \\
\hline $\mathrm{C}$ & -3.57646500 & -1.54504300 & -1.48209900 & $\mathrm{H}$ & -1.23848000 & -3.05694200 & -1.55986500 \\
\hline $\mathrm{H}$ & -2.64628500 & -1.94044100 & -1.89694200 & $\mathrm{H}$ & -0.47006500 & -1.52569900 & -1.15705300 \\
\hline $\mathrm{H}$ & -4.38666300 & -2.23230400 & -1.75105300 & $\mathrm{C}$ & -1.79312500 & -1.39624400 & -2.84952900 \\
\hline $\mathrm{H}$ & -3.78455700 & -0.58436800 & -1.96409900 & $\mathrm{H}$ & -2.05674900 & -1.98279300 & -3.73898200 \\
\hline $\mathrm{C}$ & 3.87420500 & -2.29285500 & -0.88973000 & $\mathrm{H}$ & -1.32063800 & -0.47803600 & -3.22038500 \\
\hline $\mathrm{H}$ & 4.08585200 & -2.74073200 & 0.08641000 & $\mathrm{C}$ & -3.10770000 & -1.02676500 & -2.13622600 \\
\hline $\mathrm{H}$ & 2.96513500 & -2.75031400 & -1.28751700 & $\mathrm{H}$ & -3.71398400 & -0.39793700 & -2.79651700 \\
\hline $\mathrm{H}$ & 4.70523500 & -2.55663000 & -1.55313100 & $\mathrm{H}$ & -3.69978000 & -1.92562100 & -1.93848600 \\
\hline $\mathrm{C}$ & 5.05989000 & -0.17265300 & -0.23675800 & $\mathrm{P}$ & -2.92651100 & -0.07825200 & -0.54419200 \\
\hline H & 5.90929100 & -0.51793100 & -0.83708100 & $\mathrm{C}$ & -4.57895600 & 0.82605400 & -0.27835500 \\
\hline $\mathrm{H}$ & 5.07686300 & 0.92037500 & -0.26807800 & $\mathrm{C}$ & -2.83615900 & -1.35755200 & 0.80093000 \\
\hline $\mathrm{H}$ & 5.24225500 & -0.49892000 & 0.79214600 & $\mathrm{C}$ & -3.49369000 & -2.59339000 & 0.73982500 \\
\hline $\mathrm{C}$ & 3.52088100 & -0.19836000 & -2.23679700 & $\mathrm{C}$ & -2.00419700 & -1.83188100 & 2.89907000 \\
\hline $\mathrm{H}$ & 4.35246200 & -0.48672300 & -2.88909000 & $\mathrm{C}$ & -3.38070400 & -3.46713400 & 1.82298300 \\
\hline $\mathrm{H}$ & 2.59933600 & -0.59477500 & -2.67452300 & $\mathrm{H}$ & -4.08517300 & -2.87745000 & -0.12242900 \\
\hline $\mathrm{H}$ & 3.46758200 & 0.89633800 & -2.24633100 & $\mathrm{C}$ & -2.62167900 & -3.08347900 & 2.92595200 \\
\hline $\mathrm{C}$ & 0.03404600 & -2.81223200 & -1.71476600 & $\mathrm{H}$ & -1.39892300 & -1.48819900 & 3.73469100 \\
\hline $\mathrm{O}$ & 0.06724400 & -3.68903300 & -2.45882000 & $\mathrm{H}$ & -3.87992000 & -4.43100000 & 1.80077600 \\
\hline \multirow[t]{2}{*}{$\mathrm{H}$} & 0.45241200 & 1.50853800 & -0.81331600 & $\mathrm{H}$ & -2.50651500 & -3.73403500 & 3.78632100 \\
\hline & & & & $\mathrm{N}$ & -2.10682200 & -0.99177100 & 1.86420100 \\
\hline \multirow{2}{*}{\multicolumn{4}{|c|}{$\begin{array}{l}\text { TS33 } \\
\text { imaginary frequency }=-538.95 \mathrm{~cm}^{-1}\end{array}$}} & $\mathrm{Pd}$ & -1.06632500 & 1.41262400 & -0.45506400 \\
\hline & & & & $\mathrm{H}$ & -1.42356200 & 1.64892600 & -1.96904700 \\
\hline $\mathrm{C}$ & 2.56560100 & -1.84824900 & 0.73517800 & $\mathrm{C}$ & -5.77009400 & -0.14578600 & -0.38270200 \\
\hline $\mathrm{C}$ & 2.75187200 & -3.15601100 & 1.21040800 & $\mathrm{H}$ & -5.72933600 & -0.92912200 & 0.37907000 \\
\hline $\mathrm{C}$ & 2.87963100 & -3.37569800 & 2.58158400 & $\mathrm{H}$ & -6.69899700 & 0.41342600 & -0.22580000 \\
\hline $\mathrm{C}$ & 2.82463300 & -2.28398600 & 3.44638100 & $\mathrm{H}$ & -5.83936100 & -0.61812100 & -1.36729400 \\
\hline $\mathrm{C}$ & 2.64246300 & -1.01689100 & 2.89237900 & $\mathrm{C}$ & -4.70527200 & 1.93078600 & -1.34700000 \\
\hline H & 2.81052500 & -3.98976100 & 0.51951000 & $\mathrm{H}$ & -3.87893800 & 2.64608600 & -1.29149600 \\
\hline $\mathrm{H}$ & 3.02934700 & -4.38079400 & 2.96563900 & $\mathrm{H}$ & -4.74913600 & 1.53123300 & -2.36509400 \\
\hline
\end{tabular}




\begin{tabular}{|c|c|c|c|c|c|c|c|}
\hline $\mathrm{H}$ & -5.63595900 & 2.48294300 & -1.17747200 & $\mathrm{H}$ & 5.05104100 & 0.80519400 & 1.91440600 \\
\hline $\mathrm{C}$ & -4.54828000 & 1.47183700 & 1.12125100 & $\mathrm{H}$ & 5.99567700 & 3.08846400 & 2.04701400 \\
\hline $\mathrm{H}$ & -3.72708700 & 2.18906000 & 1.21777300 & $\mathrm{H}$ & 4.96275600 & 4.92091300 & 0.67108200 \\
\hline $\mathrm{H}$ & -5.48492000 & 2.01613100 & 1.28358400 & $\mathrm{H}$ & 3.00438400 & 4.34971900 & -0.77967500 \\
\hline $\mathrm{H}$ & -4.45198900 & 0.73021100 & 1.91885200 & $\mathrm{~N}$ & 2.96812500 & 2.35962400 & -0.22921700 \\
\hline $\mathrm{C}$ & 4.11252000 & -1.06325400 & -3.19538800 & $\mathrm{P}$ & 2.66077000 & -0.29654000 & 0.27500900 \\
\hline $\mathrm{H}$ & 3.66277400 & -1.90408800 & -3.73390900 & $\mathrm{C}$ & 3.79701300 & -1.15819400 & -1.00885700 \\
\hline $\mathrm{H}$ & 3.54213200 & -0.15950200 & -3.43527700 & $\mathrm{C}$ & 3.01695400 & -1.01652600 & 1.97002800 \\
\hline $\mathrm{H}$ & 5.12631600 & -0.93135900 & -3.59175700 & $\mathrm{H}$ & 4.10390500 & -1.01931300 & 2.09720200 \\
\hline C & 4.99105900 & -2.57905300 & -1.38224500 & $\mathrm{H}$ & 2.62306700 & -0.25249200 & 2.65107100 \\
\hline $\mathrm{H}$ & 6.00212500 & -2.47419700 & -1.79478300 & $\mathrm{C}$ & 2.47059500 & -2.38802900 & 2.40198600 \\
\hline $\mathrm{H}$ & 5.09279400 & -2.75954800 & -0.30821400 & $\mathrm{H}$ & 2.79922300 & -2.51950800 & 3.43942000 \\
\hline $\mathrm{H}$ & 4.54984100 & -3.47129700 & -1.83829100 & $\mathrm{H}$ & 2.96203300 & -3.18966000 & 1.83771000 \\
\hline $\mathrm{C}$ & 4.85156700 & -0.09831600 & -0.99702300 & $\mathrm{C}$ & 0.94182900 & -2.53928300 & 2.31457600 \\
\hline $\mathrm{H}$ & 5.87431700 & 0.01490300 & -1.37611600 & $\mathrm{H}$ & 0.56949600 & -3.07864800 & 3.19311400 \\
\hline $\mathrm{H}$ & 4.31229500 & 0.83173600 & -1.20333600 & $\mathrm{H}$ & 0.46333500 & -1.55276000 & 2.35232100 \\
\hline $\mathrm{H}$ & 4.91050000 & -0.22038200 & 0.08866800 & $\mathrm{C}$ & 0.49306900 & -3.29385500 & 1.05661800 \\
\hline $\mathrm{C}$ & -0.58505900 & 1.26872000 & 1.51350500 & $\mathrm{H}$ & 0.70560600 & -4.36259300 & 1.18872800 \\
\hline $\mathrm{O}$ & -0.25720200 & 1.34851300 & 2.59964100 & $\mathrm{H}$ & 1.08601400 & -2.97587600 & 0.19454300 \\
\hline $\mathrm{C}$ & -0.11170200 & 2.78955200 & -1.96683500 & $\mathrm{C}$ & -0.99296100 & -3.11323300 & 0.73763300 \\
\hline $\mathrm{H}$ & -0.27627700 & 2.95310800 & -3.01374700 & $\mathrm{H}$ & -1.34699800 & -3.92962700 & 0.10373900 \\
\hline $\mathrm{C}$ & 0.56172800 & 2.92355200 & -0.91840700 & $\mathrm{H}$ & -1.58240100 & -3.14002700 & 1.65749900 \\
\hline C & 1.53834200 & 3.48234500 & -0.01779400 & $\mathrm{P}$ & -1.40212600 & -1.49830600 & -0.13518800 \\
\hline $\mathrm{C}$ & 1.64354800 & 4.88609900 & 0.06097500 & $\mathrm{C}$ & -2.00976600 & -2.08633700 & -1.85156200 \\
\hline $\mathrm{C}$ & 2.40508400 & 2.66668900 & 0.73306400 & $\mathrm{C}$ & -2.84529400 & -0.94860000 & 0.90459700 \\
\hline $\mathrm{C}$ & 2.61181200 & 5.46207000 & 0.87797700 & $\mathrm{C}$ & -4.16062800 & -0.72146800 & 0.48719000 \\
\hline $\mathrm{H}$ & 0.97064100 & 5.50892200 & -0.51944300 & $\mathrm{C}$ & -3.38201800 & -0.42889200 & 3.08711700 \\
\hline $\mathrm{C}$ & 3.37460300 & 3.26050400 & 1.53762400 & $\mathrm{C}$ & -5.11234500 & -0.35083400 & 1.43958900 \\
\hline $\mathrm{H}$ & 2.32657500 & 1.58358900 & 0.68895300 & $\mathrm{H}$ & -4.45099800 & -0.83100800 & -0.54884200 \\
\hline $\mathrm{C}$ & 3.47998800 & 4.65175600 & 1.61462900 & $\mathrm{C}$ & -4.72330800 & -0.20598400 & 2.76800400 \\
\hline $\mathrm{H}$ & 2.69049600 & 6.54302000 & 0.93688100 & $\mathrm{H}$ & -3.02996000 & -0.31840400 & 4.11072700 \\
\hline $\mathrm{H}$ & 4.04983500 & 2.63050200 & 2.10813500 & $\mathrm{H}$ & -6.14252100 & -0.18143000 & 1.14117600 \\
\hline $\mathrm{H}$ & 4.23588800 & 5.10502300 & 2.24860000 & $\mathrm{H}$ & -5.43302400 & 0.07314700 & 3.53972600 \\
\hline & & & & $\mathrm{N}$ & -2.46415400 & -0.78949300 & 2.18517100 \\
\hline & & & & $\mathrm{Pd}$ & 0.39074100 & 0.29391200 & -0.43655800 \\
\hline & uency $=-2$ & $33.58 \mathrm{~cm}^{-1}$ & & $\mathrm{H}$ & 1.41241900 & 1.46440400 & -0.74533300 \\
\hline $\mathrm{C}$ & 3.50734300 & 1.36866200 & 0.49577200 & $\mathrm{C}$ & 0.04799700 & 2.15699400 & -1.58056100 \\
\hline $\mathrm{C}$ & 4.61071600 & 1.60116800 & 1.32710000 & $\mathrm{C}$ & -1.08650500 & 2.05643100 & -1.06815000 \\
\hline $\mathrm{C}$ & 5.14628900 & 2.88871600 & 1.40093700 & $\mathrm{C}$ & -2.43500300 & 2.36611400 & -0.68548500 \\
\hline C & 4.57868100 & 3.90707600 & 0.63927700 & $\mathrm{C}$ & -2.74054800 & 2.66765000 & 0.65572200 \\
\hline C & 3.48673600 & 3.59112100 & -0.16854500 & $\mathrm{C}$ & -3.43577600 & 2.48783500 & -1.67044400 \\
\hline
\end{tabular}




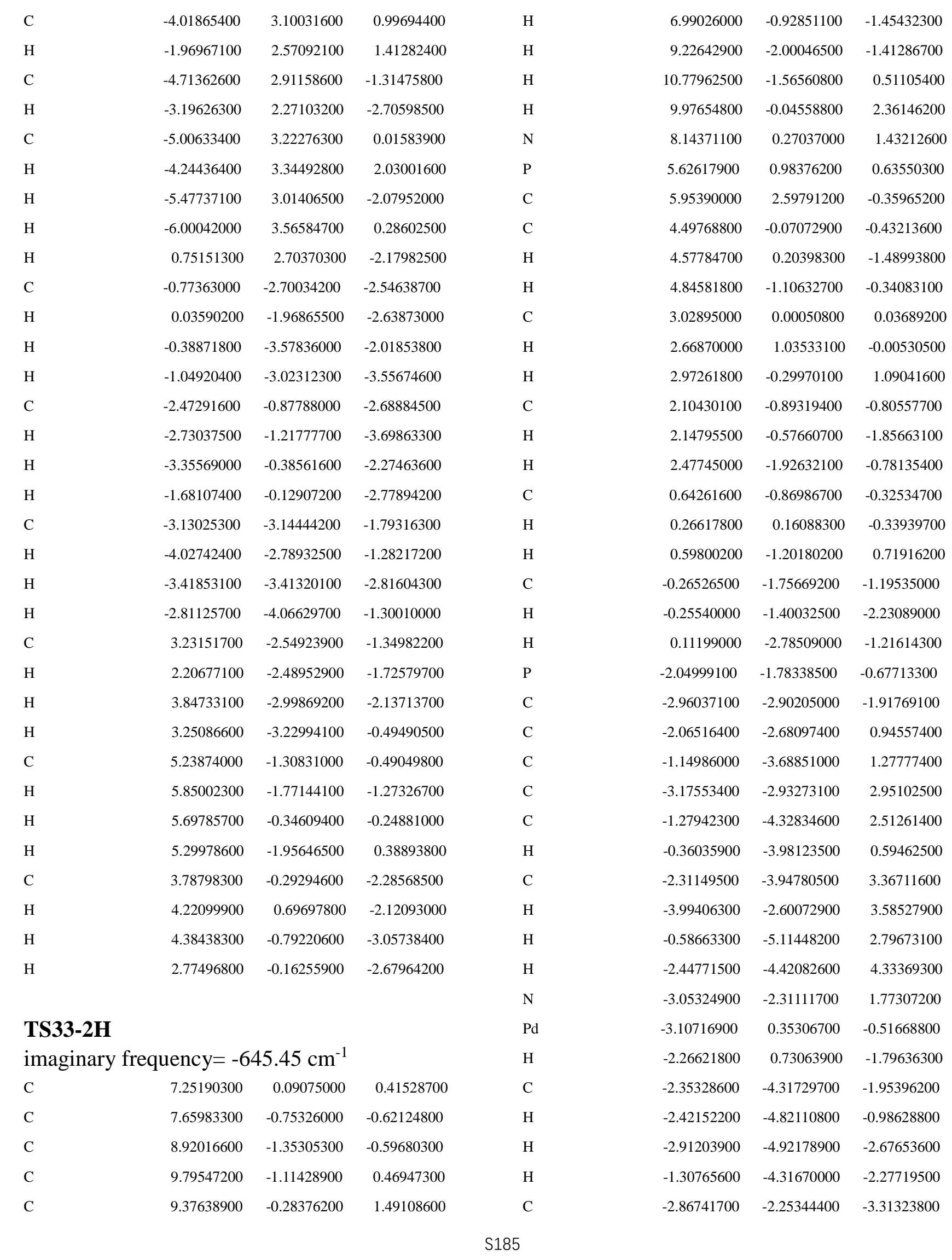




\begin{tabular}{|c|c|c|c|c|c|c|c|}
\hline $\mathrm{H}$ & -3.26536800 & -1.23402600 & -3.31817600 & $\mathrm{C}$ & 6.08114100 & 0.46219300 & -0.38017000 \\
\hline $\mathrm{H}$ & -1.84270200 & -2.23007600 & -3.69704500 & $\mathrm{C}$ & 5.97245000 & 0.37872400 & -1.76683000 \\
\hline $\mathrm{H}$ & -3.46210700 & -2.84357100 & -4.01841700 & $\mathrm{C}$ & 4.82630300 & -0.21130600 & -2.30162500 \\
\hline $\mathrm{C}$ & -4.43641000 & -2.97534900 & -1.47649500 & $\mathrm{H}$ & 5.12826800 & -0.01035700 & 1.49097200 \\
\hline $\mathrm{H}$ & -4.91192200 & -1.98925900 & -1.48968700 & $\mathrm{H}$ & 6.95947500 & 0.90419300 & 0.08164900 \\
\hline $\mathrm{H}$ & -4.98586300 & -3.61741700 & -2.17303200 & $\mathrm{H}$ & 6.75250200 & 0.75594300 & -2.42004700 \\
\hline $\mathrm{H}$ & -4.54992000 & -3.39802000 & -0.47437800 & $\mathrm{H}$ & 4.70473500 & -0.30109300 & -3.37945700 \\
\hline $\mathrm{C}$ & 4.59779800 & 3.33295500 & -0.40427200 & $\mathrm{~N}$ & 3.82088900 & -0.68499900 & -1.55600500 \\
\hline $\mathrm{H}$ & 3.86296700 & 2.80292800 & -1.01792800 & $\mathrm{P}$ & 2.44099000 & -1.31035100 & 0.65566700 \\
\hline $\mathrm{H}$ & 4.17672600 & 3.48220000 & 0.59523400 & $\mathrm{C}$ & 2.88666100 & -3.17548500 & 0.77084500 \\
\hline $\mathrm{H}$ & 4.74192700 & 4.32179300 & -0.85338100 & $\mathrm{C}$ & 2.64075800 & -0.69070600 & 2.42522100 \\
\hline C & 6.48128800 & 2.37574300 & -1.78474400 & $\mathrm{H}$ & 3.30390800 & -1.33905700 & 3.00745900 \\
\hline $\mathrm{H}$ & 6.58915700 & 3.34470900 & -2.28514600 & $\mathrm{H}$ & 3.12302900 & 0.29245300 & 2.38202000 \\
\hline $\mathrm{H}$ & 7.46640800 & 1.89938100 & -1.79382300 & $\mathrm{C}$ & 1.27310800 & -0.54643000 & 3.12748400 \\
\hline $\mathrm{H}$ & 5.80094900 & 1.77612500 & -2.39753700 & $\mathrm{H}$ & 1.42748300 & -0.41481200 & 4.20566500 \\
\hline $\mathrm{C}$ & 6.96079400 & 3.43367500 & 0.45429800 & $\mathrm{H}$ & 0.70375300 & -1.47716900 & 3.01687800 \\
\hline $\mathrm{H}$ & 7.09706500 & 4.40823500 & -0.02673900 & $\mathrm{C}$ & 0.44490400 & 0.62624400 & 2.57523300 \\
\hline $\mathrm{H}$ & 6.61124800 & 3.61750300 & 1.47535400 & $\mathrm{H}$ & 0.79934300 & 1.57014100 & 3.01081100 \\
\hline $\mathrm{H}$ & 7.95031900 & 2.96498900 & 0.49880400 & $\mathrm{H}$ & 0.62630800 & 0.69365600 & 1.49503200 \\
\hline $\mathrm{C}$ & -4.30227800 & 0.04862400 & 1.10543500 & $\mathrm{C}$ & -1.06207500 & 0.46559500 & 2.80275600 \\
\hline $\mathrm{O}$ & -5.04561000 & 0.03574000 & 1.96521500 & $\mathrm{H}$ & -1.27761000 & 0.38524000 & 3.87581000 \\
\hline C & -3.03336300 & 2.23249300 & -1.75698600 & $\mathrm{H}$ & -1.38262300 & -0.48322900 & 2.35423000 \\
\hline $\mathrm{H}$ & -2.47893500 & 2.55899500 & -2.61676800 & $\mathrm{C}$ & -1.93295200 & 1.60128800 & 2.23212800 \\
\hline $\mathrm{C}$ & -3.87765500 & 2.49512800 & -0.86851000 & $\mathrm{H}$ & -2.99095100 & 1.35406900 & 2.36639100 \\
\hline $\mathrm{C}$ & -4.89169900 & 3.15234500 & -0.09262200 & $\mathrm{H}$ & -1.75895300 & 2.52943000 & 2.78460500 \\
\hline C & -6.21540500 & 3.19025100 & -0.57552100 & P & -1.69186400 & 1.93371600 & 0.41293100 \\
\hline $\mathrm{C}$ & -4.56568000 & 3.78608800 & 1.12275600 & $\mathrm{C}$ & -3.30614900 & 2.71494900 & -0.21929600 \\
\hline C & -7.19331300 & 3.86346900 & 0.15052300 & $\mathrm{C}$ & -0.41899500 & 3.28538500 & 0.31734300 \\
\hline $\mathrm{H}$ & -6.46167200 & 2.70405200 & -1.51385600 & $\mathrm{C}$ & -0.29956400 & 4.30346600 & 1.27301200 \\
\hline $\mathrm{C}$ & -5.55407600 & 4.45679400 & 1.83569300 & $\mathrm{C}$ & 1.29693700 & 4.17825200 & -0.93988600 \\
\hline $\mathrm{H}$ & -3.54450300 & 3.75770500 & 1.48960900 & $\mathrm{C}$ & 0.67329400 & 5.28721800 & 1.08669600 \\
\hline $\mathrm{C}$ & -6.86633000 & 4.49516000 & 1.35377500 & $\mathrm{H}$ & -0.94591700 & 4.34310500 & 2.14189800 \\
\hline $\mathrm{H}$ & -8.21108100 & 3.89801000 & -0.22432700 & $\mathrm{C}$ & 1.48964500 & 5.22782700 & -0.04019300 \\
\hline $\mathrm{H}$ & -5.30198400 & 4.95259100 & 2.76751300 & $\mathrm{H}$ & 1.91168100 & 4.08546600 & -1.83221900 \\
\hline $\mathrm{H}$ & -7.63338300 & 5.01899300 & 1.91523200 & $\mathrm{H}$ & 0.78755800 & 6.08586700 & 1.81324100 \\
\hline \multirow[t]{2}{*}{$\mathrm{H}$} & 7.84100400 & 0.85586900 & 2.20911100 & $\mathrm{H}$ & 2.25890800 & 5.97108400 & -0.22047100 \\
\hline & & & & $\mathrm{N}$ & 0.36880600 & 3.23148700 & -0.76512200 \\
\hline \multirow{2}{*}{\multicolumn{4}{|c|}{$\begin{array}{l}\text { TS34 } \\
\text { imaginary frequency }=-511.52 \mathrm{~cm}^{-1}\end{array}$}} & $\mathrm{Pd}$ & -1.06487700 & 0.02137400 & -0.88037500 \\
\hline & & & & $\mathrm{H}$ & -2.25294600 & -0.72106800 & -0.13497400 \\
\hline $\mathrm{C}$ & 3.92658400 & -0.61161000 & -0.21239400 & $\mathrm{C}$ & -3.68876600 & 3.96020000 & 0.60361600 \\
\hline $\mathrm{C}$ & 5.04902800 & -0.04407600 & 0.41009700 & $\mathrm{H}$ & -2.93769900 & 4.75078900 & 0.52785500 \\
\hline
\end{tabular}




\begin{tabular}{|c|c|c|c|c|c|c|c|}
\hline $\mathrm{H}$ & -4.62996000 & 4.36633600 & 0.21717700 & $\mathrm{C}$ & -2.85099500 & -1.22099300 & -0.87887700 \\
\hline $\mathrm{H}$ & -3.84655200 & 3.72810000 & 1.66172200 & $\mathrm{C}$ & -3.74952900 & -1.99102400 & -1.63025200 \\
\hline $\mathrm{C}$ & -4.43119400 & 1.66497000 & -0.12704100 & $\mathrm{C}$ & -4.95131900 & -1.41715200 & -2.04839500 \\
\hline $\mathrm{H}$ & -4.19573700 & 0.76127200 & -0.69649500 & $\mathrm{C}$ & -5.23231900 & -0.09947300 & -1.69725000 \\
\hline $\mathrm{H}$ & -4.65373200 & 1.37604800 & 0.90489700 & $\mathrm{C}$ & -4.28960700 & 0.59524900 & -0.93960400 \\
\hline $\mathrm{H}$ & -5.34866400 & 2.09221400 & -0.54592500 & $\mathrm{H}$ & -3.52861100 & -3.01746800 & -1.89409200 \\
\hline $\mathrm{C}$ & -3.08753500 & 3.10199100 & -1.69596600 & $\mathrm{H}$ & -5.65447300 & -1.99596900 & -2.63951600 \\
\hline $\mathrm{H}$ & -2.84938500 & 2.22742100 & -2.31017200 & $\mathrm{H}$ & -6.15352300 & 0.38433700 & -2.00386900 \\
\hline $\mathrm{H}$ & -4.00777900 & 3.54682400 & -2.09001100 & $\mathrm{H}$ & -4.45854900 & 1.62448700 & -0.63743200 \\
\hline $\mathrm{H}$ & -2.28459100 & 3.83406100 & -1.81842900 & $\mathrm{~N}$ & -3.13174500 & 0.04760700 & -0.55206800 \\
\hline $\mathrm{C}$ & 1.88691700 & -3.83376300 & 1.74149600 & $\mathrm{P}$ & -1.19614900 & -1.84904400 & -0.25056700 \\
\hline $\mathrm{H}$ & 2.02762900 & -3.49690500 & 2.77332900 & $\mathrm{C}$ & -1.69045400 & -2.83766900 & 1.31484700 \\
\hline $\mathrm{H}$ & 0.84845000 & -3.63593000 & 1.45255000 & $\mathrm{C}$ & -0.81875100 & -3.06967200 & -1.62544700 \\
\hline $\mathrm{H}$ & 2.03047500 & -4.92083600 & 1.73404500 & $\mathrm{H}$ & -1.62230000 & -3.81275600 & -1.62925000 \\
\hline $\mathrm{C}$ & 4.32411500 & -3.44033300 & 1.24746300 & $\mathrm{H}$ & -0.94708100 & -2.46711200 & -2.53269900 \\
\hline $\mathrm{H}$ & 4.49540600 & -4.52150500 & 1.31968800 & $\mathrm{C}$ & 0.53086500 & -3.80419500 & -1.71277000 \\
\hline $\mathrm{H}$ & 5.06496400 & -3.03456600 & 0.55339200 & $\mathrm{H}$ & 0.48547700 & -4.39249100 & -2.63640400 \\
\hline $\mathrm{H}$ & 4.51993000 & -3.01832300 & 2.23863900 & $\mathrm{H}$ & 0.61625300 & -4.54068600 & -0.90571800 \\
\hline $\mathrm{C}$ & 2.69028700 & -3.77329700 & -0.63640500 & $\mathrm{C}$ & 1.77150000 & -2.89017600 & -1.73019300 \\
\hline $\mathrm{H}$ & 2.92964100 & -4.84348600 & -0.61918100 & $\mathrm{H}$ & 2.44611600 & -3.18390400 & -2.54213300 \\
\hline $\mathrm{H}$ & 1.65198900 & -3.66845900 & -0.96928100 & $\mathrm{H}$ & 1.47160300 & -1.85961900 & -1.95716600 \\
\hline $\mathrm{H}$ & 3.33250800 & -3.29172400 & -1.37851800 & C & 2.55304100 & -2.92588900 & -0.40958100 \\
\hline $\mathrm{C}$ & 0.43448700 & 0.79526200 & -1.99735300 & $\mathrm{H}$ & 3.13030200 & -3.85763600 & -0.35941800 \\
\hline $\mathrm{O}$ & 1.25129000 & 1.06443200 & -2.74120300 & $\mathrm{H}$ & 1.85801200 & -2.96036000 & 0.43474200 \\
\hline C & -1.95443800 & -2.16020800 & -1.11844800 & $\mathrm{C}$ & 3.50357900 & -1.73603900 & -0.24306500 \\
\hline C & -0.96286600 & -1.88396200 & -1.83992800 & $\mathrm{H}$ & 4.28625600 & -1.96206100 & 0.48604900 \\
\hline C & -3.06194400 & -2.95283400 & -0.63310100 & $\mathrm{H}$ & 3.99460900 & -1.50746900 & -1.19216600 \\
\hline C & -3.68064400 & -2.74125300 & 0.60909600 & $\mathrm{P}$ & 2.64479800 & -0.16219000 & 0.30074700 \\
\hline C & -3.49402900 & -4.01486600 & -1.45370100 & $\mathrm{C}$ & 3.25616800 & 0.09599700 & 2.09535700 \\
\hline $\mathrm{C}$ & -4.70922300 & -3.58012800 & 1.02865500 & $\mathrm{C}$ & 3.47065300 & 1.07706600 & -0.82043100 \\
\hline $\mathrm{H}$ & -3.35050700 & -1.92344100 & 1.24236500 & $\mathrm{C}$ & 4.01970200 & 2.31099400 & -0.44732300 \\
\hline $\mathrm{C}$ & -4.52225100 & -4.84827300 & -1.02382800 & $\mathrm{C}$ & 3.89520500 & 1.49785200 & -3.04899500 \\
\hline $\mathrm{H}$ & -3.02278100 & -4.17534100 & -2.41763800 & $\mathrm{C}$ & 4.52650700 & 3.15184700 & -1.44276000 \\
\hline C & -5.13169600 & -4.63407400 & 0.21506600 & $\mathrm{H}$ & 4.07365100 & 2.61509400 & 0.59022000 \\
\hline $\mathrm{H}$ & -5.18192700 & -3.41155900 & 1.99097500 & $\mathrm{C}$ & 4.46514800 & 2.74295800 & -2.77119500 \\
\hline $\mathrm{H}$ & -4.85152500 & -5.66304400 & -1.66073100 & $\mathrm{H}$ & 3.82754200 & 1.13587600 & -4.07260600 \\
\hline $\mathrm{H}$ & -5.93629000 & -5.28433800 & 0.54355700 & $\mathrm{H}$ & 4.96532600 & 4.10868500 & -1.17583600 \\
\hline \multirow[t]{2}{*}{$\mathrm{H}$} & -0.18634500 & -2.15779900 & -2.52774100 & $\mathrm{H}$ & 4.84765300 & 3.36423200 & -3.57393100 \\
\hline & & & & $\mathrm{N}$ & 3.40786700 & 0.68679400 & -2.10580000 \\
\hline \multicolumn{4}{|c|}{ TS34a } & $\mathrm{Pd}$ & 0.14492400 & 0.16062200 & 0.09585900 \\
\hline \multicolumn{4}{|c|}{ imaginary frequency $=-263.45 \mathrm{~cm}^{-1}$} & $\mathrm{H}$ & -1.38941400 & 0.62954400 & 0.04938700 \\
\hline
\end{tabular}




\begin{tabular}{|c|c|c|c|c|c|c|c|}
\hline $\mathrm{C}$ & -3.69362100 & 4.59450900 & -0.30729300 & $\mathrm{C}$ & -6.02325100 & -0.67013200 & -0.54279800 \\
\hline $\mathrm{C}$ & -2.48237800 & 3.93058800 & -0.49184500 & $\mathrm{C}$ & -6.65447900 & -1.49070600 & -1.48150600 \\
\hline $\mathrm{C}$ & -2.03340800 & 3.00920600 & 0.47331200 & $\mathrm{C}$ & -8.01290300 & -1.33542000 & -1.76621200 \\
\hline $\mathrm{C}$ & -2.81816600 & 2.75375300 & 1.61218500 & $\mathrm{C}$ & -8.76402100 & -0.35186100 & -1.11254400 \\
\hline $\mathrm{C}$ & -4.02725400 & 3.42144800 & 1.78616500 & $\mathrm{C}$ & -8.12531100 & 0.45897400 & -0.19389500 \\
\hline $\mathrm{C}$ & -4.46691800 & 4.34187700 & 0.82908800 & $\mathrm{H}$ & -6.08216300 & -2.25911500 & -1.98554500 \\
\hline $\mathrm{H}$ & -4.03219400 & 5.31065400 & -1.04953000 & $\mathrm{H}$ & -8.48947300 & -1.98640300 & -2.49289100 \\
\hline $\mathrm{H}$ & -1.87578100 & 4.12228200 & -1.37084000 & $\mathrm{H}$ & -9.81952000 & -0.21224400 & -1.31374300 \\
\hline $\mathrm{H}$ & -2.47341500 & 2.03630600 & 2.34935500 & $\mathrm{H}$ & -8.61737300 & 1.25221900 & 0.35666700 \\
\hline $\mathrm{H}$ & -4.62694900 & 3.22763800 & 2.66999800 & $\mathrm{~N}$ & -6.80608800 & 0.28050300 & 0.04536900 \\
\hline $\mathrm{H}$ & -5.40891600 & 4.86281600 & 0.97063500 & $\mathrm{P}$ & -4.22708900 & -0.65315200 & -0.01849600 \\
\hline $\mathrm{C}$ & -0.74256000 & 2.39360100 & 0.30297000 & $\mathrm{C}$ & -4.25138800 & -1.91102100 & 1.44037700 \\
\hline $\mathrm{C}$ & 0.49214100 & 2.26090100 & 0.13681200 & $\mathrm{C}$ & -3.49667700 & -1.54718200 & -1.49831300 \\
\hline $\mathrm{H}$ & 1.45473600 & 2.72977800 & 0.05237600 & $\mathrm{H}$ & -3.91156400 & -2.55752600 & -1.57659200 \\
\hline $\mathrm{C}$ & 2.76382700 & -1.12678700 & 2.90032700 & $\mathrm{H}$ & -3.84889300 & -0.98434100 & -2.37183900 \\
\hline $\mathrm{H}$ & 1.67203600 & -1.19790800 & 2.88905800 & $\mathrm{C}$ & -1.95761400 & -1.64374600 & -1.56147800 \\
\hline $\mathrm{H}$ & 3.17851000 & -2.07021700 & 2.53378400 & $\mathrm{H}$ & -1.72904400 & -2.23046200 & -2.46038500 \\
\hline $\mathrm{H}$ & 3.07729300 & -1.01834200 & 3.94464500 & $\mathrm{H}$ & -1.58035600 & -2.23877700 & -0.72210600 \\
\hline $\mathrm{C}$ & 4.79180700 & 0.18436100 & 2.20097500 & $\mathrm{C}$ & -1.18778000 & -0.31413500 & -1.64029700 \\
\hline $\mathrm{H}$ & 5.07053500 & 0.30538500 & 3.25411600 & $\mathrm{H}$ & -1.64067600 & 0.32517000 & -2.41069400 \\
\hline $\mathrm{H}$ & 5.28756000 & -0.72112000 & 1.84077900 & $\mathrm{H}$ & -1.27422300 & 0.23465400 & -0.69431900 \\
\hline $\mathrm{H}$ & 5.20714600 & 1.03151600 & 1.65130500 & $\mathrm{C}$ & 0.28847400 & -0.56201700 & -1.99427700 \\
\hline $\mathrm{C}$ & 2.60974000 & 1.35159400 & 2.71708500 & $\mathrm{H}$ & 0.32054700 & -1.13847900 & -2.92729700 \\
\hline $\mathrm{H}$ & 1.51806200 & 1.31063000 & 2.66698200 & $\mathrm{H}$ & 0.74924000 & -1.20049200 & -1.22892000 \\
\hline $\mathrm{H}$ & 2.89516100 & 1.41342100 & 3.77331400 & $\mathrm{C}$ & 1.16481100 & 0.68907400 & -2.20961100 \\
\hline $\mathrm{H}$ & 2.93217600 & 2.28350400 & 2.24578500 & $\mathrm{H}$ & 2.06726400 & 0.39359700 & -2.75453500 \\
\hline $\mathrm{C}$ & -0.44407600 & -3.52659400 & 1.89963100 & $\mathrm{H}$ & 0.65079400 & 1.41822000 & -2.84489500 \\
\hline $\mathrm{H}$ & 0.35441200 & -2.81301800 & 2.11605200 & $\mathrm{P}$ & 1.80790800 & 1.53040600 & -0.67971300 \\
\hline $\mathrm{H}$ & -0.71546200 & -4.01459100 & 2.84271900 & $\mathrm{C}$ & 3.24787000 & 2.64790600 & -1.23971400 \\
\hline $\mathrm{H}$ & -0.04884300 & -4.29981300 & 1.23563900 & $\mathrm{C}$ & 0.48269200 & 2.69786800 & -0.10865900 \\
\hline $\mathrm{C}$ & -2.76301300 & -3.90327500 & 1.02368700 & $\mathrm{C}$ & -0.34650700 & 3.40833200 & -0.98706400 \\
\hline $\mathrm{H}$ & -3.00506300 & -4.42459900 & 1.95676900 & $\mathrm{C}$ & -0.44118500 & 3.75242300 & 1.72281000 \\
\hline $\mathrm{H}$ & -3.69071700 & -3.46843800 & 0.64427100 & $\mathrm{C}$ & -1.24793600 & 4.33308900 & -0.45589200 \\
\hline $\mathrm{H}$ & -2.41584000 & -4.66168700 & 0.31506000 & $\mathrm{H}$ & -0.28790800 & 3.26261100 & -2.05953700 \\
\hline $\mathrm{C}$ & -2.23267400 & -1.81652300 & 2.33493600 & $\mathrm{C}$ & -1.29500500 & 4.51607800 & 0.92450000 \\
\hline $\mathrm{H}$ & -3.13330600 & -1.31193700 & 1.97403600 & $\mathrm{H}$ & -0.44811600 & 3.85457000 & 2.80552900 \\
\hline $\mathrm{H}$ & -2.49010000 & -2.33650200 & 3.26454700 & $\mathrm{H}$ & -1.89319000 & 4.90675700 & -1.11427000 \\
\hline \multirow[t]{2}{*}{$\mathrm{H}$} & -1.48603900 & -1.05141200 & 2.57125200 & $\mathrm{H}$ & -1.97213400 & 5.23283600 & 1.37684900 \\
\hline & & & & $\mathrm{N}$ & 0.42392600 & 2.86420100 & 1.22052000 \\
\hline \multicolumn{4}{|c|}{ TS34-2H } & $\mathrm{Pd}$ & 2.52017800 & 0.03160200 & 1.04501900 \\
\hline \multicolumn{4}{|c|}{ imaginary frequency $=-523.53 \mathrm{~cm}^{-1}$} & $\mathrm{H}$ & 3.36834300 & -0.70122500 & -0.09242400 \\
\hline
\end{tabular}




\begin{tabular}{|c|c|c|c|c|c|c|c|}
\hline $\mathrm{C}$ & 2.80361500 & 3.62795200 & -2.34261000 & \\
\hline $\mathrm{H}$ & 2.02340200 & 4.31227000 & -1.99989200 & \multicolumn{4}{|c|}{ TS35 } \\
\hline $\mathrm{H}$ & 3.66343500 & 4.23947000 & -2.63629900 & \multicolumn{4}{|c|}{ imaginary frequency $=-1180.82 \mathrm{~cm}^{-1}$} \\
\hline $\mathrm{H}$ & 2.45235700 & 3.11421800 & -3.24311700 & $\mathrm{C}$ & 1.94627300 & -2.33564400 & 0.44162200 \\
\hline $\mathrm{C}$ & 4.39177700 & 1.75937500 & -1.76715800 & $\mathrm{C}$ & 2.11315800 & -3.72726300 & 0.48456300 \\
\hline $\mathrm{H}$ & 4.73840500 & 1.04382500 & -1.01627700 & $\mathrm{C}$ & 1.52355200 & -4.47872100 & 1.50035300 \\
\hline $\mathrm{H}$ & 4.11712800 & 1.20865900 & -2.67199200 & $\mathrm{C}$ & 0.75000800 & -3.83620300 & 2.46762400 \\
\hline $\mathrm{H}$ & 5.24042200 & 2.40002800 & -2.02862300 & $\mathrm{C}$ & 0.59173400 & -2.46081500 & 2.36488200 \\
\hline $\mathrm{C}$ & 3.72944400 & 3.42871200 & -0.00002100 & $\mathrm{H}$ & 2.70341300 & -4.22121500 & -0.27696800 \\
\hline $\mathrm{H}$ & 4.07695400 & 2.75872900 & 0.79321100 & $\mathrm{H}$ & 1.66452600 & -5.55485100 & 1.53452600 \\
\hline $\mathrm{H}$ & 4.57050100 & 4.06874600 & -0.28664500 & $\mathrm{H}$ & 0.27427600 & -4.38371500 & 3.27339400 \\
\hline $\mathrm{H}$ & 2.94904300 & 4.07284000 & 0.41419800 & $\mathrm{H}$ & -0.01851400 & -1.89434200 & 3.06285000 \\
\hline $\mathrm{C}$ & -2.83400500 & -1.86653800 & 2.05239600 & $\mathrm{~N}$ & 1.17477800 & -1.75073200 & 1.38759900 \\
\hline $\mathrm{H}$ & -2.07165000 & -2.24122700 & 1.36374700 & $\mathrm{P}$ & 2.67128100 & -1.16731100 & -0.84062600 \\
\hline $\mathrm{H}$ & -2.55647500 & -0.85358200 & 2.36097800 & $\mathrm{C}$ & 4.56071800 & -1.36641900 & -0.59582400 \\
\hline $\mathrm{H}$ & -2.80839900 & -2.50500000 & 2.94247800 & $\mathrm{C}$ & 2.31081000 & -2.12773000 & -2.41974500 \\
\hline $\mathrm{C}$ & -4.60983500 & -3.34578400 & 1.02622700 & $\mathrm{H}$ & 3.13303700 & -1.90414400 & -3.10791200 \\
\hline $\mathrm{H}$ & -4.62628900 & -3.98725700 & 1.91462500 & $\mathrm{H}$ & 2.33180800 & -3.21007000 & -2.25145400 \\
\hline $\mathrm{H}$ & -5.60120600 & -3.41078400 & 0.56588500 & $\mathrm{C}$ & 0.97803300 & -1.70906600 & -3.07255900 \\
\hline $\mathrm{H}$ & -3.87875700 & -3.77595100 & 0.33591400 & $\mathrm{H}$ & 0.85381600 & -2.27413500 & -4.00548800 \\
\hline $\mathrm{C}$ & -5.25660500 & -1.39558800 & 2.48907300 & $\mathrm{H}$ & 1.03803500 & -0.65138600 & -3.35808600 \\
\hline $\mathrm{H}$ & -5.16798400 & -1.99787000 & 3.39942400 & $\mathrm{C}$ & -0.24455400 & -1.91381900 & -2.17171300 \\
\hline $\mathrm{H}$ & -5.06028200 & -0.35522800 & 2.77403000 & $\mathrm{H}$ & -0.31946400 & -2.97044100 & -1.87660500 \\
\hline $\mathrm{H}$ & -6.29445300 & -1.48340700 & 2.15251400 & $\mathrm{H}$ & -0.09521400 & -1.34225600 & -1.24564600 \\
\hline $\mathrm{C}$ & 1.53671200 & 0.78455300 & 2.62945500 & $\mathrm{C}$ & -1.57476300 & -1.47129900 & -2.79338200 \\
\hline $\mathrm{O}$ & 1.05764100 & 1.05560400 & 3.62523200 & $\mathrm{H}$ & -1.84219400 & -2.14938100 & -3.61331800 \\
\hline $\mathrm{C}$ & 4.07873100 & -1.79650400 & 1.05941000 & $\mathrm{H}$ & -1.46237600 & -0.47872600 & -3.24538800 \\
\hline $\mathrm{C}$ & 3.54233800 & -1.46064100 & 2.15047000 & $\mathrm{C}$ & -2.72490400 & -1.46440300 & -1.76409200 \\
\hline $\mathrm{C}$ & 4.99406300 & -2.45879500 & 0.16255700 & $\mathrm{H}$ & -3.69504000 & -1.50091500 & -2.26964800 \\
\hline $\mathrm{C}$ & 4.63253900 & -2.84371500 & -1.14025100 & $\mathrm{H}$ & -2.65467100 & -2.37185000 & -1.15513800 \\
\hline $\mathrm{C}$ & 6.27518300 & -2.77157500 & 0.66009100 & $\mathrm{P}$ & -2.70567000 & 0.00471000 & -0.59332800 \\
\hline $\mathrm{C}$ & 5.53784700 & -3.54292300 & -1.93105500 & $\mathrm{C}$ & -4.10670800 & 1.14797100 & -1.22187900 \\
\hline $\mathrm{H}$ & 3.64530200 & -2.59863600 & -1.52029500 & $\mathrm{C}$ & -3.40671600 & -0.67336800 & 0.99012300 \\
\hline $\mathrm{C}$ & 7.17066800 & -3.47530400 & -0.14091100 & $\mathrm{C}$ & -4.12784600 & -1.87285800 & 1.08338200 \\
\hline $\mathrm{H}$ & 6.55506000 & -2.46494100 & 1.66233400 & $\mathrm{C}$ & -3.69512000 & -0.26330800 & 3.24064600 \\
\hline $\mathrm{C}$ & 6.80577100 & -3.86026300 & -1.43323600 & $\mathrm{C}$ & -4.62942800 & -2.26581600 & 2.32499900 \\
\hline $\mathrm{H}$ & 5.25782500 & -3.84302700 & -2.93560400 & $\mathrm{H}$ & -4.31654300 & -2.48346900 & 0.20791700 \\
\hline $\mathrm{H}$ & 8.15613400 & -3.71749300 & 0.24333800 & $\mathrm{C}$ & -4.41067200 & -1.44708800 & 3.43051600 \\
\hline $\mathrm{H}$ & 7.51024300 & -4.40372300 & -2.05479500 & $\mathrm{H}$ & -3.51423600 & 0.41202900 & 4.07459100 \\
\hline $\mathrm{H}$ & 3.40337800 & -1.68808900 & 3.19173300 & $\mathrm{H}$ & -5.19213600 & -3.18983900 & 2.42060500 \\
\hline $\mathrm{H}$ & -6.35033100 & 0.90270200 & 0.71008100 & $\mathrm{H}$ & -4.78879100 & -1.70936400 & 4.41317600 \\
\hline
\end{tabular}




\begin{tabular}{|c|c|c|c|c|c|c|c|}
\hline $\mathrm{N}$ & -3.19358700 & 0.11583700 & 2.05904200 & $\mathrm{H}$ & 3.10089600 & 5.60823500 & -0.18634700 \\
\hline $\mathrm{Pd}$ & -0.54118400 & 1.20164800 & -0.29834900 & $\mathrm{H}$ & 4.55838000 & 5.52788500 & 1.82061500 \\
\hline $\mathrm{C}$ & -5.49287300 & 0.49000400 & -1.09829100 & & & & \\
\hline $\mathrm{H}$ & -5.76396800 & 0.29416900 & -0.05791700 & 43 & & & \\
\hline $\mathrm{H}$ & -6.24818600 & 1.16602000 & -1.51498900 & $\mathrm{C}$ & 2.60588800 & -2.10860200 & 0.76235300 \\
\hline $\mathrm{H}$ & -5.56334600 & -0.45101800 & -1.65397800 & $\mathrm{C}$ & 3.06571600 & -3.43309200 & 0.83375200 \\
\hline $\mathrm{C}$ & -3.83074400 & 1.48550300 & -2.70028400 & $\mathrm{C}$ & 2.79223900 & -4.20010100 & 1.96558400 \\
\hline $\mathrm{H}$ & -2.85345900 & 1.95387700 & -2.84298300 & $\mathrm{C}$ & 2.06011600 & -3.62963700 & 3.00509100 \\
\hline $\mathrm{H}$ & -3.88882800 & 0.60516400 & -3.34782700 & $\mathrm{C}$ & 1.62796300 & -2.31332300 & 2.85436500 \\
\hline $\mathrm{H}$ & -4.58739500 & 2.19619900 & -3.05124500 & $\mathrm{H}$ & 3.63646300 & -3.86244900 & 0.01865300 \\
\hline $\mathrm{C}$ & -4.06689100 & 2.43758900 & -0.37867700 & $\mathrm{H}$ & 3.14838700 & -5.22390600 & 2.03428300 \\
\hline $\mathrm{H}$ & -3.10648400 & 2.95270200 & -0.48017400 & $\mathrm{H}$ & 1.82568700 & -4.18601600 & 3.90657900 \\
\hline $\mathrm{H}$ & -4.85510700 & 3.11890700 & -0.71965300 & $\mathrm{H}$ & 1.04631600 & -1.83071800 & 3.63764400 \\
\hline $\mathrm{H}$ & -4.22874800 & 2.23515200 & 0.68342100 & $\mathrm{~N}$ & 1.88405000 & -1.57023300 & 1.76909000 \\
\hline $\mathrm{C}$ & 5.19644000 & -0.37688800 & -1.59754000 & $\mathrm{P}$ & 2.90136000 & -0.93507800 & -0.66599500 \\
\hline $\mathrm{H}$ & 5.01077400 & -0.66046600 & -2.63870700 & $\mathrm{C}$ & 4.77921900 & -0.57226200 & -0.55430000 \\
\hline $\mathrm{H}$ & 4.82599100 & 0.64259400 & -1.45110600 & $\mathrm{C}$ & 2.76023700 & -2.08421800 & -2.15284300 \\
\hline $\mathrm{H}$ & 6.28322400 & -0.36303600 & -1.45609000 & $\mathrm{H}$ & 3.52133200 & -1.77365100 & -2.87624400 \\
\hline $\mathrm{C}$ & 5.12876100 & -2.77474300 & -0.83527600 & $\mathrm{H}$ & 2.99232000 & -3.12022700 & -1.88484100 \\
\hline $\mathrm{H}$ & 6.22311300 & -2.74319500 & -0.77092400 & $\mathrm{C}$ & 1.37332700 & -2.00630800 & -2.82175600 \\
\hline $\mathrm{H}$ & 4.78818600 & -3.48996400 & -0.08149000 & $\mathrm{H}$ & 1.35575400 & -2.68730100 & -3.68296300 \\
\hline $\mathrm{H}$ & 4.87478900 & -3.16404200 & -1.82621200 & $\mathrm{H}$ & 1.23169400 & -0.99623600 & -3.22779300 \\
\hline $\mathrm{C}$ & 4.88481300 & -0.90527700 & 0.83899100 & $\mathrm{C}$ & 0.20813600 & -2.32999900 & -1.88003000 \\
\hline $\mathrm{H}$ & 5.97128600 & -0.88083300 & 0.98254700 & $\mathrm{H}$ & 0.32713000 & -3.34177200 & -1.46815700 \\
\hline $\mathrm{H}$ & 4.50126900 & 0.10113500 & 1.03812300 & $\mathrm{H}$ & 0.25282600 & -1.64669900 & -1.02056100 \\
\hline $\mathrm{H}$ & 4.47131100 & -1.58510500 & 1.59139900 & $\mathrm{C}$ & -1.17615900 & -2.21027500 & -2.53028400 \\
\hline $\mathrm{C}$ & -0.24229500 & 2.07519500 & -2.02867100 & $\mathrm{H}$ & -1.32634500 & -3.03704500 & -3.23498800 \\
\hline $\mathrm{O}$ & 0.01319500 & 2.54783300 & -3.04246900 & $\mathrm{H}$ & -1.22950300 & -1.29253800 & -3.12824000 \\
\hline $\mathrm{H}$ & 0.77922000 & -0.46435000 & 1.37702300 & $\mathrm{C}$ & -2.31631000 & -2.23496700 & -1.48969600 \\
\hline $\mathrm{C}$ & 0.23431100 & 0.74827600 & 1.65329400 & $\mathrm{H}$ & -3.25928700 & -2.55152800 & -1.94683700 \\
\hline $\mathrm{H}$ & -0.30345400 & 0.69218500 & 2.59693100 & $\mathrm{H}$ & -2.07502800 & -2.97469200 & -0.71952500 \\
\hline $\mathrm{C}$ & 0.84972200 & 1.78601200 & 1.11793400 & $\mathrm{P}$ & -2.59391200 & -0.59808600 & -0.62385100 \\
\hline $\mathrm{C}$ & 1.85133900 & 2.79036400 & 1.28217100 & $\mathrm{C}$ & -4.10978300 & 0.18506500 & -1.48962100 \\
\hline $\mathrm{C}$ & 2.69111900 & 2.75671400 & 2.42284700 & $\mathrm{C}$ & -3.24567800 & -1.04951300 & 1.05413100 \\
\hline $\mathrm{C}$ & 2.01755800 & 3.82863000 & 0.34019000 & $\mathrm{C}$ & -3.70447000 & -2.32710600 & 1.40207200 \\
\hline $\mathrm{C}$ & 3.65778700 & 3.73574400 & 2.61121300 & $\mathrm{C}$ & -3.75696700 & -0.23042100 & 3.14639500 \\
\hline $\mathrm{H}$ & 2.56540500 & 1.95720700 & 3.14597400 & $\mathrm{C}$ & -4.19374600 & -2.53842100 & 2.69224600 \\
\hline $\mathrm{C}$ & 2.98240400 & 4.80903600 & 0.53821100 & $\mathrm{H}$ & -3.69864600 & -3.14137500 & 0.68680600 \\
\hline $\mathrm{H}$ & 1.37865300 & 3.84874800 & -0.53562100 & $\mathrm{C}$ & -4.22314500 & -1.47098300 & 3.58602200 \\
\hline $\mathrm{C}$ & 3.80256800 & 4.76272700 & 1.67052900 & $\mathrm{H}$ & -3.77208900 & 0.63157800 & 3.81016300 \\
\hline $\mathrm{H}$ & 4.29759500 & 3.70649700 & 3.48751500 & $\mathrm{H}$ & -4.55350000 & -3.51977200 & 2.98677000 \\
\hline
\end{tabular}




\begin{tabular}{|c|c|c|c|c|c|c|c|}
\hline $\mathrm{H}$ & -4.59950900 & -1.58929600 & 4.59668100 & $\mathrm{H}$ & 2.32044300 & 5.48206700 & 3.30370200 \\
\hline $\mathrm{N}$ & -3.26911800 & -0.01802500 & 1.91855100 & $\mathrm{H}$ & 1.11444700 & 6.36952300 & -0.73772700 \\
\hline $\mathrm{Pd}$ & -0.66777100 & 0.97728000 & -0.43723800 & $\mathrm{H}$ & 2.13145000 & 7.07452500 & 1.41138600 \\
\hline $\mathrm{C}$ & -5.38747700 & -0.64019900 & -1.24880400 & & & & \\
\hline $\mathrm{H}$ & -5.66818800 & -0.66335300 & -0.19298900 & \multirow{2}{*}{\multicolumn{4}{|c|}{$\begin{array}{l}\text { TS35a } \\
\text { imaginary frequency }=-1193.32 \mathrm{~cm}^{-1}\end{array}$}} \\
\hline $\mathrm{H}$ & -6.21646300 & -0.18439900 & -1.80201800 & & & & \\
\hline $\mathrm{H}$ & -5.29474300 & -1.67240900 & -1.60211800 & $\mathrm{C}$ & -3.58337300 & -2.32674500 & -0.66787100 \\
\hline $\mathrm{C}$ & -3.82899100 & 0.26412800 & -3.00321100 & $\mathrm{C}$ & -3.93450200 & -3.51079800 & -1.32543200 \\
\hline $\mathrm{H}$ & -2.92685900 & 0.83893600 & -3.23128100 & $\mathrm{C}$ & -5.26425000 & -3.73660400 & -1.68943400 \\
\hline $\mathrm{H}$ & -3.73356200 & -0.72382800 & -3.46348900 & $\mathrm{C}$ & -6.23246600 & -2.77943200 & -1.39202100 \\
\hline $\mathrm{H}$ & -4.66807100 & 0.77035300 & -3.49339400 & $\mathrm{C}$ & -5.82597700 & -1.62198400 & -0.73673800 \\
\hline $\mathrm{C}$ & -4.29413100 & 1.60540800 & -0.92042100 & $\mathrm{H}$ & -3.18330700 & -4.25548600 & -1.55633400 \\
\hline $\mathrm{H}$ & -3.42089900 & 2.23375300 & -1.12354900 & $\mathrm{H}$ & -5.53724000 & -4.65424700 & -2.20125200 \\
\hline $\mathrm{H}$ & -5.16506900 & 2.07471400 & -1.39230900 & $\mathrm{H}$ & -7.27301700 & -2.91862900 & -1.66235300 \\
\hline $\mathrm{H}$ & -4.45553000 & 1.59328300 & 0.16041300 & $\mathrm{H}$ & -6.52053100 & -0.82836300 & -0.47619400 \\
\hline $\mathrm{C}$ & 5.08843400 & 0.41695500 & -1.69770600 & $\mathrm{~N}$ & -4.54618300 & -1.41874600 & -0.39971600 \\
\hline $\mathrm{H}$ & 4.94042600 & -0.02887800 & -2.68685200 & $\mathrm{P}$ & -1.84025900 & -1.86972900 & -0.12142500 \\
\hline $\mathrm{H}$ & 4.46498900 & 1.31486900 & -1.63363600 & $\mathrm{C}$ & -1.61851800 & -2.80363500 & 1.53165000 \\
\hline $\mathrm{H}$ & 6.13704600 & 0.73171100 & -1.63942200 & $\mathrm{C}$ & -0.82800800 & -2.75930300 & -1.41853800 \\
\hline $\mathrm{C}$ & 5.69389600 & -1.80249800 & -0.67357600 & $\mathrm{H}$ & -1.16519600 & -3.79878700 & -1.47344400 \\
\hline $\mathrm{H}$ & 6.74345500 & -1.48337700 & -0.66196600 & $\mathrm{H}$ & -1.10849000 & -2.28114500 & -2.36497800 \\
\hline $\mathrm{H}$ & 5.55886000 & -2.49511900 & 0.16184600 & $\mathrm{C}$ & 0.70832400 & -2.75468900 & -1.27169100 \\
\hline $\mathrm{H}$ & 5.53323900 & -2.35098800 & -1.60718800 & $\mathrm{H}$ & 1.09698500 & -3.34999800 & -2.10775700 \\
\hline $\mathrm{C}$ & 5.02582300 & 0.12949700 & 0.79554500 & $\mathrm{H}$ & 0.99900400 & -3.30098300 & -0.36701600 \\
\hline $\mathrm{H}$ & 6.07428500 & 0.44316800 & 0.86332700 & $\mathrm{C}$ & 1.38729600 & -1.38109100 & -1.28676200 \\
\hline $\mathrm{H}$ & 4.40270700 & 1.02370500 & 0.90428600 & $\mathrm{H}$ & 1.09119300 & -0.84065000 & -2.19647700 \\
\hline $\mathrm{H}$ & 4.82207900 & -0.53114700 & 1.64373300 & $\mathrm{H}$ & 1.03675200 & -0.77745100 & -0.43978100 \\
\hline $\mathrm{C}$ & -0.36076500 & 1.54188500 & -2.29864200 & $\mathrm{C}$ & 2.91875100 & -1.47799400 & -1.22481300 \\
\hline $\mathrm{O}$ & -0.10558900 & 1.86698700 & -3.36585600 & $\mathrm{H}$ & 3.28216300 & -2.08824600 & -2.06178500 \\
\hline $\mathrm{H}$ & 0.63879800 & 0.12232800 & 1.58712900 & $\mathrm{H}$ & 3.20607500 & -2.00596800 & -0.30655700 \\
\hline $\mathrm{C}$ & 0.01378300 & 1.03612700 & 1.64769900 & $\mathrm{C}$ & 3.59597600 & -0.10033500 & -1.24775400 \\
\hline $\mathrm{H}$ & -0.64871100 & 1.11799800 & 2.50879500 & $\mathrm{H}$ & 3.35109500 & 0.41827400 & -2.18201200 \\
\hline $\mathrm{C}$ & 0.32581700 & 2.12229800 & 0.88500600 & $\mathrm{H}$ & 3.23201100 & 0.50295100 & -0.41090900 \\
\hline $\mathrm{C}$ & 0.80632800 & 3.44651800 & 1.00792800 & $\mathrm{P}$ & 5.46107200 & -0.23234900 & -1.16068900 \\
\hline $\mathrm{C}$ & 1.40066800 & 3.86514300 & 2.23024400 & $\mathrm{C}$ & 6.01294200 & 1.59232500 & -1.38851300 \\
\hline $\mathrm{C}$ & 0.71752000 & 4.36838200 & -0.06634900 & $\mathrm{C}$ & 5.67138500 & -0.48235400 & 0.67872600 \\
\hline $\mathrm{C}$ & 1.86892600 & 5.16240300 & 2.37027800 & $\mathrm{C}$ & 6.89369000 & -0.98933000 & 1.15104300 \\
\hline $\mathrm{H}$ & 1.48087500 & 3.15307800 & 3.04497900 & $\mathrm{C}$ & 4.82472700 & -0.42385300 & 2.82293300 \\
\hline $\mathrm{C}$ & 1.18617300 & 5.66429800 & 0.08361600 & $\mathrm{C}$ & 7.07034700 & -1.18809900 & 2.51875800 \\
\hline $\mathrm{H}$ & 0.28013000 & 4.04010800 & -1.00264600 & $\mathrm{H}$ & 7.68669100 & -1.22870000 & 0.44930900 \\
\hline $\mathrm{C}$ & 1.76021000 & 6.06006600 & 1.29950400 & $\mathrm{C}$ & 6.01215300 & -0.89851100 & 3.37960200 \\
\hline
\end{tabular}




\begin{tabular}{|c|c|c|c|c|c|c|c|}
\hline $\mathrm{H}$ & 3.97221200 & -0.19699000 & 3.46185000 & $\mathrm{H}$ & -6.00787700 & 5.77046000 & 1.09766800 \\
\hline $\mathrm{H}$ & 8.01123100 & -1.57079500 & 2.90374100 & $\mathrm{H}$ & -1.80850200 & 6.30534200 & 0.30772800 \\
\hline $\mathrm{H}$ & 6.09804300 & -1.04214600 & 4.45193600 & $\mathrm{H}$ & -4.05794600 & 7.26378400 & 0.73528600 \\
\hline $\mathrm{N}$ & 4.64796400 & -0.21445100 & 1.51185800 & $\mathrm{C}$ & -0.73491200 & 1.42444800 & -1.21764600 \\
\hline $\mathrm{Pd}$ & -2.05388400 & 0.59754400 & -0.04450500 & $\mathrm{O}$ & 0.02221100 & 1.92444400 & -1.91410900 \\
\hline $\mathrm{C}$ & -3.94796200 & 0.83251000 & 0.99502500 & & & & \\
\hline $\mathrm{C}$ & -3.42970700 & 1.98788700 & 0.63568700 & \multirow{2}{*}{\multicolumn{4}{|c|}{$\begin{array}{l}\text { TS35b } \\
\text { imaginary frequency }=-1233.09 \mathrm{~cm}^{-1}\end{array}$}} \\
\hline $\mathrm{C}$ & 5.81328500 & 1.93157500 & -2.88038200 & & & & \\
\hline $\mathrm{H}$ & 6.35759500 & 1.23716700 & -3.52856000 & $\mathrm{C}$ & -3.47796100 & 1.31892000 & -0.56631400 \\
\hline $\mathrm{H}$ & 4.75810900 & 1.91331400 & -3.17331100 & $\mathrm{C}$ & -4.73295800 & 1.49429600 & -1.16368200 \\
\hline $\mathrm{H}$ & 6.18856400 & 2.94228100 & -3.08279300 & $\mathrm{C}$ & -5.19569100 & 2.78049100 & -1.44496700 \\
\hline $\mathrm{C}$ & 5.24068000 & 2.59357200 & -0.51420500 & $\mathrm{C}$ & -4.40452000 & 3.88473800 & -1.12718100 \\
\hline $\mathrm{H}$ & 5.65009800 & 3.60236600 & -0.65463200 & $\mathrm{C}$ & -3.16642400 & 3.65135200 & -0.54324200 \\
\hline $\mathrm{H}$ & 4.17954900 & 2.63353400 & -0.77929200 & $\mathrm{H}$ & -5.34754900 & 0.63817900 & -1.40900100 \\
\hline $\mathrm{H}$ & 5.31195500 & 2.34739300 & 0.54897100 & $\mathrm{H}$ & -6.16726700 & 2.91704400 & -1.90971100 \\
\hline $\mathrm{C}$ & 7.51731700 & 1.66950800 & -1.06272400 & $\mathrm{H}$ & -4.73074100 & 4.89772700 & -1.33330600 \\
\hline $\mathrm{H}$ & 8.09712500 & 0.95450800 & -1.65646300 & $\mathrm{H}$ & -2.48515400 & 4.45573300 & -0.28307200 \\
\hline $\mathrm{H}$ & 7.89320000 & 2.67395700 & -1.29232000 & $\mathrm{~N}$ & -2.74117700 & 2.40664700 & -0.28424800 \\
\hline $\mathrm{H}$ & 7.71793400 & 1.47749100 & -0.00440500 & $\mathrm{P}$ & -2.69734300 & -0.34420800 & -0.13861300 \\
\hline $\mathrm{C}$ & -0.32609200 & -2.26259700 & 2.18086200 & $\mathrm{C}$ & -3.67293700 & -0.82885400 & 1.44512400 \\
\hline $\mathrm{H}$ & -0.38285700 & -1.18246800 & 2.34727800 & $\mathrm{C}$ & -3.45361400 & -1.30902100 & -1.56767500 \\
\hline $\mathrm{H}$ & -0.18645300 & -2.74480700 & 3.15494500 & $\mathrm{H}$ & -4.53664400 & -1.36993800 & -1.41696000 \\
\hline $\mathrm{H}$ & 0.56542000 & -2.46800000 & 1.58381500 & $\mathrm{H}$ & -3.29081500 & -0.65474400 & -2.43213600 \\
\hline $\mathrm{C}$ & -1.52952800 & -4.32733100 & 1.33749600 & $\mathrm{C}$ & -2.91061900 & -2.70554100 & -1.92141100 \\
\hline $\mathrm{H}$ & -1.42733800 & -4.80767800 & 2.31707700 & $\mathrm{H}$ & -3.42255900 & -2.99943300 & -2.84481400 \\
\hline $\mathrm{H}$ & -2.43164000 & -4.73663600 & 0.87129000 & $\mathrm{H}$ & -3.22043300 & -3.43975900 & -1.16896000 \\
\hline $\mathrm{H}$ & -0.66354700 & -4.62450600 & 0.74012300 & $\mathrm{C}$ & -1.38447300 & -2.76390000 & -2.11637600 \\
\hline $\mathrm{C}$ & -2.80996900 & -2.46604700 & 2.44843000 & $\mathrm{H}$ & -1.13901700 & -3.36236900 & -3.00081800 \\
\hline $\mathrm{H}$ & -3.75506500 & -2.86498100 & 2.06787900 & $\mathrm{H}$ & -0.99743000 & -1.75764000 & -2.32160800 \\
\hline $\mathrm{H}$ & -2.63800700 & -2.91464800 & 3.43285200 & $\mathrm{C}$ & -0.65656400 & -3.36165600 & -0.90386400 \\
\hline $\mathrm{H}$ & -2.91725600 & -1.38686800 & 2.59215200 & $\mathrm{H}$ & -0.80058300 & -4.44930700 & -0.90810100 \\
\hline $\mathrm{H}$ & -4.20119400 & -0.24779100 & 0.21710500 & $\mathrm{H}$ & -1.12407700 & -3.00871400 & 0.02080000 \\
\hline $\mathrm{H}$ & -4.40104500 & 0.60195500 & 1.95784100 & $\mathrm{C}$ & 0.84603500 & -3.04996600 & -0.89562000 \\
\hline $\mathrm{C}$ & -3.58308500 & 3.40833400 & 0.63672000 & $\mathrm{H}$ & 1.41560100 & -3.87087100 & -0.45131900 \\
\hline $\mathrm{C}$ & -4.86153800 & 3.96770100 & 0.87742900 & $\mathrm{H}$ & 1.21379600 & -2.92097300 & -1.91653600 \\
\hline $\mathrm{C}$ & -2.48560000 & 4.27006600 & 0.42235600 & $\mathrm{P}$ & 1.34155800 & -1.48572800 & 0.00518100 \\
\hline $\mathrm{C}$ & -5.02620000 & 5.34613700 & 0.91297800 & $\mathrm{C}$ & 1.92447000 & -2.13761400 & 1.71193000 \\
\hline $\mathrm{H}$ & -5.70761500 & 3.30598600 & 1.03218200 & $\mathrm{C}$ & 2.84111100 & -1.02197300 & -1.00404100 \\
\hline $\mathrm{C}$ & -2.65710700 & 5.64789100 & 0.46638100 & $\mathrm{C}$ & 4.06757300 & -0.58756500 & -0.48927800 \\
\hline $\mathrm{H}$ & -1.50814300 & 3.84133200 & 0.22931000 & $\mathrm{C}$ & 3.60090100 & -0.79177000 & -3.16939800 \\
\hline $\mathrm{C}$ & -3.92548400 & 6.18641600 & 0.70929500 & $\mathrm{C}$ & 5.09712700 & -0.27553300 & -1.37895000 \\
\hline
\end{tabular}




$\begin{array}{lrrrrrrr}\mathrm{H} & 4.22920900 & -0.48708100 & 0.57544700 & \mathrm{H} & 2.53516200 & 2.07182400 & 2.74442800 \\ \mathrm{C} & 4.86711500 & -0.38150000 & -2.74741000 & \mathrm{C} & 5.03036200 & 3.22890500 & 0.73848900 \\ \mathrm{H} & 3.36983700 & -0.87720600 & -4.22911700 & \mathrm{H} & 4.87163600 & 3.50025600 & -1.39542300 \\ \mathrm{H} & 6.06061400 & 0.05161000 & -1.00053400 & \mathrm{H} & 4.87495300 & 2.87246300 & 2.86048900 \\ \mathrm{H} & 5.64031900 & -0.15050100 & -3.47261000 & \mathrm{H} & 6.05245800 & 3.59146300 & 0.79188100\end{array}$

2.61399800

$-0.24110900$

$-1.10734000$

$-2.32572200$

$-0.20101000$

0.30543900

0.19115400

0.97233700

0.72479200

2.48909600

0.60040800

1.93754800

0.53359000

$-0.14377600$

$-2.88343800$

2.33391100

$-2.22455400$

2.42698300

0.43389100

$-3.76947000$

1.76337400

0.99533000

$-3.21863400$

3.34146400

3.11829800

$-3.10795200$

1.60642600

$3.35888900-3.48218000$

2.60820600

2.89813500

$-3.97951800$

0.98395300

4.01680600

$-2.63279000$

1.20860600

2.27780600

$-0.96678700$

2.64686600

1.42937800

$-0.28626300$

2.76385500

2.53371900

$-1.36347000$

3.63618400

3.13012500

$-0.38050000$

2.29928100

$-3.33188200$

$-2.29459600$

1.78071400

$-2.25799900$

$-3.81601100$

$-2.45084000$

1.90648400

$-3.69573700$

$-5.19938700$

$-2.56506500$

2.72589800

$-2.98911800$

1.01823200

$-5.66793200$

$-0.69318000$

1.30876600

$-1.03284200$

2.23943100

$-5.51498300$

0.34085100

1.14929700

$-5.60826700$

$-1.31277000$

0.50431500

$-3.17120100$

0.08828900

2.57776900

$-3.41368200$

1.13991900

2.39315400

$-3.65385800$

$-0.19843000$

3.51889300

$-2.08834200$

$-1.53067700$

0.00909300

2.71369500

2.21265100

0.15017800

$-0.40641500$

3.51190600

0.91918100

2.37809700

2.31133000

0.60466300

3.05276700

2.71932200

$-0.56066000$

3.05414600

2.36456400

1.83784600

4.36686000

3.17755700

2.53673900

2.68270700

$-0.48972200$

$-1.51490700$

4.36967600

1.90051900

\section{TS35-2H}

imaginary frequency $=-954.82 \mathrm{~cm}^{-1}$

C

C

C

C

C

$\mathrm{H}$

$\mathrm{H}$

H

$\mathrm{H}$

N

P

C

C

$\mathrm{H}$

H

C

H

H

C

$\mathrm{H}$

$\mathrm{H}$

C

$\mathrm{H}$

H

C

$\mathrm{H}$

$\mathrm{H}$

P

C

C

C

C

C

\begin{tabular}{|c|c|c|}
\hline 2.35433300 & -2.19751100 & 0.71976900 \\
\hline 2.82508900 & -3.49968400 & 0.94314500 \\
\hline 2.58467800 & -4.13901700 & 2.15884800 \\
\hline 1.86081400 & -3.47314100 & 3.14875700 \\
\hline 1.39330600 & -2.19685400 & 2.86401600 \\
\hline 3.38647100 & -4.00885900 & 0.16967300 \\
\hline 2.96225200 & -5.14199100 & 2.33299700 \\
\hline 1.66072800 & -3.92925500 & 4.11187100 \\
\hline 0.81202700 & -1.62928000 & 3.58662900 \\
\hline 1.63084800 & -1.59020900 & 1.69038200 \\
\hline 2.63726200 & -1.17640200 & -0.82940800 \\
\hline 4.53772500 & -0.93670300 & -0.86509400 \\
\hline 2.30167700 & -2.46097500 & -2.16469100 \\
\hline 3.05988700 & -2.32203100 & -2.94170500 \\
\hline 2.43227400 & -3.47872600 & -1.78280800 \\
\hline 0.90615100 & -2.30243300 & -2.79785100 \\
\hline 0.78968600 & -3.05838800 & -3.58446500 \\
\hline 0.85214700 & -1.32740800 & -3.29925300 \\
\hline-0.25240600 & -2.41079300 & -1.79917100 \\
\hline-0.23577500 & -3.39175500 & -1.30442700 \\
\hline-0.10291900 & -1.66257000 & -1.00745800 \\
\hline-1.62849700 & -2.18774400 & -2.43890400 \\
\hline-1.89692300 & -3.05338200 & -3.05512000 \\
\hline-1.58600400 & -1.33505400 & -3.12667000 \\
\hline-2.75574000 & -1.97937500 & -1.40278600 \\
\hline-3.73533100 & -2.15018200 & -1.86000200 \\
\hline-2.64464300 & -2.70753000 & -0.59146200 \\
\hline-2.75467700 & -0.27167400 & -0.63549600 \\
\hline-4.10319200 & 0.73678400 & -1.56543300 \\
\hline-3.58767600 & -0.59490800 & 1.00690200 \\
\hline-4.57123700 & -1.54322200 & 1.29167600 \\
\hline-3.72354300 & 0.18581500 & 3.27281300 \\
\hline-5.12887300 & -1.61762300 & 2.57114800 \\
\hline
\end{tabular}

0.71976900

0.94314500

14875700

86401600

(300

100

200 


\begin{tabular}{|c|c|c|c|c|c|c|c|}
\hline $\mathrm{H}$ & -4.90801800 & -2.21575600 & 0.51235100 & $\mathrm{H}$ & 2.60582300 & 2.26580100 & 2.87536400 \\
\hline $\mathrm{C}$ & -4.71092300 & -0.73550000 & 3.57359800 & $\mathrm{C}$ & 2.47467500 & 5.00527000 & 0.11289200 \\
\hline $\mathrm{H}$ & -3.32715300 & 0.89920600 & 3.98600700 & $\mathrm{H}$ & 0.90253200 & 3.83746000 & -0.77065900 \\
\hline $\mathrm{H}$ & -5.89562000 & -2.35643400 & 2.78379800 & $\mathrm{C}$ & 3.38923700 & 5.10280900 & 1.16991300 \\
\hline $\mathrm{H}$ & -5.13571500 & -0.76503000 & 4.57020300 & $\mathrm{H}$ & 4.15464300 & 4.20434900 & 2.97896700 \\
\hline $\mathrm{N}$ & -3.20064600 & 0.21508500 & 2.02730800 & $\mathrm{H}$ & 2.44447200 & 5.77471500 & -0.65126400 \\
\hline $\mathrm{Pd}$ & -0.61970600 & 0.98518800 & -0.25223800 & $\mathrm{H}$ & 4.06691400 & 5.94985900 & 1.21704200 \\
\hline $\mathrm{C}$ & -5.50867600 & 0.11978300 & -1.45972900 & $\mathrm{H}$ & -2.42478800 & 0.85411400 & 1.81766500 \\
\hline $\mathrm{H}$ & -5.90214000 & 0.14200600 & -0.43981600 & & & & \\
\hline $\mathrm{H}$ & -6.19452800 & 0.70794900 & -2.07849800 & \multirow{2}{*}{\multicolumn{4}{|c|}{$\begin{array}{l}\text { TS36 } \\
\text { imaginary frequency }=-1224.01 \mathrm{~cm}^{-1}\end{array}$}} \\
\hline $\mathrm{H}$ & -5.55318600 & -0.90800300 & -1.83294300 & & & & \\
\hline $\mathrm{C}$ & -3.68049900 & 0.78260200 & -3.04897100 & $\mathrm{C}$ & 2.54590400 & 0.54330300 & 1.16931300 \\
\hline $\mathrm{H}$ & -2.68094300 & 1.19962600 & -3.19226800 & $\mathrm{C}$ & 3.39992200 & 0.66093600 & 2.27448400 \\
\hline $\mathrm{H}$ & -3.71894500 & -0.20231900 & -3.52260200 & $\mathrm{C}$ & 3.52828800 & 1.88266500 & 2.93374100 \\
\hline $\mathrm{H}$ & -4.38080700 & 1.42774000 & -3.58985800 & $\mathrm{C}$ & 2.80069500 & 2.97998500 & 2.47683800 \\
\hline $\mathrm{C}$ & -4.11054600 & 2.15884400 & -0.97258200 & $\mathrm{C}$ & 1.96115200 & 2.80487900 & 1.38256100 \\
\hline $\mathrm{H}$ & -3.12850300 & 2.63659900 & -1.03910100 & $\mathrm{H}$ & 3.96735700 & -0.19887600 & 2.60970700 \\
\hline $\mathrm{H}$ & -4.82173300 & 2.77785500 & -1.52951400 & $\mathrm{H}$ & 4.19344700 & 1.97658800 & 3.78685200 \\
\hline $\mathrm{H}$ & -4.43148300 & 2.16955700 & 0.07505700 & $\mathrm{H}$ & 2.87561500 & 3.95138600 & 2.95258800 \\
\hline $\mathrm{C}$ & 4.80508700 & -0.06023400 & -2.10875100 & $\mathrm{H}$ & 1.36097900 & 3.61595100 & 0.98619800 \\
\hline $\mathrm{H}$ & 4.55555600 & -0.57239100 & -3.04374900 & $\mathrm{~N}$ & 1.83564700 & 1.62019700 & 0.76134200 \\
\hline $\mathrm{H}$ & 4.24379500 & 0.87921100 & -2.07375600 & $\mathrm{P}$ & 2.28235700 & -1.02056000 & 0.18616200 \\
\hline $\mathrm{H}$ & 5.87102200 & 0.18893700 & -2.15470900 & $\mathrm{C}$ & 3.86042600 & -1.08077400 & -0.91283300 \\
\hline $\mathrm{C}$ & 5.37325900 & -2.22442500 & -0.95070400 & $\mathrm{C}$ & 2.59459500 & -2.31309400 & 1.52570600 \\
\hline $\mathrm{H}$ & 6.43190500 & -1.96131700 & -1.05850700 & $\mathrm{H}$ & 3.66827200 & -2.50556300 & 1.61942900 \\
\hline $\mathrm{H}$ & 5.28895700 & -2.83128500 & -0.04532200 & $\mathrm{H}$ & 2.27202000 & -1.85986300 & 2.46941200 \\
\hline $\mathrm{H}$ & 5.10825300 & -2.84298500 & -1.81342700 & $\mathrm{C}$ & 1.83947100 & -3.64872200 & 1.35425800 \\
\hline $\mathrm{C}$ & 4.92443400 & -0.14979300 & 0.40238900 & $\mathrm{H}$ & 2.17975700 & -4.30811400 & 2.16064500 \\
\hline $\mathrm{H}$ & 5.98836800 & 0.10887700 & 0.36192700 & $\mathrm{H}$ & 2.14194900 & -4.14287400 & 0.42356400 \\
\hline $\mathrm{H}$ & 4.36048800 & 0.78496900 & 0.49013200 & $\mathrm{C}$ & 0.29963900 & -3.50892100 & 1.40485200 \\
\hline $\mathrm{H}$ & 4.76691500 & -0.73612900 & 1.31372400 & $\mathrm{H}$ & -0.11902000 & -4.22707100 & 2.12004200 \\
\hline $\mathrm{C}$ & -0.27151600 & 1.74491400 & -2.06883000 & $\mathrm{H}$ & 0.04784000 & -2.51967800 & 1.80233000 \\
\hline $\mathrm{O}$ & 0.03823100 & 2.14116900 & -3.09331300 & $\mathrm{C}$ & -0.38064500 & -3.71255200 & 0.04170700 \\
\hline $\mathrm{H}$ & 0.94614800 & -0.34945200 & 1.55167400 & $\mathrm{H}$ & -0.36767200 & -4.78098800 & -0.20787100 \\
\hline $\mathrm{C}$ & 0.29149300 & 0.76384100 & 1.71081300 & $\mathrm{H}$ & 0.20937100 & -3.21330200 & -0.73377800 \\
\hline $\mathrm{H}$ & -0.10951700 & 0.81679300 & 2.72347500 & $\mathrm{C}$ & -1.83409100 & -3.21199300 & -0.04389000 \\
\hline $\mathrm{C}$ & 0.75251700 & 1.81648000 & 1.03451700 & $\mathrm{H}$ & -2.27001900 & -3.54674000 & -0.99152400 \\
\hline $\mathrm{C}$ & 1.64152500 & 2.91930600 & 1.04553700 & $\mathrm{H}$ & -2.44819900 & -3.65489100 & 0.74681700 \\
\hline $\mathrm{C}$ & 2.57665900 & 3.03558700 & 2.11121600 & $\mathrm{P}$ & -2.00184000 & -1.34904300 & -0.06442800 \\
\hline $\mathrm{C}$ & 1.61194500 & 3.92162200 & 0.04429600 & $\mathrm{C}$ & -3.77760900 & -1.02739300 & -0.69024000 \\
\hline $\mathrm{C}$ & 3.44102800 & 4.11809400 & 2.16624600 & $\mathrm{C}$ & -2.05715900 & -0.82775900 & 1.72210700 \\
\hline
\end{tabular}




\begin{tabular}{|c|c|c|c|c|c|c|c|}
\hline $\mathrm{C}$ & -2.56027200 & -1.63784700 & 2.75091300 & $\mathrm{C}$ & -1.22707700 & 3.50963500 & 0.11998100 \\
\hline $\mathrm{C}$ & -1.62933400 & 0.87943600 & 3.21107600 & $\mathrm{C}$ & -0.59377500 & 5.72869300 & -1.45735300 \\
\hline $\mathrm{C}$ & -2.58511600 & -1.14285400 & 4.05502400 & $\mathrm{H}$ & 0.58654300 & 4.36674400 & -2.63722800 \\
\hline $\mathrm{H}$ & -2.92755800 & -2.63687000 & 2.54783800 & C & -1.75634100 & 4.76399600 & 0.42919400 \\
\hline $\mathrm{C}$ & -2.10927600 & 0.14390700 & 4.29525300 & $\mathrm{H}$ & -1.47207000 & 2.63559900 & 0.71737100 \\
\hline $\mathrm{H}$ & -1.25135900 & 1.88990500 & 3.35392900 & $\mathrm{C}$ & -1.43920700 & 5.87574100 & -0.35404400 \\
\hline $\mathrm{H}$ & -2.97193100 & -1.75341800 & 4.86539000 & $\mathrm{H}$ & -0.34506300 & 6.58921400 & -2.07127000 \\
\hline $\mathrm{H}$ & -2.10887600 & 0.57037500 & 5.29289800 & $\mathrm{H}$ & -2.41854400 & 4.87288300 & 1.28335300 \\
\hline $\mathrm{N}$ & -1.59675500 & 0.41447200 & 1.95600000 & $\mathrm{H}$ & -1.84849500 & 6.85094300 & -0.10875600 \\
\hline $\mathrm{Pd}$ & -0.32686600 & -0.22243700 & -1.53594700 & $\mathrm{H}$ & 0.56118700 & 1.59437300 & -3.52056700 \\
\hline $\mathrm{C}$ & -4.82583300 & -1.85189600 & 0.07866600 & & & & \\
\hline $\mathrm{H}$ & -4.83934200 & -1.61010900 & 1.14517900 & \multirow{2}{*}{\multicolumn{4}{|c|}{$\begin{array}{l}\text { TS36a } \\
\text { imaginary frequency }=-1257.81 \mathrm{~cm}^{-1}\end{array}$}} \\
\hline $\mathrm{H}$ & -5.82194600 & -1.62517700 & -0.31834500 & & & & \\
\hline $\mathrm{H}$ & -4.67215300 & -2.92969700 & -0.03230500 & $\mathrm{C}$ & 3.23981200 & 1.64623300 & -1.12678900 \\
\hline $\mathrm{C}$ & -3.82863900 & -1.38752800 & -2.18930400 & $\mathrm{C}$ & 3.58040500 & 2.81679300 & -1.81275500 \\
\hline $\mathrm{H}$ & -3.11289100 & -0.79703200 & -2.76897800 & $\mathrm{C}$ & 4.87473100 & 2.97272800 & -2.31369400 \\
\hline $\mathrm{H}$ & -3.63573400 & -2.44871100 & -2.37704100 & $\mathrm{C}$ & 5.80860800 & 1.95709700 & -2.12249200 \\
\hline $\mathrm{H}$ & -4.83091200 & -1.16982500 & -2.57468200 & $\mathrm{C}$ & 5.41272500 & 0.82145300 & -1.42194300 \\
\hline $\mathrm{C}$ & -4.06949600 & 0.47731200 & -0.52556400 & $\mathrm{H}$ & 2.84970400 & 3.60168000 & -1.96433100 \\
\hline $\mathrm{H}$ & -3.33545400 & 1.09286400 & -1.05458600 & $\mathrm{H}$ & 5.14441200 & 3.87718800 & -2.85005900 \\
\hline $\mathrm{H}$ & -5.05778000 & 0.69878300 & -0.94405800 & $\mathrm{H}$ & 6.82019000 & 2.03556100 & -2.50454600 \\
\hline $\mathrm{H}$ & -4.07667100 & 0.78339300 & 0.52407900 & $\mathrm{H}$ & 6.09150600 & -0.00255100 & -1.22975400 \\
\hline $\mathrm{C}$ & 3.80440400 & -2.42855200 & -1.66204100 & $\mathrm{~N}$ & 4.16717100 & 0.68428100 & -0.94497500 \\
\hline $\mathrm{H}$ & 3.90147700 & -3.28213400 & -0.98377700 & $\mathrm{P}$ & 1.53073000 & 1.26582600 & -0.45079400 \\
\hline $\mathrm{H}$ & 2.87502000 & -2.54351600 & -2.22900900 & $\mathrm{C}$ & 1.36453000 & 2.33943400 & 1.11536000 \\
\hline $\mathrm{H}$ & 4.63502300 & -2.48476000 & -2.37491200 & $\mathrm{C}$ & 0.46218700 & 1.98883800 & -1.80521100 \\
\hline $\mathrm{C}$ & 5.18924100 & -0.97198200 & -0.14686500 & $\mathrm{H}$ & 0.84135400 & 2.99007500 & -2.03115300 \\
\hline $\mathrm{H}$ & 6.02066300 & -1.04899600 & -0.85739600 & $\mathrm{H}$ & 0.67676900 & 1.36411400 & -2.68073600 \\
\hline $\mathrm{H}$ & 5.29218400 & -0.01243500 & 0.36751800 & $\mathrm{C}$ & -1.06225900 & 2.08055300 & -1.59516800 \\
\hline $\mathrm{H}$ & 5.32418600 & -1.77416800 & 0.58498400 & $\mathrm{H}$ & -1.46298100 & 2.55344900 & -2.50080200 \\
\hline $\mathrm{C}$ & 3.76585900 & 0.07387600 & -1.92772500 & $\mathrm{H}$ & -1.28789400 & 2.77692900 & -0.77939300 \\
\hline $\mathrm{H}$ & 4.60657600 & 0.01425400 & -2.62854500 & $\mathrm{C}$ & -1.80070100 & 0.75809300 & -1.36388900 \\
\hline $\mathrm{H}$ & 2.84062200 & 0.02752700 & -2.50957600 & $\mathrm{H}$ & -1.52828000 & 0.04417900 & -2.15401400 \\
\hline $\mathrm{H}$ & 3.81420400 & 1.05346300 & -1.44118700 & $\mathrm{H}$ & -1.48074300 & 0.31181000 & -0.41403500 \\
\hline $\mathrm{C}$ & -0.14114100 & -1.59746100 & -2.90537100 & $\mathrm{C}$ & -3.32610000 & 0.93534200 & -1.33616700 \\
\hline $\mathrm{O}$ & -0.01783700 & -2.37284800 & -3.73883900 & $\mathrm{H}$ & -3.65931600 & 1.36954300 & -2.28762200 \\
\hline $\mathrm{H}$ & 0.88218700 & 1.58657800 & -0.28523400 & $\mathrm{H}$ & -3.58558900 & 1.66041400 & -0.55461400 \\
\hline $\mathrm{C}$ & 0.15201700 & 2.01160600 & -1.32762000 & $\mathrm{C}$ & -4.07140400 & -0.38017200 & -1.06949700 \\
\hline $\mathrm{C}$ & 0.36013500 & 1.41702100 & -2.47353500 & $\mathrm{H}$ & -3.83956100 & -1.10567600 & -1.85840100 \\
\hline $\mathrm{C}$ & -0.38711600 & 3.35534300 & -0.99489500 & $\mathrm{H}$ & -3.75270500 & -0.79724700 & -0.10983800 \\
\hline $\mathrm{C}$ & -0.07066100 & 4.47769700 & -1.77983200 & $P$ & -5.92815000 & -0.14206700 & -1.06573300 \\
\hline
\end{tabular}




\begin{tabular}{|c|c|c|c|c|c|c|c|}
\hline $\mathrm{C}$ & -6.57505600 & -1.93847600 & -0.86045600 & $\mathrm{C}$ & 5.28840000 & -1.75368900 & 0.83871300 \\
\hline $\mathrm{C}$ & -6.14843000 & 0.55806600 & 0.65245100 & $\mathrm{C}$ & 6.17298400 & -2.79554400 & 0.50785500 \\
\hline $\mathrm{C}$ & -7.34688600 & 1.22550400 & 0.95562300 & $\mathrm{C}$ & 5.74413300 & -0.68921700 & 1.63228400 \\
\hline $\mathrm{C}$ & -5.33449700 & 0.97930300 & 2.76846900 & $\mathrm{C}$ & 7.48512800 & -2.77301600 & 0.97665100 \\
\hline $\mathrm{C}$ & -7.53034400 & 1.75901000 & 2.22961100 & $\mathrm{H}$ & 5.82718600 & -3.61410100 & -0.11545200 \\
\hline $\mathrm{H}$ & -8.11568000 & 1.32627700 & 0.19585500 & $\mathrm{C}$ & 7.05562600 & -0.67935600 & 2.10699300 \\
\hline $\mathrm{C}$ & -6.50131800 & 1.63380500 & 3.16245000 & $\mathrm{H}$ & 5.06500800 & 0.11506100 & 1.89757500 \\
\hline $\mathrm{H}$ & -4.50471100 & 0.87123800 & 3.46575800 & $\mathrm{C}$ & 7.92928500 & -1.71766000 & 1.77784400 \\
\hline $\mathrm{H}$ & -8.45369100 & 2.27041400 & 2.48608000 & $\mathrm{H}$ & 8.16023400 & -3.58310700 & 0.71851900 \\
\hline $\mathrm{H}$ & -6.59366100 & 2.03693800 & 4.16585300 & $\mathrm{H}$ & 7.39367500 & 0.13817800 & 2.73637300 \\
\hline $\mathrm{N}$ & -5.15219400 & 0.44896400 & 1.55200400 & $\mathrm{H}$ & 8.95089600 & -1.70625600 & 2.14453300 \\
\hline $\mathrm{Pd}$ & 1.78044100 & -1.16752900 & -0.09116600 & $\mathrm{H}$ & 2.79997400 & -3.78385300 & 0.42212200 \\
\hline $\mathrm{C}$ & 3.88778000 & -1.80379100 & 0.36441000 & & & & \\
\hline $\mathrm{C}$ & 2.98487200 & -2.73087100 & 0.28442400 & \multirow{2}{*}{\multicolumn{4}{|c|}{$\begin{array}{l}\text { TS36b } \\
\text { imaginary frequency }=-1383.58 \mathrm{~cm}^{-1}\end{array}$}} \\
\hline $\mathrm{C}$ & -6.39320600 & -2.63192400 & -2.22636000 & & & & \\
\hline $\mathrm{H}$ & -6.90344500 & -2.08666500 & -3.02696900 & $\mathrm{C}$ & -2.69385300 & -1.24586100 & -0.97813700 \\
\hline $\mathrm{H}$ & -5.33850500 & -2.73386300 & -2.50328100 & $\mathrm{C}$ & -3.64559900 & -1.97956600 & -1.69606400 \\
\hline $\mathrm{H}$ & -6.81654700 & -3.64304500 & -2.18710700 & $\mathrm{C}$ & -4.73106800 & -1.32661900 & -2.28201300 \\
\hline $\mathrm{C}$ & -5.85694400 & -2.74007300 & 0.23705300 & $\mathrm{C}$ & -4.85474700 & 0.05450700 & -2.14408200 \\
\hline $\mathrm{H}$ & -6.32067000 & -3.72978300 & 0.33791500 & $\mathrm{C}$ & -3.88315900 & 0.73225000 & -1.41669100 \\
\hline $\mathrm{H}$ & -4.80009200 & -2.89928700 & 0.00154000 & $\mathrm{H}$ & -3.54166800 & -3.05163000 & -1.80501500 \\
\hline $\mathrm{H}$ & -5.91295400 & -2.24229200 & 1.20919700 & $\mathrm{H}$ & -5.46799100 & -1.89346500 & -2.84262900 \\
\hline $\mathrm{C}$ & -8.08114500 & -1.85722400 & -0.54548500 & $\mathrm{H}$ & -5.67868600 & 0.59995600 & -2.58999700 \\
\hline $\mathrm{H}$ & -8.62192300 & -1.27420900 & -1.29892100 & $\mathrm{H}$ & -3.91191200 & 1.80553000 & -1.26807700 \\
\hline $\mathrm{H}$ & -8.51109400 & -2.86615200 & -0.53521800 & $\mathrm{~N}$ & -2.84562000 & 0.08726100 & -0.85960700 \\
\hline $\mathrm{H}$ & -8.27096600 & -1.40967200 & 0.43456800 & $\mathrm{P}$ & -1.14872000 & -1.99627600 & -0.20136700 \\
\hline $\mathrm{C}$ & 0.08687400 & 1.88345600 & 1.85299600 & $\mathrm{C}$ & -1.83422200 & -2.85487900 & 1.36748900 \\
\hline $\mathrm{H}$ & 0.12707600 & 0.81963400 & 2.10669300 & $\mathrm{C}$ & -0.84135700 & -3.33638300 & -1.48163500 \\
\hline $\mathrm{H}$ & -0.00186700 & 2.44577600 & 2.78906600 & $\mathrm{H}$ & -1.62762500 & -4.09452900 & -1.40188100 \\
\hline $\mathrm{H}$ & -0.82284100 & 2.06452800 & 1.27592800 & $\mathrm{H}$ & -0.99171100 & -2.82576200 & -2.43987900 \\
\hline $\mathrm{C}$ & 1.29287000 & 3.84178800 & 0.79212700 & $\mathrm{C}$ & 0.53545700 & -4.02756800 & -1.51740800 \\
\hline $\mathrm{H}$ & 1.19811800 & 4.40468400 & 1.72740600 & $\mathrm{H}$ & 0.50271200 & -4.71642700 & -2.36897800 \\
\hline $\mathrm{H}$ & 2.19828100 & 4.19987400 & 0.29276200 & $\mathrm{H}$ & 0.66740200 & -4.66203400 & -0.63392000 \\
\hline $\mathrm{H}$ & 0.42872700 & 4.09579900 & 0.17245700 & $\mathrm{C}$ & 1.73090100 & -3.06293000 & -1.65841500 \\
\hline $\mathrm{C}$ & 2.58432000 & 2.05301200 & 2.01308400 & $\mathrm{H}$ & 2.40044800 & -3.40538400 & -2.45531700 \\
\hline $\mathrm{H}$ & 3.52110000 & 2.39246800 & 1.56048500 & $\mathrm{H}$ & 1.37367800 & -2.07543700 & -1.97593700 \\
\hline $\mathrm{H}$ & 2.46415400 & 2.58900800 & 2.96076000 & $\mathrm{C}$ & 2.54461200 & -2.91999600 & -0.36322800 \\
\hline $\mathrm{H}$ & 2.67359100 & 0.98719100 & 2.24839100 & $\mathrm{H}$ & 3.17268900 & -3.81038600 & -0.23614700 \\
\hline $\mathrm{H}$ & 3.75311500 & -0.53534100 & -0.24846600 & $\mathrm{H}$ & 1.86827600 & -2.90988100 & 0.49848700 \\
\hline $\mathrm{C}$ & 0.07628500 & -2.03969700 & -0.39212100 & $\mathrm{C}$ & 3.43579300 & -1.66882000 & -0.35539100 \\
\hline $\mathrm{O}$ & -0.90784500 & -2.59130900 & -0.56012800 & $\mathrm{H}$ & 4.32562200 & -1.81972400 & 0.26224800 \\
\hline
\end{tabular}




\begin{tabular}{|c|c|c|c|c|c|c|c|}
\hline $\mathrm{H}$ & 3.77876700 & -1.43346700 & -1.36634500 & $\mathrm{H}$ & 0.19126400 & -3.01263200 & 2.21028100 \\
\hline $\mathrm{P}$ & 2.58839700 & -0.12138600 & 0.25608800 & $\mathrm{H}$ & -1.01186900 & -4.06586800 & 2.96148900 \\
\hline $\mathrm{C}$ & 3.22673200 & 0.07610200 & 2.05081400 & $\mathrm{H}$ & -0.35087000 & -4.48523000 & 1.38169300 \\
\hline $\mathrm{C}$ & 3.38961200 & 1.17799600 & -0.81476600 & $\mathrm{C}$ & -3.01177200 & -3.80626500 & 1.09519300 \\
\hline $\mathrm{C}$ & 3.69285100 & 2.48212800 & -0.39860000 & $\mathrm{H}$ & -3.31212900 & -4.27825300 & 2.03756500 \\
\hline $\mathrm{C}$ & 4.06787000 & 1.64798700 & -2.96795900 & $\mathrm{H}$ & -3.88786400 & -3.28238400 & 0.70373000 \\
\hline $\mathrm{C}$ & 4.21373300 & 3.38230700 & -1.32963100 & $\mathrm{H}$ & -2.74941100 & -4.61373200 & 0.40469400 \\
\hline $\mathrm{H}$ & 3.54311800 & 2.79566400 & 0.62678200 & $\mathrm{C}$ & -2.28032800 & -1.74008300 & 2.33365200 \\
\hline $\mathrm{C}$ & 4.40606700 & 2.96280900 & -2.64336100 & $\mathrm{H}$ & -3.11492700 & -1.15748100 & 1.92995100 \\
\hline $\mathrm{H}$ & 4.20159500 & 1.27529800 & -3.98113200 & $\mathrm{H}$ & -2.61803800 & -2.18795700 & 3.27498300 \\
\hline $\mathrm{H}$ & 4.46632400 & 4.39430000 & -1.02728900 & $\mathrm{H}$ & -1.46019500 & -1.05257800 & 2.56236800 \\
\hline $\mathrm{H}$ & 4.80849100 & 3.62980700 & -3.39828700 & $\mathrm{H}$ & -1.85947300 & 0.78118300 & -0.25785300 \\
\hline $\mathrm{N}$ & 3.57287100 & 0.77698700 & -2.08271600 & & & & \\
\hline $\mathrm{Pd}$ & 0.22383700 & 0.05437400 & 0.12245300 & \multirow{2}{*}{\multicolumn{4}{|c|}{$\begin{array}{l}\text { TS36 } \\
\text { imaginary frequency }=-695.04 \mathrm{~cm}^{-1}\end{array}$}} \\
\hline $\mathrm{C}$ & -3.25935700 & 5.03881700 & 0.07665900 & & & & \\
\hline $\mathrm{C}$ & -2.22769900 & 4.13319500 & -0.16840100 & $\mathrm{C}$ & 3.05435100 & 1.00483500 & 0.73383200 \\
\hline $\mathrm{C}$ & -2.19545000 & 2.89534800 & 0.49900900 & $\mathrm{C}$ & 4.12181800 & 1.32797700 & 1.58281700 \\
\hline $\mathrm{C}$ & -3.22400500 & 2.58411100 & 1.40463500 & $\mathrm{C}$ & 4.61656200 & 2.63292800 & 1.61287500 \\
\hline $\mathrm{C}$ & -4.24777900 & 3.49854500 & 1.65378900 & $\mathrm{C}$ & 4.03819400 & 3.60254100 & 0.79432400 \\
\hline $\mathrm{C}$ & -4.27131700 & 4.72603300 & 0.98813400 & $\mathrm{C}$ & 2.95642500 & 3.23117300 & 0.00361500 \\
\hline $\mathrm{H}$ & -3.27089000 & 5.99222200 & -0.44328100 & $\mathrm{H}$ & 4.57393600 & 0.56874100 & 2.20862600 \\
\hline $\mathrm{H}$ & -1.44268000 & 4.37536100 & -0.87829500 & $\mathrm{H}$ & 5.45056900 & 2.88446800 & 2.26084900 \\
\hline $\mathrm{H}$ & -3.20414000 & 1.63284900 & 1.92909300 & $\mathrm{H}$ & 4.40562300 & 4.62249100 & 0.77388400 \\
\hline $\mathrm{H}$ & -5.02662800 & 3.25229500 & 2.36945900 & $\mathrm{H}$ & 2.44307200 & 3.94682900 & -0.63246900 \\
\hline $\mathrm{H}$ & -5.07129400 & 5.43469600 & 1.17881300 & $\mathrm{~N}$ & 2.48017200 & 1.97520600 & -0.01157500 \\
\hline $\mathrm{C}$ & -1.09648000 & 1.93564000 & 0.25067300 & $\mathrm{P}$ & 2.34963400 & -0.70850300 & 0.48513300 \\
\hline $\mathrm{C}$ & 0.19655200 & 2.04527500 & 0.24854500 & $\mathrm{C}$ & 3.74467700 & -1.57774800 & -0.52136600 \\
\hline $\mathrm{H}$ & 0.97103300 & 2.79221400 & 0.34347200 & $\mathrm{C}$ & 2.51152300 & -1.32426100 & 2.25990300 \\
\hline $\mathrm{C}$ & 2.88988700 & -1.23569000 & 2.79403700 & $\mathrm{H}$ & 3.57402500 & -1.39875800 & 2.51019500 \\
\hline $\mathrm{H}$ & 1.81421400 & -1.43848200 & 2.78334700 & $\mathrm{H}$ & 2.10715800 & -0.50747900 & 2.87025400 \\
\hline $\mathrm{H}$ & 3.41228700 & -2.10308600 & 2.38211100 & $\mathrm{C}$ & 1.81196700 & -2.63324900 & 2.67614900 \\
\hline $\mathrm{H}$ & 3.19656700 & -1.13870300 & 3.84139700 & $\mathrm{H}$ & 2.05365500 & -2.78277200 & 3.73383200 \\
\hline $\mathrm{C}$ & 4.74939000 & 0.31217500 & 2.09568700 & $\mathrm{H}$ & 2.25224000 & -3.48973400 & 2.15442400 \\
\hline $\mathrm{H}$ & 5.07268000 & 0.35928200 & 3.14182400 & $\mathrm{C}$ & 0.27952300 & -2.63639800 & 2.49150600 \\
\hline $\mathrm{H}$ & 5.31259100 & -0.49605700 & 1.62033200 & $\mathrm{H}$ & -0.20359800 & -3.05121000 & 3.38404900 \\
\hline $\mathrm{H}$ & 5.04161100 & 1.24955500 & 1.61711600 & $\mathrm{H}$ & -0.06778900 & -1.59623000 & 2.41823600 \\
\hline $\mathrm{C}$ & 2.48980300 & 1.22144900 & 2.77295500 & $\mathrm{C}$ & -0.18013300 & -3.43872700 & 1.26637200 \\
\hline $\mathrm{H}$ & 1.40705200 & 1.07094900 & 2.76008800 & $\mathrm{H}$ & -0.04238200 & -4.50758500 & 1.46740000 \\
\hline $\mathrm{H}$ & 2.81825300 & 1.25288500 & 3.81796400 & $\mathrm{H}$ & 0.45715500 & -3.20635600 & 0.40646600 \\
\hline $\mathrm{H}$ & 2.69634200 & 2.20500100 & 2.34411000 & $\mathrm{C}$ & -1.64575500 & -3.21292800 & 0.86564600 \\
\hline $\mathrm{C}$ & -0.67530200 & -3.64775800 & 2.00609000 & $\mathrm{H}$ & -1.92694800 & -3.92906100 & 0.08583600 \\
\hline
\end{tabular}




\begin{tabular}{|c|c|c|c|c|c|c|c|}
\hline $\mathrm{H}$ & -2.32355400 & -3.39708300 & 1.70530800 & $\mathrm{C}$ & 0.13125500 & 1.88834800 & -1.38688700 \\
\hline $\mathrm{P}$ & -1.94630000 & -1.54237600 & 0.09386600 & $\mathrm{C}$ & 0.19575400 & 1.09114100 & -2.43429100 \\
\hline $\mathrm{C}$ & -3.58056400 & -1.72838500 & -0.90245500 & $\mathrm{C}$ & -0.69879700 & 3.07244800 & -1.08399600 \\
\hline $\mathrm{C}$ & -2.50829400 & -0.46136600 & 1.51213700 & $\mathrm{C}$ & -1.66744500 & 3.54022600 & -1.99643400 \\
\hline $\mathrm{C}$ & -3.18196700 & -0.85938900 & 2.66720000 & $\mathrm{C}$ & -0.52481100 & 3.76626900 & 0.13057200 \\
\hline $\mathrm{C}$ & -2.65887000 & 1.81822200 & 2.25444200 & $\mathrm{C}$ & -2.42567300 & 4.67054900 & -1.70516800 \\
\hline $\mathrm{C}$ & -3.59377400 & 0.08642300 & 3.61042700 & $\mathrm{H}$ & -1.81509100 & 3.01932400 & -2.93784200 \\
\hline $\mathrm{H}$ & -3.39412200 & -1.90839700 & 2.82947800 & $\mathrm{C}$ & -1.28787200 & 4.90130200 & 0.41704900 \\
\hline $\mathrm{C}$ & -3.33792700 & 1.44466000 & 3.40071100 & $\mathrm{H}$ & 0.22851500 & 3.43219200 & 0.83833300 \\
\hline $\mathrm{H}$ & -2.40477300 & 2.84024500 & 1.99748500 & $\mathrm{C}$ & -2.23962500 & 5.35558000 & -0.49928700 \\
\hline $\mathrm{H}$ & -4.12106800 & -0.23666100 & 4.50292300 & $\mathrm{H}$ & -3.15942700 & 5.02434900 & -2.42262100 \\
\hline $\mathrm{H}$ & -3.65406000 & 2.19947400 & 4.11112700 & $\mathrm{H}$ & -1.12234600 & 5.44393900 & 1.34346800 \\
\hline $\mathrm{N}$ & -2.26710200 & 0.87149800 & 1.37345200 & $\mathrm{H}$ & -2.82604500 & 6.24258200 & -0.28182300 \\
\hline $\mathrm{Pd}$ & -0.05210900 & -0.49450400 & -1.24844000 & $\mathrm{H}$ & 0.29066700 & 1.08951800 & -3.51250400 \\
\hline $\mathrm{C}$ & -4.67621700 & -2.43721600 & -0.08545800 & $\mathrm{H}$ & -1.74056100 & 1.17641000 & 0.54880500 \\
\hline $\mathrm{H}$ & -4.96128100 & -1.87841100 & 0.81078400 & & & & \\
\hline $\mathrm{H}$ & -5.57573000 & -2.52400600 & -0.70426400 & 44 & & & \\
\hline $\mathrm{H}$ & -4.39180100 & -3.45132900 & 0.20831800 & $\mathrm{C}$ & -0.37985900 & 2.34232700 & -0.59539000 \\
\hline $\mathrm{C}$ & -3.25160400 & -2.55698800 & -2.16151700 & $\mathrm{C}$ & -1.44302300 & 3.25440700 & -0.59578500 \\
\hline $\mathrm{H}$ & -2.53936700 & -2.04454600 & -2.81264600 & $\mathrm{C}$ & -1.77524300 & 3.89430700 & -1.79058100 \\
\hline $\mathrm{H}$ & -2.86213800 & -3.55303800 & -1.92986700 & $\mathrm{C}$ & -1.04421300 & 3.60023100 & -2.93944100 \\
\hline $\mathrm{H}$ & -4.17415500 & -2.70175100 & -2.73337100 & $\mathrm{C}$ & -0.01020100 & 2.66690900 & -2.84426100 \\
\hline $\mathrm{C}$ & -4.05628300 & -0.32641400 & -1.32774700 & $\mathrm{H}$ & -1.99535500 & 3.47367900 & 0.31067400 \\
\hline $\mathrm{H}$ & -3.28186100 & 0.22101200 & -1.87541200 & $\mathrm{H}$ & -2.58784000 & 4.61411500 & -1.81818600 \\
\hline $\mathrm{H}$ & -4.91782300 & -0.43043400 & -1.99536400 & $\mathrm{H}$ & -1.26368200 & 4.08010900 & -3.88754600 \\
\hline $\mathrm{H}$ & -4.38474200 & 0.27986700 & -0.47748600 & $\mathrm{H}$ & 0.58191800 & 2.40497600 & -3.71796600 \\
\hline $\mathrm{C}$ & 3.28632200 & -3.02552100 & -0.79242700 & $\mathrm{~N}$ & 0.31948300 & 2.05088200 & -1.70367500 \\
\hline $\mathrm{H}$ & 3.22012700 & -3.61824600 & 0.12418500 & $\mathrm{P}$ & 0.24855800 & 1.50671100 & 0.93460400 \\
\hline $\mathrm{H}$ & 2.31998800 & -3.06802800 & -1.30170800 & $\mathrm{C}$ & 1.50099400 & 2.79459700 & 1.62580700 \\
\hline $\mathrm{H}$ & 4.01843200 & -3.51788300 & -1.44174400 & $\mathrm{C}$ & -1.19365600 & 1.53683600 & 2.11073100 \\
\hline $\mathrm{C}$ & 5.10622000 & -1.61255500 & 0.19456000 & $\mathrm{H}$ & -1.41562400 & 2.57492500 & 2.37334400 \\
\hline $\mathrm{H}$ & 5.82475000 & -2.13944400 & -0.44304200 & $\mathrm{H}$ & -2.05150700 & 1.18695700 & 1.53233000 \\
\hline $\mathrm{H}$ & 5.51238300 & -0.61380000 & 0.37157600 & $\mathrm{C}$ & -1.00990400 & 0.69011800 & 3.39982000 \\
\hline $\mathrm{H}$ & 5.07165200 & -2.15431600 & 1.14430800 & $\mathrm{H}$ & -1.01287700 & 1.37717700 & 4.25169200 \\
\hline $\mathrm{C}$ & 3.89142800 & -0.82227600 & -1.85670100 & $\mathrm{H}$ & -0.02401100 & 0.21214700 & 3.41599900 \\
\hline $\mathrm{H}$ & 4.60676500 & -1.35216200 & -2.49471200 & $\mathrm{C}$ & -2.07302200 & -0.39863000 & 3.66119700 \\
\hline $\mathrm{H}$ & 2.94465400 & -0.76154100 & -2.40232800 & $\mathrm{H}$ & -2.13028300 & -0.52499900 & 4.74768800 \\
\hline $\mathrm{H}$ & 4.27076800 & 0.19425000 & -1.71556500 & $\mathrm{H}$ & -3.06693000 & -0.03985500 & 3.36161200 \\
\hline $\mathrm{C}$ & 0.22807100 & -1.93894300 & -2.60458800 & $\mathrm{C}$ & -1.79804100 & -1.80196500 & 3.07051400 \\
\hline $\mathrm{O}$ & 0.39657900 & -2.70429100 & -3.43386700 & $\mathrm{H}$ & -2.17835500 & -2.53649400 & 3.78994200 \\
\hline $\mathrm{H}$ & 1.19653400 & 1.79881000 & -0.70921300 & $\mathrm{H}$ & -0.71413800 & -1.96947400 & 3.02834500 \\
\hline
\end{tabular}




\begin{tabular}{|c|c|c|c|c|c|c|c|}
\hline $\mathrm{C}$ & -2.43146300 & -2.20282900 & 1.72050200 & $\mathrm{C}$ & 1.45679000 & -2.69138400 & 0.44889300 \\
\hline $\mathrm{H}$ & -2.33399000 & -3.28804200 & 1.63921300 & $\mathrm{O}$ & 1.93902100 & -3.72506300 & 0.46867200 \\
\hline $\mathrm{H}$ & -3.50852800 & -2.01009000 & 1.72964700 & $\mathrm{C}$ & 3.33731200 & -0.72337100 & 1.99486400 \\
\hline $\mathrm{P}$ & -1.68815500 & -1.55813700 & 0.12766500 & $\mathrm{C}$ & 2.83339000 & -0.63298500 & 0.75851100 \\
\hline $\mathrm{C}$ & -1.90929400 & -3.00895400 & -1.13092000 & $\mathrm{C}$ & 3.62358100 & -0.59272500 & -0.48696200 \\
\hline $\mathrm{C}$ & -2.91156500 & -0.31986200 & -0.53120300 & $\mathrm{C}$ & 3.16254500 & 0.06423400 & -1.64354300 \\
\hline $\mathrm{C}$ & -4.05849600 & 0.10619100 & 0.15155500 & $\mathrm{C}$ & 4.86778400 & -1.25768700 & -0.54372900 \\
\hline $\mathrm{C}$ & -3.50208600 & 0.89207100 & -2.40021200 & $\mathrm{C}$ & 3.93586100 & 0.07936800 & -2.80464300 \\
\hline $\mathrm{C}$ & -4.95175300 & 0.96050800 & -0.49951400 & $\mathrm{H}$ & 2.20564800 & 0.57824600 & -1.62964600 \\
\hline $\mathrm{H}$ & -4.27149100 & -0.22101700 & 1.16159800 & $\mathrm{C}$ & 5.63621300 & -1.23376600 & -1.70396600 \\
\hline $\mathrm{C}$ & -4.67908300 & 1.35437700 & -1.80600400 & $\mathrm{H}$ & 5.22105300 & -1.80566700 & 0.32406900 \\
\hline $\mathrm{H}$ & -3.24404500 & 1.18142400 & -3.41597300 & $\mathrm{C}$ & 5.17372200 & -0.56397700 & -2.84022300 \\
\hline $\mathrm{H}$ & -5.85187200 & 1.29671900 & 0.00648600 & $\mathrm{H}$ & 3.56910700 & 0.59788200 & -3.68584100 \\
\hline $\mathrm{H}$ & -5.35552900 & 2.00073500 & -2.35531100 & $\mathrm{H}$ & 6.59196800 & -1.74885400 & -1.72589600 \\
\hline $\mathrm{N}$ & -2.63243800 & 0.08762300 & -1.78164500 & $\mathrm{H}$ & 5.77051000 & -0.55313100 & -3.74724600 \\
\hline $\mathrm{Pd}$ & 0.75265000 & -0.86136100 & 0.48975700 & $\mathrm{H}$ & 4.40704400 & -0.61228500 & 2.17315700 \\
\hline $\mathrm{H}$ & 2.72228600 & -0.89112000 & 2.87294000 & & & & \\
\hline $\mathrm{C}$ & -3.38831400 & -3.15687200 & -1.54302900 & \multicolumn{4}{|c|}{ TS37 } \\
\hline $\mathrm{H}$ & -3.74945200 & -2.29995100 & -2.11376700 & \multicolumn{4}{|c|}{ imaginary frequency $=-250.27 \mathrm{~cm}^{-1}$} \\
\hline $\mathrm{H}$ & -3.48728300 & -4.04262200 & -2.18061700 & $\mathrm{C}$ & -0.89565600 & 2.06735500 & 0.93728100 \\
\hline $\mathrm{H}$ & -4.04628100 & -3.30386200 & -0.68007300 & $\mathrm{C}$ & -0.71901100 & 3.33001000 & 1.51759100 \\
\hline $\mathrm{C}$ & -1.45194500 & -4.34391800 & -0.50789200 & $\mathrm{C}$ & -1.19297700 & 3.54475500 & 2.81315400 \\
\hline $\mathrm{H}$ & -0.45857800 & -4.30276600 & -0.05905300 & $\mathrm{C}$ & -1.82659500 & 2.49971500 & 3.48158900 \\
\hline $\mathrm{H}$ & -2.15235700 & -4.72134900 & 0.24216200 & $\mathrm{C}$ & -1.95805100 & 1.27578100 & 2.82131700 \\
\hline $\mathrm{H}$ & -1.40956700 & -5.09452600 & -1.30445100 & $\mathrm{H}$ & -0.22588300 & 4.13392600 & 0.98321400 \\
\hline $\mathrm{C}$ & -1.04280900 & -2.69696300 & -2.36803900 & $\mathrm{H}$ & -1.07157700 & 4.51445100 & 3.28683600 \\
\hline $\mathrm{H}$ & 0.02286300 & -2.66486700 & -2.11983400 & $\mathrm{H}$ & -2.21384200 & 2.62537900 & 4.48739500 \\
\hline $\mathrm{H}$ & -1.18598900 & -3.48811100 & -3.11294500 & $\mathrm{H}$ & -2.45442300 & 0.43444100 & 3.29861300 \\
\hline $\mathrm{H}$ & -1.31737700 & -1.74199400 & -2.81878700 & $\mathrm{~N}$ & -1.50023600 & 1.05791100 & 1.58478000 \\
\hline $\mathrm{C}$ & 1.96440100 & 2.35944800 & 3.02881800 & $\mathrm{P}$ & -0.36210000 & 1.59700700 & -0.77651000 \\
\hline $\mathrm{H}$ & 1.14981000 & 2.37484200 & 3.75795300 & $\mathrm{C}$ & -1.69869900 & 2.36657300 & -1.92524700 \\
\hline $\mathrm{H}$ & 2.41506800 & 1.36621900 & 3.01952400 & $\mathrm{C}$ & 1.14513300 & 2.68574200 & -1.00746600 \\
\hline $\mathrm{H}$ & 2.72573300 & 3.06556200 & 3.37890400 & $\mathrm{H}$ & 0.79499900 & 3.70423800 & -0.82073100 \\
\hline $\mathrm{C}$ & 0.83970600 & 4.18432800 & 1.72901100 & $\mathrm{H}$ & 1.82307900 & 2.44425300 & -0.18321000 \\
\hline $\mathrm{H}$ & 1.57225100 & 4.87723300 & 2.15749700 & $\mathrm{C}$ & 1.92419000 & 2.68944700 & -2.34424100 \\
\hline $\mathrm{H}$ & 0.54485900 & 4.58240700 & 0.75562900 & $\mathrm{H}$ & 2.40655900 & 3.67175600 & -2.41330900 \\
\hline $\mathrm{H}$ & -0.03149200 & 4.19629600 & 2.39104200 & $\mathrm{H}$ & 1.24373700 & 2.64164100 & -3.20084900 \\
\hline $\mathrm{C}$ & 2.70927000 & 2.88872400 & 0.67514400 & $\mathrm{C}$ & 3.02479200 & 1.62805000 & -2.49189400 \\
\hline $\mathrm{H}$ & 3.38359300 & 3.66855400 & 1.04695500 & $\mathrm{H}$ & 3.69093500 & 1.95181300 & -3.29878500 \\
\hline $\mathrm{H}$ & 3.27066900 & 1.95551300 & 0.63105000 & $\mathrm{H}$ & 3.63823000 & 1.64430700 & -1.58483300 \\
\hline $\mathrm{H}$ & 2.41501900 & 3.16175500 & -0.34147200 & $\mathrm{C}$ & 2.54989300 & 0.18448500 & -2.79437600 \\
\hline
\end{tabular}




$\begin{array}{lrrrrrrr}\mathrm{H} & 2.85837600 & -0.08577800 & -3.81015800 & \mathrm{H} & -3.23570000 & 1.01028600 & -1.11985700 \\ \mathrm{H} & 1.45490300 & 0.12893000 & -2.79826700 & \mathrm{H} & -3.25434500 & 2.62062600 & -0.39572300 \\ \mathrm{C} & 3.09536300 & -0.90461500 & -1.84973800 & \mathrm{C} & -0.72386200 & -2.75008400 & -0.59480600 \\ \mathrm{H} & 2.96832100 & -1.88020100 & -2.32885400 & \mathrm{O} & -0.82819200 & -3.89675400 & -0.50255000 \\ \mathrm{H} & 4.17194200 & -0.78070700 & -1.68820600 & \mathrm{C} & -2.50297600 & -1.74093400 & -2.42272200 \\ \mathrm{P} & 2.19899800 & -1.08791300 & -0.22731000 & \mathrm{C} & -2.29584500 & -1.56948600 & -1.10690800 \\ \mathrm{C} & 2.85284600 & -2.73797400 & 0.50188100 & \mathrm{C} & -3.33505000 & -1.54875700 & -0.05796800 \\ \mathrm{C} & 2.86505600 & 0.16600900 & 0.96526100 & \mathrm{C} & -3.04570600 & -1.89785100 & 1.27235700 \\ \mathrm{C} & 4.15036100 & 0.72166500 & 0.89864800 & \mathrm{C} & -4.66563500 & -1.21400600 & -0.38036400 \\ \mathrm{C} & 2.40119000 & 1.30416100 & 2.91301800 & \mathrm{C} & -4.05124700 & -1.94797900 & 2.23645400 \\ \mathrm{C} & 4.54629600 & 1.61587700 & 1.89456100 & \mathrm{H} & -2.02585400 & -2.13109100 & 1.55952700 \\ \mathrm{H} & 4.83546600 & 0.45967400 & 0.10044100 & \mathrm{C} & -5.66693400 & -1.25134100 & 0.58665900 \\ \mathrm{C} & 3.65601500 & 1.91645800 & 2.92367600 & \mathrm{H} & -4.91883100 & -0.91783000 & -1.39345800 \\ \mathrm{H} & 1.67702700 & 1.50718100 & 3.69832300 & \mathrm{C} & -5.36645200 & -1.62475200 & 1.89860000 \\ \mathrm{H} & 5.53565600 & 2.06249500 & 1.86752000 & \mathrm{H} & -3.80619200 & -2.24315500 & 3.25259000 \\ \mathrm{H} & 3.92459500 & 2.60459200 & 3.71831600 & \mathrm{H} & -6.68490900 & -0.99015100 & 0.31370600 \\ \mathrm{~N} & 2.00650100 & 0.45285700 & 1.95800900 & \mathrm{H} & -6.14946600 & -1.66030300 & 2.64971200 \\ \mathrm{Pd} & -0.20698500 & -0.92218000 & -0.60812800 & \mathrm{H} & -3.50556500 & -1.84153200 & -2.83627100 \\ \mathrm{H} & -686380 & -1.800 & & & \end{array}$

$\mathrm{H}$

C

$\mathrm{H}$

$\mathrm{H}$

$\mathrm{H}$

C

$\mathrm{H}$

$\mathrm{H}$

$\mathrm{H}$

C

$\mathrm{H}$

$\mathrm{H}$

$\mathrm{H}$

C

$\mathrm{H}$

$\mathrm{H}$

$\mathrm{H}$

C

$\mathrm{H}$

$\mathrm{H}$

$\mathrm{H}$

C

$\mathrm{H}$ $\begin{array}{lll}-1.68633800 & -1.81735600 & -3.13305300\end{array}$

$4.34978600 \quad-2.63021500 \quad 0.85802000$

$\begin{array}{lll}4.53766400 & -1.91280100 & 1.65988800\end{array}$

$4.69658900 \quad-3.60952100 \quad 1.20607500$

$\begin{array}{lll}4.96725300 & -2.35857100 & -0.00421000\end{array}$

2.67375200

1.65615700

3.36148300

2.90170700

2.04540800

0.98941300

2.44521700

2.11001900

$-1.58973800$

$-0.62880900$

$-1.73490400$

$-2.36915100$

$-1.53617300$

$-2.37210200$

$-1.56674500$

$-0.61586900$

$-3.08692800$

$-3.87311900$

$-0.52700800$

$-3.94761900 \quad-0.91389500$

$-3.77640000 \quad-1.37167900$

$-4.82599000 \quad-0.03704700$

$-3.04739600 \quad 1.77840100$

$-3.22488700 \quad 1.56097600$

$-3.95656500$

2.24098400

$-2.23934700 \quad 2.51204000$

$1.70446000 \quad-3.31341100$

$1.89558800 \quad-3.79915300$

$0.62388700-3.25117700$

$2.11108700 \quad-3.96806100$

$3.89192100 \quad-2.06178000$

$4.28126800 \quad-2.65399500$

$4.40000500 \quad-1.09270500$

$4.17740300 \quad-2.57820400$

$2.07036400-1.32516600$

$-3.85457900$

$-2.04060600$

$$
\begin{array}{rrr}
-3.23570000 & 1.01028600 & -1.11985700 \\
-3.25434500 & 2.62062600 & -0.39572300 \\
-0.72386200 & -2.75008400 & -0.59480600 \\
-0.82819200 & -3.89675400 & -0.50255000 \\
-2.50297600 & -1.74093400 & -2.42272200 \\
-2.29584500 & -1.56948600 & -1.10690800 \\
-3.33505000 & -1.54875700 & -0.05796800 \\
-3.04570600 & -1.89785100 & 1.27235700 \\
-4.66563500 & -1.21400600 & -0.38036400 \\
-4.05124700 & -1.94797900 & 2.23645400 \\
-2.02585400 & -2.13109100 & 1.55952700 \\
-5.66693400 & -1.25134100 & 0.58665900 \\
-4.91883100 & -0.91783000 & -1.39345800 \\
-5.36645200 & -1.62475200 & 1.89860000 \\
-3.80619200 & -2.24315500 & 3.25259000 \\
-6.68490900 & -0.99015100 & 0.31370600 \\
-6.14946600 & -1.66030300 & 2.64971200 \\
-3.50556500 & -1.84153200 & -2.83627100
\end{array}
$$

\section{TS37a}

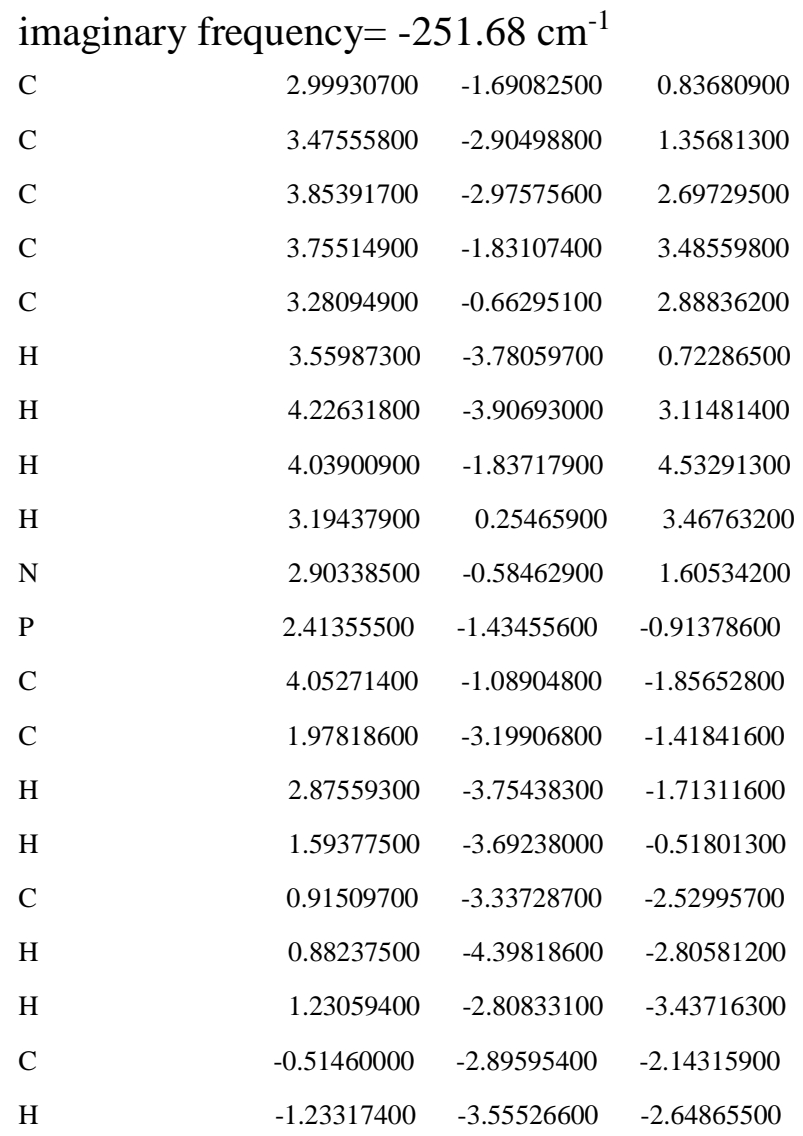




\begin{tabular}{|c|c|c|c|c|c|c|c|}
\hline $\mathrm{H}$ & -0.65915000 & -3.05820100 & -1.06853500 & $\mathrm{C}$ & 4.52966900 & 0.31326100 & -1.43435000 \\
\hline $\mathrm{C}$ & -0.86292300 & -1.44620100 & -2.51779400 & $\mathrm{H}$ & 5.42801400 & 0.58392000 & -2.00240400 \\
\hline $\mathrm{H}$ & -0.69867600 & -1.32360600 & -3.59537600 & $\mathrm{H}$ & 3.76853700 & 1.07618300 & -1.62392700 \\
\hline $\mathrm{H}$ & -0.17145100 & -0.75514600 & -2.02434100 & $\mathrm{H}$ & 4.78450900 & 0.34967300 & -0.37132300 \\
\hline $\mathrm{C}$ & -2.32795100 & -1.04140900 & -2.23633800 & $\mathrm{C}$ & -1.13314300 & 1.03793700 & 2.15970900 \\
\hline $\mathrm{H}$ & -2.60364300 & -0.21025400 & -2.89436300 & $\mathrm{O}$ & -0.94936900 & 0.95904200 & 3.27814100 \\
\hline $\mathrm{H}$ & -3.01086500 & -1.86393800 & -2.47861700 & $\mathrm{C}$ & -1.40801200 & 4.39330300 & 0.36709100 \\
\hline $\mathrm{P}$ & -2.70698900 & -0.39624700 & -0.53575700 & $\mathrm{C}$ & -0.43171400 & 3.50423800 & 0.12300200 \\
\hline $\mathrm{C}$ & -4.53224400 & 0.16060500 & -0.57636200 & $\mathrm{C}$ & -0.96910400 & 2.43083400 & -1.46433800 \\
\hline $\mathrm{C}$ & -2.67037000 & -1.81974300 & 0.64958500 & $\mathrm{O}$ & -0.79613400 & 2.72338400 & -2.56678000 \\
\hline $\mathrm{C}$ & -3.05141000 & -3.12282900 & 0.30315200 & $\mathrm{C}$ & 1.01303800 & 3.70571000 & 0.28331600 \\
\hline $\mathrm{C}$ & -2.28080700 & -2.44130800 & 2.83490000 & $\mathrm{C}$ & 1.55490100 & 4.98653000 & 0.04736700 \\
\hline $\mathrm{C}$ & -3.03288500 & -4.10980500 & 1.29016400 & $\mathrm{C}$ & 1.87029600 & 2.66896200 & 0.68693700 \\
\hline $\mathrm{H}$ & -3.35216700 & -3.36867300 & -0.70864600 & $\mathrm{C}$ & 2.90784800 & 5.22992300 & 0.26184600 \\
\hline $\mathrm{C}$ & -2.64073700 & -3.76616000 & 2.58216100 & $\mathrm{H}$ & 0.91176400 & 5.78443500 & -0.31097200 \\
\hline $\mathrm{H}$ & -1.96663000 & -2.12779000 & 3.82773100 & $\mathrm{C}$ & 3.22389500 & 2.92075900 & 0.90407700 \\
\hline $\mathrm{H}$ & -3.32115800 & -5.12858500 & 1.04996000 & $\mathrm{H}$ & 1.50041500 & 1.65795700 & 0.83338100 \\
\hline $\mathrm{H}$ & -2.61109900 & -4.50303700 & 3.37769100 & $\mathrm{C}$ & 3.74516300 & 4.19918300 & 0.69847100 \\
\hline $\mathrm{N}$ & -2.29239000 & -1.49039100 & 1.89383300 & $\mathrm{H}$ & 3.31080200 & 6.22157700 & 0.08037100 \\
\hline $\mathrm{Pd}$ & -1.31282700 & 1.46483400 & 0.15574000 & $\mathrm{H}$ & 3.86234300 & 2.10451900 & 1.22327500 \\
\hline $\mathrm{H}$ & -2.44914100 & 4.20545800 & 0.12439200 & $\mathrm{H}$ & 4.80154800 & 4.39018600 & 0.86122200 \\
\hline $\mathrm{C}$ & -5.46735700 & -0.99049300 & -0.99340000 & $\mathrm{H}$ & -1.18282300 & 5.35417600 & 0.82722100 \\
\hline $\mathrm{H}$ & -5.42527900 & -1.83070000 & -0.29551900 & & & & \\
\hline $\mathrm{H}$ & -6.49953900 & -0.62318800 & -0.99969700 & $45 b$ & & & \\
\hline $\mathrm{H}$ & -5.25075500 & -1.35992100 & -2.00015400 & $\mathrm{C}$ & 0.20425200 & 2.71942300 & 1.09406600 \\
\hline $\mathrm{C}$ & -4.67160100 & 1.32651500 & -1.57567000 & $\mathrm{C}$ & 0.19702700 & 3.73743500 & 2.04711100 \\
\hline $\mathrm{H}$ & -4.01807100 & 2.16630100 & -1.31947600 & $\mathrm{C}$ & -0.90401600 & 3.85633400 & 2.89965000 \\
\hline $\mathrm{H}$ & -4.46119600 & 1.02678000 & -2.60641000 & $\mathrm{C}$ & -1.96214300 & 2.95856500 & 2.77291600 \\
\hline $\mathrm{H}$ & -5.70321300 & 1.69384800 & -1.55094300 & $\mathrm{C}$ & -1.88082200 & 1.95199800 & 1.81098500 \\
\hline $\mathrm{C}$ & -4.90461800 & 0.65017500 & 0.83761900 & $\mathrm{H}$ & 1.03455500 & 4.42181400 & 2.12402600 \\
\hline $\mathrm{H}$ & -4.28505300 & 1.49788600 & 1.14905700 & $\mathrm{H}$ & -0.93378300 & 4.64059600 & 3.64986300 \\
\hline $\mathrm{H}$ & -5.94794100 & 0.98410800 & 0.83890400 & $\mathrm{H}$ & -2.83424000 & 3.02051200 & 3.41453400 \\
\hline $\mathrm{H}$ & -4.80595900 & -0.13750500 & 1.58937400 & $\mathrm{H}$ & -2.66902000 & 1.21566200 & 1.69520100 \\
\hline $\mathrm{C}$ & 3.71051500 & -1.07620500 & -3.35982200 & $\mathrm{~N}$ & -0.82064200 & 1.83411400 & 0.99818300 \\
\hline $\mathrm{H}$ & 3.42344700 & -2.06735600 & -3.72474300 & $\mathrm{P}$ & 1.47922800 & 2.26370000 & -0.16827700 \\
\hline $\mathrm{H}$ & 2.89868500 & -0.37681900 & -3.58738800 & $\mathrm{C}$ & 1.31959400 & 3.63433400 & -1.49305000 \\
\hline $\mathrm{H}$ & 4.58925100 & -0.76124700 & -3.93509800 & $\mathrm{C}$ & 3.11513000 & 2.48530100 & 0.70846600 \\
\hline $\mathrm{C}$ & 5.17217900 & -2.10806500 & -1.58971700 & $\mathrm{H}$ & 3.37219700 & 3.54669100 & 0.79152800 \\
\hline $\mathrm{H}$ & 6.05850100 & -1.84084600 & -2.17825200 & $\mathrm{H}$ & 2.92713100 & 2.12403800 & 1.72659200 \\
\hline $\mathrm{H}$ & 5.46970600 & -2.12306400 & -0.53742200 & $\mathrm{C}$ & 4.30517500 & 1.69095700 & 0.11854200 \\
\hline $\mathrm{H}$ & 4.89273000 & -3.12571000 & -1.88097000 & $\mathrm{H}$ & 5.14113000 & 1.82317000 & 0.81438100 \\
\hline
\end{tabular}




\begin{tabular}{|c|c|c|c|c|c|c|c|}
\hline $\mathrm{H}$ & 4.63329900 & 2.13966200 & -0.82635200 & $\mathrm{H}$ & 1.48151200 & 5.77658400 & -1.73292200 \\
\hline $\mathrm{C}$ & 4.03073200 & 0.18550100 & -0.08200600 & $\mathrm{H}$ & 0.89641000 & 5.31210500 & -0.13721000 \\
\hline $\mathrm{H}$ & 4.94966400 & -0.38358500 & 0.10101800 & $\mathrm{H}$ & 2.61749000 & 5.14244000 & -0.54714200 \\
\hline $\mathrm{H}$ & 3.31824600 & -0.14471200 & 0.68199000 & $\mathrm{C}$ & -0.11778800 & 3.57142700 & -2.04592800 \\
\hline $\mathrm{C}$ & 3.50789500 & -0.15742500 & -1.48903900 & $\mathrm{H}$ & -0.21915000 & 4.28442500 & -2.87184500 \\
\hline $\mathrm{H}$ & 4.34201000 & -0.09695000 & -2.19979300 & $\mathrm{H}$ & -0.36064600 & 2.57568000 & -2.42999800 \\
\hline $\mathrm{H}$ & 2.78481200 & 0.59428000 & -1.81246800 & $\mathrm{H}$ & -0.86216200 & 3.83531000 & -1.28883000 \\
\hline $\mathrm{C}$ & 2.85597100 & -1.53833800 & -1.64630700 & $\mathrm{C}$ & -1.75207900 & -0.88886200 & 0.06353300 \\
\hline $\mathrm{H}$ & 2.59649400 & -1.68795400 & -2.69983200 & $\mathrm{O}$ & -1.96075400 & -1.54970800 & 1.04697600 \\
\hline $\mathrm{H}$ & 3.56527100 & -2.33078400 & -1.38524200 & $\mathrm{C}$ & -2.46937300 & -0.07531900 & -2.15609400 \\
\hline$P$ & 1.27701700 & -1.84979400 & -0.68450600 & $\mathrm{C}$ & -2.82719500 & -0.47860700 & -0.92246200 \\
\hline $\mathrm{C}$ & 0.57401100 & -3.43197600 & -1.51679700 & $\mathrm{C}$ & -4.24890000 & -0.51098300 & -0.47914400 \\
\hline $\mathrm{C}$ & 1.86682100 & -2.37613400 & 1.00290700 & $\mathrm{C}$ & -4.78256900 & -1.57932100 & 0.26249400 \\
\hline $\mathrm{C}$ & 2.89146300 & -3.30885100 & 1.21709100 & $\mathrm{C}$ & -5.09760600 & 0.55324800 & -0.83622300 \\
\hline $\mathrm{C}$ & 1.56341700 & -2.09768400 & 3.26889000 & $\mathrm{C}$ & -6.13200000 & -1.58776900 & 0.61324800 \\
\hline $\mathrm{C}$ & 3.23728400 & -3.63967500 & 2.52789900 & $\mathrm{H}$ & -4.14567100 & -2.40417400 & 0.55638100 \\
\hline $\mathrm{H}$ & 3.41956800 & -3.76641300 & 0.38896300 & $\mathrm{C}$ & -6.44499900 & 0.54153100 & -0.48059300 \\
\hline $\mathrm{C}$ & 2.55684800 & -3.02849400 & 3.57845000 & $\mathrm{H}$ & -4.69227600 & 1.39828400 & -1.38554100 \\
\hline $\mathrm{H}$ & 1.01448600 & -1.58960700 & 4.05831000 & $\mathrm{C}$ & -6.96711600 & -0.53147200 & 0.24414700 \\
\hline $\mathrm{H}$ & 4.02561300 & -4.36105700 & 2.72072800 & $\mathrm{H}$ & -6.53238000 & -2.42618300 & 1.17520600 \\
\hline $\mathrm{H}$ & 2.79083000 & -3.25867700 & 4.61260000 & $\mathrm{H}$ & -7.08407700 & 1.37241800 & -0.76411600 \\
\hline $\mathrm{N}$ & 1.22493100 & -1.77188000 & 2.01619400 & $\mathrm{H}$ & -8.01636100 & -0.54222000 & 0.52321900 \\
\hline $\mathrm{Pd}$ & -0.01808900 & 0.09904500 & -0.19050600 & $\mathrm{H}$ & -3.21525600 & 0.17538400 & -2.90351700 \\
\hline $\mathrm{H}$ & -1.42592200 & -0.01069900 & -2.44760900 & & & & \\
\hline $\mathrm{C}$ & 1.68005200 & -4.43172100 & -1.91442600 & 45 & & & \\
\hline $\mathrm{H}$ & 2.23870700 & -4.80357900 & -1.05222200 & $\mathrm{C}$ & 2.06174800 & -2.50481600 & 0.94732500 \\
\hline $\mathrm{H}$ & 1.20411000 & -5.30150900 & -2.38099600 & $\mathrm{C}$ & 1.83389800 & -1.88188300 & 2.18241800 \\
\hline $\mathrm{H}$ & 2.38525000 & -4.02393600 & -2.64263600 & $\mathrm{C}$ & 1.96467100 & -2.64682500 & 3.34312300 \\
\hline $\mathrm{C}$ & -0.17790400 & -2.99016600 & -2.78794400 & $\mathrm{C}$ & 2.29996900 & -3.99445400 & 3.22947400 \\
\hline $\mathrm{H}$ & -1.03364300 & -2.35323300 & -2.55558300 & $\mathrm{C}$ & 2.48902800 & -4.52709200 & 1.95226500 \\
\hline $\mathrm{H}$ & 0.46952900 & -2.45582600 & -3.49231600 & $\mathrm{H}$ & 1.54859200 & -0.83653800 & 2.23464900 \\
\hline $\mathrm{H}$ & -0.55558500 & -3.87777400 & -3.30752200 & $\mathrm{H}$ & 1.80020700 & -2.19618600 & 4.31727700 \\
\hline $\mathrm{C}$ & -0.39434500 & -4.13414200 & -0.54586000 & $\mathrm{H}$ & 2.40777100 & -4.62529200 & 4.10558300 \\
\hline $\mathrm{H}$ & -1.20376600 & -3.48514500 & -0.21232000 & $\mathrm{H}$ & 2.74143600 & -5.57677000 & 1.82049600 \\
\hline $\mathrm{H}$ & -0.84080600 & -4.99509100 & -1.05609500 & $\mathrm{~N}$ & 2.37707900 & -3.80321300 & 0.83216500 \\
\hline $\mathrm{H}$ & 0.12309700 & -4.50805400 & 0.34201800 & $\mathrm{P}$ & 1.93651300 & -1.53768200 & -0.63207300 \\
\hline $\mathrm{C}$ & 2.31410600 & 3.32921400 & -2.63160300 & $\mathrm{C}$ & 3.68414400 & -0.85021100 & -0.94001700 \\
\hline $\mathrm{H}$ & 3.35482000 & 3.35435300 & -2.29519300 & $\mathrm{C}$ & 1.63683400 & -2.84835200 & -1.94312300 \\
\hline $\mathrm{H}$ & 2.12278200 & 2.35616600 & -3.09388400 & $\mathrm{H}$ & 2.55152500 & -2.95610800 & -2.53199800 \\
\hline $\mathrm{H}$ & 2.20703500 & 4.09053500 & -3.41248200 & $\mathrm{H}$ & 1.50787900 & -3.79120300 & -1.40260400 \\
\hline $\mathrm{C}$ & 1.59792800 & 5.03907400 & -0.93030600 & $\mathrm{C}$ & 0.44730500 & -2.54327300 & -2.87153900 \\
\hline
\end{tabular}




\begin{tabular}{|c|c|c|c|c|c|c|c|}
\hline $\mathrm{H}$ & 0.44108100 & -3.27938000 & -3.68433200 & $\mathrm{H}$ & 5.71920300 & -1.55225400 & -1.09347000 \\
\hline $\mathrm{H}$ & 0.60007500 & -1.56689700 & -3.34736800 & $\mathrm{H}$ & 4.75015800 & -2.50909100 & 0.03501400 \\
\hline C & -0.91818500 & -2.55501400 & -2.16284900 & $\mathrm{H}$ & 4.55624400 & -2.72666900 & -1.70827400 \\
\hline $\mathrm{H}$ & -1.24963400 & -3.58682600 & -1.99083600 & $\mathrm{C}$ & 4.01610400 & 0.16747600 & 0.16792400 \\
\hline $\mathrm{H}$ & -0.79350100 & -2.18562100 & -1.11580700 & $\mathrm{H}$ & 5.01921300 & 0.57114000 & -0.00884300 \\
\hline C & -2.02730100 & -1.74594300 & -2.85983800 & $\mathrm{H}$ & 3.32136400 & 1.01145800 & 0.18003100 \\
\hline $\mathrm{H}$ & -2.49718300 & -2.36622500 & -3.63233200 & $\mathrm{H}$ & 4.02017800 & -0.29227000 & 1.16034500 \\
\hline $\mathrm{H}$ & -1.58255700 & -0.89370100 & -3.38847900 & $\mathrm{C}$ & 0.44869800 & 1.34821200 & 0.38355500 \\
\hline C & -3.12668300 & -1.24313800 & -1.90560500 & $\mathrm{O}$ & 0.41699900 & 1.30287000 & 1.58625800 \\
\hline $\mathrm{H}$ & -3.95147400 & -0.81029600 & -2.47741900 & $\mathrm{C}$ & 0.91857900 & 2.53179700 & -0.42167100 \\
\hline $\mathrm{H}$ & -3.56209500 & -2.05628700 & -1.31704100 & C & 0.81722700 & 2.49776400 & -1.76305800 \\
\hline$P$ & -2.54403700 & 0.03798900 & -0.66143700 & $\mathrm{H}$ & 0.43000500 & 1.62139500 & -2.27456900 \\
\hline C & -3.45578700 & 1.65395800 & -1.09004900 & $\mathrm{H}$ & 1.11178400 & 3.34716900 & -2.37025400 \\
\hline C & -3.28905800 & -0.53203000 & 0.93989900 & $\mathrm{C}$ & 1.50936700 & 3.68582700 & 0.31127300 \\
\hline C & -2.75078200 & -0.11915800 & 2.16646200 & $\mathrm{C}$ & 2.66107100 & 4.30420900 & -0.20674000 \\
\hline C & -4.93071800 & -1.75970600 & 1.97935400 & $\mathrm{C}$ & 0.93652100 & 4.19974700 & 1.48672100 \\
\hline $\mathrm{C}$ & -3.36824000 & -0.55549300 & 3.34034900 & $\mathrm{C}$ & 3.21560000 & 5.41677200 & 0.42317000 \\
\hline $\mathrm{H}$ & -1.86692700 & 0.50845500 & 2.20410400 & $\mathrm{H}$ & 3.13240300 & 3.89931600 & -1.09751100 \\
\hline C & -4.48037900 & -1.38964300 & 3.24865400 & $\mathrm{C}$ & 1.49184100 & 5.31525400 & 2.11109300 \\
\hline $\mathrm{H}$ & -5.78913200 & -2.41771700 & 1.86504100 & $\mathrm{H}$ & 0.05291300 & 3.73506300 & 1.90822800 \\
\hline $\mathrm{H}$ & -2.98036900 & -0.25253400 & 4.30829000 & $\mathrm{C}$ & 2.63094000 & 5.92686300 & 1.58340400 \\
\hline $\mathrm{H}$ & -4.98777900 & -1.75387900 & 4.13578400 & $\mathrm{H}$ & 4.10809700 & 5.87868600 & 0.01228000 \\
\hline N & -4.35446700 & -1.34143700 & 0.84633600 & $\mathrm{H}$ & 1.03194200 & 5.70839000 & 3.01259800 \\
\hline $\mathrm{Pd}$ & -0.14772000 & -0.29912300 & -0.57648600 & $\mathrm{H}$ & 3.06341500 & 6.79161100 & 2.07715000 \\
\hline C & -4.98267400 & 1.44592600 & -1.06497400 & & & & \\
\hline $\mathrm{H}$ & -5.33926400 & 1.12470500 & -0.08383800 & $45 a$ & & & \\
\hline H & -5.46925700 & 2.39817400 & -1.30478000 & $\mathrm{C}$ & 3.36636300 & 0.70224900 & -0.05443700 \\
\hline $\mathrm{H}$ & -5.32161600 & 0.71338800 & -1.80266600 & $\mathrm{C}$ & 3.11651000 & 1.31495000 & -1.29052300 \\
\hline $\mathrm{C}$ & -3.00594300 & 2.09793300 & -2.49567400 & $\mathrm{C}$ & 3.90435000 & 2.40723000 & -1.65835600 \\
\hline $\mathrm{H}$ & -1.92983900 & 2.28880100 & -2.53752600 & $\mathrm{C}$ & 4.89247500 & 2.85320800 & -0.78287200 \\
\hline $\mathrm{H}$ & -3.26018700 & 1.36380400 & -3.26679500 & $\mathrm{C}$ & 5.05224400 & 2.18659600 & 0.43379100 \\
\hline $\mathrm{H}$ & -3.51710800 & 3.03116800 & -2.75660400 & $\mathrm{H}$ & 2.32175000 & 0.95796500 & -1.93727900 \\
\hline C & -3.06715400 & 2.72743200 & -0.05545100 & $\mathrm{H}$ & 3.74273000 & 2.90303100 & -2.61084400 \\
\hline $\mathrm{H}$ & -1.99367000 & 2.93541200 & -0.06191200 & $\mathrm{H}$ & 5.52400800 & 3.70061100 & -1.02826700 \\
\hline $\mathrm{H}$ & -3.58401000 & 3.66206900 & -0.29973600 & $\mathrm{H}$ & 5.80529100 & 2.50927700 & 1.14908300 \\
\hline $\mathrm{H}$ & -3.36342700 & 2.44593300 & 0.95930800 & $\mathrm{~N}$ & 4.31097500 & 1.13206800 & 0.79431600 \\
\hline C & 3.68404600 & -0.14845000 & -2.31222600 & $\mathrm{P}$ & 2.37476000 & -0.77674400 & 0.47778100 \\
\hline $\mathrm{H}$ & 3.45803500 & -0.83632400 & -3.13325600 & $\mathrm{C}$ & 3.33750200 & -2.27878400 & -0.19050300 \\
\hline $\mathrm{H}$ & 2.96942100 & 0.67916700 & -2.34626500 & $\mathrm{C}$ & 2.57526600 & -0.80351700 & 2.34518800 \\
\hline $\mathrm{H}$ & 4.68079100 & 0.26479200 & -2.50279800 & $\mathrm{H}$ & 3.12632000 & -1.70945100 & 2.61230600 \\
\hline C & 4.72681200 & -1.98491700 & -0.92322200 & $\mathrm{H}$ & 3.22784800 & 0.04131900 & 2.58757200 \\
\hline
\end{tabular}




\begin{tabular}{|c|c|c|c|c|c|c|c|}
\hline $\mathrm{C}$ & 1.24733400 & -0.75272400 & 3.12400800 & $\mathrm{C}$ & 4.79883200 & -2.24228300 & 0.29867700 \\
\hline $\mathrm{H}$ & 1.46712500 & -0.86834900 & 4.19215400 & $\mathrm{H}$ & 5.33068600 & -3.10444700 & -0.11967000 \\
\hline $\mathrm{H}$ & 0.63126600 & -1.61733100 & 2.84692800 & $\mathrm{H}$ & 5.32015400 & -1.33817300 & -0.02390300 \\
\hline $\mathrm{C}$ & 0.43855100 & 0.53832200 & 2.90477100 & $\mathrm{H}$ & 4.87947100 & -2.30741700 & 1.38728700 \\
\hline $\mathrm{H}$ & 0.87210100 & 1.36243700 & 3.48530100 & $\mathrm{C}$ & 3.30600300 & -2.23752500 & -1.73010000 \\
\hline $\mathrm{H}$ & 0.59125100 & 0.89746000 & 1.85897600 & $\mathrm{H}$ & 3.84684200 & -3.10688600 & -2.12012000 \\
\hline $\mathrm{C}$ & -1.07223300 & 0.42693600 & 3.18054800 & $\mathrm{H}$ & 2.28559100 & -2.28196000 & -2.12118500 \\
\hline $\mathrm{H}$ & -1.25799000 & 0.55830000 & 4.25312600 & $\mathrm{H}$ & 3.79390700 & -1.34322200 & -2.12753900 \\
\hline $\mathrm{H}$ & -1.41691200 & -0.58459100 & 2.93358300 & $\mathrm{C}$ & -0.24183100 & -0.74462700 & -1.72482600 \\
\hline $\mathrm{C}$ & -1.91536600 & 1.45870900 & 2.40820600 & $\mathrm{O}$ & 0.12936300 & 0.02438700 & -2.57650200 \\
\hline $\mathrm{H}$ & -2.95027800 & 1.43758900 & 2.75979800 & $\mathrm{C}$ & -0.84039800 & -2.10004300 & -2.02722300 \\
\hline $\mathrm{H}$ & -1.56001300 & 2.48107900 & 2.56877700 & $\mathrm{C}$ & -0.60126500 & -2.55706700 & -3.27276600 \\
\hline $\mathrm{P}$ & -1.92789800 & 1.20206400 & 0.54704800 & $\mathrm{H}$ & -0.04633300 & -1.96455700 & -3.99161500 \\
\hline $\mathrm{C}$ & -3.73013500 & 0.82086800 & 0.06862100 & $\mathrm{H}$ & -0.95309500 & -3.53559100 & -3.58317100 \\
\hline $\mathrm{C}$ & -1.59103100 & 2.89520700 & -0.13427600 & $\mathrm{C}$ & -1.66749500 & -2.86932400 & -1.05972300 \\
\hline $\mathrm{C}$ & -1.09326800 & 3.05297000 & -1.43499800 & $\mathrm{C}$ & -2.89147100 & -3.40345400 & -1.50147000 \\
\hline $\mathrm{C}$ & -1.65173200 & 5.16698700 & 0.20737000 & $\mathrm{C}$ & -1.26401700 & -3.13188900 & 0.26018200 \\
\hline $\mathrm{C}$ & -0.89371600 & 4.34914800 & -1.91417100 & $\mathrm{C}$ & -3.68165100 & -4.17965200 & -0.65419500 \\
\hline $\mathrm{H}$ & -0.85117400 & 2.19006200 & -2.04595200 & $\mathrm{H}$ & -3.22811200 & -3.19545800 & -2.51267100 \\
\hline $\mathrm{C}$ & -1.17965000 & 5.42865700 & -1.08093900 & $\mathrm{C}$ & -2.05672500 & -3.90672200 & 1.10835100 \\
\hline $\mathrm{H}$ & -1.87501400 & 5.98166200 & 0.89252700 & $\mathrm{H}$ & -0.31244400 & -2.75282000 & 0.61608800 \\
\hline $\mathrm{H}$ & -0.51564100 & 4.50931700 & -2.91948100 & $\mathrm{C}$ & -3.26726600 & -4.43396700 & 0.65453300 \\
\hline $\mathrm{H}$ & -1.03640600 & 6.45170300 & -1.41269300 & $\mathrm{H}$ & -4.62273000 & -4.58199600 & -1.01642100 \\
\hline $\mathrm{N}$ & -1.85902900 & 3.93048300 & 0.67509000 & $\mathrm{H}$ & -1.71981200 & -4.11210000 & 2.12023000 \\
\hline $\mathrm{Pd}$ & 0.03393600 & -0.17516700 & 0.15189600 & $\mathrm{H}$ & -3.88051400 & -5.04003000 & 1.31414800 \\
\hline $\mathrm{C}$ & -4.65243200 & 1.99770800 & 0.44220700 & & & & \\
\hline $\mathrm{H}$ & -4.40231900 & 2.90887200 & -0.10611400 & $45 c$ & & & \\
\hline $\mathrm{H}$ & -5.68404100 & 1.72708100 & 0.19001100 & $\mathrm{C}$ & 3.40140200 & 0.94107500 & 0.48102700 \\
\hline $\mathrm{H}$ & -4.62763100 & 2.23057400 & 1.51038700 & $\mathrm{C}$ & 4.70884300 & 0.45830400 & 0.34676200 \\
\hline $\mathrm{C}$ & -4.16394400 & -0.45250400 & 0.81918700 & $\mathrm{C}$ & 5.59685800 & 0.56930000 & 1.41863700 \\
\hline $\mathrm{H}$ & -3.52111400 & -1.30551200 & 0.59018300 & $\mathrm{C}$ & 5.16340400 & 1.16011000 & 2.60225200 \\
\hline $\mathrm{H}$ & -4.17892100 & -0.30877100 & 1.90401500 & $\mathrm{C}$ & 3.84661900 & 1.61268800 & 2.66366600 \\
\hline $\mathrm{H}$ & -5.18383300 & -0.71343900 & 0.51536300 & $\mathrm{H}$ & 5.04067000 & -0.00032400 & -0.57596000 \\
\hline $\mathrm{C}$ & -3.78965800 & 0.58019200 & -1.45134700 & $\mathrm{H}$ & 6.61401400 & 0.20111100 & 1.32376800 \\
\hline $\mathrm{H}$ & -3.19292500 & -0.28557400 & -1.75068700 & $\mathrm{H}$ & 5.82054800 & 1.26896000 & 3.45804100 \\
\hline $\mathrm{H}$ & -4.82725400 & 0.38103600 & -1.74149600 & $\mathrm{H}$ & 3.45992000 & 2.07877000 & 3.56639100 \\
\hline $\mathrm{H}$ & -3.45297400 & 1.45224700 & -2.01998100 & $\mathrm{~N}$ & 2.98857300 & 1.50127300 & 1.64099400 \\
\hline $\mathrm{C}$ & 2.64610400 & -3.56307600 & 0.30632800 & $\mathrm{P}$ & 2.08137300 & 0.87078700 & -0.84001500 \\
\hline $\mathrm{H}$ & 2.61011000 & -3.62495100 & 1.39873000 & $\mathrm{C}$ & 2.24790600 & 2.58813800 & -1.70945300 \\
\hline $\mathrm{H}$ & 1.62772700 & -3.65908000 & -0.08023900 & $\mathrm{C}$ & 2.85921400 & -0.34616700 & -2.03802900 \\
\hline $\mathrm{H}$ & 3.21331100 & -4.43166300 & -0.04619200 & $\mathrm{H}$ & 3.86805000 & 0.02294800 & -2.24200400 \\
\hline
\end{tabular}


$\mathrm{H}$

C

$\mathrm{H}$

H

C

H

$\mathrm{H}$

C

$\mathrm{H}$

H

$\mathrm{C}$

H

$\mathrm{H}$

P

C

C

$\mathrm{C}$

C

C

H

$\mathrm{C}$

$\mathrm{H}$

H

H

$\mathrm{N}$

Pd

H

C

H

H

$\mathrm{H}$

C

H

H

$\mathrm{H}$

C

H

H

H

C

H
$2.97314400 \quad-1.28047600 \quad-1.47612900 \quad \mathrm{H}$

$2.19341000 \quad-0.65413000 \quad-3.40152700$

$\begin{array}{lll}2.98163900 & -1.10858400 & -4.01407700\end{array}$

$\begin{array}{lll}1.92195400 & 0.26981000 & -3.92303700\end{array}$

$\begin{array}{lll}1.00541000 & -1.62585500 & -3.37853300\end{array}$

$\begin{array}{lll}0.88880400 & -2.03770500 & -4.38726900\end{array}$

$\begin{array}{lll}1.26157700 & -2.47855300 & -2.74311400\end{array}$

$\begin{array}{lll}-0.35188300 & -1.02883000 & -2.93487500\end{array}$

$\begin{array}{lll}-0.96313400 & -0.82939300 & -3.82214100\end{array}$

$-0.21526300 \quad-0.05540800 \quad-2.45152100$

$\begin{array}{lll}-1.16302300 & -1.94079800 & -1.99491800\end{array}$

$-2.22864900 \quad-1.70398000 \quad-2.07735200$

$\begin{array}{lll}-1.03530100 & -2.99344400 & -2.26290400\end{array}$

$\begin{array}{lll}-0.79435200 & -1.74706800 & -0.18388100\end{array}$

$\begin{array}{lll}-2.10896900 & -2.83378400 & 0.69550300\end{array}$

$0.77512100 \quad-2.69393700 \quad 0.14933000$

$\begin{array}{lll}1.55485300 & -2.38755900 & 1.27340200\end{array}$

2.16903600

$-4.43418800$

$2.68229500 \quad-3.16517400$

$-0.42414500$

1.54152800

1.27728300

$-1.56202000$

1.92083500

2.99925700

$-4.21100800$

0.67628000

2.38246200

$-5.23762400$

$-1.12561600$

3.29887900

3.86527200

$-2.95780900$

2.41106600

1.07972900

$-0.28252500$

$-4.84246500$

0.84522800

$-3.69963300 \quad-0.68551700$

0.49692400

0.41532800

$-1.21528300$

3.17372900

0.17115500

$-2.05613000 \quad-4.27863900$

0.15580800

$-1.07787500 \quad-4.74494000$

0.28537900

$-2.78847700 \quad-4.87684400$

0.70993600

$-2.32393000$

$-3.51718500$

$-4.33696700$

$-0.90270200$

$-3.64808400$

$-2.26500500$

0.44033400

$-1.26118500$

0.84172500

$-3.78078000$

$-2.25294800$

$-0.62116300$

$-4.24624300$

$-2.91092900$

0.94241200

$-1.81064200$

$-2.83336900$

2.20808700

$-1.82232600$

$-2.58404100$

$-1.82478000$

2.62724300

$-0.84863600$

1.00368700

$-3.41786000$

2.71904700

$-3.30232500 \quad 2.43439400$

$\begin{array}{lll}0.92738800 & 2.04331100 & -3.39655000\end{array}$
$\mathrm{H}$

$\mathrm{H}$

C

H

$H$

$\mathrm{H}$

C

$\mathrm{H}$

$\mathrm{H}$

$\mathrm{H}$

$\mathrm{C}$

O

C

$\mathrm{C}$

C

$\mathrm{H}$

$\mathrm{H}$

$\mathrm{H}$

$\mathrm{O}$

$\mathrm{H}$

C

C

C

C

$\mathrm{H}$

C

$\mathrm{H}$

C

H

$\mathrm{H}$

$\mathrm{H}$

H

\section{5-O}

$\mathrm{C}$

C

C

C

C

$\mathrm{H}$

H

\begin{tabular}{rrr}
0.07871200 & 2.75446900 & -2.01654500 \\
1.06074100 & 3.77185700 & -3.07930500 \\
3.52908800 & 2.67582600 & -2.55864500 \\
3.61187600 & 3.68734600 & -2.97235200 \\
4.42968400 & 2.49695800 & -1.96179300 \\
3.52809600 & 1.98188500 & -3.40286200 \\
2.27977500 & 3.70981200 & -0.65184800 \\
2.25961900 & 4.67524400 & -1.17023100 \\
1.42372600 & 3.67517700 & 0.02548900 \\
3.19226900 & 3.68128300 & -0.05062500 \\
-2.07349200 & 0.85472700 & 1.22589100 \\
-2.42129600 & 0.46581700 & 2.30965600 \\
-2.24628000 & 2.94552000 & -0.07287200 \\
-2.84743500 & 1.82036800 & 0.35711700 \\
-0.09204900 & 2.57720100 & 3.02014800 \\
0.58090400 & 3.28718100 & 3.51241900 \\
-0.18828900 & 1.67126300 & 3.62678100 \\
-1.07432400 & 3.03985200 & 2.91942600 \\
0.40002800 & 2.28459200 & 1.70111100 \\
\hline 1.37309100 & 2.04490900 & 1.75814900 \\
-4.26326000 & 1.49190600 & 0.03745000 \\
-4.73063600 & 1.66978200 & -1.27626000 \\
-5.15791600 & 1.04294400 & 1.02404700 \\
-6.06492200 & 1.42009900 & -1.59322000 \\
-4.04069500 & 1.99747400 & -2.04845100 \\
-6.49237400 & 0.79782800 & 0.70160500 \\
-4.80992600 & 0.90322000 & 2.04159900 \\
-6.94969000 & 0.98455400 & -0.60461200 \\
-6.41188200 & 1.56112100 & -2.61246100 \\
-7.17752000 & 0.46492900 & 1.47538300 \\
-7.98883200 & 0.78926700 & -0.85126100 \\
\hline & 3.68869300 & -0.62854200 \\
\hline
\end{tabular}

$\begin{array}{lll}-2.80970700 & 3.68869300 & -0.62854200\end{array}$

$\begin{array}{rrr}1.86939300 & 2.31472000 & 0.17034500 \\ 2.44089600 & 1.69189700 & 1.28528900 \\ 3.76247100 & 1.99597600 & 1.61653600 \\ 4.46413900 & 2.90024500 & 0.82278200 \\ 3.81065900 & 3.46539800 & -0.27470800 \\ 1.87240200 & 0.98077100 & 1.87286200 \\ 4.23367000 & 1.52535000 & 2.47358500\end{array}$




\begin{tabular}{|c|c|c|c|c|c|c|c|}
\hline $\mathrm{H}$ & 5.49385600 & 3.16321900 & 1.04080300 & $\mathrm{C}$ & 0.32191600 & -3.87586900 & 0.78647300 \\
\hline $\mathrm{H}$ & 4.32331700 & 4.17271200 & -0.92255000 & $\mathrm{H}$ & -0.52623200 & -3.21781600 & 0.99169400 \\
\hline $\mathrm{N}$ & 2.54240300 & 3.18564300 & -0.59582400 & $\mathrm{H}$ & -0.06117900 & -4.90142500 & 0.73580800 \\
\hline $\mathrm{P}$ & 0.08601800 & 2.02971500 & -0.28614600 & $\mathrm{H}$ & 1.01566000 & -3.83416000 & 1.63067800 \\
\hline $\mathrm{C}$ & -0.80087400 & 3.33056500 & 0.80628500 & $\mathrm{C}$ & -2.29340100 & 3.41037000 & 0.44774400 \\
\hline $\mathrm{C}$ & -0.02691100 & 2.60542500 & -2.05168800 & $\mathrm{H}$ & -2.46303800 & 3.71457600 & -0.58971300 \\
\hline $\mathrm{H}$ & -1.00927200 & 3.07087800 & -2.18045200 & $\mathrm{H}$ & -2.80875000 & 2.46556400 & 0.62041900 \\
\hline $\mathrm{H}$ & 0.73557200 & 3.37949600 & -2.18069000 & $\mathrm{H}$ & -2.76503800 & 4.16743200 & 1.08390200 \\
\hline $\mathrm{C}$ & 0.16413900 & 1.45586400 & -3.05925700 & C & -0.15593600 & 4.71699800 & 0.59857500 \\
\hline $\mathrm{H}$ & -0.05007500 & 1.83719700 & -4.06453700 & $\mathrm{H}$ & -0.68715100 & 5.44272700 & 1.22474200 \\
\hline $\mathrm{H}$ & -0.59230900 & 0.68606100 & -2.85831100 & $\mathrm{H}$ & 0.89695000 & 4.73846700 & 0.88521900 \\
\hline $\mathrm{C}$ & 1.56983200 & 0.81760600 & -3.01241700 & $\mathrm{H}$ & -0.23302100 & 5.05904800 & -0.43738200 \\
\hline $\mathrm{H}$ & 2.22381000 & 1.32795700 & -3.72763900 & $\mathrm{C}$ & -0.66467600 & 2.90115900 & 2.27994300 \\
\hline $\mathrm{H}$ & 2.02010500 & 1.01732900 & -2.03756200 & $\mathrm{H}$ & -1.19733300 & 3.62079800 & 2.91129500 \\
\hline $\mathrm{C}$ & 1.61153500 & -0.71003900 & -3.25512900 & $\mathrm{H}$ & -1.10404900 & 1.91386000 & 2.45853600 \\
\hline $\mathrm{H}$ & 2.06681800 & -0.92591600 & -4.22797800 & $\mathrm{H}$ & 0.37631600 & 2.88469800 & 2.61257600 \\
\hline $\mathrm{H}$ & 0.59403000 & -1.11488600 & -3.31175200 & $\mathrm{C}$ & -2.48045600 & -0.43735600 & -0.07221200 \\
\hline $\mathrm{C}$ & 2.42381700 & -1.46575200 & -2.18431200 & $\mathrm{O}$ & -2.29289400 & -1.58982400 & 0.27484400 \\
\hline $\mathrm{H}$ & 2.70045700 & -2.45971400 & -2.54492800 & $\mathrm{C}$ & -3.78506100 & 0.14760700 & -0.45282700 \\
\hline $\mathrm{H}$ & 3.36837600 & -0.95660400 & -1.97348100 & $\mathrm{C}$ & -3.77062800 & 1.03350600 & -1.46630300 \\
\hline $\mathrm{P}$ & 1.57096300 & -1.69508500 & -0.53368500 & $\mathrm{H}$ & -2.83960000 & 1.35114800 & -1.91985900 \\
\hline $\mathrm{C}$ & 1.01623900 & -3.53676500 & -0.54636300 & $\mathrm{H}$ & -4.68991300 & 1.43714100 & -1.87653800 \\
\hline $\mathrm{C}$ & 2.97381800 & -1.68789400 & 0.68608300 & $\mathrm{C}$ & -5.00900700 & -0.37732700 & 0.20659500 \\
\hline $\mathrm{C}$ & 2.71503200 & -1.71520300 & 2.06553500 & $\mathrm{C}$ & -6.20520000 & -0.50823200 & -0.51860900 \\
\hline $\mathrm{C}$ & 5.24583200 & -1.69884600 & 1.04796900 & $\mathrm{C}$ & -4.99931700 & -0.72005200 & 1.56854800 \\
\hline $\mathrm{C}$ & 3.79187400 & -1.75975400 & 2.95071900 & $\mathrm{C}$ & -7.36557400 & -0.95547100 & 0.10920800 \\
\hline $\mathrm{H}$ & 1.69514400 & -1.70115200 & 2.43687400 & $\mathrm{H}$ & -6.22199300 & -0.28559100 & -1.58106600 \\
\hline $\mathrm{C}$ & 5.08756400 & -1.75259800 & 2.43384800 & $\mathrm{C}$ & -6.16266400 & -1.16449800 & 2.19379600 \\
\hline $\mathrm{H}$ & 6.23896500 & -1.68292700 & 0.60429800 & $\mathrm{H}$ & -4.08440100 & -0.63208800 & 2.14705100 \\
\hline $\mathrm{H}$ & 3.62169900 & -1.79698100 & 4.02275500 & $\mathrm{C}$ & -7.34873300 & -1.28177200 & 1.46702900 \\
\hline $\mathrm{H}$ & 5.95549200 & -1.78806600 & 3.08407700 & $\mathrm{H}$ & -8.28041400 & -1.06039400 & -0.46569400 \\
\hline $\mathrm{N}$ & 4.21855900 & -1.67264100 & 0.18937600 & $\mathrm{H}$ & -6.14215100 & -1.41910900 & 3.24884600 \\
\hline $\mathrm{Pd}$ & -0.49221700 & -0.25016400 & -0.11003000 & $\mathrm{H}$ & -8.25307700 & -1.63363400 & 1.95358500 \\
\hline $\mathrm{C}$ & 2.21484900 & -4.48524900 & -0.74020300 & & & & \\
\hline $\mathrm{H}$ & 2.94480100 & -4.39662900 & 0.06864200 & 45-CO & & & \\
\hline $\mathrm{H}$ & 1.84920000 & -5.51857800 & -0.74478500 & $\mathrm{C}$ & 2.48557500 & -2.13313700 & -0.83061300 \\
\hline $\mathrm{H}$ & 2.73795000 & -4.32579100 & -1.68673900 & $\mathrm{C}$ & 2.13156800 & -1.80324400 & -2.14387600 \\
\hline $\mathrm{C}$ & 0.01483800 & -3.71337700 & -1.70592100 & $\mathrm{C}$ & 2.86296500 & -2.35898400 & -3.19645600 \\
\hline $\mathrm{H}$ & -0.86679600 & -3.07543900 & -1.58917700 & $\mathrm{C}$ & 3.92186000 & -3.21409800 & -2.90177000 \\
\hline $\mathrm{H}$ & 0.46685400 & -3.50959100 & -2.68102500 & $\mathrm{C}$ & 4.21289100 & -3.47040300 & -1.55937400 \\
\hline $\mathrm{H}$ & -0.33440700 & -4.75196600 & -1.72128800 & $\mathrm{H}$ & 1.32523500 & -1.10474400 & -2.33490200 \\
\hline
\end{tabular}




\begin{tabular}{|c|c|c|}
\hline 2.60822600 & -2.12493400 & -4.22598800 \\
\hline 4.51566100 & -3.67034300 & -3.68691600 \\
\hline 5.03834900 & -4.12412700 & -1.28655800 \\
\hline 3.51723900 & -2.94511200 & -0.54491900 \\
\hline 1.54613900 & -1.44934000 & 0.62756500 \\
\hline 0.98975000 & -3.00355400 & 1.61808000 \\
\hline 3.00313000 & -0.74004200 & 1.56911900 \\
\hline 3.74175600 & -1.54645600 & 1.53209400 \\
\hline 3.39660300 & 0.06071600 & 0.93275300 \\
\hline 2.86595800 & -0.22670800 & 3.01885400 \\
\hline 3.87944200 & -0.23831200 & 3.43773600 \\
\hline 2.29030000 & -0.92417400 & 3.63599800 \\
\hline 2.31073600 & 1.19587500 & 3.18061300 \\
\hline 2.60188600 & 1.56822800 & 4.16883800 \\
\hline 2.82296400 & 1.84277500 & 2.46199500 \\
\hline 0.77548100 & 1.33576400 & 3.03482900 \\
\hline 0.33164600 & 1.45259200 & 4.02974400 \\
\hline 0.34360000 & 0.41398700 & 2.62937100 \\
\hline 0.31070900 & 2.52257800 & 2.16912400 \\
\hline-0.72156000 & 2.78365600 & 2.42513100 \\
\hline 0.90774300 & 3.41654400 & 2.37843700 \\
\hline 0.25484000 & 2.19247000 & 0.33427100 \\
\hline-0.55955400 & 3.76414400 & -0.41671700 \\
\hline 1.98829500 & 2.28746600 & -0.32431800 \\
\hline 2.97645300 & 3.13919900 & 0.18824200 \\
\hline 3.39891200 & 1.55062100 & -1.98907500 \\
\hline 4.22556400 & 3.17201500 & -0.43373100 \\
\hline 2.78208300 & 3.76974000 & 1.04787300 \\
\hline 4.44387500 & 2.36405900 & -1.54734300 \\
\hline 3.52979300 & 0.90024400 & -2.85024700 \\
\hline 5.00905700 & 3.82125500 & -0.05497500 \\
\hline 5.39797300 & 2.36007100 & -2.06362800 \\
\hline 2.19971800 & 1.50358300 & -1.39469000 \\
\hline-0.52083700 & -0.08834600 & -0.19294200 \\
\hline-2.09895300 & 0.82417100 & 2.01630200 \\
\hline 0.25204900 & 5.02670900 & -0.05986500 \\
\hline 1.25992400 & 5.00878700 & -0.47921700 \\
\hline-0.26067900 & 5.89472800 & -0.48863800 \\
\hline 0.32026900 & 5.19722700 & 1.01911700 \\
\hline-1.98809700 & 3.93865800 & 0.13647900 \\
\hline-2.63972600 & 3.09391200 & -0.08301800 \\
\hline
\end{tabular}

H

H

C

H

H

H

C

$\mathrm{H}$

H

H

C

H

H

H

C

H

H

H

C

O

C

C

C

C

C

C

H

C

H

C

H

H

H

H

C

O

\section{TS38}

imaginary frequency $=-54.84 \mathrm{~cm}^{-1}$

C

$\mathrm{H}$

\begin{tabular}{|c|c|c|}
\hline-1.99792700 & 4.11994700 & 1.21552100 \\
\hline-2.43439800 & 4.81953800 & -0.33788200 \\
\hline-0.58922000 & 3.59463100 & -1.94933900 \\
\hline-1.16866400 & 2.72272100 & -2.25638100 \\
\hline-1.05497400 & 4.48212600 & -2.39242600 \\
\hline 0.41762700 & 3.50743900 & -2.36684200 \\
\hline-0.09366600 & -2.56738800 & 2.62717400 \\
\hline 0.28629100 & -1.86625800 & 3.37571900 \\
\hline-0.95311300 & -2.10379200 & 2.13043000 \\
\hline-0.45833100 & -3.44901000 & 3.16684300 \\
\hline 2.16293000 & -3.67373100 & 2.36265000 \\
\hline 1.78761400 & -4.57152500 & 2.86759800 \\
\hline 2.95437600 & -3.97246200 & 1.67310100 \\
\hline 2.59741700 & -3.03071600 & 3.13172800 \\
\hline 0.37640500 & -4.03377500 & 0.64978100 \\
\hline 0.04335000 & -4.90267100 & 1.22821000 \\
\hline-0.50162100 & -3.64824100 & 0.12680900 \\
\hline 1.09807400 & -4.38732600 & -0.09013800 \\
\hline-2.44879700 & 0.61610700 & -0.62140600 \\
\hline-2.74165700 & 1.10937200 & -1.67507000 \\
\hline-3.07186600 & 0.43013400 & 1.74640000 \\
\hline-3.42028600 & 0.22821100 & 0.46223800 \\
\hline-4.71572800 & -0.38355100 & 0.05926100 \\
\hline-5.45871100 & 0.08181800 & -1.03951200 \\
\hline-5.22054600 & -1.45819600 & 0.81497900 \\
\hline-6.68632300 & -0.50009800 & -1.35262100 \\
\hline-5.08187600 & 0.90261500 & -1.63735900 \\
\hline-6.44690600 & -2.03647900 & 0.49560700 \\
\hline-4.63993600 & -1.84825100 & 1.64590600 \\
\hline-7.18477600 & -1.55697300 & -0.58856400 \\
\hline-7.25580700 & -0.12343900 & -2.19673200 \\
\hline-6.82084500 & -2.86687500 & 1.08673200 \\
\hline-8.13882700 & -2.00951700 & -0.84094000 \\
\hline-3.77021300 & 0.23661100 & 2.55370600 \\
\hline-1.19127700 & -1.62790400 & -1.27347800 \\
\hline-1.51844100 & -2.41401500 & -2.03143600 \\
\hline
\end{tabular}

$\begin{array}{lll}0.76588600 & 1.59303100 & -1.92008400\end{array}$

$\begin{array}{lll}0.91927700 & 1.07364200 & -2.86387300\end{array}$ 


\begin{tabular}{|c|c|c|c|c|c|c|c|}
\hline $\mathrm{C}$ & -0.55593100 & 1.99294200 & -1.57591800 & $\mathrm{H}$ & 4.53711400 & -0.70757800 & 1.96477400 \\
\hline $\mathrm{C}$ & -1.53163300 & 1.10855400 & -2.04902500 & $\mathrm{C}$ & 4.95244500 & 2.63586300 & 1.64093600 \\
\hline $\mathrm{O}$ & -2.37030700 & 0.72024700 & -2.76842100 & $\mathrm{H}$ & 3.76766000 & 3.82190400 & 0.27594300 \\
\hline $\mathrm{C}$ & 2.35660900 & -2.07211400 & 1.15340000 & $\mathrm{H}$ & 5.91805300 & 1.14291700 & 2.86763400 \\
\hline $\mathrm{H}$ & 1.83583200 & -2.91445600 & 0.68754400 & $\mathrm{H}$ & 5.53558500 & 3.47471500 & 2.00579400 \\
\hline $\mathrm{H}$ & 3.37200100 & -2.42120300 & 1.35994200 & $\mathrm{C}$ & -3.19560300 & -0.47957300 & 0.70803400 \\
\hline $\mathrm{C}$ & 1.65322000 & -1.64250600 & 2.45901200 & $\mathrm{C}$ & -4.27418900 & -0.96229800 & 1.46569200 \\
\hline $\mathrm{H}$ & 2.39640800 & -1.24287800 & 3.15610600 & $\mathrm{C}$ & -4.25913900 & 1.54785300 & 0.41647000 \\
\hline $\mathrm{H}$ & 0.96186300 & -0.81410000 & 2.26051500 & $\mathrm{C}$ & -5.37844900 & -0.13941900 & 1.68459500 \\
\hline $\mathrm{P}$ & -1.65912700 & -1.49517200 & 0.36371500 & $\mathrm{H}$ & -4.26627200 & -1.96169500 & 1.88272400 \\
\hline $\mathrm{P}$ & 2.34276500 & -0.73507100 & -0.13223900 & $\mathrm{C}$ & -5.37376400 & 1.14647000 & 1.15155500 \\
\hline $\mathrm{C}$ & 3.42579000 & -1.41110400 & -1.56286600 & $\mathrm{H}$ & -4.20608800 & 2.54702200 & -0.00681600 \\
\hline $\mathrm{C}$ & -2.26611300 & -2.95621900 & -0.73223300 & $\mathrm{H}$ & -6.22285100 & -0.49959900 & 2.26446900 \\
\hline $\mathrm{C}$ & 2.59404700 & -2.45558200 & -2.33316900 & $\mathrm{H}$ & -6.20702800 & 1.82506800 & 1.30007100 \\
\hline $\mathrm{H}$ & 3.19053800 & -2.85196700 & -3.16258600 & $\mathrm{~N}$ & 3.20539300 & 1.83961100 & 0.18724400 \\
\hline $\mathrm{H}$ & 2.30510900 & -3.30519800 & -1.70629100 & $\mathrm{~N}$ & -3.19805200 & 0.76141500 & 0.19514400 \\
\hline $\mathrm{H}$ & 1.68533300 & -2.01729200 & -2.75702900 & $\mathrm{Pd}$ & 0.05194200 & -0.03440200 & -0.69876600 \\
\hline $\mathrm{C}$ & 4.71803900 & -2.06507100 & -1.03699600 & $\mathrm{C}$ & 0.88113500 & -2.78487800 & 3.13127800 \\
\hline $\mathrm{H}$ & 5.32989800 & -2.37709800 & -1.89074400 & $\mathrm{H}$ & 0.52831400 & -2.44458400 & 4.11289400 \\
\hline $\mathrm{H}$ & 5.32035800 & -1.37208300 & -0.44250700 & $\mathrm{H}$ & 1.55789400 & -3.62710200 & 3.32348100 \\
\hline $\mathrm{H}$ & 4.52198900 & -2.95927200 & -0.43914000 & $\mathrm{C}$ & -0.32174800 & -3.28520100 & 2.31649500 \\
\hline $\mathrm{C}$ & 3.79995200 & -0.25680800 & -2.51222500 & $\mathrm{H}$ & -0.77293200 & -4.12478200 & 2.85607300 \\
\hline $\mathrm{H}$ & 4.39048800 & 0.51630800 & -2.01411200 & $\mathrm{H}$ & 0.02326400 & -3.71035900 & 1.36894200 \\
\hline $\mathrm{H}$ & 4.40179000 & -0.65742600 & -3.33560100 & $\mathrm{C}$ & -1.41556300 & -2.21222100 & 2.08410900 \\
\hline $\mathrm{H}$ & 2.91988600 & 0.21689000 & -2.95198200 & $\mathrm{H}$ & -2.38152300 & -2.61759600 & 2.39146000 \\
\hline $\mathrm{C}$ & -1.03119600 & -3.57660800 & -1.41641300 & $\mathrm{H}$ & -1.24227600 & -1.34212900 & 2.72752300 \\
\hline $\mathrm{H}$ & -0.48813600 & -2.83322100 & -2.00642300 & $\mathrm{C}$ & -0.91584800 & 3.11108300 & -0.66391600 \\
\hline $\mathrm{H}$ & -0.33296900 & -4.02279400 & -0.70170900 & $\mathrm{C}$ & -2.01384700 & 3.92416300 & -0.98332700 \\
\hline $\mathrm{H}$ & -1.35566300 & -4.37413800 & -2.09473400 & $\mathrm{C}$ & -0.12689600 & 3.41984500 & 0.45386200 \\
\hline $\mathrm{C}$ & -3.01065600 & -4.04160000 & 0.06556700 & $\mathrm{C}$ & -2.32057900 & 5.03126600 & -0.19227600 \\
\hline $\mathrm{H}$ & -3.91309200 & -3.65682300 & 0.54808500 & $\mathrm{H}$ & -2.61323700 & 3.70365600 & -1.86262300 \\
\hline $\mathrm{H}$ & -3.33239500 & -4.82894600 & -0.62574700 & $\mathrm{C}$ & -0.44150900 & 4.52508100 & 1.24290100 \\
\hline $\mathrm{H}$ & -2.38299500 & -4.51468400 & 0.82526600 & $\mathrm{H}$ & 0.72827800 & 2.79661000 & 0.69554400 \\
\hline $\mathrm{C}$ & -3.19992000 & -2.39749600 & -1.82461200 & $\mathrm{C}$ & -1.53781500 & 5.33093700 & 0.92451000 \\
\hline $\mathrm{H}$ & -3.47598500 & -3.21189000 & -2.50427000 & $\mathrm{H}$ & -3.16373900 & 5.66342400 & -0.45354900 \\
\hline $\mathrm{H}$ & -4.12376900 & -1.98686600 & -1.40871900 & $\mathrm{H}$ & 0.17013200 & 4.75835500 & 2.10927200 \\
\hline $\mathrm{H}$ & -2.72123100 & -1.61678100 & -2.41878900 & $\mathrm{H}$ & -1.77699200 & 6.19212900 & 1.54075600 \\
\hline $\mathrm{C}$ & 3.40714400 & 0.58944500 & 0.64001600 & $\mathrm{H}$ & 1.59759800 & 2.18108700 & -1.54904700 \\
\hline $\mathrm{C}$ & 4.38004600 & 0.30369500 & 1.60919600 & & & & \\
\hline $\mathrm{C}$ & 3.96433900 & 2.82916300 & 0.67470900 & 46 & & & \\
\hline $\mathrm{C}$ & 5.16035800 & 1.34394000 & 2.11626400 & $\mathrm{C}$ & 0.66224500 & 1.57101600 & -1.83820400 \\
\hline
\end{tabular}




\begin{tabular}{|c|c|c|c|c|c|c|c|}
\hline $\mathrm{H}$ & 0.89765800 & 1.16384500 & -2.82025700 & $\mathrm{C}$ & 5.12523700 & 1.68198900 & 2.01984700 \\
\hline $\mathrm{C}$ & -0.72439200 & 1.92124100 & -1.57486400 & $\mathrm{H}$ & 4.48416200 & -0.36907600 & 2.07547400 \\
\hline $\mathrm{C}$ & -1.64667200 & 1.31775900 & -2.38053900 & $\mathrm{C}$ & 4.96278100 & 2.90989600 & 1.38441000 \\
\hline $\mathrm{O}$ & -2.38957000 & 0.91656300 & -3.17311800 & $\mathrm{H}$ & 3.87337800 & 3.93531600 & -0.17670400 \\
\hline $\mathrm{C}$ & 2.43357700 & -1.87746900 & 1.23733900 & $\mathrm{H}$ & 5.83825100 & 1.56554200 & 2.83046000 \\
\hline $\mathrm{H}$ & 1.98387800 & -2.77054700 & 0.79452200 & $\mathrm{H}$ & 5.53770500 & 3.78116200 & 1.67967600 \\
\hline $\mathrm{H}$ & 3.45841400 & -2.15535100 & 1.50065400 & $\mathrm{C}$ & -3.08667000 & -0.57405000 & 0.75069100 \\
\hline $\mathrm{C}$ & 1.64546400 & -1.43544500 & 2.48825100 & $\mathrm{C}$ & -3.86555600 & -0.66451300 & 1.91334700 \\
\hline $\mathrm{H}$ & 2.31712900 & -0.91086400 & 3.17565500 & $\mathrm{C}$ & -4.55353800 & 0.95650600 & -0.16394000 \\
\hline $\mathrm{H}$ & 0.87574900 & -0.70502800 & 2.20834300 & $\mathrm{C}$ & -5.02508200 & 0.10575200 & 2.01637400 \\
\hline $\mathrm{P}$ & -1.53919600 & -1.57767600 & 0.44047100 & $\mathrm{H}$ & -3.59355800 & -1.32536100 & 2.72625800 \\
\hline $\mathrm{P}$ & 2.39851100 & -0.61760000 & -0.12104800 & $\mathrm{C}$ & -5.38596300 & 0.93003800 & 0.95514300 \\
\hline $\mathrm{C}$ & 3.45220700 & -1.36517900 & -1.53043300 & $\mathrm{H}$ & -4.79808800 & 1.58529100 & -1.01695500 \\
\hline $\mathrm{C}$ & -2.18061300 & -2.94277100 & -0.75232500 & $\mathrm{H}$ & -5.63788800 & 0.05096800 & 2.91117500 \\
\hline $\mathrm{C}$ & 2.66904200 & -2.55174600 & -2.12627500 & $\mathrm{H}$ & -6.28311200 & 1.53892600 & 0.98822400 \\
\hline $\mathrm{H}$ & 3.22827900 & -2.96398500 & -2.97358900 & $\mathrm{~N}$ & 3.28609600 & 1.96190200 & -0.05966900 \\
\hline $\mathrm{H}$ & 2.52962000 & -3.36415000 & -1.40590900 & $\mathrm{~N}$ & -3.42776400 & 0.24068500 & -0.26425500 \\
\hline $\mathrm{H}$ & 1.68562500 & -2.24164900 & -2.49361900 & $\mathrm{Pd}$ & 0.14692300 & -0.04272400 & -0.63443800 \\
\hline $\mathrm{C}$ & 4.81985600 & -1.84885200 & -1.01010600 & $\mathrm{C}$ & 0.98051500 & -2.61095700 & 3.21762100 \\
\hline $\mathrm{H}$ & 5.39810300 & -2.24869100 & -1.85061600 & $\mathrm{H}$ & 0.55741700 & -2.24761700 & 4.16280000 \\
\hline $\mathrm{H}$ & 5.40452200 & -1.03660900 & -0.56883000 & $\mathrm{H}$ & 1.74231700 & -3.35283100 & 3.48918400 \\
\hline $\mathrm{H}$ & 4.72789900 & -2.65148100 & -0.27285900 & $\mathrm{C}$ & -0.12606100 & -3.31147200 & 2.41345700 \\
\hline $\mathrm{C}$ & 3.67021800 & -0.30010500 & -2.62176600 & $\mathrm{H}$ & -0.47181900 & -4.16984800 & 3.00004300 \\
\hline $\mathrm{H}$ & 4.17518200 & 0.59081500 & -2.24022600 & $\mathrm{H}$ & 0.28925500 & -3.74244000 & 1.49856300 \\
\hline $\mathrm{H}$ & 4.29464100 & -0.72700200 & -3.41451100 & $\mathrm{C}$ & -1.36003200 & -2.42533100 & 2.10889900 \\
\hline $\mathrm{H}$ & 2.72784700 & 0.01060400 & -3.07898600 & $\mathrm{H}$ & -2.26858700 & -3.01870300 & 2.25000700 \\
\hline $\mathrm{C}$ & -1.13843800 & -4.07529100 & -0.83360600 & $\mathrm{H}$ & -1.40783100 & -1.61704900 & 2.84774300 \\
\hline $\mathrm{H}$ & -0.14003300 & -3.69953100 & -1.08000600 & $\mathrm{C}$ & -1.17303700 & 2.95657600 & -0.59477900 \\
\hline $\mathrm{H}$ & -1.07865500 & -4.65234600 & 0.09264300 & $\mathrm{C}$ & -2.31419500 & 3.73150900 & -0.84943800 \\
\hline $\mathrm{H}$ & -1.43124400 & -4.77004400 & -1.62904200 & $\mathrm{C}$ & -0.42468800 & 3.20118900 & 0.56654300 \\
\hline $\mathrm{C}$ & -3.53172300 & -3.52362700 & -0.29873600 & $\mathrm{C}$ & -2.70780400 & 4.72429800 & 0.04675600 \\
\hline $\mathrm{H}$ & -4.32383600 & -2.77077400 & -0.29355200 & $\mathrm{H}$ & -2.88632000 & 3.57755000 & -1.76089400 \\
\hline $\mathrm{H}$ & -3.83286500 & -4.31424700 & -0.99569800 & $\mathrm{C}$ & -0.81848000 & 4.20102000 & 1.45513800 \\
\hline $\mathrm{H}$ & -3.48152400 & -3.97774100 & 0.69599300 & $\mathrm{H}$ & 0.46434500 & 2.61246900 & 0.76859100 \\
\hline $\mathrm{C}$ & -2.33456600 & -2.30572500 & -2.14597500 & $\mathrm{C}$ & -1.96186000 & 4.96201800 & 1.20224100 \\
\hline $\mathrm{H}$ & -2.68547300 & -3.06567300 & -2.85389100 & $\mathrm{H}$ & -3.58980400 & 5.32044600 & -0.16623900 \\
\hline $\mathrm{H}$ & -3.05320900 & -1.48422000 & -2.14481800 & $\mathrm{H}$ & -0.23018200 & 4.38395300 & 2.34933700 \\
\hline $\mathrm{H}$ & -1.37695200 & -1.92228300 & -2.51584200 & $\mathrm{H}$ & -2.26504900 & 5.73897800 & 1.89699600 \\
\hline $\mathrm{C}$ & 3.44439000 & 0.77293600 & 0.54889700 & $\mathrm{H}$ & 1.42115500 & 2.23439600 & -1.43537400 \\
\hline $\mathrm{C}$ & 4.35907200 & 0.59445100 & 1.59673400 & & & & \\
\hline $\mathrm{C}$ & 4.03202700 & 2.99579000 & 0.34784900 & TS39 & & & \\
\hline
\end{tabular}




\begin{tabular}{|c|c|c|c|c|c|c|c|}
\hline \multicolumn{4}{|c|}{ imaginary frequency $=-37.83 \mathrm{~cm}^{-1}$} & \multirow{2}{*}{$\begin{array}{l}\mathrm{H} \\
\mathrm{C}\end{array}$} & \multirow{2}{*}{$\begin{array}{r}-1.87242500 \\
3.94293500\end{array}$} & \multirow{2}{*}{$\begin{array}{r}2.13742200 \\
-1.50814700\end{array}$} & \multirow{2}{*}{$\begin{array}{r}0.92944500 \\
-2.39954100\end{array}$} \\
\hline $\mathrm{C}$ & -3.68665700 & 0.01217000 & 0.61409600 & & & & \\
\hline $\mathrm{C}$ & -5.06550600 & 0.02255500 & 0.37002800 & $\mathrm{H}$ & 4.46457600 & -2.06126800 & -1.61519800 \\
\hline $\mathrm{C}$ & -5.94354000 & 0.45693400 & 1.36346800 & $\mathrm{H}$ & 4.68909800 & -0.94553700 & -2.97266700 \\
\hline $\mathrm{C}$ & -5.42808700 & 0.86472600 & 2.59075900 & $\mathrm{H}$ & 3.49003200 & -2.22969500 & -3.08511200 \\
\hline $\mathrm{C}$ & -4.04781500 & 0.82417400 & 2.76311100 & $\mathrm{C}$ & 2.08660400 & 0.08012400 & -2.98622400 \\
\hline $\mathrm{H}$ & -5.45921400 & -0.31085700 & -0.58050400 & $\mathrm{H}$ & 1.40098100 & 0.85885300 & -2.63821200 \\
\hline $\mathrm{H}$ & -7.01325400 & 0.46990200 & 1.17824600 & $\mathrm{H}$ & 1.50734200 & -0.68325100 & -3.51648200 \\
\hline $\mathrm{H}$ & -6.07104500 & 1.20967100 & 3.39291000 & $\mathrm{H}$ & 2.76440000 & 0.53921800 & -3.71561500 \\
\hline $\mathrm{H}$ & -3.59469700 & 1.14626100 & 3.69711500 & $\mathrm{C}$ & 3.65307500 & 0.60837700 & -1.08403800 \\
\hline $\mathrm{N}$ & -3.19786100 & 0.41212900 & 1.80825000 & $\mathrm{H}$ & 2.96825800 & 1.31247200 & -0.60490300 \\
\hline $\mathrm{P}$ & -2.40886600 & -0.68660500 & -0.56986400 & $\mathrm{H}$ & 4.25851700 & 1.17666200 & -1.79901600 \\
\hline $\mathrm{C}$ & -3.02838700 & -0.39866400 & -2.36707000 & $\mathrm{H}$ & 4.32824500 & 0.21055900 & -0.32135200 \\
\hline $\mathrm{C}$ & -2.78860100 & -2.48393400 & -0.17719800 & $\mathrm{C}$ & -1.80477600 & -0.61664300 & -3.28487300 \\
\hline $\mathrm{H}$ & -3.79699900 & -2.68452100 & -0.55238900 & $\mathrm{H}$ & -1.42551300 & -1.64124800 & -3.23124800 \\
\hline $\mathrm{H}$ & -2.85086700 & -2.54013700 & 0.91546100 & $\mathrm{H}$ & -0.98522200 & 0.06034400 & -3.03209600 \\
\hline $\mathrm{C}$ & -1.77545700 & -3.51733600 & -0.68909800 & $\mathrm{H}$ & -2.09681500 & -0.42878300 & -4.32437800 \\
\hline $\mathrm{H}$ & -2.28445100 & -4.48192200 & -0.79987600 & $\mathrm{C}$ & -4.14140600 & -1.36362100 & -2.82250300 \\
\hline $\mathrm{H}$ & -1.43838800 & -3.25037100 & -1.69602000 & $\mathrm{H}$ & -4.42023500 & -1.11556400 & -3.85266400 \\
\hline $\mathrm{C}$ & -0.57097400 & -3.67964400 & 0.25649700 & $\mathrm{H}$ & -5.05357800 & -1.30214200 & -2.22395200 \\
\hline $\mathrm{H}$ & -0.81284400 & -4.43381500 & 1.01487900 & $\mathrm{H}$ & -3.80858900 & -2.40512600 & -2.82115600 \\
\hline $\mathrm{H}$ & -0.40898900 & -2.74324900 & 0.80473400 & $\mathrm{C}$ & -3.49041200 & 1.06455300 & -2.52000900 \\
\hline $\mathrm{C}$ & 0.72556100 & -4.07070600 & -0.45636200 & $\mathrm{H}$ & -3.73084700 & 1.25471100 & -3.57186100 \\
\hline $\mathrm{H}$ & 1.48346900 & -4.34359000 & 0.28217100 & $\mathrm{H}$ & -2.70289400 & 1.76695100 & -2.23325500 \\
\hline $\mathrm{H}$ & 0.55431800 & -4.97034000 & -1.06264900 & $\mathrm{H}$ & -4.38149500 & 1.29556400 & -1.93205800 \\
\hline $\mathrm{C}$ & 1.31967100 & -2.99072400 & -1.37996800 & $\mathrm{C}$ & 0.68365800 & 1.99464500 & 1.91849000 \\
\hline $\mathrm{H}$ & 0.65761900 & -2.78076700 & -2.22725900 & $\mathrm{O}$ & 1.55738300 & 1.96241800 & 2.70403600 \\
\hline $\mathrm{H}$ & 2.25651900 & -3.37533600 & -1.78504600 & $\mathrm{C}$ & -1.07431800 & 2.18381800 & 0.20167800 \\
\hline $\mathrm{P}$ & 1.68401700 & -1.33279400 & -0.59281300 & $\mathrm{C}$ & 0.26648200 & 2.42845500 & 0.64990900 \\
\hline $\mathrm{C}$ & 2.91075600 & -0.51734700 & -1.82908000 & $\mathrm{C}$ & -0.44410500 & 0.39450900 & 3.93455200 \\
\hline $\mathrm{C}$ & 2.80243300 & -1.84126500 & 0.80944400 & $\mathrm{H}$ & -1.24449500 & 0.42150200 & 4.67896700 \\
\hline $\mathrm{C}$ & 2.85750100 & -1.10891200 & 2.00051800 & $\mathrm{H}$ & -0.24329000 & -0.63713400 & 3.63029200 \\
\hline $\mathrm{C}$ & 4.41007100 & -3.30820600 & 1.56659000 & $\mathrm{H}$ & 0.45623300 & 0.83033400 & 4.36757100 \\
\hline $\mathrm{C}$ & 3.74340000 & -1.51260800 & 3.00136800 & $\mathrm{O}$ & -0.81859500 & 1.20427700 & 2.81064100 \\
\hline $\mathrm{H}$ & 2.23654200 & -0.23366000 & 2.13861600 & $\mathrm{H}$ & -1.58442700 & 0.77924700 & 2.30419400 \\
\hline $\mathrm{C}$ & 4.53608700 & -2.63665900 & 2.78466500 & $\mathrm{H}$ & -1.36648700 & 2.70721200 & -0.70283100 \\
\hline $\mathrm{H}$ & 5.01029900 & -4.19109800 & 1.35712100 & $\mathrm{C}$ & 1.22716400 & 3.31460500 & -0.09724400 \\
\hline $\mathrm{H}$ & 3.81174100 & -0.95573300 & 3.93130000 & $\mathrm{C}$ & 2.27038100 & 3.99657000 & 0.54969800 \\
\hline $\mathrm{H}$ & 5.23646300 & -2.98923200 & 3.53463900 & $\mathrm{C}$ & 1.05188700 & 3.52458000 & -1.47687800 \\
\hline $\mathrm{N}$ & 3.56873200 & -2.92637300 & 0.59881300 & $\mathrm{C}$ & 3.10820800 & 4.85674800 & -0.16151800 \\
\hline $\mathrm{Pd}$ & -0.24300900 & 0.27171400 & -0.15266200 & $\mathrm{H}$ & 2.43339900 & 3.87210800 & 1.61394300 \\
\hline
\end{tabular}




\begin{tabular}{|c|c|c|c|c|c|c|c|}
\hline $\mathrm{C}$ & 1.88875700 & 4.38623600 & -2.18211400 & $\mathrm{C}$ & -3.49801700 & -1.87176400 & 2.91730500 \\
\hline $\mathrm{H}$ & 0.26199700 & 3.00794800 & -2.01170600 & $\mathrm{H}$ & -3.17587000 & 0.17542500 & 2.30487600 \\
\hline $\mathrm{C}$ & 2.92381400 & 5.05757800 & -1.52863700 & $\mathrm{C}$ & -3.84160100 & -3.14851600 & 2.47159100 \\
\hline $\mathrm{H}$ & 3.90403400 & 5.37576200 & 0.36393700 & $\mathrm{H}$ & -4.39138000 & -4.29449400 & 0.72467300 \\
\hline $\mathrm{H}$ & 1.73123200 & 4.52967800 & -3.24693200 & $\mathrm{H}$ & -3.26903500 & -1.69340300 & 3.96467200 \\
\hline \multirow[t]{2}{*}{$\mathrm{H}$} & 3.57606600 & 5.72862200 & -2.07845700 & $\mathrm{H}$ & -3.88842400 & -3.99375300 & 3.15076100 \\
\hline & & & & $\mathrm{N}$ & -4.10992500 & -2.31211500 & 0.22713700 \\
\hline \multicolumn{4}{|c|}{ TS39a } & $\mathrm{Pd}$ & 0.77562600 & 0.55812000 & -0.35074400 \\
\hline \multicolumn{4}{|c|}{ imaginary frequency $=-146.78 \mathrm{~cm}^{-1}$} & $\mathrm{H}$ & 3.63006500 & 0.62290500 & 2.31901300 \\
\hline $\mathrm{C}$ & 2.48234100 & -2.77252300 & 0.20172200 & $\mathrm{C}$ & -6.63412200 & 0.05150800 & -0.64677500 \\
\hline $\mathrm{C}$ & 2.73241400 & -4.11235600 & -0.12209100 & $\mathrm{H}$ & -6.57596700 & -0.80117400 & 0.03565300 \\
\hline $\mathrm{C}$ & 3.16870200 & -4.98925200 & 0.87137300 & $\mathrm{H}$ & -7.61409500 & 0.52547900 & -0.50851000 \\
\hline $\mathrm{C}$ & 3.35083700 & -4.50926600 & 2.16704700 & $\mathrm{H}$ & -6.60039500 & -0.33538500 & -1.66944300 \\
\hline $\mathrm{C}$ & 3.06540700 & -3.17029500 & 2.41778300 & C & -5.61810500 & 2.24615900 & -1.37860100 \\
\hline $\mathrm{H}$ & 2.59282800 & -4.46732700 & -1.13623400 & $\mathrm{H}$ & -4.84389800 & 2.99896900 & -1.19714600 \\
\hline $\mathrm{H}$ & 3.36688800 & -6.02984300 & 0.63351800 & $\mathrm{H}$ & -5.53360000 & 1.91653300 & -2.41906300 \\
\hline $\mathrm{H}$ & 3.69465100 & -5.15431200 & 2.96803500 & $\mathrm{H}$ & -6.59319800 & 2.73690900 & -1.27434600 \\
\hline $\mathrm{H}$ & 3.17238800 & -2.75109000 & 3.41490500 & $\mathrm{C}$ & -5.66344600 & 1.63927700 & 1.04875400 \\
\hline $\mathrm{N}$ & 2.63672300 & -2.32598700 & 1.46541500 & $\mathrm{H}$ & -4.86812300 & 2.35498200 & 1.28514000 \\
\hline $\mathrm{P}$ & 1.95402400 & -1.49602200 & -1.04188000 & $\mathrm{H}$ & -6.62085800 & 2.16603900 & 1.13989700 \\
\hline $\mathrm{C}$ & 3.56951300 & -1.08958000 & -1.98875100 & $\mathrm{H}$ & -5.65286100 & 0.84954600 & 1.80559100 \\
\hline $\mathrm{C}$ & 0.91665100 & -2.50909700 & -2.23137400 & $\mathrm{C}$ & 3.18403100 & -0.17847300 & -3.17175000 \\
\hline $\mathrm{H}$ & 1.59455600 & -2.99597900 & -2.93954700 & $\mathrm{H}$ & 2.57031900 & -0.69918900 & -3.91274200 \\
\hline $\mathrm{H}$ & 0.45228900 & -3.30705200 & -1.64158900 & $\mathrm{H}$ & 2.64249400 & 0.71280400 & -2.83867100 \\
\hline $\mathrm{C}$ & -0.18178700 & -1.72917100 & -2.98593900 & $\mathrm{H}$ & 4.09595300 & 0.15385200 & -3.68028900 \\
\hline $\mathrm{H}$ & -0.40733300 & -2.27760100 & -3.90888900 & $\mathrm{C}$ & 4.30331600 & -2.33610200 & -2.51231800 \\
\hline $\mathrm{H}$ & 0.19543400 & -0.74954000 & -3.30587000 & $\mathrm{H}$ & 5.19114500 & -2.01459400 & -3.06818400 \\
\hline $\mathrm{C}$ & -1.47623600 & -1.55140900 & -2.18336000 & $\mathrm{H}$ & 4.64603900 & -2.98667200 & -1.70329000 \\
\hline $\mathrm{H}$ & -1.90014300 & -2.53678800 & -1.94928100 & $\mathrm{H}$ & 3.69576700 & -2.92882100 & -3.20280000 \\
\hline $\mathrm{H}$ & -1.24836200 & -1.07987100 & -1.21551900 & $\mathrm{C}$ & 4.49343900 & -0.32198800 & -1.02501400 \\
\hline $\mathrm{C}$ & -2.51793500 & -0.70592600 & -2.92558300 & $\mathrm{H}$ & 5.41722400 & -0.05045200 & -1.54819700 \\
\hline $\mathrm{H}$ & -2.70305000 & -1.15571100 & -3.91085700 & $\mathrm{H}$ & 4.02472000 & 0.59903400 & -0.66817300 \\
\hline $\mathrm{H}$ & -2.09887300 & 0.29107700 & -3.11920200 & $\mathrm{H}$ & 4.77317000 & -0.92506900 & -0.15505600 \\
\hline $\mathrm{C}$ & -3.86460000 & -0.56659500 & -2.19584300 & $\mathrm{C}$ & 1.07864700 & 1.44956600 & 1.72632800 \\
\hline $\mathrm{H}$ & -4.54946700 & 0.00261800 & -2.83181600 & $\mathrm{O}$ & 0.02307900 & 1.76320400 & 2.16120000 \\
\hline $\mathrm{H}$ & -4.31208100 & -1.54927400 & -2.02003300 & $\mathrm{C}$ & 3.54591400 & 1.62631600 & 1.92846900 \\
\hline $\mathrm{P}$ & -3.75238900 & 0.34534800 & -0.56128800 & $\mathrm{C}$ & 2.37185800 & 2.17096400 & 1.56555300 \\
\hline $\mathrm{C}$ & -5.51927000 & 1.07627100 & -0.37774300 & $\mathrm{C}$ & 0.40597500 & -0.77945800 & 3.27803900 \\
\hline $\mathrm{C}$ & -3.79162700 & -1.07696400 & 0.65268200 & $\mathrm{H}$ & 0.78897700 & -1.31143000 & 4.15378500 \\
\hline $\mathrm{C}$ & -3.45714100 & -0.82668900 & 1.99588100 & $\mathrm{H}$ & -0.18247600 & -1.45332500 & 2.64929400 \\
\hline $\mathrm{C}$ & -4.12773900 & -3.31344500 & 1.11636700 & $\mathrm{H}$ & -0.22674400 & 0.04432400 & 3.60774700 \\
\hline
\end{tabular}




\begin{tabular}{|c|c|c|c|c|c|c|c|}
\hline $\mathrm{O}$ & 1.51393200 & -0.22068300 & 2.55502700 & $\mathrm{H}$ & -1.18814900 & -1.83675100 & -4.01031900 \\
\hline $\mathrm{H}$ & 1.97609500 & -0.95847500 & 2.01104000 & $\mathrm{H}$ & -0.51339900 & -0.65029700 & -2.90809800 \\
\hline $\mathrm{C}$ & 2.25496500 & 3.55318400 & 1.01284400 & $\mathrm{C}$ & -2.45776300 & -1.37295200 & -2.32950400 \\
\hline $\mathrm{C}$ & 3.17379900 & 3.97796500 & 0.03772300 & $\mathrm{H}$ & -3.01570400 & -0.66435100 & -2.94732800 \\
\hline $\mathrm{C}$ & 1.28355400 & 4.45649700 & 1.47457600 & $\mathrm{H}$ & -3.03385900 & -2.30215700 & -2.28626600 \\
\hline $\mathrm{C}$ & 3.13078600 & 5.28112000 & -0.45437100 & $\mathrm{P}$ & -2.40293400 & -0.67204100 & -0.59713400 \\
\hline $\mathrm{H}$ & 3.91644300 & 3.28126300 & -0.33928900 & $\mathrm{C}$ & -3.92530000 & 0.49072200 & -0.55256500 \\
\hline C & 1.24660200 & 5.75952700 & 0.97978900 & C & -2.92821800 & -2.12253100 & 0.45226200 \\
\hline $\mathrm{H}$ & 0.56571200 & 4.14814700 & 2.22657700 & $\mathrm{C}$ & -2.76277800 & -2.07024900 & 1.84560600 \\
\hline C & 2.16687100 & 6.17536500 & 0.01557600 & $\mathrm{C}$ & -3.82963100 & -4.23807100 & 0.57806700 \\
\hline $\mathrm{H}$ & 3.84569400 & 5.59517500 & -1.20864000 & $\mathrm{C}$ & -3.17392200 & -3.16023500 & 2.61193000 \\
\hline $\mathrm{H}$ & 0.49770000 & 6.45157300 & 1.35200500 & $\mathrm{H}$ & -2.32496800 & -1.19212500 & 2.30770100 \\
\hline $\mathrm{H}$ & 2.13084100 & 7.18947600 & -0.36998600 & $\mathrm{C}$ & -3.71810400 & -4.27115900 & 1.96820000 \\
\hline $\mathrm{H}$ & 4.44958200 & 2.22320600 & 1.87082700 & $\mathrm{H}$ & -4.24406400 & -5.08532100 & 0.03566100 \\
\hline $\mathrm{C}$ & -0.23720400 & 2.18225100 & -0.85131200 & $\mathrm{H}$ & -3.07009900 & -3.14344300 & 3.69318100 \\
\hline \multirow[t]{2}{*}{$\mathrm{O}$} & -0.79231500 & 3.08147700 & -1.28034100 & $\mathrm{H}$ & -4.04784400 & -5.14263600 & 2.52418700 \\
\hline & & & & $\mathrm{N}$ & -3.45000700 & -3.19188100 & -0.16700800 \\
\hline \multirow{2}{*}{\multicolumn{3}{|c|}{$\begin{array}{l}\text { TS39b } \\
\text { imaginary frequency }=-146.78 \mathrm{~cm}^{-1}\end{array}$}} & & $\mathrm{Pd}$ & 0.03836000 & -0.10019700 & -0.05183600 \\
\hline & & & & $\mathrm{H}$ & 2.62973000 & 2.19793000 & 1.98361000 \\
\hline C & 3.65042000 & -0.95032300 & 0.41714500 & $\mathrm{C}$ & -5.18166400 & -0.21781600 & -1.09504100 \\
\hline $\mathrm{C}$ & 4.84871600 & -1.60826800 & 0.10720100 & $\mathrm{H}$ & -5.42484100 & -1.11797500 & -0.52394400 \\
\hline $\mathrm{C}$ & 5.83428900 & -1.75382700 & 1.08372700 & $\mathrm{H}$ & -6.03592300 & 0.46558800 & -1.02002300 \\
\hline C & 5.60855400 & -1.24303800 & 2.36032600 & $\mathrm{H}$ & -5.08448200 & -0.50174000 & -2.14613600 \\
\hline $\mathrm{C}$ & 4.38997200 & -0.62027300 & 2.60630000 & $\mathrm{C}$ & -3.58937600 & 1.72010500 & -1.41950000 \\
\hline $\mathrm{H}$ & 5.01923300 & -2.00784400 & -0.88404100 & $\mathrm{H}$ & -2.72013100 & 2.26160500 & -1.03665300 \\
\hline $\mathrm{H}$ & 6.76535400 & -2.25963900 & 0.84690300 & $\mathrm{H}$ & -3.39902700 & 1.45559400 & -2.46478200 \\
\hline $\mathrm{H}$ & 6.34886500 & -1.33026600 & 3.14771700 & $\mathrm{H}$ & -4.44130500 & 2.40974000 & -1.41220300 \\
\hline $\mathrm{H}$ & 4.15113900 & -0.21540300 & 3.58627700 & C & -4.18546400 & 0.94323300 & 0.89590300 \\
\hline $\mathrm{N}$ & 3.44120100 & -0.48392300 & 1.66526300 & $\mathrm{H}$ & -3.31554900 & 1.43607600 & 1.33364100 \\
\hline$P$ & 2.25689400 & -0.65817200 & -0.80488900 & $\mathrm{H}$ & -5.01881900 & 1.65545900 & 0.90053600 \\
\hline C & 3.04010500 & 0.53179800 & -2.10071700 & $\mathrm{H}$ & -4.46929900 & 0.10505000 & 1.53865500 \\
\hline C & 2.24452000 & -2.37393500 & -1.58039200 & $\mathrm{C}$ & 1.89171600 & 1.10716900 & -2.95467500 \\
\hline $\mathrm{H}$ & 3.25524100 & -2.57785300 & -1.94578600 & $\mathrm{H}$ & 1.41125600 & 0.34043100 & -3.56822200 \\
\hline $\mathrm{H}$ & 2.07632300 & -3.05285400 & -0.73557700 & $\mathrm{H}$ & 1.12192300 & 1.58021200 & -2.33776300 \\
\hline C & 1.23661800 & -2.70708000 & -2.69762700 & $\mathrm{H}$ & 2.29633100 & 1.86378600 & -3.63688600 \\
\hline $\mathrm{H}$ & 1.51354500 & -3.70352800 & -3.06003300 & $\mathrm{C}$ & 4.06237100 & -0.15303800 & -3.02528900 \\
\hline $\mathrm{H}$ & 1.38550300 & -2.03775200 & -3.55298500 & $\mathrm{H}$ & 4.44437300 & 0.58789600 & -3.73702200 \\
\hline C & -0.24955000 & -2.70252300 & -2.27125600 & $\mathrm{H}$ & 4.92756600 & -0.54070600 & -2.48092600 \\
\hline $\mathrm{H}$ & -0.71026100 & -3.67103600 & -2.49513500 & $\mathrm{H}$ & 3.62367400 & -0.96510100 & -3.61120900 \\
\hline $\mathrm{H}$ & -0.31243400 & -2.59644300 & -1.17907400 & $\mathrm{C}$ & 3.72884300 & 1.68294800 & -1.34147600 \\
\hline C & -1.07094300 & -1.59493900 & -2.94575400 & $\mathrm{H}$ & 4.08660000 & 2.42442100 & -2.06479100 \\
\hline
\end{tabular}




\begin{tabular}{|c|c|c|c|c|c|c|c|}
\hline $\mathrm{H}$ & 3.04340000 & 2.18894400 & -0.65651700 & $\mathrm{C}$ & -1.91368100 & -3.52285300 & -0.00251800 \\
\hline $\mathrm{H}$ & 4.59553000 & 1.33988900 & -0.76888500 & $\mathrm{H}$ & -2.37261400 & -4.51808900 & 0.03330700 \\
\hline $\mathrm{C}$ & 0.05105400 & 1.19880900 & 1.65559300 & $\mathrm{H}$ & -1.47262000 & -3.43411400 & -1.00151700 \\
\hline $\mathrm{O}$ & -1.04187800 & 0.98497100 & 2.12470400 & $\mathrm{C}$ & -0.82953900 & -3.41019700 & 1.08185600 \\
\hline $\mathrm{C}$ & 1.91360300 & 2.87104000 & 1.53368800 & $\mathrm{H}$ & -1.19010500 & -3.90449400 & 1.99303800 \\
\hline $\mathrm{C}$ & 0.63921900 & 2.53209500 & 1.28377600 & $\mathrm{H}$ & -0.68629800 & -2.35393600 & 1.34312500 \\
\hline $\mathrm{C}$ & 0.71335700 & -0.59833800 & 3.54621900 & $\mathrm{C}$ & 0.52112300 & -4.00335200 & 0.67786200 \\
\hline $\mathrm{H}$ & 1.43946200 & -0.80379800 & 4.33641400 & $\mathrm{H}$ & 1.19723900 & -3.98871900 & 1.53715900 \\
\hline $\mathrm{H}$ & 0.48791600 & -1.51142300 & 2.98737200 & $\mathrm{H}$ & 0.39136800 & -5.06149100 & 0.41235700 \\
\hline $\mathrm{H}$ & -0.20072700 & -0.20142500 & 3.98510100 & $\mathrm{C}$ & 1.22583700 & -3.31131800 & -0.50224500 \\
\hline $\mathrm{O}$ & 1.26535800 & 0.41613800 & 2.68973900 & $\mathrm{H}$ & 0.64961100 & -3.41439500 & -1.42881000 \\
\hline $\mathrm{H}$ & 2.10195400 & 0.05023400 & 2.17254600 & $\mathrm{H}$ & 2.17818300 & -3.81969500 & -0.65382700 \\
\hline $\mathrm{C}$ & -0.30904500 & 3.50580300 & 0.66291400 & $\mathrm{P}$ & 1.61020800 & -1.48698900 & -0.32609500 \\
\hline $\mathrm{C}$ & 0.05155800 & 4.16067300 & -0.52577600 & $\mathrm{C}$ & 2.74389300 & -1.18024500 & -1.85475400 \\
\hline $\mathrm{C}$ & -1.51908800 & 3.84964400 & 1.28774200 & $\mathrm{C}$ & 2.84592000 & -1.54432600 & 1.07121400 \\
\hline $\mathrm{C}$ & -0.77686000 & 5.13830600 & -1.07775700 & $\mathrm{C}$ & 3.15246800 & -0.39081600 & 1.80335100 \\
\hline $\mathrm{H}$ & 0.98434400 & 3.90053500 & -1.01693000 & $\mathrm{C}$ & 4.35815400 & -2.79010300 & 2.28335000 \\
\hline $\mathrm{C}$ & -2.33974600 & 4.83368500 & 0.73783700 & $\mathrm{C}$ & 4.11371600 & -0.46924000 & 2.81190600 \\
\hline $\mathrm{H}$ & -1.80570900 & 3.36074700 & 2.21196700 & $\mathrm{H}$ & 2.66875700 & 0.55250200 & 1.58655400 \\
\hline $\mathrm{C}$ & -1.97390000 & 5.47847200 & -0.44605200 & $\mathrm{C}$ & 4.72997900 & -1.69337000 & 3.06268100 \\
\hline $\mathrm{H}$ & -0.48528800 & 5.63419800 & -1.99870000 & $\mathrm{H}$ & 4.81545600 & -3.76416400 & 2.44525900 \\
\hline $\mathrm{H}$ & -3.26441400 & 5.10188600 & 1.23985800 & $\mathrm{H}$ & 4.37146600 & 0.41500500 & 3.38736100 \\
\hline $\mathrm{H}$ & -2.61692100 & 6.24271200 & -0.87176400 & $\mathrm{H}$ & 5.48069200 & -1.80222000 & 3.83872600 \\
\hline \multirow[t]{2}{*}{$\mathrm{H}$} & 2.25244700 & 3.87750100 & 1.31180100 & $\mathrm{~N}$ & 3.44196900 & -2.72591700 & 1.30944900 \\
\hline & & & & $\mathrm{Pd}$ & -0.33209400 & 0.19976800 & -0.21147800 \\
\hline 47 & & & & $\mathrm{H}$ & -1.75323900 & 2.46558200 & 0.52141700 \\
\hline $\mathrm{C}$ & -3.70881000 & 0.17196600 & 0.62441600 & $\mathrm{C}$ & 3.66350600 & -2.37604100 & -2.16849400 \\
\hline $\mathrm{C}$ & -5.10541300 & 0.18955900 & 0.56890700 & $\mathrm{H}$ & 4.24235200 & -2.69384200 & -1.29668000 \\
\hline $\mathrm{C}$ & -5.84796700 & 0.88252700 & 1.52498500 & $\mathrm{H}$ & 4.37314600 & -2.07671400 & -2.94884900 \\
\hline $\mathrm{C}$ & -5.19852800 & 1.56576200 & 2.55817700 & $\mathrm{H}$ & 3.11221700 & -3.24009600 & -2.54939700 \\
\hline $\mathrm{C}$ & -3.81762800 & 1.53263000 & 2.59580700 & $\mathrm{C}$ & 1.82953900 & -0.90575200 & -3.06331900 \\
\hline $\mathrm{H}$ & -5.61248100 & -0.34773400 & -0.22075400 & $\mathrm{H}$ & 1.21786600 & -0.01280900 & -2.90480900 \\
\hline $\mathrm{H}$ & -6.93207800 & 0.88721400 & 1.46717100 & $\mathrm{H}$ & 1.16575600 & -1.75051600 & -3.27920000 \\
\hline $\mathrm{H}$ & -5.74822500 & 2.11556200 & 3.31267900 & $\mathrm{H}$ & 2.44316500 & -0.73756400 & -3.95636100 \\
\hline $\mathrm{H}$ & -3.21964000 & 2.04395500 & 3.33932900 & $\mathrm{C}$ & 3.61935000 & 0.05513800 & -1.57503700 \\
\hline N & -3.13502300 & 0.85022300 & 1.65149200 & $\mathrm{H}$ & 3.03483300 & 0.91973800 & -1.25600600 \\
\hline$P$ & -2.50183400 & -0.76683300 & -0.48116800 & $\mathrm{H}$ & 4.14758100 & 0.33563400 & -2.49346600 \\
\hline $\mathrm{C}$ & -3.23883600 & -0.64677500 & -2.25732000 & $\mathrm{H}$ & 4.37312500 & -0.15265400 & -0.81114000 \\
\hline $\mathrm{C}$ & -3.00946000 & -2.46292900 & 0.17734900 & $\mathrm{C}$ & -2.07899500 & -1.05740400 & -3.19187200 \\
\hline $\mathrm{H}$ & -3.95053400 & -2.75487200 & -0.29681600 & $\mathrm{H}$ & -1.77272300 & -2.09686800 & -3.03673100 \\
\hline $\mathrm{H}$ & -3.22167300 & -2.34439200 & 1.24622700 & $\mathrm{H}$ & -1.20334800 & -0.42052500 & -3.04643400 \\
\hline
\end{tabular}




\begin{tabular}{|c|c|c|c|c|c|c|c|}
\hline $\mathrm{H}$ & -2.40604600 & -0.96352100 & -4.23357000 & $\mathrm{H}$ & 0.72612400 & 1.73320000 & -4.00264300 \\
\hline $\mathrm{C}$ & -4.43885100 & -1.56791100 & -2.54463800 & $\mathrm{~N}$ & 0.44507200 & 1.72500500 & -1.96038900 \\
\hline $\mathrm{H}$ & -4.75576600 & -1.41341500 & -3.58206800 & $\mathrm{P}$ & 0.18239100 & 1.45437600 & 0.70928100 \\
\hline $\mathrm{H}$ & -5.31282000 & -1.36761400 & -1.91807300 & $\mathrm{C}$ & 1.69603000 & 2.23090000 & 1.61763100 \\
\hline $\mathrm{H}$ & -4.18333400 & -2.62585400 & -2.44423800 & $\mathrm{C}$ & -1.30874900 & 2.05744800 & 1.66728700 \\
\hline $\mathrm{C}$ & -3.61184800 & 0.82007400 & -2.54960600 & $\mathrm{H}$ & -1.23919000 & 3.14780600 & 1.64582700 \\
\hline $\mathrm{H}$ & -3.88476600 & 0.91566800 & -3.60632700 & $\mathrm{H}$ & -2.17957400 & 1.79869000 & 1.05995500 \\
\hline $\mathrm{H}$ & -2.77290900 & 1.49584000 & -2.36462000 & $\mathrm{C}$ & -1.53405700 & 1.62117700 & 3.13685000 \\
\hline $\mathrm{H}$ & -4.46776400 & 1.16501600 & -1.96188800 & $\mathrm{H}$ & -2.05825200 & 2.45126000 & 3.62501600 \\
\hline $\mathrm{C}$ & 0.63508300 & 2.27665300 & 1.70839600 & $\mathrm{H}$ & -0.58546700 & 1.52393500 & 3.67366500 \\
\hline $\mathrm{O}$ & 1.68647600 & 2.46236300 & 2.29113900 & $\mathrm{C}$ & -2.37926600 & 0.35695100 & 3.35174000 \\
\hline $\mathrm{C}$ & -0.95572000 & 2.29008500 & -0.18741900 & $\mathrm{H}$ & -2.70683800 & 0.35567500 & 4.39690200 \\
\hline $\mathrm{C}$ & 0.38534300 & 2.37558700 & 0.24965900 & $\mathrm{H}$ & -3.29626700 & 0.44993500 & 2.76034600 \\
\hline $\mathrm{C}$ & -0.22142100 & 1.78660000 & 3.86389700 & $\mathrm{C}$ & -1.67926400 & -0.99259200 & 3.05102200 \\
\hline $\mathrm{H}$ & -1.15787200 & 1.45998500 & 4.31997200 & $\mathrm{H}$ & -1.57070200 & -1.55400600 & 3.98535800 \\
\hline $\mathrm{H}$ & 0.55884100 & 1.04475600 & 4.04246200 & $\mathrm{H}$ & -0.65683500 & -0.82620100 & 2.69095300 \\
\hline $\mathrm{H}$ & 0.09317800 & 2.74238600 & 4.28717200 & $\mathrm{C}$ & -2.41136400 & -1.90974800 & 2.05229900 \\
\hline $\mathrm{O}$ & -0.49013000 & 1.91687600 & 2.45166800 & $\mathrm{H}$ & -1.99721200 & -2.91940300 & 2.13661900 \\
\hline $\mathrm{H}$ & -2.10089800 & 0.88865000 & 1.68868700 & $\mathrm{H}$ & -3.47388200 & -1.99352600 & 2.30507800 \\
\hline $\mathrm{H}$ & -1.18899000 & 2.64399100 & -1.18578700 & $\mathrm{P}$ & -2.17567300 & -1.47698400 & 0.25473700 \\
\hline $\mathrm{C}$ & 1.43695500 & 2.94265800 & -0.66063700 & $\mathrm{C}$ & -2.85924000 & -3.00021500 & -0.69385400 \\
\hline $\mathrm{C}$ & 2.57269600 & 3.62666800 & -0.19018400 & $\mathrm{C}$ & -3.39936200 & -0.15616400 & -0.19492600 \\
\hline $\mathrm{C}$ & 1.26034400 & 2.86606400 & -2.05814300 & $\mathrm{C}$ & -4.63166800 & 0.03637800 & 0.44548100 \\
\hline $\mathrm{C}$ & 3.48023400 & 4.20696400 & -1.08011200 & $\mathrm{C}$ & -3.84561100 & 1.53164600 & -1.69871700 \\
\hline $\mathrm{H}$ & 2.74761600 & 3.71029400 & 0.87268700 & $\mathrm{C}$ & -5.48428500 & 1.03658300 & -0.02431100 \\
\hline $\mathrm{C}$ & 2.16481600 & 3.44708800 & -2.94136800 & $\mathrm{H}$ & -4.93133600 & -0.58178000 & 1.28405600 \\
\hline $\mathrm{H}$ & 0.41146200 & 2.32732500 & -2.46729500 & $\mathrm{C}$ & -5.08624700 & 1.80246000 & -1.11832400 \\
\hline $\mathrm{C}$ & 3.28586200 & 4.12517000 & -2.45688200 & $\mathrm{H}$ & -3.49719700 & 2.10248200 & -2.55621300 \\
\hline $\mathrm{H}$ & 4.34460500 & 4.73152400 & -0.68326400 & $\mathrm{H}$ & -6.44391600 & 1.20748000 & 0.45414000 \\
\hline $\mathrm{H}$ & 1.99619700 & 3.36406100 & -4.01125400 & $\mathrm{H}$ & -5.71927900 & 2.58828300 & -1.51691300 \\
\hline $\mathrm{H}$ & 3.99465400 & 4.57819500 & -3.14318600 & $\mathrm{~N}$ & -3.01469600 & 0.58225900 & -1.25043200 \\
\hline & & & & $\mathrm{Pd}$ & 0.24080200 & -0.87171300 & -0.12334400 \\
\hline 48 & & & & $\mathrm{H}$ & 3.00420900 & -1.93015300 & 0.98506900 \\
\hline $\mathrm{C}$ & 0.01962900 & 2.40428300 & -0.88174300 & $\mathrm{C}$ & -4.36991700 & -3.18503500 & -0.44316700 \\
\hline $\mathrm{C}$ & -0.49038500 & 3.70578200 & -0.97157100 & $\mathrm{H}$ & -4.96116400 & -2.35288200 & -0.83140400 \\
\hline $\mathrm{C}$ & -0.55885500 & 4.31647800 & -2.22519900 & $\mathrm{H}$ & -4.70199700 & -4.09205100 & -0.96047600 \\
\hline $\mathrm{C}$ & -0.12130900 & 3.61117200 & -3.34348000 & $\mathrm{H}$ & -4.60650800 & -3.31633300 & 0.61763100 \\
\hline $\mathrm{C}$ & 0.37348100 & 2.31856600 & -3.15664800 & $\mathrm{C}$ & -2.12963300 & -4.27631600 & -0.22649700 \\
\hline $\mathrm{H}$ & -0.83006600 & 4.24155300 & -0.09289600 & $\mathrm{H}$ & -1.04174400 & -4.19483900 & -0.27020100 \\
\hline $\mathrm{H}$ & -0.94723500 & 5.32598700 & -2.32145400 & $\mathrm{H}$ & -2.40951200 & -4.56335200 & 0.79097200 \\
\hline $\mathrm{H}$ & -0.15724400 & 4.04682100 & -4.33648900 & $\mathrm{H}$ & -2.41584000 & -5.10474000 & -0.88351300 \\
\hline
\end{tabular}




\begin{tabular}{|c|c|c|c|c|c|c|c|}
\hline $\mathrm{C}$ & -2.62023200 & -2.77112000 & -2.19982100 & $\mathrm{H}$ & -0.88063500 & 5.41976700 & -2.29192600 \\
\hline $\mathrm{H}$ & -1.55757000 & -2.70964400 & -2.44816100 & $\mathrm{H}$ & 0.03502500 & 4.18407200 & -4.28063600 \\
\hline $\mathrm{H}$ & -3.04106000 & -3.61317100 & -2.76051000 & $\mathrm{H}$ & 0.92919700 & 1.87731400 & -3.93580900 \\
\hline $\mathrm{H}$ & -3.10076600 & -1.85655700 & -2.55704900 & $\mathrm{~N}$ & 0.53336500 & 1.82868100 & -1.91287300 \\
\hline $\mathrm{C}$ & 2.19339400 & 1.24029600 & 2.69029600 & $\mathrm{P}$ & 0.12488900 & 1.50120400 & 0.72969700 \\
\hline $\mathrm{H}$ & 1.44555300 & 1.04405500 & 3.46398600 & $\mathrm{C}$ & 1.67400500 & 2.17466900 & 1.64959100 \\
\hline $\mathrm{H}$ & 2.49273300 & 0.28890100 & 2.24724900 & $\mathrm{C}$ & -1.33576300 & 2.18362400 & 1.68133400 \\
\hline $\mathrm{H}$ & 3.06987900 & 1.66867200 & 3.18937400 & $\mathrm{H}$ & -1.19409500 & 3.26712400 & 1.70048400 \\
\hline $\mathrm{C}$ & 1.31516100 & 3.56994500 & 2.28124100 & $\mathrm{H}$ & -2.21112200 & 2.00192000 & 1.05070400 \\
\hline $\mathrm{H}$ & 2.21673300 & 3.99543600 & 2.73553400 & $\mathrm{C}$ & -1.62546100 & 1.71945500 & 3.12949100 \\
\hline $\mathrm{H}$ & 0.94805900 & 4.30328400 & 1.55634800 & $\mathrm{H}$ & -2.19147500 & 2.53255400 & 3.59945300 \\
\hline $\mathrm{H}$ & 0.57529000 & 3.46172300 & 3.07752200 & $\mathrm{H}$ & -0.70207400 & 1.63601400 & 3.71216500 \\
\hline $\mathrm{C}$ & 2.82980400 & 2.50553900 & 0.60787500 & $\mathrm{C}$ & -2.45428800 & 0.43510800 & 3.27884900 \\
\hline $\mathrm{H}$ & 3.67642200 & 2.93824400 & 1.15275700 & $\mathrm{H}$ & -2.87353800 & 0.42425600 & 4.29082600 \\
\hline $\mathrm{H}$ & 3.18368300 & 1.59802300 & 0.12105500 & $\mathrm{H}$ & -3.31708900 & 0.50618500 & 2.60821700 \\
\hline $\mathrm{H}$ & 2.53034900 & 3.22420200 & -0.15951000 & $\mathrm{C}$ & -1.69685000 & -0.89563400 & 3.04407200 \\
\hline $\mathrm{C}$ & 0.76623400 & -2.51570300 & -1.01613200 & $\mathrm{H}$ & -1.56866600 & -1.40886600 & 4.00345400 \\
\hline $\mathrm{O}$ & 1.11897100 & -3.43725500 & -1.59106400 & $\mathrm{H}$ & -0.68171500 & -0.70529000 & 2.67677200 \\
\hline $\mathrm{C}$ & 3.25584100 & -1.26429400 & 0.15847500 & $\mathrm{C}$ & -2.39158500 & -1.87772300 & 2.08228400 \\
\hline $\mathrm{C}$ & 2.26697300 & -0.64254100 & -0.50173700 & $\mathrm{H}$ & -1.95443100 & -2.87301600 & 2.21077000 \\
\hline $\mathrm{C}$ & 4.70611100 & -1.12763900 & -0.07752300 & $\mathrm{H}$ & -3.45516000 & -1.97458500 & 2.32520800 \\
\hline $\mathrm{C}$ & 5.59314800 & -1.76435900 & 0.80829300 & $\mathrm{P}$ & -2.15078900 & -1.50310900 & 0.27258400 \\
\hline $\mathrm{C}$ & 5.24716600 & -0.38832100 & -1.14620200 & $\mathrm{C}$ & -2.76878400 & -3.07512200 & -0.62999500 \\
\hline $\mathrm{C}$ & 6.97311500 & -1.66359200 & 0.63957000 & $\mathrm{C}$ & -3.38815700 & -0.21337400 & -0.22429400 \\
\hline $\mathrm{H}$ & 5.19284200 & -2.34418300 & 1.63644100 & $\mathrm{C}$ & -4.65999600 & -0.07171600 & 0.34841500 \\
\hline $\mathrm{C}$ & 6.62485300 & -0.28781300 & -1.31445600 & $\mathrm{C}$ & -3.79818900 & 1.49573800 & -1.71362100 \\
\hline $\mathrm{H}$ & 4.58778600 & 0.10136700 & -1.85664300 & $\mathrm{C}$ & -5.51601400 & 0.91139000 & -0.15044000 \\
\hline $\mathrm{C}$ & 7.49430400 & -0.92342800 & -0.42228500 & $\mathrm{H}$ & -4.98371000 & -0.71220900 & 1.16102100 \\
\hline $\mathrm{H}$ & 7.63990600 & -2.16442800 & 1.33503000 & $\mathrm{C}$ & -5.08004500 & 1.71247800 & -1.20408500 \\
\hline $\mathrm{H}$ & 7.02448300 & 0.28348900 & -2.14715400 & $\mathrm{H}$ & -3.41762900 & 2.09859300 & -2.53475900 \\
\hline $\mathrm{H}$ & 8.56853800 & -0.84478000 & -0.55846200 & $\mathrm{H}$ & -6.50608900 & 1.04336000 & 0.27537600 \\
\hline \multirow[t]{2}{*}{$\mathrm{H}$} & 2.42721300 & 0.00627700 & -1.35820900 & $\mathrm{H}$ & -5.71410000 & 2.48646300 & -1.62370000 \\
\hline & & & & $\mathrm{N}$ & -2.96480700 & 0.56211900 & -1.23734000 \\
\hline \multicolumn{4}{|c|}{ TS40 } & $\mathrm{Pd}$ & 0.17306500 & -0.83524900 & -0.14796100 \\
\hline \multicolumn{4}{|c|}{ imaginary frequency $=-207.45 \mathrm{~cm}^{-1}$} & $\mathrm{H}$ & 2.92307500 & -2.09552000 & 0.84099600 \\
\hline $\mathrm{C}$ & 0.03902900 & 2.48436000 & -0.84760800 & $\mathrm{C}$ & -4.24879000 & -3.36536500 & -0.30732700 \\
\hline $\mathrm{C}$ & -0.47944800 & 3.78185200 & -0.94523700 & $\mathrm{H}$ & -4.91342800 & -2.57035700 & -0.65329000 \\
\hline $\mathrm{C}$ & -0.48578000 & 4.41321600 & -2.19066100 & $\mathrm{H}$ & -4.54561300 & -4.28526700 & -0.82324400 \\
\hline $\mathrm{C}$ & 0.02203900 & 3.73255500 & -3.29417700 & $\mathrm{H}$ & -4.42292200 & -3.52701100 & 0.76125900 \\
\hline $\mathrm{C}$ & 0.52243500 & 2.44313300 & -3.10068400 & $\mathrm{C}$ & -1.91924100 & -4.28418100 & -0.18831500 \\
\hline $\mathrm{H}$ & -0.87251100 & 4.29912300 & -0.07779400 & $\mathrm{H}$ & -0.84800500 & -4.12294400 & -0.32262400 \\
\hline
\end{tabular}




\begin{tabular}{|c|c|c|c|c|c|c|c|}
\hline $\mathrm{H}$ & -2.09944000 & -4.55965000 & 0.85475400 & $\mathrm{C}$ & -3.60045600 & 1.38340000 & 2.19203800 \\
\hline $\mathrm{H}$ & -2.19583300 & -5.14968500 & -0.80012700 & $\mathrm{H}$ & -1.36572500 & 4.44497900 & 1.83001200 \\
\hline $\mathrm{C}$ & -2.61400000 & -2.84781300 & -2.14717100 & $\mathrm{H}$ & -2.85725600 & 4.26593200 & 3.80453500 \\
\hline $\mathrm{H}$ & -1.57223100 & -2.69554000 & -2.43889400 & $\mathrm{H}$ & -4.33189100 & 2.24061600 & 4.03205900 \\
\hline $\mathrm{H}$ & -2.97970200 & -3.73336100 & -2.67871100 & $\mathrm{H}$ & -4.22559500 & 0.49452000 & 2.25120300 \\
\hline $\mathrm{H}$ & -3.19233300 & -1.98777100 & -2.49524700 & $\mathrm{~N}$ & -2.78722200 & 1.45928600 & 1.13057700 \\
\hline $\mathrm{C}$ & 2.09526000 & 1.13290600 & 2.70660000 & $\mathrm{P}$ & -0.90407400 & 2.48599800 & -0.51358200 \\
\hline $\mathrm{H}$ & 1.33012900 & 0.97565100 & 3.47249500 & $\mathrm{C}$ & -2.05908000 & 3.24089400 & -1.85490100 \\
\hline $\mathrm{H}$ & 2.32480600 & 0.16849100 & 2.24702500 & $\mathrm{C}$ & 0.33698200 & 3.84361200 & -0.11540000 \\
\hline $\mathrm{H}$ & 2.99867600 & 1.48418600 & 3.21798200 & $\mathrm{H}$ & -0.13811200 & 4.82996100 & -0.16928600 \\
\hline $\mathrm{C}$ & 1.39086300 & 3.52874800 & 2.32791700 & $\mathrm{H}$ & 0.60116900 & 3.68455300 & 0.93719600 \\
\hline $\mathrm{H}$ & 2.31910200 & 3.89133400 & 2.78376500 & $\mathrm{C}$ & 1.64936000 & 3.87528800 & -0.93087000 \\
\hline $\mathrm{H}$ & 1.06902700 & 4.29015300 & 1.61004200 & $\mathrm{H}$ & 2.21966400 & 4.73584800 & -0.56202500 \\
\hline $\mathrm{H}$ & 0.64597800 & 3.46215500 & 3.12476500 & $\mathrm{H}$ & 1.44385000 & 4.09239600 & -1.98462200 \\
\hline $\mathrm{C}$ & 2.82435400 & 2.37047700 & 0.64155100 & $\mathrm{C}$ & 2.53616500 & 2.61373000 & -0.82780800 \\
\hline $\mathrm{H}$ & 3.71845600 & 2.68550700 & 1.19124000 & $\mathrm{H}$ & 3.59054400 & 2.91677100 & -0.79656300 \\
\hline $\mathrm{H}$ & 3.07161500 & 1.45224700 & 0.10966600 & $\mathrm{H}$ & 2.33938800 & 2.11417700 & 0.12952900 \\
\hline $\mathrm{H}$ & 2.59639700 & 3.14797800 & -0.09208800 & $\mathrm{C}$ & 2.34987400 & 1.62895400 & -1.99175300 \\
\hline $\mathrm{C}$ & 0.87236900 & -2.29959400 & -1.16213700 & $\mathrm{H}$ & 2.60195100 & 2.14141000 & -2.92888300 \\
\hline $\mathrm{O}$ & 1.11241000 & -3.19514800 & -1.84773300 & $\mathrm{H}$ & 1.29487900 & 1.34827500 & -2.06987100 \\
\hline $\mathrm{C}$ & 3.20812400 & -1.40501200 & 0.04637100 & $\mathrm{C}$ & 3.21131100 & 0.35373300 & -1.92308700 \\
\hline $\mathrm{C}$ & 2.23203100 & -0.82002900 & -0.67688300 & $\mathrm{H}$ & 3.07598500 & -0.21958000 & -2.84649900 \\
\hline $\mathrm{C}$ & 4.65747600 & -1.21851200 & -0.09468800 & $\mathrm{H}$ & 4.27559000 & 0.60717500 & -1.87169100 \\
\hline C & 5.50839900 & -1.86568000 & 0.82052700 & $\mathrm{P}$ & 2.78728700 & -0.82247600 & -0.54460600 \\
\hline $\mathrm{C}$ & 5.23664200 & -0.42151000 & -1.10168200 & $\mathrm{C}$ & 3.59554700 & -2.48498800 & -1.00755800 \\
\hline $\mathrm{C}$ & 6.89137600 & -1.71920500 & 0.73922400 & $\mathrm{C}$ & 3.71292400 & -0.23419400 & 0.95289100 \\
\hline $\mathrm{H}$ & 5.07651500 & -2.48869400 & 1.59975500 & $\mathrm{C}$ & 4.96666300 & 0.38909500 & 0.89772100 \\
\hline $\mathrm{C}$ & 6.61744900 & -0.27822400 & -1.18338900 & $\mathrm{C}$ & 3.69248400 & -0.13079900 & 3.25755300 \\
\hline $\mathrm{H}$ & 4.60498900 & 0.07894700 & -1.82915500 & $\mathrm{C}$ & 5.58562700 & 0.75830000 & 2.09320700 \\
\hline $\mathrm{C}$ & 7.44993900 & -0.92440500 & -0.26306600 & $\mathrm{H}$ & 5.45625700 & 0.58594500 & -0.04892700 \\
\hline $\mathrm{H}$ & 7.53185600 & -2.22673500 & 1.45394300 & $\mathrm{C}$ & 4.93993600 & 0.49424800 & 3.29896800 \\
\hline $\mathrm{H}$ & 7.04970500 & 0.33534100 & -1.96802900 & $\mathrm{H}$ & 3.14928400 & -0.35454100 & 4.17286600 \\
\hline $\mathrm{H}$ & 8.52747600 & -0.81068400 & -0.33144500 & $\mathrm{H}$ & 6.55666300 & 1.24376000 & 2.07849100 \\
\hline \multirow[t]{2}{*}{$\mathrm{H}$} & 2.41347700 & -0.16559000 & -1.52390600 & $\mathrm{H}$ & 5.38563400 & 0.76633400 & 4.24977400 \\
\hline & & & & $\mathrm{N}$ & 3.09106800 & -0.48362900 & 2.11588000 \\
\hline \multicolumn{4}{|c|}{ TS40a } & $\mathrm{Pd}$ & 0.38532700 & -1.07181800 & -0.13470400 \\
\hline \multicolumn{4}{|c|}{ imaginary frequency $=-226.54 \mathrm{~cm}^{-1}$} & $\mathrm{H}$ & -1.60078100 & -3.46354900 & -0.59042100 \\
\hline $\mathrm{C}$ & -1.99020300 & 2.54165800 & 0.99974800 & $\mathrm{C}$ & 5.08532700 & -2.31202800 & -1.35799100 \\
\hline $\mathrm{C}$ & -1.99715700 & 3.57271500 & 1.95144000 & $\mathrm{H}$ & 5.66411700 & -1.92006700 & -0.51712700 \\
\hline $\mathrm{C}$ & -2.83984500 & 3.47614000 & 3.05910300 & $\mathrm{H}$ & 5.50674800 & -3.29035800 & -1.61439500 \\
\hline $\mathrm{C}$ & -3.66130600 & 2.35848600 & 3.18742500 & $\mathrm{H}$ & 5.23642000 & -1.65809700 & -2.22231500 \\
\hline
\end{tabular}




\begin{tabular}{|c|c|c|c|c|c|c|c|}
\hline $\mathrm{C}$ & 2.83937400 & -3.07158400 & -2.21700400 & $\mathrm{C}$ & 0.87007500 & 3.80330300 & -2.10613700 \\
\hline $\mathrm{H}$ & 1.77997100 & -3.23137900 & -1.99401800 & $\mathrm{C}$ & 1.69143800 & 3.59027000 & -3.21562400 \\
\hline $\mathrm{H}$ & 2.91379300 & -2.44380900 & -3.11031300 & $\mathrm{C}$ & 2.16619500 & 2.30551200 & -3.47123800 \\
\hline $\mathrm{H}$ & 3.27416500 & -4.04452500 & -2.47055400 & $\mathrm{C}$ & 1.79022900 & 1.26739500 & -2.62076700 \\
\hline $\mathrm{C}$ & 3.44401900 & -3.43695500 & 0.19553400 & $\mathrm{H}$ & 0.47915400 & 4.79073400 & -1.88884500 \\
\hline $\mathrm{H}$ & 2.39235300 & -3.59590000 & 0.45590200 & $\mathrm{H}$ & 1.95426300 & 4.41578700 & -3.86998000 \\
\hline $\mathrm{H}$ & 3.87279500 & -4.41135600 & -0.06289600 & $\mathrm{H}$ & 2.80668700 & 2.09966700 & -4.32180100 \\
\hline $\mathrm{H}$ & 3.96444700 & -3.06994500 & 1.08413200 & $\mathrm{H}$ & 2.12234700 & 0.24898100 & -2.78789500 \\
\hline $\mathrm{C}$ & -1.21308200 & 3.50427200 & -3.11654900 & $\mathrm{~N}$ & 0.99714400 & 1.47096200 & -1.55797500 \\
\hline $\mathrm{H}$ & -0.50124600 & 4.32211100 & -2.97286000 & $\mathrm{P}$ & -0.54230900 & 2.64451200 & 0.21139900 \\
\hline $\mathrm{H}$ & -0.65908000 & 2.61454800 & -3.43474200 & $\mathrm{C}$ & 0.36898100 & 3.65816100 & 1.54852400 \\
\hline $\mathrm{H}$ & -1.87328600 & 3.79224600 & -3.94313200 & $\mathrm{C}$ & -2.04595900 & 3.61212900 & -0.34003200 \\
\hline $\mathrm{C}$ & -2.74468000 & 4.54417200 & -1.41356700 & $\mathrm{H}$ & -1.85015000 & 4.68865300 & -0.28519100 \\
\hline $\mathrm{H}$ & -3.36623900 & 4.92554500 & -2.23292900 & $\mathrm{H}$ & -2.14614600 & 3.36247000 & -1.40273200 \\
\hline $\mathrm{H}$ & -3.39679800 & 4.39314500 & -0.54897800 & $\mathrm{C}$ & -3.37314900 & 3.26347200 & 0.36760900 \\
\hline $\mathrm{H}$ & -2.02497300 & 5.33133000 & -1.16595400 & $\mathrm{H}$ & -4.16213000 & 3.79909500 & -0.17215300 \\
\hline $\mathrm{C}$ & -3.12664300 & 2.17793600 & -2.18154400 & $\mathrm{H}$ & -3.38117000 & 3.67185600 & 1.38534600 \\
\hline $\mathrm{H}$ & -3.79676300 & 2.55997100 & -2.96097900 & $\mathrm{C}$ & -3.69331500 & 1.75596100 & 0.40544800 \\
\hline $\mathrm{H}$ & -2.67371700 & 1.25493500 & -2.55778300 & $\mathrm{H}$ & -4.77479600 & 1.60629800 & 0.30797900 \\
\hline $\mathrm{H}$ & -3.73512200 & 1.92661200 & -1.30881600 & $\mathrm{H}$ & -3.25211500 & 1.25192000 & -0.46276500 \\
\hline $\mathrm{C}$ & 0.43043400 & -1.25101400 & 1.88313000 & $\mathrm{C}$ & -3.23057400 & 1.07458900 & 1.70303400 \\
\hline $\mathrm{O}$ & 0.25040100 & -1.39975700 & 2.99612600 & $\mathrm{H}$ & -3.90049500 & 1.38255900 & 2.51627600 \\
\hline $\mathrm{C}$ & -2.24383000 & -2.62070200 & -0.33581600 & $\mathrm{H}$ & -2.23618100 & 1.42839100 & 1.99023800 \\
\hline $\mathrm{C}$ & -1.70952900 & -1.38637000 & -0.26771100 & $\mathrm{C}$ & -3.22758300 & -0.45793800 & 1.65232100 \\
\hline $\mathrm{C}$ & -3.64636700 & -2.97749300 & -0.09478300 & $\mathrm{H}$ & -3.25567500 & -0.84919100 & 2.67148500 \\
\hline $\mathrm{C}$ & -3.99875100 & -4.33919000 & -0.07195700 & $\mathrm{H}$ & -4.12628900 & -0.81590400 & 1.14396100 \\
\hline $\mathrm{C}$ & -4.65405700 & -2.01425000 & 0.11249200 & $\mathrm{P}$ & -1.78065400 & -1.32812700 & 0.80898400 \\
\hline $\mathrm{C}$ & -5.31449700 & -4.73198000 & 0.16424400 & $\mathrm{C}$ & -1.14755700 & -2.51084000 & 2.16695000 \\
\hline $\mathrm{H}$ & -3.23173000 & -5.09154600 & -0.23721200 & $\mathrm{C}$ & -2.68192200 & -2.26749800 & -0.51865800 \\
\hline $\mathrm{C}$ & -5.96718600 & -2.40909500 & 0.34249100 & $\mathrm{C}$ & -2.58837700 & -3.63394900 & -0.79562400 \\
\hline $\mathrm{H}$ & -4.40828100 & -0.95731400 & 0.07648300 & $\mathrm{C}$ & -4.12602000 & -1.93538500 & -2.28102700 \\
\hline $\mathrm{C}$ & -6.30174100 & -3.76790300 & 0.37283300 & $\mathrm{C}$ & -3.31666600 & -4.14797600 & -1.87005500 \\
\hline $\mathrm{H}$ & -5.56919900 & -5.78709300 & 0.18163900 & $\mathrm{H}$ & -1.96802100 & -4.29224100 & -0.20230900 \\
\hline $\mathrm{H}$ & -6.73737400 & -1.65855800 & 0.49302600 & $\mathrm{C}$ & -4.09927400 & -3.28633900 & -2.63278600 \\
\hline $\mathrm{H}$ & -7.32882900 & -4.07052900 & 0.55238200 & $\mathrm{H}$ & -4.72583800 & -1.22626600 & -2.84714600 \\
\hline $\mathrm{H}$ & -2.24556200 & -0.46983500 & -0.02902700 & $\mathrm{H}$ & -3.26604000 & -5.20673000 & -2.10532200 \\
\hline $\mathrm{C}$ & -0.43014100 & -1.08464900 & -1.88241800 & $\mathrm{H}$ & -4.67625900 & -3.64509100 & -3.47848900 \\
\hline \multirow[t]{2}{*}{$\mathrm{O}$} & -0.73703800 & -1.02455900 & -2.99025200 & $\mathrm{~N}$ & -3.43946700 & -1.43502600 & -1.24977700 \\
\hline & & & & $\mathrm{Pd}$ & -0.18441700 & 0.07656600 & -0.27680700 \\
\hline $49 b$ & & & & $\mathrm{H}$ & 2.31000400 & -0.56513700 & 0.55162400 \\
\hline $\mathrm{C}$ & 0.54859500 & 2.72287600 & -1.28381600 & $\mathrm{C}$ & -2.26984300 & -3.42287100 & 2.70644400 \\
\hline
\end{tabular}




\begin{tabular}{|c|c|c|c|c|c|c|c|}
\hline $\mathrm{H}$ & -2.68796200 & -4.07160900 & 1.93345500 & $\mathrm{C}$ & -1.97310400 & 3.00242200 & -0.72344000 \\
\hline $\mathrm{H}$ & -1.85602500 & -4.06654300 & 3.49080300 & $\mathrm{C}$ & -1.76600900 & 2.58712200 & -2.04565900 \\
\hline $\mathrm{H}$ & -3.09455000 & -2.86229200 & 3.15378400 & $\mathrm{C}$ & -2.60300700 & 3.10147900 & -3.03817700 \\
\hline $\mathrm{C}$ & -0.60578500 & -1.61986600 & 3.30742500 & $\mathrm{C}$ & -3.61157500 & 3.99167300 & -2.67602500 \\
\hline $\mathrm{H}$ & 0.19637100 & -0.96216900 & 2.95640400 & $\mathrm{C}$ & -3.74596800 & 4.33259800 & -1.32789600 \\
\hline $\mathrm{H}$ & -1.38030800 & -1.00029400 & 3.76906800 & $\mathrm{H}$ & -0.98576000 & 1.87350900 & -2.28925000 \\
\hline $\mathrm{H}$ & -0.19158000 & -2.25927100 & 4.09478200 & $\mathrm{H}$ & -2.47105500 & 2.80406300 & -4.07416400 \\
\hline $\mathrm{C}$ & 0.01878000 & -3.37534900 & 1.64942800 & $\mathrm{H}$ & -4.28623900 & 4.41126700 & -3.41494100 \\
\hline $\mathrm{H}$ & 0.86531600 & -2.76470200 & 1.33269500 & $\mathrm{H}$ & -4.52692200 & 5.01697200 & -1.00416000 \\
\hline $\mathrm{H}$ & 0.36256300 & -4.02581400 & 2.46146700 & $\mathrm{~N}$ & -2.94646200 & 3.85474000 & -0.36714600 \\
\hline $\mathrm{H}$ & -0.25700300 & -4.02082400 & 0.81246900 & $\mathrm{P}$ & -0.88568900 & 2.38051200 & 0.64159300 \\
\hline $\mathrm{C}$ & -0.51161800 & 3.66719300 & 2.81471600 & $\mathrm{C}$ & 0.47778600 & 3.69106400 & 0.84704200 \\
\hline $\mathrm{H}$ & -1.46254000 & 4.18366900 & 2.65395400 & $\mathrm{C}$ & -1.96639600 & 2.50583900 & 2.17338500 \\
\hline $\mathrm{H}$ & -0.72384100 & 2.65601800 & 3.17471200 & $\mathrm{H}$ & -1.70687600 & 3.42964300 & 2.69629600 \\
\hline $\mathrm{H}$ & 0.01630400 & 4.19720000 & 3.61548900 & $\mathrm{H}$ & -2.99119200 & 2.64121800 & 1.81518200 \\
\hline $\mathrm{C}$ & 0.66734700 & 5.10604500 & 1.12196000 & $\mathrm{C}$ & -1.84708300 & 1.30021900 & 3.12412400 \\
\hline $\mathrm{H}$ & 1.16556000 & 5.62818000 & 1.94718400 & $\mathrm{H}$ & -2.35120800 & 1.54672800 & 4.06605600 \\
\hline $\mathrm{H}$ & 1.33834700 & 5.15280400 & 0.26013100 & $\mathrm{H}$ & -0.79237800 & 1.13873000 & 3.37782400 \\
\hline $\mathrm{H}$ & -0.24127700 & 5.67011100 & 0.88976900 & $\mathrm{C}$ & -2.43840000 & 0.00032600 & 2.55024300 \\
\hline $\mathrm{C}$ & 1.68903500 & 2.92116600 & 1.84975200 & $\mathrm{H}$ & -3.53063800 & 0.00055800 & 2.65519800 \\
\hline $\mathrm{H}$ & 2.21811100 & 3.43624800 & 2.65952900 & $\mathrm{H}$ & -2.31226400 & 0.00031700 & 1.44332600 \\
\hline $\mathrm{H}$ & 1.50863000 & 1.88963800 & 2.16987900 & $\mathrm{C}$ & -1.84766100 & -1.29985400 & 3.12407000 \\
\hline $\mathrm{H}$ & 2.35479900 & 2.90026300 & 0.98104100 & $\mathrm{H}$ & -2.35198400 & -1.54625500 & 4.06592300 \\
\hline $\mathrm{C}$ & 0.56189100 & -1.53140300 & -1.26189500 & $\mathrm{H}$ & -0.79291900 & -1.13880800 & 3.37789800 \\
\hline $\mathrm{O}$ & -0.10405300 & -2.06441500 & -2.11861700 & $\mathrm{C}$ & -1.96736100 & -2.50531000 & 2.17316300 \\
\hline $\mathrm{C}$ & 2.77074100 & -1.30959400 & -0.09852500 & $\mathrm{H}$ & -1.70847100 & -3.42932400 & 2.69601600 \\
\hline $\mathrm{C}$ & 1.98527900 & -1.86640300 & -1.04741500 & $\mathrm{H}$ & -2.99213800 & -2.64009600 & 1.81467800 \\
\hline $\mathrm{C}$ & 4.17847100 & -1.58347700 & 0.17188700 & $\mathrm{P}$ & -0.88617000 & -2.38033400 & 0.64169700 \\
\hline $\mathrm{C}$ & 4.81191700 & -0.87275700 & 1.20989800 & $\mathrm{C}$ & 0.47709100 & -3.69100800 & 0.84775800 \\
\hline $\mathrm{C}$ & 4.93466400 & -2.52590900 & -0.55530400 & $\mathrm{C}$ & -1.97318900 & -3.00231800 & -0.72361200 \\
\hline $\mathrm{C}$ & 6.15354900 & -1.09055300 & 1.51304600 & $\mathrm{C}$ & -1.76582100 & -2.58700300 & -2.04577800 \\
\hline $\mathrm{H}$ & 4.24008700 & -0.14517100 & 1.78048900 & $\mathrm{C}$ & -3.74577300 & -4.33268200 & -1.32848400 \\
\hline $\mathrm{C}$ & 6.27322200 & -2.74314800 & -0.25107100 & $\mathrm{C}$ & -2.60255100 & -3.10142300 & -3.03849000 \\
\hline $\mathrm{H}$ & 4.47341900 & -3.09237400 & -1.35810700 & $\mathrm{H}$ & -0.98554100 & -1.87335900 & -2.28919100 \\
\hline $\mathrm{C}$ & 6.88750000 & -2.02679500 & 0.78270400 & $\mathrm{C}$ & -3.61111900 & -3.99171200 & -2.67657700 \\
\hline H & 6.62536900 & -0.53375300 & 2.31665800 & $\mathrm{H}$ & -4.52672700 & -5.01714300 & -1.00493200 \\
\hline $\mathrm{H}$ & 6.84296100 & -3.47281300 & -0.81808900 & $\mathrm{H}$ & -2.47038700 & -2.80398800 & -4.07444500 \\
\hline $\mathrm{H}$ & 7.93337000 & -2.20082300 & 1.01633200 & $\mathrm{H}$ & -4.28557200 & -4.41136100 & -3.41565500 \\
\hline \multirow[t]{2}{*}{$\mathrm{H}$} & 2.35743700 & -2.62572100 & -1.73264400 & $\mathrm{~N}$ & -2.94653300 & -3.85475400 & -0.36754700 \\
\hline & & & & $\mathrm{Pd}$ & -0.47768600 & 0.00006200 & 0.40666500 \\
\hline 49a & & & & $\mathrm{C}$ & -0.12069500 & -5.09260800 & 1.07623100 \\
\hline
\end{tabular}




\begin{tabular}{|c|c|c|}
\hline-0.75342900 & -5.41129600 & 0.24497800 \\
\hline 0.70012500 & -5.81266000 & 1.17102700 \\
\hline-0.71381200 & -5.15448800 & 1.99255700 \\
\hline 1.34068400 & -3.27881300 & 2.05600600 \\
\hline 1.77566200 & -2.28289500 & 1.92343800 \\
\hline 0.77477300 & -3.28582100 & 2.99301400 \\
\hline 2.16578400 & -3.99068100 & 2.17005100 \\
\hline 1.33810000 & -3.70362300 & -0.42972000 \\
\hline 1.81829100 & -2.73821000 & -0.60994600 \\
\hline 2.13094000 & -4.45173400 & -0.31899000 \\
\hline 0.75401600 & -3.97308400 & -1.31462000 \\
\hline 1.34169400 & 3.27894700 & 2.05509000 \\
\hline 0.77612000 & 3.28627400 & 2.99229900 \\
\hline 1.77641700 & 2.28291200 & 1.92256700 \\
\hline 2.16699000 & 3.99065600 & 2.16870800 \\
\hline-0.11980900 & 5.09275700 & 1.07546300 \\
\hline 0.70109600 & 5.81274700 & 1.16997300 \\
\hline-0.75267700 & 5.41137800 & 0.24428300 \\
\hline-0.71273900 & 5.15484300 & 1.99189500 \\
\hline 1.33843600 & 3.70344500 & -0.43067000 \\
\hline 2.13144700 & 4.45141700 & -0.32021100 \\
\hline 1.81839200 & 2.73793000 & -0.61095800 \\
\hline 0.75415800 & 3.97296000 & -1.31542400 \\
\hline 0.96523800 & -0.00008200 & -0.96049200 \\
\hline 0.63895600 & -0.00024000 & -2.12380400 \\
\hline 2.32318400 & -0.00006300 & -0.41148100 \\
\hline 3.39169100 & -0.00031700 & -1.25131200 \\
\hline 3.17075400 & -0.00053300 & -2.31752900 \\
\hline 2.42146800 & 0.00017600 & 0.66723900 \\
\hline 4.80265700 & -0.00033900 & -0.90097200 \\
\hline 5.26922800 & -0.00024600 & 0.43079400 \\
\hline 5.74784600 & -0.00042300 & -1.94683200 \\
\hline 6.63175700 & -0.00019500 & 0.69989500 \\
\hline 4.56387100 & -0.00025600 & 1.25604600 \\
\hline 7.11308400 & -0.00037800 & -1.67475700 \\
\hline 5.40024500 & -0.00053000 & -2.97642900 \\
\hline 7.55731700 & -0.00024700 & -0.35123400 \\
\hline 6.97973300 & -0.00012000 & 1.72812900 \\
\hline 7.82842100 & -0.00044500 & -2.49096800 \\
\hline 8.62132800 & -0.00018500 & -0.13539800 \\
\hline
\end{tabular}

49

C

C

C

C

C

$\mathrm{H}$

H

$\mathrm{H}$

H

N

$\mathrm{P}$

C

C

$\mathrm{H}$

$\mathrm{H}$

C

$\mathrm{H}$

H

C

H

H

C

$\mathrm{H}$

H

C

H

H

P

C

C

C

C

C

H

C

H

H

H

N

Pd

\begin{tabular}{|c|c|c|}
\hline 2.13985900 & -2.91973200 & -0.31935000 \\
\hline 2.39700400 & -2.50633000 & -1.63354700 \\
\hline 3.56358600 & -2.96042400 & -2.25250900 \\
\hline 4.42713700 & -3.78983900 & -1.54035600 \\
\hline 4.09009700 & -4.13142600 & -0.22842100 \\
\hline 1.71727300 & -1.83717800 & -2.15048400 \\
\hline 3.79390800 & -2.66307100 & -3.27116500 \\
\hline 5.34567700 & -4.16141100 & -1.98243100 \\
\hline 4.74318200 & -4.76792300 & 0.36435800 \\
\hline 2.97149800 & -3.71175300 & 0.37419400 \\
\hline 0.59529000 & -2.38017900 & 0.55565100 \\
\hline-0.67951900 & -3.75422600 & 0.22032100 \\
\hline 1.04254300 & -2.51576600 & 2.37598800 \\
\hline 0.58975200 & -3.42971200 & 2.76920600 \\
\hline 2.12438100 & -2.67513000 & 2.41088100 \\
\hline 0.61105000 & -1.30403500 & 3.22261500 \\
\hline 0.76397500 & -1.54679900 & 4.28100300 \\
\hline-0.46704600 & -1.14014400 & 3.10279600 \\
\hline 1.36675400 & -0.00749000 & 2.88150200 \\
\hline 2.36161600 & -0.01431200 & 3.34428200 \\
\hline 1.62304400 & -0.00755200 & 1.79545400 \\
\hline 0.62738400 & 1.29772000 & 3.22596500 \\
\hline 0.78863800 & 1.53927900 & 4.28337000 \\
\hline-0.45311500 & 1.14613700 & 3.11206300 \\
\hline 1.06892000 & 2.50383500 & 2.37639300 \\
\hline 0.63365800 & 3.42445100 & 2.77372400 \\
\hline 2.15321800 & 2.64710300 & 2.40293600 \\
\hline 0.60533600 & 2.37571200 & 0.55950100 \\
\hline-0.67401700 & 3.74958600 & 0.24093900 \\
\hline 2.14158100 & 2.92027800 & -0.32643000 \\
\hline 2.39099500 & 2.50801800 & -1.64240700 \\
\hline 4.08811000 & 4.13873200 & -0.24865700 \\
\hline 3.55172100 & 2.96625400 & -2.26932300 \\
\hline 1.71003800 & 1.83659800 & -2.15471500 \\
\hline 4.41726000 & 3.79852700 & -1.56298700 \\
\hline 4.74311000 & 4.77737100 & 0.33969600 \\
\hline 3.77603100 & 2.66987700 & -3.28960500 \\
\hline 5.33142400 & 4.17338200 & -2.01132100 \\
\hline 2.97518900 & 3.71512100 & 0.36163900 \\
\hline 0.27474500 & -0.00145600 & 0.21391500 \\
\hline
\end{tabular}




\begin{tabular}{|c|c|c|c|c|c|c|c|}
\hline $\mathrm{C}$ & -0.10086000 & 5.13391000 & 0.60172700 & & & & \\
\hline $\mathrm{H}$ & 0.77802100 & 5.38446800 & 0.00361100 & 49c & & & \\
\hline $\mathrm{H}$ & -0.86801900 & 5.89195700 & 0.40654900 & $\mathrm{C}$ & -2.95539300 & -2.00403900 & 0.60148900 \\
\hline $\mathrm{H}$ & 0.17648900 & 5.21522100 & 1.65616000 & $\mathrm{C}$ & -4.35285300 & -2.08434700 & 0.56259100 \\
\hline $\mathrm{C}$ & -1.91481200 & 3.45094000 & 1.10413700 & $\mathrm{C}$ & -5.04836000 & -2.52515700 & 1.69036200 \\
\hline $\mathrm{H}$ & -2.36293900 & 2.48471000 & 0.85537200 & $\mathrm{C}$ & -4.33631500 & -2.87834700 & 2.83315800 \\
\hline $\mathrm{H}$ & -1.68857000 & 3.46364600 & 2.17503200 & $\mathrm{C}$ & -2.94713300 & -2.77021300 & 2.79804300 \\
\hline $\mathrm{H}$ & -2.67182600 & 4.22208700 & 0.92350400 & $\mathrm{H}$ & -4.90180300 & -1.80927400 & -0.32897600 \\
\hline $\mathrm{C}$ & -1.05402100 & 3.72490500 & -1.25162400 & $\mathrm{H}$ & -6.13194500 & -2.59332200 & 1.67003900 \\
\hline $\mathrm{H}$ & -1.50099300 & 2.77113100 & -1.54423800 & $\mathrm{H}$ & -4.83626800 & -3.22693500 & 3.73017400 \\
\hline $\mathrm{H}$ & -1.79200800 & 4.51100500 & -1.44653400 & $\mathrm{H}$ & -2.34604700 & -3.03227300 & 3.66512000 \\
\hline $\mathrm{H}$ & -0.19165000 & 3.91854700 & -1.89645800 & $\mathrm{~N}$ & -2.27622200 & -2.34055500 & 1.72153700 \\
\hline $\mathrm{C}$ & -1.92780100 & -3.46227600 & 1.07512100 & $\mathrm{P}$ & -1.86675700 & -1.41863500 & -0.80208600 \\
\hline $\mathrm{H}$ & -1.71097200 & -3.48236000 & 2.14783200 & $\mathrm{C}$ & -1.40451800 & -3.06172600 & -1.71167300 \\
\hline $\mathrm{H}$ & -2.37488900 & -2.49477700 & 0.82943000 & $\mathrm{C}$ & -3.14771200 & -0.61219900 & -1.91212300 \\
\hline $\mathrm{H}$ & -2.68252600 & -4.23278600 & 0.88259800 & $\mathrm{H}$ & -3.93726100 & -1.35166100 & -2.07216200 \\
\hline $\mathrm{C}$ & -0.10609800 & -5.13898400 & 0.57911400 & $\mathrm{H}$ & -3.58830100 & 0.19435600 & -1.31425100 \\
\hline $\mathrm{H}$ & -0.87097400 & -5.89761200 & 0.37734600 & $\mathrm{C}$ & -2.74750300 & -0.04950700 & -3.29671600 \\
\hline $\mathrm{H}$ & 0.77616400 & -5.38600500 & -0.01552400 & $\mathrm{H}$ & -3.68983100 & 0.08762000 & -3.84089700 \\
\hline $\mathrm{H}$ & 0.16630300 & -5.22348200 & 1.63458400 & $\mathrm{H}$ & -2.18621600 & -0.79165900 & -3.87499500 \\
\hline $\mathrm{C}$ & -1.04823300 & -3.72332600 & -1.27496800 & $\mathrm{C}$ & -2.00883800 & 1.29573300 & -3.28926600 \\
\hline $\mathrm{H}$ & -1.78259500 & -4.51047500 & -1.47914900 & $\mathrm{H}$ & -2.14883100 & 1.76913900 & -4.26774500 \\
\hline $\mathrm{H}$ & -1.49563700 & -2.76940800 & -1.56649900 & $\mathrm{H}$ & -2.49930600 & 1.96173300 & -2.57346100 \\
\hline $\mathrm{H}$ & -0.18060600 & -3.91159100 & -1.91429700 & $\mathrm{C}$ & -0.49245000 & 1.22833700 & -2.99397100 \\
\hline $\mathrm{C}$ & -0.59569400 & 0.00016000 & -1.58812600 & $\mathrm{H}$ & 0.05820900 & 1.23635900 & -3.94163400 \\
\hline $\mathrm{O}$ & 0.15234400 & -0.00075700 & -2.53779700 & $\mathrm{H}$ & -0.22777800 & 0.28223100 & -2.50966300 \\
\hline $\mathrm{C}$ & -2.05848100 & 0.00203500 & -1.68537200 & $\mathrm{C}$ & 0.02236100 & 2.39261700 & -2.12867300 \\
\hline $\mathrm{C}$ & -2.89720600 & 0.00275800 & -0.62375500 & $\mathrm{H}$ & 1.09114700 & 2.54973500 & -2.30830000 \\
\hline $\mathrm{H}$ & -2.43677600 & 0.00180600 & 0.36489100 & $\mathrm{H}$ & -0.49577300 & 3.32261800 & -2.37905400 \\
\hline $\mathrm{H}$ & -2.41661300 & 0.00295900 & -2.71204400 & $\mathrm{P}$ & -0.10670800 & 2.13729300 & -0.29076000 \\
\hline $\mathrm{C}$ & -4.35302000 & 0.00476200 & -0.63603500 & $\mathrm{C}$ & 0.84407100 & 3.63145500 & 0.45187100 \\
\hline $\mathrm{C}$ & -5.11417100 & 0.00637300 & -1.82414400 & $\mathrm{C}$ & -1.86950400 & 2.52128500 & 0.17110100 \\
\hline $\mathrm{C}$ & -5.03206000 & 0.00522800 & 0.59910100 & $\mathrm{C}$ & -2.43664100 & 1.94796000 & 1.31775800 \\
\hline $\mathrm{C}$ & -6.50225800 & 0.00841900 & -1.77094000 & $\mathrm{C}$ & -3.78525200 & 3.72317300 & -0.25779000 \\
\hline $\mathrm{H}$ & -4.61900900 & 0.00605000 & -2.78997100 & $\mathrm{C}$ & -3.73817400 & 2.30574700 & 1.67297500 \\
\hline $\mathrm{C}$ & -6.42327500 & 0.00728700 & 0.65008100 & $\mathrm{H}$ & -1.86576900 & 1.24353100 & 1.91440800 \\
\hline $\mathrm{H}$ & -4.45710400 & 0.00397700 & 1.52175800 & $\mathrm{C}$ & -4.42979600 & 3.21127900 & 0.87023400 \\
\hline $\mathrm{C}$ & -7.16094300 & 0.00889100 & -0.53526400 & $\mathrm{H}$ & -4.29086400 & 4.42967400 & -0.91225900 \\
\hline $\mathrm{H}$ & -7.07708300 & 0.00967200 & -2.69168800 & $\mathrm{H}$ & -4.20070200 & 1.88541800 & 2.56105200 \\
\hline $\mathrm{H}$ & -6.93132100 & 0.00764600 & 1.60921800 & $\mathrm{H}$ & -5.44310800 & 3.51818900 & 1.10750600 \\
\hline $\mathrm{H}$ & -8.24601900 & 0.01051400 & -0.50009200 & $\mathrm{~N}$ & -2.53426700 & 3.39258700 & -0.60384100 \\
\hline
\end{tabular}




\begin{tabular}{|c|c|c|c|c|c|c|c|}
\hline $\mathrm{Pd}$ & 0.22677400 & -0.11524400 & 0.35788400 & $\mathrm{H}$ & 2.89523900 & -0.38705600 & -0.63605200 \\
\hline $\mathrm{H}$ & 4.45965400 & 0.12888200 & 1.95816700 & $\mathrm{C}$ & 1.20844100 & -2.40504000 & 2.62473900 \\
\hline $\mathrm{C}$ & 0.34887600 & 4.94824600 & -0.18371500 & $\mathrm{H}$ & 2.25577400 & -2.37095700 & 2.32328700 \\
\hline $\mathrm{H}$ & -0.72877300 & 5.08867000 & -0.08155300 & $\mathrm{H}$ & 0.97485300 & -3.41872500 & 2.96733000 \\
\hline $\mathrm{H}$ & 0.84698400 & 5.78188200 & 0.32438700 & $\mathrm{H}$ & 1.04922800 & -1.68902700 & 3.43696400 \\
\hline $\mathrm{H}$ & 0.60257200 & 5.01715000 & -1.24501900 & $\mathrm{O}$ & 0.40908700 & -2.09381500 & 1.47316700 \\
\hline $\mathrm{C}$ & 2.35288800 & 3.48775400 & 0.17025400 & $\mathrm{H}$ & -0.55955100 & -2.23987900 & 1.68072500 \\
\hline $\mathrm{H}$ & 2.79513600 & 2.62672000 & 0.67068400 & & & & \\
\hline $\mathrm{H}$ & 2.57672300 & 3.42263700 & -0.89917900 & 49-O & & & \\
\hline $\mathrm{H}$ & 2.86114800 & 4.38181300 & 0.54854400 & $\mathrm{C}$ & -3.50470700 & -0.14044500 & 1.21389700 \\
\hline $\mathrm{C}$ & 0.59722600 & 3.66683000 & 1.97335100 & $\mathrm{C}$ & -2.68763400 & 0.16039400 & 2.31144300 \\
\hline $\mathrm{H}$ & 0.90700300 & 2.74099000 & 2.46334200 & $\mathrm{C}$ & -3.25353700 & 0.79808500 & 3.41696600 \\
\hline $\mathrm{H}$ & 1.18323700 & 4.48606000 & 2.40519200 & $\mathrm{C}$ & -4.60854800 & 1.12023400 & 3.38460800 \\
\hline $\mathrm{H}$ & -0.45281200 & 3.85957500 & 2.21044900 & $\mathrm{C}$ & -5.34175000 & 0.78875700 & 2.24256400 \\
\hline $\mathrm{C}$ & -0.22470300 & -2.75495900 & -2.65742100 & $\mathrm{H}$ & -1.63353400 & -0.09790900 & 2.29375500 \\
\hline $\mathrm{H}$ & -0.47330700 & -2.01395700 & -3.42118700 & $\mathrm{H}$ & -2.64625600 & 1.04092000 & 4.28389600 \\
\hline $\mathrm{H}$ & 0.64850200 & -2.39567500 & -2.10368500 & $\mathrm{H}$ & -5.09088900 & 1.61918900 & 4.21856800 \\
\hline $\mathrm{H}$ & 0.06711700 & -3.67460700 & -3.17770500 & $\mathrm{H}$ & -6.40072300 & 1.02821500 & 2.17595500 \\
\hline $\mathrm{C}$ & -2.59329500 & -3.63079700 & -2.50688300 & $\mathrm{~N}$ & -4.81028200 & 0.17240300 & 1.18024800 \\
\hline $\mathrm{H}$ & -2.30128200 & -4.59421200 & -2.94034200 & $\mathrm{P}$ & -2.80675900 & -1.02634100 & -0.26320900 \\
\hline $\mathrm{H}$ & -3.46187600 & -3.81591700 & -1.86611500 & $\mathrm{C}$ & -3.32195000 & -2.84865100 & 0.00327900 \\
\hline $\mathrm{H}$ & -2.90387800 & -2.98713700 & -3.33406500 & $\mathrm{C}$ & -3.84985300 & -0.45377200 & -1.69011200 \\
\hline $\mathrm{C}$ & -0.94466800 & -4.11882000 & -0.68838600 & $\mathrm{H}$ & -3.88761800 & -1.29335600 & -2.39112700 \\
\hline $\mathrm{H}$ & -0.59184300 & -5.00016400 & -1.23624300 & $\mathrm{H}$ & -4.86346500 & -0.28209900 & -1.31542100 \\
\hline $\mathrm{H}$ & -0.12586200 & -3.75761300 & -0.06324400 & $\mathrm{C}$ & -3.29135900 & 0.79010600 & -2.39547300 \\
\hline $\mathrm{H}$ & -1.76154400 & -4.44336700 & -0.03826100 & $\mathrm{H}$ & -3.76709900 & 0.86969800 & -3.37969700 \\
\hline $\mathrm{C}$ & 1.97500900 & 0.29814700 & 1.23398300 & $\mathrm{H}$ & -2.22779500 & 0.62479400 & -2.59583200 \\
\hline $\mathrm{O}$ & 2.03334700 & 0.65680700 & 2.38703700 & $\mathrm{C}$ & -3.49960700 & 2.11158200 & -1.61873200 \\
\hline $\mathrm{C}$ & 4.36086400 & -0.16834300 & 0.91480400 & $\mathrm{H}$ & -4.32928600 & 2.65941000 & -2.07860300 \\
\hline $\mathrm{C}$ & 3.10939400 & -0.12093100 & 0.39304900 & $\mathrm{H}$ & -3.82699100 & 1.90577400 & -0.59455900 \\
\hline $\mathrm{C}$ & 5.59619600 & -0.55986300 & 0.25103300 & $\mathrm{C}$ & -2.28077800 & 3.04414700 & -1.57158600 \\
\hline $\mathrm{C}$ & 6.79696800 & -0.49567500 & 0.98545500 & $\mathrm{H}$ & -2.63834200 & 4.05442800 & -1.33786200 \\
\hline $\mathrm{C}$ & 5.64860100 & -0.99923900 & -1.08826800 & $\mathrm{H}$ & -1.82977000 & 3.12140400 & -2.56623000 \\
\hline $\mathrm{C}$ & 8.01031700 & -0.85486800 & 0.40447700 & $\mathrm{C}$ & -1.21489900 & 2.74066600 & -0.49432000 \\
\hline $\mathrm{H}$ & 6.76950700 & -0.15894900 & 2.01834300 & $\mathrm{H}$ & -0.63657000 & 3.65012500 & -0.30068700 \\
\hline $\mathrm{C}$ & 6.85985300 & -1.35834600 & -1.66612600 & $\mathrm{H}$ & -1.71195000 & 2.48676600 & 0.44950800 \\
\hline $\mathrm{H}$ & 4.73840600 & -1.06149400 & -1.67665900 & $\mathrm{P}$ & 0.13533500 & 1.45463900 & -0.68563600 \\
\hline $\mathrm{C}$ & 8.04412100 & -1.28695600 & -0.92241800 & $\mathrm{C}$ & 1.11129200 & 1.89837100 & -2.27671200 \\
\hline $\mathrm{H}$ & 8.92634000 & -0.79858200 & 0.98402900 & $\mathrm{C}$ & 1.18806300 & 2.01617500 & 0.75243900 \\
\hline $\mathrm{H}$ & 6.88744500 & -1.69580000 & -2.69762100 & $\mathrm{C}$ & 1.50961900 & 1.15269500 & 1.80613900 \\
\hline $\mathrm{H}$ & 8.98819500 & -1.56869000 & -1.37866800 & $\mathrm{C}$ & 2.28164400 & 3.76995000 & 1.77030000 \\
\hline
\end{tabular}




\begin{tabular}{|c|c|c|c|c|c|c|c|}
\hline $\mathrm{C}$ & 2.25136500 & 1.64867100 & 2.88179400 & $\mathrm{C}$ & 7.14328300 & -2.60383500 & 0.31393300 \\
\hline $\mathrm{H}$ & 1.18337000 & 0.11849700 & 1.78205400 & $\mathrm{H}$ & 5.23266100 & -3.45879700 & -0.13361800 \\
\hline $\mathrm{C}$ & 2.65068600 & 2.98234700 & 2.86441100 & $\mathrm{C}$ & 7.18892600 & -0.21854800 & 0.74759300 \\
\hline $\mathrm{H}$ & 2.56920800 & 4.81772000 & 1.71949600 & $\mathrm{H}$ & 5.29171400 & 0.78832300 & 0.63617200 \\
\hline $\mathrm{H}$ & 2.50854200 & 1.00265600 & 3.71605900 & $\mathrm{C}$ & 7.85558500 & -1.44050600 & 0.63172200 \\
\hline $\mathrm{H}$ & 3.22700300 & 3.41112800 & 3.67755600 & $\mathrm{H}$ & 7.66499500 & -3.55122100 & 0.22367500 \\
\hline $\mathrm{N}$ & 1.56246200 & 3.30650800 & 0.74278000 & $\mathrm{H}$ & 7.74111300 & 0.68300100 & 0.99282200 \\
\hline $\mathrm{Pd}$ & -0.34033500 & -0.83734800 & -0.46693600 & $\mathrm{H}$ & 8.92891200 & -1.48974100 & 0.78749900 \\
\hline $\mathrm{C}$ & 1.17825800 & 3.42338100 & -2.49975300 & & & & \\
\hline $\mathrm{H}$ & 1.64089400 & 3.93510000 & -1.65445300 & 49-CO & & & \\
\hline $\mathrm{H}$ & 1.78130900 & 3.61496200 & -3.39481000 & $\mathrm{C}$ & -2.93557100 & 1.51665000 & 0.77796500 \\
\hline $\mathrm{H}$ & 0.19423000 & 3.86441500 & -2.67263900 & $\mathrm{C}$ & -3.73183600 & 2.28974600 & 1.63280700 \\
\hline $\mathrm{C}$ & 0.42612600 & 1.21684500 & -3.47908100 & $\mathrm{C}$ & -4.80811500 & 1.68685000 & 2.28697700 \\
\hline $\mathrm{H}$ & 0.34973400 & 0.13285600 & -3.34369500 & $\mathrm{C}$ & -5.06576300 & 0.33718100 & 2.06114300 \\
\hline $\mathrm{H}$ & -0.57365600 & 1.61198900 & -3.67240700 & $\mathrm{C}$ & -4.22629100 & -0.35580600 & 1.18766800 \\
\hline $\mathrm{H}$ & 1.02629500 & 1.39993100 & -4.37758100 & $\mathrm{H}$ & -3.52704100 & 3.34216200 & 1.78897600 \\
\hline $\mathrm{C}$ & 2.54110400 & 1.34451700 & -2.15490500 & $\mathrm{H}$ & -5.43450200 & 2.26699900 & 2.95790700 \\
\hline $\mathrm{H}$ & 2.54597900 & 0.25890300 & -2.03540700 & $\mathrm{H}$ & -5.89063700 & -0.17153000 & 2.54852800 \\
\hline $\mathrm{H}$ & 3.08819400 & 1.57619000 & -3.07533200 & $\mathrm{H}$ & -4.38973000 & -1.41192400 & 0.98401500 \\
\hline $\mathrm{H}$ & 3.08940000 & 1.80150000 & -1.32596800 & $\mathrm{~N}$ & -3.18169500 & 0.20701100 & 0.56660800 \\
\hline $\mathrm{C}$ & -2.77973100 & -3.67910400 & -1.17799300 & $\mathrm{P}$ & -1.44704900 & 2.15068800 & -0.14717000 \\
\hline $\mathrm{H}$ & -3.21396200 & -3.37561200 & -2.13569300 & $\mathrm{C}$ & -2.25149800 & 2.58997000 & -1.84830600 \\
\hline $\mathrm{H}$ & -1.69141300 & -3.62226400 & -1.25416800 & $\mathrm{C}$ & -1.11084500 & 3.76674100 & 0.74045900 \\
\hline $\mathrm{H}$ & -3.04928700 & -4.73015700 & -1.02396300 & $\mathrm{H}$ & -1.94417500 & 4.45928800 & 0.58163800 \\
\hline $\mathrm{C}$ & -4.85417900 & -2.98895600 & 0.07734400 & $\mathrm{H}$ & -1.13588700 & 3.48624100 & 1.80065500 \\
\hline $\mathrm{H}$ & -5.10227600 & -4.04072500 & 0.26179200 & $\mathrm{C}$ & 0.22291400 & 4.49985800 & 0.47208400 \\
\hline $\mathrm{H}$ & -5.28951800 & -2.39312200 & 0.88253100 & $\mathrm{H}$ & 0.29299800 & 5.28751200 & 1.23047600 \\
\hline $\mathrm{H}$ & -5.34015400 & -2.69888100 & -0.85839300 & $\mathrm{H}$ & 0.17795400 & 5.02401500 & -0.48933500 \\
\hline $\mathrm{C}$ & -2.68534200 & -3.34654900 & 1.31547000 & $\mathrm{C}$ & 1.48963100 & 3.62193000 & 0.51797800 \\
\hline $\mathrm{H}$ & -2.93782400 & -4.40329600 & 1.45765300 & $\mathrm{H}$ & 2.31212100 & 4.17876800 & 0.98098800 \\
\hline $\mathrm{H}$ & -1.59391900 & -3.26952800 & 1.29434100 & $\mathrm{H}$ & 1.32056600 & 2.75215200 & 1.16846300 \\
\hline $\mathrm{H}$ & -3.05940600 & -2.80047900 & 2.18656700 & $\mathrm{C}$ & 1.93868900 & 3.15799000 & -0.87540400 \\
\hline $\mathrm{C}$ & 1.34845800 & -1.96491500 & -0.36614600 & $\mathrm{H}$ & 2.32835300 & 4.02675700 & -1.42076000 \\
\hline $\mathrm{O}$ & 0.49511500 & -2.85508500 & -0.38597500 & $\mathrm{H}$ & 1.07495600 & 2.81354800 & -1.44810100 \\
\hline $\mathrm{C}$ & 2.76204700 & -2.18906000 & -0.24613400 & $\mathrm{C}$ & 3.02164500 & 2.07170600 & -0.85646500 \\
\hline $\mathrm{C}$ & 3.64938400 & -1.19543300 & 0.02318300 & $\mathrm{H}$ & 3.61816000 & 2.10118900 & -1.77341000 \\
\hline $\mathrm{H}$ & 3.25331400 & -0.18888700 & 0.11941700 & $\mathrm{H}$ & 3.70545100 & 2.22582300 & -0.01771900 \\
\hline $\mathrm{H}$ & 3.06670900 & -3.23005500 & -0.32512100 & $\mathrm{P}$ & 2.43163100 & 0.30715100 & -0.66157700 \\
\hline $\mathrm{C}$ & 5.08027400 & -1.32233700 & 0.22537600 & $\mathrm{C}$ & 2.44647300 & -0.42693900 & -2.42147300 \\
\hline $\mathrm{C}$ & 5.77046400 & -2.54927000 & 0.11335200 & $\mathrm{C}$ & 3.85006300 & -0.40502300 & 0.31664900 \\
\hline $\mathrm{C}$ & 5.81374600 & -0.16053200 & 0.54551500 & $\mathrm{C}$ & 4.41392100 & -1.67153500 & 0.12754500 \\
\hline
\end{tabular}




$\begin{array}{rrrr}5.21150600 & 0.00479300 & 2.12826400 & \mathrm{C} \\ 5.43176700 & -2.08528500 & 0.98731000 & \mathrm{H} \\ 4.07513800 & -2.33101800 & -0.66088700 & \mathrm{C} \\ 5.84077600 & -1.23539300 & 2.01146900 & \mathrm{H} \\ 5.49580600 & 0.70061000 & 2.91413900 & \mathrm{C} \\ 5.89208200 & -3.06013200 & 0.85796000 & \mathrm{H} \\ 6.62435000 & -1.52109500 & 2.70506000 & \mathrm{H} \\ 4.24338600 & 0.41363700 & 1.30247400 & \mathrm{H} \\ 0.35325800 & 0.18342500 & 0.65184200 & \mathrm{H} \\ -1.36472200 & -1.68194500 & 0.02601800 & \mathrm{C} \\ 3.86291100 & -0.45813000 & -3.03100200 & \mathrm{O}\end{array}$

C

H

C

H

C

H

H

H

H

C

O

$\begin{array}{rrr}-3.84874200 & -4.39455100 & -1.48784600 \\ -3.20712800 & -2.36250900 & -1.16932100 \\ -2.42617300 & -6.10504900 & -0.53151200 \\ -0.69453900 & -5.42664000 & 0.53028200 \\ -3.56630900 & -5.74250200 & -1.25865400 \\ -4.73126300 & -4.11068300 & -2.05273700 \\ -2.20937500 & -7.15325500 & -0.35088500 \\ -4.23063000 & -6.51058000 & -1.64268600 \\ 0.61387700 & -3.77396800 & 1.14314200 \\ -0.66356900 & 0.18694400 & 2.36637500 \\ -1.10371400 & 0.28725500 & 3.41350600\end{array}$

\section{TS41}

imaginary frequency $=-32.16 \mathrm{~cm}^{-1}$

C

$1.64729700 \quad 2.77925800$

$-0.46306800$

$2.98288200 \quad 3.16856400$

$-0.67117900$

\begin{tabular}{ll}
$0.54733000 \quad-2.88599700$ \\
\hline 1.48173400 & -3.40897100
\end{tabular}

$0.93617200 \quad 3.35274700$

0.60987600

$1.95153400 \quad 1.48173400 \quad-3.40897100$

$3.58482500 \quad 4.11220100$

0.15760700

$\begin{array}{lll}3.53758000 & 2.73992600 & -1.49924900\end{array}$

$\begin{array}{lll}1.83380700 & -1.84194900 & -2.40388900\end{array}$

1.54210900

4.29066700

1.44034800

$-0.08793800$

3.04620000

0.80367300

$1.82024700 \quad-2.23954800 \quad-3.42491900$

2.86745100

4.67561200

1.21519500

$\begin{array}{lll}4.61218800 & 4.41238300 & -0.02394600\end{array}$

$\begin{array}{lll}-1.25325000 & 3.35791000 & -2.73438700\end{array}$

0.98374300

4.72191300

2.26563300

3.33736500

5.41012600

1.86194500

1.02341200

$1.82586300-1.38879100$

$\begin{array}{lll}1.68855900 & 1.28308300 & -2.05375200\end{array}$

$-3.51129400$

$3.45675400 \quad-1.65804600$

$-0.34992800$

$1.91358500 \quad-1.70213800$

$-0.95581600$

$0.84683000 \quad-2.38656400$

$-4.28220300$

$2.95080100 \quad-1.07219000$

$-1.42367500$

$0.27398200-3.29315900$

$-2.63483300$

$1.26940500 \quad-2.54421700$

$-1.01823200$

2.65377000

$-1.27678600$

$-3.07627100$

$1.49500600 \quad-3.52184000$

1.35761400

$-2.21324300$

1.78158000

$0.63010800 \quad-2.71285200$

0.62653400

$-3.00489200$

1.60024400

2.25210200

$-2.71224900$

2.16685000

$\begin{array}{lll}0.78724100 & -1.20024500 & 2.80592700\end{array}$

$\begin{array}{lll}1.58090200 & -0.87155300 \quad 3.48531000\end{array}$

$\begin{array}{lll}0.44541100 & -0.29282100 & 2.28976500\end{array}$

$\begin{array}{lll}-2.41395800 & -0.55042000 & 0.42566800\end{array}$

$\begin{array}{lll}1.66394100 & -1.47918000 & 0.10287200\end{array}$

$1.93223900 \quad-2.95938200 \quad-1.07719900$

$\begin{array}{lll}-3.67278400 & -1.80471200 & -0.31077300\end{array}$ 


\begin{tabular}{|c|c|c|c|c|c|c|c|}
\hline $\mathrm{C}$ & 0.68342300 & -3.86144300 & -1.02709200 & $\mathrm{H}$ & -5.32354400 & 4.28941000 & -0.32574500 \\
\hline $\mathrm{H}$ & 0.78742400 & -4.65022200 & -1.78028500 & $\mathrm{~N}$ & 3.82373400 & -0.20734000 & -0.91535300 \\
\hline $\mathrm{H}$ & 0.55364700 & -4.35700700 & -0.06035100 & $\mathrm{~N}$ & -3.37873700 & 1.59278800 & -0.91855300 \\
\hline $\mathrm{H}$ & -0.22658400 & -3.30066000 & -1.26108600 & $\mathrm{Pd}$ & -0.18927800 & -0.00108900 & -0.55254400 \\
\hline $\mathrm{C}$ & 3.17839300 & -3.77308700 & -0.68021800 & $\mathrm{C}$ & -0.37771500 & -1.77374700 & 3.62979700 \\
\hline $\mathrm{H}$ & 3.27980100 & -4.62787800 & -1.35866500 & $\mathrm{H}$ & -0.63693900 & -1.06651900 & 4.42779200 \\
\hline $\mathrm{H}$ & 4.09493100 & -3.18266400 & -0.75546900 & $\mathrm{H}$ & -0.04488900 & -2.69096700 & 4.13191600 \\
\hline $\mathrm{H}$ & 3.10700300 & -4.17235800 & 0.33675200 & $\mathrm{C}$ & -1.63891600 & -2.09599600 & 2.80949300 \\
\hline $\mathrm{C}$ & 2.08538800 & -2.41839700 & -2.51367300 & $\mathrm{H}$ & -2.32252100 & -2.66659700 & 3.44673400 \\
\hline $\mathrm{H}$ & 2.93654000 & -1.74214000 & -2.60631700 & $\mathrm{H}$ & -1.37615300 & -2.77454400 & 1.99370400 \\
\hline $\mathrm{H}$ & 2.23316500 & -3.26184900 & -3.19791500 & $\mathrm{C}$ & -2.40359500 & -0.85355600 & 2.27455600 \\
\hline $\mathrm{H}$ & 1.18434000 & -1.88379400 & -2.83413300 & $\mathrm{H}$ & -3.44522400 & -0.89669100 & 2.60837400 \\
\hline $\mathrm{C}$ & -3.26481500 & -3.23949700 & 0.07530500 & $\mathrm{H}$ & -1.98071600 & 0.05871000 & 2.71129100 \\
\hline $\mathrm{H}$ & -2.22853000 & -3.46574400 & -0.19101400 & & & & \\
\hline $\mathrm{H}$ & -3.40403400 & -3.43949700 & 1.14063800 & 50 & & & \\
\hline $\mathrm{H}$ & -3.90195000 & -3.94269800 & -0.47272700 & $\mathrm{C}$ & 1.78825100 & 2.60244400 & -0.39153900 \\
\hline $\mathrm{C}$ & -5.09946100 & -1.53179000 & 0.20125700 & $\mathrm{C}$ & 3.06053100 & 3.00815600 & -0.82915000 \\
\hline $\mathrm{H}$ & -5.47941500 & -0.56183700 & -0.12812900 & $\mathrm{C}$ & 1.34706700 & 3.02887600 & 0.87612000 \\
\hline $\mathrm{H}$ & -5.77068300 & -2.30094300 & -0.19764300 & $\mathrm{C}$ & 3.86322900 & 3.82105300 & -0.03073700 \\
\hline $\mathrm{H}$ & -5.17239400 & -1.57825800 & 1.29279100 & $\mathrm{H}$ & 3.41132700 & 2.69255600 & -1.80693200 \\
\hline $\mathrm{C}$ & -3.63402700 & -1.66229700 & -1.84363700 & $\mathrm{C}$ & 2.15312800 & 3.83437100 & 1.67585000 \\
\hline $\mathrm{H}$ & -4.35911900 & -2.35627200 & -2.28414000 & $\mathrm{H}$ & 0.37304300 & 2.71598600 & 1.24356700 \\
\hline $\mathrm{H}$ & -3.89050800 & -0.65186900 & -2.16960100 & $\mathrm{C}$ & 3.41375800 & 4.23618600 & 1.22394200 \\
\hline $\mathrm{H}$ & -2.64764200 & -1.90610600 & -2.24738000 & $\mathrm{H}$ & 4.83798000 & 4.13547000 & -0.39125500 \\
\hline $\mathrm{C}$ & 3.34974000 & -0.71433600 & 0.23806100 & $\mathrm{H}$ & 1.79870000 & 4.15184700 & 2.65190700 \\
\hline $\mathrm{C}$ & 4.08831000 & -0.67313700 & 1.42821100 & $\mathrm{H}$ & 4.03820100 & 4.87069100 & 1.84529300 \\
\hline $\mathrm{C}$ & 5.05976400 & 0.30516700 & -0.92397500 & $\mathrm{C}$ & 0.93829700 & 1.79253800 & -1.28968700 \\
\hline $\mathrm{C}$ & 5.36618600 & -0.11205100 & 1.41001200 & $\mathrm{H}$ & 1.42380200 & 1.46387400 & -2.20525100 \\
\hline $\mathrm{H}$ & 3.68859200 & -1.07272000 & 2.35213900 & $\mathrm{C}$ & -0.47853600 & 2.13054400 & -1.36944800 \\
\hline $\mathrm{C}$ & 5.87242100 & 0.37623800 & 0.20855600 & $\mathrm{C}$ & -1.24223200 & 1.70955200 & -2.41511800 \\
\hline $\mathrm{H}$ & 5.41431000 & 0.68077100 & -1.88178600 & $\mathrm{O}$ & -1.80348600 & 1.41371700 & -3.38045200 \\
\hline $\mathrm{H}$ & 5.95741600 & -0.06976300 & 2.31987800 & $\mathrm{H}$ & -0.95521200 & 2.82498300 & -0.68729000 \\
\hline $\mathrm{H}$ & 6.86677400 & 0.80538200 & 0.14522700 & $\mathrm{C}$ & 1.17450600 & -2.42990900 & 1.48749200 \\
\hline $\mathrm{C}$ & -3.36273600 & 1.05270600 & 0.31521000 & $\mathrm{H}$ & 0.41029600 & -3.13639900 & 1.15523800 \\
\hline $\mathrm{C}$ & -4.03026600 & 1.65399600 & 1.39116300 & $\mathrm{H}$ & 2.03043100 & -3.03484600 & 1.80353200 \\
\hline $\mathrm{C}$ & -4.08298700 & 2.71340800 & -1.12273900 & $\mathrm{C}$ & 0.62240400 & -1.57909400 & 2.65506900 \\
\hline $\mathrm{C}$ & -4.73838800 & 2.83762600 & 1.17277000 & $\mathrm{H}$ & 1.42973300 & -1.33092700 & 3.35217300 \\
\hline $\mathrm{H}$ & -4.01544200 & 1.21254100 & 2.38003100 & $\mathrm{H}$ & 0.25393400 & -0.61871900 & 2.27184100 \\
\hline $\mathrm{C}$ & -4.77701800 & 3.37758200 & -0.11004600 & $\mathrm{P}$ & -2.39568000 & -0.59466900 & 0.44299800 \\
\hline $\mathrm{H}$ & -4.08310400 & 3.09951000 & -2.13980800 & $\mathrm{P}$ & 1.58319600 & -1.43868100 & -0.03071100 \\
\hline $\mathrm{H}$ & -5.25978200 & 3.32067000 & 1.99376900 & $\mathrm{C}$ & 1.85970100 & -2.71898300 & -1.42669700 \\
\hline
\end{tabular}




\begin{tabular}{|c|c|c|c|c|c|c|c|}
\hline $\mathrm{C}$ & -3.59464900 & -1.69077600 & -0.59242700 & $\mathrm{H}$ & -5.31452900 & 2.96647000 & 2.53241500 \\
\hline $\mathrm{C}$ & 0.58457400 & -3.57053200 & -1.57370900 & $\mathrm{H}$ & -5.41124000 & 4.25366000 & 0.37288100 \\
\hline $\mathrm{H}$ & 0.69856200 & -4.23336200 & -2.43866900 & $\mathrm{~N}$ & 3.88566600 & -0.19971800 & -0.74097800 \\
\hline $\mathrm{H}$ & 0.39480600 & -4.20796000 & -0.70488800 & $\mathrm{~N}$ & -3.40402200 & 1.71459500 & -0.59514300 \\
\hline $\mathrm{H}$ & -0.29559400 & -2.94523500 & -1.75175900 & $\mathrm{Pd}$ & -0.15693900 & 0.14089200 & -0.46927200 \\
\hline $\mathrm{C}$ & 3.06256400 & -3.62718200 & -1.10933800 & $\mathrm{C}$ & -0.50774800 & -2.27822400 & 3.42683800 \\
\hline $\mathrm{H}$ & 3.17590700 & -4.36652500 & -1.91060400 & $\mathrm{H}$ & -0.74957700 & -1.68485500 & 4.31799500 \\
\hline $\mathrm{H}$ & 3.99613300 & -3.06300000 & -1.04684500 & $\mathrm{H}$ & -0.15061700 & -3.24818400 & 3.79583100 \\
\hline $\mathrm{H}$ & 2.92938800 & -4.18018200 & -0.17402200 & $\mathrm{C}$ & -1.79372700 & -2.50812800 & 2.61574400 \\
\hline $\mathrm{C}$ & 2.09774800 & -1.96474100 & -2.75106300 & $\mathrm{H}$ & -2.47242800 & -3.11142100 & 3.22839600 \\
\hline $\mathrm{H}$ & 2.96878200 & -1.31051400 & -2.69406900 & $\mathrm{H}$ & -1.57585900 & -3.12573400 & 1.74106100 \\
\hline $\mathrm{H}$ & 2.25834100 & -2.69474000 & -3.55288000 & $\mathrm{C}$ & -2.54855200 & -1.21484700 & 2.20808200 \\
\hline $\mathrm{H}$ & 1.22669500 & -1.35794100 & -3.02166300 & $\mathrm{H}$ & -3.61689300 & -1.33760800 & 2.41433300 \\
\hline $\mathrm{C}$ & -3.25140500 & -3.17835500 & -0.38226900 & $\mathrm{H}$ & -2.20809100 & -0.38607700 & 2.83957200 \\
\hline $\mathrm{H}$ & -2.19719900 & -3.39795100 & -0.57184600 & & & & \\
\hline $\mathrm{H}$ & -3.50517000 & -3.52431600 & 0.62308000 & \multirow{2}{*}{\multicolumn{4}{|c|}{$\begin{array}{l}\text { TS42 } \\
\text { imaginary frequency }=-37.28 \mathrm{~cm}^{-1}\end{array}$}} \\
\hline $\mathrm{H}$ & -3.83937600 & -3.77635700 & -1.08779100 & & & & \\
\hline $\mathrm{C}$ & -5.06577000 & -1.46025800 & -0.19888700 & $\mathrm{C}$ & 2.82759200 & -1.77415000 & 0.25921800 \\
\hline $\mathrm{H}$ & -5.40386200 & -0.44847600 & -0.43378900 & $\mathrm{C}$ & 4.07735600 & -2.13354800 & 0.78057400 \\
\hline $\mathrm{H}$ & -5.69704200 & -2.15729000 & -0.76193300 & $\mathrm{C}$ & 4.97113100 & -2.86415200 & -0.00280200 \\
\hline $\mathrm{H}$ & -5.25034200 & -1.64739500 & 0.86389400 & $\mathrm{C}$ & 4.59633300 & -3.23377300 & -1.29180600 \\
\hline $\mathrm{C}$ & -3.39200300 & -1.32512100 & -2.07470500 & $\mathrm{C}$ & 3.33340200 & -2.85314400 & -1.73634800 \\
\hline $\mathrm{H}$ & -4.05919100 & -1.93725200 & -2.69252700 & $\mathrm{H}$ & 4.35505000 & -1.85651800 & 1.78815100 \\
\hline $\mathrm{H}$ & -3.62072300 & -0.27511100 & -2.26864500 & $\mathrm{H}$ & 5.94263800 & -3.14227700 & 0.39441900 \\
\hline $\mathrm{H}$ & -2.36461800 & -1.51764600 & -2.40072400 & $\mathrm{H}$ & 5.25814200 & -3.80060100 & -1.93737800 \\
\hline $\mathrm{C}$ & 3.28822300 & -0.78795200 & 0.30993700 & $\mathrm{H}$ & 2.99361000 & -3.11297300 & -2.73582900 \\
\hline $\mathrm{C}$ & 3.92363200 & -0.91596500 & 1.55243400 & $\mathrm{~N}$ & 2.47127000 & -2.14534800 & -0.98900500 \\
\hline $\mathrm{C}$ & 5.14000600 & 0.24084700 & -0.59519400 & $\mathrm{P}$ & 1.47504400 & -0.90058000 & 1.22326100 \\
\hline $\mathrm{C}$ & 5.22507500 & -0.43342800 & 1.69645800 & $\mathrm{C}$ & 2.30202400 & 0.14880600 & 2.61021800 \\
\hline $\mathrm{H}$ & 3.42832100 & -1.38652200 & 2.39222300 & $\mathrm{C}$ & 0.85333400 & -2.45492400 & 2.07909400 \\
\hline $\mathrm{C}$ & 5.85473100 & 0.14876600 & 0.59990200 & $\mathrm{H}$ & 1.68031500 & -2.82826200 & 2.69038000 \\
\hline $\mathrm{H}$ & 5.59274600 & 0.69321700 & -1.47492900 & $\mathrm{H}$ & 0.70585000 & -3.19104300 & 1.28049000 \\
\hline $\mathrm{H}$ & 5.73841800 & -0.52232500 & 2.64923500 & $\mathrm{C}$ & -0.43597500 & -2.31538400 & 2.90501800 \\
\hline $\mathrm{H}$ & 6.87019500 & 0.52518300 & 0.66339800 & $\mathrm{H}$ & -0.42396500 & -3.07069200 & 3.69936900 \\
\hline $\mathrm{C}$ & -3.36924700 & 1.00218200 & 0.54955700 & $\mathrm{H}$ & -0.44762400 & -1.35205300 & 3.42467100 \\
\hline $\mathrm{C}$ & -4.04960400 & 1.43267800 & 1.69722600 & $\mathrm{C}$ & -1.71191600 & -2.48774300 & 2.05930400 \\
\hline $\mathrm{C}$ & -4.13611500 & 2.83561300 & -0.63720900 & $\mathrm{H}$ & -1.97984100 & -3.55061800 & 2.03053700 \\
\hline $\mathrm{C}$ & -4.78533500 & 2.61841000 & 1.65037700 & $\mathrm{H}$ & -1.50191800 & -2.20414000 & 1.02091900 \\
\hline $\mathrm{H}$ & -4.02570700 & 0.85737800 & 2.61371100 & $\mathrm{C}$ & -2.91105700 & -1.67814000 & 2.56451300 \\
\hline $\mathrm{C}$ & -4.84212400 & 3.33422100 & 0.45802200 & $\mathrm{H}$ & -3.80807200 & -1.98369600 & 2.01669500 \\
\hline $\mathrm{H}$ & -4.15188700 & 3.36125900 & -1.58981100 & $\mathrm{H}$ & -3.10416400 & -1.92105600 & 3.61772000 \\
\hline
\end{tabular}




\begin{tabular}{|c|c|c|c|c|c|c|c|}
\hline $\mathrm{C}$ & -2.76669300 & -0.14757700 & 2.45152600 & $\mathrm{C}$ & -0.61452900 & 0.15202000 & -3.06268400 \\
\hline $\mathrm{H}$ & -1.99194400 & 0.23176700 & 3.12621200 & $\mathrm{O}$ & -1.48234400 & -0.19750900 & -3.75372200 \\
\hline $\mathrm{H}$ & -3.71198800 & 0.30956700 & 2.75136500 & $\mathrm{C}$ & 1.39934500 & 0.89866600 & -1.75261900 \\
\hline $\mathrm{P}$ & -2.35547100 & 0.48819000 & 0.73878600 & $\mathrm{C}$ & 0.09975200 & 1.08916600 & -2.34062100 \\
\hline $\mathrm{C}$ & -2.84917900 & 2.34319400 & 0.79682400 & $\mathrm{C}$ & -0.17830100 & -2.79657900 & -3.24990400 \\
\hline $\mathrm{C}$ & -3.71667800 & -0.33700900 & -0.23199900 & $\mathrm{H}$ & 0.52115300 & -3.48419600 & -3.73789800 \\
\hline $\mathrm{C}$ & -3.41738600 & -1.18508900 & -1.30254400 & $\mathrm{H}$ & -0.66862700 & -3.30623800 & -2.41268200 \\
\hline $\mathrm{C}$ & -5.97438300 & -0.71415400 & -0.48643600 & $\mathrm{H}$ & -0.93497100 & -2.50067200 & -3.97969800 \\
\hline $\mathrm{C}$ & -4.46316700 & -1.81091100 & -1.98475300 & $\mathrm{O}$ & 0.49927200 & -1.61475300 & -2.82552700 \\
\hline $\mathrm{H}$ & -2.38628000 & -1.35261400 & -1.58733800 & $\mathrm{H}$ & 1.05658400 & -1.78708300 & -2.01414100 \\
\hline $\mathrm{C}$ & -5.77042200 & -1.56899600 & -1.57319600 & $\mathrm{H}$ & -0.31185100 & 2.08493300 & -2.49286600 \\
\hline $\mathrm{H}$ & -6.98064400 & -0.50561200 & -0.12922700 & $\mathrm{C}$ & 2.24730400 & 2.06628700 & -1.44208700 \\
\hline $\mathrm{H}$ & -4.25820800 & -2.47251500 & -2.82113100 & $\mathrm{C}$ & 1.72478700 & 3.32904300 & -1.10028400 \\
\hline $\mathrm{H}$ & -6.61591700 & -2.03018400 & -2.07285400 & $\mathrm{C}$ & 3.64605800 & 1.92468100 & -1.51928800 \\
\hline $\mathrm{N}$ & -4.97914700 & -0.11528800 & 0.17588300 & $\mathrm{C}$ & 2.57108000 & 4.40451200 & -0.84109100 \\
\hline $\mathrm{Pd}$ & -0.02972800 & 0.30148100 & -0.23550600 & $\mathrm{H}$ & 0.65080400 & 3.47518100 & -1.03423800 \\
\hline $\mathrm{H}$ & 1.95571300 & 0.02743600 & -2.07247200 & $\mathrm{C}$ & 4.49166200 & 3.00187900 & -1.26367300 \\
\hline $\mathrm{C}$ & -4.10148700 & 2.63424900 & 1.64696000 & $\mathrm{H}$ & 4.06789900 & 0.96165300 & -1.79463300 \\
\hline $\mathrm{H}$ & -4.95274200 & 2.02549200 & 1.33866500 & $\mathrm{C}$ & 3.95746900 & 4.24611200 & -0.92082300 \\
\hline $\mathrm{H}$ & -4.36717000 & 3.69144100 & 1.52784800 & $\mathrm{H}$ & 2.14864300 & 5.37042000 & -0.58122200 \\
\hline $\mathrm{H}$ & -3.91951600 & 2.47009400 & 2.71283600 & $\mathrm{H}$ & 5.56717300 & 2.87308000 & -1.33893100 \\
\hline $\mathrm{C}$ & -1.65402900 & 3.11635900 & 1.38832500 & $\mathrm{H}$ & 4.61454900 & 5.08744400 & -0.72403200 \\
\hline $\mathrm{H}$ & -0.74173300 & 2.97651600 & 0.79951900 & & & & \\
\hline $\mathrm{H}$ & -1.44244600 & 2.81261400 & 2.41881100 & \multirow{2}{*}{\multicolumn{4}{|c|}{$\begin{array}{l}\text { TS42a } \\
\text { imaginary frequency }=-163.28 \mathrm{~cm}^{-1}\end{array}$}} \\
\hline $\mathrm{H}$ & -1.88555300 & 4.18787200 & 1.40639900 & & & & \\
\hline $\mathrm{C}$ & -3.10219500 & 2.80735000 & -0.65071700 & $\mathrm{C}$ & -2.06655000 & 2.82395800 & 0.36543800 \\
\hline $\mathrm{H}$ & -2.25297400 & 2.58787000 & -1.30552200 & $\mathrm{C}$ & -2.52587100 & 4.12653700 & 0.12859900 \\
\hline $\mathrm{H}$ & -3.25921800 & 3.89190000 & -0.66273500 & $\mathrm{C}$ & -3.32300600 & 4.76600400 & 1.07828300 \\
\hline $\mathrm{H}$ & -3.99285400 & 2.33817400 & -1.07666400 & $\mathrm{C}$ & -3.65454500 & 4.09295000 & 2.25239700 \\
\hline $\mathrm{C}$ & 1.23006100 & 1.14558400 & 3.10335900 & $\mathrm{C}$ & -3.14525000 & 2.81211400 & 2.43525000 \\
\hline $\mathrm{H}$ & 0.36095900 & 0.64420700 & 3.53839000 & $\mathrm{H}$ & -2.26806200 & 4.64290900 & -0.78699600 \\
\hline $\mathrm{H}$ & 0.88308500 & 1.79102200 & 2.29347900 & $\mathrm{H}$ & -3.68301700 & 5.77439100 & 0.89827000 \\
\hline $\mathrm{H}$ & 1.66582600 & 1.78118100 & 3.88273900 & $\mathrm{H}$ & -4.27872800 & 4.54824200 & 3.01313300 \\
\hline $\mathrm{C}$ & 2.78598000 & -0.67614800 & 3.81981500 & $\mathrm{H}$ & -3.35246800 & 2.24925100 & 3.34149900 \\
\hline $\mathrm{H}$ & 3.25085400 & 0.00241800 & 4.54389700 & $\mathrm{~N}$ & -2.36860600 & 2.20132400 & 1.52533200 \\
\hline $\mathrm{H}$ & 3.53434900 & -1.43217100 & 3.56790300 & $\mathrm{P}$ & -1.07356300 & 1.80197800 & -0.84614600 \\
\hline $\mathrm{H}$ & 1.96225800 & -1.17979800 & 4.33335500 & $\mathrm{C}$ & -2.41793900 & 1.29668600 & -2.12790000 \\
\hline $\mathrm{C}$ & 3.46450300 & 0.97210100 & 2.01916900 & $\mathrm{C}$ & -0.06432200 & 3.18005600 & -1.63447000 \\
\hline $\mathrm{H}$ & 3.84268700 & 1.65089800 & 2.79209200 & $\mathrm{H}$ & -0.75781100 & 3.93039400 & -2.02566000 \\
\hline $\mathrm{H}$ & 3.14158200 & 1.58366200 & 1.17400800 & $\mathrm{H}$ & 0.45920400 & 3.64460600 & -0.78995700 \\
\hline $\mathrm{H}$ & 4.30343300 & 0.35692200 & 1.68757900 & $\mathrm{C}$ & 0.96552800 & 2.85145800 & -2.73696800 \\
\hline
\end{tabular}




\begin{tabular}{|c|c|c|c|c|c|c|c|}
\hline $\mathrm{H}$ & 1.33708700 & 3.82117000 & -3.08720800 & $\mathrm{C}$ & -2.91187600 & 2.47886200 & -2.97964900 \\
\hline $\mathrm{H}$ & 0.46230200 & 2.40666600 & -3.60352400 & $\mathrm{H}$ & -3.68920600 & 2.12275900 & -3.66555200 \\
\hline $\mathrm{C}$ & 2.15923500 & 1.96834600 & -2.30389000 & $\mathrm{H}$ & -3.36124800 & 3.27213400 & -2.37478300 \\
\hline $\mathrm{H}$ & 3.10157100 & 2.44771700 & -2.59201700 & $\mathrm{H}$ & -2.11772100 & 2.91368000 & -3.59272200 \\
\hline $\mathrm{H}$ & 2.18282200 & 1.90656000 & -1.20800900 & $\mathrm{C}$ & -3.60697600 & 0.68209900 & -1.36340500 \\
\hline $\mathrm{C}$ & 2.12337600 & 0.55381500 & -2.90371600 & $\mathrm{H}$ & -4.33538300 & 0.29918200 & -2.08685400 \\
\hline $\mathrm{H}$ & 2.34699500 & 0.61871000 & -3.97679700 & $\mathrm{H}$ & -3.29863600 & -0.15765400 & -0.73564500 \\
\hline $\mathrm{H}$ & 1.10724300 & 0.14645000 & -2.83090200 & $\mathrm{H}$ & -4.12254100 & 1.41522300 & -0.73625200 \\
\hline $\mathrm{C}$ & 3.09654800 & -0.44125600 & -2.25659200 & $\mathrm{C}$ & -0.21470200 & -0.98735100 & 1.79014800 \\
\hline $\mathrm{H}$ & 3.08771400 & -1.37402900 & -2.82809200 & $\mathrm{O}$ & 0.78254400 & -1.17321300 & 2.44027800 \\
\hline $\mathrm{H}$ & 4.12359500 & -0.06354500 & -2.25668000 & $\mathrm{C}$ & -2.46908800 & -1.92997100 & 1.16322700 \\
\hline $\mathrm{P}$ & 2.66650900 & -0.87670000 & -0.48802400 & $\mathrm{C}$ & -1.11668900 & -1.93639800 & 1.13253800 \\
\hline $\mathrm{C}$ & 3.22220900 & -2.70281300 & -0.33246400 & $\mathrm{C}$ & -3.35482700 & -2.91308600 & 0.54731900 \\
\hline $\mathrm{C}$ & 3.95262400 & 0.05362300 & 0.49134600 & $\mathrm{C}$ & -4.73211600 & -2.84544700 & 0.83642600 \\
\hline $\mathrm{C}$ & 3.73693800 & 0.32280500 & 1.85119600 & $\mathrm{C}$ & -2.90237800 & -3.92686400 & -0.32253800 \\
\hline $\mathrm{C}$ & 5.99897100 & 1.10693700 & 0.53878200 & $\mathrm{C}$ & -5.62601100 & -3.76130000 & 0.28750600 \\
\hline $\mathrm{C}$ & 4.72261800 & 1.00788000 & 2.56174400 & $\mathrm{H}$ & -5.09619300 & -2.06985700 & 1.50547800 \\
\hline $\mathrm{H}$ & 2.82017500 & -0.00252800 & 2.33043500 & $\mathrm{C}$ & -3.79606500 & -4.83912300 & -0.87105800 \\
\hline $\mathrm{C}$ & 5.87866200 & 1.41137700 & 1.89531900 & $\mathrm{H}$ & -1.84944100 & -3.99793800 & -0.57726700 \\
\hline $\mathrm{H}$ & 6.88319400 & 1.40700500 & -0.01968700 & $\mathrm{C}$ & -5.16018000 & -4.76175600 & -0.56732800 \\
\hline $\mathrm{H}$ & 4.58860500 & 1.22430100 & 3.61777200 & $\mathrm{H}$ & -6.68294300 & -3.69602600 & 0.52642800 \\
\hline $\mathrm{H}$ & 6.66918200 & 1.94956300 & 2.40802800 & $\mathrm{H}$ & -3.43265900 & -5.61382500 & -1.53923200 \\
\hline $\mathrm{N}$ & 5.06428100 & 0.44209500 & -0.15191600 & $\mathrm{H}$ & -5.85377900 & -5.47747800 & -0.99774100 \\
\hline $\mathrm{Pd}$ & 0.40692200 & 0.05612000 & -0.05603700 & $\mathrm{H}$ & -0.56235000 & -2.73120500 & 0.64421900 \\
\hline $\mathrm{H}$ & -2.96339000 & -1.14518300 & 1.72756100 & $\mathrm{C}$ & -0.63897000 & 0.75014400 & 3.78665300 \\
\hline $\mathrm{C}$ & 4.63469300 & -2.91328800 & -0.91299600 & $\mathrm{H}$ & 0.16614100 & 0.09070300 & 4.10804600 \\
\hline $\mathrm{H}$ & 5.37707500 & -2.27977900 & -0.42128500 & $\mathrm{H}$ & -1.40387500 & 0.80672300 & 4.56509500 \\
\hline $\mathrm{H}$ & 4.93062500 & -3.95805200 & -0.76064700 & $\mathrm{H}$ & -0.23453900 & 1.74296600 & 3.57197600 \\
\hline $\mathrm{H}$ & 4.68199000 & -2.71501600 & -1.98711200 & $\mathrm{O}$ & -1.23327600 & 0.18061800 & 2.60465700 \\
\hline $\mathrm{C}$ & 2.20510900 & -3.56637400 & -1.10344600 & $\mathrm{H}$ & -1.67456300 & 0.94933000 & 2.02191000 \\
\hline
\end{tabular}

$\mathrm{H}$

$\mathrm{H}$

$\mathrm{H}$

C

$\mathrm{H}$

$\mathrm{H}$

$\mathrm{H}$

C

$\mathrm{H}$

$\mathrm{H}$

$\mathrm{H}$ $\begin{array}{lll}1.19606300 & -3.46969300 & -0.68969200\end{array}$

$2.15898200 \quad-3.31171600 \quad-2.16718200$

$2.49437600 \quad-4.62114200 \quad-1.03350000$

$3.22190300 \quad-3.11249000 \quad 1.15221700$

$\begin{array}{lll}2.24929300 & -2.96305600 & 1.62622400\end{array}$

3.47967800

$-4.17508600$

1.22996000

3.96547000

$-2.55228100 \quad 1.72613400$

$-1.81040800$

$0.21782600-3.04686500$

$-0.99349600$

$0.60750700 \quad-3.65971300$

$-1.43268300$

$-0.63265800 \quad-2.47165700$

$-2.58500500$
TS42b

imaginary frequency $=-77.34 \mathrm{~cm}^{-1}$

$\begin{array}{lrrr}\mathrm{C} & -1.71294800 & 2.90982300 & 0.25660300 \\ \mathrm{C} & -1.71191600 & 4.30236300 & 0.09802200 \\ \mathrm{C} & -2.13126500 & 5.11287500 & 1.15287300 \\ \mathrm{C} & -2.54963900 & 4.51532400 & 2.34034700 \\ \mathrm{C} & -2.50799000 & 3.12629600 & 2.42690000 \\ \mathrm{H} & -1.39309600 & 4.74915900 & -0.83621900 \\ \mathrm{H} & -2.13508600 & 6.19316900 & 1.04462400 \\ \mathrm{H} & -2.89010800 & 5.10644400 & 3.18342700\end{array}$




\begin{tabular}{|c|c|c|c|c|c|c|c|}
\hline $\mathrm{H}$ & -2.80905700 & 2.61332900 & 3.33690200 & $\mathrm{C}$ & 5.64447700 & -2.81059200 & 1.13465800 \\
\hline $\mathrm{N}$ & -2.09306900 & 2.34221000 & 1.41904600 & $\mathrm{H}$ & 4.74652300 & -3.43616500 & 1.18834400 \\
\hline $\mathrm{P}$ & -1.23144600 & 1.72931100 & -1.09662600 & $\mathrm{H}$ & 6.51450300 & -3.47135700 & 1.22947500 \\
\hline $\mathrm{C}$ & -2.77194400 & 1.69389000 & -2.23428200 & $\mathrm{H}$ & 5.64851000 & -2.13964500 & 1.99855300 \\
\hline $\mathrm{C}$ & 0.06357400 & 2.70574300 & -2.03525100 & $\mathrm{C}$ & -2.37782500 & 0.92774100 & -3.51403500 \\
\hline $\mathrm{H}$ & -0.45320900 & 3.37972000 & -2.72642000 & $\mathrm{H}$ & -1.62568300 & 1.46250700 & -4.10174700 \\
\hline $\mathrm{H}$ & 0.57344300 & 3.33864900 & -1.30069100 & $\mathrm{H}$ & -1.99313700 & -0.07255300 & -3.28917800 \\
\hline $\mathrm{C}$ & 1.11154500 & 1.85952500 & -2.79008700 & $\mathrm{H}$ & -3.26401400 & 0.80937400 & -4.14766100 \\
\hline $\mathrm{H}$ & 1.50276700 & 2.46692100 & -3.61572500 & $\mathrm{C}$ & -3.28805400 & 3.09316400 & -2.61254000 \\
\hline $\mathrm{H}$ & 0.63504500 & 0.98955200 & -3.25943600 & $\mathrm{H}$ & -4.13478300 & 2.98382700 & -3.29954600 \\
\hline $\mathrm{C}$ & 2.28288000 & 1.39793300 & -1.91408100 & $\mathrm{H}$ & -3.64397600 & 3.65320200 & -1.74399300 \\
\hline $\mathrm{H}$ & 2.81014300 & 2.27536800 & -1.51693100 & $\mathrm{H}$ & -2.53629200 & 3.69640700 & -3.13099300 \\
\hline $\mathrm{H}$ & 1.89840000 & 0.85113900 & -1.04134000 & $\mathrm{C}$ & -3.87917800 & 0.92214900 & -1.49153600 \\
\hline $\mathrm{C}$ & 3.26966900 & 0.50775200 & -2.67956800 & $\mathrm{H}$ & -4.77402000 & 0.87300900 & -2.12214700 \\
\hline $\mathrm{H}$ & 3.61664400 & 1.04919500 & -3.57054600 & $\mathrm{H}$ & -3.57271300 & -0.10369100 & -1.26664600 \\
\hline $\mathrm{H}$ & 2.73943600 & -0.38023600 & -3.05032400 & $\mathrm{H}$ & -4.16289300 & 1.41077100 & -0.55374800 \\
\hline $\mathrm{C}$ & 4.50022800 & 0.07033600 & -1.86735700 & $\mathrm{C}$ & -0.85333000 & -1.77977800 & 1.28755200 \\
\hline $\mathrm{H}$ & 5.17142700 & -0.49114900 & -2.52431300 & $\mathrm{O}$ & 0.16613600 & -2.08909200 & 1.78521400 \\
\hline $\mathrm{H}$ & 5.04896400 & 0.94126100 & -1.49634400 & $\mathrm{C}$ & -3.35650800 & -1.82943600 & 1.22336200 \\
\hline $\mathrm{P}$ & 4.09465600 & -1.04789800 & -0.41773000 & $\mathrm{C}$ & -2.13389400 & -2.33518700 & 0.91859400 \\
\hline $\mathrm{C}$ & 5.72551500 & -2.03931400 & -0.19626100 & $\mathrm{C}$ & -0.35682200 & 0.21874500 & 3.31922400 \\
\hline $\mathrm{C}$ & 4.17902900 & 0.16071700 & 1.00715100 & $\mathrm{H}$ & -0.56780000 & 1.16527100 & 3.82617900 \\
\hline $\mathrm{C}$ & 3.64897900 & -0.22820800 & 2.25088000 & $\mathrm{H}$ & 0.61870800 & 0.28154000 & 2.82614300 \\
\hline $\mathrm{C}$ & 4.76933100 & 2.22183600 & 1.86217300 & $\mathrm{H}$ & -0.33235200 & -0.58288400 & 4.05900700 \\
\hline $\mathrm{C}$ & 3.72437700 & 0.65013300 & 3.33070900 & $\mathrm{O}$ & -1.40577500 & -0.06826900 & 2.38631200 \\
\hline $\mathrm{H}$ & 3.19058700 & -1.20622200 & 2.36096000 & $\mathrm{H}$ & -1.65434500 & 0.77460000 & 1.89423000 \\
\hline $\mathrm{C}$ & 4.29862300 & 1.90686700 & 3.13682400 & $\mathrm{C}$ & 0.40730700 & -2.15963600 & -1.36946700 \\
\hline $\mathrm{H}$ & 5.21527400 & 3.19526400 & 1.66469300 & $\mathrm{O}$ & 0.88927700 & -3.01103000 & -1.95949100 \\
\hline $\mathrm{H}$ & 3.34438100 & 0.35877100 & 4.30633000 & $\mathrm{H}$ & -2.00562000 & -3.25799800 & 0.35807900 \\
\hline $\mathrm{H}$ & 4.38073300 & 2.62479000 & 3.94661100 & $\mathrm{C}$ & -4.63946700 & -2.41146000 & 0.87210300 \\
\hline $\mathrm{N}$ & 4.71958700 & 1.37795400 & 0.82333300 & $\mathrm{C}$ & -4.78127200 & -3.54293400 & 0.03927700 \\
\hline $\mathrm{Pd}$ & -0.51794100 & -0.60056000 & -0.59827400 & $\mathrm{C}$ & -5.80270200 & -1.81045900 & 1.39714100 \\
\hline $\mathrm{H}$ & -3.38468700 & -0.92540700 & 1.82123300 & $\mathrm{C}$ & -6.04106200 & -4.05124000 & -0.24870100 \\
\hline $\mathrm{C}$ & 6.98509500 & -1.15704200 & -0.20837700 & $\mathrm{H}$ & -3.90659300 & -4.02374400 & -0.38782200 \\
\hline $\mathrm{H}$ & 6.96744700 & -0.41000300 & 0.59019500 & $\mathrm{C}$ & -7.06344400 & -2.32341400 & 1.10889800 \\
\hline H & 7.87546100 & -1.78189100 & -0.06282700 & $\mathrm{H}$ & -5.70587800 & -0.93995600 & 2.04031000 \\
\hline $\mathrm{H}$ & 7.10945400 & -0.62883100 & -1.15815500 & $\mathrm{C}$ & -7.18495100 & -3.44532600 & 0.28610400 \\
\hline $\mathrm{C}$ & 5.77890700 & -3.05873300 & -1.35279900 & $\mathrm{H}$ & -6.13805300 & -4.92127700 & -0.89025100 \\
\hline $\mathrm{H}$ & 4.90239000 & -3.71506600 & -1.35282700 & $\mathrm{H}$ & -7.94873300 & -1.85255400 & 1.52413000 \\
\hline $\mathrm{H}$ & 5.84165400 & -2.57660600 & -2.33367300 & $\mathrm{H}$ & -8.16695300 & -3.84862100 & 0.05899100 \\
\hline $\mathrm{H}$ & 6.66961300 & -3.68956300 & -1.24622500 & & & & \\
\hline
\end{tabular}


51

C

C

C

C

C

$\mathrm{H}$

H

H

H

$\mathrm{N}$

P

C

C

H

H

C

H

H

C

$\mathrm{H}$

H

C

H

$\mathrm{H}$

C

H

H

P

C

C

C

C

C

$\mathrm{H}$

C

H

H

H

N

Pd

$$
2.98560100 \quad-1.70025000 \quad-0.10342900
$$$$
\begin{array}{lll}
4.25523500 & -2.21155000 & 0.18333000
\end{array}
$$$$
5.10713700
$$$$
4.69418600
$$$$
3.43059500
$$$$
4.57563600
$$$$
6.09182400
$$$$
5.33602900
$$$$
3.01693700
$$$$
2.63870200
$$$$
1.62548300
$$$$
2.55568000
$$$$
1.20948500
$$$$
2.04718100
$$$$
1.14901600
$$$$
-0.11980500
$$$$
-0.09681800
$$$$
-0.21096000
$$$$
-1.33829900
$$$$
-1.50504600
$$$$
-1.11338900
$$$$
-2.62260500
$$$$
-3.47168900
$$$$
-2.81839400
$$$$
-2.62976200
$$$$
-1.87758800
$$$$
-3.60882400
$$$$
-2.32069900
$$$$
-2.84496400
$$$$
-3.77771100
$$$$
-3.74764600
$$$$
-5.92879200
$$$$
-4.87680700
$$$$
-2.87272700
$$$$
-5.99187500
$$$$
-6.77688600
$$$$
-4.87491900
$$$$
-6.88962600
$$$$
-4.85514100
$$$$
0.04422900
$$

$-2.61896100$

$-0.84322600$

$-2.53004700-2.17656800$

$-2.03408400 \quad-2.43634000$

$-2.29433300 \quad 1.21272800$

$-3.00891500-0.60418100$

$-2.83511000 \quad-2.99430200$

$-1.91195400 \quad-3.42927100$

$-1.64635600 \quad-1.41437900$

$-1.14670700 \quad 1.08575700$

$-0.35591900 \quad 2.57644000$

$-2.90447400 \quad 1.62890200$

$-3.29595800 \quad 2.21313800$

$-3.51269200 \quad 0.71857500$

$-3.00307600 \quad 2.39533000$

$-3.90102200$

3.02393000

$-2.16135300$

3.08977900

1.45634400

1.15741000

0.53357700

2.06976500

$-2.49386800$

1.42632800

$-2.98940900$

3.03051600

$-0.97232900$

2.31663700

$-0.68659200$

3.06094800

2.71838000

0.81653600

0.10573900

1.42949300

1.85437000

$\begin{array}{ll}-0.38311100 & -0.24601600 \\ -0.22209500 & -1.63589300\end{array}$

$-1.20695700 \quad-0.32645600$

$-0.57208400 \quad-2.37915300$

$0.16944800 \quad-2.13768100$

$-1.07722000 \quad-1.71543000$

$-1.59866900 \quad 0.23167000$

$-0.45116100 \quad-3.45837500$

$-1.36617200 \quad-2.25228400$

$-0.86925900 \quad 0.39711000$

$0.16727400 \quad-0.13823500$
H

$\mathrm{H}$

$\mathrm{H}$

C

$\mathrm{H}$

H

$\mathrm{H}$

C

$\mathrm{H}$

$\mathrm{H}$

$\mathrm{H}$

C

$\mathrm{H}$

$\mathrm{H}$

$\mathrm{H}$

C

$\mathrm{C}$

$\mathrm{H}$

$\mathrm{H}$

$\mathrm{H}$

C

H

$\mathrm{H}$

$\mathrm{H}$

C

$\mathrm{O}$

C

C

$\mathrm{C}$

H

H

H

$\mathrm{O}$

$\mathrm{H}$

H

C

$\mathrm{C}$

C

C

H

\begin{tabular}{|c|c|c|}
\hline 2.03924200 & 0.75487000 & -2.06563900 \\
\hline-4.14575600 & 1.84189800 & 2.25392900 \\
\hline-4.96595600 & 1.34724300 & 1.72880800 \\
\hline-4.44753400 & 2.87667900 & 2.45551200 \\
\hline-4.01568700 & 1.35073800 & 3.22204000 \\
\hline-1.70252200 & 2.42477500 & 2.29083900 \\
\hline-0.77005800 & 2.51041200 & 1.72463800 \\
\hline-1.51267600 & 1.81315300 & 3.17894900 \\
\hline-1.97538500 & 3.42756100 & 2.64100900 \\
\hline-3.04641500 & 2.74730000 & 0.19016500 \\
\hline-2.16741400 & 2.74658500 & -0.46087200 \\
\hline-3.22670100 & 3.78039500 & 0.50926700 \\
\hline-3.90607300 & 2.43096800 & -0.40680600 \\
\hline 1.47846100 & 0.45348200 & 3.33254500 \\
\hline 0.69536600 & -0.18715800 & 3.74956600 \\
\hline 1.00495400 & 1.19170900 & 2.68209600 \\
\hline 1.94939500 & 0.98249100 & 4.16882400 \\
\hline 3.19066200 & -1.35802100 & 3.55744500 \\
\hline 3.67318100 & -0.79868000 & 4.36671800 \\
\hline 3.96322900 & -1.98793700 & 3.10654100 \\
\hline 2.44802700 & -2.01438400 & 4.01907100 \\
\hline 3.61627800 & 0.62931300 & 2.04780200 \\
\hline 4.01597700 & 1.20553900 & 2.88961800 \\
\hline 3.19587900 & 1.34276100 & 1.33411600 \\
\hline 4.46321500 & 0.12634700 & 1.57188400 \\
\hline-0.40495400 & 0.25518100 & -3.11738800 \\
\hline-1.40408800 & 0.30203400 & -3.80732500 \\
\hline 1.27734600 & 1.38351500 & -1.61599200 \\
\hline-0.03590000 & 1.24834600 & -2.09988300 \\
\hline 0.10539800 & -1.75813900 & -4.25721400 \\
\hline 0.85628800 & -2.54915700 & -4.22367100 \\
\hline-0.88163500 & -2.16676100 & -4.03438900 \\
\hline 0.08379200 & -1.29597100 & -5.24605300 \\
\hline 0.49407100 & -0.79337100 & -3.25487800 \\
\hline 1.70771400 & -1.25822300 & -1.65147800 \\
\hline-0.72676100 & 2.08321900 & -2.07526100 \\
\hline 1.82370300 & 2.61030600 & -1.00401200 \\
\hline 1.01554800 & 3.63241700 & -0.46981800 \\
\hline 3.21930000 & 2.79513700 & -0.98332800 \\
\hline 1.58406000 & 4.78553000 & 0.06587700 \\
\hline-0.06365900 & 3.52907600 & -0.47906500 \\
\hline
\end{tabular}




\begin{tabular}{|c|c|c|c|c|c|c|c|}
\hline $\mathrm{C}$ & 3.78841000 & 3.94993700 & -0.44931200 & $\mathrm{C}$ & 0.14552800 & 4.94334500 & 1.80277700 \\
\hline $\mathrm{H}$ & 3.86269300 & 2.02837600 & -1.40955900 & $\mathrm{H}$ & 0.87335200 & 4.91426500 & -0.22115200 \\
\hline $\mathrm{C}$ & 2.97186500 & 4.95053100 & 0.08123800 & $\mathrm{C}$ & -0.02003100 & 4.19699200 & 2.96685500 \\
\hline $\mathrm{H}$ & 0.94092800 & 5.56317000 & 0.46710000 & $\mathrm{H}$ & 0.30460900 & 2.24642500 & 3.85196600 \\
\hline $\mathrm{H}$ & 4.86741400 & 4.07301300 & -0.45548600 & $\mathrm{H}$ & -0.17589100 & 5.97935400 & 1.75712500 \\
\hline \multirow[t]{2}{*}{$\mathrm{H}$} & 3.41059900 & 5.85320200 & 0.49483700 & $\mathrm{H}$ & -0.47457300 & 4.62620000 & 3.85331700 \\
\hline & & & & $\mathrm{N}$ & 0.97301300 & 2.28666600 & 1.90260000 \\
\hline \multicolumn{4}{|c|}{ TS43 } & $\mathrm{Pd}$ & 1.63594100 & -0.27228500 & -0.40541800 \\
\hline \multicolumn{4}{|c|}{ imaginary frequency $=-465.49 \mathrm{~cm}^{-1}$} & $\mathrm{H}$ & 1.92584700 & -0.25645400 & -1.96054400 \\
\hline $\mathrm{C}$ & -4.23015200 & 0.06145200 & 0.84633500 & $\mathrm{C}$ & 4.01844200 & 4.06051600 & -0.56070600 \\
\hline $\mathrm{C}$ & -5.17349100 & 1.06008800 & 1.13526600 & $\mathrm{H}$ & 3.50341800 & 4.60035600 & 0.23834200 \\
\hline $\mathrm{C}$ & -5.75933800 & 1.10278500 & 2.40036300 & $\mathrm{H}$ & 5.08696800 & 4.28529200 & -0.47110100 \\
\hline $\mathrm{C}$ & -5.39960700 & 0.14145100 & 3.34261500 & $\mathrm{H}$ & 3.68433500 & 4.45884300 & -1.52348400 \\
\hline $\mathrm{C}$ & -4.46341300 & -0.82333100 & 2.96962400 & $\mathrm{C}$ & 4.58700700 & 1.82758700 & -1.58647900 \\
\hline $\mathrm{H}$ & -5.46043500 & 1.78523600 & 0.38173200 & $\mathrm{H}$ & 4.45711000 & 0.74176400 & -1.54922800 \\
\hline $\mathrm{H}$ & -6.49223900 & 1.86786300 & 2.64065500 & $\mathrm{H}$ & 4.29133900 & 2.17519100 & -2.58078600 \\
\hline $\mathrm{H}$ & -5.83179300 & 0.13261500 & 4.33791500 & $\mathrm{H}$ & 5.65601800 & 2.03876200 & -1.47591200 \\
\hline $\mathrm{H}$ & -4.16090000 & -1.59615500 & 3.67409400 & $\mathrm{C}$ & 4.33015900 & 2.03090900 & 0.90881300 \\
\hline $\mathrm{N}$ & -3.88075500 & -0.86488100 & 1.76538300 & $\mathrm{H}$ & 4.23303300 & 0.94433000 & 1.00001700 \\
\hline$P$ & -3.31902000 & -0.13964000 & -0.76244100 & $\mathrm{H}$ & 5.39264000 & 2.27948100 & 1.00529200 \\
\hline $\mathrm{C}$ & -4.49194200 & -1.31338000 & -1.73583200 & $\mathrm{H}$ & 3.80231800 & 2.49190100 & 1.74767600 \\
\hline $\mathrm{C}$ & -3.58505800 & 1.52675100 & -1.60434800 & $\mathrm{C}$ & -3.97535200 & -1.36931700 & -3.18725700 \\
\hline $\mathrm{H}$ & -4.54276800 & 1.56177100 & -2.13333600 & $\mathrm{H}$ & -4.09709700 & -0.41469600 & -3.70977600 \\
\hline $\mathrm{H}$ & -3.62951000 & 2.29616000 & -0.82481900 & $\mathrm{H}$ & -2.91969800 & -1.65849800 & -3.22758200 \\
\hline $\mathrm{C}$ & -2.43037800 & 1.86035000 & -2.57211800 & $\mathrm{H}$ & -4.54538200 & -2.11892000 & -3.74928900 \\
\hline $\mathrm{H}$ & -2.73996900 & 2.67096000 & -3.24464100 & $\mathrm{C}$ & -5.96613800 & -0.87821200 & -1.72301100 \\
\hline $\mathrm{H}$ & -2.22341200 & 0.99694900 & -3.21627800 & $\mathrm{H}$ & -6.56242200 & -1.58522900 & -2.31310000 \\
\hline $\mathrm{C}$ & -1.13977000 & 2.27385700 & -1.84999800 & $\mathrm{H}$ & -6.37884600 & -0.86550900 & -0.71036600 \\
\hline $\mathrm{H}$ & -1.27517400 & 3.27114100 & -1.40956200 & $\mathrm{H}$ & -6.11768300 & 0.11242100 & -2.16451200 \\
\hline $\mathrm{H}$ & -0.96566900 & 1.58318400 & -1.01371000 & $\mathrm{C}$ & -4.36091200 & -2.71463900 & -1.10739600 \\
\hline $\mathrm{C}$ & 0.08187800 & 2.26343700 & -2.77553000 & $\mathrm{H}$ & -4.99179100 & -3.42186900 & -1.66002100 \\
\hline $\mathrm{H}$ & -0.13888000 & 2.83177300 & -3.68827000 & $\mathrm{H}$ & -3.32877200 & -3.07241300 & -1.15227500 \\
\hline $\mathrm{H}$ & 0.27279500 & 1.23262800 & -3.09912000 & $\mathrm{H}$ & -4.68501100 & -2.72390800 & -0.06267900 \\
\hline $\mathrm{C}$ & 1.37519600 & 2.85057000 & -2.18106600 & $\mathrm{C}$ & 1.26861100 & -0.44501700 & 1.59890600 \\
\hline $\mathrm{H}$ & 2.19185300 & 2.72705000 & -2.89992900 & $\mathrm{O}$ & 1.11710300 & -0.67929300 & 2.70050300 \\
\hline $\mathrm{H}$ & 1.26478700 & 3.92764800 & -2.02204900 & $\mathrm{C}$ & 1.72121700 & -1.99581300 & -1.99478700 \\
\hline $\mathrm{P}$ & 1.97310300 & 2.08137000 & -0.59666400 & $\mathrm{H}$ & 2.67036600 & -2.05929700 & -2.51474100 \\
\hline $\mathrm{C}$ & 3.81776300 & 2.53651200 & -0.45422900 & $\mathrm{H}$ & 0.82693300 & -1.90992600 & -2.60312200 \\
\hline $\mathrm{C}$ & 1.13378400 & 3.00244600 & 0.78105400 & $\mathrm{C}$ & 1.57950000 & -2.57078400 & -0.71883900 \\
\hline $\mathrm{C}$ & 0.73358700 & 4.34193000 & 0.68827200 & $\mathrm{C}$ & 2.74736700 & -3.16665000 & -0.00427500 \\
\hline $\mathrm{C}$ & 0.41339400 & 2.87002900 & 2.96767500 & $\mathrm{C}$ & 2.61655200 & -4.31158000 & 0.80155000 \\
\hline
\end{tabular}




\begin{tabular}{|c|c|c|c|c|c|c|c|}
\hline $\mathrm{C}$ & 4.03770600 & -2.62908400 & -0.18967900 & $\mathrm{H}$ & 0.65057600 & -4.41782400 & 1.38427400 \\
\hline $\mathrm{C}$ & 3.73562200 & -4.89259600 & 1.39747100 & $\mathrm{H}$ & 1.11188300 & -3.17131600 & 0.25589600 \\
\hline $\mathrm{H}$ & 1.64283800 & -4.75487100 & 0.96127700 & $\mathrm{C}$ & -0.98462700 & -3.14612900 & 0.79133700 \\
\hline $\mathrm{C}$ & 5.15031900 & -3.20539100 & 0.41543600 & $\mathrm{H}$ & -1.39564600 & -4.01141800 & 0.26237200 \\
\hline $\mathrm{H}$ & 4.17469300 & -1.74598500 & -0.80706200 & $\mathrm{H}$ & -1.59664500 & -3.02798200 & 1.68911100 \\
\hline $\mathrm{C}$ & 5.00387800 & -4.34286200 & 1.21348400 & $\mathrm{P}$ & -1.30530600 & -1.63207400 & -0.24684400 \\
\hline $\mathrm{H}$ & 3.61083700 & -5.78144800 & 2.00802500 & $\mathrm{C}$ & -1.48500900 & -2.30684600 & -2.04196500 \\
\hline $\mathrm{H}$ & 6.13244500 & -2.76865300 & 0.26210700 & $\mathrm{C}$ & -3.07039100 & -1.24271000 & 0.24552000 \\
\hline $\mathrm{H}$ & 5.87083400 & -4.79566100 & 1.68418700 & $\mathrm{C}$ & -3.84982100 & -0.31297400 & -0.45822100 \\
\hline $\mathrm{C}$ & 0.13619700 & -2.92453300 & -0.36568700 & $\mathrm{C}$ & -4.82430300 & -1.71481600 & 1.66694400 \\
\hline $\mathrm{O}$ & -0.74005400 & -2.98734200 & -1.20253700 & $\mathrm{C}$ & -5.17580000 & -0.11775300 & -0.07568000 \\
\hline $\mathrm{O}$ & -0.03807100 & -3.13725900 & 0.94180700 & $\mathrm{H}$ & -3.43400400 & 0.26133000 & -1.27538900 \\
\hline $\mathrm{C}$ & -1.38307900 & -3.48019000 & 1.37157800 & $\mathrm{C}$ & -5.68238800 & -0.83724000 & 1.00523500 \\
\hline $\mathrm{H}$ & -1.26860100 & -3.82164900 & 2.39969300 & $\mathrm{H}$ & -5.16938300 & -2.28749000 & 2.52541200 \\
\hline $\mathrm{H}$ & -2.03106200 & -2.60116800 & 1.32457100 & $\mathrm{H}$ & -5.79706200 & 0.59212900 & -0.61284300 \\
\hline \multirow[t]{2}{*}{$\mathrm{H}$} & -1.78421800 & -4.27519400 & 0.74120800 & $\mathrm{H}$ & -6.71047100 & -0.72138800 & 1.33238800 \\
\hline & & & & $\mathrm{N}$ & -3.55262500 & -1.91656100 & 1.29988500 \\
\hline \multicolumn{4}{|c|}{ TS43a } & $\mathrm{Pd}$ & 0.51058800 & 0.22083800 & -0.13918000 \\
\hline \multicolumn{4}{|c|}{ imaginary frequency $=-60.08 \mathrm{~cm}^{-1}$} & $\mathrm{H}$ & 1.52257600 & 1.29896900 & -0.60899000 \\
\hline $\mathrm{C}$ & 3.77102500 & 1.17196200 & 0.27662800 & $\mathrm{C}$ & -0.23804200 & -3.14987800 & -2.36842000 \\
\hline $\mathrm{C}$ & 4.86242500 & 1.39951800 & 1.12350400 & $\mathrm{H}$ & 0.67593200 & -2.55389000 & -2.30203200 \\
\hline $\mathrm{C}$ & 5.49709600 & 2.64329400 & 1.08581900 & $\mathrm{H}$ & -0.13542500 & -4.02468800 & -1.72083500 \\
\hline $\mathrm{C}$ & 5.04380100 & 3.61249600 & 0.19428100 & $\mathrm{H}$ & -0.31498800 & -3.51559600 & -3.39865400 \\
\hline $\mathrm{C}$ & 3.95350500 & 3.29997200 & -0.61838400 & $\mathrm{C}$ & -2.74380900 & -3.17959300 & -2.20175600 \\
\hline $\mathrm{H}$ & 5.22097000 & 0.63492400 & 1.80137500 & $\mathrm{H}$ & -2.76659600 & -3.59326000 & -3.21669300 \\
\hline $\mathrm{H}$ & 6.33655400 & 2.84570300 & 1.74389200 & $\mathrm{H}$ & -2.75740800 & -4.02488200 & -1.50667300 \\
\hline $\mathrm{H}$ & 5.51154600 & 4.58919700 & 0.13300000 & $\mathrm{H}$ & -3.66306300 & -2.60800400 & -2.05621500 \\
\hline $\mathrm{H}$ & 3.55716200 & 4.02286500 & -1.32712600 & $\mathrm{C}$ & -1.53210400 & -1.12723300 & -3.03216200 \\
\hline $\mathrm{N}$ & 3.32909100 & 2.11794400 & -0.56515900 & $\mathrm{H}$ & -0.65139900 & -0.48374300 & -2.92936100 \\
\hline $\mathrm{P}$ & 2.81943800 & -0.44127000 & 0.16343200 & $\mathrm{H}$ & -1.54560400 & -1.51550500 & -4.05727900 \\
\hline $\mathrm{C}$ & 3.83990000 & -1.38416100 & -1.16312400 & $\mathrm{H}$ & -2.42377600 & -0.50888200 & -2.91048000 \\
\hline $\mathrm{C}$ & 3.18565900 & -1.15121300 & 1.85898200 & $\mathrm{C}$ & 3.49955300 & -2.88426500 & -1.13948300 \\
\hline $\mathrm{H}$ & 4.27416400 & -1.25754600 & 1.92759800 & $\mathrm{H}$ & 2.43638400 & -3.07263800 & -1.29113400 \\
\hline $\mathrm{H}$ & 2.90433700 & -0.32896600 & 2.52741900 & $\mathrm{H}$ & 4.03863600 & -3.37896900 & -1.95519300 \\
\hline $\mathrm{C}$ & 2.53285900 & -2.44769400 & 2.37774600 & $\mathrm{H}$ & 3.80921500 & -3.36336600 & -0.20706500 \\
\hline $\mathrm{H}$ & 2.86215100 & -2.53455100 & 3.41937500 & $\mathrm{C}$ & 5.35427700 & -1.22343700 & -0.92130400 \\
\hline $\mathrm{H}$ & 2.95814000 & -3.31708000 & 1.86449300 & $\mathrm{H}$ & 5.88929100 & -1.81942100 & -1.66908000 \\
\hline $\mathrm{C}$ & 0.99345900 & -2.50432000 & 2.31062100 & $\mathrm{H}$ & 5.68929500 & -0.18970700 & -1.02555700 \\
\hline $\mathrm{H}$ & 0.59740700 & -2.92453300 & 3.24204400 & $\mathrm{H}$ & 5.66359900 & -1.59249100 & 0.06197000 \\
\hline $\mathrm{H}$ & 0.58718500 & -1.48671600 & 2.25127900 & $\mathrm{C}$ & 3.46674800 & -0.78276200 & -2.53285200 \\
\hline $\mathrm{C}$ & 0.49314000 & -3.36028200 & 1.13698500 & $\mathrm{H}$ & 3.68208500 & 0.28806500 & -2.58502900 \\
\hline
\end{tabular}




\begin{tabular}{|c|c|c|c|c|c|c|c|}
\hline $\mathrm{H}$ & 4.05057100 & -1.28171500 & -3.31453400 & $\mathrm{H}$ & -3.69497100 & 0.45057600 & 2.37379700 \\
\hline $\mathrm{H}$ & 2.40641000 & -0.92527200 & -2.76253600 & $\mathrm{C}$ & -1.71070800 & 1.17098900 & 2.87243400 \\
\hline $\mathrm{C}$ & -1.00796400 & 2.09921000 & 0.59467700 & $\mathrm{H}$ & -1.85438000 & 1.20258000 & 3.96040300 \\
\hline $\mathrm{C}$ & 0.31103400 & 2.49728900 & 0.45305500 & $\mathrm{H}$ & -1.07753400 & 2.03277500 & 2.62471500 \\
\hline $\mathrm{H}$ & 0.59189100 & 3.15580500 & -0.35983100 & $\mathrm{C}$ & -0.97207400 & -0.12195700 & 2.49519300 \\
\hline $\mathrm{H}$ & 0.97693200 & 2.44264700 & 1.30606000 & $\mathrm{H}$ & -1.47207100 & -0.98067100 & 2.96387900 \\
\hline $\mathrm{C}$ & -2.02952900 & 2.55224900 & -0.39587700 & $\mathrm{H}$ & -1.05322600 & -0.27252500 & 1.40983600 \\
\hline $\mathrm{C}$ & -1.74991500 & 2.46544500 & -1.77232200 & $\mathrm{C}$ & 0.51089200 & -0.09096300 & 2.88325700 \\
\hline $\mathrm{C}$ & -3.22585500 & 3.17460400 & 0.00030600 & $\mathrm{H}$ & 0.60881900 & 0.12910100 & 3.95417900 \\
\hline $\mathrm{C}$ & -2.63814000 & 2.96764700 & -2.72129900 & $\mathrm{H}$ & 0.99909800 & 0.73927500 & 2.35798300 \\
\hline $\mathrm{H}$ & -0.83269200 & 1.98369100 & -2.10085100 & $\mathrm{C}$ & 1.29585800 & -1.38631000 & 2.60858900 \\
\hline $\mathrm{C}$ & -4.11095600 & 3.68233900 & -0.95189800 & $\mathrm{H}$ & 2.34643400 & -1.23137200 & 2.87432500 \\
\hline $\mathrm{H}$ & -3.46123400 & 3.26976200 & 1.05202600 & $\mathrm{H}$ & 0.93264100 & -2.19719800 & 3.24754300 \\
\hline $\mathrm{C}$ & -3.82704600 & 3.57803900 & -2.31394600 & $\mathrm{P}$ & 1.29726600 & -1.97410500 & 0.84134300 \\
\hline $\mathrm{H}$ & -2.40283900 & 2.88022500 & -3.77789500 & $\mathrm{C}$ & 2.81319500 & -3.11627800 & 0.67787000 \\
\hline $\mathrm{H}$ & -5.02467000 & 4.16807400 & -0.62238700 & $\mathrm{C}$ & -0.16959300 & -3.10222100 & 0.67178800 \\
\hline $\mathrm{H}$ & -4.52095200 & 3.97090300 & -3.05069200 & $\mathrm{C}$ & -0.68266800 & -3.87427000 & 1.72259800 \\
\hline $\mathrm{C}$ & -1.32515600 & 1.51064100 & 1.95258000 & $\mathrm{C}$ & -1.72134800 & -3.96271400 & -0.80166700 \\
\hline $\mathrm{O}$ & -0.46183000 & 1.16979300 & 2.74318200 & $\mathrm{C}$ & -1.76577800 & -4.71773000 & 1.46863300 \\
\hline $\mathrm{O}$ & -2.63511000 & 1.41751800 & 2.21058300 & $\mathrm{H}$ & -0.25414100 & -3.83215500 & 2.71690000 \\
\hline $\mathrm{C}$ & -2.97723400 & 0.89491500 & 3.51406400 & $\mathrm{C}$ & -2.29855500 & -4.76631600 & 0.18286000 \\
\hline $\mathrm{H}$ & -4.06358400 & 0.94195300 & 3.56407600 & $\mathrm{H}$ & -2.10647700 & -3.96070100 & -1.81832200 \\
\hline $\mathrm{H}$ & -2.63255100 & -0.13639200 & 3.60626800 & $\mathrm{H}$ & -2.18244700 & -5.32586400 & 2.26573800 \\
\hline \multirow[t]{2}{*}{$\mathrm{H}$} & -2.52200800 & 1.50457900 & 4.29675300 & $\mathrm{H}$ & -3.14100800 & -5.40665100 & -0.05566300 \\
\hline & & & & $\mathrm{N}$ & -0.68605200 & -3.15086600 & -0.56434600 \\
\hline \multicolumn{4}{|c|}{ TS44 } & $\mathrm{Pd}$ & 1.26814400 & -0.25861400 & -0.85530300 \\
\hline \multicolumn{4}{|c|}{ imaginary frequency $=-666.86 \mathrm{~cm}^{-1}$} & $\mathrm{H}$ & 2.38549900 & 0.56029600 & -0.01034200 \\
\hline $\mathrm{C}$ & -3.80963300 & 0.16771300 & -0.44537300 & $\mathrm{C}$ & 2.78915200 & -4.23657100 & 1.73455500 \\
\hline $\mathrm{C}$ & -4.92433500 & -0.46346500 & 0.13021200 & $\mathrm{H}$ & 1.91185400 & -4.88051500 & 1.63179100 \\
\hline $\mathrm{C}$ & -5.56917400 & -1.48379700 & -0.56814000 & $\mathrm{H}$ & 3.67533600 & -4.86759900 & 1.60454800 \\
\hline $\mathrm{C}$ & -5.09035200 & -1.84555900 & -1.82626500 & $\mathrm{H}$ & 2.81758800 & -3.84552000 & 2.75627600 \\
\hline $\mathrm{C}$ & -3.97515100 & -1.16959900 & -2.32252400 & $\mathrm{C}$ & 4.08648500 & -2.26530100 & 0.85138100 \\
\hline $\mathrm{H}$ & -5.29726300 & -0.15533300 & 1.10064400 & $\mathrm{H}$ & 4.12441400 & -1.43480000 & 0.14063900 \\
\hline $\mathrm{H}$ & -6.43799600 & -1.97720700 & -0.14159600 & $\mathrm{H}$ & 4.18440900 & -1.85471800 & 1.86088000 \\
\hline $\mathrm{H}$ & -5.56829100 & -2.62432600 & -2.41207600 & $\mathrm{H}$ & 4.96229100 & -2.89921000 & 0.67403000 \\
\hline $\mathrm{H}$ & -3.56748300 & -1.42044800 & -3.30022700 & $\mathrm{C}$ & 2.79222000 & -3.72465300 & -0.73862200 \\
\hline $\mathrm{N}$ & -3.33935300 & -0.19793200 & -1.65564100 & $\mathrm{H}$ & 2.83273900 & -2.95040100 & -1.51160600 \\
\hline $\mathrm{P}$ & -2.84666600 & 1.57548800 & 0.30764300 & $\mathrm{H}$ & 3.66930700 & -4.36856800 & -0.86598600 \\
\hline $\mathrm{C}$ & -3.96159200 & 3.08503000 & -0.09161100 & $\mathrm{H}$ & 1.90247100 & -4.33500200 & -0.91485700 \\
\hline $\mathrm{C}$ & -3.07312300 & 1.32766800 & 2.16855300 & $\mathrm{C}$ & -3.29481600 & 4.31218000 & 0.56230100 \\
\hline $\mathrm{H}$ & -3.60294700 & 2.18824100 & 2.58788200 & $\mathrm{H}$ & -3.27803300 & 4.24522700 & 1.65564500 \\
\hline
\end{tabular}




\begin{tabular}{|c|c|c|c|c|c|c|c|}
\hline $\mathrm{H}$ & -2.26698000 & 4.44778700 & 0.20934300 & $\mathrm{C}$ & -4.25416000 & 0.55776200 & -0.21512600 \\
\hline $\mathrm{H}$ & -3.85599000 & 5.21806500 & 0.30429700 & $\mathrm{H}$ & -3.75708100 & -2.71761300 & -2.11361000 \\
\hline $\mathrm{C}$ & -5.41237000 & 2.95787200 & 0.40015300 & $\mathrm{H}$ & -5.93349700 & -1.54714100 & -2.26876100 \\
\hline $\mathrm{H}$ & -5.96772300 & 3.87075700 & 0.15169900 & $\mathrm{H}$ & -6.25583100 & 0.60609700 & -1.01499200 \\
\hline $\mathrm{H}$ & -5.93176700 & 2.12112400 & -0.07502100 & $\mathrm{H}$ & -4.32767000 & 1.48818500 & 0.33899600 \\
\hline $\mathrm{H}$ & -5.47545600 & 2.82899600 & 1.48579800 & $\mathrm{~N}$ & -3.07601800 & -0.07235600 & -0.14513800 \\
\hline $\mathrm{C}$ & -3.94522200 & 3.26344300 & -1.62248800 & $\mathrm{P}$ & -1.17782000 & -1.96355500 & -0.57603000 \\
\hline $\mathrm{H}$ & -4.51131800 & 4.16214500 & -1.89515200 & $\mathrm{C}$ & -1.49410500 & -3.34057500 & 0.71871000 \\
\hline $\mathrm{H}$ & -2.92526600 & 3.38218700 & -2.00254500 & $\mathrm{C}$ & -0.99409100 & -2.76373200 & -2.26390800 \\
\hline $\mathrm{H}$ & -4.39811100 & 2.41317800 & -2.13985000 & $\mathrm{H}$ & -1.79121200 & -3.50752800 & -2.36434900 \\
\hline C & -0.00845600 & -1.05128300 & -2.19383400 & $\mathrm{H}$ & -1.24122400 & -1.94762100 & -2.95361300 \\
\hline $\mathrm{O}$ & -0.60838900 & -1.39268200 & -3.09770000 & $\mathrm{C}$ & 0.33901200 & -3.39110900 & -2.70633600 \\
\hline $\mathrm{C}$ & 2.60051700 & 1.74677800 & -1.07149900 & $\mathrm{H}$ & 0.19137800 & -3.70336000 & -3.74635900 \\
\hline C & 1.66691000 & 1.47434900 & -2.10814300 & $\mathrm{H}$ & 0.52730800 & -4.31619400 & -2.14991600 \\
\hline C & 4.03588100 & 1.29937800 & -1.40505000 & C & 1.55822600 & -2.45378800 & -2.60007800 \\
\hline $\mathrm{O}$ & 4.37957800 & 0.96132700 & -2.51404000 & $\mathrm{H}$ & 2.12627900 & -2.46060100 & -3.53699000 \\
\hline $\mathrm{O}$ & 4.83171600 & 1.30653100 & -0.32910600 & $\mathrm{H}$ & 1.22001500 & -1.41834600 & -2.46788400 \\
\hline $\mathrm{C}$ & 6.20907700 & 0.92850700 & -0.57019400 & $\mathrm{C}$ & 2.50207700 & -2.83741100 & -1.45153000 \\
\hline $\mathrm{H}$ & 6.25896700 & -0.09962000 & -0.93394100 & $\mathrm{H}$ & 3.08871200 & -3.71536700 & -1.75000000 \\
\hline $\mathrm{H}$ & 6.65777900 & 1.59409800 & -1.30977600 & $\mathrm{H}$ & 1.91921300 & -3.15361500 & -0.58104000 \\
\hline $\mathrm{H}$ & 6.70652400 & 1.02556500 & 0.39309000 & $\mathrm{C}$ & 3.45792300 & -1.70561400 & -1.05865500 \\
\hline $\mathrm{H}$ & 2.05668100 & 1.05299100 & -3.03000300 & $\mathrm{H}$ & 4.35862600 & -2.10063800 & -0.58226500 \\
\hline $\mathrm{H}$ & 0.75651800 & 2.06122700 & -2.16915900 & $\mathrm{H}$ & 3.77367100 & -1.15267400 & -1.94673700 \\
\hline C & 2.36658900 & 2.89849800 & -0.13036700 & $\mathrm{P}$ & 2.71694100 & -0.43301100 & 0.09971000 \\
\hline C & 3.37271400 & 3.84700400 & 0.10304400 & $\mathrm{C}$ & 3.55894000 & -0.81707200 & 1.78084900 \\
\hline C & 1.09696600 & 3.09686000 & 0.43547600 & $\mathrm{C}$ & 3.46735100 & 1.12323900 & -0.60943500 \\
\hline C & 3.10971900 & 4.97399900 & 0.88348700 & C & 4.04093600 & 2.17206400 & 0.12057300 \\
\hline $\mathrm{H}$ & 4.35978200 & 3.72066300 & -0.32451500 & C & 3.75398500 & 2.27134400 & -2.59079500 \\
\hline $\mathrm{C}$ & 0.84391000 & 4.21903600 & 1.22091800 & $\mathrm{C}$ & 4.49293000 & 3.30209300 & -0.56491300 \\
\hline $\mathrm{H}$ & 0.30002500 & 2.37671600 & 0.26380800 & $\mathrm{H}$ & 4.14312400 & 2.12010200 & 1.19652800 \\
\hline $\mathrm{C}$ & 1.84932800 & 5.16245900 & 1.44794900 & $\mathrm{C}$ & 4.35058500 & 3.35824000 & -1.94857600 \\
\hline $\mathrm{H}$ & 3.89691400 & 5.70296700 & 1.04838100 & $\mathrm{H}$ & 3.61827600 & 2.27139600 & -3.67011000 \\
\hline $\mathrm{H}$ & -0.14151300 & 4.35672700 & 1.65487500 & $\mathrm{H}$ & 4.94992700 & 4.12274500 & -0.02001200 \\
\hline \multirow[t]{2}{*}{$\mathrm{H}$} & 1.65046200 & 6.03616700 & 2.06069400 & $\mathrm{H}$ & 4.68892800 & 4.21662200 & -2.51902000 \\
\hline & & & & $\mathrm{N}$ & 3.32055300 & 1.18435100 & -1.94424400 \\
\hline \multirow{2}{*}{\multicolumn{4}{|c|}{$\begin{array}{l}\text { TS44a } \\
\text { imaginary frequency }=-296.08 \mathrm{~cm}^{-1}\end{array}$}} & $\mathrm{Pd}$ & 0.21355200 & -0.09611400 & 0.18686800 \\
\hline & & & & $\mathrm{H}$ & -1.35133600 & 0.37198500 & 0.32992000 \\
\hline $\mathrm{C}$ & -2.88555700 & -1.22340500 & -0.80099800 & $\mathrm{C}$ & 3.17268500 & -2.26428400 & 2.15622600 \\
\hline C & -3.90563800 & -1.78788200 & -1.57848100 & $\mathrm{H}$ & 2.09159300 & -2.36825700 & 2.28556600 \\
\hline $\mathrm{C}$ & -5.13393600 & -1.12992000 & -1.66417200 & $\mathrm{H}$ & 3.51175800 & -3.00130900 & 1.42301600 \\
\hline C & -5.31772800 & 0.06347200 & -0.96914500 & $\mathrm{H}$ & 3.64170200 & -2.51891300 & 3.11312100 \\
\hline
\end{tabular}




\begin{tabular}{|c|c|c|c|c|c|c|c|}
\hline $\mathrm{C}$ & 5.09607000 & -0.71291700 & 1.70273000 & $\mathrm{H}$ & -3.06385200 & 5.19669900 & -2.70077300 \\
\hline $\mathrm{H}$ & 5.51734000 & -0.96799700 & 2.68187500 & & & & \\
\hline $\mathrm{H}$ & 5.52726400 & -1.40892700 & 0.97794500 & \multirow{2}{*}{\multicolumn{4}{|c|}{$\begin{array}{l}\text { TS45 } \\
\text { imaginary frequency }=-1259.79 \mathrm{~cm}^{-1}\end{array}$}} \\
\hline $\mathrm{H}$ & 5.44176500 & 0.29059100 & 1.44723400 & & & & \\
\hline $\mathrm{C}$ & 3.02435800 & 0.10295900 & 2.89793600 & $\mathrm{C}$ & 1.26075800 & 2.86217500 & -0.10242600 \\
\hline $\mathrm{H}$ & 1.93957000 & 0.02438800 & 3.01112600 & $\mathrm{C}$ & 0.38452100 & 3.94912900 & -0.18459100 \\
\hline $\mathrm{H}$ & 3.47643200 & -0.19875700 & 3.84961200 & $\mathrm{C}$ & 0.76871800 & 5.08274400 & -0.90403800 \\
\hline $\mathrm{H}$ & 3.27865600 & 1.15570300 & 2.74930800 & $\mathrm{C}$ & 2.01829300 & 5.11120400 & -1.52257900 \\
\hline $\mathrm{C}$ & -0.19156400 & -4.12666100 & 0.95026200 & $\mathrm{C}$ & 2.83733100 & 3.99121900 & -1.40858400 \\
\hline $\mathrm{H}$ & 0.62945100 & -3.47337500 & 1.25207200 & $\mathrm{H}$ & -0.58512800 & 3.91055000 & 0.29789500 \\
\hline $\mathrm{H}$ & -0.35018200 & -4.85347700 & 1.75500200 & $\mathrm{H}$ & 0.09682100 & 5.93179100 & -0.98178500 \\
\hline $\mathrm{H}$ & 0.11709200 & -4.68575700 & 0.06288100 & $\mathrm{H}$ & 2.34961200 & 5.97251300 & -2.09168600 \\
\hline $\mathrm{C}$ & -2.59899700 & -4.31981700 & 0.28288500 & $\mathrm{H}$ & 3.81349400 & 3.94464100 & -1.88256300 \\
\hline $\mathrm{H}$ & -2.73323200 & -5.07240900 & 1.06821400 & $\mathrm{~N}$ & 2.45436300 & 2.90686900 & -0.72097800 \\
\hline $\mathrm{H}$ & -3.56322100 & -3.82627400 & 0.13943000 & $\mathrm{P}$ & 0.90393800 & 1.25579400 & 0.77436100 \\
\hline $\mathrm{H}$ & -2.34084200 & -4.85849800 & -0.63424500 & $\mathrm{C}$ & 1.00022900 & 1.65886800 & 2.63365800 \\
\hline $\mathrm{C}$ & -1.90732100 & -2.63563900 & 2.02684500 & $\mathrm{C}$ & -0.88241900 & 0.94000300 & 0.35128600 \\
\hline $\mathrm{H}$ & -2.86051900 & -2.10906800 & 1.92311700 & $\mathrm{H}$ & -1.52147900 & 1.46922200 & 1.06763800 \\
\hline $\mathrm{H}$ & -2.02607300 & -3.38463300 & 2.81785500 & $\mathrm{H}$ & -1.04918500 & 1.40549100 & -0.62481700 \\
\hline $\mathrm{H}$ & -1.15535100 & -1.91200700 & 2.35790000 & $\mathrm{C}$ & -1.27045400 & -0.55053200 & 0.29106700 \\
\hline $\mathrm{C}$ & 0.63969400 & 1.92814500 & 0.94180100 & $\mathrm{H}$ & -1.00850300 & -1.04306600 & 1.23454100 \\
\hline $\mathrm{H}$ & 1.16586600 & 2.46975300 & 0.16498400 & $\mathrm{H}$ & -0.67781800 & -1.04144000 & -0.49164200 \\
\hline $\mathrm{H}$ & 1.15948100 & 1.74649600 & 1.87398200 & $\mathrm{C}$ & -2.76946900 & -0.76636700 & 0.01769500 \\
\hline $\mathrm{C}$ & -0.76489500 & 1.96450700 & 0.96317200 & $\mathrm{H}$ & -2.98813300 & -1.83634000 & 0.10956900 \\
\hline $\mathrm{C}$ & -1.37564500 & 1.61123500 & 2.31502700 & $\mathrm{H}$ & -3.35201500 & -0.27240600 & 0.80671100 \\
\hline $\mathrm{O}$ & -0.74190400 & 1.10504000 & 3.21896600 & $\mathrm{C}$ & -3.24120800 & -0.27733900 & -1.35906300 \\
\hline $\mathrm{O}$ & -2.68013700 & 1.90760000 & 2.39877000 & $\mathrm{H}$ & -2.62044400 & -0.75172800 & -2.13311000 \\
\hline $\mathrm{C}$ & -3.30571900 & 1.59454500 & 3.66362500 & $\mathrm{H}$ & -3.07797700 & 0.80475700 & -1.45105200 \\
\hline $\mathrm{H}$ & -4.33674700 & 1.93163500 & 3.56810600 & $\mathrm{C}$ & -4.71962300 & -0.58354000 & -1.65883600 \\
\hline $\mathrm{H}$ & -3.26359300 & 0.51933400 & 3.84876300 & $\mathrm{H}$ & -4.93907000 & -0.27689000 & -2.68656400 \\
\hline $\mathrm{H}$ & -2.79988700 & 2.11885000 & 4.47637700 & $\mathrm{H}$ & -4.91563500 & -1.65723800 & -1.57991800 \\
\hline $\mathrm{C}$ & -1.48449500 & 2.80823600 & -0.05242900 & $\mathrm{P}$ & -5.91430500 & 0.34191800 & -0.54622000 \\
\hline $\mathrm{C}$ & -1.31802200 & 2.56911000 & -1.42496900 & $\mathrm{C}$ & -7.52662100 & 0.33736700 & -1.59037000 \\
\hline $\mathrm{C}$ & -2.22729100 & 3.92997400 & 0.34906900 & $\mathrm{C}$ & -6.28442600 & -0.96543500 & 0.73892800 \\
\hline $\mathrm{C}$ & -1.88116000 & 3.42200600 & -2.37287000 & $\mathrm{C}$ & -6.84180800 & -0.56113600 & 1.96355900 \\
\hline $\mathrm{H}$ & -0.74935200 & 1.69990300 & -1.74632400 & $\mathrm{C}$ & -6.26298600 & -3.16605900 & 1.43073700 \\
\hline $\mathrm{C}$ & -2.78996200 & 4.78265600 & -0.60193800 & $\mathrm{C}$ & -7.13103400 & -1.51957100 & 2.93335000 \\
\hline $\mathrm{H}$ & -2.36183800 & 4.14446300 & 1.40259200 & $\mathrm{H}$ & -7.04120500 & 0.49046900 & 2.14603000 \\
\hline $\mathrm{C}$ & -2.62205700 & 4.53261700 & -1.96423300 & $\mathrm{C}$ & -6.83672400 & -2.85567000 & 2.66405000 \\
\hline $\mathrm{H}$ & -1.74398200 & 3.21580900 & -3.43025100 & $\mathrm{H}$ & -6.01002600 & -4.19617700 & 1.18509600 \\
\hline $\mathrm{H}$ & -3.35707400 & 5.64800100 & -0.27235500 & $\mathrm{H}$ & -7.57143100 & -1.22840900 & 3.88280100 \\
\hline
\end{tabular}




\begin{tabular}{|c|c|c|c|c|c|c|c|}
\hline $\mathrm{H}$ & -7.03961600 & -3.63811800 & 3.38819700 & $\mathrm{C}$ & 7.59238200 & -1.95035000 & 1.69618600 \\
\hline $\mathrm{N}$ & -5.99313800 & -2.25456300 & 0.48756400 & $\mathrm{H}$ & 8.04168000 & -3.43771700 & 0.20442600 \\
\hline $\mathrm{Pd}$ & 2.66540500 & -0.21247300 & -0.05067200 & $\mathrm{H}$ & 6.91208200 & -0.34569900 & 2.96904300 \\
\hline $\mathrm{H}$ & 3.18799000 & 1.63660200 & -0.72441300 & $\mathrm{H}$ & 8.36185100 & -2.28504800 & 2.38477800 \\
\hline $\mathrm{C}$ & -7.32895700 & 1.37419700 & -2.71492000 & $\mathrm{C}$ & 4.12420800 & -1.32272000 & -2.28382400 \\
\hline $\mathrm{H}$ & -7.10179300 & 2.36716400 & -2.31293100 & $\mathrm{O}$ & 3.75483300 & -0.77116700 & -3.30248800 \\
\hline $\mathrm{H}$ & -6.52468700 & 1.09414800 & -3.40320900 & $\mathrm{O}$ & 4.13775600 & -2.65730400 & -2.12905500 \\
\hline $\mathrm{H}$ & -8.24773500 & 1.45476500 & -3.30838500 & $\mathrm{C}$ & 3.70261300 & -3.42109200 & -3.27641400 \\
\hline $\mathrm{C}$ & -7.86812100 & -1.03573000 & -2.19213600 & $\mathrm{H}$ & 3.80738600 & -4.46469600 & -2.98406500 \\
\hline $\mathrm{H}$ & -8.81570300 & -0.97398300 & -2.74250400 & $\mathrm{H}$ & 2.66236000 & -3.18750400 & -3.51356700 \\
\hline $\mathrm{H}$ & -7.10396300 & -1.37663500 & -2.89701500 & $\mathrm{H}$ & 4.32672900 & -3.19511500 & -4.14312000 \\
\hline $\mathrm{H}$ & -7.97673700 & -1.80316400 & -1.42124300 & & & & \\
\hline $\mathrm{C}$ & -8.67741200 & 0.80921000 & -0.68111400 & \multirow{2}{*}{\multicolumn{4}{|c|}{$\begin{array}{l}\text { TS45a } \\
\text { imaginary frequency }=-1319.79 \mathrm{~cm}^{-1}\end{array}$}} \\
\hline $\mathrm{H}$ & -8.46029000 & 1.78029600 & -0.22253800 & & & & \\
\hline $\mathrm{H}$ & -9.59346000 & 0.91958900 & -1.27382600 & $\mathrm{C}$ & -1.50508300 & -2.47504800 & -0.59869100 \\
\hline $\mathrm{H}$ & -8.88759800 & 0.09224200 & 0.11783400 & $\mathrm{C}$ & -1.55967600 & -3.86416100 & -0.78116100 \\
\hline $\mathrm{C}$ & 0.52858400 & 0.40304700 & 3.39631100 & $\mathrm{C}$ & -0.73216800 & -4.48559600 & -1.71550300 \\
\hline $\mathrm{H}$ & 1.11153900 & -0.48426600 & 3.13089300 & $\mathrm{C}$ & 0.15855800 & -3.71017100 & -2.45673900 \\
\hline $\mathrm{H}$ & 0.65657700 & 0.57112200 & 4.47125000 & $\mathrm{C}$ & 0.18717100 & -2.34107300 & -2.21899100 \\
\hline $\mathrm{H}$ & -0.53014300 & 0.18728200 & 3.22505100 & $\mathrm{H}$ & -2.24610700 & -4.45819900 & -0.19071300 \\
\hline $\mathrm{C}$ & 0.13404700 & 2.86539200 & 3.03675100 & $\mathrm{H}$ & -0.78204100 & -5.56064900 & -1.86054900 \\
\hline $\mathrm{H}$ & 0.19657300 & 2.99870000 & 4.12246100 & $\mathrm{H}$ & 0.82013900 & -4.15017000 & -3.19457800 \\
\hline $\mathrm{H}$ & 0.47988000 & 3.79505800 & 2.57680800 & $\mathrm{H}$ & 0.87392300 & -1.68477500 & -2.74457700 \\
\hline $\mathrm{H}$ & -0.92383000 & 2.72573300 & 2.79367400 & $\mathrm{~N}$ & -0.61711400 & -1.75018300 & -1.32101600 \\
\hline $\mathrm{C}$ & 2.47758700 & 1.94439900 & 2.96557600 & $\mathrm{P}$ & -2.55819500 & -1.49021500 & 0.60883200 \\
\hline $\mathrm{H}$ & 2.85976000 & 2.81766500 & 2.42667200 & $\mathrm{C}$ & -4.35635300 & -1.83493700 & 0.03458400 \\
\hline $\mathrm{H}$ & 2.57661900 & 2.15057500 & 4.03692600 & $\mathrm{C}$ & -2.41383600 & -2.53267400 & 2.17038900 \\
\hline $\mathrm{H}$ & 3.11513100 & 1.08599300 & 2.73102900 & $\mathrm{H}$ & -3.33564000 & -2.35121600 & 2.73385700 \\
\hline $\mathrm{C}$ & 4.55410300 & -0.56295300 & -1.03962100 & $\mathrm{H}$ & -2.39322800 & -3.60482700 & 1.94333500 \\
\hline $\mathrm{C}$ & 4.32772300 & 0.86963600 & -1.15112200 & $\mathrm{C}$ & -1.20209200 & -2.14442100 & 3.03789300 \\
\hline $\mathrm{C}$ & 2.12764800 & -2.03689500 & 0.47183100 & $\mathrm{H}$ & -1.22597000 & -2.72737200 & 3.96738800 \\
\hline $\mathrm{O}$ & 1.80019900 & -3.07844900 & 0.80224300 & $\mathrm{H}$ & -1.29424600 & -1.09053100 & 3.32955100 \\
\hline $\mathrm{H}$ & 4.13270600 & 1.17160300 & -2.18069700 & $\mathrm{C}$ & 0.12742800 & -2.35565700 & 2.31152800 \\
\hline $\mathrm{H}$ & 5.04085500 & 1.49564700 & -0.61788000 & $\mathrm{H}$ & 0.25577900 & -3.42049300 & 2.07047700 \\
\hline $\mathrm{C}$ & 5.58791500 & -1.08632400 & -0.09704200 & $\mathrm{H}$ & 0.07515000 & -1.83218000 & 1.35084100 \\
\hline $\mathrm{C}$ & 6.41819400 & -2.17705200 & -0.41189200 & $\mathrm{C}$ & 1.37953200 & -1.84882200 & 3.03150900 \\
\hline $\mathrm{C}$ & 5.79731200 & -0.43309500 & 1.13586600 & $\mathrm{H}$ & 1.62741500 & -2.50161700 & 3.87703100 \\
\hline $\mathrm{C}$ & 7.40919300 & -2.59785000 & 0.47514800 & $\mathrm{H}$ & 1.19429500 & -0.85557800 & 3.45837700 \\
\hline $\mathrm{H}$ & 6.29590900 & -2.69948900 & -1.35086100 & $\mathrm{C}$ & 2.57993700 & -1.80580200 & 2.06337300 \\
\hline $\mathrm{C}$ & 6.77890700 & -0.86220700 & 2.02325700 & $\mathrm{H}$ & 3.52596100 & -1.75505500 & 2.60984700 \\
\hline $\mathrm{H}$ & 5.18004900 & 0.41879500 & 1.40706300 & $\mathrm{H}$ & 2.59621300 & -2.73893400 & 1.49103900 \\
\hline
\end{tabular}




\begin{tabular}{|c|c|c|c|c|c|c|c|}
\hline $\mathrm{P}$ & 2.51414500 & -0.38227100 & 0.83964400 & $\mathrm{C}$ & -0.78976800 & 2.15453000 & -0.97750100 \\
\hline $\mathrm{C}$ & 3.86858600 & 0.82415200 & 1.47137200 & $\mathrm{H}$ & 0.50133200 & 1.11894600 & -2.38510300 \\
\hline $\mathrm{C}$ & 3.27869400 & -1.06165300 & -0.71225600 & $\mathrm{H}$ & -1.22660600 & 0.55637100 & -2.31440300 \\
\hline $\mathrm{C}$ & 4.04204800 & -2.23630800 & -0.77236200 & $\mathrm{C}$ & -2.23858100 & 2.45413500 & -0.75161700 \\
\hline $\mathrm{C}$ & 3.62566400 & -0.65636100 & -2.95702600 & $\mathrm{C}$ & -3.16111700 & 2.22506500 & -1.78856400 \\
\hline $\mathrm{C}$ & 4.59666900 & -2.61999300 & -1.99436300 & $\mathrm{C}$ & -2.70437200 & 3.06174100 & 0.42725800 \\
\hline $\mathrm{H}$ & 4.22220600 & -2.83465100 & 0.11318600 & $\mathrm{C}$ & -4.50642900 & 2.56911200 & -1.64386700 \\
\hline $\mathrm{C}$ & 4.38842100 & -1.81564200 & -3.11278000 & $\mathrm{H}$ & -2.82199300 & 1.82057000 & -2.73741700 \\
\hline $\mathrm{H}$ & 3.44743900 & 0.00557900 & -3.80195700 & $\mathrm{C}$ & -4.04785600 & 3.39534700 & 0.57482900 \\
\hline $\mathrm{H}$ & 5.19206600 & -3.52549800 & -2.06472600 & $\mathrm{H}$ & -2.01008000 & 3.27906400 & 1.22822100 \\
\hline $\mathrm{H}$ & 4.80868100 & -2.07094200 & -4.07995900 & $\mathrm{C}$ & -4.95669100 & 3.14896200 & -0.45855300 \\
\hline $\mathrm{N}$ & 3.07038100 & -0.28871500 & -1.79487400 & $\mathrm{H}$ & -5.19534100 & 2.39631700 & -2.46522300 \\
\hline $\mathrm{Pd}$ & 0.35773100 & 0.91525700 & 0.37802100 & $\mathrm{H}$ & -4.38527400 & 3.85753800 & 1.49755600 \\
\hline $\mathrm{C}$ & 5.27332700 & 0.19771500 & 1.39033900 & $\mathrm{H}$ & -6.00171400 & 3.42028600 & -0.34427400 \\
\hline $\mathrm{H}$ & 5.57434000 & -0.00806400 & 0.36031700 & $\mathrm{C}$ & 0.09449000 & 3.39239200 & -0.95216400 \\
\hline $\mathrm{H}$ & 6.00012300 & 0.90087100 & 1.81248300 & $\mathrm{O}$ & -0.12348200 & 4.37212500 & -0.27117400 \\
\hline $\mathrm{H}$ & 5.35642400 & -0.73089800 & 1.96421600 & $\mathrm{O}$ & 1.14311300 & 3.28991500 & -1.79455000 \\
\hline $\mathrm{C}$ & 3.55816200 & 1.18857300 & 2.93677200 & $\mathrm{C}$ & 1.99349400 & 4.45335400 & -1.85957200 \\
\hline $\mathrm{H}$ & 2.57310200 & 1.64642800 & 3.05396200 & $\mathrm{H}$ & 2.75472700 & 4.21569100 & -2.60130900 \\
\hline $\mathrm{H}$ & 3.61900100 & 0.32613200 & 3.60709300 & $\mathrm{H}$ & 1.41518300 & 5.32789900 & -2.16435600 \\
\hline $\mathrm{H}$ & 4.29678700 & 1.91965700 & 3.28392800 & $\mathrm{H}$ & 2.44894900 & 4.64910900 & -0.88644300 \\
\hline
\end{tabular}

H

$\mathrm{H}$

H

C

H

H

H

C

H

H

H

C

H

H

H

C

O

H

C
3.82293100

2.09496700

0.60229900

2.85683900

2.60133000

0.68506800

4.59883700

2.79131900

0.94101300

3.99974000

1.87466200

$-0.45368600$

$-4.47399400$

$-1.42235800$

$-1.44500400$

$-4.15093700$

$-0.3896310$

$-1.60521300$

$-3.89080000$

$-2.07753000$

$-2.10077100$

$-5.52109800$

$-1.49290600$

$-1.76205000$

$-5.22809700$

$-0.89306200$

0.89545900

$-6.27657900$

$-0.98670000$

0.58937000

$-5.18093700$

$-1.14341300$

1.96086300

$-4.93454200$

0.15399800

0.77707300

$-4.84918000$

$-3.28236100$

0.20502900

$-5.91195700$

$-3.33394400$

$-0.06071800$

$-4.32613200$

$-3.97882900$

$-0.45605300$

$-4.75666600$

$-3.64133100$

1.23410100

0.08262400

1.80141100

2.09945300

$-0.14666300$

2.2314120

3.13418100

$-0.37111800$

$-0.28853900$

$-1.19676000$

$-0.39030400$

$1.00511700 \quad-1.77568700$ 


\begin{tabular}{|c|c|c|c|c|c|c|c|}
\hline $\mathrm{H}$ & -3.47902900 & -3.46454000 & -1.42074700 & $\mathrm{H}$ & -5.63650900 & -1.22460800 & 2.20321100 \\
\hline $\mathrm{C}$ & -1.76632000 & -2.79745800 & -2.57918100 & $\mathrm{H}$ & -5.60124900 & 0.09706400 & 1.04244800 \\
\hline $\mathrm{H}$ & -1.64460700 & -3.46767200 & -3.43726700 & $\mathrm{H}$ & -5.61728300 & -1.59090700 & 0.48392200 \\
\hline $\mathrm{H}$ & -1.42342500 & -1.81147400 & -2.91948900 & $\mathrm{C}$ & -3.20732100 & -0.00273800 & 2.45199200 \\
\hline $\mathrm{C}$ & -0.88158800 & -3.28621700 & -1.42382200 & $\mathrm{H}$ & -3.53849100 & 1.02762300 & 2.28683500 \\
\hline $\mathrm{H}$ & -0.96033300 & -4.37727000 & -1.35636300 & $\mathrm{H}$ & -3.63438500 & -0.33123900 & 3.40628100 \\
\hline $\mathrm{H}$ & -1.29191400 & -2.91111700 & -0.48150500 & $\mathrm{H}$ & -2.11801800 & 0.00043500 & 2.55863400 \\
\hline $\mathrm{C}$ & 0.59371600 & -2.87063900 & -1.56816600 & $\mathrm{C}$ & 0.97133200 & 1.74561200 & 0.97478900 \\
\hline $\mathrm{H}$ & 1.26418300 & -3.73447900 & -1.56553600 & $\mathrm{C}$ & -0.36049400 & 2.34036300 & 0.92026500 \\
\hline $\mathrm{H}$ & 0.78210600 & -2.36733300 & -2.51867700 & $\mathrm{H}$ & -0.84303000 & 2.44240300 & 1.89130600 \\
\hline $\mathrm{P}$ & 1.19358900 & -1.69108600 & -0.25978200 & $\mathrm{H}$ & -0.37516000 & 3.25261900 & 0.32127200 \\
\hline $\mathrm{C}$ & 1.75861400 & -2.87817500 & 1.15308800 & $\mathrm{C}$ & 1.98556500 & 2.31533700 & 0.02158100 \\
\hline $\mathrm{C}$ & 2.78692600 & -1.10708200 & -1.03999000 & $\mathrm{C}$ & 1.75430400 & 2.23718300 & -1.36409900 \\
\hline $\mathrm{C}$ & 3.76253400 & -0.40615800 & -0.31706300 & $\mathrm{C}$ & 3.09292200 & 3.06151500 & 0.46145500 \\
\hline $\mathrm{C}$ & 4.05145200 & -1.00763100 & -2.96266700 & $\mathrm{C}$ & 2.60123500 & 2.86268100 & -2.27644200 \\
\hline $\mathrm{C}$ & 4.93440500 & -0.02668700 & -0.96908100 & $\mathrm{H}$ & 0.90605800 & 1.66127200 & -1.72650500 \\
\hline $\mathrm{H}$ & 3.60800500 & -0.13703900 & 0.72018500 & $\mathrm{C}$ & 3.93937800 & 3.69043200 & -0.45283600 \\
\hline $\mathrm{C}$ & 5.08957100 & -0.33531700 & -2.31931800 & $\mathrm{H}$ & 3.29258900 & 3.16090900 & 1.52050400 \\
\hline $\mathrm{H}$ & 4.12159100 & -1.25649400 & -4.01946100 & $\mathrm{C}$ & 3.70206700 & 3.59280100 & -1.82385800 \\
\hline $\mathrm{H}$ & 5.70713900 & 0.51346800 & -0.43094200 & $\mathrm{H}$ & 2.40329200 & 2.77552200 & -3.34074300 \\
\hline $\mathrm{H}$ & 5.98536900 & -0.05706300 & -2.86461400 & $\mathrm{H}$ & 4.78409800 & 4.26653200 & -0.08621700 \\
\hline $\mathrm{N}$ & 2.92829000 & -1.38980400 & -2.34274700 & $\mathrm{H}$ & 4.36386000 & 4.08287700 & -2.53156100 \\
\hline $\mathrm{Pd}$ & -0.34501200 & 0.18589900 & 0.19388100 & $\mathrm{C}$ & 1.33361100 & 1.30500100 & 2.37151300 \\
\hline $\mathrm{H}$ & -1.50662500 & 1.95860400 & 0.20373000 & $\mathrm{O}$ & 0.51113900 & 1.10446000 & 3.24860900 \\
\hline $\mathrm{C}$ & 0.61887400 & -3.86808600 & 1.46408900 & $\mathrm{O}$ & 2.66067800 & 1.17477200 & 2.58661700 \\
\hline $\mathrm{H}$ & -0.30172600 & -3.35872500 & 1.76063100 & $\mathrm{C}$ & 3.04386900 & 0.92088700 & 3.95650900 \\
\hline $\mathrm{H}$ & 0.39572100 & -4.53268900 & 0.62655500 & $\mathrm{H}$ & 4.13287800 & 0.89353700 & 3.95146000 \\
\hline $\mathrm{H}$ & 0.92479800 & -4.49906800 & 2.30619000 & $\mathrm{H}$ & 2.68100500 & 1.72334400 & 4.60188900 \\
\hline $\mathrm{C}$ & 3.00861900 & -3.67595200 & 0.73046600 & $\mathrm{H}$ & 2.63410400 & -0.02810400 & 4.30447700 \\
\hline
\end{tabular}

\section{TS46}

\begin{tabular}{lrrr}
\multicolumn{4}{l}{ imaginary frequency $=-1368.71 \mathrm{~cm}^{-1}$} \\
$\mathrm{C}$ & -2.75549400 & -1.23830700 & -0.82693200 \\
$\mathrm{C}$ & -3.81777600 & -1.79757600 & -1.54768100 \\
$\mathrm{C}$ & -5.02283900 & -1.10272500 & -1.65453900 \\
$\mathrm{C}$ & -5.15290300 & 0.13571900 & -1.02965500 \\
$\mathrm{C}$ & -4.05921700 & 0.64349300 & -0.33662100 \\
$\mathrm{H}$ & -3.71094300 & -2.76409400 & -2.02366900 \\
$\mathrm{H}$ & -5.84814100 & -1.52751700 & -2.21776700 \\
$\mathrm{H}$ & -6.07288200 & 0.70675200 & -1.08551700 \\
$\mathrm{H}$ & -4.08372700 & 1.60587500 & 0.15973000
\end{tabular}




\begin{tabular}{|c|c|c|c|c|c|c|c|}
\hline $\mathrm{N}$ & -2.89996100 & -0.02946500 & -0.25511400 & $\mathrm{H}$ & 1.75344300 & -0.02514700 & 2.99150300 \\
\hline$P$ & -1.08581300 & -2.06439700 & -0.59152100 & $\mathrm{H}$ & 3.24984100 & -0.26619900 & 3.90164700 \\
\hline $\mathrm{C}$ & -1.48974100 & -3.42113700 & 0.70475000 & $\mathrm{H}$ & 3.08716300 & 1.12876400 & 2.84718000 \\
\hline $\mathrm{C}$ & -0.95469700 & -2.89389800 & -2.27099800 & $\mathrm{C}$ & -0.25441300 & -4.32885100 & 0.86203200 \\
\hline $\mathrm{H}$ & -1.72413200 & -3.66966200 & -2.34376900 & $\mathrm{H}$ & 0.63960800 & -3.76877900 & 1.14407300 \\
\hline $\mathrm{H}$ & -1.24307400 & -2.09945600 & -2.96957800 & $\mathrm{H}$ & -0.44827200 & -5.05871700 & 1.65623200 \\
\hline $\mathrm{C}$ & 0.39836100 & -3.47160100 & -2.73124600 & $\mathrm{H}$ & -0.03818000 & -4.89166500 & -0.05041500 \\
\hline $\mathrm{H}$ & 0.25363200 & -3.77653100 & -3.77382200 & $\mathrm{C}$ & -2.69946200 & -4.29688000 & 0.33309300 \\
\hline $\mathrm{H}$ & 0.62410600 & -4.39511100 & -2.18613800 & $\mathrm{H}$ & -2.83364100 & -5.06033800 & 1.10786000 \\
\hline $\mathrm{C}$ & 1.58791400 & -2.49696200 & -2.62400700 & $\mathrm{H}$ & -3.62916800 & -3.72545500 & 0.27988200 \\
\hline $\mathrm{H}$ & 2.19641200 & -2.54216600 & -3.53416000 & $\mathrm{H}$ & -2.55941100 & -4.82763000 & -0.61410000 \\
\hline $\mathrm{H}$ & 1.21741600 & -1.46573900 & -2.56478900 & $\mathrm{C}$ & -1.76845300 & -2.69074700 & 2.03397900 \\
\hline $\mathrm{C}$ & 2.48878500 & -2.79537500 & -1.41608100 & $\mathrm{H}$ & -2.67102600 & -2.07346200 & 1.97878600 \\
\hline $\mathrm{H}$ & 3.09283200 & -3.68404100 & -1.63722600 & $\mathrm{H}$ & -1.92488700 & -3.42843900 & 2.82912300 \\
\hline $\mathrm{H}$ & 1.86913700 & -3.06477100 & -0.55592000 & $\mathrm{H}$ & -0.93561900 & -2.04582800 & 2.33128700 \\
\hline $\mathrm{C}$ & 3.42046400 & -1.63199400 & -1.04938700 & $\mathrm{C}$ & 0.48006700 & 1.79414700 & 0.84249600 \\
\hline $\mathrm{H}$ & 4.34350100 & -1.99581100 & -0.58960600 & $\mathrm{C}$ & -0.98593000 & 1.76308300 & 0.83477400 \\
\hline $\mathrm{H}$ & 3.70381900 & -1.06906600 & -1.94236100 & $\mathrm{H}$ & 0.95836200 & 2.42935100 & 0.10218700 \\
\hline $\mathrm{P}$ & 2.68021500 & -0.37163700 & 0.11527900 & $\mathrm{H}$ & 0.95751000 & 1.78602300 & 1.81728200 \\
\hline $\mathrm{C}$ & 3.44863400 & -0.79827200 & 1.81867500 & $\mathrm{C}$ & -1.60693600 & 2.78731900 & -0.10738500 \\
\hline $\mathrm{C}$ & 3.46949900 & 1.19304400 & -0.52723600 & $\mathrm{C}$ & -2.24521300 & 3.94559800 & 0.36084200 \\
\hline $\mathrm{C}$ & 3.89080000 & 2.26655700 & 0.26899800 & $\mathrm{C}$ & -1.45033700 & 2.62420100 & -1.49219300 \\
\hline $\mathrm{C}$ & 3.99892300 & 2.34216700 & -2.45593100 & $\mathrm{C}$ & -2.72220300 & 4.90369200 & -0.53628300 \\
\hline $\mathrm{C}$ & 4.39685400 & 3.40876000 & -0.35417700 & $\mathrm{H}$ & -2.37353700 & 4.10329500 & 1.42544200 \\
\hline $\mathrm{H}$ & 3.83422500 & 2.22324500 & 1.34886500 & $\mathrm{C}$ & -1.92105100 & 3.58485600 & -2.38740600 \\
\hline $\mathrm{C}$ & 4.45570300 & 3.45297700 & -1.74468600 & $\mathrm{H}$ & -0.94598000 & 1.73646700 & -1.86863300 \\
\hline $\mathrm{H}$ & 4.02100200 & 2.33230800 & -3.54343400 & $\mathrm{C}$ & -2.56494800 & 4.72831300 & -1.91172700 \\
\hline $\mathrm{H}$ & 4.73871800 & 4.24878600 & 0.24301800 & $\mathrm{H}$ & -3.21144100 & 5.79462600 & -0.15366000 \\
\hline $\mathrm{H}$ & 4.84181600 & 4.32092400 & -2.26843000 & $\mathrm{H}$ & -1.78599400 & 3.43782200 & -3.45504800 \\
\hline N & 3.51731800 & 1.24186100 & -1.86845000 & $\mathrm{H}$ & -2.93657600 & 5.47642700 & -2.60516200 \\
\hline $\mathrm{Pd}$ & 0.30006900 & -0.13447100 & 0.15309700 & $\mathrm{C}$ & -1.54176600 & 1.53686500 & 2.22863100 \\
\hline $\mathrm{H}$ & -1.70777300 & 0.60826500 & 0.26201900 & $\mathrm{O}$ & -0.89927500 & 1.06810300 & 3.14773700 \\
\hline $\mathrm{C}$ & 3.08273200 & -2.26738100 & 2.12086100 & $\mathrm{O}$ & -2.85286600 & 1.83841600 & 2.33445900 \\
\hline $\mathrm{H}$ & 1.99831000 & -2.40499200 & 2.16708300 & $\mathrm{C}$ & -3.44131800 & 1.60097000 & 3.63210000 \\
\hline $\mathrm{H}$ & 3.49661200 & -2.96882600 & 1.39139800 & $\mathrm{H}$ & -2.92301300 & 2.18282400 & 4.39644500 \\
\hline $\mathrm{H}$ & 3.49025900 & -2.53893500 & 3.10086700 & $\mathrm{H}$ & -4.47914400 & 1.91980600 & 3.54382900 \\
\hline $\mathrm{C}$ & 4.98344500 & -0.64831600 & 1.80668700 & $\mathrm{H}$ & -3.38110000 & 0.54133900 & 3.88950200 \\
\hline $\mathrm{H}$ & 5.37688600 & -0.95301000 & 2.78318800 & & & & \\
\hline $\mathrm{H}$ & 5.46145400 & -1.28311800 & 1.05512200 & \multirow{2}{*}{\multicolumn{4}{|c|}{$\begin{array}{l}\text { TS46a } \\
\text { imaginary frequency }=-993.30 \mathrm{~cm}^{-1}\end{array}$}} \\
\hline $\mathrm{H}$ & 5.30407900 & 0.38059700 & 1.62946800 & & & & \\
\hline C & 2.84150300 & 0.06797100 & 2.94104300 & $\mathrm{C}$ & 3.31797900 & -0.87049600 & 1.34065400 \\
\hline
\end{tabular}




\begin{tabular}{|c|c|c|c|c|c|c|c|}
\hline $\mathrm{C}$ & 4.11343900 & -1.18376800 & 2.44978600 & $\mathrm{H}$ & -6.82147400 & -1.60388100 & -0.86837700 \\
\hline $\mathrm{C}$ & 4.77854300 & -0.17563700 & 3.14775900 & $\mathrm{H}$ & -6.01649100 & -1.80663400 & 0.68505100 \\
\hline $\mathrm{C}$ & 4.64116400 & 1.15039100 & 2.73560200 & $\mathrm{C}$ & -4.40547900 & -2.72311400 & -1.41593200 \\
\hline $\mathrm{C}$ & 3.82499000 & 1.41961100 & 1.64658900 & $\mathrm{H}$ & -3.44690500 & -2.82909000 & -1.93325500 \\
\hline $\mathrm{H}$ & 4.21705300 & -2.21647500 & 2.76008300 & $\mathrm{H}$ & -4.41664600 & -3.41147400 & -0.56584200 \\
\hline $\mathrm{H}$ & 5.40072200 & -0.42416800 & 4.00222500 & $\mathrm{H}$ & -5.18600900 & -3.05184100 & -2.11108400 \\
\hline $\mathrm{H}$ & 5.14625400 & 1.96101200 & 3.24817200 & $\mathrm{C}$ & -4.77433900 & -0.37132800 & -2.24657800 \\
\hline $\mathrm{H}$ & 3.64364400 & 2.42228600 & 1.27627200 & $\mathrm{H}$ & -3.87061600 & -0.44064500 & -2.85864300 \\
\hline $\mathrm{N}$ & 3.19327700 & 0.43193400 & 0.98491800 & $\mathrm{H}$ & -5.61898100 & -0.69744800 & -2.86412500 \\
\hline $\mathrm{P}$ & 2.37878000 & -2.08862400 & 0.27578800 & $\mathrm{H}$ & -4.92283500 & 0.67927200 & -1.98743000 \\
\hline $\mathrm{C}$ & 3.78549500 & -2.99641500 & -0.65981100 & $\mathrm{C}$ & 3.08701400 & -4.13424300 & -1.43481700 \\
\hline $\mathrm{C}$ & 1.80229300 & -3.33373200 & 1.57077700 & $\mathrm{H}$ & 2.66733100 & -4.89498500 & -0.76836200 \\
\hline $\mathrm{H}$ & 2.44749300 & -4.21749700 & 1.55568000 & $\mathrm{H}$ & 2.28211900 & -3.75704300 & -2.07427500 \\
\hline $\mathrm{H}$ & 1.90254900 & -2.88643200 & 2.56568900 & $\mathrm{H}$ & 3.81627000 & -4.63656200 & -2.08060800 \\
\hline $\mathrm{C}$ & 0.33395200 & -3.74835000 & 1.34419100 & $\mathrm{C}$ & 4.90694600 & -3.57875700 & 0.21488800 \\
\hline $\mathrm{H}$ & 0.10820900 & -4.60236900 & 1.99603200 & $\mathrm{H}$ & 5.61058100 & -4.13260500 & -0.41799300 \\
\hline $\mathrm{H}$ & 0.20823400 & -4.10958500 & 0.31486400 & $\mathrm{H}$ & 5.47909700 & -2.79846300 & 0.72473700 \\
\hline $\mathrm{C}$ & -0.66656500 & -2.61793400 & 1.61434300 & $\mathrm{H}$ & 4.53272500 & -4.28177000 & 0.96634600 \\
\hline $\mathrm{H}$ & -0.58155800 & -2.29909300 & 2.66325200 & $\mathrm{C}$ & 4.38256400 & -1.99043800 & -1.66388400 \\
\hline $\mathrm{H}$ & -0.39742600 & -1.74578200 & 1.00490000 & $\mathrm{H}$ & 5.13808600 & -2.49020100 & -2.28095200 \\
\hline $\mathrm{C}$ & -2.12128500 & -3.00859800 & 1.32537800 & $\mathrm{H}$ & 3.61848300 & -1.58858100 & -2.33735500 \\
\hline $\mathrm{H}$ & -2.41623800 & -3.82211500 & 1.99999400 & $\mathrm{H}$ & 4.87409700 & -1.14994800 & -1.16271400 \\
\hline $\mathrm{H}$ & -2.19703800 & -3.41942200 & 0.31186700 & $\mathrm{C}$ & -1.13194000 & -1.10797600 & -2.53812700 \\
\hline $\mathrm{C}$ & -3.11892800 & -1.84352100 & 1.51624700 & $\mathrm{O}$ & -1.14309500 & -1.78327800 & -3.46221900 \\
\hline $\mathrm{H}$ & -4.12067200 & -2.22873900 & 1.73292000 & $\mathrm{H}$ & 2.33228100 & 0.83156900 & 0.21351200 \\
\hline $\mathrm{H}$ & -2.81343500 & -1.25518300 & 2.38809800 & $\mathrm{C}$ & 1.32964200 & 1.66727900 & -0.62925200 \\
\hline $\mathrm{P}$ & -3.24296400 & -0.64724600 & 0.08600300 & $\mathrm{C}$ & 0.73134400 & 0.79137000 & -1.73224300 \\
\hline $\mathrm{C}$ & -4.68996800 & -1.26768000 & -0.99552000 & $\mathrm{H}$ & 1.37841400 & -0.03088400 & -2.04307000 \\
\hline $\mathrm{C}$ & -3.88802200 & 0.91798500 & 0.83376400 & $\mathrm{H}$ & 0.44530200 & 1.37908900 & -2.61238300 \\
\hline $\mathrm{C}$ & -4.39301500 & 1.03077400 & 2.13514700 & $\mathrm{C}$ & 2.31288900 & 2.69648600 & -1.11289300 \\
\hline $\mathrm{C}$ & -4.26541000 & 3.15099500 & 0.41789100 & $\mathrm{C}$ & 2.23233400 & 4.06859900 & -0.80478400 \\
\hline $\mathrm{C}$ & -4.83702100 & 2.27961100 & 2.57419200 & $\mathrm{C}$ & 3.36941500 & 2.27745000 & -1.94646400 \\
\hline $\mathrm{H}$ & -4.45387500 & 0.17117500 & 2.79308900 & $\mathrm{C}$ & 3.17456400 & 4.97158300 & -1.29853700 \\
\hline $\mathrm{C}$ & -4.77058600 & 3.36447000 & 1.70308300 & $\mathrm{H}$ & 1.42801600 & 4.43203200 & -0.17634200 \\
\hline $\mathrm{H}$ & -4.20697900 & 3.96821600 & -0.29756300 & $\mathrm{C}$ & 4.30368900 & 3.18300700 & -2.44767300 \\
\hline $\mathrm{H}$ & -5.23389100 & 2.39736500 & 3.57821100 & $\mathrm{H}$ & 3.45667100 & 1.22734200 & -2.21135200 \\
\hline $\mathrm{H}$ & -5.10682700 & 4.35146600 & 2.00317300 & $\mathrm{C}$ & 4.21493200 & 4.53709700 & -2.12190600 \\
\hline $\mathrm{N}$ & -3.82666900 & 1.96280200 & -0.01050500 & $\mathrm{H}$ & 3.08462900 & 6.02486100 & -1.04763600 \\
\hline Pd & -1.06470300 & -0.01404000 & -1.01034600 & $\mathrm{H}$ & 5.10305000 & 2.82853200 & -3.09230600 \\
\hline $\mathrm{C}$ & -6.02541900 & -1.20500100 & -0.22958800 & $\mathrm{H}$ & 4.94169300 & 5.24472700 & -2.50886500 \\
\hline $\mathrm{H}$ & -6.29711900 & -0.18044500 & 0.03653000 & $\mathrm{C}$ & 0.42103300 & 1.90832500 & 0.42820900 \\
\hline
\end{tabular}




\begin{tabular}{|c|c|c|c|c|c|c|c|}
\hline $\mathrm{O}$ & -0.66813500 & 1.24803900 & 0.57533200 & $\mathrm{C}$ & -7.74133300 & -2.14924100 & 1.22763400 \\
\hline $\mathrm{O}$ & 0.75389800 & 2.77568700 & 1.40000400 & $\mathrm{H}$ & -6.89426500 & -0.49591600 & 2.33076700 \\
\hline $\mathrm{C}$ & -0.16834000 & 2.93201200 & 2.49458900 & $\mathrm{C}$ & -7.86674800 & -2.72075000 & -0.03805600 \\
\hline $\mathrm{H}$ & 0.25822100 & 3.71805900 & 3.11737200 & $\mathrm{H}$ & -7.33377400 & -2.48619900 & -2.11858300 \\
\hline $\mathrm{H}$ & -1.15503600 & 3.22575000 & 2.13144700 & $\mathrm{H}$ & -8.18425400 & -2.62509700 & 2.09800300 \\
\hline \multirow[t]{2}{*}{$\mathrm{H}$} & -0.25712000 & 2.00342200 & 3.06377200 & $\mathrm{H}$ & -8.40841000 & -3.64877500 & -0.19010900 \\
\hline & & & & $\mathrm{N}$ & -6.59278900 & -0.91663500 & -1.00322400 \\
\hline \multicolumn{4}{|c|}{ TS46b } & $\mathrm{Pd}$ & 2.48583500 & -0.63596300 & -1.42383600 \\
\hline \multicolumn{4}{|c|}{ imaginary frequency $=-1140.90 \mathrm{~cm}^{-1}$} & $\mathrm{H}$ & 3.37274400 & 0.15677700 & 0.10219800 \\
\hline $\mathrm{C}$ & 1.69761500 & -0.24923900 & 1.83305000 & $\mathrm{C}$ & -6.24306500 & 3.93792500 & 0.24181100 \\
\hline $\mathrm{C}$ & 1.06337800 & -0.07310700 & 3.06671000 & $\mathrm{H}$ & -5.73499900 & 4.06742800 & 1.20322800 \\
\hline $\mathrm{C}$ & 1.68617500 & 0.70012500 & 4.04851400 & $\mathrm{H}$ & -5.51187600 & 4.11029000 & -0.55489500 \\
\hline $\mathrm{C}$ & 2.92614500 & 1.27587600 & 3.77756400 & $\mathrm{H}$ & -7.00367300 & 4.72348000 & 0.15653100 \\
\hline $\mathrm{C}$ & 3.49625300 & 1.06734600 & 2.52372000 & $\mathrm{C}$ & -7.62732600 & 2.41290200 & -1.21907300 \\
\hline $\mathrm{H}$ & 0.09626000 & -0.52221200 & 3.26031200 & $\mathrm{H}$ & -8.42245200 & 3.16520200 & -1.29956900 \\
\hline $\mathrm{H}$ & 1.20505000 & 0.85131300 & 5.00985300 & $\mathrm{H}$ & -6.94572900 & 2.56774100 & -2.06072500 \\
\hline $\mathrm{H}$ & 3.43976600 & 1.88527700 & 4.51286000 & $\mathrm{H}$ & -8.08505400 & 1.42711300 & -1.33748600 \\
\hline $\mathrm{H}$ & 4.45094500 & 1.49967200 & 2.24487100 & $\mathrm{C}$ & -7.93704800 & 2.41424200 & 1.27922200 \\
\hline$N$ & 2.88744900 & 0.32755500 & 1.58577400 & $\mathrm{H}$ & -7.46064400 & 2.49328100 & 2.26265100 \\
\hline $\mathrm{P}$ & 1.01152600 & -1.23980300 & 0.41384300 & $\mathrm{H}$ & -8.68146400 & 3.21618300 & 1.20590800 \\
\hline $\mathrm{C}$ & 1.15527800 & -3.06405000 & 0.94848000 & $\mathrm{H}$ & -8.47510000 & 1.46291400 & 1.23415600 \\
\hline $\mathrm{C}$ & -0.77534500 & -0.72703500 & 0.40173600 & $\mathrm{C}$ & 0.96507500 & -3.93655000 & -0.31118200 \\
\hline $\mathrm{H}$ & -1.23913000 & -0.99742200 & 1.35724400 & $\mathrm{H}$ & 1.73180700 & -3.72842500 & -1.06323800 \\
\hline $\mathrm{H}$ & -0.74540200 & 0.36725600 & 0.36378400 & $\mathrm{H}$ & 1.05451300 & -4.99134400 & -0.02919300 \\
\hline $\mathrm{C}$ & -1.62043300 & -1.27329600 & -0.76381600 & $\mathrm{H}$ & -0.01750700 & -3.80427100 & -0.77245500 \\
\hline $\mathrm{H}$ & -1.67319700 & -2.36563300 & -0.69815300 & $\mathrm{C}$ & 0.10953400 & -3.43985700 & 2.01227200 \\
\hline $\mathrm{H}$ & -1.13016900 & -1.04033700 & -1.71783400 & $\mathrm{H}$ & 0.24846600 & -4.48967000 & 2.29355100 \\
\hline $\mathrm{C}$ & -3.05402900 & -0.71150400 & -0.77614900 & $\mathrm{H}$ & 0.21625900 & -2.84705000 & 2.92582900 \\
\hline $\mathrm{H}$ & -3.63802000 & -1.27006200 & -1.51624900 & $\mathrm{H}$ & -0.91550500 & -3.33507300 & 1.64531200 \\
\hline $\mathrm{H}$ & -3.52746700 & -0.91378300 & 0.19371700 & $\mathrm{C}$ & 2.57840400 & -3.28503200 & 1.50113200 \\
\hline $\mathrm{C}$ & -3.14954000 & 0.78622300 & -1.09892000 & $\mathrm{H}$ & 2.73771600 & -2.75729300 & 2.44623000 \\
\hline $\mathrm{H}$ & -2.63890100 & 0.97354400 & -2.05499000 & $\mathrm{H}$ & 2.71999600 & -4.35400800 & 1.69419300 \\
\hline $\mathrm{H}$ & -2.61401600 & 1.37312700 & -0.34063400 & $\mathrm{H}$ & 3.35447400 & -2.97354900 & 0.79424000 \\
\hline $\mathrm{C}$ & -4.59364500 & 1.30948000 & -1.20491000 & $\mathrm{C}$ & 4.26766600 & 0.06890200 & -2.27388100 \\
\hline $\mathrm{H}$ & -4.56435300 & 2.35847200 & -1.51671300 & $\mathrm{C}$ & 4.47208500 & 0.44527600 & -0.88642100 \\
\hline $\mathrm{H}$ & -5.15099000 & 0.74958300 & -1.96186500 & $\mathrm{H}$ & 4.08526200 & 0.86276400 & -2.99178000 \\
\hline $\mathrm{P}$ & -5.53423800 & 1.24637500 & 0.41744900 & $\mathrm{H}$ & 4.78149400 & -0.81206000 & -2.64969400 \\
\hline $\mathrm{C}$ & -6.91387400 & 2.55310300 & 0.13563400 & $\mathrm{C}$ & 4.52911000 & 1.94306300 & -0.63134900 \\
\hline $\mathrm{C}$ & -6.47756300 & -0.35612000 & 0.21432200 & $\mathrm{C}$ & 5.75673600 & 2.61310200 & -0.51546000 \\
\hline $\mathrm{C}$ & -7.02850600 & -0.95865800 & 1.35776700 & $\mathrm{C}$ & 3.34737800 & 2.69636400 & -0.62900000 \\
\hline $\mathrm{C}$ & -7.26726500 & -2.06801800 & -1.11552600 & $\mathrm{C}$ & 5.79096200 & 4.00206400 & -0.38687800 \\
\hline
\end{tabular}




\begin{tabular}{|c|c|c|c|c|c|c|c|}
\hline $\mathrm{H}$ & 6.68507400 & 2.05425900 & -0.52771500 & $\mathrm{H}$ & -3.06012600 & 0.79707200 & -1.51184300 \\
\hline $\mathrm{C}$ & 3.38360700 & 4.08585400 & -0.50568000 & $\mathrm{C}$ & -4.67174300 & -0.63763300 & -1.61902000 \\
\hline $\mathrm{H}$ & 2.38974400 & 2.18912800 & -0.72582300 & $\mathrm{H}$ & -4.92252700 & -0.38259000 & -2.65357900 \\
\hline $\mathrm{C}$ & 4.60772400 & 4.74300200 & -0.37989700 & $\mathrm{H}$ & -4.83920500 & -1.71090200 & -1.48746600 \\
\hline $\mathrm{H}$ & 6.74903300 & 4.50564200 & -0.30078500 & $\mathrm{P}$ & -5.86228800 & 0.30886700 & -0.52012700 \\
\hline $\mathrm{H}$ & 2.45660300 & 4.65118000 & -0.50784900 & $\mathrm{C}$ & -7.49973600 & 0.21751200 & -1.51991200 \\
\hline $\mathrm{H}$ & 4.64053900 & 5.82360400 & -0.28193600 & $\mathrm{C}$ & -6.16640800 & -0.94647400 & 0.83280000 \\
\hline $\mathrm{C}$ & 5.39563200 & -0.54007900 & -0.16615300 & $\mathrm{C}$ & -6.71235600 & -0.50119500 & 2.04845300 \\
\hline $\mathrm{O}$ & 5.42049700 & -1.72385900 & -0.43351500 & $\mathrm{C}$ & -6.06298400 & -3.10975600 & 1.62754400 \\
\hline $\mathrm{O}$ & 6.12933100 & 0.02432900 & 0.80045400 & $\mathrm{C}$ & -6.95345800 & -1.42045100 & 3.06797300 \\
\hline $\mathrm{C}$ & 7.01828600 & -0.87148300 & 1.51192000 & $\mathrm{H}$ & -6.94017300 & 0.55148500 & 2.18555500 \\
\hline $\mathrm{H}$ & 7.72156200 & -1.33295600 & 0.81687700 & $\mathrm{C}$ & -6.62300400 & -2.75845800 & 2.85608200 \\
\hline $\mathrm{H}$ & 7.54077900 & -0.24566000 & 2.23342700 & $\mathrm{H}$ & -5.78273000 & -4.14239900 & 1.42578600 \\
\hline $\mathrm{H}$ & 6.44290100 & -1.65171000 & 2.01385700 & $\mathrm{H}$ & -7.38461200 & -1.09758600 & 4.01138400 \\
\hline $\mathrm{C}$ & 1.60492700 & -1.27598000 & -3.05111700 & $\mathrm{H}$ & -6.78802900 & -3.51125200 & 3.62020300 \\
\hline \multirow[t]{2}{*}{$\mathrm{O}$} & 1.07111300 & -1.63753800 & -3.99196300 & $\mathrm{~N}$ & -5.83981400 & -2.23689300 & 0.63684800 \\
\hline & & & & $\mathrm{Pd}$ & 2.66977700 & -0.16158500 & -0.20506100 \\
\hline 52 & & & & $\mathrm{H}$ & 3.63143000 & 1.23530300 & -1.09309500 \\
\hline $\mathrm{C}$ & 1.26326800 & 3.06284700 & -0.23150200 & $\mathrm{C}$ & -7.35040000 & 1.19575100 & -2.70295500 \\
\hline $\mathrm{C}$ & 0.24861700 & 4.00940300 & -0.42566000 & $\mathrm{H}$ & -7.12338500 & 2.21101700 & -2.36107200 \\
\hline $\mathrm{C}$ & 0.56572600 & 5.21359000 & -1.05666700 & $\mathrm{H}$ & -6.56487300 & 0.88910700 & -3.40140300 \\
\hline $\mathrm{C}$ & 1.87919500 & 5.43592500 & -1.46294800 & $\mathrm{H}$ & -8.28835000 & 1.23522000 & -3.27012300 \\
\hline $\mathrm{C}$ & 2.82428200 & 4.43648900 & -1.22569300 & $\mathrm{C}$ & -7.83008800 & -1.19220200 & -2.03710000 \\
\hline $\mathrm{H}$ & -0.76556400 & 3.81936800 & -0.09255300 & $\mathrm{H}$ & -8.78713300 & -1.17550200 & -2.57411800 \\
\hline $\mathrm{H}$ & -0.20225500 & 5.96245900 & -1.22418100 & $\mathrm{H}$ & -7.07204900 & -1.56267800 & -2.73364900 \\
\hline $\mathrm{H}$ & 2.16939900 & 6.35671000 & -1.95747500 & $\mathrm{H}$ & -7.91632300 & -1.91555100 & -1.22169000 \\
\hline $\mathrm{H}$ & 3.85917500 & 4.57037400 & -1.53148100 & $\mathrm{C}$ & -8.63584600 & 0.71765000 & -0.60740100 \\
\hline $\mathrm{N}$ & 2.52939500 & 3.27279800 & -0.63222100 & $\mathrm{H}$ & -8.42746100 & 1.71760400 & -0.21114600 \\
\hline $\mathrm{P}$ & 0.97241300 & 1.43212200 & 0.60527500 & $\mathrm{H}$ & -9.56964400 & 0.77540000 & -1.17952200 \\
\hline $\mathrm{C}$ & 1.13367000 & 1.79507200 & 2.46863600 & $\mathrm{H}$ & -8.80921400 & 0.04358600 & 0.23649800 \\
\hline $\mathrm{C}$ & -0.81291200 & 1.04823200 & 0.24303100 & $\mathrm{C}$ & 0.70495700 & 0.53779000 & 3.25056900 \\
\hline $\mathrm{H}$ & -1.44549300 & 1.59010900 & 0.95589800 & $\mathrm{H}$ & 1.29355500 & -0.34264900 & 2.97403200 \\
\hline $\mathrm{H}$ & -1.01351700 & 1.47638300 & -0.74377400 & $\mathrm{H}$ & 0.86421100 & 0.71292600 & 4.32029900 \\
\hline $\mathrm{C}$ & -1.17814500 & -0.44812300 & 0.24106000 & $\mathrm{H}$ & -0.35500000 & 0.30498000 & 3.11426400 \\
\hline $\mathrm{H}$ & -0.88349600 & -0.90689900 & 1.19146700 & $\mathrm{C}$ & 0.26434800 & 2.99217500 & 2.89552600 \\
\hline $\mathrm{H}$ & -0.60088500 & -0.95367700 & -0.54437700 & $\mathrm{H}$ & 0.38442700 & 3.15062400 & 3.97302800 \\
\hline $\mathrm{C}$ & -2.67929400 & -0.70144700 & 0.01516500 & $\mathrm{H}$ & 0.55909400 & 3.91672500 & 2.39272500 \\
\hline $\mathrm{H}$ & -2.87676100 & -1.76989000 & 0.15930500 & $\mathrm{H}$ & -0.80145800 & 2.82207700 & 2.71434000 \\
\hline $\mathrm{H}$ & -3.25115800 & -0.18297900 & 0.79607000 & $\mathrm{C}$ & 2.61778200 & 2.10138400 & 2.75117000 \\
\hline $\mathrm{C}$ & -3.19455100 & -0.28319700 & -1.36911700 & $\mathrm{H}$ & 2.97229700 & 2.97282900 & 2.19318300 \\
\hline $\mathrm{H}$ & -2.58105200 & -0.77640900 & -2.13739100 & $\mathrm{H}$ & 2.74638700 & 2.31242600 & 3.81862000 \\
\hline
\end{tabular}




\begin{tabular}{|c|c|c|c|c|c|c|c|}
\hline $\mathrm{H}$ & 3.25949800 & 1.24914200 & 2.50215700 & $\mathrm{H}$ & 3.04058900 & 2.62030600 & -0.24743600 \\
\hline $\mathrm{C}$ & 4.50359500 & -0.83630700 & -1.14133300 & $\mathrm{C}$ & 2.60991800 & 2.92394600 & 1.84120200 \\
\hline $\mathrm{C}$ & 4.46880500 & 0.58819000 & -1.58841200 & $\mathrm{H}$ & 3.02263800 & 3.93737900 & 1.91435700 \\
\hline $\mathrm{C}$ & 2.02397500 & -1.87815700 & 0.44305300 & $\mathrm{H}$ & 2.81582900 & 2.45289200 & 2.80990100 \\
\hline $\mathrm{O}$ & 1.62548500 & -2.85385100 & 0.87759800 & $\mathrm{C}$ & 1.08318300 & 3.04800600 & 1.63720600 \\
\hline $\mathrm{H}$ & 4.24612200 & 0.66240300 & -2.65237400 & $\mathrm{H}$ & 0.74697600 & 3.98486200 & 2.10183300 \\
\hline $\mathrm{H}$ & 5.35317000 & 1.15899600 & -1.30604600 & $\mathrm{H}$ & 0.87687800 & 3.15396400 & 0.56578600 \\
\hline $\mathrm{C}$ & 5.46899900 & -1.22876500 & -0.08307400 & $\mathrm{C}$ & 0.24891600 & 1.90316600 & 2.23523600 \\
\hline $\mathrm{C}$ & 6.06492600 & -2.50496300 & -0.01103900 & $\mathrm{H}$ & 0.48115800 & 1.83860200 & 3.30601800 \\
\hline $\mathrm{C}$ & 5.83260200 & -0.27443900 & 0.89688500 & $\mathrm{H}$ & 0.55461300 & 0.94410900 & 1.80361000 \\
\hline $\mathrm{C}$ & 6.97802400 & -2.80733300 & 0.99650300 & $\mathrm{C}$ & -1.28299300 & 2.08010600 & 2.11408000 \\
\hline $\mathrm{H}$ & 5.81921600 & -3.25880900 & -0.74542800 & $\mathrm{H}$ & -1.76976600 & 1.46102100 & 2.87421800 \\
\hline $\mathrm{C}$ & 6.73103900 & -0.58825200 & 1.91061600 & $\mathrm{H}$ & -1.57655900 & 3.11534200 & 2.32392300 \\
\hline $\mathrm{H}$ & 5.40832500 & 0.72459700 & 0.86884100 & $\mathrm{P}$ & -2.04484400 & 1.50255000 & 0.51852500 \\
\hline $\mathrm{C}$ & 7.31118700 & -1.85856400 & 1.96439200 & $\mathrm{C}$ & -3.93831800 & 1.68506000 & 0.71215200 \\
\hline $\mathrm{H}$ & 7.43346900 & -3.79236300 & 1.02185300 & $\mathrm{C}$ & -1.60868400 & 2.75643000 & -0.77967800 \\
\hline $\mathrm{H}$ & 6.98759100 & 0.16148700 & 2.65255200 & $\mathrm{C}$ & -1.47785600 & 4.12751500 & -0.51753400 \\
\hline $\mathrm{H}$ & 8.02139500 & -2.10232200 & 2.74820400 & $\mathrm{C}$ & -1.20761100 & 3.08538100 & -3.02748500 \\
\hline $\mathrm{C}$ & 4.03482100 & -1.79021800 & -2.23001500 & $\mathrm{C}$ & -1.19690000 & 4.99022100 & -1.57768100 \\
\hline $\mathrm{O}$ & 3.78651500 & -1.42433000 & -3.36077200 & $\mathrm{H}$ & -1.59016900 & 4.51813600 & 0.48705800 \\
\hline $\mathrm{O}$ & 3.86713100 & -3.05425400 & -1.80728600 & $\mathrm{C}$ & -1.05919400 & 4.46276200 & -2.86022600 \\
\hline $\mathrm{C}$ & 3.39646100 & -3.98763400 & -2.80948400 & $\mathrm{H}$ & -1.10437400 & 2.62722200 & -4.00846300 \\
\hline $\mathrm{H}$ & 3.34554800 & -4.95013200 & -2.30336200 & $\mathrm{H}$ & -1.08893800 & 6.05614500 & -1.40152400 \\
\hline $\mathrm{H}$ & 2.41164300 & -3.68572100 & -3.17181500 & $\mathrm{H}$ & -0.84006900 & 5.09824600 & -3.71169700 \\
\hline \multirow[t]{2}{*}{$\mathrm{H}$} & 4.09265300 & -4.02453500 & -3.64919600 & $\mathrm{~N}$ & -1.47394800 & 2.25216500 & -2.01496200 \\
\hline & & & & $\mathrm{Pd}$ & -1.36521700 & -0.75648500 & -0.13661500 \\
\hline 53 & & & & $\mathrm{C}$ & -4.35120700 & 3.14665000 & 0.96782800 \\
\hline $\mathrm{C}$ & 3.53208200 & 0.00951800 & -1.18326500 & $\mathrm{H}$ & -4.09306400 & 3.80204000 & 0.13276500 \\
\hline $\mathrm{C}$ & 4.20848700 & 0.88595500 & -2.04651100 & $\mathrm{H}$ & -5.43887500 & 3.19016400 & 1.09335700 \\
\hline $\mathrm{C}$ & 4.42043100 & 0.51269400 & -3.37340200 & $\mathrm{H}$ & -3.90437600 & 3.55230100 & 1.88118600 \\
\hline $\mathrm{C}$ & 3.96337700 & -0.73094000 & -3.80493200 & $\mathrm{C}$ & -4.41456900 & 0.81026400 & 1.88804900 \\
\hline $\mathrm{C}$ & 3.30864000 & -1.54470500 & -2.88038400 & $\mathrm{H}$ & -4.15429100 & -0.24156900 & 1.74735100 \\
\hline $\mathrm{H}$ & 4.58033800 & 1.83877200 & -1.68690600 & $\mathrm{H}$ & -4.01613400 & 1.14776100 & 2.84984400 \\
\hline $\mathrm{H}$ & 4.94375900 & 1.17814700 & -4.05410600 & $\mathrm{H}$ & -5.50649600 & 0.86986500 & 1.95210300 \\
\hline $\mathrm{H}$ & 4.10972500 & -1.06628600 & -4.82640600 & $\mathrm{C}$ & -4.58070600 & 1.17517300 & -0.59423000 \\
\hline $\mathrm{H}$ & 2.94110600 & -2.52602200 & -3.17502700 & $\mathrm{H}$ & -4.35765200 & 0.11717600 & -0.76603800 \\
\hline $\mathrm{N}$ & 3.08322600 & -1.19099300 & -1.60849500 & $\mathrm{H}$ & -5.66918500 & 1.27890100 & -0.52371800 \\
\hline $\mathrm{P}$ & 3.15193000 & 0.34243100 & 0.60891000 & $\mathrm{H}$ & -4.24816000 & 1.74235000 & -1.46826100 \\
\hline $\mathrm{C}$ & 4.72733500 & -0.35386800 & 1.46702500 & $\mathrm{C}$ & 4.70439700 & 0.12729700 & 2.93148000 \\
\hline $\mathrm{C}$ & 3.38385800 & 2.21001500 & 0.70938500 & $\mathrm{H}$ & 4.86128900 & 1.20736700 & 3.01391900 \\
\hline $\mathrm{H}$ & 4.44718700 & 2.46321200 & 0.78765700 & $\mathrm{H}$ & 3.75963500 & -0.11980100 & 3.42881900 \\
\hline
\end{tabular}




\begin{tabular}{|c|c|c|c|c|c|c|c|}
\hline $\mathrm{H}$ & 5.51075300 & -0.36035900 & 3.49241300 & $\mathrm{C}$ & 2.03727700 & -1.85807900 & -3.45115900 \\
\hline $\mathrm{C}$ & 6.04972600 & 0.07069700 & 0.80805600 & $\mathrm{H}$ & 3.23865000 & -3.43324000 & -0.18994500 \\
\hline $\mathrm{H}$ & 6.89262000 & -0.33242400 & 1.38298000 & $\mathrm{H}$ & 4.62754000 & -3.67844600 & -2.23929600 \\
\hline $\mathrm{H}$ & 6.13795600 & -0.30703200 & -0.21413100 & $\mathrm{H}$ & 3.82661100 & -2.63097600 & -4.37853400 \\
\hline $\mathrm{H}$ & 6.17363900 & 1.15822400 & 0.78063200 & $\mathrm{H}$ & 1.65289500 & -1.39780700 & -4.35838000 \\
\hline $\mathrm{C}$ & 4.61145400 & -1.89033500 & 1.43755100 & $\mathrm{~N}$ & 1.28357700 & -1.71831400 & -2.35539800 \\
\hline $\mathrm{H}$ & 5.48628300 & -2.33885700 & 1.92366300 & $\mathrm{P}$ & 0.54426100 & -1.98286000 & 0.20361200 \\
\hline $\mathrm{H}$ & 3.72074300 & -2.23195700 & 1.97557300 & C & -0.65644000 & -3.46942500 & 0.06384300 \\
\hline $\mathrm{H}$ & 4.55673200 & -2.27694400 & 0.41599700 & $\mathrm{C}$ & 1.60343900 & -2.32790000 & 1.70634700 \\
\hline $\mathrm{C}$ & -0.98157000 & -0.47981100 & -2.03517500 & $\mathrm{H}$ & 1.82830500 & -3.39977400 & 1.69786100 \\
\hline $\mathrm{O}$ & -0.70840500 & -0.50188800 & -3.14241500 & $\mathrm{H}$ & 2.55309700 & -1.81449600 & 1.51751800 \\
\hline $\mathrm{C}$ & -2.22025100 & -3.45561200 & -0.71031800 & $\mathrm{C}$ & 1.09288900 & -1.93785700 & 3.11618000 \\
\hline $\mathrm{O}$ & -3.32754100 & -3.07391800 & -0.36169500 & $\mathrm{H}$ & 1.64485800 & -2.57023100 & 3.82135900 \\
\hline $\mathrm{O}$ & -2.03492500 & -4.42972200 & -1.60866100 & $\mathrm{H}$ & 0.03920600 & -2.20463300 & 3.24595100 \\
\hline $\mathrm{C}$ & -3.23419200 & -5.02514000 & -2.15360400 & $\mathrm{C}$ & 1.31455300 & -0.47103400 & 3.52681100 \\
\hline $\mathrm{H}$ & -3.83097200 & -4.26901300 & -2.66793600 & $\mathrm{H}$ & 1.37466900 & -0.43166500 & 4.62003100 \\
\hline $\mathrm{H}$ & -2.88574200 & -5.78510100 & -2.85087500 & $\mathrm{H}$ & 2.30356500 & -0.16331900 & 3.17257000 \\
\hline $\mathrm{H}$ & -3.82924600 & -5.47332600 & -1.35572700 & C & 0.23194300 & 0.53468900 & 3.06119600 \\
\hline $\mathrm{C}$ & -0.89895200 & -2.89935700 & -0.21718000 & $\mathrm{H}$ & -0.39777700 & 0.80040300 & 3.91804700 \\
\hline $\mathrm{C}$ & -0.78098500 & -2.56850100 & 1.20142100 & $\mathrm{H}$ & -0.44468700 & 0.06697800 & 2.33733000 \\
\hline $\mathrm{C}$ & 0.47680200 & -2.59933100 & 1.87827900 & $\mathrm{C}$ & 0.76305800 & 1.84860500 & 2.45703600 \\
\hline $\mathrm{C}$ & -1.90249900 & -2.01594000 & 1.90431300 & $\mathrm{H}$ & -0.05711300 & 2.57149700 & 2.41363100 \\
\hline $\mathrm{C}$ & 0.58011600 & -2.18047700 & 3.18689900 & $\mathrm{H}$ & 1.53565100 & 2.28998000 & 3.09623700 \\
\hline $\mathrm{H}$ & 1.34982900 & -2.98271200 & 1.36632200 & $\mathrm{P}$ & 1.34938100 & 1.73550400 & 0.68849000 \\
\hline $\mathrm{C}$ & -1.75959400 & -1.57505700 & 3.24032900 & $\mathrm{C}$ & 1.49763000 & 3.56138500 & 0.11984400 \\
\hline $\mathrm{H}$ & -2.89385700 & -2.13865100 & 1.48172000 & $\mathrm{C}$ & 3.11916800 & 1.17428700 & 0.70724300 \\
\hline $\mathrm{C}$ & -0.53876100 & -1.66368300 & 3.87840000 & $\mathrm{C}$ & 3.98854200 & 1.33902600 & 1.79601800 \\
\hline $\mathrm{H}$ & 1.53703100 & -2.25037800 & 3.69458900 & $\mathrm{C}$ & 4.80149700 & 0.26381100 & -0.57602200 \\
\hline $\mathrm{H}$ & -2.63418800 & -1.20970800 & 3.76917100 & $\mathrm{C}$ & 5.31631500 & 0.92978600 & 1.66487800 \\
\hline $\mathrm{H}$ & -0.43613100 & -1.35408600 & 4.91388900 & $\mathrm{H}$ & 3.64931600 & 1.78596000 & 2.72332900 \\
\hline $\mathrm{C}$ & 0.33807000 & -3.34808400 & -0.97078900 & $\mathrm{C}$ & 5.73570600 & 0.38068400 & 0.45498900 \\
\hline H & 0.13760500 & -3.38637300 & -2.04162800 & $\mathrm{H}$ & 5.08613700 & -0.15637900 & -1.53755800 \\
\hline $\mathrm{H}$ & 1.18751200 & -2.68058200 & -0.80570300 & $\mathrm{H}$ & 6.00950000 & 1.04682900 & 2.49252900 \\
\hline \multirow[t]{2}{*}{$\mathrm{H}$} & 0.61982300 & -4.36381600 & -0.66491100 & $\mathrm{H}$ & 6.75970700 & 0.05328300 & 0.30852700 \\
\hline & & & & $\mathrm{N}$ & 3.52286000 & 0.64224300 & -0.45893100 \\
\hline \multirow{2}{*}{\multicolumn{4}{|c|}{$\begin{array}{l}\text { TS47 } \\
\text { imaginary frequency }=-24.68 \mathrm{~cm}^{-1}\end{array}$}} & $\mathrm{Pd}$ & -0.25363300 & 0.26651400 & -0.45493800 \\
\hline & & & & $\mathrm{C}$ & 2.51028900 & 4.32982400 & 0.99367800 \\
\hline $\mathrm{C}$ & 1.71466400 & -2.27044800 & -1.20648300 & $\mathrm{H}$ & 3.52375500 & 3.93134300 & 0.90416100 \\
\hline $\mathrm{C}$ & 2.91477600 & -2.98621200 & -1.12312500 & $\mathrm{H}$ & 2.53772100 & 5.37358600 & 0.66056100 \\
\hline C & 3.69392600 & -3.12448000 & -2.27347200 & $\mathrm{H}$ & 2.22987100 & 4.33759100 & 2.05159900 \\
\hline C & 3.25211200 & -2.54747000 & -3.46187300 & $\mathrm{C}$ & 0.11892500 & 4.24110400 & 0.23530500 \\
\hline
\end{tabular}




\begin{tabular}{|c|c|c|c|c|c|c|c|}
\hline $\mathrm{H}$ & -0.68478800 & 3.66821900 & -0.23137600 & $\mathrm{H}$ & -3.34387300 & 0.69218800 & -3.03534700 \\
\hline $\mathrm{H}$ & -0.16677500 & 4.42165400 & 1.27558200 & $\mathrm{H}$ & -3.44266700 & -0.98443400 & -2.47296000 \\
\hline $\mathrm{H}$ & 0.16809500 & 5.21819100 & -0.25870000 & $\mathrm{H}$ & -4.88132100 & 0.04603000 & -2.48012300 \\
\hline $\mathrm{C}$ & 1.97454400 & 3.57600800 & -1.34600800 & & & & \\
\hline $\mathrm{H}$ & 1.25801500 & 3.10033700 & -2.01863600 & $54 a$ & & & \\
\hline $\mathrm{H}$ & 2.09081800 & 4.61708500 & -1.66814700 & $\mathrm{C}$ & -3.23980900 & -0.95984400 & -0.57306300 \\
\hline $\mathrm{H}$ & 2.93832200 & 3.07665200 & -1.47137200 & $\mathrm{C}$ & -4.35608800 & -1.79607800 & -0.63845500 \\
\hline $\mathrm{C}$ & -1.56166600 & -3.49045400 & 1.30972600 & $\mathrm{C}$ & -4.37688400 & -2.82963500 & -1.57841700 \\
\hline $\mathrm{H}$ & -1.01112900 & -3.78190100 & 2.20837800 & $\mathrm{C}$ & -3.28353800 & -3.00385300 & -2.42497300 \\
\hline $\mathrm{H}$ & -2.04320400 & -2.52597000 & 1.49201000 & $\mathrm{C}$ & -2.18200300 & -2.16291900 & -2.27404800 \\
\hline $\mathrm{H}$ & -2.35578100 & -4.23105100 & 1.16335300 & $\mathrm{H}$ & -5.19337000 & -1.64919800 & 0.03244000 \\
\hline $\mathrm{C}$ & 0.08735800 & -4.81182600 & -0.05412700 & $\mathrm{H}$ & -5.23987900 & -3.48457900 & -1.65098800 \\
\hline $\mathrm{H}$ & -0.64986000 & -5.61934300 & -0.13372700 & $\mathrm{H}$ & -3.27250800 & -3.78150300 & -3.18099800 \\
\hline $\mathrm{H}$ & 0.72262900 & -4.85694500 & -0.94227800 & $\mathrm{H}$ & -1.29361200 & -2.26749900 & -2.88910500 \\
\hline $\mathrm{H}$ & 0.70347000 & -5.03100600 & 0.82352900 & $\mathrm{~N}$ & -2.16137600 & -1.17936400 & -1.36376800 \\
\hline $\mathrm{C}$ & -1.50844400 & -3.24221400 & -1.19838900 & $\mathrm{P}$ & -2.89771600 & 0.52562800 & 0.49441500 \\
\hline $\mathrm{H}$ & -2.23045500 & -4.06108500 & -1.29839200 & $\mathrm{C}$ & -4.36648800 & 1.72030300 & 0.21977500 \\
\hline $\mathrm{H}$ & -2.06376300 & -2.30257600 & -1.13395400 & $\mathrm{C}$ & -3.03590500 & -0.16862400 & 2.22917200 \\
\hline $\mathrm{H}$ & -0.89944600 & -3.21227400 & -2.10502200 & $\mathrm{H}$ & -4.08838600 & -0.25967700 & 2.51850200 \\
\hline $\mathrm{C}$ & -0.96397100 & 1.45485900 & -1.78618600 & $\mathrm{H}$ & -2.63417900 & -1.18306100 & 2.13786700 \\
\hline $\mathrm{O}$ & -1.05923200 & 2.13115000 & -2.71412800 & $\mathrm{C}$ & -2.24028100 & 0.57086000 & 3.32964400 \\
\hline $\mathrm{C}$ & -3.42424300 & 1.97497600 & -0.66901500 & $\mathrm{H}$ & -2.36309700 & -0.02030400 & 4.24419000 \\
\hline $\mathrm{O}$ & -2.96582500 & 2.54080800 & 0.31205500 & $\mathrm{H}$ & -2.69464900 & 1.54463300 & 3.54707400 \\
\hline $\mathrm{O}$ & -3.96141500 & 2.63614500 & -1.71083000 & $\mathrm{C}$ & -0.73508000 & 0.74855700 & 3.02525500 \\
\hline $\mathrm{C}$ & -3.96470900 & 4.07449700 & -1.61880400 & $\mathrm{H}$ & -0.15067900 & 0.55918100 & 3.93388000 \\
\hline $\mathrm{H}$ & -2.94287200 & 4.45734700 & -1.67831100 & $\mathrm{H}$ & -0.42676000 & -0.01259500 & 2.29964300 \\
\hline $\mathrm{H}$ & -4.54835000 & 4.41702100 & -2.47193200 & $\mathrm{C}$ & -0.37311000 & 2.14854600 & 2.49962700 \\
\hline $\mathrm{H}$ & -4.41879400 & 4.39695800 & -0.68006500 & $\mathrm{H}$ & -0.39911600 & 2.85731400 & 3.33639800 \\
\hline $\mathrm{C}$ & -3.51044800 & 0.49286600 & -0.89140500 & $\mathrm{H}$ & -1.14057800 & 2.48914400 & 1.80090800 \\
\hline $\mathrm{C}$ & -3.90411900 & -0.33697600 & 0.23257900 & $\mathrm{C}$ & 1.01994600 & 2.22934000 & 1.83761100 \\
\hline $\mathrm{C}$ & -4.56857600 & -1.57235600 & -0.00639900 & $\mathrm{H}$ & 1.43230200 & 3.23976400 & 1.93084700 \\
\hline $\mathrm{C}$ & -3.76945600 & 0.07941000 & 1.58806800 & $\mathrm{H}$ & 1.70756300 & 1.55631700 & 2.36153300 \\
\hline $\mathrm{C}$ & -5.09056500 & -2.32448000 & 1.03795600 & $\mathrm{P}$ & 1.08924700 & 1.75080600 & 0.03151800 \\
\hline $\mathrm{H}$ & -4.71266600 & -1.92344600 & -1.02030600 & $\mathrm{C}$ & 0.70855900 & 3.34923400 & -0.96801300 \\
\hline $\mathrm{C}$ & -4.27979700 & -0.68984600 & 2.62390800 & $\mathrm{C}$ & 2.90466900 & 1.55411800 & -0.30101800 \\
\hline H & -3.27212000 & 1.01572100 & 1.79908200 & $\mathrm{C}$ & 3.90697800 & 1.87695200 & 0.62264200 \\
\hline $\mathrm{C}$ & -4.94885200 & -1.89146100 & 2.35958600 & $\mathrm{C}$ & 4.45970200 & 1.13167400 & -1.94578300 \\
\hline $\mathrm{H}$ & -5.61818100 & -3.24816200 & 0.82107100 & $\mathrm{C}$ & 5.23870300 & 1.82186600 & 0.20800700 \\
\hline $\mathrm{H}$ & -4.17047000 & -0.34546900 & 3.64787100 & $\mathrm{H}$ & 3.66955100 & 2.18198100 & 1.63430800 \\
\hline $\mathrm{H}$ & -5.35970000 & -2.47980800 & 3.17432800 & $\mathrm{C}$ & 5.52624500 & 1.44739000 & -1.10172700 \\
\hline $\mathrm{C}$ & -3.79689400 & 0.03302500 & -2.29820900 & $\mathrm{H}$ & 4.63606000 & 0.82998200 & -2.97594400 \\
\hline
\end{tabular}




\begin{tabular}{|c|c|c|c|c|c|c|c|}
\hline $\mathrm{H}$ & 6.03582600 & 2.07627400 & 0.90015800 & $\mathrm{H}$ & 5.65361200 & -1.29450500 & 2.68335200 \\
\hline $\mathrm{H}$ & 6.54745000 & 1.40038700 & -1.46542400 & $\mathrm{C}$ & 1.75787500 & -3.52838900 & -1.52846700 \\
\hline $\mathrm{N}$ & 3.18059100 & 1.18310600 & -1.56112700 & $\mathrm{H}$ & 2.24220000 & -4.36814900 & -1.03024900 \\
\hline $\mathrm{Pd}$ & -0.47949200 & 0.06427000 & -0.53319200 & $\mathrm{H}$ & 0.86433800 & -3.89860800 & -2.03722200 \\
\hline $\mathrm{C}$ & 1.84225900 & 4.38178200 & -0.81516900 & $\mathrm{H}$ & 2.41884300 & -3.11756700 & -2.28984100 \\
\hline $\mathrm{H}$ & 2.77967100 & 4.03582300 & -1.25494800 & $\mathrm{C}$ & 0.27377000 & -2.96321100 & 0.43474700 \\
\hline $\mathrm{H}$ & 1.55100500 & 5.30046500 & -1.33696800 & $\mathrm{O}$ & -0.58327400 & -2.28169900 & 0.97386900 \\
\hline $\mathrm{H}$ & 2.02968500 & 4.65145300 & 0.22964900 & $\mathrm{O}$ & 0.38674700 & -4.27816800 & 0.63859900 \\
\hline $\mathrm{C}$ & -0.60561000 & 3.96969900 & -0.46472800 & $\mathrm{C}$ & -0.54157400 & -4.86971500 & 1.57160400 \\
\hline $\mathrm{H}$ & -1.42966100 & 3.25427000 & -0.51159700 & $\mathrm{H}$ & -0.26569100 & -5.92159500 & 1.62388700 \\
\hline $\mathrm{H}$ & -0.52524300 & 4.34990800 & 0.55807700 & $\mathrm{H}$ & -0.44734800 & -4.39671000 & 2.55125600 \\
\hline $\mathrm{H}$ & -0.86549400 & 4.81855300 & -1.10751100 & $\mathrm{H}$ & -1.56644200 & -4.75797300 & 1.21109600 \\
\hline $\mathrm{C}$ & 0.55315700 & 2.95835300 & -2.45179300 & & & & \\
\hline $\mathrm{H}$ & -0.29344900 & 2.28037600 & -2.60066200 & 54 & & & \\
\hline $\mathrm{H}$ & 0.37145600 & 3.86477800 & -3.04146800 & $\mathrm{C}$ & 1.21354600 & -2.84614400 & -1.23835600 \\
\hline $\mathrm{H}$ & 1.44732700 & 2.46574100 & -2.83869600 & $\mathrm{H}$ & 0.62247400 & -2.52837500 & -2.10244000 \\
\hline $\mathrm{C}$ & -4.14361700 & 2.93813700 & 1.14074600 & $\mathrm{H}$ & 2.08299600 & -3.38880600 & -1.61671800 \\
\hline $\mathrm{H}$ & -4.20999100 & 2.66882500 & 2.19905300 & $\mathrm{C}$ & 0.40285600 & -3.75120900 & -0.29281000 \\
\hline $\mathrm{H}$ & -3.17833700 & 3.42148000 & 0.96819100 & $\mathrm{H}$ & 1.09942600 & -4.36442700 & 0.28744300 \\
\hline $\mathrm{H}$ & -4.92307900 & 3.68315800 & 0.94501100 & $\mathrm{H}$ & -0.14513400 & -3.14672300 & 0.44031500 \\
\hline $\mathrm{C}$ & -5.74387400 & 1.10753700 & 0.53272000 & $\mathrm{P}$ & -2.16151600 & -1.16483800 & -0.66900000 \\
\hline $\mathrm{H}$ & -6.50750700 & 1.88977200 & 0.44982400 & $\mathrm{P}$ & 1.81750000 & -1.30090400 & -0.41409000 \\
\hline $\mathrm{H}$ & -6.01581400 & 0.32260100 & -0.17651000 & $\mathrm{C}$ & 3.06658200 & -0.50476300 & -1.62791100 \\
\hline $\mathrm{H}$ & -5.80918500 & 0.70533500 & 1.54844000 & $\mathrm{C}$ & -2.81246400 & -0.37977500 & -2.29033500 \\
\hline $\mathrm{C}$ & -4.32961000 & 2.16386800 & -1.25649500 & $\mathrm{C}$ & 2.27144600 & 0.39461500 & -2.59510200 \\
\hline $\mathrm{H}$ & -5.12900700 & 2.89118600 & -1.43806800 & $\mathrm{H}$ & 2.96724800 & 0.84716700 & -3.31056300 \\
\hline $\mathrm{H}$ & -3.38143300 & 2.64019000 & -1.52196200 & $\mathrm{H}$ & 1.53576500 & -0.17251300 & -3.17477400 \\
\hline $\mathrm{H}$ & -4.48993100 & 1.32294200 & -1.93884000 & $\mathrm{H}$ & 1.75416800 & 1.20533700 & -2.07417600 \\
\hline $\mathrm{C}$ & 0.86942300 & -1.17128000 & -1.33101700 & $\mathrm{C}$ & 3.81683200 & -1.58385200 & -2.43999000 \\
\hline $\mathrm{O}$ & 1.14269800 & -1.07373400 & -2.49255800 & $\mathrm{H}$ & 4.56138200 & -1.08006400 & -3.06680400 \\
\hline $\mathrm{C}$ & 1.37823900 & -2.44066700 & -0.49664300 & $\mathrm{H}$ & 4.33586300 & -2.29028400 & -1.78996200 \\
\hline $\mathrm{C}$ & 2.57902300 & -2.04499100 & 0.39298700 & $\mathrm{H}$ & 3.15297100 & -2.13556000 & -3.11150600 \\
\hline $\mathrm{C}$ & 3.88718300 & -2.13654200 & -0.10524700 & $\mathrm{C}$ & 4.09427100 & 0.34145600 & -0.85287500 \\
\hline $\mathrm{C}$ & 2.40304900 & -1.63918600 & 1.72597200 & $\mathrm{H}$ & 4.69110100 & -0.27014700 & -0.17273300 \\
\hline $\mathrm{C}$ & 4.98359800 & -1.86305500 & 0.71256300 & $\mathrm{H}$ & 4.77737800 & 0.79937500 & -1.57707300 \\
\hline $\mathrm{H}$ & 4.06346500 & -2.43722800 & -1.13109800 & $\mathrm{H}$ & 3.63093600 & 1.14658900 & -0.28163300 \\
\hline $\mathrm{C}$ & 3.50176500 & -1.37129100 & 2.54446200 & $\mathrm{C}$ & -1.64696800 & -0.39696000 & -3.30171000 \\
\hline $\mathrm{H}$ & 1.40575100 & -1.55463500 & 2.14176800 & $\mathrm{H}$ & -0.78703400 & 0.17046400 & -2.93367600 \\
\hline $\mathrm{C}$ & 4.79792500 & -1.48801000 & 2.04359200 & $\mathrm{H}$ & -1.31447700 & -1.41125100 & -3.54409100 \\
\hline $\mathrm{H}$ & 5.98644800 & -1.95564800 & 0.30677500 & $\mathrm{H}$ & -1.97662300 & 0.06568900 & -4.23917600 \\
\hline $\mathrm{H}$ & 3.34008900 & -1.08665600 & 3.58035500 & $\mathrm{C}$ & -4.02315200 & -1.12450100 & -2.88322600 \\
\hline
\end{tabular}




\begin{tabular}{|c|c|c|c|c|c|c|c|}
\hline $\mathrm{H}$ & -4.85540200 & -1.15628800 & -2.17800600 & $\mathrm{H}$ & 2.11825700 & 3.45254200 & 2.72264900 \\
\hline $\mathrm{H}$ & -4.35507900 & -0.59383400 & -3.78348500 & $\mathrm{C}$ & 1.18709800 & 3.56143900 & 0.02608600 \\
\hline $\mathrm{H}$ & -3.77283700 & -2.14517500 & -3.18748600 & $\mathrm{C}$ & 2.53227900 & 3.96120700 & 0.00625300 \\
\hline $\mathrm{C}$ & -3.19432400 & 1.08826000 & -2.01380000 & $\mathrm{C}$ & 0.39042200 & 3.82968400 & -1.09634400 \\
\hline $\mathrm{H}$ & -3.46709200 & 1.56707000 & -2.96146100 & $\mathrm{C}$ & 3.05729500 & 4.63009600 & -1.09901000 \\
\hline $\mathrm{H}$ & -4.05001300 & 1.15816200 & -1.33979300 & $\mathrm{H}$ & 3.17733000 & 3.74969600 & 0.85198800 \\
\hline $\mathrm{H}$ & -2.35872500 & 1.64896000 & -1.58469300 & $\mathrm{C}$ & 0.91708300 & 4.50227800 & -2.20076600 \\
\hline $\mathrm{C}$ & 2.77355800 & -2.01279900 & 1.00684100 & $\mathrm{H}$ & -0.65378400 & 3.53276100 & -1.12022700 \\
\hline $\mathrm{C}$ & 2.36013900 & -1.87263500 & 2.33406300 & $\mathrm{C}$ & 2.25172900 & 4.90626400 & -2.20541700 \\
\hline $\mathrm{C}$ & 4.54324900 & -3.35002000 & 1.61183200 & $\mathrm{H}$ & 4.09843000 & 4.93782700 & -1.09180700 \\
\hline $\mathrm{C}$ & 3.09405100 & -2.51926200 & 3.33009700 & $\mathrm{H}$ & 0.27905300 & 4.71306500 & -3.05360700 \\
\hline $\mathrm{H}$ & 1.49601600 & -1.26760800 & 2.57783900 & $\mathrm{H}$ & 2.66065500 & 5.43277400 & -3.06206600 \\
\hline $\mathrm{C}$ & 4.20919600 & -3.26985600 & 2.96572400 & $\mathrm{C}$ & -0.58899600 & -4.65935900 & -1.03204900 \\
\hline $\mathrm{H}$ & 5.40503900 & -3.92766800 & 1.28556800 & $\mathrm{H}$ & -1.02613500 & -5.35665500 & -0.30616000 \\
\hline $\mathrm{H}$ & 2.80077400 & -2.43234800 & 4.37192700 & $\mathrm{H}$ & -0.05454500 & -5.27618400 & -1.76527800 \\
\hline $\mathrm{H}$ & 4.81019100 & -3.78474000 & 3.70791000 & $\mathrm{C}$ & -1.73043700 & -3.90968900 & -1.73887200 \\
\hline $\mathrm{C}$ & -3.42282700 & -0.81282200 & 0.66092200 & $\mathrm{H}$ & -2.40786400 & -4.65462800 & -2.17118000 \\
\hline $\mathrm{C}$ & -3.01831000 & -0.81486300 & 2.00340300 & $\mathrm{H}$ & -1.34519100 & -3.34546800 & -2.59481000 \\
\hline $\mathrm{C}$ & -5.62291000 & -0.49983800 & 1.26200500 & $\mathrm{C}$ & -2.55500500 & -3.00301800 & -0.79904800 \\
\hline $\mathrm{C}$ & -3.98282600 & -0.66041600 & 2.99911200 & $\mathrm{H}$ & -3.61515100 & -3.03620700 & -1.06549000 \\
\hline $\mathrm{H}$ & -1.96980400 & -0.93541600 & 2.25905800 & $\mathrm{H}$ & -2.49464700 & -3.38050300 & 0.22830400 \\
\hline $\mathrm{C}$ & -5.31527400 & -0.49521400 & 2.62336400 & & & & \\
\hline $\mathrm{H}$ & -6.65008300 & -0.37527300 & 0.92613800 & $54 \mathrm{~b}$ & & & \\
\hline $\mathrm{H}$ & -3.69895000 & -0.66909200 & 4.04754600 & $\mathrm{C}$ & -3.39416400 & -0.27550100 & 0.68426600 \\
\hline $\mathrm{H}$ & -6.10020500 & -0.37169600 & 3.36224800 & $\mathrm{C}$ & -2.85571100 & -0.16987100 & 1.97511200 \\
\hline $\mathrm{N}$ & 3.84131200 & -2.74305400 & 0.64825400 & $\mathrm{C}$ & -3.64119400 & 0.40047900 & 2.97758600 \\
\hline $\mathrm{N}$ & -4.70547500 & -0.66230100 & 0.29977800 & $\mathrm{C}$ & -4.91826800 & 0.85751000 & 2.65692200 \\
\hline $\mathrm{Pd}$ & 0.01263500 & 0.06406900 & 0.21381500 & $\mathrm{C}$ & -5.35830300 & 0.72598000 & 1.33848600 \\
\hline $\mathrm{C}$ & 1.24130900 & 1.34469500 & 1.20151700 & $\mathrm{H}$ & -1.84871600 & -0.51451300 & 2.18632800 \\
\hline $\mathrm{O}$ & 2.26975000 & 1.11427900 & 1.77791900 & $\mathrm{H}$ & -3.25860400 & 0.48934700 & 3.98999200 \\
\hline $\mathrm{C}$ & 0.64023500 & 2.81629000 & 1.25757000 & $\mathrm{H}$ & -5.56077600 & 1.31018300 & 3.40485500 \\
\hline $\mathrm{C}$ & -0.86613900 & 2.67832300 & 1.18203400 & $\mathrm{H}$ & -6.34433900 & 1.07834100 & 1.04454900 \\
\hline $\mathrm{O}$ & -1.42740900 & 1.63459000 & 0.81922300 & $\mathrm{~N}$ & -4.61923400 & 0.16913300 & 0.37097400 \\
\hline $\mathrm{O}$ & -1.54248400 & 3.74354300 & 1.54921900 & $\mathrm{P}$ & -2.40818000 & -1.07188500 & -0.67855500 \\
\hline $\mathrm{C}$ & -2.99241100 & 3.66634100 & 1.52062500 & $\mathrm{C}$ & -2.82786200 & -2.92786300 & -0.54082800 \\
\hline $\mathrm{H}$ & -3.33505000 & 2.86672600 & 2.17918500 & $\mathrm{C}$ & -3.24869000 & -0.47614600 & -2.24866900 \\
\hline $\mathrm{H}$ & -3.33258700 & 4.63764200 & 1.87332600 & $\mathrm{H}$ & -3.61608700 & -1.35634500 & -2.78290100 \\
\hline $\mathrm{H}$ & -3.33443000 & 3.47874900 & 0.50176500 & $\mathrm{H}$ & -4.12743700 & 0.08851100 & -1.92043700 \\
\hline $\mathrm{C}$ & 1.03894900 & 3.49619300 & 2.58631700 & $\mathrm{C}$ & -2.34237100 & 0.34674000 & -3.18016600 \\
\hline $\mathrm{H}$ & 0.71422200 & 4.53793700 & 2.58408400 & $\mathrm{H}$ & -2.89882900 & 0.56904000 & -4.09865300 \\
\hline $\mathrm{H}$ & 0.57800000 & 2.98463300 & 3.43648700 & $\mathrm{H}$ & -1.48764600 & -0.26989400 & -3.48532400 \\
\hline
\end{tabular}




\begin{tabular}{|c|c|c|c|c|c|c|c|}
\hline $\mathrm{C}$ & -1.82787400 & 1.65778700 & -2.56328200 & $\mathrm{H}$ & -4.73524000 & -2.85081900 & -1.64812900 \\
\hline $\mathrm{H}$ & -2.61290900 & 2.42424600 & -2.57677700 & $\mathrm{C}$ & -2.35588100 & -3.44393300 & 0.83102300 \\
\hline $\mathrm{H}$ & -1.67011400 & 1.51197100 & -1.46853600 & $\mathrm{H}$ & -2.52695100 & -4.52506500 & 0.88056600 \\
\hline $\mathrm{C}$ & -0.53268800 & 2.20058500 & -3.18970100 & $\mathrm{H}$ & -1.29042400 & -3.26748500 & 1.00024000 \\
\hline $\mathrm{H}$ & -0.77402800 & 2.75940200 & -4.10185200 & $\mathrm{H}$ & -2.91243900 & -2.98584300 & 1.65318600 \\
\hline $\mathrm{H}$ & 0.09720400 & 1.35974500 & -3.50644000 & $\mathrm{C}$ & 0.87120700 & -0.99658600 & 0.50701500 \\
\hline $\mathrm{C}$ & 0.27744200 & 3.11573600 & -2.25583100 & $\mathrm{O}$ & 0.76926500 & -0.85226200 & 1.69015200 \\
\hline $\mathrm{H}$ & 1.12926600 & 3.53308200 & -2.79884700 & $\mathrm{C}$ & 1.83114700 & -2.01852400 & -0.21231300 \\
\hline $\mathrm{H}$ & -0.31167600 & 3.96473400 & -1.89406000 & $\mathrm{C}$ & 1.67596900 & -1.95015200 & -1.73643600 \\
\hline $\mathrm{P}$ & 0.94178200 & 2.26097000 & -0.72057300 & $\mathrm{H}$ & 0.63578000 & -2.11009300 & -2.02281700 \\
\hline $\mathrm{C}$ & 2.80842500 & 2.65188700 & -0.67653300 & $\mathrm{H}$ & 1.99564800 & -0.98028500 & -2.12090000 \\
\hline $\mathrm{C}$ & 0.22816100 & 3.25210800 & 0.68358700 & $\mathrm{H}$ & 2.26145600 & -2.73816500 & -2.20949600 \\
\hline $\mathrm{C}$ & 0.19398700 & 2.71880200 & 1.98046500 & $\mathrm{C}$ & 3.28681200 & -1.76390700 & 0.26336000 \\
\hline $\mathrm{C}$ & -0.72848200 & 5.22228300 & 1.38548100 & $\mathrm{C}$ & 4.32963900 & -1.66686600 & -0.67143400 \\
\hline $\mathrm{C}$ & -0.31548300 & 3.51082700 & 3.01034100 & $\mathrm{C}$ & 3.60596700 & -1.67696700 & 1.62928200 \\
\hline $\mathrm{H}$ & 0.54462200 & 1.71098300 & 2.17699700 & $\mathrm{C}$ & 5.64936800 & -1.48014100 & -0.25409600 \\
\hline $\mathrm{C}$ & -0.78561600 & 4.78829100 & 2.71123100 & $\mathrm{H}$ & 4.13337700 & -1.74847900 & -1.73305800 \\
\hline $\mathrm{H}$ & -1.09405100 & 6.20825200 & 1.10780000 & $\mathrm{C}$ & 4.92418900 & -1.48548200 & 2.04106500 \\
\hline $\mathrm{H}$ & -0.34724400 & 3.13254700 & 4.02780600 & $\mathrm{H}$ & 2.82391400 & -1.76016900 & 2.37229000 \\
\hline $\mathrm{H}$ & -1.19201800 & 5.43565500 & 3.48124400 & $\mathrm{C}$ & 5.95296600 & -1.38590800 & 1.10264900 \\
\hline $\mathrm{N}$ & -0.23013500 & 4.47733800 & 0.39109200 & $\mathrm{H}$ & 6.43800700 & -1.41499700 & -0.99759100 \\
\hline $\mathrm{Pd}$ & -0.28439000 & 0.14741600 & -0.63934900 & $\mathrm{H}$ & 5.14595000 & -1.42042200 & 3.10207500 \\
\hline $\mathrm{C}$ & 3.03585200 & 4.17439600 & -0.75914700 & $\mathrm{H}$ & 6.97928800 & -1.24274100 & 1.42645700 \\
\hline $\mathrm{H}$ & 2.56098200 & 4.70856200 & 0.06718200 & $\mathrm{C}$ & 1.44160900 & -3.46407700 & 0.22644800 \\
\hline $\mathrm{H}$ & 4.11314600 & 4.36924300 & -0.70848700 & $\mathrm{O}$ & 1.29473600 & -4.36609300 & -0.56812000 \\
\hline $\mathrm{H}$ & 2.67154200 & 4.60671600 & -1.69479000 & $\mathrm{O}$ & 1.33035700 & -3.60529200 & 1.55098800 \\
\hline $\mathrm{C}$ & 3.47397600 & 1.95012900 & -1.87517300 & $\mathrm{C}$ & 1.12728700 & -4.95825000 & 2.02131600 \\
\hline $\mathrm{H}$ & 3.37903000 & 0.86312900 & -1.81213700 & $\mathrm{H}$ & 1.05808900 & -4.87861100 & 3.10485300 \\
\hline $\mathrm{H}$ & 3.06946300 & 2.28292500 & -2.83644300 & $\mathrm{H}$ & 0.20926900 & -5.37276500 & 1.60115400 \\
\hline $\mathrm{H}$ & 4.54427300 & 2.18416900 & -1.87587400 & $\mathrm{H}$ & 1.97277300 & -5.58485900 & 1.73087100 \\
\hline $\mathrm{C}$ & 3.40051000 & 2.11593600 & 0.63939800 & & & & \\
\hline $\mathrm{H}$ & 3.25873000 & 1.03848900 & 0.75287400 & 54-O & & & \\
\hline $\mathrm{H}$ & 4.47940700 & 2.30544000 & 0.64618700 & $\mathrm{C}$ & 2.05653600 & 2.88175700 & -0.33223200 \\
\hline $\mathrm{H}$ & 2.97453200 & 2.61929800 & 1.51184200 & $\mathrm{H}$ & 2.56112000 & 2.40122300 & -1.17595800 \\
\hline $\mathrm{C}$ & -2.09365500 & -3.67923400 & -1.66814900 & $\mathrm{H}$ & 2.02456900 & 3.95488500 & -0.53063300 \\
\hline $\mathrm{H}$ & -2.34636800 & -3.29449700 & -2.66152800 & $\mathrm{C}$ & 2.78841700 & 2.61747900 & 0.99748300 \\
\hline $\mathrm{H}$ & -1.00808900 & -3.65364500 & -1.54731600 & $\mathrm{H}$ & 2.54897600 & 3.42866800 & 1.69122200 \\
\hline $\mathrm{H}$ & -2.39451500 & -4.73257500 & -1.64665800 & $\mathrm{H}$ & 2.41270100 & 1.70093900 & 1.46842900 \\
\hline $\mathrm{C}$ & -4.35019300 & -3.13895600 & -0.66655700 & $\mathrm{P}$ & 2.74080600 & -0.86398900 & -0.13713400 \\
\hline $\mathrm{H}$ & -4.56686400 & -4.20513300 & -0.53422100 & $\mathrm{P}$ & 0.31983800 & 2.24122700 & -0.33406700 \\
\hline $\mathrm{H}$ & -4.91021200 & -2.58678700 & 0.09194300 & $\mathrm{C}$ & -0.53676600 & 3.05850200 & -1.83386000 \\
\hline
\end{tabular}




\begin{tabular}{|c|c|c|c|c|c|c|c|}
\hline $\mathrm{C}$ & 3.22515000 & -1.51820500 & -1.87060500 & $\mathrm{H}$ & 0.84827800 & -4.04828200 & 3.24568900 \\
\hline $\mathrm{C}$ & -0.22179400 & 2.20988000 & -3.08066400 & $\mathrm{H}$ & 2.95490700 & -5.41807100 & 3.26299300 \\
\hline $\mathrm{H}$ & -0.70939500 & 2.65553100 & -3.95504400 & $\mathrm{~N}$ & -0.17783900 & 4.37335900 & 1.24890400 \\
\hline $\mathrm{H}$ & 0.85208100 & 2.17425500 & -3.29056500 & $\mathrm{~N}$ & 3.85566300 & -3.09111700 & 0.99011700 \\
\hline $\mathrm{H}$ & -0.58171400 & 1.18191300 & -2.97856700 & $\mathrm{Pd}$ & 0.32748100 & -0.10627000 & -0.30548600 \\
\hline $\mathrm{C}$ & -0.03524700 & 4.50109300 & -2.05973500 & $\mathrm{C}$ & -1.49790700 & -0.96110400 & -0.51992800 \\
\hline $\mathrm{H}$ & -0.63962400 & 4.95605600 & -2.85285400 & $\mathrm{O}$ & -0.84073600 & -1.98244000 & -0.46835100 \\
\hline $\mathrm{H}$ & -0.13166500 & 5.11017400 & -1.15895400 & $\mathrm{C}$ & -3.01226600 & -0.94504900 & -0.80126900 \\
\hline $\mathrm{H}$ & 1.00531500 & 4.52652700 & -2.39396200 & $\mathrm{C}$ & -3.64945400 & 0.18574100 & 0.01403800 \\
\hline $\mathrm{C}$ & -2.05415700 & 3.09226900 & -1.58026000 & $\mathrm{O}$ & -3.23219500 & 0.54537900 & 1.09734700 \\
\hline $\mathrm{H}$ & -2.30819200 & 3.75101300 & -0.74577500 & $\mathrm{O}$ & -4.74588100 & 0.67010100 & -0.57330200 \\
\hline $\mathrm{H}$ & -2.55906300 & 3.47512900 & -2.47408500 & $\mathrm{C}$ & -5.49650200 & 1.64398200 & 0.18769400 \\
\hline $\mathrm{H}$ & -2.46172700 & 2.10140500 & -1.37135700 & $\mathrm{H}$ & -4.87933800 & 2.52123400 & 0.39084800 \\
\hline $\mathrm{C}$ & 2.90006800 & -0.41396300 & -2.89782900 & $\mathrm{H}$ & -6.34768500 & 1.90511100 & -0.43859000 \\
\hline $\mathrm{H}$ & 1.84480200 & -0.12499000 & -2.86057100 & $\mathrm{H}$ & -5.82857300 & 1.20628500 & 1.13108300 \\
\hline $\mathrm{H}$ & 3.50740100 & 0.48473500 & -2.74963400 & $\mathrm{C}$ & -3.13706200 & -0.71835200 & -2.32633600 \\
\hline $\mathrm{H}$ & 3.11260800 & -0.78439800 & -3.90729000 & $\mathrm{H}$ & -4.19230700 & -0.69092900 & -2.59976600 \\
\hline $\mathrm{C}$ & 4.71359300 & -1.89814600 & -1.97272300 & $\mathrm{H}$ & -2.68674300 & 0.23085700 & -2.61942100 \\
\hline $\mathrm{H}$ & 4.98929100 & -2.64808200 & -1.22901700 & $\mathrm{H}$ & -2.64276300 & -1.52195700 & -2.87673500 \\
\hline $\mathrm{H}$ & 4.90390400 & -2.31344400 & -2.96939900 & $\mathrm{C}$ & -3.65372200 & -2.26554400 & -0.31689800 \\
\hline $\mathrm{H}$ & 5.36918600 & -1.03018900 & -1.85427100 & $\mathrm{C}$ & -4.23273100 & -3.17479900 & -1.20982100 \\
\hline $\mathrm{C}$ & 2.35167900 & -2.75363900 & -2.17172500 & $\mathrm{C}$ & -3.65812500 & -2.56652000 & 1.05467000 \\
\hline $\mathrm{H}$ & 2.52988400 & -3.07227900 & -3.20499600 & $\mathrm{C}$ & -4.80419000 & -4.35991200 & -0.74073500 \\
\hline $\mathrm{H}$ & 2.59609600 & -3.59365200 & -1.51727900 & $\mathrm{H}$ & -4.25072400 & -2.97571800 & -2.27459000 \\
\hline $\mathrm{H}$ & 1.28219400 & -2.54108600 & -2.07142100 & $\mathrm{C}$ & -4.22718200 & -3.75045800 & 1.51884800 \\
\hline $\mathrm{C}$ & -0.37233900 & 3.04433200 & 1.18678300 & $\mathrm{H}$ & -3.22794000 & -1.86549900 & 1.76306500 \\
\hline $\mathrm{C}$ & -0.99092100 & 2.31498900 & 2.20412800 & $\mathrm{C}$ & -4.80317200 & -4.65262300 & 0.62197000 \\
\hline $\mathrm{C}$ & -0.60823200 & 5.02083200 & 2.33660400 & $\mathrm{H}$ & -5.25141900 & -5.05190700 & -1.44770900 \\
\hline $\mathrm{C}$ & -1.42980700 & 3.00365600 & 3.33824200 & $\mathrm{H}$ & -4.22523600 & -3.96514000 & 2.58325800 \\
\hline $\mathrm{H}$ & -1.14747100 & 1.24884400 & 2.10319900 & $\mathrm{H}$ & -5.24946600 & -5.57384500 & 0.98344800 \\
\hline $\mathrm{C}$ & -1.23600900 & 4.38019000 & 3.40899900 & $\mathrm{C}$ & 4.31023200 & 2.50773800 & 0.83598300 \\
\hline $\mathrm{H}$ & -0.43873700 & 6.09508400 & 2.35389300 & $\mathrm{H}$ & 4.76050400 & 2.45516200 & 1.83526900 \\
\hline $\mathrm{H}$ & -1.91594500 & 2.46870200 & 4.14832900 & $\mathrm{H}$ & 4.70316300 & 3.42049600 & 0.37093300 \\
\hline $\mathrm{H}$ & -1.55956000 & 4.95266500 & 4.27210800 & $\mathrm{C}$ & 4.77485200 & 1.28659200 & 0.02497300 \\
\hline $\mathrm{C}$ & 2.72605500 & -2.36368100 & 0.97084200 & $\mathrm{H}$ & 5.87032500 & 1.26908200 & 0.03444200 \\
\hline $\mathrm{C}$ & 1.62045000 & -2.67356200 & 1.76978300 & $\mathrm{H}$ & 4.50135000 & 1.40303700 & -1.02939800 \\
\hline $\mathrm{C}$ & 3.91042900 & -4.15722200 & 1.79514700 & $\mathrm{C}$ & 4.27094400 & -0.05992400 & 0.58737800 \\
\hline $\mathrm{C}$ & 1.69045400 & -3.78480600 & 2.61295700 & $\mathrm{H}$ & 5.03713400 & -0.83496700 & 0.50357300 \\
\hline $\mathrm{H}$ & 0.72592200 & -2.06190900 & 1.73422100 & $\mathrm{H}$ & 4.06261400 & 0.03461900 & 1.65961000 \\
\hline $\mathrm{C}$ & 2.85601200 & -4.54555600 & 2.62575300 & & & & \\
\hline $\mathrm{H}$ & 4.83915800 & -4.72324300 & 1.77797500 & 54-CO & & & \\
\hline
\end{tabular}




\begin{tabular}{|c|c|c|c|c|c|c|c|}
\hline $\mathrm{C}$ & -0.85512500 & 2.69216500 & -0.14716900 & $\mathrm{H}$ & -4.29567500 & -2.18065400 & -2.36783900 \\
\hline $\mathrm{C}$ & -2.01990500 & 3.46656100 & -0.06670100 & $\mathrm{H}$ & -4.45276700 & -3.89673800 & -1.97944800 \\
\hline $\mathrm{C}$ & -2.30059500 & 4.36269100 & -1.09940600 & $\mathrm{H}$ & -5.06420600 & -2.68306400 & -0.85659000 \\
\hline $\mathrm{C}$ & -1.41454100 & 4.45961100 & -2.16958700 & $\mathrm{C}$ & -2.83524000 & -4.06722600 & 0.16984300 \\
\hline $\mathrm{C}$ & -0.28451500 & 3.64005600 & -2.16762600 & $\mathrm{H}$ & -1.88666900 & -4.12481800 & 0.70862400 \\
\hline $\mathrm{H}$ & -2.68923600 & 3.38901200 & 0.78189200 & $\mathrm{H}$ & -3.63846200 & -3.97493500 & 0.90581500 \\
\hline $\mathrm{H}$ & -3.19284600 & 4.98014100 & -1.05930000 & $\mathrm{H}$ & -2.97441200 & -5.02593600 & -0.34200200 \\
\hline $\mathrm{H}$ & -1.58883800 & 5.15097200 & -2.98737600 & $\mathrm{C}$ & -1.79415100 & -3.18147700 & -1.95149000 \\
\hline $\mathrm{H}$ & 0.43100200 & 3.68160400 & -2.98591700 & $\mathrm{H}$ & -0.78401900 & -3.23363300 & -1.53646800 \\
\hline $\mathrm{N}$ & -0.00798700 & 2.77134300 & -1.18808300 & $\mathrm{H}$ & -1.99427200 & -4.13862600 & -2.44571300 \\
\hline $\mathrm{P}$ & -0.29405200 & 1.54319300 & 1.19587000 & $\mathrm{H}$ & -1.80659800 & -2.41016900 & -2.72655600 \\
\hline $\mathrm{C}$ & 1.00057500 & 2.60188200 & 2.14618100 & $\mathrm{C}$ & 1.42160700 & 1.85427100 & 3.42775100 \\
\hline $\mathrm{C}$ & -1.76812800 & 1.39240600 & 2.32477200 & $\mathrm{H}$ & 0.60610300 & 1.79110300 & 4.15211300 \\
\hline $\mathrm{H}$ & -1.98274500 & 2.35432200 & 2.80077800 & $\mathrm{H}$ & 1.79453600 & 0.84947900 & 3.22238200 \\
\hline $\mathrm{H}$ & -2.60752700 & 1.18694800 & 1.65643500 & $\mathrm{H}$ & 2.23228500 & 2.41653100 & 3.90477800 \\
\hline $\mathrm{C}$ & -1.64435200 & 0.28004500 & 3.39569100 & $\mathrm{C}$ & 0.37590100 & 3.95982200 & 2.52732800 \\
\hline $\mathrm{H}$ & -1.23733600 & 0.72014400 & 4.30996000 & $\mathrm{H}$ & 1.12287600 & 4.53563800 & 3.08493400 \\
\hline $\mathrm{H}$ & -0.91380500 & -0.47574700 & 3.07780700 & $\mathrm{H}$ & 0.09239000 & 4.55083100 & 1.65284400 \\
\hline $\mathrm{C}$ & -2.96937700 & -0.42699500 & 3.73296700 & $\mathrm{H}$ & -0.49744900 & 3.85810500 & 3.17798800 \\
\hline $\mathrm{H}$ & -2.88208700 & -0.84144200 & 4.74385300 & $\mathrm{C}$ & 2.23234100 & 2.85672300 & 1.25672600 \\
\hline $\mathrm{H}$ & -3.78395100 & 0.30856400 & 3.78027500 & $\mathrm{H}$ & 2.91571800 & 3.51868600 & 1.80043100 \\
\hline $\mathrm{C}$ & -3.37511300 & -1.58508900 & 2.80414700 & $\mathrm{H}$ & 2.78270600 & 1.94363500 & 1.03191200 \\
\hline $\mathrm{H}$ & -4.22834000 & -2.09000000 & 3.27139900 & $\mathrm{H}$ & 1.96993500 & 3.34445000 & 0.31565400 \\
\hline $\mathrm{H}$ & -2.56884700 & -2.32933500 & 2.78875600 & $\mathrm{C}$ & 2.14204600 & -0.60370900 & 0.55797500 \\
\hline $\mathrm{C}$ & -3.80607800 & -1.20649500 & 1.36795400 & $\mathrm{O}$ & 2.72742500 & -0.53789700 & 1.59191900 \\
\hline $\mathrm{H}$ & -4.60446100 & -1.87598900 & 1.03712100 & $\mathrm{C}$ & 2.80531400 & -0.65491000 & -0.86891900 \\
\hline $\mathrm{H}$ & -4.25205700 & -0.20974700 & 1.32590300 & $\mathrm{C}$ & 4.15191300 & 0.09897900 & -0.83725900 \\
\hline$P$ & -2.50668800 & -1.26783600 & 0.02378400 & $\mathrm{C}$ & 5.19943700 & -0.33640800 & -0.00783600 \\
\hline $\mathrm{C}$ & -2.86714400 & -2.93353900 & -0.87531400 & $\mathrm{C}$ & 4.36022500 & 1.23058800 & -1.63921000 \\
\hline $\mathrm{C}$ & -3.14212400 & -0.01932700 & -1.20210200 & $\mathrm{C}$ & 6.41752700 & 0.34051300 & 0.01256600 \\
\hline $\mathrm{C}$ & -2.39321700 & 0.30762500 & -2.34187900 & $\mathrm{H}$ & 5.06320400 & -1.20373900 & 0.62566400 \\
\hline $\mathrm{C}$ & -4.87863600 & 1.33322900 & -1.87519400 & $\mathrm{C}$ & 5.58209100 & 1.90576200 & -1.61640000 \\
\hline $\mathrm{C}$ & -2.95620200 & 1.15930900 & -3.29169200 & $\mathrm{H}$ & 3.57765000 & 1.59589800 & -2.29262400 \\
\hline $\mathrm{H}$ & -1.39389800 & -0.08914900 & -2.48301100 & $\mathrm{C}$ & 6.61546500 & 1.46407400 & -0.79180600 \\
\hline $\mathrm{C}$ & -4.22938100 & 1.67846000 & -3.06110800 & $\mathrm{H}$ & 7.21440200 & -0.01442200 & 0.65912600 \\
\hline $\mathrm{H}$ & -5.86432000 & 1.73238900 & -1.64572300 & $\mathrm{H}$ & 5.72238700 & 2.77676600 & -2.24948800 \\
\hline $\mathrm{H}$ & -2.40759900 & 1.41399500 & -4.19336600 & $\mathrm{H}$ & 7.56618300 & 1.98816400 & -0.77647900 \\
\hline $\mathrm{H}$ & -4.70806100 & 2.33972500 & -3.77581800 & $\mathrm{C}$ & 3.09197900 & -2.14241800 & -1.21206100 \\
\hline $\mathrm{N}$ & -4.35612800 & 0.49982900 & -0.96673700 & $\mathrm{O}$ & 3.01455100 & -2.58803200 & -2.33258600 \\
\hline $\mathrm{Pd}$ & 0.04312500 & -0.82589500 & 0.52569900 & $\mathrm{O}$ & 3.44376400 & -2.86705700 & -0.13529600 \\
\hline $\mathrm{C}$ & -4.25183300 & -2.90730800 & -1.55328500 & $\mathrm{C}$ & 3.83564300 & -4.23491500 & -0.40057600 \\
\hline
\end{tabular}




$\begin{array}{lrrr}\mathrm{H} & 4.08007000 & -4.65881000 & 0.57190000 \\ \mathrm{H} & 3.01462300 & -4.78095500 & -0.86924400 \\ \mathrm{H} & 4.70382300 & -4.25248400 & -1.06189300 \\ \mathrm{C} & 0.47425500 & -2.73941600 & 0.78289800 \\ \mathrm{O} & 0.65115900 & -3.80987400 & 1.13742900 \\ \mathrm{C} & 1.83357200 & -0.12853500 & -1.93426500 \\ \mathrm{H} & 0.93815000 & -0.75750200 & -1.96674600 \\ \mathrm{H} & 1.52841000 & 0.89890900 & -1.72900600 \\ \mathrm{H} & 2.29962100 & -0.19466300 & -2.91818100 \\ & & & \end{array}$

\section{TS48}

\section{im}

$\mathrm{C}$

C

C

C

C

$\mathrm{H}$

$\mathrm{H}$

$\mathrm{H}$

$\mathrm{H}$

$\mathrm{N}$

P

C

C

$\mathrm{H}$

$\mathrm{H}$

C

$\mathrm{H}$

$\mathrm{H}$

C

$\mathrm{H}$

$\mathrm{H}$

C

$\mathrm{H}$

$\mathrm{H}$

C

$\mathrm{H}$

$\mathrm{H}$

P

C

\section{maginary frequency $=-50.54 \mathrm{~cm}^{-1}$}

$\begin{array}{lrrrr}\text { C } & -0.49975500 & 3.14740000 & -0.71736000 & \mathrm{H} \\ \mathrm{C} & -1.06307500 & 4.43151400 & -0.76145400 & \mathrm{H} \\ \mathrm{C} & -0.80156600 & 5.27905400 & -1.83771100 & \mathrm{H} \\ \mathrm{C} & 0.02288400 & 4.83212400 & -2.86615900 & \mathrm{C} \\ \mathrm{C} & 0.54152200 & 3.54593000 & -2.76618500 & \mathrm{H} \\ \mathrm{H} & -1.70139300 & 4.78265000 & 0.03811600 & \mathrm{H} \\ \mathrm{H} & -1.23584700 & 6.27375100 & -1.86575400 & \mathrm{H} \\ \mathrm{H} & 0.25866400 & 5.45503100 & -3.72181300 & \mathrm{C} \\ \mathrm{H} & 1.19171400 & 3.14512800 & -3.53988600 & \mathrm{H} \\ \mathrm{N} & 0.28630400 & 2.72615400 & -1.73282000 & \mathrm{H} \\ \mathrm{P} & -0.76507700 & 1.94998400 & 0.72094600 & \mathrm{H} \\ \mathrm{C} & 0.12088900 & 2.82345400 & 2.19351200 & \mathrm{C} \\ \mathrm{C} & -2.60718000 & 2.25693300 & 0.97954900 & \mathrm{H} \\ \mathrm{H} & -2.75149900 & 3.33226900 & 0.85431200 & \mathrm{H} \\ \mathrm{H} & -3.09023300 & 1.78389200 & 0.11665800 & \mathrm{H} \\ \mathrm{C} & -3.36046800 & 1.84972700 & 2.27150000 & \mathrm{C} \\ \mathrm{H} & -4.25604000 & 2.48293000 & 2.28839000 & \mathrm{H} \\ \mathrm{H} & -2.79310100 & 2.12973800 & 3.16474800 & \mathrm{H} \\ \mathrm{C} & -3.81944800 & 0.38939000 & 2.37449500 & \mathrm{H} \\ \mathrm{H} & -4.65691900 & 0.34578300 & 3.07976600 & \mathrm{C} \\ \mathrm{H} & -4.24335300 & 0.08780800 & 1.41162300 & \mathrm{H} \\ \mathrm{C} & -2.73729300 & -0.61648400 & 2.82863100 & \mathrm{H} \\ \mathrm{H} & -2.86893700 & -0.83311300 & 3.89532600 & \mathrm{H} \\ \mathrm{H} & -1.73991000 & -0.17502600 & 2.73534200 & \mathrm{C} \\ \mathrm{C} & -2.74942000 & -1.94026800 & 2.05288900 & \mathrm{O} \\ \mathrm{H} & -2.12884200 & -2.67862800 & 2.57005100 & \mathrm{C} \\ \mathrm{H} & -3.76051100 & -2.34949800 & 1.96895200 & \mathrm{H} \\ \mathrm{P} & -2.03644500 & -1.78211300 & 0.34067100 & \mathrm{H} \\ \mathrm{C} & -1.60967400 & -3.58659200 & -0.14104200 & \mathrm{H} \\ & & & \end{array}$

\begin{tabular}{|c|c|c|}
\hline-3.51350300 & -1.39753200 & -0.72688900 \\
\hline-3.35588500 & -0.66310400 & -1.91055900 \\
\hline-5.77604200 & -1.61925800 & -1.09759300 \\
\hline-4.47372700 & -0.42105400 & -2.71066700 \\
\hline-2.37585000 & -0.29062200 & -2.19203100 \\
\hline-5.71214400 & -0.90840100 & -2.29821800 \\
\hline-6.72487500 & -2.01089500 & -0.73709200 \\
\hline-4.37918300 & 0.13973500 & -3.63610400 \\
\hline-6.60946700 & -0.74281400 & -2.88525000 \\
\hline-4.70890200 & -1.86292600 & -0.32753400 \\
\hline-0.08970300 & -0.22549300 & 0.06673900 \\
\hline-2.77431500 & -4.54825900 & 0.16146600 \\
\hline-3.68983000 & -4.27170900 & -0.36622600 \\
\hline-2.49109100 & -5.55798100 & -0.15899800 \\
\hline-3.00303400 & -4.59786900 & 1.22975100 \\
\hline-0.36577600 & -4.00364000 & 0.66910300 \\
\hline 0.48165100 & -3.34136100 & 0.47929800 \\
\hline-0.55858100 & -4.02295600 & 1.74665600 \\
\hline-0.07443800 & -5.01942200 & 0.37706900 \\
\hline-1.27805100 & -3.62969500 & -1.64552800 \\
\hline-0.43349500 & -2.98209500 & -1.89381700 \\
\hline-1.00191300 & -4.65436100 & -1.91991400 \\
\hline-2.13731500 & -3.34782900 & -2.26173200 \\
\hline 0.18452400 & 1.82607800 & 3.37003200 \\
\hline-0.79987600 & 1.59268800 & 3.78044900 \\
\hline 0.67051400 & 0.88967000 & 3.08022100 \\
\hline 0.77581400 & 2.27154400 & 4.17832800 \\
\hline-0.57628400 & 4.12106800 & 2.64369000 \\
\hline-0.05033200 & 4.51238400 & 3.52204500 \\
\hline-0.53246600 & 4.90026600 & 1.87837700 \\
\hline-1.61876400 & 3.97287100 & 2.93351200 \\
\hline 1.56163700 & 3.15214000 & 1.77100500 \\
\hline 2.07829400 & 3.63592300 & 2.60775400 \\
\hline 2.10938500 & 2.24116800 & 1.52896100 \\
\hline 1.60706700 & 3.83532800 & 0.91757800 \\
\hline 1.81661900 & -0.36815300 & -0.86675500 \\
\hline 1.28236800 & -1.48448000 & -0.97587200 \\
\hline 1.01560700 & -0.18972900 & -3.41631400 \\
\hline 1.71996500 & -0.97546400 & -3.67972300 \\
\hline 0.88344400 & 0.50588600 & -4.24804500 \\
\hline 0.05677500 & -0.62288300 & -3.12358600 \\
\hline
\end{tabular}




\begin{tabular}{|c|c|c|c|c|c|c|c|}
\hline $\mathrm{O}$ & 1.60622700 & 0.55180300 & -2.32264400 & $\mathrm{H}$ & -0.10352600 & 4.84719200 & 0.46704300 \\
\hline $\mathrm{H}$ & 1.01216700 & 1.34835600 & -2.02001600 & $\mathrm{H}$ & -0.74779300 & 3.74125000 & -0.74201800 \\
\hline $\mathrm{C}$ & 3.31002800 & -0.17476500 & -0.51723100 & $\mathrm{C}$ & -1.79084800 & 3.71251900 & 1.15603900 \\
\hline $\mathrm{C}$ & 3.77829500 & 1.28372700 & -0.69508900 & $\mathrm{H}$ & -2.37698600 & 4.59758000 & 0.88154400 \\
\hline $\mathrm{H}$ & 3.13971400 & 1.97625200 & -0.14774600 & $\mathrm{H}$ & -1.56651500 & 3.83955500 & 2.22149300 \\
\hline $\mathrm{H}$ & 3.74937000 & 1.56223900 & -1.74905800 & C & -2.67671300 & 2.46324000 & 0.95206300 \\
\hline $\mathrm{H}$ & 4.80461900 & 1.38811000 & -0.34200400 & $\mathrm{H}$ & -3.72585900 & 2.77649300 & 0.98578700 \\
\hline C & 3.53480100 & -0.73201900 & 0.90316100 & $\mathrm{H}$ & -2.52257400 & 2.05862300 & -0.05705600 \\
\hline C & 3.35644900 & -2.10419000 & 1.14322700 & $\mathrm{C}$ & -2.48380600 & 1.35707500 & 2.00109500 \\
\hline C & 3.93710600 & 0.08391000 & 1.96697400 & $\mathrm{H}$ & -2.50787400 & 1.81918600 & 2.99818800 \\
\hline $\mathrm{C}$ & 3.56449400 & -2.64038100 & 2.41262100 & $\mathrm{H}$ & -1.48860300 & 0.90409700 & 1.89243600 \\
\hline $\mathrm{H}$ & 3.05789900 & -2.75862000 & 0.33064100 & $\mathrm{C}$ & -3.55637300 & 0.25431200 & 1.97003700 \\
\hline C & 4.14129600 & -0.45283200 & 3.24124400 & $\mathrm{H}$ & -3.40565800 & -0.40461900 & 2.83154300 \\
\hline $\mathrm{H}$ & 4.11675800 & 1.14138100 & 1.81378100 & $\mathrm{H}$ & -4.55606200 & 0.69168400 & 2.05454000 \\
\hline C & 3.95317600 & -1.81475500 & 3.47017200 & $\mathrm{P}$ & -3.50255100 & -0.83515800 & 0.44896600 \\
\hline $\mathrm{H}$ & 3.42941600 & -3.70585900 & 2.57328700 & $\mathrm{C}$ & -4.44204600 & -2.40418300 & 1.03355400 \\
\hline $\mathrm{H}$ & 4.45996600 & 0.19758600 & 4.05049600 & $\mathrm{C}$ & -4.77274300 & -0.00516200 & -0.64147900 \\
\hline $\mathrm{H}$ & 4.11915700 & -2.23213900 & 4.45864800 & $\mathrm{C}$ & -4.73907600 & -0.25295500 & -2.02335700 \\
\hline C & 4.13483200 & -1.02313600 & -1.52783000 & $\mathrm{C}$ & -6.58257900 & 1.40106500 & -0.89189800 \\
\hline $\mathrm{O}$ & 3.68997500 & -1.62009100 & -2.48438800 & $\mathrm{C}$ & -5.68917700 & 0.34990400 & -2.84724700 \\
\hline $\mathrm{O}$ & 5.43598500 & -0.97393500 & -1.21445200 & $\mathrm{H}$ & -3.97719400 & -0.90377300 & -2.44048100 \\
\hline C & 6.32228600 & -1.70466900 & -2.08954100 & $\mathrm{C}$ & -6.63533500 & 1.19623000 & -2.27147300 \\
\hline $\mathrm{H}$ & 6.25505000 & -1.31770000 & -3.10860400 & $\mathrm{H}$ & -7.29838800 & 2.06136400 & -0.40546000 \\
\hline $\mathrm{H}$ & 7.32063900 & -1.55179600 & -1.68271000 & $\mathrm{H}$ & -5.68617100 & 0.16624400 & -3.91783600 \\
\hline $\mathrm{H}$ & 6.06293100 & -2.76532300 & -2.08982500 & $\mathrm{H}$ & -7.39257500 & 1.69094000 & -2.87104600 \\
\hline & & & & $\mathrm{N}$ & -5.68134300 & 0.81951500 & -0.09063900 \\
\hline \multirow{2}{*}{\multicolumn{4}{|c|}{$\begin{array}{l}\text { TS48a } \\
\text { imaginary frequency }=-147.17 \mathrm{~cm}^{-1}\end{array}$}} & $\mathrm{Pd}$ & 0.26996200 & 0.52468100 & -0.43739600 \\
\hline & & & & $\mathrm{C}$ & -5.76783800 & -2.09975400 & 1.75139800 \\
\hline $\mathrm{C}$ & 2.16811100 & 3.30620900 & -0.64944100 & $\mathrm{H}$ & -6.45572500 & -1.53629900 & 1.11603600 \\
\hline C & 2.13328400 & 4.58650800 & -1.21104800 & $\mathrm{H}$ & -6.26069900 & -3.03936800 & 2.03207800 \\
\hline C & 3.07939300 & 4.94587100 & -2.17318400 & $\mathrm{H}$ & -5.61433700 & -1.52459000 & 2.66923800 \\
\hline C & 4.04539200 & 4.01979800 & -2.55498800 & C & -3.49515600 & -3.16504300 & 1.98410500 \\
\hline C & 4.02910700 & 2.76788400 & -1.94509800 & $\mathrm{H}$ & -2.53259300 & -3.38342000 & 1.50903200 \\
\hline $\mathrm{H}$ & 1.37410400 & 5.29897100 & -0.91557100 & $\mathrm{H}$ & -3.29685800 & -2.60917200 & 2.90630400 \\
\hline $\mathrm{H}$ & 3.05473000 & 5.93580500 & -2.61833800 & $\mathrm{H}$ & -3.95163300 & -4.11876000 & 2.27582900 \\
\hline $\mathrm{H}$ & 4.79237600 & 4.25215100 & -3.30565000 & $\mathrm{C}$ & -4.71094000 & -3.27798100 & -0.20625400 \\
\hline $\mathrm{H}$ & 4.76046400 & 2.01001500 & -2.21062500 & $\mathrm{H}$ & -3.78827100 & -3.50588700 & -0.75247600 \\
\hline $\mathrm{N}$ & 3.12101100 & 2.41980600 & -1.02176200 & $\mathrm{H}$ & -5.16049500 & -4.22961100 & 0.10216000 \\
\hline$P$ & 0.92078900 & 2.63368700 & 0.56517600 & $\mathrm{H}$ & -5.40439800 & -2.79779000 & -0.90261700 \\
\hline $\mathrm{C}$ & 1.69493600 & 3.03361700 & 2.27804400 & $\mathrm{C}$ & 0.89125500 & 2.28093300 & 3.35708200 \\
\hline C & -0.49631500 & 3.83587500 & 0.32087800 & $\mathrm{H}$ & -0.15184100 & 2.60018600 & 3.40543500 \\
\hline
\end{tabular}




\begin{tabular}{|c|c|c|c|c|c|c|c|}
\hline $\mathrm{H}$ & 0.90711000 & 1.19944100 & 3.19262400 & $\mathrm{C}$ & -0.60835200 & -0.42513200 & -1.89665400 \\
\hline $\mathrm{H}$ & 1.34022900 & 2.47617000 & 4.33768800 & $\mathrm{O}$ & -1.17917900 & -0.76607200 & -2.82707700 \\
\hline $\mathrm{C}$ & 1.67358400 & 4.54695900 & 2.55929300 & & & & \\
\hline $\mathrm{H}$ & 2.18160800 & 4.74162300 & 3.51063600 & 55 & & & \\
\hline $\mathrm{H}$ & 2.20155100 & 5.11720800 & 1.78784200 & $\mathrm{C}$ & -1.08463900 & -3.16396200 & -0.67781100 \\
\hline $\mathrm{H}$ & 0.65827800 & 4.94139200 & 2.64974500 & $\mathrm{C}$ & -1.00808300 & -4.36692700 & -1.39254900 \\
\hline $\mathrm{C}$ & 3.15240200 & 2.53483300 & 2.31047800 & $\mathrm{C}$ & -1.91655900 & -4.65347500 & -2.41025900 \\
\hline $\mathrm{H}$ & 3.55347600 & 2.69019400 & 3.31822400 & $\mathrm{C}$ & -2.92594900 & -3.73980200 & -2.73155200 \\
\hline $\mathrm{H}$ & 3.22883000 & 1.46913800 & 2.08070800 & $\mathrm{C}$ & -2.98626200 & -2.56011900 & -2.01380600 \\
\hline $\mathrm{H}$ & 3.79249700 & 3.08534800 & 1.61521700 & $\mathrm{H}$ & -0.23655800 & -5.08367600 & -1.14595600 \\
\hline $\mathrm{C}$ & 2.28802700 & -1.06797500 & -0.85061800 & $\mathrm{H}$ & -1.84263400 & -5.59341800 & -2.94889300 \\
\hline $\mathrm{O}$ & 2.52862900 & -1.02690700 & -1.99987400 & $\mathrm{H}$ & -3.64512300 & -3.93580100 & -3.51755200 \\
\hline $\mathrm{C}$ & 4.79788500 & -0.21259800 & -0.17058700 & $\mathrm{H}$ & -3.71707600 & -1.77758400 & -2.17048900 \\
\hline $\mathrm{H}$ & 5.10919400 & -0.99856000 & 0.51395800 & $\mathrm{~N}$ & -2.08289400 & -2.31514900 & -1.04336900 \\
\hline $\mathrm{H}$ & 5.34427500 & 0.70885200 & 0.04404000 & $\mathrm{P}$ & 0.08988800 & -2.54192300 & 0.65655000 \\
\hline $\mathrm{H}$ & 4.97312600 & -0.52235500 & -1.20488500 & $\mathrm{C}$ & -0.85206600 & -3.09656700 & 2.24491400 \\
\hline $\mathrm{O}$ & 3.38574600 & 0.05207800 & 0.04853600 & $\mathrm{C}$ & 1.45611100 & -3.80978200 & 0.38948500 \\
\hline $\mathrm{H}$ & 3.19371100 & 0.99113100 & -0.39225000 & $\mathrm{H}$ & 1.06660200 & -4.81269800 & 0.59629900 \\
\hline $\mathrm{C}$ & 1.96817000 & -2.21141300 & 0.13814100 & $\mathrm{H}$ & 1.66827800 & -3.75850800 & -0.68546700 \\
\hline $\mathrm{C}$ & 1.45027100 & -1.70736300 & 1.49198500 & $\mathrm{C}$ & 2.79630200 & -3.63207200 & 1.14170000 \\
\hline $\mathrm{H}$ & 0.50627100 & -1.16864100 & 1.35827700 & $\mathrm{H}$ & 3.37930000 & -4.53055400 & 0.90821300 \\
\hline $\mathrm{C}$ & 3.26530000 & -3.05830600 & 0.24311000 & $\mathrm{H}$ & 2.63277100 & -3.66898000 & 2.22445800 \\
\hline $\mathrm{C}$ & 3.90600200 & -3.26224600 & 1.47264000 & $\mathrm{C}$ & 3.61255200 & -2.37085300 & 0.76831800 \\
\hline $\mathrm{C}$ & 3.79451200 & -3.66041500 & -0.90991100 & $\mathrm{H}$ & 4.63327000 & -2.65969600 & 0.49209500 \\
\hline $\mathrm{C}$ & 5.05545700 & -4.05268100 & 1.54533300 & $\mathrm{H}$ & 3.17692900 & -1.91306800 & -0.12734700 \\
\hline $\mathrm{H}$ & 3.51628600 & -2.82018300 & 2.38161500 & $\mathrm{C}$ & 3.68036700 & -1.32590000 & 1.89510900 \\
\hline $\mathrm{C}$ & 4.94166200 & -4.44835600 & -0.83192500 & $\mathrm{H}$ & 4.38493400 & -1.67384300 & 2.66248800 \\
\hline $\mathrm{H}$ & 3.30777800 & -3.51583700 & -1.86935200 & $\mathrm{H}$ & 2.69917600 & -1.25429600 & 2.37986000 \\
\hline $\mathrm{C}$ & 5.57789100 & -4.64545400 & 0.39561900 & $\mathrm{C}$ & 4.09968500 & 0.07932100 & 1.43469700 \\
\hline $\mathrm{H}$ & 5.53716900 & -4.20667000 & 2.50611700 & $\mathrm{H}$ & 4.29564400 & 0.70115500 & 2.31294500 \\
\hline $\mathrm{H}$ & 5.33567700 & -4.91043100 & -1.73181900 & $\mathrm{H}$ & 5.01616100 & 0.05464200 & 0.83729400 \\
\hline $\mathrm{H}$ & 6.47073700 & -5.25997800 & 0.45601800 & $\mathrm{P}$ & 2.78857200 & 0.95515000 & 0.42119300 \\
\hline $\mathrm{H}$ & 2.17215000 & -1.03712200 & 1.95762300 & $\mathrm{C}$ & 2.94323100 & 2.78405600 & 0.96698000 \\
\hline $\mathrm{C}$ & 0.91573200 & -3.13053800 & -0.54652600 & $\mathrm{C}$ & 3.52164000 & 0.96715500 & -1.29459600 \\
\hline $\mathrm{O}$ & 0.64496700 & -3.13066700 & -1.72484100 & $\mathrm{C}$ & 2.70741100 & 1.29529900 & -2.39197900 \\
\hline $\mathrm{O}$ & 0.40476100 & -3.97878400 & 0.35231200 & $\mathrm{C}$ & 5.34480000 & 0.65073100 & -2.66667800 \\
\hline $\mathrm{C}$ & -0.47779900 & -4.99291400 & -0.18346100 & $\mathrm{C}$ & 3.27319200 & 1.31026000 & -3.66575500 \\
\hline $\mathrm{H}$ & -0.77302100 & -5.59752100 & 0.67227900 & $\mathrm{H}$ & 1.65948500 & 1.53591800 & -2.24073200 \\
\hline $\mathrm{H}$ & -1.34742800 & -4.52748600 & -0.65023900 & $\mathrm{C}$ & 4.62013500 & 0.97808000 & -3.81219700 \\
\hline $\mathrm{H}$ & 0.05279100 & -5.59648500 & -0.92244800 & $\mathrm{H}$ & 6.39687900 & 0.38127700 & -2.73572700 \\
\hline $\mathrm{H}$ & 1.26010200 & -2.55713000 & 2.14685300 & $\mathrm{H}$ & 2.67277400 & 1.57417100 & -4.53212700 \\
\hline
\end{tabular}




\begin{tabular}{|c|c|c|c|c|c|c|c|}
\hline $\mathrm{H}$ & 5.09969200 & 0.97115400 & -4.78564000 & $\mathrm{C}$ & -2.79263200 & 4.55833700 & 2.21292200 \\
\hline $\mathrm{N}$ & 4.81736600 & 0.64898300 & -1.43544600 & $\mathrm{H}$ & -3.78242100 & 4.67241300 & 0.31977300 \\
\hline $\mathrm{Pd}$ & 0.79313800 & -0.40790600 & 0.44352700 & $\mathrm{C}$ & -1.54363300 & 2.54448200 & 2.64929800 \\
\hline $\mathrm{C}$ & 4.40010200 & 3.27777900 & 0.90667200 & $\mathrm{H}$ & -1.51368500 & 1.08325400 & 1.07983600 \\
\hline $\mathrm{H}$ & 4.81706700 & 3.19855800 & -0.10081700 & $\mathrm{C}$ & -1.99335500 & 3.79949800 & 3.06581600 \\
\hline $\mathrm{H}$ & 4.43669100 & 4.33372700 & 1.20118200 & $\mathrm{H}$ & -3.14502700 & 5.53783100 & 2.52154200 \\
\hline $\mathrm{H}$ & 5.05683700 & 2.72696500 & 1.58600200 & $\mathrm{H}$ & -0.91684600 & 1.94449800 & 3.30224700 \\
\hline $\mathrm{C}$ & 2.40876600 & 2.88042800 & 2.40916800 & $\mathrm{H}$ & -1.72091800 & 4.18169800 & 4.04488800 \\
\hline $\mathrm{H}$ & 1.36350600 & 2.56378600 & 2.46824800 & $\mathrm{H}$ & -3.28764800 & 4.05319400 & -1.95391700 \\
\hline $\mathrm{H}$ & 2.99204100 & 2.28051300 & 3.11533000 & $\mathrm{C}$ & -4.02063100 & 0.99629100 & -0.44401400 \\
\hline $\mathrm{H}$ & 2.46282400 & 3.92194700 & 2.74734000 & $\mathrm{O}$ & -3.60948600 & -0.14712700 & -0.28158500 \\
\hline $\mathrm{C}$ & 2.06295400 & 3.65251400 & 0.04875500 & $\mathrm{O}$ & -5.28706800 & 1.33898400 & -0.23304300 \\
\hline $\mathrm{H}$ & 1.02431300 & 3.31137100 & 0.02832400 & $\mathrm{C}$ & -6.17081600 & 0.31312500 & 0.26779500 \\
\hline $\mathrm{H}$ & 2.07364400 & 4.68618400 & 0.41478400 & $\mathrm{H}$ & -7.12625600 & 0.80943700 & 0.42788000 \\
\hline $\mathrm{H}$ & 2.43848900 & 3.66756600 & -0.97850000 & $\mathrm{H}$ & -6.27492300 & -0.48955100 & -0.46564600 \\
\hline $\mathrm{C}$ & -0.01979400 & -2.59935400 & 3.44509700 & $\mathrm{H}$ & -5.78303100 & -0.09395700 & 1.20349900 \\
\hline $\mathrm{H}$ & 0.96127600 & -3.07845200 & 3.49817700 & $\mathrm{H}$ & -4.82640700 & 3.54534800 & -1.22428400 \\
\hline $\mathrm{H}$ & 0.13089000 & -1.51675700 & 3.40405900 & & & & \\
\hline $\mathrm{H}$ & -0.55189800 & -2.83748200 & 4.37361700 & 56 & & & \\
\hline $\mathrm{C}$ & -1.05435300 & -4.61778500 & 2.33853600 & $\mathrm{C}$ & 1.75454800 & 3.15316900 & -0.61835800 \\
\hline $\mathrm{H}$ & -1.63598400 & -4.85106800 & 3.23789500 & $\mathrm{C}$ & 1.68342000 & 4.53207900 & -0.36576900 \\
\hline $\mathrm{H}$ & -1.60847000 & -5.01863900 & 1.48284000 & $\mathrm{C}$ & 1.48656600 & 5.41068700 & -1.43093900 \\
\hline $\mathrm{H}$ & -0.10815100 & -5.15950900 & 2.42154200 & $\mathrm{C}$ & 1.37617700 & 4.89272500 & -2.72003000 \\
\hline $\mathrm{C}$ & -2.21901100 & -2.38652900 & 2.26916600 & $\mathrm{C}$ & 1.46345000 & 3.50986200 & -2.88258700 \\
\hline $\mathrm{H}$ & -2.69639800 & -2.56113000 & 3.24013800 & $\mathrm{H}$ & 1.79462200 & 4.91730700 & 0.64181400 \\
\hline $\mathrm{H}$ & -2.11967000 & -1.30469800 & 2.13826900 & $\mathrm{H}$ & 1.43129000 & 6.48145000 & -1.25660900 \\
\hline $\mathrm{H}$ & -2.90362900 & -2.76701200 & 1.50456700 & $\mathrm{H}$ & 1.22824400 & 5.53854800 & -3.57934300 \\
\hline $\mathrm{C}$ & -1.93465100 & 1.73372200 & -1.64690200 & $\mathrm{H}$ & 1.38219800 & 3.06596300 & -3.87279300 \\
\hline $\mathrm{O}$ & -0.79471800 & 2.10781500 & -1.49746100 & $\mathrm{~N}$ & 1.63563600 & 2.65386900 & -1.86672800 \\
\hline $\mathrm{C}$ & -1.29099400 & 0.57211300 & -3.60185500 & $\mathrm{P}$ & 2.01115100 & 1.83443900 & 0.66620700 \\
\hline $\mathrm{H}$ & -0.88043500 & 1.47419600 & -4.06080000 & $\mathrm{C}$ & 3.92665300 & 1.76003900 & 0.79286000 \\
\hline $\mathrm{H}$ & -1.78011300 & -0.04549900 & -4.35496000 & $\mathrm{C}$ & 1.50672900 & 2.68061100 & 2.27483600 \\
\hline $\mathrm{H}$ & -0.48521400 & 0.02207000 & -3.10986900 & $\mathrm{H}$ & 2.37014400 & 3.14639500 & 2.75970700 \\
\hline $\mathrm{O}$ & -2.32230400 & 0.91742600 & -2.65443400 & $\mathrm{H}$ & 0.80353400 & 3.48709200 & 2.04022200 \\
\hline $\mathrm{H}$ & -2.18935700 & -1.40857200 & -0.56501500 & $\mathrm{C}$ & 0.82593500 & 1.67690600 & 3.22809200 \\
\hline $\mathrm{C}$ & -3.14752100 & 2.20281300 & -0.81676900 & $\mathrm{H}$ & 0.76936100 & 2.10565500 & 4.23585600 \\
\hline $\mathrm{C}$ & -3.92171500 & 3.19449400 & -1.71966500 & $\mathrm{H}$ & 1.45177400 & 0.78018600 & 3.31793400 \\
\hline $\mathrm{H}$ & -4.20868000 & 2.70763700 & -2.65358400 & $\mathrm{C}$ & -0.58350000 & 1.28149400 & 2.74803500 \\
\hline $\mathrm{C}$ & -2.70650300 & 2.80677700 & 0.53152700 & $\mathrm{H}$ & -1.32402600 & 1.99057500 & 3.14093100 \\
\hline $\mathrm{C}$ & -3.15285900 & 4.06316800 & 0.95725800 & $\mathrm{H}$ & -0.61730600 & 1.37924700 & 1.65542900 \\
\hline $\mathrm{C}$ & -1.89302700 & 2.05361900 & 1.39270500 & $\mathrm{C}$ & -0.97706900 & -0.15281700 & 3.11339100 \\
\hline
\end{tabular}




\begin{tabular}{|c|c|c|c|c|c|c|c|}
\hline $\mathrm{H}$ & -0.95122300 & -0.28707400 & 4.20230900 & $\mathrm{H}$ & 4.05784500 & 0.05679600 & -0.58213800 \\
\hline $\mathrm{H}$ & -0.22073900 & -0.83637700 & 2.70507600 & $\mathrm{H}$ & 4.16739700 & 1.63238500 & -1.38930100 \\
\hline C & -2.36700500 & -0.58498000 & 2.61320800 & $\mathrm{C}$ & -1.01366800 & 0.58844200 & -2.13807200 \\
\hline $\mathrm{H}$ & -2.53910800 & -1.63454200 & 2.87152400 & $\mathrm{O}$ & -1.17539000 & 1.23849600 & -3.05957600 \\
\hline $\mathrm{H}$ & -3.14861500 & -0.01066400 & 3.11957900 & $\mathrm{C}$ & 1.02344000 & -2.48307700 & -0.80012500 \\
\hline$P$ & -2.60709300 & -0.44675800 & 0.76499900 & C & 1.07215700 & -1.33236200 & -1.74291600 \\
\hline C & -3.94726200 & -1.73111900 & 0.33764700 & $\mathrm{C}$ & 0.43735900 & -3.74694700 & -1.46308200 \\
\hline C & -3.40913600 & 1.20580500 & 0.48542700 & $\mathrm{O}$ & 0.39790700 & -3.93113600 & -2.65704400 \\
\hline C & -4.28634500 & 1.81693400 & 1.39126200 & $\mathrm{O}$ & 0.00221900 & -4.61115100 & -0.53562400 \\
\hline C & -3.64510000 & 2.94780000 & -1.01002600 & $\mathrm{C}$ & -0.47327300 & -5.88199300 & -1.03942600 \\
\hline C & -4.84916300 & 3.04950400 & 1.05541000 & $\mathrm{H}$ & -1.32107900 & -5.73034000 & -1.71045400 \\
\hline $\mathrm{H}$ & -4.53746600 & 1.34938200 & 2.33621900 & $\mathrm{H}$ & 0.32675500 & -6.39349500 & -1.57775500 \\
\hline C & -4.52539500 & 3.62999400 & -0.16863800 & $\mathrm{H}$ & -0.77008800 & -6.44922500 & -0.15897200 \\
\hline $\mathrm{H}$ & -3.36161800 & 3.36110200 & -1.97532700 & $\mathrm{H}$ & 0.85446100 & -1.56495300 & -2.78228100 \\
\hline $\mathrm{H}$ & -5.53078500 & 3.54372600 & 1.74098200 & $\mathrm{H}$ & 1.84421500 & -0.58521800 & -1.58317200 \\
\hline $\mathrm{H}$ & -4.94011800 & 4.58675700 & -0.46760300 & $\mathrm{C}$ & 2.31132700 & -2.79223300 & -0.03913100 \\
\hline $\mathrm{N}$ & -3.09714200 & 1.76988300 & -0.69214400 & $\mathrm{C}$ & 3.49791700 & -2.95820000 & -0.76726500 \\
\hline Pd & -0.61828000 & -0.66906800 & -0.72019900 & $\mathrm{C}$ & 2.32240000 & -2.97027600 & 1.34783300 \\
\hline $\mathrm{H}$ & 0.25897700 & -2.23860100 & 0.05181700 & $\mathrm{C}$ & 4.68172600 & -3.29257700 & -0.10836500 \\
\hline C & -5.21514500 & -1.54980400 & 1.19247300 & $\mathrm{H}$ & 3.49255900 & -2.83960900 & -1.84702500 \\
\hline $\mathrm{H}$ & -5.68569500 & -0.57683400 & 1.02718400 & $\mathrm{C}$ & 3.50841700 & -3.30196600 & 2.00403700 \\
\hline $\mathrm{H}$ & -5.94717600 & -2.31709600 & 0.91630100 & $\mathrm{H}$ & 1.40387000 & -2.85616100 & 1.91761700 \\
\hline $\mathrm{H}$ & -5.01660200 & -1.66282300 & 2.26290600 & $\mathrm{C}$ & 4.68995500 & -3.46356500 & 1.27705900 \\
\hline C & -3.35285400 & -3.13419200 & 0.57591700 & $\mathrm{H}$ & 5.59570600 & -3.42103200 & -0.67991800 \\
\hline $\mathrm{H}$ & -2.42803100 & -3.29517100 & 0.01230900 & $\mathrm{H}$ & 3.50824000 & -3.43738700 & 3.08128000 \\
\hline $\mathrm{H}$ & -3.14574400 & -3.32997700 & 1.63253500 & $\mathrm{H}$ & 5.61177400 & -3.72362700 & 1.78792200 \\
\hline $\mathrm{H}$ & -4.07590700 & -3.88837600 & 0.24592100 & & & & \\
\hline $\mathrm{C}$ & -4.29505000 & -1.57211300 & -1.15607100 & 57 & & & \\
\hline $\mathrm{H}$ & -3.42279900 & -1.74200100 & -1.79580200 & $\mathrm{C}$ & -0.20506800 & 2.95257900 & -0.77059500 \\
\hline $\mathrm{H}$ & -5.05592500 & -2.31164400 & -1.42916700 & $\mathrm{C}$ & -1.33589800 & 3.63901800 & -1.23536200 \\
\hline $\mathrm{H}$ & -4.69755300 & -0.58143700 & -1.38470700 & $\mathrm{C}$ & -1.22966600 & 4.39451200 & -2.40488900 \\
\hline C & 4.26641400 & 0.87947600 & 2.01187600 & $\mathrm{C}$ & -0.01027200 & 4.44546800 & -3.07328700 \\
\hline $\mathrm{H}$ & 3.96365500 & 1.34460100 & 2.95579200 & $\mathrm{C}$ & 1.06031100 & 3.72574800 & -2.53925500 \\
\hline $\mathrm{H}$ & 3.79650800 & -0.10748000 & 1.94646800 & $\mathrm{H}$ & -2.28375600 & 3.59282200 & -0.71604000 \\
\hline $\mathrm{H}$ & 5.35115500 & 0.72662500 & 2.06117500 & $\mathrm{H}$ & -2.09182700 & 4.93612600 & -2.78260700 \\
\hline C & 4.59646300 & 3.13625900 & 0.94262000 & $\mathrm{H}$ & 0.11392300 & 5.02165000 & -3.98397100 \\
\hline $\mathrm{H}$ & 5.68123300 & 3.00533300 & 1.03970600 & $\mathrm{H}$ & 2.03289100 & 3.73307600 & -3.02608100 \\
\hline $\mathrm{H}$ & 4.41935800 & 3.77477900 & 0.07287100 & $\mathrm{~N}$ & 0.96750500 & 2.99375200 & -1.42491600 \\
\hline $\mathrm{H}$ & 4.25834300 & 3.67348000 & 1.83464400 & $\mathrm{P}$ & -0.20333100 & 1.84895300 & 0.73997300 \\
\hline $\mathrm{C}$ & 4.44346000 & 1.07681900 & -0.48836000 & $\mathrm{C}$ & 1.06790800 & 2.64431900 & 1.96430400 \\
\hline $\mathrm{H}$ & 5.53792200 & 1.01369400 & -0.45523400 & $\mathrm{C}$ & -1.87993200 & 2.29460100 & 1.46504700 \\
\hline
\end{tabular}




\begin{tabular}{|c|c|c|c|c|c|c|c|}
\hline $\mathrm{H}$ & -1.92107700 & 3.38426000 & 1.38311700 & $\mathrm{H}$ & 1.68989400 & 0.71203000 & 2.80581600 \\
\hline $\mathrm{H}$ & -2.61477700 & 1.90155800 & 0.75515500 & $\mathrm{H}$ & 2.00906400 & 2.10988000 & 3.83592100 \\
\hline $\mathrm{C}$ & -2.31370300 & 1.92033900 & 2.90280000 & $\mathrm{C}$ & 0.52213300 & 3.99718700 & 2.46918000 \\
\hline $\mathrm{H}$ & -3.09838700 & 2.63901400 & 3.16960900 & $\mathrm{H}$ & 1.28131700 & 4.46248200 & 3.10754000 \\
\hline $\mathrm{H}$ & -1.51062700 & 2.09581200 & 3.62310300 & $\mathrm{H}$ & 0.32581200 & 4.68856900 & 1.64350000 \\
\hline $\mathrm{C}$ & -2.89594300 & 0.51608800 & 3.09252300 & $\mathrm{H}$ & -0.38591700 & 3.90330100 & 3.06742600 \\
\hline $\mathrm{H}$ & -3.38464700 & 0.48156700 & 4.07253100 & $\mathrm{C}$ & 2.43959300 & 2.90957600 & 1.31156400 \\
\hline $\mathrm{H}$ & -3.69983900 & 0.38370300 & 2.36135900 & $\mathrm{H}$ & 3.08236600 & 3.37303400 & 2.06910100 \\
\hline $\mathrm{C}$ & -1.88618500 & -0.65148100 & 2.99119400 & $\mathrm{H}$ & 2.93658200 & 1.99733000 & 0.98243900 \\
\hline $\mathrm{H}$ & -1.69754300 & -1.05843700 & 3.99096800 & $\mathrm{H}$ & 2.37492100 & 3.58617300 & 0.46026800 \\
\hline $\mathrm{H}$ & -0.91456100 & -0.29773800 & 2.63136800 & $\mathrm{C}$ & 0.28196400 & -1.48932500 & -1.94351300 \\
\hline $\mathrm{C}$ & -2.35231100 & -1.80507100 & 2.09129100 & $\mathrm{O}$ & 0.38941600 & -1.93956000 & -2.98637300 \\
\hline $\mathrm{H}$ & -1.72241000 & -2.68382100 & 2.26795700 & $\mathrm{C}$ & 4.44753300 & -0.52501600 & -0.09451700 \\
\hline $\mathrm{H}$ & -3.37423900 & -2.10277800 & 2.34795000 & $\mathrm{C}$ & 5.25977400 & 0.13929500 & 0.83214600 \\
\hline $\mathrm{P}$ & -2.20829500 & -1.49692700 & 0.25404600 & $\mathrm{C}$ & 5.00796100 & -0.95683800 & -1.30566400 \\
\hline $\mathrm{C}$ & -2.51237400 & -3.25095300 & -0.47579400 & $\mathrm{C}$ & 6.60607100 & 0.37819300 & 0.55142900 \\
\hline $\mathrm{C}$ & -3.70594400 & -0.54773300 & -0.29429800 & $\mathrm{H}$ & 4.84151100 & 0.46292400 & 1.78156500 \\
\hline $\mathrm{C}$ & -4.98378500 & -0.67097000 & 0.26951700 & $\mathrm{C}$ & 6.35386300 & -0.71994400 & -1.58406900 \\
\hline $\mathrm{C}$ & -4.48067000 & 0.93811900 & -1.87648100 & $\mathrm{H}$ & 4.39540500 & -1.49251800 & -2.02572600 \\
\hline $\mathrm{C}$ & -6.03810400 & 0.05583300 & -0.28578200 & $\mathrm{C}$ & 7.15570600 & -0.04875300 & -0.65818600 \\
\hline $\mathrm{H}$ & -5.15870400 & -1.31580700 & 1.12347800 & $\mathrm{H}$ & 7.22502600 & 0.89306300 & 1.28022300 \\
\hline $\mathrm{C}$ & -5.78589800 & 0.87499100 & -1.38437100 & $\mathrm{H}$ & 6.77709900 & -1.06312900 & -2.52340500 \\
\hline $\mathrm{H}$ & -4.24016900 & 1.56678000 & -2.73081200 & $\mathrm{H}$ & 8.20313400 & 0.13563200 & -0.87649800 \\
\hline $\mathrm{H}$ & -7.03740600 & -0.02167700 & 0.13185800 & $\mathrm{C}$ & 2.09638200 & 0.01731800 & -0.77553300 \\
\hline $\mathrm{H}$ & -6.57676600 & 1.45368200 & -1.84996300 & $\mathrm{H}$ & 2.23667600 & 1.09011000 & -0.70260700 \\
\hline $\mathrm{N}$ & -3.45888800 & 0.25301600 & -1.34715400 & $\mathrm{H}$ & 2.31952400 & -0.27095000 & -1.80160400 \\
\hline $\mathrm{Pd}$ & 0.01622600 & -0.36994700 & -0.36945200 & $\mathrm{C}$ & 2.96085300 & -0.77090900 & 0.20953500 \\
\hline $\mathrm{C}$ & -3.71676200 & -3.94409500 & 0.19384900 & $\mathrm{H}$ & 2.77654200 & -0.44051300 & 1.23532600 \\
\hline $\mathrm{H}$ & -4.64444200 & -3.37968200 & 0.06712700 & $\mathrm{C}$ & 2.71460000 & -2.27648200 & 0.17988500 \\
\hline $\mathrm{H}$ & -3.86702200 & -4.91928300 & -0.28294900 & $\mathrm{O}$ & 2.24109200 & -2.89767600 & -0.75532100 \\
\hline $\mathrm{H}$ & -3.55975300 & -4.12947800 & 1.25957900 & $\mathrm{O}$ & 3.13366500 & -2.85352100 & 1.31493300 \\
\hline $\mathrm{C}$ & -1.25054600 & -4.10549900 & -0.23517500 & $\mathrm{C}$ & 3.08597800 & -4.29491100 & 1.35029900 \\
\hline $\mathrm{H}$ & -0.34513300 & -3.67762100 & -0.67080000 & $\mathrm{H}$ & 2.05847800 & -4.64783900 & 1.23679600 \\
\hline $\mathrm{H}$ & -1.06374100 & -4.26886600 & 0.83157400 & $\mathrm{H}$ & 3.69928200 & -4.71304500 & 0.54930500 \\
\hline $\mathrm{H}$ & -1.39917600 & -5.09228900 & -0.68778900 & $\mathrm{H}$ & 3.48358000 & -4.57505300 & 2.32462800 \\
\hline
\end{tabular}

\section{TS49}

imaginary frequency $=-241.43 \mathrm{~cm}^{-1}$

$\begin{array}{llll}\mathrm{C} & -0.96929900 & 2.82108700 & -0.72993600 \\ \mathrm{C} & -2.02742800 & 3.71537700 & -0.93195900 \\ \mathrm{C} & -2.08222500 & 4.43499500 & -2.12812900\end{array}$




\begin{tabular}{|c|c|c|c|c|c|c|c|}
\hline $\mathrm{C}$ & -1.08493700 & 4.24323000 & -3.07998800 & $\mathrm{C}$ & -0.83099800 & -4.37263200 & -0.37062300 \\
\hline $\mathrm{C}$ & -0.06557500 & 3.33324600 & -2.79112400 & $\mathrm{H}$ & 0.13521400 & -3.87703200 & -0.47256300 \\
\hline $\mathrm{H}$ & -2.79949200 & 3.85557400 & -0.18530700 & $\mathrm{H}$ & -0.93681700 & -4.70654200 & 0.66579400 \\
\hline $\mathrm{H}$ & -2.89273700 & 5.13534400 & -2.30717000 & $\mathrm{H}$ & -0.80572600 & -5.27240500 & -0.99510700 \\
\hline $\mathrm{H}$ & -1.08999200 & 4.78086500 & -4.02223000 & $\mathrm{C}$ & -1.94872600 & -3.23219200 & -2.33831100 \\
\hline $\mathrm{H}$ & 0.73546200 & 3.15404900 & -3.50467600 & $\mathrm{H}$ & -1.01844900 & -2.74646700 & -2.63616800 \\
\hline $\mathrm{N}$ & -0.00628100 & 2.63567000 & -1.65216000 & $\mathrm{H}$ & -2.00251900 & -4.19125900 & -2.86522900 \\
\hline $\mathrm{P}$ & -0.72860700 & 1.76427100 & 0.78347600 & $\mathrm{H}$ & -2.78284500 & -2.61617900 & -2.68557600 \\
\hline $\mathrm{C}$ & 0.47484200 & 2.79428200 & 1.88293900 & $\mathrm{C}$ & 1.06418000 & 1.85593800 & 2.95648600 \\
\hline $\mathrm{C}$ & -2.39110900 & 1.94313000 & 1.63058700 & $\mathrm{H}$ & 0.30151200 & 1.44953300 & 3.62602300 \\
\hline $\mathrm{H}$ & -2.57344300 & 3.01925600 & 1.69140500 & $\mathrm{H}$ & 1.60282600 & 1.01345100 & 2.51313500 \\
\hline $\mathrm{H}$ & -3.12647000 & 1.54408000 & 0.92427200 & $\mathrm{H}$ & 1.77511500 & 2.41440900 & 3.57619800 \\
\hline $\mathrm{C}$ & -2.64621200 & 1.34625600 & 3.03537900 & $\mathrm{C}$ & -0.24539400 & 3.97969400 & 2.55432500 \\
\hline $\mathrm{H}$ & -3.48323500 & 1.91632300 & 3.45620500 & $\mathrm{H}$ & 0.49350500 & 4.58118800 & 3.09586100 \\
\hline $\mathrm{H}$ & -1.80204700 & 1.53589100 & 3.70598500 & $\mathrm{H}$ & -0.72115100 & 4.63780700 & 1.81989800 \\
\hline $\mathrm{C}$ & -3.02767300 & -0.13918300 & 3.08099500 & $\mathrm{H}$ & -0.99879700 & 3.66639600 & 3.28091400 \\
\hline $\mathrm{H}$ & -3.50400100 & -0.33600000 & 4.04777700 & $\mathrm{C}$ & 1.62184800 & 3.36265600 & 1.02390700 \\
\hline $\mathrm{H}$ & -3.80744300 & -0.31320500 & 2.33197700 & $\mathrm{H}$ & 2.34485600 & 3.85029400 & 1.68790800 \\
\hline $\mathrm{C}$ & -1.86232900 & -1.13939600 & 2.88847000 & $\mathrm{H}$ & 2.15364900 & 2.59332600 & 0.46316300 \\
\hline $\mathrm{H}$ & -1.62452400 & -1.60336200 & 3.85216900 & $\mathrm{H}$ & 1.27013700 & 4.11029800 & 0.30995800 \\
\hline $\mathrm{H}$ & -0.94886200 & -0.61835500 & 2.58152100 & $\mathrm{C}$ & 1.08918700 & -1.57597300 & -1.33350900 \\
\hline $\mathrm{C}$ & -2.14421800 & -2.26874200 & 1.88342200 & $\mathrm{O}$ & 1.44917700 & -2.36411100 & -2.09858600 \\
\hline $\mathrm{H}$ & -1.42098500 & -3.07606600 & 2.03618600 & $\mathrm{C}$ & 4.68646300 & -0.05765600 & -0.46146800 \\
\hline $\mathrm{H}$ & -3.13502500 & -2.70312500 & 2.05428000 & $\mathrm{C}$ & 5.56411800 & 1.00189400 & -0.20808500 \\
\hline $\mathrm{P}$ & -1.93226600 & -1.81233200 & 0.08851900 & $\mathrm{C}$ & 5.10881900 & -1.11527700 & -1.28021200 \\
\hline $\mathrm{C}$ & -2.01861100 & -3.49552000 & -0.82062600 & $\mathrm{C}$ & 6.84377400 & 1.00893100 & -0.76596200 \\
\hline $\mathrm{C}$ & -3.46004500 & -0.93023600 & -0.48595600 & $\mathrm{H}$ & 5.24843800 & 1.82404800 & 0.42907500 \\
\hline $\mathrm{C}$ & -4.75596200 & -1.20014600 & -0.02359500 & $\mathrm{C}$ & 6.38805000 & -1.10810600 & -1.83547600 \\
\hline $\mathrm{C}$ & -4.25126600 & 0.63132100 & -1.98422200 & $\mathrm{H}$ & 4.43932500 & -1.94832600 & -1.47939200 \\
\hline $\mathrm{C}$ & -5.82819600 & -0.50433000 & -0.58442000 & $\mathrm{C}$ & 7.25832500 & -0.04568800 & -1.58037900 \\
\hline $\mathrm{H}$ & -4.93247200 & -1.93657400 & 0.75237600 & $\mathrm{H}$ & 7.51549000 & 1.83751800 & -0.56239800 \\
\hline $\mathrm{C}$ & -5.57481000 & 0.42838400 & -1.58817900 & $\mathrm{H}$ & 6.70600200 & -1.93380900 & -2.46494800 \\
\hline $\mathrm{H}$ & -4.00817000 & 1.35259900 & -2.76080700 & $\mathrm{H}$ & 8.25388600 & -0.04091800 & -2.01345400 \\
\hline $\mathrm{H}$ & -6.84202500 & -0.69370200 & -0.24483300 & $\mathrm{C}$ & 2.21483200 & 0.12703700 & -0.95550300 \\
\hline $\mathrm{H}$ & -6.37939000 & 0.98719100 & -2.05463800 & $\mathrm{H}$ & 1.81885300 & 1.13867300 & -1.04245300 \\
\hline $\mathrm{N}$ & -3.21371000 & -0.02292400 & -1.44707000 & $\mathrm{H}$ & 2.58856800 & -0.14653100 & -1.93928700 \\
\hline $\mathrm{Pd}$ & 0.03581000 & -0.43834400 & -0.25544600 & $\mathrm{C}$ & 3.27557600 & -0.05373100 & 0.13774700 \\
\hline $\mathrm{C}$ & -3.33164000 & -4.24129400 & -0.50449100 & $\mathrm{H}$ & 3.21700200 & 0.79037100 & 0.82937500 \\
\hline $\mathrm{H}$ & -4.21299300 & -3.70081900 & -0.85805600 & $\mathrm{C}$ & 3.11421000 & -1.30124200 & 1.00964300 \\
\hline $\mathrm{H}$ & -3.31447300 & -5.20668100 & -1.02240700 & $\mathrm{O}$ & 2.51911500 & -2.31840900 & 0.70403900 \\
\hline $\mathrm{H}$ & -3.45230200 & -4.45287300 & 0.56225300 & $\mathrm{O}$ & 3.75185700 & -1.13811300 & 2.17651200 \\
\hline
\end{tabular}




\begin{tabular}{|c|c|c|c|c|c|c|c|}
\hline $\mathrm{C}$ & 3.78531200 & -2.28551400 & 3.05116800 & $\mathrm{H}$ & -2.44530000 & 5.90902900 & 1.44889000 \\
\hline $\mathrm{H}$ & 2.77272400 & -2.57436100 & 3.34165200 & $\mathrm{H}$ & -0.41485700 & 3.28950900 & 4.19808400 \\
\hline $\mathrm{H}$ & 4.27115900 & -3.12676400 & 2.55266800 & $\mathrm{H}$ & -1.72784600 & 5.39292700 & 3.78555200 \\
\hline \multirow[t]{2}{*}{$\mathrm{H}$} & 4.35994700 & -1.97017800 & 3.92069100 & $\mathrm{~N}$ & -1.60374500 & 4.21507400 & 0.62535000 \\
\hline & & & & $\mathrm{Pd}$ & -0.65767000 & -0.23239100 & 0.07205400 \\
\hline 58 & & & & $\mathrm{C}$ & 0.71978100 & 4.26562400 & -1.90007200 \\
\hline $\mathrm{C}$ & -2.47438600 & -2.29737500 & 1.08419600 & $\mathrm{H}$ & 0.32463700 & 4.89422500 & -1.09832100 \\
\hline $\mathrm{C}$ & -3.19371600 & -3.15019100 & 1.92196100 & $\mathrm{H}$ & 1.65517400 & 4.71605500 & -2.25059700 \\
\hline $\mathrm{C}$ & -2.53521500 & -3.76934200 & 2.98716800 & $\mathrm{H}$ & 0.01443600 & 4.29203400 & -2.73392600 \\
\hline $\mathrm{C}$ & -1.17982000 & -3.51482200 & 3.18786400 & $\mathrm{C}$ & 1.45687800 & 1.96369300 & -2.61973100 \\
\hline $\mathrm{C}$ & -0.52685400 & -2.63436500 & 2.32710700 & $\mathrm{H}$ & 1.71782600 & 0.94612900 & -2.31864000 \\
\hline $\mathrm{H}$ & -4.24951900 & -3.32517300 & 1.74860800 & $\mathrm{H}$ & 0.68613900 & 1.90386800 & -3.39503600 \\
\hline $\mathrm{H}$ & -3.07451000 & -4.43864000 & 3.65037100 & $\mathrm{H}$ & 2.34611400 & 2.40820900 & -3.07981700 \\
\hline $\mathrm{H}$ & -0.63420300 & -3.97388900 & 4.00491300 & $\mathrm{C}$ & 2.14691100 & 2.88653400 & -0.37280300 \\
\hline $\mathrm{H}$ & 0.51726700 & -2.37750300 & 2.46990100 & $\mathrm{H}$ & 2.42090000 & 1.90629100 & 0.01794200 \\
\hline $\mathrm{N}$ & -1.15852800 & -2.04104300 & 1.30259400 & $\mathrm{H}$ & 3.04441000 & 3.30542000 & -0.83976700 \\
\hline $\mathrm{P}$ & -3.02760800 & -1.30408900 & -0.37638900 & $\mathrm{H}$ & 1.88256600 & 3.52927100 & 0.47142700 \\
\hline $\mathrm{C}$ & -3.33799400 & -2.60671900 & -1.74209800 & $\mathrm{C}$ & -3.81683500 & -1.87181500 & -3.01026600 \\
\hline $\mathrm{C}$ & -4.67398600 & -0.59838700 & 0.16160300 & $\mathrm{H}$ & -4.77099800 & -1.35820400 & -2.85883100 \\
\hline $\mathrm{H}$ & -5.46074400 & -1.35681000 & 0.08902300 & $\mathrm{H}$ & -3.08307900 & -1.14265400 & -3.36563600 \\
\hline $\mathrm{H}$ & -4.53885700 & -0.38328400 & 1.22830000 & $\mathrm{H}$ & -3.96543900 & -2.60415500 & -3.81170400 \\
\hline $\mathrm{C}$ & -5.11270300 & 0.70393700 & -0.54834300 & $\mathrm{C}$ & -4.38439900 & -3.65718500 & -1.33229700 \\
\hline $\mathrm{H}$ & -5.99170000 & 1.07040400 & -0.00655500 & $\mathrm{H}$ & -4.53066000 & -4.35930600 & -2.16125900 \\
\hline $\mathrm{H}$ & -5.46121800 & 0.48376800 & -1.56425900 & $\mathrm{H}$ & -4.06398500 & -4.24329800 & -0.46686900 \\
\hline $\mathrm{C}$ & -4.03002600 & 1.80116100 & -0.59517800 & $\mathrm{H}$ & -5.36051300 & -3.21348800 & -1.11353800 \\
\hline $\mathrm{H}$ & -4.49658400 & 2.78938700 & -0.52217300 & $\mathrm{C}$ & -1.98824500 & -3.29410400 & -2.02749200 \\
\hline $\mathrm{H}$ & -3.39035200 & 1.71184400 & 0.29195400 & $\mathrm{H}$ & -2.10837500 & -4.00063200 & -2.85625600 \\
\hline $\mathrm{C}$ & -3.18237000 & 1.74778600 & -1.87768200 & $\mathrm{H}$ & -1.21730800 & -2.57172400 & -2.31458900 \\
\hline $\mathrm{H}$ & -3.78894300 & 2.12436900 & -2.71149000 & $\mathrm{H}$ & -1.62379200 & -3.85955400 & -1.16433500 \\
\hline $\mathrm{H}$ & -2.94510000 & 0.71153700 & -2.13113800 & $\mathrm{C}$ & 1.23236000 & -0.31713700 & 0.73099000 \\
\hline $\mathrm{C}$ & -1.87935800 & 2.55293000 & -1.85300700 & $\mathrm{O}$ & 1.57412500 & 0.09435700 & 1.81024300 \\
\hline $\mathrm{H}$ & -1.41959000 & 2.51190400 & -2.84479300 & $\mathrm{C}$ & 2.07187900 & -1.24368400 & -0.14635300 \\
\hline $\mathrm{H}$ & -2.07608200 & 3.60291500 & -1.61595100 & $\mathrm{H}$ & 1.53886100 & -2.20140200 & -0.19199600 \\
\hline $\mathrm{P}$ & -0.54395400 & 2.04050200 & -0.65131100 & $\mathrm{H}$ & 2.06537500 & -0.87160500 & -1.17325700 \\
\hline $\mathrm{C}$ & 1.02208000 & 2.82915300 & -1.42141300 & $\mathrm{C}$ & 3.51656300 & -1.46047800 & 0.35999100 \\
\hline $\mathrm{C}$ & -0.90857200 & 3.08936600 & 0.84666600 & $\mathrm{H}$ & 3.49838700 & -1.62602000 & 1.44099300 \\
\hline $\mathrm{C}$ & -0.45882300 & 2.71330800 & 2.11968300 & $\mathrm{C}$ & 4.43687100 & -0.27562800 & 0.07714200 \\
\hline $\mathrm{C}$ & -1.87909900 & 5.00665000 & 1.66872900 & $\mathrm{C}$ & 4.90441000 & 0.52769500 & 1.12366400 \\
\hline $\mathrm{C}$ & -0.75137400 & 3.54965300 & 3.19888100 & $\mathrm{C}$ & 4.82591800 & 0.02404200 & -1.23710200 \\
\hline $\mathrm{H}$ & 0.11218600 & 1.80195100 & 2.26011100 & $\mathrm{C}$ & 5.74046400 & 1.61517200 & 0.86304700 \\
\hline $\mathrm{C}$ & -1.47722600 & 4.71760400 & 2.97411500 & $\mathrm{H}$ & 4.61023700 & 0.30227000 & 2.14453100 \\
\hline
\end{tabular}




\begin{tabular}{|c|c|c|c|c|c|c|c|}
\hline $\mathrm{C}$ & 5.66333800 & 1.10929100 & -1.49706900 & $\mathrm{H}$ & -2.12307400 & 3.68026600 & -1.72882300 \\
\hline $\mathrm{H}$ & 4.48879200 & -0.60574300 & -2.05681700 & $\mathrm{P}$ & -0.19323400 & 2.51950800 & -0.83887100 \\
\hline $\mathrm{C}$ & 6.12175400 & 1.90894800 & -0.44723000 & $\mathrm{C}$ & 1.54170200 & 3.17537900 & -1.26023000 \\
\hline $\mathrm{H}$ & 6.09863200 & 2.22768200 & 1.68520200 & $\mathrm{C}$ & -0.74012300 & 3.49308700 & 0.64165800 \\
\hline $\mathrm{H}$ & 5.96677000 & 1.32320800 & -2.51779900 & $\mathrm{C}$ & -0.47474900 & 3.02751200 & 1.93684700 \\
\hline $\mathrm{H}$ & 6.77913100 & 2.74945700 & -0.64870500 & $\mathrm{C}$ & -1.78174500 & 5.37815400 & 1.44211400 \\
\hline $\mathrm{C}$ & 4.07984600 & -2.72249200 & -0.29383500 & $\mathrm{C}$ & -0.88486400 & 3.81007300 & 3.01784600 \\
\hline $\mathrm{O}$ & 3.65498500 & -3.21494900 & -1.31985900 & $\mathrm{H}$ & 0.02460300 & 2.07737400 & 2.09496800 \\
\hline $\mathrm{O}$ & 5.12019900 & -3.20214500 & 0.40228200 & $\mathrm{C}$ & -1.54995800 & 5.00891200 & 2.76908400 \\
\hline $\mathrm{C}$ & 5.78255900 & -4.34899700 & -0.17022000 & $\mathrm{H}$ & -2.30596200 & 6.30157900 & 1.20675000 \\
\hline $\mathrm{H}$ & 6.58489800 & -4.59768700 & 0.52285500 & $\mathrm{H}$ & -0.69294800 & 3.48288200 & 4.03532600 \\
\hline $\mathrm{H}$ & 5.08440900 & -5.18303600 & -0.26990100 & $\mathrm{H}$ & -1.88928400 & 5.64491400 & 3.57996400 \\
\hline $\mathrm{H}$ & 6.18644400 & -4.10132300 & -1.15432200 & $\mathrm{~N}$ & -1.38549300 & 4.64296000 & 0.39624600 \\
\hline & & & & Pd & -0.80275200 & 0.19088400 & -0.54694300 \\
\hline $58 \mathbf{a}$ & & & & $\mathrm{C}$ & 1.52371300 & 4.70464900 & -1.45117400 \\
\hline $\mathrm{C}$ & -3.31229400 & -1.63877200 & 1.02567700 & $\mathrm{H}$ & 1.18758600 & 5.22797800 & -0.55302200 \\
\hline $\mathrm{C}$ & -2.72371300 & -1.35332100 & 2.26550100 & $\mathrm{H}$ & 2.54209300 & 5.04200000 & -1.67511400 \\
\hline $\mathrm{C}$ & -3.54534800 & -1.30490600 & 3.39324000 & $\mathrm{H}$ & 0.88734600 & 5.01932200 & -2.28255600 \\
\hline $\mathrm{C}$ & -4.91262400 & -1.52639000 & 3.24191100 & $\mathrm{C}$ & 1.99839700 & 2.48654800 & -2.56094300 \\
\hline $\mathrm{C}$ & -5.40636700 & -1.78543400 & 1.96125000 & $\mathrm{H}$ & 2.01953300 & 1.39646100 & -2.46494100 \\
\hline $\mathrm{H}$ & -1.65920400 & -1.15891300 & 2.34423500 & $\mathrm{H}$ & 1.35746800 & 2.74060300 & -3.41128500 \\
\hline $\mathrm{H}$ & -3.12288000 & -1.09025500 & 4.37026900 & $\mathrm{H}$ & 3.01320800 & 2.81701000 & -2.80785100 \\
\hline $\mathrm{H}$ & -5.58723700 & -1.49367500 & 4.09100600 & $\mathrm{C}$ & 2.49430200 & 2.81829500 & -0.10405800 \\
\hline $\mathrm{H}$ & -6.46883200 & -1.95273900 & 1.79963300 & $\mathrm{H}$ & 2.54541300 & 1.74325500 & 0.08449200 \\
\hline $\mathrm{N}$ & -4.62880700 & -1.84513200 & 0.87360200 & $\mathrm{H}$ & 3.50746700 & 3.14928400 & -0.35566600 \\
\hline $\mathrm{P}$ & -2.27121800 & -1.74695100 & -0.50747400 & $\mathrm{H}$ & 2.20799200 & 3.31636500 & 0.82677600 \\
\hline $\mathrm{C}$ & -1.78586200 & -3.58344200 & -0.65899200 & $\mathrm{C}$ & -1.04705900 & -3.77409300 & -1.99838400 \\
\hline $\mathrm{C}$ & -3.48438800 & -1.41390900 & -1.90138500 & $\mathrm{H}$ & -1.68173300 & -3.53907900 & -2.85875900 \\
\hline $\mathrm{H}$ & -3.69089800 & -2.36268400 & -2.40295100 & $\mathrm{H}$ & -0.13736800 & -3.17060800 & -2.06342600 \\
\hline $\mathrm{H}$ & -4.42083000 & -1.11177800 & -1.42274300 & $\mathrm{H}$ & -0.74533200 & -4.82263200 & -2.09405600 \\
\hline $\mathrm{C}$ & -2.99749000 & -0.37324100 & -2.92603800 & $\mathrm{C}$ & -3.03902000 & -4.48059700 & -0.61516600 \\
\hline $\mathrm{H}$ & -3.69292700 & -0.36776200 & -3.77383600 & $\mathrm{H}$ & -2.72245200 & -5.52555600 & -0.70855400 \\
\hline $\mathrm{H}$ & -2.02800100 & -0.68707200 & -3.33224300 & $\mathrm{H}$ & -3.58691800 & -4.37816000 & 0.32426900 \\
\hline $\mathrm{C}$ & -2.87732200 & 1.05050100 & -2.35506800 & $\mathrm{H}$ & -3.73399600 & -4.27716100 & -1.43407400 \\
\hline $\mathrm{H}$ & -3.86660700 & 1.52137000 & -2.29390900 & $\mathrm{C}$ & -0.85977400 & -3.95225600 & 0.51569000 \\
\hline $\mathrm{H}$ & -2.58937700 & 0.99190600 & -1.27837100 & $\mathrm{H}$ & -0.59594300 & -5.01260900 & 0.44002400 \\
\hline $\mathrm{C}$ & -1.89053600 & 1.97276600 & -3.09244800 & $\mathrm{H}$ & 0.07615400 & -3.38906900 & 0.50165700 \\
\hline $\mathrm{H}$ & -2.38879500 & 2.41983900 & -3.96084100 & $\mathrm{H}$ & -1.34699200 & -3.80213200 & 1.48362500 \\
\hline $\mathrm{H}$ & -1.06309200 & 1.37456800 & -3.49324000 & $\mathrm{C}$ & 0.72160700 & -0.48051100 & 0.52760300 \\
\hline $\mathrm{C}$ & -1.32813900 & 3.10661000 & -2.21486100 & $\mathrm{O}$ & 0.69650300 & -0.35489000 & 1.72092700 \\
\hline $\mathrm{H}$ & -0.77373000 & 3.81994700 & -2.83019900 & $\mathrm{C}$ & 1.80840400 & -1.16270100 & -0.28579100 \\
\hline
\end{tabular}




\begin{tabular}{|c|c|c|c|c|c|c|c|}
\hline $\mathrm{H}$ & 2.17721500 & -0.44590900 & -1.02491700 & $\mathrm{H}$ & -2.90132400 & -2.68061900 & -3.20651900 \\
\hline $\mathrm{H}$ & 1.34008700 & -1.96788300 & -0.85883100 & $\mathrm{C}$ & -1.19766700 & -4.15440900 & -0.55561600 \\
\hline $\mathrm{C}$ & 2.96616400 & -1.73606800 & 0.56850300 & $\mathrm{H}$ & -2.08133900 & -4.51333600 & -0.02340600 \\
\hline $\mathrm{H}$ & 2.58151700 & -1.93788300 & 1.57529900 & $\mathrm{H}$ & -0.80317500 & -4.98682800 & -1.14883300 \\
\hline $\mathrm{C}$ & 4.14079900 & -0.77900200 & 0.70408900 & $\mathrm{H}$ & -0.44150200 & -3.89326800 & 0.18950400 \\
\hline $\mathrm{C}$ & 4.95333300 & -0.48358700 & -0.40089800 & $\mathrm{C}$ & 0.22741300 & 1.53813500 & -3.19958600 \\
\hline $\mathrm{C}$ & 4.40815700 & -0.15250900 & 1.92626600 & $\mathrm{H}$ & 0.50236700 & 0.57335300 & -2.76004300 \\
\hline $\mathrm{C}$ & 6.01149600 & 0.41635000 & -0.28313900 & $\mathrm{H}$ & -0.80612100 & 1.46933100 & -3.55279500 \\
\hline $\mathrm{H}$ & 4.76809900 & -0.97041900 & -1.35624600 & $\mathrm{H}$ & 0.85863600 & 1.69853400 & -4.08126700 \\
\hline $\mathrm{C}$ & 5.46625700 & 0.75163200 & 2.04521600 & $\mathrm{C}$ & 0.06119100 & 4.03254000 & -2.88365300 \\
\hline $\mathrm{H}$ & 3.78799100 & -0.37563600 & 2.79031000 & $\mathrm{H}$ & 0.17387800 & 4.87323500 & -2.19684100 \\
\hline $\mathrm{C}$ & 6.27020300 & 1.03811300 & 0.94153500 & $\mathrm{H}$ & 0.73087000 & 4.19294600 & -3.73699900 \\
\hline $\mathrm{H}$ & 6.63964200 & 0.62601700 & -1.14395100 & $\mathrm{H}$ & -0.96064500 & 4.02742200 & -3.27378000 \\
\hline $\mathrm{H}$ & 5.66503200 & 1.22506000 & 3.00212800 & $\mathrm{C}$ & 1.92098700 & 2.73946000 & -1.79962100 \\
\hline $\mathrm{H}$ & 7.09789800 & 1.73464200 & 1.03489000 & $\mathrm{H}$ & 2.53368200 & 2.86083900 & -2.70035400 \\
\hline $\mathrm{C}$ & 3.35848800 & -3.11393100 & 0.01184000 & $\mathrm{H}$ & 2.13371100 & 3.57795600 & -1.13328800 \\
\hline $\mathrm{O}$ & 2.72485600 & -3.72278800 & -0.82898200 & $\mathrm{H}$ & 2.23178800 & 1.81309200 & -1.30969300 \\
\hline $\mathrm{O}$ & 4.45632600 & -3.58367800 & 0.61010300 & $\mathrm{C}$ & -3.16034300 & -2.05803100 & 0.87578300 \\
\hline $\mathrm{C}$ & 4.88758000 & -4.89854200 & 0.19839200 & $\mathrm{C}$ & -2.97530400 & -1.76389300 & 2.22882300 \\
\hline $\mathrm{H}$ & 5.78565300 & -5.10327200 & 0.77891400 & $\mathrm{C}$ & -5.13471400 & -3.16003000 & 1.29421200 \\
\hline $\mathrm{H}$ & 4.11155100 & -5.63680600 & 0.41208300 & $\mathrm{C}$ & -3.94044800 & -2.20438300 & 3.13886900 \\
\hline $\mathrm{H}$ & 5.10752400 & -4.90809000 & -0.87110900 & $\mathrm{H}$ & -2.09177100 & -1.23572700 & 2.56190500 \\
\hline & & & & $\mathrm{C}$ & -5.04035900 & -2.91483300 & 2.66709400 \\
\hline $58 b$ & & & & $\mathrm{H}$ & -5.97836200 & -3.71166900 & 0.88555300 \\
\hline $\mathrm{C}$ & -3.15936400 & -0.43360700 & -1.47822100 & $\mathrm{H}$ & -3.82766400 & -1.99574000 & 4.19844500 \\
\hline $\mathrm{H}$ & -2.55416500 & 0.02971000 & -2.26276800 & $\mathrm{H}$ & -5.81178400 & -3.27479900 & 3.33984200 \\
\hline $\mathrm{H}$ & -3.87294600 & -1.10484900 & -1.96137100 & $\mathrm{C}$ & -0.03334200 & 3.45676900 & 0.69971000 \\
\hline $\mathrm{C}$ & -3.91092700 & 0.61875500 & -0.64108700 & $\mathrm{C}$ & -0.07687100 & 3.03520900 & 2.03499700 \\
\hline $\mathrm{H}$ & -4.77581600 & 0.13833800 & -0.17403400 & $\mathrm{C}$ & 0.67526700 & 5.55792300 & 1.31951800 \\
\hline $\mathrm{H}$ & -3.28145800 & 0.97117900 & 0.18489300 & $\mathrm{C}$ & 0.26571400 & 3.94209800 & 3.03919200 \\
\hline $\mathrm{P}$ & -0.62628800 & 2.31809600 & -0.65230500 & $\mathrm{H}$ & -0.37227500 & 2.02026600 & 2.28133800 \\
\hline $\mathrm{P}$ & -2.00693400 & -1.45575400 & -0.44871500 & $\mathrm{C}$ & 0.65319300 & 5.23044300 & 2.67690400 \\
\hline $\mathrm{C}$ & -1.54526000 & -2.98627900 & -1.49965900 & $\mathrm{H}$ & 0.97279800 & 6.55272600 & 0.99501800 \\
\hline $\mathrm{C}$ & 0.43452000 & 2.70112300 & -2.20494800 & $\mathrm{H}$ & 0.23034900 & 3.64584900 & 4.08356000 \\
\hline $\mathrm{C}$ & -0.32756800 & -2.61615600 & -2.36866700 & $\mathrm{H}$ & 0.92883200 & 5.96894500 & 3.42257900 \\
\hline $\mathrm{H}$ & -0.05223500 & -3.47589700 & -2.98981900 & $\mathrm{~N}$ & -4.22218300 & -2.73918600 & 0.41238300 \\
\hline $\mathrm{H}$ & -0.54446200 & -1.78159000 & -3.04333200 & $\mathrm{~N}$ & 0.33327900 & 4.70050100 & 0.35024700 \\
\hline $\mathrm{H}$ & 0.54232700 & -2.34497100 & -1.76419800 & $\mathrm{Pd}$ & -0.18910800 & -0.12943400 & 0.26921600 \\
\hline $\mathrm{C}$ & -2.70496000 & -3.41786000 & -2.42299300 & $\mathrm{C}$ & 0.56522900 & -1.66020300 & 1.34269300 \\
\hline $\mathrm{H}$ & -2.42012000 & -4.35110100 & -2.92232000 & $\mathrm{O}$ & 0.09004900 & -1.96600000 & 2.40535000 \\
\hline $\mathrm{H}$ & -3.62362900 & -3.59326900 & -1.86063600 & $\mathrm{C}$ & -4.37734600 & 1.82886700 & -1.46037900 \\
\hline
\end{tabular}




\begin{tabular}{|c|c|c|c|c|c|c|c|}
\hline $\mathrm{H}$ & -5.00991300 & 2.45465400 & -0.81819000 & $\mathrm{H}$ & 1.71796000 & -2.63677600 & -0.18604000 \\
\hline $\mathrm{H}$ & -5.01602500 & 1.49633000 & -2.28820600 & $\mathrm{~N}$ & -0.28882500 & -2.29649000 & 0.21129300 \\
\hline $\mathrm{C}$ & -3.23669000 & 2.69535400 & -2.01875100 & $\mathrm{P}$ & -2.79412700 & -1.56655000 & 0.35165000 \\
\hline $\mathrm{H}$ & -3.68309700 & 3.56288500 & -2.51753000 & $\mathrm{C}$ & -3.92619900 & -2.60813500 & -0.78854400 \\
\hline $\mathrm{H}$ & -2.70378200 & 2.15655400 & -2.80921800 & $\mathrm{C}$ & -3.67878500 & -1.24886300 & 1.97026800 \\
\hline $\mathrm{C}$ & -2.25786700 & 3.21213400 & -0.94079900 & $\mathrm{H}$ & -4.29459600 & -2.11205000 & 2.24496900 \\
\hline $\mathrm{H}$ & -1.96627000 & 4.24568900 & -1.14650100 & $\mathrm{H}$ & -2.86595900 & -1.19595200 & 2.70447700 \\
\hline $\mathrm{H}$ & -2.75185700 & 3.23146400 & 0.03768200 & $\mathrm{C}$ & -4.51249300 & 0.05182400 & 2.06649000 \\
\hline $\mathrm{C}$ & 1.92398200 & -2.22736100 & 0.89588900 & $\mathrm{H}$ & -4.78540100 & 0.16523400 & 3.12158900 \\
\hline $\mathrm{H}$ & 1.71742700 & -3.09790000 & 0.26433700 & $\mathrm{H}$ & -5.45970200 & -0.06541900 & 1.52678900 \\
\hline $\mathrm{H}$ & 2.42426300 & -2.59488000 & 1.79704500 & $\mathrm{C}$ & -3.79189500 & 1.32345700 & 1.57372400 \\
\hline $\mathrm{C}$ & 2.84760000 & -1.26762600 & 0.10302600 & $\mathrm{H}$ & -4.15314900 & 2.19342900 & 2.13359300 \\
\hline $\mathrm{H}$ & 2.35327000 & -1.05475700 & -0.85384500 & $\mathrm{H}$ & -2.72291400 & 1.24240800 & 1.80634000 \\
\hline $\mathrm{C}$ & 2.85424900 & 0.08572300 & 0.80167400 & $\mathrm{C}$ & -4.00551700 & 1.56718200 & 0.07030400 \\
\hline $\mathrm{O}$ & 1.80885300 & 0.72361200 & 0.98693800 & $\mathrm{H}$ & -5.02482200 & 1.94524600 & -0.08152900 \\
\hline $\mathrm{O}$ & 4.02616900 & 0.53740800 & 1.18483900 & $\mathrm{H}$ & -3.96428800 & 0.61590600 & -0.46280000 \\
\hline $\mathrm{C}$ & 4.06717500 & 1.83015700 & 1.84356800 & $\mathrm{C}$ & -3.04463500 & 2.53335300 & -0.62911900 \\
\hline $\mathrm{H}$ & 5.12050800 & 2.00591700 & 2.05147700 & $\mathrm{H}$ & -3.33706600 & 2.60536500 & -1.68068100 \\
\hline $\mathrm{H}$ & 3.66421600 & 2.59743700 & 1.18133100 & $\mathrm{H}$ & -3.10360500 & 3.54278300 & -0.20851900 \\
\hline $\mathrm{H}$ & 3.48623800 & 1.79514300 & 2.76666400 & $\mathrm{P}$ & -1.21665300 & 2.12245600 & -0.62857500 \\
\hline $\mathrm{C}$ & 4.20391400 & -1.88673700 & -0.18727100 & $\mathrm{C}$ & -0.57700500 & 3.06211500 & -2.17032500 \\
\hline $\mathrm{C}$ & 4.60530100 & -2.06820900 & -1.51579200 & $\mathrm{C}$ & -0.63122400 & 3.07435100 & 0.86289300 \\
\hline $\mathrm{C}$ & 5.05362600 & -2.31693200 & 0.84182500 & $\mathrm{C}$ & 0.08092900 & 2.46221200 & 1.89945500 \\
\hline $\mathrm{C}$ & 5.83052200 & -2.66688400 & -1.81513600 & $\mathrm{C}$ & -0.59174800 & 5.10602700 & 1.94377700 \\
\hline $\mathrm{H}$ & 3.95660800 & -1.74108500 & -2.32518000 & $\mathrm{C}$ & 0.47165200 & 3.23502400 & 2.99562500 \\
\hline $\mathrm{C}$ & 6.27777500 & -2.91337900 & 0.54457200 & $\mathrm{H}$ & 0.31383400 & 1.40476100 & 1.85177100 \\
\hline $\mathrm{H}$ & 4.76820000 & -2.17830400 & 1.88093600 & $\mathrm{C}$ & 0.13459100 & 4.58543700 & 3.01820300 \\
\hline $\mathrm{C}$ & 6.66900200 & -3.09127800 & -0.78474800 & $\mathrm{H}$ & -0.88411000 & 6.15357400 & 1.92811700 \\
\hline $\mathrm{H}$ & 6.12640200 & -2.80101200 & -2.85107600 & $\mathrm{H}$ & 1.02989600 & 2.78712900 & 3.81197400 \\
\hline $\mathrm{H}$ & 6.92682200 & -3.24004900 & 1.35131900 & $\mathrm{H}$ & 0.41840700 & 5.22508300 & 3.84724000 \\
\hline \multirow[t]{2}{*}{$\mathrm{H}$} & 7.62184300 & -3.55829600 & -1.01395400 & $\mathrm{~N}$ & -0.97186200 & 4.37318200 & 0.89191900 \\
\hline & & & & $\mathrm{Pd}$ & -0.70722100 & -0.19884900 & -0.42935800 \\
\hline 58-O & & & & $\mathrm{C}$ & -1.28000500 & 4.42442600 & -2.35529600 \\
\hline $\mathrm{C}$ & -1.43011000 & -2.73327500 & 0.80681700 & $\mathrm{H}$ & -1.15799600 & 5.06697000 & -1.48208500 \\
\hline $\mathrm{C}$ & -1.43899400 & -3.88243400 & 1.59698400 & $\mathrm{H}$ & -0.83139500 & 4.92390700 & -3.22145100 \\
\hline $\mathrm{C}$ & -0.25862100 & -4.61873500 & 1.73373200 & $\mathrm{H}$ & -2.34760100 & 4.32187000 & -2.56365600 \\
\hline $\mathrm{C}$ & 0.89010100 & -4.18996200 & 1.07306200 & $\mathrm{C}$ & -0.86237900 & 2.15775000 & -3.38803400 \\
\hline $\mathrm{C}$ & 0.83907000 & -3.01410500 & 0.32485100 & $\mathrm{H}$ & -0.33098100 & 1.20463600 & -3.32718600 \\
\hline $\mathrm{H}$ & -2.34839300 & -4.20015100 & 2.09441800 & $\mathrm{H}$ & -1.93221200 & 1.95253000 & -3.50580000 \\
\hline $\mathrm{H}$ & -0.24261400 & -5.51853300 & 2.34116700 & $\mathrm{H}$ & -0.53248500 & 2.67129000 & -4.29821300 \\
\hline $\mathrm{H}$ & 1.82412800 & -4.73593500 & 1.13925900 & $\mathrm{C}$ & 0.93832900 & 3.31112300 & -2.04282700 \\
\hline
\end{tabular}




\begin{tabular}{|c|c|c|c|c|c|c|c|}
\hline $\mathrm{H}$ & 1.51364000 & 2.38469400 & -2.00692400 & 58-CO & & & \\
\hline $\mathrm{H}$ & 1.27780200 & 3.85960000 & -2.92830600 & $\mathrm{C}$ & 3.89560500 & -1.20538200 & -0.44193600 \\
\hline $\mathrm{H}$ & 1.18068600 & 3.92118100 & -1.16793300 & $\mathrm{C}$ & 3.84924400 & -0.86833200 & -1.79943400 \\
\hline $\mathrm{C}$ & -5.11427800 & -1.73640900 & -1.24241000 & $\mathrm{C}$ & 5.02581000 & -0.93661100 & -2.54868800 \\
\hline $\mathrm{H}$ & -5.73563100 & -1.40638900 & -0.40444000 & $\mathrm{C}$ & 6.20359000 & -1.32832900 & -1.91615500 \\
\hline $\mathrm{H}$ & -4.78904900 & -0.85575900 & -1.80303300 & $\mathrm{C}$ & 6.15569100 & -1.62960500 & -0.55281900 \\
\hline $\mathrm{H}$ & -5.75277000 & -2.32862100 & -1.90748400 & $\mathrm{H}$ & 2.92153400 & -0.53482600 & -2.24952100 \\
\hline $\mathrm{C}$ & -4.46270900 & -3.87345100 & -0.09573600 & $\mathrm{H}$ & 5.01983900 & -0.68605000 & -3.60543500 \\
\hline $\mathrm{H}$ & -5.12291800 & -4.40686700 & -0.78919000 & $\mathrm{H}$ & 7.14069000 & -1.39683800 & -2.45874800 \\
\hline $\mathrm{H}$ & -3.66197800 & -4.56402300 & 0.18044600 & $\mathrm{H}$ & 7.05491300 & -1.93095900 & -0.02008300 \\
\hline $\mathrm{H}$ & -5.05326800 & -3.64546100 & 0.79709000 & $\mathrm{~N}$ & 5.03280800 & -1.57178300 & 0.17213900 \\
\hline $\mathrm{C}$ & -3.08544800 & -3.00075900 & -2.02006100 & $\mathrm{P}$ & 2.36014800 & -1.18682000 & 0.61038700 \\
\hline $\mathrm{H}$ & -3.71779200 & -3.54597100 & -2.72961900 & $\mathrm{C}$ & 2.16418600 & -3.01866500 & 1.16888800 \\
\hline $\mathrm{H}$ & -2.68527400 & -2.12225100 & -2.53624200 & $\mathrm{C}$ & 3.03204000 & -0.26830000 & 2.09697200 \\
\hline $\mathrm{H}$ & -2.24718100 & -3.65334500 & -1.75839100 & $\mathrm{H}$ & 3.98692400 & -0.76467200 & 2.29235200 \\
\hline $\mathrm{C}$ & 1.19491400 & 0.04509500 & -1.03216400 & $\mathrm{H}$ & 3.28432500 & 0.73251700 & 1.73188200 \\
\hline $\mathrm{O}$ & 1.43547500 & -0.26474900 & -2.17117000 & $\mathrm{C}$ & 2.22352600 & -0.15344300 & 3.41001700 \\
\hline $\mathrm{C}$ & 2.25054800 & 0.43181900 & -0.00570200 & $\mathrm{H}$ & 2.96422500 & 0.00713800 & 4.20257900 \\
\hline $\mathrm{H}$ & 2.10126800 & 1.49629500 & 0.20377600 & $\mathrm{H}$ & 1.73389000 & -1.09977400 & 3.66257000 \\
\hline $\mathrm{H}$ & 2.03677600 & -0.07803200 & 0.93849800 & $\mathrm{C}$ & 1.20674800 & 0.99788500 & 3.49159100 \\
\hline $\mathrm{C}$ & 3.70512300 & 0.17651300 & -0.46124300 & $\mathrm{H}$ & 1.01148600 & 1.19938600 & 4.55041300 \\
\hline $\mathrm{H}$ & 3.81171700 & 0.48061300 & -1.50602700 & $\mathrm{H}$ & 1.68578000 & 1.90324700 & 3.10710500 \\
\hline $\mathrm{C}$ & 4.11012800 & -1.29144300 & -0.34774800 & $\mathrm{C}$ & -0.15045100 & 0.76145500 & 2.78172300 \\
\hline $\mathrm{C}$ & 4.21644200 & -2.09158700 & -1.49292000 & $\mathrm{H}$ & -0.92351500 & 0.58995200 & 3.53866700 \\
\hline $\mathrm{C}$ & 4.36178800 & -1.86568000 & 0.90795200 & $\mathrm{H}$ & -0.11442800 & -0.16122200 & 2.18920400 \\
\hline $\mathrm{C}$ & 4.56374000 & -3.44046600 & -1.38569300 & $\mathrm{C}$ & -0.63614400 & 1.90954600 & 1.87183300 \\
\hline $\mathrm{H}$ & 4.02477000 & -1.65701500 & -2.46940300 & $\mathrm{H}$ & -1.71578800 & 1.81266700 & 1.71319300 \\
\hline $\mathrm{C}$ & 4.71518200 & -3.21180600 & 1.01426800 & $\mathrm{H}$ & -0.48018800 & 2.88615800 & 2.34316800 \\
\hline $\mathrm{H}$ & 4.29957000 & -1.25153100 & 1.80276300 & $\mathrm{P}$ & 0.07613100 & 1.87751000 & 0.15404100 \\
\hline $\mathrm{C}$ & 4.81569100 & -4.00403400 & -0.13296000 & $\mathrm{C}$ & -0.85223100 & 3.22602700 & -0.84892900 \\
\hline $\mathrm{H}$ & 4.64811800 & -4.04640000 & -2.28291700 & $\mathrm{C}$ & 1.80038200 & 2.56253200 & 0.20775600 \\
\hline $\mathrm{H}$ & 4.92649400 & -3.63710700 & 1.99128300 & $\mathrm{C}$ & 2.23045700 & 3.53174500 & 1.12412600 \\
\hline $\mathrm{H}$ & 5.10268600 & -5.04838200 & -0.05245700 & $\mathrm{C}$ & 3.83222700 & 2.59418200 & -0.87581600 \\
\hline $\mathrm{C}$ & 4.64415400 & 1.04440400 & 0.37567500 & $\mathrm{C}$ & 3.53035700 & 4.02775200 & 1.01785300 \\
\hline $\mathrm{O}$ & 4.37592400 & 1.48337300 & 1.47700000 & $\mathrm{H}$ & 1.56828600 & 3.90531900 & 1.89645100 \\
\hline $\mathrm{O}$ & 5.81082100 & 1.23829000 & -0.25301900 & $\mathrm{C}$ & 4.35087800 & 3.55188600 & -0.00292500 \\
\hline $\mathrm{C}$ & 6.80125300 & 1.99256900 & 0.47673000 & $\mathrm{H}$ & 4.43928500 & 2.19437600 & -1.68409900 \\
\hline $\mathrm{H}$ & 7.66813800 & 2.04606100 & -0.18006800 & $\mathrm{H}$ & 3.88976800 & 4.77648300 & 1.71721600 \\
\hline $\mathrm{H}$ & 6.42635700 & 2.99278400 & 0.70456800 & $\mathrm{H}$ & 5.36709000 & 3.91163700 & -0.12435200 \\
\hline \multirow[t]{2}{*}{$\mathrm{H}$} & 7.05427900 & 1.48372000 & 1.40945800 & $\mathrm{~N}$ & 2.59032000 & 2.10202900 & -0.77615700 \\
\hline & & & & $\mathrm{Pd}$ & 0.24512600 & -0.42063500 & -0.68110800 \\
\hline
\end{tabular}




\begin{tabular}{|c|c|c|c|c|c|c|c|}
\hline $\mathrm{C}$ & -0.46273100 & 4.63904300 & -0.36854400 & $\mathrm{H}$ & -6.57459200 & 2.70703100 & -1.32705900 \\
\hline $\mathrm{H}$ & 0.58492200 & 4.87672300 & -0.56230900 & $\mathrm{H}$ & -5.51132600 & 1.72563900 & 2.72341600 \\
\hline $\mathrm{H}$ & -1.07097000 & 5.36540700 & -0.91883400 & $\mathrm{H}$ & -6.61779400 & 3.24999600 & 1.09905000 \\
\hline $\mathrm{H}$ & -0.66606000 & 4.79366400 & 0.69641400 & $\mathrm{C}$ & -4.70057800 & -2.43944200 & 0.03983200 \\
\hline $\mathrm{C}$ & -2.37433200 & 3.06443800 & -0.67942800 & $\mathrm{O}$ & -4.19110200 & -3.02877600 & 0.97217700 \\
\hline $\mathrm{H}$ & -2.73874400 & 2.07330700 & -0.94304000 & $\mathrm{O}$ & -5.88000200 & -2.75599100 & -0.50752200 \\
\hline $\mathrm{H}$ & -2.70796500 & 3.30032400 & 0.33502900 & $\mathrm{C}$ & -6.58290500 & -3.85815300 & 0.10519700 \\
\hline $\mathrm{H}$ & -2.87016700 & 3.77325000 & -1.35170700 & $\mathrm{H}$ & -6.80054300 & -3.63371300 & 1.15159500 \\
\hline $\mathrm{C}$ & -0.44932900 & 3.05158600 & -2.32764300 & $\mathrm{H}$ & -7.50399700 & -3.96775700 & -0.46507300 \\
\hline $\mathrm{H}$ & -0.78501500 & 2.09262800 & -2.72764200 & $\mathrm{H}$ & -5.98292500 & -4.76902000 & 0.05035300 \\
\hline $\mathrm{H}$ & -0.91869100 & 3.84651100 & -2.91832100 & & & & \\
\hline $\mathrm{H}$ & 0.63288400 & 3.12857300 & -2.46668900 & \multirow{2}{*}{\multicolumn{4}{|c|}{$\begin{array}{l}\text { TS50 } \\
\text { imaginary frequency }=-134.76 \mathrm{~cm}^{-1}\end{array}$}} \\
\hline $\mathrm{C}$ & 0.73234200 & -3.20879800 & 1.71017000 & & & & \\
\hline $\mathrm{H}$ & 0.53216700 & -2.59709600 & 2.59401300 & $\mathrm{C}$ & 1.96514800 & -3.10232800 & 0.88441300 \\
\hline $\mathrm{H}$ & -0.02507700 & -2.97127600 & 0.95570700 & $\mathrm{C}$ & 2.59665400 & -4.35394600 & 0.88830100 \\
\hline $\mathrm{H}$ & 0.58984400 & -4.25560200 & 2.00139600 & $\mathrm{C}$ & 3.62350600 & -4.61649500 & 1.79456500 \\
\hline $\mathrm{C}$ & 3.19255300 & -3.40778700 & 2.24965300 & $\mathrm{C}$ & 4.00501800 & -3.62515900 & 2.69628900 \\
\hline $\mathrm{H}$ & 3.07435000 & -4.47339400 & 2.47824500 & $\mathrm{C}$ & 3.32823900 & -2.41235400 & 2.65292500 \\
\hline $\mathrm{H}$ & 4.21631500 & -3.24325600 & 1.90724300 & $\mathrm{H}$ & 2.29150500 & -5.12477300 & 0.19291800 \\
\hline $\mathrm{H}$ & 3.04710100 & -2.85976700 & 3.18376500 & $\mathrm{H}$ & 4.11574700 & -5.58420500 & 1.79481000 \\
\hline $\mathrm{C}$ & 2.36443200 & -3.94121200 & -0.04997200 & $\mathrm{H}$ & 4.79879300 & -3.78524500 & 3.41727200 \\
\hline $\mathrm{H}$ & 2.18623200 & -4.97696000 & 0.25996500 & $\mathrm{H}$ & 3.57231200 & -1.60369000 & 3.33677900 \\
\hline $\mathrm{H}$ & 1.66542600 & -3.72788400 & -0.86242400 & $\mathrm{~N}$ & 2.33984000 & -2.16214700 & 1.77722500 \\
\hline $\mathrm{H}$ & 3.38096000 & -3.88475500 & -0.44609700 & $\mathrm{P}$ & 0.56005300 & -2.66055000 & -0.28773400 \\
\hline $\mathrm{C}$ & -1.75433000 & -0.35775000 & -1.33040800 & $\mathrm{C}$ & 1.29963700 & -2.92035400 & -2.05150800 \\
\hline $\mathrm{O}$ & -2.16171600 & 0.14011600 & -2.33743100 & $\mathrm{C}$ & -0.50584700 & -4.16909800 & 0.07935900 \\
\hline $\mathrm{C}$ & -2.56844200 & -1.21249500 & -0.36568600 & $\mathrm{H}$ & 0.10127400 & -5.05794700 & -0.11656100 \\
\hline $\mathrm{H}$ & -2.35775600 & -0.90349600 & 0.66174200 & $\mathrm{H}$ & -0.67328900 & -4.13781700 & 1.16279400 \\
\hline $\mathrm{H}$ & -2.17105300 & -2.23169400 & -0.44399700 & $\mathrm{C}$ & -1.85923600 & -4.29557500 & -0.64309100 \\
\hline $\mathrm{C}$ & -4.08812700 & -1.21547600 & -0.64528600 & $\mathrm{H}$ & -2.22664000 & -5.30971000 & -0.44962300 \\
\hline $\mathrm{H}$ & -4.25573800 & -1.31345300 & -1.72125400 & $\mathrm{H}$ & -1.70976600 & -4.24433200 & -1.72747500 \\
\hline $\mathrm{C}$ & 0.38428400 & -1.83543600 & -2.06591800 & $\mathrm{C}$ & -2.91259400 & -3.25418000 & -0.20443800 \\
\hline $\mathrm{O}$ & 0.53351300 & -2.49068200 & -2.98841100 & $\mathrm{H}$ & -3.69976800 & -3.73519600 & 0.38786800 \\
\hline $\mathrm{C}$ & -4.79379200 & 0.04695500 & -0.15865300 & $\mathrm{H}$ & -2.43805700 & -2.52884800 & 0.46928100 \\
\hline $\mathrm{C}$ & -5.43038800 & 0.90332300 & -1.06406400 & $\mathrm{C}$ & -3.55226000 & -2.49177500 & -1.37307400 \\
\hline $\mathrm{C}$ & -4.82904200 & 0.35567300 & 1.21014200 & $\mathrm{H}$ & -4.27211900 & -3.14156300 & -1.88656500 \\
\hline $\mathrm{C}$ & -6.08122600 & 2.05399900 & -0.61332200 & $\mathrm{H}$ & -2.77994000 & -2.25091500 & -2.11474000 \\
\hline $\mathrm{H}$ & -5.41620200 & 0.66948400 & -2.12478400 & $\mathrm{C}$ & -4.25841500 & -1.20157000 & -0.93289600 \\
\hline $\mathrm{C}$ & -5.47899800 & 1.50531000 & 1.66025600 & $\mathrm{H}$ & -4.90739800 & -0.82961400 & -1.72992000 \\
\hline $\mathrm{H}$ & -4.37437600 & -0.32302700 & 1.92804000 & $\mathrm{H}$ & -4.90650200 & -1.36748500 & -0.06756900 \\
\hline $\mathrm{C}$ & -6.10509500 & 2.35936100 & 0.74842600 & $\mathrm{P}$ & -3.06178200 & 0.15463700 & -0.45350300 \\
\hline
\end{tabular}




\begin{tabular}{|c|c|c|c|}
\hline $\mathrm{C}$ & -3.29950200 & 1.49077900 & -1.80565200 \\
\hline $\mathrm{C}$ & -3.87428600 & 0.89335500 & 1.05503400 \\
\hline $\mathrm{C}$ & -3.21007000 & 1.87385000 & 1.80991900 \\
\hline $\mathrm{C}$ & -5.70047400 & 0.96534300 & 2.45726400 \\
\hline $\mathrm{C}$ & -3.85816300 & 2.42005200 & 2.91767100 \\
\hline $\mathrm{H}$ & -2.21064800 & 2.19141800 & 1.53719300 \\
\hline $\mathrm{C}$ & -5.13072500 & 1.95975000 & 3.25264100 \\
\hline $\mathrm{H}$ & -6.68733500 & 0.56938500 & 2.68795100 \\
\hline $\mathrm{H}$ & -3.37368800 & 3.18977900 & 3.51179400 \\
\hline $\mathrm{H}$ & -5.66946300 & 2.35432400 & 4.10786300 \\
\hline $\mathrm{N}$ & -5.09600700 & 0.44523900 & 1.38204400 \\
\hline $\mathrm{Pd}$ & -0.57655800 & -0.61574300 & -0.00708300 \\
\hline $\mathrm{C}$ & -4.75712000 & 1.98050100 & -1.88091900 \\
\hline $\mathrm{H}$ & -5.07403100 & 2.45560800 & -0.94869000 \\
\hline $\mathrm{H}$ & -4.84680000 & 2.72445500 & -2.68132900 \\
\hline $\mathrm{H}$ & -5.46295200 & 1.17599200 & -2.10488700 \\
\hline $\mathrm{C}$ & -2.88160300 & 0.85635900 & -3.14740600 \\
\hline $\mathrm{H}$ & -1.84138300 & 0.51398300 & -3.12502200 \\
\hline $\mathrm{H}$ & -3.51628300 & 0.00991200 & -3.42792100 \\
\hline $\mathrm{H}$ & -2.96701300 & 1.60420900 & -3.94421900 \\
\hline $\mathrm{C}$ & -2.37592700 & 2.68604000 & -1.50679500 \\
\hline $\mathrm{H}$ & -1.32659200 & 2.39090700 & -1.42328400 \\
\hline $\mathrm{H}$ & -2.45866300 & 3.41557900 & -2.32087700 \\
\hline $\mathrm{H}$ & -2.65744500 & 3.19749900 & -0.58243900 \\
\hline $\mathrm{C}$ & 0.38708500 & -2.15690800 & -3.03474800 \\
\hline $\mathrm{H}$ & -0.63058600 & -2.55646400 & -3.05380100 \\
\hline $\mathrm{H}$ & 0.32576800 & -1.09394800 & -2.78381500 \\
\hline $\mathrm{H}$ & 0.79677800 & -2.24628900 & -4.04736200 \\
\hline $\mathrm{C}$ & 1.36548300 & -4.39942100 & -2.47820000 \\
\hline $\mathrm{H}$ & 1.74785600 & -4.44932400 & -3.50387600 \\
\hline $\mathrm{H}$ & 2.04879900 & -4.98962200 & -1.86183000 \\
\hline $\mathrm{H}$ & 0.38667900 & -4.88526400 & -2.47536800 \\
\hline $\mathrm{C}$ & 2.71469200 & -2.31206600 & -2.11095000 \\
\hline $\mathrm{H}$ & 3.08962800 & -2.39116100 & -3.13739300 \\
\hline $\mathrm{H}$ & 2.73083500 & -1.25271600 & -1.84643700 \\
\hline $\mathrm{H}$ & 3.42261600 & -2.83882300 & -1.46519800 \\
\hline $\mathrm{C}$ & 0.83118600 & 0.77719200 & 0.64332800 \\
\hline $\mathrm{O}$ & -0.05005000 & 1.60486600 & 0.79347800 \\
\hline $\mathrm{C}$ & 0.39182200 & 0.18132400 & 3.22751500 \\
\hline $\mathrm{H}$ & -0.27539100 & 1.02359200 & 3.04285500 \\
\hline $\mathrm{H}$ & 0.90566000 & 0.31510200 & 4.18188300 \\
\hline
\end{tabular}

\begin{tabular}{|c|c|c|c|}
\hline $\mathrm{H}$ & -0.18478900 & -0.74827300 & 3.22580400 \\
\hline $\mathrm{O}$ & 1.37965000 & 0.16802500 & 2.18034400 \\
\hline $\mathrm{H}$ & 1.72471400 & -0.81751000 & 1.98100100 \\
\hline $\mathrm{C}$ & 2.13110900 & 0.92348300 & -0.11143700 \\
\hline $\mathrm{H}$ & 1.87871400 & 0.96852900 & -1.17347000 \\
\hline $\mathrm{H}$ & 2.73963000 & 0.03227400 & 0.03624400 \\
\hline $\mathrm{C}$ & 2.93730300 & 2.18540900 & 0.28689400 \\
\hline $\mathrm{H}$ & 3.05744200 & 2.20988600 & 1.37416100 \\
\hline $\mathrm{C}$ & 2.29460400 & 3.49351500 & -0.16087100 \\
\hline $\mathrm{C}$ & 1.86084600 & 4.43200800 & 0.78159100 \\
\hline $\mathrm{C}$ & 2.14350200 & 3.78083700 & -1.52534800 \\
\hline $\mathrm{C}$ & 1.27470700 & 5.63075000 & 0.37204600 \\
\hline $\mathrm{H}$ & 1.98557900 & 4.22610000 & 1.84140800 \\
\hline $\mathrm{C}$ & 1.55790300 & 4.97840300 & -1.93538500 \\
\hline $\mathrm{H}$ & 2.50115900 & 3.07440600 & -2.27064100 \\
\hline $\mathrm{C}$ & 1.11957500 & 5.90580900 & -0.98719900 \\
\hline $\mathrm{H}$ & 0.94428900 & 6.35059400 & 1.11503300 \\
\hline $\mathrm{H}$ & 1.45214900 & 5.19124200 & -2.99509100 \\
\hline $\mathrm{H}$ & 0.66733700 & 6.83977500 & -1.30682600 \\
\hline $\mathrm{C}$ & 4.33323200 & 2.05669000 & -0.32812000 \\
\hline $\mathrm{O}$ & 4.61145500 & 1.31836700 & -1.25232300 \\
\hline $\mathrm{O}$ & 5.20327000 & 2.87951600 & 0.27227300 \\
\hline $\mathrm{C}$ & 6.53421100 & 2.90084800 & -0.28459000 \\
\hline $\mathrm{H}$ & 7.09037400 & 3.61706500 & 0.31838800 \\
\hline $\mathrm{H}$ & 6.98870400 & 1.90934700 & -0.22545400 \\
\hline $\mathrm{H}$ & 6.50292000 & 3.21839600 & -1.32904900 \\
\hline
\end{tabular}

\section{TS50a} imaginary frequency $=-109.40 \mathrm{~cm}^{-1}$

$\begin{array}{lrrr}\mathrm{C} & -1.38906200 & 3.43536400 & 0.96015300 \\ \mathrm{C} & -1.39903000 & 4.78814600 & 1.32499300 \\ \mathrm{C} & -2.07531100 & 5.18551000 & 2.47867600 \\ \mathrm{C} & -2.73089300 & 4.22462200 & 3.24638700 \\ \mathrm{C} & -2.65850500 & 2.89695900 & 2.83683300 \\ \mathrm{H} & -0.88785100 & 5.52417800 & 0.71663500 \\ \mathrm{H} & -2.09088700 & 6.23136500 & 2.76959900 \\ \mathrm{H} & -3.27355400 & 4.49079700 & 4.14664800 \\ \mathrm{H} & -3.12903100 & 2.10340700 & 3.41138400 \\ \mathrm{~N} & -2.00234300 & 2.51216500 & 1.72979700 \\ \mathrm{P} & -0.56369800 & 2.79812100 & -0.58336700 \\ \mathrm{C} & -1.71238000 & 3.39016300 & -2.00015400\end{array}$




\begin{tabular}{|c|c|c|c|c|c|c|c|}
\hline $\mathrm{C}$ & 0.95862300 & 3.89053300 & -0.65659100 & $\mathrm{H}$ & -0.09931400 & 3.72517300 & -3.46164200 \\
\hline $\mathrm{H}$ & 0.65459000 & 4.86148400 & -1.06095400 & $\mathrm{H}$ & -0.68844200 & 2.04977200 & -3.41277900 \\
\hline $\mathrm{H}$ & 1.26103700 & 4.06339600 & 0.38240900 & $\mathrm{H}$ & -1.66681900 & 3.31891600 & -4.16093700 \\
\hline $\mathrm{C}$ & 2.16172700 & 3.33986800 & -1.45063000 & $\mathrm{C}$ & -2.06094500 & 4.88712100 & -1.92590200 \\
\hline $\mathrm{H}$ & 2.80495000 & 4.19354300 & -1.69907200 & $\mathrm{H}$ & -2.66379800 & 5.14946000 & -2.80235300 \\
\hline $\mathrm{H}$ & 1.83211600 & 2.92715000 & -2.41152600 & $\mathrm{H}$ & -2.65440800 & 5.13262400 & -1.04157400 \\
\hline $\mathrm{C}$ & 2.99769000 & 2.29622200 & -0.70035200 & $\mathrm{H}$ & -1.17701900 & 5.53232000 & -1.94650000 \\
\hline $\mathrm{H}$ & 3.36356800 & 2.72489000 & 0.24234300 & $\mathrm{C}$ & -3.00815700 & 2.56073600 & -1.92728900 \\
\hline $\mathrm{H}$ & 2.36224600 & 1.44101600 & -0.41976200 & $\mathrm{H}$ & -3.68798700 & 2.87835000 & -2.72546500 \\
\hline $\mathrm{C}$ & 4.18412400 & 1.79555500 & -1.53299400 & $\mathrm{H}$ & -2.81389800 & 1.49429000 & -2.06688100 \\
\hline $\mathrm{H}$ & 4.80427600 & 2.65827600 & -1.81323600 & $\mathrm{H}$ & -3.53551100 & 2.69714700 & -0.97725200 \\
\hline $\mathrm{H}$ & 3.80923200 & 1.37058800 & -2.47426700 & $\mathrm{C}$ & -1.27998400 & -1.04641800 & 0.27717600 \\
\hline $\mathrm{C}$ & 5.07731900 & 0.76528800 & -0.82120400 & $\mathrm{O}$ & -0.57509900 & -1.96228900 & 0.53242600 \\
\hline $\mathrm{H}$ & 5.95578800 & 0.57578200 & -1.44478500 & $\mathrm{C}$ & -0.43036100 & -0.18663000 & 2.78490800 \\
\hline $\mathrm{H}$ & 5.43208600 & 1.15315400 & 0.13862700 & $\mathrm{H}$ & 0.16005500 & -1.06017500 & 2.50376900 \\
\hline $\mathrm{P}$ & 4.23955200 & -0.88629200 & -0.52396300 & $\mathrm{H}$ & -0.87470100 & -0.34912200 & 3.77071600 \\
\hline $\mathrm{C}$ & 5.69532000 & -2.12855100 & -0.69919400 & $\mathrm{H}$ & 0.21664900 & 0.69446300 & 2.80188200 \\
\hline $\mathrm{C}$ & 3.97705600 & -0.84164700 & 1.32668700 & $\mathrm{O}$ & -1.47932300 & -0.02927500 & 1.81664600 \\
\hline $\mathrm{C}$ & 3.18345500 & -1.84085700 & 1.92011600 & $\mathrm{H}$ & -1.69912600 & 0.97131200 & 1.66800100 \\
\hline $\mathrm{C}$ & 4.31027900 & 0.16155500 & 3.37911800 & $\mathrm{C}$ & -2.66035500 & -0.92667100 & -0.30619200 \\
\hline $\mathrm{C}$ & 2.99621100 & -1.83505800 & 3.30109500 & $\mathrm{H}$ & -2.57858500 & -1.15551400 & -1.37147700 \\
\hline $\mathrm{H}$ & 2.72548900 & -2.60762100 & 1.30334500 & $\mathrm{H}$ & -3.01322600 & 0.09851000 & -0.21798300 \\
\hline $\mathrm{C}$ & 3.57194200 & -0.81056900 & 4.05409100 & $\mathrm{C}$ & -3.65106000 & -1.91139100 & 0.37758600 \\
\hline $\mathrm{H}$ & 4.76694500 & 0.98483500 & 3.92605200 & $\mathrm{H}$ & -3.57673700 & -1.80008900 & 1.46308800 \\
\hline $\mathrm{H}$ & 2.41204900 & -2.61600600 & 3.78066000 & $\mathrm{C}$ & -3.40221300 & -3.36891300 & 0.01183800 \\
\hline $\mathrm{H}$ & 3.45623900 & -0.76385400 & 5.13225800 & $\mathrm{C}$ & -2.99451600 & -4.28743000 & 0.98539200 \\
\hline $\mathrm{N}$ & 4.51654400 & 0.15083900 & 2.05571000 & $\mathrm{C}$ & -3.58215200 & -3.80812700 & -1.30832500 \\
\hline $\mathrm{Pd}$ & 0.01508100 & 0.44868100 & -0.89066600 & $\mathrm{C}$ & -2.76121600 & -5.62127400 & 0.64650700 \\
\hline $\mathrm{C}$ & 6.93194500 & -1.73943200 & 0.12838400 & $\mathrm{H}$ & -2.86206900 & -3.95945000 & 2.01300700 \\
\hline $\mathrm{H}$ & 6.70692500 & -1.69273700 & 1.19773700 & $\mathrm{C}$ & -3.34866400 & -5.14029300 & -1.64671500 \\
\hline $\mathrm{H}$ & 7.72280600 & -2.48668900 & -0.01409700 & $\mathrm{H}$ & -3.92195800 & -3.11085100 & -2.07065400 \\
\hline $\mathrm{H}$ & 7.34189800 & -0.77040200 & -0.17072800 & $\mathrm{C}$ & -2.93560700 & -6.04970400 & -0.66981700 \\
\hline $\mathrm{C}$ & 6.05094500 & -2.17782700 & -2.19964900 & $\mathrm{H}$ & -2.44556700 & -6.32429400 & 1.41144000 \\
\hline $\mathrm{H}$ & 5.18891700 & -2.46784200 & -2.80940900 & $\mathrm{H}$ & -3.49493400 & -5.46984600 & -2.67092900 \\
\hline $\mathrm{H}$ & 6.42501500 & -1.21986000 & -2.57458300 & $\mathrm{H}$ & -2.75556900 & -7.08734500 & -0.93363900 \\
\hline $\mathrm{H}$ & 6.84186400 & -2.91914400 & -2.36458300 & $\mathrm{C}$ & -5.06579200 & -1.49430600 & -0.03520800 \\
\hline $\mathrm{C}$ & 5.20150000 & -3.52248600 & -0.26783300 & $\mathrm{O}$ & -5.32153100 & -0.79013300 & -0.99105200 \\
\hline $\mathrm{H}$ & 4.30382100 & -3.82499700 & -0.81816500 & $\mathrm{O}$ & -5.97847300 & -2.02584100 & 0.78632500 \\
\hline $\mathrm{H}$ & 5.98144400 & -4.26582900 & -0.47143000 & $\mathrm{C}$ & -7.35780400 & -1.77023500 & 0.44365400 \\
\hline $\mathrm{H}$ & 4.98185900 & -3.56692100 & 0.80265900 & $\mathrm{H}$ & -7.94493300 & -2.26624600 & 1.21492600 \\
\hline $\mathrm{C}$ & -0.98698300 & 3.09961800 & -3.33013900 & $\mathrm{H}$ & -7.55562900 & -0.69619100 & 0.43568700 \\
\hline
\end{tabular}




\begin{tabular}{|c|c|c|c|c|c|c|c|}
\hline $\mathrm{H}$ & -7.58560900 & -2.18473300 & -0.54062700 & $\mathrm{H}$ & -0.37080200 & 5.03167600 & 2.22471100 \\
\hline $\mathrm{C}$ & 0.65423600 & -1.05396600 & -2.02823500 & $\mathrm{H}$ & -2.47493000 & 5.92155100 & 3.27887800 \\
\hline \multirow[t]{2}{*}{$\mathrm{O}$} & 0.97518500 & -1.85135200 & -2.77766200 & $\mathrm{~N}$ & -3.82753200 & 3.51025200 & 1.33885700 \\
\hline & & & & $\mathrm{Pd}$ & -1.58186600 & -0.35433700 & -0.17485800 \\
\hline 59 & & & & $\mathrm{C}$ & -3.65022500 & 3.78765800 & -2.22656200 \\
\hline $\mathrm{C}$ & -0.32936200 & -3.28140600 & 1.33631100 & $\mathrm{H}$ & -3.35484600 & 4.54116300 & -1.49165900 \\
\hline $\mathrm{C}$ & -0.38029500 & -4.49499700 & 2.02883200 & $\mathrm{H}$ & -3.55564100 & 4.23836700 & -3.22170400 \\
\hline $\mathrm{C}$ & 0.59925700 & -4.81479000 & 2.97001300 & $\mathrm{H}$ & -4.70952300 & 3.56361800 & -2.07090900 \\
\hline $\mathrm{C}$ & 1.64582800 & -3.92214300 & 3.22728800 & $\mathrm{C}$ & -3.18733400 & 1.51872300 & -3.23099300 \\
\hline $\mathrm{C}$ & 1.66678100 & -2.72031000 & 2.54362800 & $\mathrm{H}$ & -2.57591000 & 0.61080300 & -3.18893200 \\
\hline $\mathrm{H}$ & -1.18859300 & -5.18662900 & 1.82671700 & $\mathrm{H}$ & -4.23936100 & 1.23146000 & -3.14037500 \\
\hline $\mathrm{H}$ & 0.55080000 & -5.76068600 & 3.50095500 & $\mathrm{H}$ & -3.05568300 & 1.96691100 & -4.22276000 \\
\hline $\mathrm{H}$ & 2.42061700 & -4.14742000 & 3.95030600 & $\mathrm{C}$ & -1.29159200 & 2.92677100 & -2.38301700 \\
\hline $\mathrm{H}$ & 2.41441900 & -1.94806000 & 2.67619700 & $\mathrm{H}$ & -0.62483500 & 2.06160800 & -2.31194400 \\
\hline $\mathrm{N}$ & 0.69470700 & -2.44694900 & 1.64898800 & $\mathrm{H}$ & -1.18513900 & 3.35750900 & -3.38565900 \\
\hline $\mathrm{P}$ & -1.52056500 & -2.60471900 & 0.04300200 & $\mathrm{H}$ & -0.94918500 & 3.67849300 & -1.66664400 \\
\hline $\mathrm{C}$ & -1.04308400 & -3.66908800 & -1.48546900 & $\mathrm{C}$ & -2.01723000 & -3.26633200 & -2.61247700 \\
\hline $\mathrm{C}$ & -3.11296100 & -3.33280800 & 0.73477300 & $\mathrm{H}$ & -3.04061700 & -3.59265200 & -2.40742100 \\
\hline $\mathrm{H}$ & -3.12540500 & -4.41570400 & 0.57014100 & $\mathrm{H}$ & -2.02374800 & -2.18398800 & -2.77032300 \\
\hline $\mathrm{H}$ & -3.06392400 & -3.17252200 & 1.81859000 & $\mathrm{H}$ & -1.70183800 & -3.74588000 & -3.54618200 \\
\hline $\mathrm{C}$ & -4.42156400 & -2.70502900 & 0.19830600 & $\mathrm{C}$ & -1.11347900 & -5.18866300 & -1.26176700 \\
\hline $\mathrm{H}$ & -5.24149800 & -3.33235600 & 0.56626100 & $\mathrm{H}$ & -0.86422800 & -5.69900500 & -2.19905900 \\
\hline $\mathrm{H}$ & -4.45251300 & -2.79668800 & -0.89223300 & $\mathrm{H}$ & -0.39626500 & -5.53568000 & -0.51132900 \\
\hline $\mathrm{C}$ & -4.64875000 & -1.22919700 & 0.61182600 & $\mathrm{H}$ & -2.11326200 & -5.52675700 & -0.97312200 \\
\hline $\mathrm{H}$ & -5.43851400 & -1.16959800 & 1.37042600 & $\mathrm{C}$ & 0.38850900 & -3.26743400 & -1.89059500 \\
\hline $\mathrm{H}$ & -3.73682500 & -0.85011800 & 1.08953100 & $\mathrm{H}$ & 0.66322300 & -3.78059500 & -2.81874600 \\
\hline $\mathrm{C}$ & -4.99831700 & -0.29610400 & -0.56388200 & $\mathrm{H}$ & 0.46816800 & -2.19054500 & -2.06773600 \\
\hline $\mathrm{H}$ & -6.06247800 & -0.40165100 & -0.81062500 & $\mathrm{H}$ & 1.12922800 & -3.55385100 & -1.13489500 \\
\hline $\mathrm{H}$ & -4.44776200 & -0.62006200 & -1.45462000 & $\mathrm{C}$ & 1.98731400 & 0.96703900 & 0.32690100 \\
\hline $\mathrm{C}$ & -4.69988000 & 1.19099100 & -0.28623600 & $\mathrm{O}$ & 1.55680500 & 2.07971300 & 0.14937200 \\
\hline $\mathrm{H}$ & -5.28297200 & 1.83059600 & -0.95475600 & $\mathrm{C}$ & 1.05114900 & 1.01967500 & 2.51477600 \\
\hline $\mathrm{H}$ & -4.99693200 & 1.47862500 & 0.72625600 & $\mathrm{H}$ & 1.53463300 & 1.98221300 & 2.68596500 \\
\hline $\mathrm{P}$ & -2.88934000 & 1.64993800 & -0.45857700 & $\mathrm{H}$ & 1.09295200 & 0.40735700 & 3.41623800 \\
\hline $\mathrm{C}$ & -2.76259400 & 2.53287700 & -2.15071700 & $\mathrm{H}$ & 0.01616300 & 1.17675000 & 2.20373500 \\
\hline $\mathrm{C}$ & -2.70715100 & 3.03733500 & 0.77031100 & $\mathrm{O}$ & 1.77058700 & 0.27675100 & 1.49867000 \\
\hline $\mathrm{C}$ & -1.43545500 & 3.54879300 & 1.07607800 & $\mathrm{H}$ & 0.70585000 & -1.51780300 & 1.20064900 \\
\hline $\mathrm{C}$ & -3.71922200 & 4.51305100 & 2.21920400 & $\mathrm{C}$ & 2.82526300 & 0.16798600 & -0.64235900 \\
\hline $\mathrm{C}$ & -1.34028700 & 4.60634100 & 1.98081500 & $\mathrm{H}$ & 2.76395000 & 0.65335400 & -1.61614200 \\
\hline $\mathrm{H}$ & -0.54234200 & 3.12907000 & 0.62508400 & $\mathrm{H}$ & 2.40420500 & -0.83731300 & -0.74811700 \\
\hline $\mathrm{C}$ & -2.50354900 & 5.10243300 & 2.56767400 & $\mathrm{C}$ & 4.30451300 & 0.06146200 & -0.19550300 \\
\hline $\mathrm{H}$ & -4.65022500 & 4.86272600 & 2.66098300 & $\mathrm{H}$ & 4.35649300 & -0.41653300 & 0.78795800 \\
\hline
\end{tabular}




\begin{tabular}{|c|c|c|c|c|c|c|c|}
\hline $\mathrm{C}$ & 4.99304900 & 1.42026600 & -0.09478800 & $\mathrm{C}$ & -1.02031100 & -0.14788500 & 3.31790700 \\
\hline $\mathrm{C}$ & 5.41239700 & 1.91150800 & 1.14634300 & $\mathrm{H}$ & -1.28333200 & 0.28785800 & 4.28961400 \\
\hline $\mathrm{C}$ & 5.20782600 & 2.19646900 & -1.24230600 & $\mathrm{H}$ & -1.30724600 & -1.20360100 & 3.38219800 \\
\hline $\mathrm{C}$ & 6.02960300 & 3.16009100 & 1.24410600 & $\mathrm{C}$ & -1.87138800 & 0.58941000 & 2.25770000 \\
\hline $\mathrm{H}$ & 5.26234700 & 1.31206300 & 2.04119100 & $\mathrm{H}$ & -2.88587500 & 0.73864000 & 2.64269900 \\
\hline $\mathrm{C}$ & 5.82561800 & 3.44261800 & -1.14524600 & $\mathrm{H}$ & -1.44037800 & 1.58838500 & 2.12662500 \\
\hline $\mathrm{H}$ & 4.90117500 & 1.82079400 & -2.21489900 & $\mathrm{P}$ & -1.95149900 & -0.14954800 & 0.52309400 \\
\hline $\mathrm{C}$ & 6.23652300 & 3.92845200 & 0.09822600 & $\mathrm{C}$ & -3.56729200 & -1.20212300 & 0.55676700 \\
\hline $\mathrm{H}$ & 6.35218700 & 3.52834100 & 2.21351700 & $\mathrm{C}$ & -2.48660100 & 1.33394500 & -0.47158600 \\
\hline $\mathrm{H}$ & 5.98901700 & 4.03327900 & -2.04162300 & $\mathrm{C}$ & -2.95737000 & 2.54099600 & 0.06913900 \\
\hline $\mathrm{H}$ & 6.71861000 & 4.89851100 & 0.17192100 & $\mathrm{C}$ & -2.68894500 & 2.16579200 & -2.61462800 \\
\hline $\mathrm{C}$ & 5.05461300 & -0.83767000 & -1.17866100 & $\mathrm{C}$ & -3.28317900 & 3.59042600 & -0.79041500 \\
\hline $\mathrm{O}$ & 4.69743600 & -1.06599700 & -2.31541500 & $\mathrm{H}$ & -3.07688400 & 2.66336200 & 1.13940100 \\
\hline $\mathrm{O}$ & 6.17524000 & -1.32640600 & -0.62307200 & $\mathrm{C}$ & -3.14306100 & 3.40687500 & -2.16375100 \\
\hline $\mathrm{C}$ & 7.01464200 & -2.10916600 & -1.49715100 & $\mathrm{H}$ & -2.57470900 & 1.97283900 & -3.67983700 \\
\hline $\mathrm{H}$ & 7.86909100 & -2.40742300 & -0.89119400 & $\mathrm{H}$ & -3.64469100 & 4.53381500 & -0.39006300 \\
\hline $\mathrm{H}$ & 6.47442300 & -2.98535900 & -1.86303500 & $\mathrm{H}$ & -3.38074400 & 4.19661600 & -2.86949600 \\
\hline \multirow[t]{2}{*}{$\mathrm{H}$} & 7.33859400 & -1.50874900 & -2.34993000 & $\mathrm{~N}$ & -2.36323200 & 1.15764700 & -1.80098300 \\
\hline & & & & $\mathrm{Pd}$ & -0.04007100 & -1.25177000 & -0.55407900 \\
\hline 60 & & & & $\mathrm{C}$ & -4.79376200 & -0.42928400 & 1.07316900 \\
\hline $\mathrm{C}$ & 2.44054100 & 1.40144900 & 0.26714700 & $\mathrm{H}$ & -5.00071100 & 0.46134200 & 0.47221900 \\
\hline $\mathrm{C}$ & 2.99164800 & 2.06477800 & 1.37490200 & $\mathrm{H}$ & -5.67913800 & -1.07499200 & 1.01906700 \\
\hline $\mathrm{C}$ & 3.11378300 & 3.45416100 & 1.34772200 & $\mathrm{H}$ & -4.68997400 & -0.12113000 & 2.11839200 \\
\hline $\mathrm{C}$ & 2.69454100 & 4.14761100 & 0.21501300 & $\mathrm{C}$ & -3.30190100 & -2.42091400 & 1.46189500 \\
\hline $\mathrm{C}$ & 2.17026800 & 3.40704800 & -0.84626500 & $\mathrm{H}$ & -2.41823700 & -2.97663800 & 1.13208300 \\
\hline $\mathrm{H}$ & 3.32311700 & 1.51366400 & 2.24690600 & $\mathrm{H}$ & -3.16066700 & -2.13511500 & 2.50940500 \\
\hline $\mathrm{H}$ & 3.53428600 & 3.98312300 & 2.19880400 & $\mathrm{H}$ & -4.16122300 & -3.10205800 & 1.42327900 \\
\hline $\mathrm{H}$ & 2.76878700 & 5.22854500 & 0.15024800 & $\mathrm{C}$ & -3.85084100 & -1.69314900 & -0.87510500 \\
\hline $\mathrm{H}$ & 1.83542800 & 3.90525900 & -1.75457700 & $\mathrm{H}$ & -3.00011900 & -2.24377600 & -1.28311000 \\
\hline N & 2.03497800 & 2.07822300 & -0.82590400 & $\mathrm{H}$ & -4.72109200 & -2.36150900 & -0.85986300 \\
\hline$P$ & 2.14345800 & -0.43697600 & 0.13380800 & $\mathrm{H}$ & -4.06785800 & -0.86572300 & -1.55521500 \\
\hline $\mathrm{C}$ & 3.67516800 & -0.99278300 & -0.88621600 & $\mathrm{C}$ & 3.68544200 & -2.53310500 & -0.94097700 \\
\hline $\mathrm{C}$ & 2.49578800 & -1.11853900 & 1.85033300 & $\mathrm{H}$ & 3.90594300 & -2.98863100 & 0.02985300 \\
\hline $\mathrm{H}$ & 2.97082200 & -2.09119800 & 1.69099800 & $\mathrm{H}$ & 2.72877000 & -2.92677500 & -1.29598100 \\
\hline $\mathrm{H}$ & 3.21986600 & -0.51361200 & 2.40731400 & $\mathrm{H}$ & 4.46710500 & -2.86313500 & -1.63598400 \\
\hline $\mathrm{C}$ & 1.19727400 & -1.32940700 & 2.65086000 & $\mathrm{C}$ & 4.99679600 & -0.48606200 & -0.28205200 \\
\hline $\mathrm{H}$ & 1.40906400 & -1.95048100 & 3.53177900 & $\mathrm{H}$ & 5.84092000 & -0.83924400 & -0.88794800 \\
\hline $\mathrm{H}$ & 0.51098700 & -1.90657100 & 2.01868000 & $\mathrm{H}$ & 5.04300100 & 0.60667200 & -0.25792300 \\
\hline $\mathrm{C}$ & 0.50724100 & -0.02681700 & 3.09507600 & $\mathrm{H}$ & 5.15144100 & -0.85624600 & 0.73735200 \\
\hline $\mathrm{H}$ & 0.98923000 & 0.33420900 & 4.01284800 & $\mathrm{C}$ & 3.50834700 & -0.45661200 & -2.32109700 \\
\hline $\mathrm{H}$ & 0.68351500 & 0.75202900 & 2.34659900 & $\mathrm{H}$ & 4.35212200 & -0.79676100 & -2.93482500 \\
\hline
\end{tabular}




\begin{tabular}{|c|c|c|c|c|c|c|c|}
\hline $\mathrm{H}$ & 2.58452400 & -0.82918800 & -2.77423300 & $\mathrm{H}$ & -3.45664000 & -0.40209900 & -3.53738800 \\
\hline $\mathrm{H}$ & 3.47762900 & 0.63466800 & -2.35297400 & $\mathrm{H}$ & -3.67741300 & 3.83827000 & -2.74928600 \\
\hline $\mathrm{C}$ & -0.24432000 & -2.79272300 & -1.69951900 & $\mathrm{H}$ & -4.08379200 & 1.90985900 & -4.32939900 \\
\hline \multirow[t]{2}{*}{$\mathrm{O}$} & -0.33115300 & -3.73312000 & -2.36852000 & $\mathrm{~N}$ & -2.80809800 & 0.26822600 & -1.69941300 \\
\hline & & & & $\mathrm{Pd}$ & -0.10048200 & -0.78308900 & 0.03564700 \\
\hline 62 & & & & $\mathrm{C}$ & -3.96160900 & 1.17586700 & 2.52513200 \\
\hline $\mathrm{C}$ & 3.10016300 & 0.95486400 & -0.59959900 & $\mathrm{H}$ & -4.42068300 & 1.82694600 & 1.77259100 \\
\hline $\mathrm{C}$ & 3.88497000 & 1.96886000 & -0.02544200 & $\mathrm{H}$ & -4.77560000 & 0.71637300 & 3.10004900 \\
\hline $\mathrm{C}$ & 4.40784000 & 2.97499300 & -0.83770000 & $\mathrm{H}$ & -3.39117000 & 1.80062900 & 3.21847700 \\
\hline $\mathrm{C}$ & 4.14716400 & 2.94448500 & -2.20531400 & $\mathrm{C}$ & -2.41456200 & -0.76652800 & 2.98037500 \\
\hline $\mathrm{C}$ & 3.36691800 & 1.89493900 & -2.69466700 & $\mathrm{H}$ & -1.79265600 & -1.55054800 & 2.53843600 \\
\hline $\mathrm{H}$ & 4.09074300 & 1.97981700 & 1.03801000 & $\mathrm{H}$ & -1.78302800 & -0.15408500 & 3.63197000 \\
\hline $\mathrm{H}$ & 5.01391900 & 3.76695500 & -0.40546600 & $\mathrm{H}$ & -3.17451800 & -1.24212700 & 3.61323300 \\
\hline $\mathrm{H}$ & 4.53270500 & 3.70614400 & -2.87580700 & $\mathrm{C}$ & -4.02149000 & -0.85397700 & 1.06368800 \\
\hline $\mathrm{H}$ & 3.14136000 & 1.82617300 & -3.75797800 & $\mathrm{H}$ & -3.45761400 & -1.61047500 & 0.51279400 \\
\hline $\mathrm{N}$ & 2.84810500 & 0.93454900 & -1.92541900 & $\mathrm{H}$ & -4.70326000 & -1.37516100 & 1.74658800 \\
\hline $\mathrm{P}$ & 2.25114200 & -0.42286200 & 0.33340800 & $\mathrm{H}$ & -4.63828400 & -0.29160700 & 0.35463800 \\
\hline $\mathrm{C}$ & 3.41065000 & -1.91857300 & -0.00383200 & $\mathrm{C}$ & 2.85819200 & -3.11406200 & 0.79976100 \\
\hline $\mathrm{C}$ & 2.59304700 & -0.04067800 & 2.14868000 & $\mathrm{H}$ & 2.95346200 & -2.97394400 & 1.88196200 \\
\hline $\mathrm{H}$ & 2.78570900 & -1.00860800 & 2.62143000 & $\mathrm{H}$ & 1.80444600 & -3.30013000 & 0.56898600 \\
\hline $\mathrm{H}$ & 3.50427200 & 0.54987500 & 2.29227600 & $\mathrm{H}$ & 3.42978600 & -4.01451800 & 0.54177400 \\
\hline $\mathrm{C}$ & 1.38541700 & 0.60879000 & 2.84873300 & $\mathrm{C}$ & 4.87156600 & -1.63850200 & 0.38738300 \\
\hline $\mathrm{H}$ & 1.54449300 & 0.59114200 & 3.93611400 & $\mathrm{H}$ & 5.49179900 & -2.51628300 & 0.16398000 \\
\hline $\mathrm{H}$ & 0.51503600 & -0.02444200 & 2.64630400 & $\mathrm{H}$ & 5.28691700 & -0.79155400 & -0.16831600 \\
\hline $\mathrm{C}$ & 1.08077300 & 2.05285200 & 2.40993600 & $\mathrm{H}$ & 4.98092500 & -1.42966400 & 1.45720000 \\
\hline $\mathrm{H}$ & 1.78302600 & 2.72903300 & 2.91521600 & $\mathrm{C}$ & 3.32176100 & -2.26389500 & -1.50260200 \\
\hline $\mathrm{H}$ & 1.27816700 & 2.16274100 & 1.33818300 & $\mathrm{H}$ & 3.90879600 & -3.17040200 & -1.69868000 \\
\hline $\mathrm{C}$ & -0.36766000 & 2.51825800 & 2.69389600 & $\mathrm{H}$ & 2.28564900 & -2.45606400 & -1.79724100 \\
\hline $\mathrm{H}$ & -0.35055400 & 3.56373400 & 3.02624800 & $\mathrm{H}$ & 3.70741900 & -1.46165000 & -2.13577400 \\
\hline $\mathrm{H}$ & -0.78442800 & 1.95237900 & 3.53652600 & $\mathrm{C}$ & -1.07148400 & -2.57123300 & -0.50144000 \\
\hline $\mathrm{C}$ & -1.32692100 & 2.47112800 & 1.47985500 & $\mathrm{O}$ & -0.93511900 & -3.71266600 & -0.12505900 \\
\hline $\mathrm{H}$ & -2.24785000 & 3.00866600 & 1.73160100 & $\mathrm{H}$ & -2.52049600 & -0.72272600 & -1.49247000 \\
\hline $\mathrm{H}$ & -0.84522000 & 3.01780700 & 0.66031200 & $\mathrm{O}$ & -2.14176800 & -2.38225800 & -1.52366100 \\
\hline $\mathrm{P}$ & -1.69448600 & 0.76669200 & 0.74939600 & $\mathrm{C}$ & -2.86860200 & -3.55753500 & -1.90593100 \\
\hline $\mathrm{C}$ & -3.10438000 & 0.07085800 & 1.88497600 & $\mathrm{H}$ & -2.18382100 & -4.34800900 & -2.22427600 \\
\hline $\mathrm{C}$ & -2.56307400 & 1.27218000 & -0.78998300 & $\mathrm{H}$ & -3.47294200 & -3.94391400 & -1.07707600 \\
\hline $\mathrm{C}$ & -2.88138300 & 2.57757300 & -1.20463900 & $\mathrm{H}$ & -3.52409100 & -3.27940200 & -2.73654400 \\
\hline $\mathrm{C}$ & -3.33201300 & 0.48364800 & -2.92509800 & & & & \\
\hline $\mathrm{C}$ & -3.42584100 & 2.82322400 & -2.45777500 & 65 & & & \\
\hline $\mathrm{H}$ & -2.71082000 & 3.40139100 & -0.52355500 & $\mathrm{C}$ & -3.39704800 & 0.22060200 & -0.80427900 \\
\hline $\mathrm{C}$ & -3.66217000 & 1.75315100 & -3.34403600 & $\mathrm{C}$ & -4.51577000 & -0.32632600 & -1.44908400 \\
\hline
\end{tabular}




\begin{tabular}{|c|c|c|c|c|c|c|c|}
\hline $\mathrm{C}$ & -5.40726800 & 0.50746900 & -2.12336100 & $\mathrm{H}$ & 4.28430800 & -3.11686700 & -0.06244600 \\
\hline $\mathrm{C}$ & -5.17273000 & 1.88090500 & -2.13933000 & $\mathrm{C}$ & 2.07074100 & -2.92817100 & 1.61068600 \\
\hline $\mathrm{C}$ & -4.05064700 & 2.35861800 & -1.46998000 & $\mathrm{H}$ & 1.08972300 & -2.55024700 & 1.90964400 \\
\hline $\mathrm{H}$ & -4.69596500 & -1.39405800 & -1.41742500 & $\mathrm{H}$ & 1.92681300 & -3.69589200 & 0.84529800 \\
\hline $\mathrm{H}$ & -6.27468500 & 0.08850700 & -2.62468000 & $\mathrm{H}$ & 2.52338300 & -3.41691100 & 2.48133300 \\
\hline $\mathrm{H}$ & -5.83829300 & 2.56620800 & -2.65237900 & $\mathrm{C}$ & 3.17286100 & -0.78486600 & 2.29015900 \\
\hline $\mathrm{H}$ & -3.82045000 & 3.42081000 & -1.44416400 & $\mathrm{H}$ & 2.22011700 & -0.32390800 & 2.56890800 \\
\hline $\mathrm{N}$ & -3.18788400 & 1.55561400 & -0.82839700 & $\mathrm{H}$ & 3.56837200 & -1.30620700 & 3.16934600 \\
\hline $\mathrm{P}$ & -2.10931600 & -0.78284500 & 0.11276700 & $\mathrm{H}$ & 3.88111200 & 0.01296100 & 2.04261900 \\
\hline $\mathrm{C}$ & -2.94825700 & -1.03716700 & 1.82383200 & $\mathrm{C}$ & -2.05260800 & -2.01505600 & 2.60995400 \\
\hline $\mathrm{C}$ & -2.27912500 & -2.46487400 & -0.72954700 & $\mathrm{H}$ & -2.00168400 & -3.00270700 & 2.13977100 \\
\hline $\mathrm{H}$ & -2.96198700 & -3.09484400 & -0.15191400 & $\mathrm{H}$ & -1.03402200 & -1.62669300 & 2.71011100 \\
\hline $\mathrm{H}$ & -2.74076300 & -2.31758800 & -1.71093400 & $\mathrm{H}$ & -2.45937200 & -2.15513400 & 3.61830200 \\
\hline $\mathrm{C}$ & -0.92221800 & -3.16821600 & -0.90855700 & $\mathrm{C}$ & -4.38270000 & -1.58696700 & 1.75138100 \\
\hline $\mathrm{H}$ & -1.08752700 & -4.24753700 & -1.01986600 & $\mathrm{H}$ & -4.77118400 & -1.71673900 & 2.76847900 \\
\hline $\mathrm{H}$ & -0.32643300 & -3.04171600 & 0.00059400 & $\mathrm{H}$ & -5.05924900 & -0.90454600 & 1.22932100 \\
\hline $\mathrm{C}$ & -0.12998900 & -2.65337000 & -2.12188300 & $\mathrm{H}$ & -4.43795100 & -2.56510900 & 1.26362600 \\
\hline $\mathrm{H}$ & -0.60542300 & -3.03398600 & -3.03399900 & $\mathrm{C}$ & -2.96030000 & 0.32632200 & 2.54155500 \\
\hline $\mathrm{H}$ & -0.21283700 & -1.56070400 & -2.16879500 & $\mathrm{H}$ & -3.37233500 & 0.20482800 & 3.55026200 \\
\hline $\mathrm{C}$ & 1.36529800 & -3.05090200 & -2.10447100 & $\mathrm{H}$ & -1.95103200 & 0.73810900 & 2.63174500 \\
\hline $\mathrm{H}$ & 1.64703500 & -3.48429400 & -3.07079400 & $\mathrm{H}$ & -3.58230800 & 1.05951100 & 2.01832100 \\
\hline $\mathrm{H}$ & 1.53311800 & -3.84742200 & -1.37045200 & $\mathrm{C}$ & -0.24224700 & 2.34496200 & 0.59750700 \\
\hline $\mathrm{C}$ & 2.34678700 & -1.88547300 & -1.85371400 & $\mathrm{O}$ & -1.33735500 & 2.97483600 & 0.30580600 \\
\hline $\mathrm{H}$ & 3.37513300 & -2.25957100 & -1.90277100 & $\mathrm{H}$ & 2.01665700 & 1.69581100 & 0.14646600 \\
\hline $\mathrm{H}$ & 2.23405500 & -1.15902100 & -2.66835200 & $\mathrm{O}$ & 0.61822700 & 3.13581200 & 1.25694000 \\
\hline $\mathrm{P}$ & 2.08537700 & -0.86699000 & -0.29287700 & $\mathrm{C}$ & 0.21726600 & 4.48674800 & 1.63329600 \\
\hline $\mathrm{C}$ & 2.99682500 & -1.79033200 & 1.13442700 & $\mathrm{H}$ & 0.03353200 & 5.09440800 & 0.74514200 \\
\hline $\mathrm{C}$ & 3.38344300 & 0.47061100 & -0.68395400 & $\mathrm{H}$ & -0.68560500 & 4.45639000 & 2.24432800 \\
\hline $\mathrm{C}$ & 4.61568600 & 0.39108000 & -1.33747600 & $\mathrm{H}$ & 1.05277100 & 4.88368300 & 2.20873600 \\
\hline $\mathrm{C}$ & 3.70950100 & 2.82202500 & -0.39686800 & $\mathrm{H}$ & -2.01888300 & 2.32632600 & -0.15067700 \\
\hline $\mathrm{C}$ & 5.38743000 & 1.54117900 & -1.51793200 & & & & \\
\hline $\mathrm{H}$ & 4.96736600 & -0.56279300 & -1.71040200 & 66 & & & \\
\hline $\mathrm{C}$ & 4.93800000 & 2.77462000 & -1.03495700 & $\mathrm{C}$ & -0.98473000 & 3.82308900 & 0.44349300 \\
\hline $\mathrm{H}$ & 3.26208600 & 3.72513700 & 0.00063600 & $\mathrm{C}$ & -0.42876000 & 2.58060800 & 0.79242300 \\
\hline $\mathrm{H}$ & 6.34043800 & 1.47722500 & -2.03449100 & $\mathrm{C}$ & 0.97834500 & 2.44409500 & 0.78527800 \\
\hline $\mathrm{H}$ & 5.52070000 & 3.67949900 & -1.16054700 & $\mathrm{C}$ & 1.75549900 & 3.56662400 & 0.45252500 \\
\hline $\mathrm{N}$ & 2.99408900 & 1.69286900 & -0.25983100 & $\mathrm{C}$ & 1.18720800 & 4.78198200 & 0.08098000 \\
\hline $\mathrm{Pd}$ & 0.11810000 & 0.31033400 & 0.20559900 & $\mathrm{C}$ & -0.19986500 & 4.91054600 & 0.07066100 \\
\hline $\mathrm{C}$ & 4.36135700 & -2.38086200 & 0.74347200 & $\mathrm{H}$ & -2.06369300 & 3.94414400 & 0.48624100 \\
\hline $\mathrm{H}$ & 5.08233000 & -1.61300200 & 0.44723100 & $\mathrm{H}$ & 2.83598200 & 3.49982200 & 0.52567500 \\
\hline $\mathrm{H}$ & 4.78926800 & -2.89967100 & 1.60931200 & $\mathrm{H}$ & 1.82385000 & 5.62477100 & -0.16854200 \\
\hline
\end{tabular}




\begin{tabular}{|c|c|c|c|c|c|c|c|}
\hline $\mathrm{H}$ & -0.66723800 & 5.85392400 & -0.19306700 & $\mathrm{C}$ & 4.14733700 & 1.21780200 & -0.95617400 \\
\hline $\mathrm{C}$ & 1.68902200 & 1.19930300 & 1.28171700 & $\mathrm{H}$ & 4.85223200 & 0.80989500 & -0.23032900 \\
\hline $\mathrm{H}$ & 2.67796500 & 1.48318500 & 1.65443800 & $\mathrm{H}$ & 4.71419900 & 1.44711900 & -1.86509700 \\
\hline $\mathrm{C}$ & -1.37665100 & 1.51480200 & 1.30461800 & $\mathrm{H}$ & 3.76147700 & 2.16218000 & -0.57617100 \\
\hline $\mathrm{H}$ & -0.96550600 & 1.05296200 & 2.20910500 & $\mathrm{C}$ & 2.05016000 & 0.91619600 & -2.31548900 \\
\hline $\mathrm{H}$ & -2.30016900 & 2.00652500 & 1.61776300 & $\mathrm{H}$ & 2.61820100 & 1.26210300 & -3.18642600 \\
\hline $\mathrm{H}$ & 1.14710100 & 0.78892000 & 2.13708200 & $\mathrm{H}$ & 1.28765800 & 0.21628400 & -2.67264200 \\
\hline$P$ & 1.95827800 & -0.30540500 & 0.17691400 & $\mathrm{H}$ & 1.55079700 & 1.78420100 & -1.87803900 \\
\hline$P$ & -1.88748200 & 0.04291700 & 0.24896100 & $\mathrm{C}$ & 2.99192700 & -2.91617800 & 0.76945500 \\
\hline $\mathrm{C}$ & -2.94913600 & -0.93236700 & 1.53629700 & $\mathrm{H}$ & 3.53546200 & -2.94525100 & -0.17545500 \\
\hline $\mathrm{C}$ & -2.93251200 & 0.73865000 & -1.20401800 & $\mathrm{H}$ & 3.53350300 & -3.55209900 & 1.47841600 \\
\hline $\mathrm{C}$ & 2.88941700 & -1.49941400 & 1.37211000 & $\mathrm{H}$ & 2.00595800 & -3.35942400 & 0.61050200 \\
\hline $\mathrm{C}$ & 3.02092200 & 0.23191200 & -1.32634800 & $\mathrm{C}$ & 4.30535600 & -0.99476600 & 1.71896400 \\
\hline $\mathrm{C}$ & -3.88215500 & -1.94899000 & 0.85031200 & $\mathrm{H}$ & 4.72732400 & -1.64626800 & 2.49213600 \\
\hline $\mathrm{H}$ & -4.72325000 & -1.46688700 & 0.34717100 & $\mathrm{H}$ & 4.98055200 & -1.03480800 & 0.86159800 \\
\hline $\mathrm{H}$ & -3.36377600 & -2.58632200 & 0.12897800 & $\mathrm{H}$ & 4.31236700 & 0.02252600 & 2.12097000 \\
\hline $\mathrm{H}$ & -4.30311100 & -2.60845300 & 1.61736500 & $\mathrm{C}$ & 2.06171200 & -1.61102400 & 2.67289200 \\
\hline $\mathrm{C}$ & -1.94873800 & -1.71617500 & 2.41504200 & $\mathrm{H}$ & 2.09032800 & -0.70072600 & 3.27675900 \\
\hline $\mathrm{H}$ & -2.50200200 & -2.27299900 & 3.17982000 & $\mathrm{H}$ & 1.01840500 & -1.87314100 & 2.47304300 \\
\hline $\mathrm{H}$ & -1.37317300 & -2.43961900 & 1.82854600 & $\mathrm{H}$ & 2.48700200 & -2.41261000 & 3.28615400 \\
\hline $\mathrm{H}$ & -1.24634500 & -1.06274100 & 2.94137900 & $\mathrm{Pd}$ & -0.02565200 & -1.37904500 & -0.61600200 \\
\hline $\mathrm{C}$ & -3.80019700 & -0.02261300 & 2.44816200 & $\mathrm{H}$ & 1.16272200 & -2.22544900 & -1.18301400 \\
\hline $\mathrm{H}$ & -4.40856800 & -0.65710900 & 3.10215700 & $\mathrm{C}$ & -1.10488900 & -2.79947100 & -1.45505900 \\
\hline $\mathrm{H}$ & -3.19171800 & 0.61082300 & 3.09775100 & $\mathrm{O}$ & -1.60120600 & -3.70335600 & -1.94019400 \\
\hline
\end{tabular}

$\mathrm{H}$

$\mathrm{H}$

$\mathrm{H}$

C

$\mathrm{H}$

$\mathrm{H}$

$\mathrm{H}$

C

$\mathrm{H}$

H

$\mathrm{H}$

C

$\mathrm{H}$

$\mathrm{H}$

H
$-4.48605700$

$-4.13482300$

$-4.60560000$

$-4.89876900$

$-3.85021700$

$-1.99085500$

$-2.52968700$

$-1.66096600$

$-1.10277900$

$-3.44124300$

$-3.99677100$

$-2.61559600$

$-4.11451600$

3.64994300

4.44662500

2.91364000

4.10006300
$0.61647200 \quad 1.88799100$

$1.58021100-0.73246600$

$2.04575600-1.60571200$

$0.97122600-0.24245000$

$2.38771700 \quad-0.05251700$

$1.60762300-2.06967300$

$1.90733000 \quad-2.97567000$

$2.51452300-1.56501200$

$1.05084900 \quad-2.38526500$

$-0.40245900 \quad-2.11124500$

$0.04454700 \quad-2.94317700$

$-0.96909600 \quad-2.54929800$

$-1.09704000 \quad-1.61020400$

$-0.99220500 \quad-2.02072300$

$-1.44443400 \quad-1.42570600$

$-1.76202700 \quad-2.26825800$

$\begin{array}{ll}-0.65775900 & -2.96187600\end{array}$

\section{TS51}

imaginary frequency $=-446.96 \mathrm{~cm}^{-1}$

C

C

C

C

C

C

$\mathrm{H}$

H

$\mathrm{H}$

$\mathrm{H}$

C

$\mathrm{H}$

C

H

$\begin{array}{lrr}2.55226200 & 3.68689600 & 0.11195100 \\ 2.18948500 & 2.40765300 & 0.56834300 \\ 3.15922200 & 1.38392100 & 0.53228500 \\ 4.44448600 & 1.68624900 & 0.05080200 \\ 4.77790900 & 2.95157000 & -0.42337200 \\ 3.81908300 & 3.96309400 & -0.39433800 \\ 1.82581700 & 4.49200000 & 0.17589500 \\ 5.20705200 & 0.91246700 & 0.06589600 \\ 5.78094900 & 3.14930500 & -0.78784300 \\ 4.06078200 & 4.96430200 & -0.73683100 \\ 2.92900500 & 0.00843200 & 1.11602800 \\ 3.89940600 & -0.42016400 & 1.37721000 \\ 0.83150300 & 2.24983500 & 1.21919700 \\ 0.93130300 & 1.67394100 & 2.14650400\end{array}$




\begin{tabular}{|c|c|c|c|c|c|c|c|}
\hline $\mathrm{H}$ & 0.49838400 & 3.24274400 & 1.52411400 & $\mathrm{H}$ & 4.58709600 & -3.06982500 & -0.51468500 \\
\hline $\mathrm{H}$ & 2.36927200 & 0.08638600 & 2.05381200 & $\mathrm{H}$ & 5.08323700 & -2.20127200 & -1.96241700 \\
\hline $\mathrm{P}$ & 2.00242700 & -1.33040400 & 0.17175100 & $\mathrm{H}$ & 5.00339000 & -1.34877200 & -0.42555600 \\
\hline $\mathrm{P}$ & -0.65508500 & 1.44837100 & 0.36615900 & $\mathrm{C}$ & 2.42979000 & -2.91641900 & -2.18171100 \\
\hline $\mathrm{C}$ & -1.94358800 & 1.66373300 & 1.79937500 & $\mathrm{H}$ & 1.41013900 & -2.68676700 & -2.50279900 \\
\hline $\mathrm{C}$ & -1.06159700 & 2.55516500 & -1.14672200 & $\mathrm{H}$ & 3.02734000 & -3.06321800 & -3.08835700 \\
\hline $\mathrm{C}$ & 2.01506600 & -2.73457900 & 1.50149000 & $\mathrm{H}$ & 2.42301400 & -3.86265700 & -1.64137000 \\
\hline $\mathrm{C}$ & 3.05109100 & -1.75236000 & -1.38249400 & $\mathrm{C}$ & -1.77163700 & 0.42863700 & 2.71004300 \\
\hline $\mathrm{Pd}$ & -0.28438600 & -0.90468800 & -0.38030400 & $\mathrm{H}$ & -1.97808600 & -0.50120700 & 2.17278000 \\
\hline $\mathrm{H}$ & 0.03368200 & -2.33692800 & -0.89396300 & $\mathrm{H}$ & -0.76504500 & 0.35919800 & 3.13512200 \\
\hline $\mathrm{C}$ & -1.57192700 & -2.35921700 & -1.57276100 & $\mathrm{H}$ & -2.47255100 & 0.49865800 & 3.54993100 \\
\hline $\mathrm{C}$ & -2.51863400 & -1.72329100 & -1.06856700 & $\mathrm{C}$ & -1.74050100 & 2.93510400 & 2.65359400 \\
\hline $\mathrm{C}$ & -3.86053800 & -1.35523700 & -0.71972300 & $\mathrm{H}$ & -2.56576500 & 2.99949700 & 3.37189000 \\
\hline $\mathrm{C}$ & -4.67132800 & -0.64804500 & -1.63027000 & $\mathrm{H}$ & -0.81650200 & 2.91706700 & 3.23575100 \\
\hline $\mathrm{C}$ & -4.40912900 & -1.78922900 & 0.50457700 & $\mathrm{H}$ & -1.75901200 & 3.85305200 & 2.06057800 \\
\hline $\mathrm{C}$ & -5.99896800 & -0.37507100 & -1.31171400 & $\mathrm{C}$ & -3.38736900 & 1.68190800 & 1.26391700 \\
\hline $\mathrm{H}$ & -4.25954200 & -0.33254100 & -2.58254200 & $\mathrm{H}$ & -3.59763800 & 0.85657100 & 0.58499100 \\
\hline $\mathrm{C}$ & -5.73953700 & -1.51532600 & 0.80798700 & $\mathrm{H}$ & -4.07479300 & 1.59005500 & 2.11262200 \\
\hline $\mathrm{H}$ & -3.79075100 & -2.34774100 & 1.19976400 & $\mathrm{H}$ & -3.62572600 & 2.62182300 & 0.76028900 \\
\hline $\mathrm{C}$ & -6.53539500 & -0.80462500 & -0.09473900 & $\mathrm{C}$ & -1.27855100 & 4.03606400 & -0.78550200 \\
\hline $\mathrm{H}$ & -6.61961600 & 0.16481800 & -2.01995300 & $\mathrm{H}$ & -1.42520300 & 4.61155300 & -1.70692700 \\
\hline $\mathrm{H}$ & -6.15774800 & -1.86040400 & 1.74836800 & $\mathrm{H}$ & -2.16686900 & 4.18792600 & -0.16680700 \\
\hline $\mathrm{H}$ & -7.57246000 & -0.59273200 & 0.14587600 & $\mathrm{H}$ & -0.42177500 & 4.47477400 & -0.26781700 \\
\hline $\mathrm{H}$ & -1.24883200 & -3.15250000 & -2.22039100 & $\mathrm{C}$ & -2.30770700 & 2.01228900 & -1.87531000 \\
\hline $\mathrm{C}$ & 1.69843800 & -4.10838200 & 0.88039000 & $\mathrm{H}$ & -3.22681300 & 2.11670200 & -1.30069800 \\
\hline $\mathrm{H}$ & 1.49995600 & -4.81966600 & 1.68984900 & $\mathrm{H}$ & -2.43654700 & 2.57456100 & -2.80751300 \\
\hline $\mathrm{H}$ & 0.80999700 & -4.08697100 & 0.24228200 & $\mathrm{H}$ & -2.18189700 & 0.95883800 & -2.14067300 \\
\hline $\mathrm{H}$ & 2.53675600 & -4.50575800 & 0.30393200 & $\mathrm{C}$ & 0.11788700 & 2.41972500 & -2.13680900 \\
\hline $\mathrm{C}$ & 3.34934300 & -2.84000300 & 2.27255800 & $\mathrm{H}$ & 0.27252600 & 1.37424200 & -2.42165800 \\
\hline $\mathrm{H}$ & 3.54931500 & -1.96115700 & 2.88982100 & $\mathrm{H}$ & -0.12507800 & 2.97777200 & -3.04850000 \\
\hline $\mathrm{H}$ & 3.28845200 & -3.69715400 & 2.95231500 & $\mathrm{H}$ & 1.05671900 & 2.81171000 & -1.74755700 \\
\hline $\mathrm{H}$ & 4.20828000 & -3.00692700 & 1.61934100 & & & & \\
\hline $\mathrm{C}$ & 0.89312300 & -2.39158000 & 2.50653500 & \multirow{2}{*}{\multicolumn{4}{|c|}{$\begin{array}{l}\text { TS51a } \\
\text { imaginary frequency }=-626.85 \mathrm{~cm}^{-1}\end{array}$}} \\
\hline $\mathrm{H}$ & -0.08990000 & -2.38527700 & 2.02699200 & & & & \\
\hline $\mathrm{H}$ & 0.88052400 & -3.15003100 & 3.29756500 & $\mathrm{P}$ & -0.65665200 & 1.58454400 & -0.10420400 \\
\hline $\mathrm{H}$ & 1.04224500 & -1.42239600 & 2.99186900 & $\mathrm{C}$ & -0.60018600 & 2.50057700 & -1.79044200 \\
\hline $\mathrm{C}$ & 3.01735200 & -0.51013300 & -2.30227900 & $\mathrm{Pd}$ & -2.84392700 & 0.57595100 & 0.07598600 \\
\hline $\mathrm{H}$ & 3.50444200 & 0.36100000 & -1.86601600 & $\mathrm{H}$ & -3.18790300 & 1.87824400 & 0.89859800 \\
\hline $\mathrm{H}$ & 3.54008800 & -0.75485800 & -3.23388400 & $\mathrm{C}$ & 0.64933500 & 3.38904500 & -1.95745500 \\
\hline $\mathrm{H}$ & 1.99093300 & -0.23485000 & -2.56178400 & $\mathrm{H}$ & 1.58218600 & 2.83156600 & -1.83210900 \\
\hline $\mathrm{C}$ & 4.50947100 & -2.11062200 & -1.03334200 & $\mathrm{H}$ & 0.65372000 & 3.79655300 & -2.97460200 \\
\hline
\end{tabular}

C

H

$\mathrm{H}$

H

C

H

H

H

C
TS51a

imaginary frequency $=-626.85 \mathrm{~cm}^{-1}$

$\mathrm{P}$

C

$\mathrm{Pd}$

$\mathrm{H}$

C

H

H 


\begin{tabular}{|c|c|c|c|c|c|c|c|}
\hline $\mathrm{H}$ & 0.65799900 & 4.23814300 & -1.27127000 & $\mathrm{H}$ & 4.06696200 & 0.58007200 & 1.49851600 \\
\hline $\mathrm{C}$ & -1.87570200 & 3.35527800 & -1.95195800 & $\mathrm{P}$ & 5.04285400 & -1.25421100 & 0.26404500 \\
\hline $\mathrm{H}$ & -2.78029200 & 2.74102400 & -1.89671700 & $\mathrm{C}$ & 6.51027700 & -0.02202200 & 0.05426800 \\
\hline $\mathrm{H}$ & -1.95656700 & 4.14730700 & -1.20629700 & $\mathrm{C}$ & 4.47344600 & -1.92691600 & -1.45128800 \\
\hline $\mathrm{H}$ & -1.85817500 & 3.83257100 & -2.93793900 & $\mathrm{C}$ & 6.42051200 & 0.98496200 & -1.10538000 \\
\hline $\mathrm{C}$ & -0.59719000 & 1.43899700 & -2.91488600 & $\mathrm{H}$ & 6.46789300 & 0.49489900 & -2.08147700 \\
\hline $\mathrm{H}$ & -1.43891100 & 0.74429000 & -2.83546000 & $\mathrm{H}$ & 5.50375600 & 1.58362700 & -1.06629000 \\
\hline $\mathrm{H}$ & -0.69776400 & 1.95513000 & -3.87567400 & $\mathrm{H}$ & 7.26542100 & 1.68391600 & -1.05584800 \\
\hline $\mathrm{H}$ & 0.33058800 & 0.86342900 & -2.95546600 & $\mathrm{C}$ & 6.63514800 & 0.75765100 & 1.38442200 \\
\hline $\mathrm{C}$ & -2.54407000 & -1.07850300 & -1.02795200 & $\mathrm{H}$ & 6.58374500 & 0.09627500 & 2.25651300 \\
\hline $\mathrm{O}$ & -2.37745700 & -2.00228800 & -1.67056600 & $\mathrm{H}$ & 7.60728400 & 1.26333900 & 1.41481000 \\
\hline $\mathrm{C}$ & -4.74983700 & 1.24773400 & 1.09697800 & $\mathrm{H}$ & 5.87121800 & 1.53351800 & 1.49336600 \\
\hline $\mathrm{C}$ & -5.05109600 & 0.14203000 & 0.59203300 & $\mathrm{C}$ & 7.79531800 & -0.86990500 & -0.08997700 \\
\hline $\mathrm{C}$ & -0.20126600 & 2.71305200 & 1.37726800 & $\mathrm{H}$ & 8.67019600 & -0.20832900 & -0.09162000 \\
\hline $\mathrm{C}$ & -0.95699400 & 4.05323500 & 1.29390100 & $\mathrm{H}$ & 7.90646600 & -1.56891800 & 0.74471300 \\
\hline $\mathrm{H}$ & -2.03954800 & 3.91571100 & 1.20010300 & $\mathrm{H}$ & 7.82112700 & -1.44599700 & -1.01636400 \\
\hline $\mathrm{H}$ & -0.78160800 & 4.60791200 & 2.22204800 & $\mathrm{C}$ & 3.80575700 & -0.92089400 & -2.40687600 \\
\hline $\mathrm{H}$ & -0.61573900 & 4.68446600 & 0.47066700 & $\mathrm{H}$ & 3.52604300 & -1.43214600 & -3.33714200 \\
\hline $\mathrm{C}$ & 1.31964600 & 2.97172000 & 1.43698300 & $\mathrm{H}$ & 2.88605900 & -0.50381800 & -1.98549100 \\
\hline $\mathrm{H}$ & 1.52489300 & 3.63603200 & 2.28362700 & $\mathrm{H}$ & 4.46213300 & -0.09078700 & -2.67588300 \\
\hline $\mathrm{H}$ & 1.88047100 & 2.05023500 & 1.60375900 & $\mathrm{C}$ & 5.67339200 & -2.57817700 & -2.16808600 \\
\hline $\mathrm{H}$ & 1.70477400 & 3.45942600 & 0.53997200 & $\mathrm{H}$ & 6.37956000 & -1.83644100 & -2.54940500 \\
\hline $\mathrm{C}$ & -0.62951500 & 1.99845600 & 2.67873800 & $\mathrm{H}$ & 6.21685800 & -3.26683300 & -1.51338200 \\
\hline $\mathrm{H}$ & -0.35593600 & 2.63558300 & 3.52723900 & $\mathrm{H}$ & 5.31328800 & -3.15665500 & -3.02781200 \\
\hline $\mathrm{H}$ & -1.71123400 & 1.84051800 & 2.72091800 & $\mathrm{C}$ & 3.45659900 & -3.04578400 & -1.12255900 \\
\hline $\mathrm{H}$ & -0.13632900 & 1.03459800 & 2.81072500 & $\mathrm{H}$ & 2.56801000 & -2.66285000 & -0.61199700 \\
\hline $\mathrm{C}$ & 0.71703900 & 0.31421300 & -0.26554600 & $\mathrm{H}$ & 3.12653700 & -3.52588700 & -2.05273000 \\
\hline $\mathrm{H}$ & 0.40898300 & -0.31564000 & -1.10638900 & $\mathrm{H}$ & 3.90003800 & -3.81671800 & -0.48578400 \\
\hline $\mathrm{H}$ & 1.58795800 & 0.87342000 & -0.61013600 & $\mathrm{C}$ & -5.78552800 & -1.01713000 & 0.16393200 \\
\hline $\mathrm{C}$ & 1.07704900 & -0.56797600 & 0.92170800 & $\mathrm{C}$ & -6.68611000 & -0.90925100 & -0.91416800 \\
\hline $\mathrm{C}$ & 2.41434600 & -0.74721800 & 1.35200600 & $\mathrm{C}$ & -5.63606500 & -2.24595500 & 0.83613300 \\
\hline $\mathrm{C}$ & 0.05399800 & -1.28407800 & 1.56990700 & $\mathrm{C}$ & -7.42748600 & -2.02038600 & -1.30605400 \\
\hline $\mathrm{C}$ & 2.64539500 & -1.59950900 & 2.44992200 & $\mathrm{H}$ & -6.80276900 & 0.04049300 & -1.42597900 \\
\hline $\mathrm{C}$ & 0.30726400 & -2.13067600 & 2.64273200 & $\mathrm{C}$ & -6.38664000 & -3.34664800 & 0.43498400 \\
\hline $\mathrm{H}$ & -0.97226700 & -1.15492200 & 1.23654700 & $\mathrm{H}$ & -4.94591300 & -2.32401900 & 1.67026200 \\
\hline $\mathrm{C}$ & 1.61969600 & -2.27998300 & 3.09386200 & $\mathrm{C}$ & -7.27940000 & -3.23759600 & -0.63564500 \\
\hline $\mathrm{H}$ & 3.66882500 & -1.73846100 & 2.78370600 & $\mathrm{H}$ & -8.12432800 & -1.93502900 & -2.13365400 \\
\hline $\mathrm{H}$ & -0.50802400 & -2.66318300 & 3.12314200 & $\mathrm{H}$ & -6.27702300 & -4.29083300 & 0.95871300 \\
\hline $\mathrm{H}$ & 1.84338500 & -2.92901900 & 3.93490000 & $\mathrm{H}$ & -7.86063300 & -4.10032200 & -0.94545300 \\
\hline $\mathrm{C}$ & 3.62704100 & -0.06812900 & 0.73520800 & $\mathrm{H}$ & -5.02372400 & 2.09242300 & 1.70090400 \\
\hline $\mathrm{H}$ & 3.37055400 & 0.58038000 & -0.10409700 & & & & \\
\hline
\end{tabular}


67

C

C

C

C

C

C

H

$\mathrm{H}$

$\mathrm{H}$

$\mathrm{H}$

C

$\mathrm{H}$

C

$\mathrm{H}$

H

$\mathrm{H}$

P

P

C

C

C

C

Pd

$\mathrm{H}$

C

C

C

C

C

C

$\mathrm{H}$

C

$\mathrm{H}$

C

H

H

H

$\mathrm{H}$

C

H

$$
2.98447700
$$

2.46822300

3.32498600

4.65023000

5.13344300

4.28908400

2.35318900

5.32762100

6.16437400

4.64815900

2.91341100

3.80690300

1.08697000

1.06691600

0.91544000

2.39334000

1.74380300

$-0.52521000$

$-1.75914000$

$-0.77380500$

1.42536400

2.70292600

$-0.45647200$

$-0.96906200$

$-1.88253000$

$-2.23084100$

$-3.55240700$

$-4.69924700$

$-3.72427300$

$-5.96929500$

$-4.58175600$

$-4.99517400$

$-2.85516200$

$-6.12127100$

$-6.84119800$

$-5.11103500$

$-7.11156100$

$-2.50699200$

0.21560400

$-0.04633200$
3.24370300

0.15795600

2.02629000

0.63519100

0.90167200

0.66724000

1.05245100

0.22635200

2.25878900

$-0.27286900$

3.36646000

$-0.31144100$

4.12692300

0.17802200

0.20730300

2.33636400

0.30234300

$-0.60336700$

4.32454600

$-0.67359300$

$-0.43398900$

1.24985600

$-1.02093500$

1.48220900

2.04291500

1.26265800

1.38514500

2.13667400

3.05126300

1.64574300

$-0.27756300$

2.19937100

$-1.50041900$

0.23804800

1.59880800

0.37373300

1.82329100

1.84645700

$2.90148800 \quad-1.01815300$

$-3.00670700$

1.39388700

$-2.01518600 \quad-1.34473900$

$-0.60077500-0.45850200$

$-0.56945300-3.13062900$

$-0.94891200 \quad-2.67709800$

$-0.70148200 \quad-1.39872800$

$-0.94022800 \quad-0.81459000$

$-0.41721600 \quad-1.44819400$

$-1.70330900$

0.35834000

$-0.64710800 \quad-0.92292900$

$0.17212500-2.35228900$

$-1.94093700 \quad 0.87354500$

$-2.13262100 \quad 0.84755100$

$-1.40803700 \quad 0.23839200$

$-0.23686400 \quad-1.42323300$

$-2.54615200 \quad 1.76756200$

$-1.59011100 \quad 0.64427400$

$-1.57189300 \quad-3.31950900$

$-3.79217100$

0.84124800

1.54173900
$\mathrm{H}$

$\mathrm{H}$

C

$\mathrm{H}$

$\mathrm{H}$

H

C

$\mathrm{H}$

$\mathrm{H}$

$\mathrm{H}$

C

H

$\mathrm{H}$

$\mathrm{H}$

C

$\mathrm{H}$

H

H

C

H

H

H

C

H

H

H

C

H

H

H

C

H

H

H

C

$\mathrm{H}$

H

H

C

$\mathrm{H}$

H

\begin{tabular}{|c|c|c|}
\hline-0.66516100 & -3.14759400 & 0.73507700 \\
\hline 0.41248000 & -4.25484100 & -0.12649600 \\
\hline 2.62893300 & -3.95182200 & 1.56085700 \\
\hline 3.52665300 & -3.42975500 & 1.90627900 \\
\hline 2.38541000 & -4.70805400 & 2.31646400 \\
\hline 2.87202200 & -4.48558900 & 0.63969900 \\
\hline 1.03268400 & -2.46196700 & 2.78542200 \\
\hline 0.23222200 & -1.71865600 & 2.72848100 \\
\hline 0.66272900 & -3.29313500 & 3.39595700 \\
\hline 1.88000200 & -2.02657200 & 3.32138000 \\
\hline 2.66170200 & -0.80396000 & -2.30528100 \\
\hline 3.15006900 & 0.07895600 & -1.88959500 \\
\hline 3.18453300 & -1.06987000 & -3.23143100 \\
\hline 1.63427800 & -0.53531300 & -2.56947700 \\
\hline 4.17393000 & -2.40253400 & -1.09768200 \\
\hline 4.28457900 & -3.25242700 & -0.42308800 \\
\hline 4.62761100 & -2.68517200 & -2.05474800 \\
\hline 4.75779100 & -1.56957600 & -0.70320400 \\
\hline 1.97786900 & -3.18911900 & -2.03668700 \\
\hline 0.91238400 & -2.98779600 & -2.19250400 \\
\hline 2.42669800 & -3.34386800 & -3.02441600 \\
\hline 2.07778800 & -4.12900600 & -1.48919100 \\
\hline-1.67725200 & 0.53644300 & 2.69421000 \\
\hline-1.96502800 & -0.34468000 & 2.11694600 \\
\hline-0.67852900 & 0.37290800 & 3.11020000 \\
\hline-2.36671900 & 0.62676000 & 3.54109600 \\
\hline-1.41570300 & 3.02546000 & 2.75641700 \\
\hline-2.21325100 & 3.12065500 & 3.50184300 \\
\hline-0.48269900 & 2.88960700 & 3.30768000 \\
\hline-1.36643200 & 3.97393100 & 2.21734900 \\
\hline-3.20603900 & 1.99422300 & 1.34371000 \\
\hline-3.49297500 & 1.24218000 & 0.60829000 \\
\hline-3.88199600 & 1.89195500 & 2.19999500 \\
\hline-3.37540300 & 2.98710300 & 0.92123000 \\
\hline-0.67915400 & 4.34536000 & -0.48131600 \\
\hline-0.69739000 & 5.03428400 & -1.33311400 \\
\hline-1.52855800 & 4.60199900 & 0.15630000 \\
\hline 0.24012500 & 4.54531100 & 0.07300200 \\
\hline-2.12964000 & 2.73401200 & -1.73200100 \\
\hline-2.98243400 & 2.96823800 & -1.09611900 \\
\hline-2.15240300 & 3.43122300 & -2.57739400 \\
\hline
\end{tabular}


68

$\begin{array}{rrr}-2.25827100 & 1.72749000 & -2.13232000 \\ 0.33145500 & 2.64728200 & -2.06833800 \\ 0.25950500 & 1.63668200 & -2.48014800 \\ 0.19404800 & 3.35293700 & -2.89533100 \\ 1.33918900 & 2.78402700 & -1.67730200\end{array}$

$$
2.84280900
$$$$
2.40738500
$$$$
3.32020700
$$$$
4.62758100
$$$$
5.03153200
$$$$
4.12429200
$$$$
2.17086500
$$$$
5.36019000
$$$$
6.05044100
$$$$
4.41868300
$$$$
2.98953800
$$$$
3.91926600
$$$$
1.06410800
$$$$
1.13195100
$$$$
0.86266700
$$$$
2.48177400
$$$$
1.89246400
$$$$
-0.55536200
$$$$
-1.70733300
$$$$
-0.94703700
$$$$
1.82853700
$$$$
2.89212300
$$$$
-0.46017700
$$$$
-1.33022000
$$$$
-2.26572000
$$$$
-2.33548800
$$$$
-3.59220800
$$$$
-4.79637400
$$$$
-3.64767100
$$$$
-6.00085600
$$$$
-4.78338200
$$$$
-4.85467800
$$$$
-2.73692400
$$$$
-6.03737400
$$

$-0.06986100$

0.48440600

0.53449900

0.06198700

1.40635300

2.60925700

$-0.51033000$

3.66298800

4.34707100

$-0.58707700$

$-0.07057300$

0.61466400

0.17907400

2.72719000

4.61866900

$-0.11566600$

$-0.86510500$

$-1.00905800$

1.20102400

$-0.63344400 \quad 1.45213500$

2.24740200

1.18674900

$1.61569500 \quad 2.07731700$

3.25304700

1.55927900

$0.06853000 \quad 2.15055400$

0.31751000

$1.69111500 \quad 0.38494000$

1.81255800

1.94259700

$3.05007300 \quad-0.92892000$

$-2.78105500 \quad 1.63481900$

$-1.89722400 \quad-1.24706900$

$-0.66441700-0.46017700$

$-0.15091600-3.30093600$

$-0.33200500 \quad-2.78039800$

$-0.53171900-1.45696200$

$-0.81150100 \quad-0.73280600$

$-0.20475100 \quad-1.14837800$

$-1.69763300 \quad 0.35873800$

$-0.49181200 \quad-0.51016600$

$0.50252000-1.97146700$

$-1.98948300 \quad 0.99279200$

$-2.17156500$

0.71270500

0.56142100
$-1.36587500$
$\mathrm{H}$

$\mathrm{H}$

$\mathrm{H}$

$\mathrm{H}$

C

$\mathrm{H}$

$\mathrm{H}$

H

C

$\mathrm{H}$

$\mathrm{H}$

$\mathrm{H}$

C

$\mathrm{H}$

$\mathrm{H}$

$\mathrm{H}$

C

$\mathrm{H}$

H

$\mathrm{H}$

H

C

H

H

H

C

H

H

H

C

$\mathrm{H}$

H

H

C

$\mathrm{H}$

H

H

C

H

H

H

C

\begin{tabular}{|c|c|c|}
\hline-6.91403900 & -0.01207400 & -0.84951300 \\
\hline-4.87004700 & -2.68709000 & 1.82487900 \\
\hline-6.97695200 & -1.60858600 & 1.05792900 \\
\hline-3.16076000 & -0.34476000 & -3.40246400 \\
\hline 0.77265900 & -3.83205800 & 1.23906900 \\
\hline 0.77728000 & -4.63399500 & 1.98573200 \\
\hline-0.23420700 & -3.40521700 & 1.23237100 \\
\hline 0.96362600 & -4.29290300 & 0.26899900 \\
\hline 3.18476200 & -3.48351000 & 1.84925200 \\
\hline 3.98876300 & -2.78631700 & 2.10106500 \\
\hline 3.08844300 & -4.17690800 & 2.69264100 \\
\hline 3.49471200 & -4.07342200 & 0.98406300 \\
\hline 1.37943200 & -2.16412100 & 2.97865800 \\
\hline 0.46096600 & -1.58009700 & 2.87618900 \\
\hline 1.17065100 & -2.97684800 & 3.68305800 \\
\hline 2.14827900 & -1.53731400 & 3.43716100 \\
\hline 2.62138600 & -0.81392500 & -2.31728700 \\
\hline 2.94018300 & 0.17959800 & -1.99322000 \\
\hline 3.18175800 & -1.06330100 & -3.22578000 \\
\hline 1.56107800 & -0.76423900 & -2.58577100 \\
\hline 4.41366500 & -1.99150100 & -1.00973700 \\
\hline 4.68326800 & -2.71527200 & -0.23971800 \\
\hline 4.88562400 & -2.32050900 & -1.94244700 \\
\hline 4.85417500 & -1.02996500 & -0.75570800 \\
\hline 2.42162000 & -3.25878400 & -1.79605900 \\
\hline 1.35729200 & -3.28733600 & -2.02873500 \\
\hline 2.95322100 & -3.45006600 & -2.73501500 \\
\hline 2.65352800 & -4.08809500 & -1.12393200 \\
\hline-1.53355500 & 0.48721200 & 2.71103700 \\
\hline-1.85851400 & -0.37061700 & 2.11675200 \\
\hline-0.49887800 & 0.32476400 & 3.02624200 \\
\hline-2.14568400 & 0.51649100 & 3.61971500 \\
\hline-1.33977400 & 2.97553800 & 2.89154000 \\
\hline-2.08912300 & 3.00781800 & 3.69044800 \\
\hline-0.36852400 & 2.84613000 & 3.37386600 \\
\hline-1.35459400 & 3.95045800 & 2.39938400 \\
\hline-3.18840500 & 1.96839400 & 1.54971700 \\
\hline-3.50642300 & 1.26510400 & 0.78060400 \\
\hline-3.80164300 & 1.78192800 & 2.43865100 \\
\hline-3.41280800 & 2.98325400 & 1.21566500 \\
\hline-0.77330000 & 4.47781400 & -0.37129700 \\
\hline
\end{tabular}




\begin{tabular}{|c|c|c|c|c|c|c|c|}
\hline $\mathrm{H}$ & -0.91910700 & 5.18786700 & -1.19314000 & $\mathrm{C}$ & -2.38003900 & -0.77316200 & -1.53057300 \\
\hline $\mathrm{H}$ & -1.52449000 & 4.70980000 & 0.38786000 & $\mathrm{C}$ & -3.63816500 & -0.85284500 & -0.76320000 \\
\hline $\mathrm{H}$ & 0.21172700 & 4.67647000 & 0.05113300 & $\mathrm{C}$ & -4.78064400 & -0.14742000 & -1.19331400 \\
\hline $\mathrm{C}$ & -2.38043500 & 2.95796900 & -1.49441800 & $\mathrm{C}$ & -3.74302700 & -1.63476600 & 0.40103100 \\
\hline $\mathrm{H}$ & -3.13806000 & 3.27858800 & -0.78010100 & $\mathrm{C}$ & -5.98029300 & -0.23763600 & -0.49200100 \\
\hline $\mathrm{H}$ & -2.44107200 & 3.63667200 & -2.35334900 & $\mathrm{H}$ & -4.72725700 & 0.48387900 & -2.07442300 \\
\hline $\mathrm{H}$ & -2.62628100 & 1.95884100 & -1.84625200 & $\mathrm{C}$ & -4.94636100 & -1.72822600 & 1.09872400 \\
\hline C & 0.03554700 & 2.79828400 & -2.09591300 & $\mathrm{H}$ & -2.87924800 & -2.18514800 & 0.76151900 \\
\hline $\mathrm{H}$ & -0.14416900 & 1.82387600 & -2.55862100 & $\mathrm{C}$ & -6.07020800 & -1.03024100 & 0.65527900 \\
\hline $\mathrm{H}$ & -0.12673400 & 3.56258600 & -2.86450500 & $\mathrm{H}$ & -6.84728900 & 0.31482900 & -0.84126600 \\
\hline $\mathrm{H}$ & 1.08269700 & 2.84783900 & -1.79570000 & $\mathrm{H}$ & -5.00508200 & -2.34790700 & 1.98831900 \\
\hline C & -0.83373900 & -2.44463700 & -1.18252400 & $\mathrm{H}$ & -7.00692300 & -1.09943600 & 1.19933600 \\
\hline \multirow[t]{2}{*}{$\mathrm{O}$} & -1.13978700 & -3.45746100 & -1.61095300 & $\mathrm{H}$ & -3.14431000 & -0.29639000 & -3.45912700 \\
\hline & & & & $\mathrm{C}$ & 0.54409200 & -3.81788900 & 1.27632500 \\
\hline \multirow{2}{*}{\multicolumn{3}{|c|}{$\begin{array}{l}\text { TS52 } \\
\text { imaginary frequency }=-261.03 \mathrm{~cm}^{-1}\end{array}$}} & & $\mathrm{H}$ & 0.51389900 & -4.61173800 & 2.03106600 \\
\hline & & & & $\mathrm{H}$ & -0.44055300 & -3.34354300 & 1.26534200 \\
\hline C & 3.04787800 & 3.41745900 & -0.04514000 & $\mathrm{H}$ & 0.70724900 & -4.29312300 & 0.30859900 \\
\hline C & 2.52465300 & 2.23349700 & 0.50197800 & $\mathrm{C}$ & 2.96958500 & -3.57802000 & 1.88901300 \\
\hline C & 3.35017800 & 1.08718200 & 0.53758700 & $\mathrm{H}$ & 3.81002400 & -2.91825600 & 2.12309400 \\
\hline C & 4.66643100 & 1.19405400 & 0.05763900 & $\mathrm{H}$ & 2.84108400 & -4.24733500 & 2.74731300 \\
\hline $\mathrm{C}$ & 5.15829100 & 2.36742500 & -0.50734200 & $\mathrm{H}$ & 3.24350600 & -4.20128500 & 1.03534200 \\
\hline $\mathrm{C}$ & 4.33563000 & 3.48972800 & -0.56868800 & C & 1.22598600 & -2.15507200 & 2.99475600 \\
\hline $\mathrm{H}$ & 2.43944900 & 4.31610900 & -0.03387600 & $\mathrm{H}$ & 0.34223000 & -1.52218000 & 2.87680100 \\
\hline $\mathrm{H}$ & 5.33733900 & 0.34830700 & 0.16523800 & $\mathrm{H}$ & 0.96746500 & -2.94574200 & 3.70762500 \\
\hline $\mathrm{H}$ & 6.18125200 & 2.40931500 & -0.86761000 & $\mathrm{H}$ & 2.02539100 & -1.56555900 & 3.45059600 \\
\hline $\mathrm{H}$ & 4.70166900 & 4.42320000 & -0.98433300 & $\mathrm{C}$ & 2.56321100 & -0.93873000 & -2.30926700 \\
\hline C & 2.92493200 & -0.20319000 & 1.20748100 & $\mathrm{H}$ & 2.95450800 & 0.03318800 & -1.99961800 \\
\hline $\mathrm{H}$ & 3.81705400 & -0.76607900 & 1.49656600 & $\mathrm{H}$ & 3.09724400 & -1.23930100 & -3.21802800 \\
\hline $\mathrm{C}$ & 1.17844600 & 2.30136300 & 1.19228800 & $\mathrm{H}$ & 1.50661000 & -0.81737000 & -2.56903200 \\
\hline $\mathrm{H}$ & 1.20688700 & 1.70425700 & 2.10920000 & $\mathrm{C}$ & 4.27391200 & -2.21389000 & -0.98532800 \\
\hline $\mathrm{H}$ & 1.01902400 & 3.33166400 & 1.51474600 & $\mathrm{H}$ & 4.49636300 & -2.94711400 & -0.20953900 \\
\hline $\mathrm{H}$ & 2.39685900 & 0.02464100 & 2.13674800 & $\mathrm{H}$ & 4.72641700 & -2.57857600 & -1.91436100 \\
\hline$P$ & 1.79343800 & -1.42579200 & 0.32510000 & $\mathrm{H}$ & 4.77329700 & -1.27994200 & -0.73667500 \\
\hline$P$ & -0.43207300 & 1.75270300 & 0.37752900 & $\mathrm{C}$ & 2.19886300 & -3.35942900 & -1.76102000 \\
\hline C & -1.61977600 & 1.97895400 & 1.89123300 & $\mathrm{H}$ & 1.12882000 & -3.32434800 & -1.96608200 \\
\hline C & -0.75739800 & 3.04081100 & -1.01460000 & $\mathrm{H}$ & 2.70052500 & -3.58755000 & -2.70811200 \\
\hline C & 1.64875500 & -2.81472500 & 1.66277700 & $\mathrm{H}$ & 2.39543800 & -4.19560300 & -1.08615900 \\
\hline $\mathrm{C}$ & 2.76201900 & -2.02635300 & -1.22858000 & $\mathrm{C}$ & -1.46815200 & 0.70327600 & 2.74660100 \\
\hline $\mathrm{Pd}$ & -0.43157700 & -0.64192100 & -0.42206000 & $\mathrm{H}$ & -1.78140200 & -0.18965600 & 2.19741200 \\
\hline $\mathrm{H}$ & -1.31554500 & -0.50301700 & -3.35658300 & $\mathrm{H}$ & -0.44298900 & 0.55602400 & 3.09963100 \\
\hline C & -2.27109600 & -0.50347900 & -2.84207300 & $\mathrm{H}$ & -2.10393400 & 0.78970000 & 3.63516900 \\
\hline
\end{tabular}




\begin{tabular}{|c|c|c|c|c|c|c|c|}
\hline $\mathrm{C}$ & -1.28251600 & 3.20198200 & 2.77171000 & $\mathrm{Pd}$ & 0.53819700 & 0.11498700 & -0.76858500 \\
\hline $\mathrm{H}$ & -2.04243900 & 3.27758500 & 3.55788500 & $\mathrm{C}$ & 2.54748500 & -1.09772000 & -1.88292400 \\
\hline $\mathrm{H}$ & -0.31624200 & 3.11467300 & 3.27352600 & $\mathrm{H}$ & 1.96790800 & -0.84388100 & -2.76466200 \\
\hline $\mathrm{H}$ & -1.29995600 & 4.14319300 & 2.21729500 & $\mathrm{C}$ & 2.95449000 & -0.12498100 & -1.01164300 \\
\hline $\mathrm{C}$ & -3.09123900 & 2.09405400 & 1.44983200 & $\mathrm{C}$ & 2.22790300 & 1.16111600 & -1.18066700 \\
\hline $\mathrm{H}$ & -3.38012900 & 1.33267600 & 0.72432500 & $\mathrm{O}$ & 2.57882700 & 2.25679900 & -1.49971900 \\
\hline $\mathrm{H}$ & -3.72898600 & 1.96555300 & 2.33176200 & $\mathrm{C}$ & -0.17005400 & -3.15720200 & 0.74593500 \\
\hline $\mathrm{H}$ & -3.31519600 & 3.07911100 & 1.03565400 & $\mathrm{C}$ & 0.41335700 & -2.28039800 & 1.87883100 \\
\hline $\mathrm{C}$ & -0.58575800 & 4.49970700 & -0.54873900 & $\mathrm{H}$ & 0.90891600 & -2.92726900 & 2.61264100 \\
\hline $\mathrm{H}$ & -0.69475300 & 5.15985100 & -1.41689800 & $\mathrm{H}$ & 1.16044100 & -1.57312300 & 1.50402100 \\
\hline $\mathrm{H}$ & -1.35398000 & 4.79001900 & 0.17232600 & $\mathrm{H}$ & -0.35949200 & -1.71569000 & 2.40519700 \\
\hline $\mathrm{H}$ & 0.39134000 & 4.70734800 & -0.10953300 & $\mathrm{C}$ & 0.99353000 & -3.88717900 & 0.04874700 \\
\hline $\mathrm{C}$ & -2.17210500 & 2.89829900 & -1.61501800 & $\mathrm{H}$ & 1.58258900 & -4.41635200 & 0.80653700 \\
\hline $\mathrm{H}$ & -2.95451700 & 3.25452400 & -0.94550200 & $\mathrm{H}$ & 0.65318400 & -4.63347200 & -0.67282400 \\
\hline $\mathrm{H}$ & -2.21828000 & 3.51218800 & -2.52214500 & $\mathrm{H}$ & 1.66144700 & -3.19242700 & -0.46386100 \\
\hline $\mathrm{H}$ & -2.39716200 & 1.87103500 & -1.90028900 & $\mathrm{C}$ & -1.10216200 & -4.21510200 & 1.36961700 \\
\hline $\mathrm{C}$ & 0.25580700 & 2.71495000 & -2.13741100 & $\mathrm{H}$ & -1.58107100 & -4.85730300 & 0.63008000 \\
\hline $\mathrm{H}$ & 0.09785600 & 1.70673400 & -2.53290100 & $\mathrm{H}$ & -0.50436800 & -4.86166000 & 2.02280100 \\
\hline $\mathrm{H}$ & 0.10587900 & 3.42013200 & -2.96304100 & $\mathrm{H}$ & -1.87525300 & -3.76760800 & 1.99230600 \\
\hline $\mathrm{H}$ & 1.29481800 & 2.79693100 & -1.81656400 & $\mathrm{C}$ & -2.83150800 & -0.83194300 & 1.55120100 \\
\hline $\mathrm{C}$ & -1.10529700 & -2.27170600 & -1.16314500 & $\mathrm{C}$ & -2.72826400 & 0.57793100 & 1.63709600 \\
\hline \multirow[t]{2}{*}{$\mathrm{O}$} & -1.35135400 & -3.35644300 & -1.47853800 & $\mathrm{C}$ & -3.18063700 & -1.55295400 & 2.70534200 \\
\hline & & & & $\mathrm{C}$ & -2.97692900 & 1.18074100 & 2.88198900 \\
\hline 69 & & & & $\mathrm{C}$ & -3.37917000 & -0.93860400 & 3.93806100 \\
\hline $\mathrm{C}$ & -2.72270000 & -1.59004800 & 0.24503600 & $\mathrm{H}$ & -3.33876600 & -2.62320600 & 2.62646600 \\
\hline $\mathrm{H}$ & -3.24348900 & -1.03806300 & -0.54133800 & $\mathrm{C}$ & -3.27283300 & 0.44623900 & 4.02695600 \\
\hline $\mathrm{H}$ & -3.24536600 & -2.54619300 & 0.34109800 & $\mathrm{H}$ & -2.98767600 & 2.26094400 & 2.95475500 \\
\hline$P$ & -1.02007500 & -1.94130000 & -0.48260600 & $\mathrm{H}$ & -3.64640000 & -1.53294900 & 4.80607700 \\
\hline $\mathrm{C}$ & -1.50974000 & -2.85466800 & -2.11781300 & $\mathrm{H}$ & -3.45515500 & 0.95827800 & 4.96646200 \\
\hline $\mathrm{C}$ & -2.05945500 & -4.27898600 & -1.90499700 & $\mathrm{C}$ & -2.56265000 & 1.46502300 & 0.41254900 \\
\hline $\mathrm{H}$ & -2.41247500 & -4.66611700 & -2.86804200 & $\mathrm{H}$ & -3.11194300 & 2.39509700 & 0.58388500 \\
\hline $\mathrm{H}$ & -1.30159000 & -4.97432200 & -1.53904500 & $\mathrm{H}$ & -3.04340500 & 0.98692400 & -0.44247800 \\
\hline $\mathrm{H}$ & -2.91073400 & -4.30298800 & -1.21884900 & $\mathrm{P}$ & -0.87967600 & 1.99574900 & -0.27370500 \\
\hline $\mathrm{C}$ & -0.28370900 & -2.91244900 & -3.05155200 & $\mathrm{C}$ & -1.40256200 & 2.89890700 & -1.90033300 \\
\hline $\mathrm{H}$ & -0.56059900 & -3.43405300 & -3.97483100 & $\mathrm{C}$ & -0.15877000 & 3.23090100 & 1.02599800 \\
\hline $\mathrm{H}$ & 0.04695500 & -1.90762800 & -3.32934500 & $\mathrm{C}$ & -2.25686500 & 4.15611000 & -1.63788000 \\
\hline $\mathrm{H}$ & 0.56180700 & -3.44782200 & -2.61532200 & $\mathrm{H}$ & -1.68430700 & 4.95392700 & -1.16061900 \\
\hline $\mathrm{C}$ & -2.59951900 & -2.02770400 & -2.83588000 & $\mathrm{H}$ & -2.60649200 & 4.54508000 & -2.60102700 \\
\hline $\mathrm{H}$ & -2.33124200 & -0.97214700 & -2.92651200 & $\mathrm{H}$ & -3.14621300 & 3.95468700 & -1.03404000 \\
\hline $\mathrm{H}$ & -2.72475300 & -2.41982100 & -3.85120700 & $\mathrm{C}$ & -2.21650100 & 1.91268300 & -2.76502900 \\
\hline $\mathrm{H}$ & -3.57303500 & -2.09929200 & -2.34467600 & $\mathrm{H}$ & -1.65761400 & 0.99147900 & -2.95346200 \\
\hline
\end{tabular}




\begin{tabular}{|c|c|c|c|c|c|c|c|}
\hline $\mathrm{H}$ & -3.19005100 & 1.65841700 & -2.33828500 & $\mathrm{C}$ & 0.07811000 & -3.65298800 & 1.33282000 \\
\hline H & -2.40875800 & 2.38116000 & -3.73641600 & $\mathrm{H}$ & -0.10569000 & -4.42993100 & 2.08376200 \\
\hline C & -0.15936000 & 3.29254500 & -2.72458000 & $\mathrm{H}$ & -0.69392500 & -2.88650900 & 1.45193300 \\
\hline $\mathrm{H}$ & 0.41622200 & 2.41527600 & -3.02712800 & $\mathrm{H}$ & -0.04911300 & -4.10919100 & 0.34966200 \\
\hline $\mathrm{H}$ & -0.50007800 & 3.79621700 & -3.63627500 & $\mathrm{C}$ & 1.53137100 & -2.44506200 & 2.96305500 \\
\hline $\mathrm{H}$ & 0.51299200 & 3.97217500 & -2.20339200 & $\mathrm{H}$ & 0.89081100 & -1.56269500 & 3.04879800 \\
\hline $\mathrm{C}$ & -1.23528500 & 4.12242000 & 1.68176200 & $\mathrm{H}$ & 1.16276000 & -3.18422700 & 3.68266300 \\
\hline $\mathrm{H}$ & -0.72586300 & 4.82315100 & 2.35259200 & $\mathrm{H}$ & 2.54339800 & -2.17294300 & 3.27405400 \\
\hline $\mathrm{H}$ & -1.79460100 & 4.71860500 & 0.95941500 & $\mathrm{Pd}$ & -0.28860300 & -0.42937600 & -0.23686100 \\
\hline H & -1.94207200 & 3.56330700 & 2.28893600 & $\mathrm{C}$ & -3.36271700 & 0.73265000 & -2.64795100 \\
\hline $\mathrm{C}$ & 0.89308700 & 4.17852800 & 0.41389500 & $\mathrm{H}$ & -2.42598400 & 1.01892800 & -3.11365300 \\
\hline $\mathrm{H}$ & 0.43608700 & 4.93325800 & -0.23082900 & $\mathrm{C}$ & -3.40463900 & -0.05691500 & -1.56143100 \\
\hline $\mathrm{H}$ & 1.37840000 & 4.71591700 & 1.23666400 & $\mathrm{C}$ & -2.12138300 & -0.61677100 & -1.07094600 \\
\hline $\mathrm{H}$ & 1.66573200 & 3.66371600 & -0.15136400 & $\mathrm{O}$ & -1.82045800 & -1.80208600 & -1.08687600 \\
\hline $\mathrm{C}$ & 0.51443100 & 2.36268100 & 2.11287100 & $\mathrm{C}$ & 2.34778200 & -2.38016400 & -1.43866800 \\
\hline $\mathrm{H}$ & 1.36124800 & 1.79788500 & 1.71130800 & $\mathrm{C}$ & 2.30580700 & -1.20451500 & -2.44283000 \\
\hline $\mathrm{H}$ & 0.89710700 & 3.01772600 & 2.90423200 & $\mathrm{H}$ & 2.60588800 & -1.57302300 & -3.43070700 \\
\hline $\mathrm{H}$ & -0.18133900 & 1.65718800 & 2.57543100 & $\mathrm{H}$ & 1.29724800 & -0.78876500 & -2.53587000 \\
\hline $\mathrm{C}$ & 3.89721400 & -0.27767600 & 0.11931100 & $\mathrm{H}$ & 2.98413200 & -0.39415100 & -2.16834400 \\
\hline $\mathrm{C}$ & 4.23933000 & -1.54605500 & 0.62291400 & $\mathrm{C}$ & 1.33853000 & -3.44878800 & -1.90872000 \\
\hline $\mathrm{C}$ & 4.51792800 & 0.86087200 & 0.66434700 & $\mathrm{H}$ & 1.57481900 & -3.72065400 & -2.94378400 \\
\hline $\mathrm{C}$ & 5.18375500 & -1.67032000 & 1.63706000 & $\mathrm{H}$ & 1.39211000 & -4.36434300 & -1.31524300 \\
\hline $\mathrm{H}$ & 3.76881900 & -2.43842200 & 0.22455600 & $\mathrm{H}$ & 0.30731300 & -3.08410800 & -1.89184200 \\
\hline $\mathrm{C}$ & 5.46433700 & 0.73023800 & 1.67930400 & $\mathrm{C}$ & 3.76525200 & -2.98475400 & -1.43913800 \\
\hline H & 4.28305500 & 1.84573700 & 0.27576900 & $\mathrm{H}$ & 3.86594100 & -3.83729200 & -0.76670700 \\
\hline $\mathrm{C}$ & 5.79844000 & -0.53284600 & 2.16915800 & $\mathrm{H}$ & 3.99148500 & -3.34048800 & -2.45108200 \\
\hline $\mathrm{H}$ & 5.44128200 & -2.65505400 & 2.01435300 & $\mathrm{H}$ & 4.53000500 & -2.25022700 & -1.18246600 \\
\hline $\mathrm{H}$ & 5.94426200 & 1.61641300 & 2.08228000 & $\mathrm{C}$ & 3.76573900 & 0.48349700 & 0.27608700 \\
\hline $\mathrm{H}$ & 6.53509500 & -0.63323100 & 2.96012600 & $\mathrm{C}$ & 3.13103900 & 1.74488100 & 0.38159500 \\
\hline \multirow[t]{2}{*}{$\mathrm{H}$} & 2.94958000 & -2.10360700 & -1.84290400 & $\mathrm{C}$ & 4.98794100 & 0.39870300 & -0.41057900 \\
\hline & & & & $\mathrm{C}$ & 3.77316400 & 2.85626800 & -0.18993300 \\
\hline 69-O & & & & $\mathrm{C}$ & 5.58233700 & 1.50482600 & -1.01130300 \\
\hline $\mathrm{C}$ & 3.26304900 & -0.75963700 & 0.98012200 & $\mathrm{H}$ & 5.50325300 & -0.55605800 & -0.44662600 \\
\hline $\mathrm{H}$ & 2.95365900 & -0.50432400 & 1.99707800 & $\mathrm{C}$ & 4.96890400 & 2.74927100 & -0.89528900 \\
\hline $\mathrm{H}$ & 4.08731000 & -1.47176400 & 1.08431900 & $\mathrm{H}$ & 3.34973300 & 3.84325900 & -0.04424700 \\
\hline$P$ & 1.78928400 & -1.67149900 & 0.25883400 & $\mathrm{H}$ & 6.52823600 & 1.39834900 & -1.53282100 \\
\hline $\mathrm{C}$ & 1.49151100 & -3.07046700 & 1.55064600 & $\mathrm{H}$ & 5.42819500 & 3.63595900 & -1.32057400 \\
\hline $\mathrm{C}$ & 2.53462400 & -4.20260700 & 1.49671500 & $\mathrm{C}$ & 1.88820800 & 1.97752600 & 1.22621900 \\
\hline $\mathrm{H}$ & 2.35565600 & -4.88734700 & 2.33392500 & $\mathrm{H}$ & 1.91959000 & 2.99847200 & 1.61907600 \\
\hline $\mathrm{H}$ & 2.46562800 & -4.79165400 & 0.58006200 & $\mathrm{H}$ & 1.91466800 & 1.31847600 & 2.09637000 \\
\hline $\mathrm{H}$ & 3.55961600 & -3.83333100 & 1.59622100 & $P$ & 0.13703800 & 1.74551500 & 0.54609600 \\
\hline
\end{tabular}




\begin{tabular}{|c|c|c|c|c|c|c|c|}
\hline $\mathrm{C}$ & -0.93859700 & 2.05019500 & 2.11756400 & $\mathrm{C}$ & -3.63890400 & 1.04608600 & -0.62109900 \\
\hline $\mathrm{C}$ & -0.11944200 & 3.11996800 & -0.77226500 & $\mathrm{C}$ & -2.79664100 & 2.17407100 & -0.49935600 \\
\hline $\mathrm{C}$ & -0.86244200 & 3.50111600 & 2.63444600 & $\mathrm{C}$ & -3.01517500 & 3.26541400 & -1.35791000 \\
\hline $\mathrm{H}$ & -1.32922400 & 4.21175200 & 1.94946600 & $\mathrm{C}$ & -3.99965800 & 3.25388200 & -2.34187600 \\
\hline $\mathrm{H}$ & -1.40860700 & 3.56195800 & 3.58263700 & $\mathrm{C}$ & -4.83107900 & 2.14260200 & -2.45896800 \\
\hline $\mathrm{H}$ & 0.16150800 & 3.83030300 & 2.83364600 & $\mathrm{H}$ & -5.35468600 & 0.24040600 & -1.64197500 \\
\hline $\mathrm{C}$ & -0.44750000 & 1.09839700 & 3.23083700 & $\mathrm{H}$ & -2.41551100 & 4.16142000 & -1.23606900 \\
\hline $\mathrm{H}$ & -0.42852300 & 0.05843300 & 2.89210100 & $\mathrm{H}$ & -4.13442700 & 4.11762100 & -2.98516500 \\
\hline $\mathrm{H}$ & 0.53831400 & 1.36383700 & 3.62076000 & $\mathrm{H}$ & -5.62964800 & 2.12235100 & -3.19372200 \\
\hline $\mathrm{H}$ & -1.14760200 & 1.15558700 & 4.07139600 & $\mathrm{C}$ & -1.80798100 & 2.34128100 & 0.63707100 \\
\hline $\mathrm{C}$ & -2.41054300 & 1.68597100 & 1.82924600 & $\mathrm{H}$ & -1.78552800 & 3.39894400 & 0.90408300 \\
\hline $\mathrm{H}$ & -2.51529500 & 0.63639800 & 1.54253200 & $\mathrm{C}$ & -3.60040000 & -0.10966800 & 0.35580200 \\
\hline $\mathrm{H}$ & -2.99220900 & 1.83886500 & 2.74516800 & $\mathrm{H}$ & -3.51904600 & 0.28150800 & 1.37273400 \\
\hline $\mathrm{H}$ & -2.86197100 & 2.29975300 & 1.05025200 & $\mathrm{H}$ & -4.55490500 & -0.64173700 & 0.31478900 \\
\hline $\mathrm{C}$ & 0.51721200 & 4.47382000 & -0.39547300 & $\mathrm{H}$ & -2.17467300 & 1.82063500 & 1.52649800 \\
\hline $\mathrm{H}$ & 0.27980200 & 5.19335000 & -1.18733200 & $\mathrm{P}$ & 0.00970200 & 1.81860100 & 0.56380000 \\
\hline $\mathrm{H}$ & 0.13048800 & 4.88313800 & 0.53887100 & $\mathrm{P}$ & -2.24297700 & -1.41141900 & 0.24902500 \\
\hline $\mathrm{H}$ & 1.60187400 & 4.42538800 & -0.32815200 & $\mathrm{C}$ & -2.70718800 & -2.52286900 & 1.76294400 \\
\hline $\mathrm{C}$ & -1.62043800 & 3.36015400 & -1.02196500 & $\mathrm{C}$ & -2.56360000 & -2.32619200 & -1.41973300 \\
\hline $\mathrm{H}$ & -2.09172200 & 3.90329100 & -0.19945900 & $\mathrm{C}$ & 0.53324900 & 2.30967000 & 2.37797600 \\
\hline $\mathrm{H}$ & -1.72589100 & 3.98243400 & -1.91826100 & $\mathrm{C}$ & 0.81855400 & 2.96322500 & -0.75712500 \\
\hline $\mathrm{H}$ & -2.17573100 & 2.43844700 & -1.19493600 & $\mathrm{Pd}$ & 0.26966200 & -0.65169100 & 0.25699600 \\
\hline $\mathrm{C}$ & 0.53265000 & 2.59632300 & -2.07130400 & $\mathrm{H}$ & 1.46016100 & -0.80757100 & -2.15677100 \\
\hline $\mathrm{H}$ & 0.05599400 & 1.67345700 & -2.41484500 & $\mathrm{C}$ & 2.54389200 & -0.83607200 & -2.10534400 \\
\hline $\mathrm{H}$ & 0.41841900 & 3.35152900 & -2.85743700 & $\mathrm{C}$ & 3.19365200 & -0.66169900 & -0.93905600 \\
\hline $\mathrm{H}$ & 1.60087400 & 2.40057400 & -1.95033700 & $\mathrm{C}$ & 4.67575300 & -0.74453700 & -0.83044000 \\
\hline $\mathrm{C}$ & -4.64709700 & -0.44403100 & -0.84116400 & $\mathrm{C}$ & 5.35909900 & -1.74060100 & -1.55022300 \\
\hline $\mathrm{C}$ & -5.68263700 & 0.49895200 & -0.71196600 & $\mathrm{C}$ & 5.42010700 & 0.15800000 & -0.05302200 \\
\hline $\mathrm{C}$ & -4.82236000 & -1.72708400 & -0.29594900 & $\mathrm{C}$ & 6.74943500 & -1.82066700 & -1.50910800 \\
\hline $\mathrm{C}$ & -6.87499500 & 0.15630700 & -0.07875000 & $\mathrm{H}$ & 4.79525800 & -2.46745700 & -2.12773200 \\
\hline $\mathrm{H}$ & -5.54523300 & 1.50423300 & -1.09850800 & $\mathrm{C}$ & 6.81123800 & 0.07752600 & -0.01826700 \\
\hline $\mathrm{C}$ & -6.01764000 & -2.06320000 & 0.33864200 & $\mathrm{H}$ & 4.91619900 & 0.93027600 & 0.51562800 \\
\hline $\mathrm{H}$ & -4.03918500 & -2.47135800 & -0.39290600 & $\mathrm{C}$ & 7.48014300 & -0.90931800 & -0.74468400 \\
\hline $\mathrm{C}$ & -7.04632100 & -1.12588900 & 0.44806500 & $\mathrm{H}$ & 7.26002500 & -2.60016800 & -2.06626100 \\
\hline $\mathrm{H}$ & -7.66678300 & 0.89367900 & 0.01116200 & $\mathrm{H}$ & 7.37398000 & 0.78849300 & 0.57892400 \\
\hline $\mathrm{H}$ & -6.14655700 & -3.06255700 & 0.74271100 & $\mathrm{H}$ & 8.56338700 & -0.97234600 & -0.70945900 \\
\hline $\mathrm{H}$ & -7.97436400 & -1.39074000 & 0.94512200 & $\mathrm{H}$ & 3.08145800 & -0.99371400 & -3.03418400 \\
\hline \multirow[t]{2}{*}{$\mathrm{H}$} & -4.27737800 & 1.06988100 & -3.12355100 & $\mathrm{C}$ & 2.02479100 & 2.68375400 & 2.49346900 \\
\hline & & & & $\mathrm{H}$ & 2.26598800 & 2.77245400 & 3.55899000 \\
\hline 69-CO & & & & $\mathrm{H}$ & 2.68886200 & 1.92882600 & 2.07576600 \\
\hline $\mathrm{C}$ & -4.65494600 & 1.06825600 & -1.59162500 & $\mathrm{H}$ & 2.24070900 & 3.65350200 & 2.03869100 \\
\hline
\end{tabular}




\begin{tabular}{|c|c|c|c|c|c|c|c|}
\hline $\mathrm{C}$ & -0.28360800 & 3.49419800 & 2.94350500 & $\mathrm{H}$ & -0.82064400 & -1.37908600 & -2.36456900 \\
\hline $\mathrm{H}$ & -1.33853700 & 3.25732300 & 3.09529300 & $\mathrm{H}$ & -2.07443100 & -1.91245500 & -3.49206700 \\
\hline $\mathrm{H}$ & 0.12892400 & 3.74464200 & 3.92703500 & $\mathrm{H}$ & -2.31326500 & -0.44159500 & -2.54059700 \\
\hline $\mathrm{H}$ & -0.21326200 & 4.39489700 & 2.32832000 & $\mathrm{C}$ & 2.38984200 & -0.41913200 & 0.31169900 \\
\hline $\mathrm{C}$ & 0.26837100 & 1.06520600 & 3.25117900 & $\mathrm{O}$ & 2.87093000 & -0.24174700 & 1.39998800 \\
\hline $\mathrm{H}$ & 0.90821600 & 0.22854300 & 2.96098100 & $\mathrm{C}$ & 0.88975100 & -2.52718000 & 0.20114700 \\
\hline $\mathrm{H}$ & 0.48694700 & 1.30913500 & 4.29722400 & $\mathrm{O}$ & 1.34126600 & -3.57414900 & 0.23396600 \\
\hline $\mathrm{H}$ & -0.77790600 & 0.74712700 & 3.20710100 & & & & \\
\hline $\mathrm{C}$ & 2.35475600 & 2.82605600 & -0.80670400 & \multirow{2}{*}{\multicolumn{4}{|c|}{$\begin{array}{l}\text { TS53 } \\
\text { imaginary frequency }=-87.63 \mathrm{~cm}^{-1}\end{array}$}} \\
\hline $\mathrm{H}$ & 2.67473300 & 1.81384100 & -1.04857200 & & & & \\
\hline $\mathrm{H}$ & 2.72016200 & 3.47326900 & -1.61236100 & $\mathrm{C}$ & -3.04871200 & 0.62290200 & 0.24164100 \\
\hline $\mathrm{H}$ & 2.84774600 & 3.14417600 & 0.10984200 & $\mathrm{H}$ & -2.98376000 & 1.29048500 & -0.61963300 \\
\hline $\mathrm{C}$ & 0.27874300 & 2.50762400 & -2.13174500 & $\mathrm{H}$ & -4.10558500 & 0.36079500 & 0.34330700 \\
\hline $\mathrm{H}$ & -0.80134600 & 2.60979600 & -2.22886600 & $\mathrm{P}$ & -2.13939700 & -0.93527100 & -0.32735900 \\
\hline $\mathrm{H}$ & 0.74393600 & 3.12082400 & -2.91195900 & $\mathrm{C}$ & -2.98297800 & -1.28500800 & -2.03218600 \\
\hline $\mathrm{H}$ & 0.54344800 & 1.46512900 & -2.33259700 & $\mathrm{C}$ & -4.47970100 & -1.63712000 & -1.91853000 \\
\hline $\mathrm{C}$ & 0.48742500 & 4.44921200 & -0.50524400 & $\mathrm{H}$ & -4.90493000 & -1.68878300 & -2.92745500 \\
\hline $\mathrm{H}$ & 0.95283100 & 4.81997200 & 0.41174300 & $\mathrm{H}$ & -4.64574400 & -2.60990900 & -1.45171400 \\
\hline $\mathrm{H}$ & 0.88971100 & 5.04264100 & -1.33393600 & $\mathrm{H}$ & -5.05141100 & -0.88477600 & -1.36782800 \\
\hline $\mathrm{H}$ & -0.58204900 & 4.65794200 & -0.45232500 & $\mathrm{C}$ & -2.24338500 & -2.42230700 & -2.76917300 \\
\hline $\mathrm{C}$ & -2.85525800 & -1.61583400 & 3.00587800 & $\mathrm{H}$ & -2.74864700 & -2.60601900 & -3.72411700 \\
\hline $\mathrm{H}$ & -1.98702500 & -0.96640500 & 3.15120100 & $\mathrm{H}$ & -1.21099400 & -2.14225200 & -2.99699800 \\
\hline $\mathrm{H}$ & -3.75709400 & -0.99952400 & 2.97791000 & $\mathrm{H}$ & -2.23728700 & -3.36436600 & -2.22019800 \\
\hline $\mathrm{H}$ & -2.93341300 & -2.25288400 & 3.89368900 & $\mathrm{C}$ & -2.83827900 & -0.02305500 & -2.90824000 \\
\hline $\mathrm{C}$ & -4.02290200 & -3.30355300 & 1.57184900 & $\mathrm{H}$ & -1.79360000 & 0.28930400 & -2.98369600 \\
\hline $\mathrm{H}$ & -4.26627400 & -3.81468400 & 2.51054200 & $\mathrm{H}$ & -3.18502300 & -0.25743700 & -3.92077300 \\
\hline $\mathrm{H}$ & -4.86945300 & -2.65324000 & 1.33417000 & $\mathrm{H}$ & -3.43799200 & 0.81947400 & -2.55447800 \\
\hline $\mathrm{H}$ & -3.94797300 & -4.07207800 & 0.79956000 & $\mathrm{Pc}$ & 0.26887200 & -0.59884300 & -0.64249900 \\
\hline $\mathrm{C}$ & -1.56500500 & -3.51581300 & 2.05844500 & $\mathrm{C}$ & 0.76522800 & -2.68452000 & -0.70206200 \\
\hline $\mathrm{H}$ & -0.64660200 & -2.99551300 & 2.34534800 & $\mathrm{H}$ & 0.36873000 & -3.02456500 & -1.65454600 \\
\hline $\mathrm{H}$ & -1.85549800 & -4.14642000 & 2.90615400 & $\mathrm{C}$ & 2.06881500 & -2.08412300 & -0.68914900 \\
\hline $\mathrm{H}$ & -1.34449600 & -4.18246100 & 1.22312600 & $\mathrm{C}$ & 2.29793200 & -1.24519600 & -1.77069700 \\
\hline $\mathrm{C}$ & -4.05572000 & -2.50470400 & -1.76805800 & $\mathrm{O}$ & 2.77612200 & -0.86333800 & -2.76902300 \\
\hline $\mathrm{H}$ & -4.12454600 & -3.04546500 & -2.71898200 & $\mathrm{C}$ & -2.62032700 & -2.29308700 & 0.95931500 \\
\hline $\mathrm{H}$ & -4.60360400 & -3.08641900 & -1.02597200 & $\mathrm{C}$ & -1.67581400 & -2.09561500 & 2.16590300 \\
\hline $\mathrm{H}$ & -4.56354900 & -1.55224000 & -1.90884400 & $\mathrm{H}$ & -1.91579600 & -2.83877000 & 2.93527300 \\
\hline $\mathrm{C}$ & -1.90161200 & -3.71807100 & -1.43671000 & $\mathrm{H}$ & -0.62857100 & -2.22803600 & 1.88248200 \\
\hline $\mathrm{H}$ & -2.37404900 & -4.41827800 & -0.74425600 & $\mathrm{H}$ & -1.77886000 & -1.10526400 & 2.61520000 \\
\hline $\mathrm{H}$ & -2.00811300 & -4.13937800 & -2.44278300 & $\mathrm{C}$ & -2.42335300 & -3.71448800 & 0.39159900 \\
\hline $\mathrm{H}$ & -0.83289700 & -3.68678200 & -1.22037300 & $\mathrm{H}$ & -2.52279200 & -4.43081700 & 1.21518600 \\
\hline $\mathrm{C}$ & -1.90264900 & -1.45358300 & -2.51146800 & $\mathrm{H}$ & -3.18505200 & -3.97237400 & -0.34768300 \\
\hline
\end{tabular}




\begin{tabular}{|c|c|c|c|c|c|c|c|}
\hline $\mathrm{H}$ & -1.44350600 & -3.87184600 & -0.05815000 & $\mathrm{C}$ & 1.74337100 & 1.20354300 & 1.94719700 \\
\hline $\mathrm{C}$ & -4.08122200 & -2.20047900 & 1.44988700 & $\mathrm{H}$ & 1.73605400 & 0.15928800 & 1.62349800 \\
\hline $\mathrm{H}$ & -4.80868200 & -2.30014600 & 0.64383200 & $\mathrm{H}$ & 2.55055100 & 1.31450000 & 2.68091500 \\
\hline $\mathrm{H}$ & -4.25948500 & -3.02341700 & 2.15137200 & $\mathrm{H}$ & 0.80011800 & 1.41248400 & 2.45617100 \\
\hline $\mathrm{H}$ & -4.28899700 & -1.27796900 & 1.98735800 & $\mathrm{C}$ & 3.08527500 & -2.16784100 & 0.39470000 \\
\hline $\mathrm{C}$ & -2.59969900 & 1.36215500 & 1.49016400 & $\mathrm{C}$ & 2.72968900 & -2.56713500 & 1.69300400 \\
\hline $\mathrm{C}$ & -1.59821900 & 2.36286400 & 1.47016200 & $\mathrm{C}$ & 4.43640900 & -1.91586700 & 0.10273500 \\
\hline $\mathrm{C}$ & -3.30121000 & 1.14950600 & 2.68906000 & $\mathrm{C}$ & 3.70776700 & -2.71408900 & 2.67411000 \\
\hline $\mathrm{C}$ & -1.34332000 & 3.07925700 & 2.65162600 & $\mathrm{H}$ & 1.69258900 & -2.76458100 & 1.94254600 \\
\hline $\mathrm{C}$ & -3.01121800 & 1.84576800 & 3.85921200 & $\mathrm{C}$ & 5.40977800 & -2.05931200 & 1.08969600 \\
\hline $\mathrm{H}$ & -4.12573300 & 0.44610300 & 2.70129900 & $\mathrm{H}$ & 4.73463700 & -1.62821400 & -0.90164300 \\
\hline $\mathrm{C}$ & -2.01643400 & 2.81974500 & 3.84189400 & $\mathrm{C}$ & 5.04886900 & -2.45788600 & 2.37787500 \\
\hline $\mathrm{H}$ & -0.62166000 & 3.88894700 & 2.62977900 & $\mathrm{H}$ & 3.42101300 & -3.02818200 & 3.67286500 \\
\hline $\mathrm{H}$ & -3.57950500 & 1.64864200 & 4.76274200 & $\mathrm{H}$ & 6.45057800 & -1.86905100 & 0.84758300 \\
\hline $\mathrm{H}$ & -1.78899700 & 3.39722300 & 4.73238400 & $\mathrm{H}$ & 5.80760400 & -2.57350500 & 3.14534200 \\
\hline $\mathrm{C}$ & -0.90861100 & 2.81171500 & 0.19816400 & $\mathrm{H}$ & 0.53276200 & -3.32921200 & 0.13710400 \\
\hline $\mathrm{H}$ & -0.60197400 & 3.85562200 & 0.31169400 & & & & \\
\hline $\mathrm{H}$ & -1.62747400 & 2.79915700 & -0.62311700 & 70 & & & \\
\hline$P$ & 0.57522400 & 1.87753200 & -0.49408800 & $\mathrm{C}$ & -3.06980100 & 0.49304100 & 0.00461100 \\
\hline $\mathrm{C}$ & 0.88626900 & 2.88470700 & -2.12316100 & $\mathrm{H}$ & -2.97979400 & 1.01330200 & -0.95073300 \\
\hline $\mathrm{C}$ & 2.00382500 & 2.16960600 & 0.76806900 & $\mathrm{H}$ & -4.11387900 & 0.17531900 & 0.08048100 \\
\hline $\mathrm{C}$ & 1.50765300 & 4.27510700 & -1.88742100 & $\mathrm{P}$ & -2.05510800 & -1.08211900 & -0.24899200 \\
\hline $\mathrm{H}$ & 2.53728700 & 4.22093200 & -1.52844900 & $\mathrm{C}$ & -2.80229300 & -1.75921900 & -1.90058600 \\
\hline $\mathrm{H}$ & 1.53095700 & 4.81036200 & -2.84386800 & $\mathrm{C}$ & -4.27152700 & -2.20893600 & -1.77231500 \\
\hline $\mathrm{H}$ & 0.92685000 & 4.88746200 & -1.19159600 & $\mathrm{H}$ & -4.65159700 & -2.44754100 & -2.77237600 \\
\hline $\mathrm{C}$ & -0.45647300 & 3.09089500 & -2.85889500 & $\mathrm{H}$ & -4.38207100 & -3.11007600 & -1.16551900 \\
\hline $\mathrm{H}$ & -1.02115500 & 2.16296900 & -2.97346400 & $\mathrm{H}$ & -4.92017200 & -1.43015000 & -1.36138500 \\
\hline $\mathrm{H}$ & -1.09502600 & 3.83286600 & -2.37328000 & $\mathrm{C}$ & -1.95065200 & -2.93481900 & -2.42285600 \\
\hline $\mathrm{H}$ & -0.24365500 & 3.46497400 & -3.86633900 & $\mathrm{H}$ & -2.41229200 & -3.32296400 & -3.33783100 \\
\hline $\mathrm{C}$ & 1.80191400 & 2.06445600 & -3.04872400 & $\mathrm{H}$ & -0.94130900 & -2.60338900 & -2.67995000 \\
\hline $\mathrm{H}$ & 1.33709600 & 1.11909400 & -3.33651700 & $\mathrm{H}$ & -1.87585500 & -3.76440500 & -1.71876800 \\
\hline H & 1.98828000 & 2.63635300 & -3.96509000 & $\mathrm{C}$ & -2.72047200 & -0.64290900 & -2.96345800 \\
\hline $\mathrm{H}$ & 2.77130000 & 1.84040300 & -2.60139400 & $\mathrm{H}$ & -1.70713700 & -0.24043000 & -3.04309500 \\
\hline $\mathrm{C}$ & 2.10538000 & 3.60298700 & 1.32842100 & $\mathrm{H}$ & -2.98162200 & -1.06872300 & -3.93845700 \\
\hline $\mathrm{H}$ & 2.89648800 & 3.61835500 & 2.08707500 & $\mathrm{H}$ & -3.41639100 & 0.17943600 & -2.77928000 \\
\hline $\mathrm{H}$ & 2.36951600 & 4.34205400 & 0.57327500 & $\mathrm{Pd}$ & 0.28756500 & -0.66635200 & -0.50406100 \\
\hline $\mathrm{H}$ & 1.18973000 & 3.92941500 & 1.82163300 & $\mathrm{C}$ & 0.93554900 & -2.63897200 & -0.37289100 \\
\hline $\mathrm{C}$ & 3.35504300 & 1.80083900 & 0.12570900 & $\mathrm{H}$ & 0.61839200 & -3.20049700 & -1.24854600 \\
\hline $\mathrm{H}$ & 3.65244100 & 2.49794900 & -0.66089800 & $\mathrm{C}$ & 2.25936300 & -2.01046500 & -0.43785000 \\
\hline $\mathrm{H}$ & 4.13205000 & 1.83067700 & 0.89800700 & $\mathrm{C}$ & 2.68044800 & -1.67741500 & -1.68743200 \\
\hline $\mathrm{H}$ & 3.35515000 & 0.78963700 & -0.28760200 & $\mathrm{O}$ & 3.09235100 & -1.48572000 & -2.75445100 \\
\hline
\end{tabular}




\begin{tabular}{|c|c|c|c|c|c|c|c|}
\hline $\mathrm{C}$ & -2.51267300 & -2.23270500 & 1.23071200 & $\mathrm{C}$ & 1.77959600 & 3.87885200 & 0.95998800 \\
\hline $\mathrm{C}$ & -1.65355200 & -1.76187700 & 2.42552000 & $\mathrm{H}$ & 2.55051500 & 4.06515700 & 1.71691400 \\
\hline $\mathrm{H}$ & -1.87910800 & -2.38752100 & 3.29690100 & $\mathrm{H}$ & 2.01018500 & 4.51228200 & 0.10361900 \\
\hline $\mathrm{H}$ & -0.58535500 & -1.85226900 & 2.21188500 & $\mathrm{H}$ & 0.83278100 & 4.20870800 & 1.38775600 \\
\hline $\mathrm{H}$ & -1.85581700 & -0.72373000 & 2.69891800 & $\mathrm{C}$ & 3.18947600 & 1.96848000 & 0.15366000 \\
\hline $\mathrm{C}$ & -2.17971400 & -3.70727400 & 0.92266700 & $\mathrm{H}$ & 3.53312000 & 2.56109700 & -0.69712200 \\
\hline $\mathrm{H}$ & -2.29484200 & -4.28636300 & 1.84598000 & $\mathrm{H}$ & 3.89826300 & 2.13390100 & 0.97293500 \\
\hline $\mathrm{H}$ & -2.86108100 & -4.14089900 & 0.18715500 & $\mathrm{H}$ & 3.24805700 & 0.90967600 & -0.11095000 \\
\hline $\mathrm{H}$ & -1.15826100 & -3.85424300 & 0.57276900 & $\mathrm{C}$ & 1.46008100 & 1.58825200 & 1.92189800 \\
\hline $\mathrm{C}$ & -4.00234000 & -2.17574000 & 1.63408000 & $\mathrm{H}$ & 1.53764600 & 0.50835200 & 1.76833800 \\
\hline $\mathrm{H}$ & -4.67274900 & -2.50163700 & 0.83853000 & $\mathrm{H}$ & 2.18858800 & 1.86428800 & 2.69355500 \\
\hline $\mathrm{H}$ & -4.15135500 & -2.85435700 & 2.48169500 & $\mathrm{H}$ & 0.46400200 & 1.81252800 & 2.30941500 \\
\hline $\mathrm{H}$ & -4.31707300 & -1.18737100 & 1.96303300 & $\mathrm{C}$ & 3.22088000 & -1.87668100 & 0.70181700 \\
\hline $\mathrm{C}$ & -2.76857000 & 1.46491500 & 1.13324200 & $\mathrm{C}$ & 2.78150800 & -2.03920400 & 2.02395800 \\
\hline $\mathrm{C}$ & -1.85164600 & 2.53533200 & 0.99671300 & $\mathrm{C}$ & 4.58352900 & -1.63126100 & 0.46588200 \\
\hline $\mathrm{C}$ & -3.53972900 & 1.39835700 & 2.30582800 & $\mathrm{C}$ & 3.68655300 & -1.95875700 & 3.08208000 \\
\hline $\mathrm{C}$ & -1.74991500 & 3.46889200 & 2.04147600 & $\mathrm{H}$ & 1.73503500 & -2.22776800 & 2.23685900 \\
\hline $\mathrm{C}$ & -3.39860900 & 2.31183800 & 3.34706600 & $\mathrm{C}$ & 5.48217900 & -1.54334900 & 1.52716200 \\
\hline $\mathrm{H}$ & -4.30148200 & 0.63252300 & 2.39446800 & $\mathrm{H}$ & 4.95683800 & -1.52454600 & -0.54990000 \\
\hline $\mathrm{C}$ & -2.49090800 & 3.35909600 & 3.21463700 & $\mathrm{C}$ & 5.03777800 & -1.70735900 & 2.84038000 \\
\hline $\mathrm{H}$ & -1.09685900 & 4.32635800 & 1.91689400 & $\mathrm{H}$ & 3.33025800 & -2.09279600 & 4.09881600 \\
\hline $\mathrm{H}$ & -4.01572900 & 2.22102200 & 4.23536400 & $\mathrm{H}$ & 6.53233400 & -1.35852000 & 1.32399800 \\
\hline $\mathrm{H}$ & -2.38262500 & 4.10241800 & 3.99817000 & $\mathrm{H}$ & 5.73891900 & -1.64594600 & 3.66643400 \\
\hline $\mathrm{C}$ & -1.08315100 & 2.80709500 & -0.28048700 & $\mathrm{H}$ & 0.76214100 & -3.17748300 & 0.55333600 \\
\hline
\end{tabular}

\section{TS54}

$\begin{array}{lrrr}\text { imaginary frequency }=-403.12 \mathrm{~cm}^{-1} & \\ \mathrm{C} & -3.01868900 & 3.55349600 & -0.49761800 \\ \mathrm{C} & -2.62993500 & 2.24405500 & -0.82959300 \\ \mathrm{C} & -3.45745200 & 1.17299600 & -0.41041700 \\ \mathrm{C} & -4.64578000 & 1.48069200 & 0.27526900 \\ \mathrm{C} & -4.99748500 & 2.78215500 & 0.62029300 \\ \mathrm{C} & -4.16598700 & 3.83188800 & 0.23913000 \\ \mathrm{H} & -2.42819000 & 4.38424000 & -0.86828300 \\ \mathrm{H} & -5.33563800 & 0.67769200 & 0.51226000 \\ \mathrm{H} & -5.92459000 & 2.97188100 & 1.15194000 \\ \mathrm{H} & -4.42617600 & 4.85965500 & 0.47188500 \\ \mathrm{C} & -3.21777500 & -0.27683400 & -0.78925100 \\ \mathrm{H} & -4.18277300 & -0.79313200 & -0.79536700 \\ \mathrm{C} & -1.46776200 & 2.07220200 & -1.79016500 \\ \mathrm{H} & -1.72900900 & 1.29375400 & -2.50945500\end{array}$




\begin{tabular}{|c|c|c|c|c|c|c|c|}
\hline $\mathrm{H}$ & -1.36660900 & 2.99542100 & -2.36906400 & $\mathrm{H}$ & 1.75360600 & 2.79052600 & -4.61863300 \\
\hline $\mathrm{H}$ & -2.83840400 & -0.33064900 & -1.81331800 & $\mathrm{H}$ & 0.44356000 & 3.50676800 & -3.68529100 \\
\hline $\mathrm{P}$ & -2.01967900 & -1.38090100 & 0.18847000 & $\mathrm{H}$ & 2.10373200 & 3.53093700 & -3.06100900 \\
\hline$P$ & 0.27195300 & 1.57892300 & -1.23423000 & $\mathrm{C}$ & 2.52332800 & 0.85571900 & -2.80304000 \\
\hline $\mathrm{C}$ & 1.15426100 & 1.54792100 & -2.95613300 & $\mathrm{H}$ & 2.41193200 & -0.16908200 & -2.44226900 \\
\hline $\mathrm{C}$ & 0.92987000 & 3.04371400 & -0.17484500 & $\mathrm{H}$ & 3.01863500 & 0.82000000 & -3.78118300 \\
\hline $\mathrm{C}$ & -2.40839400 & -3.09303300 & -0.61604600 & $\mathrm{H}$ & 3.19126200 & 1.37242400 & -2.11408900 \\
\hline $\mathrm{C}$ & -2.61322700 & -1.33972200 & 2.02235200 & $\mathrm{C}$ & 0.56562700 & 4.45019000 & -0.68922700 \\
\hline $\mathrm{Pd}$ & 0.37937500 & -0.60700400 & 0.05785500 & $\mathrm{H}$ & 0.95177500 & 4.65915900 & -1.68658100 \\
\hline $\mathrm{H}$ & 1.66498200 & -2.98420200 & 0.51244000 & $\mathrm{H}$ & -0.50926700 & 4.62851200 & -0.69048200 \\
\hline $\mathrm{C}$ & 1.54578400 & -2.13202400 & 1.16777500 & $\mathrm{H}$ & 1.00819700 & 5.18817100 & -0.00979600 \\
\hline $\mathrm{C}$ & 2.46915100 & -1.06812100 & 1.12475000 & $\mathrm{C}$ & 2.46352300 & 2.94410700 & -0.05427500 \\
\hline $\mathrm{C}$ & -1.31937600 & -4.10884200 & -0.21270500 & $\mathrm{H}$ & 2.96882200 & 3.23484400 & -0.97897100 \\
\hline $\mathrm{H}$ & -1.55413500 & -5.07788600 & -0.66913600 & $\mathrm{H}$ & 2.79935500 & 3.63611600 & 0.72650600 \\
\hline $\mathrm{H}$ & -0.33454000 & -3.80188900 & -0.56700400 & $\mathrm{H}$ & 2.80302600 & 1.94605800 & 0.22916100 \\
\hline $\mathrm{H}$ & -1.26660400 & -4.26329100 & 0.86637700 & $\mathrm{C}$ & 0.30972400 & 2.86043200 & 1.22853400 \\
\hline $\mathrm{C}$ & -3.78982400 & -3.67250600 & -0.25459000 & $\mathrm{H}$ & 0.63697900 & 1.92881800 & 1.69738000 \\
\hline $\mathrm{H}$ & -4.61019800 & -2.97119000 & -0.43233900 & $\mathrm{H}$ & 0.64248400 & 3.68173500 & 1.87406900 \\
\hline $\mathrm{H}$ & -3.97642400 & -4.55164700 & -0.88278000 & $\mathrm{H}$ & -0.78301500 & 2.88158600 & 1.20493000 \\
\hline $\mathrm{H}$ & -3.83787900 & -4.00695000 & 0.78361300 & $\mathrm{C}$ & 3.53135600 & -1.06319300 & 0.08045200 \\
\hline $\mathrm{C}$ & -2.35258900 & -2.90881600 & -2.15003600 & $\mathrm{O}$ & 3.54800300 & -2.22039600 & -0.70304000 \\
\hline $\mathrm{H}$ & -1.46756800 & -2.34877800 & -2.46621700 & $\mathrm{O}$ & 4.36992400 & -0.21084700 & -0.11576200 \\
\hline $\mathrm{H}$ & -2.31052500 & -3.89517500 & -2.62577700 & $\mathrm{C}$ & 1.30056300 & -2.73692800 & -3.37733900 \\
\hline $\mathrm{H}$ & -3.23636000 & -2.39924400 & -2.54158400 & $\mathrm{H}$ & 0.27664900 & -2.75554900 & -3.74622900 \\
\hline $\mathrm{C}$ & -2.14381200 & 0.01933000 & 2.58873700 & $\mathrm{H}$ & 1.77438900 & -3.69950000 & -3.58492700 \\
\hline $\mathrm{H}$ & -2.65506300 & 0.85992000 & 2.11689700 & $\mathrm{H}$ & 1.85581100 & -1.92703600 & -3.85398600 \\
\hline $\mathrm{H}$ & -2.36462900 & 0.05542300 & 3.66219500 & $\mathrm{O}$ & 1.24226700 & -2.56486700 & -1.94077600 \\
\hline $\mathrm{H}$ & -1.06770000 & 0.16836800 & 2.46754500 & $\mathrm{H}$ & 2.15466900 & -2.53193800 & -1.54275700 \\
\hline $\mathrm{C}$ & -4.13512300 & -1.45714300 & 2.24196800 & $\mathrm{H}$ & 0.66651900 & -1.57233800 & -1.24728500 \\
\hline $\mathrm{H}$ & -4.54274600 & -2.41625200 & 1.92363500 & $\mathrm{C}$ & 4.71922600 & -2.35591400 & -1.54827500 \\
\hline $\mathrm{H}$ & -4.33228100 & -1.36413900 & 3.31672100 & $\mathrm{H}$ & 4.74884400 & -1.56800000 & -2.30302100 \\
\hline $\mathrm{H}$ & -4.69169600 & -0.66239200 & 1.74974300 & $\mathrm{H}$ & 5.62123600 & -2.30165900 & -0.93838700 \\
\hline $\mathrm{C}$ & -1.93225300 & -2.47574100 & 2.81214500 & $\mathrm{H}$ & 4.63112200 & -3.34003400 & -2.00864500 \\
\hline $\mathrm{H}$ & -0.85199700 & -2.50380200 & 2.66042400 & $\mathrm{H}$ & 1.06038800 & -2.37087300 & 2.10431100 \\
\hline $\mathrm{H}$ & -2.11391900 & -2.32074900 & 3.88180100 & C & 2.63384600 & -0.16667800 & 2.31756500 \\
\hline $\mathrm{H}$ & -2.33802000 & -3.45754200 & 2.55667300 & $\mathrm{C}$ & 1.63473300 & -0.14374800 & 3.31443000 \\
\hline $\mathrm{C}$ & 0.29174200 & 0.70326600 & -3.92259200 & $\mathrm{C}$ & 3.78010300 & 0.62198200 & 2.54334400 \\
\hline $\mathrm{H}$ & -0.05604700 & -0.22964000 & -3.46742500 & $\mathrm{C}$ & 1.76285600 & 0.63023400 & 4.46470000 \\
\hline $\mathrm{H}$ & -0.58174300 & 1.24959000 & -4.28643500 & $\mathrm{H}$ & 0.73923800 & -0.74155300 & 3.20392700 \\
\hline $\mathrm{H}$ & 0.89157100 & 0.44298900 & -4.80251500 & $\mathrm{C}$ & 3.90827800 & 1.39150600 & 3.70039400 \\
\hline $\mathrm{C}$ & 1.36923000 & 2.93072100 & -3.60114700 & $\mathrm{H}$ & 4.57880800 & 0.63305200 & 1.81711200 \\
\hline
\end{tabular}




$\begin{array}{llll}\mathrm{C} & 2.90395000 & 1.40777900 & 4.66659300 \\ \mathrm{H} & 0.96969800 & 0.61906500 & 5.20652000 \\ \mathrm{H} & 4.80988000 & 1.97984000 & 3.84307500 \\ \mathrm{H} & 3.00954300 & 2.00944300 & 5.56396100\end{array}$

\section{TS54a} imaginary frequency $=-194.11 \mathrm{~cm}^{-1}$

C

C

C

$\mathrm{C}$

C

C

$\mathrm{H}$

$\mathrm{H}$

H

$\mathrm{H}$

C

$\mathrm{H}$

C

$\mathrm{H}$

$\mathrm{H}$

$\mathrm{H}$$$
2.46703300
$$

$$
3.36494400 \quad 3.16439800
$$$$
2.88249700
$$$$
3.53985100
$$$$
2.07783500
$$$$
0.82681200
$$$$
0.74941000
$$$$
4.63165500
$$$$
5.06600600
$$$$
1.8281640
$$$$
4.42174200
$$$$
3.05529900
$$$$
2.91630800
$$$$
4.14045900
$$$$
5.17172300
$$$$
5.91394700
$$$$
-0.19050600
$$$$
1.71764100
$$$$
4.75596300
$$$$
3.27254700
$$$$
3.92624200
$$$$
-0.41571900
$$$$
-0.96577800
$$$$
4.21366700
$$$$
1.80158900
$$$$
2.38122700
$$$$
1.75814600
$$$$
1.95987400
$$$$
1.92542000
$$$$
3.41879100
$$$$
2.99798300
$$$$
1.92432100
$$$$
-0.13144700
$$$$
-1.68489400
$$$$
2.11211700
$$$$
-0.05491300
$$$$
2.63595600
$$$$
3.33378700
$$$$
-0.54430400
$$$$
2.09279700
$$$$
-2.87108400
$$$$
-2.54086000
$$

0.09070700

$$
-0.20634900
$$$$
-1.03850900 \quad-2.55891300
$$$$
-0.97186900
$$$$
-2.03349500
$$$$
-0.83142200 \quad-2.48428300
$$$$
-2.74284200
$$$$
-1.17370400 \quad-1.39630300
$$$$
-4.18803700
$$$$
-0.90904100-1.18481900
$$$$
-4.75825500
$$$$
0.29615100 \quad-1.62515800
$$$$
-5.01230500
$$$$
-1.87621700 \quad-0.58485500
$$$$
-6.12489100
$$$$
0.52349300 \quad-1.47431800
$$$$
-4.12936700
$$$$
1.05622300 \quad-2.07760700
$$$$
\begin{array}{lll}
-6.37791400 & -1.64343000 & -0.43400900
\end{array}
$$$$
-4.59524600
$$$$
-2.82867400
$$$$
-0.26817300
$$$$
-6.93704000
$$

$-0.44273500 \quad-0.87655800$

\begin{tabular}{|c|c|c|}
\hline-6.55482000 & 1.45886400 & -1.81881800 \\
\hline-7.00685500 & -2.40363400 & 0.01864700 \\
\hline-8.00065000 & -0.26166300 & -0.75780800 \\
\hline-2.51581100 & -0.33465400 & -3.31926100 \\
\hline 1.37317400 & -4.20799100 & 1.41113500 \\
\hline 1.27420100 & -4.75316600 & 2.35735700 \\
\hline 0.37009400 & -4.07089300 & 0.99756700 \\
\hline 1.94003900 & -4.84976600 & 0.73329200 \\
\hline 3.54790700 & -3.16490300 & 2.10247300 \\
\hline 4.06489900 & -2.27574100 & 2.47128600 \\
\hline 3.53429500 & -3.88927700 & 2.92538600 \\
\hline 4.14530400 & -3.60005000 & 1.29932700 \\
\hline 1.38060600 & -2.17389100 & 2.85843300 \\
\hline 0.32139600 & -2.01248500 & 2.64298200 \\
\hline 1.45609400 & -2.80703200 & 3.75037100 \\
\hline 1.82306000 & -1.20539300 & 3.10924400 \\
\hline 2.42509800 & -1.45886100 & -2.57677800 \\
\hline 3.18254700 & -0.68645300 & -2.45152100 \\
\hline 2.59366100 & -1.93960800 & -3.54746700 \\
\hline 1.44865400 & -0.96361000 & -2.61467600 \\
\hline 3.86620800 & -3.18157700 & -1.42814100 \\
\hline 3.89897900 & -4.05190200 & -0.76769800 \\
\hline 4.13702500 & -3.52969200 & -2.43196300 \\
\hline 4.64270500 & -2.48032500 & -1.10970300 \\
\hline 1.42564600 & -3.61223200 & -1.86418300 \\
\hline 0.40960500 & -3.20843100 & -1.86289900 \\
\hline 1.64453100 & -3.95795300 & -2.88112200 \\
\hline 1.44154300 & -4.48753400 & -1.21525200 \\
\hline-0.13836400 & 1.79954800 & 3.38731900 \\
\hline-0.10272600 & 0.73369900 & 3.13591600 \\
\hline 0.88042000 & 2.13665200 & 3.59511700 \\
\hline-0.70559200 & 1.90612700 & 4.31893600 \\
\hline-0.67030200 & 4.12927100 & 2.63276900 \\
\hline-0.98936200 & 4.28933900 & 3.66959200 \\
\hline 0.36412800 & 4.47698700 & 2.55649200 \\
\hline-1.29604500 & 4.76783900 & 2.00505400 \\
\hline-2.32807900 & 2.27416600 & 2.29501400 \\
\hline-2.49840800 & 1.21599600 & 2.08007200 \\
\hline-2.73709700 & 2.48358100 & 3.29028600 \\
\hline-2.90923800 & 2.85433600 & 1.57768500 \\
\hline-0.00745800 & 4.77024500 & -0.66381400 \\
\hline
\end{tabular}




\begin{tabular}{|c|c|c|c|c|c|c|c|}
\hline $\mathrm{H}$ & -0.29389800 & 5.34561000 & -1.55223100 & $\mathrm{C}$ & -0.34606500 & 2.78154700 & 1.73173400 \\
\hline $\mathrm{H}$ & -0.41797200 & 5.28863500 & 0.20319200 & $\mathrm{C}$ & -1.34024100 & -2.67976400 & -2.52697300 \\
\hline $\mathrm{H}$ & 1.08001400 & 4.80738400 & -0.59695700 & $\mathrm{C}$ & -0.80537500 & -3.18277700 & 0.55782100 \\
\hline $\mathrm{C}$ & -2.08172700 & 3.39204700 & -0.91907100 & $\mathrm{Pd}$ & 0.24530600 & 0.10384300 & -0.55691100 \\
\hline $\mathrm{H}$ & -2.53331100 & 3.98082100 & -0.11707400 & $\mathrm{H}$ & 4.38403000 & 2.28295400 & -0.17939700 \\
\hline $\mathrm{H}$ & -2.35084600 & 3.87798500 & -1.86447300 & $\mathrm{C}$ & 4.34703800 & 1.60244600 & 0.66245900 \\
\hline $\mathrm{H}$ & -2.53437000 & 2.39564200 & -0.91429100 & $\mathrm{C}$ & 3.66337300 & 0.43742100 & 0.60907300 \\
\hline $\mathrm{C}$ & 0.00362800 & 2.71675100 & -2.11629400 & $\mathrm{C}$ & 0.08408700 & -2.97187300 & -3.04663700 \\
\hline $\mathrm{H}$ & -0.41215000 & 1.71910400 & -2.28875200 & $\mathrm{H}$ & 0.03860600 & -3.23240300 & -4.11125300 \\
\hline $\mathrm{H}$ & -0.28655400 & 3.35420200 & -2.96027100 & $\mathrm{H}$ & 0.74422600 & -2.10975300 & -2.92117100 \\
\hline $\mathrm{H}$ & 1.09245100 & 2.63665600 & -2.11936000 & $\mathrm{H}$ & 0.55081400 & -3.80956200 & -2.52766300 \\
\hline $\mathrm{C}$ & -2.06716400 & -1.98623500 & -0.35676900 & $\mathrm{C}$ & -2.17023200 & -3.97579800 & -2.59179600 \\
\hline $\mathrm{O}$ & -1.75442400 & -3.11153900 & -0.22717700 & $\mathrm{H}$ & -3.17735200 & -3.85407400 & -2.18247200 \\
\hline $\mathrm{C}$ & -2.59614400 & -2.08858300 & 2.30014800 & $\mathrm{H}$ & -2.28368700 & -4.27921600 & -3.63987700 \\
\hline $\mathrm{H}$ & -3.64849100 & -2.36981200 & 2.29611900 & $\mathrm{H}$ & -1.68354000 & -4.80221900 & -2.06975500 \\
\hline $\mathrm{H}$ & -2.35585700 & -1.53219600 & 3.20632400 & $\mathrm{C}$ & -1.99489000 & -1.63863800 & -3.46455600 \\
\hline $\mathrm{H}$ & -1.95763900 & -2.96811800 & 2.20163500 & $\mathrm{H}$ & -1.54684200 & -0.64489600 & -3.35284600 \\
\hline $\mathrm{O}$ & -2.40327500 & -1.19299900 & 1.16985900 & $\mathrm{H}$ & -1.86529900 & -1.95655000 & -4.50568400 \\
\hline \multirow[t]{2}{*}{$\mathrm{H}$} & -1.51365400 & -0.68909900 & 1.21999000 & $\mathrm{H}$ & -3.06979800 & -1.54193400 & -3.29259900 \\
\hline & & & & $\mathrm{C}$ & -0.52890900 & -2.38991200 & 1.85682400 \\
\hline \multicolumn{4}{|c|}{ TS54b } & $\mathrm{H}$ & -1.38893300 & -1.79778300 & 2.17670600 \\
\hline \multicolumn{4}{|c|}{ imaginary frequency $=-81.61 \mathrm{~cm}^{-1}$} & $\mathrm{H}$ & -0.29334600 & -3.09736000 & 2.66111300 \\
\hline $\mathrm{C}$ & -3.91552000 & 1.24942900 & 2.20091600 & $\mathrm{H}$ & 0.33206000 & -1.72450700 & 1.74272000 \\
\hline $\mathrm{C}$ & -3.30871000 & 0.74030400 & 1.03820200 & $\mathrm{C}$ & -1.91306800 & -4.21784000 & 0.83064100 \\
\hline $\mathrm{C}$ & -3.43252500 & -0.64884400 & 0.77624900 & $\mathrm{H}$ & -2.15583800 & -4.82493800 & -0.04238900 \\
\hline $\mathrm{C}$ & -4.14543700 & -1.43683600 & 1.69786600 & $\mathrm{H}$ & -1.56695300 & -4.90194100 & 1.61505300 \\
\hline $\mathrm{C}$ & -4.69507700 & -0.91944700 & 2.86625300 & $\mathrm{H}$ & -2.83128300 & -3.75764800 & 1.19475600 \\
\hline $\mathrm{C}$ & -4.57989000 & 0.44465400 & 3.12059100 & $\mathrm{C}$ & 0.49137900 & -3.92766200 & 0.17734300 \\
\hline $\mathrm{H}$ & -3.90066100 & 2.32084900 & 2.36894500 & $\mathrm{H}$ & 1.29377400 & -3.23990000 & -0.10183300 \\
\hline $\mathrm{H}$ & -4.30805300 & -2.48478300 & 1.46936900 & $\mathrm{H}$ & 0.83200700 & -4.50115000 & 1.04753400 \\
\hline $\mathrm{H}$ & -5.23691100 & -1.56879700 & 3.54680600 & $\mathrm{H}$ & 0.33511100 & -4.64265000 & -0.63430700 \\
\hline $\mathrm{H}$ & -5.03082500 & 0.88632300 & 4.00377300 & $\mathrm{C}$ & -1.62564600 & 3.07873500 & -2.55357200 \\
\hline $\mathrm{C}$ & -2.99399300 & -1.31865200 & -0.51458100 & $\mathrm{H}$ & -1.24313400 & 2.11005300 & -2.89046000 \\
\hline $\mathrm{H}$ & -3.63956700 & -2.18623100 & -0.68514600 & $\mathrm{H}$ & -2.70388600 & 2.98560000 & -2.40258500 \\
\hline $\mathrm{C}$ & -2.72041200 & 1.74671100 & 0.06363100 & $\mathrm{H}$ & -1.47564600 & 3.79644200 & -3.36820700 \\
\hline $\mathrm{H}$ & -2.93175800 & 1.41946800 & -0.95727400 & $\mathrm{C}$ & -1.60725500 & 4.86849500 & -0.80616800 \\
\hline $\mathrm{H}$ & -3.24965200 & 2.69514600 & 0.19915100 & $\mathrm{H}$ & -1.71957300 & 5.55621000 & -1.65303300 \\
\hline $\mathrm{H}$ & -3.17166000 & -0.63378100 & -1.34782600 & $\mathrm{H}$ & -2.60938500 & 4.67771200 & -0.41251200 \\
\hline $\mathrm{P}$ & -1.19955600 & -1.86368700 & -0.78394100 & $\mathrm{H}$ & -1.03165500 & 5.39544200 & -0.04230500 \\
\hline $\mathrm{P}$ & -0.86052300 & 2.13862000 & -0.00939300 & $\mathrm{C}$ & 0.54913300 & 3.96299100 & -1.69478400 \\
\hline $\mathrm{C}$ & -0.89326600 & 3.59096400 & -1.29101900 & $\mathrm{H}$ & 1.10305500 & 3.10038800 & -2.06760800 \\
\hline
\end{tabular}




\begin{tabular}{|c|c|c|c|c|c|c|c|}
\hline $\mathrm{H}$ & 0.51201700 & 4.70990900 & -2.49691100 & 71 & & & \\
\hline $\mathrm{H}$ & 1.11457500 & 4.40129400 & -0.87165100 & $\mathrm{C}$ & -2.93413600 & 3.46994900 & -1.05824300 \\
\hline $\mathrm{C}$ & -1.30166900 & 3.78949700 & 2.39732400 & $\mathrm{C}$ & -2.55278100 & 2.12499400 & -1.20814300 \\
\hline $\mathrm{H}$ & -1.38260600 & 4.72678500 & 1.84693600 & $\mathrm{C}$ & -3.39986100 & 1.12433500 & -0.67010900 \\
\hline $\mathrm{H}$ & -2.30395000 & 3.38697300 & 2.53698800 & $\mathrm{C}$ & -4.58662900 & 1.52990300 & -0.03389700 \\
\hline $\mathrm{H}$ & -0.91384200 & 4.03194600 & 3.39420300 & $\mathrm{C}$ & -4.92807900 & 2.86818700 & 0.13457800 \\
\hline $\mathrm{C}$ & 1.05471200 & 3.42025900 & 1.63915200 & $\mathrm{C}$ & -4.08708500 & 3.85186000 & -0.37949000 \\
\hline $\mathrm{H}$ & 1.02784800 & 4.41298400 & 1.18268100 & $\mathrm{H}$ & -2.33065600 & 4.24069300 & -1.52496900 \\
\hline $\mathrm{H}$ & 1.45331000 & 3.54674400 & 2.65308300 & $\mathrm{H}$ & -5.28164400 & 0.77223900 & 0.31190100 \\
\hline $\mathrm{H}$ & 1.75707900 & 2.79555800 & 1.07913200 & $\mathrm{H}$ & -5.85461700 & 3.13424200 & 0.63350800 \\
\hline $\mathrm{C}$ & -0.24253500 & 1.52532200 & 2.62613700 & $\mathrm{H}$ & -4.34131400 & 4.90321000 & -0.28900100 \\
\hline $\mathrm{H}$ & 0.49636500 & 0.81658500 & 2.24026900 & $\mathrm{C}$ & -3.17816400 & -0.36358500 & -0.87064000 \\
\hline $\mathrm{H}$ & 0.07941400 & 1.82838200 & 3.62994100 & $\mathrm{H}$ & -4.15298500 & -0.86216700 & -0.86828000 \\
\hline $\mathrm{H}$ & -1.19734400 & 1.00549800 & 2.72783600 & $\mathrm{C}$ & -1.36047600 & 1.82087400 & -2.09674500 \\
\hline $\mathrm{C}$ & 3.01863500 & 0.01947900 & -0.65098500 & $\mathrm{H}$ & -1.58389700 & 0.93855500 & -2.69974900 \\
\hline $\mathrm{O}$ & 2.34553700 & -0.99029200 & -0.84979400 & $\mathrm{H}$ & -1.23963800 & 2.64700500 & -2.80459200 \\
\hline $\mathrm{C}$ & 4.61962800 & 0.34808300 & -2.44014400 & $\mathrm{H}$ & -2.75128100 & -0.54185400 & -1.86011300 \\
\hline $\mathrm{H}$ & 5.44232100 & 0.42138000 & -1.72680000 & $\mathrm{P}$ & -2.05098200 & -1.36442100 & 0.27654700 \\
\hline $\mathrm{H}$ & 4.79822300 & 1.02109400 & -3.27927800 & $\mathrm{P}$ & 0.36226400 & 1.44823800 & -1.40757400 \\
\hline $\mathrm{H}$ & 4.51074100 & -0.68269500 & -2.78917600 & $\mathrm{C}$ & 1.32748700 & 1.13466700 & -3.04863300 \\
\hline $\mathrm{O}$ & 3.38970000 & 0.79956400 & -1.80408700 & $\mathrm{C}$ & 0.96811200 & 3.04244900 & -0.52279800 \\
\hline $\mathrm{H}$ & 2.20733300 & 0.87993900 & -2.68842000 & $\mathrm{C}$ & -2.27221700 & -3.13904700 & -0.45527100 \\
\hline $\mathrm{C}$ & 1.18872600 & 0.32804900 & -4.46087400 & $\mathrm{C}$ & -2.81660000 & -1.23656700 & 2.04126200 \\
\hline $\mathrm{H}$ & 1.83586500 & 0.90467300 & -5.12164500 & $\mathrm{Pd}$ & 0.31988200 & -0.45729100 & 0.21332400 \\
\hline $\mathrm{H}$ & 0.15464100 & 0.40855400 & -4.79019800 & $\mathrm{H}$ & 1.63730100 & -2.82932700 & 0.77857400 \\
\hline $\mathrm{H}$ & 1.49714700 & -0.71632200 & -4.41569200 & $\mathrm{C}$ & 1.51884100 & -1.99180200 & 1.45094100 \\
\hline $\mathrm{O}$ & 1.27148100 & 0.95050000 & -3.14412900 & $\mathrm{C}$ & 2.41731200 & -0.92963000 & 1.41485900 \\
\hline $\mathrm{H}$ & 0.60568100 & 0.59600300 & -2.36962200 & $\mathrm{C}$ & -1.16065700 & -4.05837200 & 0.09303800 \\
\hline $\mathrm{C}$ & 3.56109500 & -0.46893800 & 1.78740100 & $\mathrm{H}$ & -1.34702200 & -5.07985500 & -0.25918800 \\
\hline $\mathrm{C}$ & 3.72081000 & -1.85964100 & 1.66513300 & $\mathrm{H}$ & -0.18267600 & -3.75373100 & -0.28284500 \\
\hline $\mathrm{C}$ & 3.36723100 & 0.07571100 & 3.06800600 & $\mathrm{H}$ & -1.13154900 & -4.09251800 & 1.18381400 \\
\hline $\mathrm{C}$ & 3.69789300 & -2.67673900 & 2.79435000 & $\mathrm{C}$ & -3.64815900 & -3.76833500 & -0.16134000 \\
\hline $\mathrm{H}$ & 3.87776000 & -2.30314500 & 0.68828500 & $\mathrm{H}$ & -4.48517500 & -3.12283300 & -0.44233900 \\
\hline $\mathrm{C}$ & 3.33858200 & -0.74426300 & 4.19530400 & $\mathrm{H}$ & -3.74139400 & -4.69002600 & -0.74718800 \\
\hline $\mathrm{H}$ & 3.22519100 & 1.14653800 & 3.17714800 & $\mathrm{H}$ & -3.76063600 & -4.04568600 & 0.88877800 \\
\hline $\mathrm{C}$ & 3.50555200 & -2.12365700 & 4.06210100 & $\mathrm{C}$ & -2.09196500 & -3.05459700 & -1.98803800 \\
\hline $\mathrm{H}$ & 3.83791500 & -3.74786200 & 2.68367200 & $\mathrm{H}$ & -1.17438900 & -2.52858700 & -2.26580500 \\
\hline $\mathrm{H}$ & 3.18374900 & -0.30525600 & 5.17625700 & $\mathrm{H}$ & -2.01177100 & -4.07426600 & -2.38144400 \\
\hline $\mathrm{H}$ & 3.48609600 & -2.76300400 & 4.93941000 & $\mathrm{H}$ & -2.94235800 & -2.58491100 & -2.48874400 \\
\hline \multirow[t]{2}{*}{$\mathrm{H}$} & 4.87647700 & 1.89109900 & 1.56327600 & $\mathrm{C}$ & -2.48180200 & 0.18269200 & 2.55705200 \\
\hline & & & & $\mathrm{H}$ & -2.99664600 & 0.96166700 & 1.99265400 \\
\hline
\end{tabular}




$\begin{array}{lrrrrrrr}\mathrm{H} & -2.80014900 & 0.26426900 & 3.60327400 & \mathrm{O} & 1.43423900 & -2.88542200 & -1.91472800 \\ \mathrm{H} & -1.40951500 & 0.39651900 & 2.52517500 & \mathrm{H} & 2.24924900 & -2.75967900 & -1.40165400 \\ \mathrm{C} & -4.34423500 & -1.43343000 & 2.11576000 & \mathrm{H} & 0.49741200 & -1.29742800 & -1.00765400 \\ \mathrm{H} & -4.64941500 & -2.45780900 & 1.90637500 & \mathrm{C} & 4.86578500 & -2.29215300 & -1.02432000 \\ \mathrm{H} & -4.67282800 & -1.19895100 & 3.13520100 & \mathrm{H} & 4.85371100 & -1.58784400 & -1.85810500 \\ \mathrm{H} & -4.88932700 & -0.77061100 & 1.44502300 & \mathrm{H} & 5.74940900 & -2.10749000 & -0.41188900 \\ \mathrm{C} & -2.15254500 & -2.27191200 & 2.97085500 & \mathrm{H} & 4.85865800 & -3.32154900 & -1.38173600 \\ \mathrm{H} & -1.06186100 & -2.20850100 & 2.95741700 & \mathrm{H} & 0.93833900 & -2.19122600 & 2.34136200 \\ \mathrm{H} & -2.48040700 & -2.08463700 & 3.99984700 & \mathrm{C} & 2.44375700 & 0.08613600 & 2.51355200 \\ \mathrm{H} & -2.43791600 & -3.29676500 & 2.72260200 & \mathrm{C} & 1.32939300 & 0.20426700 & 3.37347300 \\ \mathrm{C} & 0.51886500 & 0.14134800 & -3.91409300 & \mathrm{C} & 3.55475600 & 0.91269800 & 2.77924100 \\ \mathrm{H} & 0.21637200 & -0.74629300 & -3.35029100 & \mathrm{C} & 1.31339500 & 1.11207000 & 4.42972200 \\ \mathrm{H} & -0.36609400 & 0.59518800 & -4.36655600 & \mathrm{H} & 0.46221200 & -0.42980000 & 3.23324200 \\ \mathrm{H} & 1.15738000 & -0.19890100 & -4.73718900 & \mathrm{C} & 3.53828300 & 1.81279500 & 3.84400500 \\ \mathrm{C} & 1.57643100 & 2.41106700 & -3.87631600 & \mathrm{H} & 4.43349600 & 0.85094700 & 2.15443800 \\ \mathrm{H} & 2.00509000 & 2.12348200 & -4.84348000 & \mathrm{C} & 2.42082900 & 1.92582200 & 4.67160700 \\ \mathrm{H} & 0.65923600 & 2.96961800 & -4.08499600 & \mathrm{H} & 0.43736000 & 1.17479100 & 5.06829400 \\ \mathrm{H} & 2.29106100 & 3.08261800 & -3.39628700 & \mathrm{H} & 4.41407700 & 2.42800800 & 4.02722400 \\ \mathrm{C} & 2.67661300 & 0.46557600 & -2.71919000 & \mathrm{H} & 2.41596400 & 2.63002500 & 5.49765900\end{array}$

$\mathrm{H}$

$\mathrm{H}$

H

C

H

$\mathrm{H}$

H

C

H

H

H

C

H

$\mathrm{H}$

H

C

O

O

C

H

H

H
2.51645000

3.24308200

3.29335600

0.64988000

1.14367100

$-0.41895900$

1.01065300

2.48950000

3.05573400

2.78259400

2.79969200

0.25638100

$-0.52639300$

$0.34170900-3.65032400$

$1.04380900-2.03130400$

$4.35775600 \quad-1.26030300$

$4.43373500 \quad-2.22884100$

$4.51064000 \quad-1.40578400$

$5.19102800-0.64598500$

$2.96132400-0.28984100$

$3.08819000-1.21617700$

3.77685600

0.38141700

2.02506600

0.17874600

3.05409000

0.84980500

0.56168100

2.20754700

1.47241000

0.54045300

3.96479600

1.39007400

$-0.83246600$

3.05016500

0.75718700

3.54277900

$-0.95428400$

0.42152100

3.66910400

$-2.15781600$

$-0.21904800$

4.32395200

$-0.05022800$

0.20661200

1.73847700

$-3.54506700 \quad-3.14507000$

$0.79553400 \quad-3.69228400 \quad-3.67512200$

$2.19332200 \quad-4.52800000 \quad-2.97134200$

$2.40492600 \quad-2.94729600 \quad-3.77936900$
TS55

imaginary frequency $=-386.71 \mathrm{~cm}^{-1}$

$\begin{array}{lrrr}\mathrm{C} & -4.53433100 & 0.88718700 & -0.86755600 \\ \mathrm{C} & -3.37663900 & 0.90409600 & -0.07082400 \\ \mathrm{C} & -2.67968300 & 2.12437000 & 0.07956500 \\ \mathrm{C} & -3.18766800 & 3.26739500 & -0.56101900 \\ \mathrm{C} & -4.31845100 & 3.22530800 & -1.37198000 \\ \mathrm{C} & -4.99746000 & 2.01942100 & -1.53154400 \\ \mathrm{H} & -5.09964500 & -0.03692700 & -0.95016200 \\ \mathrm{H} & -2.70690700 & 4.22482000 & -0.38843100 \\ \mathrm{H} & -4.67882800 & 4.13157300 & -1.84828500 \\ \mathrm{H} & -5.89549000 & 1.96485500 & -2.13875600 \\ \mathrm{C} & -1.50688700 & 2.28977000 & 1.02554900 \\ \mathrm{H} & -1.42900700 & 3.34283400 & 1.31276100 \\ \mathrm{C} & -3.04956800 & -0.35145000 & 0.71158400 \\ \mathrm{H} & -2.76928000 & -0.08520400 & 1.73635400 \\ \mathrm{H} & -3.96773100 & -0.93580300 & 0.79865700 \\ \mathrm{H} & -1.70642000 & 1.73771600 & 1.94760200 \\ \mathrm{P} & 0.22773100 & 1.73555900 & 0.53891400 \\ \mathrm{P} & -1.69470800 & -1.56031400 & 0.19293900 \\ \mathrm{C} & -1.80390900 & -2.78654900 & 1.68700500\end{array}$




\begin{tabular}{|c|c|c|c|}
\hline $\mathrm{C}$ & -2.29371400 & -2.34996400 & -1.45491600 \\
\hline $\mathrm{C}$ & 1.22447900 & 2.19592700 & 2.12869000 \\
\hline $\mathrm{C}$ & 0.75171000 & 2.79909500 & -0.97122900 \\
\hline $\mathrm{Pd}$ & 0.54848100 & -0.57612400 & 0.01794500 \\
\hline $\mathrm{H}$ & 2.02489700 & -0.02425200 & -0.14513800 \\
\hline $\mathrm{C}$ & 5.85096400 & -0.48535700 & -2.07830300 \\
\hline $\mathrm{C}$ & 4.50729600 & -0.81256100 & -1.92167300 \\
\hline $\mathrm{C}$ & 4.04686000 & -1.30608900 & -0.68545300 \\
\hline $\mathrm{C}$ & 4.94552400 & -1.45756500 & 0.38816400 \\
\hline $\mathrm{C}$ & 6.28744300 & -1.12737800 & 0.21834600 \\
\hline $\mathrm{C}$ & 6.74056500 & -0.64093300 & -1.01095200 \\
\hline $\mathrm{H}$ & 6.20665000 & -0.11317900 & -3.03379200 \\
\hline $\mathrm{H}$ & 3.81168600 & -0.70175000 & -2.74734200 \\
\hline $\mathrm{H}$ & 4.58658200 & -1.84157800 & 1.33753400 \\
\hline $\mathrm{H}$ & 6.98089300 & -1.25215000 & 1.04389500 \\
\hline $\mathrm{H}$ & 7.78781300 & -0.38489200 & -1.13835300 \\
\hline $\mathrm{C}$ & 2.67417800 & -1.70870800 & -0.52963500 \\
\hline $\mathrm{C}$ & 1.61356500 & -2.37888800 & -0.44791700 \\
\hline $\mathrm{H}$ & 1.24528400 & -3.38080100 & -0.56111300 \\
\hline $\mathrm{C}$ & -1.15716200 & -4.14610900 & 1.36017200 \\
\hline $\mathrm{H}$ & -1.74859700 & -4.72975500 & 0.65151300 \\
\hline $\mathrm{H}$ & -0.14098600 & -4.04293600 & 0.97426000 \\
\hline $\mathrm{H}$ & -1.09222200 & -4.73306700 & 2.28336000 \\
\hline $\mathrm{C}$ & -0.99403300 & -2.13506400 & 2.82952400 \\
\hline $\mathrm{H}$ & -1.02832900 & -2.78440700 & 3.71186700 \\
\hline $\mathrm{H}$ & 0.05476800 & -1.99557500 & 2.55088000 \\
\hline $\mathrm{H}$ & -1.40082900 & -1.16422800 & 3.12861900 \\
\hline $\mathrm{C}$ & -3.24110300 & -3.04548800 & 2.18937200 \\
\hline $\mathrm{H}$ & -3.19778700 & -3.78743200 & 2.99479100 \\
\hline $\mathrm{H}$ & -3.71090000 & -2.15276200 & 2.60810900 \\
\hline $\mathrm{H}$ & -3.89621100 & -3.44905700 & 1.41444800 \\
\hline $\mathrm{C}$ & -3.69445800 & -2.98321200 & -1.34694700 \\
\hline $\mathrm{H}$ & -4.02369600 & -3.29096500 & -2.34596300 \\
\hline $\mathrm{H}$ & -3.69496500 & -3.87783700 & -0.71850000 \\
\hline $\mathrm{H}$ & -4.44744400 & -2.29204800 & -0.95980200 \\
\hline $\mathrm{C}$ & -2.30007200 & -1.22774300 & -2.51784700 \\
\hline $\mathrm{H}$ & -2.52535000 & -1.67324000 & -3.49372500 \\
\hline $\mathrm{H}$ & -3.04506000 & -0.45728300 & -2.32530900 \\
\hline $\mathrm{H}$ & -1.32058500 & -0.74545800 & -2.59299800 \\
\hline $\mathrm{C}$ & -1.29983900 & -3.41874000 & -1.96045300 \\
\hline $\mathrm{H}$ & -1.68646400 & -3.82510100 & -2.90197500 \\
\hline
\end{tabular}

\begin{tabular}{lrrr}
$\mathrm{H}$ & -0.32164600 & -2.98350600 & -2.17688800 \\
$\mathrm{H}$ & -1.17178400 & -4.25926100 & -1.27746400 \\
$\mathrm{C}$ & 2.28113600 & 2.76612600 & -1.15756900 \\
$\mathrm{H}$ & 2.80876100 & 3.32894400 & -0.38393200 \\
$\mathrm{H}$ & 2.67897500 & 1.74727700 & -1.18207700 \\
$\mathrm{H}$ & 2.52232000 & 3.23419900 & -2.11866800 \\
$\mathrm{C}$ & 0.29877300 & 4.27076700 & -0.88769300 \\
$\mathrm{H}$ & 0.70528800 & 4.79516400 & -0.02210100 \\
$\mathrm{H}$ & 0.65545600 & 4.79457900 & -1.78171500 \\
$\mathrm{H}$ & -0.78599000 & 4.36807000 & -0.87503000 \\
$\mathrm{C}$ & 0.09498200 & 2.14084000 & -2.20605900 \\
$\mathrm{H}$ & 0.35422800 & 2.72128500 & -3.09919400 \\
$\mathrm{H}$ & 0.45670600 & 1.11819200 & -2.35048400 \\
$\mathrm{H}$ & -0.99458000 & 2.11276000 & -2.13176300 \\
$\mathrm{C}$ & 2.63588500 & 1.57651100 & 2.07260100 \\
$\mathrm{H}$ & 3.22415800 & 1.92419000 & 1.22225800 \\
$\mathrm{H}$ & 3.17693400 & 1.85755100 & 2.98336300 \\
$\mathrm{H}$ & 2.59097700 & 0.48518200 & 2.03319700 \\
$\mathrm{C}$ & 1.34874500 & 3.71707300 & 2.34978500 \\
$\mathrm{H}$ & 1.81019600 & 3.89302500 & 3.32819800 \\
$\mathrm{H}$ & 1.98698600 & 4.19553400 & 1.60361600 \\
$\mathrm{H}$ & 0.38130300 & 4.22734600 & 2.35822000 \\
$\mathrm{H}$ & 0.49962500 & 1.57723000 & 3.34643100 \\
$\mathrm{H}$ & -0.43803700 & 2.08497200 & 3.58626500 \\
$\mathrm{H}$ & 0.30003200 & 0.51055500 & 3.208660000 \\
$\mathrm{H}$ & 1.14790200 & 1.67696100 & 4.22397100 \\
\hline & & & \\
$\mathrm{H}$ & &
\end{tabular}

\section{TS55a}

$\begin{array}{lrrr}\text { imaginary frequency }=-572.47 \mathrm{~cm}^{-1} & \\ \mathrm{P} & 0.89114300 & 0.51878700 & 0.70376500 \\ \mathrm{C} & 0.82099500 & 1.25057100 & 2.47811600 \\ \mathrm{Pd} & 2.63872300 & -1.15552100 & 0.68407600 \\ \mathrm{H} & 3.58965100 & 0.00642800 & 0.11976000 \\ \mathrm{C} & -0.06524400 & 2.50808600 & 2.58222800 \\ \mathrm{H} & -1.09015600 & 2.33014000 & 2.24366800 \\ \mathrm{H} & -0.12339100 & 2.80881700 & 3.63428800 \\ \mathrm{H} & 0.34047100 & 3.35601100 & 2.02675000 \\ \mathrm{C} & 2.25240900 & 1.58057200 & 2.95372000 \\ \mathrm{H} & 2.88836700 & 0.68941700 & 2.96349600 \\ \mathrm{H} & 2.73939400 & 2.34207400 & 2.34292600 \\ \mathrm{H} & 2.20496800 & 1.96292000 & 3.97942400\end{array}$




\begin{tabular}{|c|c|c|c|c|c|c|c|}
\hline $\mathrm{C}$ & 0.24820100 & 0.16823600 & 3.42226700 & $\mathrm{H}$ & -6.29083500 & 1.52229500 & 1.76916400 \\
\hline $\mathrm{H}$ & 0.80404300 & -0.77284300 & 3.36414400 & $\mathrm{H}$ & -4.83328200 & 2.33246600 & 1.16550900 \\
\hline $\mathrm{H}$ & 0.33527400 & 0.52822200 & 4.45299300 & $\mathrm{H}$ & -6.39642500 & 3.14687800 & 1.09730200 \\
\hline $\mathrm{H}$ & -0.80950200 & -0.03647700 & 3.24117200 & $\mathrm{C}$ & -5.75940100 & 2.67598300 & -1.47070000 \\
\hline $\mathrm{C}$ & 1.62254700 & -2.68610800 & 1.44852600 & $\mathrm{H}$ & -5.81856200 & 2.29026900 & -2.49461600 \\
\hline $\mathrm{O}$ & 1.05958400 & -3.56594200 & 1.89983100 & $\mathrm{H}$ & -6.44029800 & 3.53266400 & -1.40443900 \\
\hline $\mathrm{C}$ & 4.94161600 & -1.01721400 & 0.00338900 & $\mathrm{H}$ & -4.74862900 & 3.05984900 & -1.30291200 \\
\hline $\mathrm{C}$ & 4.50141000 & -2.12881600 & 0.40738700 & $\mathrm{C}$ & -7.68982500 & 1.37281400 & -0.60718800 \\
\hline $\mathrm{C}$ & 1.11459200 & 1.85538000 & -0.65210200 & $\mathrm{H}$ & -8.22992600 & 2.31649800 & -0.46242400 \\
\hline $\mathrm{C}$ & 2.24277800 & 2.83179800 & -0.26986000 & $\mathrm{H}$ & -7.91560700 & 1.01015700 & -1.61480900 \\
\hline $\mathrm{H}$ & 3.18680300 & 2.31621000 & -0.06418400 & $\mathrm{H}$ & -8.09381800 & 0.65383300 & 0.10722900 \\
\hline $\mathrm{H}$ & 2.42206000 & 3.50092700 & -1.11864200 & $\mathrm{C}$ & -4.42716500 & -0.89896900 & 1.79198800 \\
\hline $\mathrm{H}$ & 1.99186900 & 3.45944000 & 0.58802300 & $\mathrm{H}$ & -4.53112400 & -1.67477000 & 2.56162700 \\
\hline $\mathrm{C}$ & -0.19608000 & 2.63369500 & -0.89281900 & $\mathrm{H}$ & -3.36659000 & -0.85338700 & 1.52809200 \\
\hline $\mathrm{H}$ & -0.01331400 & 3.39130200 & -1.66288400 & $\mathrm{H}$ & -4.70829000 & 0.05272000 & 2.24812100 \\
\hline $\mathrm{H}$ & -0.99066800 & 1.98194400 & -1.26018300 & $\mathrm{C}$ & -6.75512600 & -1.47113300 & 1.04347700 \\
\hline $\mathrm{H}$ & -0.55256100 & 3.15399500 & -0.00248900 & $\mathrm{H}$ & -7.14704500 & -0.61019000 & 1.59112300 \\
\hline $\mathrm{C}$ & 1.51680700 & 1.14501000 & -1.96421100 & $\mathrm{H}$ & -7.42611200 & -1.68074500 & 0.20446000 \\
\hline $\mathrm{H}$ & 1.64780700 & 1.90567700 & -2.74208100 & $\mathrm{H}$ & -6.79778800 & -2.33310700 & 1.72065000 \\
\hline $\mathrm{H}$ & 2.46581100 & 0.61018000 & -1.86237800 & $\mathrm{C}$ & -4.79922500 & -2.60192900 & -0.00951200 \\
\hline $\mathrm{H}$ & 0.75944300 & 0.44001600 & -2.30954700 & $\mathrm{H}$ & -3.76628500 & -2.54167400 & -0.36345100 \\
\hline $\mathrm{C}$ & -0.82498700 & -0.21726300 & 0.49071100 & $\mathrm{H}$ & -4.84142900 & -3.37797700 & 0.76540600 \\
\hline $\mathrm{H}$ & -0.87519200 & -1.00108900 & 1.25319400 & $\mathrm{H}$ & -5.42122300 & -2.93043000 & -0.84729400 \\
\hline $\mathrm{H}$ & -1.51676900 & 0.56171700 & 0.81307200 & $\mathrm{C}$ & 5.85946100 & -0.06561700 & -0.58004300 \\
\hline $\mathrm{C}$ & -1.24471500 & -0.78605700 & -0.85711200 & $\mathrm{C}$ & 6.16425000 & 1.14806400 & 0.06217000 \\
\hline $\mathrm{C}$ & -2.46048200 & -0.42209700 & -1.48666200 & $\mathrm{C}$ & 6.49498000 & -0.40458500 & -1.78933400 \\
\hline $\mathrm{C}$ & -0.42495300 & -1.75033400 & -1.47209900 & $\mathrm{C}$ & 7.10605000 & 2.00590000 & -0.49679300 \\
\hline $\mathrm{C}$ & -2.75675100 & -1.00114200 & -2.73653700 & $\mathrm{H}$ & 5.67355500 & 1.40228800 & 0.99657200 \\
\hline $\mathrm{C}$ & -0.74735300 & -2.32396400 & -2.69650800 & $\mathrm{C}$ & 7.43706500 & 0.46288900 & -2.33719200 \\
\hline $\mathrm{H}$ & 0.50336900 & -2.04234900 & -0.98816400 & $\mathrm{H}$ & 6.25091100 & -1.33948500 & -2.28272300 \\
\hline $\mathrm{C}$ & -1.92194400 & -1.93246800 & -3.34152000 & $\mathrm{C}$ & 7.74216100 & 1.66518800 & -1.69481000 \\
\hline $\mathrm{H}$ & -3.68499000 & -0.71909800 & -3.22370900 & $\mathrm{H}$ & 7.34846300 & 2.93864400 & 0.00210400 \\
\hline $\mathrm{H}$ & -0.08876800 & -3.06229100 & -3.14380200 & $\mathrm{H}$ & 7.93089200 & 0.20017700 & -3.26712700 \\
\hline $\mathrm{H}$ & -2.19071400 & -2.35807300 & -4.30343600 & $\mathrm{H}$ & 8.47535600 & 2.33843700 & -2.12772000 \\
\hline $\mathrm{C}$ & -3.46224100 & 0.57316600 & -0.92448300 & $\mathrm{H}$ & 4.75067600 & -3.14103500 & 0.67008800 \\
\hline $\mathrm{H}$ & -3.17284000 & 0.95259000 & 0.05717800 & & & & \\
\hline $\mathrm{H}$ & -3.48419900 & 1.43885900 & -1.59355900 & 72 & & & \\
\hline$P$ & -5.27260700 & -0.02298300 & -0.90174700 & $\mathrm{C}$ & -3.70644700 & -2.27800500 & -1.28326000 \\
\hline $\mathrm{C}$ & -6.17400700 & 1.61477400 & -0.42436000 & $\mathrm{C}$ & -3.13268400 & -1.35938500 & -0.38651300 \\
\hline $\mathrm{C}$ & -5.30048500 & -1.26053500 & 0.57729200 & $\mathrm{C}$ & -3.62797400 & -0.03408600 & -0.38007200 \\
\hline $\mathrm{C}$ & -5.90180400 & 2.17367200 & 0.98235000 & $\mathrm{C}$ & -4.66286600 & 0.30310200 & -1.26806100 \\
\hline
\end{tabular}




\begin{tabular}{|c|c|c|c|c|c|c|c|}
\hline $\mathrm{C}$ & -5.19424800 & -0.61289600 & -2.17131800 & $\mathrm{H}$ & -0.78533800 & -1.16148200 & 3.30354600 \\
\hline $\mathrm{C}$ & -4.70843200 & -1.91859500 & -2.18006100 & $\mathrm{C}$ & -0.69854100 & -3.84159600 & 2.54751700 \\
\hline $\mathrm{H}$ & -3.37753200 & -3.31202900 & -1.26201000 & $\mathrm{H}$ & -0.28137000 & -4.30454300 & 3.44853900 \\
\hline $\mathrm{H}$ & -5.08041500 & 1.30460500 & -1.22880100 & $\mathrm{H}$ & -1.69636400 & -3.48240900 & 2.80857000 \\
\hline $\mathrm{H}$ & -5.99536200 & -0.31516100 & -2.84035500 & $\mathrm{H}$ & -0.79628000 & -4.63029200 & 1.79797800 \\
\hline $\mathrm{H}$ & -5.12301500 & -2.65963500 & -2.85604000 & $\mathrm{C}$ & -0.52432500 & -4.32497400 & -1.03461800 \\
\hline $\mathrm{C}$ & -3.17233500 & 1.01677100 & 0.60938300 & $\mathrm{H}$ & -0.35069800 & -4.84307200 & -1.98425600 \\
\hline $\mathrm{H}$ & -3.91535600 & 1.81831500 & 0.65811700 & $\mathrm{H}$ & -0.10465200 & -4.95287700 & -0.24477900 \\
\hline $\mathrm{C}$ & -2.15005800 & -1.89441800 & 0.64017100 & $\mathrm{H}$ & -1.60411000 & -4.26757100 & -0.89169600 \\
\hline $\mathrm{H}$ & -2.29413700 & -1.38680400 & 1.59882400 & $\mathrm{C}$ & -0.32142300 & -2.14783800 & -2.32830800 \\
\hline $\mathrm{H}$ & -2.39945600 & -2.94380000 & 0.81596900 & $\mathrm{H}$ & -0.09664600 & -2.72994100 & -3.22900600 \\
\hline $\mathrm{H}$ & -3.12202100 & 0.58334100 & 1.61213800 & $\mathrm{H}$ & -1.39287500 & -1.94794100 & -2.31980500 \\
\hline$P$ & -1.47928400 & 1.78148600 & 0.35063000 & $\mathrm{H}$ & 0.20688400 & -1.19330600 & -2.40904800 \\
\hline$P$ & -0.26377300 & -1.87943600 & 0.44331800 & $\mathrm{C}$ & 1.68071900 & -3.17053600 & -1.26162800 \\
\hline $\mathrm{C}$ & 0.25364000 & -2.70281100 & 2.11320100 & $\mathrm{H}$ & 1.84639400 & -3.61596100 & -2.24896200 \\
\hline $\mathrm{C}$ & 0.16467300 & -2.94535100 & -1.09744700 & $\mathrm{H}$ & 2.24584300 & -2.23969300 & -1.21631600 \\
\hline $\mathrm{C}$ & -1.29320600 & 2.93458900 & 1.87950700 & $\mathrm{H}$ & 2.09160500 & -3.86044500 & -0.52517100 \\
\hline $\mathrm{C}$ & -1.54998500 & 2.77412900 & -1.28756800 & $\mathrm{C}$ & -0.33467200 & 3.72264500 & -1.36174700 \\
\hline $\mathrm{Pd}$ & 0.50177900 & 0.30696500 & 0.32629900 & $\mathrm{H}$ & -0.40808500 & 4.55628100 & -0.66016300 \\
\hline $\mathrm{H}$ & 2.63362900 & 0.29482800 & -1.65493100 & $\mathrm{H}$ & 0.61190800 & 3.20054400 & -1.18229900 \\
\hline $\mathrm{C}$ & 6.50891600 & 1.09898200 & -2.11845800 & $\mathrm{H}$ & -0.28313800 & 4.15087200 & -2.36912000 \\
\hline $\mathrm{C}$ & 5.14835000 & 0.82277500 & -1.99930900 & $\mathrm{C}$ & -2.84507300 & 3.58476100 & -1.48687800 \\
\hline $\mathrm{C}$ & 4.58715100 & 0.47537500 & -0.75691600 & $\mathrm{H}$ & -3.00366200 & 4.33611000 & -0.71206500 \\
\hline $\mathrm{C}$ & 5.43073000 & 0.42423600 & 0.36948500 & $\mathrm{H}$ & -2.78448500 & 4.11305200 & -2.44552300 \\
\hline $\mathrm{C}$ & 6.78847000 & 0.70006000 & 0.24929400 & $\mathrm{H}$ & -3.72763300 & 2.94444700 & -1.53267700 \\
\hline $\mathrm{C}$ & 7.33346900 & 1.03781500 & -0.99431700 & $\mathrm{C}$ & -1.41988700 & 1.74630400 & -2.43496500 \\
\hline $\mathrm{H}$ & 6.92421700 & 1.36109000 & -3.08674000 & $\mathrm{H}$ & -1.41297200 & 2.28221500 & -3.39126400 \\
\hline $\mathrm{H}$ & 4.50961700 & 0.86987800 & -2.87794200 & $\mathrm{H}$ & -0.48538400 & 1.18125000 & -2.36107300 \\
\hline $\mathrm{H}$ & 5.02279300 & 0.17022700 & 1.34325100 & $\mathrm{H}$ & -2.24898600 & 1.03644300 & -2.45796400 \\
\hline $\mathrm{H}$ & 7.42659200 & 0.65447700 & 1.12665700 & $\mathrm{C}$ & 0.19572500 & 3.32710100 & 2.01557900 \\
\hline $\mathrm{H}$ & 8.39383400 & 1.25304700 & -1.08315200 & $\mathrm{H}$ & 0.56618600 & 3.90870500 & 1.17017200 \\
\hline $\mathrm{C}$ & 3.14640200 & 0.17523900 & -0.69761300 & $\mathrm{H}$ & 0.32533300 & 3.93910300 & 2.91574100 \\
\hline $\mathrm{C}$ & 2.41842900 & -0.20094800 & 0.37507300 & $\mathrm{H}$ & 0.83546200 & 2.44340400 & 2.12990200 \\
\hline $\mathrm{H}$ & 2.86458900 & -0.47168300 & 1.33093100 & $\mathrm{C}$ & -2.16457900 & 4.20361500 & 1.83465600 \\
\hline $\mathrm{C}$ & 1.68416400 & -3.27301100 & 2.03885100 & $\mathrm{H}$ & -2.06747600 & 4.73497900 & 2.78856400 \\
\hline $\mathrm{H}$ & 1.71992200 & -4.21038700 & 1.47966500 & $\mathrm{H}$ & -1.85527000 & 4.89573000 & 1.04843800 \\
\hline $\mathrm{H}$ & 2.40012200 & -2.57912800 & 1.59552100 & $\mathrm{H}$ & -3.22654200 & 3.97717100 & 1.69830800 \\
\hline $\mathrm{H}$ & 2.02152600 & -3.49442000 & 3.05751700 & $\mathrm{C}$ & -1.67422000 & 2.12131400 & 3.13784700 \\
\hline $\mathrm{C}$ & 0.21105200 & -1.60035100 & 3.19390000 & $\mathrm{H}$ & -2.74930800 & 1.93778700 & 3.21148600 \\
\hline $\mathrm{H}$ & 0.47458700 & -2.04395200 & 4.16056800 & $\mathrm{H}$ & -1.14774300 & 1.16273800 & 3.18588400 \\
\hline $\mathrm{H}$ & 0.91973700 & -0.79408700 & 2.98768800 & $\mathrm{H}$ & -1.38608300 & 2.69476900 & 4.02577400 \\
\hline
\end{tabular}


73

C

C

C

C

C

C

H

H

H

H

C

H

C

H

H

H

P

P

C

C

C

C

Pd

H

C

C

C

C

C

C

H

H

H

H

H

C

C

H

C
$2.22911800 \quad 3.43718400 \quad-1.72522800$

2.27658800

2.38595300

$-0.79451000$

3.18113800

$1.32429500-1.03373100$

3.99198100

1.37597000

$2.40753000 \quad-3.10986900$

3.90109300

3.00595100

3.44858300

$-2.88066700$

1.59849000

4.74271100

4.29509500

$-1.52477600$

0.60696000

$-2.32880600$

4.54535700

2.41074000

$-3.98329400$

2.93542300

4.28193000

3.42606700

0.21165800

$-3.57246300$

$-0.03577400$

4.41646500

$-0.21680100$

$-0.21723000$

1.50358200

2.52081200

0.50624900

2.10525100

2.11586800

1.32182000

1.37407200

3.58340100

0.72924500

3.45846000

0.62571200

0.97478900

2.22309300

$-1.23000100$

0.10146700

$-0.19037500$

1.72662000

0.76506500

$-0.47867100$

2.09038500

2.64275900

$-1.40350300$

2.73316200

$-0.34478900$

3.06758200

$-2.27998700$

1.48795300

2.27658000

$-2.13038900$

$-1.60304000$

$-0.23343000$

$-0.72438200$

0.47537700

$-2.45128800$

$-1.32160200$

$-1.56422300$

$-6.35660600$

$-1.39954100$

$-2.34638100$

$-4.98241900$

$-1.36616600$

$-2.11630300$

$-4.46801800$

$-1.07535900$

$-0.84017700$

$-5.37732100$

$-0.82069700$

0.20379400

$-6.74914200$

$-0.85451400$

$-0.02599000$

$-7.24512300$

$-1.14300800$

$-1.30154400$

$-6.73248600$

$-1.62748300$

$-3.33920300$

$-4.29453600$

$-1.56897400$

$-2.93361100$

$-5.01255500$

$-0.60490600$

1.20374800

$-7.43646000$

$-0.65974700$

0.79187100

$-8.31626600$

$-1.17031000$

$-1.47621500$

$-3.00402600$

$-1.05204500$

$-0.66269700$

$-2.32042800$

$-0.71253700$

0.44310600

$-2.82146300$

$-0.4871360$

1.37927100

$-1.73587200$

1.3518220

3.14893500

\begin{tabular}{|c|c|c|}
\hline-2.64563100 & 1.63158500 & 2.61674600 \\
\hline-1.61225000 & 0.26764200 & 3.08588600 \\
\hline-1.88421800 & 1.60427300 & 4.20490200 \\
\hline 0.71985800 & 1.52399500 & 3.43498700 \\
\hline 0.48536300 & 1.56996000 & 4.50400600 \\
\hline 0.90356400 & 0.47490300 & 3.18635500 \\
\hline 1.64245100 & 2.09110000 & 3.28831700 \\
\hline-0.61389900 & 3.59432800 & 2.95687500 \\
\hline-0.64371500 & 3.71963600 & 4.04532900 \\
\hline 0.22951600 & 4.18641700 & 2.59040400 \\
\hline-1.53761500 & 4.02109200 & 2.56079500 \\
\hline-1.07915800 & 4.24134200 & -0.38932100 \\
\hline-1.82738700 & 4.72712100 & -1.02543100 \\
\hline-1.12993600 & 4.71877500 & 0.58978400 \\
\hline-0.10682800 & 4.45799400 & -0.82625100 \\
\hline-1.27084100 & 2.14286300 & -1.76672800 \\
\hline-1.91690100 & 2.70973200 & -2.44686800 \\
\hline-0.24952100 & 2.20775600 & -2.15068700 \\
\hline-1.59118900 & 1.09926200 & -1.79600800 \\
\hline-2.86654700 & 2.59601400 & 0.12579000 \\
\hline-3.50754800 & 3.07410400 & -0.62364500 \\
\hline-3.18852300 & 1.56023100 & 0.21342800 \\
\hline-3.04560500 & 3.11139200 & 1.07258400 \\
\hline 1.68856000 & -3.55246900 & -1.49952600 \\
\hline 2.29312300 & -4.21654600 & -0.87763200 \\
\hline 0.66347700 & -3.56328600 & -1.12517600 \\
\hline 1.66320500 & -3.98857000 & -2.50446000 \\
\hline 3.68699900 & -2.24439400 & -2.21769200 \\
\hline 4.36103600 & -2.86692300 & -1.62903800 \\
\hline 3.59322400 & -2.71253900 & -3.20420300 \\
\hline 4.15602000 & -1.27452600 & -2.37209500 \\
\hline 1.38516700 & -1.29963900 & -2.55655100 \\
\hline 1.33850700 & -1.80406300 & -3.52866700 \\
\hline 0.35999500 & -1.20828600 & -2.18062300 \\
\hline 1.77883000 & -0.29464000 & -2.72244900 \\
\hline 2.07572800 & -3.33161000 & 2.02488000 \\
\hline 1.71996300 & -4.01967300 & 1.25571800 \\
\hline 2.57896700 & -3.93354500 & 2.78968700 \\
\hline 1.21060400 & -2.86103200 & 2.50056700 \\
\hline 4.35394400 & -2.98990400 & 1.01952700 \\
\hline 4.83647500 & -3.44730100 & 1.89079800 \\
\hline
\end{tabular}

C

$\mathrm{H}$

C

$\mathrm{H}$

$\mathrm{H}$

$\mathrm{H}$

C

$\mathrm{H}$

$\mathrm{H}$

H

C

H

$\mathrm{H}$

H

C

H

H

H

C

H

H

C

H

H

H

C

H

H

H

C

H

H

H

C

H 


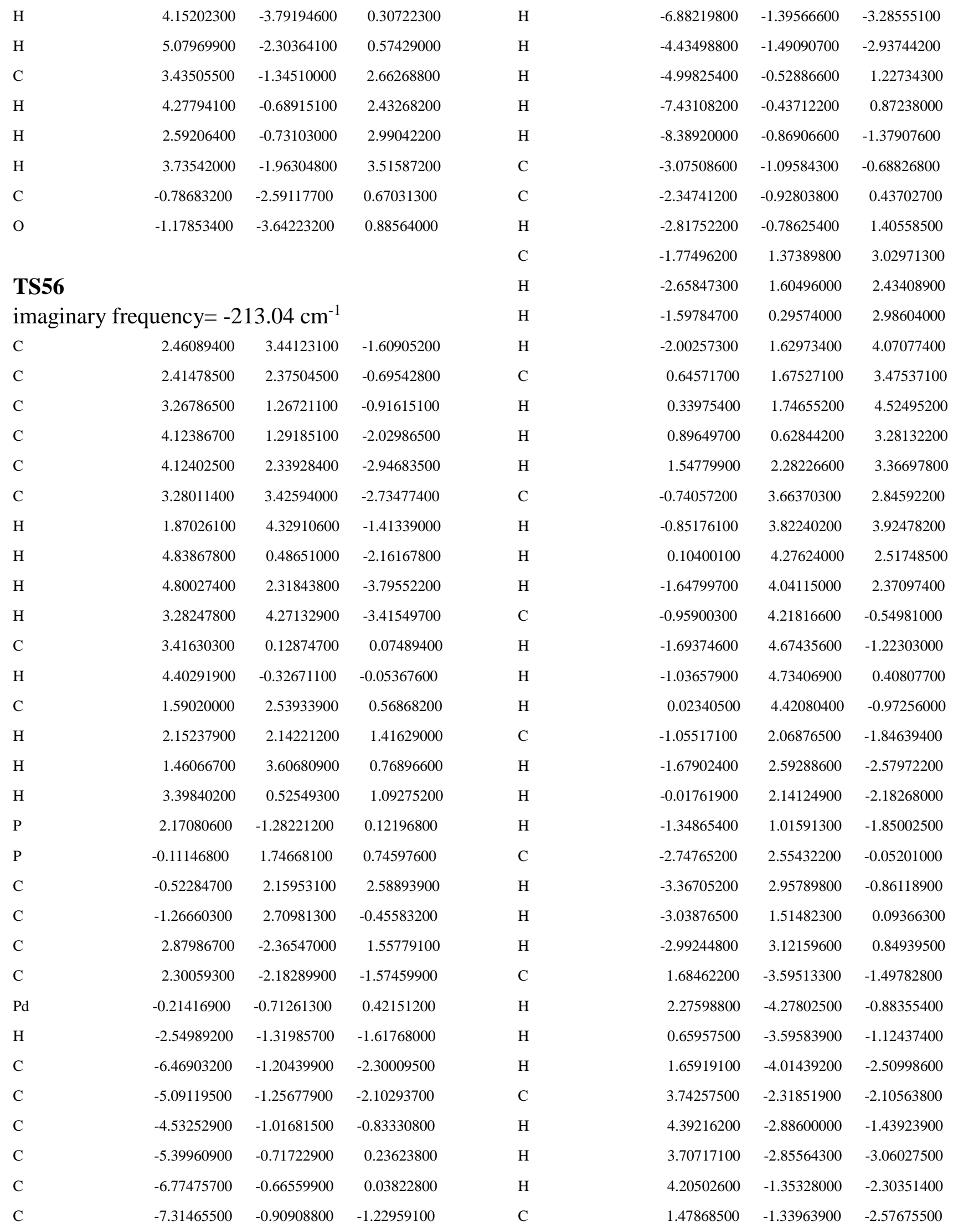




\begin{tabular}{|c|c|c|c|c|c|c|c|}
\hline $\mathrm{H}$ & 1.51733200 & -1.82022500 & -3.56120600 & $\mathrm{H}$ & -5.39993700 & -1.54273400 & -1.41247600 \\
\hline $\mathrm{H}$ & 0.42678800 & -1.27220300 & -2.28012200 & $\mathrm{C}$ & -5.97604400 & -0.56044000 & 2.29451200 \\
\hline $\mathrm{H}$ & 1.86968100 & -0.32544900 & -2.68588600 & $\mathrm{H}$ & -3.91504100 & -1.04753300 & 2.61059800 \\
\hline $\mathrm{C}$ & 1.82015400 & -3.39241700 & 2.00385600 & $\mathrm{C}$ & -7.02879400 & -0.48200200 & 1.37411500 \\
\hline $\mathrm{H}$ & 1.49737000 & -4.05550300 & 1.20021600 & $\mathrm{H}$ & -7.63516800 & -0.77631900 & -0.67492000 \\
\hline $\mathrm{H}$ & 2.24813300 & -4.01908100 & 2.79427800 & $\mathrm{H}$ & -6.14265600 & -0.29210800 & 3.33294300 \\
\hline $\mathrm{H}$ & 0.93700600 & -2.89955700 & 2.41899700 & $\mathrm{H}$ & -8.00961600 & -0.15100300 & 1.70124900 \\
\hline $\mathrm{C}$ & 4.18165100 & -3.10304300 & 1.18358200 & $\mathrm{C}$ & -3.20711700 & -1.82041800 & 0.04739600 \\
\hline $\mathrm{H}$ & 4.57913200 & -3.58467300 & 2.08423400 & $\mathrm{H}$ & -3.18687000 & -2.08902300 & -1.00789900 \\
\hline $\mathrm{H}$ & 4.02035800 & -3.89023300 & 0.44469400 & $\mathrm{C}$ & -2.05704700 & -2.00662900 & 0.75492400 \\
\hline $\mathrm{H}$ & 4.95860600 & -2.42902900 & 0.81150000 & $\mathrm{C}$ & -0.84472300 & -2.58717200 & 0.10917400 \\
\hline $\mathrm{C}$ & 3.17730600 & -1.44987600 & 2.76647200 & $\mathrm{O}$ & -0.61795700 & -3.67937200 & -0.31856100 \\
\hline $\mathrm{H}$ & 4.05072600 & -0.81191300 & 2.61122800 & $\mathrm{H}$ & -1.97979300 & -1.78385300 & 1.81548800 \\
\hline $\mathrm{H}$ & 2.32449400 & -0.81964500 & 3.03266000 & $\mathrm{C}$ & -1.27262700 & 2.66602400 & 0.65540500 \\
\hline $\mathrm{H}$ & 3.39386300 & -2.08218100 & 3.63432600 & $\mathrm{C}$ & -0.63865500 & 2.21062000 & 1.99055500 \\
\hline $\mathrm{C}$ & -1.00241900 & -2.46964600 & 0.49333900 & $\mathrm{H}$ & -1.14231600 & 2.72279100 & 2.81898200 \\
\hline \multirow[t]{2}{*}{$\mathrm{O}$} & -1.31712900 & -3.56882700 & 0.64935900 & $\mathrm{H}$ & -0.75919800 & 1.13251000 & 2.14137500 \\
\hline & & & & $\mathrm{H}$ & 0.42606300 & 2.44584300 & 2.05206400 \\
\hline 74 & & & & $\mathrm{C}$ & -2.75902200 & 2.25762700 & 0.67236800 \\
\hline $\mathrm{C}$ & 1.27097100 & 2.60758500 & -1.01784100 & $\mathrm{H}$ & -3.22803000 & 2.67086400 & 1.57284800 \\
\hline $\mathrm{H}$ & 1.68422100 & 2.12713800 & -1.90812600 & $\mathrm{H}$ & -3.31173100 & 2.64819400 & -0.18539000 \\
\hline $\mathrm{H}$ & 1.03758300 & 3.63640500 & -1.30854400 & $\mathrm{H}$ & -2.89042100 & 1.17222500 & 0.70457300 \\
\hline $\mathrm{P}$ & -0.35241100 & 1.69769200 & -0.72282700 & $\mathrm{C}$ & -1.17302600 & 4.19951000 & 0.54852100 \\
\hline $\mathrm{C}$ & -1.22352000 & 1.89538000 & -2.43084600 & $\mathrm{H}$ & -1.60865300 & 4.58947800 & -0.37221700 \\
\hline $\mathrm{C}$ & -1.71190900 & 3.32272200 & -2.74015200 & $\mathrm{H}$ & -1.72220700 & 4.64791000 & 1.38474500 \\
\hline $\mathrm{H}$ & -2.08471000 & 3.35675000 & -3.77072800 & $\mathrm{H}$ & -0.14376800 & 4.55267700 & 0.61823700 \\
\hline $\mathrm{H}$ & -2.53340300 & 3.63336300 & -2.09094600 & $\mathrm{C}$ & 2.32222000 & 2.62732800 & 0.07376200 \\
\hline $\mathrm{H}$ & -0.91143000 & 4.06455100 & -2.66320200 & $\mathrm{C}$ & 3.19806100 & 1.54588400 & 0.32918800 \\
\hline $\mathrm{C}$ & -2.41198200 & 0.91208700 & -2.49551300 & $\mathrm{C}$ & 2.51814700 & 3.82660300 & 0.78039500 \\
\hline $\mathrm{H}$ & -2.89045000 & 0.99269000 & -3.47846100 & $\mathrm{C}$ & 4.22079700 & 1.72456300 & 1.27773600 \\
\hline $\mathrm{H}$ & -2.07088700 & -0.12203200 & -2.37719100 & $\mathrm{C}$ & 3.51425600 & 3.97215600 & 1.74135900 \\
\hline $\mathrm{H}$ & -3.17534500 & 1.10912300 & -1.74096900 & $\mathrm{H}$ & 1.89177000 & 4.68121400 & 0.54496600 \\
\hline $\mathrm{C}$ & -0.22105100 & 1.47003400 & -3.52833700 & $\mathrm{C}$ & 4.37590800 & 2.90689000 & 1.99472200 \\
\hline $\mathrm{H}$ & 0.23951900 & 0.50097800 & -3.31130000 & $\mathrm{H}$ & 4.93082800 & 0.91865400 & 1.43975700 \\
\hline $\mathrm{H}$ & -0.75961000 & 1.36945900 & -4.47722300 & $\mathrm{H}$ & 3.63236700 & 4.91672500 & 2.26288200 \\
\hline $\mathrm{H}$ & 0.56933100 & 2.20790400 & -3.68815700 & $\mathrm{H}$ & 5.17900800 & 3.00274900 & 2.71856000 \\
\hline $\mathrm{Pd}$ & -0.14730900 & -0.75749400 & -0.13361700 & $\mathrm{C}$ & 3.22076100 & 0.26246900 & -0.47957600 \\
\hline $\mathrm{C}$ & -4.49075300 & -1.34476400 & 0.53809700 & $\mathrm{H}$ & 4.25169100 & -0.09729200 & -0.49062200 \\
\hline $\mathrm{C}$ & -5.56165600 & -1.26077500 & -0.37550500 & $\mathrm{H}$ & 2.96634900 & 0.46929100 & -1.52426000 \\
\hline $\mathrm{C}$ & -4.71930700 & -0.98629100 & 1.88398600 & $\mathrm{P}$ & 2.17620900 & -1.26938800 & -0.09152500 \\
\hline $\mathrm{C}$ & -6.81941800 & -0.83238400 & 0.03865800 & $\mathrm{C}$ & 2.75417000 & -2.42397600 & -1.53655900 \\
\hline
\end{tabular}




\begin{tabular}{|c|c|c|c|c|c|c|c|}
\hline $\mathrm{C}$ & 2.71561800 & -1.90971300 & 1.63641900 & $\mathrm{H}$ & 1.68903000 & -1.34209200 & 3.02796200 \\
\hline $\mathrm{C}$ & 4.23609900 & -2.21703900 & -1.92493200 & $\mathrm{H}$ & 2.44039100 & -2.78830800 & 3.70176700 \\
\hline $\mathrm{H}$ & 4.92163700 & -2.36621500 & -1.08685400 & $\mathrm{H}$ & 3.45992100 & -1.49077500 & 3.09373200 \\
\hline $\mathrm{H}$ & 4.49230000 & -2.96221300 & -2.68622900 & $\mathrm{Pd}$ & -0.04674500 & -0.72473000 & 0.04031800 \\
\hline $\mathrm{H}$ & 4.43088400 & -1.23593900 & -2.36367800 & $\mathrm{C}$ & -5.61461100 & -0.56036200 & -0.46520100 \\
\hline $\mathrm{C}$ & 1.87078200 & -2.07006100 & -2.75223900 & $\mathrm{C}$ & -6.30279600 & 0.65166800 & -0.24806600 \\
\hline $\mathrm{H}$ & 0.81617100 & -2.28058400 & -2.55654300 & $\mathrm{C}$ & -6.36140600 & -1.71075900 & -0.80125900 \\
\hline $\mathrm{H}$ & 1.96799900 & -1.02072900 & -3.04857200 & $\mathrm{C}$ & -7.68753800 & 0.71716800 & -0.36347100 \\
\hline $\mathrm{H}$ & 2.18343500 & -2.67765300 & -3.60903600 & $\mathrm{H}$ & -5.73907400 & 1.54377300 & 0.01152200 \\
\hline $\mathrm{C}$ & 2.54846400 & -3.91472100 & -1.20094800 & $\mathrm{C}$ & -7.74353200 & -1.64289600 & -0.91555300 \\
\hline $\mathrm{H}$ & 1.53085100 & -4.13566100 & -0.88016900 & $\mathrm{H}$ & -5.85945700 & -2.65744900 & -0.97192800 \\
\hline $\mathrm{H}$ & 2.74283800 & -4.49645800 & -2.10945200 & $\mathrm{C}$ & -8.40996000 & -0.43051800 & -0.69768100 \\
\hline $\mathrm{H}$ & 3.25291100 & -4.26712200 & -0.44360800 & $\mathrm{H}$ & -8.20338900 & 1.65686800 & -0.19415900 \\
\hline $\mathrm{C}$ & 4.23160500 & -2.18532500 & 1.70400200 & $\mathrm{H}$ & -8.30819300 & -2.53294600 & -1.17416500 \\
\hline $\mathrm{H}$ & 4.49922500 & -2.44643000 & 2.73408400 & $\mathrm{H}$ & -9.49083500 & -0.38385300 & -0.78851500 \\
\hline $\mathrm{H}$ & 4.52471300 & -3.02641900 & 1.07064100 & $\mathrm{C}$ & -4.16958000 & -0.56283100 & -0.33319600 \\
\hline $\mathrm{H}$ & 4.83580300 & -1.31883800 & 1.42432600 & $\mathrm{H}$ & -3.72586400 & 0.39060900 & -0.06602500 \\
\hline $\mathrm{C}$ & 1.95274800 & -3.19393800 & 2.02316600 & $\mathrm{C}$ & -3.32583000 & -1.61305300 & -0.50963400 \\
\hline $\mathrm{H}$ & 2.14249800 & -4.03710200 & 1.36122700 & $\mathrm{C}$ & -1.89982800 & -1.52741000 & -0.34681600 \\
\hline $\mathrm{H}$ & 2.27232500 & -3.48605300 & 3.02985200 & $\mathrm{O}$ & -1.14494400 & -2.49817100 & -0.48551900 \\
\hline $\mathrm{H}$ & 0.87415400 & -3.02508900 & 2.06301100 & $\mathrm{H}$ & -3.69119200 & -2.60054200 & -0.78308500 \\
\hline $\mathrm{C}$ & 2.31972800 & -0.83036000 & 2.66938000 & $\mathrm{C}$ & 2.78956000 & -2.08999600 & -1.56195500 \\
\hline $\mathrm{H}$ & 1.24923500 & -0.60464500 & 2.61710900 & $\mathrm{C}$ & 2.33242800 & -1.02699000 & -2.58756200 \\
\hline $\mathrm{H}$ & 2.52790200 & -1.21687100 & 3.67360200 & $\mathrm{H}$ & 2.60801900 & -1.36347000 & -3.59387100 \\
\hline \multirow[t]{2}{*}{$\mathrm{H}$} & 2.86839900 & 0.10273800 & 2.55146400 & $\mathrm{H}$ & 1.24643100 & -0.89378800 & -2.56732500 \\
\hline & & & & $\mathrm{H}$ & 2.80163300 & -0.05467700 & -2.42092300 \\
\hline 74-O & & & & $\mathrm{C}$ & 2.01719200 & -3.39508000 & -1.84894700 \\
\hline $\mathrm{C}$ & 3.64026100 & -0.17529200 & 0.65203600 & $\mathrm{H}$ & 2.16488600 & -3.65980200 & -2.90231300 \\
\hline $\mathrm{H}$ & 3.40279200 & 0.08029300 & 1.68790800 & $\mathrm{H}$ & 2.38085800 & -4.23610500 & -1.25366500 \\
\hline $\mathrm{H}$ & 4.59378500 & -0.71078100 & 0.68317800 & $\mathrm{H}$ & 0.94185700 & -3.28895400 & -1.68295600 \\
\hline $\mathrm{P}$ & 2.30977400 & -1.40978200 & 0.16912200 & $\mathrm{C}$ & 4.29866400 & -2.34244000 & -1.74845100 \\
\hline $\mathrm{C}$ & 2.51015700 & -2.74916400 & 1.54281700 & $\mathrm{H}$ & 4.70249800 & -3.06960500 & -1.04298300 \\
\hline $\mathrm{C}$ & 3.79874400 & -3.58329600 & 1.41010100 & $\mathrm{H}$ & 4.46124100 & -2.74239900 & -2.75605300 \\
\hline $\mathrm{H}$ & 3.88849800 & -4.23421200 & 2.28769800 & $\mathrm{H}$ & 4.88303500 & -1.42454900 & -1.67415400 \\
\hline $\mathrm{H}$ & 3.78693500 & -4.22970900 & 0.53028400 & $\mathrm{C}$ & 3.80757900 & 1.09634500 & -0.15230500 \\
\hline $\mathrm{H}$ & 4.70117200 & -2.96556100 & 1.37542200 & $\mathrm{C}$ & 2.94029700 & 2.20489500 & -0.02066000 \\
\hline $\mathrm{C}$ & 1.28414800 & -3.68702200 & 1.52534500 & $\mathrm{C}$ & 4.94286400 & 1.22511400 & -0.96930000 \\
\hline $\mathrm{H}$ & 1.40599300 & -4.43984000 & 2.31272300 & $\mathrm{C}$ & 3.25507800 & 3.38629000 & -0.71352200 \\
\hline $\mathrm{H}$ & 0.35767000 & -3.14233500 & 1.72661400 & $\mathrm{C}$ & 5.22047400 & 2.39275300 & -1.67509300 \\
\hline $\mathrm{H}$ & 1.16167000 & -4.21550800 & 0.57942600 & $\mathrm{H}$ & 5.64387700 & 0.39829500 & -1.02856600 \\
\hline $\mathrm{C}$ & 2.52853600 & -2.03359100 & 2.91246300 & $\mathrm{C}$ & 4.36641200 & 3.48585000 & -1.54628600 \\
\hline
\end{tabular}




\begin{tabular}{|c|c|c|c|c|c|c|c|}
\hline $\mathrm{H}$ & 2.62195900 & 4.25885800 & -0.58065800 & $\mathrm{H}$ & 3.03014700 & 4.10637100 & -0.80977900 \\
\hline $\mathrm{H}$ & 6.10862000 & 2.45402400 & -2.29601800 & $\mathrm{H}$ & 5.75556400 & 0.01401000 & -0.92087400 \\
\hline $\mathrm{H}$ & 4.57475200 & 4.41593700 & -2.06558100 & $\mathrm{H}$ & 6.40868400 & 1.93626800 & -2.30132200 \\
\hline $\mathrm{C}$ & 1.77695100 & 2.24352300 & 0.95095700 & $\mathrm{H}$ & 5.02156100 & 4.01823500 & -2.24909900 \\
\hline $\mathrm{H}$ & 1.60822200 & 3.28644400 & 1.22747400 & $\mathrm{C}$ & 3.65537000 & -0.30894400 & 0.71564300 \\
\hline $\mathrm{H}$ & 2.04996300 & 1.73247200 & 1.87949600 & $\mathrm{H}$ & 4.57542900 & -0.88419600 & 0.85226700 \\
\hline $\mathrm{P}$ & 0.05804700 & 1.56121900 & 0.55960900 & $\mathrm{C}$ & 2.01035300 & 2.28504300 & 0.83462100 \\
\hline $\mathrm{C}$ & -0.78990800 & 1.89634000 & 2.26709100 & $\mathrm{H}$ & 2.22311900 & 1.80364200 & 1.79475100 \\
\hline $\mathrm{C}$ & -0.62284400 & 2.65603400 & -0.86895800 & $\mathrm{H}$ & 1.96221300 & 3.35456000 & 1.04498400 \\
\hline $\mathrm{C}$ & -0.44609000 & 3.27111300 & 2.88159100 & $\mathrm{H}$ & 3.35807900 & 0.02786800 & 1.71139100 \\
\hline $\mathrm{H}$ & -0.71428700 & 4.10811400 & 2.23395800 & $\mathrm{P}$ & 2.29821000 & -1.51646600 & 0.21161500 \\
\hline $\mathrm{H}$ & -1.01564500 & 3.38464600 & 3.81090500 & $\mathrm{P}$ & 0.21785900 & 1.78559400 & 0.48878700 \\
\hline $\mathrm{H}$ & 0.60966800 & 3.36302000 & 3.14579000 & $\mathrm{C}$ & -0.58412400 & 2.50651100 & 2.10137400 \\
\hline $\mathrm{C}$ & -0.29337600 & 0.79047100 & 3.22480000 & $\mathrm{C}$ & -0.30980600 & 2.75590500 & -1.08758600 \\
\hline $\mathrm{H}$ & -0.55618700 & -0.20602500 & 2.85785400 & $\mathrm{C}$ & 2.22691600 & -2.67903500 & 1.75194900 \\
\hline $\mathrm{H}$ & 0.78778200 & 0.82533500 & 3.38717600 & $\mathrm{C}$ & 2.98667900 & -2.43983500 & -1.33223200 \\
\hline $\mathrm{H}$ & -0.76834600 & 0.92810500 & 4.20279400 & $\mathrm{Pd}$ & -0.11608400 & -0.63717900 & 0.01381200 \\
\hline $\mathrm{C}$ & -2.31943400 & 1.76887200 & 2.16104000 & $\mathrm{H}$ & -4.52331800 & -0.93249300 & 1.53033300 \\
\hline $\mathrm{H}$ & -2.61390200 & 0.82677700 & 1.69331800 & $\mathrm{C}$ & -4.50865800 & -0.68034000 & 0.47101900 \\
\hline $\mathrm{H}$ & -2.74486100 & 1.77949400 & 3.17082800 & $\mathrm{C}$ & -3.28420000 & -0.48571100 & -0.08977600 \\
\hline $\mathrm{H}$ & -2.77082700 & 2.60048500 & 1.61492300 & $\mathrm{C}$ & 1.00210300 & -3.61437100 & 1.63864000 \\
\hline $\mathrm{C}$ & -0.56427900 & 4.16475500 & -0.55717800 & $\mathrm{H}$ & 0.96873100 & -4.25705600 & 2.52571000 \\
\hline $\mathrm{H}$ & -0.83323300 & 4.72126300 & -1.46215000 & $\mathrm{H}$ & 0.06545000 & -3.04947400 & 1.61072000 \\
\hline $\mathrm{H}$ & -1.27774300 & 4.45148300 & 0.21964900 & $\mathrm{H}$ & 1.03932200 & -4.26741100 & 0.76561200 \\
\hline $\mathrm{H}$ & 0.42809000 & 4.50611500 & -0.25429500 & $\mathrm{C}$ & 3.50184100 & -3.51944100 & 1.95658500 \\
\hline $\mathrm{C}$ & -2.07542000 & 2.28073900 & -1.21780900 & $\mathrm{H}$ & 4.40549500 & -2.90594700 & 2.01995800 \\
\hline $\mathrm{H}$ & -2.78003100 & 2.51257600 & -0.41753800 & $\mathrm{H}$ & 3.41673300 & -4.06319200 & 2.90477600 \\
\hline $\mathrm{H}$ & -2.38023000 & 2.86260100 & -2.09498100 & $\mathrm{H}$ & 3.64418300 & -4.26516200 & 1.17161300 \\
\hline $\mathrm{H}$ & -2.16425000 & 1.22404300 & -1.47816500 & $\mathrm{C}$ & 2.01255500 & -1.79645400 & 3.00307400 \\
\hline $\mathrm{C}$ & 0.22599000 & 2.33670800 & -2.12165100 & $\mathrm{H}$ & 1.17204400 & -1.10658500 & 2.88357500 \\
\hline $\mathrm{H}$ & 0.18085500 & 1.27245500 & -2.37013500 & $\mathrm{H}$ & 1.77875600 & -2.44727700 & 3.85285300 \\
\hline $\mathrm{H}$ & -0.18792100 & 2.89268100 & -2.97074300 & $\mathrm{H}$ & 2.90370800 & -1.22576600 & 3.27758100 \\
\hline \multirow[t]{2}{*}{$\mathrm{H}$} & 1.27242900 & 2.62027500 & -2.01902100 & $\mathrm{C}$ & 2.69284800 & -1.52990100 & -2.54734100 \\
\hline & & & & $\mathrm{H}$ & 3.16304500 & -0.54813200 & -2.45301800 \\
\hline 74-CO & & & & $\mathrm{H}$ & 3.09031100 & -2.00331200 & -3.45262000 \\
\hline $\mathrm{C}$ & 3.59432200 & 3.18042900 & -0.87412700 & $\mathrm{H}$ & 1.61960300 & -1.38093900 & -2.69785100 \\
\hline $\mathrm{C}$ & 3.16983200 & 2.07340400 & -0.11827400 & $\mathrm{C}$ & 4.50386900 & -2.71239500 & -1.27220000 \\
\hline $\mathrm{C}$ & 3.94663500 & 0.89399100 & -0.15766800 & $\mathrm{H}$ & 4.79969900 & -3.28730900 & -0.39362100 \\
\hline $\mathrm{C}$ & 5.11264300 & 0.88758200 & -0.94196700 & $\mathrm{H}$ & 4.78728500 & -3.29677000 & -2.15505900 \\
\hline $\mathrm{C}$ & 5.49949300 & 1.98205800 & -1.71020500 & $\mathrm{H}$ & 5.09007000 & -1.79502800 & -1.29941400 \\
\hline $\mathrm{C}$ & 4.72836900 & 3.14194200 & -1.67965700 & $\mathrm{C}$ & 2.27107100 & -3.79037500 & -1.53983400 \\
\hline
\end{tabular}




\begin{tabular}{|c|c|c|c|c|c|c|c|}
\hline $\mathrm{H}$ & 1.18318800 & -3.70873500 & -1.53330300 & $\mathrm{C}$ & -0.76244200 & -1.99881900 & -1.25519000 \\
\hline $\mathrm{H}$ & 2.55711400 & -4.18610900 & -2.52087100 & $\mathrm{O}$ & -1.15618700 & -2.65240600 & -2.10712300 \\
\hline $\mathrm{H}$ & 2.56369500 & -4.53390600 & -0.79514400 & & & & \\
\hline $\mathrm{C}$ & -0.29149700 & 1.49243100 & 3.23061200 & \multirow{2}{*}{\multicolumn{4}{|c|}{$\begin{array}{l}\text { TS57 } \\
\text { imaginary frequency }=-102.30 \mathrm{~cm}^{-1}\end{array}$}} \\
\hline $\mathrm{H}$ & -0.74766600 & 0.52016900 & 3.04116000 & & & & \\
\hline $\mathrm{H}$ & 0.78132400 & 1.35332900 & 3.39696000 & $\mathrm{C}$ & -0.51850300 & 2.79386500 & 0.52059400 \\
\hline $\mathrm{H}$ & -0.70797300 & 1.88169600 & 4.16688700 & $\mathrm{H}$ & -1.28185600 & 2.57423400 & 1.26915800 \\
\hline $\mathrm{C}$ & -0.01160400 & 3.87707800 & 2.53130700 & $\mathrm{H}$ & -0.13070000 & 3.78641200 & 0.76708000 \\
\hline $\mathrm{H}$ & -0.59452800 & 4.23112200 & 3.38897600 & $\mathrm{P}$ & 0.85659700 & 1.55397400 & 0.89505700 \\
\hline $\mathrm{H}$ & 1.02732400 & 3.81641000 & 2.86286800 & $\mathrm{C}$ & 1.07726600 & 1.80569200 & 2.80240500 \\
\hline $\mathrm{H}$ & -0.08456900 & 4.64069600 & 1.75496800 & $\mathrm{C}$ & 1.63493700 & 3.18996900 & 3.18981200 \\
\hline $\mathrm{C}$ & -2.11065500 & 2.65869600 & 1.94801900 & $\mathrm{H}$ & 1.60740200 & 3.28713100 & 4.28151900 \\
\hline $\mathrm{H}$ & -2.59914200 & 1.75829800 & 1.57333700 & $\mathrm{H}$ & 2.67422900 & 3.32419700 & 2.88303400 \\
\hline $\mathrm{H}$ & -2.53807000 & 2.87291700 & 2.93407300 & $\mathrm{H}$ & 1.04301400 & 4.01376800 & 2.78049600 \\
\hline $\mathrm{H}$ & -2.37404800 & 3.49546600 & 1.29644200 & $\mathrm{C}$ & 2.00092200 & 0.70606500 & 3.36786700 \\
\hline $\mathrm{C}$ & -0.14000100 & 4.28127100 & -0.94786500 & $\mathrm{H}$ & 2.10581400 & 0.85205400 & 4.44889200 \\
\hline $\mathrm{H}$ & -0.86833900 & 4.71377000 & -0.25740800 & $\mathrm{H}$ & 1.57107000 & -0.28752800 & 3.21201000 \\
\hline $\mathrm{H}$ & 0.85985900 & 4.57450500 & -0.61949100 & $\mathrm{H}$ & 3.00322600 & 0.72276400 & 2.93838300 \\
\hline $\mathrm{H}$ & -0.30745600 & 4.74616000 & -1.92621100 & $\mathrm{C}$ & -0.30041000 & 1.62900400 & 3.47951500 \\
\hline $\mathrm{C}$ & -1.77723200 & 2.44807400 & -1.44669700 & $\mathrm{H}$ & -0.78636000 & 0.69702800 & 3.17716800 \\
\hline $\mathrm{H}$ & -2.48847600 & 2.72168400 & -0.66754300 & $\mathrm{H}$ & -0.15468800 & 1.58543500 & 4.56457200 \\
\hline $\mathrm{H}$ & -2.03790100 & 3.01649100 & -2.34689800 & $\mathrm{H}$ & -0.98090600 & 2.46166000 & 3.28505500 \\
\hline $\mathrm{H}$ & -1.90654400 & 1.38943700 & -1.67899900 & $\mathrm{Pd}$ & 0.24547500 & -0.80322400 & 0.54387000 \\
\hline $\mathrm{C}$ & 0.55034100 & 2.23338600 & -2.26138400 & $\mathrm{C}$ & 3.06197400 & -1.97460100 & -0.67228600 \\
\hline $\mathrm{H}$ & 0.49211400 & 1.14316500 & -2.34565900 & $\mathrm{C}$ & 4.44339200 & -1.72537300 & -0.56037400 \\
\hline $\mathrm{H}$ & 0.16051100 & 2.65900300 & -3.19335900 & $\mathrm{C}$ & 2.55308500 & -2.36454200 & -1.92646400 \\
\hline $\mathrm{H}$ & 1.59955600 & 2.51286700 & -2.18233200 & $\mathrm{C}$ & 5.28459800 & -1.85431500 & -1.66296600 \\
\hline $\mathrm{C}$ & -2.08109300 & -0.63897100 & 0.71457400 & $\mathrm{H}$ & 4.86205100 & -1.45383400 & 0.40457300 \\
\hline $\mathrm{O}$ & -2.01572100 & -0.96497100 & 1.87873000 & $\mathrm{C}$ & 3.39499200 & -2.49030500 & -3.02827300 \\
\hline $\mathrm{C}$ & -5.80654000 & -0.59723000 & -0.17367600 & $\mathrm{H}$ & 1.49202900 & -2.56128400 & -2.04487500 \\
\hline $\mathrm{C}$ & -6.95474500 & -0.84350100 & 0.60732800 & $\mathrm{C}$ & 4.76321400 & -2.23374800 & -2.90169700 \\
\hline $\mathrm{C}$ & -5.97276500 & -0.28477700 & -1.54050400 & $\mathrm{H}$ & 6.34829200 & -1.66746800 & -1.55297200 \\
\hline $\mathrm{C}$ & -8.22639600 & -0.77996800 & 0.04555000 & $\mathrm{H}$ & 2.98461200 & -2.78919400 & -3.98791000 \\
\hline $\mathrm{H}$ & -6.83842300 & -1.08621500 & 1.66006200 & $\mathrm{H}$ & 5.41811500 & -2.33470400 & -3.76141100 \\
\hline $\mathrm{C}$ & -7.24256400 & -0.22226100 & -2.09854500 & $\mathrm{C}$ & 2.21537900 & -1.88111500 & 0.52987500 \\
\hline $\mathrm{H}$ & -5.10696000 & -0.09207600 & -2.16618200 & $\mathrm{H}$ & 2.72342800 & -1.54430600 & 1.42847400 \\
\hline $\mathrm{C}$ & -8.37217700 & -0.46937700 & -1.30798800 & $\mathrm{C}$ & 1.14701000 & -2.83021600 & 0.71443200 \\
\hline $\mathrm{H}$ & -9.10073400 & -0.97245600 & 0.65893300 & $\mathrm{C}$ & 0.42497300 & -2.75785200 & 1.87899300 \\
\hline $\mathrm{H}$ & -7.35960900 & 0.01771000 & -3.15065800 & $\mathrm{O}$ & -0.01742600 & -2.93654300 & 2.94156600 \\
\hline $\mathrm{H}$ & -9.36260800 & -0.41961100 & -1.74995600 & $\mathrm{H}$ & 0.87878400 & -3.59876600 & -0.00415900 \\
\hline $\mathrm{H}$ & -3.15589500 & -0.23494900 & -1.13381500 & $\mathrm{C}$ & 2.39381100 & 2.20300700 & -0.06377500 \\
\hline
\end{tabular}




\begin{tabular}{|c|c|c|c|c|c|c|c|}
\hline $\mathrm{C}$ & 2.23767800 & 1.69710500 & -1.51604100 & $\mathrm{H}$ & -3.56195000 & -1.75546500 & -3.50237500 \\
\hline $\mathrm{H}$ & 3.09212200 & 2.04650300 & -2.10746800 & $\mathrm{H}$ & -4.52592500 & -1.79388900 & -2.03165200 \\
\hline $\mathrm{H}$ & 2.22341700 & 0.60583500 & -1.56536300 & $\mathrm{H}$ & -3.89477900 & -0.26069400 & -2.64310100 \\
\hline $\mathrm{H}$ & 1.33003800 & 2.07704300 & -1.99109400 & $\mathrm{C}$ & -2.14764800 & -3.06741700 & -1.57261100 \\
\hline $\mathrm{C}$ & 3.68505800 & 1.61231500 & 0.53401600 & $\mathrm{H}$ & -3.00504100 & -3.55459600 & -1.10209200 \\
\hline $\mathrm{H}$ & 4.51587500 & 1.82191500 & -0.14925000 & $\mathrm{H}$ & -2.02885200 & -3.51487700 & -2.56617800 \\
\hline $\mathrm{H}$ & 3.93784000 & 2.06219700 & 1.49703300 & $\mathrm{H}$ & -1.25312300 & -3.31192400 & -0.99232300 \\
\hline $\mathrm{H}$ & 3.63193100 & 0.53044900 & 0.65332600 & $\mathrm{C}$ & -1.18924800 & -0.97788600 & -2.58090700 \\
\hline $\mathrm{C}$ & 2.54381900 & 3.73800800 & -0.08023800 & $\mathrm{H}$ & -0.21873300 & -1.10587000 & -2.09071900 \\
\hline $\mathrm{H}$ & 2.65141100 & 4.16925600 & 0.91562000 & $\mathrm{H}$ & -1.15442300 & -1.51074500 & -3.53871900 \\
\hline $\mathrm{H}$ & 3.45264700 & 3.98673400 & -0.64033200 & $\mathrm{H}$ & -1.31558000 & 0.08450300 & -2.79646300 \\
\hline $\mathrm{H}$ & 1.71848000 & 4.23912400 & -0.58314000 & & & & \\
\hline $\mathrm{C}$ & -1.16552000 & 2.84707200 & -0.85126600 & 75 & & & \\
\hline $\mathrm{C}$ & -2.27621600 & 2.04400900 & -1.20455100 & $\mathrm{C}$ & -0.60334500 & 2.64765500 & 0.92678600 \\
\hline $\mathrm{C}$ & -0.74715700 & 3.83708700 & -1.75609200 & $\mathrm{H}$ & -1.32530400 & 2.27633600 & 1.65636400 \\
\hline $\mathrm{C}$ & -2.90714700 & 2.27926400 & -2.43805200 & $\mathrm{H}$ & -0.23332500 & 3.59680700 & 1.32529200 \\
\hline $\mathrm{C}$ & -1.35763800 & 4.02646300 & -2.99279200 & $\mathrm{P}$ & 0.83158100 & 1.41813600 & 1.04216200 \\
\hline $\mathrm{H}$ & 0.05611300 & 4.50715100 & -1.47134000 & $\mathrm{C}$ & 1.11149700 & 1.34828100 & 2.95497500 \\
\hline $\mathrm{C}$ & -2.45244200 & 3.23848600 & -3.33817200 & $\mathrm{C}$ & 1.57581000 & 2.68441000 & 3.56718600 \\
\hline $\mathrm{H}$ & -3.80320300 & 1.71951200 & -2.68526200 & $\mathrm{H}$ & 1.57576900 & 2.59019000 & 4.65942700 \\
\hline $\mathrm{H}$ & -1.00054100 & 4.80391900 & -3.66078600 & $\mathrm{H}$ & 2.59261800 & 2.94867100 & 3.26914200 \\
\hline $\mathrm{H}$ & -2.96912700 & 3.38779500 & -4.28098400 & $\mathrm{H}$ & 0.91268900 & 3.51759300 & 3.31785300 \\
\hline $\mathrm{C}$ & -2.91812100 & 1.05441600 & -0.25366200 & $\mathrm{C}$ & 2.13513400 & 0.24538000 & 3.29945300 \\
\hline $\mathrm{H}$ & -3.96876800 & 0.93473300 & -0.53226500 & $\mathrm{H}$ & 2.28517600 & 0.23118500 & 4.38500700 \\
\hline $\mathrm{H}$ & -2.92197500 & 1.47265500 & 0.75579800 & $\mathrm{H}$ & 1.76262000 & -0.74010800 & 3.00613000 \\
\hline$P$ & -2.22746000 & -0.68582100 & -0.00923600 & $\mathrm{H}$ & 3.10987800 & 0.39951800 & 2.83631100 \\
\hline $\mathrm{C}$ & -3.56397800 & -1.39009500 & 1.20477600 & $\mathrm{C}$ & -0.22159500 & 0.93612600 & 3.61639700 \\
\hline $\mathrm{C}$ & -2.35094500 & -1.54615200 & -1.73177300 & $\mathrm{H}$ & -0.62989100 & 0.03296600 & 3.15319900 \\
\hline $\mathrm{C}$ & -4.95094300 & -1.59037900 & 0.56134600 & $\mathrm{H}$ & -0.03758900 & 0.71315000 & 4.67329400 \\
\hline $\mathrm{H}$ & -4.96951000 & -2.44036200 & -0.12400900 & $\mathrm{H}$ & -0.97856600 & 1.72401900 & 3.58588500 \\
\hline $\mathrm{H}$ & -5.67434000 & -1.80646500 & 1.35615600 & $\mathrm{Pd}$ & 0.26355500 & -0.82051000 & 0.32701700 \\
\hline $\mathrm{H}$ & -5.31462900 & -0.70742200 & 0.02851700 & $\mathrm{C}$ & 3.09168500 & -1.67385500 & -0.91034200 \\
\hline $\mathrm{C}$ & -3.71389400 & -0.40125500 & 2.38378700 & $\mathrm{C}$ & 4.46877700 & -1.56846100 & -0.64132200 \\
\hline $\mathrm{H}$ & -2.75041800 & -0.12607300 & 2.82289000 & $\mathrm{C}$ & 2.67465400 & -1.66755600 & -2.25515800 \\
\hline $\mathrm{H}$ & -4.24549500 & 0.51195500 & 2.10514100 & $\mathrm{C}$ & 5.39481100 & -1.44965200 & -1.67651600 \\
\hline $\mathrm{H}$ & -4.30177800 & -0.88541500 & 3.17150500 & $\mathrm{H}$ & 4.81636300 & -1.59636300 & 0.38823900 \\
\hline $\mathrm{C}$ & -3.09013600 & -2.73377400 & 1.79167500 & $\mathrm{C}$ & 3.59997300 & -1.54899800 & -3.28908300 \\
\hline $\mathrm{H}$ & -2.23400600 & -2.59877400 & 2.45154500 & $\mathrm{H}$ & 1.61824400 & -1.74416300 & -2.49622300 \\
\hline $\mathrm{H}$ & -3.89960200 & -3.15984500 & 2.39539900 & $\mathrm{C}$ & 4.96409500 & -1.43771900 & -3.00413400 \\
\hline $\mathrm{H}$ & -2.83455500 & -3.47280100 & 1.02980700 & $\mathrm{H}$ & 6.45309100 & -1.37653200 & -1.44557300 \\
\hline $\mathrm{C}$ & -3.66725300 & -1.31701900 & -2.50289800 & $\mathrm{H}$ & 3.25751500 & -1.54287300 & -4.31941700 \\
\hline
\end{tabular}




\begin{tabular}{|c|c|c|c|c|c|c|c|}
\hline $\mathrm{H}$ & 5.68408100 & -1.34882400 & -3.81154300 & $\mathrm{H}$ & -2.65763700 & -0.39440100 & 2.84072100 \\
\hline $\mathrm{C}$ & 2.14398400 & -1.84219200 & 0.21732300 & $\mathrm{H}$ & -4.27356700 & 0.04989400 & 2.25669500 \\
\hline $\mathrm{H}$ & 2.62662200 & -1.82558000 & 1.19208000 & $\mathrm{H}$ & -4.04735100 & -1.41385400 & 3.20509600 \\
\hline C & 1.10158800 & -2.87767200 & 0.05581700 & C & -2.65296200 & -2.93660500 & 1.60019400 \\
\hline C & 0.69237400 & -3.59177900 & 1.12878900 & $\mathrm{H}$ & -1.73523600 & -2.70838400 & 2.14777400 \\
\hline $\mathrm{O}$ & 0.37560900 & -4.20427300 & 2.05560900 & $\mathrm{H}$ & -3.30306400 & -3.50619300 & 2.27427000 \\
\hline $\mathrm{H}$ & 0.89717700 & -3.33266700 & -0.90923400 & $\mathrm{H}$ & -2.40627800 & -3.59067400 & 0.76013600 \\
\hline $\mathrm{C}$ & 2.30463700 & 2.29539600 & 0.16552800 & $\mathrm{C}$ & -3.86948000 & -1.02334500 & -2.48164000 \\
\hline $\mathrm{C}$ & 2.11172300 & 2.04706700 & -1.34788100 & $\mathrm{H}$ & -3.86303000 & -1.36678100 & -3.52289400 \\
\hline H & 2.91984500 & 2.54724100 & -1.89454300 & $\mathrm{H}$ & -4.68164800 & -1.54424800 & -1.97564000 \\
\hline $\mathrm{H}$ & 2.15475200 & 0.98318700 & -1.59055300 & $\mathrm{H}$ & -4.10263900 & 0.04085000 & -2.50216800 \\
\hline $\mathrm{H}$ & 1.16492300 & 2.44713100 & -1.71804700 & $\mathrm{C}$ & -2.23288000 & -2.83239700 & -1.92451500 \\
\hline $\mathrm{C}$ & 3.64360000 & 1.67569800 & 0.60684400 & $\mathrm{H}$ & -3.00486100 & -3.40755300 & -1.40837500 \\
\hline $\mathrm{H}$ & 4.43292400 & 2.03801500 & -0.06159700 & $\mathrm{H}$ & -2.23524100 & -3.15377000 & -2.97229700 \\
\hline $\mathrm{H}$ & 3.92006200 & 1.97104500 & 1.62208200 & $\mathrm{H}$ & -1.26254000 & -3.10220900 & -1.49981000 \\
\hline $\mathrm{H}$ & 3.64189700 & 0.58861000 & 0.54053900 & $\mathrm{C}$ & -1.41311800 & -0.60526000 & -2.72737300 \\
\hline C & 2.38543000 & 3.81441700 & 0.42321900 & $\mathrm{H}$ & -0.40409900 & -0.77196900 & -2.33614000 \\
\hline $\mathrm{H}$ & 2.50573200 & 4.06361600 & 1.47803500 & $\mathrm{H}$ & -1.45241500 & -1.00386100 & -3.74828200 \\
\hline $\mathrm{H}$ & 3.26450200 & 4.20233500 & -0.10413200 & $\mathrm{H}$ & -1.57361400 & 0.47304500 & -2.78408300 \\
\hline $\mathrm{H}$ & 1.52363400 & 4.35859800 & 0.04090000 & & & & \\
\hline $\mathrm{C}$ & -1.31440400 & 2.91335400 & -0.38941900 & \multirow{2}{*}{\multicolumn{4}{|c|}{$\begin{array}{l}\text { TS58 } \\
\text { imaginary frequency }=-30.27 \mathrm{~cm}^{-1}\end{array}$}} \\
\hline $\mathrm{C}$ & -2.43765100 & 2.16439100 & -0.81474800 & & & & \\
\hline $\mathrm{C}$ & -0.94475500 & 4.03802800 & -1.14601600 & C & 4.63262800 & -1.14012200 & -0.94628400 \\
\hline $\mathrm{C}$ & -3.12423000 & 2.57656200 & -1.96895700 & C & 3.58182400 & -0.86034600 & -0.05607100 \\
\hline $\mathrm{C}$ & -1.61383300 & 4.41037400 & -2.30894400 & C & 2.97841200 & -1.93826100 & 0.63637800 \\
\hline $\mathrm{H}$ & -0.13090600 & 4.66419500 & -0.79944300 & C & 3.47010000 & -3.23322800 & 0.39969300 \\
\hline $\mathrm{C}$ & -2.71728700 & 3.67091400 & -2.72650200 & C & 4.48278600 & -3.49595400 & -0.51849000 \\
\hline $\mathrm{H}$ & -4.02408400 & 2.04564100 & -2.26286600 & C & 5.07133200 & -2.43584500 & -1.20227500 \\
\hline $\mathrm{H}$ & -1.29252800 & 5.28760300 & -2.86159600 & $\mathrm{H}$ & 5.15136600 & -0.31389700 & -1.42079600 \\
\hline $\mathrm{H}$ & -3.27562000 & 3.95820000 & -3.61189700 & $\mathrm{H}$ & 3.07626800 & -4.05945000 & 0.97987700 \\
\hline $\mathrm{C}$ & -3.02466600 & 1.02685600 & -0.00548200 & $\mathrm{H}$ & 4.82796400 & -4.51407500 & -0.66883600 \\
\hline $\mathrm{H}$ & -4.08075400 & 0.91083100 & -0.26881500 & $\mathrm{H}$ & 5.88607000 & -2.60688900 & -1.89886800 \\
\hline $\mathrm{H}$ & -3.00731400 & 1.29073000 & 1.05444700 & C & 1.95141300 & -1.74346600 & 1.73829100 \\
\hline$P$ & -2.22783200 & -0.68054500 & -0.06207300 & $\mathrm{H}$ & 2.05983000 & -2.55680900 & 2.46229800 \\
\hline $\mathrm{C}$ & -3.37442800 & -1.64674700 & 1.16534000 & C & 3.27273100 & 0.59274700 & 0.24443300 \\
\hline $\mathrm{C}$ & -2.48578500 & -1.31233100 & -1.86650300 & $\mathrm{H}$ & 3.14470000 & 0.70867800 & 1.32381100 \\
\hline $\mathrm{C}$ & -4.76166400 & -2.00318400 & 0.59670400 & $\mathrm{H}$ & 4.14522800 & 1.19634200 & -0.02262600 \\
\hline $\mathrm{H}$ & -4.71073800 & -2.74822300 & -0.19972200 & $\mathrm{H}$ & 2.17984000 & -0.82344200 & 2.28036000 \\
\hline $\mathrm{H}$ & -5.36950500 & -2.43446000 & 1.40076300 & $\mathrm{P}$ & 0.09978800 & -1.59046300 & 1.35421000 \\
\hline $\mathrm{H}$ & -5.29997700 & -1.12869100 & 0.22065700 & $\mathrm{P}$ & 1.74919100 & 1.44673500 & -0.47998000 \\
\hline $\mathrm{C}$ & -3.59192300 & -0.78632900 & 2.43090400 & C & 1.99917100 & 3.21554300 & 0.27874200 \\
\hline
\end{tabular}




\begin{tabular}{|c|c|c|c|c|c|c|c|}
\hline $\mathrm{C}$ & 2.04875900 & 1.50388900 & -2.38629000 & $\mathrm{H}$ & 0.86661600 & 5.04753600 & 0.49102200 \\
\hline $\mathrm{C}$ & -0.59437200 & -1.28658200 & 3.13690500 & $\mathrm{H}$ & 0.46849800 & 4.18227900 & -0.99103400 \\
\hline $\mathrm{C}$ & -0.42088000 & -3.33982500 & 0.74484300 & $\mathrm{C}$ & 3.50083900 & 1.77662100 & -2.82606300 \\
\hline $\mathrm{Pd}$ & -0.46925500 & 0.28858700 & -0.12606400 & $\mathrm{H}$ & 3.88388500 & 2.73620800 & -2.47864500 \\
\hline $\mathrm{H}$ & -3.19367500 & 0.20875500 & 0.13639500 & $\mathrm{H}$ & 4.18516700 & 0.99333600 & -2.50352400 \\
\hline $\mathrm{C}$ & -2.57726800 & 0.06120900 & -0.74451400 & $\mathrm{H}$ & 3.53283700 & 1.79358100 & -3.92204600 \\
\hline $\mathrm{C}$ & -2.04158700 & 1.24971700 & -1.35296900 & $\mathrm{C}$ & 1.14196200 & 2.57140700 & -3.03253500 \\
\hline $\mathrm{C}$ & -2.07099100 & -0.85032300 & 3.03086200 & $\mathrm{H}$ & 1.50088200 & 3.58671500 & -2.84617800 \\
\hline $\mathrm{H}$ & -2.45441400 & -0.63778400 & 4.03612300 & $\mathrm{H}$ & 1.14458800 & 2.42634400 & -4.11912600 \\
\hline $\mathrm{H}$ & -2.17957000 & 0.05616500 & 2.43162800 & $\mathrm{H}$ & 0.10580000 & 2.51077700 & -2.69411700 \\
\hline $\mathrm{H}$ & -2.70553300 & -1.62297500 & 2.59474900 & $\mathrm{C}$ & 1.63470500 & 0.11291700 & -2.91798300 \\
\hline $\mathrm{C}$ & -0.49835600 & -2.49420800 & 4.09107200 & $\mathrm{H}$ & 0.58841900 & -0.11030400 & -2.69001800 \\
\hline $\mathrm{H}$ & 0.51768300 & -2.89109200 & 4.17073100 & $\mathrm{H}$ & 1.75701900 & 0.09314000 & -4.00765700 \\
\hline $\mathrm{H}$ & -0.79857300 & -2.17430200 & 5.09632700 & $\mathrm{H}$ & 2.24756800 & -0.68799000 & -2.49851700 \\
\hline $\mathrm{H}$ & -1.16685300 & -3.30729100 & 3.80234100 & $\mathrm{C}$ & -2.33673500 & 2.57917200 & -0.99283300 \\
\hline $\mathrm{C}$ & 0.20294600 & -0.12771700 & 3.77855900 & $\mathrm{O}$ & -3.27217900 & 2.56229700 & 0.45947200 \\
\hline $\mathrm{H}$ & 0.33600100 & 0.71717900 & 3.09478100 & $\mathrm{O}$ & -2.19356500 & 3.68055800 & -1.39981800 \\
\hline $\mathrm{H}$ & -0.33884800 & 0.23484600 & 4.65942400 & $\mathrm{C}$ & -1.39110400 & 3.67562400 & 3.37512100 \\
\hline $\mathrm{H}$ & 1.19470300 & -0.43892500 & 4.11655200 & $\mathrm{H}$ & -0.89549200 & 3.27225400 & 4.26198500 \\
\hline $\mathrm{C}$ & -0.02321400 & -3.40188600 & -0.74773700 & $\mathrm{H}$ & -2.34977700 & 4.10348700 & 3.67007900 \\
\hline $\mathrm{H}$ & 1.05455300 & -3.29530500 & -0.89277600 & $\mathrm{H}$ & -0.76917100 & 4.45123300 & 2.91867200 \\
\hline $\mathrm{H}$ & -0.31866000 & -4.37665500 & -1.15383900 & $\mathrm{O}$ & -1.67907700 & 2.61642800 & 2.44275700 \\
\hline $\mathrm{H}$ & -0.53106100 & -2.63153200 & -1.33128100 & $\mathrm{H}$ & -2.63569500 & 2.63456100 & 1.25492700 \\
\hline $\mathrm{C}$ & 0.23704000 & -4.52432600 & 1.48155100 & $\mathrm{H}$ & -0.87139900 & 2.13955800 & 2.19510000 \\
\hline $\mathrm{H}$ & 0.01687300 & -4.54647900 & 2.54870800 & $\mathrm{C}$ & -3.03465000 & -1.05681200 & -1.60126500 \\
\hline $\mathrm{H}$ & -0.15684700 & -5.45254000 & 1.05116100 & $\mathrm{C}$ & -2.41634000 & -1.40241900 & -2.81842800 \\
\hline $\mathrm{H}$ & 1.31743800 & -4.55401100 & 1.35458700 & $\mathrm{C}$ & -4.19337600 & -1.76387000 & -1.22820800 \\
\hline $\mathrm{C}$ & -1.94811400 & -3.50015200 & 0.86183300 & $\mathrm{C}$ & -2.93388800 & -2.41469000 & -3.62287200 \\
\hline $\mathrm{H}$ & -2.48820900 & -2.64968900 & 0.44832900 & $\mathrm{H}$ & -1.51879200 & -0.88225300 & -3.13933800 \\
\hline $\mathrm{H}$ & -2.25471000 & -4.38574300 & 0.29340600 & $\mathrm{C}$ & -4.71154300 & -2.77764800 & -2.03242600 \\
\hline $\mathrm{H}$ & -2.26843800 & -3.65508100 & 1.89530600 & $\mathrm{H}$ & -4.69876200 & -1.50322600 & -0.30131900 \\
\hline $\mathrm{C}$ & 2.20275200 & 3.06134900 & 1.80324400 & $\mathrm{C}$ & -4.08302900 & -3.10871300 & -3.23399700 \\
\hline $\mathrm{H}$ & 1.46076900 & 2.39675400 & 2.26035300 & $\mathrm{H}$ & -2.43941700 & -2.66328400 & -4.55725600 \\
\hline $\mathrm{H}$ & 3.19383900 & 2.68054500 & 2.06074400 & $\mathrm{H}$ & -5.61065500 & -3.30276800 & -1.72411600 \\
\hline $\mathrm{H}$ & 2.10612800 & 4.04433300 & 2.27869400 & $\mathrm{H}$ & -4.48570300 & -3.89558000 & -3.86413700 \\
\hline $\mathrm{C}$ & 3.20388100 & 3.99611300 & -0.28249000 & $\mathrm{H}$ & -1.72029700 & 1.21609300 & -2.39109300 \\
\hline $\mathrm{H}$ & 3.33894300 & 4.91191500 & 0.30581400 & $\mathrm{C}$ & -4.23573700 & 3.64828100 & 0.50547500 \\
\hline $\mathrm{H}$ & 4.14033500 & 3.43472200 & -0.22066600 & $\mathrm{H}$ & -3.76710000 & 4.58711300 & 0.20492200 \\
\hline $\mathrm{H}$ & 3.05197300 & 4.30318800 & -1.31916400 & $\mathrm{H}$ & -5.04622400 & 3.39424000 & -0.17688800 \\
\hline $\mathrm{C}$ & 0.72225500 & 4.04893700 & 0.06020900 & $\mathrm{H}$ & -4.61296800 & 3.70554900 & 1.52733500 \\
\hline $\mathrm{H}$ & -0.14145500 & 3.59582500 & 0.55262300 & & & & \\
\hline
\end{tabular}


TS58a

imaginary frequency $=-152.10 \mathrm{~cm}^{-1}$

$\mathrm{Pd}$

P

$\mathrm{P}$

C

C

C

C

C

C

C

C

C

C

C

C

$\mathrm{H}$

$\mathrm{H}$

$\mathrm{H}$

$\mathrm{H}$

$\mathrm{H}$

$\mathrm{H}$

$\mathrm{H}$

$\mathrm{H}$

C

$\mathrm{O}$

$\mathrm{O}$

C

$\mathrm{H}$

$\mathrm{H}$

$\mathrm{H}$

$\mathrm{H}$

C

C

C

C

C

C

C

C

$-1.34553900 \quad 3.07439500$

$1.07277200 \quad 3.60478500$

$3.48611400-2.03055000$

$1.14164800 \quad-3.16881900$

$1.57496700 \quad 2.38123400$

2.73183900

1.94590900

$-0.57573200$

0.11933800

1.85849700

$-0.57159600$

1.26507200

0.76517600

0.87299100

$-0.02951000$

1.26956300

1.98407800

1.44400800

1.43522700

3.46155800

$-1.23130000$

0.15765200

3.31140800

2.50766100

2.16523800

1.66420400

3.40637100

2.29794900

$-1.56299200$

1.22394200

$-0.60267100$

1.73462600

0.32448300

3.01486400

0.53459500

$-1.48889100$

$-0.38313900$

$-1.56010400$

$-0.22191300$

0.53737800

$-1.55544300$

0.34469500

$-1.23886500$

1.29653100

$-1.76367400$

$-0.35708900$

0.48139200

$-1.60648700$

$-0.16094800$

$-1.35279000$

0.43599300

1.05741700

5.08078700

3.1747290

2.53976100

0.33753000

3.45833100

4.31745700

$-1.19625200$

$-2.31340100$

3.40463900

$-1.96956900$

1.92701100

2.12414500

$-3.99519500$

$-2.67426600$ $\begin{array}{lll}0.44637100 & 0.06367300 & -0.68229800\end{array}$

$0.28576000 \quad 2.37378200 \quad-0.09927600$

$\begin{array}{lll}1.90556900 & -1.60033300 & 0.06813800\end{array}$
0.63222900

$-1.35813700$

$-0.96492900$

0.87890200

1.30602300

1.45411300

2.55415500

3.77823500

4.91248000

4.85589300

3.66790000

2.49574000

1.93671900

0.88586300

0.78156700

1.68093500

3.84514500

5.83348200

5.73223600

3.65302700

$-2.56161400$

$-3.29361100$

$-3.06345800$

$-4.36374600$

$-4.44955300$

$-5.11102900$

$-4.46695300$

$-2.32269100$

$-0.91914600$

$-1.58659400$

$-2.70799100$

1.53036500

$-0.52254200$

1.46031700

1.72742400

1.76196400
$0.52832700 \quad-4.07593100 \quad-0.21083500$

$4.72313300 \quad-2.39729600 \quad-0.11704200$

$3.20662000 \quad-3.18476400 \quad-1.94796500$

$3.82381400 \quad-0.77027100 \quad-1.79350900$

$\begin{array}{lll}2.93194500 & 3.71060900 & -2.45838800\end{array}$

$\begin{array}{lll}3.18769900 & 3.42439600 & -0.74210000\end{array}$

$\begin{array}{lll}2.62371400 & 2.10210700 & -1.79010600\end{array}$

$0.82712400 \quad 4.09746400 \quad-3.45233400$

$\begin{array}{lll}0.37100800 & 2.42790400 & -3.07179700\end{array}$

$\begin{array}{lll}-0.70952100 & 3.75982900 & -2.65895400\end{array}$

$\begin{array}{lll}1.64285600 & 5.67284800 & -1.63282700\end{array}$

$0.04843700 \quad 5.49983100 \quad-0.90906200$

$\begin{array}{lll}1.50439500 & 5.23205700 & 0.06828500\end{array}$

$\begin{array}{lll}-2.93233400 & 2.26258400 \quad 1.86485700\end{array}$

$\begin{array}{lll}-2.15087600 & 1.04174000 & 0.84425300\end{array}$

$\begin{array}{lll}-1.34164000 & 1.62791400 \quad 2.30162800\end{array}$

$\begin{array}{lll}-2.18154000 & 4.58600100 & 1.93028700\end{array}$

$\begin{array}{lll}-0.54390800 & 4.13999600 & 2.38688800\end{array}$

$\begin{array}{lll}-0.81792800 & 5.18676800 & 0.99171000\end{array}$

$\begin{array}{lll}-3.31312000 & 3.58202600 & -0.10851400\end{array}$

$\begin{array}{lll}-2.02543900 & 4.30867600 & -1.06418900\end{array}$

$-2.38823400 \quad 2.58297500 \quad-1.24182400$

$\begin{array}{lll}-0.08135300 & -4.84564500 & 0.27647400\end{array}$

$\begin{array}{lll}-0.12535400 & -3.51972000 & -0.89075500\end{array}$

$\begin{array}{lll}1.27616700 & -4.59260500 & -0.81233000\end{array}$

$\begin{array}{lll}-0.56681000 & -3.54555800 & 2.15346400\end{array}$

$\begin{array}{lll}0.30219700 & -2.07853600 & 2.61220500\end{array}$

$\begin{array}{lll}-0.73233100 & -2.06519800 & 1.18619100\end{array}$

$\begin{array}{lll}1.57631600 & -4.78987000 & 2.24769600\end{array}$

$2.88851900-4.48009900 \quad 1.11505100$

$\begin{array}{lll}2.62722400 & -3.39667700 & 2.49127700\end{array}$

$4.71914500 \quad-0.96566800 \quad-2.39545600$

$3.01042900 \quad-0.49641100 \quad-2.47248500$

$4.03858800 \quad 0.10045100 \quad-1.16740600$

$\begin{array}{lll}4.01757600 & -3.23046100 & -2.68391400\end{array}$

$3.17363200 \quad-4.15542200 \quad-1.44856900$

$\begin{array}{lll}2.26903800 & -3.05519600 & -2.49902300\end{array}$

$5.54155900 \quad-2.67764300 \quad-0.79047400$

$\begin{array}{lll}5.08029100 & -1.55842700 & 0.48471500\end{array}$

$4.54502600 \quad-3.24510400 \quad 0.54724600$

$\begin{array}{lll}-2.27645000 & -1.10155000 & -1.61385200\end{array}$ 


\begin{tabular}{|c|c|c|c|c|c|c|c|}
\hline $\mathrm{H}$ & -1.87984300 & -2.02898000 & -1.22957100 & $\mathrm{C}$ & -3.95816200 & -1.26893400 & -0.46194700 \\
\hline $\mathrm{C}$ & -3.50188500 & -0.59853300 & -1.28055600 & $\mathrm{C}$ & -2.71414200 & -1.47226500 & -0.96948200 \\
\hline $\mathrm{H}$ & -3.80420100 & 0.32982800 & -1.76134300 & $\mathrm{C}$ & 4.03336200 & -3.06847900 & -0.51528700 \\
\hline $\mathrm{C}$ & -4.46553500 & -1.17231700 & -0.36655200 & $\mathrm{H}$ & 4.69828000 & -3.65346200 & 0.13154800 \\
\hline $\mathrm{C}$ & -4.21367600 & -2.33597000 & 0.39413800 & $\mathrm{H}$ & 3.02085000 & -3.46745300 & -0.40652400 \\
\hline $\mathrm{C}$ & -5.71999200 & -0.53727900 & -0.23856400 & $\mathrm{H}$ & 4.35531400 & -3.24347900 & -1.54370700 \\
\hline $\mathrm{C}$ & -5.18625600 & -2.84159400 & 1.24568800 & $\mathrm{C}$ & 5.52750800 & -1.06226900 & -0.52812700 \\
\hline $\mathrm{H}$ & -3.25485000 & -2.83910500 & 0.32204600 & $\mathrm{H}$ & 5.73180400 & -0.06048600 & -0.14295900 \\
\hline $\mathrm{C}$ & -6.69243400 & -1.04874900 & 0.61402200 & $\mathrm{H}$ & 6.28735700 & -1.72798800 & -0.10110800 \\
\hline $\mathrm{H}$ & -5.92423400 & 0.35788100 & -0.81962900 & $\mathrm{H}$ & 5.67817000 & -1.05111600 & -1.60926800 \\
\hline $\mathrm{C}$ & -6.42765600 & -2.20181800 & 1.35679900 & $\mathrm{C}$ & 4.00996000 & -1.47731800 & 1.41062800 \\
\hline $\mathrm{H}$ & -4.98396800 & -3.73541400 & 1.82716600 & $\mathrm{H}$ & 3.05551100 & -1.87317900 & 1.76984700 \\
\hline $\mathrm{H}$ & -7.65369500 & -0.55256500 & 0.70006700 & $\mathrm{H}$ & 4.81659500 & -2.05382700 & 1.87886000 \\
\hline \multirow[t]{2}{*}{$\mathrm{H}$} & -7.18476200 & -2.60328400 & 2.02327800 & $\mathrm{H}$ & 4.09906700 & -0.44917100 & 1.77276200 \\
\hline & & & & $\mathrm{C}$ & 1.31461000 & 0.05813400 & -3.21881000 \\
\hline \multicolumn{4}{|c|}{ TS58b } & $\mathrm{H}$ & 1.53489100 & 1.11961000 & -3.10824600 \\
\hline \multicolumn{4}{|c|}{ imaginary frequency $=-61.51 \mathrm{~cm}^{-1}$} & $\mathrm{H}$ & 1.11895300 & -0.13477700 & -4.28049700 \\
\hline $\mathrm{C}$ & 1.12105300 & 4.28636900 & -0.45381600 & $\mathrm{H}$ & 0.39392500 & -0.15980600 & -2.66686100 \\
\hline $\mathrm{C}$ & 1.66749900 & 3.04669500 & -0.07542800 & $\mathrm{C}$ & 3.73845200 & -0.56048800 & -3.57428300 \\
\hline $\mathrm{C}$ & 2.56042600 & 2.41179700 & -0.97256200 & $\mathrm{H}$ & 4.53599500 & -1.28031400 & -3.37202500 \\
\hline $\mathrm{C}$ & 2.84263100 & 3.04498500 & -2.19677200 & $\mathrm{H}$ & 3.50193600 & -0.63602700 & -4.64246900 \\
\hline $\mathrm{C}$ & 2.25864200 & 4.25125300 & -2.56875900 & $\mathrm{H}$ & 4.13356300 & 0.44413800 & -3.40281200 \\
\hline $\mathrm{C}$ & 1.38829200 & 4.88214800 & -1.68241700 & $\mathrm{C}$ & 2.01970400 & -2.31088700 & -3.02914900 \\
\hline $\mathrm{H}$ & 0.48883100 & 4.81654300 & 0.25032800 & $\mathrm{H}$ & 1.15398000 & -2.58734100 & -2.42361600 \\
\hline $\mathrm{H}$ & 3.56260200 & 2.58343800 & -2.86668700 & $\mathrm{H}$ & 1.72579300 & -2.38599300 & -4.08304500 \\
\hline $\mathrm{H}$ & 2.50570500 & 4.70505700 & -3.52346900 & $\mathrm{H}$ & 2.81344500 & -3.04077800 & -2.86859200 \\
\hline $\mathrm{H}$ & 0.94338500 & 5.84114900 & -1.92917500 & $\mathrm{C}$ & 1.72878800 & 0.65939500 & 3.83419500 \\
\hline $\mathrm{C}$ & 3.36148600 & 1.16735400 & -0.64423500 & $\mathrm{H}$ & 2.09666600 & -0.13064100 & 3.17358100 \\
\hline $\mathrm{H}$ & 4.29112300 & 1.21129600 & -1.21692600 & $\mathrm{H}$ & 2.38726500 & 1.52670300 & 3.73914100 \\
\hline $\mathrm{C}$ & 1.38459200 & 2.54124200 & 1.32797700 & $\mathrm{H}$ & 1.82227100 & 0.29997600 & 4.86540100 \\
\hline $\mathrm{H}$ & 2.28494200 & 2.06936700 & 1.72718600 & $\mathrm{C}$ & -0.12865000 & 2.23763800 & 4.40182300 \\
\hline $\mathrm{H}$ & 1.17945700 & 3.40129400 & 1.97290000 & $\mathrm{H}$ & 0.18636200 & 2.06798400 & 5.43843300 \\
\hline $\mathrm{H}$ & 3.65814900 & 1.18804500 & 0.40957800 & $\mathrm{H}$ & 0.36299100 & 3.15331200 & 4.06167400 \\
\hline $\mathrm{P}$ & 2.64259300 & -0.57248100 & -0.85691200 & $\mathrm{H}$ & -1.20670800 & 2.41081800 & 4.42030800 \\
\hline $\mathrm{P}$ & 0.03259900 & 1.24734300 & 1.64756900 & $\mathrm{C}$ & -0.59742900 & -0.19686100 & 4.03356400 \\
\hline $\mathrm{C}$ & 0.24780100 & 1.00376400 & 3.55844900 & $\mathrm{H}$ & -0.25965700 & -1.13151700 & 3.58174900 \\
\hline $\mathrm{C}$ & -1.63413000 & 2.14115200 & 1.27426100 & $\mathrm{H}$ & -0.48414900 & -0.30049800 & 5.11936600 \\
\hline $\mathrm{C}$ & 4.13005500 & -1.58018700 & -0.12547500 & $\mathrm{H}$ & -1.66176700 & -0.07979100 & 3.82648300 \\
\hline $\mathrm{C}$ & 2.47104300 & -0.85875600 & -2.75406700 & $\mathrm{C}$ & -1.76165200 & 3.58700500 & 1.79422200 \\
\hline $\mathrm{Pd}$ & 0.40131800 & -0.63070300 & 0.21429400 & $\mathrm{H}$ & -1.77800900 & 3.64808900 & 2.88225100 \\
\hline $\mathrm{H}$ & -4.16054700 & -1.63418600 & 0.54276000 & $\mathrm{H}$ & -0.96751600 & 4.24020700 & 1.43177500 \\
\hline
\end{tabular}




\begin{tabular}{|c|c|c|c|c|c|c|c|}
\hline $\mathrm{H}$ & -2.70867200 & 4.00523000 & 1.43264900 & $\mathrm{C}$ & 4.86785300 & -3.13607700 & -0.71791800 \\
\hline $\mathrm{C}$ & -2.79393200 & 1.30569800 & 1.85086700 & $\mathrm{C}$ & 5.33264100 & -1.98041500 & -1.33929900 \\
\hline $\mathrm{H}$ & -2.86636700 & 1.39152700 & 2.93808600 & $\mathrm{H}$ & 5.18731600 & 0.14713500 & -1.42449800 \\
\hline $\mathrm{H}$ & -3.73959100 & 1.67065500 & 1.43310300 & $\mathrm{H}$ & 3.51040900 & -3.93616300 & 0.72130000 \\
\hline $\mathrm{H}$ & -2.69949200 & 0.24907500 & 1.58579500 & $\mathrm{H}$ & 5.33719800 & -4.09629600 & -0.90761300 \\
\hline $\mathrm{C}$ & -1.77349500 & 2.15294700 & -0.26603400 & $\mathrm{H}$ & 6.16713600 & -2.01878800 & -2.03242500 \\
\hline $\mathrm{H}$ & -1.76753500 & 1.13792000 & -0.67243000 & $\mathrm{C}$ & 2.05966700 & -1.83314500 & 1.51079800 \\
\hline $\mathrm{H}$ & -2.73243800 & 2.61349500 & -0.53276000 & $\mathrm{H}$ & 2.19573000 & -2.71059400 & 2.15020700 \\
\hline $\mathrm{H}$ & -0.98135400 & 2.72487500 & -0.75272300 & $\mathrm{C}$ & 3.25399700 & 0.73826300 & 0.32537200 \\
\hline $\mathrm{C}$ & -1.64744700 & -2.18362000 & -0.31007100 & $\mathrm{H}$ & 3.13491600 & 0.71910500 & 1.41108300 \\
\hline $\mathrm{O}$ & -0.54257300 & -2.47402100 & -0.80564500 & $\mathrm{H}$ & 4.08187900 & 1.42452800 & 0.12612100 \\
\hline $\mathrm{C}$ & -2.64560300 & -4.14412100 & 0.73031800 & $\mathrm{H}$ & 2.17008100 & -0.96020600 & 2.15968600 \\
\hline $\mathrm{H}$ & -3.58831400 & -4.00055800 & 0.20138700 & $\mathrm{P}$ & 0.22020900 & -1.79458600 & 1.02883300 \\
\hline $\mathrm{H}$ & -2.83515300 & -4.54997800 & 1.72457600 & $\mathrm{P}$ & 1.67600000 & 1.57785200 & -0.28204500 \\
\hline $\mathrm{H}$ & -1.99445000 & -4.81107500 & 0.15807100 & $\mathrm{C}$ & 1.78414900 & 3.20162500 & 0.77159700 \\
\hline $\mathrm{O}$ & -1.99960800 & -2.85194900 & 0.92941400 & $\mathrm{C}$ & 2.00389100 & 1.95923800 & -2.14228400 \\
\hline $\mathrm{H}$ & -0.75336700 & -2.95295100 & 1.64492000 & $\mathrm{C}$ & -0.56195600 & -2.08845100 & 2.77776100 \\
\hline $\mathrm{C}$ & 0.96416600 & -4.13995700 & 1.65616600 & $\mathrm{C}$ & -0.06761400 & -3.33929800 & -0.08652600 \\
\hline $\mathrm{H}$ & 0.49196700 & -4.96132500 & 2.19423000 & $\mathrm{Pd}$ & -0.49468700 & 0.31514000 & -0.06070100 \\
\hline $\mathrm{H}$ & 1.99227500 & -4.01757900 & 1.98989900 & $\mathrm{H}$ & -3.20103300 & 0.40129100 & 0.37697000 \\
\hline $\mathrm{H}$ & 0.90968900 & -4.27960500 & 0.57641100 & $\mathrm{C}$ & -2.65605400 & 0.24033600 & -0.54968200 \\
\hline $\mathrm{O}$ & 0.23675100 & -2.92382600 & 2.02746600 & $\mathrm{C}$ & -2.06868000 & 1.39937100 & -1.16021000 \\
\hline $\mathrm{H}$ & 0.60431800 & -2.06897900 & 1.53178300 & $\mathrm{C}$ & -2.08281200 & -1.83758100 & 2.71614000 \\
\hline $\mathrm{C}$ & -5.07098800 & -0.59207200 & -1.11136400 & $\mathrm{H}$ & -2.50728900 & -1.98084900 & 3.71728300 \\
\hline $\mathrm{C}$ & -6.27576000 & -0.44129300 & -0.39428100 & $\mathrm{H}$ & -2.31388700 & -0.81913300 & 2.39870200 \\
\hline $\mathrm{C}$ & -5.00655700 & -0.08146200 & -2.42576500 & $\mathrm{H}$ & -2.59571400 & -2.52399400 & 2.04163700 \\
\hline $\mathrm{C}$ & -7.37324300 & 0.19941300 & -0.96252600 & $\mathrm{C}$ & -0.31642400 & -3.49315300 & 3.36362900 \\
\hline $\mathrm{H}$ & -6.34318300 & -0.83234500 & 0.61773700 & $\mathrm{H}$ & 0.74241000 & -3.76534900 & 3.38784900 \\
\hline $\mathrm{C}$ & -6.10349300 & 0.55691300 & -2.99120700 & $\mathrm{H}$ & -0.67585700 & -3.51255800 & 4.39958500 \\
\hline $\mathrm{H}$ & -4.09826100 & -0.19039000 & -3.00997200 & $\mathrm{H}$ & -0.86028400 & -4.26895600 & 2.82154500 \\
\hline $\mathrm{C}$ & -7.29027700 & 0.70111800 & -2.26290000 & $\mathrm{C}$ & 0.05707100 & -1.05637200 & 3.74810000 \\
\hline $\mathrm{H}$ & -8.29196500 & 0.30582500 & -0.39416900 & $\mathrm{H}$ & 0.06432500 & -0.04348400 & 3.32983200 \\
\hline $\mathrm{H}$ & -6.03899300 & 0.94203700 & -4.00421900 & $\mathrm{H}$ & -0.53208600 & -1.03425900 & 4.67234200 \\
\hline $\mathrm{H}$ & -8.14490700 & 1.19913800 & -2.71031500 & $\mathrm{H}$ & 1.08326200 & -1.30689300 & 4.02752800 \\
\hline \multirow[t]{2}{*}{$\mathrm{H}$} & -2.43677400 & -1.10867800 & -1.95352300 & $\mathrm{C}$ & 0.36184200 & -2.91741700 & -1.50973500 \\
\hline & & & & $\mathrm{H}$ & 1.41901800 & -2.64456800 & -1.56003500 \\
\hline 76 & & & & $\mathrm{H}$ & 0.20383200 & -3.76051100 & -2.19295100 \\
\hline $\mathrm{C}$ & 4.75230500 & -0.75769800 & -1.01356900 & $\mathrm{H}$ & -0.23482200 & -2.07755400 & -1.87400300 \\
\hline $\mathrm{C}$ & 3.66985000 & -0.64871700 & -0.12408400 & $\mathrm{C}$ & 0.71309600 & -4.60988200 & 0.30464400 \\
\hline $\mathrm{C}$ & 3.16150200 & -1.83026300 & 0.46760700 & $\mathrm{H}$ & 0.53757000 & -4.92933400 & 1.33231800 \\
\hline $\mathrm{C}$ & 3.81303200 & -3.04326800 & 0.18580500 & $\mathrm{H}$ & 0.37762300 & -5.42517400 & -0.34740200 \\
\hline
\end{tabular}




\begin{tabular}{|c|c|c|c|c|c|c|c|}
\hline $\mathrm{H}$ & 1.78325600 & -4.50228500 & 0.14841900 & $\mathrm{C}$ & -4.36119000 & -1.53764600 & -0.85234500 \\
\hline $\mathrm{C}$ & -1.56593700 & -3.69507100 & -0.10655500 & $\mathrm{C}$ & -3.44753500 & -2.15671900 & -3.40642900 \\
\hline $\mathrm{H}$ & -2.20145500 & -2.82980400 & -0.28492700 & $\mathrm{H}$ & -1.95564500 & -0.65298600 & -3.09337800 \\
\hline $\mathrm{H}$ & -1.74549200 & -4.40462500 & -0.92219000 & $\mathrm{C}$ & -5.00122100 & -2.52642900 & -1.59794500 \\
\hline $\mathrm{H}$ & -1.88434600 & -4.18432700 & 0.81781100 & $\mathrm{H}$ & -4.72958000 & -1.29196500 & 0.14078400 \\
\hline $\mathrm{C}$ & 1.93232100 & 2.79611800 & 2.25668400 & $\mathrm{C}$ & -4.54623200 & -2.84175200 & -2.87929600 \\
\hline $\mathrm{H}$ & 1.22205500 & 2.01171000 & 2.54306800 & $\mathrm{H}$ & -3.08989100 & -2.39115100 & -4.40472200 \\
\hline $\mathrm{H}$ & 2.93855300 & 2.44900400 & 2.50258600 & $\mathrm{H}$ & -5.85957700 & -3.04454500 & -1.18078100 \\
\hline $\mathrm{H}$ & 1.73766700 & 3.67167000 & 2.88749900 & $\mathrm{H}$ & -5.04460000 & -3.60809700 & -3.46468100 \\
\hline $\mathrm{C}$ & 2.95396200 & 4.13604500 & 0.40597800 & $\mathrm{H}$ & -1.79998700 & 1.34975000 & -2.21176100 \\
\hline $\mathrm{H}$ & 3.00246500 & 4.95120400 & 1.13842700 & $\mathrm{C}$ & -3.56183400 & 4.24345600 & 0.84902200 \\
\hline $\mathrm{H}$ & 3.92336100 & 3.63057400 & 0.42658000 & $\mathrm{H}$ & -2.70994300 & 4.90440800 & 1.01158200 \\
\hline $\mathrm{H}$ & 2.82499900 & 4.59624000 & -0.57595600 & $\mathrm{H}$ & -4.15816400 & 4.60089600 & 0.01315100 \\
\hline $\mathrm{C}$ & 0.46470500 & 3.98523700 & 0.63971300 & $\mathrm{H}$ & -4.17462900 & 4.14439800 & 1.74457900 \\
\hline $\mathrm{H}$ & -0.37432300 & 3.40017600 & 1.02648800 & & & & \\
\hline $\mathrm{H}$ & 0.53390100 & 4.90609300 & 1.23220800 & \multirow{2}{*}{\multicolumn{4}{|c|}{$\begin{array}{l}\text { TS59 } \\
\text { imaginary frequency }=-247.58 \mathrm{~cm}^{-1}\end{array}$}} \\
\hline $\mathrm{H}$ & 0.23154400 & 4.27008300 & -0.38583600 & & & & \\
\hline $\mathrm{C}$ & 3.45152000 & 2.36074100 & -2.48644400 & $\mathrm{C}$ & 4.74242700 & -0.65761900 & -1.05079300 \\
\hline $\mathrm{H}$ & 3.78465700 & 3.25829700 & -1.96445000 & $\mathrm{C}$ & 3.66755300 & -0.57595300 & -0.14917200 \\
\hline $\mathrm{H}$ & 4.16214800 & 1.56010700 & -2.28584300 & $\mathrm{C}$ & 3.19156600 & -1.77122900 & 0.44224500 \\
\hline $\mathrm{H}$ & 3.50662200 & 2.57224400 & -3.56088500 & $\mathrm{C}$ & 3.86439800 & -2.96898700 & 0.14504900 \\
\hline $\mathrm{C}$ & 1.06233700 & 3.07165400 & -2.64492100 & $\mathrm{C}$ & 4.90972000 & -3.03490500 & -0.77187600 \\
\hline $\mathrm{H}$ & 1.35575300 & 4.05958800 & -2.28004900 & $\mathrm{C}$ & 5.34387400 & -1.86608800 & -1.39082400 \\
\hline $\mathrm{H}$ & 1.11672400 & 3.10877000 & -3.73929800 & $\mathrm{H}$ & 5.15485700 & 0.25839100 & -1.46018700 \\
\hline $\mathrm{H}$ & 0.01831500 & 2.90560100 & -2.37525400 & $\mathrm{H}$ & 3.58584900 & -3.87118900 & 0.67819300 \\
\hline $\mathrm{C}$ & 1.66797700 & 0.65063100 & -2.89434800 & $\mathrm{H}$ & 5.39605800 & -3.98419100 & -0.97341700 \\
\hline $\mathrm{H}$ & 0.62125000 & 0.36428500 & -2.75473300 & $\mathrm{H}$ & 6.17117300 & -1.88292300 & -2.09337400 \\
\hline $\mathrm{H}$ & 1.83754700 & 0.79902700 & -3.96748700 & $\mathrm{C}$ & 2.10351400 & -1.80439500 & 1.49953800 \\
\hline $\mathrm{H}$ & 2.29360000 & -0.18530800 & -2.57073800 & $\mathrm{H}$ & 2.26736200 & -2.68317200 & 2.13042800 \\
\hline $\mathrm{C}$ & -2.36028000 & 2.75885800 & -0.82464300 & $\mathrm{C}$ & 3.22749200 & 0.79993100 & 0.31149500 \\
\hline $\mathrm{O}$ & -3.10642500 & 2.89554700 & 0.52632400 & $\mathrm{H}$ & 3.11170600 & 0.77005400 & 1.39753600 \\
\hline $\mathrm{O}$ & -2.20078000 & 3.79487200 & -1.39841700 & $\mathrm{H}$ & 4.04262200 & 1.50203300 & 0.11438100 \\
\hline $\mathrm{C}$ & -1.74140700 & 2.32916700 & 3.66312000 & $\mathrm{H}$ & 2.20407100 & -0.93358500 & 2.15293300 \\
\hline $\mathrm{H}$ & -1.44760800 & 1.55843000 & 4.37721100 & $\mathrm{P}$ & 0.25702000 & -1.80002000 & 1.04154800 \\
\hline $\mathrm{H}$ & -2.65390400 & 2.81167200 & 4.01454600 & $\mathrm{P}$ & 1.63103700 & 1.61733700 & -0.28200500 \\
\hline $\mathrm{H}$ & -0.94416600 & 3.06925100 & 3.55624300 & $\mathrm{C}$ & 1.73167100 & 3.24651400 & 0.76370000 \\
\hline $\mathrm{O}$ & -2.06100100 & 1.71377900 & 2.39544700 & $\mathrm{C}$ & 1.92588400 & 2.00010200 & -2.14755900 \\
\hline $\mathrm{H}$ & -2.64171900 & 2.39938300 & 1.36415300 & $\mathrm{C}$ & -0.49415200 & -2.13088600 & 2.79679900 \\
\hline $\mathrm{H}$ & -1.31065600 & 1.20330700 & 2.00809100 & $\mathrm{C}$ & -0.01126100 & -3.33887600 & -0.08642500 \\
\hline $\mathrm{C}$ & -3.25062800 & -0.84130000 & -1.36561900 & $\mathrm{Pd}$ & -0.51358000 & 0.30844500 & -0.03022400 \\
\hline $\mathrm{C}$ & -2.80763800 & -1.16966500 & -2.66110900 & $\mathrm{H}$ & -3.25009700 & 0.33168300 & 0.38546600 \\
\hline
\end{tabular}




\begin{tabular}{|c|c|c|c|c|c|c|c|}
\hline $\mathrm{C}$ & -2.68701400 & 0.20454300 & -0.53581200 & $\mathrm{H}$ & 3.39835000 & 2.63293000 & -3.58881400 \\
\hline $\mathrm{C}$ & -2.10867500 & 1.38233700 & -1.10758500 & $\mathrm{C}$ & 0.96331800 & 3.10012300 & -2.63852400 \\
\hline $\mathrm{C}$ & -2.02196100 & -1.92059600 & 2.75659900 & $\mathrm{H}$ & 1.25174000 & 4.09243000 & -2.28172500 \\
\hline $\mathrm{H}$ & -2.42792900 & -2.06627700 & 3.76501600 & $\mathrm{H}$ & 1.00160000 & 3.13431700 & -3.73370400 \\
\hline $\mathrm{H}$ & -2.28696200 & -0.91311300 & 2.43082800 & $\mathrm{H}$ & -0.07519400 & 2.92521700 & -2.35368200 \\
\hline $\mathrm{H}$ & -2.52477600 & -2.62753700 & 2.09599400 & $\mathrm{C}$ & 1.59528700 & 0.68589700 & -2.89185500 \\
\hline $\mathrm{C}$ & -0.20600300 & -3.53337300 & 3.36834700 & $\mathrm{H}$ & 0.55506200 & 0.38566200 & -2.73362900 \\
\hline $\mathrm{H}$ & 0.85945300 & -3.77929100 & 3.37698700 & $\mathrm{H}$ & 1.74355200 & 0.83472000 & -3.96807700 \\
\hline $\mathrm{H}$ & -0.55177800 & -3.57056800 & 4.40855400 & $\mathrm{H}$ & 2.23848000 & -0.14056700 & -2.57828800 \\
\hline $\mathrm{H}$ & -0.73710700 & -4.31791900 & 2.82612500 & $\mathrm{C}$ & -2.44518900 & 2.73512200 & -0.73452500 \\
\hline $\mathrm{C}$ & 0.11291000 & -1.09050600 & 3.76593100 & $\mathrm{O}$ & -3.11868100 & 2.84151500 & 0.58471700 \\
\hline $\mathrm{H}$ & 0.09997100 & -0.07738900 & 3.34830300 & $\mathrm{O}$ & -2.31216600 & 3.76335300 & -1.34103300 \\
\hline $\mathrm{H}$ & -0.46848800 & -1.08121400 & 4.69542600 & $\mathrm{C}$ & -1.70121000 & 2.27507200 & 3.59776400 \\
\hline $\mathrm{H}$ & 1.14584700 & -1.32330800 & 4.03572300 & $\mathrm{H}$ & -1.30110300 & 1.52843000 & 4.28250300 \\
\hline $\mathrm{C}$ & 0.39776700 & -2.89472600 & -1.50902200 & $\mathrm{H}$ & -2.61933200 & 2.69239100 & 4.01089300 \\
\hline $\mathrm{H}$ & 1.44855600 & -2.59950800 & -1.56530200 & $\mathrm{H}$ & -0.96839300 & 3.06333100 & 3.41578000 \\
\hline $\mathrm{H}$ & 0.25162000 & -3.73466700 & -2.19873700 & $\mathrm{O}$ & -2.07196200 & 1.61234500 & 2.35929400 \\
\hline $\mathrm{H}$ & -0.21929700 & -2.06479700 & -1.86175600 & $\mathrm{H}$ & -2.60769200 & 2.26409100 & 1.49219800 \\
\hline $\mathrm{C}$ & 0.79602100 & -4.59885400 & 0.28393300 & $\mathrm{H}$ & -1.32517400 & 1.12666200 & 1.89793200 \\
\hline $\mathrm{H}$ & 0.63423300 & -4.93378800 & 1.30893300 & $\mathrm{C}$ & -3.23096700 & -0.88557100 & -1.37501700 \\
\hline $\mathrm{H}$ & 0.47176700 & -5.41270400 & -0.37557600 & $\mathrm{C}$ & -2.76272300 & -1.17687800 & -2.67040800 \\
\hline $\mathrm{H}$ & 1.86274500 & -4.46824800 & 0.12168300 & $\mathrm{C}$ & -4.32165000 & -1.62916900 & -0.88585800 \\
\hline $\mathrm{C}$ & -1.50337200 & -3.71977000 & -0.09669300 & $\mathrm{C}$ & -3.35843500 & -2.17460200 & -3.43791700 \\
\hline $\mathrm{H}$ & -2.15484100 & -2.86186500 & -0.25374000 & $\mathrm{H}$ & -1.92730200 & -0.62131800 & -3.08621900 \\
\hline $\mathrm{H}$ & -1.68071700 & -4.41861900 & -0.92195800 & $\mathrm{C}$ & -4.91811200 & -2.62783100 & -1.65396200 \\
\hline $\mathrm{H}$ & -1.80303100 & -4.22941300 & 0.82297100 & $\mathrm{H}$ & -4.70975200 & -1.41256200 & 0.10661800 \\
\hline $\mathrm{C}$ & 1.91175900 & 2.84900700 & 2.24750100 & $\mathrm{C}$ & -4.43762100 & -2.90680500 & -2.93444800 \\
\hline $\mathrm{H}$ & 1.22389500 & 2.05025700 & 2.54876500 & $\mathrm{H}$ & -2.98200000 & -2.37981800 & -4.43574800 \\
\hline $\mathrm{H}$ & 2.92891300 & 2.52292600 & 2.47666400 & $\mathrm{H}$ & -5.76227500 & -3.18221000 & -1.25484900 \\
\hline $\mathrm{H}$ & 1.71073900 & 3.72248200 & 2.87937300 & $\mathrm{H}$ & -4.90174900 & -3.68092700 & -3.53748800 \\
\hline $\mathrm{C}$ & 2.88001500 & 4.19912700 & 0.37754600 & $\mathrm{H}$ & -1.84065200 & 1.36440300 & -2.15982500 \\
\hline $\mathrm{H}$ & 2.92468000 & 5.01850900 & 1.10554900 & $\mathrm{C}$ & -3.65229400 & 4.15933000 & 0.90563100 \\
\hline $\mathrm{H}$ & 3.85802600 & 3.71019000 & 0.38732900 & $\mathrm{H}$ & -2.84599700 & 4.88956000 & 0.97812000 \\
\hline $\mathrm{H}$ & 2.73005700 & 4.65229400 & -0.60456800 & $\mathrm{H}$ & -4.34588300 & 4.44945200 & 0.11894500 \\
\hline $\mathrm{C}$ & 0.39780900 & 4.00758900 & 0.64744200 & $\mathrm{H}$ & -4.17708800 & 4.04211800 & 1.85357500 \\
\hline $\mathrm{H}$ & -0.42929000 & 3.40418300 & 1.03141800 & & & & \\
\hline $\mathrm{H}$ & 0.45336800 & 4.92529900 & 1.24624700 & 77 & & & \\
\hline $\mathrm{H}$ & 0.15097200 & 4.29477700 & -0.37419000 & $\mathrm{C}$ & 4.71780400 & -0.65677200 & -1.10047200 \\
\hline $\mathrm{C}$ & 3.36316400 & 2.41859500 & -2.51409900 & $\mathrm{C}$ & 3.65292000 & -0.58341500 & -0.18618700 \\
\hline $\mathrm{H}$ & 3.69461800 & 3.31871700 & -1.99552500 & $\mathrm{C}$ & 3.18153600 & -1.78522300 & 0.39584500 \\
\hline $\mathrm{H}$ & 4.08520600 & 1.62524200 & -2.32655800 & $\mathrm{C}$ & 3.84518200 & -2.98149500 & 0.07281000 \\
\hline
\end{tabular}




\begin{tabular}{|c|c|c|c|c|c|c|c|}
\hline $\mathrm{C}$ & 4.87851000 & -3.03861700 & -0.85806000 & $\mathrm{H}$ & 1.83404200 & -4.47934000 & 0.04352600 \\
\hline $\mathrm{C}$ & 5.31069000 & -1.86245400 & -1.46438600 & $\mathrm{C}$ & -1.52999900 & -3.69907100 & -0.10539100 \\
\hline $\mathrm{H}$ & 5.12992500 & 0.26326900 & -1.50085500 & $\mathrm{H}$ & -2.17505500 & -2.83074300 & -0.23031100 \\
\hline $\mathrm{H}$ & 3.56918400 & -3.88996000 & 0.59670100 & $\mathrm{H}$ & -1.72833800 & -4.37545900 & -0.94441300 \\
\hline $\mathrm{H}$ & 5.35816400 & -3.98687300 & -1.07947600 & $\mathrm{H}$ & -1.81939900 & -4.22815400 & 0.80653000 \\
\hline $\mathrm{H}$ & 6.13026800 & -1.87216400 & -2.17605900 & $\mathrm{C}$ & 1.95696100 & 2.84029300 & 2.25314700 \\
\hline $\mathrm{C}$ & 2.11108000 & -1.83008400 & 1.47029000 & $\mathrm{H}$ & 1.27639900 & 2.03751500 & 2.56048800 \\
\hline $\mathrm{H}$ & 2.28581400 & -2.71609000 & 2.08811600 & $\mathrm{H}$ & 2.97816400 & 2.51436500 & 2.46373000 \\
\hline $\mathrm{C}$ & 3.22098700 & 0.78834000 & 0.29480400 & $\mathrm{H}$ & 1.76486900 & 3.70949700 & 2.89370400 \\
\hline $\mathrm{H}$ & 3.11189000 & 0.74559900 & 1.38115600 & $\mathrm{C}$ & 2.90214100 & 4.19277400 & 0.37437400 \\
\hline $\mathrm{H}$ & 4.03769300 & 1.48953400 & 0.10058500 & $\mathrm{H}$ & 2.96292000 & 5.00919300 & 1.10453100 \\
\hline $\mathrm{H}$ & 2.22382500 & -0.96684200 & 2.13177800 & $\mathrm{H}$ & 3.87696700 & 3.69740600 & 0.36816400 \\
\hline $\mathrm{P}$ & 0.25700200 & -1.81828100 & 1.04486700 & $\mathrm{H}$ & 2.74064200 & 4.65036700 & -0.60377300 \\
\hline$P$ & 1.62337700 & 1.62257100 & -0.27564200 & $\mathrm{C}$ & 0.42368600 & 4.01820900 & 0.68273700 \\
\hline $\mathrm{C}$ & 1.75329600 & 3.24628000 & 0.77449900 & $\mathrm{H}$ & -0.40415700 & 3.42021700 & 1.07342700 \\
\hline $\mathrm{C}$ & 1.89398100 & 2.01263700 & -2.14356700 & $\mathrm{H}$ & 0.49510200 & 4.93167000 & 1.28631500 \\
\hline $\mathrm{C}$ & -0.45985500 & -2.18771700 & 2.80553100 & $\mathrm{H}$ & 0.16333800 & 4.31344400 & -0.33316800 \\
\hline $\mathrm{C}$ & -0.03441700 & -3.33179400 & -0.11024800 & $\mathrm{C}$ & 3.33047100 & 2.41504400 & -2.53055900 \\
\hline $\mathrm{Pd}$ & -0.52894100 & 0.31117800 & -0.00058900 & $\mathrm{H}$ & 3.68320900 & 3.30544000 & -2.00931100 \\
\hline $\mathrm{H}$ & -3.28726000 & 0.35637700 & 0.39686000 & $\mathrm{H}$ & 4.04412100 & 1.60990800 & -2.36330300 \\
\hline $\mathrm{C}$ & -2.71798200 & 0.24108800 & -0.52222100 & $\mathrm{H}$ & 3.34983300 & 2.63950300 & -3.60359400 \\
\hline $\mathrm{C}$ & -2.11508000 & 1.41569100 & -1.06218700 & $\mathrm{C}$ & 0.93807700 & 3.12724400 & -2.61438900 \\
\hline $\mathrm{C}$ & -1.98699100 & -1.96677500 & 2.80112200 & $\mathrm{H}$ & 1.24504700 & 4.11377600 & -2.25701800 \\
\hline $\mathrm{H}$ & -2.37342100 & -2.12828000 & 3.81479400 & $\mathrm{H}$ & 0.96200800 & 3.16675600 & -3.70983400 \\
\hline $\mathrm{H}$ & -2.25443200 & -0.95314900 & 2.49673500 & $\mathrm{H}$ & -0.09885200 & 2.96587700 & -2.31662000 \\
\hline $\mathrm{H}$ & -2.50649100 & -2.65979100 & 2.13856200 & $\mathrm{C}$ & 1.53636900 & 0.70629100 & -2.88890100 \\
\hline $\mathrm{C}$ & -0.17239700 & -3.60531700 & 3.33981000 & $\mathrm{H}$ & 0.49520400 & 0.41757600 & -2.71572200 \\
\hline $\mathrm{H}$ & 0.89087300 & -3.86018600 & 3.32065400 & $\mathrm{H}$ & 1.66850000 & 0.85873900 & -3.96671000 \\
\hline $\mathrm{H}$ & -0.49676100 & -3.66258900 & 4.38604000 & $\mathrm{H}$ & 2.17547800 & -0.12881800 & -2.59005000 \\
\hline $\mathrm{H}$ & -0.72151200 & -4.37331200 & 2.79188100 & $\mathrm{C}$ & -2.45288100 & 2.77292000 & -0.66148000 \\
\hline $\mathrm{C}$ & 0.17795100 & -1.17676100 & 3.78652200 & $\mathrm{O}$ & -3.08148800 & 2.87049100 & 0.63165000 \\
\hline $\mathrm{H}$ & 0.18848600 & -0.15759200 & 3.38434400 & $\mathrm{O}$ & -2.31050700 & 3.78960100 & -1.29400100 \\
\hline $\mathrm{H}$ & -0.39462800 & -1.16871900 & 4.72150400 & $\mathrm{C}$ & -1.59580000 & 2.20452400 & 3.59332600 \\
\hline $\mathrm{H}$ & 1.20743100 & -1.43844400 & 4.04279000 & $\mathrm{H}$ & -1.14489700 & 1.45465100 & 4.23962100 \\
\hline $\mathrm{C}$ & 0.35674100 & -2.86110200 & -1.52931500 & $\mathrm{H}$ & -2.50622500 & 2.58962300 & 4.05126300 \\
\hline $\mathrm{H}$ & 1.40924700 & -2.57446100 & -1.59590500 & $\mathrm{H}$ & -0.89356800 & 3.00971300 & 3.37631900 \\
\hline $\mathrm{H}$ & 0.19181800 & -3.68481600 & -2.23416000 & $\mathrm{O}$ & -2.00810800 & 1.53480200 & 2.36291200 \\
\hline $\mathrm{H}$ & -0.25805000 & -2.01885900 & -1.85564800 & $\mathrm{H}$ & -2.51500500 & 2.16570000 & 1.63371300 \\
\hline $\mathrm{C}$ & 0.76883400 & -4.60509400 & 0.22043400 & $\mathrm{H}$ & -1.27500100 & 1.05885600 & 1.81861800 \\
\hline $\mathrm{H}$ & 0.62111500 & -4.96034100 & 1.24059100 & $\mathrm{C}$ & -3.24889600 & -0.84374300 & -1.37553000 \\
\hline $\mathrm{H}$ & 0.42787100 & -5.40262900 & -0.45055900 & $\mathrm{C}$ & -2.77539700 & -1.11485100 & -2.67335300 \\
\hline
\end{tabular}




\begin{tabular}{|c|c|c|c|c|c|c|c|}
\hline $\mathrm{C}$ & -4.33529400 & -1.60146000 & -0.89865900 & $\mathrm{C}$ & 2.70216800 & -0.85377400 & -0.52064600 \\
\hline $\mathrm{C}$ & -3.36126700 & -2.10772500 & -3.45456700 & $\mathrm{C}$ & 1.83624500 & -1.84334600 & -1.02639000 \\
\hline $\mathrm{H}$ & -1.94527800 & -0.54578900 & -3.08128800 & $\mathrm{C}$ & 2.35247900 & 1.60405100 & 2.76589200 \\
\hline $\mathrm{C}$ & -4.92256000 & -2.59455300 & -1.68083800 & $\mathrm{H}$ & 2.77913400 & 1.71845000 & 3.76962500 \\
\hline $\mathrm{H}$ & -4.72801300 & -1.39958300 & 0.09515400 & $\mathrm{H}$ & 2.37886800 & 0.54385900 & 2.50869600 \\
\hline $\mathrm{C}$ & -4.43622600 & -2.85424800 & -2.96319200 & $\mathrm{H}$ & 3.00020200 & 2.14298500 & 2.07341900 \\
\hline $\mathrm{H}$ & -2.98123600 & -2.29708100 & -4.45416900 & $\mathrm{C}$ & 0.93324700 & 3.61922300 & 3.24592000 \\
\hline $\mathrm{H}$ & -5.76410300 & -3.15959000 & -1.29127200 & $\mathrm{H}$ & -0.05514900 & 4.08810400 & 3.23533700 \\
\hline $\mathrm{H}$ & -4.89318700 & -3.62393700 & -3.57728500 & $\mathrm{H}$ & 1.28801100 & 3.64768800 & 4.28303900 \\
\hline $\mathrm{H}$ & -1.84463100 & 1.41481300 & -2.11378500 & $\mathrm{H}$ & 1.61519200 & 4.23579200 & 2.65724300 \\
\hline $\mathrm{C}$ & -3.62411700 & 4.18074400 & 0.95499700 & $\mathrm{C}$ & 0.08357200 & 1.33454000 & 3.79156200 \\
\hline $\mathrm{H}$ & -2.82544400 & 4.92076500 & 1.01833500 & $\mathrm{H}$ & -0.11016000 & 0.31649700 & 3.44124000 \\
\hline $\mathrm{H}$ & -4.33220700 & 4.47215300 & 0.18058700 & $\mathrm{H}$ & 0.64359700 & 1.26438500 & 4.73107600 \\
\hline \multirow[t]{2}{*}{$\mathrm{H}$} & -4.13420700 & 4.06076300 & 1.91091300 & $\mathrm{H}$ & -0.87588200 & 1.80361400 & 4.02338500 \\
\hline & & & & $\mathrm{C}$ & 0.22170600 & 2.78470900 & -1.58834100 \\
\hline \multicolumn{4}{|c|}{ TS60 } & $\mathrm{H}$ & -0.86458000 & 2.67668400 & -1.63932500 \\
\hline \multicolumn{4}{|c|}{ imaginary frequency $=-204.72 \mathrm{~cm}^{-1}$} & $\mathrm{H}$ & 0.51703400 & 3.53459200 & -2.33184900 \\
\hline $\mathrm{C}$ & -4.49752700 & 1.53443900 & -1.10304200 & $\mathrm{H}$ & 0.68528500 & 1.83803600 & -1.88129600 \\
\hline $\mathrm{C}$ & -3.45787100 & 1.27685600 & -0.19247700 & $\mathrm{C}$ & 0.15064700 & 4.65951100 & 0.07326300 \\
\hline $\mathrm{C}$ & -2.75974400 & 2.37941200 & 0.35956600 & $\mathrm{H}$ & 0.34431900 & 5.01514500 & 1.08575700 \\
\hline $\mathrm{C}$ & -3.18133600 & 3.67348100 & 0.00681000 & $\mathrm{H}$ & 0.65174600 & 5.35207000 & -0.61348700 \\
\hline $\mathrm{C}$ & -4.19251200 & 3.90740500 & -0.92045500 & $\mathrm{H}$ & -0.91567000 & 4.73247500 & -0.12667000 \\
\hline $\mathrm{C}$ & -4.85043800 & 2.82278100 & -1.49380000 & $\mathrm{C}$ & 2.23691400 & 3.31832700 & -0.18861900 \\
\hline $\mathrm{H}$ & -5.08394000 & 0.70222600 & -1.47713600 & $\mathrm{H}$ & 2.70777100 & 2.33898400 & -0.26227100 \\
\hline $\mathrm{H}$ & -2.73072600 & 4.52349500 & 0.50730100 & $\mathrm{H}$ & 2.56170600 & 3.90211900 & -1.05734100 \\
\hline $\mathrm{H}$ & -4.48203300 & 4.92497500 & -1.16326000 & $\mathrm{H}$ & 2.62000600 & 3.82629000 & 0.70008800 \\
\hline $\mathrm{H}$ & -5.66077600 & 2.97381400 & -2.20002700 & $\mathrm{C}$ & -2.56164200 & -2.41604300 & 2.25697800 \\
\hline $\mathrm{C}$ & -1.70041300 & 2.25052800 & 1.43887200 & $\mathrm{H}$ & -1.75487400 & -1.75461700 & 2.59020400 \\
\hline $\mathrm{H}$ & -1.69932900 & 3.17345300 & 2.02653500 & $\mathrm{H}$ & -3.51270400 & -1.90635300 & 2.42837300 \\
\hline $\mathrm{C}$ & -3.29620200 & -0.14397900 & 0.31350400 & $\mathrm{H}$ & -2.55546100 & -3.30123500 & 2.90356800 \\
\hline $\mathrm{H}$ & -3.14749400 & -0.10557700 & 1.39513200 & $\mathrm{C}$ & -3.67997100 & -3.57863100 & 0.34652400 \\
\hline $\mathrm{H}$ & -4.24370200 & -0.66912500 & 0.15944500 & $\mathrm{H}$ & -3.91577500 & -4.36652000 & 1.07197700 \\
\hline $\mathrm{H}$ & -1.97595500 & 1.44966200 & 2.12991300 & $\mathrm{H}$ & -4.54192200 & -2.90641500 & 0.31297500 \\
\hline$P$ & 0.11742000 & 1.87972000 & 1.03384600 & $\mathrm{H}$ & -3.57690300 & -4.05963400 & -0.62809600 \\
\hline$P$ & -1.92575500 & -1.31104400 & -0.26698600 & $\mathrm{C}$ & -1.21922400 & -3.87021200 & 0.73669700 \\
\hline $\mathrm{C}$ & -2.38671900 & -2.86704300 & 0.78780700 & $\mathrm{H}$ & -0.30346600 & -3.42708200 & 1.13577400 \\
\hline $\mathrm{C}$ & -2.25617600 & -1.63700300 & -2.13553900 & $\mathrm{H}$ & -1.46901900 & -4.74253900 & 1.35317900 \\
\hline $\mathrm{C}$ & 0.91016800 & 2.15222600 & 2.77294000 & $\mathrm{H}$ & -1.00211300 & -4.22971000 & -0.26887000 \\
\hline $\mathrm{C}$ & 0.69862700 & 3.24357700 & -0.19226600 & $\mathrm{C}$ & -3.74061700 & -1.75156700 & -2.53506300 \\
\hline Pd & 0.47495100 & -0.39501100 & 0.01828400 & $\mathrm{H}$ & -4.26181900 & -2.56045000 & -2.02223800 \\
\hline $\mathrm{H}$ & 3.19686700 & -1.06246400 & 0.42372300 & $\mathrm{H}$ & -4.28765800 & -0.82482400 & -2.36784200 \\
\hline
\end{tabular}




\begin{tabular}{|c|c|c|c|c|c|c|}
\hline-3.79418800 & -1.96236900 & -3.60973900 & $\mathrm{C}$ & -4.83540800 & 0.54459800 & -0.50373400 \\
\hline-1.52856000 & -2.91752100 & -2.59120100 & $\mathrm{C}$ & -5.61519100 & 1.44194600 & 0.22040000 \\
\hline-2.02338900 & -3.82304300 & -2.23132700 & $\mathrm{C}$ & -5.05491500 & 2.64698100 & 0.63888500 \\
\hline-1.54730500 & -2.95952900 & -3.68661200 & $\mathrm{H}$ & -3.32072400 & 3.89847100 & 0.58484800 \\
\hline-0.48393300 & -2.95915100 & -2.27883300 & $\mathrm{H}$ & -5.29993600 & -0.35976400 & -0.88298900 \\
\hline-1.64299300 & -0.42788200 & -2.87743200 & $\mathrm{H}$ & -6.65526800 & 1.21215700 & 0.42895000 \\
\hline-0.56139500 & -0.36219200 & -2.71655400 & $\mathrm{H}$ & -5.64754600 & 3.37307500 & 1.18633800 \\
\hline-1.80603800 & -0.54393700 & -3.95546200 & $\mathrm{C}$ & -2.73987100 & -0.19549200 & -1.67105300 \\
\hline-2.09561100 & 0.51938100 & -2.57286400 & $\mathrm{H}$ & -3.46581900 & -0.81105300 & -2.21082900 \\
\hline 1.85538600 & -3.24983500 & -0.58648300 & $\mathrm{C}$ & -1.55681100 & 2.50660800 & -0.84098900 \\
\hline 2.44081000 & -3.45726800 & 0.65279800 & $\mathrm{H}$ & -1.38860100 & 2.21876300 & -1.88471400 \\
\hline 1.45839100 & -4.18335600 & -1.24833900 & $\mathrm{H}$ & -1.58071000 & 3.59773300 & -0.83861900 \\
\hline 0.97282700 & -2.35305200 & 3.64059900 & $\mathrm{H}$ & -2.17205400 & 0.34519800 & -2.43197200 \\
\hline 0.51999000 & -1.56797700 & 4.24335000 & $\mathrm{P}$ & -1.47641000 & -1.38413100 & -0.93568900 \\
\hline 1.79380500 & -2.80520500 & 4.20367200 & $\mathrm{P}$ & 0.06544600 & 2.02734900 & 0.00256700 \\
\hline 0.22481000 & -3.10933600 & 3.39201300 & $\mathrm{C}$ & 1.27292900 & 2.97746800 & -1.17617700 \\
\hline 1.51584200 & -1.73311500 & 2.45345600 & $\mathrm{C}$ & -0.01185800 & 2.81846400 & 1.75498100 \\
\hline 1.93507000 & -2.41951600 & 1.87412200 & $\mathrm{C}$ & -0.94327000 & -2.36160500 & -2.51380400 \\
\hline 0.80526100 & -1.02060600 & 1.46513100 & $\mathrm{C}$ & -2.42213300 & -2.49468800 & 0.31529100 \\
\hline 3.41662200 & 0.12884200 & -1.35433800 & $\mathrm{Pd}$ & 0.46325700 & -0.42151600 & 0.09493000 \\
\hline 3.02809700 & 0.46696500 & -2.66515400 & $\mathrm{H}$ & 0.70783700 & -1.97244300 & 0.29082400 \\
\hline 4.60043500 & 0.69671300 & -0.84532300 & $\mathrm{C}$ & 2.61287400 & 3.26808200 & -0.47834300 \\
\hline 3.78970400 & 1.34751200 & -3.42837700 & $\mathrm{H}$ & 2.52102500 & 4.04751500 & 0.28167000 \\
\hline 2.13110000 & 0.03516400 & -3.09924600 & $\mathrm{H}$ & 3.04501600 & 2.37777600 & -0.02180900 \\
\hline 5.36603800 & 1.57242800 & -1.61272100 & $\mathrm{H}$ & 3.32930300 & 3.62699500 & -1.22631700 \\
\hline 4.92537800 & 0.43579000 & 0.15874100 & $\mathrm{C}$ & 1.53351900 & 2.03149800 & -2.36937800 \\
\hline 4.96123100 & 1.90509200 & -2.90673700 & $\mathrm{H}$ & 2.19672800 & 2.53022000 & -3.08583200 \\
\hline 3.47463600 & 1.59311900 & -4.43810400 & $\mathrm{H}$ & 2.01738400 & 1.10237100 & -2.05525300 \\
\hline 6.28062900 & 1.98989000 & -1.20269100 & $\mathrm{H}$ & 0.61367900 & 1.77760100 & -2.90639700 \\
\hline 5.55652500 & 2.58476400 & -3.50841800 & $\mathrm{C}$ & 0.71887100 & 4.31138500 & -1.72390900 \\
\hline 1.54860600 & -1.79398800 & -2.07228600 & $\mathrm{H}$ & 1.50875700 & 4.79418000 & -2.31052300 \\
\hline 2.65976700 & -4.84797900 & 1.00128000 & $\mathrm{H}$ & -0.13309800 & 4.17588800 & -2.39389300 \\
\hline 1.70842300 & -5.37316900 & 1.10252300 & $\mathrm{H}$ & 0.43051600 & 5.01061400 & -0.93568600 \\
\hline 3.25760700 & -5.33113000 & 0.22806000 & $\mathrm{C}$ & -0.26284300 & 4.33764100 & 1.70932900 \\
\hline 3.20073100 & -4.83002600 & 1.94710900 & $\mathrm{H}$ & -0.41878400 & 4.70240900 & 2.73101600 \\
\hline & & & $\mathrm{H}$ & 0.58891500 & 4.88522600 & 1.29782700 \\
\hline & & & $\mathrm{H}$ & -1.15222900 & 4.60659700 & 1.13358800 \\
\hline quency $=-51$ & $16.05 \mathrm{~cm}^{-1}$ & & $\mathrm{C}$ & -1.15409400 & 2.11498600 & 2.52389400 \\
\hline-3.73332000 & 2.93230300 & 0.30858000 & $\mathrm{H}$ & -1.13933600 & 2.46673400 & 3.56203000 \\
\hline-2.92298500 & 2.02619700 & -0.39690800 & $\mathrm{H}$ & -2.14193700 & 2.32603000 & 2.11703800 \\
\hline-3.48462500 & 0.79461400 & -0.80034800 & $\mathrm{H}$ & -1.00919600 & 1.03095500 & 2.54572500 \\
\hline \multicolumn{7}{|c|}{ S305 } \\
\hline
\end{tabular}




\begin{tabular}{|c|c|c|c|c|c|c|c|}
\hline $\mathrm{C}$ & 1.27974400 & 2.53723000 & 2.55344300 & $\mathrm{H}$ & 6.67905200 & 1.39333900 & -0.36378400 \\
\hline $\mathrm{H}$ & 1.20212000 & 3.05477900 & 3.51675600 & $\mathrm{H}$ & 6.83494400 & -0.03894000 & -2.39134000 \\
\hline $\mathrm{H}$ & 1.37903800 & 1.47278200 & 2.77571200 & $\mathrm{C}$ & 1.41992600 & -1.93887800 & 2.61884100 \\
\hline $\mathrm{H}$ & 2.18701300 & 2.89734900 & 2.06806100 & $\mathrm{O}$ & 1.06578200 & -1.04024600 & 3.35570700 \\
\hline $\mathrm{C}$ & -1.62627800 & -3.77680100 & 0.62871300 & $\mathrm{O}$ & 1.31519200 & -3.24517400 & 2.90402300 \\
\hline $\mathrm{H}$ & -1.60568200 & -4.47236600 & -0.21361200 & $\mathrm{C}$ & 0.77001800 & -3.56490700 & 4.20465900 \\
\hline $\mathrm{H}$ & -0.59913200 & -3.57533900 & 0.94479100 & $\mathrm{H}$ & 0.77990100 & -4.65186500 & 4.26264900 \\
\hline $\mathrm{H}$ & -2.12210100 & -4.29368300 & 1.45833200 & $\mathrm{H}$ & 1.39116600 & -3.12920100 & 4.98941200 \\
\hline $\mathrm{C}$ & -3.83210400 & -2.90485500 & -0.15763000 & $\mathrm{H}$ & -0.24767200 & -3.17920800 & 4.29355700 \\
\hline $\mathrm{H}$ & -3.82494000 & -3.47077300 & -1.08990200 & & & & \\
\hline $\mathrm{H}$ & -4.27850500 & -3.54788200 & 0.60921900 & 78 & & & \\
\hline $\mathrm{H}$ & -4.49253700 & -2.04758900 & -0.27484300 & $\mathrm{C}$ & -3.67116800 & 2.87922600 & 0.61901200 \\
\hline $\mathrm{C}$ & -2.55119100 & -1.66600800 & 1.61414400 & $\mathrm{C}$ & -2.94031200 & 1.98718700 & -0.18500000 \\
\hline $\mathrm{H}$ & -3.10937700 & -2.25299900 & 2.35308400 & $\mathrm{C}$ & -3.57241700 & 0.79923900 & -0.61488000 \\
\hline $\mathrm{H}$ & -1.57280600 & -1.42869300 & 2.04181300 & $\mathrm{C}$ & -4.90429200 & 0.56948700 & -0.23082900 \\
\hline $\mathrm{H}$ & -3.09298000 & -0.73032100 & 1.45828900 & $\mathrm{C}$ & -5.60166500 & 1.44960200 & 0.59194500 \\
\hline $\mathrm{C}$ & 0.28075600 & -3.24916200 & -2.21057600 & $\mathrm{C}$ & -4.97565600 & 2.61603300 & 1.02646900 \\
\hline $\mathrm{H}$ & 0.09160000 & -3.99525100 & -1.43744400 & $\mathrm{H}$ & -3.21123900 & 3.81755700 & 0.91451900 \\
\hline $\mathrm{H}$ & 0.56050600 & -3.78595800 & -3.12411400 & $\mathrm{H}$ & -5.41945100 & -0.30503800 & -0.61538700 \\
\hline $\mathrm{H}$ & 1.13871900 & -2.64535600 & -1.90474800 & $\mathrm{H}$ & -6.63026700 & 1.23770100 & 0.86612400 \\
\hline $\mathrm{C}$ & -2.06885700 & -3.24080300 & -3.09554000 & $\mathrm{H}$ & -5.50497000 & 3.33009100 & 1.64941200 \\
\hline $\mathrm{H}$ & -1.73148400 & -3.65410300 & -4.05286200 & $\mathrm{C}$ & -2.92204300 & -0.16886900 & -1.58035100 \\
\hline $\mathrm{H}$ & -2.31150500 & -4.08628000 & -2.44822300 & $\mathrm{H}$ & -3.69614000 & -0.78194600 & -2.05124900 \\
\hline $\mathrm{H}$ & -2.98743400 & -2.68194000 & -3.29501200 & $\mathrm{C}$ & -1.59071300 & 2.44719600 & -0.69832200 \\
\hline $\mathrm{C}$ & -0.51043000 & -1.33246700 & -3.58437900 & $\mathrm{H}$ & -1.47104100 & 2.15184900 & -1.74580800 \\
\hline $\mathrm{H}$ & -1.35223100 & -0.77440200 & -4.00165700 & $\mathrm{H}$ & -1.60541600 & 3.53825700 & -0.70016400 \\
\hline $\mathrm{H}$ & 0.22728000 & -0.62283500 & -3.19748400 & $\mathrm{H}$ & -2.44322400 & 0.39193100 & -2.38798700 \\
\hline $\mathrm{H}$ & -0.04290800 & -1.87087000 & -4.41623500 & $\mathrm{P}$ & -1.57491400 & -1.32881600 & -0.96440600 \\
\hline $\mathrm{C}$ & 2.70893000 & -0.53683500 & 1.00668100 & $\mathrm{P}$ & 0.08639900 & 1.99513500 & 0.05699000 \\
\hline $\mathrm{C}$ & 2.05829500 & -1.75068800 & 1.27017500 & $\mathrm{C}$ & 1.21082900 & 3.02487600 & -1.14111500 \\
\hline $\mathrm{H}$ & 2.64816000 & 0.21699400 & 1.78320900 & $\mathrm{C}$ & 0.07725900 & 2.69967300 & 1.84755400 \\
\hline $\mathrm{H}$ & 2.42732800 & -2.66033100 & 0.80516400 & $\mathrm{C}$ & -1.11273900 & -2.25062300 & -2.59544900 \\
\hline $\mathrm{C}$ & 3.79187300 & -0.39274200 & 0.02501000 & $\mathrm{C}$ & -2.43114200 & -2.50819600 & 0.29197100 \\
\hline $\mathrm{C}$ & 3.90339000 & -1.20717100 & -1.11913800 & $\mathrm{Pd}$ & 0.49851200 & -0.34914100 & 0.00254100 \\
\hline $\mathrm{C}$ & 4.80934200 & 0.54817500 & 0.27673100 & $\mathrm{H}$ & 1.06219800 & -2.14122100 & 0.41100500 \\
\hline $\mathrm{C}$ & 4.98695100 & -1.07541400 & -1.98293400 & $\mathrm{C}$ & 2.56682700 & 3.34865300 & -0.48969400 \\
\hline $\mathrm{H}$ & 3.13937300 & -1.94718300 & -1.33514800 & $\mathrm{H}$ & 2.47549800 & 4.09944700 & 0.29875700 \\
\hline $\mathrm{C}$ & 5.89847900 & 0.67154600 & -0.58326700 & $\mathrm{H}$ & 3.05772300 & 2.46473300 & -0.08477600 \\
\hline $\mathrm{H}$ & 4.75495400 & 1.16580900 & 1.16919400 & $\mathrm{H}$ & 3.23076000 & 3.76434000 & -1.25632100 \\
\hline $\mathrm{C}$ & 5.98818900 & -0.13557600 & -1.71904300 & $\mathrm{C}$ & 1.45322900 & 2.13500300 & -2.37967900 \\
\hline $\mathrm{H}$ & 5.05564900 & -1.70990300 & -2.86131300 & $\mathrm{H}$ & 2.07096000 & 2.68580900 & -3.09849900 \\
\hline
\end{tabular}




\begin{tabular}{|c|c|c|c|c|c|c|c|}
\hline $\mathrm{H}$ & 1.97874800 & 1.21256700 & -2.12094100 & $\mathrm{H}$ & -0.30995800 & -1.68005600 & -4.52171500 \\
\hline $\mathrm{H}$ & 0.52136200 & 1.87291100 & -2.89135400 & $\mathrm{C}$ & 2.49744300 & -0.50987300 & 0.81601000 \\
\hline $\mathrm{C}$ & 0.58011800 & 4.35519600 & -1.61350900 & $\mathrm{C}$ & 2.01875600 & -1.91502900 & 1.02761200 \\
\hline $\mathrm{H}$ & 1.33253800 & 4.89429700 & -2.20009200 & $\mathrm{H}$ & 2.56325000 & 0.06142000 & 1.73507800 \\
\hline $\mathrm{H}$ & -0.28327400 & 4.21027800 & -2.26638000 & $\mathrm{H}$ & 2.69810700 & -2.67304800 & 0.63018100 \\
\hline $\mathrm{H}$ & 0.28740100 & 5.00962400 & -0.78907200 & $\mathrm{C}$ & 3.62960300 & -0.29410600 & -0.10775700 \\
\hline $\mathrm{C}$ & -0.23168800 & 4.20999600 & 1.86569900 & $\mathrm{C}$ & 3.75705300 & -0.98902500 & -1.32800000 \\
\hline $\mathrm{H}$ & -0.35485000 & 4.53084300 & 2.90621100 & $\mathrm{C}$ & 4.66550600 & 0.58755400 & 0.25993000 \\
\hline $\mathrm{H}$ & 0.58106500 & 4.80318600 & 1.43904100 & $\mathrm{C}$ & 4.86158900 & -0.79316200 & -2.15329800 \\
\hline $\mathrm{H}$ & -1.15412900 & 4.46574000 & 1.33963200 & $\mathrm{H}$ & 2.98869300 & -1.69373300 & -1.63252600 \\
\hline $\mathrm{C}$ & -0.99273500 & 1.92171900 & 2.64779300 & $\mathrm{C}$ & 5.77492300 & 0.77692500 & -0.56160200 \\
\hline $\mathrm{H}$ & -0.95980000 & 2.26661600 & 3.68787900 & $\mathrm{H}$ & 4.60625600 & 1.10943600 & 1.21159300 \\
\hline $\mathrm{H}$ & -2.00647600 & 2.07454600 & 2.28083900 & $\mathrm{C}$ & 5.87468000 & 0.09254400 & -1.77492700 \\
\hline $\mathrm{H}$ & -0.77803500 & 0.84942500 & 2.65420900 & $\mathrm{H}$ & 4.93827400 & -1.33889300 & -3.08896400 \\
\hline $\mathrm{C}$ & 1.41824200 & 2.44966700 & 2.57059600 & $\mathrm{H}$ & 6.56619400 & 1.45215300 & -0.25044400 \\
\hline $\mathrm{H}$ & 1.37072800 & 2.94509000 & 3.54710800 & $\mathrm{H}$ & 6.73915700 & 0.23809100 & -2.41505100 \\
\hline $\mathrm{H}$ & 1.56427500 & 1.38574600 & 2.76208400 & $\mathrm{C}$ & 1.60775500 & -2.21310300 & 2.46275700 \\
\hline $\mathrm{H}$ & 2.28759100 & 2.84787000 & 2.04792100 & $\mathrm{O}$ & 1.11620400 & -1.39925400 & 3.21664900 \\
\hline $\mathrm{C}$ & -1.60374800 & -3.79344000 & 0.49655800 & $\mathrm{O}$ & 1.84286600 & -3.49427900 & 2.76449100 \\
\hline $\mathrm{H}$ & -1.63011200 & -4.45388600 & -0.37316000 & $\mathrm{C}$ & 1.49608100 & -3.90332800 & 4.10898800 \\
\hline $\mathrm{H}$ & -0.55795600 & -3.59521600 & 0.74960000 & $\mathrm{H}$ & 1.75339700 & -4.95951900 & 4.16426300 \\
\hline $\mathrm{H}$ & -2.03376000 & -4.35113100 & 1.33626400 & $\mathrm{H}$ & 2.06984500 & -3.32595600 & 4.83625800 \\
\hline $\mathrm{C}$ & -3.86480000 & -2.91462100 & -0.10374700 & $\mathrm{H}$ & 0.42943100 & -3.75211300 & 4.28611700 \\
\hline $\mathrm{H}$ & -3.91554100 & -3.42187100 & -1.06836700 & & & & \\
\hline $\mathrm{H}$ & -4.25083600 & -3.60977400 & 0.65073400 & 79 & & & \\
\hline $\mathrm{H}$ & -4.54205000 & -2.06171200 & -0.12362600 & $\mathrm{C}$ & 3.96932300 & 2.99527700 & -0.19523000 \\
\hline $\mathrm{C}$ & -2.47834900 & -1.74374900 & 1.63528900 & $\mathrm{C}$ & 3.31336700 & 1.85567200 & 0.30132200 \\
\hline $\mathrm{H}$ & -2.98436300 & -2.36814200 & 2.38131700 & $\mathrm{C}$ & 3.92283400 & 0.59496900 & 0.09996300 \\
\hline $\mathrm{H}$ & -1.47621500 & -1.51870300 & 2.01206300 & $\mathrm{C}$ & 5.13433500 & 0.54141000 & -0.60904000 \\
\hline $\mathrm{H}$ & -3.03341600 & -0.80613000 & 1.55952600 & $\mathrm{C}$ & 5.73455800 & 1.67595700 & -1.14795700 \\
\hline $\mathrm{C}$ & 0.15381300 & -3.10164000 & -2.36617900 & $\mathrm{C}$ & 5.14693600 & 2.91930700 & -0.93358500 \\
\hline $\mathrm{H}$ & 0.01853000 & -3.88714500 & -1.62101000 & $\mathrm{H}$ & 3.57446400 & 3.97500000 & 0.04726600 \\
\hline $\mathrm{H}$ & 0.43221300 & -3.58857500 & -3.30772600 & $\mathrm{H}$ & 5.64884800 & -0.40841500 & -0.70206300 \\
\hline $\mathrm{H}$ & 0.99797600 & -2.47584200 & -2.06083400 & $\mathrm{H}$ & 6.67105200 & 1.58963200 & -1.68979000 \\
\hline $\mathrm{C}$ & -2.23478800 & -3.14701000 & -3.15373700 & $\mathrm{H}$ & 5.61711900 & 3.82641300 & -1.29981400 \\
\hline $\mathrm{H}$ & -1.92723900 & -3.53202800 & -4.13305700 & $\mathrm{C}$ & 3.42122200 & -0.67006500 & 0.76677100 \\
\hline $\mathrm{H}$ & -2.43348800 & -4.01167200 & -2.51673100 & $\mathrm{H}$ & 4.25207900 & -1.37405100 & 0.86366300 \\
\hline $\mathrm{H}$ & -3.17305500 & -2.60510600 & -3.30361100 & $\mathrm{C}$ & 2.12072700 & 2.05655400 & 1.21705000 \\
\hline $\mathrm{C}$ & -0.75745200 & -1.18113900 & -3.65464200 & $\mathrm{H}$ & 2.19370000 & 1.36113200 & 2.05580200 \\
\hline $\mathrm{H}$ & -1.63364200 & -0.63936200 & -4.01983800 & $\mathrm{H}$ & 2.19878200 & 3.05707900 & 1.64799800 \\
\hline $\mathrm{H}$ & -0.02662700 & -0.45691500 & -3.28060500 & $\mathrm{H}$ & 3.10179300 & -0.43757800 & 1.78560100 \\
\hline
\end{tabular}




\begin{tabular}{|c|c|c|c|c|c|c|c|}
\hline$P$ & 1.97294600 & -1.61736000 & 0.02596300 & $\mathrm{H}$ & 1.53705800 & -1.05552200 & -2.89175900 \\
\hline$P$ & 0.30561900 & 1.89120900 & 0.69254700 & $\mathrm{H}$ & 3.12594300 & -0.42561800 & -2.42775700 \\
\hline $\mathrm{C}$ & -0.51035800 & 2.49068100 & 2.36069800 & $\mathrm{C}$ & 0.37694100 & -3.75282000 & 1.03859400 \\
\hline $\mathrm{C}$ & 0.01005500 & 3.19332200 & -0.70472100 & $\mathrm{H}$ & 0.45118400 & -4.30769700 & 0.10306100 \\
\hline $\mathrm{C}$ & 1.65812400 & -2.96015300 & 1.37515500 & $\mathrm{H}$ & 0.19788800 & -4.48390400 & 1.83526700 \\
\hline $\mathrm{C}$ & 2.68157400 & -2.41390200 & -1.58644600 & $\mathrm{H}$ & -0.50155300 & -3.10414000 & 0.99024700 \\
\hline $\mathrm{Pd}$ & -0.27659000 & -0.39016100 & -0.28328800 & $\mathrm{C}$ & 2.83412700 & -3.93962500 & 1.55673600 \\
\hline $\mathrm{C}$ & -2.04906300 & 2.35013700 & 2.35697500 & $\mathrm{H}$ & 2.61845500 & -4.59351000 & 2.40965500 \\
\hline $\mathrm{H}$ & -2.54276400 & 2.84624400 & 1.52205800 & $\mathrm{H}$ & 2.97747800 & -4.58368700 & 0.68671100 \\
\hline $\mathrm{H}$ & -2.37056700 & 1.30851300 & 2.37575700 & $\mathrm{H}$ & 3.77840800 & -3.43164500 & 1.77278200 \\
\hline $\mathrm{H}$ & -2.43137800 & 2.80932700 & 3.27567100 & $\mathrm{C}$ & 1.41118200 & -2.23109700 & 2.71503600 \\
\hline $\mathrm{C}$ & 0.02619800 & 1.59839700 & 3.50433200 & $\mathrm{H}$ & 2.31793200 & -1.78373200 & 3.13027300 \\
\hline $\mathrm{H}$ & -0.56053500 & 1.80027700 & 4.40714300 & $\mathrm{H}$ & 0.64676400 & -1.45375400 & 2.62166700 \\
\hline $\mathrm{H}$ & -0.07716500 & 0.53127400 & 3.28386200 & $\mathrm{H}$ & 1.05050800 & -2.95909000 & 3.44991600 \\
\hline $\mathrm{H}$ & 1.07025700 & 1.80183500 & 3.75295400 & $\mathrm{C}$ & -0.88331400 & -1.63371300 & -1.65177100 \\
\hline $\mathrm{C}$ & -0.17807900 & 3.96271000 & 2.69026600 & $\mathrm{O}$ & -1.28076400 & -2.25594300 & -2.52503700 \\
\hline $\mathrm{H}$ & -0.55519400 & 4.17889800 & 3.69634800 & $\mathrm{C}$ & -3.09365300 & 0.41488900 & -1.58715400 \\
\hline $\mathrm{H}$ & 0.89246700 & 4.18205500 & 2.70237400 & $\mathrm{H}$ & -3.24511900 & -0.42217100 & -2.26849700 \\
\hline $\mathrm{H}$ & -0.66913300 & 4.66054100 & 2.00930600 & $\mathrm{H}$ & -2.45351100 & 1.12979800 & -2.11390000 \\
\hline $\mathrm{C}$ & 0.84974300 & 4.48100700 & -0.57042500 & $\mathrm{C}$ & -2.45926000 & 0.02127100 & -0.25411100 \\
\hline $\mathrm{H}$ & 0.48149400 & 5.20272300 & -1.30858700 & $\mathrm{H}$ & -2.45649200 & 0.88150300 & 0.40151200 \\
\hline $\mathrm{H}$ & 0.77147200 & 4.95624800 & 0.40836500 & $\mathrm{C}$ & -3.02801500 & -1.16905300 & 0.44674000 \\
\hline $\mathrm{H}$ & 1.89895000 & 4.30702900 & -0.79589500 & $\mathrm{C}$ & -2.69551500 & -1.41322900 & 1.79723800 \\
\hline $\mathrm{C}$ & 0.40714100 & 2.50093500 & -2.02680000 & $\mathrm{C}$ & -3.90980500 & -2.07514000 & -0.17768800 \\
\hline $\mathrm{H}$ & 0.28211000 & 3.20855500 & -2.85471800 & $\mathrm{C}$ & -3.21589600 & -2.50337900 & 2.49030000 \\
\hline $\mathrm{H}$ & 1.45256300 & 2.17915600 & -2.02079100 & $\mathrm{H}$ & -2.01907500 & -0.73559200 & 2.30803000 \\
\hline $\mathrm{H}$ & -0.21553200 & 1.62553800 & -2.23215700 & $\mathrm{C}$ & -4.43247800 & -3.16490700 & 0.51708900 \\
\hline $\mathrm{C}$ & -1.47281700 & 3.61525000 & -0.76191900 & $\mathrm{H}$ & -4.22018700 & -1.92010700 & -1.20493900 \\
\hline $\mathrm{H}$ & -1.63598100 & 4.16356700 & -1.69713800 & $\mathrm{C}$ & -4.08749300 & -3.38836600 & 1.85183800 \\
\hline $\mathrm{H}$ & -2.18260300 & 2.79071100 & -0.74043200 & $\mathrm{H}$ & -2.94783300 & -2.65802300 & 3.53128700 \\
\hline $\mathrm{H}$ & -1.73208000 & 4.29643600 & 0.05200400 & $\mathrm{H}$ & -5.12102100 & -3.83733900 & 0.01419900 \\
\hline $\mathrm{C}$ & 1.87602900 & -3.65671900 & -2.01867600 & $\mathrm{H}$ & -4.50074300 & -4.23559600 & 2.39002900 \\
\hline $\mathrm{H}$ & 2.06159700 & -4.51437500 & -1.36831800 & $\mathrm{C}$ & -4.42113400 & 1.13210700 & -1.35341700 \\
\hline $\mathrm{H}$ & 0.80056100 & -3.49220000 & -2.06787900 & $\mathrm{O}$ & -4.58406100 & 1.99221500 & -0.51089000 \\
\hline $\mathrm{H}$ & 2.20099000 & -3.94195000 & -3.02572000 & $\mathrm{O}$ & -5.36781400 & 0.71803200 & -2.20641600 \\
\hline $\mathrm{C}$ & 4.15801800 & -2.84861700 & -1.46406900 & $\mathrm{C}$ & -6.64890900 & 1.37731800 & -2.09115600 \\
\hline $\mathrm{H}$ & 4.33374900 & -3.54938400 & -0.64634000 & $\mathrm{H}$ & -6.54126800 & 2.44832400 & -2.27507200 \\
\hline $\mathrm{H}$ & 4.44412400 & -3.35523600 & -2.39271200 & $\mathrm{H}$ & -7.28141900 & 0.91751200 & -2.84888000 \\
\hline $\mathrm{H}$ & 4.83196600 & -2.00254400 & -1.34731700 & $\mathrm{H}$ & -7.06431900 & 1.22413100 & -1.09306700 \\
\hline
\end{tabular}

C $\quad 2.57470600 \quad-1.33320300 \quad-2.68666400$

$\mathrm{H} \quad 3.00138700 \quad-1.72645400 \quad-3.61656000$

\section{TS62}




\begin{tabular}{|c|c|c|c|c|c|c|c|}
\hline \multicolumn{4}{|c|}{ imaginary frequency $=-119.11 \mathrm{~cm}^{-1}$} & $\mathrm{H}$ & 0.63141700 & 3.20078000 & -2.73684300 \\
\hline $\mathrm{C}$ & 4.26082400 & 2.79620700 & -0.11985800 & $\mathrm{H}$ & 1.80539300 & 2.18550100 & -1.89210800 \\
\hline $\mathrm{C}$ & 3.52828100 & 1.69238600 & 0.34888000 & $\mathrm{H}$ & 0.15090100 & 1.60368300 & -2.14177100 \\
\hline $\mathrm{C}$ & 4.01098200 & 0.39481300 & 0.05664000 & $\mathrm{C}$ & -1.16771900 & 3.56276600 & -0.69735900 \\
\hline $\mathrm{C}$ & 5.19284100 & 0.27478100 & -0.69328900 & $\mathrm{H}$ & -1.30286900 & 4.14748400 & -1.61476400 \\
\hline $\mathrm{C}$ & 5.87462400 & 1.38021800 & -1.19461800 & $\mathrm{H}$ & -1.85826400 & 2.72139600 & -0.74662500 \\
\hline $\mathrm{C}$ & 5.40360800 & 2.65700600 & -0.90265400 & $\mathrm{H}$ & -1.48078000 & 4.20039200 & 0.13272200 \\
\hline $\mathrm{H}$ & 3.95424400 & 3.79342900 & 0.17639700 & $\mathrm{C}$ & 1.54028800 & -3.55232600 & -2.23025700 \\
\hline $\mathrm{H}$ & 5.62107900 & -0.70912800 & -0.84833100 & $\mathrm{H}$ & 1.69322800 & -4.46088500 & -1.64302900 \\
\hline $\mathrm{H}$ & 6.78409800 & 1.24170700 & -1.77053400 & $\mathrm{H}$ & 0.48002300 & -3.30488000 & -2.21836000 \\
\hline $\mathrm{H}$ & 5.93795800 & 3.53766700 & -1.24462300 & $\mathrm{H}$ & 1.80254500 & -3.79332700 & -3.26664800 \\
\hline $\mathrm{C}$ & 3.42107500 & -0.85589400 & 0.67805400 & $\mathrm{C}$ & 3.90362600 & -2.90504900 & -1.78900400 \\
\hline $\mathrm{H}$ & 4.20326300 & -1.61807600 & 0.74200700 & $\mathrm{H}$ & 4.09861700 & -3.67982000 & -1.04636500 \\
\hline $\mathrm{C}$ & 2.38025800 & 1.95092600 & 1.30530600 & $\mathrm{H}$ & 4.09585500 & -3.34453100 & -2.77427600 \\
\hline $\mathrm{H}$ & 2.45361200 & 1.25739700 & 2.14722400 & $\mathrm{H}$ & 4.62590800 & -2.10305500 & -1.65502400 \\
\hline $\mathrm{H}$ & 2.50898400 & 2.95027500 & 1.72920000 & $\mathrm{C}$ & 2.31181500 & -1.21959300 & -2.75961500 \\
\hline $\mathrm{H}$ & 3.12473300 & -0.63950000 & 1.70668500 & $\mathrm{H}$ & 2.65074400 & -1.54832300 & -3.74870400 \\
\hline $\mathrm{P}$ & 1.90496800 & -1.70939500 & -0.04872600 & $\mathrm{H}$ & 1.27376900 & -0.88812700 & -2.85666800 \\
\hline $\mathrm{P}$ & 0.56568100 & 1.83607900 & 0.78708700 & $\mathrm{H}$ & 2.92117900 & -0.35985400 & -2.46887500 \\
\hline $\mathrm{C}$ & -0.26907200 & 2.39159800 & 2.44361700 & $\mathrm{C}$ & 0.28475700 & -3.83772800 & 0.96599600 \\
\hline $\mathrm{C}$ & 0.31626200 & 3.16035200 & -0.59252100 & $\mathrm{H}$ & 0.21830300 & -4.27610000 & -0.03017000 \\
\hline $\mathrm{C}$ & 1.62803300 & -3.12511500 & 1.23325200 & $\mathrm{H}$ & 0.17624000 & -4.65454700 & 1.68852400 \\
\hline $\mathrm{C}$ & 2.44779000 & -2.39511000 & -1.76562500 & $\mathrm{H}$ & -0.56607500 & -3.16537700 & 1.10494000 \\
\hline $\mathrm{Pd}$ & -0.16613000 & -0.39789400 & -0.16787500 & $\mathrm{C}$ & 2.76424400 & -4.16781200 & 1.23841900 \\
\hline $\mathrm{C}$ & -1.80070200 & 2.21033100 & 2.38287900 & $\mathrm{H}$ & 2.59930200 & -4.86010900 & 2.07206700 \\
\hline $\mathrm{H}$ & -2.27844700 & 2.74079800 & 1.55893300 & $\mathrm{H}$ & 2.78632400 & -4.76631400 & 0.32527500 \\
\hline $\mathrm{H}$ & -2.08432200 & 1.15766000 & 2.32809300 & $\mathrm{H}$ & 3.75168200 & -3.72112300 & 1.38525400 \\
\hline $\mathrm{H}$ & -2.23194100 & 2.60242200 & 3.31110600 & $\mathrm{C}$ & 1.53682100 & -2.48381700 & 2.63670700 \\
\hline $\mathrm{C}$ & 0.25515500 & 1.47276700 & 3.57185400 & $\mathrm{H}$ & 2.50169900 & -2.12870000 & 3.00702600 \\
\hline $\mathrm{H}$ & -0.34823400 & 1.64397000 & 4.47008000 & $\mathrm{H}$ & 0.82361800 & -1.65373700 & 2.66106200 \\
\hline $\mathrm{H}$ & 0.16355700 & 0.41138600 & 3.31819600 & $\mathrm{H}$ & 1.18228700 & -3.24058000 & 3.34488400 \\
\hline $\mathrm{H}$ & 1.29348200 & 1.67832200 & 3.84257500 & $\mathrm{C}$ & -1.22453100 & -1.61211200 & -1.18873200 \\
\hline $\mathrm{C}$ & 0.03476700 & 3.85647500 & 2.82115900 & $\mathrm{O}$ & -1.70348400 & -2.39466900 & -1.88680400 \\
\hline $\mathrm{H}$ & -0.35524000 & 4.04192100 & 3.82859400 & $\mathrm{C}$ & -3.13606300 & 0.42582000 & -1.71336100 \\
\hline $\mathrm{H}$ & 1.10357600 & 4.08552700 & 2.84754200 & $\mathrm{H}$ & -3.42320700 & -0.39835300 & -2.36781100 \\
\hline $\mathrm{H}$ & -0.45662600 & 4.56677100 & 2.15304300 & $\mathrm{H}$ & -2.35977500 & 0.99404100 & -2.23747000 \\
\hline $\mathrm{C}$ & 1.14300600 & 4.44779200 & -0.40440300 & $\mathrm{C}$ & -2.61517500 & -0.03944900 & -0.36252800 \\
\hline $\mathrm{H}$ & 0.84453000 & 5.16163600 & -1.18096200 & $\mathrm{H}$ & -2.34492000 & 0.80877000 & 0.25463300 \\
\hline $\mathrm{H}$ & 0.97609600 & 4.93277300 & 0.55826400 & $\mathrm{C}$ & -3.39968800 & -1.04397000 & 0.40433200 \\
\hline $\mathrm{H}$ & 2.20940400 & 4.27250900 & -0.52954300 & $\mathrm{C}$ & -2.98912300 & -1.42306700 & 1.69882800 \\
\hline $\mathrm{C}$ & 0.75498800 & 2.48812700 & -1.91291900 & $\mathrm{C}$ & -4.56321800 & -1.63855300 & -0.11837100 \\
\hline
\end{tabular}




\begin{tabular}{|c|c|c|c|c|c|c|c|}
\hline $\mathrm{C}$ & -3.71716000 & -2.34313500 & 2.44710300 & $\mathrm{H}$ & -1.77258000 & 2.91489600 & 1.45061700 \\
\hline $\mathrm{H}$ & -2.07661100 & -1.00045500 & 2.11095900 & $\mathrm{H}$ & -1.49122300 & 1.36325500 & 2.25475800 \\
\hline $\mathrm{C}$ & -5.29526300 & -2.55644200 & 0.63566700 & $\mathrm{H}$ & -1.75162800 & 2.82469800 & 3.21197900 \\
\hline $\mathrm{H}$ & -4.92356000 & -1.37011900 & -1.10535600 & $\mathrm{C}$ & 0.80154100 & 1.90749200 & 3.56714900 \\
\hline $\mathrm{C}$ & -4.87768800 & -2.91495600 & 1.91755800 & $\mathrm{H}$ & 0.20313100 & 2.14713500 & 4.45311700 \\
\hline $\mathrm{H}$ & -3.38139700 & -2.61532700 & 3.44336600 & $\mathrm{H}$ & 0.74494800 & 0.82432900 & 3.42247100 \\
\hline $\mathrm{H}$ & -6.19718200 & -2.99166000 & 0.21610600 & $\mathrm{H}$ & 1.83608600 & 2.17465900 & 3.79818400 \\
\hline $\mathrm{H}$ & -5.44883900 & -3.63183700 & 2.49896300 & $\mathrm{C}$ & 0.50717100 & 4.19549700 & 2.61310100 \\
\hline $\mathrm{C}$ & -4.31395400 & 1.38287600 & -1.53016600 & $\mathrm{H}$ & 0.10075300 & 4.47036600 & 3.59349700 \\
\hline $\mathrm{O}$ & -4.33989900 & 2.27452500 & -0.70649900 & $\mathrm{H}$ & 1.57604500 & 4.42909300 & 2.63006300 \\
\hline $\mathrm{O}$ & -5.29500800 & 1.12941500 & -2.40660900 & $\mathrm{H}$ & 0.02403100 & 4.83709300 & 1.87392200 \\
\hline $\mathrm{C}$ & -6.43849700 & 2.01192600 & -2.34205600 & $\mathrm{C}$ & 1.82315200 & 4.51221500 & -0.52280900 \\
\hline $\mathrm{H}$ & -6.13105600 & 3.04331400 & -2.52622400 & $\mathrm{H}$ & 1.71604200 & 5.14619000 & -1.41089100 \\
\hline $\mathrm{H}$ & -7.11628700 & 1.66579700 & -3.12070100 & $\mathrm{H}$ & 1.55830400 & 5.12091100 & 0.34261800 \\
\hline \multirow[t]{2}{*}{$\mathrm{H}$} & -6.90982700 & 1.94700800 & -1.35929500 & $\mathrm{H}$ & 2.87856200 & 4.24701000 & -0.44704300 \\
\hline & & & & $\mathrm{C}$ & 1.34225500 & 2.51675800 & -1.96927700 \\
\hline 80a & & & & $\mathrm{H}$ & 1.24400400 & 3.19023400 & -2.82879900 \\
\hline $\mathrm{C}$ & 4.68736900 & 2.10540400 & -0.44909500 & $\mathrm{H}$ & 2.37741900 & 2.17194500 & -1.93280600 \\
\hline $\mathrm{C}$ & 3.79628300 & 1.23731000 & 0.20512200 & $\mathrm{H}$ & 0.70298700 & 1.64534900 & -2.15411600 \\
\hline $\mathrm{C}$ & 3.91081400 & -0.15648500 & -0.02900300 & $\mathrm{C}$ & -0.54185700 & 3.73013000 & -0.86087700 \\
\hline $\mathrm{C}$ & 4.92091300 & -0.59426100 & -0.90304500 & $\mathrm{H}$ & -0.62041500 & 4.31244700 & -1.78635100 \\
\hline $\mathrm{C}$ & 5.77158000 & 0.28292800 & -1.56926400 & $\mathrm{H}$ & -1.24190700 & 2.89306100 & -0.94163700 \\
\hline $\mathrm{C}$ & 5.65438500 & 1.65118900 & -1.34005900 & $\mathrm{H}$ & -0.88563700 & 4.37301300 & -0.04747500 \\
\hline $\mathrm{H}$ & 4.63887500 & 3.16694500 & -0.22763200 & $\mathrm{C}$ & 0.78319900 & -4.03123200 & -1.72321900 \\
\hline $\mathrm{H}$ & 5.07464100 & -1.65694400 & -1.04438600 & $\mathrm{H}$ & 0.74252600 & -4.83676300 & -0.98653500 \\
\hline $\mathrm{H}$ & 6.53606000 & -0.10510600 & -2.23491300 & $\mathrm{H}$ & -0.19657300 & -3.56526600 & -1.80529200 \\
\hline $\mathrm{H}$ & 6.32397700 & 2.35549300 & -1.82348100 & $\mathrm{H}$ & 1.00599400 & -4.49522800 & -2.69093400 \\
\hline $\mathrm{C}$ & 3.11909300 & -1.19868600 & 0.75004200 & $\mathrm{C}$ & 3.21608100 & -3.82241200 & -1.25522900 \\
\hline $\mathrm{H}$ & 3.77429000 & -2.05032400 & 0.96080100 & $\mathrm{H}$ & 3.16011200 & -4.56668000 & -0.45963500 \\
\hline $\mathrm{C}$ & 2.84511400 & 1.84853400 & 1.21380100 & $\mathrm{H}$ & 3.39525900 & -4.36308200 & -2.19144900 \\
\hline $\mathrm{H}$ & 2.83702300 & 1.24859600 & 2.12727200 & $\mathrm{H}$ & 4.09026600 & -3.19991500 & -1.07612200 \\
\hline $\mathrm{H}$ & 3.21623700 & 2.83673400 & 1.50274200 & $\mathrm{C}$ & 2.03816500 & -2.03902600 & -2.60653900 \\
\hline $\mathrm{H}$ & 2.83802200 & -0.78638500 & 1.72015300 & $\mathrm{H}$ & 2.31988300 & -2.60722400 & -3.50037700 \\
\hline $\mathrm{P}$ & 1.49111400 & -1.94010200 & 0.11893300 & $\mathrm{H}$ & 1.08910900 & -1.54072000 & -2.81477400 \\
\hline $\mathrm{P}$ & 1.02795800 & 2.00563900 & 0.75069100 & $\mathrm{H}$ & 2.80550600 & -1.27906200 & -2.44139000 \\
\hline $\mathrm{C}$ & 0.23977400 & 2.69910200 & 2.36458000 & $\mathrm{C}$ & -0.53799500 & -3.48477600 & 1.37574500 \\
\hline $\mathrm{C}$ & 0.92163100 & 3.27403200 & -0.68745000 & $\mathrm{H}$ & -0.65137000 & -4.10638600 & 0.48787900 \\
\hline $\mathrm{C}$ & 0.91336800 & -3.01596400 & 1.61061900 & $\mathrm{H}$ & -0.85492800 & -4.08229500 & 2.23798900 \\
\hline $\mathrm{C}$ & 1.90764900 & -3.01882200 & -1.41761300 & $\mathrm{H}$ & -1.22437600 & -2.63816200 & 1.29032700 \\
\hline $\mathrm{Pd}$ & -0.02586800 & -0.13091200 & -0.13045100 & $\mathrm{C}$ & 1.82230500 & -4.23745100 & 1.85533800 \\
\hline $\mathrm{C}$ & -1.28098900 & 2.43785400 & 2.30048200 & $\mathrm{H}$ & 1.50755200 & -4.72558100 & 2.78475000 \\
\hline
\end{tabular}




\begin{tabular}{|c|c|c|c|c|c|c|c|}
\hline $\mathrm{H}$ & 1.74311100 & -4.98042900 & 1.05946300 & $\mathrm{H}$ & -4.13607400 & 4.67504700 & -2.42823500 \\
\hline $\mathrm{H}$ & 2.87543900 & -3.96643200 & 1.97500800 & $\mathrm{H}$ & -5.87992500 & 2.88027200 & -2.36915000 \\
\hline $\mathrm{C}$ & 0.90932400 & -2.13595000 & 2.87916200 & $\mathrm{C}$ & -1.52662900 & 2.62035300 & 0.85249000 \\
\hline $\mathrm{H}$ & 1.91298100 & -1.86950900 & 3.22031600 & $\mathrm{H}$ & -1.35107400 & 3.66188800 & 1.13645400 \\
\hline $\mathrm{H}$ & 0.32663100 & -1.22141400 & 2.73310700 & $\mathrm{C}$ & -3.58778300 & 0.36179400 & 0.80314500 \\
\hline $\mathrm{H}$ & 0.43563700 & -2.69885300 & 3.69068600 & $\mathrm{H}$ & -3.29484700 & 0.68651600 & 1.80480000 \\
\hline $\mathrm{C}$ & -1.40867100 & -1.01175300 & -1.22502000 & $\mathrm{H}$ & -4.58978700 & -0.06486100 & 0.90519600 \\
\hline $\mathrm{O}$ & -1.40174500 & -1.72198100 & -2.18396500 & $\mathrm{H}$ & -1.82751500 & 2.11026400 & 1.76825700 \\
\hline $\mathrm{C}$ & -3.33317300 & 0.47756600 & -1.91851200 & $\mathrm{P}$ & 0.19602700 & 1.91828500 & 0.50094600 \\
\hline $\mathrm{H}$ & -3.94365200 & -0.23771500 & -2.47191300 & $\mathrm{P}$ & -2.39926200 & -1.04899500 & 0.40864100 \\
\hline $\mathrm{H}$ & -2.59083000 & 0.87719500 & -2.62083000 & $\mathrm{C}$ & -2.74493900 & -2.23018700 & 1.89745400 \\
\hline $\mathrm{C}$ & -2.63581300 & -0.19762800 & -0.71503600 & $\mathrm{C}$ & -3.04738200 & -1.80721100 & -1.24134300 \\
\hline $\mathrm{H}$ & -2.31407500 & 0.61765400 & -0.04905700 & $\mathrm{C}$ & 1.02293100 & 2.15189000 & 2.23436900 \\
\hline $\mathrm{C}$ & -3.54822800 & -1.11441200 & 0.09433600 & $\mathrm{C}$ & 0.96544200 & 3.10099100 & -0.81530700 \\
\hline $\mathrm{C}$ & -3.96942100 & -0.72192100 & 1.37226300 & $\mathrm{Pd}$ & 0.09552800 & -0.41791000 & -0.03004800 \\
\hline $\mathrm{C}$ & -4.01445900 & -2.32533700 & -0.43875100 & $\mathrm{C}$ & -1.64402100 & -3.30946800 & 1.94076600 \\
\hline $\mathrm{C}$ & -4.84485300 & -1.52529300 & 2.10493200 & $\mathrm{H}$ & -1.64172700 & -3.93740400 & 1.04919200 \\
\hline $\mathrm{H}$ & -3.63434700 & 0.22631300 & 1.78224300 & $\mathrm{H}$ & -0.64959800 & -2.86549900 & 2.04159700 \\
\hline $\mathrm{C}$ & -4.88814900 & -3.12626700 & 0.29637600 & $\mathrm{H}$ & -1.81431900 & -3.95815000 & 2.80817300 \\
\hline $\mathrm{H}$ & -3.69485800 & -2.64403400 & -1.42669200 & $\mathrm{C}$ & -2.64428400 & -1.41252700 & 3.20498200 \\
\hline $\mathrm{C}$ & -5.30362400 & -2.72974200 & 1.56993900 & $\mathrm{H}$ & -2.63712600 & -2.10778000 & 4.05195000 \\
\hline $\mathrm{H}$ & -5.17144000 & -1.20580100 & 3.08995600 & $\mathrm{H}$ & -1.72444400 & -0.82464200 & 3.26067600 \\
\hline $\mathrm{H}$ & -5.24524700 & -4.06017100 & -0.12691200 & $\mathrm{H}$ & -3.49575500 & -0.74326100 & 3.35111700 \\
\hline $\mathrm{H}$ & -5.98439500 & -3.35490300 & 2.13950100 & $\mathrm{C}$ & -4.12838800 & -2.90822400 & 1.86559900 \\
\hline $\mathrm{C}$ & -4.17367100 & 1.65392400 & -1.44457000 & $\mathrm{H}$ & -4.26918100 & -3.46819800 & 2.79787900 \\
\hline $\mathrm{O}$ & -3.87212300 & 2.36538100 & -0.50418000 & $\mathrm{H}$ & -4.94845500 & -2.18698600 & 1.79950800 \\
\hline $\mathrm{O}$ & -5.25222500 & 1.82779800 & -2.21100000 & $\mathrm{H}$ & -4.22867900 & -3.62137100 & 1.04482000 \\
\hline $\mathrm{C}$ & -6.08921000 & 2.95951300 & -1.88203700 & $\mathrm{C}$ & -4.58137500 & -1.86753200 & -1.37333300 \\
\hline $\mathrm{H}$ & -5.51994300 & 3.88781600 & -1.96333800 & $\mathrm{H}$ & -4.82781000 & -2.33470200 & -2.33429400 \\
\hline $\mathrm{H}$ & -6.90097300 & 2.93920700 & -2.60711800 & $\mathrm{H}$ & -5.05456000 & -2.46514700 & -0.59318200 \\
\hline \multirow[t]{2}{*}{$\mathrm{H}$} & -6.47476500 & 2.85988700 & -0.86547400 & $\mathrm{H}$ & -5.03731900 & -0.87861800 & -1.37754600 \\
\hline & & & & $\mathrm{C}$ & -2.48382800 & -0.91316600 & -2.37145800 \\
\hline 80 & & & & $\mathrm{H}$ & -2.82088400 & -1.30650100 & -3.33803000 \\
\hline $\mathrm{C}$ & -4.79938500 & 1.70896000 & -0.92358700 & $\mathrm{H}$ & -2.82705900 & 0.12106100 & -2.29703000 \\
\hline $\mathrm{C}$ & -3.65426100 & 1.55737900 & -0.12347300 & $\mathrm{H}$ & -1.38865100 & -0.90886100 & -2.37940400 \\
\hline $\mathrm{C}$ & -2.67876700 & 2.58262800 & -0.13737500 & $\mathrm{C}$ & -2.48567400 & -3.23036100 & -1.43308900 \\
\hline $\mathrm{C}$ & -2.89511800 & 3.69129100 & -0.97341600 & $\mathrm{H}$ & -2.72317200 & -3.56238800 & -2.45093100 \\
\hline $\mathrm{C}$ & -4.01191200 & 3.80068800 & -1.79720900 & $\mathrm{H}$ & -1.40226500 & -3.26177000 & -1.31093100 \\
\hline $\mathrm{C}$ & -4.98076600 & 2.80135200 & -1.76627200 & $\mathrm{H}$ & -2.93793000 & -3.95160400 & -0.74725400 \\
\hline $\mathrm{H}$ & -5.58628400 & 0.96466400 & -0.85893600 & $\mathrm{C}$ & 2.50002200 & 2.96456700 & -0.88354500 \\
\hline $\mathrm{H}$ & -2.18825800 & 4.51210700 & -0.96020500 & $\mathrm{H}$ & 2.99126500 & 3.40679200 & -0.01327500 \\
\hline
\end{tabular}




\begin{tabular}{|c|c|c|c|c|c|c|c|}
\hline $\mathrm{H}$ & 2.84540200 & 1.93846100 & -0.99804600 & $\mathrm{O}$ & 0.64701800 & -2.79948400 & -0.36630300 \\
\hline $\mathrm{H}$ & 2.84981000 & 3.51704700 & -1.76309600 & $\mathrm{O}$ & 1.86184900 & -4.65396500 & -0.74925500 \\
\hline $\mathrm{C}$ & 0.66005700 & 4.59347000 & -0.55800800 & $\mathrm{C}$ & 0.67694400 & -5.48267000 & -0.70475100 \\
\hline $\mathrm{H}$ & 1.12687700 & 4.97190200 & 0.35157800 & $\mathrm{H}$ & 0.00701500 & -5.22393700 & -1.52645800 \\
\hline $\mathrm{H}$ & 1.07174600 & 5.16769600 & -1.39553200 & $\mathrm{H}$ & 1.03994500 & -6.50278400 & -0.81491200 \\
\hline $\mathrm{H}$ & -0.40338000 & 4.82118300 & -0.51199500 & $\mathrm{H}$ & 0.16491500 & -5.35612300 & 0.25029900 \\
\hline $\mathrm{C}$ & 0.35424500 & 2.68166500 & -2.17329000 & & & & \\
\hline $\mathrm{H}$ & 0.73040700 & 3.36034100 & -2.94783900 & 80-0 & & & \\
\hline $\mathrm{H}$ & 0.64179000 & 1.66697100 & -2.45142700 & $\mathrm{C}$ & -5.18483900 & 0.56685300 & -0.79895500 \\
\hline $\mathrm{H}$ & -0.73549700 & 2.75057100 & -2.17673500 & $\mathrm{C}$ & -4.06959400 & 0.39615500 & 0.03733000 \\
\hline $\mathrm{C}$ & 2.42156000 & 1.50795700 & 2.24054400 & $\mathrm{C}$ & -3.50115400 & 1.53656600 & 0.65461800 \\
\hline $\mathrm{H}$ & 3.09224100 & 1.90586700 & 1.47981200 & $\mathrm{C}$ & -4.09758000 & 2.78539100 & 0.41125900 \\
\hline $\mathrm{H}$ & 2.88690900 & 1.68816200 & 3.21656900 & $\mathrm{C}$ & -5.18213800 & 2.94175800 & -0.44787000 \\
\hline $\mathrm{H}$ & 2.35156300 & 0.42697500 & 2.10662300 & $\mathrm{C}$ & -5.73153600 & 1.81964900 & -1.06276300 \\
\hline $\mathrm{C}$ & 1.14616300 & 3.62707800 & 2.66727100 & $\mathrm{H}$ & -5.65540800 & -0.31148900 & -1.22966100 \\
\hline $\mathrm{H}$ & 1.47555900 & 3.65459200 & 3.71236400 & $\mathrm{H}$ & -3.72884500 & 3.65805900 & 0.93872300 \\
\hline $\mathrm{H}$ & 1.89337300 & 4.16750600 & 2.08312800 & $\mathrm{H}$ & -5.60941100 & 3.92667000 & -0.60758400 \\
\hline $\mathrm{H}$ & 0.20116000 & 4.17446200 & 2.61625800 & $\mathrm{H}$ & -6.59419800 & 1.91064800 & -1.71519700 \\
\hline $\mathrm{C}$ & 0.17826300 & 1.39118000 & 3.27930800 & $\mathrm{C}$ & -2.40364300 & 1.45029000 & 1.70223500 \\
\hline $\mathrm{H}$ & -0.78707100 & 1.86147000 & 3.48245700 & $\mathrm{H}$ & -2.53496200 & 2.27877600 & 2.40509700 \\
\hline $\mathrm{H}$ & 0.01161200 & 0.35352700 & 2.97501300 & $\mathrm{C}$ & -3.61310400 & -1.01717000 & 0.33335800 \\
\hline $\mathrm{H}$ & 0.72841900 & 1.37305200 & 4.22669500 & $\mathrm{H}$ & -3.44394700 & -1.12774900 & 1.40788500 \\
\hline $\mathrm{C}$ & 1.90487200 & -0.44571700 & -0.87843800 & $\mathrm{H}$ & -4.41293400 & -1.71817600 & 0.07602200 \\
\hline $\mathrm{O}$ & 1.97569900 & -0.21053100 & -2.05717800 & $\mathrm{H}$ & -2.53449900 & 0.53746100 & 2.28724600 \\
\hline $\mathrm{C}$ & 3.05606800 & -2.63637300 & -0.70412000 & $\mathrm{P}$ & -0.55756900 & 1.43384100 & 1.27868900 \\
\hline $\mathrm{H}$ & 3.82512600 & -3.23439700 & -0.20710900 & $\mathrm{P}$ & -2.01911100 & -1.62237200 & -0.44932700 \\
\hline $\mathrm{H}$ & 3.32242500 & -2.60505900 & -1.76703600 & $\mathrm{C}$ & -1.81787700 & -3.37823600 & 0.31949900 \\
\hline $\mathrm{C}$ & 3.02401800 & -1.20920900 & -0.12170700 & $\mathrm{C}$ & -2.31877200 & -1.70867100 & -2.34446600 \\
\hline $\mathrm{H}$ & 2.73656600 & -1.27957200 & 0.92968800 & $\mathrm{C}$ & 0.21368600 & 1.32169000 & 3.04610300 \\
\hline $\mathrm{C}$ & 4.39348400 & -0.54606800 & -0.21048200 & $\mathrm{C}$ & -0.17380100 & 3.10857400 & 0.41642900 \\
\hline $\mathrm{C}$ & 5.14600700 & -0.38643200 & 0.96187800 & $\mathrm{Pd}$ & 0.07032600 & -0.37243700 & -0.07957300 \\
\hline $\mathrm{C}$ & 4.95321500 & -0.14948000 & -1.43440100 & $\mathrm{C}$ & -0.37078200 & -3.86395700 & 0.08401100 \\
\hline $\mathrm{C}$ & 6.42849000 & 0.16183300 & 0.91678600 & $\mathrm{H}$ & -0.11382900 & -3.94267800 & -0.97318400 \\
\hline $\mathrm{H}$ & 4.73076500 & -0.69995000 & 1.91634100 & $\mathrm{H}$ & 0.36015600 & -3.20581000 & 0.56391400 \\
\hline $\mathrm{C}$ & 6.23401500 & 0.40294700 & -1.47666300 & $\mathrm{H}$ & -0.25798000 & -4.86114800 & 0.52563100 \\
\hline $\mathrm{H}$ & 4.38497600 & -0.26080000 & -2.35165500 & $\mathrm{C}$ & -2.02692300 & -3.26259400 & 1.84633600 \\
\hline $\mathrm{C}$ & 6.97489100 & 0.56050000 & -0.30365400 & $\mathrm{H}$ & -1.71945300 & -4.20511100 & 2.31265700 \\
\hline $\mathrm{H}$ & 6.99823900 & 0.27532900 & 1.83413700 & $\mathrm{H}$ & -1.41842600 & -2.46893700 & 2.29041500 \\
\hline $\mathrm{H}$ & 6.65394800 & 0.70793100 & -2.43042100 & $\mathrm{H}$ & -3.07286400 & -3.09791500 & 2.11833700 \\
\hline $\mathrm{H}$ & 7.97210900 & 0.98805000 & -0.34129300 & $\mathrm{C}$ & -2.81331400 & -4.41758100 & -0.22923300 \\
\hline $\mathrm{C}$ & 1.72919300 & -3.34772800 & -0.58272700 & $\mathrm{H}$ & -2.70188400 & -5.34872800 & 0.33873600 \\
\hline
\end{tabular}




\begin{tabular}{|c|c|c|c|c|c|c|c|}
\hline $\mathrm{H}$ & -3.85468500 & -4.09845300 & -0.12328400 & $\mathrm{H}$ & 5.45501200 & -0.70605400 & 0.07176400 \\
\hline $\mathrm{H}$ & -2.63006400 & -4.65746100 & -1.27857500 & $\mathrm{H}$ & 4.46450800 & -1.67384100 & -1.00277200 \\
\hline $\mathrm{C}$ & -3.70196200 & -2.25839900 & -2.74478100 & $\mathrm{C}$ & 3.46718600 & 0.11244700 & -0.23698900 \\
\hline $\mathrm{H}$ & -3.76898000 & -2.27178500 & -3.83900200 & $\mathrm{H}$ & 3.46806600 & 0.69424600 & 0.68577700 \\
\hline $\mathrm{H}$ & -3.87497000 & -3.27735100 & -2.39668000 & $\mathrm{C}$ & 3.88522600 & 1.02905800 & -1.38924600 \\
\hline $\mathrm{H}$ & -4.51646600 & -1.62813700 & -2.38461300 & $\mathrm{C}$ & 4.55001700 & 2.22757400 & -1.09530400 \\
\hline $\mathrm{C}$ & -2.18155400 & -0.26393900 & -2.87916300 & $\mathrm{C}$ & 3.68324500 & 0.67298600 & -2.72975900 \\
\hline $\mathrm{H}$ & -2.33718900 & -0.27370900 & -3.96438700 & $\mathrm{C}$ & 5.00740900 & 3.05479200 & -2.12131700 \\
\hline $\mathrm{H}$ & -2.91606900 & 0.41726600 & -2.44455400 & $\mathrm{H}$ & 4.71979400 & 2.51019100 & -0.05941800 \\
\hline $\mathrm{H}$ & -1.18290000 & 0.14106500 & -2.68957400 & $\mathrm{C}$ & 4.13565800 & 1.50518200 & -3.75493200 \\
\hline $\mathrm{C}$ & -1.21816700 & -2.56439500 & -3.00672400 & $\mathrm{H}$ & 3.17582700 & -0.25465800 & -2.97734800 \\
\hline $\mathrm{H}$ & -1.29688000 & -2.45061200 & -4.09409500 & $\mathrm{C}$ & 4.79805700 & 2.69669100 & -3.45409700 \\
\hline $\mathrm{H}$ & -0.21285000 & -2.24663400 & -2.71482500 & $\mathrm{H}$ & 5.52709100 & 3.97664300 & -1.87837700 \\
\hline $\mathrm{H}$ & -1.32427900 & -3.62929800 & -2.78694000 & $\mathrm{H}$ & 3.97310900 & 1.21874900 & -4.78950900 \\
\hline $\mathrm{C}$ & 1.34419200 & 3.35916500 & 0.37753500 & $\mathrm{H}$ & 5.15151400 & 3.34063900 & -4.25340000 \\
\hline $\mathrm{H}$ & 1.75151900 & 3.58995900 & 1.36489800 & $\mathrm{C}$ & 4.05164900 & -1.99051200 & 1.06553800 \\
\hline $\mathrm{H}$ & 1.89383400 & 2.51843500 & -0.04808600 & $\mathrm{O}$ & 2.98374900 & -1.93817200 & 1.65223600 \\
\hline $\mathrm{H}$ & 1.53692900 & 4.22693000 & -0.26323700 & $\mathrm{O}$ & 5.02070600 & -2.86443300 & 1.34330900 \\
\hline $\mathrm{C}$ & -0.84493800 & 4.32790300 & 1.08120200 & $\mathrm{C}$ & 4.75090100 & -3.80718300 & 2.40419700 \\
\hline $\mathrm{H}$ & -0.49569100 & 4.50368400 & 2.09889800 & $\mathrm{H}$ & 3.88802700 & -4.42644700 & 2.15001500 \\
\hline $\mathrm{H}$ & -0.59306200 & 5.21785900 & 0.49321000 & $\mathrm{H}$ & 5.65013800 & -4.41532900 & 2.48602900 \\
\hline $\mathrm{H}$ & -1.93084600 & 4.25614500 & 1.09711600 & $\mathrm{H}$ & 4.55497200 & -3.27886700 & 3.33949000 \\
\hline $\mathrm{C}$ & -0.68482800 & 2.97526900 & -1.03584500 & & & & \\
\hline $\mathrm{H}$ & -0.47498200 & 3.90872700 & -1.57068000 & 80-CO & & & \\
\hline $\mathrm{H}$ & -0.17615400 & 2.16422400 & -1.56536000 & $\mathrm{C}$ & -5.43645700 & 0.62905100 & -0.25268300 \\
\hline $\mathrm{H}$ & -1.76130900 & 2.79787600 & -1.08537900 & $\mathrm{C}$ & -4.17872600 & 0.12205200 & -0.61966000 \\
\hline $\mathrm{C}$ & 1.73171800 & 1.06959300 & 2.95176700 & $\mathrm{C}$ & -3.90185100 & -1.24251800 & -0.36702000 \\
\hline $\mathrm{H}$ & 2.25978200 & 1.87167800 & 2.43334800 & $\mathrm{C}$ & -4.90951700 & -2.02485500 & 0.22284800 \\
\hline $\mathrm{H}$ & 2.13615600 & 1.02217400 & 3.96969200 & $\mathrm{C}$ & -6.13079900 & -1.49293300 & 0.62670700 \\
\hline $\mathrm{H}$ & 1.95706600 & 0.11886600 & 2.46462200 & $\mathrm{C}$ & -6.39731000 & -0.14812300 & 0.38805800 \\
\hline $\mathrm{C}$ & -0.03585000 & 2.57828400 & 3.90426300 & $\mathrm{H}$ & -5.69142000 & 1.64843800 & -0.51912300 \\
\hline $\mathrm{H}$ & 0.28942500 & 2.36781800 & 4.92953200 & $\mathrm{H}$ & -4.75543900 & -3.09275800 & 0.32118300 \\
\hline $\mathrm{H}$ & 0.54195100 & 3.43757000 & 3.55668200 & $\mathrm{H}$ & -6.87780700 & -2.13503000 & 1.08246100 \\
\hline $\mathrm{H}$ & -1.08945300 & 2.86609900 & 3.95710300 & $\mathrm{H}$ & -7.35584200 & 0.28487600 & 0.65593700 \\
\hline $\mathrm{C}$ & -0.40698100 & 0.09684400 & 3.75482600 & $\mathrm{C}$ & -2.67952100 & -1.94693500 & -0.92522300 \\
\hline $\mathrm{H}$ & -1.45281600 & 0.24692000 & 4.03413900 & $\mathrm{H}$ & -2.95774400 & -2.98590100 & -1.11984500 \\
\hline $\mathrm{H}$ & -0.32524300 & -0.80683000 & 3.14414600 & $\mathrm{C}$ & -3.25143300 & 1.01162700 & -1.42416200 \\
\hline $\mathrm{H}$ & 0.14723100 & -0.08654100 & 4.68183300 & $\mathrm{H}$ & -2.83502800 & 0.44112600 & -2.25836400 \\
\hline $\mathrm{C}$ & 2.04833300 & -0.39195700 & -0.44737300 & $\mathrm{H}$ & -3.83716300 & 1.81795100 & -1.87357800 \\
\hline $\mathrm{O}$ & 1.76685000 & -1.33157200 & -1.17486500 & $\mathrm{H}$ & -2.44011400 & -1.51440400 & -1.89853000 \\
\hline $\mathrm{C}$ & 4.43808500 & -1.07847800 & -0.08381600 & $\mathrm{P}$ & -0.97762100 & -2.05946600 & -0.09430400 \\
\hline
\end{tabular}




\begin{tabular}{|c|c|c|c|c|c|c|c|}
\hline$P$ & -1.75025000 & 1.79747400 & -0.60161700 & $\mathrm{H}$ & -2.37068500 & -1.32206500 & 2.37362000 \\
\hline $\mathrm{C}$ & -0.88649100 & 2.63592600 & -2.10993300 & $\mathrm{C}$ & 1.39671300 & -3.29588700 & -1.10465000 \\
\hline $\mathrm{C}$ & -2.48520100 & 3.12327900 & 0.59944300 & $\mathrm{H}$ & 1.60046300 & -3.68779800 & -0.10790200 \\
\hline $\mathrm{C}$ & -0.10619400 & -3.17713500 & -1.41470000 & $\mathrm{H}$ & 1.85212100 & -3.98379800 & -1.82592200 \\
\hline $\mathrm{C}$ & -1.22140400 & -3.02055400 & 1.56778100 & $\mathrm{H}$ & 1.90030100 & -2.33519700 & -1.22074300 \\
\hline $\mathrm{Pd}$ & 0.04150900 & 0.18312200 & 0.43252700 & $\mathrm{C}$ & -0.71237500 & -4.59412300 & -1.49984400 \\
\hline $\mathrm{C}$ & 0.45805800 & 3.25215200 & -1.66954700 & $\mathrm{H}$ & -0.21990800 & -5.13119800 & -2.31847100 \\
\hline $\mathrm{H}$ & 0.34328900 & 4.07709600 & -0.96625800 & $\mathrm{H}$ & -0.55029500 & -5.17454600 & -0.59003700 \\
\hline $\mathrm{H}$ & 1.11972100 & 2.50757500 & -1.21863900 & $\mathrm{H}$ & -1.78238800 & -4.59234900 & -1.72304700 \\
\hline $\mathrm{H}$ & 0.96780800 & 3.65014600 & -2.55464800 & $\mathrm{C}$ & -0.23011500 & -2.50236000 & -2.79961700 \\
\hline $\mathrm{C}$ & -0.56355000 & 1.52591700 & -3.13471500 & $\mathrm{H}$ & -1.23977500 & -2.55542900 & -3.21318400 \\
\hline $\mathrm{H}$ & 0.06293900 & 1.94843500 & -3.92826200 & $\mathrm{H}$ & 0.08619700 & -1.45520400 & -2.77819700 \\
\hline $\mathrm{H}$ & -0.00342100 & 0.70403400 & -2.67703300 & $\mathrm{H}$ & 0.42840300 & -3.02590000 & -3.50133700 \\
\hline $\mathrm{H}$ & -1.45352300 & 1.11559000 & -3.61870200 & $\mathrm{C}$ & 1.82954600 & -0.70866900 & 1.15484800 \\
\hline $\mathrm{C}$ & -1.74404100 & 3.71547500 & -2.79731800 & $\mathrm{O}$ & 1.87724800 & -1.23557800 & 2.22937300 \\
\hline $\mathrm{H}$ & -1.22023200 & 4.06562900 & -3.69450100 & $\mathrm{C}$ & 3.08213500 & -0.44732700 & 0.28331800 \\
\hline $\mathrm{H}$ & -2.71823600 & 3.33901900 & -3.12211300 & $\mathrm{H}$ & 2.77239200 & -0.42811700 & -0.76356000 \\
\hline $\mathrm{H}$ & -1.90471100 & 4.58651400 & -2.15865600 & $\mathrm{C}$ & 4.11416400 & -1.58107500 & 0.49658000 \\
\hline $\mathrm{C}$ & -3.77238700 & 3.78874900 & 0.06922700 & $\mathrm{H}$ & 3.63737900 & -2.55666100 & 0.35652900 \\
\hline $\mathrm{H}$ & -4.05758400 & 4.58736300 & 0.76354100 & $\mathrm{H}$ & 4.51286900 & -1.55730600 & 1.51059700 \\
\hline $\mathrm{H}$ & -3.64372700 & 4.24588500 & -0.91330100 & $\mathrm{C}$ & 5.29189400 & -1.50407200 & -0.46127500 \\
\hline $\mathrm{H}$ & -4.60987400 & 3.09668600 & 0.02503200 & $\mathrm{O}$ & 6.44809200 & -1.63968300 & -0.13986400 \\
\hline $\mathrm{C}$ & -2.81308000 & 2.37536900 & 1.91212200 & $\mathrm{O}$ & 4.88000200 & -1.30455100 & -1.73553700 \\
\hline $\mathrm{H}$ & -3.28238400 & 3.07375800 & 2.61457500 & $\mathrm{C}$ & 5.93533900 & -1.26035300 & -2.71903500 \\
\hline $\mathrm{H}$ & -3.51044700 & 1.54878100 & 1.75263600 & $\mathrm{H}$ & 5.44064100 & -1.08543700 & -3.67391800 \\
\hline $\mathrm{H}$ & -1.91627100 & 1.97738400 & 2.39492500 & $\mathrm{H}$ & 6.63413200 & -0.45274100 & -2.49118500 \\
\hline $\mathrm{C}$ & -1.48187500 & 4.25665900 & 0.89837900 & $\mathrm{H}$ & 6.48004300 & -2.20677700 & -2.73513400 \\
\hline $\mathrm{H}$ & -1.87867100 & 4.85255600 & 1.72811400 & $\mathrm{C}$ & 3.66681400 & 0.91915400 & 0.62899900 \\
\hline $\mathrm{H}$ & -0.49604300 & 3.90846500 & 1.20125200 & $\mathrm{C}$ & 3.82433900 & 1.88783500 & -0.37240000 \\
\hline $\mathrm{H}$ & -1.36290500 & 4.93300600 & 0.04876000 & $\mathrm{C}$ & 4.10200600 & 1.21027600 & 1.93111300 \\
\hline $\mathrm{C}$ & 0.03635500 & -3.81773900 & 1.97442400 & $\mathrm{C}$ & 4.40215600 & 3.12492800 & -0.07945200 \\
\hline $\mathrm{H}$ & 0.19309000 & -4.69091000 & 1.33710100 & $\mathrm{H}$ & 3.51921800 & 1.66081400 & -1.39026600 \\
\hline $\mathrm{H}$ & 0.94368300 & -3.21940300 & 1.99620200 & $\mathrm{C}$ & 4.67438100 & 2.44745800 & 2.22373100 \\
\hline $\mathrm{H}$ & -0.12344100 & -4.19128600 & 2.99222200 & $\mathrm{H}$ & 3.99003300 & 0.47136000 & 2.71947700 \\
\hline $\mathrm{C}$ & -2.40300000 & -4.01112000 & 1.52898100 & $\mathrm{C}$ & 4.82431800 & 3.40844600 & 1.22026600 \\
\hline $\mathrm{H}$ & -2.37991700 & -4.68701500 & 0.67147100 & $\mathrm{H}$ & 4.53020700 & 3.86081300 & -0.86764200 \\
\hline $\mathrm{H}$ & -2.35512200 & -4.63294600 & 2.42989700 & $\mathrm{H}$ & 5.00806400 & 2.65956900 & 3.23476400 \\
\hline $\mathrm{H}$ & -3.36046000 & -3.49680400 & 1.55006100 & $\mathrm{H}$ & 5.27590600 & 4.36869800 & 1.44947000 \\
\hline $\mathrm{C}$ & -1.51115900 & -1.94545300 & 2.63949200 & $\mathrm{C}$ & 0.76726000 & 1.65111700 & 1.54317300 \\
\hline $\mathrm{H}$ & -1.74689300 & -2.44338500 & 3.58732000 & $\mathrm{O}$ & 1.01419000 & 2.40896400 & 2.35969700 \\
\hline $\mathrm{H}$ & -0.64297900 & -1.30356500 & 2.80865500 & & & & \\
\hline
\end{tabular}


TS63

imaginary frequency $=-132.18 \mathrm{~cm}^{-1}$

C

$\mathrm{C}$

C

C

C

C

$\mathrm{H}$

$\mathrm{H}$

$\mathrm{H}$

$\mathrm{H}$

C

$\mathrm{H}$

C

$\mathrm{H}$

$\mathrm{H}$

$\mathrm{H}$

$\mathrm{P}$

$\mathrm{P}$

C

C

C

C

Pd

C

C

$\mathrm{H}$

$\mathrm{H}$

$\mathrm{H}$

C

$\mathrm{H}$

$\mathrm{H}$

$\mathrm{H}$

C

$\mathrm{H}$

$\mathrm{H}$

$\mathrm{H}$

C

$\mathrm{H}$

H

3.85190500
$4.40672100 \quad 2.62993400$

1.44453800

$-0.75940000$

4.13153600

0.22988700

$-0.24386500$

4.92894700

0.28067000

$-0.92019200$

5.42340100

5.16214300

1.47193800

$-2.07770300$

$2.66338000-1.9272160$

$-1.92721600$

$\begin{array}{lll}4.26936900 & 3.55323500 & -0.20685900\end{array}$

5.20291100

$-0.65067600$

6.03481400

5.56675400

$1.46131400 \quad-3.49588900$

$3.60464100-2.28602100$

$3.76646300 \quad-1.14391500 \quad-0.38673200$

4.48366000

$-1.86935600$

3.13419000

1.53161400

$-0.78368600$

1.09197300

3.35986100

0.63934500

1.68080500

3.55574500

3.89562200

2.37843700

1.64245000

0.69893900

2.02612100

1.24078300

$\begin{array}{rr}-1.84883100 & -0.61932400 \\ 1.65805300 & 1.25026200\end{array}$

1.11785500

0.68960100

2.02468700

3.15067100

3.20031900

0.23224000

2.22939700

$-3.56829000$

0.24086100

1.77429500

$-2.07645800$

0.30052900

$-0.36121600$

$-2.51273400$

0.38051100

$-3.19615700$

0.22142300

$-0.14534500$

0.83019700

$-4.19849000$

0.40760700

0.90558100

$-5.09034600$

1.04216000

$0.12581400 \quad-3.49649500$

0.86277600

0.39706000

$-4.51028600$

$-0.54333100$

$3.14839000 \quad-4.56894500 \quad-0.48445300$

4.14279900

3.28666500

2.72083400

$-4.15881400$

$-0.68407200$

$-5.45585800 \quad 0.14629600$

2.81309000

$-4.9106920$

$-1.42916600$

$-3.31545500 \quad 1.65028800$

2.29972700

$-2.49940300$

2.17089800

2.70364400

$-4.22372700$

2.25443700

3.87938400

$-3.07765900$

1.62354300

1.40441000

2.18763700

$-0.67735400 \quad-3.05747100$

$0.06286700 \quad-2.87855800$

$\begin{array}{lll}1.24968900 & -0.74491200 & -4.14112700\end{array}$
$\mathrm{H}$

$\mathrm{H}$

H

C

$\mathrm{H}$

$\mathrm{H}$

H

C

$\mathrm{H}$

$\mathrm{H}$

$\mathrm{H}$

C

H

$\mathrm{H}$

$\mathrm{H}$

C

$\mathrm{H}$

H

$\mathrm{H}$

C

$\mathrm{H}$

$\mathrm{H}$

$\mathrm{H}$

C

H

H

H

C

H

H

$\mathrm{H}$

C

O

C

H

H

H

O

H

\begin{tabular}{|c|c|c|}
\hline 0.47481400 & -0.31164400 & -2.61066300 \\
\hline 2.99371400 & -2.60376700 & -3.29271100 \\
\hline 3.32605300 & -3.58725700 & -2.95798100 \\
\hline 2.71966200 & -2.69693900 & -4.35062300 \\
\hline 3.84147600 & -1.92029700 & -3.24419600 \\
\hline 0.57304900 & -3.01260900 & -2.76393200 \\
\hline-0.29977600 & -2.73448300 & -2.16708900 \\
\hline 0.29135600 & -2.94448200 & -3.82161700 \\
\hline 0.81573900 & -4.06029200 & -2.56777400 \\
\hline 1.91185100 & 0.92115600 & 3.88962500 \\
\hline 1.68166500 & -0.08256100 & 3.52046300 \\
\hline 2.99210000 & 1.06741900 & 3.81383600 \\
\hline 1.65663300 & 0.94999600 & 4.95498500 \\
\hline 1.68918000 & 3.38692700 & 3.59612500 \\
\hline 1.74931700 & 3.40262000 & 4.69101000 \\
\hline 2.69686100 & 3.57875900 & 3.21731500 \\
\hline 1.04616000 & 4.21832000 & 3.30198000 \\
\hline-0.35701900 & 1.95133800 & 3.59767000 \\
\hline-0.81597200 & 0.99562600 & 3.34290100 \\
\hline-0.40662500 & 2.06715600 & 4.68711900 \\
\hline-0.96279500 & 2.74579900 & 3.16032200 \\
\hline 1.49985700 & 4.49285800 & 0.45375400 \\
\hline 1.34049100 & 4.92597800 & 1.44067900 \\
\hline 2.57103600 & 4.35479900 & 0.31012800 \\
\hline 1.17178500 & 5.23756400 & -0.28138900 \\
\hline-0.79131000 & 3.50021000 & 0.53864300 \\
\hline-0.91958200 & 3.97817700 & 1.51278800 \\
\hline-1.18460900 & 4.19420900 & -0.21203100 \\
\hline-1.41629800 & 2.60509600 & 0.51000200 \\
\hline 0.81552100 & 2.79578500 & -1.25462600 \\
\hline 0.29524100 & 1.85803500 & -1.46822500 \\
\hline 0.36891700 & 3.58048100 & -1.87683800 \\
\hline 1.85462200 & 2.67525000 & -1.56481900 \\
\hline-2.29562400 & -0.87913100 & 0.38153100 \\
\hline-1.56470700 & -1.63308800 & -0.25651900 \\
\hline-3.34255300 & -2.58404200 & 1.80583100 \\
\hline-4.31562300 & -2.50532600 & 1.32338600 \\
\hline-3.46934700 & -2.79776100 & 2.86790100 \\
\hline-2.73338600 & -3.35074300 & 1.32195600 \\
\hline-2.67378700 & -1.28405600 & 1.73200500 \\
\hline-1.53670800 & -1.35396300 & 2.50942700 \\
\hline
\end{tabular}

$\begin{array}{lll}0.47481400 & -0.31164400 & -2.61066300\end{array}$

$3.32605300 \quad-3.58725700 \quad-2.95798100$

$2.71966200 \quad-2.69693900 \quad-4.35062300$

$\begin{array}{lll}3.84147600 & -1.92029700 & -3.24419600\end{array}$

$0.57304900-3.0126090--2.76393200$

$0.29135600 \quad-2.94448200 \quad-3.82161700$

$\begin{array}{lll}0.81573900 & -4.06029200 & -2.56777400\end{array}$

$\begin{array}{lll}1.68166500 & -0.08256100 & 3.52046300\end{array}$

$1.65663300 \quad 0.94999600 \quad 4.95498500$

$\begin{array}{lll}1.68918000 & 3.38692700 & 3.59612500\end{array}$

$1.74931700 \quad 3.40262000 \quad 4.69101000$

3.21731500

$\begin{array}{lll}1.04616000 & 4.21832000 & 3.30198000\end{array}$

$\begin{array}{lll}-0.81597200 & 0.99562600 & 3.34290100\end{array}$

$\begin{array}{lll}-0.40662500 & 2.06715600 \quad 4.68711900\end{array}$

$\begin{array}{lll}-0.96279500 & 2.74579900 & 3.16032200\end{array}$

$1.34049100 \quad 4.92597800 \quad 1.44067900$

$\begin{array}{lll}2.57103600 & 4.35479900 & 0.31012800\end{array}$

$\begin{array}{lll}1.17178500 & 5.23756400 & -0.28138900\end{array}$

$-0.91958200 \quad 3.97817700 \quad 1.51278800$

$\begin{array}{lll}-1.18460900 & 4.19420900 & -0.21203100\end{array}$

$\begin{array}{lll}-1.41629800 & 2.60509600 & 0.51000200\end{array}$

$0.29524100 \quad 1.85803500 \quad-1.46822500$

$\begin{array}{lll}0.36891700 & 3.58048100 & -1.87683800\end{array}$

$\begin{array}{rrr}1.85462200 & 2.67525000 & -1.56481900 \\ -2.29562400 & -0.87913100 & 0.38153100 \\ -1.56470700 & -1.63308800 & -0.25651900 \\ -3.34255300 & -2.58404200 & 1.80583100 \\ -4.31562300 & -2.50532600 & 1.32338600 \\ -3.46934700 & -2.79776100 & 2.86790100 \\ -2.73338600 & -3.35074300 & 1.32195600 \\ -2.67378700 & -1.28405600 & 1.73200500 \\ -1.53670800 & -1.35396300 & 2.50942700\end{array}$ 


\begin{tabular}{|c|c|c|c|c|c|c|c|}
\hline $\mathrm{C}$ & -0.23651300 & -2.56258800 & 3.75137100 & $\mathrm{C}$ & -4.74151100 & -0.92320400 & -1.55871200 \\
\hline $\mathrm{H}$ & -0.99326600 & -2.66523100 & 4.52879400 & $\mathrm{C}$ & -5.38765600 & -0.22553100 & -2.57259000 \\
\hline $\mathrm{H}$ & 0.73908700 & -2.39903100 & 4.20649200 & $\mathrm{C}$ & -5.24536700 & 1.15946200 & -2.63798200 \\
\hline $\mathrm{H}$ & -0.21759200 & -3.43713600 & 3.10061800 & $\mathrm{C}$ & -4.45417200 & 1.80183100 & -1.69261700 \\
\hline $\mathrm{O}$ & -0.57359800 & -1.36150300 & 2.99837400 & $\mathrm{C}$ & -3.75725700 & 1.11066200 & -0.68259100 \\
\hline $\mathrm{H}$ & 0.07138400 & -1.08610100 & 2.21551900 & $\mathrm{H}$ & -4.11265700 & -1.98880700 & 0.64705400 \\
\hline $\mathrm{H}$ & -3.19196700 & 1.03930400 & 0.57899900 & $\mathrm{H}$ & -3.41589100 & -0.60824400 & 1.46071800 \\
\hline $\mathrm{C}$ & -4.63673100 & -0.29912700 & -0.23708200 & $\mathrm{H}$ & -3.13783000 & 1.54480700 & 1.33336500 \\
\hline C & -5.64676300 & 0.29454900 & 0.52888700 & $\mathrm{H}$ & -3.49459100 & 2.94494900 & 0.34929500 \\
\hline C & -4.96597000 & -1.35961500 & -1.09536200 & $\mathrm{H}$ & -4.90439300 & -1.99413900 & -1.47923000 \\
\hline $\mathrm{C}$ & -6.96345800 & -0.16095000 & 0.43850300 & $\mathrm{H}$ & -6.01886700 & -0.75366300 & -3.28041700 \\
\hline $\mathrm{H}$ & -5.40455700 & 1.12823800 & 1.18135400 & $\mathrm{H}$ & -5.76401500 & 1.73646100 & -3.39723300 \\
\hline $\mathrm{C}$ & -6.28182700 & -1.81588500 & -1.18369700 & $\mathrm{H}$ & -4.38568100 & 2.88583500 & -1.72025700 \\
\hline $\mathrm{H}$ & -4.19378000 & -1.83338300 & -1.69676800 & $\mathrm{C}$ & 2.31867900 & 0.41462700 & 1.02027200 \\
\hline $\mathrm{C}$ & -7.28371100 & -1.21754600 & -0.41551600 & $\mathrm{O}$ & 2.29498600 & 1.00296200 & 2.04352000 \\
\hline $\mathrm{H}$ & -7.73887900 & 0.31474800 & 1.03134800 & $\mathrm{O}$ & 2.21134200 & -1.23683100 & 1.19580500 \\
\hline $\mathrm{H}$ & -6.52451600 & -2.63489300 & -1.85420800 & $\mathrm{C}$ & 2.45092500 & -1.69566400 & 2.56414100 \\
\hline $\mathrm{H}$ & -8.30809900 & -1.57009200 & -0.48686100 & $\mathrm{H}$ & 2.28447300 & -2.77125100 & 2.56454700 \\
\hline $\mathrm{C}$ & -2.69258200 & 0.75774500 & -1.49229500 & $\mathrm{H}$ & 3.49314800 & -1.46729200 & 2.77913500 \\
\hline $\mathrm{H}$ & -1.61876300 & 0.96849800 & -1.42929100 & $\mathrm{H}$ & 1.78908900 & -1.18352100 & 3.26138300 \\
\hline $\mathrm{H}$ & -2.82516000 & 0.03292600 & -2.29739700 & $\mathrm{H}$ & 1.26739400 & -1.42406800 & 0.87924500 \\
\hline $\mathrm{C}$ & -3.38562500 & 2.06184300 & -1.84831300 & $\mathrm{C}$ & -2.04326300 & 4.33957900 & 2.11369900 \\
\hline $\mathrm{O}$ & -3.81764700 & 2.85407200 & -1.03609700 & $\mathrm{C}$ & -0.91892800 & 2.29151900 & 3.01606100 \\
\hline $\mathrm{O}$ & -3.42226300 & 2.24445600 & -3.17716900 & $\mathrm{C}$ & 0.43278300 & 4.04412100 & 1.86359200 \\
\hline C & -4.02153300 & 3.47958500 & -3.62162000 & $\mathrm{C}$ & -1.64972800 & 4.64153400 & -1.39704700 \\
\hline $\mathrm{H}$ & -3.46761600 & 4.33543600 & -3.22928600 & $\mathrm{C}$ & 0.66398100 & 3.67097400 & -1.47086800 \\
\hline $\mathrm{H}$ & -3.97056200 & 3.45357200 & -4.70915100 & $\mathrm{C}$ & -1.19226500 & 2.44528400 & -2.55511600 \\
\hline \multirow[t]{2}{*}{$\mathrm{H}$} & -5.05838700 & 3.54068400 & -3.28446600 & $\mathrm{C}$ & -2.78572400 & -4.27646600 & -0.84742200 \\
\hline & & & & $\mathrm{C}$ & -1.65076600 & -2.45677400 & -2.18643800 \\
\hline \multirow{2}{*}{\multicolumn{4}{|c|}{$\begin{array}{l}\text { TS63a } \\
\text { imaginary frequency }=-131.81 \mathrm{~cm}^{-1}\end{array}$}} & $\mathrm{C}$ & -0.28421600 & -4.03426300 & -0.84652100 \\
\hline & & & & $\mathrm{C}$ & -3.17301000 & -3.45164100 & 2.60726900 \\
\hline $\mathrm{Pd}$ & -0.39613500 & 0.00277100 & 0.34345000 & $\mathrm{C}$ & -0.72570100 & -3.92225500 & 2.44474900 \\
\hline$P$ & -1.13528400 & 2.27317300 & 0.23509000 & $\mathrm{C}$ & -1.52292300 & -1.71933400 & 3.33259100 \\
\hline$P$ & -1.66792600 & -1.97756500 & 0.55236100 & $\mathrm{H}$ & -0.79501400 & 2.83203200 & 3.96219200 \\
\hline $\mathrm{C}$ & -0.83664400 & 3.33445200 & -1.34122200 & $\mathrm{H}$ & -1.85595100 & 1.73108800 & 3.08610300 \\
\hline $\mathrm{C}$ & -0.92318300 & 3.31037200 & 1.85367200 & $\mathrm{H}$ & -0.09849400 & 1.57612400 & 2.92295200 \\
\hline $\mathrm{C}$ & -1.79733700 & -2.82546400 & 2.28989300 & $\mathrm{H}$ & 0.61648400 & 4.43011100 & 2.87300300 \\
\hline $\mathrm{C}$ & -1.62337100 & -3.26660200 & -0.86984400 & $\mathrm{H}$ & 1.26752300 & 3.38351700 & 1.61350400 \\
\hline C & -3.01529600 & 1.96285800 & 0.32957400 & $\mathrm{H}$ & 0.44218700 & 4.90248000 & 1.18784000 \\
\hline $\mathrm{C}$ & -3.40005200 & -1.17001500 & 0.52209600 & $\mathrm{H}$ & -1.80053200 & 4.89776800 & 3.02562800 \\
\hline $\mathrm{C}$ & -3.91220500 & -0.29639900 & -0.60818600 & $\mathrm{H}$ & -2.14937600 & 5.06840900 & 1.30781600 \\
\hline
\end{tabular}




\begin{tabular}{|c|c|c|c|c|c|c|c|}
\hline $\mathrm{H}$ & -3.01496300 & 3.86845400 & 2.28021400 & $\mathrm{H}$ & 1.31322600 & 0.04572800 & -1.49374300 \\
\hline $\mathrm{H}$ & -0.88274300 & 2.96069400 & -3.47207600 & $\mathrm{H}$ & 2.64294400 & 0.78206400 & -2.41623300 \\
\hline $\mathrm{H}$ & -0.66784700 & 1.48507100 & -2.51663400 & $\mathrm{C}$ & 2.90928700 & -1.28238600 & -1.97650500 \\
\hline $\mathrm{H}$ & -2.25805900 & 2.23831900 & -2.63413200 & $\mathrm{O}$ & 2.61771700 & -2.31469600 & -1.40242600 \\
\hline $\mathrm{H}$ & -1.52759900 & 5.09994200 & -2.38570500 & $\mathrm{O}$ & 3.70276500 & -1.22486400 & -3.05246500 \\
\hline $\mathrm{H}$ & -2.72115700 & 4.48333500 & -1.24678800 & $\mathrm{C}$ & 4.22976300 & -2.48431700 & -3.52205800 \\
\hline $\mathrm{H}$ & -1.30691100 & 5.37292100 & -0.66043600 & $\mathrm{H}$ & 3.41580000 & -3.17308400 & -3.75662600 \\
\hline $\mathrm{H}$ & 0.82589500 & 4.19425000 & -2.42039600 & $\mathrm{H}$ & 4.80354100 & -2.24342400 & -4.41536300 \\
\hline $\mathrm{H}$ & 1.03958200 & 4.31455100 & -0.67562400 & $\mathrm{H}$ & 4.87243700 & -2.93090900 & -2.76026500 \\
\hline $\mathrm{H}$ & 1.26665700 & 2.75969300 & -1.49733800 & $\mathrm{H}$ & 2.77058700 & 1.80740800 & -0.38574000 \\
\hline $\mathrm{H}$ & -0.20954100 & -4.62434300 & -1.76766100 & & & & \\
\hline $\mathrm{H}$ & 0.57667900 & -3.36129900 & -0.82743100 & \multirow{2}{*}{\multicolumn{4}{|c|}{$\begin{array}{l}\text { TS63b } \\
\text { imaginary frequency }=-183.86 \mathrm{~cm}^{-1}\end{array}$}} \\
\hline $\mathrm{H}$ & -0.20620100 & -4.73472200 & -0.01443500 & & & & \\
\hline $\mathrm{H}$ & -1.54390700 & -3.15178400 & -3.02756200 & $\mathrm{Pc}$ & 0.29239300 & -0.10344100 & -0.51115900 \\
\hline $\mathrm{H}$ & -2.57648000 & -1.89968100 & -2.32892700 & $\mathrm{P}$ & 0.41298200 & 2.27079000 & -0.36730500 \\
\hline $\mathrm{H}$ & -0.81702100 & -1.74885600 & -2.22958700 & $\mathrm{P}$ & 2.97191100 & -1.20878500 & -0.59831300 \\
\hline $\mathrm{H}$ & -2.74773300 & -4.88674300 & -1.75744300 & $\mathrm{C}$ & -0.61462100 & 3.34443700 & 0.87488300 \\
\hline $\mathrm{H}$ & -2.71782900 & -4.96380100 & 0.00021600 & $\mathrm{C}$ & 0.25494000 & 3.04859600 & -2.13936600 \\
\hline $\mathrm{H}$ & -3.76840500 & -3.79852400 & -0.82152200 & $\mathrm{C}$ & 3.80464500 & -1.61637700 & -2.31442300 \\
\hline $\mathrm{H}$ & -1.55097700 & -2.15447300 & 4.33863400 & $\mathrm{C}$ & 3.37024300 & -2.63474700 & 0.65078300 \\
\hline $\mathrm{H}$ & -0.54576700 & -1.25169400 & 3.18557200 & $\mathrm{C}$ & -2.04126800 & 3.57542400 & 0.34401000 \\
\hline $\mathrm{H}$ & -2.27236400 & -0.92324100 & 3.30361700 & $\mathrm{C}$ & -0.71925900 & 2.53161000 & 2.18223100 \\
\hline $\mathrm{H}$ & -0.63043300 & -4.18521200 & 3.50464900 & $\mathrm{C}$ & -0.00982900 & 4.73066000 & 1.18639200 \\
\hline $\mathrm{H}$ & -0.99392700 & -4.83522100 & 1.90924400 & $\mathrm{C}$ & 1.30691900 & 2.37009700 & -3.03920700 \\
\hline $\mathrm{H}$ & 0.25997300 & -3.60589600 & 2.09063900 & $\mathrm{C}$ & 0.47788900 & 4.57225600 & -2.19152900 \\
\hline $\mathrm{H}$ & -3.11058000 & -3.96218500 & 3.57556700 & $\mathrm{C}$ & -1.12521600 & 2.70888400 & -2.74119000 \\
\hline $\mathrm{H}$ & -3.96459500 & -2.70394200 & 2.69445300 & $\mathrm{C}$ & 3.48167400 & -3.04808400 & -2.78459900 \\
\hline $\mathrm{H}$ & -3.48220300 & -4.19460200 & 1.86946300 & $\mathrm{C}$ & 5.33698200 & -1.43140500 & -2.35487400 \\
\hline $\mathrm{C}$ & 2.98568300 & 0.73814400 & -0.30883000 & $\mathrm{C}$ & 3.17668500 & -0.64408400 & -3.33231800 \\
\hline $\mathrm{C}$ & 4.49928200 & 0.60533000 & -0.10354000 & $\mathrm{C}$ & 2.55835900 & -3.89649200 & 0.28276200 \\
\hline $\mathrm{C}$ & 5.12517600 & -0.63476600 & 0.10023800 & $\mathrm{C}$ & 4.85853400 & -3.01424000 & 0.75759600 \\
\hline $\mathrm{C}$ & 5.27759900 & 1.77071900 & -0.10603800 & $\mathrm{C}$ & 2.85699000 & -2.18330800 & 2.03519000 \\
\hline $\mathrm{C}$ & 6.50757300 & -0.69966200 & 0.28037700 & $\mathrm{C}$ & 2.22083500 & 2.69404600 & 0.04185500 \\
\hline $\mathrm{H}$ & 4.54116400 & -1.54855000 & 0.10864300 & $\mathrm{C}$ & 4.10743500 & 0.22907400 & -0.09454900 \\
\hline $\mathrm{C}$ & 6.65962000 & 1.70071600 & 0.06914100 & $\mathrm{C}$ & 3.83590600 & 1.02022700 & 1.16736000 \\
\hline $\mathrm{H}$ & 4.80378000 & 2.73766500 & -0.25374400 & $\mathrm{C}$ & 4.61973500 & 0.70555500 & 2.29326200 \\
\hline $\mathrm{C}$ & 7.27754500 & 0.46480400 & 0.26444200 & $\mathrm{C}$ & 4.50717300 & 1.38466000 & 3.50138600 \\
\hline $\mathrm{H}$ & 6.98307200 & -1.66376100 & 0.43369300 & $\mathrm{C}$ & 3.60376800 & 2.44091300 & 3.59702500 \\
\hline $\mathrm{H}$ & 7.25026800 & 2.61138200 & 0.05506800 & $\mathrm{C}$ & 2.85783600 & 2.80023600 & 2.47862400 \\
\hline $\mathrm{H}$ & 8.35251200 & 0.40933100 & 0.40500900 & $\mathrm{C}$ & 2.94028800 & 2.11539400 & 1.25187600 \\
\hline $\mathrm{C}$ & 2.40734700 & 0.10081900 & -1.59631900 & $\mathrm{H}$ & 2.33167800 & 2.67289800 & -2.80528200 \\
\hline
\end{tabular}




\begin{tabular}{|c|c|c|c|c|c|c|c|}
\hline $\mathrm{H}$ & 1.12054900 & 2.66054800 & -4.07936700 & $\mathrm{H}$ & 3.50797000 & 3.00866800 & 4.51757600 \\
\hline $\mathrm{H}$ & 1.23606200 & 1.28250200 & -2.98100100 & $\mathrm{H}$ & 2.21626300 & 3.66898700 & 2.55437600 \\
\hline $\mathrm{H}$ & 1.42690800 & 4.88289400 & -1.74366200 & $\mathrm{C}$ & -1.70826900 & -0.84302000 & -1.22013400 \\
\hline $\mathrm{H}$ & 0.50336600 & 4.88327400 & -3.24277700 & $\mathrm{O}$ & -1.76672700 & -0.61768700 & -2.41217700 \\
\hline $\mathrm{H}$ & -0.33148700 & 5.13003500 & -1.71639100 & $\mathrm{O}$ & -0.85202100 & -2.15803700 & -0.82512700 \\
\hline $\mathrm{H}$ & -1.15278700 & 3.08664000 & -3.77061500 & $\mathrm{C}$ & -0.50189700 & -3.00363100 & -1.93993600 \\
\hline $\mathrm{H}$ & -1.29703100 & 1.63207000 & -2.77658100 & $\mathrm{H}$ & 0.42550700 & -3.52006700 & -1.69453900 \\
\hline $\mathrm{H}$ & -1.95297000 & 3.17100300 & -2.20538700 & $\mathrm{H}$ & -0.86318500 & -2.74739900 & 0.46239900 \\
\hline $\mathrm{H}$ & 0.96911000 & 4.68992600 & 1.66235500 & $\mathrm{O}$ & -0.74100400 & -3.07641500 & 1.46818800 \\
\hline $\mathrm{H}$ & 0.07784200 & 5.35981600 & 0.30088900 & $\mathrm{C}$ & -1.72941600 & -4.03630000 & 1.95826000 \\
\hline $\mathrm{H}$ & -0.68080000 & 5.24661900 & 1.88339700 & $\mathrm{H}$ & -1.69029100 & -4.89925100 & 1.29362600 \\
\hline $\mathrm{H}$ & -2.06597300 & 4.32500700 & -0.44973300 & $\mathrm{H}$ & -0.66164900 & -2.21008900 & 2.05115900 \\
\hline $\mathrm{H}$ & -2.66292600 & 3.95434000 & 1.16302600 & $\mathrm{O}$ & -0.56696200 & -0.88050300 & 2.61404400 \\
\hline $\mathrm{H}$ & -2.50539500 & 2.66043400 & -0.02216400 & $\mathrm{H}$ & -0.19100300 & -0.28510200 & 1.92613500 \\
\hline $\mathrm{H}$ & -1.23265000 & 3.13431500 & 2.94127400 & $\mathrm{C}$ & -0.03853700 & -0.58237300 & 3.92342100 \\
\hline $\mathrm{H}$ & -1.30878800 & 1.62404100 & 2.02859000 & $\mathrm{H}$ & 1.05190100 & -0.65705200 & 3.93740400 \\
\hline $\mathrm{H}$ & 0.26036400 & 2.25797500 & 2.58394300 & $\mathrm{H}$ & -0.37232100 & -2.38897900 & -2.83063000 \\
\hline $\mathrm{H}$ & 1.81017800 & -1.87269800 & 1.98391100 & $\mathrm{H}$ & -1.30374100 & -3.72899100 & -2.11638000 \\
\hline $\mathrm{H}$ & 3.43253100 & -1.36103600 & 2.45665100 & $\mathrm{H}$ & -2.72443200 & -3.58658000 & 1.97010800 \\
\hline $\mathrm{H}$ & 2.92171700 & -3.02854400 & 2.73166200 & $\mathrm{H}$ & -1.41224600 & -4.33523400 & 2.95714600 \\
\hline $\mathrm{H}$ & 2.85671400 & -4.34486100 & -0.66375900 & $\mathrm{H}$ & -0.34948000 & 0.41924400 & 4.22697400 \\
\hline $\mathrm{H}$ & 2.71434100 & -4.65256100 & 1.06190800 & $\mathrm{H}$ & -0.46760600 & -1.31403100 & 4.60903900 \\
\hline $\mathrm{H}$ & 1.48707900 & -3.68518300 & 0.25041100 & $\mathrm{C}$ & -2.91590200 & -0.88103800 & -0.26351000 \\
\hline $\mathrm{H}$ & 5.50206500 & -2.15669400 & 0.97242700 & $\mathrm{H}$ & -2.55712800 & -1.08222300 & 0.74736300 \\
\hline $\mathrm{H}$ & 5.22741100 & -3.49606600 & -0.15128300 & $\mathrm{C}$ & -3.81035000 & -2.08270200 & -0.70980100 \\
\hline $\mathrm{H}$ & 4.99295800 & -3.73028800 & 1.57778700 & $\mathrm{H}$ & -3.20408700 & -2.98807500 & -0.81706700 \\
\hline $\mathrm{H}$ & 2.41311900 & -3.27621100 & -2.73363600 & $\mathrm{H}$ & -4.27522500 & -1.86833500 & -1.67258700 \\
\hline $\mathrm{H}$ & 4.02897500 & -3.80610300 & -2.22028700 & $\mathrm{C}$ & -4.86883500 & -2.38234500 & 0.33557700 \\
\hline $\mathrm{H}$ & 3.78395400 & -3.15075200 & -3.83351400 & $\mathrm{O}$ & -4.61705100 & -2.60712600 & 1.50807000 \\
\hline $\mathrm{H}$ & 5.86166600 & -2.05368200 & -1.62741700 & $\mathrm{O}$ & -6.10045100 & -2.38477100 & -0.17631200 \\
\hline $\mathrm{H}$ & 5.69811600 & -1.71996600 & -3.34932600 & $\mathrm{C}$ & -7.17278500 & -2.66623300 & 0.74999800 \\
\hline $\mathrm{H}$ & 5.64023200 & -0.39342900 & -2.19848900 & $\mathrm{H}$ & -8.08314700 & -2.64730500 & 0.15329400 \\
\hline $\mathrm{H}$ & 3.60731900 & -0.82815600 & -4.32372600 & $\mathrm{H}$ & -7.03028500 & -3.64628100 & 1.20992400 \\
\hline $\mathrm{H}$ & 3.37435400 & 0.40199200 & -3.08604900 & $\mathrm{H}$ & -7.20463000 & -1.90247300 & 1.52964600 \\
\hline $\mathrm{H}$ & 2.09375600 & -0.77937500 & -3.40671600 & $\mathrm{C}$ & -3.77939200 & 0.36959200 & -0.22643700 \\
\hline $\mathrm{H}$ & 5.13085700 & -0.14914700 & -0.04319100 & $\mathrm{C}$ & -4.27469000 & 0.80838600 & 1.01041600 \\
\hline $\mathrm{H}$ & 4.07605300 & 0.88799600 & -0.96817700 & $\mathrm{C}$ & -4.21993400 & 1.00614000 & -1.39668500 \\
\hline $\mathrm{H}$ & 2.76036300 & 2.40805800 & -0.86133400 & $\mathrm{C}$ & -5.18679100 & 1.86316300 & 1.08002300 \\
\hline $\mathrm{H}$ & 2.28255500 & 3.78454400 & 0.09465500 & $\mathrm{H}$ & -3.95948100 & 0.30708200 & 1.92155200 \\
\hline $\mathrm{H}$ & 5.35611200 & -0.08861200 & 2.20620200 & $\mathrm{C}$ & -5.12336100 & 2.06729600 & -1.32410900 \\
\hline $\mathrm{H}$ & 5.13516100 & 1.11010400 & 4.34337600 & $\mathrm{H}$ & -3.85222400 & 0.67169100 & -2.36045400 \\
\hline
\end{tabular}




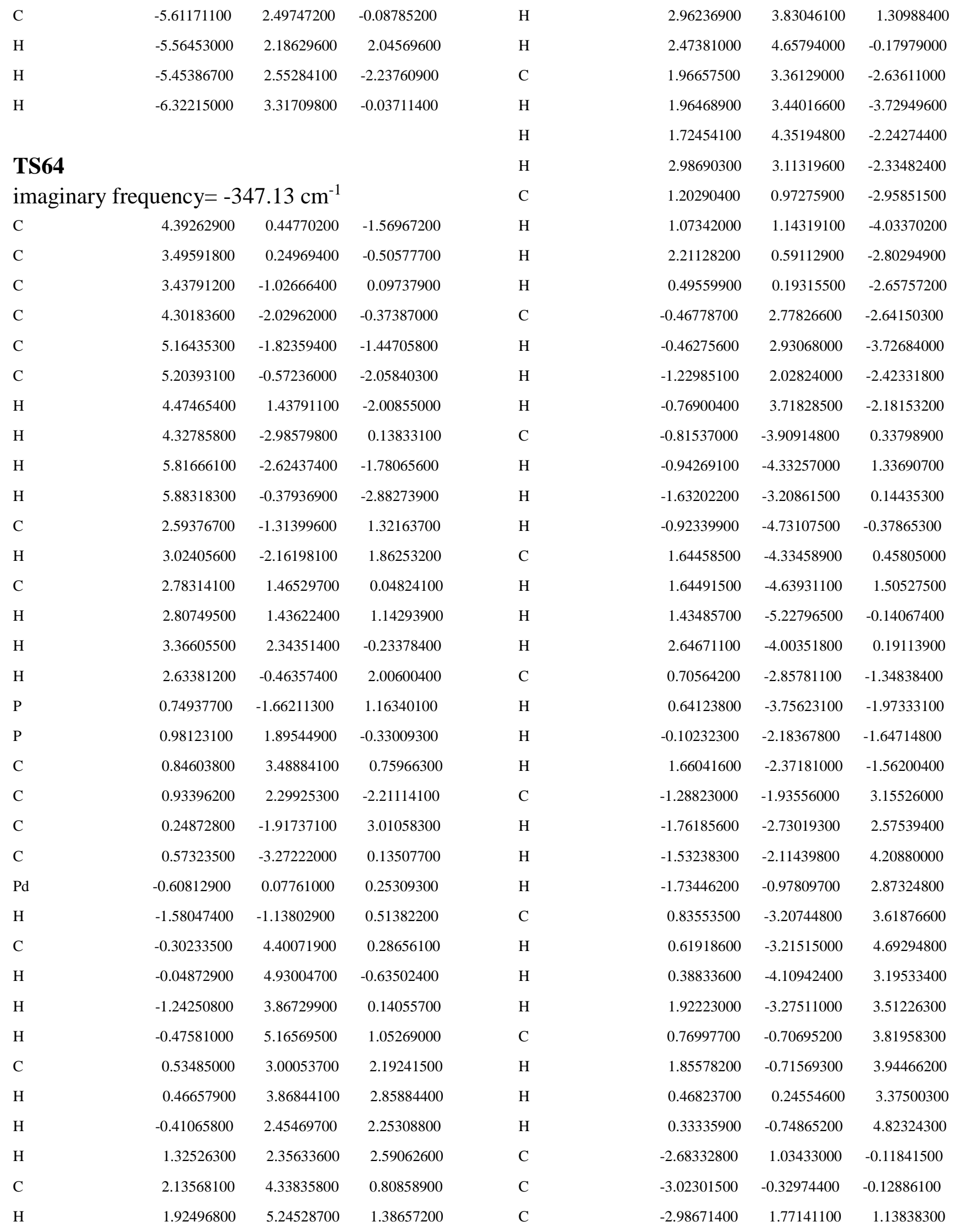




\begin{tabular}{|c|c|c|c|c|c|c|c|}
\hline $\mathrm{O}$ & -2.97527300 & 1.28071500 & 2.25464400 & $\mathrm{C}$ & 0.66441500 & -1.91515100 & 2.94672500 \\
\hline $\mathrm{O}$ & -3.32565600 & 3.04902200 & 0.88540300 & $\mathrm{C}$ & 1.07403400 & -3.19501000 & 0.04894700 \\
\hline $\mathrm{C}$ & -3.71744100 & 3.83389200 & 2.03305700 & $\mathrm{Pd}$ & -0.56548200 & 0.03880600 & 0.29697300 \\
\hline $\mathrm{H}$ & -4.57238400 & 3.37225000 & 2.53063400 & $\mathrm{H}$ & -1.81468200 & -1.33949100 & 0.39690900 \\
\hline $\mathrm{H}$ & -2.89023300 & 3.91418400 & 2.74146200 & $\mathrm{C}$ & -0.82852900 & 4.26190600 & 0.34614900 \\
\hline $\mathrm{H}$ & -3.98408700 & 4.81246200 & 1.63705400 & $\mathrm{H}$ & -0.66625400 & 4.80194400 & -0.58986600 \\
\hline $\mathrm{H}$ & -2.68865400 & 1.60740200 & -1.03599600 & $\mathrm{H}$ & -1.70790400 & 3.62706000 & 0.23826700 \\
\hline $\mathrm{C}$ & -3.32303200 & -1.14667000 & -1.32014100 & $\mathrm{H}$ & -1.05869800 & 5.01379800 & 1.10993300 \\
\hline $\mathrm{C}$ & -2.90811100 & -0.80005400 & -2.61842200 & $\mathrm{C}$ & 0.23086000 & 2.97789200 & 2.23331900 \\
\hline $\mathrm{C}$ & -4.09904600 & -2.30569700 & -1.14458200 & $\mathrm{H}$ & 0.07852900 & 3.83751900 & 2.89625400 \\
\hline $\mathrm{C}$ & -3.26462900 & -1.59057500 & -3.70667300 & $\mathrm{H}$ & -0.63482300 & 2.31904100 & 2.33259300 \\
\hline $\mathrm{H}$ & -2.30368200 & 0.08666000 & -2.77896400 & $\mathrm{H}$ & 1.11171900 & 2.44452200 & 2.60501200 \\
\hline $\mathrm{C}$ & -4.45899000 & -3.09448200 & -2.23625000 & $\mathrm{C}$ & 1.61495300 & 4.48619900 & 0.79618800 \\
\hline $\mathrm{H}$ & -4.43362700 & -2.57989200 & -0.14732400 & $\mathrm{H}$ & 1.30893400 & 5.36845600 & 1.36936800 \\
\hline $\mathrm{C}$ & -4.04094100 & -2.73938100 & -3.51935500 & $\mathrm{H}$ & 2.50536700 & 4.08616400 & 1.28586800 \\
\hline $\mathrm{H}$ & -2.94080800 & -1.31126800 & -4.70451200 & $\mathrm{H}$ & 1.89145100 & 4.83288100 & -0.20180500 \\
\hline $\mathrm{H}$ & -5.06651100 & -3.98111900 & -2.08490900 & $\mathrm{C}$ & 1.37396600 & 3.54022800 & -2.62106900 \\
\hline $\mathrm{H}$ & -4.31915600 & -3.35146300 & -4.37166000 & $\mathrm{H}$ & 1.33066500 & 3.62749300 & -3.71249000 \\
\hline \multirow[t]{2}{*}{$\mathrm{H}$} & -3.44152900 & -0.70246000 & 0.80422400 & $\mathrm{H}$ & 0.98416600 & 4.47406500 & -2.20864200 \\
\hline & & & & $\mathrm{H}$ & 2.42880400 & 3.45746400 & -2.34885500 \\
\hline 81 & & & & $\mathrm{C}$ & 0.99804400 & 1.06750100 & -2.97667800 \\
\hline $\mathrm{C}$ & 4.25142000 & 0.99485300 & -1.72038100 & $\mathrm{H}$ & 0.77842300 & 1.22537500 & -4.03885600 \\
\hline $\mathrm{C}$ & 3.45175400 & 0.64182500 & -0.61965300 & $\mathrm{H}$ & 2.06494600 & 0.87091700 & -2.88396400 \\
\hline $\mathrm{C}$ & 3.63014000 & -0.63397100 & -0.04030400 & $\mathrm{H}$ & 0.45928200 & 0.17052700 & -2.65707000 \\
\hline $\mathrm{C}$ & 4.60352400 & -1.48890300 & -0.58298800 & $\mathrm{C}$ & -0.93740000 & 2.56583500 & -2.57551600 \\
\hline $\mathrm{C}$ & 5.36453700 & -1.13450300 & -1.69373700 & $\mathrm{H}$ & -0.97042700 & 2.82729400 & -3.63923400 \\
\hline $\mathrm{C}$ & 5.18338900 & 0.12022800 & -2.27193400 & $\mathrm{H}$ & -1.54300000 & 1.66727900 & -2.44774000 \\
\hline $\mathrm{H}$ & 4.15746600 & 1.99203200 & -2.14114600 & $\mathrm{H}$ & -1.40482700 & 3.38137000 & -2.02539800 \\
\hline $\mathrm{H}$ & 4.79247400 & -2.44401100 & -0.10334600 & $\mathrm{C}$ & -0.25081600 & -3.95745800 & 0.24953700 \\
\hline $\mathrm{H}$ & 6.10834600 & -1.82176600 & -2.08418800 & $\mathrm{H}$ & -0.34728500 & -4.38449600 & 1.25013800 \\
\hline $\mathrm{H}$ & 5.78137000 & 0.42937500 & -3.12335400 & $\mathrm{H}$ & -1.12957400 & -3.34001200 & 0.04331100 \\
\hline $\mathrm{C}$ & 2.90399600 & -1.06386200 & 1.21545400 & $\mathrm{H}$ & -0.27953900 & -4.79142400 & -0.46081200 \\
\hline $\mathrm{H}$ & 3.45238900 & -1.88047300 & 1.69423000 & $\mathrm{C}$ & 2.24393100 & -4.15162000 & 0.35622000 \\
\hline $\mathrm{C}$ & 2.58177100 & 1.72055200 & -0.00686300 & $\mathrm{H}$ & 2.25300000 & -4.49813000 & 1.39024000 \\
\hline $\mathrm{H}$ & 2.64392000 & 1.67525500 & 1.08556300 & $\mathrm{H}$ & 2.14433500 & -5.03659600 & -0.28256000 \\
\hline $\mathrm{H}$ & 3.01679000 & 2.68188900 & -0.28527200 & $\mathrm{H}$ & 3.21280600 & -3.70604100 & 0.13181600 \\
\hline $\mathrm{H}$ & 2.89674400 & -0.23991800 & 1.93333600 & $\mathrm{C}$ & 1.15610100 & -2.75651100 & -1.43095000 \\
\hline $\mathrm{P}$ & 1.10286200 & -1.58144100 & 1.09607100 & $\mathrm{H}$ & 1.16304000 & -3.65023800 & -2.06574900 \\
\hline $\mathrm{P}$ & 0.72968600 & 1.93176700 & -0.31638800 & $\mathrm{H}$ & 0.28814200 & -2.15488900 & -1.71571500 \\
\hline $\mathrm{C}$ & 0.42972100 & 3.49227100 & 0.79048000 & $\mathrm{H}$ & 2.06186400 & -2.18656600 & -1.64931400 \\
\hline $\mathrm{C}$ & 0.53703300 & 2.31692800 & -2.19268300 & $\mathrm{C}$ & -0.86715300 & -2.03486800 & 3.10056000 \\
\hline
\end{tabular}




\begin{tabular}{|c|c|c|c|c|c|c|c|}
\hline $\mathrm{H}$ & -1.29271900 & -2.84831000 & 2.50945400 & $\mathrm{H}$ & 2.97597100 & 4.41169000 & 0.05634900 \\
\hline $\mathrm{H}$ & -1.09724900 & -2.24523400 & 4.15143400 & $\mathrm{H}$ & 5.71129600 & 0.42749000 & -0.81126700 \\
\hline $\mathrm{H}$ & -1.37688200 & -1.10239600 & 2.83945400 & $\mathrm{H}$ & 6.37341100 & 2.57605500 & -1.78726800 \\
\hline $\mathrm{C}$ & 1.34443600 & -3.17164300 & 3.52430300 & $\mathrm{H}$ & 4.99150500 & 4.61086900 & -1.32840300 \\
\hline $\mathrm{H}$ & 1.14963700 & -3.21134900 & 4.60222200 & $\mathrm{C}$ & 3.59509900 & -0.19616500 & 0.72645300 \\
\hline $\mathrm{H}$ & 0.95183400 & -4.09525200 & 3.09352800 & $\mathrm{H}$ & 4.52839100 & -0.76134800 & 0.77879000 \\
\hline $\mathrm{H}$ & 2.43079600 & -3.16254300 & 3.39444900 & $\mathrm{C}$ & 1.86208900 & 2.25674100 & 1.19570000 \\
\hline $\mathrm{C}$ & 1.11432000 & -0.68908200 & 3.77381800 & $\mathrm{H}$ & 2.04977500 & 1.57117900 & 2.02497600 \\
\hline $\mathrm{H}$ & 2.19992500 & -0.62995700 & 3.88665900 & $\mathrm{H}$ & 1.76982100 & 3.24838800 & 1.64429700 \\
\hline $\mathrm{H}$ & 0.74729100 & 0.25107300 & 3.35241200 & $\mathrm{H}$ & 3.29562600 & -0.01962800 & 1.76220400 \\
\hline $\mathrm{H}$ & 0.69329300 & -0.77709000 & 4.78120700 & $\mathrm{P}$ & 2.29388900 & -1.37319400 & 0.05161300 \\
\hline $\mathrm{C}$ & -2.59265100 & 0.67212900 & 0.02203200 & $\mathrm{P}$ & 0.10441000 & 1.79587400 & 0.65378800 \\
\hline $\mathrm{C}$ & -2.82245400 & -0.80562300 & 0.09519000 & $\mathrm{C}$ & -0.83200500 & 2.33528900 & 2.27901800 \\
\hline $\mathrm{C}$ & -3.03961900 & 1.39501900 & 1.24299600 & $\mathrm{C}$ & -0.34768900 & 2.92650000 & -0.84421300 \\
\hline $\mathrm{O}$ & -2.91411200 & 0.96859900 & 2.38142000 & $\mathrm{C}$ & 2.17268200 & -2.67313400 & 1.47761400 \\
\hline $\mathrm{O}$ & -3.63870500 & 2.56597500 & 0.95190900 & $\mathrm{C}$ & 3.10798400 & -2.12091500 & -1.53717900 \\
\hline $\mathrm{C}$ & -4.15525900 & 3.30717900 & 2.07879700 & $\mathrm{Pd}$ & -0.10453700 & -0.56536800 & -0.23023400 \\
\hline $\mathrm{H}$ & -4.86880500 & 2.69752200 & 2.63618500 & $\mathrm{C}$ & -6.31699600 & 1.49255800 & -1.68268800 \\
\hline $\mathrm{H}$ & -3.34325300 & 3.60681600 & 2.74505400 & $\mathrm{C}$ & -5.00976200 & 1.03069800 & -1.84741500 \\
\hline $\mathrm{H}$ & -4.64525600 & 4.18050600 & 1.65113500 & $\mathrm{C}$ & -4.57977800 & -0.15304700 & -1.23191100 \\
\hline $\mathrm{H}$ & -2.84479100 & 1.15890700 & -0.91284200 & $\mathrm{C}$ & -5.49979600 & -0.87201300 & -0.45247400 \\
\hline $\mathrm{C}$ & -3.31905400 & -1.51808000 & -1.14128500 & $\mathrm{C}$ & -6.80534200 & -0.41086400 & -0.28380000 \\
\hline $\mathrm{C}$ & -2.68284600 & -1.37958100 & -2.38262300 & $\mathrm{C}$ & -7.21779700 & 0.77510000 & -0.89498400 \\
\hline $\mathrm{C}$ & -4.45066200 & -2.33758500 & -1.04656100 & $\mathrm{H}$ & -6.63224800 & 2.40734300 & -2.17606200 \\
\hline $\mathrm{C}$ & -3.17652000 & -2.03647300 & -3.50917100 & $\mathrm{H}$ & -4.32222400 & 1.58726500 & -2.47956300 \\
\hline $\mathrm{H}$ & -1.79630400 & -0.75665600 & -2.46697100 & $\mathrm{H}$ & -5.19693800 & -1.80733500 & 0.00711600 \\
\hline $\mathrm{C}$ & -4.94593300 & -2.99501600 & -2.17443700 & $\mathrm{H}$ & -7.50469500 & -0.98362000 & 0.31833400 \\
\hline $\mathrm{H}$ & -4.95295600 & -2.45583200 & -0.08989400 & $\mathrm{H}$ & -8.23557100 & 1.13072300 & -0.76649000 \\
\hline $\mathrm{C}$ & -4.31080300 & -2.84574300 & -3.40753300 & $\mathrm{C}$ & -2.32254100 & 1.93543900 & 2.28189700 \\
\hline $\mathrm{H}$ & -2.67552700 & -1.91897600 & -4.46542300 & $\mathrm{H}$ & -2.88054600 & 2.32474900 & 1.42854500 \\
\hline $\mathrm{H}$ & -5.82778900 & -3.62213300 & -2.08687100 & $\mathrm{H}$ & -2.44634700 & 0.85439200 & 2.34573200 \\
\hline $\mathrm{H}$ & -4.69492700 & -3.35767400 & -4.28434000 & $\mathrm{H}$ & -2.77973900 & 2.36625500 & 3.18026800 \\
\hline \multirow[t]{2}{*}{$\mathrm{H}$} & -3.40366800 & -1.06480400 & 0.98496500 & $\mathrm{C}$ & -0.17010800 & 1.59766900 & 3.46643700 \\
\hline & & & & $\mathrm{H}$ & -0.77698300 & 1.77898900 & 4.36014800 \\
\hline 82 & & & & $\mathrm{H}$ & -0.14575100 & 0.51596700 & 3.31149100 \\
\hline $\mathrm{C}$ & 3.51963100 & 3.51177700 & -0.20898700 & $\mathrm{H}$ & 0.83745900 & 1.95578300 & 3.68994700 \\
\hline $\mathrm{C}$ & 3.06537400 & 2.27371200 & 0.27606800 & $\mathrm{C}$ & -0.74933400 & 3.85859900 & 2.51835400 \\
\hline $\mathrm{C}$ & 3.86566700 & 1.13118000 & 0.04649600 & $\mathrm{H}$ & -1.14241700 & 4.06309700 & 3.52057500 \\
\hline $\mathrm{C}$ & 5.05190200 & 1.27974700 & -0.69083300 & $\mathrm{H}$ & 0.26575700 & 4.26203600 & 2.48973600 \\
\hline $\mathrm{C}$ & 5.44945800 & 2.50387100 & -1.22240700 & $\mathrm{H}$ & -1.36704500 & 4.41994200 & 1.81392000 \\
\hline $\mathrm{C}$ & 4.67886300 & 3.63520200 & -0.97014400 & $\mathrm{C}$ & 0.18892900 & 4.36814200 & -0.74700700 \\
\hline
\end{tabular}




\begin{tabular}{|c|c|c|c|c|c|c|c|}
\hline $\mathrm{H}$ & -0.20981300 & 4.93469900 & -1.59637700 & $\mathrm{H}$ & -2.49158400 & 0.46064300 & 0.33865600 \\
\hline $\mathrm{H}$ & -0.12322000 & 4.88630500 & 0.15959800 & $\mathrm{C}$ & -2.45796300 & -1.55097400 & 0.93747400 \\
\hline $\mathrm{H}$ & 1.27250100 & 4.40716500 & -0.82221700 & $\mathrm{O}$ & -2.16231900 & -1.38496500 & 2.11241200 \\
\hline $\mathrm{C}$ & 0.27493700 & 2.25818100 & -2.09050400 & $\mathrm{O}$ & -2.89302400 & -2.74151300 & 0.46559200 \\
\hline $\mathrm{H}$ & 0.05633500 & 2.87286000 & -2.97157100 & $\mathrm{C}$ & -3.05996900 & -3.78667300 & 1.44723200 \\
\hline $\mathrm{H}$ & 1.36175000 & 2.17070600 & -2.00869500 & $\mathrm{H}$ & -3.77988800 & -3.47987000 & 2.20860000 \\
\hline $\mathrm{H}$ & -0.13999800 & 1.26029700 & -2.26723700 & $\mathrm{H}$ & -3.42880800 & -4.64873500 & 0.89319800 \\
\hline $\mathrm{C}$ & -1.87581300 & 3.00746500 & -1.02301900 & $\mathrm{H}$ & -2.10777700 & -4.01589400 & 1.93038200 \\
\hline $\mathrm{H}$ & -2.08265200 & 3.51611700 & -1.97174400 & & & & \\
\hline $\mathrm{H}$ & -2.35914800 & 2.03283000 & -1.06885100 & \multirow{2}{*}{\multicolumn{4}{|c|}{$\begin{array}{l}\text { TS65 } \\
\text { imaginary frequency }=-150.15 \mathrm{~cm}^{-1}\end{array}$}} \\
\hline $\mathrm{H}$ & -2.35327000 & 3.59481600 & -0.23538000 & & & & \\
\hline $\mathrm{C}$ & 2.56232600 & -3.52333600 & -1.87416500 & $\mathrm{C}$ & 3.56746600 & 3.52909900 & -0.25830700 \\
\hline $\mathrm{H}$ & 2.93761500 & -4.28232500 & -1.18382900 & $\mathrm{C}$ & 3.12260400 & 2.30566900 & 0.26986300 \\
\hline $\mathrm{H}$ & 1.47592100 & -3.58492600 & -1.89607800 & $\mathrm{C}$ & 3.88115300 & 1.14230400 & 0.00039700 \\
\hline $\mathrm{H}$ & 2.91736700 & -3.79565200 & -2.87442100 & $\mathrm{C}$ & 5.04878300 & 1.26945300 & -0.77003300 \\
\hline $\mathrm{C}$ & 4.64235000 & -2.25323700 & -1.42851200 & $\mathrm{C}$ & 5.44954900 & 2.48340600 & -1.32170900 \\
\hline $\mathrm{H}$ & 4.96181200 & -2.83348000 & -0.56044400 & $\mathrm{C}$ & 4.69764300 & 3.62642900 & -1.06566300 \\
\hline $\mathrm{H}$ & 5.00373500 & -2.77842300 & -2.31953700 & $\mathrm{H}$ & 3.04244400 & 4.43991900 & 0.00840800 \\
\hline $\mathrm{H}$ & 5.14440700 & -1.28842600 & -1.40661900 & $\mathrm{H}$ & 5.68974600 & 0.40541600 & -0.90518300 \\
\hline $\mathrm{C}$ & 2.77144100 & -1.14896400 & -2.69063600 & $\mathrm{H}$ & 6.35825200 & 2.53720000 & -1.91272000 \\
\hline $\mathrm{H}$ & 3.26078200 & -1.49833900 & -3.60697500 & $\mathrm{H}$ & 5.00571200 & 4.59195300 & -1.45407200 \\
\hline $\mathrm{H}$ & 1.69679800 & -1.10219200 & -2.88859600 & $\mathrm{C}$ & 3.58514200 & -0.19308600 & 0.65505400 \\
\hline $\mathrm{H}$ & 3.12634100 & -0.13363100 & -2.49295500 & $\mathrm{H}$ & 4.51423400 & -0.76667300 & 0.72568000 \\
\hline $\mathrm{C}$ & 1.13196200 & -3.76462700 & 1.14714100 & $\mathrm{C}$ & 1.97630300 & 2.32556800 & 1.26112500 \\
\hline $\mathrm{H}$ & 1.40831900 & -4.38614900 & 0.29576900 & $\mathrm{H}$ & 2.24070200 & 1.70422000 & 2.12049800 \\
\hline $\mathrm{H}$ & 1.04258800 & -4.42678500 & 2.01571600 & $\mathrm{H}$ & 1.87334600 & 3.34246900 & 1.64919800 \\
\hline $\mathrm{H}$ & 0.14239600 & -3.33795600 & 0.96396300 & $\mathrm{H}$ & 3.25049000 & -0.03001700 & 1.68179300 \\
\hline $\mathrm{C}$ & 3.52587900 & -3.34238000 & 1.78870100 & $\mathrm{P}$ & 2.29401900 & -1.37417800 & -0.04402200 \\
\hline $\mathrm{H}$ & 3.40043600 & -4.00566800 & 2.65212700 & $\mathrm{P}$ & 0.23274300 & 1.75882600 & 0.81702400 \\
\hline $\mathrm{H}$ & 3.88448300 & -3.95772300 & 0.96034900 & $\mathrm{C}$ & -0.65629500 & 2.07421200 & 2.50728000 \\
\hline $\mathrm{H}$ & 4.30574700 & -2.62234100 & 2.05117200 & $\mathrm{C}$ & -0.37943100 & 2.97096600 & -0.54906300 \\
\hline $\mathrm{C}$ & 1.66022200 & -1.93907800 & 2.73762100 & $\mathrm{C}$ & 2.34733500 & -2.81050500 & 1.24482700 \\
\hline $\mathrm{H}$ & 2.35019500 & -1.17740500 & 3.10999800 & $\mathrm{C}$ & 2.93988000 & -1.93075100 & -1.77179200 \\
\hline $\mathrm{H}$ & 0.68007900 & -1.48319700 & 2.56980300 & $\mathrm{Pd}$ & -0.00137200 & -0.57242200 & -0.11005300 \\
\hline $\mathrm{H}$ & 1.54056700 & -2.67343400 & 3.54190300 & $\mathrm{C}$ & -5.94309800 & 1.88393600 & -2.05059300 \\
\hline $\mathrm{C}$ & -0.52514500 & -2.05634200 & -1.40168800 & $\mathrm{C}$ & -4.77277000 & 1.12916600 & -2.15089100 \\
\hline $\mathrm{O}$ & -0.80895100 & -2.82595000 & -2.19558800 & $\mathrm{C}$ & -4.57661800 & -0.00818200 & -1.35508300 \\
\hline $\mathrm{C}$ & -3.14856100 & -0.64184200 & -1.41241500 & $\mathrm{C}$ & -5.58947100 & -0.37724500 & -0.45600500 \\
\hline $\mathrm{H}$ & -3.15512200 & -1.68214600 & -1.74638400 & $\mathrm{C}$ & -6.75739000 & 0.37835600 & -0.35115800 \\
\hline $\mathrm{H}$ & -2.67925100 & -0.05608900 & -2.20924300 & $\mathrm{C}$ & -6.93793800 & 1.51263700 & -1.14576600 \\
\hline $\mathrm{C}$ & -2.32145800 & -0.50090900 & -0.12406600 & $\mathrm{H}$ & -6.07885200 & 2.75582500 & -2.68385300 \\
\hline
\end{tabular}




\begin{tabular}{|c|c|c|c|c|c|c|c|}
\hline $\mathrm{H}$ & -4.00929500 & 1.41846600 & -2.86932400 & $\mathrm{H}$ & 1.17842300 & -4.22476900 & 0.00070900 \\
\hline $\mathrm{H}$ & -5.47058200 & -1.26979500 & 0.14904000 & $\mathrm{H}$ & 1.28813900 & -4.62757800 & 1.71165800 \\
\hline $\mathrm{H}$ & -7.53295400 & 0.07474700 & 0.34600200 & $\mathrm{H}$ & 0.21741700 & -3.32596600 & 1.19699300 \\
\hline $\mathrm{H}$ & -7.85018400 & 2.09602700 & -1.06617900 & $\mathrm{C}$ & 3.68522200 & -3.57768200 & 1.21830600 \\
\hline $\mathrm{C}$ & -2.07765300 & 1.47618100 & 2.49261200 & $\mathrm{H}$ & 3.69438700 & -4.28811500 & 2.05255200 \\
\hline $\mathrm{H}$ & -2.71504700 & 1.89745600 & 1.71287700 & $\mathrm{H}$ & 3.81556800 & -4.15647800 & 0.30153500 \\
\hline $\mathrm{H}$ & -2.05294200 & 0.38893800 & 2.39650700 & $\mathrm{H}$ & 4.55397100 & -2.92525900 & 1.34422800 \\
\hline $\mathrm{H}$ & -2.55696600 & 1.70579700 & 3.45140100 & $\mathrm{C}$ & 2.14215700 & -2.20659800 & 2.65213700 \\
\hline $\mathrm{C}$ & 0.13604800 & 1.33124800 & 3.60740900 & $\mathrm{H}$ & 2.99525500 & -1.61682100 & 2.99635900 \\
\hline $\mathrm{H}$ & -0.45534300 & 1.34721500 & 4.52924400 & $\mathrm{H}$ & 1.23611400 & -1.59602200 & 2.70364800 \\
\hline $\mathrm{H}$ & 0.30487300 & 0.28072100 & 3.35379900 & $\mathrm{H}$ & 2.01629000 & -3.02911500 & 3.36458600 \\
\hline $\mathrm{H}$ & 1.09512800 & 1.80218000 & 3.83695600 & $\mathrm{C}$ & -0.77482100 & -2.04215800 & -1.04878900 \\
\hline $\mathrm{C}$ & -0.74164300 & 3.56786400 & 2.87855100 & $\mathrm{O}$ & -1.09064300 & -2.93533600 & -1.70579200 \\
\hline $\mathrm{H}$ & -1.13690600 & 3.64848900 & 3.89767500 & $\mathrm{C}$ & -3.29653600 & -0.82190200 & -1.49419700 \\
\hline $\mathrm{H}$ & 0.22845900 & 4.07252100 & 2.87343200 & $\mathrm{H}$ & -3.52905000 & -1.85019200 & -1.78456100 \\
\hline $\mathrm{H}$ & -1.42337200 & 4.11871700 & 2.22701100 & $\mathrm{H}$ & -2.70781800 & -0.39107400 & -2.31065100 \\
\hline $\mathrm{C}$ & 0.06986200 & 4.43201400 & -0.34835600 & $\mathrm{C}$ & -2.44872800 & -0.84131300 & -0.21846300 \\
\hline $\mathrm{H}$ & -0.35456900 & 5.03532400 & -1.15917200 & $\mathrm{H}$ & -2.38600100 & 0.13269900 & 0.25648400 \\
\hline $\mathrm{H}$ & -0.27836400 & 4.86286600 & 0.59095100 & $\mathrm{C}$ & -2.67641400 & -1.87047600 & 0.84295800 \\
\hline $\mathrm{H}$ & 1.15096700 & 4.54480000 & -0.40331300 & $\mathrm{O}$ & -2.07391000 & -1.88544500 & 1.90812700 \\
\hline $\mathrm{C}$ & 0.20882300 & 2.44570200 & -1.87813300 & $\mathrm{O}$ & -3.56949500 & -2.80987800 & 0.49149800 \\
\hline $\mathrm{H}$ & -0.10374700 & 3.10672500 & -2.69494600 & $\mathrm{C}$ & -3.81127600 & -3.85218300 & 1.46238200 \\
\hline $\mathrm{H}$ & 1.30137800 & 2.42281600 & -1.86924000 & $\mathrm{H}$ & -4.17694400 & -3.42259800 & 2.39698400 \\
\hline $\mathrm{H}$ & -0.15401500 & 1.43794300 & -2.10622400 & $\mathrm{H}$ & -4.56361100 & -4.49722000 & 1.01150800 \\
\hline $\mathrm{C}$ & -1.91609100 & 2.95244100 & -0.65013500 & $\mathrm{H}$ & -2.89186600 & -4.40819600 & 1.65731500 \\
\hline $\mathrm{H}$ & -2.21187300 & 3.53273900 & -1.53150900 & & & & \\
\hline $\mathrm{H}$ & -2.32604900 & 1.94995300 & -0.77989900 & $83 a$ & & & \\
\hline $\mathrm{H}$ & -2.39951400 & 3.41517800 & 0.21317600 & $\mathrm{C}$ & 4.32320200 & 2.88918300 & -0.36776400 \\
\hline $\mathrm{C}$ & 2.29684400 & -3.25647800 & -2.22673000 & $\mathrm{C}$ & 3.58616900 & 1.84652300 & 0.21901400 \\
\hline $\mathrm{H}$ & 2.63121400 & -4.10732200 & -1.62907600 & $\mathrm{C}$ & 4.01601900 & 0.51218400 & 0.01203000 \\
\hline $\mathrm{H}$ & 1.20840800 & -3.23639300 & -2.22738600 & $\mathrm{C}$ & 5.19579800 & 0.30876000 & -0.72466700 \\
\hline $\mathrm{H}$ & 2.61374600 & -3.44657900 & -3.25829700 & $\mathrm{C}$ & 5.89697400 & 1.35155600 & -1.32340200 \\
\hline $\mathrm{C}$ & 4.47102000 & -2.11958900 & -1.81436700 & $\mathrm{C}$ & 5.44821300 & 2.65828000 & -1.15381800 \\
\hline $\mathrm{H}$ & 4.83069800 & -2.86548300 & -1.10411100 & $\mathrm{H}$ & 4.03071100 & 3.91472000 & -0.16579500 \\
\hline $\mathrm{H}$ & 4.74112000 & -2.47012800 & -2.81673100 & $\mathrm{H}$ & 5.60843600 & -0.69081500 & -0.79006900 \\
\hline $\mathrm{H}$ & 5.00958700 & -1.18985300 & -1.64880900 & $\mathrm{H}$ & 6.80158500 & 1.14346900 & -1.88589600 \\
\hline $\mathrm{C}$ & 2.54144400 & -0.81407900 & -2.76258900 & $\mathrm{H}$ & 5.98908900 & 3.49277200 & -1.58872800 \\
\hline $\mathrm{H}$ & 2.90211600 & -1.08109500 & -3.76245400 & $\mathrm{C}$ & 3.38675500 & -0.68168500 & 0.71070300 \\
\hline $\mathrm{H}$ & 1.45569600 & -0.69292700 & -2.82051500 & $\mathrm{H}$ & 4.17311000 & -1.41700200 & 0.90949400 \\
\hline $\mathrm{H}$ & 2.98164000 & 0.15062200 & -2.49806900 & $\mathrm{C}$ & 2.47713500 & 2.22868300 & 1.17810800 \\
\hline $\mathrm{C}$ & 1.18463300 & -3.79731300 & 1.00406000 & $\mathrm{H}$ & 2.57647500 & 1.64240400 & 2.09582400 \\
\hline
\end{tabular}




\begin{tabular}{|c|c|c|c|c|c|c|c|}
\hline $\mathrm{H}$ & 2.61168500 & 3.27354100 & 1.47208200 & $\mathrm{H}$ & -1.30649600 & 3.88539100 & -1.96151600 \\
\hline $\mathrm{H}$ & 3.00752500 & -0.37231500 & 1.68656200 & $\mathrm{H}$ & -1.68878200 & 2.42961700 & -1.04195000 \\
\hline $\mathrm{P}$ & 1.94135000 & -1.69421700 & 0.00844700 & $\mathrm{H}$ & -1.59654400 & 4.00159900 & -0.22772800 \\
\hline$P$ & 0.67924200 & 1.99931000 & 0.68525800 & $\mathrm{C}$ & 1.68048600 & -3.70955800 & -2.03829100 \\
\hline $\mathrm{C}$ & -0.23760300 & 2.58327200 & 2.27497100 & $\mathrm{H}$ & 1.93067300 & -4.55952200 & -1.39882400 \\
\hline $\mathrm{C}$ & 0.37062400 & 3.18094500 & -0.79708800 & $\mathrm{H}$ & 0.60545600 & -3.54753700 & -2.01444000 \\
\hline $\mathrm{C}$ & 1.73850300 & -3.03889500 & 1.37850400 & $\mathrm{H}$ & 1.95133200 & -3.99268500 & -3.06195200 \\
\hline $\mathrm{C}$ & 2.49317600 & -2.44803500 & -1.67556500 & $\mathrm{C}$ & 3.98671400 & -2.83499500 & -1.69841900 \\
\hline $\mathrm{Pd}$ & -0.00381200 & -0.33683100 & -0.03828900 & $\mathrm{H}$ & 4.27549900 & -3.49170500 & -0.87614100 \\
\hline $\mathrm{C}$ & -5.76419000 & 2.18908800 & -1.67356600 & $\mathrm{H}$ & 4.17711200 & -3.38127200 & -2.62899000 \\
\hline $\mathrm{C}$ & -4.69427000 & 1.30873100 & -1.83462600 & $\mathrm{H}$ & 4.63846900 & -1.96588500 & -1.70254100 \\
\hline $\mathrm{C}$ & -4.79499000 & -0.02955200 & -1.42510400 & $\mathrm{C}$ & 2.23448800 & -1.35897500 & -2.73922500 \\
\hline $\mathrm{C}$ & -6.00104300 & -0.46998200 & -0.86301300 & $\mathrm{H}$ & 2.59441300 & -1.71911200 & -3.70972100 \\
\hline $\mathrm{C}$ & -7.07452600 & 0.40797600 & -0.70487900 & $\mathrm{H}$ & 1.16734600 & -1.14208700 & -2.83733600 \\
\hline $\mathrm{C}$ & -6.95796700 & 1.74010900 & -1.10469800 & $\mathrm{H}$ & 2.76073900 & -0.42703300 & -2.51511700 \\
\hline $\mathrm{H}$ & -5.67220900 & 3.21907600 & -2.00541900 & $\mathrm{C}$ & 0.43506000 & -3.83409300 & 1.15826600 \\
\hline $\mathrm{H}$ & -3.77922500 & 1.65819900 & -2.30883600 & $\mathrm{H}$ & 0.36963600 & -4.30046800 & 0.17586300 \\
\hline $\mathrm{H}$ & -6.09973600 & -1.50654400 & -0.55677900 & $\mathrm{H}$ & 0.38975400 & -4.63164600 & 1.90861400 \\
\hline $\mathrm{H}$ & -8.00537900 & 0.04853900 & -0.27625200 & $\mathrm{H}$ & -0.44057100 & -3.19749000 & 1.30325700 \\
\hline $\mathrm{H}$ & -7.79511000 & 2.42107000 & -0.98517900 & $\mathrm{C}$ & 2.93419100 & -4.01282800 & 1.42898400 \\
\hline $\mathrm{C}$ & -1.72831600 & 2.19561500 & 2.17323500 & $\mathrm{H}$ & 2.80459200 & -4.67486200 & 2.29273100 \\
\hline $\mathrm{H}$ & -2.24009600 & 2.67560000 & 1.33720400 & $\mathrm{H}$ & 2.99452900 & -4.64828500 & 0.54315300 \\
\hline $\mathrm{H}$ & -1.85520100 & 1.11235900 & 2.09566200 & $\mathrm{H}$ & 3.89412600 & -3.50493900 & 1.55961400 \\
\hline $\mathrm{H}$ & -2.23818200 & 2.51679100 & 3.08909100 & $\mathrm{C}$ & 1.61125600 & -2.32427800 & 2.74306200 \\
\hline $\mathrm{C}$ & 0.35824100 & 1.80273200 & 3.46948700 & $\mathrm{H}$ & 2.54774400 & -1.87285600 & 3.07981200 \\
\hline $\mathrm{H}$ & -0.28192600 & 1.96074900 & 4.34428300 & $\mathrm{H}$ & 0.82664500 & -1.56099800 & 2.72936600 \\
\hline $\mathrm{H}$ & 0.38992300 & 0.72415400 & 3.28403200 & $\mathrm{H}$ & 1.32649200 & -3.06718400 & 3.49610900 \\
\hline $\mathrm{H}$ & 1.36022200 & 2.14507100 & 3.74099500 & $\mathrm{C}$ & -1.25774300 & -1.63325000 & -0.84732700 \\
\hline $\mathrm{C}$ & -0.11135200 & 4.09255400 & 2.55652200 & $\mathrm{O}$ & -1.25869700 & -2.59540800 & -1.54917500 \\
\hline $\mathrm{H}$ & -0.54868200 & 4.30708100 & 3.53861400 & $\mathrm{C}$ & -3.61834600 & -0.96649900 & -1.60046900 \\
\hline $\mathrm{H}$ & 0.92816500 & 4.43303400 & 2.58709400 & $\mathrm{H}$ & -3.94115800 & -2.00678500 & -1.68292600 \\
\hline $\mathrm{H}$ & -0.65270000 & 4.69840900 & 1.82621300 & $\mathrm{H}$ & -3.09070900 & -0.72527300 & -2.52910300 \\
\hline $\mathrm{C}$ & 1.03994200 & 4.56324100 & -0.67280400 & $\mathrm{C}$ & -2.59259600 & -0.87499300 & -0.44285200 \\
\hline $\mathrm{H}$ & 0.78031100 & 5.15921600 & -1.55564600 & $\mathrm{H}$ & -2.38912500 & 0.18393700 & -0.22279800 \\
\hline $\mathrm{H}$ & 0.70850300 & 5.12040400 & 0.20425600 & $\mathrm{C}$ & -2.96256300 & -1.48305900 & 0.90994100 \\
\hline $\mathrm{H}$ & 2.12735900 & 4.49182600 & -0.64761200 & $\mathrm{O}$ & -2.30834000 & -1.25468800 & 1.91223200 \\
\hline $\mathrm{C}$ & 0.93470500 & 2.46376200 & -2.04563200 & $\mathrm{O}$ & -4.00095700 & -2.31092600 & 0.85245800 \\
\hline $\mathrm{H}$ & 0.77869500 & 3.10047900 & -2.92448000 & $\mathrm{C}$ & -4.38552200 & -2.94073700 & 2.09941500 \\
\hline $\mathrm{H}$ & 2.00577700 & 2.26621600 & -1.96420000 & $\mathrm{H}$ & -4.66320800 & -2.18050300 & 2.83155300 \\
\hline $\mathrm{H}$ & 0.42676500 & 1.51071600 & -2.22895800 & $\mathrm{H}$ & -5.23723200 & -3.57123300 & 1.85151700 \\
\hline $\mathrm{C}$ & -1.14450000 & 3.37891100 & -1.00301400 & $\mathrm{H}$ & -3.55896600 & -3.53853300 & 2.48743500 \\
\hline
\end{tabular}




\begin{tabular}{|c|c|c|c|}
\hline 83 & & & \\
\hline $\mathrm{C}$ & -4.11318300 & 1.75364500 & 2.41633300 \\
\hline $\mathrm{C}$ & -3.60925200 & 1.07389800 & 1.29450900 \\
\hline $\mathrm{C}$ & -3.72010800 & -0.33391800 & 1.24273600 \\
\hline $\mathrm{C}$ & -4.37155900 & -0.98247600 & 2.30632300 \\
\hline $\mathrm{C}$ & -4.83939400 & -0.29864400 & 3.42514100 \\
\hline $\mathrm{C}$ & -4.70181500 & 1.08592500 & 3.48595900 \\
\hline $\mathrm{H}$ & -4.06063200 & 2.83845700 & 2.43853600 \\
\hline $\mathrm{H}$ & -4.56711200 & -2.04591000 & 2.23733400 \\
\hline $\mathrm{H}$ & -5.33540700 & -0.84199500 & 4.22315400 \\
\hline $\mathrm{H}$ & -5.07920000 & 1.64478400 & 4.33649900 \\
\hline $\mathrm{C}$ & -3.36618000 & -1.12919900 & -0.00023000 \\
\hline $\mathrm{H}$ & -4.03101100 & -1.99617200 & -0.05093700 \\
\hline $\mathrm{C}$ & -3.10385200 & 1.91212900 & 0.14225900 \\
\hline $\mathrm{H}$ & -3.54567300 & 1.56215400 & -0.79772300 \\
\hline $\mathrm{H}$ & -3.46806200 & 2.93165800 & 0.27917300 \\
\hline $\mathrm{H}$ & -3.59756300 & -0.52595600 & -0.87927300 \\
\hline$P$ & -1.65092300 & -1.82962400 & -0.41688100 \\
\hline$P$ & -1.26621600 & 2.02110300 & -0.23654900 \\
\hline $\mathrm{C}$ & -1.33767100 & 3.16314300 & -1.80161100 \\
\hline $\mathrm{C}$ & -0.48846900 & 2.91564300 & 1.28488000 \\
\hline $\mathrm{C}$ & -2.06660000 & -2.64230800 & -2.12663000 \\
\hline $\mathrm{C}$ & -1.26917300 & -3.15017100 & 0.93807900 \\
\hline $\mathrm{Pd}$ & 0.03606500 & -0.15928200 & -0.67077800 \\
\hline $\mathrm{C}$ & 5.91624800 & -0.62739500 & 3.03830500 \\
\hline $\mathrm{C}$ & 4.97286800 & -1.10747800 & 2.13024700 \\
\hline $\mathrm{C}$ & 5.18401600 & -0.99826800 & 0.74743800 \\
\hline $\mathrm{C}$ & 6.37001400 & -0.40671400 & 0.29638800 \\
\hline $\mathrm{C}$ & 7.31981900 & 0.06896800 & 1.20250500 \\
\hline $\mathrm{C}$ & 7.09398200 & -0.03599900 & 2.57546600 \\
\hline $\mathrm{H}$ & 5.73894400 & -0.72644700 & 4.10519600 \\
\hline $\mathrm{H}$ & 4.07119100 & -1.59097400 & 2.50258000 \\
\hline $\mathrm{H}$ & 6.55384300 & -0.32492700 & -0.77125900 \\
\hline $\mathrm{H}$ & 8.23810700 & 0.51737300 & 0.83480100 \\
\hline $\mathrm{H}$ & 7.83328400 & 0.33170900 & 3.28048800 \\
\hline $\mathrm{C}$ & 0.00526100 & 3.87689100 & -2.05152100 \\
\hline $\mathrm{H}$ & 0.17564900 & 4.68719900 & -1.33824900 \\
\hline $\mathrm{H}$ & 0.85227400 & 3.18974800 & -2.01994900 \\
\hline $\mathrm{H}$ & -0.02422400 & 4.33168300 & -3.04887800 \\
\hline $\mathrm{C}$ & -1.61379300 & 2.22058700 & -2.99377100 \\
\hline
\end{tabular}

\begin{tabular}{|c|c|c|}
\hline-1.69691900 & 2.81395900 & -3.91177800 \\
\hline-0.80532600 & 1.49665300 & -3.13468200 \\
\hline-2.55380800 & 1.67047200 & -2.88277800 \\
\hline-2.45269300 & 4.22937600 & -1.75079400 \\
\hline-2.37083900 & 4.85804700 & -2.64499100 \\
\hline-3.45557600 & 3.79615900 & -1.76050100 \\
\hline-2.37012700 & 4.88911800 & -0.88391000 \\
\hline-1.18804500 & 4.24356100 & 1.62988900 \\
\hline-0.76701100 & 4.63886000 & 2.56184500 \\
\hline-1.03195900 & 5.00260800 & 0.85879700 \\
\hline-2.26314200 & 4.13003500 & 1.78718800 \\
\hline-0.58818700 & 1.93995000 & 2.48126300 \\
\hline-0.05884600 & 2.37881400 & 3.33520800 \\
\hline-1.61347400 & 1.74551000 & 2.79238700 \\
\hline-0.11246000 & 0.97930100 & 2.25602700 \\
\hline 1.01464100 & 3.18939700 & 1.06817500 \\
\hline 1.42105100 & 3.61554300 & 1.99253600 \\
\hline 1.56382300 & 2.27005000 & 0.85860600 \\
\hline 1.21510500 & 3.89822100 & 0.26558200 \\
\hline-0.27056700 & -4.22422400 & 0.46001700 \\
\hline-0.70860600 & -4.88667600 & -0.29021300 \\
\hline 0.65243500 & -3.81656600 & 0.05694500 \\
\hline-0.01253400 & -4.84780300 & 1.32377600 \\
\hline-2.52997400 & -3.88993500 & 1.43480300 \\
\hline-3.09807600 & -4.36512300 & 0.63372600 \\
\hline-2.20669000 & -4.68476200 & 2.11607900 \\
\hline-3.19307300 & -3.24185500 & 2.00124500 \\
\hline-0.65621800 & -2.37219800 & 2.12375700 \\
\hline-0.47407500 & -3.06661500 & 2.95197200 \\
\hline 0.29956800 & -1.91338200 & 1.85395100 \\
\hline-1.32147600 & -1.58520900 & 2.49064300 \\
\hline-0.78429500 & -3.11540900 & -2.83746400 \\
\hline-0.21920100 & -3.85216100 & -2.26946800 \\
\hline-1.07394500 & -3.57399700 & -3.78981600 \\
\hline-0.11564800 & -2.28128300 & -3.05736100 \\
\hline-3.03671500 & -3.83506700 & -1.99804400 \\
\hline-3.29718900 & -4.17378100 & -3.00738300 \\
\hline-2.58737700 & -4.68575300 & -1.48189800 \\
\hline-3.97480800 & -3.57886800 & -1.49814900 \\
\hline-2.71525000 & -1.56898400 & -3.03126700 \\
\hline-3.73743100 & -1.31768800 & -2.73795900 \\
\hline
\end{tabular}




\begin{tabular}{|c|c|c|c|c|c|c|c|}
\hline $\mathrm{H}$ & -2.11994900 & -0.65245000 & -3.06997000 & $\mathrm{C}$ & 7.20812100 & 1.10407400 & 1.22420600 \\
\hline $\mathrm{H}$ & -2.76791400 & -1.96655800 & -4.05054900 & $\mathrm{C}$ & 5.90778600 & 0.60969100 & 1.31994400 \\
\hline $\mathrm{C}$ & 1.59588100 & -1.47376700 & -0.83444200 & $\mathrm{C}$ & 5.53304200 & -0.55697700 & 0.63834400 \\
\hline $\mathrm{O}$ & 1.75108700 & -2.55463600 & -1.32691500 & $\mathrm{C}$ & 6.49181900 & -1.22284100 & -0.13724100 \\
\hline $\mathrm{C}$ & 4.14926000 & -1.53107500 & -0.22473800 & $\mathrm{C}$ & 7.79488300 & -0.73133400 & -0.23158700 \\
\hline $\mathrm{H}$ & 4.56107100 & -1.59310100 & -1.23551600 & $\mathrm{C}$ & 8.15500600 & 0.43432000 & 0.44593500 \\
\hline $\mathrm{H}$ & 3.86604300 & -2.55098200 & 0.05366300 & $\mathrm{H}$ & 7.48519200 & 2.00550300 & 1.76262600 \\
\hline $\mathrm{C}$ & 2.84983800 & -0.70081800 & -0.25116600 & $\mathrm{H}$ & 5.18014500 & 1.12791000 & 1.94132700 \\
\hline $\mathrm{H}$ & 2.57166900 & -0.41716500 & 0.77421000 & $\mathrm{H}$ & 6.21756800 & -2.13520300 & -0.65954100 \\
\hline $\mathrm{C}$ & 2.85756100 & 0.58615800 & -1.03334700 & $\mathrm{H}$ & 8.52967600 & -1.26276300 & -0.82903200 \\
\hline $\mathrm{O}$ & 1.78566900 & 1.13808200 & -1.32921400 & $\mathrm{H}$ & 9.16914800 & 0.81533000 & 0.37461300 \\
\hline $\mathrm{O}$ & 4.02894000 & 1.05126000 & -1.39504200 & $\mathrm{C}$ & -1.48301300 & -3.86666400 & 1.89635500 \\
\hline $\mathrm{C}$ & 4.06290000 & 2.26635000 & -2.18881300 & $\mathrm{H}$ & -1.90815100 & -4.65144300 & 1.26722300 \\
\hline $\mathrm{H}$ & 3.63800900 & 3.09359600 & -1.61870300 & $\mathrm{H}$ & -0.50286700 & -3.59079900 & 1.49568800 \\
\hline $\mathrm{H}$ & 5.11731900 & 2.43947900 & -2.39289600 & $\mathrm{H}$ & -1.32192400 & -4.30495900 & 2.88793900 \\
\hline \multirow[t]{2}{*}{$\mathrm{H}$} & 3.50368700 & 2.12067800 & -3.11454000 & $\mathrm{C}$ & -1.87760400 & -1.75258200 & 3.16231100 \\
\hline & & & & $\mathrm{H}$ & -1.87187300 & -2.31180000 & 4.10480300 \\
\hline 83-0 & & & & $\mathrm{H}$ & -0.85051000 & -1.43720100 & 2.95345200 \\
\hline $\mathrm{C}$ & -5.44314400 & 0.40797200 & -1.04850100 & $\mathrm{H}$ & -2.48659300 & -0.85769500 & 3.32516600 \\
\hline $\mathrm{C}$ & -4.33177400 & 0.58138400 & -0.20671700 & $\mathrm{C}$ & -3.82909700 & -3.16109800 & 2.45677900 \\
\hline $\mathrm{C}$ & -3.69243800 & 1.84229200 & -0.17489700 & $\mathrm{H}$ & -3.72364600 & -3.78867000 & 3.34917300 \\
\hline $\mathrm{C}$ & -4.23430200 & 2.87802200 & -0.95542900 & $\mathrm{H}$ & -4.50776100 & -2.34543600 & 2.71945200 \\
\hline $\mathrm{C}$ & -5.32425900 & 2.68486300 & -1.79978100 & $\mathrm{H}$ & -4.30643700 & -3.77008600 & 1.68656400 \\
\hline $\mathrm{C}$ & -5.93010100 & 1.43179000 & -1.85591900 & $\mathrm{C}$ & -4.01738600 & -3.48110400 & -1.09617900 \\
\hline $\mathrm{H}$ & -5.95157100 & -0.55248100 & -1.05082300 & $\mathrm{H}$ & -4.17412800 & -3.94924100 & -2.07484000 \\
\hline $\mathrm{H}$ & -3.81847300 & 3.87601400 & -0.86957200 & $\mathrm{H}$ & -3.94366100 & -4.28753900 & -0.36244200 \\
\hline $\mathrm{H}$ & -5.70945600 & 3.51356600 & -2.38533200 & $\mathrm{H}$ & -4.91281000 & -2.89347200 & -0.87432800 \\
\hline $\mathrm{H}$ & -6.79170900 & 1.26003400 & -2.49323100 & $\mathrm{C}$ & -2.83371100 & -1.62564000 & -2.32672600 \\
\hline $\mathrm{C}$ & -2.56683400 & 2.17275100 & 0.78883300 & $\mathrm{H}$ & -2.90065500 & -2.19460500 & -3.26111400 \\
\hline $\mathrm{H}$ & -2.61174800 & 3.24190200 & 1.01993100 & $\mathrm{H}$ & -3.70879500 & -0.97875300 & -2.26971300 \\
\hline $\mathrm{C}$ & -3.98187700 & -0.56048800 & 0.72315600 & $\mathrm{H}$ & -1.94417700 & -0.99154400 & -2.38902200 \\
\hline $\mathrm{H}$ & -3.85998900 & -0.18328400 & 1.74488800 & $\mathrm{C}$ & -1.52288200 & -3.53507400 & -1.42837500 \\
\hline $\mathrm{H}$ & -4.83062600 & -1.24753900 & 0.76109000 & $\mathrm{H}$ & -1.65127500 & -3.98672200 & -2.41860300 \\
\hline $\mathrm{H}$ & -2.73559900 & 1.65014700 & 1.73295900 & $\mathrm{H}$ & -0.58269100 & -2.97629900 & -1.44151000 \\
\hline $\mathrm{P}$ & -0.74178500 & 1.81989000 & 0.41880500 & $\mathrm{H}$ & -1.42248600 & -4.34967800 & -0.71166900 \\
\hline $\mathrm{P}$ & -2.43735000 & -1.58645100 & 0.43654400 & $\mathrm{C}$ & 1.25100800 & 2.92141300 & -1.30494100 \\
\hline $\mathrm{C}$ & -2.43098900 & -2.65909800 & 2.03997500 & $\mathrm{H}$ & 1.68273700 & 3.57024100 & -0.53885200 \\
\hline $\mathrm{C}$ & -2.73922000 & -2.62442000 & -1.15044800 & $\mathrm{H}$ & 1.76442200 & 1.95992300 & -1.29621200 \\
\hline $\mathrm{C}$ & 0.08297300 & 2.56691200 & 1.99758700 & $\mathrm{H}$ & 1.47410500 & 3.38001600 & -2.27451300 \\
\hline $\mathrm{C}$ & -0.27719600 & 2.78917100 & -1.17383500 & $\mathrm{C}$ & -0.89461200 & 4.20077600 & -1.24259100 \\
\hline $\mathrm{Pd}$ & -0.24903100 & -0.47089200 & 0.22227300 & $\mathrm{H}$ & -0.56310200 & 4.85509100 & -0.43599800 \\
\hline
\end{tabular}




\begin{tabular}{|c|c|c|c|c|c|c|c|}
\hline $\mathrm{H}$ & -0.57773800 & 4.66232200 & -2.18450300 & $\mathrm{H}$ & 4.69834500 & -3.25455400 & 0.29816000 \\
\hline $\mathrm{H}$ & -1.98211200 & 4.17921100 & -1.24996700 & $\mathrm{H}$ & 6.97100800 & -2.53146600 & -0.27315200 \\
\hline $\mathrm{C}$ & -0.80157100 & 1.95094700 & -2.36095700 & $\mathrm{H}$ & 7.56957800 & -0.10741500 & -0.09785100 \\
\hline $\mathrm{H}$ & -0.55285400 & 2.46827700 & -3.29450200 & $\mathrm{C}$ & 2.58115000 & -1.83877200 & 1.10652200 \\
\hline $\mathrm{H}$ & -0.32809400 & 0.96504800 & -2.39144900 & $\mathrm{H}$ & 2.76111000 & -2.86103300 & 1.44544100 \\
\hline $\mathrm{H}$ & -1.88568300 & 1.81756400 & -2.33271800 & $\mathrm{C}$ & 3.30874900 & 1.12569700 & 1.31044500 \\
\hline $\mathrm{C}$ & 1.55585900 & 2.12007800 & 2.08680100 & $\mathrm{H}$ & 2.78300000 & 0.70326500 & 2.17057300 \\
\hline $\mathrm{H}$ & 2.14937300 & 2.44639200 & 1.23145400 & $\mathrm{H}$ & 3.90190800 & 1.95847200 & 1.69880900 \\
\hline $\mathrm{H}$ & 2.00215700 & 2.56231300 & 2.98460700 & $\mathrm{H}$ & 2.25984100 & -1.28291900 & 1.99071500 \\
\hline $\mathrm{H}$ & 1.63516500 & 1.03327500 & 2.17730700 & $\mathrm{P}$ & 0.98283600 & -1.94911900 & 0.08967300 \\
\hline $\mathrm{C}$ & 0.02241100 & 4.10730000 & 2.04425600 & $\mathrm{P}$ & 1.92795900 & 1.87644800 & 0.27383700 \\
\hline $\mathrm{H}$ & 0.38882300 & 4.43960400 & 3.02226100 & $\mathrm{C}$ & 1.16372900 & 3.11239600 & 1.54947300 \\
\hline $\mathrm{H}$ & 0.65785400 & 4.57249100 & 1.28782800 & $\mathrm{C}$ & 2.80669000 & 2.81613800 & -1.16701300 \\
\hline $\mathrm{H}$ & -0.99301700 & 4.49779200 & 1.93442400 & $\mathrm{C}$ & -0.03458100 & -3.04326500 & 1.32338700 \\
\hline $\mathrm{C}$ & -0.64487900 & 2.00486300 & 3.23974500 & $\mathrm{C}$ & 1.37070500 & -2.93084700 & -1.53667600 \\
\hline $\mathrm{H}$ & -1.64205300 & 2.43043700 & 3.37490700 & $\mathrm{Pd}$ & 0.01422900 & 0.29383900 & -0.49974800 \\
\hline $\mathrm{H}$ & -0.72655300 & 0.91480000 & 3.21130200 & $\mathrm{C}$ & -7.14296000 & 1.50376400 & -1.42320000 \\
\hline $\mathrm{H}$ & -0.06354200 & 2.26653200 & 4.13062400 & $\mathrm{C}$ & -5.93701900 & 0.80867300 & -1.51263100 \\
\hline $\mathrm{C}$ & 1.72141000 & -0.78430200 & -0.02361100 & $\mathrm{C}$ & -5.63739400 & -0.23045800 & -0.61906300 \\
\hline $\mathrm{O}$ & 1.40867200 & -1.96356100 & -0.01223800 & $\mathrm{C}$ & -6.58140800 & -0.56502800 & 0.35899100 \\
\hline $\mathrm{C}$ & 4.11554900 & -1.07926100 & 0.73097400 & $\mathrm{C}$ & -7.79285300 & 0.12318200 & 0.44668500 \\
\hline $\mathrm{H}$ & 4.06768400 & -2.13659600 & 0.46337900 & $\mathrm{C}$ & -8.07516100 & 1.16240200 & -0.44080700 \\
\hline $\mathrm{H}$ & 3.74631100 & -0.98367600 & 1.75843200 & $\mathrm{H}$ & -7.36038800 & 2.30153300 & -2.12725800 \\
\hline $\mathrm{C}$ & 3.14289100 & -0.29100800 & -0.19787700 & $\mathrm{H}$ & -5.23079400 & 1.06434000 & -2.30081400 \\
\hline $\mathrm{H}$ & 3.17906800 & 0.77296700 & 0.02823100 & $\mathrm{H}$ & -6.36773200 & -1.37375300 & 1.05215300 \\
\hline $\mathrm{C}$ & 3.49182000 & -0.40686400 & -1.69676000 & $\mathrm{H}$ & -8.51714600 & -0.15511200 & 1.20669800 \\
\hline $\mathrm{O}$ & 3.49728200 & 0.54131500 & -2.44756900 & $\mathrm{H}$ & -9.01754900 & 1.69742400 & -0.37361000 \\
\hline $\mathrm{O}$ & 3.75090900 & -1.67010500 & -2.04861100 & $\mathrm{C}$ & -0.16018000 & 3.68745600 & 1.00135000 \\
\hline $\mathrm{C}$ & 4.10418700 & -1.87312800 & -3.43735600 & $\mathrm{H}$ & -0.03712100 & 4.22168300 & 0.05787300 \\
\hline $\mathrm{H}$ & 5.01961800 & -1.32707400 & -3.67292300 & $\mathrm{H}$ & -0.91175700 & 2.90489200 & 0.87859500 \\
\hline $\mathrm{H}$ & 4.25380700 & -2.94618000 & -3.54320100 & $\mathrm{H}$ & -0.55483400 & 4.40617400 & 1.72890800 \\
\hline \multirow[t]{2}{*}{$\mathrm{H}$} & 3.29780200 & -1.52613500 & -4.08634600 & $\mathrm{C}$ & 0.82943600 & 2.32394100 & 2.83737800 \\
\hline & & & & $\mathrm{H}$ & 0.24486800 & 2.97557400 & 3.49664400 \\
\hline 83-CO & & & & $\mathrm{H}$ & 0.21746800 & 1.44126200 & 2.63517400 \\
\hline $\mathrm{C}$ & 5.58094600 & 0.46720500 & 0.48771100 & $\mathrm{H}$ & 1.72226900 & 2.03002500 & 3.39511000 \\
\hline $\mathrm{C}$ & 4.25229300 & 0.08647300 & 0.74211700 & $\mathrm{C}$ & 2.10924300 & 4.27432300 & 1.91419300 \\
\hline $\mathrm{C}$ & 3.90953800 & -1.28091100 & 0.63141400 & $\mathrm{H}$ & 1.64670500 & 4.86090600 & 2.71626400 \\
\hline $\mathrm{C}$ & 4.92231300 & -2.19365300 & 0.28787900 & $\mathrm{H}$ & 3.07862700 & 3.93284100 & 2.28821100 \\
\hline $\mathrm{C}$ & 6.22160700 & -1.79208900 & -0.00902400 & $\mathrm{H}$ & 2.28109700 & 4.95405900 & 1.07699600 \\
\hline $\mathrm{C}$ & 6.55495500 & -0.44415400 & 0.08978100 & $\mathrm{C}$ & 4.14828200 & 3.46024600 & -0.75994900 \\
\hline $\mathrm{H}$ & 5.87289200 & 1.49877500 & 0.65318100 & $\mathrm{H}$ & 4.52804900 & 4.03244700 & -1.61412400 \\
\hline
\end{tabular}




$\begin{array}{cccccccc}\mathrm{H} & 4.05773700 & 4.15250300 & 0.07842300 & \mathrm{C} & -2.83666700 & 0.10635600 & 1.11084900 \\ \mathrm{H} & 4.90269600 & 2.71478000 & -0.51875100 & \mathrm{O} & -1.85471500 & 0.70000300 & 1.53287000 \\ \mathrm{C} & 3.07630900 & 1.76334700 & -2.26445100 & \mathrm{O} & -3.79410300 & -0.38094200 & 1.89489200 \\ \mathrm{H} & 3.58598300 & 2.24499300 & -3.10694900 & \mathrm{C} & -3.67800200 & -0.10967200 & 3.31018700 \\ \mathrm{H} & 3.72128100 & 0.95582500 & -1.90780700 & \mathrm{H} & -3.58259300 & 0.96403900 & 3.48052400 \\ \mathrm{H} & 2.14983800 & 1.32149900 & -2.64241000 & \mathrm{H} & -4.59687800 & -0.49145100 & 3.75120800 \\ \mathrm{C} & 1.91520700 & 3.92895300 & -1.75431100 & \mathrm{H} & -2.80816800 & -0.62419000 & 3.72349600 \\ \mathrm{H} & 2.41102200 & 4.32729600 & -2.64678300 & \mathrm{C} & -0.74053800 & 1.70751900 & -1.65712900 \\ \mathrm{H} & 0.93266400 & 3.58052300 & -2.07080000 & \mathrm{O} & -1.21400600 & 2.41288100 & -2.42053400\end{array}$

$\mathrm{H}$

C

H

$\mathrm{H}$

H

C

H

$\mathrm{H}$

H

C

H

$\mathrm{H}$

H

C

H

$\mathrm{H}$

$\mathrm{H}$

C

H

$\mathrm{H}$

H

C

H

$\mathrm{H}$

H

C

O

C

H

H

C

$\mathrm{H}$
$1.78019100 \quad 4.76449600 \quad-1.06409900$

$\begin{array}{lll}0.14949900 & -3.72250800 & -2.04945000\end{array}$

$\begin{array}{lll}-0.09604600 & -4.57082300 & -1.40681700\end{array}$

$\begin{array}{lll}-0.73366400 & -3.10304500 & -2.18898700\end{array}$

$0.41430300 \quad-4.13550700 \quad-3.02970900$

$2.54203000 \quad-3.92156300 \quad-1.38241800$

$2.43175300 \quad-4.59976000 \quad-0.53307700$

$2.58180200 \quad-4.54124300 \quad-2.28523900$

$3.49761000 \quad-3.40847200 \quad-1.30460300$

$\begin{array}{lll}1.75387200 & -1.87439900 & -2.59371400\end{array}$

$2.04310700 \quad-2.38545100-3.51943000$

$\begin{array}{lll}0.91164700 & -1.21604500 & -2.82279200\end{array}$

$2.60327400 \quad-1.26440500 \quad-2.27435100$

$\begin{array}{lll}-1.49627200 & -3.20972000 & 0.86162700\end{array}$

$\begin{array}{lll}-1.59721100 & -3.61523500 & -0.14367300\end{array}$

$\begin{array}{lll}-1.99764900 & -3.90174700 & 1.54782700\end{array}$

$-2.04476800 \quad-2.26985100 \quad 0.91395200$

$\begin{array}{lll}0.57061900 & -4.45117300 & 1.51569600\end{array}$

$0.00092400 \quad-4.96687400 \quad 2.29712200$

$0.50401000 \quad-5.06161600 \quad 0.61302700$

$1.61213600 \quad-4.43614100 \quad 1.84616100$

$\begin{array}{lll}-0.06281900 & -2.31659000 & 2.68950900\end{array}$

$\begin{array}{lll}0.89852500 & -2.34637700 & 3.20733000\end{array}$

$-0.38041700$

$-0.78575900$

$-1.27329700$

2.59260000

$-2.82529100 \quad 3.33781100$

$-1.79021600$

$-1.86421300$

$-0.60898100 \quad-1.10877700$

$-4.32792700$

$-1.26966600-2.10622000$

$-0.98617600 \quad-0.74326400$

$\begin{array}{lll}-4.35058300 & -1.89092500 & -0.13030900\end{array}$

$\begin{array}{lll}-4.18627700 & -1.30764000 & -1.77921300\end{array}$

$-3.08796600$

$-0.13871600 \quad-0.37031800$

$-3.23137600$
TS66b

imaginary frequency $=-130.87 \mathrm{~cm}^{-1}$

Pd

$0.56341500 \quad-0.04031000$

$-0.43089800$

$0.31807100 \quad 2.41171900 \quad-0.58213000$

$2.73003600 \quad-1.26727200 \quad-0.45016500$

$\begin{array}{lll}-0.60734100 & 3.58974000 & 0.65039800\end{array}$

$\begin{array}{lll}0.03530700 & 3.04886900 & -2.39478400\end{array}$

$3.33707200 \quad-1.88292600 \quad-2.19480700$

$3.28659900 \quad-2.51460900 \quad 0.91838400$

$\begin{array}{lll}-2.08905000 & 3.75658600 & 0.25959700\end{array}$

$\begin{array}{lll}-0.55600000 & 2.88976900 & 2.02569300\end{array}$

$\begin{array}{lll}-0.00720200 & 5.00641400 & 0.78053700\end{array}$

$\begin{array}{lll}1.09120800 & 2.39963900 & -3.31356200\end{array}$

$0.12805800 \quad 4.57779600 \quad-2.56090800$

$\begin{array}{lll}-1.34007000 & 2.55839100 & -2.89458200\end{array}$

$2.94985700 \quad-3.35202200 \quad-2.44793900$

$\begin{array}{lll}4.85501500 & -1.73515500 & -2.43811600\end{array}$

$2.59717500 \quad-1.01733700 \quad-3.23476700$

$2.50859800 \quad-3.84267500 \quad 0.79121000$

$4.79557800 \quad-2.82381800 \quad 0.92321600$

$\begin{array}{lll}2.87699900 & -1.90749100 & 2.27843600\end{array}$

$2.16525900 \quad 2.83684800 \quad-0.34925600$

$\begin{array}{lll}3.89410100 & 0.23313600 & -0.27980700\end{array}$

$3.77048000 \quad 1.20935800 \quad 0.87262200$

$\begin{array}{lll}4.62812600 & 1.00254500 & 1.96987500\end{array}$

$4.66675800 \quad 1.85451400 \quad 3.06782200$

$\begin{array}{lll}3.84735200 & 2.98113700 & 3.07467000\end{array}$

$3.03082100 \quad 3.23222400 \quad 1.97680500$

$\begin{array}{lll}2.95807000 & 2.37319300 & 0.86391900\end{array}$

$2.09635200 \quad 2.80236600 \quad-3.16175300$

$\begin{array}{lll}0.82167800 & 2.60755400 & -4.35531800\end{array}$ 


\begin{tabular}{|c|c|c|c|c|c|c|c|}
\hline $\mathrm{H}$ & 1.11742300 & 1.31506900 & -3.19315400 & $\mathrm{H}$ & 2.45640000 & 4.15081200 & 1.96875400 \\
\hline $\mathrm{H}$ & 1.06518000 & 4.98897800 & -2.17480400 & $\mathrm{C}$ & -1.28360200 & -1.33131000 & -1.14150800 \\
\hline $\mathrm{H}$ & 0.08775100 & 4.81791300 & -3.63011400 & $\mathrm{O}$ & -1.14064500 & -1.22455600 & -2.33107800 \\
\hline $\mathrm{H}$ & -0.70252700 & 5.10439500 & -2.08639800 & $\mathrm{O}$ & -0.75474200 & -2.70322000 & -0.54397300 \\
\hline $\mathrm{H}$ & -1.48148900 & 2.89925400 & -3.92703500 & $\mathrm{H}$ & -0.84828300 & -2.88721200 & 0.53999200 \\
\hline $\mathrm{H}$ & -1.39373300 & 1.46695200 & -2.89693800 & $\mathrm{C}$ & -2.51782300 & -0.95950900 & -0.30263200 \\
\hline $\mathrm{H}$ & -2.17444100 & 2.95050900 & -2.31072600 & $\mathrm{O}$ & -0.80017800 & -3.03132000 & 1.86034800 \\
\hline $\mathrm{H}$ & 1.04231300 & 5.01120200 & 1.07186300 & $\mathrm{H}$ & -0.67525400 & -2.13260500 & 2.28601700 \\
\hline $\mathrm{H}$ & -0.10043800 & 5.58724200 & -0.13707000 & $\mathrm{O}$ & -0.48176100 & -0.57147600 & 2.68277900 \\
\hline $\mathrm{H}$ & -0.55723200 & 5.54483700 & 1.56148500 & $\mathrm{H}$ & 0.06157800 & -0.14557000 & 1.98443800 \\
\hline $\mathrm{H}$ & -2.21681900 & 4.33285900 & -0.65872000 & $\mathrm{C}$ & -3.48064400 & -2.15758500 & -0.32594400 \\
\hline $\mathrm{H}$ & -2.60097600 & 4.30721400 & 1.05768500 & $\mathrm{O}$ & -3.72813700 & -2.81610500 & -1.31242400 \\
\hline $\mathrm{H}$ & -2.61031700 & 2.80531700 & 0.14675500 & $\mathrm{O}$ & -4.05968100 & -2.33812000 & 0.87214900 \\
\hline $\mathrm{H}$ & -1.06391900 & 3.51613800 & 2.76921800 & $\mathrm{C}$ & -5.16992800 & -3.26738800 & 0.88978100 \\
\hline $\mathrm{H}$ & -1.06651000 & 1.92279300 & 1.99673500 & $\mathrm{H}$ & -4.85585100 & -4.25021600 & 0.53353400 \\
\hline $\mathrm{H}$ & 0.46798900 & 2.72828100 & 2.37205600 & $\mathrm{H}$ & -5.49485000 & -3.31192900 & 1.92805800 \\
\hline $\mathrm{H}$ & 1.79921200 & -1.73424400 & 2.31892200 & $\mathrm{H}$ & -5.97231500 & -2.88918900 & 0.25376100 \\
\hline $\mathrm{H}$ & 3.38145700 & -0.96898000 & 2.50361900 & $\mathrm{H}$ & -2.23707500 & -0.74496800 & 0.73010000 \\
\hline $\mathrm{H}$ & 3.12868700 & -2.62105900 & 3.07251300 & $\mathrm{C}$ & -3.26460100 & 0.24215200 & -0.93371500 \\
\hline $\mathrm{H}$ & 2.78259800 & -4.42222800 & -0.08932800 & $\mathrm{H}$ & -2.58844800 & 1.09671000 & -0.89087800 \\
\hline $\mathrm{H}$ & 2.73680400 & -4.46107900 & 1.66761800 & $\mathrm{H}$ & -3.42597200 & 0.01518300 & -1.99032100 \\
\hline $\mathrm{H}$ & 1.42981800 & -3.67636000 & 0.78738800 & $\mathrm{C}$ & -4.58843000 & 0.55875400 & -0.26578200 \\
\hline $\mathrm{H}$ & 5.41787800 & -1.92646500 & 0.97507700 & $\mathrm{C}$ & -5.78845300 & 0.37430300 & -0.96533100 \\
\hline $\mathrm{H}$ & 5.10001300 & -3.39776700 & 0.04463500 & $\mathrm{C}$ & -4.64725300 & 1.03762500 & 1.05166000 \\
\hline $\mathrm{H}$ & 5.03456900 & -3.43339500 & 1.80296800 & $\mathrm{C}$ & -7.01693800 & 0.66739400 & -0.36810800 \\
\hline $\mathrm{H}$ & 1.90552400 & -3.55567400 & -2.20177600 & $\mathrm{H}$ & -5.76010700 & 0.00503000 & -1.98726500 \\
\hline $\mathrm{H}$ & 3.58333300 & -4.05056000 & -1.89725100 & $\mathrm{C}$ & -5.87205000 & 1.33144100 & 1.65020300 \\
\hline $\mathrm{H}$ & 3.08410600 & -3.57202100 & -3.51346600 & $\mathrm{H}$ & -3.72943700 & 1.18115500 & 1.61688400 \\
\hline $\mathrm{H}$ & 5.46162800 & -2.27401600 & -1.70783600 & $\mathrm{C}$ & -7.06166300 & 1.14752800 & 0.94099300 \\
\hline $\mathrm{H}$ & 5.08972300 & -2.15200400 & -3.42473300 & $\mathrm{H}$ & -7.93601700 & 0.52511700 & -0.92885800 \\
\hline $\mathrm{H}$ & 5.18063500 & -0.69249200 & -2.45042400 & $\mathrm{H}$ & -5.89905600 & 1.70606700 & 2.66938400 \\
\hline $\mathrm{H}$ & 2.90511000 & -1.32315500 & -4.24197100 & $\mathrm{H}$ & -8.01517000 & 1.37999800 & 1.40549300 \\
\hline $\mathrm{H}$ & 2.84196600 & 0.04277900 & -3.13411700 & $\mathrm{C}$ & -0.04823500 & -0.15153200 & 3.98856400 \\
\hline $\mathrm{H}$ & 1.51152800 & -1.12860400 & -3.16334400 & $\mathrm{H}$ & -0.17142000 & 0.92811700 & 4.10570000 \\
\hline $\mathrm{H}$ & 4.91727500 & -0.14952400 & -0.28979200 & $\mathrm{H}$ & -0.68697600 & -0.66081200 & 4.71210100 \\
\hline $\mathrm{H}$ & 3.75763300 & 0.75292300 & -1.23211300 & $\mathrm{H}$ & 0.99576200 & -0.42446400 & 4.17112300 \\
\hline $\mathrm{H}$ & 2.62609800 & 2.42643700 & -1.24770900 & $\mathrm{C}$ & -0.76308000 & -3.86396900 & -1.42016900 \\
\hline $\mathrm{H}$ & 2.26414200 & 3.92373200 & -0.43219900 & $\mathrm{H}$ & -1.76303500 & -4.30020300 & -1.43739500 \\
\hline $\mathrm{H}$ & 5.30568100 & 0.15404500 & 1.94559100 & $\mathrm{H}$ & -0.49023600 & -3.51870400 & -2.41526000 \\
\hline $\mathrm{H}$ & 5.34720100 & 1.65522900 & 3.88995500 & $\mathrm{H}$ & -0.01757900 & -4.56052900 & -1.03740300 \\
\hline $\mathrm{H}$ & 3.87068700 & 3.68052400 & 3.90477700 & $\mathrm{C}$ & -1.70268200 & -3.86041800 & 2.61452400 \\
\hline
\end{tabular}




\begin{tabular}{|c|c|c|c|c|c|c|c|}
\hline $\mathrm{H}$ & -2.67657000 & -3.37843300 & 2.72517000 & $\mathrm{C}$ & -1.33484200 & -3.89491000 & 0.94655800 \\
\hline $\mathrm{H}$ & -1.81779600 & -4.79999600 & 2.07156300 & $\mathrm{C}$ & -1.70868700 & -1.95449400 & 2.44679200 \\
\hline \multirow[t]{2}{*}{$\mathrm{H}$} & -1.26777100 & -4.07202400 & 3.59470600 & $\mathrm{C}$ & 0.25377200 & 4.64031200 & 1.25265000 \\
\hline & & & & $\mathrm{C}$ & 0.61492500 & 2.30863700 & 2.13228400 \\
\hline \multicolumn{4}{|c|}{ TS66a } & $\mathrm{C}$ & 2.17046900 & 3.26028800 & 0.42503000 \\
\hline \multicolumn{4}{|c|}{ imaginary frequency $=-218.17 \mathrm{~cm}^{-1}$} & $\mathrm{C}$ & -0.36763000 & 4.78216700 & -2.09581000 \\
\hline $\mathrm{Pd}$ & -0.51764800 & -0.02307600 & -0.43478700 & $\mathrm{C}$ & 1.13265900 & 2.90937000 & -2.82436000 \\
\hline $\mathrm{P}$ & -2.34841500 & -1.49734300 & -0.21672800 & $\mathrm{C}$ & -1.33788500 & 2.69654500 & -3.08993000 \\
\hline $\mathrm{P}$ & -0.36158200 & 2.34392900 & -0.47007300 & $\mathrm{H}$ & -3.36441600 & -1.65876900 & -3.86755600 \\
\hline $\mathrm{C}$ & -2.30760600 & -2.73488700 & 1.25318800 & $\mathrm{H}$ & -3.40789000 & -0.28497500 & -2.76509500 \\
\hline $\mathrm{C}$ & -3.16132800 & -2.25947000 & -1.79570200 & $\mathrm{H}$ & -1.86736800 & -1.07485300 & -3.11801400 \\
\hline $\mathrm{C}$ & -0.22407300 & 3.24957400 & -2.17071700 & $\mathrm{H}$ & -2.75830900 & -3.85611000 & -3.19824600 \\
\hline $\mathrm{C}$ & 0.69685300 & 3.21155600 & 0.87913200 & $\mathrm{H}$ & -1.37655200 & -3.51413400 & -2.14583800 \\
\hline $\mathrm{C}$ & -3.65664200 & -0.16864000 & 0.18920600 & $\mathrm{H}$ & -2.77474500 & -4.40794400 & -1.52997000 \\
\hline $\mathrm{C}$ & -2.20333800 & 2.65443400 & -0.05346300 & $\mathrm{H}$ & -5.03426200 & -2.98304300 & -2.59592100 \\
\hline $\mathrm{C}$ & -2.84780000 & 2.10217600 & 1.21040300 & $\mathrm{H}$ & -4.93109900 & -3.17981200 & -0.84869400 \\
\hline $\mathrm{C}$ & -2.93241100 & 2.97621300 & 2.31085300 & $\mathrm{H}$ & -5.24835000 & -1.58540300 & -1.54948500 \\
\hline $\mathrm{C}$ & -3.57348800 & 2.64130900 & 3.49796300 & $\mathrm{H}$ & -1.57953700 & -2.64713000 & 3.28739500 \\
\hline $\mathrm{C}$ & -4.19536200 & 1.39936500 & 3.60409900 & $\mathrm{H}$ & -0.72597400 & -1.53809300 & 2.20291800 \\
\hline $\mathrm{C}$ & -4.15987300 & 0.53430700 & 2.51674800 & $\mathrm{H}$ & -2.34546600 & -1.13894600 & 2.78842600 \\
\hline $\mathrm{C}$ & -3.48865500 & 0.83810700 & 1.31645000 & $\mathrm{H}$ & -3.57664000 & -3.89043500 & 2.57032400 \\
\hline $\mathrm{H}$ & -2.36373800 & 3.73750200 & -0.07259300 & $\mathrm{H}$ & -4.43551900 & -2.55020800 & 1.81516900 \\
\hline $\mathrm{H}$ & -2.71743500 & 2.25205200 & -0.92835700 & $\mathrm{H}$ & -4.06032700 & -4.00751300 & 0.88213100 \\
\hline $\mathrm{H}$ & -3.76513400 & 0.35585600 & -0.76458700 & $\mathrm{H}$ & -1.19131400 & -4.47395500 & 1.86667300 \\
\hline $\mathrm{H}$ & -4.60217900 & -0.69165100 & 0.35576700 & $\mathrm{H}$ & -1.70957400 & -4.58554000 & 0.19050000 \\
\hline $\mathrm{H}$ & -2.50476000 & 3.96876500 & 2.21903300 & $\mathrm{H}$ & -0.35374100 & -3.53647900 & 0.62375500 \\
\hline $\mathrm{H}$ & -3.61039100 & 3.35411100 & 4.31580500 & $\mathrm{H}$ & 2.79315600 & 3.54192300 & 1.28169100 \\
\hline $\mathrm{H}$ & -4.72787100 & 1.11833200 & 4.50729100 & $\mathrm{H}$ & 2.53222200 & 2.28978000 & 0.07022500 \\
\hline $\mathrm{H}$ & -4.69395200 & -0.40904900 & 2.58581000 & $\mathrm{H}$ & 2.34207200 & 4.00399300 & -0.35719200 \\
\hline $\mathrm{C}$ & 1.51563500 & -1.78023000 & -1.20891300 & $\mathrm{H}$ & 1.23278200 & 2.75208000 & 2.92258100 \\
\hline $\mathrm{O}$ & 1.17347200 & -2.87722800 & -1.45347900 & $\mathrm{H}$ & -0.40214100 & 2.22455600 & 2.51959000 \\
\hline $\mathrm{O}$ & 1.35381800 & -0.68603500 & -2.53300900 & $\mathrm{H}$ & 0.99644700 & 1.30213400 & 1.94048500 \\
\hline $\mathrm{C}$ & 1.01572900 & -1.32198600 & -3.79999900 & $\mathrm{H}$ & 0.87873500 & 4.99381400 & 2.08125800 \\
\hline $\mathrm{H}$ & 0.56751900 & -0.55808800 & -4.43514000 & $\mathrm{H}$ & 0.37099000 & 5.35158300 & 0.43404600 \\
\hline $\mathrm{H}$ & 1.94893200 & -1.67809600 & -4.23445800 & $\mathrm{H}$ & -0.78069500 & 4.68468400 & 1.59479200 \\
\hline $\mathrm{H}$ & 0.32632100 & -2.15071400 & -3.63533100 & $\mathrm{H}$ & -1.13539400 & 3.00934800 & -4.12063000 \\
\hline $\mathrm{H}$ & 0.54817600 & -0.17279100 & -2.12586100 & $\mathrm{H}$ & -1.38320000 & 1.60203300 & -3.07274600 \\
\hline $\mathrm{C}$ & -4.67911800 & -2.51006400 & -1.67263600 & $\mathrm{H}$ & -2.32609100 & 3.08355800 & -2.82876600 \\
\hline $\mathrm{C}$ & -2.93195500 & -1.25315900 & -2.94537800 & $\mathrm{H}$ & 1.17241900 & 3.36430600 & -3.82096600 \\
\hline $\mathrm{C}$ & -2.46779600 & -3.58353200 & -2.17674000 & $\mathrm{H}$ & 1.98222900 & 3.29299200 & -2.25827500 \\
\hline $\mathrm{C}$ & -3.67856500 & -3.31898100 & 1.64010100 & $\mathrm{H}$ & 1.27252400 & 1.83201800 & -2.94613600 \\
\hline
\end{tabular}




\begin{tabular}{|c|c|c|c|c|c|c|c|}
\hline $\mathrm{H}$ & -0.44232100 & 5.18509100 & -3.11288800 & C & 2.83151400 & 1.89060100 & 1.23928900 \\
\hline $\mathrm{H}$ & -1.26549400 & 5.09864100 & -1.55717900 & $\mathrm{H}$ & 3.27516300 & 0.99646500 & 1.68462900 \\
\hline $\mathrm{H}$ & 0.49889200 & 5.25394200 & -1.62761000 & $\mathrm{H}$ & 3.08648200 & 2.72420000 & 1.90073500 \\
\hline $\mathrm{C}$ & 5.04652800 & -0.73156900 & -0.12803900 & $\mathrm{H}$ & 4.15577000 & -0.45359100 & 0.41370100 \\
\hline $\mathrm{C}$ & 5.35477800 & 0.61635500 & -0.36066400 & $\mathrm{P}$ & 2.40395100 & -1.38905300 & -0.91787700 \\
\hline $\mathrm{C}$ & 5.75940600 & -1.43027700 & 0.85571900 & P & 0.96651300 & 1.56686100 & 1.45168700 \\
\hline $\mathrm{C}$ & 6.34996800 & 1.25498800 & 0.37857700 & $\mathrm{C}$ & 0.84681400 & 1.71014600 & 3.38186500 \\
\hline $\mathrm{H}$ & 4.82584600 & 1.16387300 & -1.13785800 & $\mathrm{C}$ & 0.01862700 & 3.02577300 & 0.61793400 \\
\hline C & 6.75700500 & -0.79246000 & 1.59456500 & $\mathrm{C}$ & 3.03964600 & -3.10039400 & -0.28074000 \\
\hline $\mathrm{H}$ & 5.54091600 & -2.47959700 & 1.03299100 & $\mathrm{C}$ & 2.11055500 & -1.44844000 & -2.81949200 \\
\hline $\mathrm{C}$ & 7.05163900 & 0.55140900 & 1.36028000 & $\mathrm{Pd}$ & 0.44364700 & -0.47159400 & 0.32877300 \\
\hline $\mathrm{H}$ & 6.58536200 & 2.29656300 & 0.18183000 & $\mathrm{C}$ & -3.07576500 & -0.54772600 & -0.05765000 \\
\hline $\mathrm{H}$ & 7.30858900 & -1.34745700 & 2.34737400 & $\mathrm{C}$ & 1.83187000 & -4.05268900 & -0.15125600 \\
\hline $\mathrm{H}$ & 7.83013100 & 1.04602300 & 1.93291400 & $\mathrm{H}$ & 2.14934400 & -4.97982200 & 0.34230700 \\
\hline C & 3.95715800 & -1.41428200 & -0.92482500 & $\mathrm{H}$ & 1.02962100 & -3.59786400 & 0.43673400 \\
\hline $\mathrm{H}$ & 4.11542400 & -2.49459200 & -0.96315800 & $\mathrm{H}$ & 1.40531300 & -4.32620400 & -1.11690200 \\
\hline $\mathrm{H}$ & 3.94780400 & -1.03416500 & -1.95097700 & $\mathrm{C}$ & 4.11869200 & -3.77085000 & -1.15108100 \\
\hline $\mathrm{C}$ & 2.53548000 & -1.14797900 & -0.30880900 & $\mathrm{H}$ & 4.97389900 & -3.11540700 & -1.33970200 \\
\hline $\mathrm{H}$ & 2.34715600 & -0.07656700 & -0.25318200 & $\mathrm{H}$ & 4.50072800 & -4.65889100 & -0.63255800 \\
\hline $\mathrm{C}$ & 2.38193100 & -1.70269000 & 1.11681300 & $\mathrm{H}$ & 3.72285800 & -4.10646200 & -2.11173500 \\
\hline $\mathrm{O}$ & 1.98033000 & -1.03970100 & 2.04494200 & $\mathrm{C}$ & 3.62555800 & -2.88320000 & 1.13346500 \\
\hline $\mathrm{O}$ & 2.74858900 & -2.98609100 & 1.18614700 & $\mathrm{H}$ & 2.96146000 & -2.28400000 & 1.76651200 \\
\hline $\mathrm{C}$ & 2.65999500 & -3.59725600 & 2.49627200 & $\mathrm{H}$ & 3.76730600 & -3.85646000 & 1.61776900 \\
\hline $\mathrm{H}$ & 3.01178800 & -4.61848400 & 2.36172600 & $\mathrm{H}$ & 4.60279300 & -2.39464900 & 1.11149200 \\
\hline $\mathrm{H}$ & 3.29019400 & -3.05582400 & 3.20406300 & $\mathrm{C}$ & 1.42491600 & -0.11099300 & -3.18174100 \\
\hline \multirow[t]{2}{*}{$\mathrm{H}$} & 1.62608500 & -3.58544700 & 2.84540600 & $\mathrm{H}$ & 2.05997700 & 0.75451700 & -2.98019400 \\
\hline & & & & $\mathrm{H}$ & 1.19081000 & -0.10918300 & -4.25304800 \\
\hline \multirow{2}{*}{\multicolumn{4}{|c|}{$\begin{array}{l}\text { TS66 } \\
\text { imaginary frequency }=-171.19 \mathrm{~cm}^{-1}\end{array}$}} & $\mathrm{H}$ & 0.48538700 & 0.01454600 & -2.63522500 \\
\hline & & & & $\mathrm{C}$ & 3.37468100 & -1.60896100 & -3.68563800 \\
\hline C & 3.74776700 & 3.49854500 & -0.43975000 & $\mathrm{H}$ & 3.89377200 & -2.55276500 & -3.51389000 \\
\hline $\mathrm{C}$ & 3.47356700 & 2.15835800 & -0.11110000 & $\mathrm{H}$ & 3.08051500 & -1.58887600 & -4.74194600 \\
\hline $\mathrm{C}$ & 3.95257400 & 1.14872700 & -0.98447300 & $\mathrm{H}$ & 4.08692700 & -0.79654700 & -3.53890700 \\
\hline C & 4.62618600 & 1.54463900 & -2.15373800 & $\mathrm{C}$ & 1.12587000 & -2.58859300 & -3.15477700 \\
\hline $\mathrm{C}$ & 4.82586400 & 2.87936500 & -2.49199900 & $\mathrm{H}$ & 0.23355700 & -2.56444100 & -2.52426300 \\
\hline C & 4.39138900 & 3.87045100 & -1.61593400 & $\mathrm{H}$ & 0.80116800 & -2.47560200 & -4.19602700 \\
\hline $\mathrm{H}$ & 3.48261100 & 4.27304800 & 0.27204800 & $\mathrm{H}$ & 1.59086000 & -3.57446400 & -3.07388300 \\
\hline $\mathrm{H}$ & 5.04376100 & 0.77882900 & -2.79906800 & $\mathrm{C}$ & 1.90501300 & 0.75549600 & 3.98454400 \\
\hline H & 5.35183900 & 3.13617700 & -3.40618000 & $\mathrm{H}$ & 1.86474900 & -0.24675500 & 3.54645100 \\
\hline $\mathrm{H}$ & 4.57744500 & 4.91913200 & -1.82569500 & $\mathrm{H}$ & 2.92327600 & 1.13378300 & 3.86635200 \\
\hline $\mathrm{C}$ & 3.94730100 & -0.33297900 & -0.65223700 & $\mathrm{H}$ & 1.72103400 & 0.65346000 & 5.06000000 \\
\hline $\mathrm{H}$ & 4.78069400 & -0.80581000 & -1.18154200 & $\mathrm{C}$ & 1.11071400 & 3.12282600 & 3.94072300 \\
\hline
\end{tabular}




\begin{tabular}{|c|c|c|c|c|c|c|c|}
\hline $\mathrm{H}$ & 1.18249000 & 3.06349700 & 5.03339100 & $\mathrm{H}$ & -5.63007200 & -0.93056100 & -2.41245300 \\
\hline $\mathrm{H}$ & 2.04646200 & 3.56139100 & 3.58342600 & $\mathrm{C}$ & -5.15515100 & 2.91301200 & -2.29397800 \\
\hline $\mathrm{H}$ & 0.29636600 & 3.81348500 & 3.71270400 & $\mathrm{H}$ & -3.14287500 & 2.49221000 & -1.65903300 \\
\hline $\mathrm{C}$ & -0.54753500 & 1.25496600 & 3.86171600 & $\mathrm{C}$ & -6.38479600 & 2.37655300 & -2.68092000 \\
\hline $\mathrm{H}$ & -0.79768100 & 0.25362300 & 3.51041500 & $\mathrm{H}$ & -7.50053900 & 0.56600600 & -3.03496700 \\
\hline $\mathrm{H}$ & -0.55653400 & 1.23664400 & 4.95807100 & $\mathrm{H}$ & -5.01421200 & 3.98977400 & -2.27187000 \\
\hline $\mathrm{H}$ & -1.33907800 & 1.93382000 & 3.54413200 & $\mathrm{H}$ & -7.20372400 & 3.03358700 & -2.95781600 \\
\hline $\mathrm{C}$ & 0.58820600 & 4.44052900 & 0.83901900 & $\mathrm{C}$ & -4.40861200 & -1.16172800 & 0.38316100 \\
\hline $\mathrm{H}$ & 0.64262400 & 4.72488800 & 1.88954400 & $\mathrm{O}$ & -4.80878600 & -2.24794600 & 0.01231300 \\
\hline $\mathrm{H}$ & 1.57346900 & 4.56161400 & 0.39387000 & $\mathrm{O}$ & -5.08238600 & -0.33782900 & 1.18978500 \\
\hline $\mathrm{H}$ & -0.07496000 & 5.16004700 & 0.34338100 & $\mathrm{C}$ & -6.40327000 & -0.77714800 & 1.57841900 \\
\hline $\mathrm{C}$ & -1.44502500 & 3.01646900 & 1.10302900 & $\mathrm{H}$ & -6.78050800 & -0.00438800 & 2.24608000 \\
\hline $\mathrm{H}$ & -1.54844800 & 3.42909700 & 2.10932300 & $\mathrm{H}$ & -7.03952900 & -0.86932400 & 0.69610100 \\
\hline $\mathrm{H}$ & -2.04642500 & 3.64509500 & 0.43639200 & $\mathrm{H}$ & -6.34860800 & -1.74074000 & 2.08883300 \\
\hline $\mathrm{H}$ & -1.88067200 & 2.01381100 & 1.09696800 & & & & \\
\hline $\mathrm{C}$ & 0.03759900 & 2.72501000 & -0.89686700 & 84 & & & \\
\hline $\mathrm{H}$ & -0.40710100 & 1.74869300 & -1.11626400 & $\mathrm{C}$ & -1.37016900 & 3.68862500 & 0.31766800 \\
\hline $\mathrm{H}$ & -0.54494200 & 3.49060000 & -1.42383600 & $\mathrm{C}$ & -0.70946800 & 2.51881100 & 0.73511700 \\
\hline $\mathrm{H}$ & 1.04969300 & 2.73704000 & -1.30739800 & $\mathrm{C}$ & 0.70868100 & 2.52040900 & 0.75500400 \\
\hline $\mathrm{C}$ & -1.93942400 & -1.51663100 & 0.25992000 & $\mathrm{C}$ & 1.37357800 & 3.69783700 & 0.36722000 \\
\hline $\mathrm{O}$ & -1.10550200 & -1.96123300 & -0.52628100 & $\mathrm{C}$ & 0.70119500 & 4.83465500 & -0.07109800 \\
\hline $\mathrm{C}$ & -2.38698500 & -3.67722200 & 1.30401700 & $\mathrm{C}$ & -0.69134300 & 4.82932900 & -0.09940700 \\
\hline $\mathrm{H}$ & -3.29694500 & -3.79888400 & 0.71759800 & $\mathrm{H}$ & -2.45655500 & 3.70361800 & 0.33812900 \\
\hline $\mathrm{H}$ & -2.52053000 & -4.09188700 & 2.30374000 & $\mathrm{H}$ & 2.45610100 & 3.72961700 & 0.43980600 \\
\hline $\mathrm{H}$ & -1.53203800 & -4.12990000 & 0.79903100 & $\mathrm{H}$ & 1.26040700 & 5.71902700 & -0.36206700 \\
\hline $\mathrm{O}$ & -2.15684900 & -2.23896500 & 1.49973600 & $\mathrm{H}$ & -1.24526100 & 5.70815800 & -0.41613600 \\
\hline $\mathrm{H}$ & -1.02498100 & -2.09769500 & 2.33337000 & $\mathrm{C}$ & 1.55393200 & 1.38354100 & 1.30137700 \\
\hline $\mathrm{C}$ & 0.55685700 & -3.07015000 & 3.41554900 & $\mathrm{H}$ & 2.49240500 & 1.80225800 & 1.68088800 \\
\hline $\mathrm{H}$ & -0.12647200 & -3.45695100 & 4.17113600 & $\mathrm{C}$ & -1.57890700 & 1.39272700 & 1.26232500 \\
\hline $\mathrm{H}$ & 1.47159700 & -2.71822100 & 3.88971100 & $\mathrm{H}$ & -1.11324700 & 0.96392500 & 2.15634400 \\
\hline $\mathrm{H}$ & 0.77784600 & -3.82379800 & 2.65957300 & $\mathrm{H}$ & -2.52576100 & 1.82942800 & 1.59326800 \\
\hline $\mathrm{O}$ & -0.09501100 & -1.91050700 & 2.81789700 & $\mathrm{H}$ & 1.04091700 & 0.94775700 & 2.16269200 \\
\hline $\mathrm{H}$ & 0.43228900 & -1.38561700 & 2.06067400 & $\mathrm{P}$ & 1.93123700 & -0.14151900 & 0.22644000 \\
\hline $\mathrm{H}$ & -2.94201600 & 0.37807500 & 0.50348400 & $\mathrm{P}$ & -1.94221200 & -0.15028900 & 0.21132000 \\
\hline $\mathrm{C}$ & -3.12057100 & -0.24428500 & -1.58206100 & $\mathrm{C}$ & -2.91270500 & -1.17158300 & 1.54091400 \\
\hline $\mathrm{H}$ & -2.16023900 & 0.19378000 & -1.86828800 & $\mathrm{C}$ & -3.11138500 & 0.44289300 & -1.20221800 \\
\hline $\mathrm{H}$ & -3.20676100 & -1.19905900 & -2.10840200 & $\mathrm{C}$ & 2.99218200 & -1.17670500 & 1.47484000 \\
\hline $\mathrm{C}$ & -4.25907600 & 0.67516000 & -1.97544500 & $\mathrm{C}$ & 3.03658400 & 0.49069200 & -1.21911300 \\
\hline $\mathrm{C}$ & -5.49593900 & 0.14712700 & -2.37270800 & $\mathrm{C}$ & -3.66890800 & -2.33848500 & 0.87506600 \\
\hline $\mathrm{C}$ & -4.10179200 & 2.06720000 & -1.94529300 & $\mathrm{H}$ & -4.57935200 & -2.00312600 & 0.37320000 \\
\hline $\mathrm{C}$ & -6.55159200 & 0.99135700 & -2.72159100 & $\mathrm{H}$ & -3.04526800 & -2.87020400 & 0.15048600 \\
\hline
\end{tabular}




\begin{tabular}{|c|c|c|c|c|c|c|c|}
\hline $\mathrm{H}$ & -3.97282700 & -3.05557300 & 1.64783500 & $\mathrm{C}$ & 2.18808100 & -1.29311700 & 2.79002400 \\
\hline C & -1.83292100 & -1.78629900 & 2.46077400 & $\mathrm{H}$ & 2.17865300 & -0.36095100 & 3.36188400 \\
\hline $\mathrm{H}$ & -2.31741600 & -2.41163200 & 3.22131400 & $\mathrm{H}$ & 1.15484200 & -1.60390500 & 2.60907500 \\
\hline $\mathrm{H}$ & -1.13928200 & -2.41241000 & 1.89080400 & $\mathrm{H}$ & 2.65523000 & -2.05472900 & 3.42610700 \\
\hline $\mathrm{H}$ & -1.24999000 & -1.02683500 & 2.99225900 & $\mathrm{Pd}$ & -0.00862200 & -1.36939100 & -0.61266200 \\
\hline C & -3.90179100 & -0.37035800 & 2.41069500 & $\mathrm{C}$ & 0.00831600 & -2.97099300 & -1.67897400 \\
\hline $\mathrm{H}$ & -4.40919000 & -1.05644700 & 3.10089800 & $\mathrm{O}$ & 0.01470700 & -3.93947500 & -2.31507900 \\
\hline $\mathrm{H}$ & -3.40456800 & 0.38618800 & 3.02389800 & & & & \\
\hline $\mathrm{H}$ & -4.67465500 & 0.12561800 & 1.81861800 & 85 & & & \\
\hline C & -4.34573800 & 1.24251800 & -0.75069100 & $\mathrm{C}$ & -0.97534600 & 3.87761700 & 0.33523600 \\
\hline $\mathrm{H}$ & -4.86493900 & 1.64253200 & -1.63110800 & $\mathrm{C}$ & -0.43089000 & 2.64504500 & 0.73592100 \\
\hline $\mathrm{H}$ & -5.06436100 & 0.61956600 & -0.21065400 & $\mathrm{C}$ & 0.97781500 & 2.49602800 & 0.71763900 \\
\hline $\mathrm{H}$ & -4.08840000 & 2.09334000 & -0.11350900 & $\mathrm{C}$ & 1.75744900 & 3.59847700 & 0.32764600 \\
\hline C & -2.23648000 & 1.31643400 & -2.13189000 & $\mathrm{C}$ & 1.19831800 & 4.80244900 & -0.09145300 \\
\hline $\mathrm{H}$ & -2.82320400 & 1.59085100 & -3.01771600 & $\mathrm{C}$ & -0.18726600 & 4.94164100 & -0.09384600 \\
\hline $\mathrm{H}$ & -1.89933700 & 2.23809600 & -1.65767500 & $\mathrm{H}$ & -2.05196700 & 4.01051000 & 0.38295000 \\
\hline $\mathrm{H}$ & -1.35338600 & 0.76593900 & -2.47093700 & $\mathrm{H}$ & 2.83812900 & 3.52080300 & 0.38771600 \\
\hline C & -3.56771000 & -0.77074200 & -2.04065400 & $\mathrm{H}$ & 1.84088300 & 5.62634000 & -0.38736400 \\
\hline $\mathrm{H}$ & -4.01253500 & -0.40651100 & -2.97501700 & $\mathrm{H}$ & -0.65190900 & 5.87499900 & -0.39759800 \\
\hline $\mathrm{H}$ & -2.72308000 & -1.41715200 & -2.29592200 & $\mathrm{C}$ & 1.69010500 & 1.26471200 & 1.24396000 \\
\hline $\mathrm{H}$ & -4.32292100 & -1.37566100 & -1.53742300 & $\mathrm{H}$ & 2.68151900 & 1.56101600 & 1.60168800 \\
\hline $\mathrm{C}$ & 3.70920200 & -0.71832400 & -1.90133800 & $\mathrm{C}$ & -1.38371300 & 1.60606800 & 1.29457400 \\
\hline $\mathrm{H}$ & 4.52710300 & -1.12940300 & -1.30318400 & $\mathrm{H}$ & -0.95949000 & 1.18232200 & 2.21119700 \\
\hline $\mathrm{H}$ & 2.99075600 & -1.51713500 & -2.10774800 & $\mathrm{H}$ & -2.30104100 & 2.11692300 & 1.59675700 \\
\hline $\mathrm{H}$ & 4.13852600 & -0.39534000 & -2.85794000 & $\mathrm{H}$ & 1.14790600 & 0.88850500 & 2.11489300 \\
\hline C & 4.11948000 & 1.52416800 & -0.85739800 & $\mathrm{P}$ & 1.90528400 & -0.29407400 & 0.18380200 \\
\hline $\mathrm{H}$ & 4.85706900 & 1.13811400 & -0.15229700 & $\mathrm{P}$ & -1.86530200 & 0.08299100 & 0.28032500 \\
\hline $\mathrm{H}$ & 4.65924100 & 1.80906300 & -1.76982100 & $\mathrm{C}$ & -2.89971400 & -0.86762300 & 1.60519800 \\
\hline $\mathrm{H}$ & 3.69673800 & 2.43939300 & -0.44145100 & $\mathrm{C}$ & -2.95095700 & 0.76302800 & -1.15398400 \\
\hline $\mathrm{C}$ & 2.06035500 & 1.12376900 & -2.23770800 & $\mathrm{C}$ & 2.89209300 & -1.40033600 & 1.43209700 \\
\hline $\mathrm{H}$ & 2.62324500 & 1.45149800 & -3.12115700 & $\mathrm{C}$ & 3.02218700 & 0.20611500 & -1.30557700 \\
\hline $\mathrm{H}$ & 1.30788500 & 0.39770100 & -2.56116000 & $\mathrm{C}$ & -3.65865500 & -2.03889800 & 0.94535500 \\
\hline $\mathrm{H}$ & 1.54238600 & 1.99489900 & -1.82978400 & $\mathrm{H}$ & -4.55935300 & -1.69577300 & 0.43201800 \\
\hline C & 3.15036500 & -2.60704700 & 0.91472200 & $\mathrm{H}$ & -3.02386400 & -2.57064000 & 0.22830200 \\
\hline $\mathrm{H}$ & 3.72645300 & -2.63808700 & -0.01155100 & $\mathrm{H}$ & -3.98042400 & -2.73489100 & 1.73092400 \\
\hline $\mathrm{H}$ & 3.67717500 & -3.22532800 & 1.65271100 & $\mathrm{C}$ & -1.86460600 & -1.47954700 & 2.57617400 \\
\hline $\mathrm{H}$ & 2.17634300 & -3.06155600 & 0.71483400 & $\mathrm{H}$ & -2.39095000 & -2.07564300 & 3.33157100 \\
\hline $\mathrm{C}$ & 4.38120400 & -0.60073300 & 1.80414500 & $\mathrm{H}$ & -1.17375600 & -2.13772600 & 2.04190200 \\
\hline $\mathrm{H}$ & 4.83757000 & -1.19155600 & 2.60902100 & $\mathrm{H}$ & -1.28153600 & -0.72350100 & 3.11368200 \\
\hline $\mathrm{H}$ & 5.06015500 & -0.64686500 & 0.94927700 & $\mathrm{C}$ & -3.88622100 & -0.00261000 & 2.41263100 \\
\hline $\mathrm{H}$ & 4.33614700 & 0.43661900 & 2.15118900 & $\mathrm{H}$ & -4.40621400 & -0.64330000 & 3.13587600 \\
\hline
\end{tabular}




\begin{tabular}{|c|c|c|c|c|c|c|c|}
\hline $\mathrm{H}$ & -3.39273000 & 0.78907200 & 2.98441800 & $\mathrm{C}$ & -1.16565800 & -3.74269900 & -2.00769400 \\
\hline $\mathrm{H}$ & -4.65107300 & 0.45726000 & 1.78199200 & $\mathrm{H}$ & -2.10124400 & -4.24716700 & -2.30945300 \\
\hline $\mathrm{C}$ & -4.00759400 & 1.79824600 & -0.72714800 & $\mathrm{H}$ & -0.58889400 & -3.56040100 & -2.93184200 \\
\hline $\mathrm{H}$ & -4.51267500 & 2.18012500 & -1.62331600 & $\mathrm{H}$ & -0.58807400 & -4.47168100 & -1.41140200 \\
\hline $\mathrm{H}$ & -4.77816000 & 1.35771500 & -0.08933100 & & & & \\
\hline $\mathrm{H}$ & -3.58497300 & 2.66020400 & -0.20477400 & 86 & & & \\
\hline $\mathrm{C}$ & -1.95805600 & 1.41114700 & -2.14824000 & $\mathrm{C}$ & -4.75028300 & 1.15019800 & -1.12522500 \\
\hline $\mathrm{H}$ & -2.52384300 & 1.81669900 & -2.99628600 & $\mathrm{C}$ & -3.63400300 & 1.17820200 & -0.27059900 \\
\hline $\mathrm{H}$ & -1.38345700 & 2.22943000 & -1.71040000 & $\mathrm{C}$ & -2.88666900 & 2.37556200 & -0.18233300 \\
\hline $\mathrm{H}$ & -1.25805700 & 0.66612300 & -2.53626600 & $\mathrm{C}$ & -3.29549900 & 3.47409700 & -0.95846100 \\
\hline $\mathrm{C}$ & -3.65038900 & -0.39501800 & -1.90065600 & $\mathrm{C}$ & -4.37838600 & 3.41377100 & -1.83082800 \\
\hline $\mathrm{H}$ & -3.98476400 & -0.02188600 & -2.87731800 & $\mathrm{C}$ & -5.11697500 & 2.23596200 & -1.91450800 \\
\hline $\mathrm{H}$ & -2.97390400 & -1.24361300 & -2.04802500 & $\mathrm{H}$ & -5.35838600 & 0.25156900 & -1.15872000 \\
\hline $\mathrm{H}$ & -4.53896000 & -0.74463900 & -1.37008300 & $\mathrm{H}$ & -2.77278800 & 4.41776700 & -0.84543500 \\
\hline $\mathrm{C}$ & 3.63063400 & -1.04911300 & -1.96307800 & $\mathrm{H}$ & -4.65687500 & 4.28731500 & -2.41277300 \\
\hline $\mathrm{H}$ & 4.40637900 & -1.50776500 & -1.34413000 & $\mathrm{H}$ & -5.98390400 & 2.16791600 & -2.56498800 \\
\hline $\mathrm{H}$ & 2.86944900 & -1.80042800 & -2.18705700 & $\mathrm{C}$ & -1.78286100 & 2.57828700 & 0.83465700 \\
\hline $\mathrm{H}$ & 4.10210900 & -0.75414500 & -2.90846400 & $\mathrm{H}$ & -1.70123400 & 3.64796800 & 1.04572200 \\
\hline $\mathrm{C}$ & 4.16436000 & 1.18481500 & -0.97097700 & $\mathrm{C}$ & -3.39559300 & -0.02843200 & 0.61336600 \\
\hline $\mathrm{H}$ & 4.87880600 & 0.78171600 & -0.25196600 & $\mathrm{H}$ & -3.11755200 & 0.29700300 & 1.62160600 \\
\hline $\mathrm{H}$ & 4.71839800 & 1.40184700 & -1.89240000 & $\mathrm{H}$ & -4.34868800 & -0.54894100 & 0.71956300 \\
\hline $\mathrm{H}$ & 3.79453000 & 2.13704000 & -0.59261300 & $\mathrm{H}$ & -2.08803700 & 2.10768400 & 1.77206300 \\
\hline $\mathrm{C}$ & 2.07716400 & 0.87358200 & -2.33003600 & $\mathrm{P}$ & -0.02906800 & 1.90994400 & 0.55133000 \\
\hline $\mathrm{H}$ & 2.65322300 & 1.14516700 & -3.22356900 & $\mathrm{P}$ & -2.08614400 & -1.34611700 & 0.21703700 \\
\hline $\mathrm{H}$ & 1.27865800 & 0.18868500 & -2.62813500 & $\mathrm{C}$ & -2.42670200 & -2.52426400 & 1.72738000 \\
\hline $\mathrm{H}$ & 1.61967500 & 1.78539000 & -1.93778900 & $\mathrm{C}$ & -2.69600200 & -2.14037800 & -1.43234200 \\
\hline $\mathrm{C}$ & 2.93241300 & -2.85755300 & 0.92491300 & $\mathrm{C}$ & 0.73467000 & 2.36492900 & 2.27388000 \\
\hline $\mathrm{H}$ & 3.44591600 & -2.95908800 & -0.03242700 & $\mathrm{C}$ & 0.68868700 & 3.05544700 & -0.82626500 \\
\hline $\mathrm{H}$ & 3.47211000 & -3.46891700 & 1.65906900 & $\mathrm{C}$ & -1.98506100 & -3.97457600 & 1.44462600 \\
\hline $\mathrm{H}$ & 1.92599800 & -3.26334500 & 0.80488600 & $\mathrm{H}$ & -2.66678200 & -4.48465700 & 0.75904700 \\
\hline $\mathrm{C}$ & 4.33347900 & -0.92308200 & 1.69821300 & $\mathrm{H}$ & -0.96614200 & -4.03503000 & 1.06196500 \\
\hline $\mathrm{H}$ & 4.76545400 & -1.53221700 & 2.50185100 & $\mathrm{H}$ & -2.00930800 & -4.52906600 & 2.39118400 \\
\hline $\mathrm{H}$ & 4.97631200 & -1.04391100 & 0.82341400 & $\mathrm{C}$ & -1.55642000 & -1.97923200 & 2.88132200 \\
\hline $\mathrm{H}$ & 4.38537000 & 0.12052100 & 2.02423900 & $\mathrm{H}$ & -1.73332300 & -2.58362100 & 3.78013900 \\
\hline $\mathrm{C}$ & 2.12793500 & -1.40847600 & 2.77648700 & $\mathrm{H}$ & -0.49303000 & -2.03665500 & 2.63537600 \\
\hline $\mathrm{H}$ & 2.22101700 & -0.46528800 & 3.32187600 & $\mathrm{H}$ & -1.80885400 & -0.94441300 & 3.13559700 \\
\hline $\mathrm{H}$ & 1.06679100 & -1.63531800 & 2.64353600 & $\mathrm{C}$ & -3.89756000 & -2.56337400 & 2.19547600 \\
\hline $\mathrm{H}$ & 2.55359500 & -2.19040600 & 3.41636000 & $\mathrm{H}$ & -3.98323400 & -3.30359100 & 3.00021800 \\
\hline $\mathrm{Pd}$ & -0.01144600 & -1.36434700 & -0.55944700 & $\mathrm{H}$ & -4.23971800 & -1.60989100 & 2.60561900 \\
\hline $\mathrm{H}$ & 1.05924300 & -2.32424800 & -1.15501300 & $\mathrm{H}$ & -4.58831900 & -2.86557500 & 1.40371500 \\
\hline $\mathrm{O}$ & -1.48033600 & -2.57254400 & -1.30644300 & $\mathrm{C}$ & -4.17393000 & -2.57397500 & -1.40712400 \\
\hline
\end{tabular}




\begin{tabular}{|c|c|c|c|c|c|c|c|}
\hline $\mathrm{H}$ & -4.46877000 & -2.89935000 & -2.41253200 & $\mathrm{H}$ & 2.16134800 & -4.52814700 & -2.21448200 \\
\hline H & -4.33622000 & -3.42113400 & -0.73443100 & $\mathrm{H}$ & 2.92071500 & -4.22116600 & -0.62039800 \\
\hline H & -4.85741800 & -1.77419000 & -1.11472700 & $\mathrm{C}$ & 2.27313300 & -0.33527500 & -0.46159300 \\
\hline $\mathrm{C}$ & -2.46219800 & -1.07990900 & -2.53454300 & $\mathrm{H}$ & 2.35035200 & -0.05049100 & -1.51486700 \\
\hline $\mathrm{H}$ & -2.69974600 & -1.52599300 & -3.50837200 & $\mathrm{C}$ & 3.41559500 & -0.55709700 & 0.21252000 \\
\hline $\mathrm{H}$ & -3.08094700 & -0.19105700 & -2.41763300 & $\mathrm{H}$ & 3.36222900 & -0.90620600 & 1.24424700 \\
\hline $\mathrm{H}$ & -1.41307700 & -0.76871500 & -2.55669200 & $\mathrm{C}$ & 4.79809300 & -0.37574700 & -0.27503100 \\
\hline $\mathrm{C}$ & -1.84182500 & -3.36420000 & -1.82614700 & $\mathrm{C}$ & 5.86653500 & -0.58960500 & 0.61642300 \\
\hline $\mathrm{H}$ & -2.16868300 & -3.69661500 & -2.81976500 & $\mathrm{C}$ & 5.11960900 & 0.00681500 & -1.59251200 \\
\hline $\mathrm{H}$ & -0.78164400 & -3.11907500 & -1.88736300 & $\mathrm{C}$ & 7.19269200 & -0.42733200 & 0.21788200 \\
\hline $\mathrm{H}$ & -1.96368400 & -4.20928100 & -1.14870500 & $\mathrm{H}$ & 5.64435300 & -0.89006300 & 1.63789600 \\
\hline $\mathrm{C}$ & 2.23106300 & 3.07086400 & -0.78877900 & $\mathrm{C}$ & 6.44308200 & 0.17029400 & -1.99229300 \\
\hline $\mathrm{H}$ & 2.61246100 & 3.67609700 & 0.03842200 & $\mathrm{H}$ & 4.32307300 & 0.17116400 & -2.31228900 \\
\hline $\mathrm{H}$ & 2.66293500 & 2.07274400 & -0.71177900 & $\mathrm{C}$ & 7.49024000 & -0.04437000 & -1.09069500 \\
\hline $\mathrm{H}$ & 2.59707900 & 3.52866000 & -1.71633000 & $\mathrm{H}$ & 7.99453300 & -0.60127500 & 0.93086900 \\
\hline C & 0.20446500 & 4.51831900 & -0.78724600 & $\mathrm{H}$ & 6.66112900 & 0.46411600 & -3.01600500 \\
\hline $\mathrm{H}$ & 0.42020800 & 5.01503200 & 0.16037500 & $\mathrm{H}$ & 8.52190500 & 0.08283400 & -1.40632000 \\
\hline $\mathrm{H}$ & 0.72610900 & 5.07812000 & -1.57351200 & & & & \\
\hline $\mathrm{H}$ & -0.86182500 & 4.61006000 & -0.98999600 & & & & \\
\hline $\mathrm{C}$ & 0.22704400 & 2.42058200 & -2.15876700 & \multicolumn{4}{|c|}{ M06-L Geometry optimization } \\
\hline H & 0.59494000 & 3.02822500 & -2.99527600 & & & & \\
\hline $\mathrm{H}$ & 0.61664300 & 1.40639600 & -2.27710400 & \multirow{2}{*}{\multicolumn{4}{|c|}{$\begin{array}{l}\text { TS4 } \\
\text { imaginary frequency }=-289.33 \mathrm{~cm}^{-1}\end{array}$}} \\
\hline $\mathrm{H}$ & -0.86332000 & 2.37520500 & -2.23510000 & & & & \\
\hline $\mathrm{C}$ & 2.16061900 & 1.79129200 & 2.38316000 & $\mathrm{C}$ & 2.79089200 & -1.72043000 & -0.61828500 \\
\hline H & 2.84804100 & 2.20291700 & 1.64494600 & $\mathrm{H}$ & 2.90586600 & -1.92808500 & -1.68893800 \\
\hline H & 2.55712900 & 2.02758200 & 3.37872800 & $\mathrm{H}$ & 3.78730600 & -1.79435000 & -0.16930600 \\
\hline $\mathrm{H}$ & 2.15731300 & 0.70801100 & 2.26230500 & $\mathrm{C}$ & 1.84010400 & -2.73983200 & 0.00010600 \\
\hline $\mathrm{C}$ & 0.77241100 & 3.87408700 & 2.58154000 & $\mathrm{H}$ & 2.37180700 & -3.69349600 & 0.08526900 \\
\hline $\mathrm{H}$ & 1.11765400 & 4.01623400 & 3.61338500 & $\mathrm{H}$ & 1.58301900 & -2.45401200 & 1.03023200 \\
\hline $\mathrm{H}$ & 1.47000100 & 4.41113400 & 1.93445800 & $\mathrm{C}$ & 0.57492700 & -2.96720700 & -0.83070000 \\
\hline $\mathrm{H}$ & -0.20880000 & 4.35240400 & 2.50453100 & $\mathrm{H}$ & 0.15439800 & -3.96096400 & -0.64407500 \\
\hline $\mathrm{C}$ & -0.10483100 & 1.65918700 & 3.36362500 & $\mathrm{H}$ & 0.82607900 & -2.94933800 & -1.89834200 \\
\hline $\mathrm{H}$ & -1.08934400 & 2.11222200 & 3.51066000 & $\mathrm{P}$ & -0.74615600 & -1.72169200 & -0.56826300 \\
\hline $\mathrm{H}$ & -0.23186100 & 0.59523900 & 3.14693200 & $\mathrm{P}$ & 2.30607600 & 0.04531200 & -0.43521200 \\
\hline $\mathrm{H}$ & 0.42551700 & 1.73796000 & 4.31999400 & $\mathrm{C}$ & 3.23976800 & 0.88617300 & -1.84090900 \\
\hline $\mathrm{Pd}$ & 0.28857400 & -0.56985200 & 0.05867600 & $\mathrm{C}$ & -1.95960300 & -2.01828600 & -1.97345100 \\
\hline $\mathrm{C}$ & 1.02594000 & -2.45810000 & 0.03029700 & $\mathrm{C}$ & 2.45929300 & 0.58407000 & -3.12223100 \\
\hline $\mathrm{O}$ & 1.22603800 & -3.09211600 & 1.05218700 & $\mathrm{H}$ & 2.95572600 & 1.05538200 & -3.97717000 \\
\hline $\mathrm{O}$ & 1.33563000 & -2.98421600 & -1.19230700 & $\mathrm{H}$ & 2.40337300 & -0.49006100 & -3.33537700 \\
\hline C & 1.97744900 & -4.27396800 & -1.16998600 & $\mathrm{H}$ & 1.43655800 & 0.97513500 & -3.06901500 \\
\hline $\mathrm{H}$ & 1.33711300 & -5.02731400 & -0.70285500 & $\mathrm{C}$ & 4.67228300 & 0.36482800 & -1.94940000 \\
\hline
\end{tabular}




$\begin{array}{lrrrrrrr}\mathrm{H} & 5.18489500 & 0.89978400 & -2.75635900 & \mathrm{C} & -2.55602000 & 2.80205000 & 0.09990100 \\ \mathrm{H} & 5.23857800 & 0.51871900 & -1.02746800 & \mathrm{C} & -2.88520400 & 4.14682800 & -0.15130300 \\ \mathrm{H} & 4.71212500 & -0.70015900 & -2.19359700 & \mathrm{C} & -3.56287300 & 1.90697200 & 0.48880000 \\ \mathrm{C} & 3.25588900 & 2.39429400 & -1.60082300 & \mathrm{C} & -4.19806900 & 4.57595500 & -0.01591100 \\ \mathrm{H} & 3.71746000 & 2.65886000 & -0.64300400 & \mathrm{H} & -2.10667300 & 4.83944300 & -0.45727700 \\ \mathrm{H} & 3.83930100 & 2.88144000 & -2.38896300 & \mathrm{C} & -4.87109900 & 2.34978900 & 0.62587500 \\ \mathrm{H} & 2.24814400 & 2.81715100 & -1.63815400 & \mathrm{H} & -3.30441700 & 0.86821600 & 0.68971700 \\ \mathrm{C} & -1.28512300 & -1.52973000 & -3.25763500 & \mathrm{C} & -5.19359800 & 3.68157200 & 0.37277500 \\ \mathrm{H} & -1.02763900 & -0.46641100 & -3.19335200 & \mathrm{H} & -4.44503100 & 5.61311200 & -0.22008800 \\ \mathrm{H} & -0.37465300 & -2.08804600 & -3.49998100 & \mathrm{H} & -5.64516100 & 1.65044900 & 0.92834200 \\ \mathrm{H} & -1.97228100 & -1.65585200 & -4.10075100 & \mathrm{H} & -6.21957000 & 4.02230900 & 0.47318000 \\ \mathrm{C} & -2.29064000 & -3.50874300 & -2.06603700 & \mathrm{C} & 0.07479700 & 2.60661100 & -0.09387900 \\ \mathrm{H} & -2.71258700 & -3.89369000 & -1.13130700 & \mathrm{H} & 0.93491100 & 3.24254300 & -0.01035900 \\ \mathrm{H} & -3.03866600 & -3.66641600 & -2.84966800 & \mathrm{H} & -1.63896200 & 0.56055400 & -0.27991400 \\ \mathrm{H} & -1.41999800 & -4.11646700 & -2.32862100 & & & \end{array}$

$\begin{array}{lll}-1.41999800 & -4.11646700 & -2.32862100\end{array}$

$\begin{array}{lll}-3.24031400 & -1.21867200 & -1.74499600\end{array}$

$\begin{array}{lll}-3.91600100 & -1.39134600 & -2.58948100\end{array}$

$\begin{array}{lll}-3.76261800 & -1.51353400 & -0.83125700\end{array}$

$\begin{array}{lll}-3.04928500 & -0.14185800 & -1.69353900\end{array}$

3.18258600

2.60026900

5.06900300

3.31655000

1.60023100

4.57790500

6.04784000

2.89181900

5.17051200

$-1.59001400$

$-1.30205000$

$-3.18574400$

$-2.00083700$

$-0.54960700$

$-2.96355300$

$-3.92977100$

$-1.79244600$

$-3.52902100$

4.39521400

$-2.51368200$

$-0.05507800$

$-1.17144600$
$0.51170900 \quad 1.11656500$

$1.39048700 \quad 2.03216000$

$0.30246300 \quad 2.40179100$

$1.73349500 \quad 3.17598800$

$1.77924700 \quad 1.85545400$

$1.18305100 \quad 3.36422500$

$-0.15801200 \quad 2.52048100$

$2.41305900 \quad 3.90881100$

$1.42027500 \quad 4.24154100$

$-2.25752400 \quad 0.97211400$

$-3.45169200 \quad 1.63637600$

$-1.69905200 \quad 2.52622600$

$-3.75308400 \quad 2.80267900$

$-4.13916000 \quad 1.26310300$

$-2.86343400 \quad 3.25898900$

$-0.97224600 \quad 2.84929600$

$-4.67158800 \quad 3.34311900$

$-3.05958700 \quad 4.16382900$

$-0.03432100 \quad 1.29927900$

$-1.38771500 \quad 1.41659700$

$0.47945200-0.35264000$

$2.42969900 \quad-0.02143500$

\section{TS6}

imaginary frequency $=-1026.39 \mathrm{~cm}^{-1}$

C

$\mathrm{H}$

$\mathrm{H}$

C

$\mathrm{H}$

$\mathrm{H}$

C

$\mathrm{H}$

$\mathrm{H}$

$\mathrm{P}$

$\mathrm{P}$

C

C

C

$\mathrm{H}$

$\mathrm{H}$

$\mathrm{H}$

C

$\mathrm{H}$

$\mathrm{H}$

$\mathrm{H}$

C

$\mathrm{H}$

$\mathrm{H}$

$$
\begin{array}{ccc}
-0.20100400 & -2.96067300 & 0.31184100 \\
-0.57948000 & -3.37401000 & -0.63247700 \\
0.43255400 & -3.72258800 & 0.77477100 \\
-1.36113800 & -2.62405400 & 1.25297000 \\
-1.63247100 & -3.52694000 & 1.80980100 \\
-1.03674500 & -1.89832700 & 2.01151200 \\
-2.61698300 & -2.11925300 & 0.53883000 \\
-3.47301000 & -2.10674200 & 1.22229500 \\
-2.88502800 & -2.81792400 & -0.26430700 \\
-2.38795800 & -0.47000900 & -0.25007500 \\
0.90886700 & -1.54991900 & -0.09650300 \\
1.99594600 & -2.22036600 & -1.47948400 \\
-3.57179700 & -0.43940300 & -1.71381000 \\
1.19904700 & -2.09506600 & -2.77883200 \\
1.79561100 & -2.46676400 & -3.61940900 \\
0.27292200 & -2.68198100 & -2.75647400 \\
0.93342400 & -1.05124500 & -2.98588800 \\
2.38419700 & -3.67566700 & -1.22353600 \\
3.10218800 & -3.99962000 & -1.98533300 \\
2.85742700 & -3.80819400 & -0.24501100 \\
1.52591700 & -4.35161100 & -1.27989300 \\
3.25118700 & -1.35405700 & -1.55672900 \\
3.91158000 & -1.51665700 & -0.69978200 \\
3.81635600 & -1.60391100 & -2.46135300 \\
& & \\
\hline
\end{array}
$$




\begin{tabular}{|c|c|c|c|c|c|c|c|}
\hline $\mathrm{H}$ & 3.01099100 & -0.28646600 & -1.59725000 & $\mathrm{H}$ & 2.34902500 & 3.49265700 & 1.25973700 \\
\hline $\mathrm{C}$ & -2.91610900 & -1.31115000 & -2.78733800 & $\mathrm{C}$ & 4.35117500 & 2.61667800 & -1.94052500 \\
\hline $\mathrm{H}$ & -1.93022300 & -0.92356100 & -3.06581900 & $\mathrm{H}$ & 2.52880800 & 1.70605300 & -2.64420200 \\
\hline $\mathrm{H}$ & -2.79411100 & -2.35159200 & -2.46445500 & $\mathrm{C}$ & 4.97697000 & 3.28860100 & -0.89119900 \\
\hline $\mathrm{H}$ & -3.54214400 & -1.32386900 & -3.68555700 & $\mathrm{H}$ & 4.73989600 & 4.12684300 & 1.07824300 \\
\hline $\mathrm{C}$ & -4.95849900 & -0.96751500 & -1.36051500 & $\mathrm{H}$ & 4.90570500 & 2.38203100 & -2.84432500 \\
\hline $\mathrm{H}$ & -5.45763400 & -0.34202700 & -0.61285200 & $\mathrm{H}$ & 6.02163800 & 3.57269300 & -0.97316600 \\
\hline $\mathrm{H}$ & -5.59272500 & -0.96294000 & -2.25375000 & $\mathrm{H}$ & -0.73429400 & 3.55585900 & -0.74963800 \\
\hline $\mathrm{H}$ & -4.93480800 & -1.99758700 & -0.99020000 & & & & \\
\hline $\mathrm{C}$ & -3.66050800 & 1.00393100 & -2.20817100 & \multirow{2}{*}{\multicolumn{4}{|c|}{$\begin{array}{l}\text { TS16 } \\
\text { imaginary frequency }=-678.49 \mathrm{~cm}^{-1}\end{array}$}} \\
\hline $\mathrm{H}$ & -4.23170400 & 1.03966000 & -3.14153000 & & & & \\
\hline $\mathrm{H}$ & -4.17300700 & 1.65305000 & -1.48956800 & $\mathrm{C}$ & -2.99540500 & 1.60954300 & -0.90714600 \\
\hline $\mathrm{H}$ & -2.66876000 & 1.42626500 & -2.40594700 & $\mathrm{H}$ & -2.97062000 & 1.74228500 & -1.99610300 \\
\hline $\mathrm{C}$ & 2.02019800 & -1.50885300 & 1.37163200 & $\mathrm{H}$ & -4.04706200 & 1.61325700 & -0.60103500 \\
\hline $\mathrm{C}$ & 2.78855400 & -0.36806800 & 1.62400300 & $\mathrm{C}$ & -2.23307900 & 2.74239500 & -0.22537500 \\
\hline $\mathrm{C}$ & 2.88099900 & -2.58460500 & 3.20086300 & $\mathrm{H}$ & -2.86956200 & 3.63303100 & -0.23398900 \\
\hline $\mathrm{C}$ & 3.64768900 & -0.37177700 & 2.71650700 & $\mathrm{H}$ & -2.06668400 & 2.51880900 & 0.83768800 \\
\hline $\mathrm{H}$ & 2.71288400 & 0.49716000 & 0.96897700 & $\mathrm{C}$ & -0.91695400 & 3.09977400 & -0.90941400 \\
\hline $\mathrm{C}$ & 3.69295200 & -1.50157500 & 3.52686900 & $\mathrm{H}$ & -0.57018300 & 4.08295900 & -0.57549800 \\
\hline $\mathrm{H}$ & 2.88729900 & -3.48414300 & 3.81356700 & $\mathrm{H}$ & -1.05739500 & 3.14388500 & -1.99628400 \\
\hline $\mathrm{H}$ & 4.26490300 & 0.49637800 & 2.93171100 & $\mathrm{P}$ & 0.46244200 & 1.94742800 & -0.57303300 \\
\hline $\mathrm{H}$ & 4.34131100 & -1.54591100 & 4.39589900 & $\mathrm{P}$ & -2.39540600 & -0.08382500 & -0.53679800 \\
\hline $\mathrm{C}$ & -3.16859100 & 0.71577400 & 0.94457500 & $\mathrm{C}$ & -3.27975300 & -1.16864800 & -1.79452400 \\
\hline $\mathrm{C}$ & -4.29150100 & 0.46704600 & 1.73152700 & $\mathrm{C}$ & 1.72429800 & 2.28889500 & -1.92584100 \\
\hline $\mathrm{C}$ & -3.03657900 & 2.89686700 & 1.80361400 & $\mathrm{C}$ & -2.42128800 & -1.15756400 & -3.06159400 \\
\hline $\mathrm{C}$ & -4.78242800 & 1.46459400 & 2.57081900 & $\mathrm{H}$ & -2.90059100 & -1.75955400 & -3.84093300 \\
\hline $\mathrm{H}$ & -4.78349500 & -0.49949500 & 1.68528100 & $\mathrm{H}$ & -2.30148400 & -0.14433600 & -3.46401500 \\
\hline $\mathrm{C}$ & -4.14888300 & 2.70267000 & 2.60950700 & $\mathrm{H}$ & -1.41859700 & -1.56546700 & -2.88918800 \\
\hline $\mathrm{H}$ & -2.48138200 & 3.83059300 & 1.78409100 & $\mathrm{C}$ & -4.68098000 & -0.63550700 & -2.09849100 \\
\hline $\mathrm{H}$ & -5.65502900 & 1.27503400 & 3.18822200 & $\mathrm{H}$ & -5.19310700 & -1.34470400 & -2.75791900 \\
\hline $\mathrm{H}$ & -4.50134200 & 3.50056000 & 3.25287500 & $\mathrm{H}$ & -5.28340800 & -0.52293700 & -1.19226400 \\
\hline $\mathrm{N}$ & 2.05983700 & -2.60337700 & 2.14514000 & $\mathrm{H}$ & -4.65807100 & 0.32711500 & -2.61670400 \\
\hline $\mathrm{N}$ & -2.57533900 & 1.92482200 & 1.01051900 & $\mathrm{C}$ & -3.39710500 & -2.58415000 & -1.23058200 \\
\hline $\mathrm{Pd}$ & -0.24295900 & 0.44366500 & -0.47386400 & $\mathrm{H}$ & -4.07607500 & -2.62164600 & -0.37290300 \\
\hline $\mathrm{C}$ & -0.32903400 & 2.59037600 & -0.44609700 & $\mathrm{H}$ & -3.80277800 & -3.24858400 & -2.00058900 \\
\hline $\mathrm{C}$ & 0.90267100 & 2.16153900 & -0.55605300 & $\mathrm{H}$ & -2.43414000 & -2.99582800 & -0.91798300 \\
\hline $\mathrm{H}$ & -1.50117400 & 2.08930600 & 0.31149000 & $\mathrm{C}$ & 1.23524600 & 1.56612600 & -3.18312700 \\
\hline $\mathrm{C}$ & 2.28079700 & 2.55481500 & -0.68023600 & $\mathrm{H}$ & 1.18700600 & 0.47971700 & -3.03506200 \\
\hline $\mathrm{C}$ & 2.91753700 & 3.24565500 & 0.36700000 & $\mathrm{H}$ & 0.24165900 & 1.90444800 & -3.49974900 \\
\hline $\mathrm{C}$ & 3.02056500 & 2.23617700 & -1.83217000 & $\mathrm{H}$ & 1.92194500 & 1.76216200 & -4.01401400 \\
\hline $\mathrm{C}$ & 4.25742400 & 3.59739300 & 0.26189200 & $\mathrm{C}$ & 1.83012900 & 3.79628900 & -2.16601500 \\
\hline
\end{tabular}




\begin{tabular}{|c|c|c|c|c|c|c|c|}
\hline $\mathrm{H}$ & 2.09062000 & 4.33984300 & -1.25182900 & $\mathrm{H}$ & -0.33467400 & -2.82304100 & -1.38444800 \\
\hline $\mathrm{H}$ & 2.61642200 & 3.99222800 & -2.90249400 & $\mathrm{H}$ & -0.42326900 & -2.92618500 & 0.47061000 \\
\hline $\mathrm{H}$ & 0.90370100 & 4.22128900 & -2.56131600 & C & 1.41965900 & -2.15763000 & -0.35428900 \\
\hline C & 3.08900500 & 1.74443100 & -1.51216300 & $\mathrm{C}$ & 2.11916000 & -2.15072000 & -1.70412900 \\
\hline $\mathrm{H}$ & 3.79291900 & 1.87331400 & -2.34189900 & $\mathrm{O}$ & 1.53584200 & -2.28936500 & -2.75733900 \\
\hline $\mathrm{H}$ & 3.50076000 & 2.27622700 & -0.64908400 & $\mathrm{O}$ & 3.43167100 & -1.91473400 & -1.58747400 \\
\hline $\mathrm{H}$ & 3.06112700 & 0.67497500 & -1.27268400 & $\mathrm{C}$ & 4.13393300 & -1.82042900 & -2.84054300 \\
\hline C & -3.20010600 & -0.42875300 & 1.07784400 & $\mathrm{H}$ & 3.70510300 & -1.02346000 & -3.45297400 \\
\hline C & -2.53498100 & -1.14610500 & 2.07357100 & $\mathrm{H}$ & 5.16622100 & -1.59792600 & -2.58048300 \\
\hline C & -5.08609000 & -0.23935600 & 2.36273700 & $\mathrm{H}$ & 4.06401400 & -2.76218200 & -3.38696800 \\
\hline C & -3.20640800 & -1.41491800 & 3.26304100 & & & & \\
\hline $\mathrm{H}$ & -1.51059600 & -1.47799700 & 1.91343500 & \multirow{2}{*}{\multicolumn{4}{|c|}{$\begin{array}{l}\text { TS19 } \\
\text { imaginary frequency }=-1073.13 \mathrm{~cm}^{-1}\end{array}$}} \\
\hline $\mathrm{C}$ & -4.50871700 & -0.95448900 & 3.41111300 & & & & \\
\hline $\mathrm{H}$ & -6.10207800 & 0.14154100 & 2.44590200 & C & -0.06341200 & 3.09297200 & -0.38550300 \\
\hline $\mathrm{H}$ & -2.71923600 & -1.96994300 & 4.05916300 & $\mathrm{H}$ & -0.58114100 & 3.41596300 & 0.52766900 \\
\hline $\mathrm{H}$ & -5.07036500 & -1.13925100 & 4.32099600 & $\mathrm{H}$ & 0.62821600 & 3.88772100 & -0.67607900 \\
\hline $\mathrm{C}$ & 1.18804300 & 2.59840900 & 0.98464700 & $\mathrm{C}$ & -1.06023700 & 2.85000700 & -1.52854900 \\
\hline $\mathrm{C}$ & 2.07520600 & 1.81330100 & 1.72581300 & $\mathrm{H}$ & -1.19516900 & 3.78652200 & -2.07911400 \\
\hline $\mathrm{C}$ & 1.34394700 & 4.32387800 & 2.48044000 & $\mathrm{H}$ & -0.63276400 & 2.14772700 & -2.25436200 \\
\hline $\mathrm{C}$ & 2.61344100 & 2.33911300 & 2.89482000 & C & -2.44089400 & 2.36523600 & -1.08099700 \\
\hline $\mathrm{H}$ & 2.33215200 & 0.80999000 & 1.39271200 & $\mathrm{H}$ & -3.10732000 & 2.27011000 & -1.94547700 \\
\hline C & 2.23652000 & 3.61935700 & 3.28517100 & $\mathrm{H}$ & -2.89985400 & 3.10862100 & -0.41764300 \\
\hline $\mathrm{H}$ & 1.02698600 & 5.32851300 & 2.75325100 & $\mathrm{P}$ & -2.36258500 & 0.76749400 & -0.16915900 \\
\hline $\mathrm{H}$ & 3.30694200 & 1.75290000 & 3.49142200 & $P$ & 0.96056100 & 1.62032900 & 0.03038200 \\
\hline $\mathrm{H}$ & 2.62173900 & 4.06664900 & 4.19565700 & C & 2.04271000 & 2.16619700 & 1.46759200 \\
\hline $\mathrm{N}$ & -4.45503600 & 0.02571200 & 1.21592700 & $\mathrm{C}$ & -3.42557200 & 0.98397100 & 1.36850500 \\
\hline $\mathrm{N}$ & 0.82483000 & 3.83625400 & 1.34899700 & C & 1.16646400 & 2.14124900 & 2.72022500 \\
\hline Pd & -0.04176700 & -0.32613400 & -0.44662700 & $\mathrm{H}$ & 1.76557600 & 2.40678200 & 3.59881400 \\
\hline $\mathrm{H}$ & 1.57482900 & -0.47118200 & -0.35277300 & $\mathrm{H}$ & 0.34670200 & 2.86630200 & 2.66091500 \\
\hline $\mathrm{C}$ & 2.16435000 & -2.34015200 & 0.92870100 & $\mathrm{H}$ & 0.73078200 & 1.14867400 & 2.89062800 \\
\hline $\mathrm{C}$ & 1.71823000 & -1.69680000 & 2.08904900 & $\mathrm{C}$ & 2.61091700 & 3.56469900 & 1.23619400 \\
\hline $\mathrm{C}$ & 3.24499900 & -3.22206600 & 1.01876300 & $\mathrm{H}$ & 3.31687400 & 3.80946100 & 2.03764100 \\
\hline $\mathrm{C}$ & 2.34522400 & -1.91264400 & 3.31069100 & $\mathrm{H}$ & 3.15185700 & 3.63645000 & 0.28676200 \\
\hline $\mathrm{H}$ & 0.88146800 & -0.99874000 & 2.01613200 & $\mathrm{H}$ & 1.83331800 & 4.33432900 & 1.24382500 \\
\hline $\mathrm{C}$ & 3.86655200 & -3.44345400 & 2.24429100 & C & 3.18010400 & 1.15392000 & 1.60845600 \\
\hline $\mathrm{H}$ & 3.60133000 & -3.73748300 & 0.13296800 & $\mathrm{H}$ & 3.90757200 & 1.24337300 & 0.79626200 \\
\hline $\mathrm{C}$ & 3.42475700 & -2.78924000 & 3.39050600 & $\mathrm{H}$ & 3.71497900 & 1.33406100 & 2.54824800 \\
\hline $\mathrm{H}$ & 1.99408200 & -1.39199200 & 4.19748500 & $\mathrm{H}$ & 2.81680400 & 0.11992400 & 1.62704200 \\
\hline $\mathrm{H}$ & 4.70486100 & -4.13117300 & 2.29955100 & $\mathrm{C}$ & -2.69683000 & 1.98933900 & 2.26190900 \\
\hline $\mathrm{H}$ & 3.92069300 & -2.95941300 & 4.34112500 & $\mathrm{H}$ & -1.69411800 & 1.63132100 & 2.52196100 \\
\hline C & 0.05158200 & -2.51637700 & -0.41639300 & $\mathrm{H}$ & -2.60816600 & 2.98005300 & 1.80076100 \\
\hline
\end{tabular}




\begin{tabular}{|c|c|c|c|c|c|c|c|}
\hline $\mathrm{H}$ & -3.25553400 & 2.11897200 & 3.19474000 & $\mathrm{H}$ & 3.41172900 & -2.61284300 & 1.49475600 \\
\hline C & -4.82510000 & 1.48164600 & 1.01936500 & C & 3.77198300 & -3.18476800 & -1.83026000 \\
\hline $\mathrm{H}$ & -5.38019800 & 0.75531700 & 0.41650700 & $\mathrm{H}$ & 2.18014900 & -3.06223100 & -3.28009700 \\
\hline $\mathrm{H}$ & -5.39773300 & 1.63887400 & 1.93986100 & $\mathrm{H}$ & 5.11551500 & -3.21066200 & -0.15176200 \\
\hline $\mathrm{H}$ & -4.81330300 & 2.43691700 & 0.48311600 & $\mathrm{H}$ & 4.53208500 & -3.46189800 & -2.55501000 \\
\hline $\mathrm{C}$ & -3.49090300 & -0.36557700 & 2.08380800 & $\mathrm{C}$ & 0.80994100 & -1.70258800 & 2.41635800 \\
\hline $\mathrm{H}$ & -4.06447500 & -0.25662100 & 3.01031500 & $\mathrm{O}$ & -0.15114100 & -1.35095700 & 3.09126000 \\
\hline $\mathrm{H}$ & -3.99563700 & -1.12998100 & 1.48197000 & $\mathrm{O}$ & 2.05526900 & -1.75509800 & 2.92485700 \\
\hline $\mathrm{H}$ & -2.49126500 & -0.72896300 & 2.35118000 & $\mathrm{C}$ & 2.16540400 & -1.33590400 & 4.29293300 \\
\hline $\mathrm{C}$ & 2.09860600 & 1.57143000 & -1.41695100 & $\mathrm{H}$ & 3.21786600 & -1.44694300 & 4.54738100 \\
\hline $\mathrm{C}$ & 2.96023500 & 0.48686400 & -1.60816300 & $\mathrm{H}$ & 1.85163400 & -0.29456600 & 4.40108200 \\
\hline C & 2.86814100 & 2.61507100 & -3.30678100 & $\mathrm{H}$ & 1.54253800 & -1.95935100 & 4.93595700 \\
\hline C & 3.81592500 & 0.50501400 & -2.70447300 & & & & \\
\hline $\mathrm{H}$ & 2.96149400 & -0.34957300 & -0.91352000 & \multirow{2}{*}{\multicolumn{4}{|c|}{$\begin{array}{l}\text { TS34 } \\
\text { imaginary frequency }=-629.62 \mathrm{~cm}^{-1}\end{array}$}} \\
\hline $\mathrm{C}$ & 3.77105900 & 1.58918400 & -3.57344400 & & & & \\
\hline $\mathrm{H}$ & 2.79995600 & 3.47775000 & -3.96712700 & $\mathrm{C}$ & -2.72984100 & -2.08999000 & -0.45438700 \\
\hline $\mathrm{H}$ & 4.49914900 & -0.32263600 & -2.87456100 & C & -2.99379600 & -3.46279300 & -0.39060800 \\
\hline $\mathrm{H}$ & 4.41677700 & 1.64046100 & -4.44409300 & $\mathrm{C}$ & -3.55924100 & -4.10077700 & -1.48927900 \\
\hline $\mathrm{C}$ & -3.30331700 & -0.47017900 & -1.15931000 & C & -3.86260300 & -3.35294400 & -2.62039900 \\
\hline $\mathrm{C}$ & -4.41411600 & -0.23731700 & -1.96602000 & C & -3.57937500 & -1.99178300 & -2.59855100 \\
\hline $\mathrm{C}$ & -3.40083900 & -2.75983500 & -1.62684300 & $\mathrm{H}$ & -2.77917100 & -4.02711700 & 0.51148300 \\
\hline $\mathrm{C}$ & -5.01458400 & -1.30828800 & -2.62447300 & $\mathrm{H}$ & -3.77039800 & -5.16584600 & -1.45578400 \\
\hline $\mathrm{H}$ & -4.80941000 & 0.76897000 & -2.07092200 & $\mathrm{H}$ & -4.30846300 & -3.80800200 & -3.49850500 \\
\hline $\mathrm{C}$ & -4.50457100 & -2.59155000 & -2.45290000 & $\mathrm{H}$ & -3.80567900 & -1.36883000 & -3.46264900 \\
\hline $\mathrm{H}$ & -2.95121400 & -3.73310800 & -1.44951500 & $\mathrm{~N}$ & -3.01766000 & -1.36624500 & -1.55869700 \\
\hline $\mathrm{H}$ & -5.87750600 & -1.14160000 & -3.26182100 & $P$ & -1.97537800 & -1.10497400 & 0.90876700 \\
\hline $\mathrm{H}$ & -4.94714600 & -3.44661500 & -2.95102900 & C & -3.53221400 & -0.32858600 & 1.68342200 \\
\hline $\mathrm{N}$ & 2.04452700 & 2.61932700 & -2.25401600 & C & -1.50429700 & -2.43212600 & 2.11913000 \\
\hline $\mathrm{N}$ & -2.82268700 & -1.72086500 & -1.01736700 & $\mathrm{H}$ & -2.39205600 & -2.85733000 & 2.60644500 \\
\hline Pd & -0.37162300 & -0.31050900 & 0.29839000 & $\mathrm{H}$ & -1.06578400 & -3.23929900 & 1.51864600 \\
\hline $\mathrm{C}$ & -0.67840400 & -2.48421900 & 0.64703600 & $\mathrm{C}$ & -0.46796400 & -2.01845100 & 3.16872300 \\
\hline C & 0.69011800 & -2.08625200 & 0.98270800 & $\mathrm{H}$ & -0.26672900 & -2.89563400 & 3.79275800 \\
\hline $\mathrm{H}$ & -1.70786000 & -1.79983700 & -0.21817400 & $\mathrm{H}$ & -0.88963800 & -1.27042900 & 3.85066500 \\
\hline $\mathrm{H}$ & -1.31293500 & -2.67338500 & 1.51259100 & C & 0.84441700 & -1.48852700 & 2.57672000 \\
\hline $\mathrm{H}$ & -0.72059300 & -3.28173100 & -0.09763400 & $\mathrm{H}$ & 1.70503600 & -1.96318300 & 3.06654500 \\
\hline C & 1.79348600 & -2.47599700 & 0.06973700 & $\mathrm{H}$ & 0.90543200 & -1.79138500 & 1.51904300 \\
\hline C & 1.49032300 & -2.61761100 & -1.30146100 & C & 0.99443600 & 0.02490700 & 2.67787900 \\
\hline $\mathrm{C}$ & 3.12661400 & -2.69648300 & 0.45525500 & $\mathrm{H}$ & 0.94936500 & 0.32584200 & 3.73199400 \\
\hline C & 2.45473000 & -2.96925700 & -2.23287700 & $\mathrm{H}$ & 0.13968600 & 0.50872400 & 2.18197200 \\
\hline $\mathrm{H}$ & 0.47857400 & -2.40842700 & -1.64664300 & C & 2.28517300 & 0.56478100 & 2.06757200 \\
\hline $\mathrm{C}$ & 4.09573800 & -3.04070900 & -0.48590100 & $\mathrm{H}$ & 2.33259300 & 1.65429500 & 2.18373100 \\
\hline
\end{tabular}




\begin{tabular}{|c|c|c|c|c|c|c|c|}
\hline $\mathrm{H}$ & 3.16378800 & 0.16230000 & 2.58597600 & C & -1.06297800 & 2.32577400 & -1.00704300 \\
\hline $\mathrm{P}$ & 2.41655000 & 0.23556900 & 0.26218000 & $\mathrm{C}$ & -1.46903300 & 1.30595600 & -1.62310200 \\
\hline $\mathrm{C}$ & 3.70840300 & 1.42802500 & -0.40456300 & $\mathrm{C}$ & -1.06755400 & 3.70605400 & -0.60459800 \\
\hline $\mathrm{C}$ & 3.19221300 & -1.42188000 & 0.09831000 & $\mathrm{C}$ & -0.37044800 & 4.17700100 & 0.51701800 \\
\hline $\mathrm{C}$ & 4.12349100 & -1.91770800 & 1.01459800 & $\mathrm{C}$ & -1.85528500 & 4.60014800 & -1.35137600 \\
\hline $\mathrm{C}$ & 3.35761700 & -3.30811400 & -1.19891700 & $\mathrm{C}$ & -0.45893300 & 5.51133000 & 0.88776100 \\
\hline $\mathrm{C}$ & 4.68063200 & -3.17230500 & 0.78632700 & $\mathrm{H}$ & 0.23605700 & 3.48392900 & 1.09824100 \\
\hline $\mathrm{H}$ & 4.41252900 & -1.34092800 & 1.88856600 & $\mathrm{C}$ & -1.93738400 & 5.93279100 & -0.97281500 \\
\hline $\mathrm{C}$ & 4.29367600 & -3.88407400 & -0.34276300 & $\mathrm{H}$ & -2.39250900 & 4.23753000 & -2.22227500 \\
\hline $\mathrm{H}$ & 3.01935600 & -3.83188100 & -2.09139200 & $\mathrm{C}$ & -1.24056000 & 6.39225100 & 0.14321400 \\
\hline $\mathrm{H}$ & 5.40534600 & -3.58470800 & 1.48172300 & $\mathrm{H}$ & 0.08398400 & 5.86569700 & 1.75840700 \\
\hline $\mathrm{H}$ & 4.70184900 & -4.86642000 & -0.55605500 & $\mathrm{H}$ & -2.54513200 & 6.61709000 & -1.55632100 \\
\hline $\mathrm{N}$ & 2.81249000 & -2.10746200 & -0.98900100 & $\mathrm{H}$ & -1.30334300 & 7.43711500 & 0.43065000 \\
\hline $\mathrm{Pd}$ & 0.33731600 & 0.43892000 & -0.81258200 & $\mathrm{H}$ & -2.17886400 & 0.59275900 & -2.02169300 \\
\hline $\mathrm{H}$ & 0.32480500 & 1.87383000 & -0.09973200 & & & & \\
\hline $\mathrm{C}$ & 5.00347900 & 1.32453500 & 0.40032400 & \multirow{2}{*}{\multicolumn{4}{|c|}{$\begin{array}{l}\text { TS35 } \\
\text { imaginary frequency }=-964.11 \mathrm{~cm}^{-1}\end{array}$}} \\
\hline $\mathrm{H}$ & 5.45193700 & 0.32886100 & 0.33258100 & & & & \\
\hline $\mathrm{H}$ & 5.73526700 & 2.03581500 & 0.00348600 & $\mathrm{C}$ & 1.10806700 & 2.41524100 & 0.04827100 \\
\hline $\mathrm{H}$ & 4.85594700 & 1.56828000 & 1.45794600 & $\mathrm{C}$ & 0.86205800 & 3.73003800 & 0.45611300 \\
\hline $\mathrm{C}$ & 3.15104300 & 2.84888000 & -0.31991600 & $\mathrm{C}$ & -0.05960300 & 4.51512200 & -0.22864600 \\
\hline $\mathrm{H}$ & 2.22966400 & 2.96170000 & -0.90109800 & $\mathrm{C}$ & -0.74381700 & 3.98021000 & -1.31698800 \\
\hline $\mathrm{H}$ & 2.94802700 & 3.16208300 & 0.71020600 & $\mathrm{C}$ & -0.46591600 & 2.67314400 & -1.68225200 \\
\hline $\mathrm{H}$ & 3.88687500 & 3.54973500 & -0.72738900 & $\mathrm{H}$ & 1.38669400 & 4.13202100 & 1.31707900 \\
\hline $\mathrm{C}$ & 3.95603400 & 1.05909500 & -1.86778800 & $\mathrm{H}$ & -0.24728700 & 5.53662800 & 0.08898600 \\
\hline $\mathrm{H}$ & 3.03280200 & 1.10428600 & -2.45606600 & $\mathrm{H}$ & -1.47808300 & 4.55696700 & -1.86860400 \\
\hline $\mathrm{H}$ & 4.66823800 & 1.76507200 & -2.30714600 & $\mathrm{H}$ & -0.97037500 & 2.17486200 & -2.50753400 \\
\hline $\mathrm{H}$ & 4.37537000 & 0.05440500 & -1.97515400 & $\mathrm{~N}$ & 0.43312100 & 1.93655900 & -1.02030100 \\
\hline $\mathrm{C}$ & -3.17046400 & 0.11648200 & 3.10042900 & $\mathrm{P}$ & 2.22741800 & 1.18169300 & 0.87946200 \\
\hline $\mathrm{H}$ & -3.02548800 & -0.73352400 & 3.77467100 & $\mathrm{C}$ & 3.93488000 & 1.88297100 & 0.46071200 \\
\hline $\mathrm{H}$ & -2.26307600 & 0.73236400 & 3.12171100 & $\mathrm{C}$ & 1.97811700 & 1.64769000 & 2.66170200 \\
\hline $\mathrm{H}$ & -3.98550500 & 0.72071200 & 3.51502600 & $\mathrm{H}$ & 2.90248800 & 1.36704700 & 3.18075300 \\
\hline $\mathrm{C}$ & -4.70711200 & -1.30099000 & 1.73300500 & $\mathrm{H}$ & 1.87611200 & 2.73198900 & 2.79494400 \\
\hline $\mathrm{H}$ & -5.54113100 & -0.84474900 & 2.27912500 & $\mathrm{C}$ & 0.78826500 & 0.91188000 & 3.28392600 \\
\hline $\mathrm{H}$ & -5.06925200 & -1.55577600 & 0.73239000 & $\mathrm{H}$ & 0.70508200 & 1.18589300 & 4.34296200 \\
\hline $\mathrm{H}$ & -4.45763600 & -2.23485600 & 2.24939200 & $\mathrm{H}$ & 0.98484700 & -0.16878600 & 3.26546600 \\
\hline $\mathrm{C}$ & -3.90603200 & 0.89489700 & 0.84838500 & $\mathrm{C}$ & -0.52072000 & 1.18486800 & 2.56139800 \\
\hline $\mathrm{H}$ & -4.82170300 & 1.34775800 & 1.24629300 & $\mathrm{H}$ & -0.75828200 & 2.25956600 & 2.59466400 \\
\hline $\mathrm{H}$ & -3.11693500 & 1.65361700 & 0.87578000 & $\mathrm{H}$ & -0.37913000 & 0.94894700 & 1.49534200 \\
\hline $\mathrm{H}$ & -4.08943900 & 0.63434700 & -0.19926900 & $\mathrm{C}$ & -1.72461100 & 0.38252300 & 3.03129300 \\
\hline $\mathrm{C}$ & 0.24836300 & -1.27250900 & -1.85457800 & $\mathrm{H}$ & -2.07388200 & 0.74335200 & 4.00582300 \\
\hline $\mathrm{O}$ & 0.07785800 & -2.23095400 & -2.45741000 & $\mathrm{H}$ & -1.43870000 & -0.66772500 & 3.18363400 \\
\hline
\end{tabular}




\begin{tabular}{|c|c|c|c|c|c|c|c|}
\hline $\mathrm{C}$ & -2.86035200 & 0.47778900 & 2.00992900 & $\mathrm{O}$ & 0.42139100 & -2.96841400 & 2.66313000 \\
\hline $\mathrm{H}$ & -3.82107000 & 0.17585600 & 2.44297500 & $\mathrm{H}$ & 0.46747500 & 0.63695600 & -1.35814100 \\
\hline $\mathrm{H}$ & -2.97006700 & 1.52775800 & 1.71342200 & $\mathrm{C}$ & 0.35707400 & -0.61400800 & -1.81966500 \\
\hline $\mathrm{P}$ & -2.54096500 & -0.52307700 & 0.47746100 & $\mathrm{H}$ & -0.18636200 & -0.59011500 & -2.76083300 \\
\hline $\mathrm{C}$ & -3.76250800 & -1.95854800 & 0.59580800 & $\mathrm{C}$ & 1.24948200 & -1.45859800 & -1.34000800 \\
\hline $\mathrm{C}$ & -3.24358200 & 0.49318600 & -0.88674300 & $\mathrm{C}$ & 2.53404900 & -2.05368400 & -1.47215100 \\
\hline $\mathrm{C}$ & -4.11839200 & 1.56782900 & -0.68872700 & $\mathrm{C}$ & 3.34534700 & -1.70423800 & -2.57492400 \\
\hline $\mathrm{C}$ & -3.36097600 & 0.75030100 & -3.16808900 & $\mathrm{C}$ & 3.02806400 & -2.96721000 & -0.52148100 \\
\hline $\mathrm{C}$ & -4.59996900 & 2.25946400 & -1.79565300 & $\mathrm{C}$ & 4.60678700 & -2.25830500 & -2.71837000 \\
\hline $\mathrm{H}$ & -4.44097100 & 1.84798700 & 0.30964800 & $\mathrm{H}$ & 2.96171100 & -0.99569400 & -3.30403900 \\
\hline $\mathrm{C}$ & -4.21216700 & 1.84828100 & -3.06578400 & $\mathrm{C}$ & 4.28969400 & -3.52163900 & -0.67354900 \\
\hline $\mathrm{H}$ & -3.05019200 & 0.38249200 & -4.14542300 & $\mathrm{H}$ & 2.40234400 & -3.22286700 & 0.32968400 \\
\hline $\mathrm{H}$ & -5.28086800 & 3.09614300 & -1.66629800 & $\mathrm{C}$ & 5.07876900 & -3.16725000 & -1.76916000 \\
\hline $\mathrm{H}$ & -4.56933600 & 2.35350100 & -3.95754700 & $\mathrm{H}$ & 5.22575900 & -1.98808900 & -3.56809200 \\
\hline $\mathrm{N}$ & -2.86907200 & 0.08977200 & -2.11501100 & $\mathrm{H}$ & 4.66445500 & -4.22840900 & 0.05977200 \\
\hline $\mathrm{Pd}$ & -0.24189400 & -1.36228600 & 0.08732200 & $\mathrm{H}$ & 6.06808000 & -3.60047700 & -1.88338200 \\
\hline $\mathrm{C}$ & -5.21098300 & -1.48080900 & 0.53617600 & & & & \\
\hline $\mathrm{H}$ & -5.45234700 & -1.02233600 & -0.42770000 & \multirow{2}{*}{\multicolumn{4}{|c|}{$\begin{array}{l}\text { TS51 } \\
\text { imaginary frequency }=-635.48 \mathrm{~cm}^{-1}\end{array}$}} \\
\hline $\mathrm{H}$ & -5.88336800 & -2.33510600 & 0.67126000 & & & & \\
\hline $\mathrm{H}$ & -5.45033800 & -0.75876400 & 1.32548000 & $\mathrm{C}$ & 2.40906400 & 3.67645600 & 0.16629800 \\
\hline $\mathrm{C}$ & -3.49959100 & -2.67807400 & 1.91894400 & $\mathrm{C}$ & 2.07148200 & 2.39735300 & 0.62973300 \\
\hline $\mathrm{H}$ & -2.46194400 & -3.01875400 & 1.99956000 & $\mathrm{C}$ & 3.06966600 & 1.40774700 & 0.64870100 \\
\hline $\mathrm{H}$ & -3.72381500 & -2.05331900 & 2.78990200 & $\mathrm{C}$ & 4.36149100 & 1.74223900 & 0.22132100 \\
\hline $\mathrm{H}$ & -4.14005400 & -3.56403000 & 1.98410000 & $\mathrm{C}$ & 4.67233400 & 3.00616000 & -0.25974700 \\
\hline $\mathrm{C}$ & -3.46725000 & -2.89818600 & -0.57320900 & $\mathrm{C}$ & 3.68201500 & 3.98329800 & -0.29302200 \\
\hline $\mathrm{H}$ & -2.44159700 & -3.28180200 & -0.52399900 & $\mathrm{H}$ & 1.64787500 & 4.45438600 & 0.18555900 \\
\hline $\mathrm{H}$ & -4.15114000 & -3.75387200 & -0.54355400 & $\mathrm{H}$ & 5.14328600 & 0.98745500 & 0.27993100 \\
\hline $\mathrm{H}$ & -3.58914600 & -2.39950700 & -1.53948100 & $\mathrm{H}$ & 5.68318600 & 3.23177100 & -0.58441700 \\
\hline $\mathrm{C}$ & 4.93788900 & 0.87155100 & 1.02218500 & $\mathrm{H}$ & 3.90553100 & 4.98505200 & -0.64637100 \\
\hline $\mathrm{H}$ & 4.88770300 & 0.79800000 & 2.11383900 & $\mathrm{C}$ & 2.83895200 & 0.03331900 & 1.20166000 \\
\hline $\mathrm{H}$ & 4.77595500 & -0.12947100 & 0.60768300 & $\mathrm{H}$ & 3.80358300 & -0.39444400 & 1.49525200 \\
\hline $\mathrm{H}$ & 5.95799100 & 1.17999500 & 0.76756700 & $\mathrm{C}$ & 0.70644300 & 2.20281600 & 1.22475800 \\
\hline $\mathrm{C}$ & 4.21461600 & 3.26884500 & 1.03119400 & $\mathrm{H}$ & 0.78095000 & 1.64377600 & 2.16832300 \\
\hline $\mathrm{H}$ & 5.23673000 & 3.57454100 & 0.77817100 & $\mathrm{H}$ & 0.32704800 & 3.19008500 & 1.50463800 \\
\hline $\mathrm{H}$ & 3.54562900 & 4.02927100 & 0.61538800 & $\mathrm{H}$ & 2.24111500 & 0.07571300 & 2.12227600 \\
\hline $\mathrm{H}$ & 4.13357900 & 3.29522300 & 2.12265500 & $\mathrm{P}$ & 1.98447500 & -1.26622000 & 0.18489800 \\
\hline $\mathrm{C}$ & 4.04361500 & 1.90975600 & -1.06437600 & $\mathrm{P}$ & -0.70233700 & 1.35878900 & 0.33783800 \\
\hline $\mathrm{H}$ & 5.06570200 & 2.16928700 & -1.36176900 & $\mathrm{C}$ & -2.02724600 & 1.54952900 & 1.70364700 \\
\hline $\mathrm{H}$ & 3.80980100 & 0.93254100 & -1.50404600 & $\mathrm{C}$ & -1.08210000 & 2.43902200 & -1.16456900 \\
\hline $\mathrm{H}$ & 3.37501100 & 2.65369800 & -1.51136300 & $\mathrm{C}$ & 1.95283500 & -2.68726600 & 1.45335500 \\
\hline $\mathrm{C}$ & 0.16634200 & -2.37055000 & 1.70920800 & $\mathrm{C}$ & 3.10384100 & -1.60608600 & -1.30154100 \\
\hline
\end{tabular}




\begin{tabular}{|c|c|c|c|c|c|c|c|}
\hline $\mathrm{Pd}$ & -0.25669900 & -0.92110500 & -0.45569900 & $\mathrm{H}$ & -2.06654200 & -0.61769000 & 1.99284300 \\
\hline $\mathrm{H}$ & 0.17346700 & -2.33418600 & -1.01407700 & $\mathrm{H}$ & -0.89968500 & 0.21730800 & 3.03382200 \\
\hline $\mathrm{C}$ & -1.44262700 & -2.30669100 & -1.72866500 & $\mathrm{H}$ & -2.62614400 & 0.32634200 & 3.38642000 \\
\hline $\mathrm{C}$ & -2.40611500 & -1.72343600 & -1.18263500 & $\mathrm{C}$ & -1.84989100 & 2.78636000 & 2.59086700 \\
\hline $\mathrm{C}$ & -3.71684000 & -1.37030900 & -0.75402800 & $\mathrm{H}$ & -2.70700700 & 2.84596400 & 3.27054000 \\
\hline $\mathrm{C}$ & -4.57234600 & -0.62182200 & -1.58233000 & $\mathrm{H}$ & -0.95445800 & 2.74410000 & 3.21531400 \\
\hline $\mathrm{C}$ & -4.18012800 & -1.81291900 & 0.49942000 & $\mathrm{H}$ & -1.82898000 & 3.72022800 & 2.02065300 \\
\hline $\mathrm{C}$ & -5.85172800 & -0.30316800 & -1.14787400 & $\mathrm{C}$ & -3.43526300 & 1.59662500 & 1.11405400 \\
\hline $\mathrm{H}$ & -4.22351300 & -0.29956600 & -2.55857000 & $\mathrm{H}$ & -3.62520300 & 0.79171900 & 0.40490000 \\
\hline $\mathrm{C}$ & -5.46482100 & -1.49662300 & 0.91685100 & $\mathrm{H}$ & -4.16273500 & 1.49258500 & 1.92731100 \\
\hline $\mathrm{H}$ & -3.52238100 & -2.40639100 & 1.12899000 & $\mathrm{H}$ & -3.64034000 & 2.55030300 & 0.61896400 \\
\hline $\mathrm{C}$ & -6.29867500 & -0.73217600 & 0.10121100 & $\mathrm{C}$ & -1.34110500 & 3.89765100 & -0.79813400 \\
\hline $\mathrm{H}$ & -6.50719300 & 0.27547200 & -1.79129400 & $\mathrm{H}$ & -1.47860800 & 4.48748400 & -1.71150600 \\
\hline $\mathrm{H}$ & -5.81752100 & -1.84639100 & 1.88223800 & $\mathrm{H}$ & -2.24664600 & 4.02148800 & -0.19613600 \\
\hline $\mathrm{H}$ & -7.30073100 & -0.47993400 & 0.43431000 & $\mathrm{H}$ & -0.50591800 & 4.34688700 & -0.25155400 \\
\hline $\mathrm{H}$ & -1.11091800 & -3.05904300 & -2.41970400 & $\mathrm{C}$ & -2.27683300 & 1.86121900 & -1.92708100 \\
\hline $\mathrm{C}$ & 1.75404300 & -4.04414700 & 0.78090800 & $\mathrm{H}$ & -3.22484000 & 1.95906400 & -1.39743500 \\
\hline $\mathrm{H}$ & 1.50823200 & -4.78579500 & 1.54861500 & $\mathrm{H}$ & -2.37679700 & 2.39161200 & -2.88098300 \\
\hline $\mathrm{H}$ & 0.93079200 & -4.04367200 & 0.05792900 & $\mathrm{H}$ & -2.11802000 & 0.80075900 & -2.15734000 \\
\hline $\mathrm{H}$ & 2.66043000 & -4.39322200 & 0.27915900 & $\mathrm{C}$ & 0.12576200 & 2.32790100 & -2.10371500 \\
\hline $\mathrm{C}$ & 3.21381300 & -2.74945100 & 2.32252800 & $\mathrm{H}$ & 0.31153100 & 1.28122700 & -2.37344800 \\
\hline $\mathrm{H}$ & 3.30248800 & -1.89279400 & 2.99508500 & $\mathrm{H}$ & -0.09272600 & 2.87251500 & -3.02905700 \\
\hline $\mathrm{H}$ & 3.15971500 & -3.64251400 & 2.95399500 & $\mathrm{H}$ & 1.04577500 & 2.73941400 & -1.68527600 \\
\hline $\mathrm{H}$ & 4.13556500 & -2.82429500 & 1.73880500 & & & & \\
\hline $\mathrm{C}$ & 0.74681800 & -2.40884300 & 2.35905900 & \multicolumn{4}{|c|}{ TS55 } \\
\hline $\mathrm{H}$ & -0.19825600 & -2.45710300 & 1.80421500 & \multicolumn{4}{|c|}{ imaginary frequency $=-503.35 \mathrm{~cm}^{-1}$} \\
\hline $\mathrm{H}$ & 0.71042600 & -3.15909000 & 3.15695400 & $\mathrm{C}$ & -4.41763200 & 1.07871400 & -0.90931300 \\
\hline $\mathrm{H}$ & 0.80555000 & -1.42632100 & 2.84153600 & $\mathrm{C}$ & -3.29006100 & 1.02109200 & -0.07943800 \\
\hline $\mathrm{C}$ & 3.03007000 & -0.36160900 & -2.19710200 & $\mathrm{C}$ & -2.54983400 & 2.20014500 & 0.13717900 \\
\hline $\mathrm{H}$ & 3.42294900 & 0.54241900 & -1.72901700 & $\mathrm{C}$ & -2.99887300 & 3.38625600 & -0.45981900 \\
\hline $\mathrm{H}$ & 3.61452300 & -0.54736100 & -3.10479200 & $\mathrm{C}$ & -4.10230300 & 3.41964000 & -1.30207000 \\
\hline $\mathrm{H}$ & 1.99632100 & -0.16620700 & -2.50376300 & $\mathrm{C}$ & -4.81439000 & 2.24960500 & -1.53939300 \\
\hline $\mathrm{C}$ & 4.54636200 & -1.88614500 & -0.87961300 & $\mathrm{H}$ & -5.00784900 & 0.17396100 & -1.04660500 \\
\hline $\mathrm{H}$ & 4.64314800 & -2.84386000 & -0.35888200 & $\mathrm{H}$ & -2.49154300 & 4.31756700 & -0.22271800 \\
\hline $\mathrm{H}$ & 5.17932000 & -1.94164400 & -1.77163600 & $\mathrm{H}$ & -4.41849100 & 4.35935400 & -1.74384300 \\
\hline $\mathrm{H}$ & 4.96406300 & -1.10379800 & -0.23982900 & $\mathrm{H}$ & -5.69210200 & 2.25462200 & -2.17764000 \\
\hline $\mathrm{C}$ & 2.59697800 & -2.77860300 & -2.14584400 & $\mathrm{C}$ & -1.39001800 & 2.25971400 & 1.09358800 \\
\hline $\mathrm{H}$ & 1.58229900 & -2.60384300 & -2.51665500 & $\mathrm{H}$ & -1.24033000 & 3.29645400 & 1.42141900 \\
\hline $\mathrm{H}$ & 3.24433000 & -2.86735200 & -3.02486000 & $\mathrm{C}$ & -3.03933500 & -0.26394200 & 0.65808300 \\
\hline $\mathrm{H}$ & 2.62117800 & -3.73881500 & -1.63021300 & $\mathrm{H}$ & -2.80457200 & -0.05340200 & 1.71057500 \\
\hline $\mathrm{C}$ & -1.89182400 & 0.29534600 & 2.57308300 & $\mathrm{H}$ & -3.97855900 & -0.82604500 & 0.68059200 \\
\hline
\end{tabular}




\begin{tabular}{|c|c|c|c|c|c|c|c|}
\hline $\mathrm{H}$ & -1.62848700 & 1.68865700 & 1.99766300 & $\mathrm{H}$ & -2.98816600 & -0.25865500 & -2.33027400 \\
\hline$P$ & 0.28316400 & 1.61899700 & 0.59370300 & $\mathrm{H}$ & -1.24944000 & -0.53500700 & -2.50729000 \\
\hline $\mathrm{P}$ & -1.72387500 & -1.48056800 & 0.15763200 & $\mathrm{C}$ & -1.28287800 & -3.22817500 & -2.02342400 \\
\hline $\mathrm{C}$ & -1.93480100 & -2.72746500 & 1.58190100 & $\mathrm{H}$ & -1.63865200 & -3.59606600 & -2.99208100 \\
\hline $\mathrm{C}$ & -2.27753200 & -2.18210900 & -1.51053600 & $\mathrm{H}$ & -0.29590200 & -2.78701900 & -2.18932500 \\
\hline $\mathrm{C}$ & 1.29624700 & 1.95174000 & 2.16693300 & $\mathrm{H}$ & -1.17314800 & -4.09660200 & -1.37150000 \\
\hline $\mathrm{C}$ & 0.86065300 & 2.69932100 & -0.84833600 & $\mathrm{C}$ & 2.37986300 & 2.63749400 & -1.00159500 \\
\hline $\mathrm{Pd}$ & 0.52349100 & -0.64339400 & 0.02594300 & $\mathrm{H}$ & 2.90613500 & 3.15577700 & -0.19533900 \\
\hline $\mathrm{H}$ & 1.99799500 & -0.04900500 & -0.15189200 & $\mathrm{H}$ & 2.75986500 & 1.61094500 & -1.05538700 \\
\hline $\mathrm{C}$ & 5.54848100 & -0.15539200 & -2.02468900 & $\mathrm{H}$ & 2.65747200 & 3.13249000 & -1.93898100 \\
\hline $\mathrm{C}$ & 4.23381000 & -0.54967100 & -1.82407100 & $\mathrm{C}$ & 0.43656500 & 4.16321800 & -0.71888600 \\
\hline $\mathrm{C}$ & 3.89763100 & -1.32501600 & -0.70182300 & $\mathrm{H}$ & 0.80980000 & 4.64253000 & 0.18845600 \\
\hline $\mathrm{C}$ & 4.89212000 & -1.68576700 & 0.21992600 & $\mathrm{H}$ & 0.84044200 & 4.72309600 & -1.56926000 \\
\hline $\mathrm{C}$ & 6.20545200 & -1.28285700 & 0.00893800 & $\mathrm{H}$ & -0.64664900 & 4.27774800 & -0.75163500 \\
\hline $\mathrm{C}$ & 6.53524300 & -0.51977300 & -1.10900900 & $\mathrm{C}$ & 0.21567500 & 2.09578500 & -2.10320700 \\
\hline $\mathrm{H}$ & 5.80662500 & 0.43554400 & -2.89776000 & $\mathrm{H}$ & 0.47421400 & 2.70698900 & -2.97525000 \\
\hline $\mathrm{H}$ & 3.45580200 & -0.27128100 & -2.53107100 & $\mathrm{H}$ & 0.58096600 & 1.07718000 & -2.28270600 \\
\hline $\mathrm{H}$ & 4.62578600 & -2.27992300 & 1.08878500 & $\mathrm{H}$ & -0.87754700 & 2.05910200 & -2.03536800 \\
\hline $\mathrm{H}$ & 6.97411900 & -1.56684400 & 0.72094700 & $\mathrm{C}$ & 2.64567900 & 1.22865700 & 2.08915800 \\
\hline $\mathrm{H}$ & 7.56289700 & -0.20750100 & -1.26754700 & $\mathrm{H}$ & 3.24203100 & 1.49994800 & 1.21404700 \\
\hline $\mathrm{C}$ & 2.54709500 & -1.77293700 & -0.50597000 & $\mathrm{H}$ & 3.23330600 & 1.48262500 & 2.97829700 \\
\hline $\mathrm{C}$ & 1.52562000 & -2.50036200 & -0.37894200 & $\mathrm{H}$ & 2.51258700 & 0.14137700 & 2.07793200 \\
\hline $\mathrm{H}$ & 1.17168500 & -3.50859500 & -0.49371200 & $\mathrm{C}$ & 1.52028600 & 3.44265000 & 2.42266500 \\
\hline $\mathrm{C}$ & -1.38192700 & -4.10031700 & 1.20726500 & $\mathrm{H}$ & 1.99199500 & 3.56852200 & 3.40333200 \\
\hline $\mathrm{H}$ & -2.00358400 & -4.61009400 & 0.46583900 & $\mathrm{H}$ & 2.18561100 & 3.90072300 & 1.68617100 \\
\hline $\mathrm{H}$ & -0.35728300 & -4.04485700 & 0.83133900 & $\mathrm{H}$ & 0.58611400 & 4.01325700 & 2.44316300 \\
\hline $\mathrm{H}$ & -1.36357200 & -4.73332100 & 2.10113300 & $\mathrm{C}$ & 0.53250200 & 1.35177400 & 3.35457200 \\
\hline $\mathrm{C}$ & -1.10250900 & -2.16530800 & 2.74012600 & $\mathrm{H}$ & -0.35459600 & 1.92823400 & 3.63013300 \\
\hline $\mathrm{H}$ & -1.19759600 & -2.81851500 & 3.61477000 & $\mathrm{H}$ & 0.23564000 & 0.31380900 & 3.16476200 \\
\hline $\mathrm{H}$ & -0.03952800 & -2.09644600 & 2.47942500 & $\mathrm{H}$ & 1.19039100 & 1.34519500 & 4.22997800 \\
\hline $\mathrm{H}$ & -1.44087300 & -1.16757100 & 3.04431200 & & & & \\
\hline $\mathrm{C}$ & -3.38221400 & -2.89841900 & 2.05507800 & & & & \\
\hline $\mathrm{H}$ & -3.41177400 & -3.69472800 & 2.80662000 & & & & \\
\hline $\mathrm{H}$ & -3.77827800 & -1.99955000 & 2.53377600 & & & & \\
\hline $\mathrm{H}$ & -4.06581300 & -3.18796800 & 1.25218400 & & & & \\
\hline $\mathrm{C}$ & -3.67693100 & -2.79102400 & -1.45135500 & & & & \\
\hline $\mathrm{H}$ & -3.99942800 & -3.06385100 & -2.46232100 & & & & \\
\hline $\mathrm{H}$ & -3.70104500 & -3.70456300 & -0.84868700 & & & & \\
\hline $\mathrm{H}$ & -4.42572600 & -2.09914500 & -1.05188700 & & & & \\
\hline $\mathrm{C}$ & -2.23228800 & -1.02235500 & -2.51318500 & & & & \\
\hline $\mathrm{H}$ & -2.39725200 & -1.41893900 & -3.52105300 & & & & \\
\hline
\end{tabular}

